\title{
EFFECTS OF SUPERSATURATION OF DISSOLVED GASES ON THE FISHERY OF THE BIGHORN RIVER DOWNSTREAM OF THE YELLOWTAIL AFTERBAY DAM
}

\author{
Completion Report \\ April 1991
}

\author{
Prepared for: \\ U.S. Department of the Interior \\ Bureau of Reclamation \\ Missouri Basin \\ Region 6
}

\begin{abstract}
by:
Robert G. White, Principal Investigator Glenn Phillips, Co-Principal Investigator George Liknes, Project Biologist Jim Brammer, Graduate Assistant William Connor, Graduate Assistant Larry Fidler, Graduate Assistant Tommy Williams, Graduate Assistant W. Patrick Dwyer, Cooperator
\end{abstract}

\author{
U.S. Fish and Wildlife service \\ Montana Cooperative Fishery Research Unit \\ Montana state University \\ Bozeman, MT \\ and \\ Montana Department of Fish, Wildlife and Parks \\ Helena, MT
}


The Yellowtail Afterbay Dam serves as a reregulation facility below Yellowtail Dam and power plant to provide uniform daily discharges to the Bighorn River. Afterbay Dam has, for many years, produced supersaturated levels of dissolved gases. Water spilling over the dam entrains air and forces it into solution as it plunges into the pool below. Accompanying this supersaturation is high incidence of external symptoms of gas bubble trauma (GBT) in rainbow and brown trout populations downstream of the dam, particularly among brown trout larger than $356 \mathrm{~mm}$ total length. All sizes of brown trout appeared more susceptible to GBT than rainbow trout, and high gas levels were thought to negatively influence recruitment of rainbow trout.

An attempt to solve the gas supersaturation problem below Afterbay Dam by installing deflector plates (flip-lips) was unsuccessful due to structural impacts to the dam. Since there was no clear understanding of how reservoir operations, flows, and other ambient conditions affected dissolved gas levels and the incidence of GBT in fish, this study was undertaken. Because of the relatively small size of the Bighorn River compared to other rivers with gas supersaturation problems, this study site presented the opportunity to increase our understanding of the relationship between operation and design of dams and GBT. Specific objectives are provided on page 9.

Bighorn River trout populations are large. The population 
is dominated by brown trout, with abundance estimates of Age 1 and older fish as high as $7774 / \mathrm{km}$ in the $6 \mathrm{~km}$ immediately below Afterbay Dam. Brown trout outnumbered rainbow trout by about 10 to 1.

Incidence of GBT in rainbow and brown trout was not uniformly distributed in space or time. Incidence among brown trout was always largest $(28-65 \%)$ in the $1.9 \mathrm{~km}$ immediately below Afterbay Dam. External symptoms of GBT were confined to about the first $8 \mathrm{~km}$ below the dam which is approximately the point where $\mathrm{N}_{2}$ and Ar supersaturation dissipates. Although incidence diminished with distance downstream, the pattern of occurrence was similar in each study reach. Gas bubble trauma incidence among all brown trout varied more than two-fold between years, with incidence among large trout (>356 mm) almost always greater (68-88\%) than for all size groups combined. Peak incidence of GBT usually occurred between mid-April and early June. Although incidence usually increased as flow and delta $\mathrm{P}$ increased, GBT incidence was not directly related to discharge.

Similar patterns, but lower GBT incidence, occurred among rainbow trout except during the spring spawning period when incidence among rainbow trout usually exceeded that of brown trout downstream of river kilometer (Rkm) 1.9. Highest incidence of GBT always coincided with the spawning period and was related to movement into shallow side channel spawning areas. A catchability study indicated that minor symptoms of GBT did not remove brown and rainbow trout from the fishery. Laboratory studies of the physiology of GBT were conducted 
concurrently with field observations for use in interpreting these observations and for predicting consequences of flow patterns from Afterbay Dam. Gas bubble trauma is an acute condition involving various forms of bubble growth, both internal and external to the fish. Theoretical models were developed to establish thresholds for bubble growth in trout. These models apply to: 1) bubble growth in the vascular system of trout; 2) bubble growth in the environmental water that can occur in the buccal cavity and between gill lamella; 3) sub-dermal bubbles that occur on external skin surfaces such as the opercular flaps, between fin rays and in the lining of the mouth; and 4) over inflation of the swimbladder.

We developed theoretical models describing bubble growth based on a review of scientific literature and a two phase experimental program. The literature review resulted in the compilation of a data base containing over 1000 records of supersaturation data on salmonids. Various filters based on fish length, species, total gas pressure (TGP), partial pressure of oxygen $\left(\mathrm{pO}_{2}\right)$, and other criteria were applied to the data base. These operations established the existence of GBT mortality thresholds and identified relationships between other experimental parameters. This analysis suggested that a lower threshold occurs at a water TGP of $110 \%$ ( 1.1 Atmospheres) and a higher threshold occurs at 115-118\%. However, it was not established that the apparent mortality thresholds correspond to thresholds for bubble growth predicted by the theoretical models. To determine if a relationship exists between mortality thresholds and thresholds for bubble growth, we examined the 
physiological response of adult rainbow trout exposed to supersaturated water. We found that arterial $\mathrm{pO}_{2}$, hematocrit and blood pressure yield unique responses to bubble growth over specific ranges of water TGP. Results also indicated that the lower mortality threshold identified from the database is associated with a combination of sub-dermal bubble growth in the mouth and extracorporeal bubbles growing between the gill lamella.

The second phase of experimental studies included measurement of blood $\mathrm{pO}_{2}$, hematocrit and $\mathrm{pH}$ along with microscopic studies of intravascular and extracorporeal bubble growth in gills. The results confirmed the source of mortality for the lower threshold at a water TGP of 110-112\%. In addition, the data demonstrated that the upper TGP threshold is 115-119\%.

The transition from a lower TGP threshold to an upper threshold appears to involve a shift in the bubble related mechanisms that lead to death. At the lower threshold, subdermal bubbles in the lining of the mouth and extracorporeal bubbles in gill lamella appear to block the exchange of respiratory gases. At a water TGP of $115 \%$, however, the extracorporeal bubbles are fewer and even disappear and time to mortality increases. This appears to be the result of the larger bubbles being more easily dislodged by respiratory water flow. At water TGP levels between 116 and 119\%, intravascular bubble formation begins and time to mortality again decreases with increasing TGP. At these TGP levels, sub-dermal bubbles in the lining of the mouth are smaller or absent. Apparently the rapid death caused by intravascular bubbles at these higher 
levels of TGP does not allow time for sub-dermal bubbles to develop. This sequence correlates with data from the literature which suggest that during this transition there is a range of TGP that offers some relief to the fish. Results confirmed, as predicted by the theoretical model, that intravascular bubble growth thresholds are dependent on water $\mathrm{pO}_{2}$.

To determine how species and size influence susceptibility to GBT, laboratory bioassays were conducted on juvenile brown and rainbow trout $(53-130 \mathrm{~mm}$ total length). No significant mortality occurred in the $112 \%$ saturation treatment except during two tests when TGP exceeded 113\%, indicating that a critical threshold exists between 113 and $117 \%$ saturation. This is slightly higher than the initial threshold level identified in physiology studies with adult trout (110-112\%). Exposure to $112 \%$ dissolved gas saturation had no effect on growth of either species.

As juvenile trout grew they became more susceptible to $125 \%$ dissolved gas supersaturation. Total mortality and mortality rate were less when fish were small. Daily mortality of brown trout was always significantly greater than for rainbow trout of similar size. The slightly higher juvenile trout GBT threshold identified in bioassays seems reasonable considering that smaller juveniles are less sensitive to gas supersaturation than larger trout.

The frequency at which emphysema occurred by body region also varied with fish size and smaller fish died with different external symptoms. Larger fish were more likely to die faster than smaller fish and with no external symptoms. Recovery of juvenile trout varied according to external symptom severity. 
Fish that did not recover usually had severe exopthalmia.

Juvenile brown txout, repeatedly exposed to $118 \%$ gas supersaturated water, given 30 d to recover between exposures, developed more severe symptoms with each exposure. Surviving fish developed new symptoms more often than recurring symptoms. Mortality of juvenile brown trout was significantly greater during the first and second exposure to gas supersaturated water than during the third exposure, but fish did not appear to acclimate to supersaturated water. Some individuals, however, were more tolerant of high dissolved gas levels. None of six fish surviving two exposures died when exposed a third time. Selection for tolerance to high dissolved gas levels may help explain why Bighorn River trout can survive at gas levels reported to be lethal in other situations. Bacterial challenges indicated that juvenile brown trout exposed to $118 \%$ TGP for 5-d were more susceptible to infection by Aeromonas hydrophila than non-exposed fish.

Dissolved gas saturation in the Bighorn River varied longitudinally and between years. Near the dam, mean hyperberic pressure was $111 \mathrm{mmHg}$ (TGP 116\%) and ranged from 35-153 $\mathrm{mmHg}$ (TGP 105-123\%). Gas saturation was lowest in December and January, gradually increasing to maximum in mid-June and July. Downstream (Rkm 4.8-14.5) the pattern was similar but high gas levels occurred as early as April. Although annual mean gas levels declined downstream, maximum and minimum gas pressures (16 and $191 \mathrm{mmHg}$; TGP 102 and 128\%) occurred at Rkm 14.5. Downstream sites were also the most variable. Nitrogen and argon were the principal components of hyperbaric pressure downstream to $\mathrm{Rkm}$ 
2.4. Oxygen made up an increasingly greater proportion of TGP, and oxygen pressures became more variable, with distance downstream. Oxygen levels as high as $222 \%$ saturation and 312 mmHg were measured at $\mathrm{Rkm}$ 14.5. Pattern and variability of oxygen pressures was directly related to photosynthesis. Increased oxygen tension is beneficial to fish since it results in higher threshold levels for cardiovascular system bubble growth and swim bladder overinflation.

Hyperbaric pressure did not affect brown trout and rainbow trout egg incubation success. Although rainbow trout embryos develop during a period of substantially higher hyperbaric pressure compared to brown trout embryos (often exceeding the threshold for bubble growth in water), no difference in survival to hatching was observed. The internal pressure of salmonid eggs and hydrostatic pressure of the water above the average rainbow trout redd in the Bighorn River provides about 80-120 $\mathrm{mmHg}$ compensating pressure. This lowers uncompensated pressures to under $110 \% \mathrm{TGP}$. If differences in rainbow trout year class strength are influenced by dissolved gas levels, the controlling mechanism operates after hatching.

Results of trout fry field bioassays were inconsistent and difficult to interpret. Most inconsistencies were probably due to our use of point measurements of hyperbaric pressure to describe exposure conditions: large diurnal fluctuations occurred at all locations sometime during the study. Also use of all or no hydrostatic pressure in rearing boxes could change threshold levels as much as $18 \mathrm{mmHg}$ and account for most of the variation in some experiments. In general, high fry mortality occurred at 
delta $\mathrm{P}^{\prime} \mathrm{s}$ of $120 \mathrm{~mm}$ H (TGP 118\%) or more, and often at mean delta $\mathrm{P}^{\prime} \mathrm{s}$ above $110 \mathrm{mmHg}$ (TGP 116\%). Delta $\mathrm{P}^{\prime} \mathrm{S}$ of $80-95 \mathrm{mmHg}$ (TGP 112$114 \%$ ) pose minimal threat to fry. The longer the delta $P$ was above the cardiovascular system threshold, the higher the cumulative mortality. Fry closer to Afterbay Dam are at greatest risk. Hyperbaric pressures that approximate or are below the threshold for bubble formation in environmental water pose minimal risk to fry. Swimming and feeding ability of fry can, however, be affected by sub-lethal levels of hyperbaric pressure. Excess buoyancy in as many as $50 \%$ of the fry in groups held at Rkm 0.6 was observed. Free-ranging fry with equilibrium problems would have greatly reduced probability of survival due to increased vulnerability to predation and excessive energy expenditures necessary to maintain a normal swimming position. Since brown trout fry emerge from the gravel earlier, they are larger than rainbow trout fry by June when hyperbaric pressures are high in the Bighorn River. This larger size may improve survival of brown trout fry since it allows them to occupy deeper water where compensating pressures are greater.

Differences in habitat use between brown trout and rainbow trout did not account for the higher incidence of GBT observed in the adult brown trout population and the large difference in relative abundance between the two species. High levels of gas supersaturation did not have a detectable influence on the distribution of either trout species. Snorkel surveys indicated that trout were concentrated along river banks during winter and spring and used bank and midchannel areas during summer and fall. Although gas levels remained high, the percentage of trout 
exhibiting external symptoms of GBT decreased during periods of midchannel use. Seasonal trout movement appeared to be related to changes in availability of energetically suitable habitat created by aquatic vegetation. It appears that vegetation in midchannel areas created usable habitat with adequate depth to provide hydrostatic compensation, reducing the incidence of GBT. Radio telemetry data collected during gas supersaturation manipulation tests (delta $\mathrm{P}$ values $63 \mathrm{mmHg}$ to $123 \mathrm{mmHg}$; TGP 109$118 \%$ ) indicated that adult brown and rainbow trout did not actively avoid high dissolved gas levels. Trout movements were restricted to localized areas and sounding by trout to avoid high hyperbaric pressures was not detected by pressure sensitive radio transmitters.

To determine if aquatic macroinvertebrates were adversely affected by dissolved gas supersaturation in the Bighorn River, benthic and drift samples were taken near the dam (Rkm 2.4) where high incidence of GBT occurred in trout, and downstream (Rkm 14.5) where incidence was low. Community composition was similar between sites and taxa were representative of those commonly found below deep-release impoundments. Community structure was similar between sites during April (before high dissolved gas supersaturation), but differed strongly during late summer and fall. Summer temperature differences between sites coincided with the period of highest gas saturation levels confounding interpretation. Eight invertebrate taxa which differed in abundance between sites and were considered the best candidates for being affected by gas supersaturation were tested in controlled bioassays. Susceptibility differed between taxa, but 
al were negatively affected by mean delta $\mathrm{P}$ levels of $181 \mathrm{mmHg}$ (TGP 127\%) or greater. Baetis tricaudatis was the most susceptible with adverse effects apparent at delta p levels of $113 \mathrm{mmHg}$ (TGP 117\%). Ephemerella inermis and Tricorthyodes minutus appeared to have susceptibility thresholds near a delta $P$ of $120 \mathrm{mmHg}$ (TGP 118\%). Since these levels commonly occur below Afterbay Dam, at least these three species appear to be negatively impacted. Invertebrate sensitivity to high gas saturation levels was expressed through increased buoyancy, which could cause involuntary drift. Total inveretebrate densities were greater at Rkm 14.5, however densities at Rkm 2.4 were not low, ranging from 32,658 to 41,761 organisms per square meter. These high invertebrate densities indicate that hyperbaric pressures are not having an important impact on trout food supply.

Longnose dace and mountain whitefish are found in the Bighorn River in low abundance compared to trout. Laboratory bioassays showed mountain whitefish to be less tolerant of high hyperbaric pressures compared to longnose dace. Mottled sculpin, an important forage species for brown trout in most Montana rivers, does not occur in the Bighorn River. To determine if this species could survive if introduced, we conducted bioassays to examine susceptibility to GBT. Results indicate that mottled sculpin are more tolerant than trout or dace to dissolved gas supersaturation.

Gas tension changes in the Bighorn River result from variations in dam operational patterns, discharge, river water surface elevation, water temperature, and weather conditions. 
Flow and operational pattern tests demonstrated that the dissolved gas supersaturation problem is primarily a result of sluiceway discharge; delta $P$ varied with sluicegate openings. Consequently, the highest gas pressures and most severe GBT in trout are found along the right bank, just downstream from Afterbay Dam.

When only radial gates were used to discharge water, delta p's remained low. Avoiding use of the sluicegates in the midrange $(1,500-2,000 \mathrm{cfs})$ would reduce the problem, since this discharge pattern produced the maximum dissolved gas levels. Minimizing use of sluiceway gate openings between 25 and $75 \%$ would not eliminate hyperbaric pressures above trout cardiovascular threshold levels, but it would reduce time of exposure to extreme gas pressures. Both larger and smaller sluicegate openings coincided with lower hyperbaric pressures. Maximumizing discharge through the radial gates would benefit the fishery. During peak runoff, almost all discharge could and should be routed through radial gates. Reducing gas tensions by operational change would require a more consistent discharge via radial gates. Automation of radial gates would allow shifts in flow patterns not easily accomplished at present. However, solutions other than operational changes that eliminate or substantially reduce gas supersaturation and allow compliance with water quality standards should be persued. 


\section{ACKNOWLEDGEMENTS}

The authors greatfully acknowledge the following individuals for their assistance in field data collection and laboratory work: Patricia Bigelow, Randy Elliot, steve Hiebert, Beth MacConnell, Hiram Perez, Bruce Roberts, Mike Vaughn, Art Viola, and Kevin Weibe. Research technicians, Cal sprague and Scott Sanford provided high quality assistance in all aspects of the study. Mr. Charlie Smith, U.S.F.W.S. Fish Technology center, provided laboratory space for bioassay studies. Dr. Vickie Bokum, D.V.M., performed the sergical implantation of radio transmitters. Special thanks to Dr. Dan Gustafson for his help in identifying aquatic invertebrates and his willingness to provide computer and statistical advice. Additional statistical consultation was provided by Drs. Carol Bittenger, Dalton Burkhalter, Dan Goodman, Randall Ryti, and Mr. Milo Adkison. Dr. Jack Griffith, Idaho state University, assisted in design of the habitat use study. Wade Fredenberg, Montana Department Fish Wildlife and Parks, was instrumental in initiating the study and assisted in field work. Steve Leathe served as the first project biologist. Funding was provided by the U.S. Department of Interior, Bureau of Reclamation. We are indebted to the excellent support and cooporation of Dr. Jim LaBounty and Mr. Dean Loomis in administering the contract. Mr. Dennis Christensen, Project Manager Yellowtail Dam and Power plant, and Harold Hergenvider, Tim Felchle, and Gary Butterfield were always willing to accomodate research needs. Finally, special thanks to Ms. Cindee Woodberry for her skills and patience in preparing and editing this report. 
TABLE OF CONTENTS

$\underline{\text { Page }}$

Executive Summary . . . . . . . . . . . . . . . . .

Acknowledgements ...., ...., . . . . . . . . , . xili

List of Tables ........................ . xix

List of Figures .......................... . xxix

1.0 Introduction ..................... . . . 1

1.1 Dissolved Gas Supersaturation . . . . . . . . . . 2

1.2 The Bighorn River and the Yellowtail Dam Afterbay * 4

1.3 Relevant Literature . . . . . . . . . . . 5

1.4 Bighorn River Project objectives . . . . . . . . 9

2.0 Field and Laboratory Studies . . . . . . . . . . . . 11

2.1 Physical and Physiological Parameters Associated with GBT . . . . . . . . . . . . . . . . . 11

2.1.1 Phase 1 -- Theoretical Studies...... . 12

a Vascular System Bubble Growth . . . . . 16

b Swimbladder Overinflation ........ 18

c Water Bubbles in Environmental Water. . . 19

d Unknown Parameters . . . . . . . . 20

e Sub-dermal Bubble Growth. . . . . . . 21

f Results of Theoretical Studies . . . . . 22

2.1.2 Phase 2 -- Literature Evidence for GBT Thresholds ............. . . 28

a Methods and Materials . . . . . . . 28

b Results ................ 30

c Discussion.............. . . 48 
2.1.3 Phase 3 - Experimental Studies . . .

2.1.3.1 Part 1: Physiological parameters and $\mathrm{GBT} . . . . . . . . . . .652$

a Materials and Methods . . . . . . 52

b Results.............. 59

C Discussion ........... . . 86

2.1.3.2 Part 2: Juvenile Trout and GBT . . . 97

a Introduction .......... . . 97

b Methods............. . . 97

c Results and Discussion . . . . . 115

2.1 Summary: Physical and Physiological Parameters Associated with GBT. . . . . . . . . . . 135

2.2 Recovery from symptoms of GBT ........... . 140

a Introduction ................. 140

b Methods.................... 141

c Results and Discussion ........... . 142

a summary .................. . 153

2.3 Bighorn River studies... . . . . . . . . . . 154

2.3.1 Description of Study Area . . . . . . . . 154

2.3 .2 Water Chemistry . . . . . . . . . . 157

a Methods . . . . . . . . . . . . 157

b Results and Discussion.......... 157

2.3 .3 population structure .......... . 161

a Methods ............... 161

b Results ............. 161

2.3.4 Dissolved Gas Levels, Water Temperature

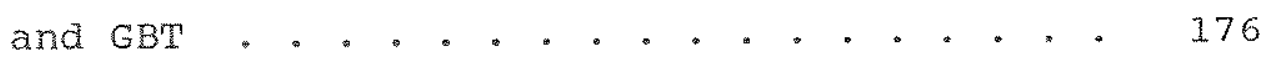


a Methods . . . . . . . . . . 176

b Results ................. 178

2.3.5. Symptoms of GBT in Relation to Dissolved

Gas Levels . . . . . . . . . . . 197

2.3.5.1. Embryo Incubation Tests..... . 197

a Methods ............ . . 197

b Results . . . . . . . . . . . 198

c Discussion . . . . . . . . . . 204

2.3 .5 .2 Fry Survival Tests . . . . . . . 211

a Methods.............. 211

b Results and Discussion . . . . . . 211

$2 \cdot 3.5 .3$ Adult Symptoms, Frequency and

Severity Indices . . . . . . . 267

a Methods ............. . . 267

b Results and Discussion . . . . . 268

2.3.5.4 Relationship Between GBT Thresholds and GBT Incidence on the Bighorn River . 286

a Methods . . . . . . . . . . 286

b Results and Discussion . . . . . . 287

2.3 .5 .5 Changes in GBT Symptoms Through Time Among Tagged Trout . . . . . . 316

a Methods ............. 316

b Results and Discussion . . . . . 316

2.4 Behavior and Habitat . . . . . . . . . . . 327

2.4.1 Predation Susceptibility . . . . . . . 327

a Methods ............. . 327

b Results and Discussion......... 332 xvi 
2.4 .2 Habitat Use . . . . . . . . . .

2.4.2.1 Macrohabitat ........... 343

a Methods . . . . . . . . . . 344

b Results . . . . . . . . . . 345

2.4 .2 .2 Microhabitat . . . . . . . 353

a Methods............. 353

b Results............ . . 359

2.4 .2 .3 Radio Telemetry . . . . . . . . 373

a Methods... . . . . . . . . 373

b Results............. . . 387

2.4 .2 .4 Discussion . . . . . . . . . . 400

2.5 Impacts of GBT on Trout catchability . . . . . . . 412

2.6 Impacts on Invertebrate and Forage Fish Communities 414

2.6.1 Aquatic Invertebrates . . . . . . . . . 414

a Methods . . . . . . . . . . . 414

b Results .............. . . 4 425

c Discussion............. . . 473

d Conclusions . . . . . . . . . . 487

2.6 .2 Forage Fish . . . . . . . . . . . 489

2.6 .2 .1 Dace .............. 489

a Methods............ . . 489

b Results and Discussion . . . . . 490

2.6 .2 .2 Mountain Whitefish . . . . . . . 495

2.6 .2 .3 sculpin ............ . 496

a Methods . . . . . . . . . . 4 496

b Results............ . . 497

2.7 Dam Operational Characteristics and River Dissolved Gas Levels . . . . . . . . . . . . . . 
2.7.1 River Discharge .. . . . . . . . 505

2.7.2 Afterbay Reservoir Gas Monitoring . . . . . 506

2.7.3 Afterbay Operation Monitoring . . . . . . 510

a Flow Tests........... 510

b Dye Tests . . . . . . . . . . 525

c Downstream Gas Measurements . . . . . 526

d Cross River Gas Measurements . . . . . . 532

e Target Gas Level Recommendations . . . . 541

f Operation Options . . . . . . . . . 542

Literature Cited . . . . . . . . . . . . . . . 555

Appendix A: Afterbay Gates . . . . . . . . . . . 572

Appendix B: Bighorn River water Quality . . . . . . . . . 574

Appendix C: Trout Population Statistics . . . . . . . . . 581

Appendix D: Rainbow Trout Embryo Development Rates . . . 600

Appendix E: Incidence of Gas Bubble Trauma . . . . . . . . 602

Appendix F: Severity of Gas Bubble Trauma in Trout . . . . 628

Appendix G: Monte Carlo Simulations . . . . . . . . . . 642

Appendix H: Calibration of Pressure-Sensitive Radio

Transmitters..... . . . . . . . . . 648

Appendix I: Gas Levels . . . . . . . . . . . . . . 653

Appendix J: Fish Movement Maps . . . . . . . . . . . 662

Appendix K: Gas Saturation Trends During Sampling Periods. 680

Appendix L: Invertebrate Gas Bubble Trauma Symptoms . . . 683

Appendix M: Invertebrate Bioassay Results . . . . . . . 690

Appendix N: Bighorn River Delta $P$ Frequencies Related to Bioassay Delta P's........... . 706 
Table

2.1.1 Definition of symbols used in threshold equations . . . . . . . . . . . . . . . 15

2.1.2. Survey of intravascular gill bubbles at death of trout for experimental series A, B, C and 4 . . . 85

2.1.3. Mean delta $\mathrm{P}$ values $(\mathrm{mm})$ for 30-day exposure tests

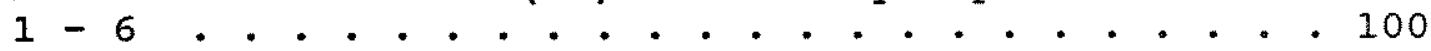

2.1.4. Mean dissolved oxygen concentrations and water temperatures during tests $1-6$. . . . . . . . 108

2.1.5. Pre-treatment mean lengths of juvenile rainbow and brown trout computed from a random sample of fish from the rearing tanks... . . . . . . . . . 109

2.1.6. Mean weight and number of juvenile rainbow and brown trout exposed to high, medium, and control levels of gas supersaturation during tests 1, 2,

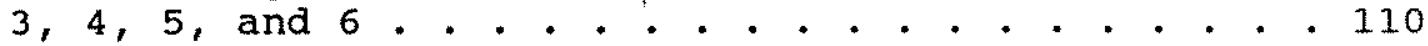

2.1.7. Intra- and interspecific mortality comparisons for juvenile brown (BT) and rainbow trout (RBT) exposed to the high gas treatment during tests 1 6 . . . . . . . . . . . . . . . . . . . 112

2.1.8. Sample sizes used to test for the effects of gas supersaturated water on juvenile brown (BT) and rainbow trout (RBT) growth . . . . . . . . . 112

2.1.9. Breslow rank analysis of medium gas treatment percent cumulative mortality for juvenile brown (BT) and rainbow trout (RBT) during tests $1,2,3$,

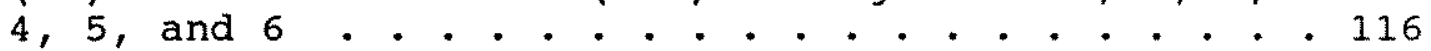

2.1.10. Breslow rank analysis of high gas treatment percent cumulative mortality for juvenile brown (BT) and rainbow trout (RBT) during tests 1, 2, 3, 4,5 , and 6 . . . . . . . . . . . . . . . 120

2.1.11. Breslow rank analysis of high gas treatment percent cumulative mortality for juvenile brown (BT) and rainbow trout (RBT) exposed to the high gas treatment during tests $1,2,3,4,5$, and 6.123

2.1.12. Breslow rank analysis comparing the rate mortality for juvenile brown (BT) and rainbow trout (RBT) of similar size in the high gas treatment 
2.1.13. Effect of gas supersaturated water on the growth of control (104\%) and medium treatment (112\%)

brown and rainbow trout from tests $1,3,5$, and 6 and the associated p-values calculated using the Man-Whitney nomparametric rank test (alpha $=$ $0.05)$

2.1.14. Two sample t-test analysis of the number of A. hydrophila identified in kidney samples of

juvenile brown trout challenged with bacteria and unchallenged fish ................. 134

2.1.15. Two sample t-test analysis of the number of A. hydrophila identified in kidney samples of juvenile brown trout (196 $\pm 13 \mathrm{~mm}$ and $74.5 \pm$ 17.39 ) not exposed to gas supersaturated water (control) and fish (198 $\pm 12 \mathrm{~mm}$ and $75.4 \pm 16.2 \mathrm{~g}$ ) with GBT (test) from exposure to $118 \%$ supersaturated water for 120-h . . . . . . . 135

2.2.1. Gas bubble trauma description and other criteria used to rank laboratory recovery data . . . . . . 142

2.2.2. Recovery of juvenile rainbow trout $(228 \pm 17 \mathrm{~mm}$ and $124.6 \pm 30.7 \mathrm{~g})$ exposed to $117 \%$ gas supersaturated water for 9 d February $1-9,1987,143$

2.2.3. Recovery of juvenile brown trout $(133 \pm 21 \mathrm{~mm}$ and $27.5 \pm 9.5 \mathrm{~g})$ by severity level, following exposure to various levels and durations of gas supersaturation ....... . . . . . . . 145

2.2.4. GBT rating, mortality and percent recovery of juvenile brown trout (fish size on day 1 of testing $=164 \pm 17 \mathrm{~mm}$ and $43.2 \pm 14.4 \mathrm{~g})$ by GBT rating exposed to $118 \%$ gas supersaturated water for $120 \mathrm{~h}$, three times and given $30 \mathrm{~d}$ to recover between exposures June 6 - September 18, 1987 • . . 148

2.2.5. Gas bubble trauma rating for juvenile brown trout (fish size on day 1 of testing $=164 \pm 17 \mathrm{~mm}$ and $43.2 \pm 14.4 \mathrm{~g})$ by number and percent composition after exposure to $118 \%$ gas supersaturated water for $120-\mathrm{h}$ three times at $30-\mathrm{d}$ intervals from June 6 - September 18, 1987 . . . . . . . . . . . 151

2.2.6. Comparison of GBT severity in juvenile brown trout (fish size on day 1 of testing $=164 \pm 17 \mathrm{~mm}$ and $43.2 \pm 14.4 \mathrm{~g}$ ) exposed to $118 \%$ gas supersaturated water three times at 30-d intervals June 6 september 18, 1987 using Fisher"s Exact Test . . . 152

2.3.1. Mean and ranges of water quality parameters from samples collected 17 May 1985 - 1 June 1988 near the gagehouse (Rkm 0.6) below Afterbay Dam on the Bighorn River $x$ 158 
2.3.2. Dissolved metal concentrations from seven samples collected 16 June 1985 - 1 August 1986 near the gagehouse (Rkm 0.6) below Afterbay Dam on the Bighorn River

2.3.3. Spring rainbow trout population estimates by age group from Rkm 0-15.4 (RM 0-9.6) below Afterbay Dam on the Bighorn River during 1985-87 (MCFRU and MDFWP data) .................. . 163

2.3.4. The average length ( $\mathrm{mm}$ ) and weight $(\mathrm{g})$ of rainbow trout by age group sampled during spring population estimates from Rkm 0-15.4 (RM 0-9.6) below Afterbay Dam on the Bighorn River during 1985-87 (MCFRU and MDFWP data) . . . . . . . . . 164

2.3.5. Fall rainbow trout population estimates by age group from $\mathrm{kkm} \mathrm{0-15.4} \mathrm{(RM} \mathrm{0-9.6)} \mathrm{below} \mathrm{Afterbay}$ Dam on the Bighorn River during 1985-87 (MCFRU and MDFWP data) .................. 165

2.3.6. The average length (mm) and weight $(\mathrm{g})$ at age of rainbow trout sampled during fall population estimates from Rkm 0-15.4 (RM 0-9.6) below Afterbay Dam on the Bighorn River during 1985-87 (MCFRU and MDFWP data) . . . . . . . . . . 166

2.3.7. Spring brown trout population estimates by age group from $\mathrm{Rkm} 0-6.1$ (RM $0-3.8$ ) below Afterbay Dam on the Bighorn River during 1985-87 (MCFRU and MDFWP data) . . . . . . . . . . . . . 167

2.3.8. The average length (mm) and weight (g) at age of brown trout sampled during spring population estimates from Rkm 0-6.1 (RM 0-3.8) below Afterbay Dam on the Bighorn River during 1985-87 (MCFRU and MDFWP data)

2.3.9. Fall brown trout population estimates by age group from Rkm 0-6.1 (RM 0-3.8) below Afterbay Dam on the Bighorn River during 1985-87 (MCFRU and MDFWP data) MCFRU and MDFW 169

2.3.10. The average length (mm) and weight ( $g$ ) at age of brown trout sampled during fall population estimates from Rkm 0-6.1 (RM 0-3.8) below Afterbay Dam on the Bighorn River during 1985-87 (MCFRU and MDFWP data)

2.3.11. Spring brown trout population estimates by age group from $\mathrm{Rkm} 3.9-15.4$ (RM 2.4-9.6) below Afterbay Dam on the Bighorn River during 1985-87 (MCFRU and MDFWP data) . . . . . . . . . . . 172 
2.3.12. The average length (mm) and weight (g) at age of brown trout sampled during spring population estimates from Rkm 3.9-15.4 (RM 2.4-9.6) below Afterbay Dam on the Bighorn River during 1985-87 (MCRRU and MDFWP data)

2.3.13. Fall brown trout population estimates by age group from Rkm 3.9-15.4 (RM 2.4-9.6) below Afterbay Dam on the Bighorn River during 1985-87 (MCFRU and MDFWP data) .............. . 174

2.3.14. The average length (mm) and weight (g) of brown trout sampled during fall population estimates from Rkm 3.9-15.4 (RM 2.4-9.6) below Afterbay Dam on the Bighorn River during 1985-87 (MCFRU and MDFWP data)

2.3.15. Mountain whitefish population estimates from the upper Bighorn River below Afterbay Dam during March 1986 and April 1987 (MCFRU and MDFWP data) . . 175

2.3.16. Mean and range of hyperbaric pressure (mmHg) at gas monitoring sites on the Bighorn River, Montana

2.3.17. Mean and range of barometric pressure (mmHg) at gas monitoring sites on the Bighorn River, Montana ................... . 188

2.3.18. Mean and range of oxygen pressure (mmHg) at gas monitoring sites on the Bighorn River, Montana . . 191

2.3.19. Mean and range of weekly water temperatures at gas monitoring sites on the Bighorn River, Montana

2.3.20. Proportions of live brown trout fry with obvious buoyancy which affected swiming ability during May and June 1987 on the Bighorn River. . . . . 237

2.3.21. Comparison of severity of gas bubble trauma (GBT) among brown trout (BR) and rainbow trout (RB) with external symptoms of GBT collected from three sections of the Bighorn River, March 1985 - April 1988. $I=$ minor, $I I=$ serious and $I I I=$ severe symptoms..................... 277

2.3.22. Progression of GBT among recaptured brown and rainbow trout below Afterbay Dam in the Bighorn River 1985-1988................. 318

2.3.23. Mean condition factors (K) of brown trout tagged in the Bighorn River below Afterbay Dam for each size group and each GBT code category. GBT code: $0=$ no external symptoms, 1 = minor, 2 = serious and 3 = severe symptoms: $\mathbb{N}=$ sample size . . . . 319 xxis 
2.3.24. Mean condition factors (K) of rainbow trout tagged in the Bighorn River below Afterbay Dam for each size group and each gas code category. GBT code: $0=$ no external symptoms, $1=$ minor, $2=$ serious and $3=$ severe symptoms. $N=$ sample size .320

2.3.25. The percentages of affected body parts on tagged brown trout exhibiting GBT when captured in the Bighorn River below Afterbay Dam, 1985-1988. GBT code: $0=$ no symptoms, $1=$ minor, $2=$ serious, and 3 = severe symptoms

2.3.26. The percentages of affected body parts on tagged rainbow trout exhibiting GBT when captured in the Bighorn River below Afterbay Dam, 1985-1988. GBT code: $0=$ no symptoms, $1=$ minor, $2=$ serious . . 324

2.4.1. Number of survivors (beginning number $=25$ ), ratios of instantaneous predation rates (dp), and chi-square statistic corrected for continuity $\left(\mathrm{X}^{2} \mathrm{c}\right)$ for predation tests with juvenile rainbow trout prey $(77 \pm 5 \mathrm{~mm})$ after exposure for $30 \mathrm{~d}$ to $112 \%$ gas supersaturated water . . . . . . . . 333

2.4.2. Number of survivors (beginning number $=25$ ), ratios of instantaneous predation rates (dp), and chi-square statistic corrected for continuity $\left(\mathrm{X}^{2} \mathrm{C}\right)$ for predation tests with juvenile rainbow trout prey $(45 \mathrm{~mm})$ after exposure for 30 d to $124 \%$ gas supersaturated water... . . . . . . . . 334

2.4.3. Number of survivors (beginning number $=25$ ), ratios of instantaneous predation rates (dp), and chi-square statistic corrected for continuity $\left(\mathrm{X}^{2} \mathrm{c}\right)$ for predation tests with juvenile brown trout prey (69 $\pm 7 \mathrm{~mm}$ ) after exposure for $30 \mathrm{~d}$ to $110 \%$ gas supersaturated water . . . . . . . . 335

2.4.4. Number of survivors (beginning number $=25$ ), ratios of instantaneous predation rates (dp), and chi-square statistic corrected for continuity $\left(\mathrm{X}^{2} \mathrm{c}\right)$ for predation tests with juvenile brown trout prey ( $56 \pm 7 \mathrm{~mm}$ ) after exposure for $30 \mathrm{~d}$ to $112 \%$ gas supersāturated water . . . . . . . . 336

2.4.5. Number of survivors (beginning number $=25$ ), ratios of instantaneous predation rates (dp), and chi-square statistic corrected for continuity $\left(\mathrm{X}^{2} \mathrm{c}\right)$ for predation tests with juvenile brown trout prey $(56 \pm 5 \mathrm{~mm}$ ) after exposure for $13 \mathrm{~h}$ to $130 \%$ gas supersaturated water . . . . . . . . 337

2.4.6. Ratio of instantaneous predation rates (dP) of juvenile brown trout exposed to $118 \%$ gas supersaturated water and non-exposed fish 
2.4.7. Ratio of instantaneous predation rates (dP) of juvenile rainbow trout exposed to $118 \%$ gas

supersaturated water and non-exposed fish.... 340

2.4.8. Correlation among trout abundance in sections 1 and 2 snorkel lane counts and day number (26 May 17 october) 1987, Bighorn River, Montana ( $N=27$ for both sections) .................. . 349

2.4.9. Correlation among trout abundance in sections 1 and 2 snorkel lane counts and day number 130 January - 23 November) 1988, Bighorn River, Montana ( $N=7$ for section $1, N=6$ for section 2) . . 352

2.4.10. Correlation among percentage of total trout in the middle snorkel lane in sections 1 and 2 and day number, 1987 and 1988 , Bighorn River, Montana $(N=27$ for $1987 ; N=7$ for section $1, N=6$ for section 2 for 1988) ................. 353

2.4.11. Correlations among trout number in snorkel lanes and discharge, visibility, water temperature, above water light level, and total gas pressure, sections 1 and 2, 1987, Bighorn River, Montana $(\mathrm{N}=27)$. The upper triangular matrix indicates simple significance levels ${ }^{\mathrm{a}}$.......... . . 356

2.4.12. Correlations among trout number in snorkel lanes and discharge, visibility, water temperature, above water 1 ight level, and total gas pressure, sections 1 and 2, 1988, Bighorn River, Montana $(N=7$ for section $1, N=6$ for section 2). The upper triangular matrix indicates simple significance levelsa

2.4.13. Comparison of microhabitat use between rainbow trout size groups using analysis of variance, 1987, Bighorn River, Montana ( $1=$ rainbow trout less than $15 \mathrm{~cm}, \mathrm{~N}=23 ; 2$ =rainbow trout $15-40 \mathrm{~cm}$, $\mathrm{N}=65 ; 3=$ rainbow trout greater than $40 \mathrm{~cm}, \mathrm{~N}=27 ; *$ $\mathrm{P}<0.05, * * \mathrm{P}<0.01) . . . . . . . .360$

2.4.14. Comparison of microhabitat use between brown trout size groups using analysis of variance, 1987, Bighorn River, Montana ( $1=b r o w n$ trout less than $15 \mathrm{~cm}, \mathrm{~N}=33 ; 2=$ brown trout $15-40 \mathrm{~cm}, \mathrm{~N}=141$; $3=$ brown trout greater than $40 \mathrm{~cm}, \mathrm{~N}=44 ; * \mathrm{P}<0.05$,

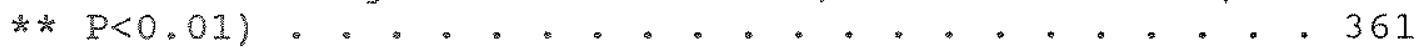

2.4.15. Discriminant analysis of use of selected microhabitat variables by brown trout and rainbow trout <15 cm TL, June - October 1987, Bighorn River, Montana (brown trout $N=33$, rainbow trout $\mathrm{N}=23$; JK=jackknife validation) ......... 362 
2.4.16. Discriminant analysis of use of selected

microhabitat variables by brown trout and rainbow trout $>15 \mathrm{~cm} \mathrm{TL}$, June - October 1987, Bighorn

River, Montana (June: brown trout $N=71$, rainbow trout $\mathrm{N}=33$; July: brown trout $\mathrm{N}=92$, rainbow trout $\mathrm{N}=41$; September/October: brown trout $\mathrm{N}=22$, rainbow trout $\mathrm{N}=18$; JK=jackknife validation) . . . . . . 363

2.4.17. Comparisons between brown trout and rainbow trout $>15 \mathrm{~cm}$ TL use of selected microhabitat variables, $t$ test for equality of means, June 1987, Bighorn River, Montana (brown trout $N=71$, rainbow trout $\mathrm{N}=33$ )

2.4.18. Comparisons between brown trout and rainbow trout $>15 \mathrm{~cm}$ TL use of selected microhabitat variables, $t$ test for equality of means, July 1987, Bighorn River, Montana (brown trout $\mathrm{N}=92$, rainbow trout $\mathrm{N}=41$ )

2.4.19. Comparisons between brown trout and rainbow trout $>15 \mathrm{~cm}$ TL use of selected microhabitat variables, $t$ test for equality of means, september - october 1987, Bighorn River, Montana (brown trout $N=22$, rainbow trout $\mathrm{N}=18$

2.4.20. Principal component analysis for all brown trout and rainbow trout using selected habitat variables, June - october 1987. Bighorn River, Montana

2.4.21. Principal component analysis for all brown trout and rainbow trout $<15 \mathrm{~cm}$ TL using selected habitat variables, June - October 1987, Bighorn River, Montana

2.4.22. Principal component analysis for brown trout and rainbow trout $>15 \mathrm{~cm}$ TL using selected habitat variables, June 1987, Bighorn River, Montana . . . . 371

2.4.23. Principal component analysis for brown trout and rainbow trout $>15 \mathrm{~cm} \mathrm{TL}$ using selected habitat variables, July 1987, Bighorn River, Montana . . . 373

2.4.24. Principal component analysis for brown trout and rainbow trout $>15 \mathrm{~cm}$ TL using selected habitat variables, September - October 1987, Bighorn River, Montana

2.4.25. Specifications of eight pressure-sensitive radio transmitters implanted in brown trout and rainbow trout, 1987, Bighorn River, Montana . . . . . . 377

2.4.26. Specifications of three pressure sensitive radio transmitters implanted in brown trout and rainbow trout, 1988, Bighorn River, Montana . . . . . . 377 
2.4.27. Specifications of ten location radio transmitters implanted in brown trout and rainbow trout, 1987, Bighorn River, Montana... . . . . . . . . 378

2.4.28. Length and weight of brown trout and rainbow trout implanted with pressure-sensitive radio transmitters, 1987 and 1988, Bighorn River, Montana (Percent of trout weight = transmitter weight in water as a percentage of trout weight) . . 379

2.4.29. Length and weight of brown trout and rainbow trout implanted with location radio transmitters, 1987, Bighorn River, Montana (Percent of trout weight = transmitter water as a percentage of trout weight) . . ............ . 380

2.4.30. Configurations of Afterbay Dam sluice gate and radial gates for manipulating gas levels during telemetry studies, 1987 and 1988, Bighorn River, Montana . . . . . . . . . . . . . . 383

2.4.31. Number of locations per day and daily range of fish 255 (brown trout) implanted with pressure sensitive radio transmitter, $19 \mathrm{July}-28 \mathrm{July}$ 1988, Bighorn River, Montana. . . . . . . . . 389

2.4 .32 . Number of locations per day and daily range of fish 270 (brown trout) implanted with pressure sensitive radio transmitter, 19 July - 28 July 1988, Bighorn River, Montana . . . . . . . . . 389

2.4.33. Number of locations per day and daily range of fish 280 (rainbow trout) implanted with pressure sensitive radio transmitter, 19 July - 28 July 1988, Bighorn River, Montana . . . . . . . . 390

2.4.34. Number of locations and duration of monitoring (days) of trout implanted with radio transmitters, 8 August - 11 September 1987, Bighorn River, Montana

2.6.1. Aquatic macroinvertebrate taxa collected in benthic and drift samples, 2.4 and 14.5 river kilometers below Yellowtail Afterbay Dam in the Bighorn River, 1986 and 1987 . . . . . . . . . 426

2.6.2. Relative abundance of benthic invertebrates, showing the number of samples each taxa was found in, the mean per sample, standard deviation, range, and percent of all organisms . . . . . . 428

2.6.3. Principal components analysis of the 14

"taxa/groups a which varied most in abundance among 107 benthic samples collected at $\mathrm{Rkm} 2.4$ and $\mathrm{Rkm}$ 14.5 , Bighorn River, 1986 and 1987 . . . . . . . . 429 
2.6.4. Estimated density $\left(\# / \mathrm{m}^{2}\right)$ for taxa/groups ${ }^{\mathrm{a}}$ commonly occurring in benthic invertebrate samples. Results are provided by site for collection period 1 (August 23-24, 1986), period 2 (April 13-14, 1987) and period 3 (September 22-23, 1987), Bighorn River, MT

2.6.5. Mean drift densities $\left(\# / \mathrm{m}^{3}\right)$ and standard deviations at $\mathrm{Rkm} 2.4$ and 14.5 , for those taxa most commonly present in the drift during collection periods 2 and 3 , Bighorn River

2.6.6. Mean water depths, water velocities ( $M=0.6$ depth; $\underline{B}=3$ to $5 \mathrm{~cm}$ above the substrate), and dry weight of vegetation (DWV), measured for each benthic sample, according to site and collection period. Ranges are in parentheses. . . . . . . . 446

2.6.7. Mean water depths, water velocities $(M=0.6$ depth; $\underline{B}=3$ to $5 \mathrm{~cm}$ above the substrate), and dry weight of vegetation (DWV) associated with benthic samples collected in April 1987 in each riffle subsection. Ranges are in parentheses . . . 448

2.6.8. Mean and standard deviation of periphytic AFDW $\left(\mathrm{g} / \mathrm{cm}^{2} \cdot 10^{-3}\right)$ and Chlorophyll a concentration $(\mu \mathrm{g} / \mathrm{g}$ of blotted wet weight) at $\mathrm{Rkm} 2.4$ and 14.5 for April and September 1987 sampling periods. Data are also presented by subsection for April 1987 • 449

2.6.9. Means, standard deviations (S.D.), and ranges of Delta $\mathrm{P}$ (mmHg), dissolved oxygen $(\mathrm{mg} / \mathrm{L})$, and temperature $\left({ }^{\circ} \mathrm{C}\right)$ during bioassay $\# 1$, August 2 to 9. 1988

2.6.10. Water chemistry measurements taken the day following gas supersaturation bioassays, Bighorn River, Montana 1988 . . . . . . . . . . . . .

2.6.11. Means, standard deviations (S.D.), and ranges for Delta $\mathrm{P}$ (mmHg), dissolved oxygen (mg/L), and temperature $\left({ }^{\circ} \mathrm{C}\right)$ during bioassay \#2, september 8 to 15,1988 . . . . . . . . . . . . . . . . . . 467

2.6.12. The percentages of mottled sculpin in groups 1 and 2 with external GBT symptoms and the severity of the symptoms on days 20,49, and 72 of the bioassay near the right bank at Rkm 0.6 (gagehouse) on the Bighorn River, Montana, February - May 1988

2.7.1. Gas pressures (mmHg) measured at selected sites on Afterbay Reservoir and the irrigation canal below Afterbay Dam, 3 september, 1986 
2.7.2. Gas tensions (mmHg) measured at the boat ramp on Afterbay Reservoir from $8 \mathrm{May}$ - $30 \mathrm{July}, 1987$. . 509

2.7.3. Discharge equations for the sluiceway gates of Afterbay Dam on the Bighorn River . . . . . . . 512

2.7.3. Results of downriver gas dissipation test conducted on the Bighorn River downstream from the Afterbay Dam on 29 March 1985 . . . . . . . . 527

2.7.5. Mean barometric pressure (B.P.) and Afterbay Dam gate openings (in feet) during cross-river transect measurements in 1986 . . . . . . . . 533

2.7.6. Mean barometric pressure (mmHg) during measurements of cross-river transects in 1987 on the Bighorn River, Montana below Afterbay Dam . . 537 


\section{LIST OF FIGURES}

Figure

Page

2.1.1. Theoretical TGP thresholds for arterial bubble, water bubble, and swimbladder overinflation as a

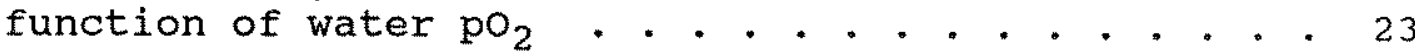

2.1.2. Theoretical delta $\mathrm{p}$ thresholds for arterial bubble, water bubble, and swimbladder overinflation as a function of water $\mathrm{pO}_{2}$. • . . . . . . . . . . . . . . . . . 24

2.1.3. Ratio of Arterial to water $\mathrm{po}_{2}$ for Rainbow Trout . . 26

2.1.4. Time to mortality (20-70\%) of salmonids larger and smaller than $50 \mathrm{~mm}$ as a function of water total gas pressure. Data from published literature. Negative times correspond to experiments with no mortality . . . . . . . . . . . . . . .

2.1.5. Time to mortality $(20-70 \%)$ of salmonids at total gas pressures ranging from 1.08 to 1.50 atms as a function of fish length. Data from published literature. Negative times correspond to experiments with no mortality

2.1.6. Time to mortality $(20-70 \%)$ of steelhead trout and coho and chinook salmon $<50 \mathrm{~mm}$ in length as a function of total gas pressure. Data from published literature. Negative times correspond to experiments with no mortality . . . . . . . .

2.1.7. Time to mortality $(20-70 \%)$ of steelhead trout and coho and chinook salmon $>50 \mathrm{~mm}$ in length as a function of total gas pressure. Data from published literature. Negative times correspond to experiments with no mortality . . . . . . . .

2.1.8. Time to mortality $(20-70 \%)$ of sockeye salmon $>50$ $\mathrm{mm}$ in length as a function of total gas pressure. Data from published literature. Negative times correspond to experiments with no mortality . . .

2.1.9. Time to mortality $(20-70 \%)$ of coho salmon $>50 \mathrm{~mm}$ in length as a function of total gas pressure. Data from published literature. Negative times correspond to experiments with no mortality . . .

2.1.10. Time to mortality $(20-70 \%)$ of steelhead trout $>$ $50 \mathrm{~mm}$ in length as a function of total gas pressure. Data from published literature. Negative times correspond to experiments with no mortality 
2.1.11. Time to mortality $(20-70 \%)$ of cutthroat trout $>$ $50 \mathrm{~mm}$ in length as a function of total gas pressure. Data from published Iiterature. Negative times comespond to experiments with no

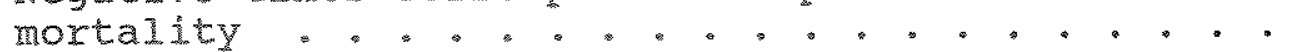

2.1.12. Intluence of water $\mathrm{po}_{2}$ (mmHg) on time to $50 \%$ mortality of steelhead trout. Data from Nebeker et a1. 1979

2.1.13. Influence of water $\mathrm{po}_{2}$ (mmHg) on time to $25 \%$ and $50 \%$ mortality of coho salmon exposed to TGP of $1.193 \mathrm{~atm}$. Data from Rucker 1975 . . . . . . 44 44

2.1.14. Time to mortality $(20-70 \%)$ of salmonids smaller and larger than $50 \mathrm{~mm}$ as a function of depthcorrected total gas pressure...........

2.1.15. Time to 50\% mortality of steelhead crout at water total gas pressures with and without correction for depth. Data from knittel et al. 1980 . . . .

2.1.16. Schematic of experimental system designed to test effects of dissolved gas supersaturation on trout

2.1.17. Schematic of experimental container and placement of swimbladder and dorsal aorta cannula . . . . 55

2.1.18. Comparison of time to mortality of cannulated and uncannulated rainbow trout as a function of water total gas pressure..................

2.1.19. Variation in arterial blood $\mathrm{po}_{2}$ with time in rainbow trout exposed to water TGP of 1.10 atms with a $\mathrm{pO}_{2}$ of $170 \mathrm{mmHg}$...............

2.1.20. Relative severity of gas bubble trauma symptoms at time of death of six cannulated $(1-6)$ and six noncannulated $(7-12)$ rainbow trout exposed to water TGP of 1.10 atms with a $\mathrm{pO}_{2}$ of $175 \mathrm{mmHg}$. .

2.1.21. Arterial blood hematocrit and pH variation with time in rainbow trout exposed to water TGP of 1.10 atms with a $\mathrm{po}_{2}$ of $170 \mathrm{mmHg}$. . . . . . . . .

2.1.22. Variation in arterial blood $\mathrm{pO}_{2}$ with time in rainbow trout exposed to water TGP of 1.12 atms with a $\mathrm{pO}_{2}$ of $175 \mathrm{mmHg} . . . . . . . . . . . .$.

2.1.23. Arterial blood hematocrit and $\mathrm{pH}$ variation with time in rainbow trout exposed to water TGP of 1.12 atms with a $\mathrm{pO}_{2}$ of $175 \mathrm{mmHg}$ 
2.1.24. Relative severity of gas bubble trauma symptoms at time of death of six cannulated (1-6) and six noncannulated $(7-12)$ trout exposed to water TGP of 1.12 atms with a $\mathrm{pO}_{2}$ of $175 \mathrm{mmHg}$. . . . . . . .

2.1.25. Extracorporeal bubbles between gill lamella of rainbow trout resulting from dissolved gas supersaturation

2.1.26. Subdurmal bubbles on the upper part of the mouth of a rainbow trout exposed to TGP of 1.12 atms . . . 70

2.1.27. Subdurmal bubbles lining the entrances to the gills of a rainbow trout exposed to TGP of 1.12 atms

2.1.28. Variation in arterial blood $\mathrm{pO}_{2}$ with time in rainbow trout exposed to water TGP of $1.15 \mathrm{atms}$ with a $\mathrm{pO}_{2}$ of $183 \mathrm{mmHg}$. . . . . . . . . . . . . .

2.1.29. Arterial blood hematocrit and $\mathrm{pH}$ variation with time in rainbow trout exposed to water TGP of 1.15

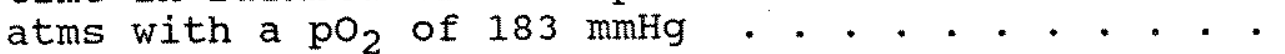

2.1.30. Relative severity of gas bubble trauma symptoms at time of death of six cannulated $(1-6)$ and six noncannulated $(7-12)$ rainbow trout exposed to water TGP of 1.15 atms with a $\mathrm{pO}_{2}$ of $183 \mathrm{mmHg} . . \quad 74$

2.1.31. Vascular bubbles in filamental arteries of primary gill lamella of rainbow trout . . . . . . 75

2.1.32. Vascular bubbles in filamental arteries of primary gill lamella of rainbow trout . . . . . .

2.1.33. Relative severity of gas bubble trauma symptoms at time of death of six cannulated $(1-6)$ and six noncannulated $(7-12)$ rainbow trout exposed to water TGP of 1.17 atms with a $\mathrm{pO}_{2}$ of $195 \mathrm{mmHg}$. . 77

2.1.34. Arterial blood hematocrit and $\mathrm{pH}$ variation with time in rainbow trout exposed to water TGP of 1.17 atms with a $\mathrm{pO}_{2}$ of $195 \mathrm{mmHg}$. . . . . . . . .

2.1.35. Variation in arterial blood $\mathrm{po}_{2}$ with time in rainbow trout exposed to water TGP of 1.17 atms with a $\mathrm{pO}_{2}$ of $195 \mathrm{mmHg}$. . . . . . . . . . .

2.1.36. Variation in arterial blood $\mathrm{po}_{2}$ with time in rainbow trout exposed to water TGP of 1.19 atms

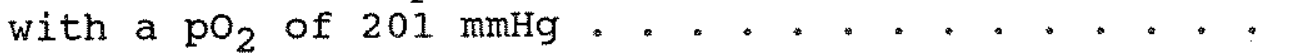

2.1.37. Relative severity of gas bubble trauma symptoms at time of death of six cannulated $(1-6)$ and six noncannulated $(7-12)$ rainbow trout exposed to water TGP of 1.19 atms with a $\mathrm{pO}_{2}$ of $201 \mathrm{mmHg}$ 
2.1.38. Arterial blood hematocrit and $\mathrm{pH}$ variation with

time in rainbow trout exposed to water TGP of 1.19

atms with a $\mathrm{pO}_{2}$ of $201 \mathrm{mmHg}$. . . . . . . . .

2.1.39. Influence of increasing levels of water $\mathrm{pO}_{2}$ on

time to mortality of rainbow trout while

maintaining a constant TGP of 1.15 atms . . . . . 84

2.1.40. Total gas pressure thresholds for bubble growth

in arterial blood of rainbow trout and coho salmon

as a function of $\mathrm{pO}_{2}$ and nucleus radius . . . . . 88

2.1.41. Influence of total gas pressure and water $\mathrm{po}_{2}$ on swimbladder inflation of rainbow trout... . . . . 93

2.1.42. Rainbow trout swimbladder pneumatic duct release pressure as a function of fish weight . . . . . . 95

2.1.43. Laboratory apparatus used to test effects of gas supersaturation on juvenile brown and rainbow

trout

2.1.44. Delta $P$ of water for the high (HT) (mean " $P=155$

$\mathrm{mm}$ and mean percent total saturation $=124.3)$,

medium (MT) (mean ' $\mathrm{P}=82 \mathrm{~mm}$ and mean percent

total saturation $=112.8)$, and control treatments (CT) (mean ${ }^{A} \mathrm{P}=16 \mathrm{~mm}$ and mean percent total

saturation $=102.5)$, test 1, March $15-$ April 15, 1986 ................... 101

2.1.45. Delta $P$ of water for the high (HT) (mean ${ }^{\wedge} P=153$

$\mathrm{mm}$ and mean percent total saturation $=124.0)$, medium (MT) (mean ' $\mathrm{P}=78 \mathrm{~mm}$ and mean percent

total saturation $=112.3)$, and control treatments (CT) (mean ${ }^{\wedge} \mathrm{P}=38 \mathrm{~mm}$ and mean percent total

saturation $=105.9)$, test 2 , May 18 - June 11,

1986

2.1.46. Delta $P$ of water for the high (HT) (mean " $P=162$

$\mathrm{mm}$ and mean percent total saturation $=125.4)$,

medium (MT) (mean ' $P=69 \mathrm{~mm}$ and mean percent

total saturation $=110.8)$, and control treatments

(CT) (mean ${ }^{\wedge} P=29 \mathrm{~mm}$ and mean percent total

saturation $=104.5)$, test 3 , July 17 - August 17 ,

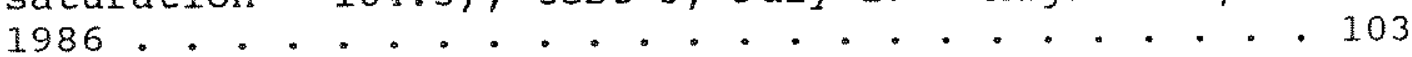

2.1.47. Delta $P$ of water for the high (HT) (mean ${ }^{\wedge} P=167$

$\mathrm{mm}$ and mean percent total saturation $=126.1)$,

medium (MT) (mean ${ }^{\wedge} \mathrm{P}=83 \mathrm{~mm}$ and mean percent

total saturation $=113.0)$, and control treatments

(CT) (mean ${ }^{\wedge} \mathrm{P}=20 \mathrm{~mm}$ and mean percent total

saturation $=103.3)$, test 4 , September 28 -

october 29, 1986 ................. . 104 
2.1.48. Delta $P$ of water for the high (HT) (mean " $P=160$ $\mathrm{mm}$ and mean percent total saturation $=125.0)$, medium (MT) (mean " $P=72 \mathrm{~mm}$ and mean percent total saturation $=111.2)$, and control treatments (CT) (mean ${ }^{\wedge} \mathrm{P}=26 \mathrm{~mm}$ and mean percent total saturation $=104.1)$, test 5 , January 9 - February 9,1987 . . . . . . . . . . . . . . 105

2.1.49. Delta $P$ of water for the high (HT) (mean ${ }^{\wedge} P=160$ $\mathrm{mm}$ and mean percent total saturation $=125.1)$, medium (MT) (mean ' $\mathrm{P}=84 \mathrm{~mm}$ and mean percent total saturation $=113.2)$, and control treatments (CT) (mean ' $\mathrm{P}=25 \mathrm{~mm}$ and mean percent total saturation $=103.9)$, test 5, February 19 - March 19,1987 . . . . . . . . . . . . . 106

2.1.50. Comparison of medium treatment (MT) juvenile brown (BT) and rainbow trout (RBT) daily mortality and associated ' $P$ values by day during test 2, May

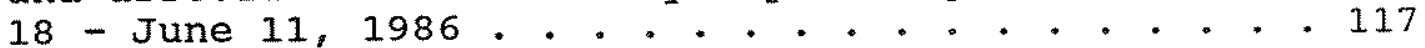

2.1.51. Comparison of medium treatment (MT) juvenile brown (BT) and rainbow trout (RBT) daily mortality and associated $P$ values by day during test 4 , September 28 - October 29,1986

2.1.52. High treatment (125\%) juvenile brown trout percent cumulative mortality by day, during tests $1,2,3,4,5$, and $6(\mathrm{BT} 1=35.2 \mathrm{~mm}$ and $0.4 \mathrm{~g}, \mathrm{BT} 2$ $=53.1 \mathrm{~mm}$ and $1.3 \mathrm{~g}, \mathrm{BT} 3=62.2 \mathrm{~mm}$ and $2.9 \mathrm{~g}, \mathrm{BT} 4$ $=90.7 \mathrm{~mm}$ and $8.3 \mathrm{~g}$, BT5 $=126.3 \mathrm{~mm}$ and $22.9 \mathrm{~g}$,

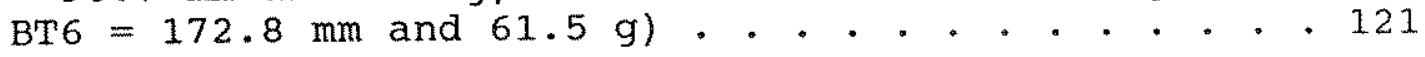

2.1.53. High treatment (125\%) juvenile rainbow trout percent cumulative mortality by day, during tests $1,2,3,4,5$, and 6 (RBT1 $=34.0 \mathrm{~mm}$ and $0.4 \mathrm{~g}$, RBT2 $=70.5 \mathrm{~mm}$ and $3.4 \mathrm{~g}, \operatorname{RBT} 3=90.6 \mathrm{~mm}$ and 8.9 $\mathrm{g}, \mathrm{RBT} 4=130.5 \mathrm{~mm}$ and $26.3 \mathrm{~g}, \mathrm{RBT5}=194.1 \mathrm{~mm}$ and $79.3 \mathrm{~g}, \mathrm{RBT6}=178.7 \mathrm{~mm}$ and $60.5 \mathrm{~g}) . . . . . . .122$

2.1.54. High treatment juvenile brown (mean beginning length and weight $=35 \mathrm{~mm}$ and $0.4 \mathrm{~g}$ ) and rainbow trout (mean beginning length and weight $=34 \mathrm{~mm}$ and $0.4 \mathrm{~g}$ ) percent cumulative mortality by day

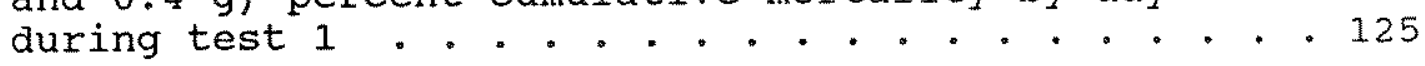

2.1.55. High treatment juvenile brown (mean beginning length and weight $=62 \mathrm{~mm}$ and $2.9 \mathrm{~g}$ ) and rainbow trout (mean beginning length and weight $=71 \mathrm{~mm}$ and $3.4 \mathrm{~g}$ ) percent cumulative mortality by day during tests 3 and 2 , respectively . . . . . . 126 
2.1.56. High treatment juvenile brown (mean beginning length and weight $=91 \mathrm{~mm}$ and $8.3 \mathrm{~g}$ ) and rainbow trout (mean beginning length and weight $=90 \mathrm{~mm}$ and $8.9 \mathrm{~g})$ percent cumulative mortality by day during tests 4 and 3 , respectively . . . . . . . 127

2.1.57. High treatment juvenile brown (mean beginning length and weight $=173 \mathrm{~mm}$ and $61.5 \mathrm{~g}$ ) and rainbow trout (mean beginning length and weight $=179 \mathrm{~mm}$ and $60.5 \mathrm{~g})$ percent cumulative mortality by day

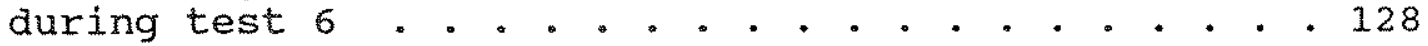

2.2.1. Comparison of GBT severity and recovery rate of juvenile rainbow trout $(228 \pm 17 \mathrm{~mm}$ and $125 \pm 31$ g) by 2-d intervals February 9 - March 12, 1987 (See Table 2.2.1 for severity definitions) . . . . . 143

2.2.2. Percent cumulative recovery for juvenile brown trout (fish size on day 1 of testing $=164 \pm 17 \mathrm{~mm}$ and $43.2 \pm 14.4 \mathrm{~g})$ from GBT calculated by combining GBT rating groups 1, 2, and 3 (see Table 2.2 .1 ) from three $120-\mathrm{h}$ exposure tests conducted June 6 - September 18, 1987 . . . . . . . . . . . . 149

2.3.1. Map of the study area showing GBT electrofishing sections, early life history study sites, satellite relay stations, side-channel index and access sites... . . . . . . . . . . 156

2.3.2. Spring rainbow trout population estimates by length group from Rkm 0-15.4 (RM 0-9.6) below Afterbay Dam on the Bighorn River, 1985-87 (MCFRU and MDFWP data) ............... 163

2.3.3. Fall rainbow trout population estimates by length group from Rkm 0-15.4 (RM $0-9.6)$ below Afterbay Dam on the Bighorn River, 1985-87 (MCFRU and MDFWP data) ................... . 165

2.3.4. Spring brown trout population estimates by length group from Rkm 0-6.I (RM $0-3.8$ ) below Afterbay Dam on the Bighorn River, 1985-87 (MCFRU and MDFWP data)

2.3.5. Fall brown trout population estimates by length group from Rkm 0-6.1 (RM 0-3.8) below Afterbay Dam on the Bighorn River, 1985-87 (MCFRU and MDFWP data)

2.3.6. Spring brown trout population estimates by length group from Rkm 3.9-15.4 (RM 2.4-9.6) below Afterbay Dam on the Bighorn River, 1985-87 (MCFRU and MDFWP data) ................ . 172 
2.3.7. Fall brown trout population estimates by length group from Rkm 3.9-15.4 (RM 2.4-9.6) below

Afterbay Dam on the Bighorn River, 1985-87 (MCFRU and MDFWP data) . . . . . . . . . . . . 174

2.3.8. Delta $\mathrm{p}$ (mmHg) measured by Bouck gasometers near the right bank at Rkm 0.6 (gagehouse) between A) January - June and B) July - December from 19851988 on the Bighorn River, Montana... . . . . . 179

2.3.9. Delta p. (mmHg) measured by Bouck gasometers near the right bank at $\mathrm{Rkm} 2.4$ (RM 1.5) between A) January - June and B) July - December from 19851988 on the Bighorn River, Montana . . . . . . . 181

2.3.10. Delta $\mathrm{P}$ ( $\mathrm{mmHg}$ ) measured by Bouck gasometers near the right bank at $\mathrm{Rkm} 4.8$ (RM 3.0) between $A$ ) January - June and B) July - December from 19861988 on the Bighorn River, Montana . . . . . . . 183

2.3.11. Delta $\mathrm{P}$ ( $\mathrm{mmHg}$ ) measured by Bouck gasometers near the right bank at $\mathrm{Rkm} 8.0$ (RM 5.0) between A) January - June and B) July - December from 19851988 on the Bighorn River, Montana....... . . 184

2.3.12. Delta $\mathrm{P}$ (mmHg) measured by Bouck gasometers near the right bank at $\mathrm{Rkm} 14.5$ (RM 9.0) between A) January - June and B) July - December from 19851988 on the Bighorn River, Montana... . . . . . 185

2.3.13. Delta $\mathrm{p}$ (mmHg) measured by Bouck gasometers near the right bank at $\mathrm{Rkm} 19.3$ (RM 12.0) from 18 December, 1987 - 3 May, 1988 on the Bighorn River, Montana . . . . . . . . . . . . . . . 187

2.3.14. Mean weekly absolute barometric pressure measured at Fort Smith, Montana (elevation about $1001 \mathrm{~m}$ ) from April, 1985 - March, 1986 and at Rkm 0.6 (elevation about $965 \mathrm{~m}$ ) from March 1986 December, 1988 on the Bighorn River, Montana . . . 187

2.3.15. Mean weekly oxygen pressure ( $\mathrm{mmHg}$ ) calculated from modified winkler samples collected between 1985 and 1988 at Rkm 0.6 (gagehouse), Bighorn River, Montana............... . . 189

2.3.16. Mean weekly oxygen pressure (mmHg) calculated from modified Winkler samples collected between 1985 and 1988 at Rkm 2.4 (RM 1.5), Bighorn River, Montana

2.3.17. Mean weekly oxygen pressure (mmHg) calculated from modified winkler samples collected between 1985 and 1988 at Rkm 4.8 (RM 3.0), Bighorn River, Montana 
2.3.18. Mean weekly oxygen pressure (mmHg) calculated

from modified Winkler samples collected between

1985 and 1988 at Rkm 8.0 (RM 5.0), Bighorn River,

Montana

2.3.19. Mean weekly oxygen pressure (mmHg) calculated

from modified winkler samples collected between

1985 and 1988 at Rkm 14.5 (RM 9.0), Bighorn River,

Montana

2.3.20. Mean weekly water temperature of the Bighorn

River, Montana from 1985-1988 at A) Rkm 0.6

(gagehouse), B) $\mathrm{Rkm} \mathrm{2.4,} \mathrm{C)} \mathrm{Rkm} \mathrm{8.0,} \mathrm{and} \mathrm{D)} \mathrm{Rkm}$

$14.5 . . . . . . . . . . . . . . . .194$

2.3.21. Mean weekly water temperatures of the forebay

epilimnion of Bighom Lake, the Bighorn River at

$\mathrm{Rkm} 0.6$ (gagehouse), and Rkm 4.8 in 1987 reported

via the Hydromet system . . . . . . . . . . 196

2.3.22. Brown trout embryo survival in A) 1985, B) 1986 ,

and C) 1987 associated with Bouck delta p's (mmHg)

during embryo incubation tests in D) 1985-1986,

E) 1986-1987, and F) 1987-1988 at early life history

sites on the Bighorn River and in Afterbay

Reservoir, Montana............... . . 201

2.3.23. Rainbow trout embryo survival made in A) 1985,

B) 1987, and C) 1988 associated with Bouck delta p's

(mmHg) during embryo incubation tests in D) 1985 ,

E) 1987, and F) 1988 at early life history sites on

the Bighorn River and in Afterbay Reservoir,

Montana

205

2.3.24. Hyperbaric pressure (mmHg) greater than the

threshold for bubble growth in environmental water

during embryo incubation experiments for brown

trout in A) 1985-86, B) 1986-87, and C) 1987-88 and

for rainbow trout in D) 1985, E) 1987, and F) 1988 at

early life history sites on the Bighorn River and

in Afterbay Reservoir, Montana.......... . . 208

2.3.25. Cumulative mortality by day among rainbow trout

fry held at $2.4,8.0$, and $14.5 \mathrm{~km}$ below Afterbay

Dam on the Bighorn River, July 1985 - January

1986

2.3.26. The environmental water (EW) threshold for bubble growth and mean delta $P^{\prime} \mathrm{s}$ (mmHg) at $2.4,8.0$, and

$14.5 \mathrm{~km}$ downstream from Afterbay Dam during

rainbow trout survival tests, July 1985 - January

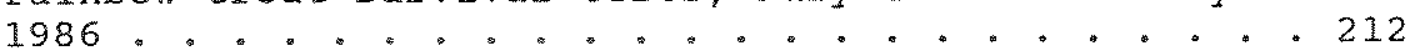


2.3.27. The environmental water (EW) threshold for bubble growth and delta $\mathrm{P}$ (mmHg) at $\mathrm{Rkm} 0.6$, and cumulative percent mortality among rainbow trout fry reared at $\mathrm{kkm} 14.5$ and moved to $0.6 \mathrm{~km}$ below Afterbay Dam on the Bighorn River, October November 1985

2.3.28. Percent cumulative mortality by day among rainbow trout fry reared at $\mathrm{Rkm} 8.0$ and held at $\mathrm{Rkm} 0.6$ and 8.0 on the Bighorn River below Afterbay Dam, November - December 1985... . . . . . . . . 215

2.3.29. The environmental water threshold for bubble growth and delta $\mathrm{P}$ 's (mmHg) measured at $\mathrm{Rkm} 0.6$ and 8.0 below Afterbay Dam on the Bighorn River, Montana, during rainbow trout survival tests, November - December 1985... . . . . . . . 217

2.3.30. Percent cumulative mortality by day among rainbow trout fry reared at $\mathrm{Rkm} 2.4$ and held at $\mathrm{Rkm} 0.6$ and 2.4 on the Bighorn River below Afterbay Dam, January - February 1986 . . . . . . . . . . 217

2.3.31. The environmental water threshold for bubble growth and delta $P^{\prime} \mathrm{s}$ (mmHg) measured at Rkm 0.6 and 2.4 below Afterbay Dam on the Bighorn River, Montana, during rainbow trout survival tests, January - February 1986 . . . . . . . . . . 219

2.3.32. Percent cumulative mortality by day of brown trout fry reared in non-supersaturated water (control) and those held at Rkm 0.6 (test) on the Bighorn River below Afterbay Dam, March - April 1987 . . . . . . . . . . . . . . . . 220

2.3.33. Environmental water (EW) and cardiovascular (CV) thresholds for bubble growth and delta $\mathrm{P}^{\prime} \mathrm{s}$ (mmHg) measured at Rkm 0.6 below Afterbay Dam on the Bighorn River, Montana, during brown trout fry survival tests, March - April 1987 . . . . . . 220

2.3.34. Percent cumulative mortality by day of brown trout fry reared at $\mathrm{Rkm} 2.4$ and held at $\mathrm{Rkm} 0.6$ and 2.4 on the Bighorn River below Afterbay Dam, March - June, 1987 . . . . . . . . . . . 222

2.3.35. Environmental watex (EW) and cardiovascular (CV) thresholds for bubble growth and delta P's (mmHg) measured at Rkm 2.4 (A) and 0.6 (B) below Afterbay Dam on the Bighorn River, Montana, during brown trout fry survival tests, March - June 1987 . . . 223

2.3.36. Percent cumulative mortality by day of brown trout fry reared at $\mathrm{Rkm} 2.4$ and held at $\mathrm{Rkm} 0.6$ and 2.4 on the Bighorn River below Afterbay Dam, June -

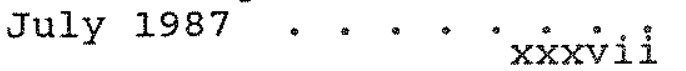


2.3.37. Environmental water (EW) and cardiovascular (CV) thresholds for bubble growth and delta $\mathrm{P}^{\prime} \mathrm{s}$ (mmHg) measured at Rkm 2.4 (A) and 0.6 (B) below Afterbay Dam on the Bighorn River, Montana during brown trout fry tests, June - July 1987 . . . . . . . 225

2.3.38. Percent cumulative mortality by day of brown trout fry reared at $\mathrm{Rkm} 8.0$ and held at $\mathrm{Rkm} 0.6$ and 8.0 on the Bighorn River below Afterbay Dam, March - June 1987 . . . . . . . . . . . . 227

2.3.39. Environmental water (EW) and cardiovascular (CV) thresholds for bubble growth and delta $P^{\prime} s$ (mmH) measured at $\mathrm{Rkm} 8.0$ (A) and 0.6 (B) below Afterbay Dam on the Bighorn River, Montana, during brown trout fry survival tests, March - June 1987 . . . 228

2.3.40. Percent cumulative mortality by day of brown trout fry reared at $\mathrm{Rkm} 8.0$ and held at $\mathrm{Rkm} 0.6$ and 8.0 on the Bighorn River below Afterbay Dam, June - August $1987 . . . . . . . . . . . . .229$

2.3.41. Environmental water (EW) and cardiovascular (CV) thresholds for bubble growth and delta p's (mmHg) measured at Rkm 8.0 (A) and 0.6 (B) below Afterbay Dam on the Bighorn River, Montana, during brown trout fry survival tests, June - August 1987. . . . 230

2.3 .42 . Percent cumulative mortality by day among brown trout fry reared at $\mathrm{Rkm} 14.5$ and held at $\mathrm{Rkm} 0.6$ and 14.5 on the Bighorn River below Afterbay Dam, April - June 1987 . . . . . . . . . . . 232

2.3.43. Environmental water (EW) and cardiovascular (CV) thresholds for bubble growth and delta $\mathrm{P}^{\prime} \mathrm{s}$ (mmHg) measured at Rkm 14.5 (A) and 0.6 (B) below Afterbay Dam on the Bighorn River, Montana, during brown trout fry survival tests, April - June 1987 . 233

2.3.44. Percent cumulative mortality by day of brown trout fry reared at $\mathrm{Rkm} 14.5$ and held at $\mathrm{Rkm} 0.6$ and 14.5 on the Bighorn River below Afterbay Dam, June - October 1987 . . . . . . . . . . . . 234

2.3.45. Environmental water (EW) and cardiovascular (CV) thresholds for bubble growth and delta $\mathrm{P}^{\prime} \mathrm{s}$ (mmHg) measured at Rkm 14.5 (A) and 0.6 (B) below Afterbay Dam on the Bighorn River, Montana, during brown trout fry survival tests, June - October $1987 . . . . . . . . . . . . . . .235$

2.3.46. Percent cumulative mortality by day among rainbow trout fry reared and held at $\mathrm{Rkm} 2.4$ on the Bighorn River below Afterbay Dam, July - August 1987 
2.3.47. Environmental water (EW) and cardiovascular (CV) thresholds for bubble growth and delta $P^{\prime s}$ (mmHg) measured at $\mathrm{Rkm} 2.4$ below Afterbay Dam on the Bighorn River, Montana, during rainbow trout fry

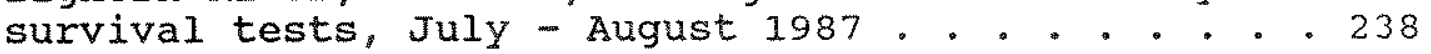

2.3.48. Percent cumulative mortality by day among rainbow trout fry reared at $\mathrm{Rkm} 2.4$ and held at $\mathrm{Rkm} 0.6$ and 2.4 on the Bighorn River below Afterbay Dam, August - October 1987 . . . . . . . . . . . 239

2.3.49. Environmental water (EW) and cardiovascular (CV) thresholds for bubble growth and delta p's (mmHg) measured at $\mathrm{Rkm} 2.4$ (A) and 0.6 (B) below Afterbay Dam on the Bighorn River, Montana, during rainbow trout fry survival tests, August - October 1987 . . 240

2.3.50. Percent cumulative mortality by day among rainbow trout fry reared at Rkm 2.4 and held at $\mathrm{Rkm} 0.6$ and 2.4 on the Bighorn River below Afterbay Dam, October 1987 - January 1988 . . . . . . . . . 242

2.3.51. Environmental water (EW) and cardiovascular (CV) thresholds for bubble growth and delta $P^{\prime} \mathrm{s}$ (mmHg) measured at $\mathrm{Rkm} 2.4$ (A) and 0.6 (B) below Afterbay Dam on the Bighorn River, Montana, during rainbow trout fry survival tests, October 1987 - January 1988 . . . . . . . . . . . . . . 243

2.3.52. Percent cumulative mortality by day among rainbow trout fry reared and held at $\mathrm{Rkm} 8.0$ on the Bighorn River below Afterbay Dam, July - August 1987 . . . . . . . . . . . . . . . . 244

2.3.53. Environmental water (EW) and cardiovascular (CV) thresholds for bubble growth and delta P's (mmHg) measured at $\mathrm{Rkm} 8.0$ below Afterbay Dam on the Bighorn River, Montana, during rainbow trout fry survival tests, July - August 1987 . . . . . . . 244

2.3.54. Percent cumulative mortality by day among rainbow trout fry reared at $\mathrm{Rkm} 8.0$ and held at $\mathrm{Rkm} 0.6$ and 8.0 on the Bighorn Rivex below Afterbay Dam, August - October 1987 . . . . . . . . . . 2 246

2.3.55. Environmental water (EW) and cardiovascular (CV) thresholds for bubble growth and delta $\mathrm{P}^{\prime} \mathrm{s}$ (mmHg) measured at $\mathrm{Rkm} 8.0$ (A) and 0.6 (B) below Afterbay Dam on the Bighorn River, Montana, during rainbow trout fry survival tests, August - October 1987 . 247

2.3.56. Percent cumulative mortality by day among rainbow trout fry reared at $\mathrm{Rkm} 8.0$ and held at $\mathrm{Rkm} 0.6$ and 8.0 on the Bighorn River below Afterbay Dam, October 1987 - January 1988 . . . . . . . . . 248 
2.3.57. Environmental water (EW) and cardiovascular (CV) thresholds for bubble growth and delta p's (mmHg) measured at Rkm 8.0 (A) and 0.6 (B) below Afterbay Dam on the Bighorn River, Montana, during rainbow trout fry survival tests, october 1987 - January

2.3.58. Percent cumulative mortality by day among rainbow trout fry reared and held at Rkm 14.5 on the Bighorn River below Afterbay Dam, July - October

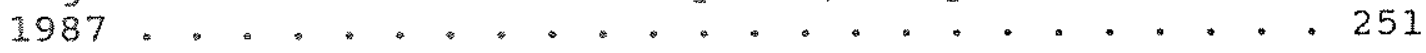

2.3.59. Environmental water (EW) and cardiovascular (CV) thresholds for bubble growth and delta $\mathrm{P}^{\prime} \mathrm{s}$ (mmHg) measured at Rkm 14.5 below Afterbay Dam on the Bighorn River, Montana, during rainbow trout fry survival tests, July - october 1987 . . . . . . 251

2.3.60. Percent cumulative mortality by day among brown trout fry reared and held at $\mathrm{Rkm} 2.4,8.0$, and 14.5 and a control group held at $\mathrm{Rkm} 19.3$ on the Bighorn River below Afterbay Dam, January - July

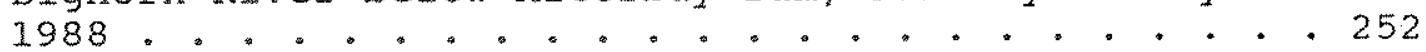

2.3.61. Environmental water (EW) and cardiovascular (CV) thresholds for bubble growth and delta $P^{\prime} s$ (mmHg) measured for the control group (Rkm 19.3) during brown trout fry survival tests, January - May 1988 on the Bighorn River, Montana ......... 253

2.3.62. Mean delta $P$, mean environmental water (EW) and cardiovascular (CV) thresholds for bubble growth (mmHg) measured for the control group and Rkm 2.4, 8.0 , and 14.5 study sites during brown trout fry survival tests, January - May 1988, on the Bighorn River, Montana................. . 254

2.3.63. Environmental water (EW) and cardiovascular (CV) thresholds for bubble growth and delta $\mathrm{P}^{\prime} \mathrm{s}$ (mmHg) measured at Rkm 2.A below Afterbay Dam on the Bighorn River, Montana during brown trout fry survival tests, January - July 1988 . ..... . 254

2.3.64. Environmental water (EW) and cardiovascular (CV) thresholds for bubble growth and delta $\mathrm{P}^{\prime} \mathrm{s}$ (mmHg) measured at Rkm 8.0 below Afterbay Dam on the Bighorn River, Montana, during brown trout fry survival tests, February - July 1988 . . . . . . 256

2.3.65. Environmental water (EW) and cardiovascular (CV) thresholds for bubble growth and delta $\mathrm{P}^{\prime} \mathrm{s}$ (mmHg) measured at Rkm 14.5 (D) below Afterbay Dam on the Bighorn River, Montana, during brown trout fry survival tests, March - July 1988 . . . . . . . 256 
2.3.66. Percent cumulative mortality by day among brown trout fry reared at $\mathrm{Rkm} 2.4,8.0$, and 14.5 and held at Rkm 4.8 on the Bighorn River below Afterbay Dam, July - September 1988 . . . . . . 257

2.3.67. Environmental water (EW) and cardiovascular (CV) thresholds for bubble growth and delta P's (mmHg) measured while test groups from Rkm 2.4, Rkm 8.0, and $\mathrm{Rkm} 14.5$ were held $4.8 \mathrm{Rkm}$ below Afterbay Dam on the Bighorn River, Montana, during brown trout fry survival tests, July - September 1988 . . . . 257

2.3.68. Percent cumulative mortality by day among rainbow trout fry reared at $\mathrm{Rkm} 2.4,8.0$, and 14.5 and held at Rkm 4.8 on the Bighorn River below Afterbay Dam, July - September 1988 . . . . . . . 259

2.3.69. Environmental water (EW) and cardiovascular (CV) thresholds for bubble growth and delta P's (mmHg) measured while test groups from Rkm 2.4, Rkm 8.0, and $\mathrm{Rkm} 14.5$ were held $4.8 \mathrm{Rkm}$ below Afterbay Dam on the Bighorn River, Montana, during rainbow trout fry survival tests, July - September 1988 . . 259

2.3.70. Uncorrected delta $\mathrm{P}$ (mmHg) measured at $\mathrm{Rkm} 4.8$ by a Common Sensing tensionometer during rainbow trout bioassays below Afterbay Dam on the Bighorn River, Montana, July - September 1988 . . . . . . 260

2.3.71. Corrected delta $\mathrm{P}$ (mmHg) measured by a common Sensing tensionometer at $\mathrm{Rkm} 0.6$ and 4.8 below Afterbay Dam on the Bighorn River, Montana, from July - September 1987 . . . . . . . . . . . 262

2.3.72. Percent incidence in gas bubble trauma (GBT) among brown trout in sections 1,2 and 3 , Bighorn River, March 1985 - April 1987 . . . . . . . . 270

2.3.73. Percent incidence of gas bubble trauma (GBT) among brown trout $\geq 356 \mathrm{~mm}$ in total length in sections 1, 2 and 3, Bighorn River, March 1985 April 1987 .................. . 271

2.3.74. Percent incidence of gas bubble trauma (GBT) among rainbow trout in sections 1,2 and 3 , Bighorn River, March 1985 - April 1987 . . . . . . 272

2.3.75. Percent incidence in gas bubble trauma (GBT) among rainbow trout $\geq 356 \mathrm{~mm}$ in total length in sections 1, 2 and 3, Bighorn River, March 1985 April 1987 . . . . . . . . . . . . . . 273

2.3.76. Mean weekly discharge (cfs) of the Bighorn River below Afterbay Dam measured at the st. Xavier gagehouse from 1985-88............. . 275 
2.3.77. Bouck gasometer measurements of delta $p$ and the trout cardiovascular threshold for gas bubble trauma in section 1, Bighorn River, October 1985 -

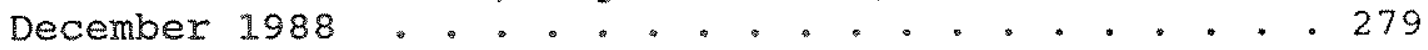

2.3.78. Bouck gasometer measurements of delta $\mathrm{P}$ and the trout cardiovascular threshold for gas bubble trauma in section 2, Bighorn River, October 1985 December 1988

2.3.79. Bouck gasometer measurements of delta $\mathrm{P}$ and the trout cardiovascular threshold for gas bubble trauma in section 3, Bighorn River, october 1985 December 1988

2.3.80. Threshold TGP for bubble formation in the cardiovascular system for trout in section 3 at the watex surface, $0.5 \mathrm{~m}$, and $1.0 \mathrm{~m}$ below the surface during 1987 ............... . 284

2.3.81. Relationship between delta $\mathrm{P}, \mathrm{pO}_{2}$, and cardiovascular (arterial DP), skin blister (water DP) and swimbladder thresholds, January 1987 . . . . 288

2.3.82. Relationship between percent of trout with GBT, delta $\mathrm{P}, \mathrm{pO}_{2}$, and cardiovascular (arterial DP), skin bilister (water DP), and swimbladder thresholds, February 1987 . . . . . . . . . . 289

2.3.83. Relationship between percent of trout with GBT, delta $\mathrm{P}_{8} \mathrm{pO}_{2}$, and cardiovascular (arterial DP), skin blister (water DP), and swimbladder thresholds, March 1987 . . . . . . . . . . . . 291

2.3.84. Relationship between percent of trout with GBT, delta $\mathrm{P}, \mathrm{pO}_{2}$, and cardiovascular (arterial DP), skin blister (water DP), and swimbladder thresholds, April 1987 . . . . . . . . . . 292

2.3.85. Relationship between percent of trout with GBT, delta $P, \mathrm{pO}_{2}$, and cardiovascular (arterial DP), skin blister (water DP), and swimbladder thresholds, May 1987 . . . . . . . . . . . . . 294

2.3.86. Relationship between delta $P, \mathrm{pO}_{2}$, and cardiovascular (arterial DP), skin blister (water DP), and swimbladder thresholds, June 1987 . . . . 295

2.3.87. Relationship between percent of trout with GBT, delta $\mathrm{P}, \mathrm{pO}_{2}$, and cardiovascular (arterial DP), skin blister (water DP), and swimbladder thresholds, July 1987

2.3.88. Relationship between delta $\mathrm{P}, \mathrm{pO}_{2}$, and cardiovascular (arterial DP), skin blister (water DP), and swimbladder thresholds, August 1987 . . . 298 
2.3.89. Relationship between percent of trout with GBT, delta $\mathrm{P}, \mathrm{pO}_{2}$, and cardiovascular (arterial DP), skin blister (water DP), and swimbladder thresholds, september 1987 . . . . . . . . . . . 299

2.3.90. Relationship between delta $\mathrm{p}, \mathrm{pO}_{2}$, and cardiovascular (arterial DP), skin blister (water DP), and swimbladder thresholds, October 1987 . . 301

2.3.91. Relationship between percent of trout with GBT, delta $\mathrm{P}, \mathrm{pO}_{2}$, and cardiovascular (arterial DP), skin blister (water DP), and swimbladder

thresholds, November 1987 . . . . . . . . . . 302

2.3.92. Relationship between percent of trout with GBT, delta $\mathrm{P}, \mathrm{pO}_{2}$, and cardiovascular (arterial DP), skin blister (water DP), and swimbladder thresholds, December 1987 . . . . . . . . . . . 304

2.3.93. Relationship between delta $\mathrm{P}, \mathrm{pO}_{2}$, and cardiovascular (arterial DP), skin blister (water DP), and swimbladder thresholds, January 1988 . . 306

2.3.94. Relationship between percent of trout with GBT, delta $\mathrm{P}, \mathrm{pO}_{2}$, and cardiovascular (arterial DP), skin blister (water DP), and swimbladder thresholds, February 1988 . . . . . . . . . 307

2.3.95. Relationship between percent of trout with GBT, delta $\mathrm{P}, \mathrm{pO}_{2}$, and cardiovascular (arterial DP), skin blister (water DP), and swimbladder thresholds, March 1988 . . . . . . . . . . . 308

2.3.96. Relationship between percent of trout with GBT, delta $\mathrm{P}, \mathrm{pO}_{2}$, and cardiovascular (arterial DP), skin blister (water DP), and swimbladder

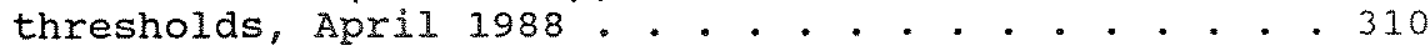

2.3.97. Dissolved gas and bubble growth thresholds calculated from Bouck gasometer and Winkler dissolved oxygen data. $\mathrm{CV}=$ cardiovascular threshold, $\mathrm{SB}=$ swimbladder threshold; $\mathrm{EW}=\mathrm{skin}$ blister threshold ............... . . 312

2.3.98. Mean GBT symptom rating and percent of rainbow trout affected when bubble growth thresholds calculated from Bouck and winkler data are exceeded.

2.3.99. Mean GBT symptom rating and percent of brown trout affected when bubble growth thresholds calculated from Bouck and winkler data are exceeded ....................... . 314 
2.3.100. Required compensation depths at Rkm 0.6 to avoid exceeding cardiovascular and skin blister

thresholds calculated from Bouck and Winkler data . 315

2.4.1. Flow diagram for tests to determine relative

vulnerability to predation between fish exposed to

gas supersaturated water and unexposed fish . . . 327

2.4.2. Artificial study section used in the second series

of predation tests May 1-21, 1987 . . . . . . . 330

2.4.3. Apparatus and water level system used to induce

external symptoms of GBT in rainbow trout for

predation tests May 16-21, 1987 . . . . . . . . 332

2.4.4. Ratio of instantaneous predation rates (dp) by

rainbow trout predators on juvenile brown trout

exposed 118\% gas supersaturated water for $4-7 \mathrm{~d}$

and control fish (dp > 1 signifies greater

predation on brown trout exposed to gas

supersaturation).............. . 340

2.4.5. Number of rainbow trout and brown trout counted in section 1 snorkel lanes, 26 May - 17 October 1987 , Bighorn River, Montana.... . . . . . . . 347

2.4.6. Number of rainbow trout and brown trout counted in section 2 snorkel lanes, 26 May - 17 october 1987, Bighorn River, Montana.... . . . . . . . . 348

2.4.7. Number of rainbow trout and brown trout in section 1 snorkel lanes, 30 January - 23 November 1988, Bighorn River, Montana............ . 350

2.4.8. Number of rainbow trout and brown trout counted in section 2 snorkel lanes, 30 January - 18 september 1988, Bighorn River, Montana..... . . . . . 351

2.4.9. Percentage of total rainbow trout and brown trout counted in midale lane of snorkel sections 1 and 2, 1987, Bighorn River, Montana . . . . . . . . 354

2.4.10. Percentage of total rainbow trout and brown trout counted in middle lane of snorkel sections 1 and 2, 1988, Bighorn River, Montana ... . . . . . 355

2.4.11. Principal component I versus principal component II for brown trout and rainbow trout of ali size groups using the variables water depth, fish depth, substrate, vegetation height, velocity, and cover, June - October 1987, Bighorn River, Montana $(0=$ brown trout, 1 =rainbow trout) . . . . . . . 367 
2.4.12. Principal component $I$ versus principal component II for brown trout and rainbow trout of all size groups using the variables water depth, fish depth, substrate, vegetation height, velocity, and cover, June - October 1987, Bighorn River, Montana (1=size group $1,<15 \mathrm{~cm} T L ; 2=$ size group 2, 15-40 $\mathrm{cm} \mathrm{TL}$; 3=size group 3, >40 cm TL) . . . . . . . 369

2.4.13. Principal component I versus principal component II for brown trout and rainbow trout $<15 \mathrm{~cm} \mathrm{TL}$ using the variables water depth, fish depth, substrate, vegetation height, velocity, and cover, June - October 1987, Bighorn River, Montana ( 0 =brown trout, 1 =rainbow trout) ........ . 370

2.4.14. Principal component I versus principal component II for brown trout and rainbow trout $>15 \mathrm{~cm}$ TL using the vaxiables water depth, fish depth, substrate, vegetation height, velocity, and cover, June 1987, Bighorn River, Montana ( $0=$ brown trout, $1=$ rainbow trout) .............. . 372

2.4.15. Principal component I versus principal component II for brown trout and rainbow trout $>15 \mathrm{~cm} \mathrm{TL}$ using the variables water depth, fish depth, substrate, vegetation height, velocity, and cover, July 1987, Bighorn River, Montana (0=brown trout, $1=$ rainbow trout) .............. . 374

2.4.16. Principal component I versus principal component II for brown trout and rainbow trout $>15 \mathrm{~cm}$ TL using the variables water depth, fish depth, substrate, vegetation height, velocity, and cover, September - october 1987, Bighorn River, Montana ( 0 =brown trout, $1=$ rainbow trout) . . . . . . . 376

2.4.17. Delta $\mathrm{P}$ levels (mmHg) measured at satelite station Rkm 0.6 showing three different gas regimes created by changes in Afterbay Dam operation, 5 August - 11 september 1987, Bighorn River, Montana... . . . . . . . . . . . . 384

2.4.18. Discharge $\left(\mathrm{m}^{3} / \mathrm{s}\right)$ during 1987 radio telemetry study, 5 August - 11 september 1987, Bighorn River, Montana... . . . . . . . . . . 385

2.4.19. Delta $\mathrm{P}$ levels (mmig) measured at Rkm 0.6 showing five periods of dissolved gas levels created by changes in Afterbay Dam operation, $18 \mathrm{July}-29$ July 1988, Bighorn River, Montana . . . . . . . 386

2.4.20. Discharge $\left(\mathrm{m}^{3} / \mathrm{s}\right)$ during 1988 radio telemetry study, 18 July - 29 July 1987, Bighorn River, Montana 
2.4.21. Fish depth determined from pressure-sensitive radio transmitters, 19 July - 28 July 1988, Bighorn River, Montana (bars represent confidence interval for depth from measured pulse repetition). Fish 280 , rainbow trout; fish 270 and 255 , brown trout.............. . 391

2.4.22. Fish depth determined from pressure-sensitive radio transmitters at delta $P$ levels encountered during monitoring period, 19 July - 28 July 1988, Bighorn River, Montana (bars represent confidence interval for depth from measured pulse repetition). Fish 280, rainbow trout; fish 270 and 255 , brown trout............. . . 392

2.4.23. Fish depth determined from pressure-sensitive radio transmitters at total gas pressures encountered during monitoring period, 19 July -28 July 1988, Bighorn River, Montana (bars represent confidence interval for depth from measured pulse repetition). Fish 280 , rainbow trout; fish 270 and 255 , brown trout............. . . 393

2.4.24. Fish depth determined from pressure-sensitive radio transmitters at light levels recorded at time of observation, $19 \mathrm{July}-28 \mathrm{July} 1988$, Bighorn River, Montana (bars represent confidence interval for depth from measured pulse repetition). Fish 280, rainbow trout; fish 270 and 255 , brown trout............. . . 394

2.4.25. Fish depth determined from pressure-sensitive radio transmitters at various times of day, data combined from 19 July - 28 July 1988, Bighorn River, Montana (bars represent confidence interval for depth from measured pulse repetition). Fish 280, rainbow trout: fish 270 and 255 , brown trout . 395

2.4.26. Distance traveled per day by radio-tagged trout, 8 August - 11 September 1987, Bighorn River, Montana (each point is an individual trout, positive distance represents movement upstream, negative distance represents movement downstream) . 397

2.4.27. Daily range (m) of fish 270 (brown trout), 19 July - 28 July 1988, Bighorn River, Montana . . . 398

2.4.28. Daily range ( $m$ ) of fish 280 (rainbow trout), 19 July - 28 July 1988, Bighorn River, Montana . . . 399

2.4.29. Daily range (m) of fish 255 (brown trout), 19 July - 28 July 1988, Bighorn River, Montana . . . 401

2.6.1. Bighorn River study area, illustrating channel characteristics at invertebrate collection sites

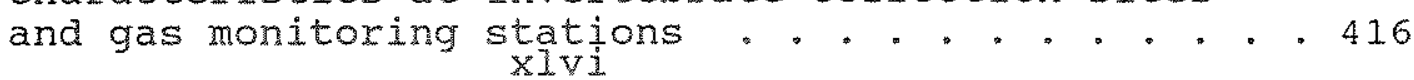


2.6.2. Invertebrate collection sites illustrating how riffles were divided into subsections in April 1987

2.6.3. Gas supersaturation system used in aquatic invertebrate bioassay tests . . . . . . . . . . . 422

2.6.4. Principal components (PC) of vectors 1 and 2 , generated from benthic samples collected at Rkm 2.4 and 14.5 during all sampling periods. . . . . 430

2.6.5. Principal components (PC) of vectors 1 and 3 , generated from benthic samples collected at $\mathrm{Rkm}$ 2.4 and 14.5 during all sampling periods . . . . . 431

2.6.6. Mean percent of total invertebrate abundance comprised by the nine most common taxa, at Rkm 2.4 and 14.5, Bighorn River, August 1986 . . . . . . . 438

2.6.7. Mean percent of total invertebrate abundance comprised by the five most common taxa, at Rkm 2.4 and 14.5, Bighorn River, September 1987 . . . . . 440

2.6.8. Mean percent of total invertebrate abundance comprised by the five most common taxa, at Rkm 2.4 and 14.5, Bighorn River, April 1987 . . . . . . . 441

2.6.9. Mean percent of Baetis tricaudatis, Chironomidae larvae and pupae, and Simulium larvae collected at $\mathrm{Rkm} 14.5$, in benthic and drift samples, Bighorn River, April 1987

2.6.10. Mean percent of nine taxa collected at Rkm 14.5 in benthic and drift samples, Bighorn River, september 1987

2.6.11. Mean weekly, Bighorn River water temperatures $\left({ }^{\circ} \mathrm{C}\right)$ at $\mathrm{Rkm} 2.4$ and $14.5,1986 . .^{\circ} \cdot$. $^{\circ} \cdot$. $^{\circ} 450$

2.6.12. Mean weekly Bighorn River water temperatures $\left({ }^{\circ} \mathrm{C}\right)$ at $\mathrm{Rkm} \mathrm{2.4}$ and $14.5,1987$. . . . . . . . . . 450

2.6.13. Delta $P$ levels (mmHg) measured two or three times weekly on the Bighorn River, at Rkm 2.4 and 14.5 , from January 1 through June 30 (A) and from July 1 through December 31 (B) 1986 . . . . . . . . 452

2.6.14. Mean monthly delta $\mathrm{p}$ levels (mmHg) at $\mathrm{Rkm} 2.4$ and 14.5, Bighorn River, 1986 . . . . . . . . . . 453

2.6.15. Delta $\mathrm{P}$ levels (mmig) measured two or three times weekly on the Bighorn river, at $\mathrm{Rkm} 2.4$ and 14.5 , from January 1 through June 30 (A) and from July 1 through December 31 (B), 1987 
2.6.16. Mean monthly delta $p$ levels (mmig) at $R \mathrm{~km} 2.4$ and 14.5, Bighorn River, 1987 . . . . . . . . . 455

2.6.17. Percent of mean monthly delta $P$ levels comprised of oxygen at Rkm 2.4 and 14.5, Bighorn River, 1986

2.6.18. percent of mean monthly delta $p$ levels comprised of oxygen at Rkm 2.4 and 14.5, Bighorn River, 1987

2.6.19. Comparison of mean delta p levels (mmHg) in all test tanks during bioassays 1 and $2 .{ }^{\circ} .{ }^{\circ} . .^{\circ} .460$

2.6.20. Susceptibility of four taxa to stranding at six delta $\mathrm{p}$ levels used in bioassay \#1. $N=20$. . . . 462

2.6.21. Gas-related mortalities for four taxa tested at six delta $P$ levels during bioassay \#1. $N=20 . .4463$

2.6.22. Susceptibility of four taxa to stranding at six delta $P$ levels used in bioassay $\# 2 . N=20$. . . 468

2.6.23. Gas-related mortalities for four taxa tested at six delta $p$ levels during bioassay \#2. $N=20$. . . 469

2.6.24. Cumulative mortality by day among four groups of longnose dace during bioassays at Fort Smith, Montana in 1988. No mortality occurred in groups 1 and 2. Mean delta $P$ for groups $1,2,3$ and 4 were $-33,104,163$, and $204 \mathrm{mmHg}$, respectively . . 491

2.6.25. Hyperbaric gas pressures (mmg) which each group of longnose dace were exposed to during bioassays at Fort Smith, Montana, September and October 1988

2.6.26. Oxygen pressures (mmg) which each group of longnose dace was exposed to during bioassays at Fort Smith, Montana, September and October 1988

2.6.27. Nitrogen and argon gas pressures (mmH) which each group of longnose dace was exposed to during bioassays at Fort Smith, Montana, September and October 1988

2.6.28. Trout cardiovascular (CV) system GBT threshold delta $P$ applied to longnose dace groups 1 and 2 compared to the delta $p$ that group 2 was exposed to during bioassay work at Fort Smith, Montana, September and octobex 1988. Group 1 delta $P$ remained hypobaric. (The threshold assumes no hydrostatic compensation) 
2.6.29. Trout cardiovascular (CV) system GBT threshold delta $p$ applied to longnose dace groups 3 and 4 compared to the delta $P^{\prime}$ s measured during bioassay work at Fort Smith, Montana, september and october 1988. (The threshold assumes no hydrostatic compensation)

2.6.30. Cumulative daily mortality in two groups of mottled sculpin held near the right bank at Rkm 0.6 (gagehouse) on the Bighorn River, Montana, February - May 1988 . . . . . . . . . . . .

2.6.31. The maximum cardiovascular system (CV) (rainbow trout) and environmental water thresholds (EW) for bubble growth and delta P's (common sensing) at $\mathrm{Rkm} 0.6$ on the Bighorn River in 1988 for days $0-30$ (thresholds incorporate hydrostatic compensation) 499

2.6.32. The maximum cardiovascular system (CV) (rainbow trout) and environmental water thresholds (EW) for bubble growth and delta P's (common sensing) at $\mathrm{Rkm} 0.6$ on the Bighorn River in 1988 for days 3060 (thresholds incorporate hydrostatic compensation

2.6.33. The maximum cardiovascular system (CV) (rainbow trout) and environmental water thresholds (EW) for bubble growth and the delta P's (common sensing) at $\mathrm{Rkm} 0.6$ on the Bighorn River in 1988 for days 60-72 (thresholds incorporate hydrostatic compensation)

2.7.1. Mean weekly discharge (cfs) of the Bighorn River below Afterbay Dam measured at the St. Xavier gagehouse from 1985-88. . . . . . . . . . 506

2.7.2. Delta $P$, oxygen pressure (mmH), and sluiceway discharge (cfs) measured on the right bank (Rkm 0.6 ) on 17 october 1985 (top) and the relationship between delta $\mathrm{P}$ and discharge from the sluiceway (bottom) ............... . . 511

2.7.3. Calculated sluiceway and observed river discharge (A), delta $\mathrm{P}$ and oxygen pressures (mmHg) (B), $\mathrm{N}_{2}+$ Ar pressures (C), sIuicegate openings (D), and the relationship between delta $\mathrm{P}$ and total sluiceway discharge (E) on 15 october 1986. Water temperature $=14.2 \mathrm{C} . . . . . . . . . .513$

2.7.4. Delta $P(A)$, oxygen pressure (mmHg) (B), $\mathrm{N}_{2}+\mathrm{Ar}$ pressure (mmig) (C), and river-Afterbay Reservoir water elevations measured at $\mathrm{Rkm} 0.6$ using models TBO and TGO Common sensing tensionometers on the Bighorn River, 9 July 1986 . . . . . . . . . . 517 
2.7.5. Delta $P$ (muHg) (A), oxygen (OP) and $\mathrm{N}_{2}+A \mathrm{Ar}$ (NP) pressure (B) at Rkm 0.6 and 4.8 , river discharge (C), water temperature (D), mean daily Afterbay Dam gate openings (E), and Afterbay Reservoir river water elevations (F) on the Bighorn River from 6 August - 4 September 1987.......... 519

2.7.6. Delta $\mathrm{P}$ and barometric pressure (mmig) (A), oxygen and $\mathrm{N}_{2}+\mathrm{Ar}$ pressure (mmig) (B), river discharge and water temperature (C), mean daily Afterbay Dam gate openings (ft) (D), and Afterbay Reservoir - river water elevations (ft) (E) at Rkm 0.6 on the Bighorn Rivex from 25-31 July 1988 . . . . . . 524

2.7.7. Downriver survey of delta $P(A)$ and oxygen and nitrogen-argon pressures (B) on the Bighorn River downstream from Afterbay Dam, 18 July 1986.

$(\mathrm{Q}=6262 \mathrm{cfs}$ : sluiceway gates closed, opening of radial gates $1,3,+5=3.0$ ft, mean opening of radial gates $2+4=2.44 \mathrm{ft}$, day $=$ primarily overcast)

2.7.8. Downriver survey of delta $P$ (mmHg) on 16 August (A), and 22 August 1987 (B), and the corresponding oxygen and $\mathrm{N}_{2}+\mathrm{Ar}$ pressure (mmig) (C\&D) in the Bighom River downstream from Afterbay Dam in 1987

2.7.9. Cross-river measuments of delta $P$ at $R \mathrm{~km} 0.6$ on 31 July, 4 September, and 14 October 1986 . . . . 533

2.7.10. Cross-river transects of delta $P$ (mmHg) at $\mathrm{Rkm}$ 0.6 (A), Rkm 4.8 (C), and Rkm 14.9 (E), and oxygen and $\mathrm{N}_{2}+$ Ar pressures (mmHg) at Rkm 0.6 (B), Rkm 4.8 (D), and Rkm 14.9 (F) ............ 538

2.7.11. Mean daily opening of sluiceway gates (A \& B), spillway radial gates (C\& D) on Afterbay Dam, and Afterbay Reservoir and river elevations (ft) ( $E$ \&

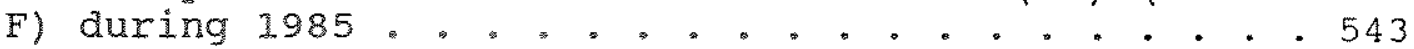

2.7.12. Mean daily opening of sluiceway gates (A \& B), spillway radial gates (C\& D) on Afterbay Dam, and Afterbay Reservoir and river elevations (ft) (E \& F) during 1986................... 544

2.7.13. Mean daily opening of sluiceway gates $(A \& B)$, spillway radial gates (C $\&$ D) on Afterbay Dam, and Afterbay Reservoir and river elevations (ft) ( $E$ \& F) during $1987 . . . . . . . . . . . . . . . .5545$

2.7.14. Mean da11y opening of sluiceway gates (A), spillway radial gates (B) on Afterbay Dam, and Afterbay Reservoir and river elevations (ft) (C) $r m 6^{\prime \prime}$ during the first half of 1988 ......... . . 546 
2.7.15. Uncorrected mean daily delta $\mathrm{P}$ (mmHg) measured at Rkm 0.6 (gagehouse) by the Common Sensing

tensionometer from 1985-1987 (Hydromet archive

files data) ............... . 550

2.7.16. The frequency of occurrence of Afterbay Reservoir minimum elevations from 30 March 1985 - 5 October 1988. Arrow designates elevation below which spillway discharge is not possible . . . . . . . 552 


\subsection{INTRODUCTION}

Gas Bubble Trauma (GBT) is a condition that arises in fish and other aquatic organisms when water becomes supersaturated with dissolved atmospheric gases. The condition can produce a variety of physiological symptoms that are often fatal. The major symptoms that characterize GBT in fish include:

1.) Bubble formation in the cardiovascular system.

2.) Overinflation of the swim bladder, intestinal and peritoneal cavities.

3.) Sub-dermal emphysema on body surfaces including the lining of the mouth.

4.) Extracorporeal bubble formation in gill lamella.

5.) Emphysema in muscle, internal organs and the spinal cord.

6.) Exopthalmia or pop-eye.

In general, these symptoms involve the growth of gas bubbles in one form or another, both internal and external to the animal. The activation of symptoms, however, is not an easily demonstrated cause and effect relationship. This is because internally, bubbles can grow in all body compartments and produce disruptions of neurological, cardiovascular, respiratory, osmoregulatory and other physiological functions (Weitkamp and Katz 1980; Stroud et al. 1975). Externally, they can block the flow of respiratory water through the gills (Shirahata 1966 and Jensen 1980). Bubbles may also increase susceptibility of fish to other stresses such as bacterial, viral and fungal infections (Weitkamp 1976; Nebeker et al. 1976; Sniesko 1974; Meekin and 
Turner 1974). Although not demonstrated, it is probable that most internal and external symptoms weaken fish, especially larval and juvenile life stages, and increase their susceptibility to predation.

\subsection{Dissolved Gas supersaturation}

Natural bodies of water contain dissolved atmospheric gases that are usually in equilibrium with the atmosphere. That is, the partial pressures of the dissolved gas components (oxygen, nitrogen, water vapor, argon, etc.) are the same as the partial pressures of their respective atmospheric components. occasionally, due to human and natural causes, the dissolved gases are thrown into a state of disequilibrium with respect to atmospheric gases. When this disequilibrium involves a dissolved gas partial pressure which exceeds that of the atmospheric component, water is supersaturated with that dissolved gas. This in itself may not create a problem for aquatic organisms. However, a unique condition arises when the sum of the partial pressures of all dissolved gases, (i.e. total dissolved gas pressure, henceforth referred to as Total Gas Pressure or TGP) exceeds atmospheric pressure. When this occurs, there is the potential for dissolved gases to diffuse into microscopic nucleation sites or hollow cavities and form bubbles (Harvey 1951; Hills 1977; Weitkamp and Katz 1980; Fidler 1985). These bubbles can form in both the environmental water and within the organisms that inhabit that water.

Expressed in a mathematical form, the necessary condition for bubble growth and overinflation of body cavities is: 


$$
\begin{aligned}
& \mathrm{TGP}=\mathrm{pO}_{2}+\mathrm{pN}_{2}+\mathrm{pH}_{2} \mathrm{O}+\mathrm{petc}_{\mathrm{e}}>\mathrm{P}_{\text {atms }} \\
& \text { TGP = total gas pressure } \\
& \mathrm{pO}_{2}=\text { the partial pressure of dissolved oxygen. } \\
& \mathrm{pN}_{2}=\text { the partial pressure of dissolved nitrogen. } \\
& \mathrm{pH}_{2} \mathrm{O}=\text { the partial pressure of water vapor. } \\
& \text { petc = the partial pressure of all other dissolved gases. } \\
& \text { Patms = atmospheric pressure. }
\end{aligned}
$$

Alternatively, this relationship can be expressed in terms of the difference between the total gas pressure and atmospheric pressure or delta P (DP). The necessary condition for bubble growth is then:

$$
\mathrm{DP}=\left(\mathrm{TGP}-\mathrm{P}_{\mathrm{atm}}\right)>0
$$

Perhaps the most widely known form of supersaturation is that produced by hydroelectric dams. Water spilling over dams entrains air as it plunges into pools at the base of the dam. The air, in the form of bubbles, is forced into solution under hydrostatic pressure and increases water dissolved gas tensions. Coutant and Genoway (1968), Beiningen and Ebel (1970), Bouck et al. (1970), Boyer (1974), Dell (1975), Meekin and Turner (1974), Dawley et al. (1976), Ebel (1969, 1971\& 1979), Ebel et al. (1971), Ebel et al. (1973), Ebel et al. (1975), Ebel et al. (1979), Blahm et al. (1973), Blahm et al. (1975), Stroud and Nebeker (1976) and Weitkamp (1974\& 1976) report the effects of this form of supersaturation on $f i s h$ in the columbia River system of the United States. Supersaturation resulting from other dams and hydroelectric installations are described by colt (1984), Berg (1984), Heggberget (1984) and Alderdice and Jensen (1985b). 


\subsection{The Bighorn River and the Yellowtail Afterbay Dam}

The Yellowtail Afterbay Dam on the Bighorn River of Montana has, for many years, produced supersaturated levels of dissolved gases. Accompanying this supersaturation has been high incidence of symptoms of GBT in the rainbow and brown trout populations of the river. The Afterbay Dam serves as a reregulating facility below Yellowtail Dam to provide uniform daily discharges into the Bighorn River. The presence of GBT in trout below the Afterbay Dam was first documented in 1973 (Swedberg 1973). The Bureau of Reclamation and Montana Department of Fish, Wildlife and Parks (MDFWP) subsequently concluded that gas entrainment occurs when water passes through gates in the Afterbay Dam, particularly the sluiceway gates (Bureau of Reclamation 1973). However, they surmised that no practical modifications in the Afterbay operation would preclude gas levels from exceeding $110 \%$ criterion for total dissolved gases to protect freshwater aquatic life (EPA 1976).

A fish kill observed in 1979 was considered to be the result of dissolved gas supersaturation (Porter and viel 1980). Subsequent studies by the U. S. Fish and Wildlife Service demonstrated the longitudinal extent and biological severity of the problem. From February-August 1981,33 and $11 \%$ of the brown and rainbow trout, respectively, exhibited external symptoms of GBT (Curry and Curry 1981). GBT in brown trout during 1981 peaked in June when $72 \%$ of those captured were affected (Curry and Curry 1981). Most (90\%) of the traumatized fish were found in the first 8 river kilometers ( $\mathrm{rkm}$ ) below Afterbay dam (Porter and Viel 1980). 
In fall 1982, the Bureau of Reclamation attempted to solve the problem by installing deflector plates (flip lips) on the face of the dam. However, turbulence resulting from these structures caused rocks to be pulled into the afterbay stilling basin and threatened to erode the base of the dam. Consequently, the flip lips were removed in July 1983.

More recent monitoring by the MDFWP confirmed that the problem persists and that large brown trout, those $>356 \mathrm{~mm}(14.0$ inches), are the most visibly affected segment of the population and that brown trout are more susceptible to GBT than rainbow trout. Additionally, poor reproductive success of rainbow trout in the mainstem Bighorn River may be due to dissolved gas supersaturation (Fredenberg 1985). Based on available data, there was no clear understanding of how reservoir operations, flows and other ambient conditions affected dissolved gas levels and the incidence of GBT in fishes. Furthermore, it was not known why adult brown trout exhibit more severe external symptoms of GBT than adult rainbow trout, nor why rainbow trout recruitment was suppressed.

\subsection{Relevant Literature}

The definitive, although slightly dated, review of GBT in fish is that of Weitkamp and Katz (1980). Their review examines almost 200 papers and describes in detail the many symptoms of GBT. In most cases, the conditions that produce the symptoms are also specified. The more recent review by colt et al. (1986) adds further to the understanding of GBT, its symptoms, causes and treatment. 
Gas bubble trauma research has focused on the identification of symptoms and time to mortality as the primary response of fish to supersaturation (Blahm et al. 1973; Blahm et al. 1975; Dawley and Ebel 1975; Dawley et al. 1976; Knittel et al. 1980; Meekin and Turner 1974; Nebeker and Brett 1976; Nebeker et al. 1979; Nebeker et al. 1978; Rucker 1974; Jensen et al. 1985; Stroud et al. 1975; Weitkamp 1976). There have been few attempts to identify causes of mortality or lethal thresholds. Stroud and Nebeker (1976), stroud et al. (1975) and Meekin and Turner (1974) provide a correlation of various symptoms of GBT with water TGP and time to mortality. Knittel et al. (1980), studying steelhead trout, offer the only description of a threshold for mortality. Many studies of gas supersaturation have been conducted on the Columbia River system in the United states. But little has been done to increase our understanding of the relationship between the physical causes of GBT and the physiological effects. For example, it is not known which symptoms lead to observed mortalities. Bubble growth in the vascular system is often cited as a cause (Weitkamp and Katz 1980), yet other symptoms, such as extracorporeal bubbles in the gills and sub-dermal bubbles on the skin and in the mouth, are often present at the same time (stroud and Nebeker 1976; stroud et al. 1975; Meekin and Turner 1974). It is not clear whether the various symptoms act in concert or at different levels of water TGP (or DP). It is also unknown how other factors, such as $\mathrm{pO}_{2}$, affect the onset of GBT. We do not understand the consequences of repeated exposure to dissolved gas supersaturation, a topic relevant to the Bighorn River, nor is it known how long it takes fish to recover from various levels and 
durations of exposure to dissolved gas supersaturation.

There has been no attempt to determine if fish behavior or habitat selection affects susceptibility to GBT. For example, we do not know if fish that prefer deeper water survive better than fish that inhabit shallow water. Nor has it been conclusively established whether fish seek deeper water to avoid dissolved gas supersaturation. Dissolved gas supersaturation is known to affect aquatic invertebrate communities (Nebeker 1976; Fickeisen and Montgomery 1975). This could either increase or decrease available food for fish. High supersaturation could kill invertebrates or simply increase their buoyancy, causing them to drift more readily, thus making them more susceptible to predation.

The only significant attempt to relate mortality data to water parameters, species and fish size is that of Jensen et al. (1986). In this work, data records from the literature [that include water $\mathrm{TGP}, \mathrm{pO}_{2}$, time to $50 \%$ mortality and other parameters (Jensen et al. 1985)] were incorporated into a generalized surface response analysis (Schnute and McKinnell 1984) in which the authors assume there is a single cause of mortality in fish exposed to supersaturated water. Unfortunately, these workers did not consider that different GBT symptoms may have their own lethal thresholds. Furthermore, these thresholds may be mediated by physical parameters (e.g. TGP or $\mathrm{pO}_{2}$ ) and expressed differently depending on fish size or species.

Fidler (1985) derived equations that describe thresholds for various forms of bubble growth in fish exposed to supersaturated 
water. The resulting equations contain physical parameters related to the environmental water that include TGP, $\mathrm{po}_{2}$, temperature and depth. Other physical parameters in the equations account for barometric pressure, the vapor pressure of water and the surface tensions of water and $\mathrm{fish}$ blood.

Physiological parameters include the ratio of the partial pressure of oxygen in blood to the partial pressure of oxygen in the environmental water, the system pressure where bubble growth occurs and the size of nucleation sites from which bubble growth begins. The equations were originally derived to describe the growth of bubbles in the cardiovascular systems of fish. A slight modification to the equations allowed them to be applied to the problem of swimbladder overinflation in fish. A further simplification of the equations yielded a description of thresholds for bubble growth in the environmental water. Unknowns in the equations include the size of nucleation sites, system pressures where bubbles form, and the partial pressure of oxygen within the vascular system. It is known that fish that have died as a result of exposure to supersaturated water have bubbles in the swimbladder and cardiovascular system. However, it has not been demonstrated that thresholds for mortality are directly related to thresholds for bubble growth.

The above points out many of the gaps that existed in our knowledge of dissolved gas supersaturation and its effects on fish and other aquatic organisms. To understand and ultimately solve the problem of GBT in Bighorn River fish, it was necessary to better quantify the effects of dissolved gas supersaturation on fish. 


\subsection{Bighorn River Project objectives}

The Bighorn River and the Yellowtail Afterbay Dam are small in comparison to other western hydroelectric projects where gas supersaturation problems exist (e.g., the columbia River).

Consequently, the dam and river present a unique environment for examining the relationships between the effects of dam design and operation on dissolved gas supersaturation and GBT in aquatic organisms. In 1985, the Montana Cooperative Fisheries Research Unit, with funds from the U.S. Bureau of Reclamation, initiated this study of dissolved gas supersaturation and GBT in fish of the Bighorn River below the Yellowtail Afterbay Dam. The objectives of the study were to:

1.) Determine the important physical and physiological parameters associated with GBT in fish, and establish the relationship between these parameters and the severity of GBT symptoms.

2.) Examine the relationship between dissolved gas levels on the Bighorn River and symptoms of GBT in adult and juvenile trout.

3.) Determine the effects of behavior and habitat selection on susceptibility of trout to dissolved gas supersaturation.

4.) Assess the impact of dissolved gas supersaturation on invertebrate and forage fish communities in the Bighorn River.

5.) Determine the impact of GBT on trout catchability. 
6.) Examine potential mechanisms which might explain the higher incidence of GBT symptoms in brown as compared to rainbow trout in the Bighorn River.

7.) For various 1 ife stages of rainbow and brown trout, determine safe levels of exposure to dissolved gas supersaturation.

8.) Examine the relationship between dam operational characteristics and dissolved gas levels on the Bighorn River. 


\subsection{FIELD AND LABORATORY STUDIES}

The experiments and research performed to address each of the eight project objectives are presented in the following sections of this report. Physiology studies and mathematical modeling were performed in the Department of zoology at the University of British columbia. Field studies on the Bighorn River and laboratory studies at the U.S. Fish and Wildife Service (USFWS) Fish Technology Center at Bozeman, Montana, were conducted by the USFWS Montana Cooperative Fishery Research Unit, Biology Department, Montana state University. This research was done in cooperation with the Montana state Department of Fish, Wildlife and Parks.

\subsection{Physical and Physiological parameters Associated with GBT}

The theoretical threshold equations of Fidler (1983) could provide a practical method of predicting the occurrence of various GBT symptoms in fish based on water TGP (or DP) and the partial pressure of dissolved oxygen $\left(\mathrm{po}_{2}\right)$. Therefore, a goal of this study was to determine the unknown physiological parameters needed to complete the equations. It was also necessary to establish that these forms of bubble growth are responsible for physiological stress and mortality in trout. Thus, the study involved an examination of the physiological response of fish to supersaturation and bubble growth including a correlation between mortality and bubble growth thresholds. The work was performed in three phases: 
Phase 1) A theoretical analysis expanded the threshold equations of Fidler (1985) and improved their usefulness in terms of relating mathematical parameters to measurable physiological parameters.

Phase 2) GBT data from the literature were compiled into a database and examined for evidence of thresholds for mortality in fish exposed to dissolved gas supersaturation.

Phase 3) Laboratory studies provided a clearer definition of the physiological parameters contained in the threshold equations. Experiments also allowed us to relate physiological symptoms of stress and mortality to thresholds for mortality derived from literature data analysis and theoretically predicted bubble growth thresholds.

other work completed at the University of British Columbia, allowed us to describe the effects of dissolved gas supersaturation on the overinflation of swimbladders in both adult and juvenile fish.

\subsubsection{Phase 1 -- Theoretical studies}

The bubble growth threshold equations of Fidler (1988) are shown as Equations 1,2 and 3. Table 2.1.1 gives a definition of the terms appearing in the equations. 
EQUATION 1: Threshold criteria for Bubble Growth in the vascular system.

$\mathrm{TGP}_{\mathrm{CV}} \geq \mathrm{P}_{\mathrm{atm}}+\mathrm{Ps}+\mathrm{o} \cdot \mathrm{g} \cdot \mathrm{h}+\frac{2 \cdot \sigma}{\mathrm{r}_{\mathrm{O}}}+\mathrm{pO}_{2} \cdot(1-\mathrm{F})$

where the subscript $\mathrm{CV}$ refers to the vascular system.

EQUATION 2: Threshold criteria for overinflation of the swimbladder.

$\mathrm{TGP}_{\mathrm{SB}} \geq \mathrm{P}_{\mathrm{atm}}+\mathrm{Ps}+\mathrm{o} \cdot \mathrm{g} \cdot \mathrm{h}+\mathrm{pO}_{2} \cdot(1-\mathrm{F})$

where the subscript SB refers to the swimbladder.

EQUATION 3: Threshold criteria for Bubble Growth in Environmental water.

$\mathrm{TGP}_{\mathrm{EW}} \geq \mathrm{P}_{\mathrm{atm}}+\mathrm{PS}+0 \cdot \mathrm{g} \cdot \mathrm{h}+\frac{2 \cdot \sigma}{\mathrm{r}_{\mathrm{O}}}$

where the subscript EW refers to environmental water

Alternatively, the threshold equations can be written in terms of delta $P$ (DP). 
EQUATION 1': Threshold criteria for Bubble Growth in the vascular system.

$\mathrm{DP}_{\mathrm{CV}} \geq \mathrm{Ps}+\mathrm{o} \cdot \mathrm{g} \cdot \mathrm{h}+\frac{2 \cdot \sigma}{\mathrm{r}_{\mathrm{O}}}+\mathrm{pO}_{2} \cdot(1-\mathrm{F})$

where DP indicates the difference between TGP and barometric pressure and the subscript $\mathrm{CV}$ refers to the vascular system.

EQUATION 2': Threshold criteria for overinflation of the swimbladder.

$$
\mathrm{DP}_{\mathrm{SB}} \geq \mathrm{PS}+0 \cdot \mathrm{g} \cdot \mathrm{h}+\mathrm{pO}_{2} \cdot(1-\mathrm{F})
$$

where the subscript SB refers to the swimbladder.

EQUATION 3': Threshold criteria for Bubble Growth in Environmental water.

$\mathrm{DP}_{\mathrm{EW}} \geq \mathrm{Ps}+0 \cdot \mathrm{g} \cdot \mathrm{h}+\frac{2 \cdot \sigma}{\mathrm{r}_{\mathrm{O}}}$

where the subscript EW refers to environmental water. 
Table 2.1.1 Definition of symbols used in threshold equations.

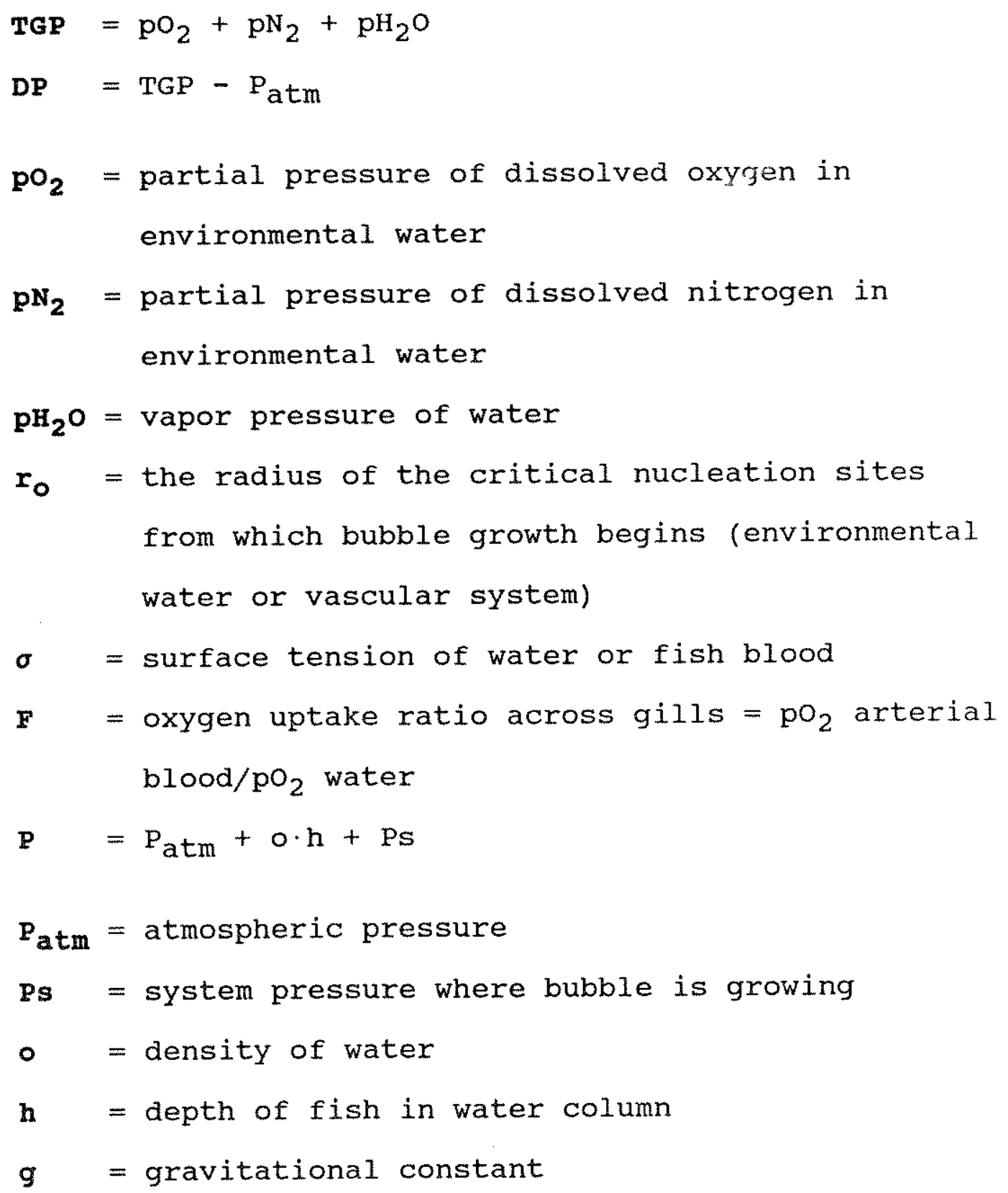


In general, the equations predict that TGP or DP thresholds for bubble growth increase with increasing water depth (h), system pressure (Ps), and barometric pressure ( $\mathrm{P}_{\text {atm }}$ ); thresholds decrease as nucleation site radius $\left(r_{0}\right)$ increases. For vascular system bubble growth and swimbladder overinflation, the system pressure (Ps) is defined as arterial blood pressure. In the case of bubble growth in the environmental water, Ps is zero. In Equations 1 and 2 (or $1^{\prime}$ and $2^{\prime}$ ), the effect of water $\mathrm{pO}_{2}$ is dependent on the oxygen uptake ratio (F) across the gill. In general, the effect of $\mathrm{pO}_{2}$ on threshold TGP diminishes as $\mathrm{F}$ increases.

Physical parameters contained in the equations (water temperature, depth, $\mathrm{pO}_{2}$ and surface tension) are either definable, measurable or are controlled for many situations in which the equations can be applied. The surface tension of water is defined as a function of temperature (Perry and chilton 1983; Reid et al. 1977). The surface tension of rainbow trout blood, at a temperature of $7^{\circ} \mathrm{C}$ was determined by Fidler (1985), and found to be close to that of water. Thus, the principal unknown parameters in the equations are physiological. These consist of the ratio of arterial $\mathrm{po}_{2}$ to water $\mathrm{po}_{2}$, the vascular system pressure where initial bubble growth begins, and the size of critical nucleation sites. To examine these parameters in more detail, the threshold equations will be considered individually.

\subsection{1.a Vascular System Bubble Growth}

Vascular system bubble growth commonly occurs in fish exposed to dissolved gas supersaturation. It is considered to be the most lethal of all GBT symptoms (Stroud et al. 1975; Bouck 
1980). Although not explicit in the threshold equation, there is a strong coupling of the nucleation site location with vascular system pressure and oxygen uptake ratio. The system pressure (Ps) and oxygen uptake ratio (F) are specifically those at the location of the nucleation site. Fidler (1985) has pointed out that the most likely location for initial intravascular bubble formation is the arterial side of the circulatory system. This is because venous levels of dissolved oxygen are on the order of 20 - $30 \mathrm{mmHg}$; whereas arterial values are 100 - $130 \mathrm{mmHg}$ (Holeton and Randall 1967a, 1967b; Randall 1970; Davis and Cameron 1971; Sovio et al. 1981; Thomas and Hughes 1982; Wood and Jackson 1980; Wood et al. 1984). Because of the lower venous $\mathrm{po}_{2}$, the effective TGP for venous blood is significantly reduced from that of arterial blood. This indicates that much higher levels of water TGP are required before the thresholds for bubble growth in venous blood are reached. Thus, the $F$ term in Equation 1 applies primarily to arterial blood.

In general, the location of nucleation sites in the vascular system is unknown. Therefore, it is not possible to define Ps and $F$ directly. Furthermore, the size of nucleation sites in physiological systems are unknown, and this leaves the $r_{0}$ term in the equation without definition.

As pointed out earlier, the threshold for intravascular bubble growth is dependent on water $\mathrm{po}_{2} \cdot$ This dependency is a result of differences between blood and water $\mathrm{po}_{2}$ caused by oxygen transport resistances at the gill (Randall 1970; Randall and Daxboeck 1984; Piiper and Scheid 1984). The ratio of blood $\mathrm{pO}_{2}$ to water $\mathrm{pO}_{2}$ has not been determined explicitly. However, 
there may be sufficient data in the literature to make reasonable estimates of this ratio at the dorsal aorta. With $\mathrm{F}$ defined at the dorsal aorta, there remains the problem of determining an appropriate value of $\mathrm{F}$ at nucleation sites.

\subsection{1.b Swimbladder overinflation}

The swimbladder of most fish is highly vascularized with arterial blood (Steen 1970). Thus, there is the potential for dissolved gases to overinflate this organ (Fidler 1985; Shrimpton et al. 1988). Overinflation of the swimbladder and other body cavities accompanied by occasional mortality are reported by Shirahata (1966); Kral (1983); Bowser et al. (1983); Cornacchia and Colt (1984); Johnson and Katavic (1984); Kolbeinshavn and Wallace (1985); and Shrimpton et al. (1988). Equation 2 was derived as a special case of Equation 1. Basically, the swimbladder acts as an extremely large nucleation site. As such, surface tension effects are small which permits the $2 \sigma / r_{0}$ term to be dropped from the general threshold equation. Equation 1 reduces to Equation 2 where the only physiological unknown is $F$, the ratio of arterial $\mathrm{pO}_{2}$ to water $\mathrm{po}_{2}$. As pointed out above, there may be enough data in the literature to define this term for arterial blood at the dorsal aorta.

An additional consideration in the effects of dissolved gas supersaturation on swimbladder overinflation is the degree to which overinflation occurs. This is important from the standpoint that an overinflated swimbladder can lead to buoyancy problems for fish and, as reported by Jensen et al. (1985), there have been observed cases of swimbladder rupture in small fish 
exposed to dissolved gas supersaturation. Fidler (1985)

hypothesized that for physostome fish, such as trout, there should be a relationship between the size of a fish and the maximum pressure attained in the swimbladder before gas is released through the pneumatic duct (a small duct connecting the swimbladder to the esophagus, enabling gas to be vented from the swimbladder). The size of the duct, coupled with surface tension forces, establish the pneumatic duct release pressure through the relationship:

$$
\mathrm{DRP}=2 \cdot \sigma / \mathrm{R}_{\mathrm{PD}}
$$

where $D R P$ is the duct release pressure and $R_{P D}$ is the effective radius of the pneumatic duct.

Thus, Fidler (1985) suggested that small fish will have a much higher duct release pressure than large fish and therefore will sustain a much higher degree of overbuoyancy. This hypothesis also implies that small fish will be more susceptible to swimbladder rupture and perhaps to predation. Shrimpton et al. (1988) experimentally examined the swimbladder overinflation threshold equation (Equation 2), and the relationship between the limits of swimbladder overinflation and fish size in rainbow trout. He also examined the behavior of fish experiencing swimbladder overinflation and their tendency to seek depth as a means of compensating for overbuoyancy (Shrimpton et al. 1989).

\subsection{1.c Water Bubbles in Environmental water}

Equation 3 is another special case of Equation 1 where, for bubble growth in the environmental water, $F$ is equal to 1.0 . 
This reduces Equation 1 to Equation 3 where the only physiological unknown is the size of nuclei from which bubble growth begins. In general, this dimension is not known for physiological systems.

Situations in which the equation can be applied include extracorporeal bubble growth in the buccal cavity or extracorporeal bubble growth between gill lamellae. In the first case, it is observed that a single bubble in the buccal cavity of larval fish can block respiratory water flow and cause death (Shirahata 1966; Jensen 1980). Extracorporeal bubbles growing between gill lamellae are observed in fish exposed to gas supersaturated water (Weitkamp and Katz 1980). However, it has not been established that these bubbles are lethal to fish.

\subsection{1.d Unknown Parameters}

Gill oxygen uptake ratio (F): Many measurements of arterial blood $\mathrm{pO}_{2}$ from the dorsal aorta of fish have been reported. In most cases water $\mathrm{pO}_{2}$ is also reported. To use this information for determining appropriate values of $F$, it was tabulated for rainbow trout (Fidler 1988). Only data obtained for resting fish were selected for this tabulation in order to limit variation in the data and to yield results applicable to the experimental phases of this study. The data were converted to $F$ values and a mean and standard deviations were calculated.

Effective Nuclei Radius $\left(r_{0}\right)$ : In Equation 1, nucleation site radius, as contained in the $2 \sigma / r_{0}$ portion of the equation, is a pressure term like Ps. Since both of these parameters are unknown and difficult to determine, the two can be combined into a 
single unknown pressure parameter. To retain surface tension explicitly in the equation, the parameters are combined in Equation 4 so that an effective radius, $R_{O}$, accounts for both $P S$ and $r_{0}$

EQUATION 4:

$\mathrm{TGP}_{\mathrm{CV}} \geq \mathrm{P}_{\mathrm{atm}}+\mathrm{o} \cdot \mathrm{g} \cdot \mathrm{h}+\frac{2 \sigma}{\mathrm{R}_{\mathrm{O}}}+\mathrm{po}_{2} \cdot(1-\mathrm{F})$

EQUATION 4 ':

$\mathrm{DP}_{\mathrm{CV}} \geq 0 \cdot \mathrm{g} \cdot \mathrm{h}+\frac{2 \cdot \sigma}{\mathrm{R}_{\mathrm{O}}}+\mathrm{po}_{2} \cdot(1-\mathrm{F})$

This combination leaves only one unknown on the right side of the equation. Thus, if a threshold for cardiovascular bubble growth can be determined for known levels of water $\mathrm{DP}$ and $\mathrm{pO}_{2}$ (or just DP in the case of Equation 3), the $R_{0}$ and $r_{0}$ terms can be back-calculated from the equations. The equations can then be completed and used to predict thresholds of bubble growth for a wide range of conditions. Experimental methods that were used to determining bubble growth thresholds are described later.

\subsection{1.e Sub-dermal Bubble Growth}

The literature offers many examples of sub-dermal bubbles or blisters growing on the external body surfaces of fish exposed to supersaturated water. These observations include bubbles on opercular flaps, between fin rays, and in the lining of the mouth (Weitkamp and Katz 1980). It is hypothesized that the threshold equations apply to these forms of bubble growth also. To select 
the appropriate equation, it is necessary to establish if dissolved gases are transported to these bubbles by blood flow through the circulatory system, or as a result of direct diffusion from the water. Kirsch and Nonnote (1977) show that oxygen transport to the skin of rainbow trout is primarily by direct diffusion from the environmental water. Furthermore, sub-dermal bubble growth appears to originate just below the epithelium tissue layer (Nebeker and Brett 1976). Therefore, it is reasonable to assume that $\mathrm{po}_{2}$ and $\mathrm{pN}_{2}$ levels are not significantly reduced from that of water and that Equation 3 is appropriate.

Aside from the dimensions of nucleation sites, the other unknown parameter is the appropriate form of surface tension. At the inception of bubble growth, growth rate is controlled by extracellular fluid surface tension forces (Vann and Clark 1975; Meisel et al. 1981; Yang and Liang 1972). The surface tension of extracellular fluid is essentially that of water (Altman and Dittmer 1961). Hence, Equations 3 or $3^{\prime}$ are used as the combined water and sub-dermal bubble growth threshold equations in subsequent discussion and analyses. The experimental validation of Equations 3 and $3^{\prime \prime}$ as threshold equations for skin blistering will be described later.

\subsection{1.f Results of Theoretical studies}

Threshold Equations: Equations 2, 3, and 4 are plotted in Figure 2.1.1 and Equations $2^{\prime}, 3^{\prime}$ and $4^{\prime}$ in Figure 2.1.2. Plots are based on an effective nuclei radius of $10 \mu \mathrm{m}$, a water depth of $0.0 \mathrm{~m}$, a water temperature of $12^{\circ} \mathrm{C}$, an $\mathrm{F}$ of 0.79 (see below) 


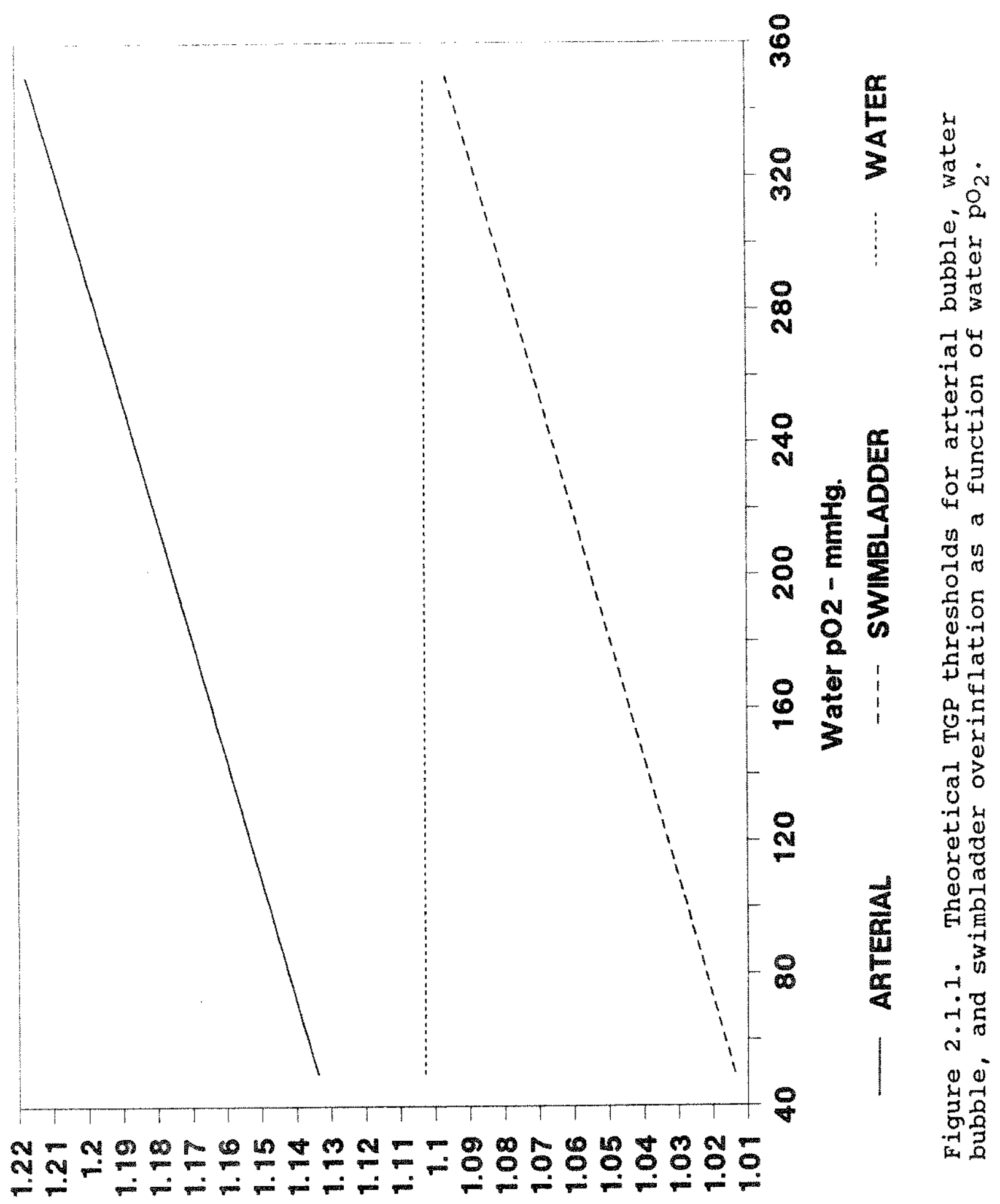

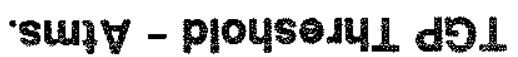




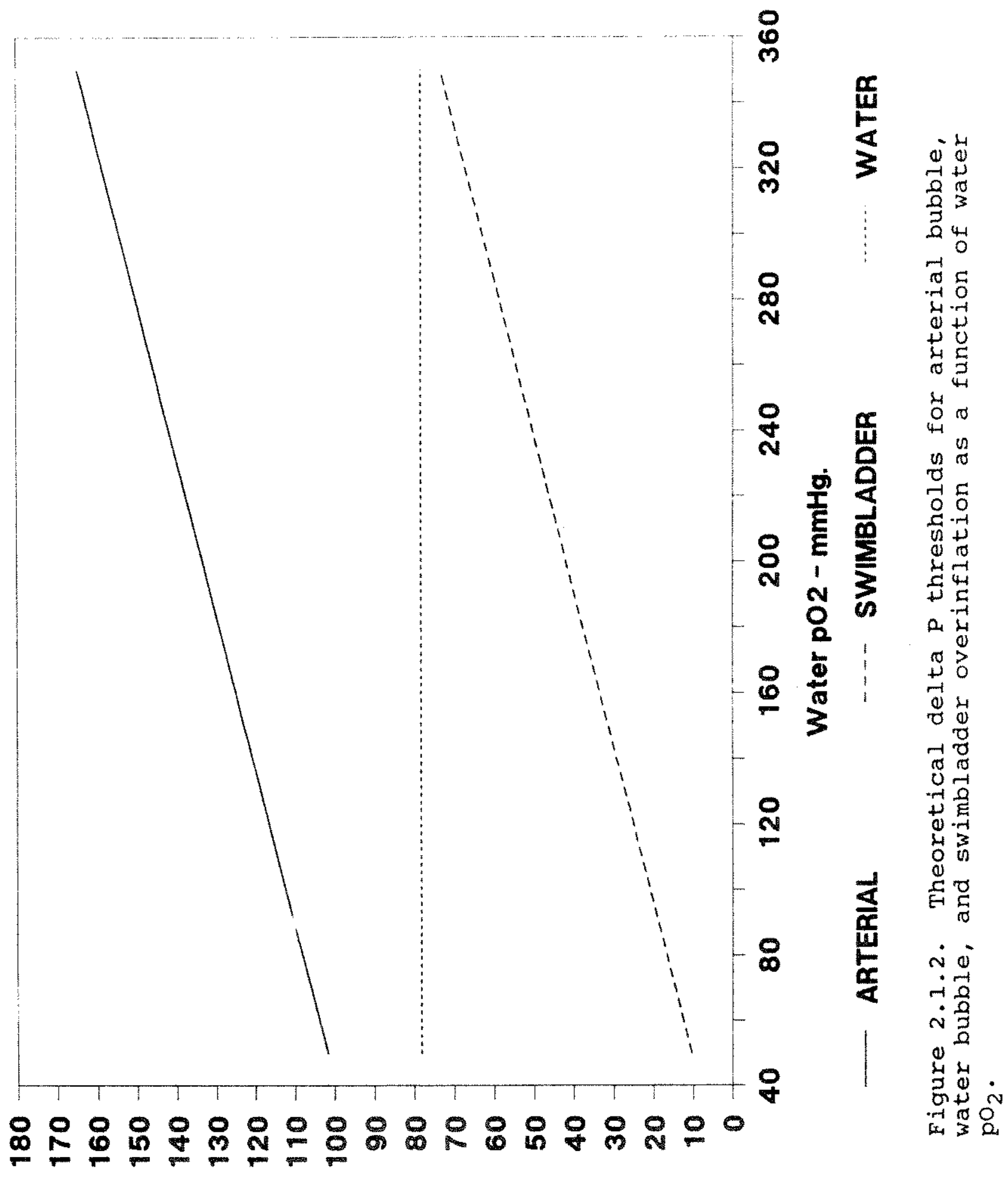

"Bнuи - ployses41 d Ej०a 
and atmospheric pressure at sea level. Rationale for the selection of $R_{0}$ will be described later. The figure clearly shows a separation of the various theoretical thresholds and the dependency of the thresholds for swimbladder overinflation and intravascular bubble growth on water $\mathrm{po}_{2}$. Interestingly, Equations 2 and 4 (or $2^{\prime}$ and $4^{\prime}$ ) now contain water $\mathrm{pO}_{2}$ as the only independent dissolved gas parameter. The other physical and physiological parameters are as in the original equations. Thus, specification of water $\mathrm{pO}_{2}$ and temperature is sufficient to define the DP thresholds for swimbladder overinflation and intravascular bubble growth. Although this may appear odd at first, it should be remembered that TGP is made up of the vapor pressure of water plus the partial pressures of dissolved oxygen and dissolved nitrogen as defined in Table 2.1.1. By specifying water $\mathrm{pO}_{2}$, water TGP is defined by the threshold equation, from which $\mathrm{pN}_{2}$ is determined by:

$$
\mathrm{pN}_{2}=\mathrm{TGP}-\mathrm{pO}_{2}-\mathrm{pH}_{2} \mathrm{O} \quad \text { Equation } 5
$$

With the specification of temperature, the vapor pressure of water is determined, and in turn, $\mathrm{pN}_{2}$. Thus, the definition of $\mathrm{pN}_{2}$ is implicit in Equations 1 through $4^{\prime}$.

Oxygen Uptake Ratio: Figure 2.1 .3 shows the gill oxygen uptake ratios (F) calculated from the data of Fidler (1988) and plotted as a function of water $\mathrm{po}_{2}$. It is clear that $\mathrm{F}$ is quite variable and that the highest variability occurs where water $\mathrm{po}_{2}$ levels are hypoxic for fish (Holeton and Randall 1967a,b; Thomas and Hughes 1982). Also, at the two extremes of water $\mathrm{pO}_{2}$ (less than $70 \mathrm{mmHg}$ and greater than $350 \mathrm{mmHg}$ ), the ratio of arterial $\mathrm{pO}_{2}$ to water $\mathrm{pO}_{2}$ appears to decline. Between these two 


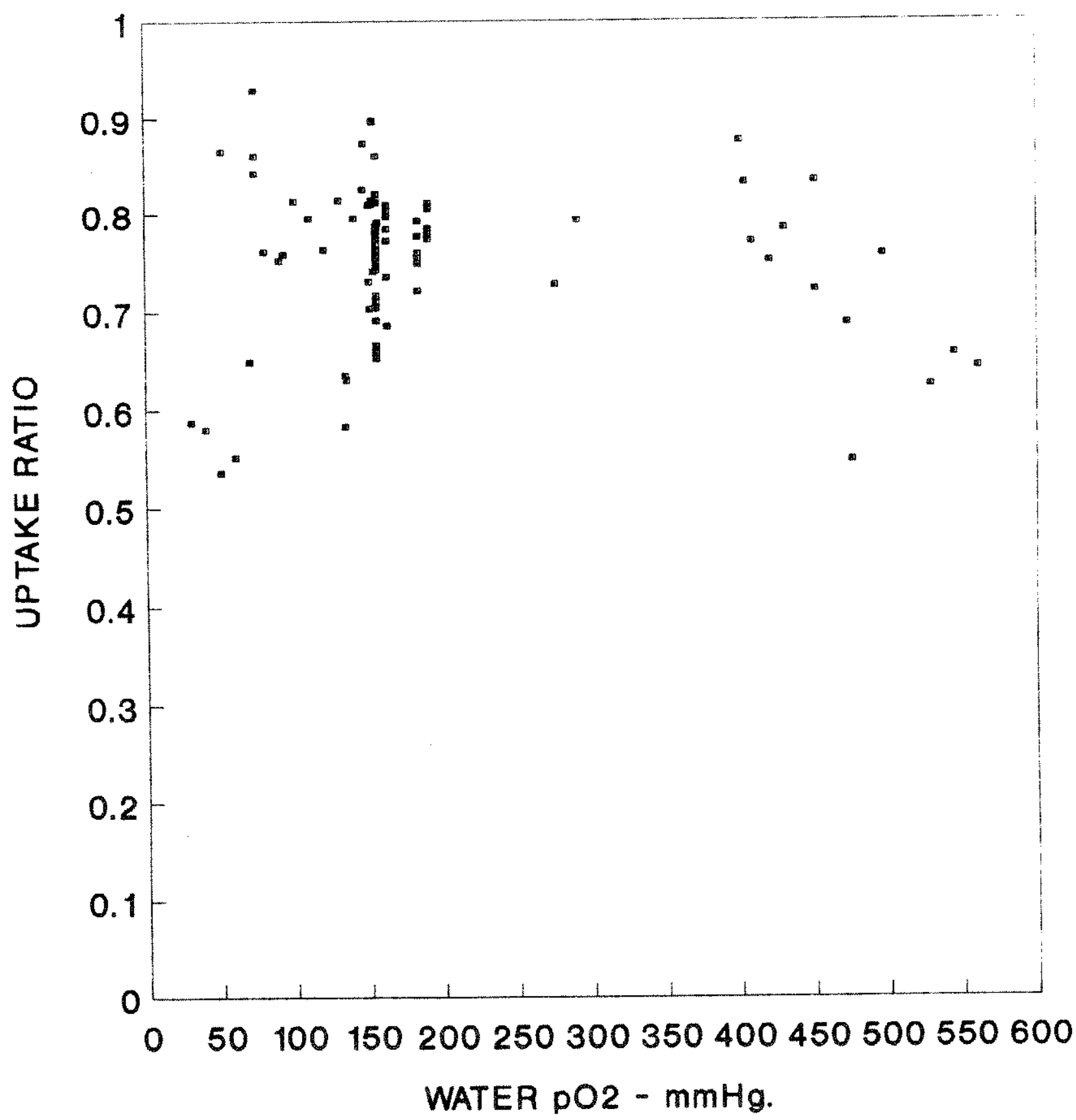

Figure 2.1.3. Ratio of arterial to water $\mathrm{pO}_{2}$ for rainbow trout. 
values, $F$ is estimated to have a mean of 0.79 with a standard deviation of 0.045 . Usually when fish are exposed to supersaturated water, $\mathrm{pO}_{2}$ is elevated along with other atmospheric gases (Weitkamp and Katz 1980; Colt et al. 1986). The maximum $\mathrm{pO}_{2}$ reported in the 1 iterature is about $390 \mathrm{mmHg}$ (Renfro 1963). Cases of supersaturation when $\mathrm{po}_{2}$ is low usually occur in well water. However, in these situations oxygen partial pressures less than $70 \mathrm{mmHg}$ have not been reported in the literature. Therefore, in subsequent analyses, the value of 0.79 for $F$ will be assumed as representative of most situations involving supersaturation and GBT.

Effective Nuclei Radius $\left(x_{0}\right)$ : With the above definition of $\mathrm{F}$, the bubble growth threshold equations (Equations 2, $2^{\prime}, 3,3^{\prime}$, 4 and $4^{\prime}$ ) are reduced to forms containing an effective nuclei radius as the only unknown dependent variable. In the case of thresholds for swimbladder overinflation, Equation 2 is complete without a nuclei radius. Based on the above, Phases 2 and 3 were required to define the effective nuclei radius in the intravascular and water/sub-dermal bubble growth threshold equations. In phase 2 we examined data from the literature to determine if there are thresholds for time to mortality associated with GBT and if mortality thresholds were correlated with bubble growth thresholds as predicted by Equations 2,3 and 4 (or $2^{\prime}, 3^{\prime}$ and $4^{\prime}$ ). In Phase 3 we experimentally examined the relationships between predicted bubble growth thresholds, observed bubble growth thresholds, and physiological parameters that relate the two to mortality. 


\subsubsection{Phase 2 - Literature Evidence for GBT Thresholds}

A literature review of dissolved gas supersaturation and GBT in fish was conducted to build a database of documented response to supersaturation that could be analyzed for threshold information and to extract other relationships that may exist between reported parameters.

We anticipated that gaps in literature data from one series of experiments would be filled by literature data from other experiments. With a more complete description of response, the chances of identifying relationships between parameters and mortality thresholds would be enhanced.

\subsection{2.a Methods and Materials}

The literature review was restricted to five fish species: chinook, coho, and sockeye salmon (oncorhynchus tshawytcha, o. kisutch, ㅇ. nerka) as well as steelhead and cutthroat trout (ㅇ. mykiss, ㅇ. clarki). This restriction was based on two factors: 1) the abundance of data on these species compared to the relatively limited data on other species (Weitkamp and Katz 1980; Colt et al. 1986), and 2) the physiological similarity of these fish to each other, to the trout of the Bighorn River, and to the rainbow trout used in the U.B.C. laboratory experimental work.

The review included a re-examination of the data reported by Jensen et al. (1985) as well as additional data. In particular, Jensen and co-workers did not include in their database experiments in which the fish did not die. clearly, all data are important to establishing GBT mortality thresholds.

Data sources: Literature sources and information are 
summarized in Appendix C of Fidler (1988). Several recording errors found in the database of Jensen et al. (1985) were corrected. When sufficient data were available, results such as time to $50 \%$ mortality were interpreted.

Methods of analysis: The GBT database was analyzed on computer using relational database and spreadsheet programs. Program choice depended on the need for numerical or filtering operations. For example, TGP is sometimes calculated with and without the vapor pressure of water. For consistency, both for reporting and from the standpoint of the physics of the processes involved (Fidler 1985), all TGP data were corrected to include the vapor pressure of water. In addition, where information permitted, oxygen to nitrogen ratios were converted to the partial pressures of oxygen and nitrogen. The relational database program was used to filter only those data useful for establishing relationships between mortality thresholds and other parameters.

Preliminary Filtering: Numerous sorting and filtering operations were performed on the database to determine if mortality thresholds or other useful relationships were present. For example, salmon and trout eggs are highly resistant to supersaturation and mortalities occur only at very high levels of TGP (Alderdice and Jensen 1985a; Rucker 1975b). Thus, the database was filtered to include records only for hatched fish. often experimenters, believing that dissolved nitrogen is the sole cause of GBT, failed to record TGP. Thus, the database was filtered to include only those records in which TGP was reported. Finally we restricted the database to include only 
those records where the mortality level was between $20 \%$ and $70 \%$ and where fish size was known (Figure 2.1.4).

\subsection{2b Results}

One thousand thirteen data records were developed from the literature including about 500 entries from the database of Jensen et al. (1985). In general, these data show that time to mortality for fish larger than $50 \mathrm{~mm}$ is shorter than for smaller fish. Also, there is strong indication of a threshold for mortality at a TGP of 1.1 atms. The negative times shown in the figure correspond to experiments where mortalities were not observed. The absolute value of the negative entries is based on the duration of the experiment divided by -10 .

Unique Data sets: Before considering the database further, it is important to examine six distinct data records that appear repeatedly throughout the analyses that follow. First, the datum point shown by the solid black circle at $1182 \mathrm{~h}$ (Figure 2.1.5) is unique in that the level of TGP is low (1.13 atms), the depth is $1 \mathrm{~m}$ and the fish are near $50 \mathrm{~mm}$ in length. These data yield a time to $50 \%$ mortality almost two orders of magnitude greater than data at similar water TGP but slightly shallower depths. Next, five data sets, shown by the five triangular symbols in the figure, correspond to a water TGP of 1.19 atms and $\mathrm{pO}_{2}$ levels above $271 \mathrm{mmHg}$. Again, the times to mortality are considerably above those of data at similar TGP but lower water $\mathrm{pO}_{2}$ levels. The importance of these data will be explained in the discussion that follows. 


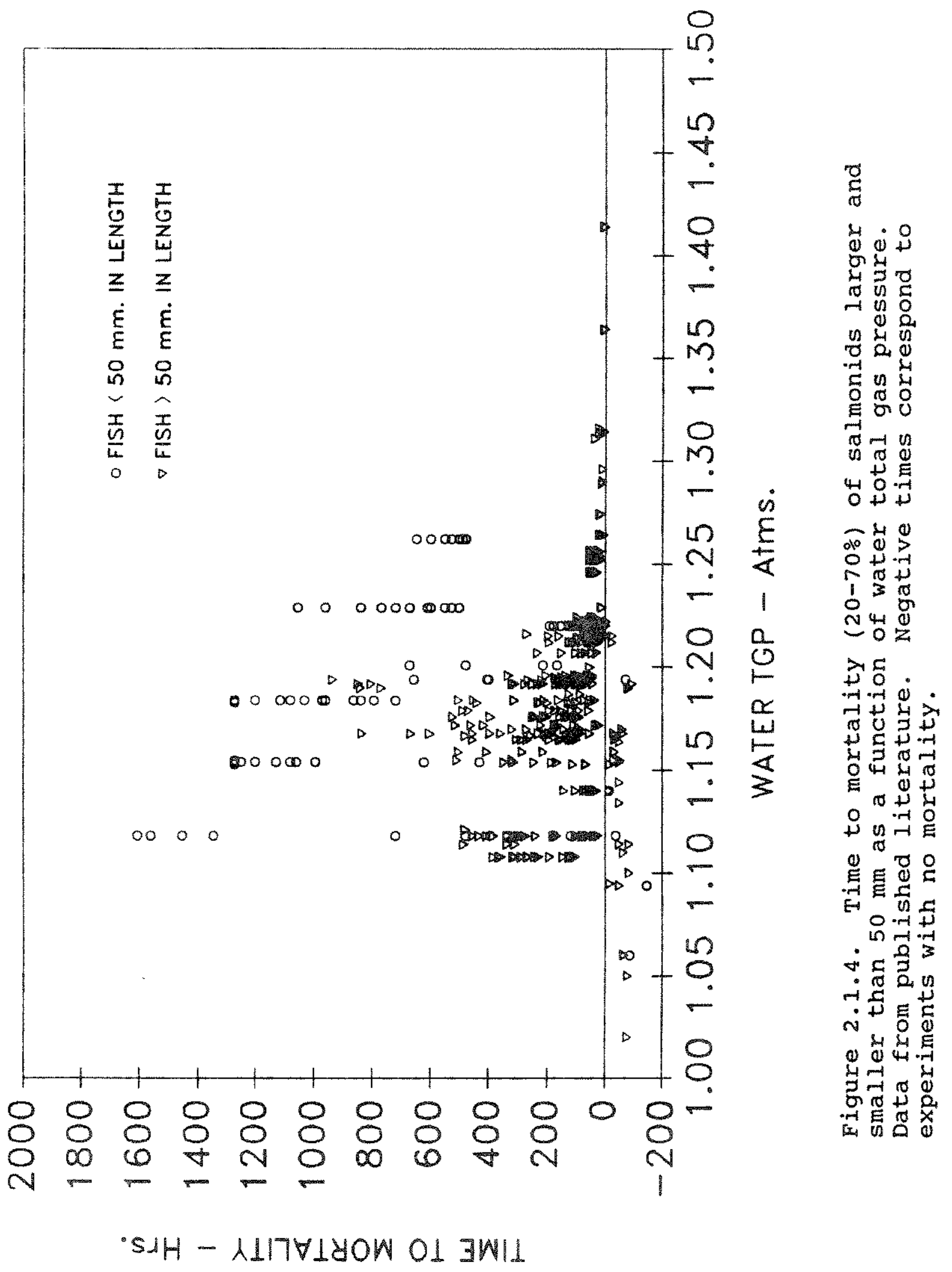




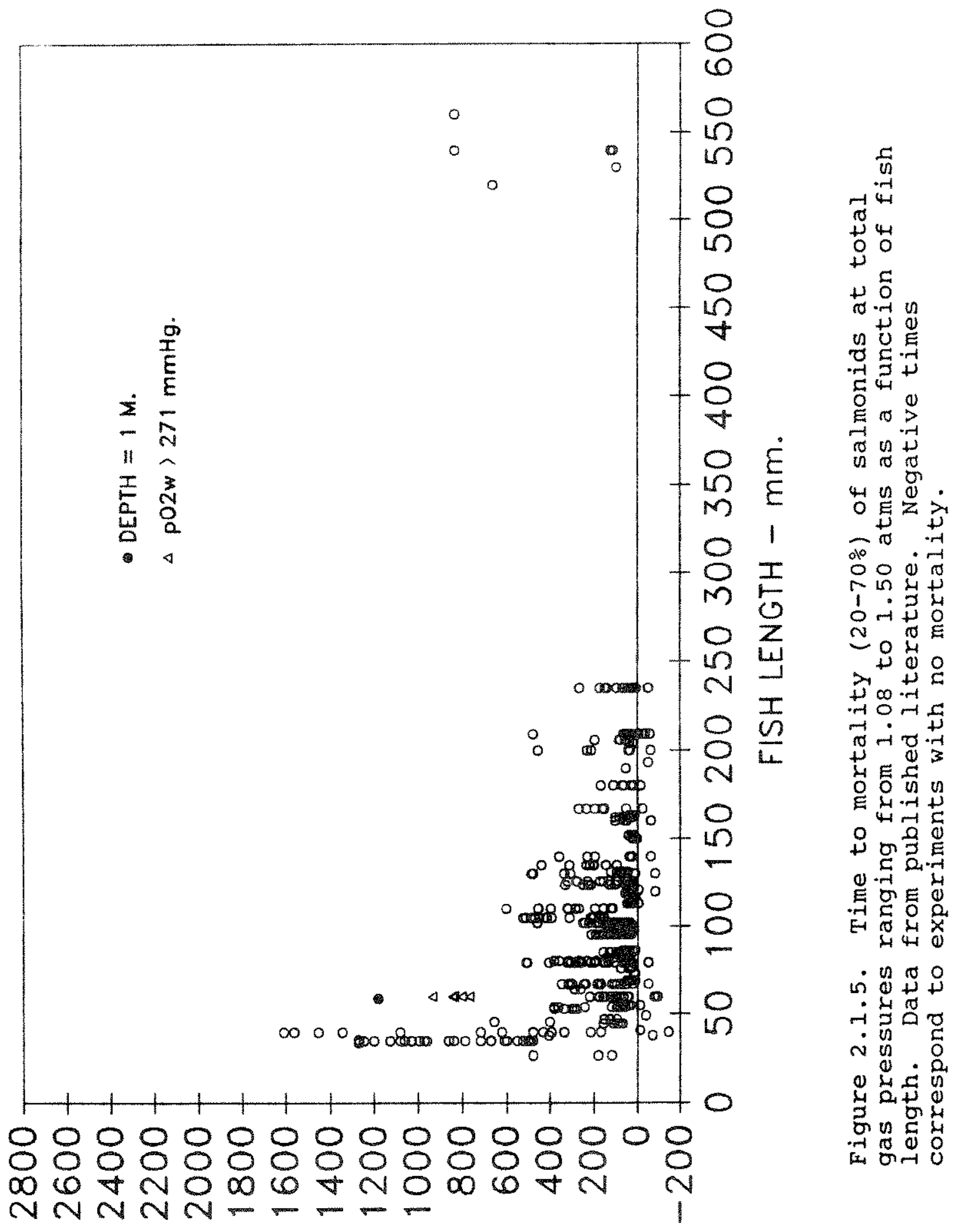

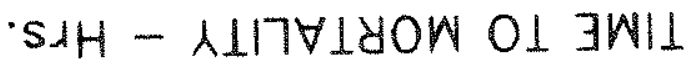


Effect of Fish size: To illustrate the effect of fish size on mortality, the time to mortality $(20-70 \%)$ is plotted against fish length (Figure 2.1.5). Except for the six data sets mentioned above, it is clear that a difference exists between the times to mortality for fish less than $50 \mathrm{~mm}$ in length and for those greater than $50 \mathrm{~mm}$. When this relationship is examined in more detail, it is found that the difference exists independent of water TGP. These results indicate that $50 \mathrm{~mm}$ is a critical length regarding time to mortality for these five species.

Effect of Fish species: The data sets were next filtered according to species. The intent of this separation was to determine if differences in thresholds or other parametric relationships exist based on species.

Fish Less Than $50 \mathrm{~mm}$ : Figure 2.1 .6 summarizes time to mortality as a function of TGP for fish less than $50 \mathrm{~mm}$ in length. At this level of filtering, the database contains information on steelhead trout and chinook and coho salmon only. clearly, a mortality threshold exists for all three species at a water TGP of about 1.12 atms. For steelhead trout, time to mortality is relatively short between 1.10 and 1.15 atms compared to that just above 1.15 atms. As TGP increases beyond 1.15 atms, time to mortality declines to levels comparable to those below 1.15 atms. The opposite response is observed in chinook salmon where mortality levels below 1.15 atms are high in comparison to those above 1.15 atms. For coho salmon, there is no trend in time to mortality above or below 1.15 atms.

Chinook Salmon Greater Than 50 mm: The response of larger chinook salmon exposed to supersaturated water as indicated by 


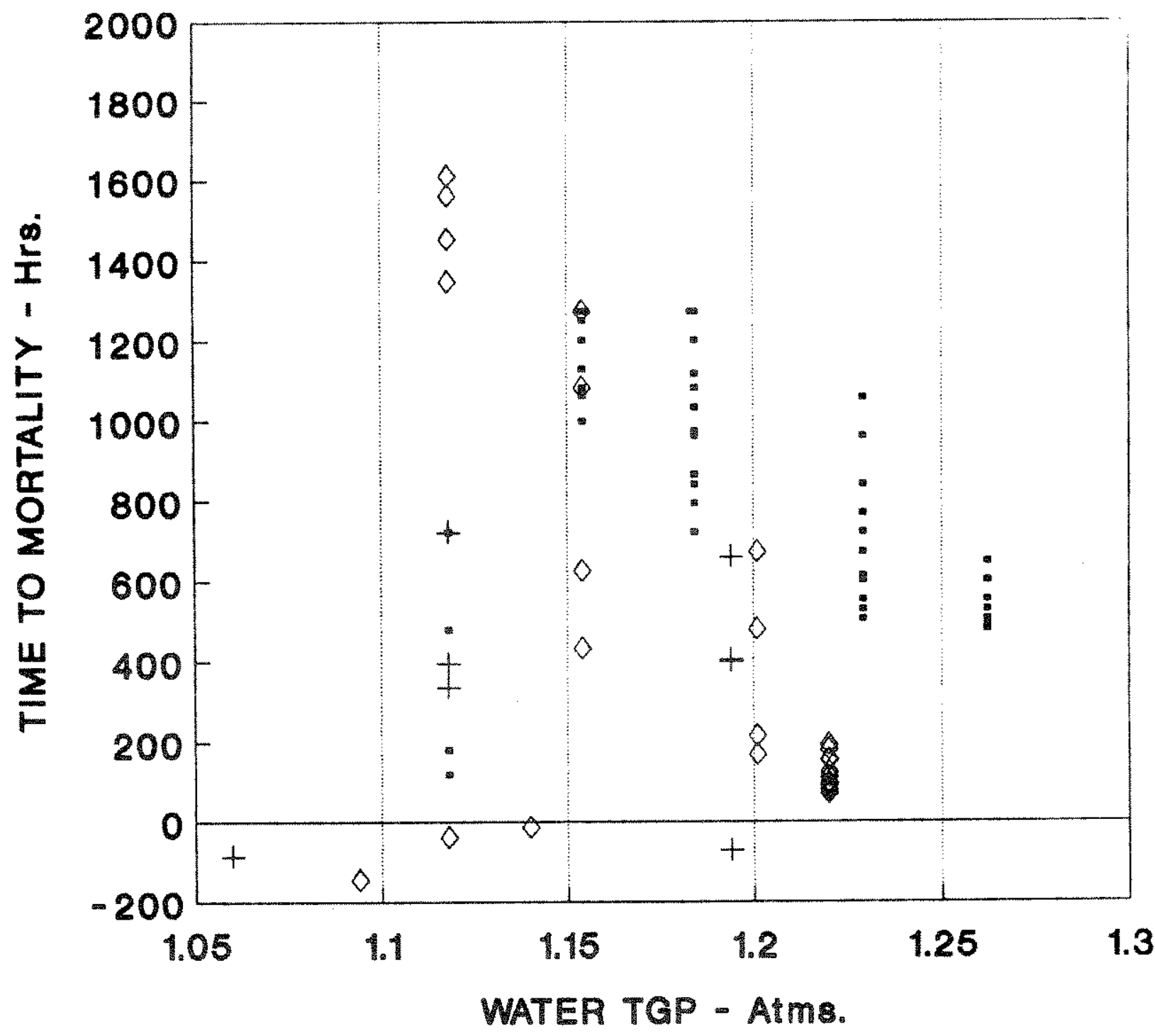

- STEELHEAD + COHO $\diamond$ CHINOOK

Figure 2.1.6. Time to moxtality (20-70\%) of steelhead trout and coho and chinook salmon $<50 \mathrm{~mm}$ in length as a function of total gas pressure. Data from published literature. Negative times correspond to experiments with no mortality. 
time to mortality versus water TGP is shown in Figure 2.1 .7 . clearly, a lower threshold for mortality exists at 1.11 atms. From this threshold, time to mortality decreases as TGP increases to 1.18 atms. At 1.18 atms, time to mortality suddenly increases to levels comparable to those at 1.11 atms. Above 1.18 atms, time to mortality again declines with increasing prp, similar to that between 1.1 and 1.18 atms. Due to the similarity of response at 1.1 atms and 1.18 atms, there may be another threshold at a TGP of 1.18 atms for larger chinook salmon. If so, it is not clear why the mechanism that is responsible for mortality between 1.1 and 1.18 atms suddenly becomes less effective at a TGP of 1.18 atms. In fact, the data suggest a transition to another mechanism of mortality at 1.18 atms and above. Another interesting feature of the data is that at both 1.11 atms and 1.18 atms there is a minimum time to mortality of about $100 \mathrm{~h}$.

Sockeye Salmon Greater Than $50 \mathrm{~mm}$ : Although there is no evidence of a mortality threshold near the 1.1 atms TGP, a somewhat similar response to that of chinook is observed in sockeye salmon (Figure 2.1.8). This lack of a threshold may be due to the sparseness of data in the region between 1.1 and 1.17 atms. There is, however, strong indication of a mortality threshold at a TGP of 1.17 atms. This is particularly evident from the clustering of both positive and negative data near 1.17 atms. Also apparent at this threshold is a minimum time to mortality of about $100 \mathrm{~h}$. This is similar to that observed with chinook salmon as described above.

Coho Salmon Greater whan $50 \mathrm{~mm}$ : Although there were 


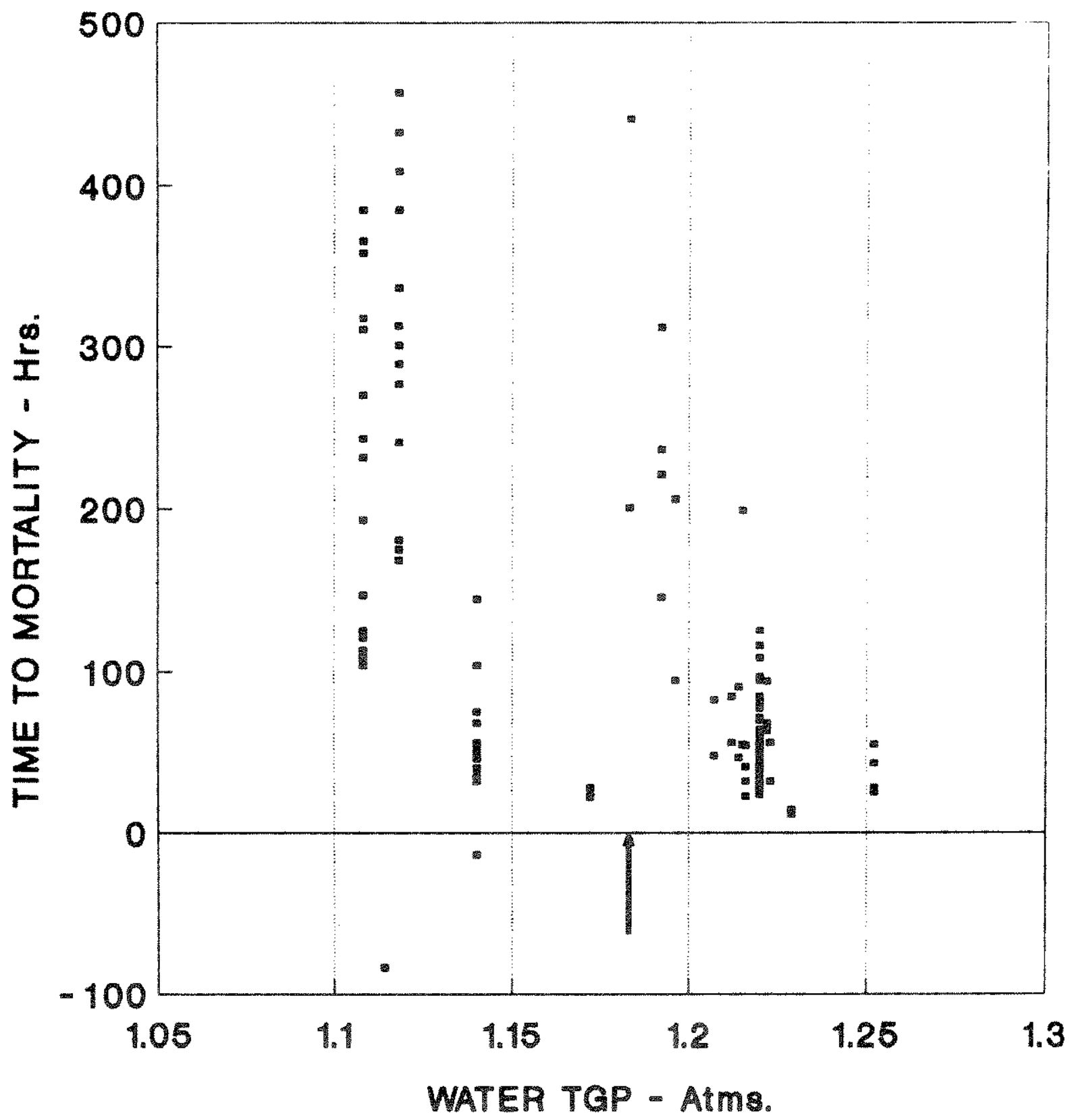

Figure 2.1.7. Time to mortality $(20-70 \%)$ of steelhead trout and coho and chinook salmon $>50 \mathrm{~mm}$ in length as a function of total gas pressure. Data from published literature. Negative times correspond to experiments with no mortality. 


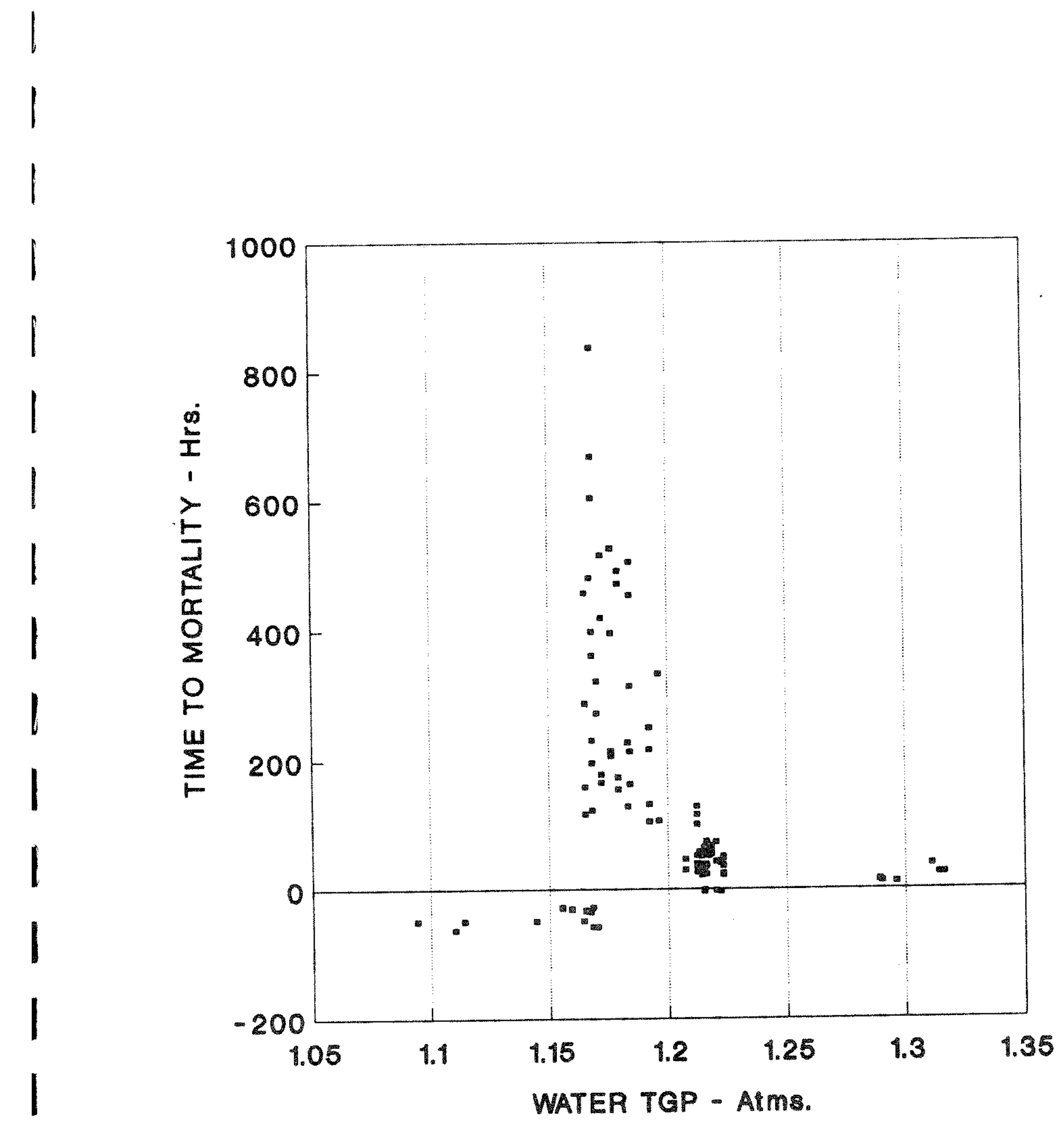

Figure 2.1.8. Time to mortality (20-70\%) of sockeye salmon $>50 \mathrm{~mm}$ in length as a function of total gas pressure. Data from published literature. Negative times correspond to experiments with no mortality. 
mortalities among coho salmon at 1.12 and 1.175 atms, there is nothing to suggest that these are thresholds (Figure 2.1.9). An aspect of these data is the relative scarcity of information in the vicinity of 1.1 to 1.17 atms TGP. Also, as described earlier, the five data sets with water $\mathrm{pO}_{2}$ above $271 \mathrm{mmHg}$ are higher than all other times to mortality shown in the figure. Steelhead Trout Greater Than $50 \mathrm{~mm}:$ For steelhead trout there are sufficient positive and negative data just at and above a water TGP of 1.15 atms to suggest this is a threshold for this species (Figure 2.1.10). Again, a minimum time to mortality is evident at 1.15 atms. As water TGP approaches 1.1 atms, from lower values, the combination of negative times to mortality and of the sudden increase to positive times at a TGP of 1.1 atms suggest this is the lower mortality threshold.

Cutthroat Trout Greater Than $50 \mathrm{~mm}$ : For cutthroat there is no information in the database below a water TGP of 1.15 atms. Available data suggest a threshold near 1.15 atms (Figure 2.1.11). Once again, there is strong indication of a minimum time to mortality at 1.15 atms.

Effects of water Dissolved oxygen Tension $\left(\mathrm{po}_{2}\right)$ : As indicated in the theoretical equations, thresholds for vascular bubble growth are dependent on the partial pressure of dissolved oxygen in the water. To search for such a dependency, the database was filtered to restrict the records to those containing information on water TGP, $\mathrm{pO}_{2}$, depth and fish length.

Two distinct sets of data that exhibit $\mathrm{pO}_{2}$ dependency were found. The first involves the experiments of Nebeker et al. (1979). In four experimental series, water TGP was held 


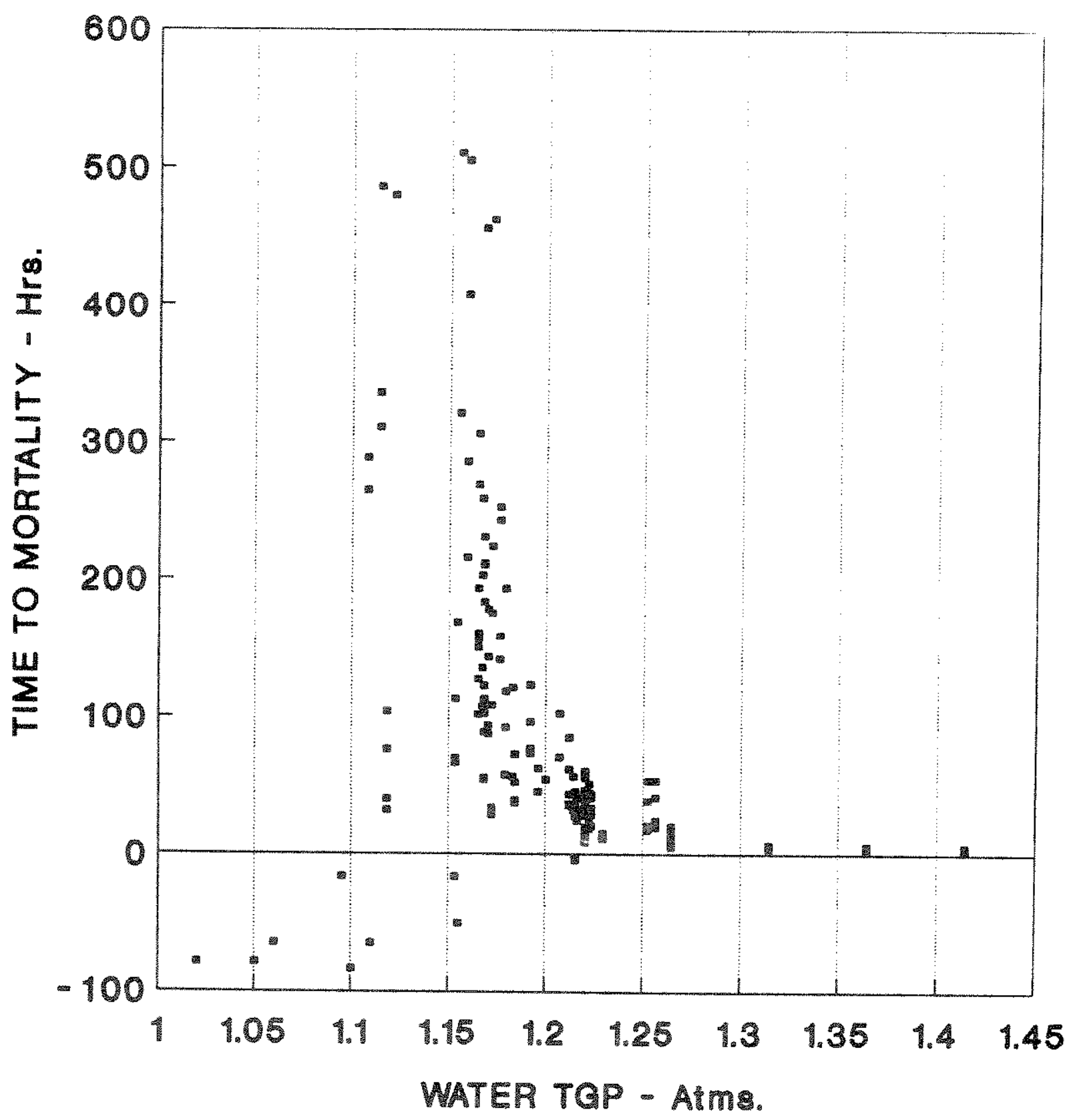

Figure 2.1.10. Time to mortality $(20-70 \%)$ of steelhead trout $>50 \mathrm{~mm}$ in length as a function of total gas pressure. Data from published literature. Negative times correspond to experiments with no mortality. 


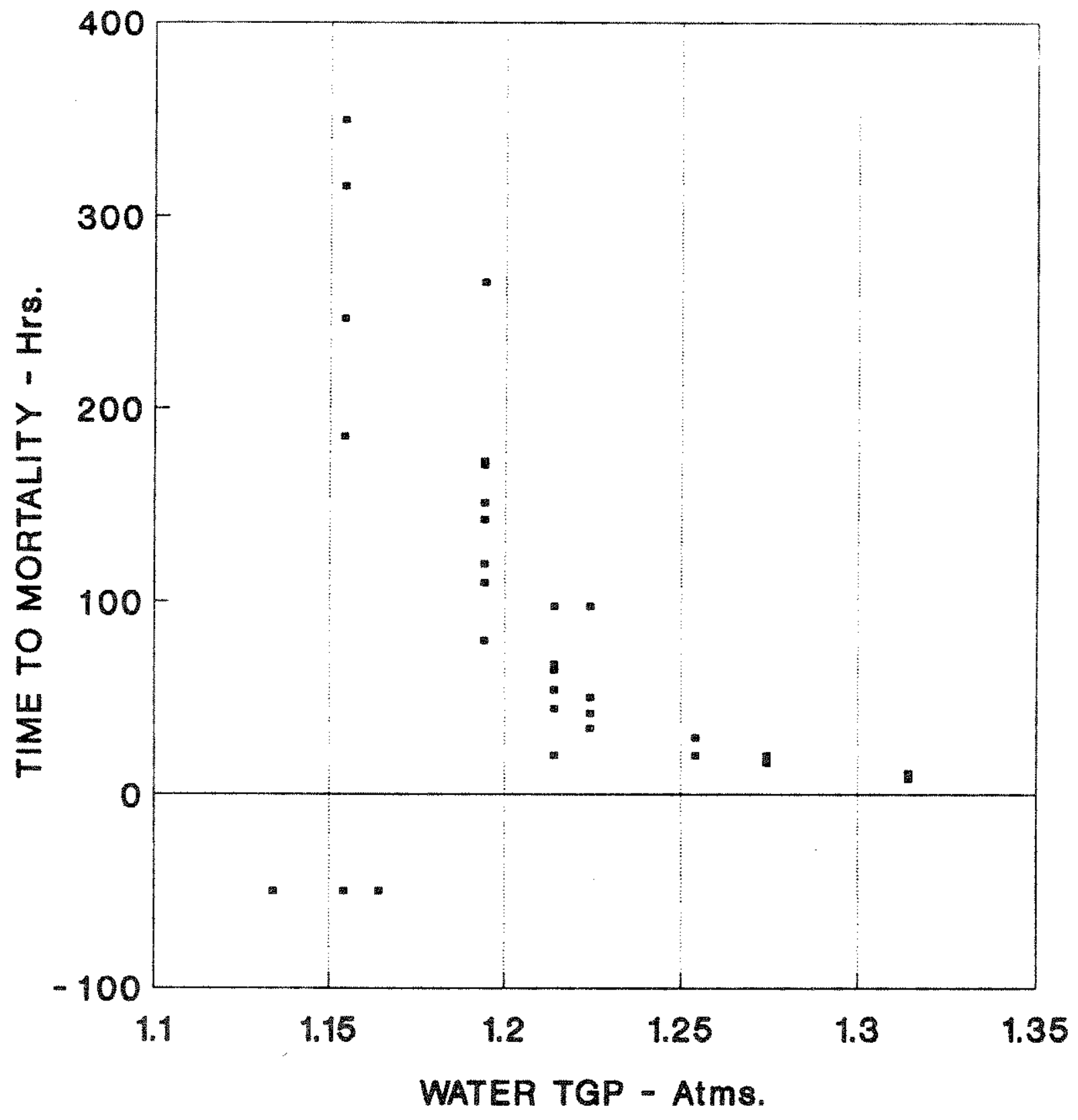

Figure 2.1.11. Time to mortality $(20-70 \%)$ of cutthroat trout $>50 \mathrm{~mm}$ in length as a function of total gas pressure. Data from published literature. Negative times correspond to experiments with no mortality. 
constant at $1.264,1.314,1.364$ and 1.414 atms while water $\mathrm{po}_{2}$ was varied. At each TGP it is evident there is an increase in time to $50 \%$ mortality with increasing water $\mathrm{po}_{2}$ (Figure 2.1.12). The most dramatic increase occurs at the lower TGP of 1.264 atms. Although the data in this range of TGP levels show time to mortality is dependent on water $\mathrm{pO}_{2}$, there is nothing to suggest the existence of thresholds.

perhaps the most intriguing set of records are those of Rucker et al. (1975a). These data are for a water TGP of 1.193 atms and water $\mathrm{pO}_{2}$ varying between 80 and $400 \mathrm{mmHg}$. Time to mortality is less than $220 \mathrm{~h}$ for water $\mathrm{pO}_{2}$ levels between 80 and $249 \mathrm{mmHg}$ (Figure 2.1.13). As water $\mathrm{pO}_{2}$ increases between these two values, the time to $25 \%$ and $50 \%$ mortality also increases gradually. However, between 249 and $271 \mathrm{mmHg}$ there is almost a five-fold increase in time to $25 \%$ mortality. The $50 \%$ level of mortality was not reached in an exposure period of $1000 \mathrm{~h}$ once water $\mathrm{pO}_{2}$ rose above $249 \mathrm{mmHg}$ (negative times in the figure). Nor was a $25 \%$ mortality reached during the experiment at water $\mathrm{pO}_{2}$ levels above $300 \mathrm{mmHg}$. These data strongly suggest the existence of a TGP threshold at 1.193 atms that is dependent on water $\mathrm{po}_{2}$. Further, the threshold is located between a water $\mathrm{pO}_{2}$ of $249 \mathrm{mmHg}$ and $271 \mathrm{mmHg}$. It is this response that made these particular data unique.

Compensation Depth: Theoretical thresholds for all forms of bubble growth should increase with increasing water depth. If there is sufficient depth, and fish use that depth, the effects of supersaturation can be avoided or reduced. Shrimpton et al. (1989) showed that small fish exposed to supersaturated water 


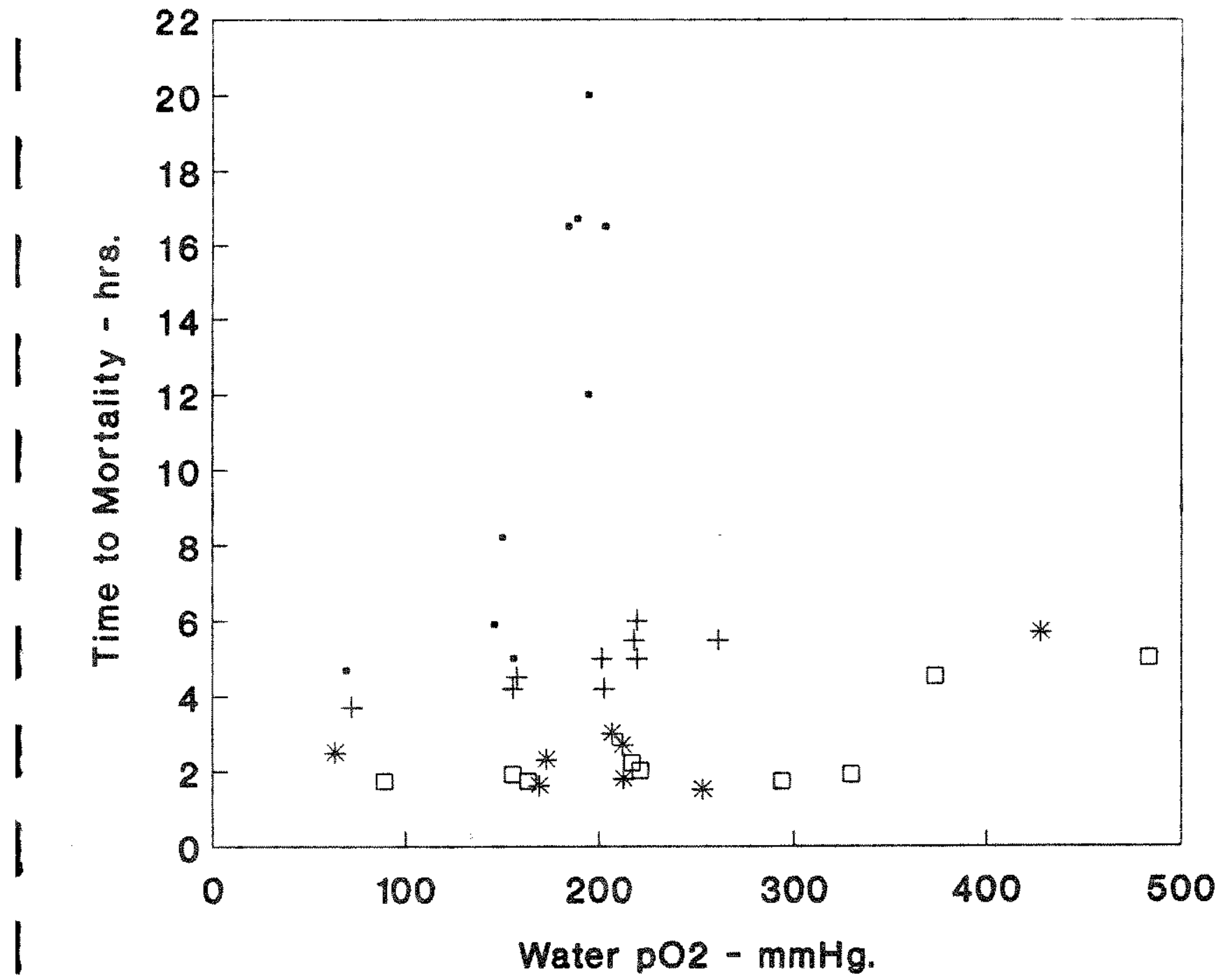

$$
\begin{array}{ll}
-T G P=1.264 \text { Atms. } & +\quad \text { TGP }=1.314 \text { Atms. } \\
\text { * TGP }=1.384 \text { Atms. } & \square \quad \text { TGP }=1.414 \text { Atms. }
\end{array}
$$

Figure 2.1.12. Influence of water $\mathrm{pO}_{2}$ (mmHg) on time to $50 \%$ mortality of steelhead trout. Data from Nebeker et al. 1979. 


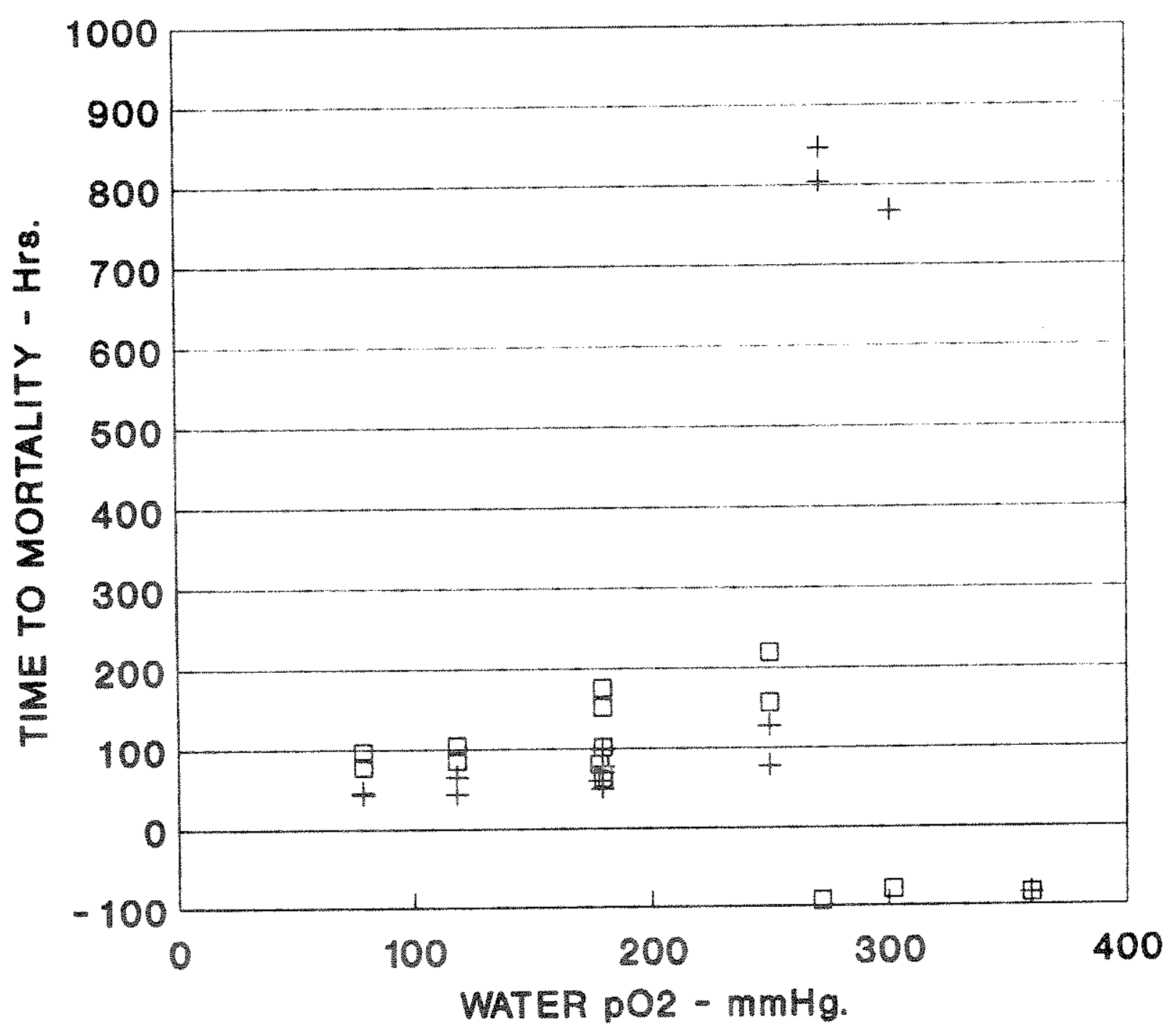

- $50 \%$ Mortality $+25 \%$ Mortality

Figure 2.1.13. Influence of water $\mathrm{pO}_{2}$ (mmHg) on time to $25 \%$ and $50 \%$ mortality of coho salmon exposed to TGP of $1.193 \mathrm{~atm}$. Data from Rucker 1975. 
seek depth to overcome excess buoyancy induced by swimbladder overinflation. Because large fish do not experience the same degree of over buoyancy (Shrimpton et al. 1989), they do not seek depth as a means of compensating for supersaturation. To examine this effect, database TGP's were corrected for depth. That is, TGP was reduced by the hydrostatic head reported in the data. The correction formula used is:

$\mathrm{TGP}_{\text {corr }}=\mathrm{TGP}_{\text {uncorr }}-\left(73.1 \cdot \mathrm{h} / \mathrm{P}_{\text {atms }}\right)$

where, TGP is in atmospheres, Patms is in mmHg and $h$ is in meters. The constant, 73.1, combines the density of water and the gravitational constant to yield units of $\mathrm{mmHg} / \mathrm{m}$.

The correction produces a horizontal shift of the data to the left on the TGP axis (Figure 2.1.14; see Figure 2.1.6 to compare the corrected data with uncorrected data). With the correction of TGP for depth, the apparent lower threshold is shifted from 1.12 atms to 1.1 atms. This corresponds to the lower threshold of mortality for fish greater than $50 \mathrm{~mm}$.

In the data records, depths range from near zero to about $4 \mathrm{~m}$. There is no restriction on movement of fish within the depth indicated, with the exception of data presented by Knittel et al. (1980). In these experiments steelhead trout were exposed to supersaturated water in cages held at specific depths (Figure 2.1.15). TGP ranged from about 1.19 atms to 1.41 atms. Depth correction reduces data scatter and suggests that the response is hyperbolic or asymptotic in form. Further, the corrected data suggest a TGP threshold near 1.19 atms. This threshold is above that indicated in the rest of the steelhead data (Figure 2.1.10). 
FISH LESS THAN $60 \mathrm{~mm}$.

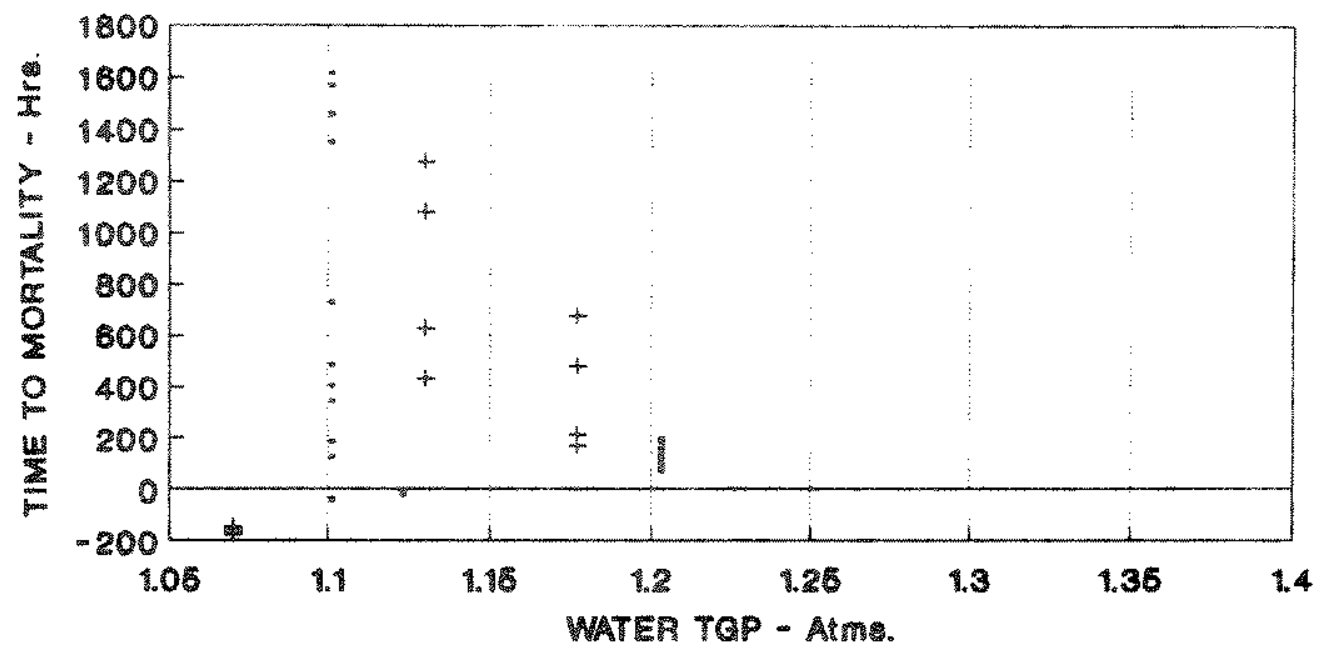

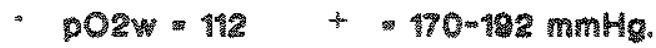

FISN OREATER THAM 30 mT.

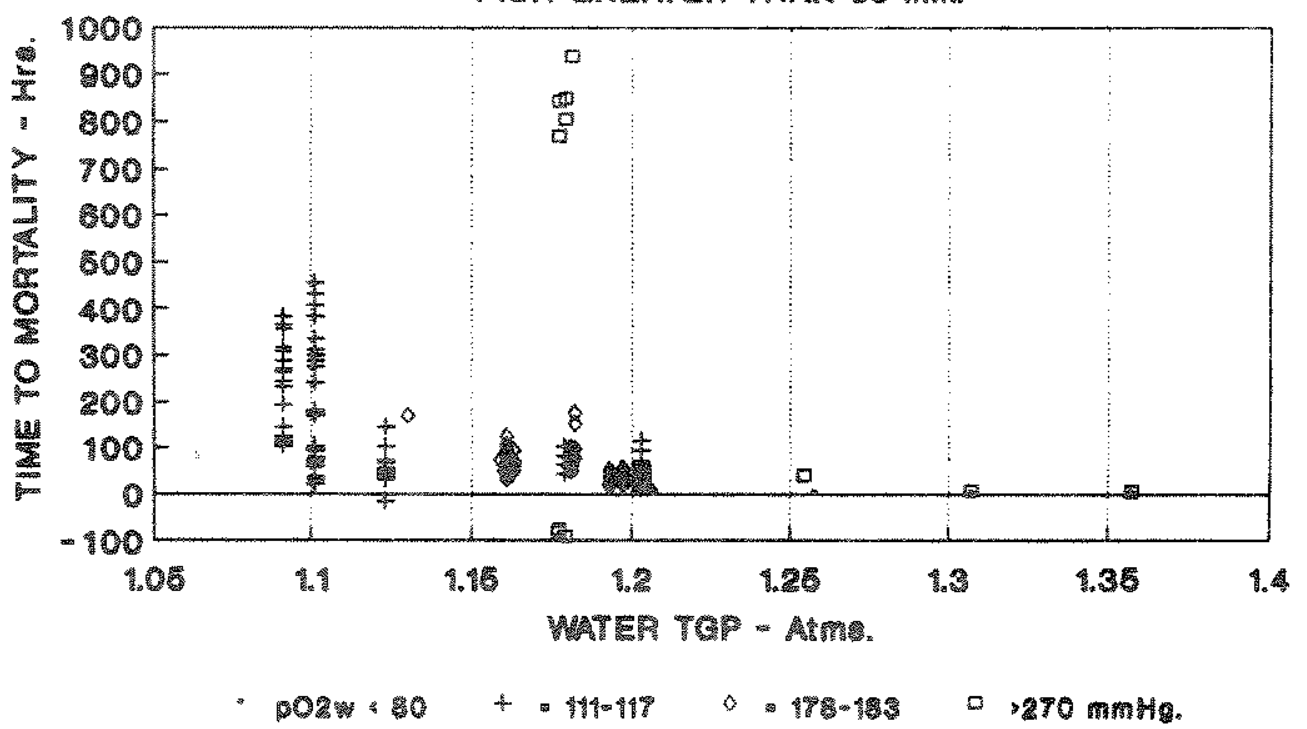

ำง

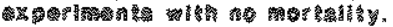

Figure 2.1.14. Time to mortality (20-70\%) of salmonids smaller and laxger than $50 \mathrm{~mm}$ as a function of depth-corrected total gas pressure. 


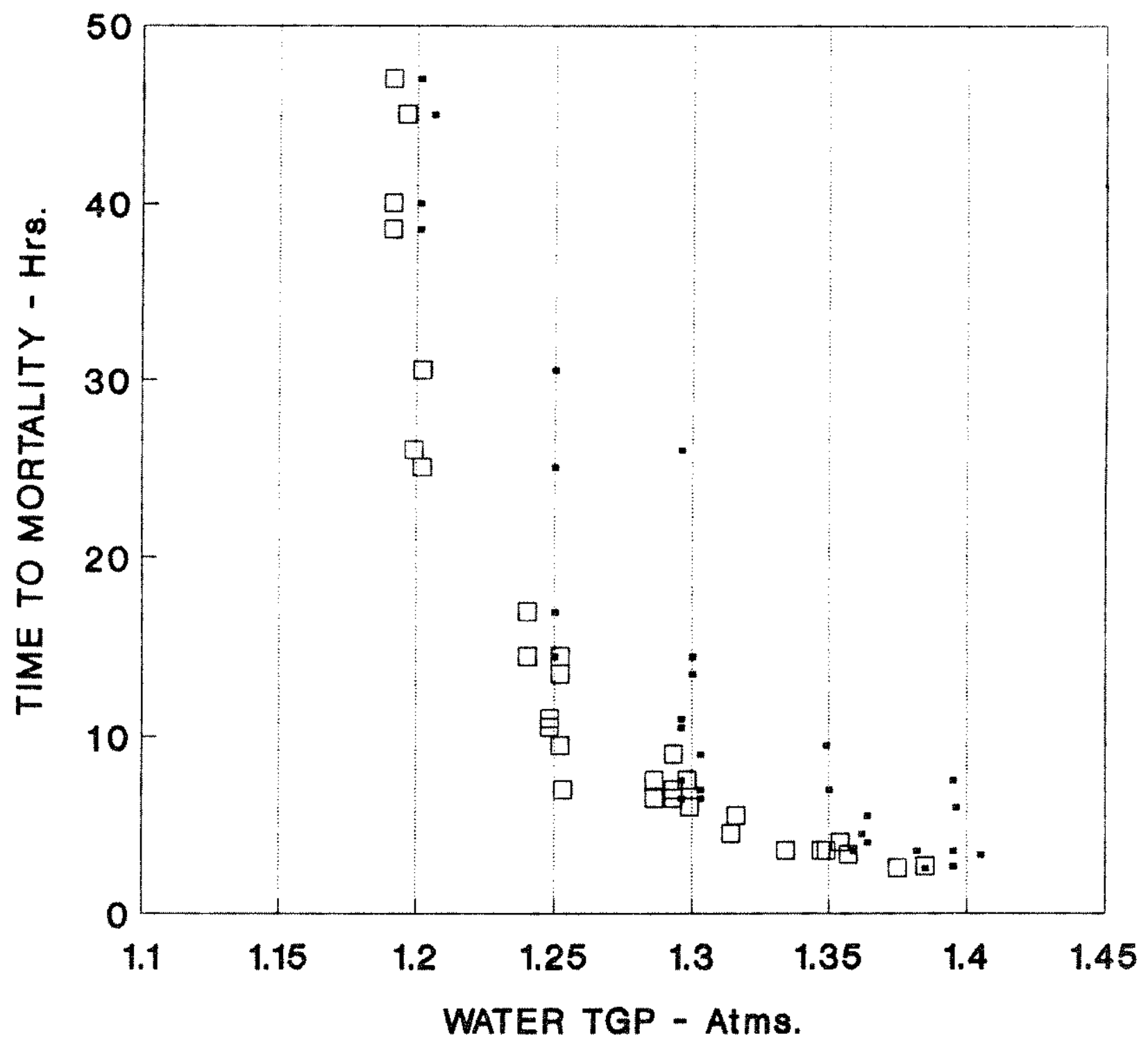

Figure 2.1.15. Time to 50\% mortality of steelhead trout at water total gas pressures with and without correction for depth. Data from Knittel et al. 1980. 
However, water $\mathrm{pO}_{2}$ was not reported.

\section{$2.1 .2 . c$ Discussion}

From this analysis, it is clear that the response to supersaturated water by chinook, coho and sockeye salmon and steelhead and cutthroat trout is highly dependent on fish size. The difference in time to mortality between fish greater than 50 $\mathrm{mm}$ and those less than $50 \mathrm{~mm}$ suggests a discontinuous relationship between time to mortality and fish size. Although not conclusive for all species examined, the database analysis suggests that two TGP and $\mathrm{po}_{2}$ related mortality thresholds may be involved in the response of fish to supersaturation. A lower threshold is apparent at a TGP of about 1.1 atms, while a second threshold may exist in the TGP range of 1.15 to 1.17 atms. The evidence for the two thresholds is strongest for chinook salmon longer than $50 \mathrm{~mm}$ (Figure 2.1 .7 ). The evidence for a higher threshold near a TGP of 1.15 atms is particularly good for sockeye salmon and cutthroat trout (Figures 2.1 .8 and 2.1 .11 , respectively). The data also suggest that the higher threshold varies slightly with fish species.

For fish shorter than $50 \mathrm{~mm}$, the only mortality threshold that is apparent, is one at a TGP of 1.12 atms. However, if depth corrections are applied to this threshold (assuming small fish seek compensation depth), the threshold for zero depth is shifted to 1.1 atms (Figure 2.1.14). This places the apparent lower threshold at about the same TGP as for fish longer than $50 \mathrm{~mm}$ with TGP uncorrected for depth.

Although there is evidence for two TGP thresholds for fish 
longer than $50 \mathrm{~mm}$, the physiological consequences of these thresholds is not clear. Nor is it clear which lethal symptoms are associated with each threshold. The data for chinook salmon longer than $50 \mathrm{~mm}$ suggest that two separate mortality mechanisms are operating over two different ranges of TGP. Furthermore, at a TGP of 1.17 atms, there appears to be a transition from one mechanism of mortality to another. This is accompanied by a dramatic increase in time to mortality. We do not understand these mechanisms nor the reasons for the apparent transition from one to the other. However, experimental work (Phase 3) yielded further insight into these mechanisms and the nature of the apparent transition and will be described later.

An indication of the cause of mortality at the higher TGP threshold is suggested in the studies of stroud and Nebeker (1976) on steelhead trout. In this work, symptoms of GBT were recorded for fish exposed to various levels of water TGP. It was noted that vascular bubbles in the gill arteries did not appear until water TGP rose above 1.15 atms. Meekin and Turner (1974) made similar observations; however, they reported only dissolved nitrogen concentrations and not TGP.

A much stronger correlation of time to mortality with water TGP, correcting TGP for depth, was reported by knittel et al. (1980). However, their experimental TGP levels were always at or above the upper TGP threshold indicated in the database, thus their data are for a single threshold. Although the correction of TGP for fish restrained to specific depths is valid, such restrictions can seldom be achieved. 


\subsubsection{Phase 3 - Experimental studies}

Theoretical models that describe the thresholds for bubble growth and swimbladder overinflation were reviewed in an earlier section. Equations 2 and $4,\left(2^{\prime}\right.$ and $\left.4^{\prime \prime}\right)$ predict that swimbladder overinflation and vascular system bubble growth thresholds are dependent on water TGP (or delta $\mathrm{P}$ ), water $\mathrm{pO}_{2}$, water depth, and the effective size of nucleation sites in the vascular system. In the case of bubble growth in the vascular system and in the environmental water, the principal unknown is the size of nucleation sites. The model describing thresholds for bubble growth in the environmental water may also be applicable to subdermal bubble growth on the external body of fish. However, it is not known if this form of bubble growth can produce mortality.

Data from the literature indicate that there may be two thresholds for mortality in fish exposed to supersaturated water. The higher threshold (near 1.15 to 1.17 atms TGP) appears to correlate with the appearance of intravascular bubbles in gill lamellae (Stroud and Nebeker 1976). The lower threshold (near 1.1. atms) also appears in the database but is not associated with specific physiological symptoms nor with the theoretical thresholds for bubble growth.

Thus, theory predicts multiple thresholds for bubble growth in fish exposed to supersaturated water, and data from the Iiterature suggest the existence of two thresholds for mortality in fish exposed to supersaturated water. There is, however, no clear association of the theoretical bubble growth thresholds and 
the observed thresholds for mortality. Mortality may be caused by bubble growth or other physiological insults resulting from supersaturation. Thus, there arose the need to experimentally study the relationship between thresholds for bubble growth and physiological parameters that may establish whether or not bubbles are responsible for death in fish.

Experimental studies were carried out in two parts. Part one, performed at the University of British Columbia, involved detailed physiological studies of fish exposed to supersaturated water. These studies examined an array of physiological parameters as indicators of thresholds for bubble growth. In addition to intravascular bubbles, we examined thresholds for water bubble and sub-dermal bubble growth. We measured arterial $\mathrm{pO}_{2}, \mathrm{pH}$, hematocrit levels and microscopically examined gill lamellae and other tissues. Observations were correlated with the severity of GBT symptoms, water TGP, and $\mathrm{pO}_{2}$. other studies at the University of British Columbia, which were not a part of this program, provided further insight into the thresholds for swimbladder overinflation and the effects of overbuoyancy on juvenile fish.

Part two of laboratory threshold and susceptibility studies was performed at the U.S.F.W.S. Fish Technology center at Bozeman, Montana. These studies focused on TGP thresholds for mortality in juvenile Bighorn River rainbow and brown trout as a function of species and size. The effects of GBT on growth and disease resistance were also examined. 


\subsubsection{Part 1: Physiological Parameters and GBT \\ 2.1.3.1a Materials and Methods}

Production of supersaturated water: A packed column that employed turbulent mixing of gas and water under pressure was used to produce supersaturated water (Fidler 1983). The column consisted of a plastic pipe, $2.5 \mathrm{~m}$ long by $200 \mathrm{~mm}$ inside diameter, filled with $1.5 \mathrm{~cm}$ Norton Intalox saddles (Figure 2.1.16). The Intalox saddles produce turbulence thereby enhancing the gas exchange process. The column was mounted vertically and sealed at both ends except for water and gas ports. Water was supplied to the column via two independent sources. The main supply entered at the top of the column and fell over the packing material while a continuous flow of air (or preselected gas mixtures) passed upward through the packing. The second supply, or make-up water, entered at the bottom of the column and mixed with the outflow supply of supersaturated water. A float valve at the foot of the column controlled make-up water flow and assured a uniform discharge from the column. The makeup supply also allowed fine tuning of the system TGP. Pressure regulators upstream in the raw water supply lines insured uniform inlet water flow.

The gas supply to the column was atmospheric air under pressure or a pressurized mixture of oxygen, nitrogen and water vapor; a three-way valve in the gas supply system allowed switching between air and the preselected gas mixtures. The mixture ratio of oxygen to nitrogen was controlled by a gas flow mixing system with a $\mathrm{pO}_{2}$ monitoring control loop. A weighted relief valve at the top of the column controlled pressure within 


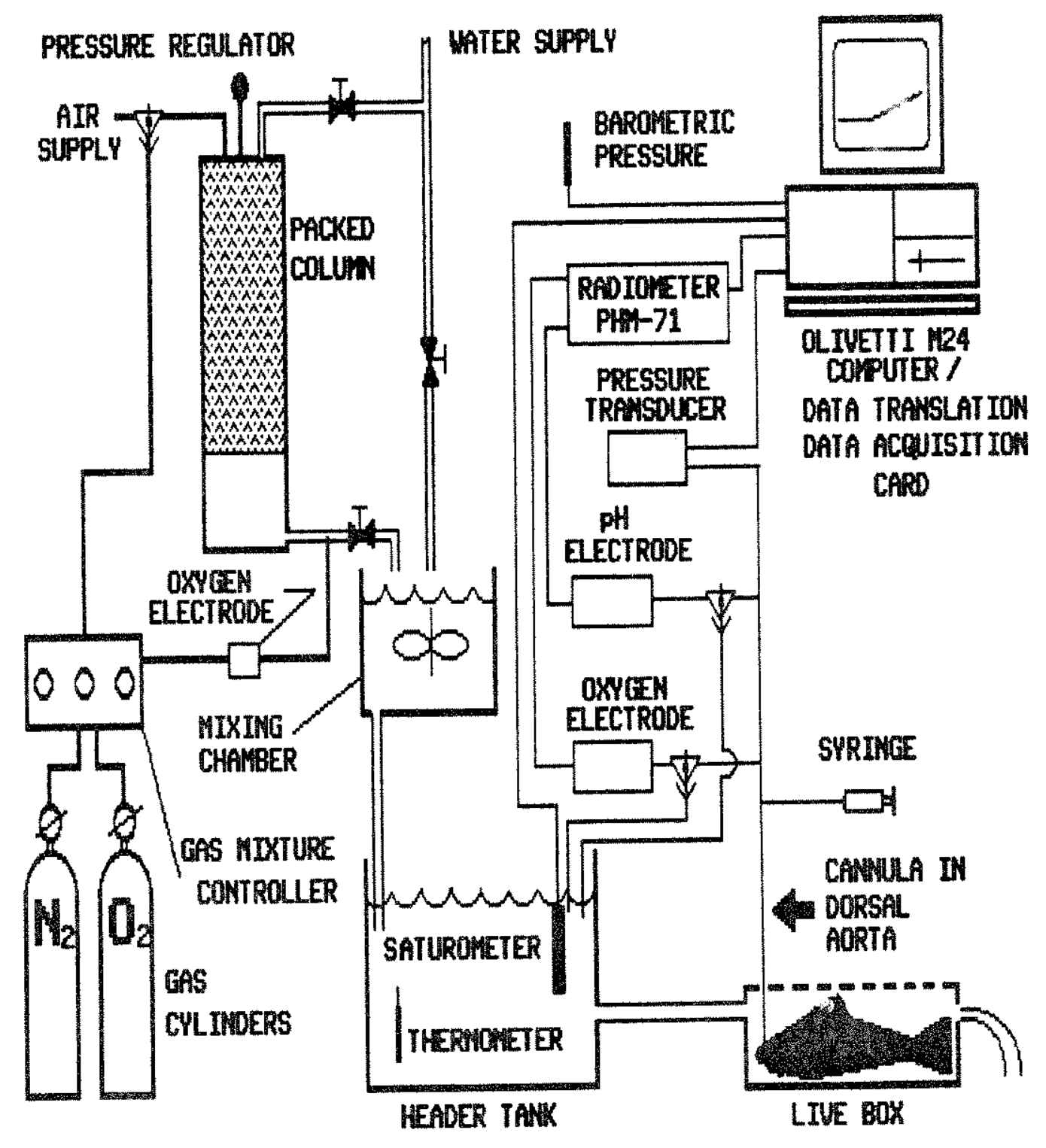

Figure 2.1.16. Schematic of experimental system designed to test effects of dissolved gas supersaturation on trout. 
the column. Column pressure was maintained at a precise level by adjusting the weight of the relief valve.

Water from the column flowed into a mixing chamber, then into a head tank, and finally into the chamber containing the fish (Figure 2.1.16). Water $\mathrm{pH}$, temperature, delta $\mathrm{P}$ and $\mathrm{pO}_{2}$ were measured in the head tank at a point near the discharge to the live boxes (Figure 2.1.17). Water depth in the box was no more than $10 \mathrm{~cm}$ and was open to the atmosphere to prevent hydrostatic head buildup.

Water Dissolved Gas Measurements: Water dissolved gas tensions were determined by measuring TGP and $\mathrm{pO}_{2} \cdot$ TGP was measured continuously with a Model 300 Nova Tech Saturometer. Before each experiment, the instrument output was set to zero and barometric pressure recorded. All subsequent measurements of TGP were corrected for changes in barometric pressure occurring during the experiment. The sensor portion of the instrument was placed in the head tank supplying water to the live boxes (Figure 2.1.16). The sensor was shaken continuously during experiments by a mechanical shaker. This prevented bubble formation on the silastic tubing membrane of the instrument -- a problem shown to reduce instrument sensitivity (Bouck, 1982).

Dissolved oxygen was monitored continuously with a thermostated Radiometer Model E5046 oxygen electrode connected to a Radiometer PHM 71 signal conditioner and meter. The system was calibrated before and at times during each experiment with a low standard consisting of water that had been completely degassed of oxygen and a high standard consisting of water that had been equilibrated with atmospheric air. 


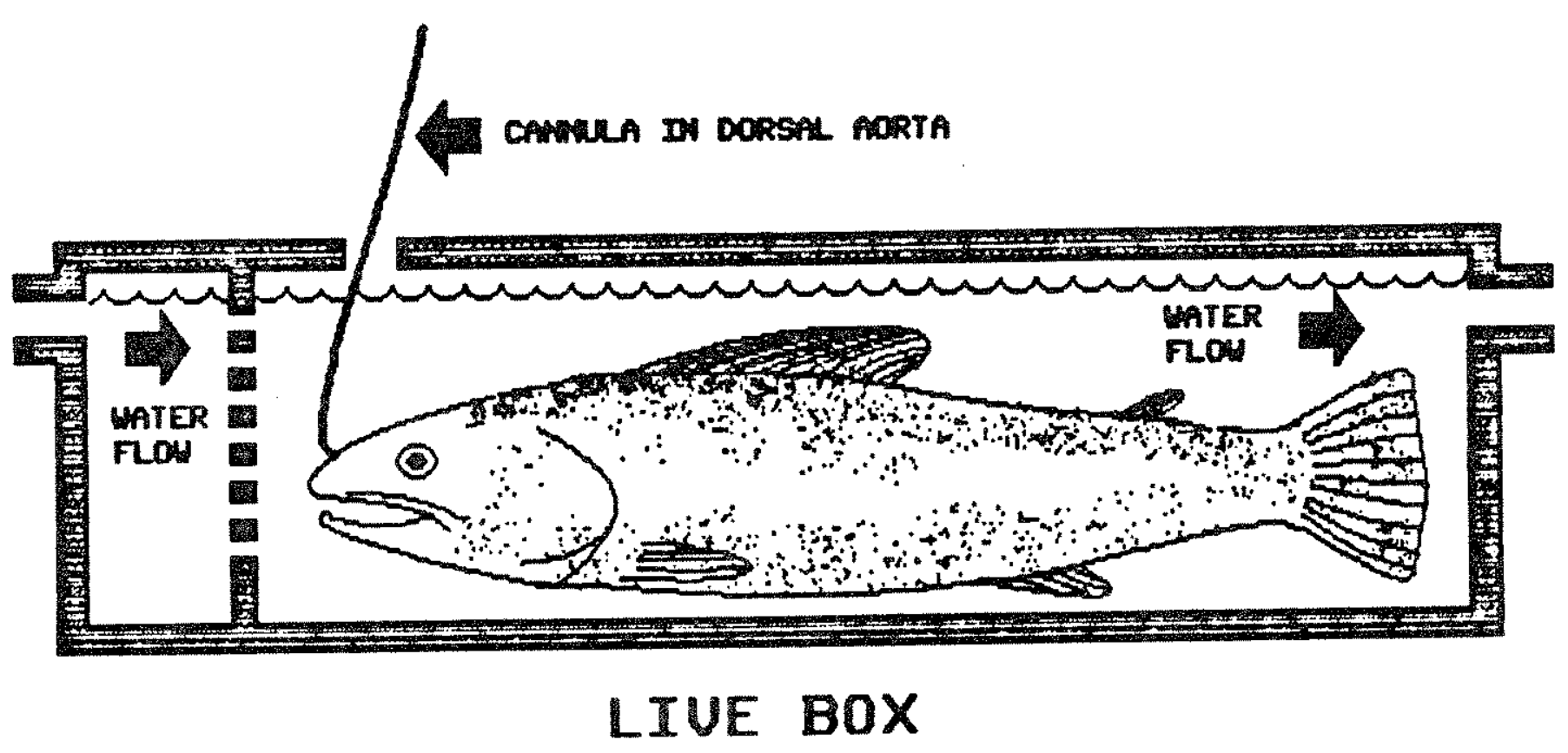

Figure 2.1.17. Schematic of experimental container and placement of swimbladder and dorsal aorta cannula. 
water pH and Temperature: Water $\mathrm{pH}$ was measured with a Radiometer G279/G2 glass capillary electrode and F497 calomel electrode connected to a Radiometer PHM 71 signal conditioner and meter. Before and following each experimental series, the pH electrode was calibrated using Radiometer precision phosphate buffers $\$ 1500$ and $\$ 1510$. Water pH was measured at prescribed intervals during each experimental series. Water temperature was measured with a mercury thermometer.

Computerized Data Acquisition system: As shown in Figure 2.1.16, most measurements were recorded and stored digitally on computer via a Data Translation DT-2801 analog to digital card.

Experimental Animals: Rainbow trout, weighing between $220 \mathrm{~g}$ and $850 \mathrm{~g}$ were obtained from West Creek Trout Farms of Aldergrove British columbia. The fish were maintained in outdoor tanks at water temperatures ranging from 5 to $15^{\circ} \mathrm{C}$, depending on the time of year. The animals were fed commercial trout food once weekly, ad Iibitum. When necessary, fish were acclimated to the experimental temperature by temperature changes of no more than $0.5^{\circ} \mathrm{C}$ per day. The maximum temperature acclimation required was $4^{\circ} \mathrm{C}$ (i.e. from $5^{\circ} \mathrm{C}$ to $9^{\circ} \mathrm{C}$ ).

surgery procedures: For experiments during which blood parameters were measured, fish were fitted with dorsal aorta cannula using techniques described in Sovio et al. (1972) and Fidler (1988). Before committing an animal to an experimental series, blood $\mathrm{pO}_{2}$ was measured. If the arterial $\mathrm{pO}_{2}$ was not above $90 \mathrm{mmHg}$, the animal was not used. This was a precaution against failure of the animal to fully recover from surgery and as a screen against animals of poor health. 


\section{Arterial Blood Measurements}

Partial pressure of oxygen. Arterial blood $\mathrm{po}_{2}$ was measured with a thermostated Radiometer E5046 oxygen electrode connected to a Radiometer PHM 71 signal conditioner (details in Fidler 1988).

Arterial Blood pH. Blood pH was measured with a Radiometer G279/G2 glass capillary pH electrode and $\mathrm{K} 497$ calomel electrode thermostated to the experimental water temperature. The electrical output of the electrode was connected to a Radiometer PHM 71 signal conditioner and meter (Fidler 1988).

Hematocrit: Hematocrit was determined from blood drawn into microhematocrit tubes. Samples were taken directly from the dorsal aorta cannula immediately after blood had been drawn into the $\mathrm{pH} / \mathrm{pO}_{2}$ sampling syringe (Fidler 1988). Except for hematocrit and $\mathrm{pH}$ samples, any blood removed from the fish was returned to the animal or replaced with an equal volume of cortland saline. Blood removal was minimized to obtain accurate hematocrit assays and to reduce any effect of sampling on the intravascular bubble growth process.

physiological studies: Physiological studies involved several experimental series, each having different objectives and. different experimental techniques.

Correlation of Physiological Measurements with GBT Symptoms (Series 1-5): The objective of these experiments was to correlate physiological data with bubble growth thresholds and mortality. During exposure to supersaturated water, blood $\mathrm{po}_{2}$ ' hematocrit and $\mathrm{pH}$ were monitored in groups of $12 \mathrm{fish}$ ( $\mathrm{six}$ cannulated and six un-cannulated). The five series consisted of 
exposure to water TGP levels of $1.10,1.12,1.15,1.17$, and 1.19 atms, and corresponding $\mathrm{pO}_{2}$ levels of $170,175,183,195$, and 201 mmHg. After death, we also examined each fish to assess the severity of each of the following GBT symptoms:

1.) Extra-corporeal bubble in gill lamellae (bubbles observed in the water between gill lamellae -- not internal to the animal).

2.) Intravascular bubbles in gill lamellae (bubbles located in the filamental arteries formed within the blood medium) .

3.) Sub-dermal bubbles in buccal cavity (bubbles forming obvious blisters forcing the separation of the epithelium tissue layer from the underlying tissue).

4.) Sub-dermal bubbles on the opercula (as above).

5.) Sub-dermal bubbles on the fins (as above).

To obtain a relative evaluation of symptom severity, an arbitrary scale ranging from 0 to 3 was used. A value of 0 indicated that the particular symptom was absent while a value of 3 indicated the symptom was of maximum severity. Unaided visual examinations were made for external symptoms (sub-dermal blisters on the body, fins and in the mouth). Examination for extra-corporeal and intravascular gill bubbles was done microscopically after excising 4 to 6 samples of gill tissue from each side of the animal. Samples were placed on glass slips that had been cooled to the experimental water temperature, covered with a cooled glass slip and quickly placed under the microscope for examination. Glass slips and covers were to prevent bubble formation due to temperature changes. A 
dissecting microscope equipped with a Leitz photographic system was used to examine and photograph gill and other tissue samples. Vascular Bubble Growth Threshold Dependency on Water $\mathrm{pO}_{2}$

(Series $A, B, C$ and 4): These experiments were designed to determine if vascular system bubble growth thresholds are dependent on water $\mathrm{pO}_{2}$ and if such a $\mathrm{po}_{2}$ bubble growth dependency is similar to that shown in the data of Rucker (1975a). In these experiments, water TGP was held constant at 1.15 atms, while $\mathrm{po}_{2}$ was varied. Water $\mathrm{pO}_{2}$ was $100 \mathrm{mmHg}$ in series $A$ and $B, 125 \mathrm{mmHg}$ in series $C$ and $183 \mathrm{mmHg}$ in series 4. In addition, we observed if intravascular bubbles were present on the gill lamellae of the experimental fish at death.

\section{$2 \cdot 1 \cdot 3 \cdot 1 b$ Results}

\section{Correlation of Physiological Measurements with GBT Symptoms}

(Series 1-5): The individual experimental series will be described in the order of increasing water TGP. In the following figures and tables, Fish No's 1 through 6 are the cannulated fish while Fish No's 7 through 12 are the uncannulated fish.

In general, cannulated fish did not live as long as uncannulated fish exposed to supersaturated water (Figure $2.1 .18)$. Times to mortality did not vary greatly in the TGP range of 1.10 through 1.17 atms. However, at a TGP of 1.19 atms, time to mortality was one third to one half of that at lower TGP. Both cannulated and uncannulated fish showed similar signs of GBT throughout the experiments. Decreased survival of the cannulated fish may have been due to the stress of cannulation, increased susceptibility to bubble growth caused by handing and surgery, 


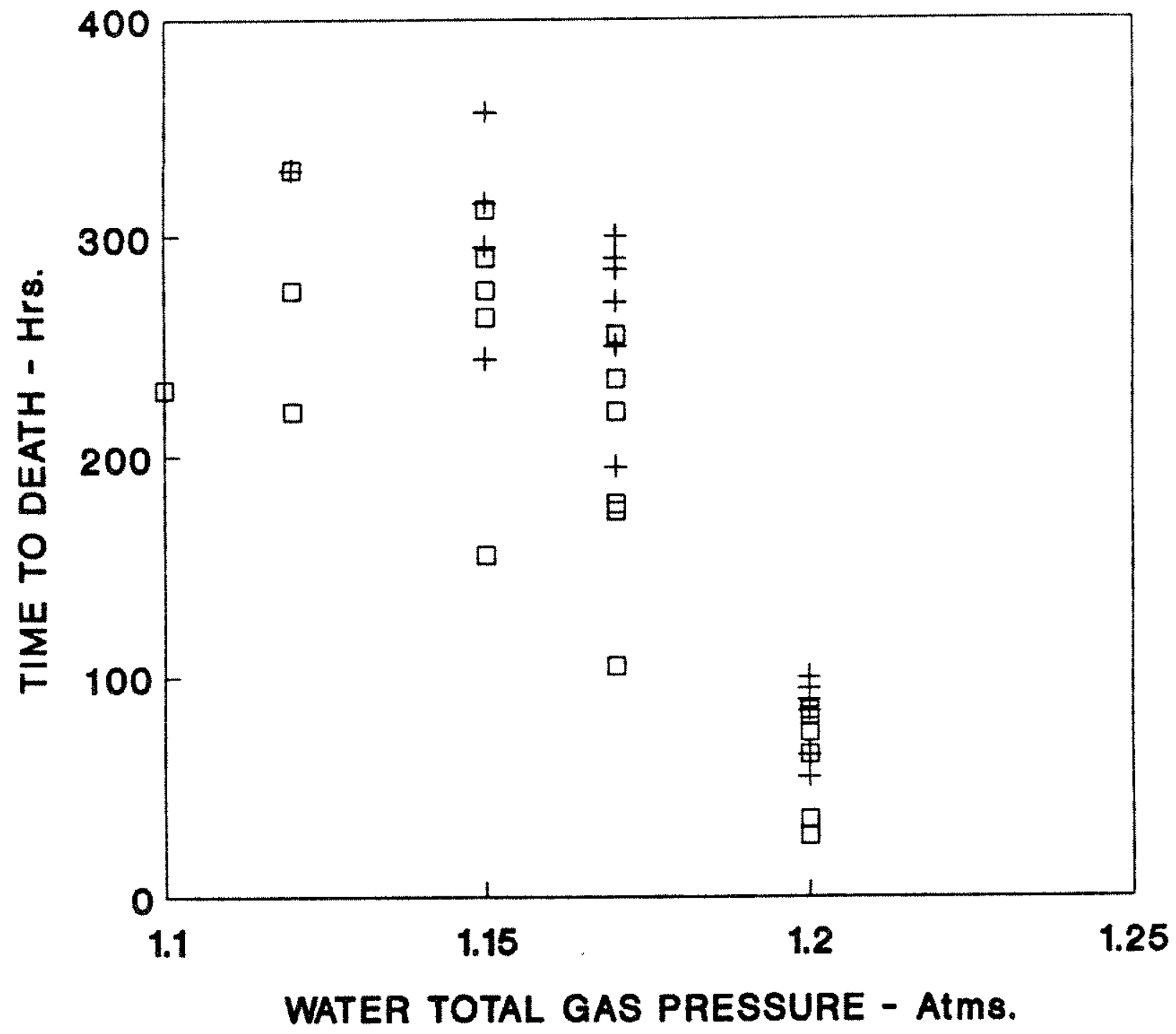

CANNULATED FISH + UN-CANNULATED FISH

Figure 2.1.18. Comparison of time to mortality of cannulated and uncannulated rainbow trout as a function of water total gas pressure. 
or the presence of the cannula.

Series $1-$ - water $T G P=1.10$ atms; $\mathrm{pO}_{2} \mathrm{w}=170 \mathrm{mmHg}:$ In this series of experiments, only two fish showed external signs of GBT. Of these, one fish (Fish 4) died early and exhibited a decline in arterial $\mathrm{pO}_{2}$ which suggested a blockage of oxygen transport. Furthermore, this was the only fish to exhibit a number of extracorporeal bubbles between gill lamellae. All other blood parameters were normal. Arterial $\mathrm{po}_{2}$ declined but not enough to suggest acute hypoxia. Except for Fish 4 , only small changes occurred in arterial $\mathrm{po}_{2}$ (Figure 2.1.19). Although three fish died at various times during the experiment, Fish 4 was the only fish showing severe signs of GBT. Fish 8 showed bubble growth between gill lamellae, but there was no evidence of hypoxia. This fish lived without signs of stress for the full $362 \mathrm{~h}$ of the experiment (Figure 2.1.20).

Little change in either arterial blood $\mathrm{pH}$ or hematocrit occurred over the $362 \mathrm{~h}$ of exposure (Figure 2.1.21). Most fish were alive at the end of the experiment and were sacrificed to make the necessary measurements. This procedure was followed in each of the series when fish remained alive through the end of the experiment.

Series $3-$ Water $T G P=1.12$ atms; $\mathrm{pO}_{2} \underline{\mathrm{W}}=175 \mathrm{mmHg}$ : Arterial $\mathrm{pO}_{2}$ declined in all but two fish ( 1 and 6) suggesting that oxygen transport was impeded (Figure 2.1.22). Furthermore, GBT related mortality occurred in all but three of the cannulated fish and in one of the uncannulated fish (Figure 2.1.18).

Arterial blood $\mathrm{pH}$ also declined in the fish which experienced a blood $\mathrm{pO}_{2}$ decline (Figure 2.1.23). However, 


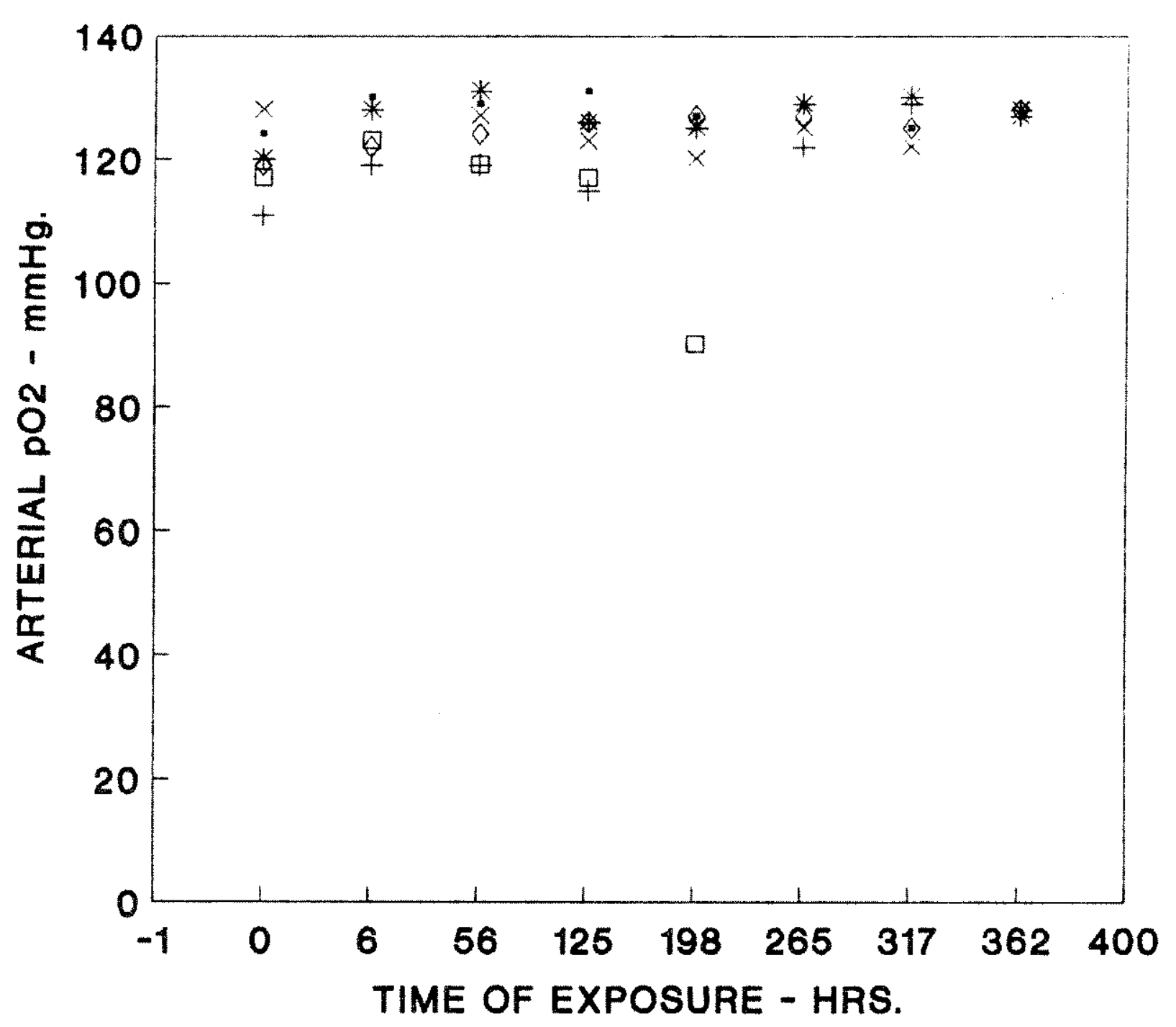

$\begin{array}{lllll}- \text { FISH } 1 & + \text { FISH } 2 & * & \text { FISH } 3 \\ \square \text { FISH } 4 & \times \text { FISH } 5 & \diamond & \text { FISH } 6\end{array}$

Figure 2.1.19. Variation in arterial blood $\mathrm{pO}_{2}$ with time in rainbow trout exposed to water TGP of 1.10 atms with a $\mathrm{pO}_{2}$ of $170 \mathrm{mmHg}$. 

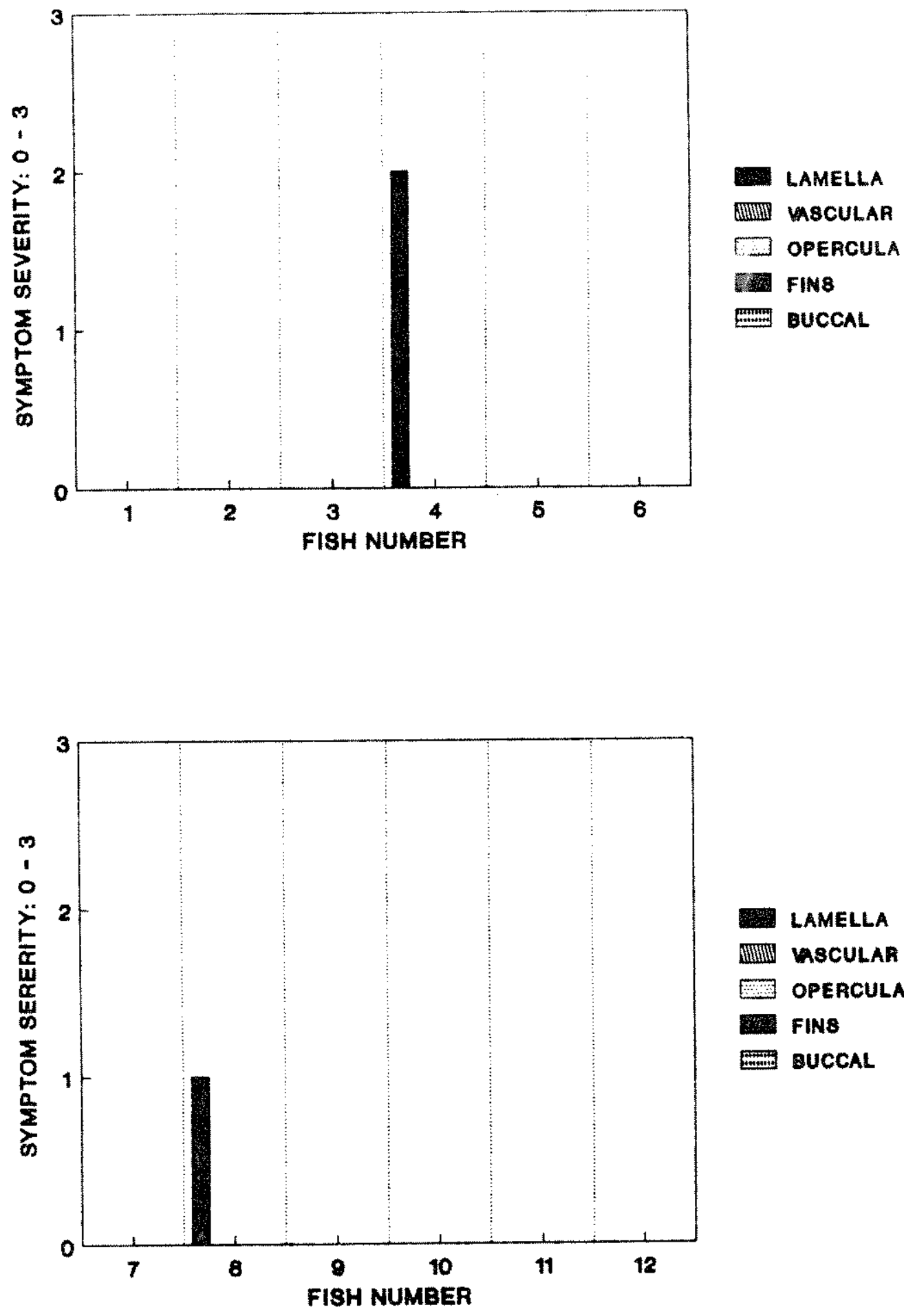

W.7. LAMELLA

HIIIII ISCULAR

OPERCULA

Fins

BUCCAL

Figure 2.1.20. Relative severity of gas bubble trauma symptoms at time of death of six cannulated $(1-6)$ and six noncannulated $(7-12)$ rainbow trout exposed to water TGP of 1.10 atms with a $\mathrm{pO}_{2}$ of 175 $\mathrm{mmHg}$. 


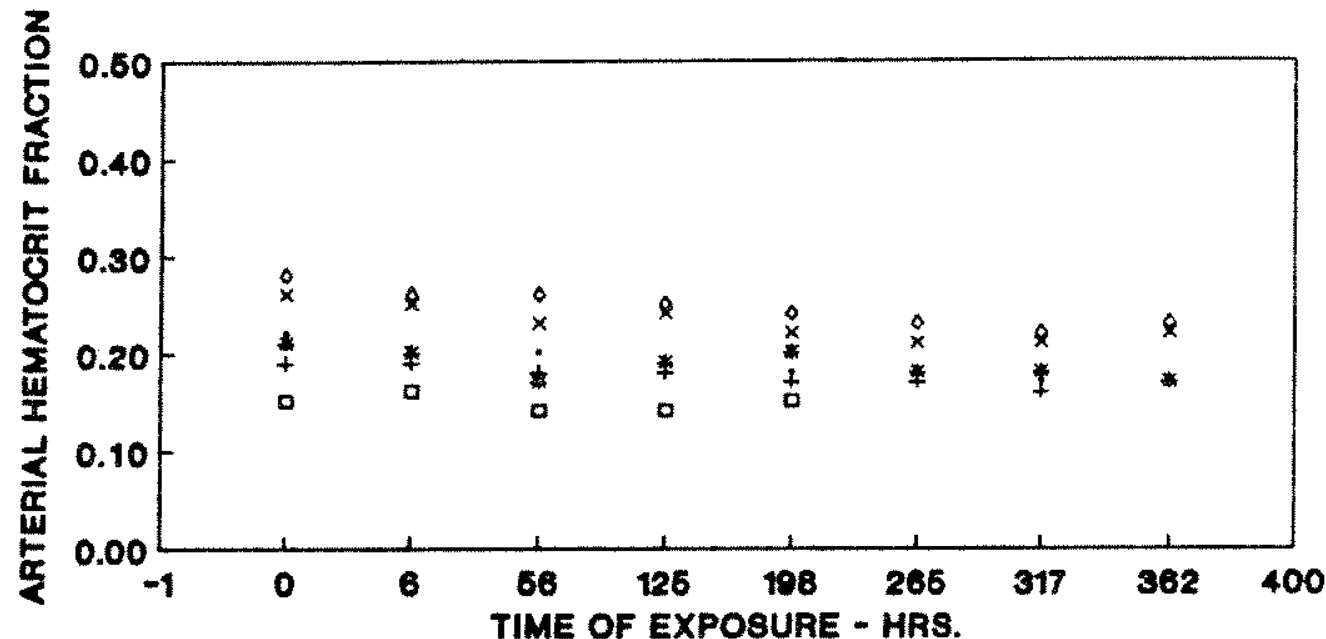

- F18H 1

+ FI8H 2

- F18H 3

- FI8H 4

$\times$ Fien 5

- FI8H 6

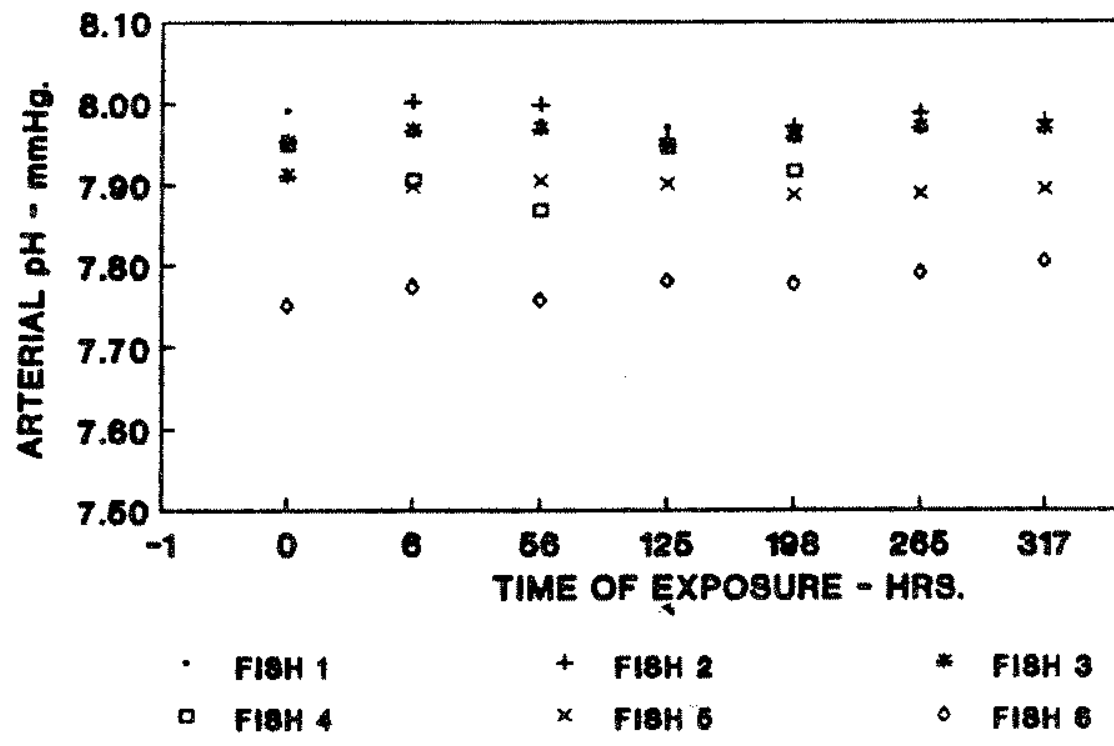

Figure 2.1.21. Arterial blood hematocrit and $\mathrm{pH}$ variation with time in rainbow trout exposed to water TGP of 1.10 atms with a $\mathrm{pO}_{2}$ of $170 \mathrm{mmHg}$. 


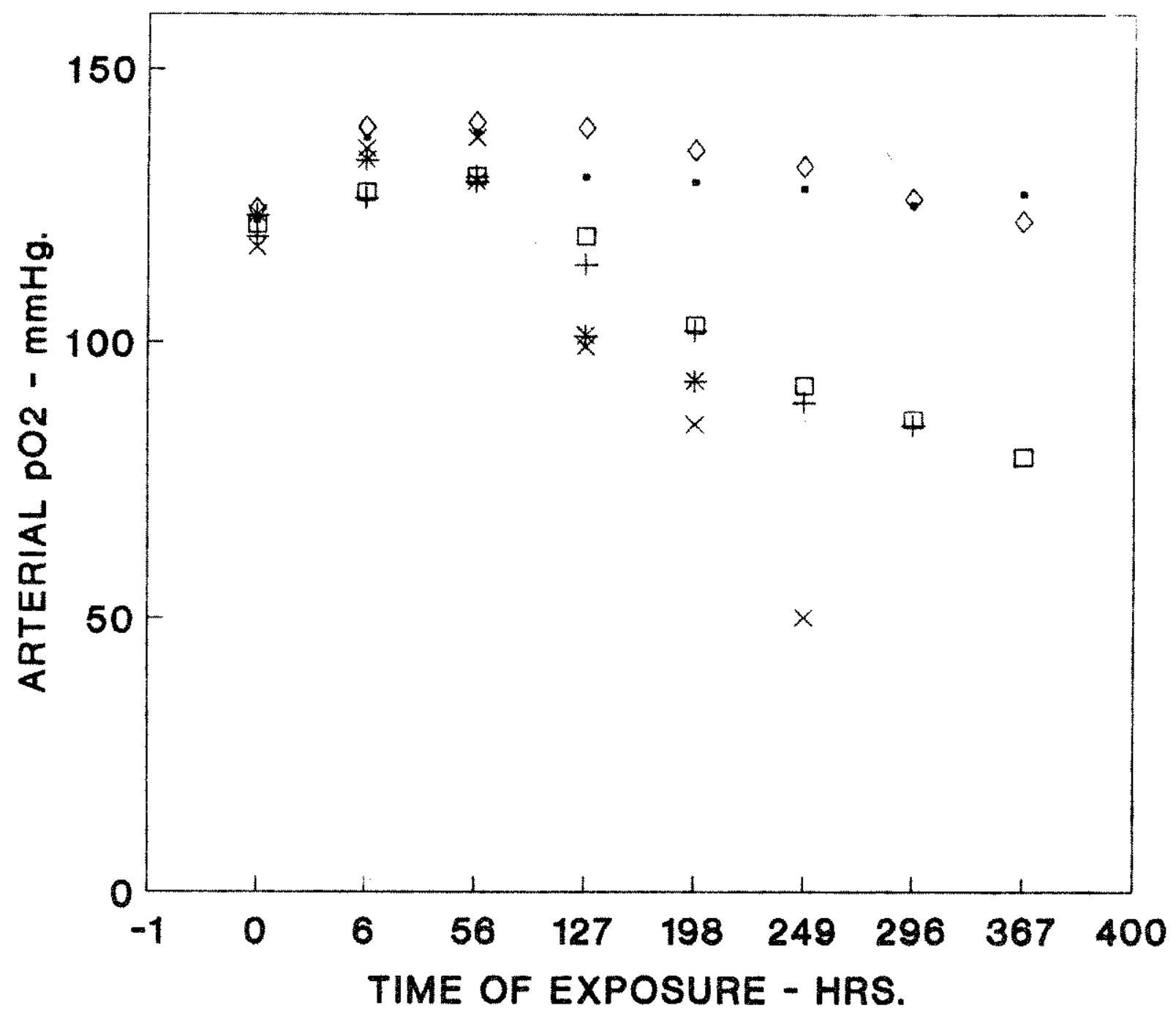

- FISH 1

+ FISH 2

* FISH 4

FISH 4

$\times$ FISH 5

$\diamond$ FISH 6

Figure 2.1.22. Variation in arterial blood $\mathrm{pO}_{2}$ with time in rainbow trout exposed to water TGP of 1.12 atms with a $\mathrm{pO}_{2}$ of $175 \mathrm{mmHg}$. 


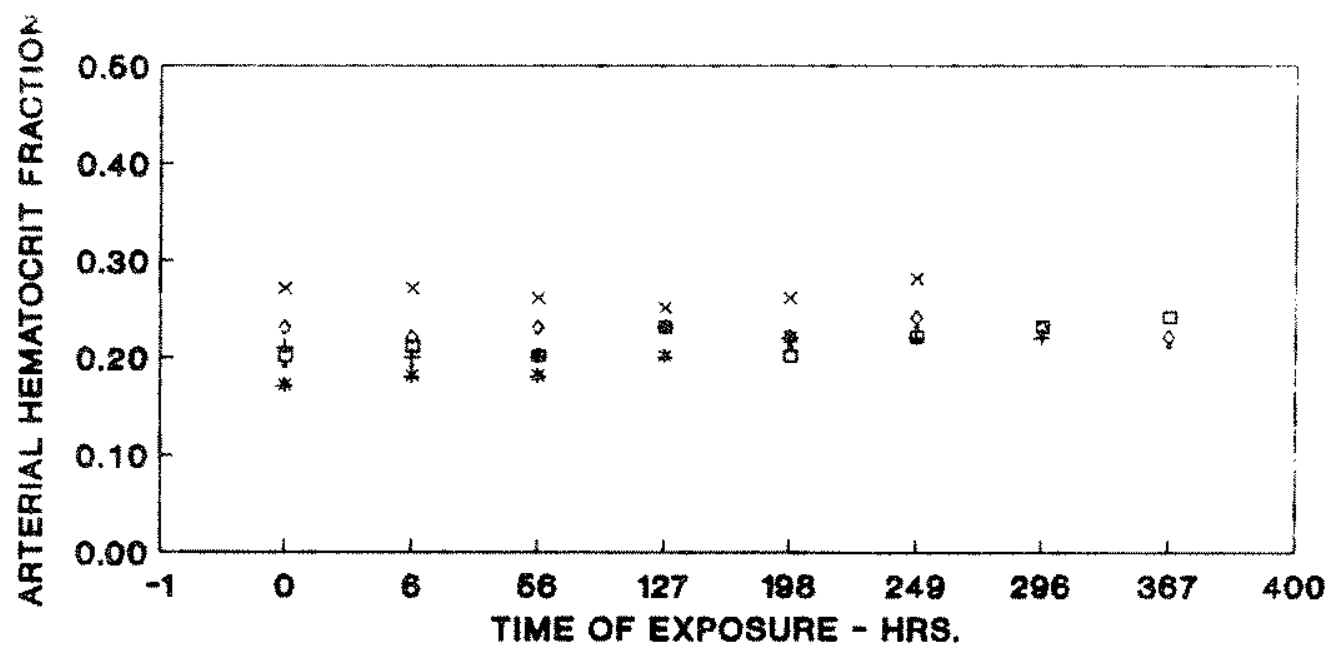

- FISH 1

+ FisH 2

* FISH 3

- FISH 4

$\times$ F18H 6

- FI8H $O$

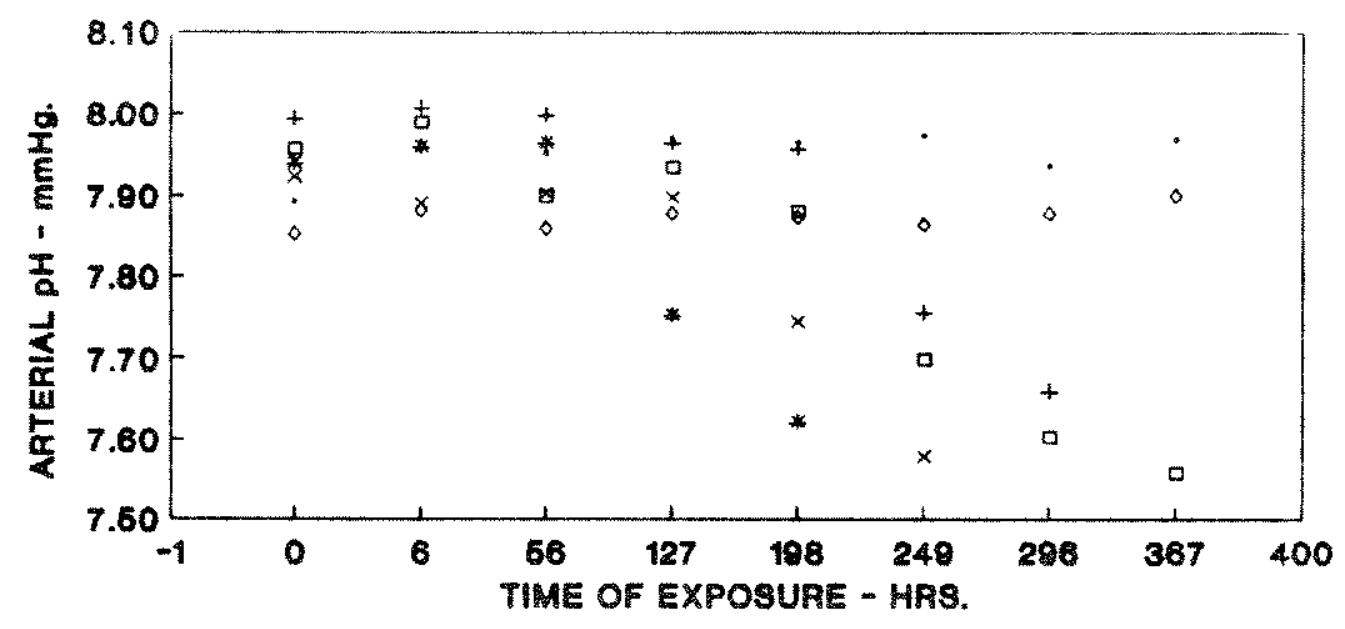
- FISH 1
+ FISH 2
* FISH 3
- FISH 4
$\times$ FISH 6
- FISH 8

Figure 2.1.23. Arterial blood hematocrit and $\mathrm{pH}$ variation with time in rainbow trout exposed to water TGP of 1.12 atms with a $\mathrm{pO}_{2}$ of $175 \mathrm{mmHg}$. 
arterial hematocrit did not change in any of the cannulated fish. Both cannulated and uncannulated fish that died showed external blisters on the opercula and fins, but the degree varied considerably from fish to fish (Figure 2.1.24). All but fish 1 had extracorporeal bubbles between gill lamellae and blisters in the buccal cavity (e.g. Figure 2.1.25). The bubbles shown in Figure 2.1.25 are typical of those assigned a number one severity level and appear to be randomly distributed throughout the primary and secondary lamellae. Those between secondary lamellae were persistent and difficult to dislodge with a needle. There was no evidence of intravascular bubbles in these gill lamellae. The same was true for all fish examined in this series. Figure 2.1.26 shows large blisters on the upper part of the mouth of one of the experimental fish tested at a TGP of 1.12 atms and Figure 2.1 .27 (same fish) shows blisters lining the entrances to the gills. These blisters (severity level 3) were of a size and number that would suggest interference with water flow to the gills. During first $6 \mathrm{~h}$ of the experiment, arterial $\mathrm{po}_{2}$ was elevated over pre-exposure levels. This reflects the higher levels of dissolved oxygen in supersaturated water. This observation was common to all experiments where experimental water $\mathrm{pO}_{2}$ was higher than pre-exposure holding water.

Series $4--$ water $T G P=1.15$ atms; $\mathrm{pO}_{2} \mathrm{~W}=183 \mathrm{mmHg}:$ In this series cannulated fish had varying rates of declining arterial $\mathrm{pO}_{2}$ (Figure 2.1.28). At the time of measurement, none of the levels appeared acute; however, all but one of the cannulated fish and one of the uncannulated fish died during the experiment. All fish showed signs of GBT and, as in experimental 


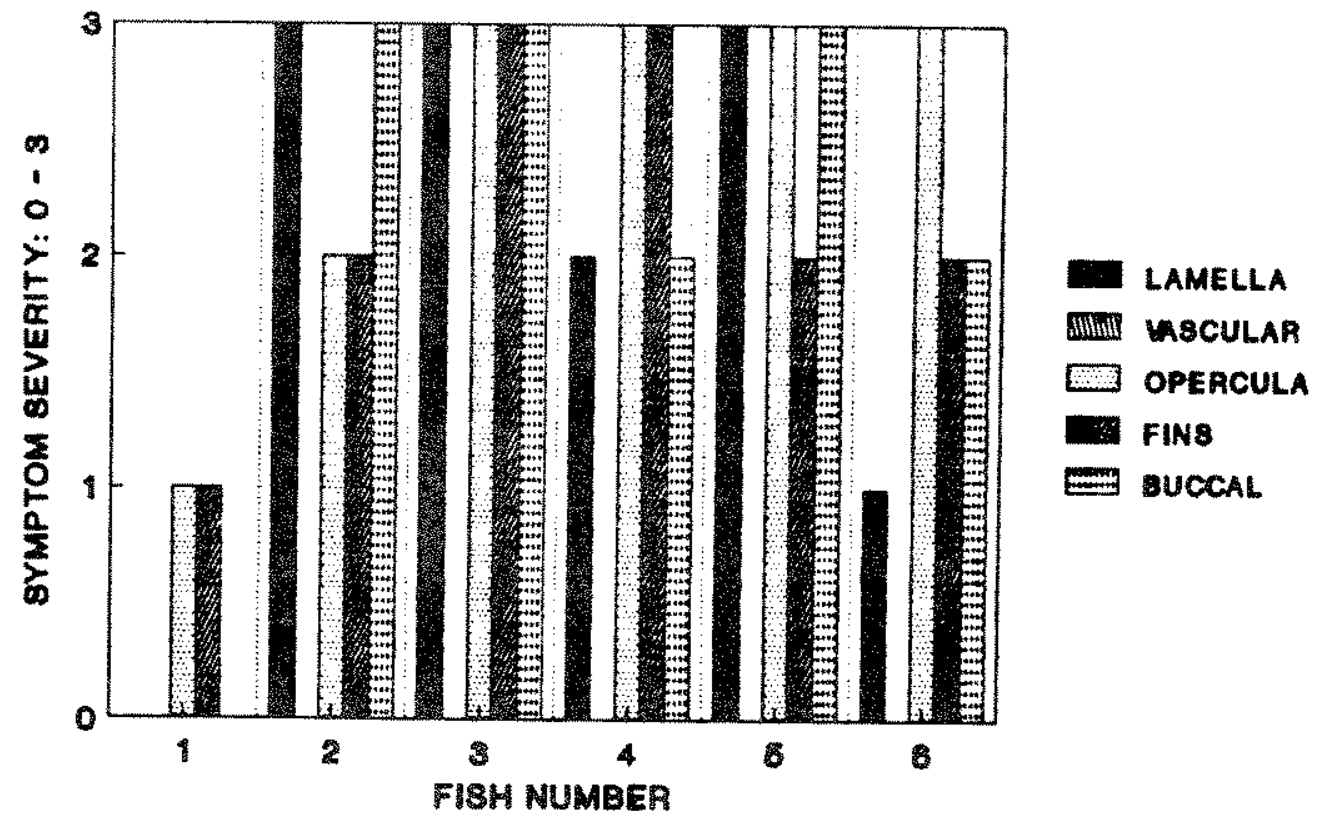

BEVERITY OF SYMPTOMS AT DEATH WTER TOP - 12 Atms. DOZW - 176 mMHg.
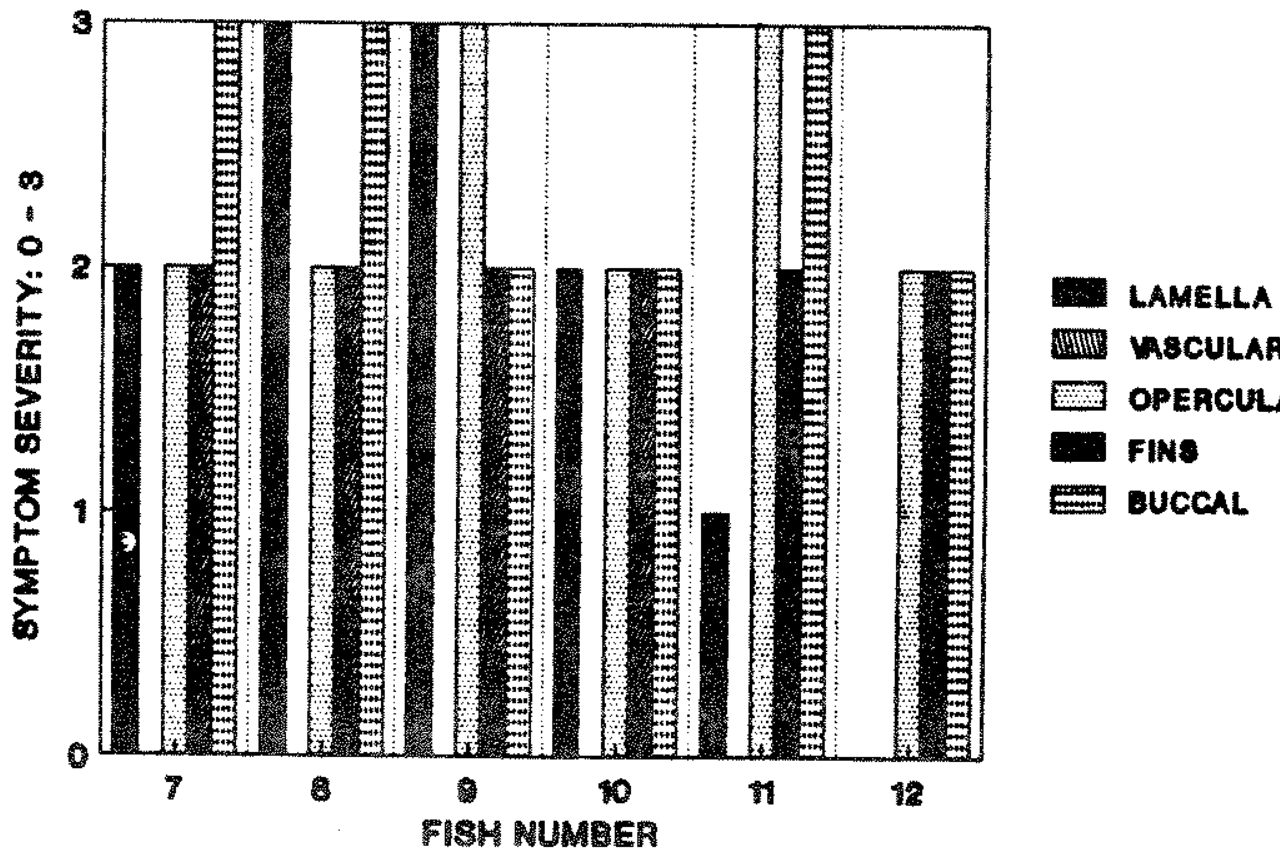

ATIII MSCULAA

OPERCULA

FINe

BUCCAL

Figure 2.1.24. Relative severity of gas bubble trauma symptoms at time of death of six cannulated $(1-6)$ and six noncannulated (7-12) trout exposed to water TGP of 1.12 atms with a $\mathrm{pO}_{2}$ of $175 \mathrm{mmHg}$. 


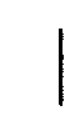




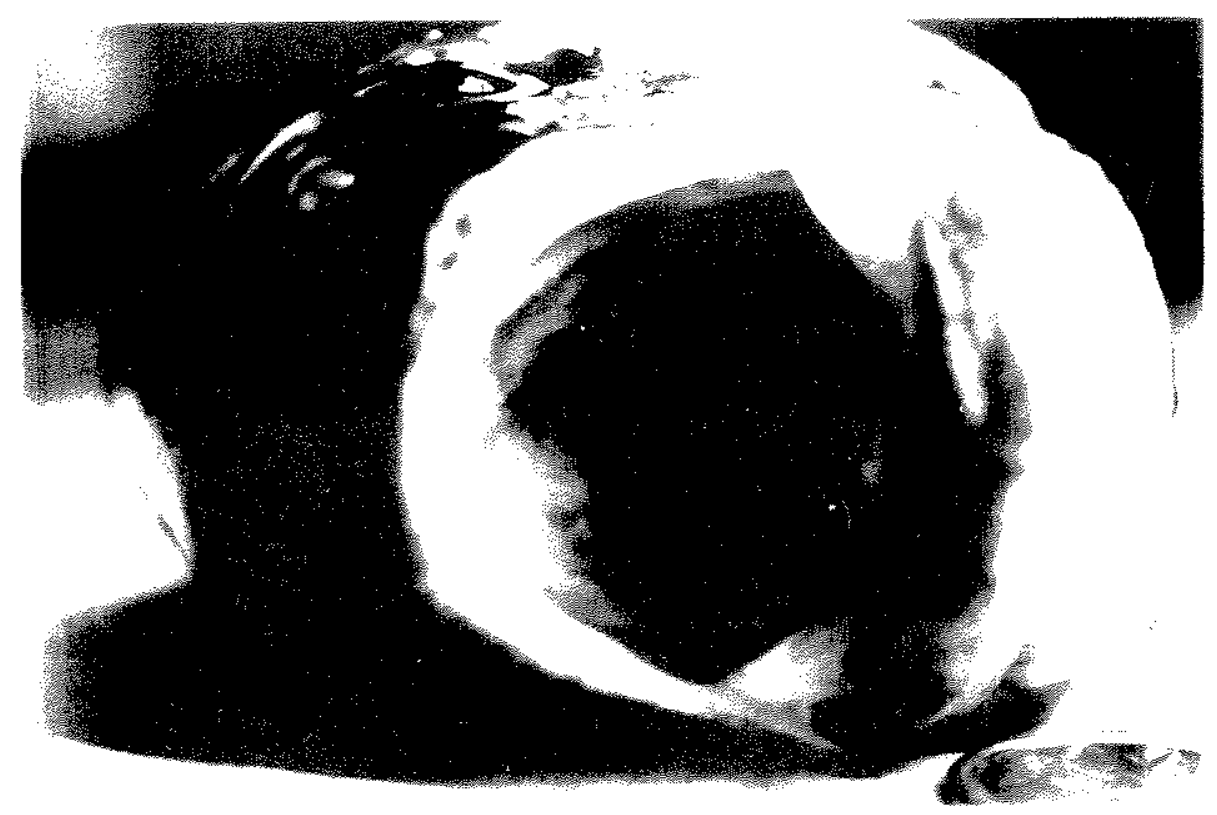

Figure 2.1.26. Subdurmal bubbles on the upper part of the mouth of a rainbow trout exposed to TGP of 1. 12 atms.

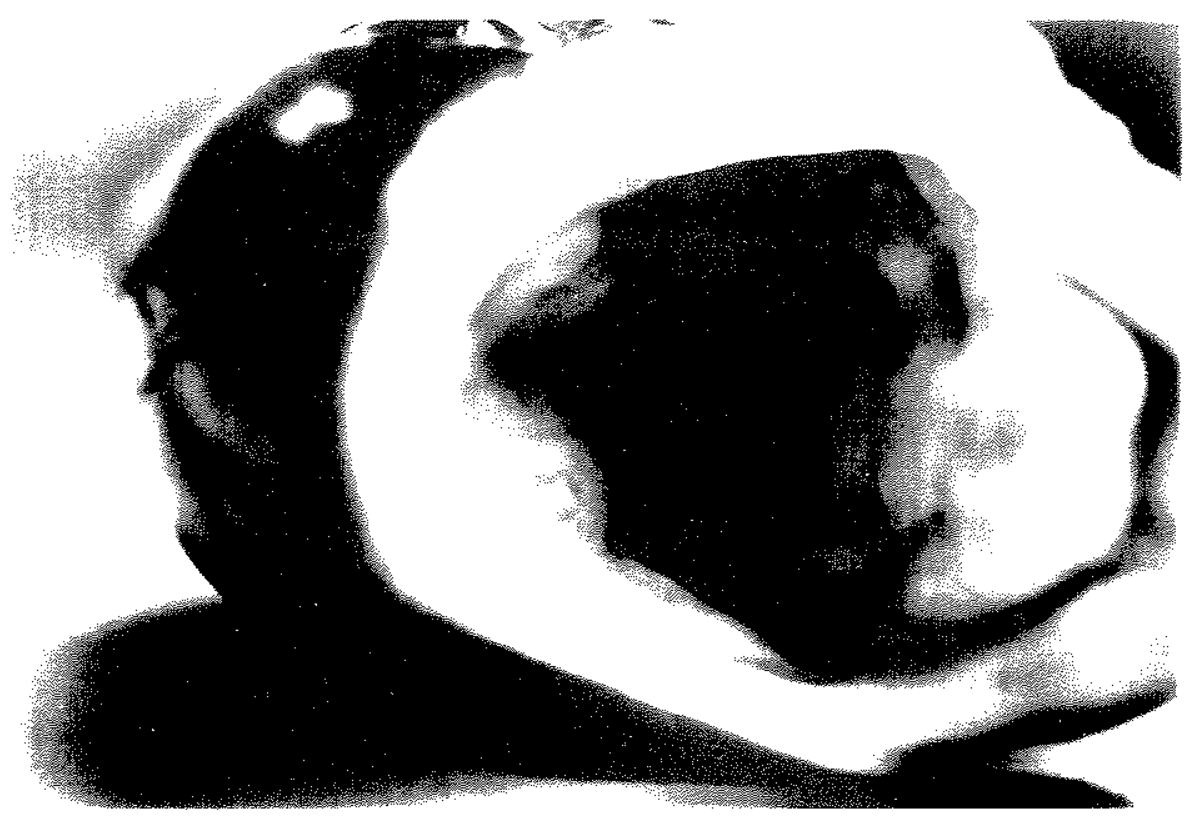

Figure 2.1.27. Subdurmal bubbles lining the entrances to the gills of a rainbow trout exposed to TGP of 1.12 atms. 

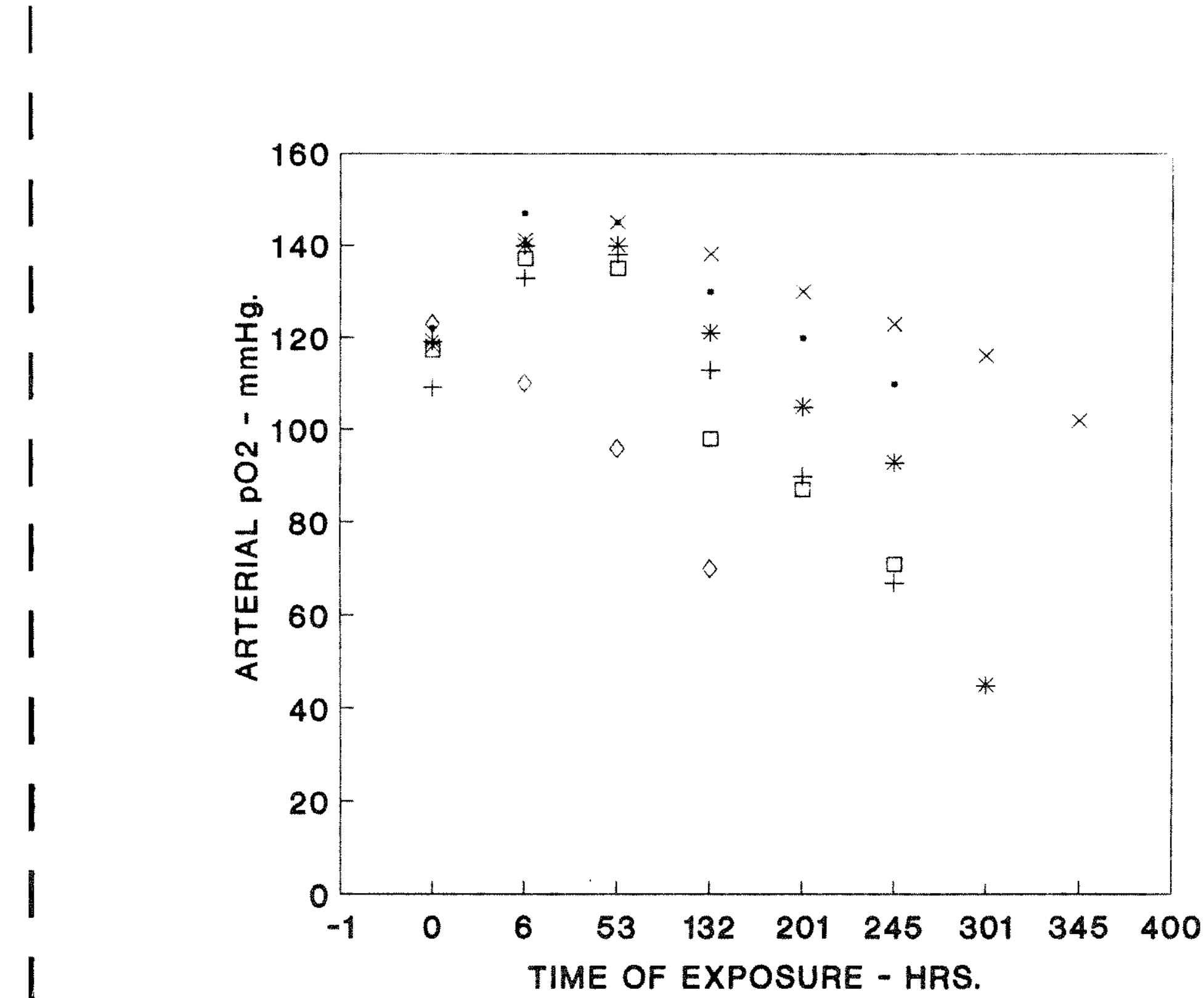

- FISH 1

+ FISH 2

* FISH 3

ㅁ $\mathrm{FISH} 4$

$\times$ FISH 5

$\diamond$ FISH 6

Figure 2.1.28. Variation in arterial blood $\mathrm{pO}_{2}$ with time in rainbow trout exposed to water TGP of 1.15 atms with a $\mathrm{pO}_{2}$ of $183 \mathrm{mmHg}$. 
Series 3, time to mortality was correlated with severity of symptoms. All cannulated fish showed a decline in arterial pH (Figure 2.1.29), which corresponded closely to declining $\mathrm{pO}_{2}$. Extracorporeal bubbles were present in varying degrees throughout the gills of all fish. Blood hematocrit varied little in cannulated fish except for a $67 \%$ increase in Fish No. 3. Fishes 4 and 10 were the first to show signs of intravascular bubble growth in gill arteries (Figure 2.1.30): however, times to mortality were not much different from those for fish without vascular bubbles. Microscope photographs taken of gill lamellae from some of these fish and from fish in series at higher levels of TGP (Figures 2.1.31 and 2.1.32) show vascular bubbles in the filamental arteries of the primary lamellae. of all the gill lamellae examined microscopically, only one fish, exposed to a water TGP of 1.19 atms, showed the presence of bubbles in the secondary lamellae. These bubbles had grown into the region from the filamental arteries of the primary lamellae. With few exceptions, bubbles appear to start at the distal ends of the filamental arteries and grow toward the base of the primary lamellae.

Series 2 - water $T G P=1.17$ atms, $\mathrm{pO}_{2} \underline{\mathrm{W}}=195 \mathrm{mmHg}:$ Every fish in series 2 experiments had intravascular bubble growth in gill lamellae (Figure 2.1.33). Increases in hematocrit occurred in all cannulated fish (Figure 2.1.34). Arterial blood pH and $\mathrm{pO}_{2}$ dropped dramatically (Figures 2.1 .34 and 2.1 .35 , respectively), and all fish died during the experiment (Figure 2.1.18). 

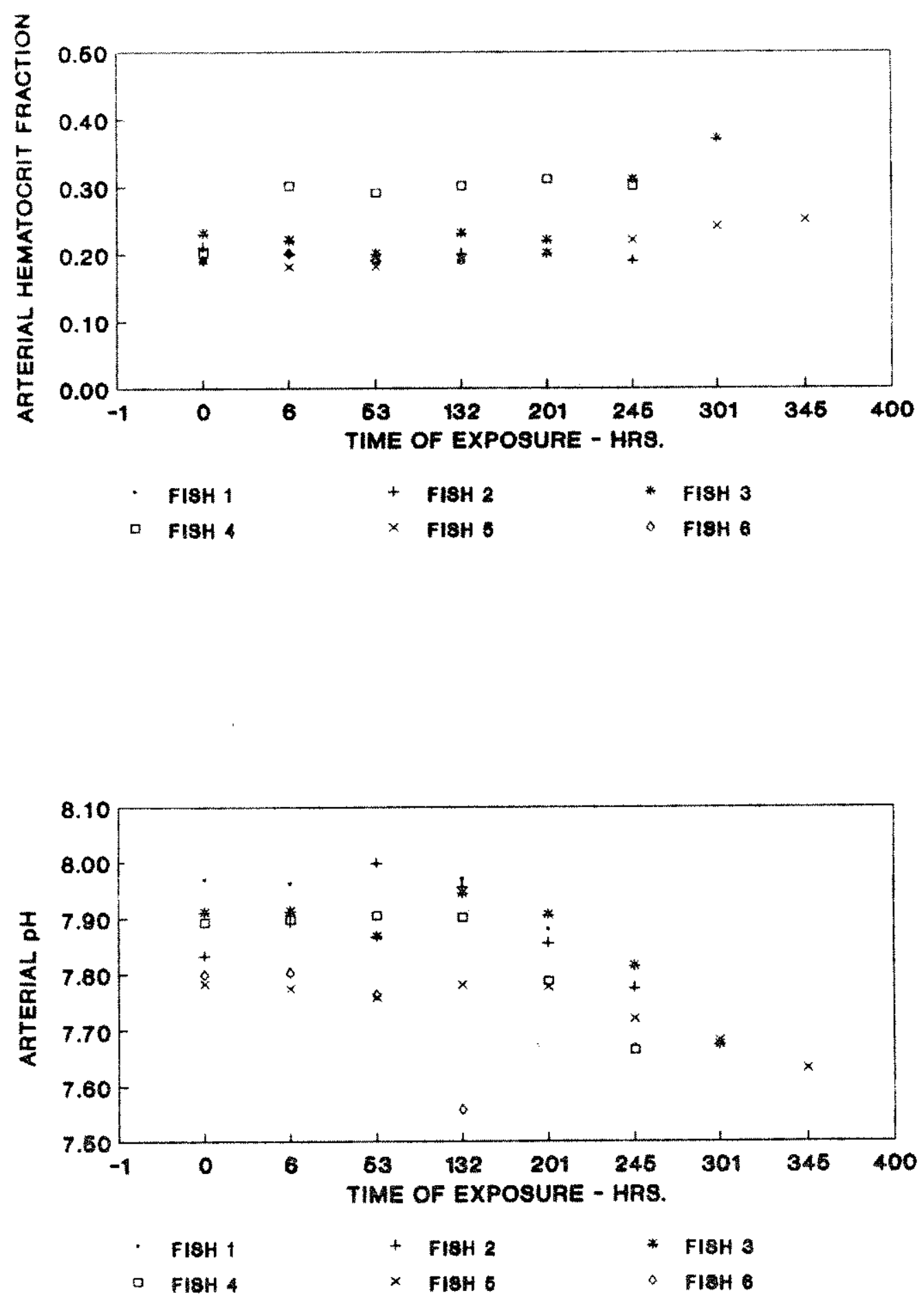

Figure 2.1.29. Arterial blood hematocrit and $\mathrm{pH}$ variation with time in rainbow trout exposed to water TGP of 1.15 atms with a $\mathrm{pO}_{2}$ of $183 \mathrm{mmHg}$. 

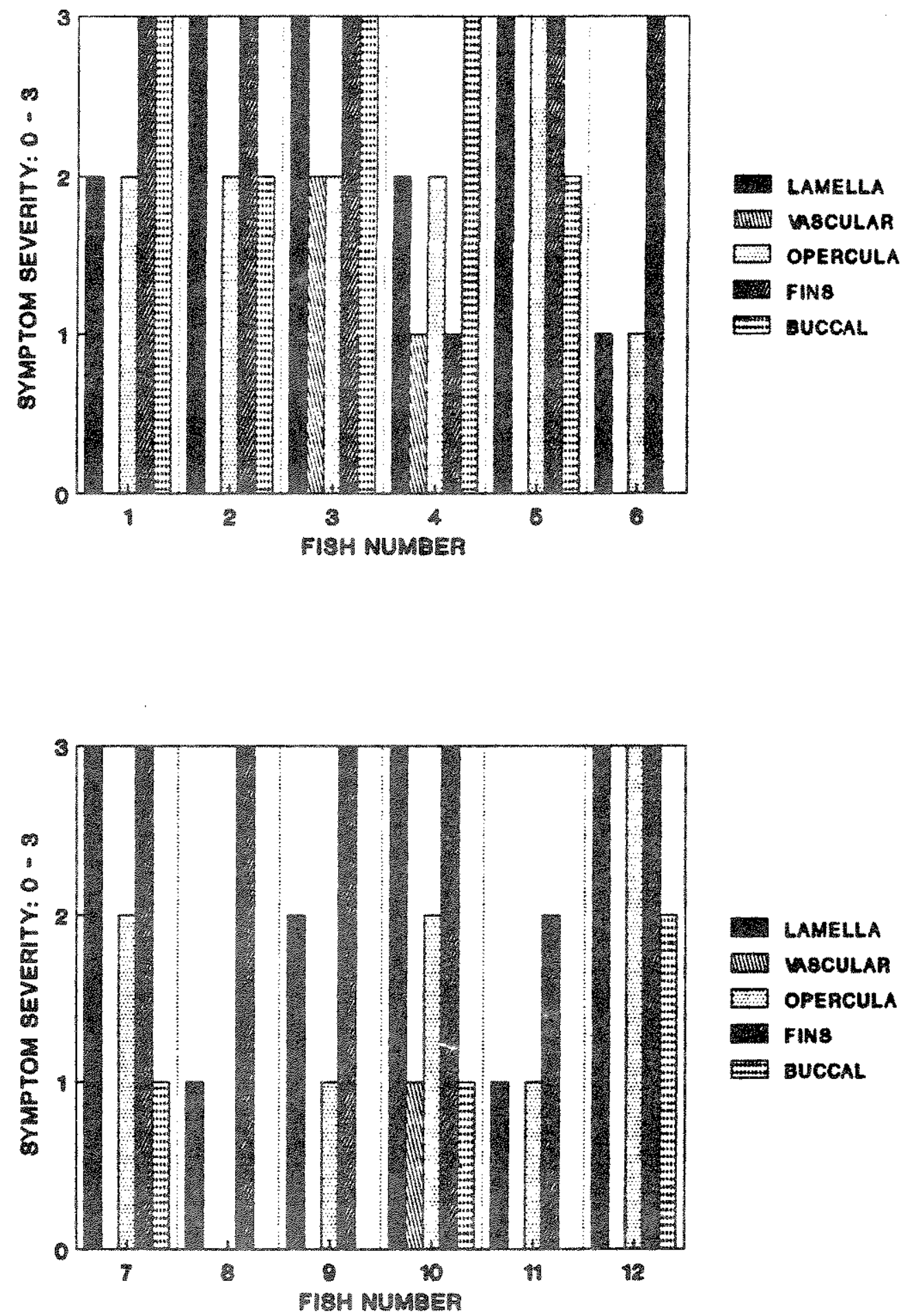

Figure 2.1.30. Relative severity of gas bubble trauma symptoms at time of death of six cannulated (1-6) and six noncannulated $(7-12)$ rainbow trout exposed to water TGP of 1.15 atms with a $\mathrm{pO}_{2}$ of 183 mmig. 

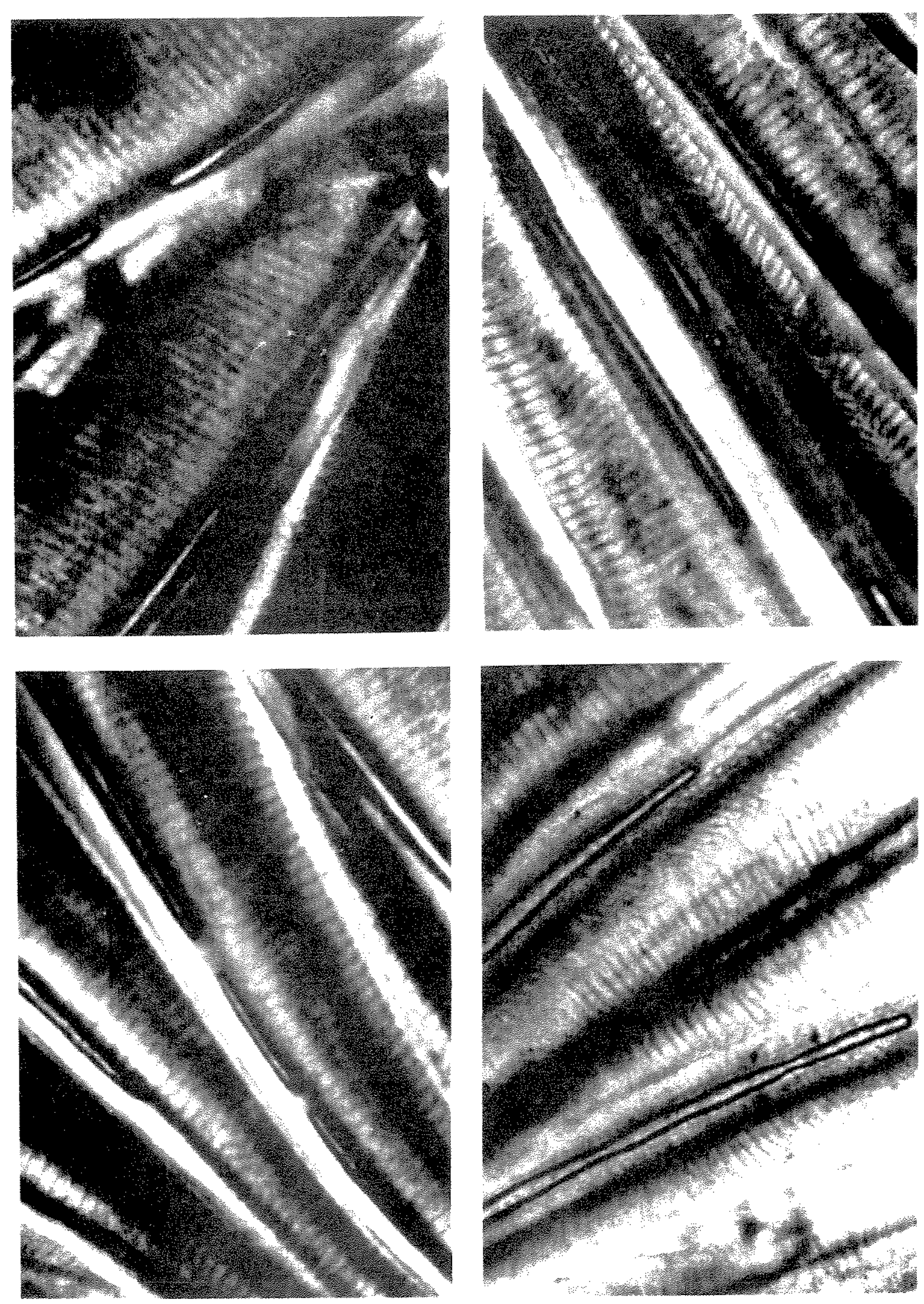

Figure 2.1.31. Vascular bubbles in filamental arteries of primary gill lamellae of rainbow trout. 

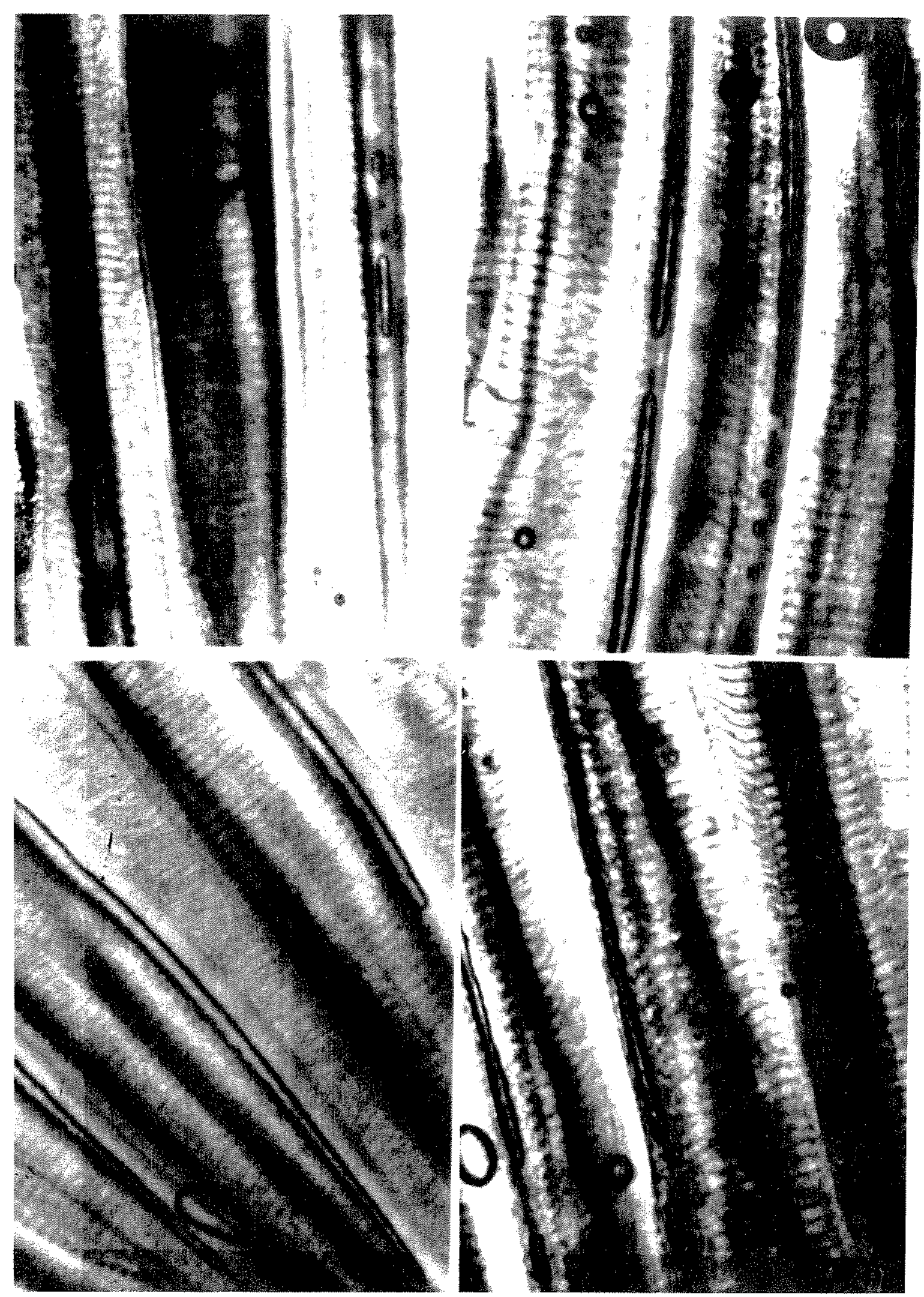

Figure 2.1.32. Vascular bubbles in filamental arteries of primary gill lamellae of rainbow trout. 

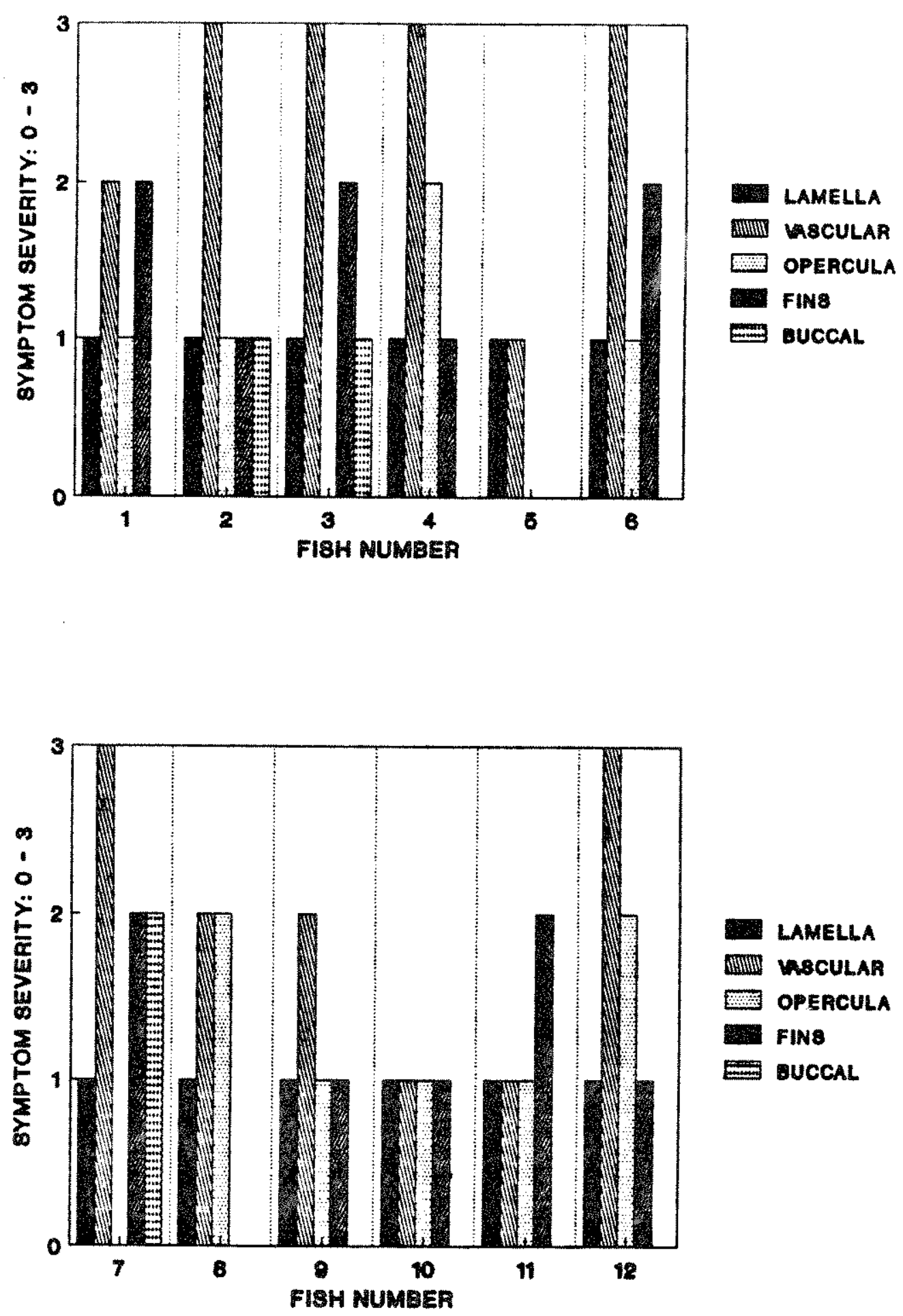

Figure 2.1.33. Relative severity of gas bubble trauma symptoms at time of death of $s i x$ cannulated $(1-6)$ and six noncannulated (7-12) rainbow trout exposed to water TGP of 1.17 atms with a $\mathrm{pO}_{2}$ of $195 \mathrm{mmHg}$. 


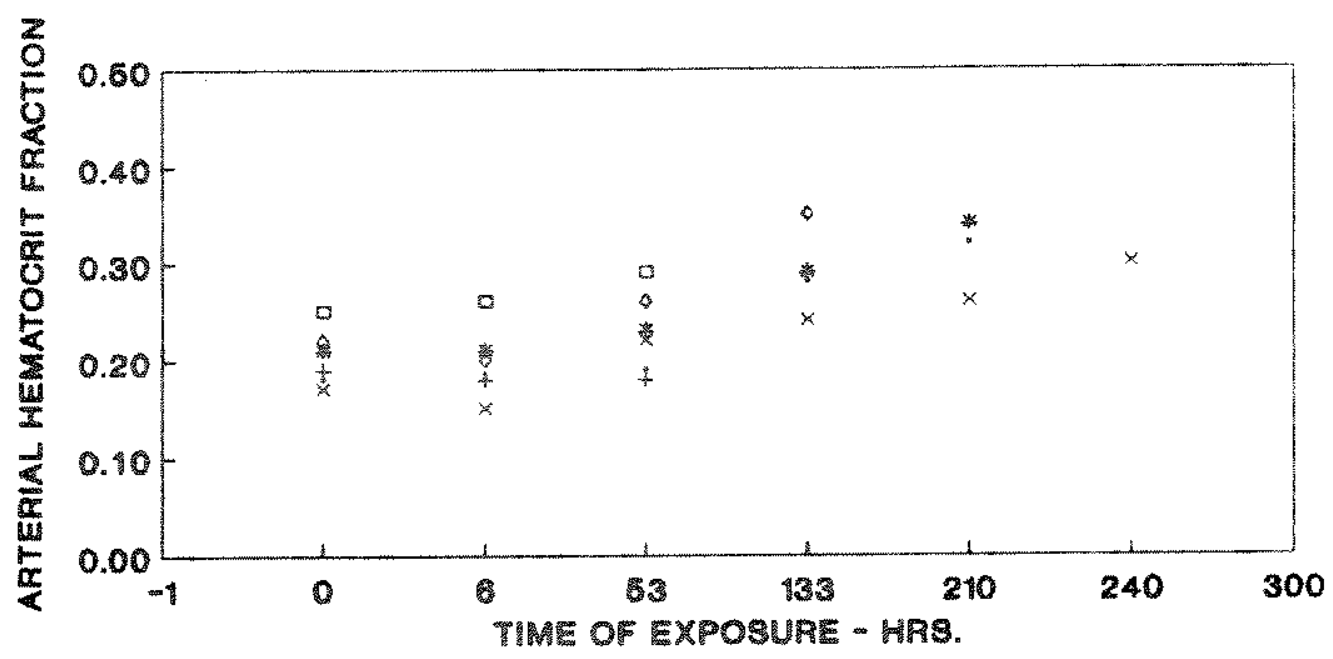

\begin{tabular}{|c|c|c|c|c|}
\hline Fas? & + & Froh 2 & $*$ & FI8H 3 \\
\hline 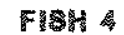 & $x$ & 5183 5 & 0 & $\mathrm{FlBH}$ \\
\hline
\end{tabular}

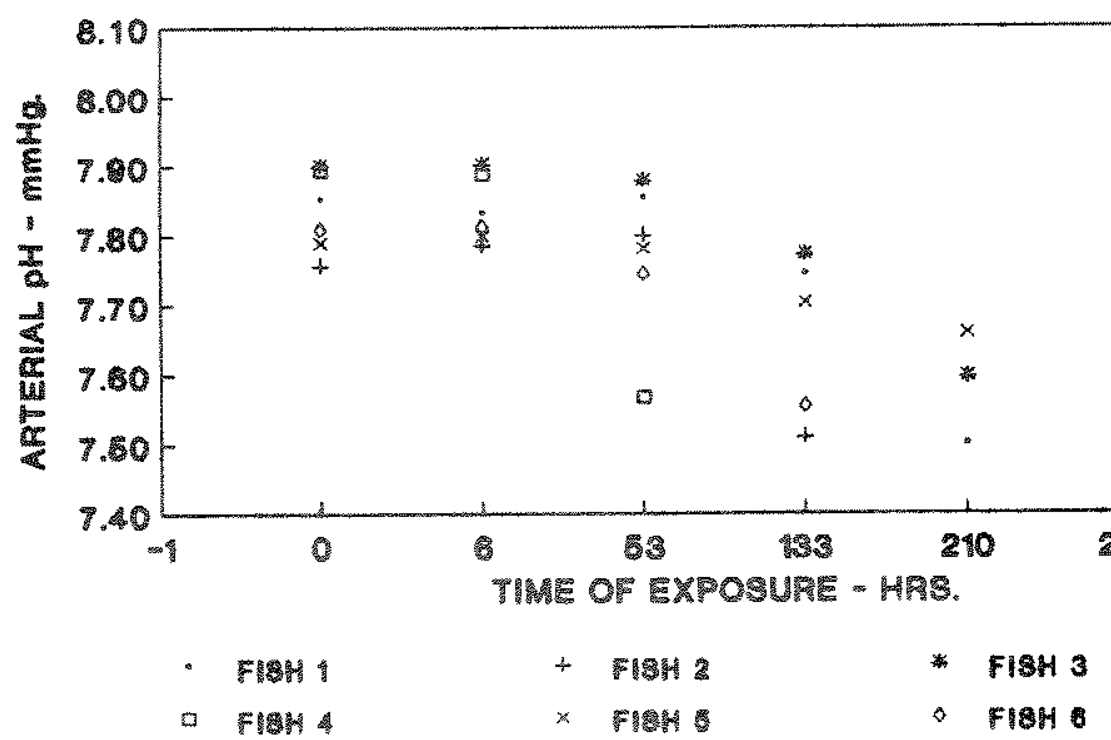

Figure 2.1.34. Arterial blood hematocrit and $\mathrm{pH}$ variation with time in rainbow trout exposed to water TGP of 1.17 atms with a $\mathrm{pO}_{2}$ of $195 \mathrm{mmHg}$. 


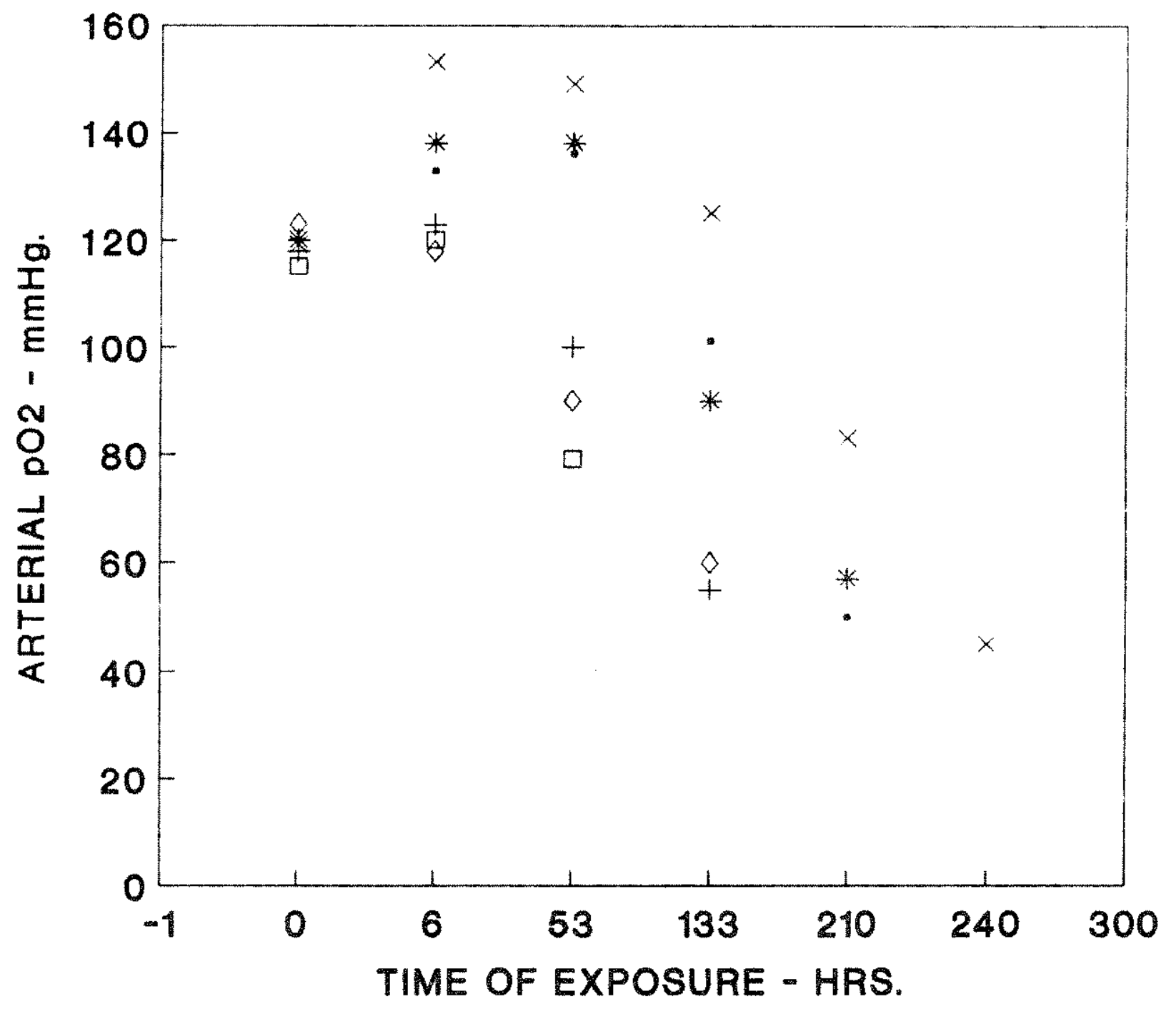

FISH 1

$+\quad$ FISH 2

* FISH 3

FISH 4

$\times$ FISH 5

FISH 6

Figure 2.1.35. Variation in arterial blood $\mathrm{po}_{2}$ with time in rainbow trout exposed to water TGP of 1.17 atms with a $\mathrm{pO}_{2}$ of $195 \mathrm{mmHg}$. 
External signs of GBT, such as blistering of tissue, were not as pronounced as in the experiments at lower TGP levels. Presumably, this is because fish died from other causes before these symptoms could become well developed. Also, the number of extracorporeal bubbles wexe larger and fewer than at lower levels of TGP.

Series 5 - Water TGP $=1.19$ atms; $\mathrm{pO}_{2} \mathrm{~W}=201 \mathrm{mmHg}:$ At a TGP of 1.19 atms, intravascular bubble growth was rapid and the time of survival of all fish declined dramatically (Figure 2.1.18). Arterial $\mathrm{pO}_{2}$ declined rapidly for all cannulated fish (Figure 2.1.36) and presumably for the uncannulated fish as well. There also was a high level of intravascular bubble formation in the gills of all fish (Figure 2.1.37). Arterial pH dropped and hematocrit rose more rapidly than in any of the other experiments (Figure 2.1.38). The trend in fewer but larger sized extracorporeal bubbles in the gills continued. Few external blisters were present in these fish - presumably because of the short time of survival.

Series A, B, C, and series 4-- Water TGP $=1.15$ atms; $\mathrm{pO}_{2} \mathrm{w}$ $=100,125$ and $183 \mathrm{mmHg}):$ At constant water TGP (1.15 atms), increased water $\mathrm{pO}_{2}$ resulted in increased time to mortality of experimental trout (Figure 2.1.39). Furthermore, a marked transition occurred between $\mathrm{a} \mathrm{pO}_{2}$ of 100 and $125 \mathrm{mmHg}$.

These results are similar to those reported for coho salmon at a water TGP of 1.19 atms (Rucker 1975a). In that case, the transition in time to mortality occurred at a water $\mathrm{po}_{2}$ of 249 $275 \mathrm{mmHg}$. Rucker concluded that the strong transition in time to mortality represents a threshold for the cause of mortality. our 


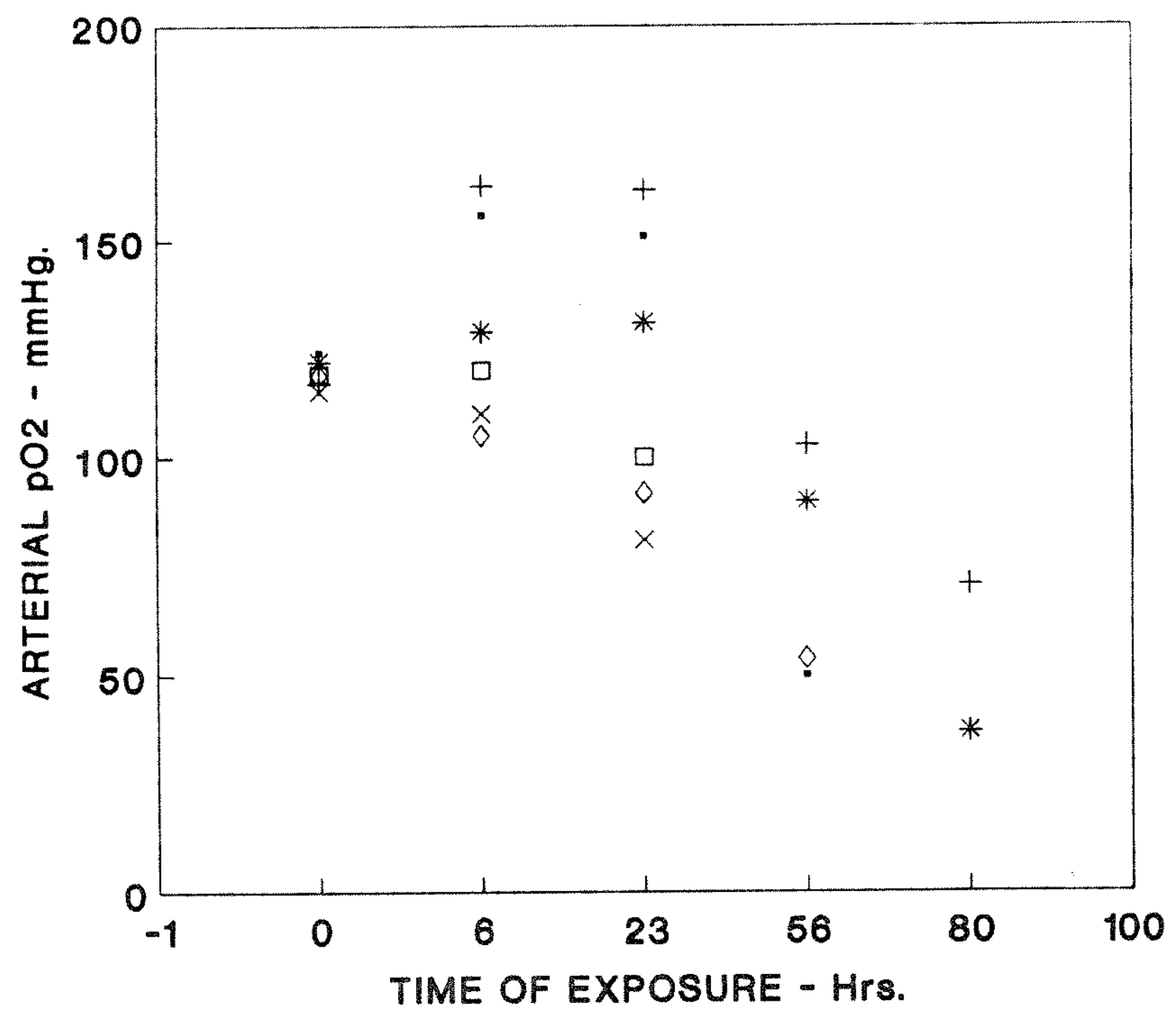

$\begin{array}{lll}- \text { FISH }_{1} & + \text { FISH } 2 & * \text { FISH } 3 \\ \square \text { FISH } 4 & \times \text { FISH } 5 & \diamond \text { FISH } 6\end{array}$

Figure 2.1.36. Variation in arterial blood $\mathrm{pO}_{2}$ with time in rainbow trout exposed to water TGP of 1.19 atms with a $\mathrm{pO}_{2}$ of $201 \mathrm{mmHg}$. 


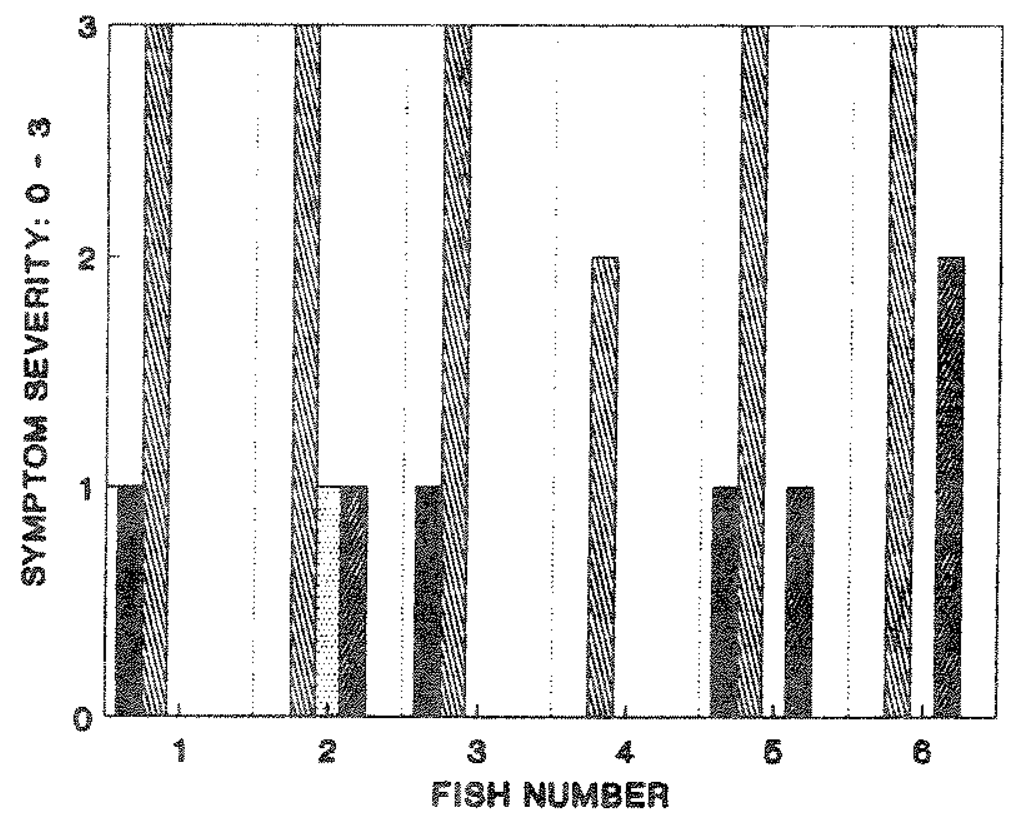

WA LAMELLA

DiIIII VASCULAR

OP OPERCULA

FINB

SUCCAL

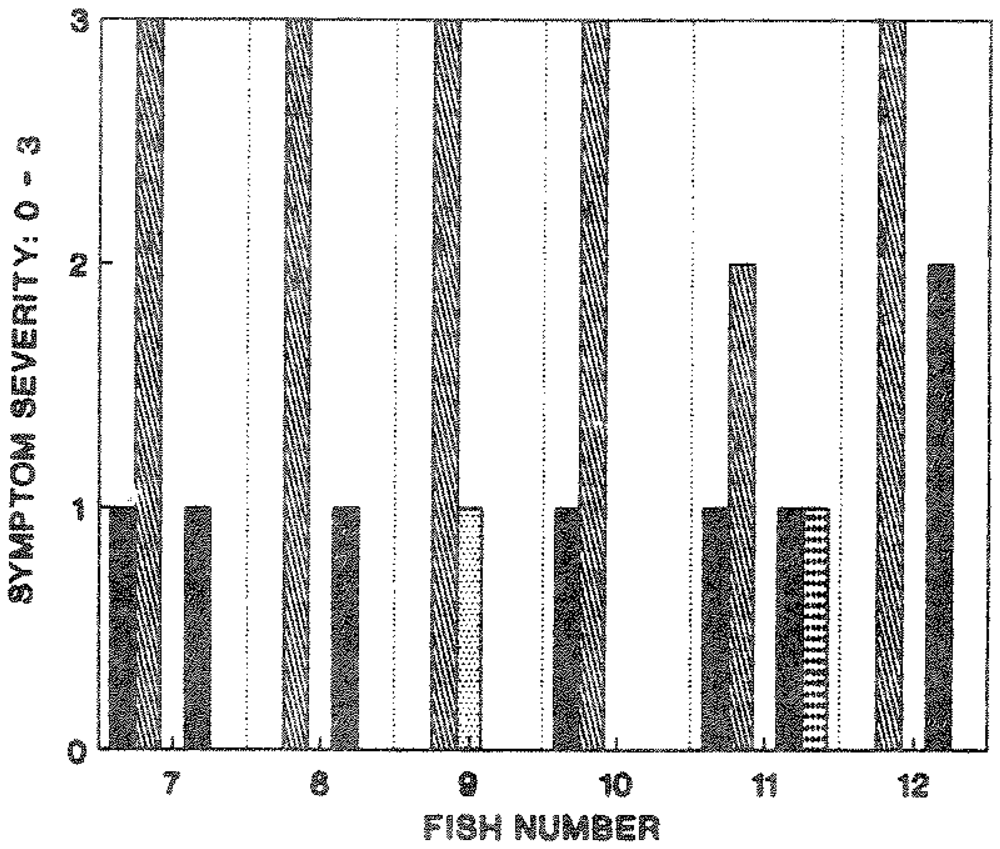

WAMELLA

(1)iII SCULAR

OPERCULA

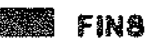

BUCCAL

Figure 2.1.37. Relative severity of gas bubble trauma symptoms at time os death of six cannulated (1-6) and $s i x$ noncannulated $(7-12)$ rainbow trout exposed to water TGP of 1.19 atms with a $\mathrm{pO}_{2}$ of $201 \mathrm{mmHg}$. 


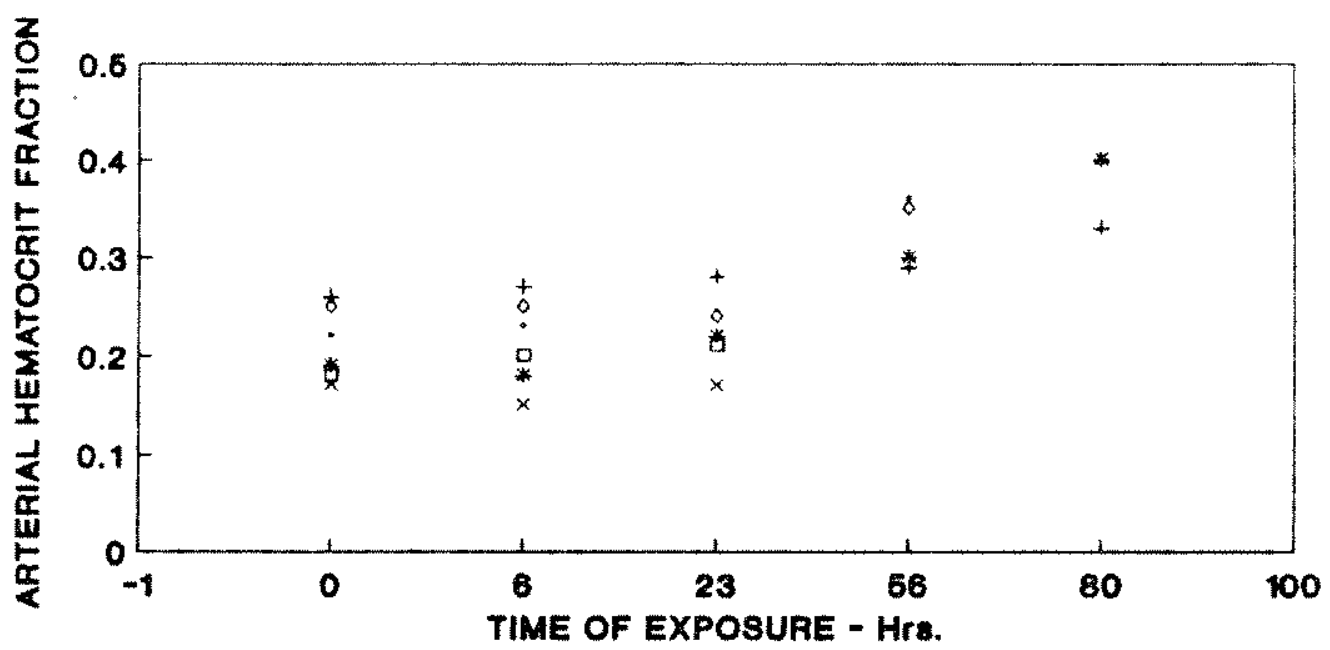

- F18H 1

$+\mathrm{F} 18 \mathrm{H} 2$

* FI8H 3

- $\mathrm{F} 18 \mathrm{H} 4$

$\times$ FI8H 8

- F18H 6

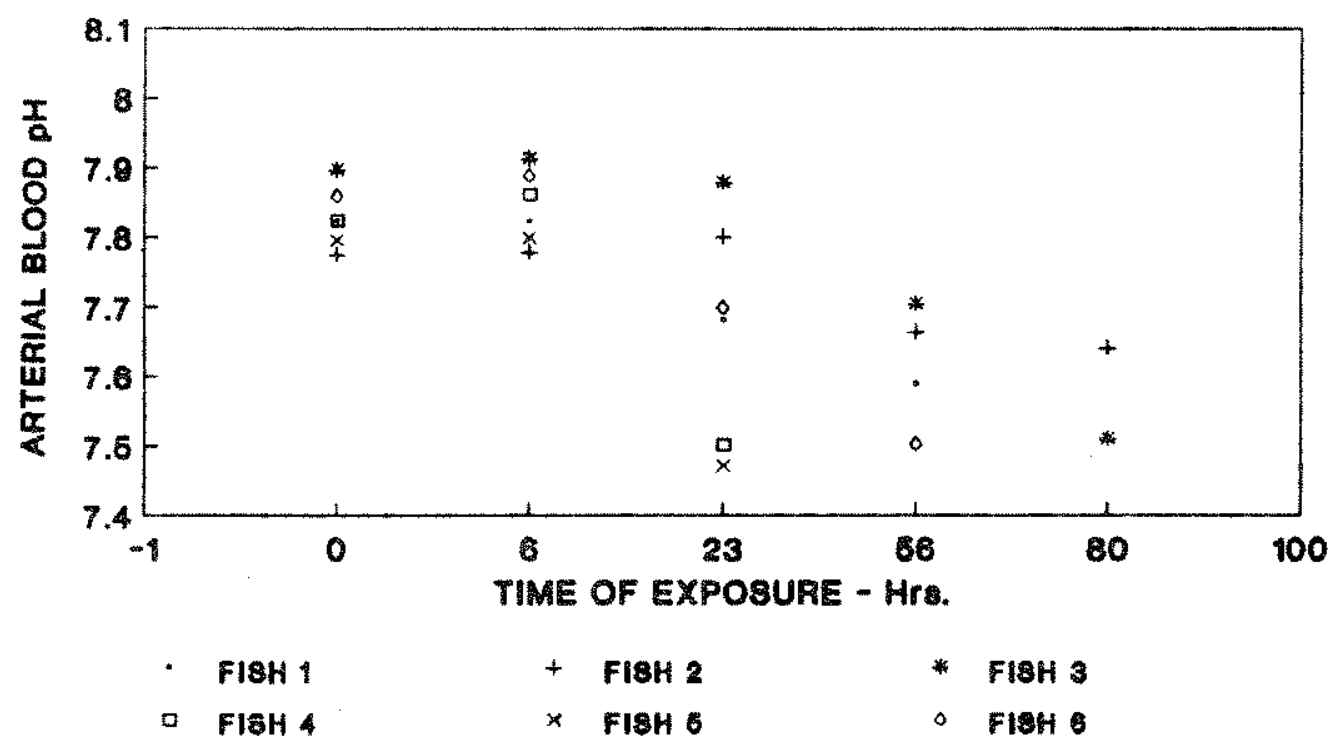

Figure 2.1.38. Arterial blood hematocrit and $\mathrm{pH}$ variation with time in rainbow trout exposed to water TGP of 1.19 atms with a $\mathrm{pO}_{2}$ of $201 \mathrm{mmHg}$. 


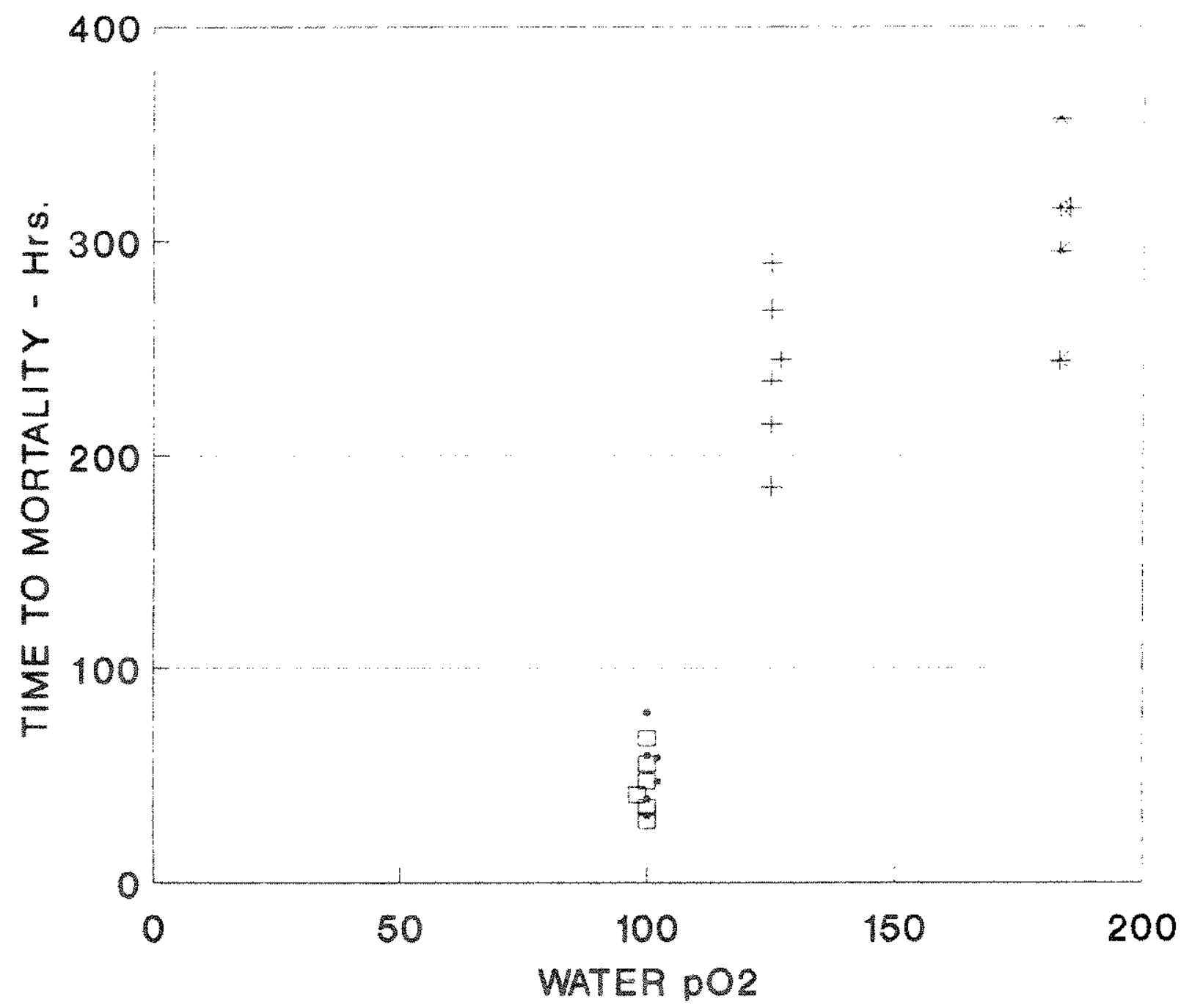

+ Series C

- Series A
$*$ Series 4

$\square$ Series $B$

Note: One lish in series 4 did not die in 400 hours.

Figure 2.1.39. Intuence of increasing levels of water $\mathrm{pO}_{2}$ on time to mortality of rainbow trout while maintaining a constant TGP of 1.15 atms. 
data (Table 2.1 .2 ) confirm that the transition in time to mortality relates well with the presence of intravascular gill bubbles.

Table 2.1.2. Survey of intravascular gill bubbles at death of trout for experimental series A, B, C and 4 .

FISH NO. BUBBLES?a po $_{2}^{\mathrm{b}}$ FISH NO. BUBBLES? $\mathrm{pO}_{2}$

\begin{tabular}{|c|c|c|c|c|c|}
\hline $\mathrm{A} 1$ & + & 100 & $\mathrm{Cl}$ & - & 125 \\
\hline $\mathrm{A} 2$ & + & 100 & $\mathrm{C} 2$ & - & 125 \\
\hline A 3 & + & 100 & $c 3$ & - & 125 \\
\hline A4 & + & 100 & $\mathrm{C} 4$ & + & 125 \\
\hline A 5 & - & 100 & $\mathrm{C} 5$ & - & 125 \\
\hline A6 6 & + & 100 & $\mathrm{C} 6$ & - & 125 \\
\hline BI & - & 100 & $4-7$ & - & 183 \\
\hline $\mathrm{B} 2$ & + & 100 & $4-8$ & - & 183 \\
\hline B3 & + & 100 & $4-9$ & - & 183 \\
\hline B4 & + & 100 & $4-10$ & + & 183 \\
\hline B5 & + & 100 & $4-11$ & - & 183 \\
\hline B6 & + & 100 & $4-12$ & - & 183 \\
\hline
\end{tabular}

$\mathrm{a}+$ indicates bubbles present; - indicates bubbles absent.

b $\mathrm{pO}_{2}$ in $\mathrm{mmHg}$. 


\subsubsection{1c Dilscussion:}

Experimental results confirm the existence of bubble growth thresholds and refine the definition. In addition, these results add to the understanding of bubble growth and the physiological response of fish to this growth.

Extracorporeal and Sub-dermal Bubble Growth Thresholds: Experimental studies showed that the 1.10 to 1.12 atms TGP threshold applies not only to sub-dermal bubble growth in external epithelium tissue, but also to the growth of extracorporeal bubbles between gill lamellae. It was not possible, however, to demonstrate that sub-dermal bubble growth in the mouth, extra-corporeal bubble growth in the gill lamellae, or the two acting in concert was the primary or contributing cause of mortality at the lower TGP levels. Data did show that, in this range of $\mathrm{TGP}$, arterial $\mathrm{pO}_{2}$ and $\mathrm{pH}$ declined with time of exposure, indicating a progressive blockage of the respiratory pathway. The only two apparent mechanical means for this blockage are the sub-dermal bubbles in the mouth and extracorporeal bubbles in the gills. Thus, there is at least a relationship between mortality, blockage of respiratory pathways and the two forms of bubble growth in this narrow range of water TGP.

We were not able to define the lower bubble growth threshold more precisely than the TGP range of 1.10 to 1.12 atms. This was due to the variability in the response of the trout. Because the time to mortality is quite long in this range of $T G P$, factors such as fish health, handling stress and water quality probably contributed to observed variability. Using this range (1.10 to 
1.12 atms) for the extracorporeal and sub-dermal bubble growth thresholds, the effective critical radius of nuclei, back calculated from Equation 4 , is $12-14 \mu \mathrm{m}$.

Intravascular Bubble Growth Thresholds: Results showed the existence of a threshold for intravascular bubble growth in fish exposed to supersaturated water. This conclusion is based primarily on the observation of bubbles in the gill vasculature and the substantial changes in hematocrit. A precise definition of this threshold, however, cannot be obtained due to the large variability in the response of the trout coupled with unknown variations in stress produced by sub-dermal and extracorporeal bubble growth. Experimental results showed the intravascular bubble growth threshold to be in the TGP range of 1.15 to 1.19 atms for water $\mathrm{pO}_{2}$ between 185 and $201 \mathrm{mmHg}$. If only uncannulated fish are considered, the threshold appears to be in a TGP range of 1.16 to 1.19 atms.

The dependence of intravascular bubble growth thresholds on water $\mathrm{pO}_{2}$ can be obtained by combining the thresholds indicated in the experimental series 1 through 5 with the data of Rucker (1975a); only data for uncannulated fish were used (Figure 2.1.40). We have also plotted the theoretical predictions of Equation 4 for various sizes of nucleation sites. The theoretical curves are for an oxygen uptake ratio of 0.79 , a water temperature of 5 to $12^{\circ} \mathrm{C}$ and sea level atmospheric pressure. The experimental thresholds relate well to theoretical thresholds based on an effective nuclei radius of 12 to $14 \mu \mathrm{m}$. This observation is important in terms of the size of arteries in which the bubbles were found. This radius is approximately twice 


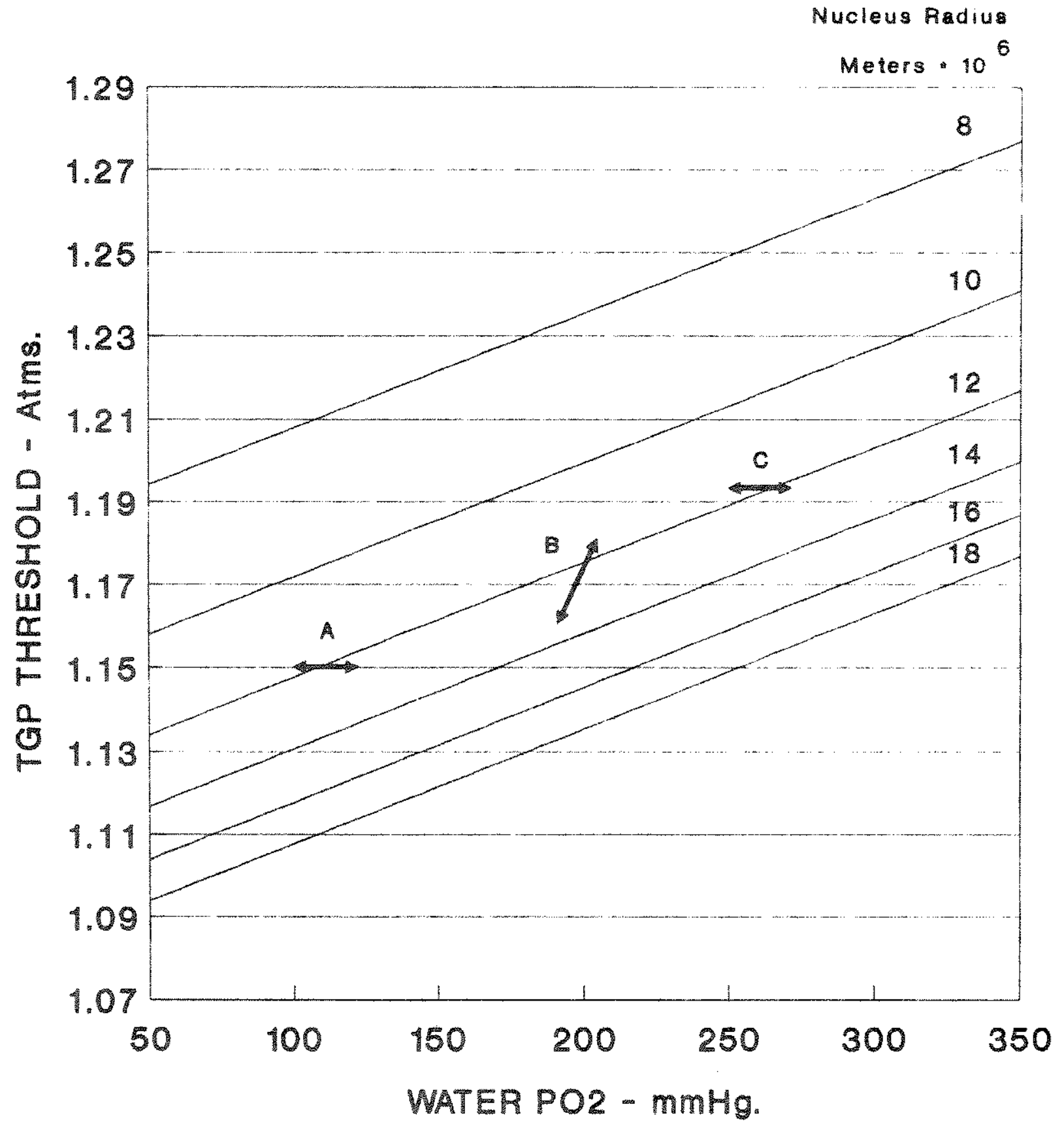

Water Depth $=0.0 \mathrm{M}$. F 0.79

Water Temp $=5-15$ deg. C.

IA 8 - Rainbow Troutl Fidler (1888)

Atmospheric Presure $760 \mathrm{mmHg}$. (c - Coho Salmonl Rucker (1975)

Figure 2.1.40. Total gas pressure thresholds for bubble growth in arterial blood of rainbow trout and coho salmon as a function of $\mathrm{pO}_{2}$ and nucleus radius. 
that of rainbow trout red blood cells (Heming 1984; Mott 1957 and Smith et al. 1952). Arteries of the secondary lamellae have characteristic widths comparable to the diameter of red blood cells. This implies that nuclei and bubbles could not exist in the secondary lamellae. As discussed above, with the exception of a single fish exposed to the highest level of TG?, all

lamellae examined had bubbles only in the filamental arteries (Figures 2.1 .31 and 2.1.32). In the one gill segment that had a bubble in a secondary lamellae, the origin was in the much larger filamental artery. Thus, the size of nuclei is consistent with the size of gill blood vessels in which bubbles were found. Bubbles probably also grow in other locations within the fish. In supplemental experimental studies, bubbles were found in muscle, heart and liver tissues. Smith (1988) and Nebeker et al. (1976) and Dawley and Ebel (1975) have also shown intravascular bubbles to be present in these organs. As with the gill vascular bubbles, the indicated size of the critical nuclei is larger than the characteristic dimensions of capillary beds. Thus, these nuclei would have to exist in vessels the size of arterioles or larger. Considering the variation of blood pressure and $\mathrm{pO}_{2}$ in the circulatory system, the most likely bubble locations are the arterioles just upstream of the capillary beds.

Bubble Growth at Low TGP: Unique physiological characteristics were observed with each form of bubble growth. In many cases these characteristics relate to time to mortality for each bubble growth threshold.

Sub-dermal Bubble Growth: This form of bubble growth took 
considerable time to develop at the lower levels of water TGP ( 1.12 to 1.15 atms). However, based on the size of bubbles, blisters in the buccal cavity appeax to grow more quickly than blisters formed on the opercular flaps or other external surfaces. All sub-dermal bubbles were present at TGP levels well below the thresholds for intravascular bubble growth. This implies that the gas transport for this form of bubble growth is directly from the environmental water and does not involve the circulatory system. Otherwise, one would expect sub-dermal bubbles only at levels of TGP that initiate intravascular bubble growth. Based on the thresholds indicated for this form of bubble growth, nuclei are again about 12 - $14 \mu \mathrm{m}$ in radius, as calculated from Equation 3 (3').

Extracorporeal Bubble Growth Between Gill Lamellae: These bubbles appear to develop early during exposure to supersaturated water and bubble size appears to increase with increasing TGP. Although extracorporeal bubbles were larger at higher levels of TGP, there were fewer present or they were entirely absent once TGP levels rose above 1.15 atms. Presumably, this was because larger bubbles could be more easily dislodged by the respiratory water flow. This observation offers an explanation for the two thresholds noted in the database analysis for chinook salmon longer than $50 \mathrm{~mm}$ (Figure 2.1.17). In these experiments a transition from one mechanism of mortality to another occurred at a water TGP of 1.15 atms; there was a sharp dip in the time to mortality at this TGP. Thus, if extracorporeal bubbles are no longer present above a TGP of 1.15 atms, sub-dermal bubbles alone may not be enough to cause mortality. Alternatively, if a TGP of 
1.15 is the threshold for intravascular bubble growth, this mechanism of mortality may become effective above 1.15 atms and involve different times to mortality. Thus, a transition from one mechanism of mortality to another may occur.

It is reasonable to assume that particles that serve as nucleation sites free in the environmental water may be carried into gill lamellae by respiratory water flow. For example, water below dam spillways is often filled with bubbles. Many water sources carry a high loading of large silt particles. Thus, nucleation sites of considerable size could be carried into gill lamellae and initiate extracorporeal bubble growth at very low levels of TGP.

Bubble Growth at High TGP Levels: Bubble formation in the gill filamental arteries occurred above a water TGP of 1.15 to 1.16 atms. Results confirm that, at TGP levels above 1.15 atms, arterial $\mathrm{pO}_{2}$ and $\mathrm{pH}$ levels decline as TGP levels increase. At TGP levels near 1.15 atms, extracorporeal bubbles are still present in gill lamellae and may account for a portion of the $\mathrm{pO}_{2}$ and $\mathrm{pH}$ response. However, sub-dermal blisters were few or nonexistent at the higher levels of TGP. Also, extra-corporeal bubbles became larger but less numerous as water TGP increased, presumably because larger bubbles are more easily dislodged by the respiratory water flow. At TGP above 1.19 atms, the number of extracorporeal bubbles were so few that it is difficult to see how they would contribute to the declining arterial $\mathrm{po}_{2}$.

Swimbladder overinflation: Shrimpton et al. (1989) examined the response of rainbow trout swimbladders to gas supersaturation. One facet of these experiments was the 
determination of the threshold in terms of water TGP and $\mathrm{pO}_{2}$ for swimbladder overinflation.

Swimbladder overinflation Thresholds: In the shrimpton et al. (1989) experiments, the swimbladders of various sized rainbow trout were cannulated and swimbladder pressures were monitored under a range of dissolved gas supersurations. plotted results show a threshold for swimbladder inflation occuring at the TGP between fish exhibiting increased swimbladder pressure and those exhibiting decreasing swimbladder pressure. This threshold is slightly higher than the theoretical threshold curve developed from Equation 2 (Figure 2.1.41)\% The reason for this difference is unknown. The theoretical curve uses an F of 0.79 , a system pressure of $0.0 \mathrm{mmHg}$, and a temperature of $10^{\circ} \mathrm{C}$. If the value of $F$ is increased from 0.79 to 0.85 , the theoretical threshold corresponds closely to that indicated by the experimental data. Pneumatic Duct Release Pressure Thresholds: Shrimpton et al. (1989) also experimentally examined the maximum swimbladder pressures that could be attained before gas was vented from the swimbladder via the pneumatic duct. For large fish, pressures were monitored by way of an indwelling cannula inserted into the swimbladder. For small fish that could not be cannulated a hyperbaric chamber was used to establish pneumatic duct release pressures.

The results of these experiments indicate that pneumatic duct release pressure is a function of fish size, and that small fish sustain a much larger degree of swimbladder overinflation than do large fish (Figure 2.1.42). In fact, the swimbladder of many of the smaller fish ruptured. Juvenile physostome fish such 


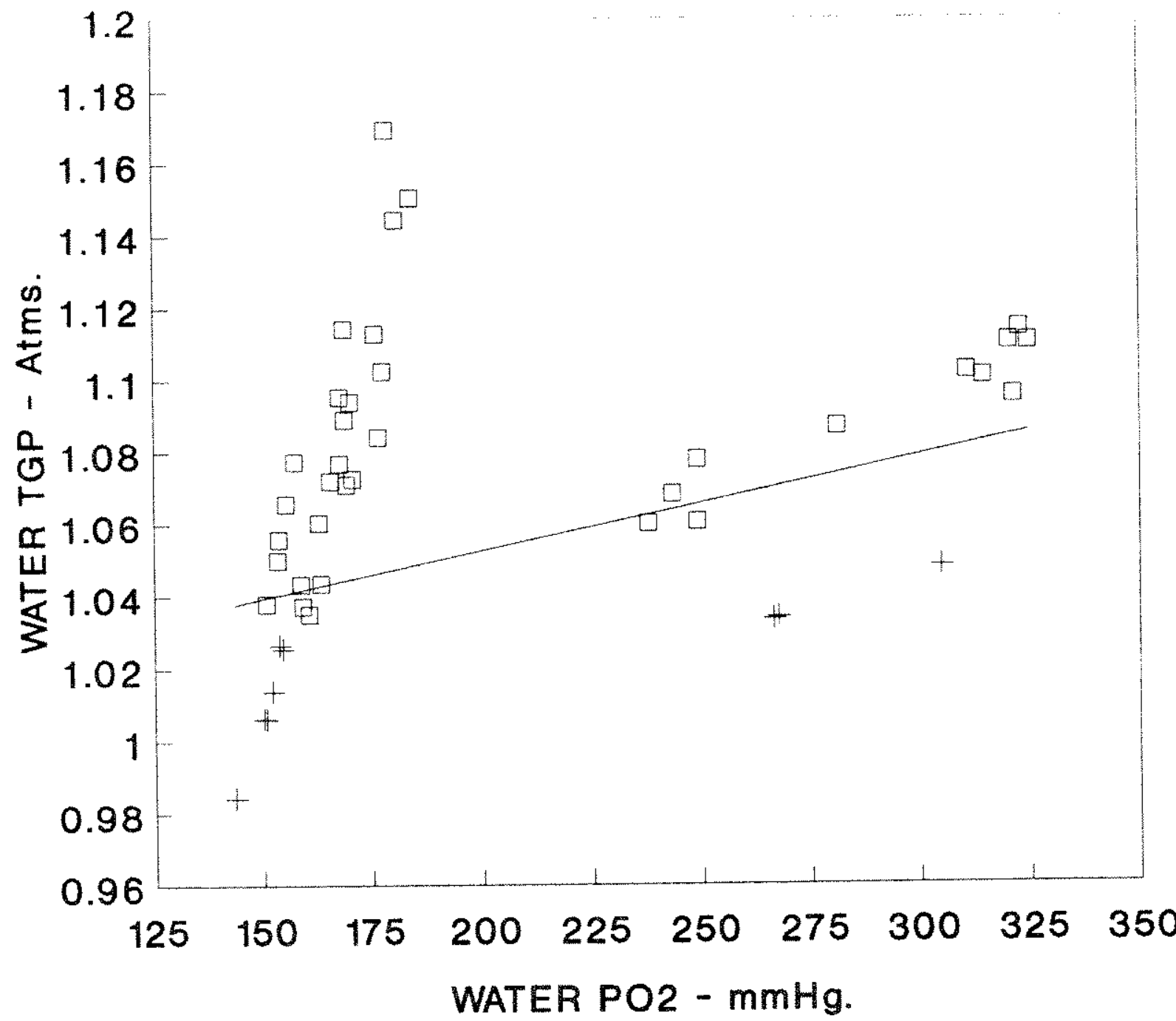

INFLATION + DEFLATION

THEORY

Experimental Data: Shrimpton, Randall and Fidler (1988)

Theory: Equation 2

Figure 2.1.41. Influence of total gas pressure and water $\mathrm{pO}_{2}$ on swimbladder inflation of rainbow trout. 
as rainbow and brown trout are very susceptible to swimbladder overinflation. This may include actual swimbladder rupture and increased susceptibility to predation. In the case of the Bighorn River where rainbow and brown trout spawn at very different times of the year, the water dissolved gas tension existing at the time of emergence may be critical to survival. In fact, the susceptibility to swimbladder overinflation combined with the difference in time of emergence and the level of dissolved gas supersaturation existing on the river may explain the suppressed recruitment of rainbow trout on the Bighorn River. Behavioral effects of swimbladder overinflation: Experiments by Shrimpton et al. (1989) also examined the behavioral response of rainbow trout to swimbladder overinflation. That is, the experiments were designed to determine if rainbow trout, exposed to dissolved gas supersaturation, used water depth to reduce buoyancy. The experiments involved exposing individual fish of various sizes to dissolved gas supersaturation in a water column approximately 2.5 $\mathrm{m}$ deep. One side of the water column was constructed of clear plexiglas. Fish were monitored continuously using a video camera and the depth of the fish in the column was recorded over a $24-\mathrm{h}$ period.

Shrimpton et al. (1989) found that fish larger than $50 \mathrm{~g}$ did not use depth to compensate for swimbladder overinflation. This is consistent with the results of the pneumatic duct release pressure experiments in which larger fish showed less susceptability to swimbladder overinflation. Although there was evidence of overbuoyancy in larger fish, they compensated by 


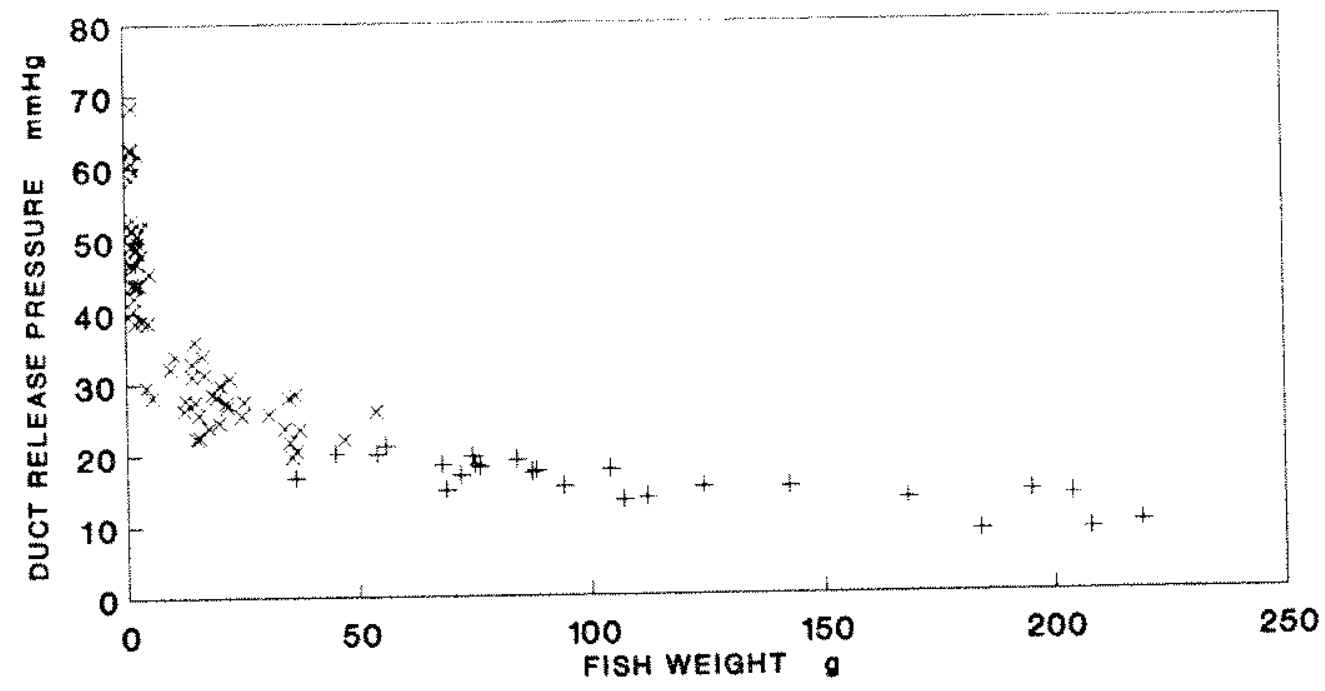

+ SWIMBLADDER CANNULA $\times$ HYPERBARIC CHAMBER

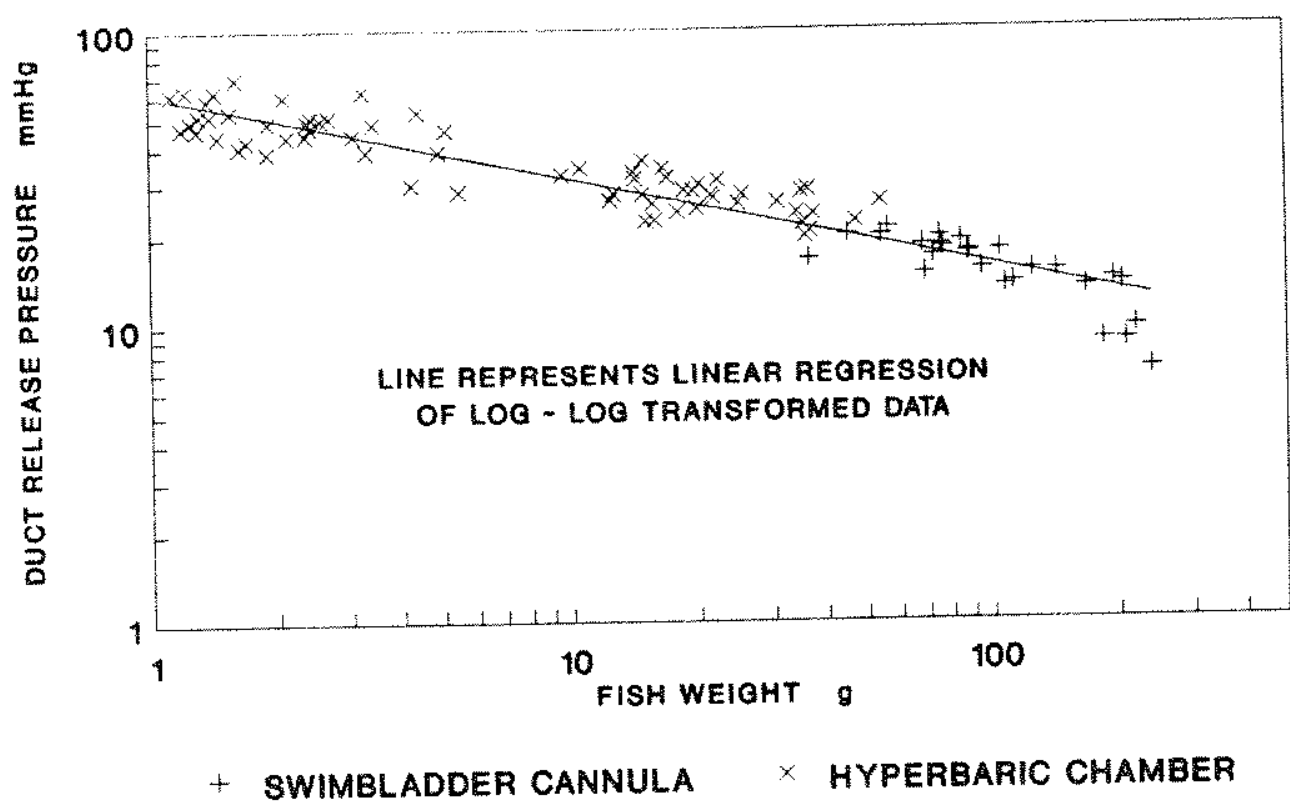

Figure 2.1.42. Rainbow trout swimbladder pneumatic duct release pressure as a function of fish weight. 
swimming in a head down position. Smaller fish were consistently observed at greater depths and appeared to be using hydrostatic pressure to compensate for swimbladder overinflation.

on the Bighorn River, the availability of enough water depth to compensate for a given level of supersaturation may be an important factor in the survival of juvenile fish. This factor will be considered in later sections of this report. 


\subsubsection{Part 2: Juvenile Trout and GBT \\ $2 \cdot 1 \cdot 3 \cdot 2 \cdot a$ Introduction}

Based upon the need for better information on effects of gas supersaturated water on juvenile brown and rainbow trout in the Bighorn River, part two physiological studies were designed to:

(1) determine the relationship between juvenile brown and rainbow trout size and susceptibility to GBT;

(2) compare the relative sensitivity of juvenile brown and rainbow trout to GBT;

(3) investigate the sublethal effects of exposure to gas supersaturated water on fish growth and susceptibility to microbial invasion.

\section{$2 \cdot 1 \cdot 3 \cdot 2 \cdot b$ Methods}

\section{Gas supersaturated Water production and Measurement}

This research was conducted in laboratory facilities at the United states Fish and Wildlife Service, Bozeman Fish Technology center. Gas supersaturated water was produced by a method similar to that described by Bouck and King (1983). An air compressor was used to force air into a water pipeline. Increased turbulence and pressure forced air into solution (Figure 2.1.43). Six 30-d exposure tests were conducted in nine fiberglas tanks $(57 \mathrm{~cm}$ long, $33 \mathrm{~cm}$ deep, $43 \mathrm{~cm}$ wide), each subdivided into two equal sections by a wooden frame covered by $400.0 \mathrm{~cm}^{2}$ of plastic screening. Water depth was $22.0 \mathrm{~cm}$. Equal flow of water was provided through PVC pipe to each side of each 


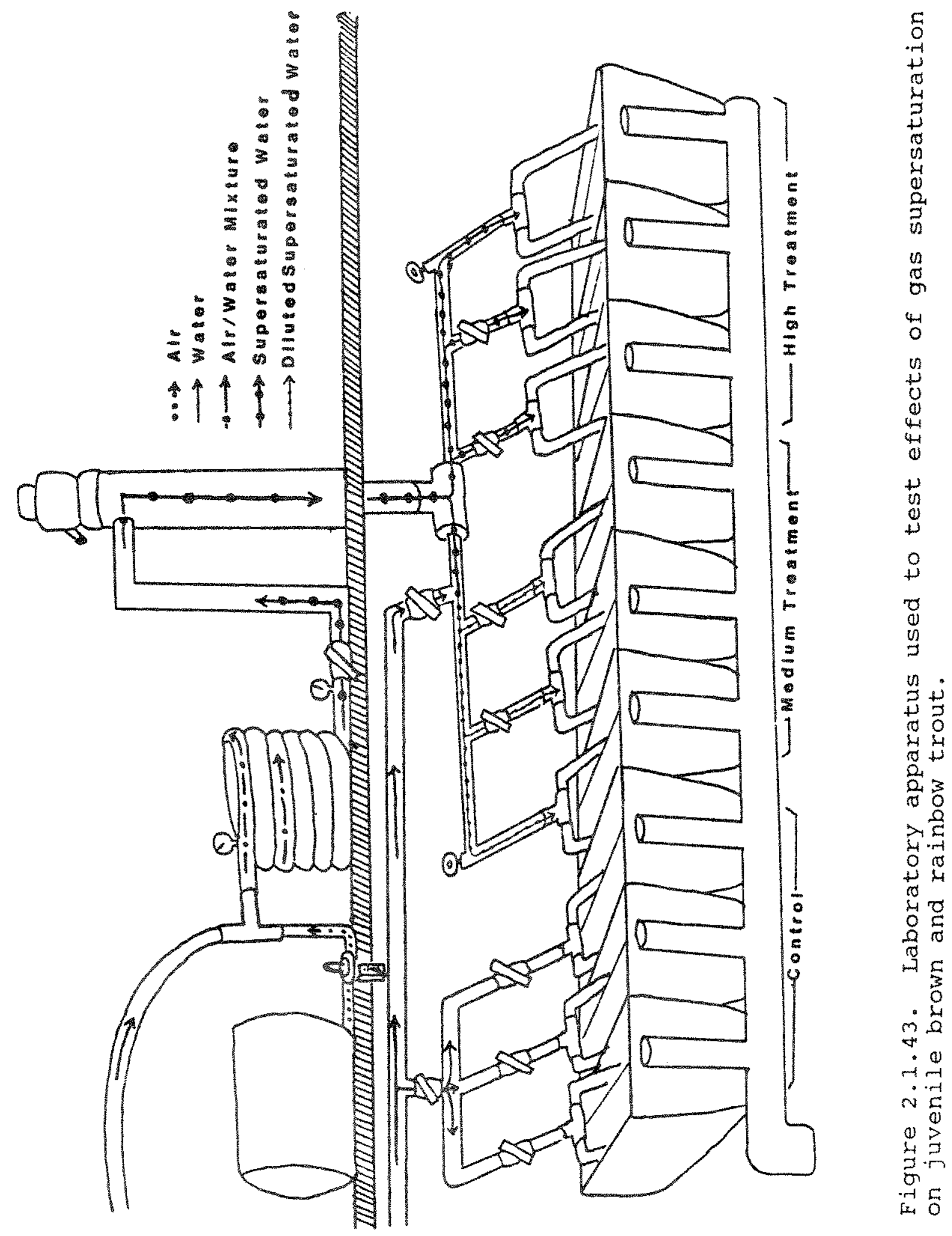


tank. Mixing was complete throughout the tank; no gradient of gas levels was present.

T'wo levels of gas supersaturated water were produced (mean of $\operatorname{six}$ tests $=112 \pm 1.0 \%$ and $125 \pm 0.7 \%$ ) by mixing artificially supersaturated water with 102 - 104\% gas supersaturated spring water. Daily monitoring of delta $P$ (pressure in mrin in excess of atmospheric pressure) was accomplished using a Weis saturometer for the control (mean of six tests $=104 \pm 1.2 \%$ ) and Bouck gasometers (Bouck 1982) for the two test treatments; meters were calibrated weekly by taking measurements in the same treatment tank with both kinds of instruments. Dissolved oxygen (DO) was measured using a XSI dissolved oxygen meter, standardized weekly by Winkler's procedure (APHA 1976). Temperature was measured in degrees centigrade $\left({ }^{\circ} \mathrm{C}\right)$ using a digital thermometer calibrated with a mercury thermometer. Gas measurements were taken daily from one tank of each gas treatment. Daily temperature and dissolved oxygen (DO) measurements were taken on both sides of each tank. A rotation schedule ensured equal distribution of measurements. Barometric pressure was measured daily using a Princo Nova, fortin-type mercury barometer.

Gas levels fluctuated somewhat during the first three tests in the medium gas treatment and during tests 2 and 3 of the high gas treatment. The problem was corrected in subsequent tests by modifying the measuring equipment and by installing a constant differential pressure regulator on the air compressor (Table 2.1 .3$, Figures 2.1.44-2.1.49).

Dissolved oxygen concentrations and water temperature were relatively constant between tests except during test 4 when 
Table 2.1.3. Mean delta $P$ values (mm) for 30-day exposure tests $1-6$.

Test Treatment Delta $\mathrm{P}$ values $\pm \mathrm{SD}$

\begin{tabular}{|c|c|c|}
\hline 1 & High & $155 \pm 6$ \\
\hline 2 & High & $153 \pm 15$ \\
\hline 3 & High & $162 \pm 13$ \\
\hline 4 & High & $167 \pm 6$ \\
\hline 5 & High & $160 \pm 6$ \\
\hline 6 & High & $160 \pm 9$ \\
\hline 1 & Medium & $82 \pm 20$ \\
\hline 2 & Medium & $78 \pm 22$ \\
\hline 3 & Medium & $69 \pm 15$ \\
\hline 4 & Medium & $83 \pm 7$ \\
\hline 5 & Medium & $72 \pm 11$ \\
\hline 6 & Medium & $84 \pm$ \\
\hline 1 & Control & $16 \pm$ \\
\hline 2 & control & $38 \pm$ \\
\hline 3 & control & $29 \pm$ \\
\hline 4 & Control & $20 \pm$ \\
\hline 5 & control & $26 \pm$ \\
\hline 6 & Control & $25 \pm 5$ \\
\hline
\end{tabular}




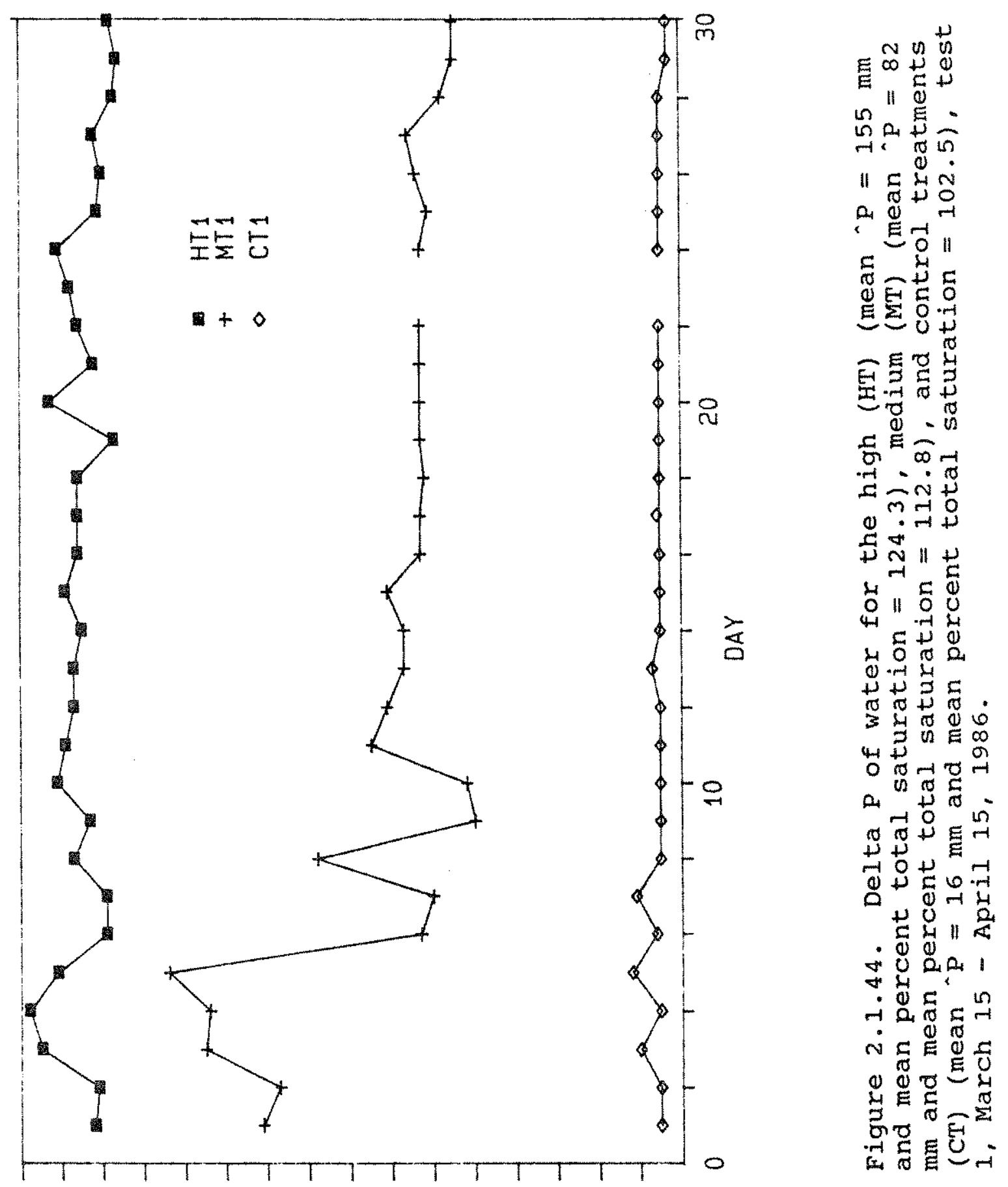

(ww) d $\forall 17 \exists 0$ 


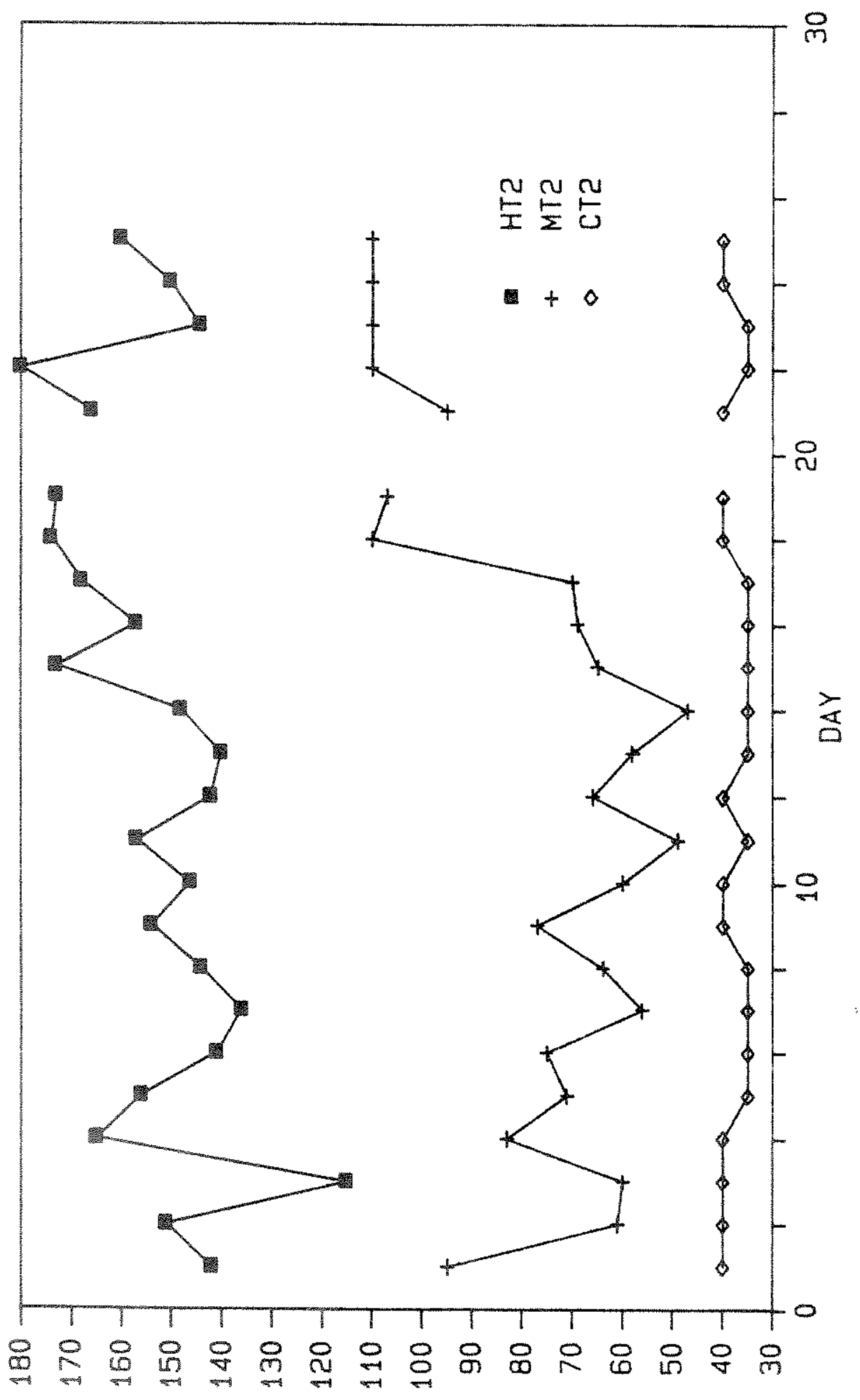

\begin{tabular}{lll}
$\infty$ & 0 & 0 \\
\hdashline & 0 & 0 \\
11 & 0
\end{tabular} ใิ ris $\|$ 대 a. 0 "Eन 5 .

बैस E ${ }^{2} 0 .-1$ 틈 톨. पr 0 进吉

됴요 O

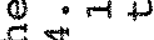

ग्ञ

$4{ }^{-1}$ द्व

क्ष.+14

Cक⿻

숭

(4) $\rightarrow$ s

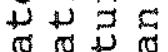

3400

ป

40

, 1000

- 100

त)

\&

1) 요요

0 क

+U 0

5 4 II

เं 005

* म

-i ह ह

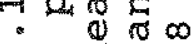

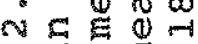

0 \% $E$

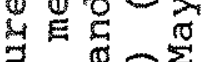

Gुष 证曷U

(ww) d $\forall 17 \exists 0$ 


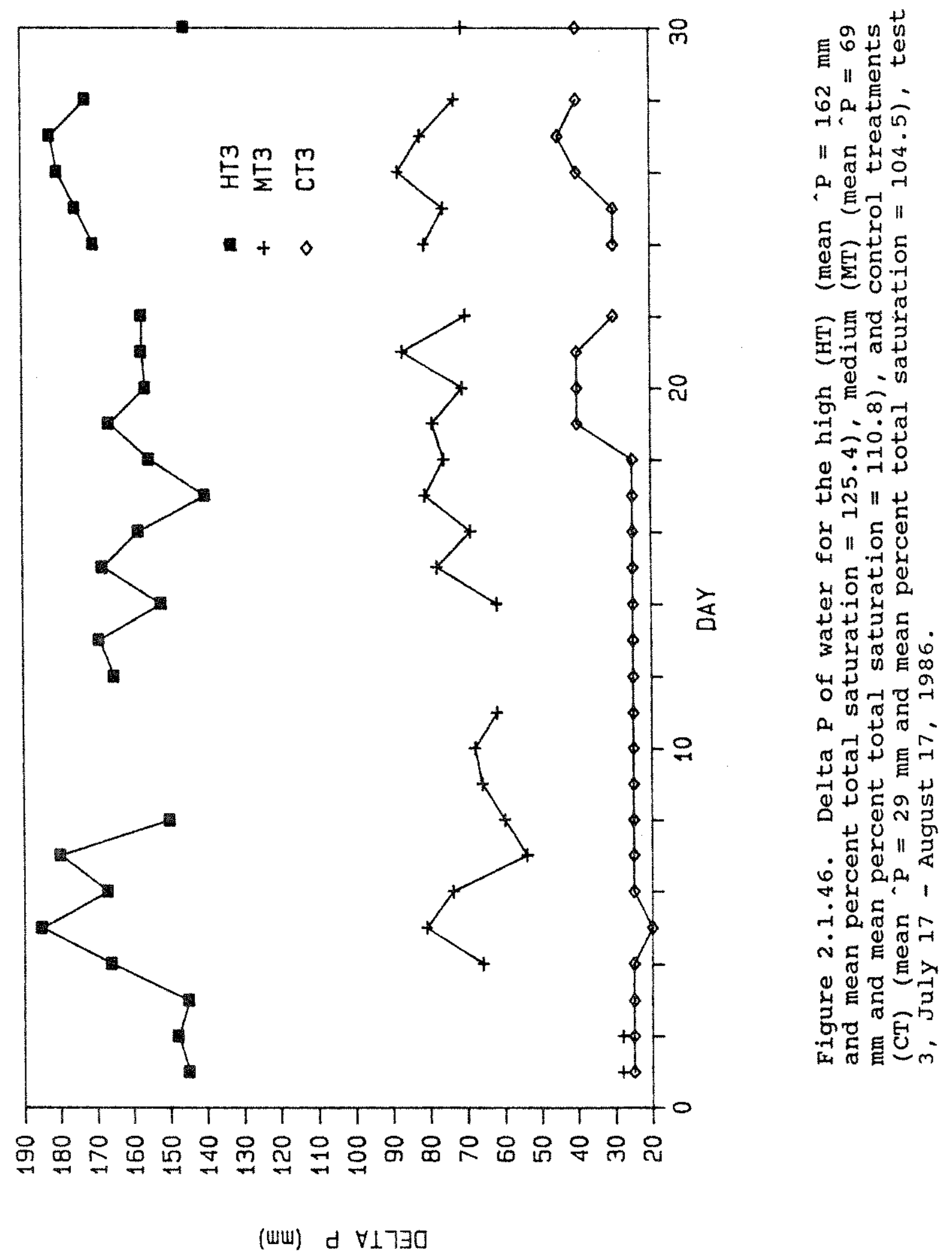




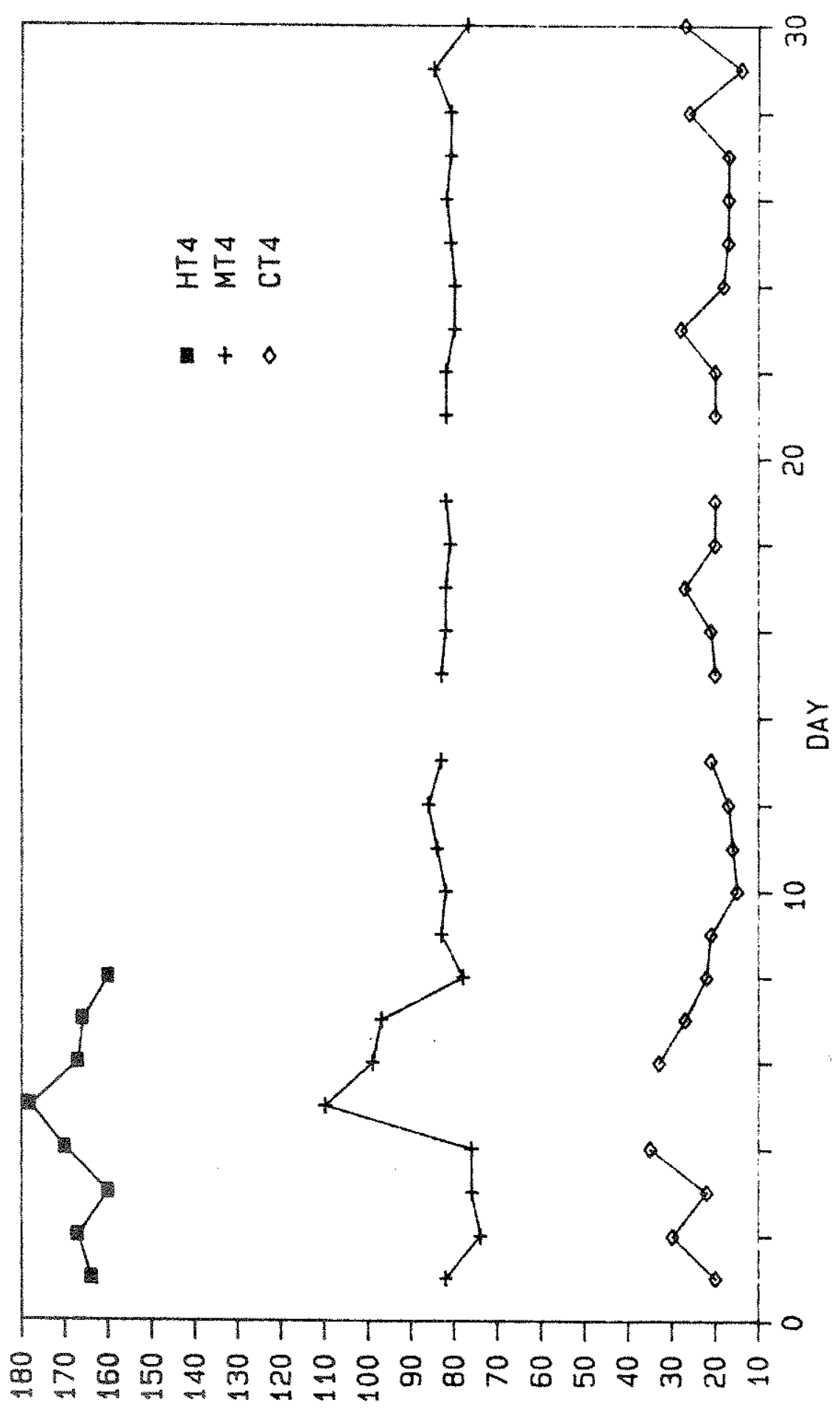

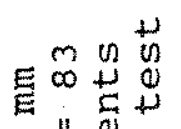
$\therefore \stackrel{0}{E}$.

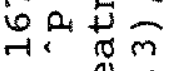

$\| \varepsilon \dot{D}^{0} \dot{0}$

a.

" छ엉

范点

Q턷ㄷㅇ

E $\Sigma 0$.

—

䛼点焉

赑 0

뎽 $\overline{0}$

- -10

c $-m+$

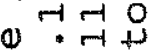

造

त्

4 동

에 11.00

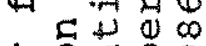
4000

$+ \pm E$

$340 \stackrel{0}{0} N$

का की

०

a $4 \pi$

-1 0

तै

廿

닝유.

$+0$

$\pm \| \infty$

- $00 \quad N$

$\sim 0 \Omega \infty$

- 40 द

- 0 的 5

․

NE

- Q

岁至 0

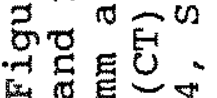




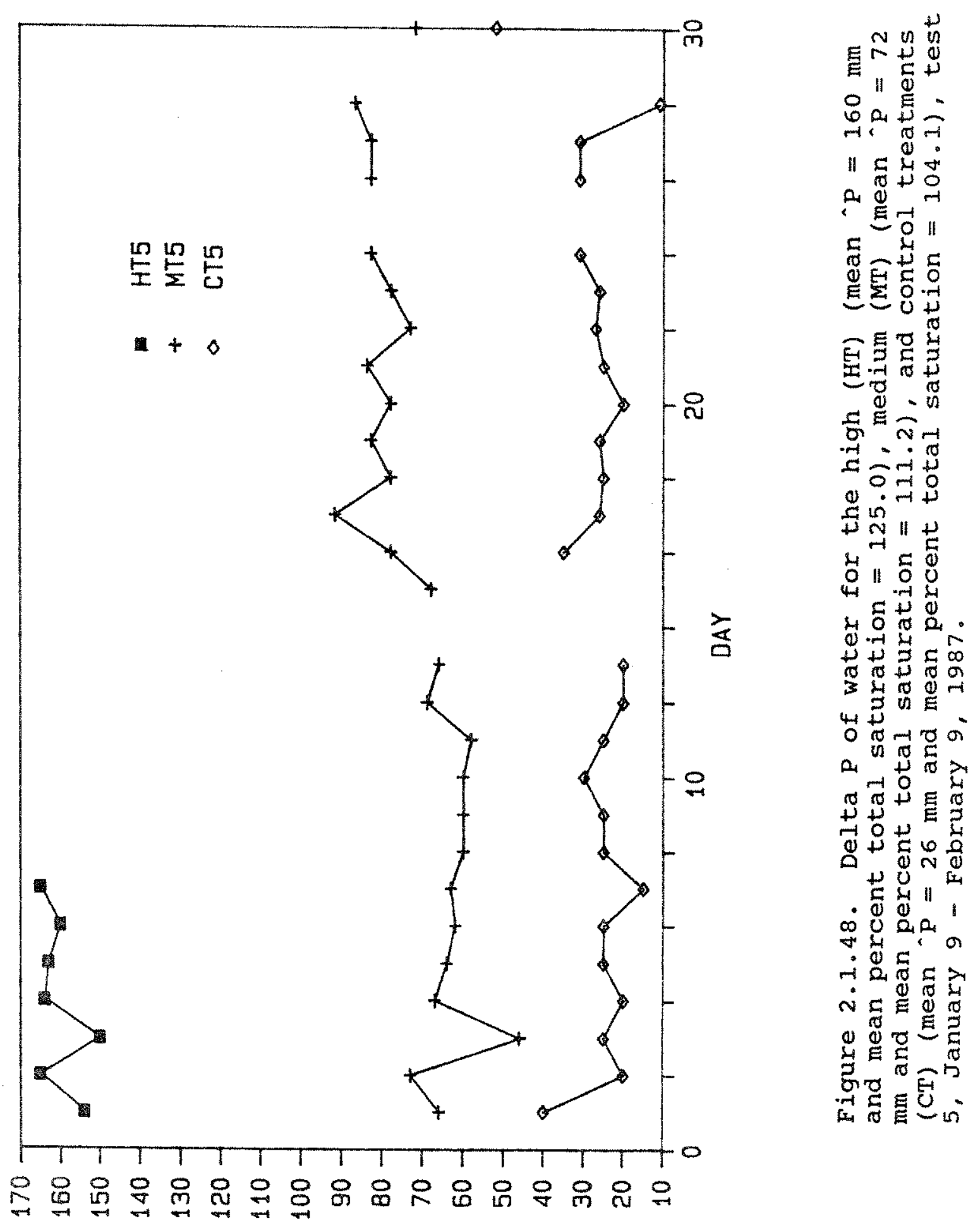

(ww) d $\forall 1730$ 

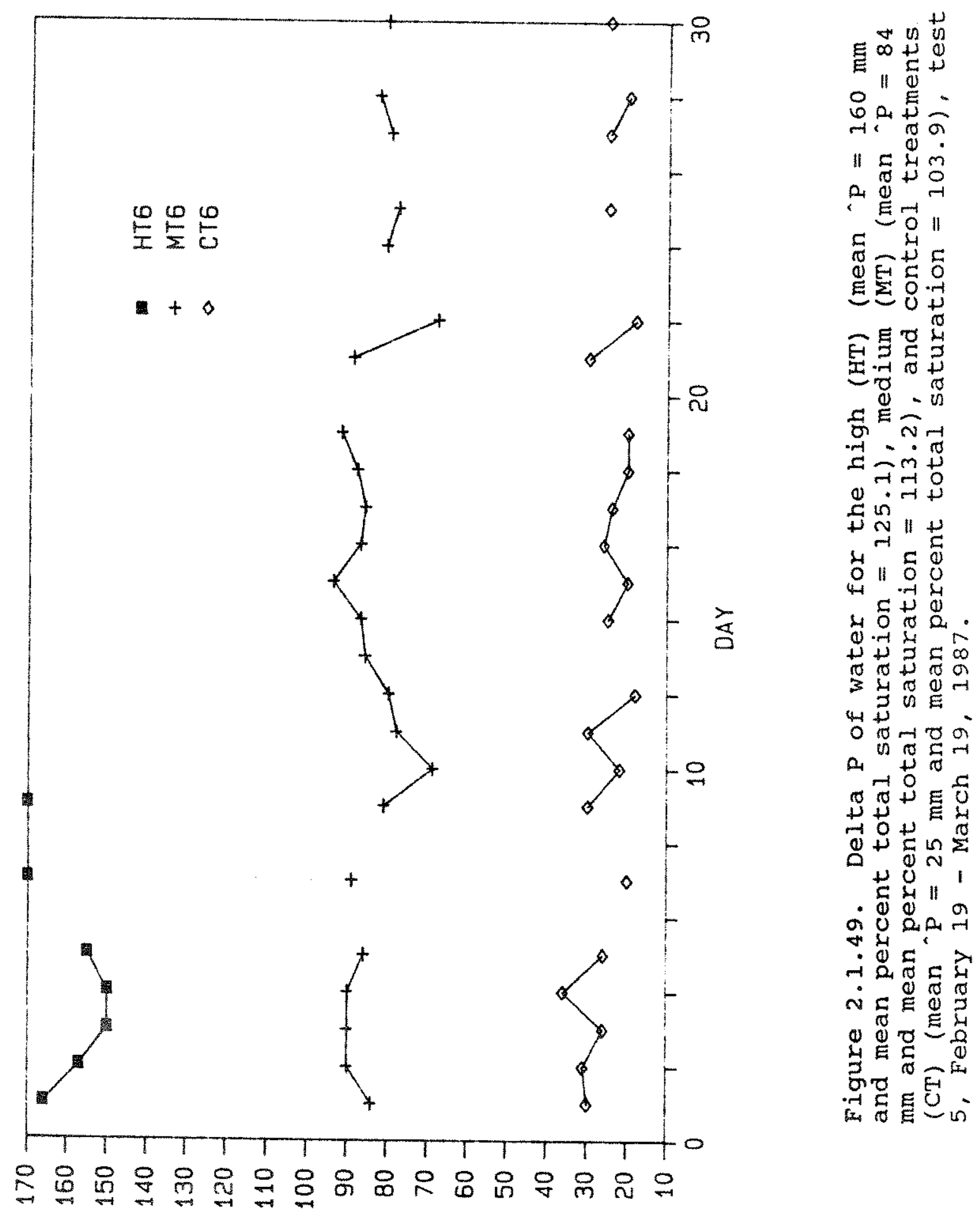

(wu) d $\forall 17 \exists 0$ 
temperature was lower (Table 2.1.4). This problem was corrected by using more warm spring water in the system.

After each 30-d test, values for daily delta $P$, Do, water temperature, and barometric pressure were used in Colt's formulas (1984) to calculate mean $30-d$ gas levels.

\section{Fish Care}

Brown trout eggs were obtained from wild stock of the Bighorn River in early December, 1985. At the same time, domestic Shasta strain rainbow trout were spawned at the Ennis Fish Hatchery.

Eggs were water hardened, treated with 100 ppm alpha caps-7 betadine and transported to the Bozeman Fish Technology center where they were incubated in Heath trays supplied with spring water $\left(19.0 \mathrm{~L} / \mathrm{min}\right.$ at $\left.10 \pm 1^{\circ} \mathrm{C}\right)$. Eggs hatched in approximately 30 d. At the end of January, fish were transferred to $1.8 \mathrm{~m}$ circular fiberglass tanks supplied with $42-49 \mathrm{I} / \mathrm{min}$ of $10 \pm 1^{\circ} \mathrm{C}$ spring water.

\section{Test Fish selection}

Mean fish length was determined before each experiment by randomly selecting 25 - 100 fish, measuring each to the nearest 1 mm, and returning them to the rearing tanks (Table 2.1.5). Fish of each species were then randomly selected from the rearing tanks, weighed, and placed into each experimental tank (Table 2.1.6). The number of fish used varied by test because as fish grew the number of fish each tank could support decreased (Table 
Table 2.1.4. Mean dissolved oxygen concentrations and water temperatures during tests $1-6$.

\begin{tabular}{llrr}
\hline Test & Treatment & $\begin{array}{c}\text { Dissolved oxygen } \\
(\mathrm{mg} / 1)\end{array}$ & $\begin{array}{c}\text { Temperature } \\
\left({ }^{\circ}\right)\end{array}$ \\
1 & High & $10.5 \pm 0.4$ & $10.2 \pm 0.4$ \\
2 & High & $11.4 \pm 0.4$ & $9.7 \pm 0.4$ \\
3 & High & $11.3 \pm 0.3$ & $9.7 \pm 0.3$ \\
4 & High & $11.6 \pm 0.4$ & $8.6 \pm 0.1$ \\
5 & High & $10.7 \pm 0.8$ & $10.4 \pm 0.3$ \\
6 & $10.6 \pm 0.5$ & $11.0 \pm 0.1$ \\
1 & Medium & $9.6 \pm 0.3$ & $10.2 \pm 0.4$ \\
2 & Medium & $10.6 \pm 0.4$ & $9.8 \pm 0.2$ \\
3 & Medium & $9.9 \pm 0.3$ & $10.0 \pm 0.2$ \\
4 & Medium & $10.4 \pm 0.4$ & $8.1 \pm 0.1$ \\
5 & Medium & $9.4 \pm 0.3$ & $10.0 \pm 0.4$ \\
6 & Medium & $9.5 \pm 0.4$ & $10.2 \pm 0.5$ \\
& Control & $8.9 \pm 0.2$ & $10.1 \pm 0.2$ \\
1 & Control & $9.6 \pm 0.4$ & $10.1 \pm 0.5$ \\
2 & Control & $9.2 \pm 0.2$ & $10.0 \pm 0.2$ \\
3 & Control & $9.4 \pm 0.4$ & $7.9 \pm 0.1$ \\
4 & Control & $8.6 \pm 0.3$ & $9.8 \pm 0.3$ \\
5 & Control & $8.8 \pm 0.3$ & $9.9 \pm 0.6$ \\
6 & & & \\
& & &
\end{tabular}

2.1.5). Fish size increased with each test, except rainbow trout used in test 6 were smaller than rainbow trout used in test 5 .

\section{Gas supersaturation Bioassay Data Analysis}

The influence of fish length and weight on susceptibility of juvenile brown and rainbow trout to GBT was evaluated and relative sensitivity was compared. Percent cumulative mortality was calculated for control, medium, and high treatment fish of both species for all tests. Where:

Pexcent Cumulative Mortality $=$ (Day $x$ mortality) + (Day $x . . n$ mortality) $x 100$ fish number at day 0 
Table 2.1.5. Pre-treatment mean lengths of juvenile rainbow and brown trout computed from a random sample of fish from the rearing tanks.

\begin{tabular}{|c|c|c|c|}
\hline Species & Test & $\begin{array}{c}\text { Mean total length } \\
(\mathrm{mm} \pm \mathrm{SD})\end{array}$ & $\begin{array}{c}\text { Number of } f i s h \\
\text { measured }\end{array}$ \\
\hline Brown & 1 & $35 *$ & 25 \\
\hline Brown & 2 & $53 *$ & 25 \\
\hline Brown & 3 & $62 \pm 6$ & 102 \\
\hline Brown & 4 & $91 \pm 8$ & 73 \\
\hline Brown & 5 & $126 \pm 6$ & 100 \\
\hline Brown & 6 & $173 \pm 12$ & 90 \\
\hline Rainbow & 1 & $34 *$ & 25 \\
\hline Rainbow & 2 & $71 *$ & 25 \\
\hline Rainbow & 3 & $91 \pm 11$ & 95 \\
\hline Rainbow & 4 & $131 \pm 14$ & 39 \\
\hline Rainbow & 5 & $194 \pm 20$ & 100 \\
\hline Rainbow & 6 & $179 \pm 14$ & 90 \\
\hline
\end{tabular}

* Data not available for standard deviation calculations 
Table 2.1.6. Mean weight and number of juvenile rainbow and brown trout exposed to high, medium, and control levels of gas supersaturation during tests $1,2,3,4,5$, and 6 .

\begin{tabular}{|c|c|c|c|c|}
\hline Species & $\begin{array}{c}\text { Exposure } \\
\text { test }\end{array}$ & $\begin{array}{c}\text { Gas } \\
\text { treatment }\end{array}$ & $\begin{array}{c}\text { Total } \\
\text { number of fish } \\
\text { exposed }\end{array}$ & $\begin{array}{c}\text { Mean weight } \\
(g / f i s h)\end{array}$ \\
\hline Brown & 1 & High & 150 & 0.3 \\
\hline Brown & 1 & Medium & 150 & 0.3 \\
\hline Brown & 1 & Control & 150 & 0.3 \\
\hline Brown & 2 & High & 75 & 1.3 \\
\hline Brown & 2 & Medium & 75 & 1.2 \\
\hline Brown & 2 & Control & 75 & 1.2 \\
\hline Brown & 3 & High & 75 & 2.9 \\
\hline Brown & 3 & Medium & 75 & 2.9 \\
\hline Brown & 3 & control & 75 & 2.9 \\
\hline Brown & 4 & High & 75 & 8.3 \\
\hline Brown & 4 & Medium & 75 & 8.3 \\
\hline Brown & 4 & control & 75 & 7.9 \\
\hline Brown & 5 & High & 45 & 22.9 \\
\hline Brown & 5 & Medium & 45 & 23.0 \\
\hline Brown & 5 & control & 45 & 21.3 \\
\hline Brown & 6 & High & 30 & 61.5 \\
\hline Brown & 6 & Medium & 30 & 59.7 \\
\hline Brown & 6 & control & 30 & 59.7 \\
\hline Rainbow & 1 & High & 150 & 0.4 \\
\hline Rainbow & 1 & Medium & 150 & 0.4 \\
\hline Rainbow & 1 & control & 150 & 0.4 \\
\hline Rainbow & 2 & High & 75 & 3.4 \\
\hline Rainbow & 2 & Medium & 75 & 3.6 \\
\hline Rainbow & 2 & control & 75 & 3.6 \\
\hline Raimbow & 3 & $\mathrm{High}$ & 75 & 8.9 \\
\hline Rainbow & 3 & Medium & 75 & 8.4 \\
\hline Rainbow & 3 & Control & 75 & 8.2 \\
\hline Rainbow & 4 & High & 45 & 26.3 \\
\hline Rainbow & 4 & Medium & 45 & 26.7 \\
\hline Rainbow & 4 & Control & 45 & 26.1 \\
\hline Rainbow & 5 & High & 45 & 79.3 \\
\hline Rainbow & 5 & Medium & 45 & 89.5 \\
\hline Rainbow & 5 & Control & 45 & 83.5 \\
\hline Rainbow & 6 & High & 30 & 60.5 \\
\hline Rainbow & 6 & Medium & 30 & 60.3 \\
\hline Rainbow & 6 & Control & 30 & 57.8 \\
\hline
\end{tabular}


Significant differences in mortality were tested by comparing mortality of control fish to mortality of medium and high gas treatment fish using Biomedical Data processing (BMDP) software (Dixon et al. 1983). BMDP employs the non-parametric Breslow rank test to generate p-values. The effect of size on susceptibility to GBT was then determined by comparing intraspecific percent cumulative mortality from the high treatment of the six tests (Table 2.1.7). Relative sensitivity of juvenile brown and rainbow trout was compared by testing for significant difference in percent cumulative mortality between high treatment rainbow and brown trout of similar size (Table $2.1 .7)$.

Growth of Fish Exposed to sublethal Levels of Gas supersaturation

Brown and rainbow trout juveniles from control and medium gas treatment tests $1,3,5$, and 6 were used to determine the effect of exposure to sublethal levels of gas supersaturation on fish growth. Weights of juvenile brown and rainbow trout from any high treatment or from medium treatments of tests 2 and 4 were not used to test for sublethal effects on growth because mortality attributable to GBT occurred in these tests.

Growth effects were examined by comparing control fish weight to treatment fish weight. Fish weights taken on days 1 and 30 from tests $1,3,5$, and 6 were pooled to provide a sample size of 12 mean weights (four tests a three replicates/test) (Table 2.1.8). Average day 1 and 30 control and medium treatment weights were calculated using these 12 weights and comparisons were made using the Mann-Whitney nonparametric rank test (Hintze 1987). 
Table 2.1.7. Intra- and interspecific mortality comparisons for juvenile brown (BT) and rainbow trout (RBT) exposed to the high gas treatment during tests $1-6$.

\begin{tabular}{|c|c|c|}
\hline $\begin{array}{l}\text { Brown trout } \\
\text { mortality } \\
\text { comparison }\end{array}$ & $\begin{array}{l}\text { Rainbow trout } \\
\text { mortality } \\
\text { comparison }\end{array}$ & $\begin{array}{c}\text { Interspecific } \\
\text { mortality } \\
\text { comparison }\end{array}$ \\
\hline $\mathrm{BT} 1$ vs $\mathrm{BT} 2$ & RBT1 vs RBT2 & BT1 VS RBT1 \\
\hline BT2 vs BT3 & RBT2 vs RBT3 & BT3 vs RBT2 \\
\hline BT3 vs BT4 & RBT3 vs RBT4 & BT4 VS RBT3 \\
\hline BT4 vS BT5 & RBT4 VS RBT6 & BT5 vS RBT4 \\
\hline BT5 vs BT6 & RBT6 VS RBT5 & BT6 VS RBT6 \\
\hline
\end{tabular}

Table 2.1.8. Sample sizes used to test for the effects of gas supersaturated water on juvenile brown (BT) and rainbow trout (RBT) growth.

\begin{tabular}{|c|c|c|c|c|c|c|}
\hline \multirow{2}{*}{ Test } & \multirow{2}{*}{ Treatment } & \multirow{2}{*}{$\begin{array}{l}\text { Number } \\
\text { of } \\
\text { samples }\end{array}$} & \multicolumn{4}{|c|}{$\begin{array}{l}\text { Number of fish } \\
\text { weighed/sample }\end{array}$} \\
\hline & & & $\mathrm{BTDAYI}^{\mathrm{a}}$ & $\mathrm{BTDA} \times 30^{\mathrm{b}}$ & RBTDAY $1^{c}$ & $\operatorname{RBTDAY} 30^{\mathrm{d}}$ \\
\hline 1 & Control & 3 & 150 & 139 & 150 & 145 \\
\hline 3 & Control & 3 & 75 & 73 & 75 & 71 \\
\hline 5 & Control & 3 & 45 & 45 & 30 & 28 \\
\hline 6 & Control & 3 & 30 & 29 & 30 & 26 \\
\hline 1 & Medium & 3 & 150 & 140 & 150 & 145 \\
\hline 3 & Medium & 3 & 75 & 66 & 75 & 69 \\
\hline 5 & Medium & 3 & 45 & 45 & 30 & 27 \\
\hline 6 & Medium & 3 & 30 & 30 & 30 & 30 \\
\hline \multicolumn{7}{|c|}{$\begin{array}{l}\text { a Brown trout we } \\
\text { b Brown trout we } \\
\text { c Rainbow trout } \\
\text { d Rainbow trout }\end{array}$} \\
\hline
\end{tabular}

Bacterial chal1enge

The effects of GBT on the susceptibility of juvenile rainbow and brom trout to the pathogen Aeromonas hydrophila was tested. A. hydrophila CDC strain III A-20 was obtained from the Wyoming Department of Game and Fish, Laramie, WY. Test protocol involved: 1) antiserum production; 2) pre-treatment serial 
dilution; 3) challenge application; 4) post-treatment serial dilution; 5) fluorescent antibody technique application; 6) enumeration and statistical analysis of bacterial infection of the kidney using a two-sample t-test (Hintze 1987).

Preparation of the antigen was completed using the method of Garvey et al. (1977). Five hundred milliliters of rripticate soy Broth (TSB) were inoculated with broth culture of A. hydrophila that had incubated at $28^{\circ} \mathrm{C}$ for $18 \mathrm{~h}$. At the end of the incubation period, an equal volume of $0.6 \%$ formalinized saline was added. This preparation was stored at room temperature for 4 d. Sterility of the bacterial suspension was checked by inoculating a plate of Triptic Soy Agar (TSA), incubating for 48 $h$ at $28^{\circ} \mathrm{C}$, and examining the plate for growth.

Bacteria were collected by centrifugation at approximately $3000 \mathrm{rpm}$ for $0.5 \mathrm{~h}$. The supernatant fluid was discarded and the bacteria resuspended in $100 \mathrm{ml}$ of $0.85 \%$ saline. At $4-\mathrm{d}$ intervals, a $2.5 \mathrm{~kg}$ rabbit was intravenously injected with 0.5 , $1.0,2.0,3.0,4.0,6.0$, and $6.0 \mathrm{ml}$ of the $\underline{A}$. hydrophila antigen preparation diluted $50 \%$ with physiological saline. The rabbit was rested for $6 \mathrm{~d}$ after the last injection. A small serum sample was then obtained by venous puncture and its titer was tested by the agglutination reaction. specificity of the antiserum was confirmed by testing it against $\underline{A}$. salmonicida and yersinia ruckeri. The rabbit was bled at 3 -week intervals. Whole blood was centrifuged at $3000 \mathrm{rpm}$ for $15 \mathrm{~min}$ and the serum was removed with a syringe. The antiserum was preserved with $0.2 \%$ sodium azide and frozen.

Inoculum was prepared independently for two replicate 
challenges. Seventy-two hours prior to the challenge a brown trout (approximately $180 \mathrm{~mm}$ ) was injected with $1.0 \mathrm{cc}$ of a $1.0 \mathrm{x}$ $10^{8}$ suspension of $\mathrm{A}$. hydrophila and placed in $20^{\circ} \mathrm{C}$ water. Mortality occurred within $24 \mathrm{~h}$. The kidney of the dead fish was punctured with an inoculating loop which was then used to inoculate a test tube of TSB. The TSB was incubated at $20^{\circ} \mathrm{C}$ for $24 \mathrm{~h}$. One tenth milliliter of this suspension was used to inoculate $200 \mathrm{ml}$ of $\mathrm{TSB}$ which was then incubated at $37^{\circ} \mathrm{C}$ for 12 h.

A pre-treatment serial dilution was made in triplicate to determine the viability of challenge inoculum. One-tenth milliliter of the $12-\mathrm{h}$ challenge inoculum of $\mathrm{A}$. hydrophila was pipetted into $9.9 \mathrm{ml}$ of distilled water to give $1: 10^{2}$ dilution. one milliliter of this suspension was transferred to a second test tube containing $9.0 \mathrm{ml}$ of distilled water to give $1: 10^{3}$ dilution. This procedure was followed for three more suspensions until a tube with a dilution factor of $1: 10^{6}$ was achieved. One hundred microliters of the $1: 10^{6}$ dilution were pipetted onto petri dishes containing TSA and incubated at $20^{\circ} \mathrm{C}$ for $48 \mathrm{~h}$. Colonies were counted after incubation to verify inoculum viability and to insure that there were approximately $1.1 \times 10.0^{8}$ viable cells in the challenge inoculum.

Six juvenile brown trout $(180-210 \mathrm{~mm})$ were placed in each of five plastic buckets containing $10.0 \mathrm{~L}$ of $15.9^{\circ} \mathrm{C}$ water with $7.4 \mathrm{mg} / \mathrm{L}$ DO. Three fish in each bucket had GBT as a result of exposure to $118 \%$ gas supersaturated water for $120 \mathrm{~h}$ with no recovery time. The remaining three fish had no previous exposure to gas supersaturated water. Nine milliliters of 12 -h challenge 
inoculum of $\underline{A}$. hydrophila were introduced into four of the buckets. The fifth bucket was treated with $9.0 \mathrm{ml}$ of sterile TSB. The challenge times ranged from $10-40 \mathrm{~min}$. After the challenge was complete, fish from each bucket were placed into a separate tank that received $2.4 \mathrm{~L} / \mathrm{min}$ of $15.9^{\circ} \mathrm{C}$ water with 7.6 mg/L oxygen. The negative control treatment was removed at 20 min.

After $96 \mathrm{~h}$, fish in each bucket were euthanized with MS-222 and refrigerated at $0^{\circ} \mathrm{C}$. Fish were measured and weighed, and kidneys were removed and homogenized in $5 \mathrm{ml}$ of distilled water using a tissue homogenizer. One milliliter of homogenized solution was pipetted onto a circle $(d=1.5 \mathrm{~cm})$ on a fluorescent antibody technique slide and heat fixed. This procedure was followed once for each of the $30 \mathrm{fish}$. One-tenth milliliter of rabbit antiserum was then applied to the slide for $1 \mathrm{~h}$, then rinsed with physiological saline. Commercially prepared fluoroscein-conjugated goat antisera to rabbit immunoglobulins was applied to the slide for $0.5 \mathrm{~h}$ and then rinsed off with physiological saline.

A fluorescent phase contrast microscope (400x objective) was used to count all bacteria in the circle of each slide. The number of bacteria counted on control and treatment slides were compared using the two sample t-test (Hintze 1987).

\section{$2 \cdot 1 \cdot 3 \cdot 2 \cdot c$ Results and Discussion \\ Brown and Rainbow Trout Mortality}

Significant mortality of medium treatment fish in the $30-d$ bioassays occurred only during tests 2 and 4 (Table 2.1.9). No 
Table 2.1.9. Breslow rank analysis of medium gas treatment percent cumulative mortality for juvenile brown (BT) and rainbow trout (RBT) during tests $1,2,3,4,5$, and 6 .

Mortality Comparison

Control treatment Medium treatment p-value
species Test outcome Species Test

\begin{tabular}{llllll}
\hline $\mathrm{BT}$ & 1 & $=$ & $\mathrm{BT}$ & 1 & 0.6287 \\
$\mathrm{BT}$ & 2 & $<$ & $\mathrm{BT}$ & 2 & $<0.0001 *$ \\
$\mathrm{BT}$ & 3 & $=$ & $\mathrm{BT}$ & 3 & 0.1583 \\
$\mathrm{BT}$ & 4 & $<$ & $\mathrm{BT}$ & 4 & $0.0001 *$ \\
$\mathrm{BT}$ & 5 & $=$ & $\mathrm{BT}$ & 5 &.--0 \\
$\mathrm{BT}$ & 6 & $=$ & $\mathrm{BT}$ & 6 & 0.3999 \\
$\mathrm{RBT}$ & 1 & $=$ & $\mathrm{RBT}$ & 1 & $0.0001 *$ \\
$\mathrm{RBT}$ & 2 & $<$ & $\mathrm{RBT}$ & 2 & 0.3173 \\
$\mathrm{RBT}$ & 3 & $=$ & $\mathrm{RBT}$ & 3 & 0.0351 \\
$\mathrm{RBT}$ & 4 & $<$ & $\mathrm{RBT}$ & 4 & -0.3255 \\
$\mathrm{RBT}$ & 5 & $=$ & $\mathrm{RBT}$ & 5 & \\
$\mathrm{RBT}$ & 6 & $=$ & $\mathrm{RBT}$ & 6 &
\end{tabular}

* Statistically different at alpha $=0.05$

brown trout mortality occurred in control or medium treatments during tests 5 or 6 and no rainbow trout mortality occurred in test 5.

Approximately $30 \%$ of mortality in test 2 occurred when delta $P$ values were higher than planned for $10 \mathrm{~d}$ (Figure 2.1.50). During test 4, all mortality of both species in the medium treatment coincided with a $3-d$ period of elevated delta $P$ values (Figure 2.1.51).

Research has shown that critical levels of gas supersaturation exist for different species of fish (Weitkamp and Katz 1980). The critical level is defined as the maximum level. of supersaturation that allows for survival and propagation of 


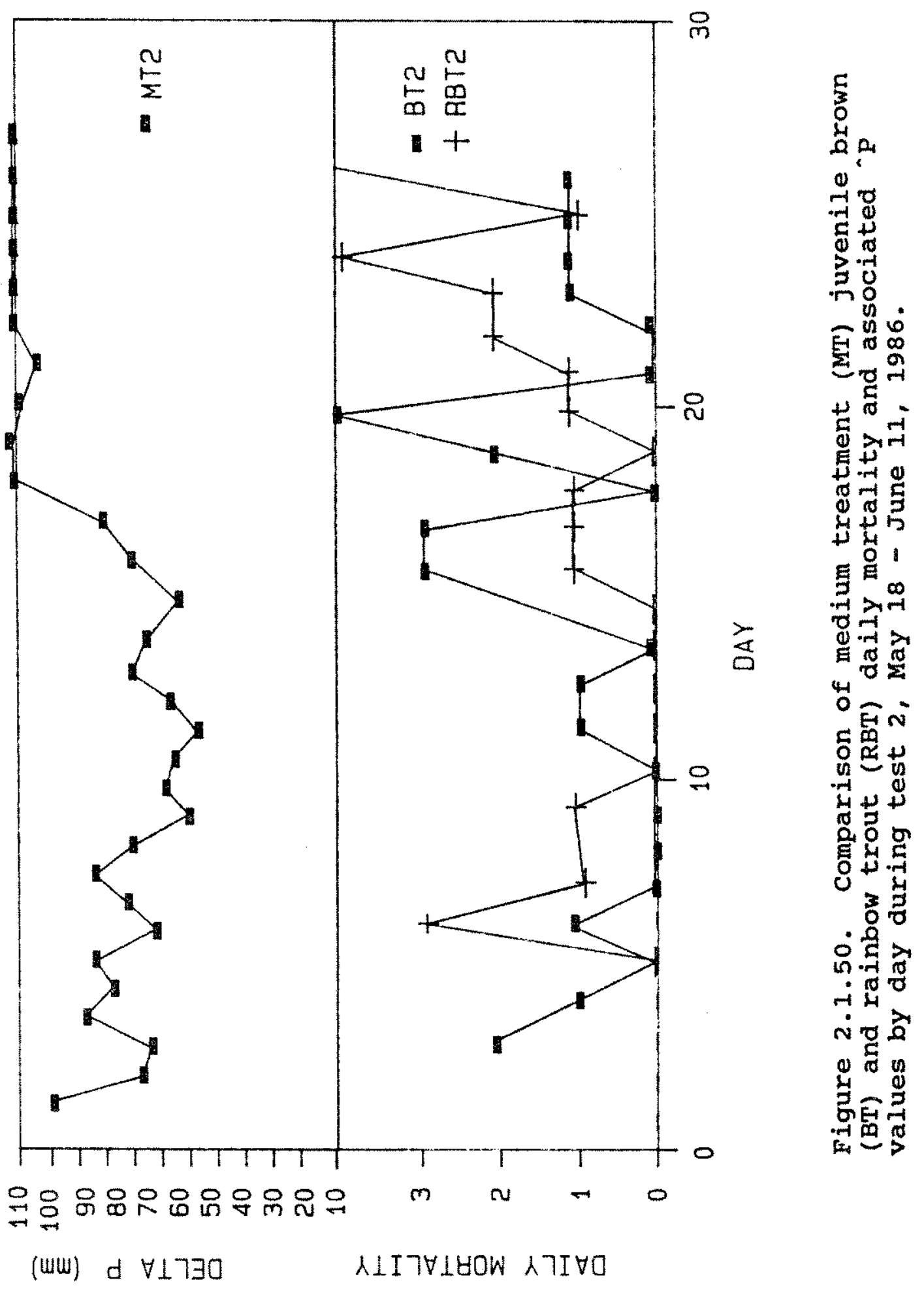




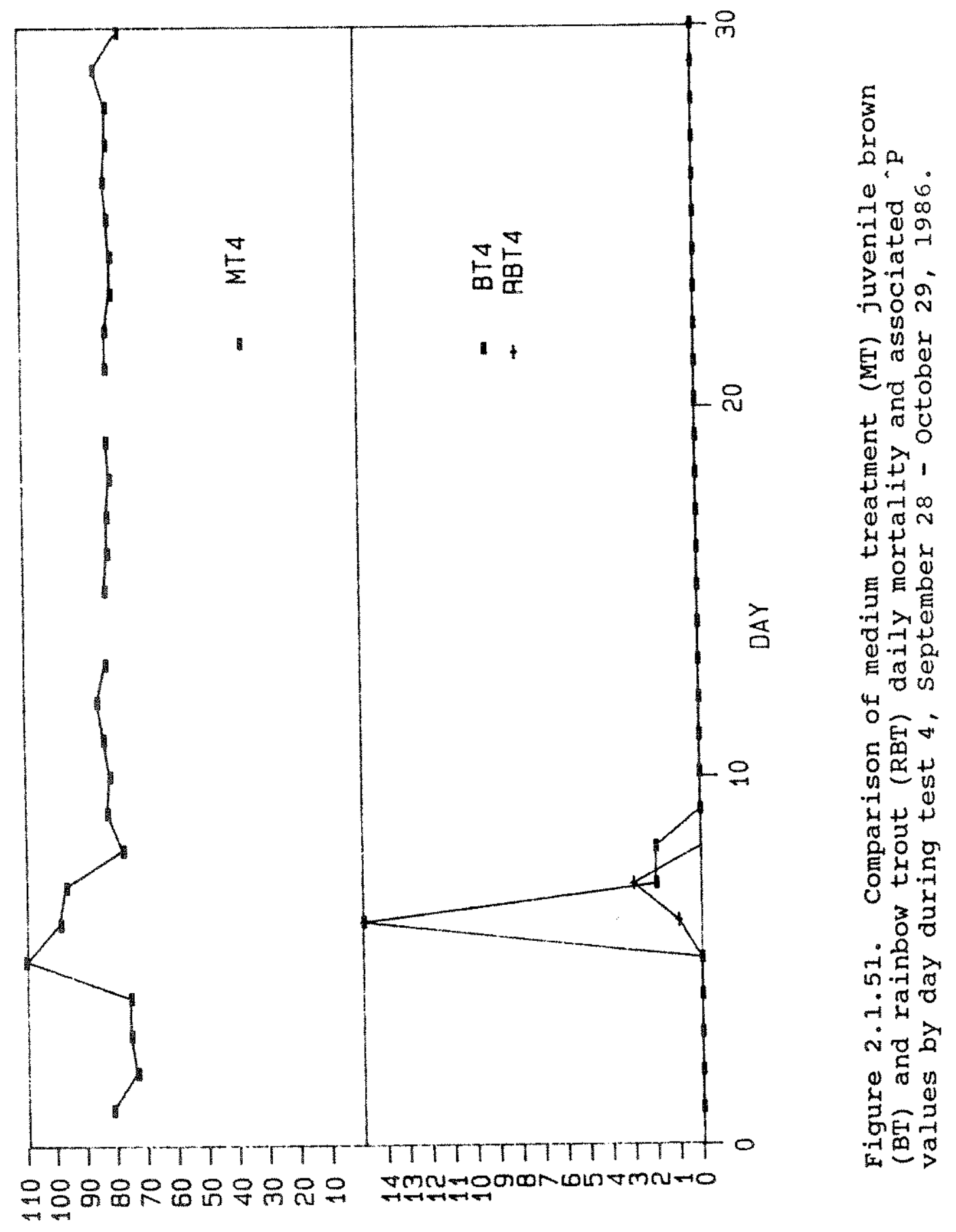

(ww) $d \forall 1790 \quad \wedge \perp I 7 \forall$ HOW $\wedge 7 \mathrm{I} \forall 0$ 
aquatic biota. Cutthroat, rainbow, and steelhead trout fry 19.5 $\mathrm{cm})$ were tolerant to water containing $110 \%$ saturation of nitrogen, but died when exposed to $115 \%$ nitrogen supersaturation (Rucker and Hodgeboom 1953). Juvenile steelhead (180-200 mm) survived exposure to $115 \%$ gas supersaturation, but $120 \%$ supersaturation caused $100 \%$ mortality (Stroud and Nebeker 1976). Apparently a tolerance threshold for juvenile steelhead trout $(180-200 \mathrm{~mm})$ exists between 115 and $120 \%$ supersaturation. Data from this study indicate a similar threshold exists for juvenile brown and rainbow trout. Critical levels of gas supersaturation for brown and rainbow trout were surpassed during tests 2 and 4 . The critical threshold required to cause acute mortality in juvenile rainbow and brown trout (53-131 mm) appears to be between 113-117\%. Medium treatment levels never exceeded the critical threshold long enough to cause mortality in tests 1, 3 , 5 , and 7 .

Mortality of rainbow and brown trout of the high gas treatment was significantly greater than that of control fish in all cases except for test 1 rainbow trout (Table 2.1.10).

Several test 1 control treatment rainbow trout fry died and high treatment rainbow trout mortality was low. The relevance of these data is discussed later.

\section{Influence of Length and weight on susceptibility to GBT}

Length and weight of juvenile brown and rainbow trout had a significant influence on susceptioility to supersaturated water. During test 1 , percent cumulative mortality for both species exposed to the high gas treatment was significantly less 
Table 2.1.10. Breslow rank analysis of high gas treatment percent cumulative mortality for juvenile brown (BT) and rainbow trout (RBT) during tests $1,2,3,4,5$, and 6 .

Mortality Comparison

Control treatment High treatment

species test species test p-value

outcome

\begin{tabular}{|c|c|c|c|c|c|c|}
\hline $\mathrm{BT}$ & 1 & $<$ & $\mathrm{BT}$ & 1 & $<$ & $0.0001 *$ \\
\hline $\mathrm{BT}$ & 2 & $<$ & $\mathrm{BT}$ & 2 & $<$ & $0.0001 *$ \\
\hline BT & 3 & $<$ & $\mathrm{BT}$ & 3 & $<$ & $0.0001 *$ \\
\hline $\mathrm{BT}$ & 4 & $<$ & $\mathrm{BT}$ & 4 & $<$ & $0.0001 *$ \\
\hline BT & 5 & $<$ & $\mathrm{BT}$ & 5 & $<$ & $0.0001 *$ \\
\hline $\mathrm{BT}$ & 6 & $<$ & $\mathrm{BT}$ & 6 & $<$ & $0.0001 *$ \\
\hline RBT & 1 & $=$ & RBT & 1 & & 0.7760 \\
\hline RBT & 2 & $<$ & RBT & 2 & $<$ & $0.0001 *$ \\
\hline RBT & 3 & $<$ & RBT & 3 & $<$ & $0.0001 *$ \\
\hline RBT & 4 & $<$ & RBT & 4 & $<$ & $0.0001 *$ \\
\hline RBT & 5 & $<$ & RBT & 5 & $<$ & $0.0001 *$ \\
\hline $\mathrm{RBT}$ & 6 & $<$ & RBT & 6 & $<$ & $0.0001 *$ \\
\hline
\end{tabular}

* Statistically different at alpha $=0.05$

for small fish than for large fish (Table 2.1.11, Figures 2.1 .52 and 2.1.53). However, there was no statistical difference in brown trout mortality between tests 4 and 5 or rainbow trout mortality between tests 4 and 6 (Table 2.1.11).

Available literature indicates that fish become more sensitive to gas supersaturated water as they increase in size. Meekin and Turner (1974) found large juvenile chinook salmon exposed to $124 \%$ supersaturation suffered greater mortality than did smaller fish of these species. Rucker (1976) tested two size groups of coho salmon $(3.8-6.0 \mathrm{~cm}$ and $8.0-10.0 \mathrm{~cm})$ in $119 \%$ gas supersaturated water and concluded that larger fingerlings 


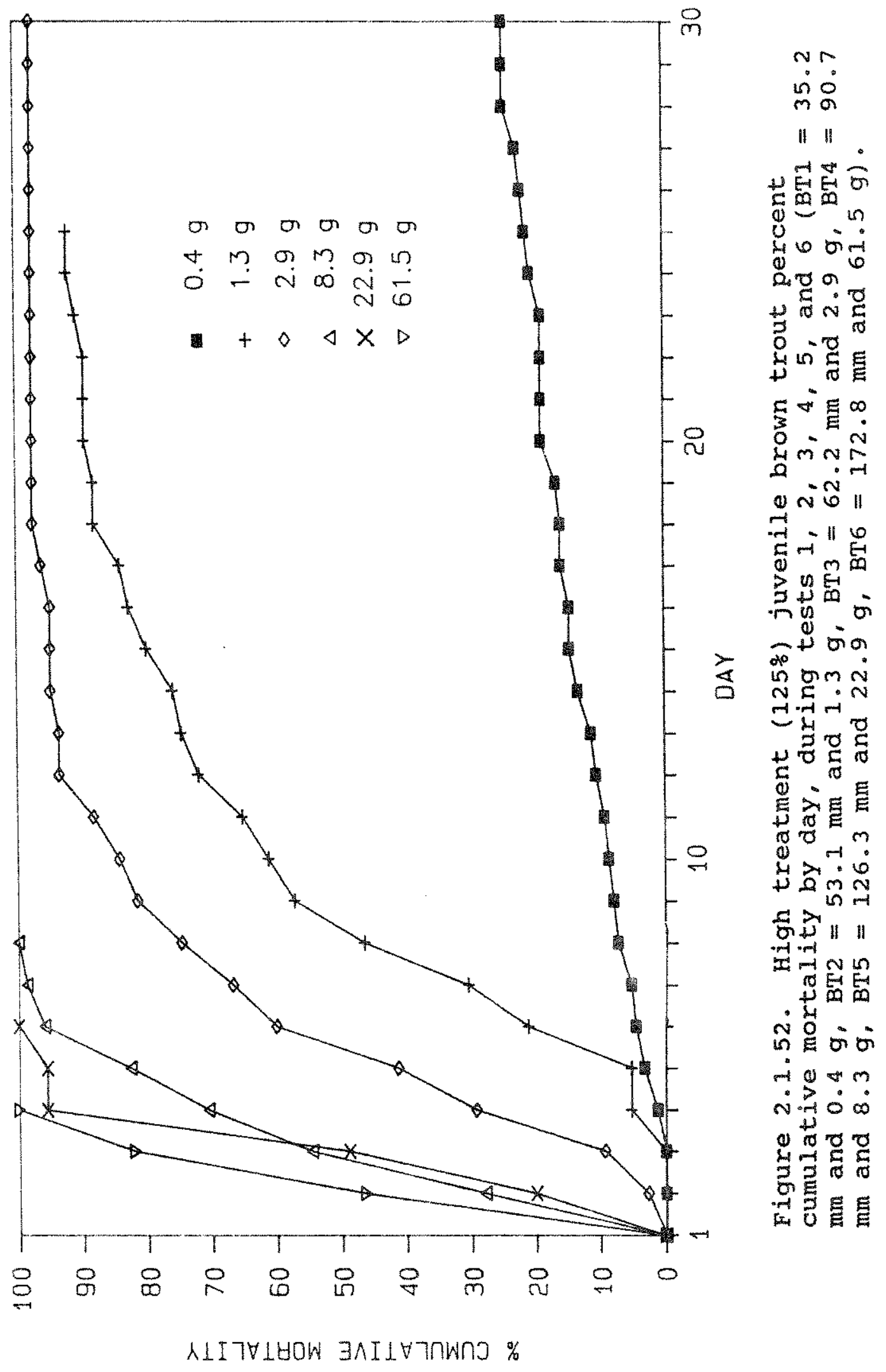




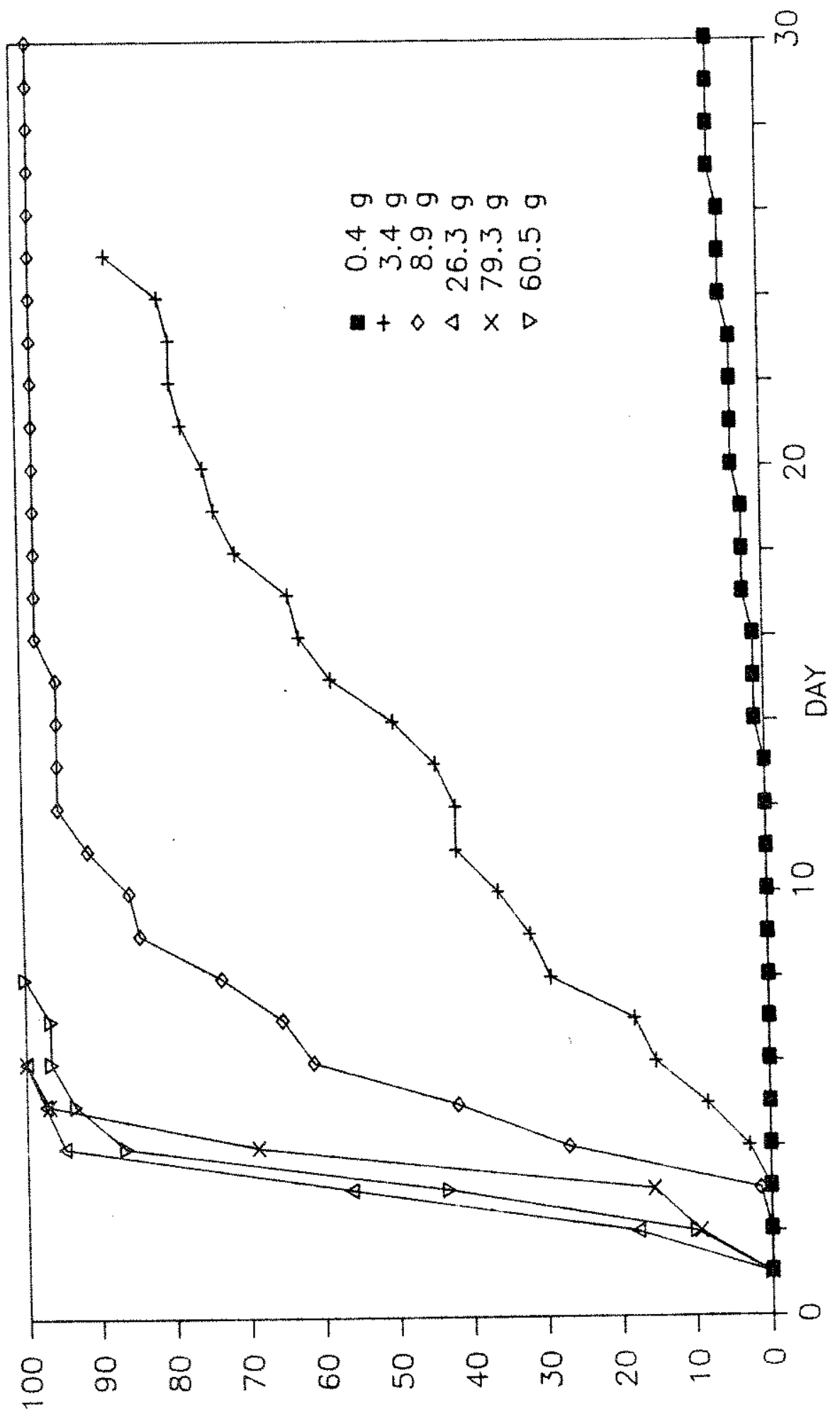

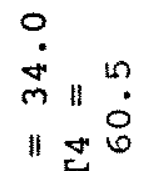

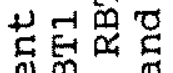

0 क

米企朂

an

$+\underset{\infty}{\infty}$

ว

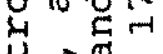

in 11

强

虽苗

省品

:

- $v i$ o

- $-m$ ?

न

()

군

ํㅐㅇㅛ

아 +5

유

กั

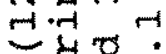

- 늘용

+口

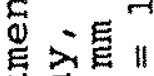

声入

ชั

\& > $0^{\circ}$

कूم

否㓡

- $\begin{aligned} & -1 \\ & \text { f }\end{aligned}$

नु

. 䓵察

$m$ 잉

in

- $0+\frac{\pi}{0}$

$\dot{\sim} \cdot \vec{O}$ E

$0+0$

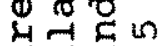

ว

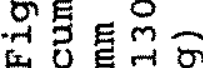

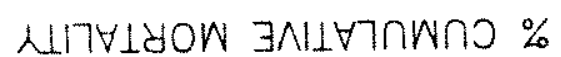


Table 2.1.11. Breslow rank analysis of high gas treatment percent cumulative mortality for juvenile brown (BT) and rainbow trout (RBT) exposed to the high gas treatment during tests 1,2 , $3,4,5$, and 6 .

\section{Mortality comparison outcome}

$$
\text { p-value }
$$

$\begin{array}{ll}\text { BT1 } 1<\text { BT2 } & <0.0001 * \\ \text { BT2 }<\text { BT3 } & <0.0001 * \\ \text { BT3 } 3 \text { BT4 } & <0.0001 * \\ \text { BT4 } 4 \text { BT5 } & 0.7009 \\ \text { BT5 } 5 \text { BT6 } & 0.0019 * \\ \text { RBT1 }<\text { RBT2 } & <0.0001 * \\ \text { RBT2 }<\text { RBT3 } & <0.0001 * \\ \text { RBT3 }<\text { RBT4 } & <0.0001 * \\ \text { RBT4 }=\text { RBT6 } & 0.1778 \\ \text { RBT6 }<\text { RBT5 } & 0.0295 *\end{array}$

* Statistically different at alpha $=0.05$

were more subject to harm than smaller fish. Jensen et al. (1986) predicted that larger anadromous juvenile salmonids should be more sensitive to effects of gas supersaturated water than are smaller fish.

The basis for increased susceptibility to gas supersaturated water with size remains unclear. The mechanics of bubble formation (emboli) may offer an explanation for increasing sensitivity to gas supersaturated water with increasing fish size. Harvey et al. (1944) explained the mechanism of emboli formation in the blood stream of vertebrates. Undissolved gases in the blood stream begin to form emboli when they adhere to "nucleation sites" (rough or irregular regions in the vascular system or body tissue). As the delta $\mathrm{P}$ of the blood increases, the radius of a gas bubble on the nucleation site expands as gas diffuses into it. If supersaturated conditions continue long 
enough at a high enough level, the buoyant force of the bubble becomes sufficient for it to dislodge from the nucleation site.

Fidler (1985) hypothesized that increased susceptibility is closely associated with the radius and number of nucleation sites in a fish. Two reasons were given as support for this hypothesis. The gas level threshold required for bubble formation is lower for large nucleation sites. Consequently, smaller fish (with smaller nucleations sites) would suffer less severe emboli formation than a larger fish at a given percent TGP. Secondly, larger fish may have greater discontinuity in tissue and vascular wall structure. This may provided a greater number of nucleation sites and result in more emboli.

our results generally support Fidler's (1985) theory with results of test 4 being the only exception. Total gas pressure was $1 \%$ higher during test 4 than in any other test and may have influenced mortality.

\section{species Influence on susceptibility to Gas supersaturation}

Mortality rate of brown trout exposed to high gas levels (125\%) was significantly greater than that of rainbow trout of similar size (Table 2.1.12). The difference in susceptibility was greatest when fish were smallest (Figure 2.1.54) and became progressively less evident as fish grew (Figures 2.1.55, 2.1.56, and 2.1 .57 .

Variations in susceptibility to GBT have been reported for different species of fish. Coho salmon (ㅇ․ kisutch) were more tolerant to water supersaturated with nitrogen in the presence of decreasing water temperatures than were chinook salmon or 


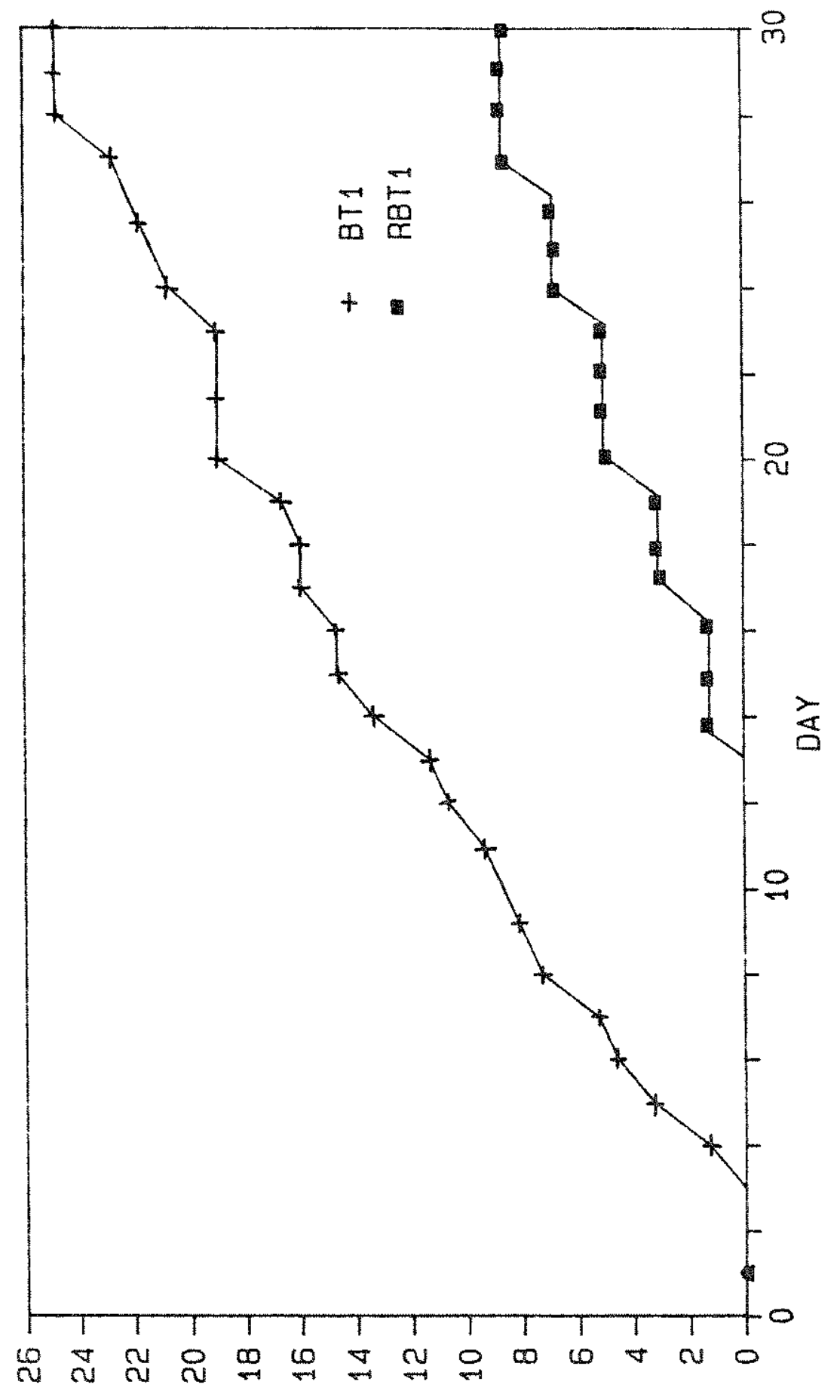

至点

-7

o

岁苦

ᄃㄴ

ชิ

胥芯

है क

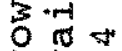

\& ${ }^{2} 0$

0

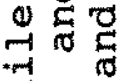

ธิธ

可

ํํㅇ.

崖

㟧山药

से

o ह

幽的是

है। यू

两 동구

..- कु

$\therefore 0$

เง

-

들

$\sim \prod_{. \rightarrow-1}$

过它

3 ठ․न

कृ

的車员

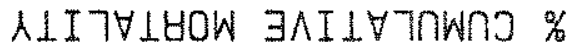




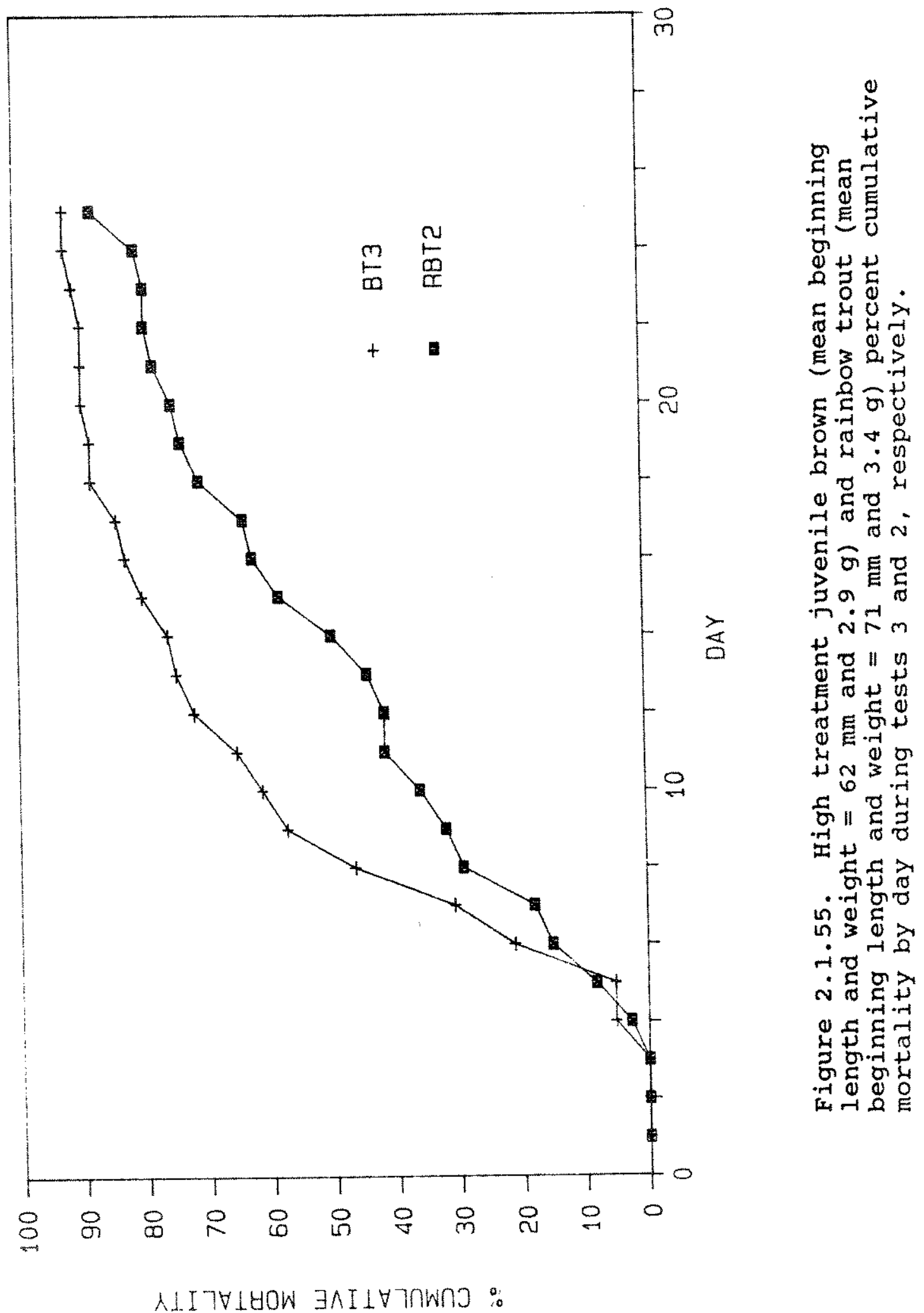




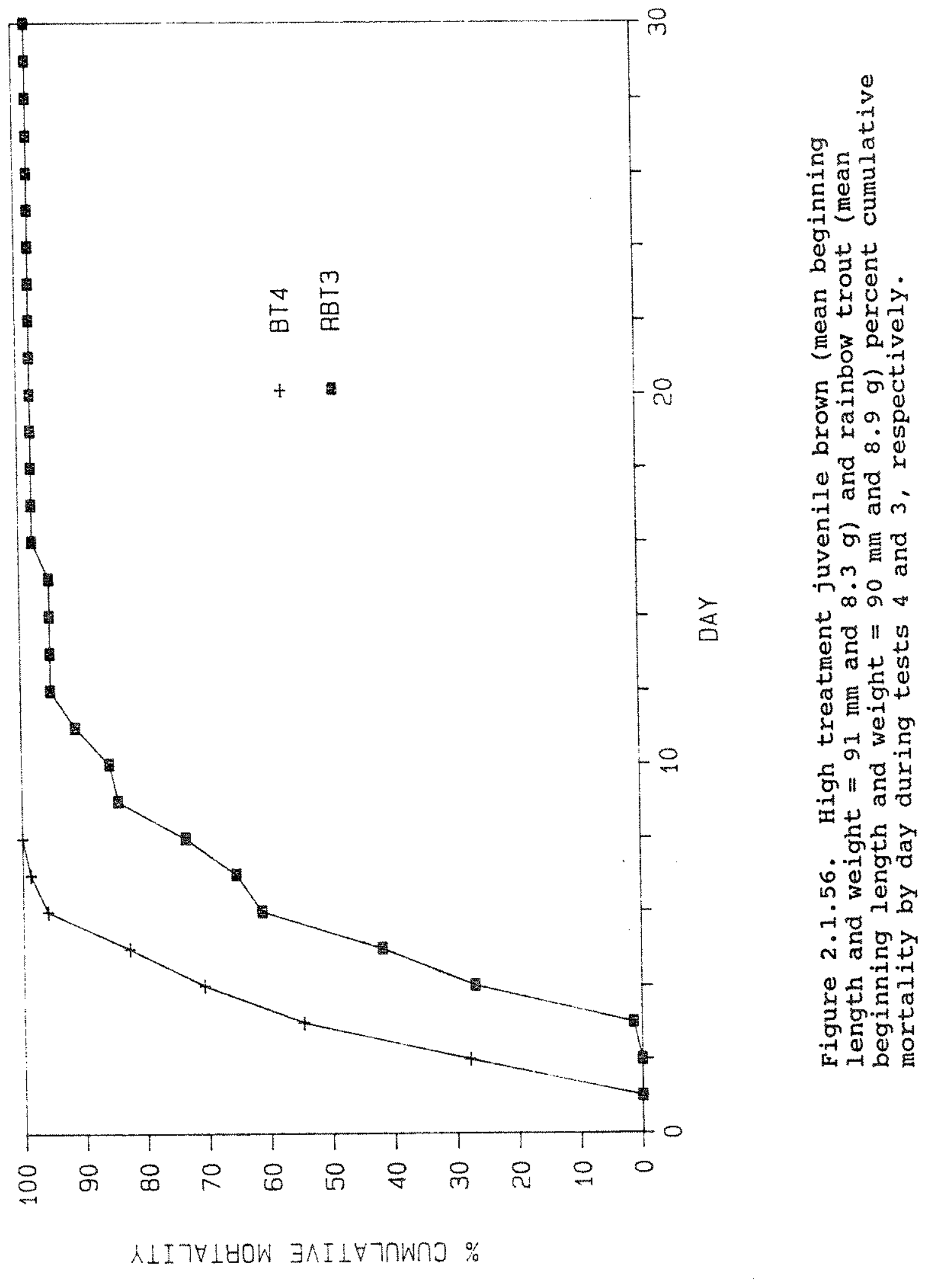




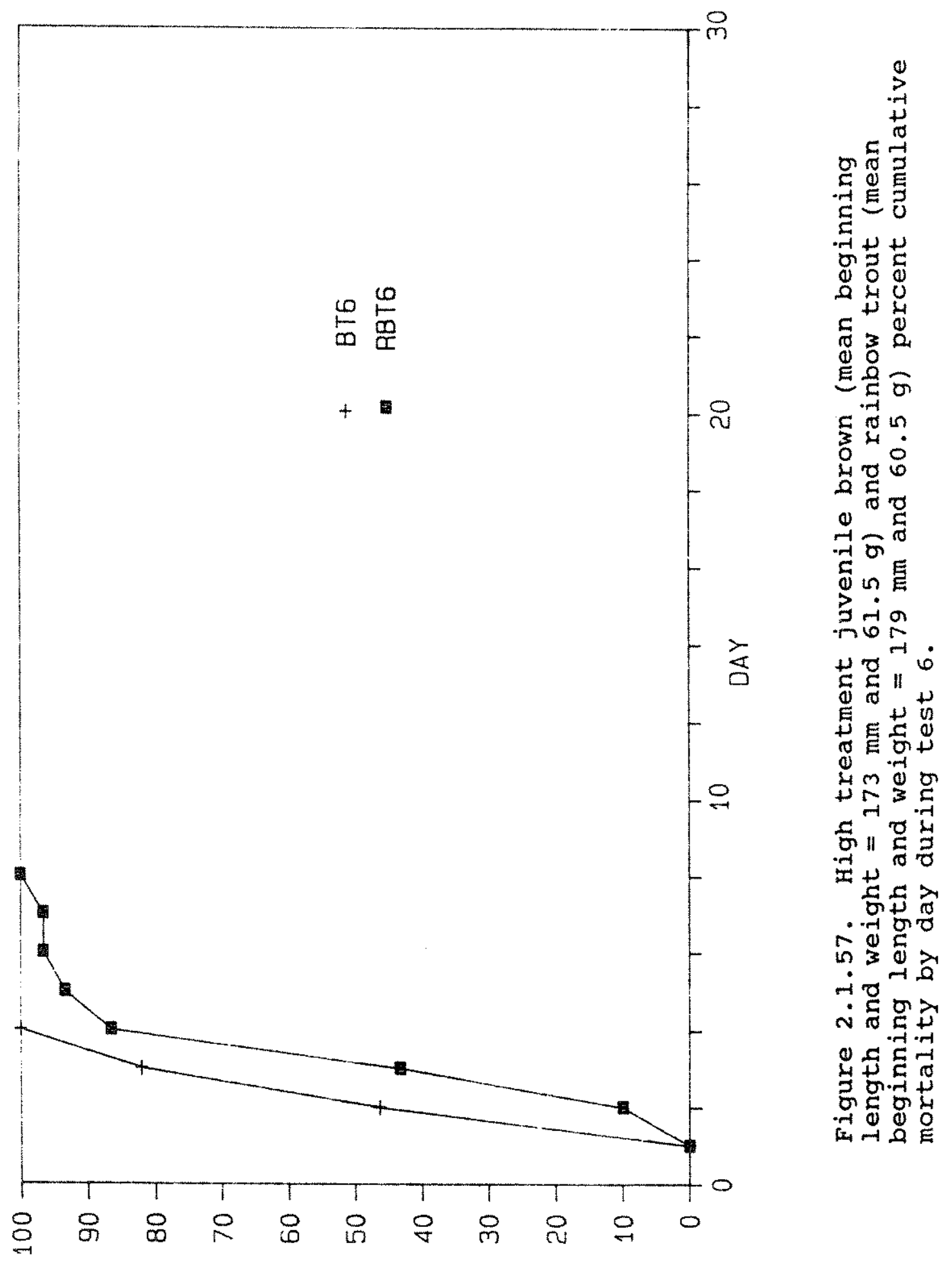

$\wedge \perp I 7 \forall \perp$ HOW $\exists \wedge I \perp \forall 7 \cap W \cap 3 \%$ 
Table 2.1.12. Breslow rank analysis comparing the rate mortality for juvenile brown (BT) and rainbow trout (RBT) of similar size in the high gas treatment.

Mortality comparison

$\mathrm{p}$-value outcome

$\begin{array}{rlrl}\mathrm{BT} 1 & >\mathrm{RBT} 1 & <0.0001 * \\ \mathrm{BT} 3 & >\mathrm{RBT} 2 & <0.0001 * \\ \mathrm{BT} 4 & >\mathrm{RBT3} & <0.0001 * \\ \mathrm{BT6}>\mathrm{RBT} 6 & <0.0002 *\end{array}$

* statistically different at alpha $=0.05$

steelhead trout (Ebel et al. 1971). Coho salmon were also more tolerant $\left(\mathrm{LC}_{50}=116.2 \%\right)$ of gas supersaturated water than were steelhead trout $\left(\mathrm{LC}_{50}=114.0 \%\right)$ or sockeye salmon $\left(\mathrm{LC}_{50}=\right.$ 113.9\%), (Nebeker and Brett 1976). Fickeisen and Montgomery (1978) found the median time to death caused by exposure to gas supersaturated water to be longest for whitefish (Prosopium williamsoni), followed in descending order by cutthroat trout (Salmo clarki), large scaled sucker (Catostomous macrocheilus), and torrent sculpin (Cottus rhotheus). Thorn et al. (1978) exposed rainbow and brown trout to $122 \%, 127 \%$, and $129 \%$ gas supersaturated water for $7 \mathrm{~h}$. They reported higher brown trout mortality in the $122 \%$ exposure test, while rainbow trout mortality was larger at the two higher gas levels tested. Mean time to $50 \%$ mortality at four lethal concentrations of gas supersaturated water was longer for speckled dace (Rhinicthys osculus) than cutthroat trout (Nebeker et al. 1980). Sea bass (Dicentrarchu labrax) and striped mullet (Mugil cephalus) had 
similar mortality responses when exposed to $127.4 \%$ and $129.6 \%$ gas supersaturated water (Gray et al. 1985). Differences in the anatomy of rainbow and brown trout may help provide an explanation for interspecific variation in mortality. According to Fidler (1985), emboli form faster in a fish with more rough areas in the walls of the vascular system. Perhaps, during the earliest life stages tested, brown trout vascular tissue was more irregular than that of rainbow trout. Irregularities in vascular tissue of rainbow trout may increase with fish size explaining the similarity in mortality of the species in later tests. Thorn et al. (1978) suggested that differences in interspecific mortality may be related to the capacity of the gas bladder. Increased capillary area on the bladder would facilitate passage of gas from the blood stream to the gas bladder, where it could be passed through the pneumatic duct. The vascular structure or functioning of the pneumatic duct may differ between brown and rainbow trout. A conclusive explanation for differences in interspecific mortality remains unknown.

\section{Sublethal Effects of Exposure to Gas supersaturated water}

Growth: Exposure of juvenile brown and rainbow trout to $112 \%$ gas supersaturated water (medium treatment) did not affect growth. Beginning and final mean weights of medium treatment fish from tests $1,3,5$, and 6 were not significantly different from control fish (Table 2.1.13). Growth data from medium treatment tests 2 and 4 were not used because mortality, which may have been related to fish size, occurred and could have biased results. 


\begin{tabular}{|c|c|c|c|}
\hline 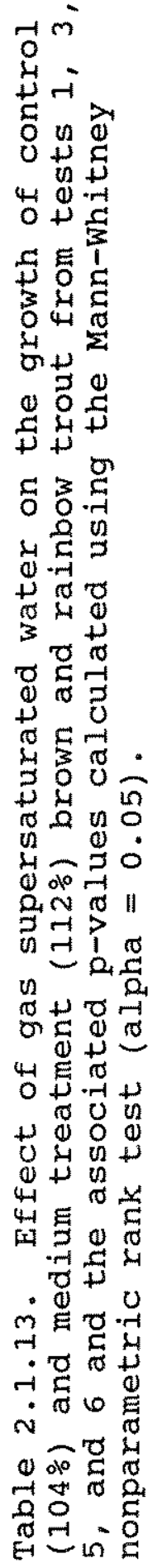 & 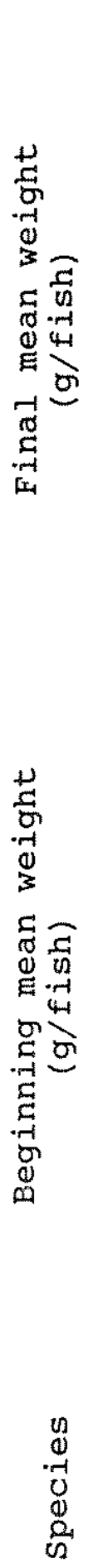 & 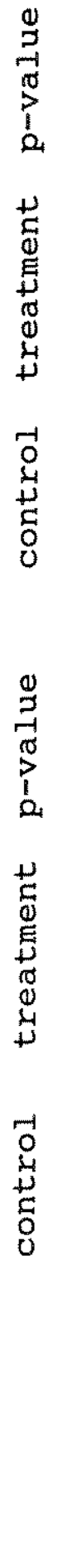 & 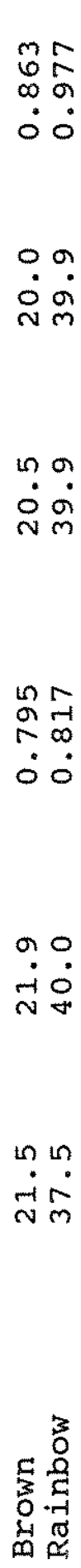 \\
\hline
\end{tabular}


Some workers have shown that exposure to gas supersaturated water affects growth of surviving fish. Brown and rainbow trout reared in $120 \%$ gas supersaturated water grew more slowly than those reared in $108 \%$ gas supersaturated water for 36 weeks (Poston et al. 1973). Northern squawfish exposed to $120 \%$ gas supersaturated water for $12 \mathrm{~d}$ consumed an average of $12 \mathrm{~g} / \mathrm{d}$ less food than did squawfish exposed to $100 \%$ saturation (Bentley et al. 1976). Yearling lake trout growth and food conversion was reduced during exposure to sublethal levels of gas supersaturation (Thorn et al. 1978). Stress experienced by fish during exposure to gas supersaturated water may be tied to decreased fish growth. Stress induced by unfavorable environmental conditions affects fish production traits (Piper et al. 1982). Exposure to sublethal levels of supersaturated water may cause improper functioning of the gastrointestinal tract. stroud and Nebeker (1976) found that food was held in the stomach of juvenile steelhead trout up to $54 \mathrm{~h}$ at $120 \%$ TGP and $93 \mathrm{~h}$ at 115\%. They determined that this time period was longer than would be expected, possibly because of decreased peristalic movement of the gut. Poorer growth of fishes exposed to gas supersaturated water in these investigations may be due to inefficient food digestion attributable to gas supersaturated water.

other researchers report that gas supersaturation does not affect fish growth. Juvenile steelhead trout surviving 40-d exposure to gas supersaturated water with delta-P from 150 - 180 mm showed no significant decrease in growth (Nebeker et al. 1978). Channel catfish (Ictalurus punctatus) surviving exposure 
to 104.7 - $115 \%$ TGP for $35 \mathrm{~d}$ showed no reduced growth or signs of GBT, yet some died (Colt et al. 1985). The response appeared to be all or nothing; fish either died or showed no growth effects. During our tests, $112 \%$ TGP did not impair feeding behavior, physiological growth factors, or cause significant mortality in juvenile brown or rainbow trout within the length and weight range tested. However, some mortality occurred in 30-d bioassays when percent TGP surpassed $112 \%$. The "all or nothing response" identified by colt et al. (1985) seems applicable to this study.

Disease Resistance: Significantly more bacteria were identified in kidney samples of fish challenged with bacteria than in unchallenged fish (Table 2.1.14). Juvenile brown trout with GBT from exposure to $118 \%$ gas supersaturated water challenged with bacteria for 20 - $40 \mathrm{~min}$ had more bacteria in kidney samples than fish similarly challenged but not exposed to gas supersaturated water (Table 2.1.15). However, this difference was significant only for the 30-min challenge (Table 2.1.15). Results of the second replicate test were discarded because control fish died from a Pseudomonas sp. infection unrelated to experimental procedure.

Bacteria of the genus Aeromonas sp. are ubiquitous microbes of water and are commonly found in the alimentary tract of healthy salmonids (Trust and Sparrow 1974). However, under conditions of stress, $\underline{A}$. hydrophila can become an opportunistic pathogen. Thorpe and Roberts (1972) described a septicemic outbreak in spawning brown trout of Loch Leven, Scotland. Grayish skin ulcerations, abdominal distention, swelling and discoloration of internal organs, secretion of a yellow mucous in 
Table 2.1.14. Two sample t-test analysis of the number of $\underline{A}$. hydrophila identified in kidney samples of juvenile brown trout challenged with bacteria and unchallenged fish.

\begin{tabular}{ccccc}
\hline & \multicolumn{4}{c}{ Comparison } \\
\cline { 3 - 5 } $\begin{array}{c}\text { Challenge } \\
\text { duration (min) }\end{array}$ & $\begin{array}{c}\text { chlienged } \\
\text { fish } \\
\text { (w/bacteria) }\end{array}$ & $\begin{array}{c}\text { unchallenged } \\
\text { fish } \\
\text { (nocteria) }\end{array}$ & p-value \\
\hline 10 & no GBT & $>$ & no GBT & $0.0039 *$ \\
20 & no GBT & $>$ & no GBT & $0.0010 *$ \\
30 & no GBT & $>$ & no GBT & $0.0000 *$ \\
40 & no GBT & $>$ & no GBT & $0.0000 *$ \\
10 & GBT & $>$ & GBT & $0.0003 *$ \\
20 & GBT & $>$ & GBT & $0.0001 *$ \\
30 & GBT & $>$ & GBT & $0.0000 *$ \\
40 & GBT & $>$ & GBT & $0.0000 *$
\end{tabular}

* Significantly more $\underline{A}$. hydrophila in juvenile brown trout challenged with bacteria than in unchallenged fish at alpha $=$ 0.05

the intestine and subsequent swelling of the anal vent are common in infected fish during epizootics of motile aeromonad septicemia (Piper et al. 1982).

Trust (1986) speculated that bacterial infection may result from penetration of the thick mucosal layer of the skin. Post (1983) hypothesized that soon after a bacterium enters the tissue or blood stream of a fish, it is phagocytized and transported to the spleen or anterior kidney. Macrophages may break the bacterium into separate antigenic components. B-cells and plasma cells may produce antibodies specific to these antigens.

It appears that stress and increased systemic access can facilitate bacterial disease. During our tests, stress caused by GBT may have impaired the defense (macrophages) and immune system (antibody production) functioning. The blistering of the Table 2.1.15. Two sample t-test analysis of the number of $\underline{A}$. 
Table 2.1.15. Two sample t-test analysis of the number of $\mathrm{A}$. hydrophila identified in kidney samples of juvenile brown trout $(196 \pm 13 \mathrm{~mm}$ and $74.5 \pm 17.3 \mathrm{~g})$ not exposed to gas supersaturated water (control) and fish $(198 \pm 12 \mathrm{~mm}$ and $75.4 \pm 16.2 \mathrm{~g})$ with GBT (test) from exposure to $118 \%$ supersaturated water for $120 \mathrm{~h}$.

\begin{tabular}{cccc}
\hline \multirow{2}{*}{$\begin{array}{c}\text { Challenge duration } \\
\text { (min) }\end{array}$} & \multicolumn{2}{c}{$\begin{array}{c}\text { Mean number of } \\
\text { bacterium/kidney }\end{array}$} & p-value \\
\cline { 2 - 3 } 10 & control & test & 0.4234 \\
20 & $58 \pm 9$ & $53 \pm 3$ & 0.0672 \\
30 & $70 \pm 3$ & $65 \pm 4$ & $0.0145 *$ \\
40 & $85 \pm 4$ & $92 \pm 3$ & 0.0535
\end{tabular}

* Significantly more $\underline{A}$. hydrophila in juvenile brown with GBT at alpha $=0.05$

epidermis common to emphysema may have provided bacteria open passage to capillary beds. Severe exopthalmia characterized by freely bleeding open eye wounds also may have provided bacteria direct access to the bloodstream of the fish.

\subsection{Summary: Physical and Phsyiological Parameters Associated with GBT}

Experimental studies confirm the existence of bubble growth thresholds associated with various forms of bubble growth and swimbladder overinflation in fish exposed to supersaturated water. These thresholds correlate with mortality thresholds observed during our experiments as well as those derived from the literature. Furthermore, with the definition of a gill oxygen uptake ratio (F) and a critical nucleation site radius ( $r_{0}$ ), these thresholds can be predicted using the bubble growth threshold equations (Equations 2 through $4^{\prime}$ ). An adult trout mortality threshold occurring at water TGP 
levels between 1.10 and 1.12 atms corresponds to thresholds for the growth of extracorporeal bubbles on the gills and sub-dermal bubbles in the buccal cavity. A mortality threshold occurring at water TGP levels of 1.15 to 1.18 atms correlates with thresholds for intravascular bubble growth occurring in the TGP range of 1.16 to 1.19 atms.

The transition from a lower TGP mortality threshold (1.101.12 atms) to an upper threshold (1.16 - 1.19 atms) appears to involve a shift in the bubble related mechanisms that lead to death. At the lower threshold, sub-dermal bubbles in the lining of the mouth and extracorporeal bubbles in gill lamellae appear to block the exchange of respiratory gases. At a water TGP of 1.15 atms, however, the extracorporeal bubbles become fewer and even disappear and time to mortality increases. This appears to be the result of the larger bubbles being more easily dislodged by respiratory water flow. At water TGP levels between 1.16 and 1.19 atms, intravascular bubble formation begins and time to mortality again decreases with increasing TGP. At these TGP levels, sub-dermal bubbles in the lining of the mouth are smaller or are absent entirely. Apparently the rapid death caused by intravascular bubbles at these higher levels of TGP does not allow time for sub-dermal bubbles to develop. This sequence correlates with data from the literature which suggest that during this transition there is a region of TGP that offers some relief to the fish.

It should be noted that these experimental studies, as well as most of those reported in the literature, involve fish that are cestrained. Because extracorporeal bubbles between gill 
lamellae appeared to be easily dislodged at TGP levels above 1.15 atms, it might be expected that free swimming fish would be able to remove these bubbles by burst swimming. If this were the case, a mortality threshold at 1.10 atms may not be observed. As pointed out above, mortality at the 1.1 atms threshold appears to require both extracorporeal bubble growth between gill lamellae and blistering within the buccal cavity. The absence of a mortality threshold at 1.1 atms appears to be the case for trout populations on the Bighorn River. The various experiments conducted on the river that support this conclusion will be described in a later section of this report.

Using the threshold equations to back calculate an effective nuclei radius, it is found that a radius of 12 to $14 \mu \mathrm{m}$ is common to all forms of bubble growth. Using $12 \mu \mathrm{m}$ as an effective nucleation radius, the theoretical threshold equations can be written in a final form. These are shown in terms of TGP thresholds as Equations $5,6 \& 7$ and delta $\mathrm{p}$ thresholds in Equations $5^{\prime}, 6^{\prime} \& 7^{\prime}$. The oxygen uptake ratio, $F$, has been taken as 0.79 for intravascular bubble growth and 0.85 for swimbladder overinflation.

EQUATION 5: Threshold Criteria for Bubble Growth in the vascular system $\mathrm{TGP}_{\mathrm{CV}} \geq \mathrm{P}_{\mathrm{Atm}}+73.1 \cdot \mathrm{h}+0.21 \cdot \mathrm{pO}_{2}+83.0$ 
EQUATION 6: Threshola criteria for Overinflation of the swimbladder

$\operatorname{TGP}_{\mathrm{SB}} \geq \mathrm{P}_{\mathrm{Atm}}+73.1 \cdot \mathrm{h}+0.15 \cdot \mathrm{pO}_{2}$

EQUATION 7: Threshold criteria for Bubble Growth in Environmental water

$\mathrm{TGP}_{\mathrm{EW}} \geq \mathrm{P}_{\mathrm{Atm}}+73.1 \cdot \mathrm{h}+83.0$

EQUATION 5\%: Threshold Criteria for Bubble Growth in the Vascular system

$\mathrm{DP}_{\mathrm{CV}} \geq 73.1 \cdot \mathrm{h}+0.21 \cdot \mathrm{pO}_{2}+83.0$

EQUATION 6: Threshold criteria for Overinflation of the S: 1 mbladder

$\mathrm{DP}_{\mathrm{SB}} \geq 73.1 \cdot \mathrm{h}+0.15 \cdot \mathrm{pO}_{2}$

EQUATION 7: Threshold Criteria for Bubble Growth in gnvirronmental mater

$$
\mathrm{Dp}_{\mathrm{EW}} \geq 73.1 \cdot \mathrm{h}+83.0
$$

Part two experimental studies indicate that the critical threshold required to cause acute mortality in juvenile rainbow and brown trout $(53.1-130.5 \mathrm{~mm})$ was between $113-117 \%$ TGP. This threshold level is slightly higher than the initial 
threshold level found in the part one experiments with adult trout (110-112\% TGP). This slightly higher juvenile trout GBT threshold seems reasonable considering the observation that smaller juveniles were less sensitive to nitrogen supersatuation than larger juvenile trout.

Part two studies examining interspecific and sublethal effects of GBT on juvenile trout found that:

1) Daily mortality of brown trout exposed to high gas supersaturation levels (125\%) was significantly greater than that of rainbow trout of similar size during all comparisons. The difference in susceptibility was most evident when fish were smallest $(0.4 \mathrm{~g})$. Differences in percent cumulative mortality between species were less in subsequent tests when fish were larger $(8.3-60.5$ g).

2) Exposure to $112 \%$ gas supersaturated water did not affect growth of juvenile brown and rainbow trout.

3) Bacterial challenges indicated that GBT may increase the susceptibility of brown trout to $\underline{\mathbb{A}}$. hydrophila infection. 


\subsection{Recovery trom symptoms of GBT \\ 2.2. Introduction}

An early study on effects of gas supersaturated water on marine fishes showed external signs of GBT regressed within $24 \mathrm{~h}$ after removal to unsaturated water (Gorham 1901). Information pertaining to brown and rainbow trout recovery from GBT is scarce. Subsequent to extended periods of low percent total gas pressure (TGP), emphysema was superseded by dark patches of scar tissue on brown trout of the Bighorn River. However, the time needed for this recovery is uncertain.

Repeated exposure may influence the time of recovery after initial exposure to gas supersaturated water. Weitkamp and Katz (1980) implied that intermittent exposure may increase the level of supersaturation fish can tolerate by increasing the time over which a specific exposure accumulates. Alternatively, scar tissue formed during recovery from prior exposure could accelerate formation of GBT symptoms by providing nucleation sites with largex surface area.

The goal of GBT recovery research was to gain a better understanding of the ability of rainbow and brown trout to recover from exposure to gas supersaturated water. Specific objectives were: (1) evaluate the ability of juvenile brown and rainbow trout to recover from GBT at various levels of severity; and (2) detemine the effect of repeated exposure to gas supersaturated water on juvenile brown trout. 


\section{$2.2 \mathrm{~b}$ Methods}

Recovery tests were conducted in laboratory facilities at the United States Fish and Wildlife Service, Bozeman Fish Technology Center. Supersaturated water was produced as described in section $2.1 .3 .2 . b$.

\section{Single Exposure Recovery Tests}

Both rainbow and brown trout were placed into two, $1.2 \mathrm{~m}$ diameter tanks and exposed to 111 to $117 \%$ supersaturated water for periods long enough to develop external symptoms of GBT. This time period ranged from 5 to 30 d. Affected fish were removed from treatment tanks, symptoms recorded, and each fish assigned a GBT rating of $0,1,2$, or 3 (Table 2.2 .1 ). Fish were then transferred to $104 \%$ supersaturated water and recovery was monitored every other day for $30 \mathrm{~d}$.

\section{Repeated Exposure Recovery Tests}

A second series of recovery tests was conducted to investigate the effects of repeated exposure of juvenile brown and rainbow trout to gas supersaturated water. The procedure was identical to single recovery tests except fish were exposed initialiy, survivors were given $30 \mathrm{~d}$ to recover and then were exposed a second and third time, with a 30-d recovery period in between. One additional GBT rating (4) was used to describe fish that did not survive repeat exposure to gas supersaturated water (Table 2.2.1). Fisher's Exact Test (Hintze 1987) was used to test for changes in symptom severity between exposures. 
Table 2.2.1. Gas bubble trauma description and other criteria used to rank laboratory recovery data.

Description and GBT status

Rating

-No visible external symptoms. Fish have developed no external symptoms of GBT during exposure to gas supersaturated water or have lost all previously

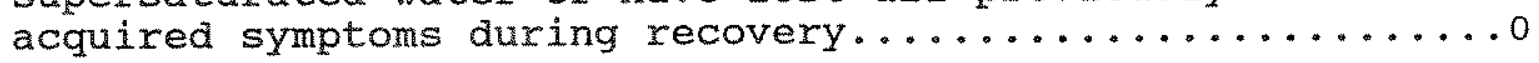

-Emphysema present in one to four of the following regions: Buccal cavity, mandible, maxillary, left opercle, right opercle, left eye, right eye, head, left lateral line, right lateral line, left pectoral fin, right pectoral fin, anal fin, adipose fin, caudal fin,

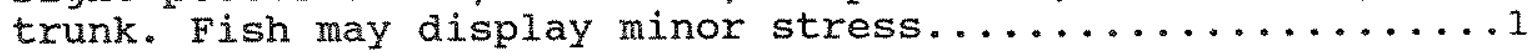

-Emphysema present on more than four of the above regions. Mild exopthalmia in one or both eyes, but no evidence of impaired swimming. Impaired vision combined with reduced appetite may reduce feeding activity and

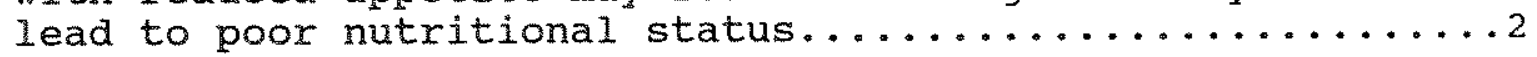

-Severe emphysema and exopthalmia in one or both eyes. Eyes may be cloudy and hemorrhaging. May lack response to visual stimuli. Noticeable swimming impairment. obvious loss of equilibrium, spastic swimming may be evident. Blinded fish physiologically deteriorate (embedded scales and weight loss) and may starve.

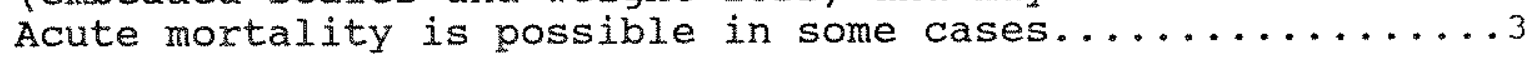

-Fish that died during the second or third exposure to gas supersaturated water.......................

\subsection{Results and Discussion}

\section{Recovery from a single Exposure}

Juvenile brown and rainbow trout recovery (loss of external symptoms) from GBT varied with severity of external symptoms. Less severely affected rainbow trout recovered more often and faster (Table 2.2 .2 and Figure 2.2.1). Five of six rainbow trout that did not recover within 30 d exhibited eye damage characterized by severe unilateral and bilateral exopthalmia, hemorrhaging, cloudiness, corneal cataracts, or bubbles in the 
Table 2.2.2. Recovery of juvenile rainbow trout $(228 \pm 17 \mathrm{~mm}$ and $124.6 \pm 30.7 \mathrm{~g}$ ) exposed to $117 \%$ gas supersaturated water for $9 d$ February $1-9,1987$.

\begin{tabular}{cccc}
\hline $\begin{array}{c}\text { GBT } \\
\text { rating }\end{array}$ & $\begin{array}{c}\text { Beginning } \\
\text { number }\end{array}$ & $\begin{array}{c}\text { Number } \\
\text { dead by day 30 }\end{array}$ & $\begin{array}{c}\text { Number with symptoms } \\
\text { remaining after 30 d }\end{array}$ \\
\hline 1 & 5 & 0 & 0 \\
2 & 7 & 0 & 5 \\
\hline
\end{tabular}

a

see Table 2.2.1

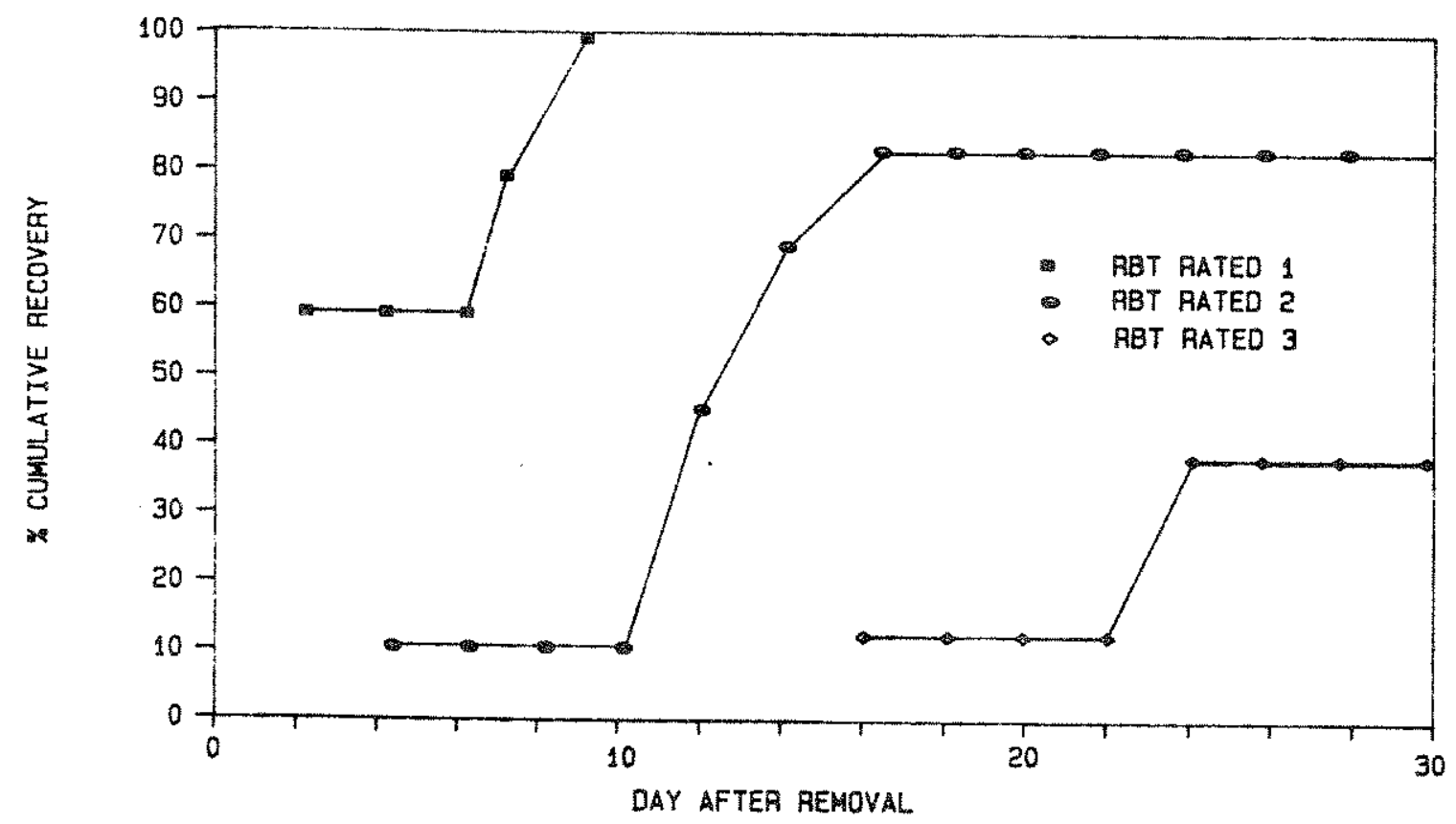

Figure 2.2.1. Comparison of GBT severity and recovery rate of juvenile rainbow trout $(228 \pm 17 \mathrm{~mm}$ and $125 \pm 31 \mathrm{~g})$ by 2d intervals February 9 - March 12, 1987 (See Table 2.2.1 for severity definitions). 
eye orbit.

Juvenile brown trout with emphysema (GBT rating 1; Table 2.2.1) and mild exopthalmia recovered within $30 \mathrm{~d}$. However, brown trout with widespread emphysema and severe exopthalmia (GBT rating 3) recovered less often (Table 2.2.3). Exopthalmia developed during recovery in the two fish that did not recover from 17-d exposure to $111 \%$ gas supersaturated water (Table $2.2 .3)$. Two brown trout rated 3 after exposure to $117 \%$ TGP for 5 d died soon after transfer to a recovery tank.

Recovery from GBT has been documented for several species. Emphysema and exopthalmia regressed in scup (stenotomus chrysops) within $24 \mathrm{~h}$ of removal from shallow tanks to tanks with pressure equal to 16 feet of water (Gorham 1901). Cutthroat, rainbow, and steelhead trout fingerlings raised in hatchery troughs at Puyallup Hatchery of the Washington state Department of Game showed high incidence of GBT symptoms. Transfer of fingerlings to an outside pond eliminated all external symptoms (Rucker and Hodgeboom 1953). Impaired swimming ability of juvenile chinook salmon exposed to $120 \%$ supersaturation was rectified within $2 \mathrm{~h}$ after removal to $100 \%$ saturated water (Schiewe 1974). Eratic swimming and yolk sac emboli were alleviated in coho salmon sac fry using a recompression chamber (Adams 1974). Dawley et al. (1976) determined that 2 weeks was an adequate recovery period for juvenile chinook with GBT acquired from exposure to 110-120\% supersaturation for $120 \mathrm{~d}$. Ninety-one to $95 \%$ of juvenile chinook salmon surviving $10-20-\mathrm{d}$ exposure in $1-4 \mathrm{~m}$ of water of 120-131\% gas supersaturation lost all external symptoms of GBT after $20 \mathrm{~d}$ in an unsupersaturated environment (Weitkamp 1976). Apparently, 
Table 2.2.3. Recovery of juvenile brown trout (133 $\pm 21 \mathrm{~mm}$ and $27.5 \pm 9.5 \mathrm{~g})$ by severity level, following exposure to various levels and durations of gas supersaturation.

\begin{tabular}{cccccc}
\hline $\begin{array}{c}\text { GBTa } \\
\text { rating }\end{array}$ & $\begin{array}{c}\text { Beginning } \\
\text { number }\end{array}$ & $\begin{array}{c}\text { Exposure } \\
\text { duration } \\
\text { (days) }\end{array}$ & $\begin{array}{c}\text { Gas } \\
\text { level } \\
\text { (\%TGP) }\end{array}$ & $\begin{array}{c}\text { Number dead } \\
\text { after } \\
30 \mathrm{~d}\end{array}$ & $\begin{array}{c}\text { Number } \\
\text { retaining } \\
\text { symptoms } \\
\text { after } 30 \mathrm{~d}\end{array}$ \\
\hline 1 & 15 & 17 & 111 & 0 & 0 \\
1 & 10 & 30 & 111 & 0 & 0 \\
2 & 3 & 5 & 117 & 0 & 0 \\
2 & 2 & 30 & 111 & 0 & 0 \\
3 & 2 & 5 & 117 & 2 & 2 \\
3 & 3 & 17 & 111 & 0 & 0 \\
\hline
\end{tabular}

a see Table 2.2.1 for rating description

most fish with GBT will recover if moved to an unsaturated environment.

Eye damage in surviving salmonids may be the principal permanent effect of exposure to sublethal doses of gas supersaturated water. Dawley et al. (1976) removed chinook salmon with GBT to a non-supersaturated environment, and $95 \%$ of the fish recovered. The remaining 5\% suffered hemorrhaging or bubbles in the orbit of the eye. The degree to which this condition could affect the well being of the fish depends on the severity of the eye damage.

Physical degradation of the eye components from exopthalmia can be irreversible. Corneal lesion formation can be a precursor to loss of eye function (Hoffert and Fromm 1965). Machado et al. (1987) reported that juvenile rainbow trout exposed to $116.9 \pm$ $0.7 \%$ nitrogen and $110.1 \pm 4.6 \%$ oxygen supersaturation experienced eye damage characterized by formation of space between the 
capillary layer of the choroid and the pigment epithelial layer of the retina, and degenerative vacuolization of the optic nerve, musculature, adjacent connective tissue, and glandular tissues of the fish. Two brown trout and one rainbow trout that eventually died were removed from gas supersaturated water during the "convulsive stage". This stage usually preceded death by a few minutes and is characterized by side-swimming, abnormal buoyancy, whirling movements accompanied by inactivity, and spasmodic convulsions (Machado et al. 1987). At this point emboli formation in the vascular system was probably at an irreversible stage.

The occasional formation of exopthalmia during recovery was perplexing. This symptom was originally attributed to frequent handling of fish for rating, sharp blows against recovery tank walls, and exposure to the low level of supersaturation in the recovery tanks. However, post-exposure eye damage has been documented and interpreted differently. Bouck et al. (1976) reported perforation of the cornea, lens loss, and acute necrosis of the eyeball in adult sockeye salmon after removal from the supersaturated water of the columbia River. The capture process and holding environment were dismissed as primary factors responsible for post-capture eye damage. The researchers hypothesized that the main cause of this problem was previous exposure to gas supersaturated water, a theory they supported with data from other work completed on the columbia River. A definitive explanation for post-exposure eye damage is still unavailable. 


\section{Efrects of Repeated Exposure}

Recovery and sensitization: The recovery (loss of external symptoms) of juvenile brown trout from three $30-\mathrm{d}$ exposures to $118 \%$ gas supersaturated water was influenced by severity of external symptoms. Most fish rated 1 (Table 2.2.1) after exposure recovered (Table 2.2.4). Two of $12 \mathrm{fish}$ rated 1 after the first exposure did not totally recover due to eye damage incurred during the recovery period. Fifty percent of fish rated 2 after the first two exposures recovered. Fish that did not totally recover had permanent eye damage. Both fish rated 3 after initial exposure died during recovery (Table 2.2.4). One of these fish was in the "convulsive stage" when it was removed from gas supersaturated water. The second fish suffered from bilateral exopthalmia, impaired swimming, and severe emphysema. Permanent eye damage prohibited recovery of fish rated 3 after the second exposure. One of two fish that had no external symptoms after the second exposure was killed accidently when the recovery tank was cleaned. The third 30-d recovery period was affected when water temperature increased to $23^{\circ} \mathrm{C}$ for $24 \mathrm{~h}$. Three of the six survivors died from a pseudomonad sp. infection. One of the three surviving fish was rated 1 and totally recovered within $30 \mathrm{~d}$, while one rated 2 and one rated 3 did not recover because of permanent eye damage (Table 2.2.4).

Overall, recovery of juvenile brown trout from GBT decreased with each exposure (Figure 2.2.2). Extreme eye damage (often occurring after previous less severe eye damage) and progression to the convulsive stage were primary reasons for lack of recovery. 


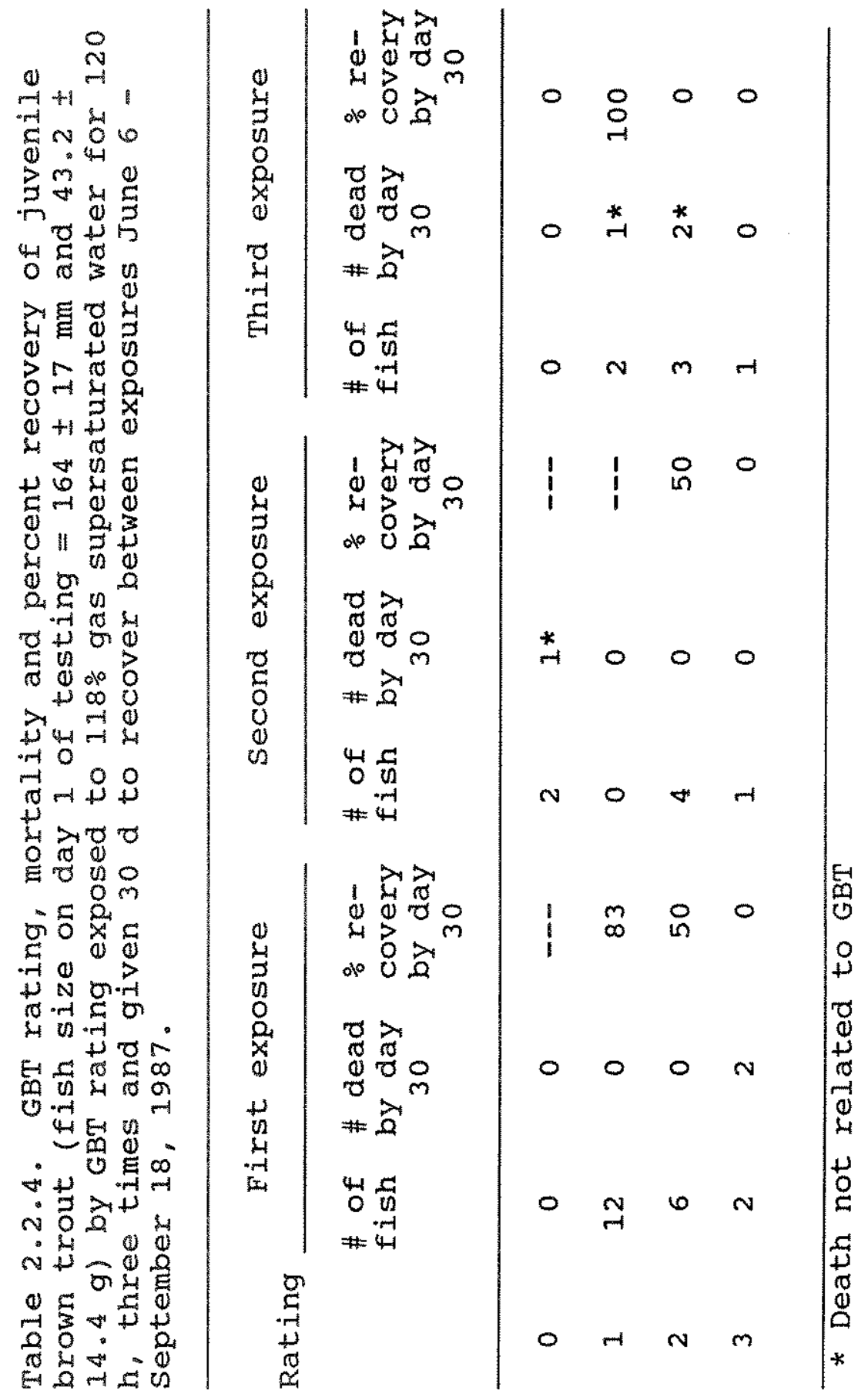




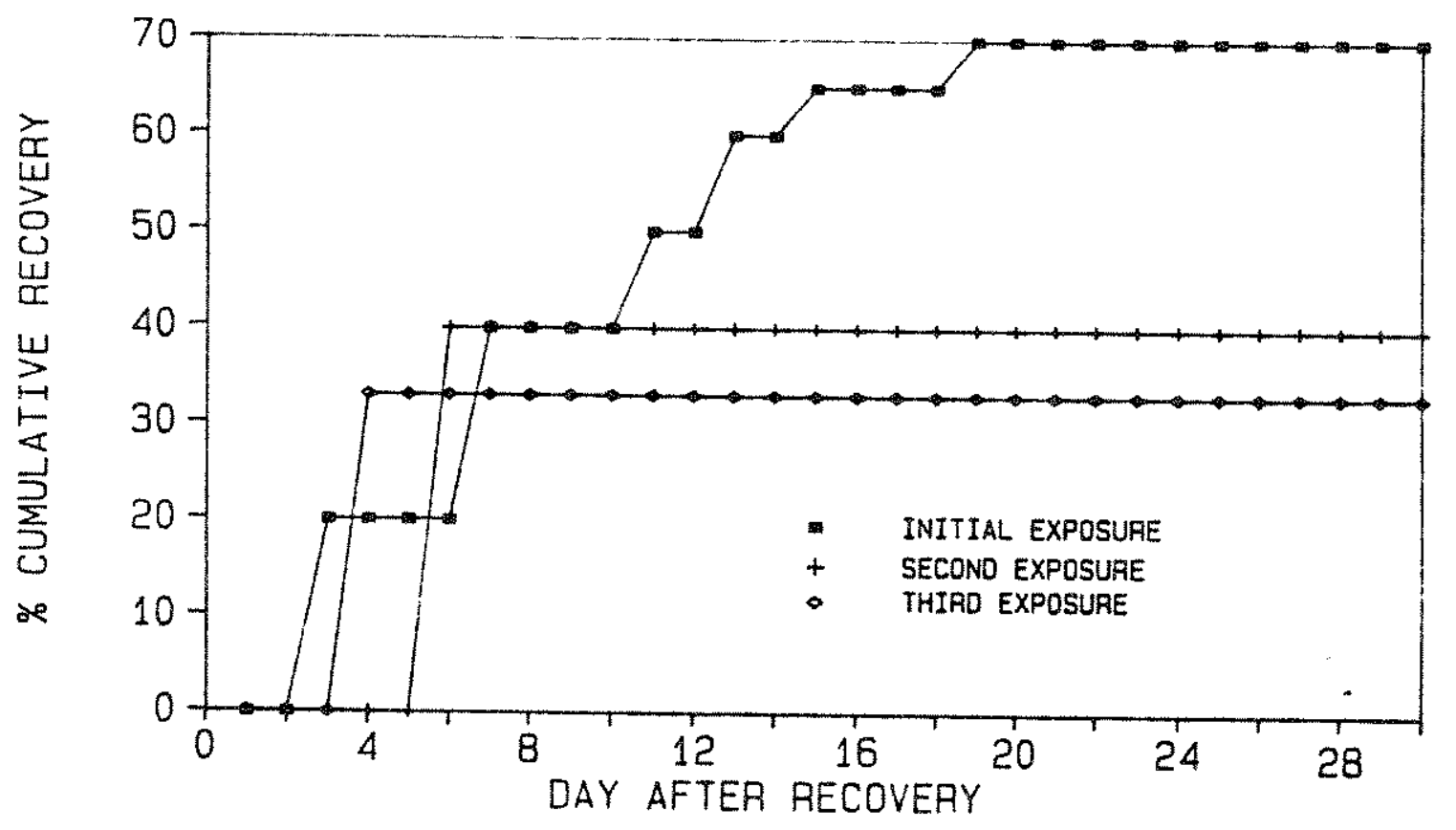

Figure 2.2.2. Percent cumulative recovery for juvenile brown trout (fish size on day 1 of testing $=164 \pm 17 \mathrm{~mm}$ and $43.2 \pm 14.4 \mathrm{~g}$ ) from $\mathrm{GBT}$ calculated by combining GBT rating groups 1,2 , and 3 (see Table 2.2.1) from three 120-h exposure tests conducted June 6 - September 18 , 1987. 
Increased symptom severity in fish surviving an initial exposure to gas supersaturated water was noted by Gorham (1901). He subjected scup to a sequence of decompression - recompression - decompression three to four times for 30-min intervals. Eye bubbles from initial decompression grew during later decompression. Some juvenile brown trout in our study may have become sensitized to GBT.

Acclimization and Tolerance to Repeated Exposure: Different proportions of GBT rating were observed in the three progressive exposures. Mortality (rating 4; Table 2.2.1) of juvenile brown trout was significantly greater during the first and second exposure to gas supersaturated water than during the third exposure (Tables 2.2 .5 and 2.2 .6 ). A significantly greater proportion of fish were rated 1 after the first exposure than in subsequent exposures (Tables 2.2 .5 and 2.2 .6 ). The proportion of fish rated 2 increased significantly from exposure 1 to exposure 3 (Tables 2.2 .5 and 2.2 .6 ). The proportion of fish rated 3 increased with each exposure, however the change was not significant (Tables 2.2 .5 and 2.2 .6 )

Repeated exposure to gas supersaturated water may reveal juvenile salmonids are able to acclimate to gas supersaturation. Meekin and Turner (1974) attempted to acclimate juvenile summer chinook salmon by conditioning them in progressively higher levels of water supersaturated with nitrogen for varying periods of time. High mortality occurred when nitrogen saturation was increased to $135 \%$, regardless of conditioning; chinook salmon survived exposure to $135 \%$ TGP longer when removed to water of $100 \%$ saturation for $8 \mathrm{~h} / \mathrm{d}$ than if exposed continuously. 
Table 2.2.5. Gas bubble trauma rating for juvenile brown trout (fish size on day 1 of testing $=164 \pm 17 \mathrm{~mm}$ and $43.2 \pm 14.4 \mathrm{~g}$ ) by number and percent composition after exposure to $11 \overline{8} \%$ gas supersaturated water for $120 \mathrm{~h}$ three times at 30-d intervals from June 6 - September 18, 1987.

\begin{tabular}{|c|c|c|c|c|c|c|}
\hline $\begin{array}{l}\mathrm{GBT}^{\mathrm{a}} \\
\text { rating }\end{array}$ & \multicolumn{2}{|c|}{$\begin{array}{l}\text { First } \\
\text { exposure }\end{array}$} & \multicolumn{2}{|c|}{$\begin{array}{c}\text { Second } \\
\text { exposure }\end{array}$} & \multicolumn{2}{|c|}{$\begin{array}{l}\text { Third } \\
\text { exposure }\end{array}$} \\
\hline 0 & 0 & 0 & 2 & 12 & 0 & 0 \\
\hline 1 & 12 & 24 & 0 & 0 & 2 & 33 \\
\hline 2 & 6 & 12 & 4 & 23 & 3 & 50 \\
\hline 3 & 2 & 4 & 1 & 6 & 1 & 17 \\
\hline 4 & 30 & 60 & 10 & 59 & 0 & 0 \\
\hline
\end{tabular}

a see Table 2.2 .1 for rating description

Weitkamp (1976) exposed juvenile chinook to $125 \%$ TGP for a sequence of three exposure/non-exposure periods. When the sequence was $16^{-h}$ exposure and 8 -h recovery, juvenile chinook developed external signs of GBT and were twice as likely to die as fish exposed for $8 \mathrm{~h}$, then given $16 \mathrm{~h}$ to recover. Chinook salmon, coho salmon, steelhead trout, rainbow trout, mountain whitefish, and largemouth bass (Micropterus salmoides) exposed to $110-130 \%$ nitrogen for $8 \mathrm{~h}$ and given $16 \mathrm{~h}$ to recover lived longer than fish exposed constantly to similar nitrogen supersaturations (Blahm et al. 1976). Although these investigations show that relief from supersaturated conditions can extend the survival time of most fish, they do not document acclimation.

Juvenile brown trout in this study did not appear to acclimate to gas supersaturated water. External symptom severity did not decrease with repeated exposuce (Table 2.2.6). However, some brown trout were more tolerant of high dissolved gas levels. 
Table 2.2.6. Comparison of GBT severity in juvenile brown trout (fish size on day 1 of testing $=164 \pm 17 \mathrm{~mm}$ and $43.2 \pm 14.4 \mathrm{~g}$ ) exposed to $118 \%$ gas supersaturated water three times at $30-d$ intervals June 6 - September 18, 1987 using Fisher's Exact Test.

GBT rating ${ }^{\mathrm{a}}$

comparison

p-value

Exposure 1 vs Exposure 2
$0=0$
0.067
$1>1$
$0.017 *$
$2=2$
0.921
$3=3$
0.831
$4=4$
0.581

Exposure 2 Vs Exposure 3
$0=0$
0.554
$1=1$
0.059
$2=2$
0.239
$3=3$
0.941
$4>4$
$0.013 *$

Exposure 3 vs Exposure 1
$0=0$
0.067
$1=1$
0.842
$2>2$
$0.046 *$
$3=3$
0.972
$4<4$
$0.007 *$

* Statistically significant at alpha $=0.05$

a see Table 2.2 .1 for rating description

None of the six fish remaining after two exposures died when exposed a third time.

Tolerance to gas supersaturation was demonstrated by Thorn et al. (1978) who exposed bluegill sunfish to supersaturations ranging from 113-124\%. Survivors of these tests were subjected to $124 \%$ then $127 \%$ gas supersaturated water but showed no external GBT symptoms or mortality.

Tolerance is described in the literature as a heritable trait. Gray et al. (1982) showed that carp (Cyprinus carpio) and 
black bullhead (Ictulurus melas) of the Columbia River were more tolerant of gas supersaturation than those from Italy. The researchers suggested that this difference may be due to genetic adaptation traceable to historical supersaturation of columbia River water. Cramer and McIntyre (1975) hypothesized that a selection for fall chinook salmon with the phenotypes that promoted greatest resistance to GBT occurred in the columbia River. A similar selection may be occurring in the Bighorn River below Yellowtail Afterbay Dam.

\section{2d summary}

Juvenile rainbow and brown trout recovery from GBT varied with external symptom severity. Fish of both species with less severe symptoms recovered more often than fish with more severe symptoms.

Non-recovering fish exhibited eye damage characterized by severe unilateral and bilateral exopthalmia, hemorrhaging, cloudiness or cataracts of the cornea, or bubbles in the orbit of the eye.

The condition of fish subjected to intermittent exposure worsened with each exposure leading to poorer recovery. The development of new symptoms with each exposure and recurrence and progression of prior symptoms was responsible for poorer recovery with each additional exposure.

Some fish may become sensitized to GBT, while other individuals may be tolerant to exposure to gas supersaturated water. 


\subsection{Bighorn River studies}

\subsubsection{Description of study Area}

Yellowtail and Afterbay Dams, located in south-central Moncana on the Bighorn River, lie approximately 43 air miles southeast of Billings on the crow Indian Reservation (see front cover). Facility construction occurred from 1963-1966 (WPRS 1980). The principal uses of Yellowtail Dam include power generation, irrigation, flood control and fish and wildlife enhancement. Located at the base of Yellowtail Dam, the powerhouse turbines are operated in a peaking mode. They can generate a maximum of 250-MW. This thin-arch concrete structure is $160 \mathrm{~m}$ (525 ft) high and $442 \mathrm{~m}$ (1,450 ft) long at the crest. Afterbay Dam serves as a reregulating facility $3.5 \mathrm{~km}(2.2 \mathrm{miles})$ downstream of the main dam; it consists of an earthfill

embankment with concrete spillway, sluiceway and diversion works. The height of Afterbay Dam is $22 \mathrm{~m}(72 \mathrm{ft})$ and it has an overall crest length of $414.5 \mathrm{~m}(1,360 \mathrm{ft})$. The spillway has a discharge capacity of $566.4 \mathrm{~m}^{3} / \mathrm{s}(20,000 \mathrm{cfs})$, and is $49.4 \mathrm{~m}$ (162 ft) wide; flows are controlled by five, $9.1 \mathrm{~m} \times 4.1 \mathrm{~m}(30 \times 13.5 \mathrm{ft}$ ) radial gates and by the sluiceway, which is $10.4 \mathrm{~m}$ (34 ft) wide: its discharge is adjusted by three, $3 \times 2.4 \mathrm{~m}(10 \times 8 \mathrm{ft})$ slide gates (Appendix A). The height from the streambed to the maximum controlled water surface is $16.1 \mathrm{~m}$ (53 ft). The sluiceway gates can release water $6.9 \mathrm{~m}(22.5 \mathrm{ft})$ lower than the radial gates. Gate openings are continually adjusted by the automated sluiceway slide gates, maintaining a relatively uniform discharge to the river.

The study area extends approximately $19.3 \mathrm{~km}$ (12 miles) 
downstream of Afterbay Dam to Bighorn Access (Figure 2.3.1). The gradient is approximately $1.9 \mathrm{~m}$ per river kilometer (Rkm) (6.3 feet/river mile) (RM) (Stevenson 1975) and the river channel. braids in several areas, forming numerous islands. Cottonwoods dominate this portion of the riverbottom. Public access sites are located immediately downstream of Afterbay Dam, at Rkm 5.4 (RM 3.4; Lind Access) and at Rkm 19.3 (RM 12.0; Bighorn Access). Soap creek, which empties into the river about $15.6 \mathrm{Rkm}$ (9.7 RM) downstream of Afterbay Dam, is the largest tributary in the study area.

We established an electrofishing section that extended from Afterbay Dam to Rkm 3.9 (RM 2.4) to obtain population estimates in the upper reach of the study area. A previously established section, $\operatorname{Rkm} 3.9-15.4$ (RM 2.4-9.6), provided additional fish population statistics gathered by the MDFWP. Subsections established within the electrofishing sections at Rkm $0-1.9$ (RM $0-1.2), \operatorname{Rkm} 3.9-6.1$ (RM 2.4-3.8) and $\mathrm{Rkm} 12.2-15.4$ (RM 7.6-9.6) allowed monitoring GBT incidence among salmonids under different dissolved gas regimes. Experiments to monitor early life history stages of trout were conducted at Rkm 2.4 (RM 1.5), Rkm 8.0 (RM 5.0) and Rkm 14.5 (RM 9.0). 


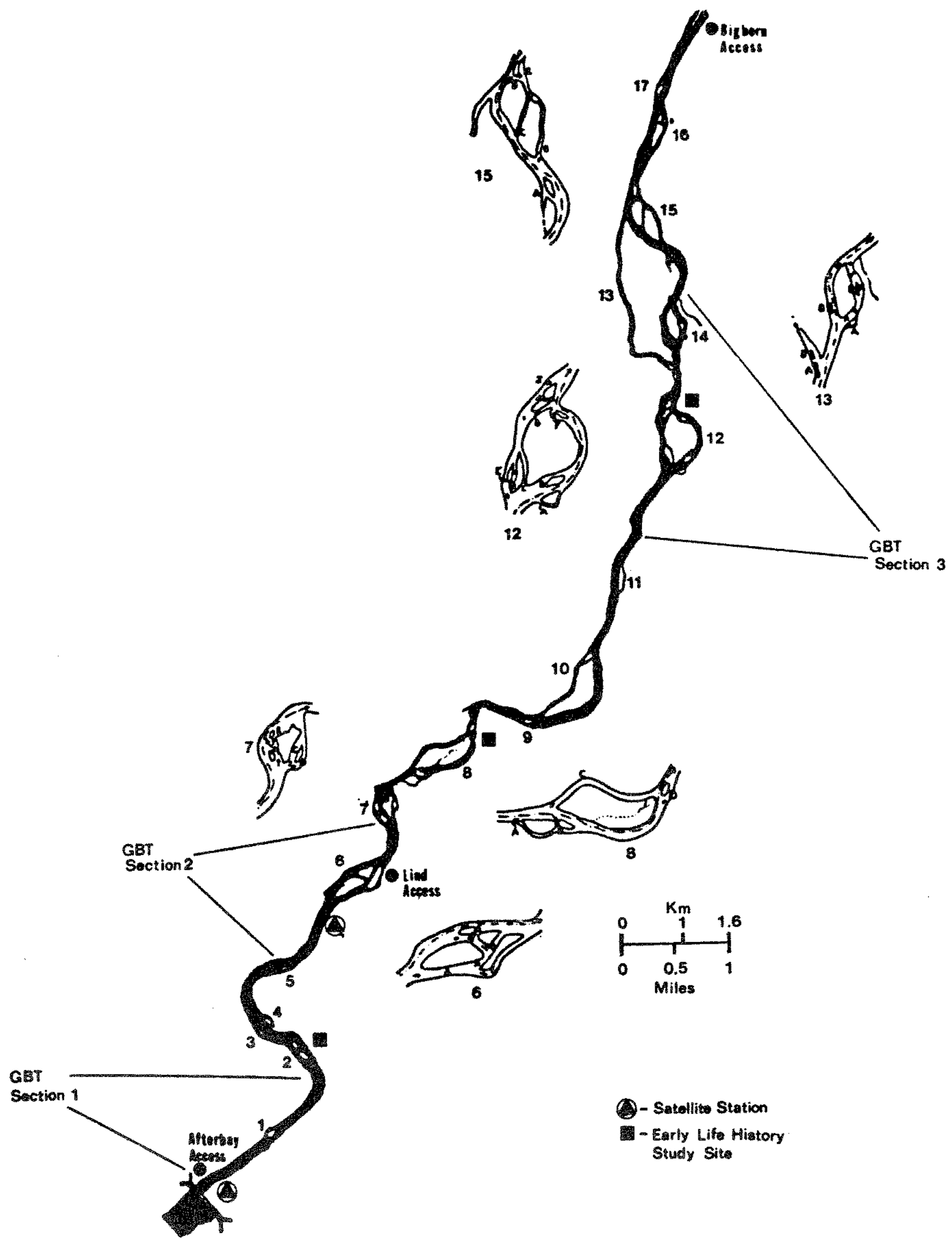

Figure 2.3.1. Map of the study area showing GBT electrofishing sections, early life history study sites, satellite relay stations, side-channel index and access sites. 


\subsection{2 water Chemistry}

\subsection{2a Methods}

Water samples for analysis of common ions and nutrients were collected at the Saint Xavier gagehouse $0.6 \mathrm{~km}$ downstream from Afterbay Dam every 2 to 4 weeks from 17 May 1985 - 1 June 1988. Metals samples were obtained on seven occasions between 18 June 1985 - 1 August 1986. Nutrient and metal samples were preserved with sulfuric and nitric acid, respectively. Samples collected after 2 February 1986 were delivered unfrozen to either Northern Engineering and Testing, Inc. or Energy Laboratories in Billings, MT, for analysis.

\subsection{2b Results and Discussion}

Water discharged from Bighorn Lake was hard and had relatively high alkalinity, conductivity, and total dissolved solid levels (Table 2.3.1; Appendix B). Sodium and calcium were the cations found in the highest concentrations. Magnesium concentrations were about two-thirds lower on a milligrams/liter basis. However, when compared on a milliequivalent/liter basis, magnesium concentrations were 60 and $65 \%$ of calcium and sodium, respectively. Potassium levels were low and usually close to detection limits. Principal anions were sulfate and bicarbonate. chloride was the only other anion present at any significant level. In all years of the study, sulfate was present in higher concentrations than any other anion or cation and was higher than the $250 \mathrm{mg} / \mathrm{L}$ recommended for total dissolved solids in domestic water supplies (EPA 1976). Mean sulfate concentrations were highest in 1988 at $309 \mathrm{mg} / \mathrm{L}$. In 1986 and 1987 , mean sulfate 
Table 2.3.1. Mean and ranges of water quality parameters from samples collected 17 May 1985 - 1 June 1988 near the gagehouse (Rkm 0.6 ) below Afterbay Dam on the Bighorn River.

\begin{tabular}{|c|c|c|c|c|}
\hline Parameter & Mean & Range & $\mathrm{meq} / \mathrm{L}$ & $\begin{array}{l}\text { Sample } \\
\text { size }\end{array}$ \\
\hline $\begin{array}{l}\text { Total Hardness } \\
\left(\mathrm{mg} / \mathrm{L} \text { as } \mathrm{CaCO}_{3}\right)\end{array}$ & 291 & $173-390$ & 5.81 & 43 \\
\hline $\begin{array}{l}\text { Total Alkalinity } \\
\left(\mathrm{mg} / \mathrm{L} \text { as } \mathrm{CaCO}_{3}\right)\end{array}$ & 156 & $80-205$ & 3.13 & 43 \\
\hline $\begin{array}{l}\text { Bicarbonate Alkalinity } \\
\left(\mathrm{mg} / \mathrm{I} \mathrm{HCO}_{3}\right)\end{array}$ & 191 & $98-251$ & 3.12 & 43 \\
\hline Calcium (mg/L) & 73 & $46-100$ & 3.64 & 43 \\
\hline Magnesium (mg/L) & 26 & $14-35$ & 2.20 & 43 \\
\hline Sodium $(\mathrm{mg} / \mathrm{L})$ & 78 & $37-96$ & 3.38 & 43 \\
\hline Potassium (mg/L) & 4 & $1-7$ & 0.09 & 43 \\
\hline Chloride (mg/L) & $11^{\mathrm{a}}$ & $<1-22$ & 0.32 & 43 \\
\hline Fluoride (mg/L) & 0.4 & $0.1-0.6$ & 0.02 & 43 \\
\hline Nitrate + Nitrite $(\mathrm{mg} / \mathrm{L})$ & 0.53 & $0.05-0.96$ & 0.04 & 42 \\
\hline Sulfate $(\mathrm{mg} / \mathrm{L})$ & 279 & $114-367$ & 5.81 & 43 \\
\hline Total Dissolved Solids (mg/L) & 579 & $344-784$ & & 37 \\
\hline Conductivity (micromhos/cm) & 812 & $400-1081$ & & 42 \\
\hline $\mathrm{pH}$ & 8.0 & $6.7-8.4$ & & 42 \\
\hline $\begin{array}{l}\text { Total Phosphorus } \\
\text { as } \mathrm{P}(\mathrm{mg} / \mathrm{L})\end{array}$ & $0.06^{a}$ & $<0.01-0.28$ & & 41 \\
\hline $\begin{array}{l}\text { Ortho-Phosphate } \\
\text { as } \mathrm{P}(\mathrm{mg} / \mathrm{L})\end{array}$ & $0.04^{\mathrm{a}}$ & $<0.01-0.18^{b}$ & & 42 \\
\hline $\begin{array}{l}\text { Ammonia Nitrogen } \\
\text { as } N(\mathrm{mg} / \mathrm{L})\end{array}$ & $0.20^{2}$ & $0.10-0.65^{b}$ & & 42 \\
\hline $\begin{array}{l}\text { Total Kjeldahl } \\
\text { Nitrogen as } N(\mathrm{mg} / \mathrm{L})\end{array}$ & $0.48^{\mathrm{a}}$ & $<0.20-1.52^{b}$ & & 42 \\
\hline
\end{tabular}


levels were $264-265 \mathrm{mg} / \mathrm{L}$, or $31 \mathrm{mg} / \mathrm{L}$ lower than 1985 levels. Anion and cation levels during 1987 were approximately the same as found in 1968 and 1969 by wright and Soltero (1973). The mean pH was alkaline and varied only 0.4 units.

Mean total phosphorus and kjeldahl nitrogen concentrations during the study were 0.06 and $0.48 \mathrm{mg} / \mathrm{L}$, respectively. The mean total phosphorus level exceeded Montana water quality criteria of $0.10 \mathrm{mg} / \mathrm{L}$ for aquatic life on five sampling dates (DHES 1986). These elevated levels included $0.13 \mathrm{mg} / \mathrm{L}$ in both April and October 1986, and $0.18 \mathrm{mg} / \mathrm{L}$ in June, $0.28 \mathrm{mg} / \mathrm{L}$ in October, and $0.11 \mathrm{mg} / \mathrm{L}$ in December 1987. Wright and Soltero (1973) found similar total phosphorus levels in 1968-1969. The large biomass of rooted macrophytes and cladaphora in the Bighorn River is probably related to these high nutrient levels. Ortho-phosphate averaged $0.04 \mathrm{mg} / \mathrm{L}$ and was below detection limits $(0.01$ or 0.02 $\mathrm{mg} / \mathrm{L}$ ) on 11 sampling dates. The mean ammonia concentration, which was $0.20 \mathrm{mg} / \mathrm{L}$, was usually below detection limits and unionized ammonia was always less than $0.02 \mathrm{mg} / \mathrm{L}$. However, if periods of high water temperature coincide with elevated $\mathrm{pH}$ levels, un-ionized ammonia concentrations may approach this level. Concentrations of both ortho-phosphate and ammonia were similar to those measured in 1968 and 1969 (Wright and soltero 1973). The mean total kjeldahl nitrogen during the study was higher than the total organic nitrogen measured in 1968 and 1969 (Wright and Soltero 1973); the highest annual mean level was recorded in 1985. However, the maximum level (1.52 $\mathrm{mg} / \mathrm{L}$ ) occurred in october 1987 and coincided with high levels of total dissolved solids, total phosphorus, and ortho-phosphate. 
Concentrations of dissolved or total recoverable metals, with the exception of manganese, were lower than EPA guidelines (EPA 1976 and 1980) for protection of freshwater aquatic life on all sampling dates during the study (Table 2.3.2; Appendix B) . Maximum iron and manganese levels in 1986 were substantially higher than 1985 values. Manganese remained below or near detection limits except on 5 April 1986 when concentrations were $80 \mathrm{ug} / \mathrm{L}$. Wright and soltero (1973) reported similarly low metal concentrations in 1968 and 1969, but their range of concentrations was much greater and detection limits much lower. Metals analyses were discontinued after the August sampling date because of the low levels found and since previous researchers were unable to find any synergistic effect between supersaturation and copper and zinc (Garton and Nebeker 1977).

Table 2.3.2. Dissolved metal concentrations from seven samples collected 16 June 1985 - 1 August 1986 near the gagehouse (Rkm $0.6)$ below Afterbay Dam on the Bighorn River.

Dissolved Metals (ug/L) Mean Range

$\begin{array}{ccc}\mathrm{Cu} & 20^{\mathrm{a}} & - \\ \mathrm{Fe} & 56^{\mathrm{b}} & 40-90^{\mathrm{c}} \\ \mathrm{Pb} & 20^{\mathrm{a}} & - \\ \mathrm{Mn} & 29^{\mathrm{b}} & <20-80 \\ \mathrm{Zn} & 23^{\mathrm{b}} & <20-40\end{array}$

all values below detection limits.

$b$ values below detection limits considered to be equal to detection limit.

$c$ change in detection limits. 


\subsubsection{Population structure}

\subsection{3a Methods}

Boat-mounted, fixed electrode electrofishing systems were used by MCFRU and MDFWP personnel to conduct fish population estimates. Multiple mark and recapture trips were made to obtain adequate sample sizes; procedures were those of Vincent (1971 and 1974), adapted for computer analysis. Population estimates were made for various fish length groupings. At least four (but usually seven or more) recaptured trout were included within each $1.3 \mathrm{~cm}(0.5 \mathrm{in})$ length interval. After reading scales, an estimate for each age was obtained by a summation of the proportions found in each size group. Population estimates were calculated using Chapman's modification of the Peterson formula (Ricker 1975) :

$$
\begin{aligned}
& N=\frac{(M+1)(C+1)}{R+1} \\
& \text { where: } N=\text { Population estimate } \\
& M=\text { Number of fish marked } \\
& C=\text { Number of fish in the recapture } \\
& \text { sample } \\
& \mathrm{R}=\text { Number of marked fish in the } \\
& \text { recapture sample (C) }
\end{aligned}
$$

\subsection{3b Results}

The high trout population levels in the Bighorn River demonstrate the importance of the fishery resource (Appendix C). Although gas supersaturation in the river affects trout and other fish populations, it is difficult to isolate effects of supersaturation from other factors operating concurrently at the 
population level. Consequently, we did not relate population statistics directly to effects of gas supersaturation.

since sample sizes of rainbow trout were relatively low, all mark-recapture data for the first $15.4 \mathrm{Rkm}$ (9.6 river miles) below Afterbay Dam were combined to calculate population estimates. Combining the data resulted in problems with interpretation because of a mixture of hatchery and wild fish. Fish from 1979 river plants, as well as those from 1981-83, could have been present in the river during the study. An additional source of hatchery rainbow trout was downstream movement of some of the 20-40,000 fish planted annually in Afterbay Reservoir. In general, the number of wild rainbow trout increased in a downstream direction.

spring rainbow trout population estimates were made during spawning periods and are inflated for spawning-sized fish. The estimates do, however, provide information on expected spring population levels in the upper river. The estimated number of age II and older rainbow trout in the spring varied from $258( \pm 31) / \mathrm{km}$ in 1986 to $462( \pm 62) / \mathrm{km}$ in 1985 (Table 2.3 .3$)$. When the 1985-86 population estimates were compared by length group, we observed larger numbers in all size groups greater than $267 \mathrm{~mm}$ (10.5 in) (Figure 2.3.2). However, population structure for age II and older fish remained constant between years. The only year that we obtained a spring estimate of age I fish was 1987. Age I densities were almost three times larger than any other peak in abundance (Figure 2.3.2). The mean length of age III and older fish remained relatively constant in 1985 and 1986, but decreased for all ages in 1987 (Table 2.3.4). The mean length of age II 
Table 2.3.3. Spring rainbow trout population estimates by age group from Rkm 0-15.4 (RM 0-9.6) below Afterbay Dam on the Bighorn River during 1985-87 (MCFRU and MDFWP data).

\begin{tabular}{lccc} 
& Number $/ \mathrm{km}$ & $(80 \%$ Confidence intervals) \\
\cline { 2 - 4 } Age & 1985 & 1986 & 1987 \\
\hline I & - & - & $509( \pm 142)$ \\
II & $111( \pm 40)$ & $40( \pm 12)^{a}$ & $111( \pm 25)$ \\
III & $160( \pm 36)$ & $119( \pm 26)$ & $73( \pm 14)$ \\
IV & $104( \pm 25)$ & $71( \pm 12)$ & $123( \pm 17)$ \\
VI & $76( \pm 19)$ & $21( \pm 6)$ & $29( \pm 5)$ \\
VII & $9( \pm 2)$ & $6( \pm 2)$ & $2( \pm .5)$ \\
Total: & $2( \pm .6)$ & $1( \pm .2)$ & - \\
Age II \& older & $462( \pm 62)$ & & $338( \pm 34)$ \\
Age I \& older & - & $258( \pm 31)$ & $847( \pm 146)$ \\
\end{tabular}

a underestimate

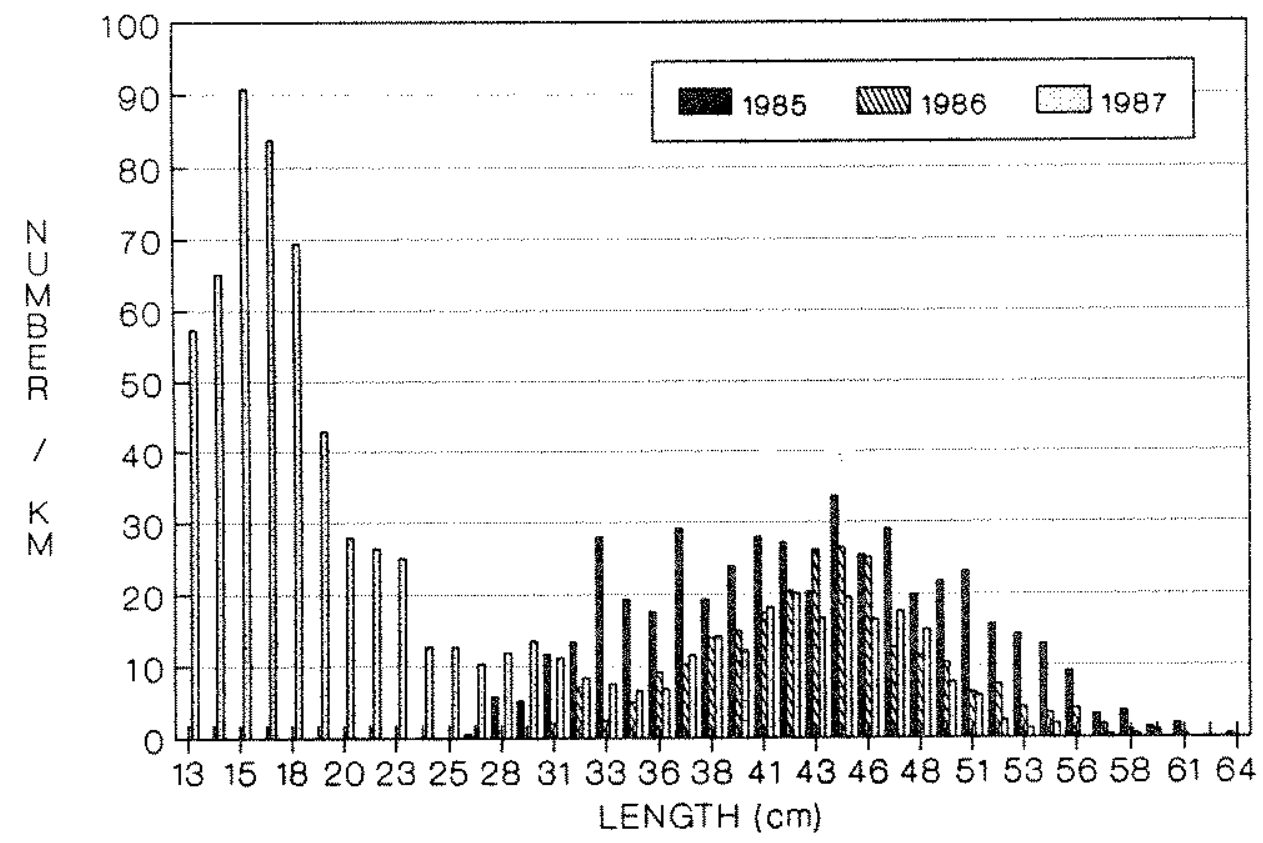

Figure 2.3.2. Spring rainbow trout population estimates by length group from Rkm 0-15.4 (RM 0-9.6) below Afterbay Dam on the Bighorn River, 1985-87 (MCFRU and MDFWP data). 
Table 2.3.4. The average length (mm) and weight ( $\mathrm{g}$ ) of rainbow trout by age group sampled during spring population estimates from Rkm 0-15.4 (RM 0-9.6) below Afterbay Dam on the Bighorn River during 1985-87 (MCFRU and MDFWP data).

\begin{tabular}{|c|c|c|c|c|c|c|}
\hline \multirow[t]{2}{*}{ Age } & \multicolumn{2}{|c|}{1985} & \multicolumn{2}{|c|}{1986} & \multicolumn{2}{|c|}{1987} \\
\hline & Length & wt & Length & wt & Length & wt \\
\hline $\bar{I}$ & - & - & - & - & 170 & 45 \\
\hline $\bar{I} I$ & 333 & 454 & 318 & 426 & 259 & 163 \\
\hline III & 414 & 826 & 414 & 912 & 353 & 522 \\
\hline IV & 485 & 1216 & 483 & 1166 & 439 & 862 \\
\hline V & 533 & 1515 & 538 & 1610 & 498 & 1098 \\
\hline VI & 559 & 1678 & 561 & 1851 & 551 & 1665 \\
\hline VII & 564 & 1606 & 589 & 2204 & - & - \\
\hline
\end{tabular}

fish declined from 1985-87. Mean condition factors calculated using metric measurements were $1.10,1.15$, and 1.02 in 1985 , 1986, and 1987, respectively. The lower condition of rainbow trout in spring 1987 was consistent for all sizes except for fish $>585 \mathrm{~mm}$.

Fall rainbow trout estimates, considered more reliable than the spring estimates, showed an annual increase in total numbers of age II and older fish from 1985-1987 (Table 2.3.5). Most of the increase in 1987 was in age II fish; however, the 1985 estimate appeared to underestimated age II fish. When the same data were examined by length group, we could not clearly differentiate yearlings (age I) from older age groups in 1987 (Figure 2.3.3). The lack of discernible age groups in 1987 length data resulted from relatively good growth and a large average size (254 m; $10 \mathrm{in}$ ) among age I fish but a small average size (340 mm; $13.3 \mathrm{in}$ ) for age II fish (Table 2.3.6). The distinct length group separation of age I fish (216 mm; 8.5 in) and older age groups in 1986, was due to slower growth in 
Table 2.3.5. Fall rainbow trout population estimates by age group from Rkm 0-15.4 (RM $0-9.6$ ) below Afterbay Dam on the Bighorn River during 1985-87 (MCFRU and MDFWP data).

\begin{tabular}{lccc}
\hline & Number $/ \mathrm{km}$ & $(80 \%$ Confidence intervals) \\
\cline { 2 - 4 } Age & 1985 & 1986 & 1987 \\
\hline I & - & $180( \pm 49)$ & $151( \pm 61)$ \\
II & $112( \pm 40)$ & $86( \pm 19)$ & $249( \pm 64)$ \\
III & $91( \pm 34)$ & $147( \pm 29)$ & $143( \pm 37)$ \\
IV & $45( \pm 17)$ & $53( \pm 11)$ & $7( \pm 11)$ \\
VI & $16( \pm 6)$ & $7( \pm 1)$ & $1( \pm 0.1)$ \\
VII & - & $3( \pm 1)$ & - \\
Total: & - & - & $457( \pm 75)$ \\
Age II \& older & $264( \pm 56)$ a & $296( \pm 36)$ & $608( \pm 96)$ \\
Age I \& older & - & $476( \pm 61)$ & fish \\
a underestimate because entire length range for all age II fish &
\end{tabular}

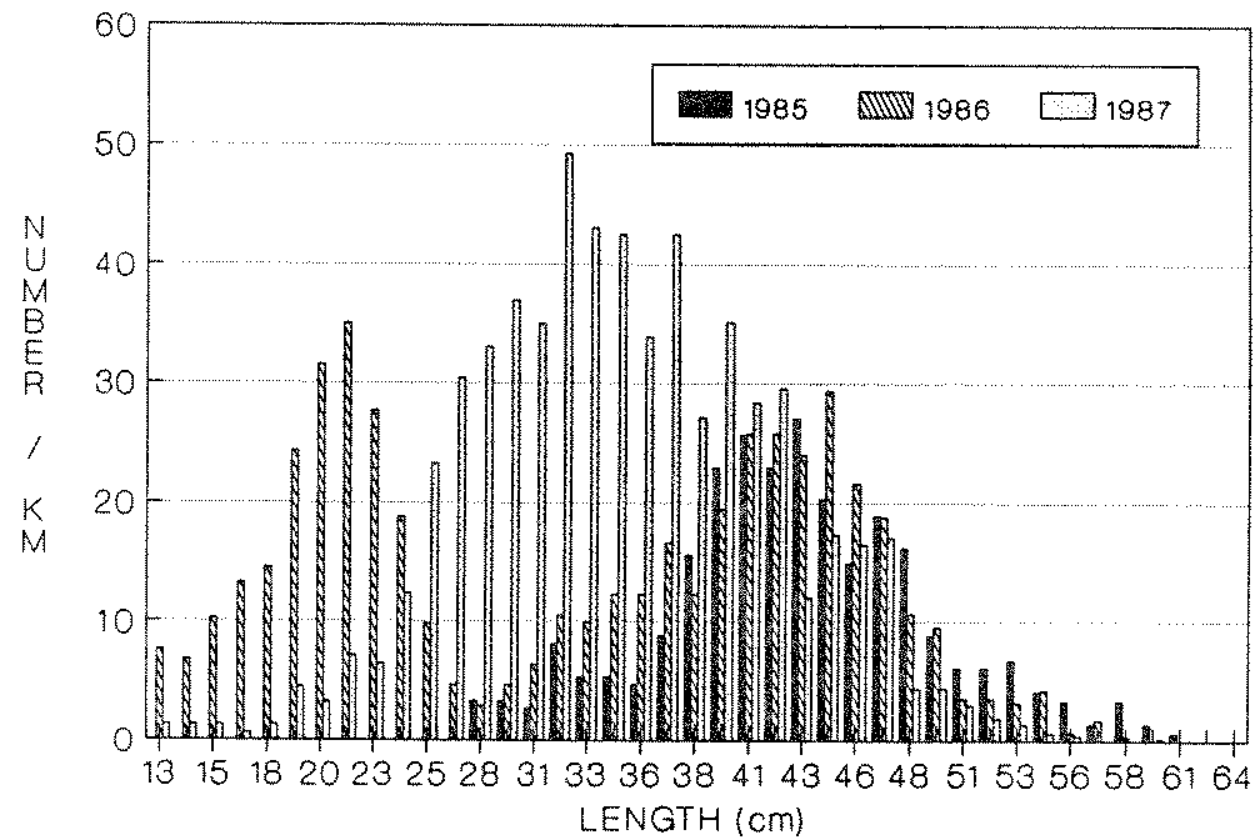

Figure 2.3.3. Fall rainbow trout population estimates by length group from Rkm 0-15.4 (RM $0-9.6$ ) below Afterbay Dam on the Bighorn River, 1985-87 (MCFRU and MDFWP data). 
Table 2.3.6. The average length ( $\mathrm{mm}$ ) and weight (g) at age of rainbow trout sampled during fall population estimates from Rkm $0-15.4$ (RM 0-9.6) below Afterbay Dam on the Bighorn River during 1985-87 (MCFRU and MDFWP data).

\begin{tabular}{|c|c|c|c|c|c|c|}
\hline \multirow[t]{2}{*}{ Agge } & \multicolumn{2}{|c|}{1985} & \multicolumn{2}{|c|}{1986} & \multicolumn{2}{|c|}{1987} \\
\hline & Length & $w t$ & Length & wt & Length & wt \\
\hline$I$ & 236 & 200 & 208 & 122 & 254 & 245 \\
\hline II & 386 & 748 & 338 & 463 & 340 & 481 \\
\hline III & 450 & 1139 & 424 & 844 & 414 & 767 \\
\hline IV & 493 & 1402 & 495 & 1089 & 470 & 1048 \\
\hline V & 561 & 1914 & 541 & 1506 & 523 & 1415 \\
\hline VI & - & - & 554 & 1606 & 572 & 1901 \\
\hline VII & - & - & - & - & - & - \\
\hline
\end{tabular}

both age I and II fish. The number of yearling rainbow trout remained relatively constant with point estimates varying from $151-180 / \mathrm{km}$ in 1986 and 1987 (Table 2.3.5). The number of Age V and older rainbow trout declined from 1985 levels. This decline occurred in fish $483 \mathrm{~mm}$ (19 in) and longer (Figure 2.3.3). The average annual mortality rate for age II and older rainbow trout varied from 20-30\%. The mean condition factors for all rainbow trout calculated using metric measurements were $1.26,1.11$, and 1.17 in 1985, 1986, and 1987, respectively. The condition factor for rainbow trout of all lengths was consistently higher in 1985 than in other years. Only 1986 showed lower condition factors in the fall than in the spring.

Brown trout sample sizes were large enough to estimate population abundance in the $\mathrm{Rkm} 3.9-15.4$ section (MDFWP electrofishing section) and in the $\mathrm{Rkm} 0-3.8$ section.

In the upper (Rkm 0-3.8) section, spring estimates of age II and older brown trout numbers increased annually from $2257( \pm 452)$ $/ \mathrm{km}$ in 1985 to $5483( \pm 491) / \mathrm{km}$ in 1987 (Table 2.3.7). Spring 
Table 2.3.7. Spring brown trout population estimates by age group from Rkm 0-6.I (RM 0-3.8) below Afterbay Dam on the Bighorn River during 1985-87 (MCFRU and MDFWP data).

\begin{tabular}{|c|c|c|c|}
\hline \multirow[b]{2}{*}{ Age } & \multirow[t]{2}{*}{ Number / km } & \multicolumn{2}{|c|}{ ( $80 \%$ Confidence intervals) } \\
\hline & & 1986 & 1987 \\
\hline $\mathrm{I}$ & $4425( \pm 1058)$ & - & - \\
\hline II & $1326( \pm 405)$ & $990( \pm 228)^{a}$ & $1623( \pm 323)$ \\
\hline III & $696( \pm 196)$ & $1333( \pm 281)$ & $2719( \pm 344)$ \\
\hline IV & $209( \pm 43)$ & $413( \pm 63)$ & $1016( \pm 133)$ \\
\hline $\mathrm{V}$ & $26( \pm 6)$ & $49( \pm 10)$ & $125( \pm 19)$ \\
\hline VI & $=$ & $2( \pm \cdot 3)$ & - \\
\hline \multicolumn{4}{|l|}{ Total: } \\
\hline Age II \& older & $2257( \pm 452)$ & $2787( \pm 367)$ & $5483( \pm 491)$ \\
\hline Age I \& older & $6682( \pm 1151)$ & - & - \\
\hline
\end{tabular}

brown trout abundance averaged 10.3 times (range 4.9-16.2) more than spring or fall rainbow trout abundance. The mean length at age in the spring was always less for brown trout (Table 2.3.8) than for rainbow trout (Table 2.3.4). Although hatchery rainbow trout influenced this, the consistently slower growth rate among young-of-the-year brown trout that emerge from the gravel earlier in the season was not expected. Both species had decreased growth rates at age II and III in the 1986 and 1987 estimates, and the annual increase in abundance of age II and older fish is displayed in length frequencies, but these concurrent changes can be easily misinterpreted (Figure 2.3.4).

Fall brown trout population estimates in the upper $3.8 \mathrm{Rkm}$ show even larger increases in numbers over the course of the study. Point estimates increased from $1815( \pm 274) / \mathrm{km}$ in 1985 to $6458( \pm 508) / \mathrm{km}$ in 1987 for fish age II and older (Table 2.3.9). The fall brown trout numbers averaged 13 times (range 3.9-19.1) 
Table 2.3.8. The average length $(\mathrm{mm})$ and weight (g) at age of brown trout sampled during spring population estimates from $\mathrm{Rkm}$ $0-6.1$ (RM 0-3.8) below Afterbay Dam on the Bighorn River during 1985-87 (MCFRU and MDFWP data).

\begin{tabular}{|c|c|c|c|c|c|c|}
\hline \multirow[t]{2}{*}{ Age } & \multicolumn{2}{|c|}{1985} & \multicolumn{2}{|c|}{1986} & \multicolumn{2}{|c|}{1987} \\
\hline & Length & wt & Length & wt & Length & wt \\
\hline$I$ & 137 & 27 & - & - & - & - \\
\hline II & 282 & 231 & 241 & 168 & 213 & 118 \\
\hline III & 386 & 553 & 371 & 544 & 323 & 349 \\
\hline$I V$ & 445 & 816 & 467 & 807 & 404 & 540 \\
\hline $\mathrm{V}$ & 521 & 1234 & 531 & 1197 & 490 & 767 \\
\hline VI & & & 546 & 1166 & & \\
\hline
\end{tabular}

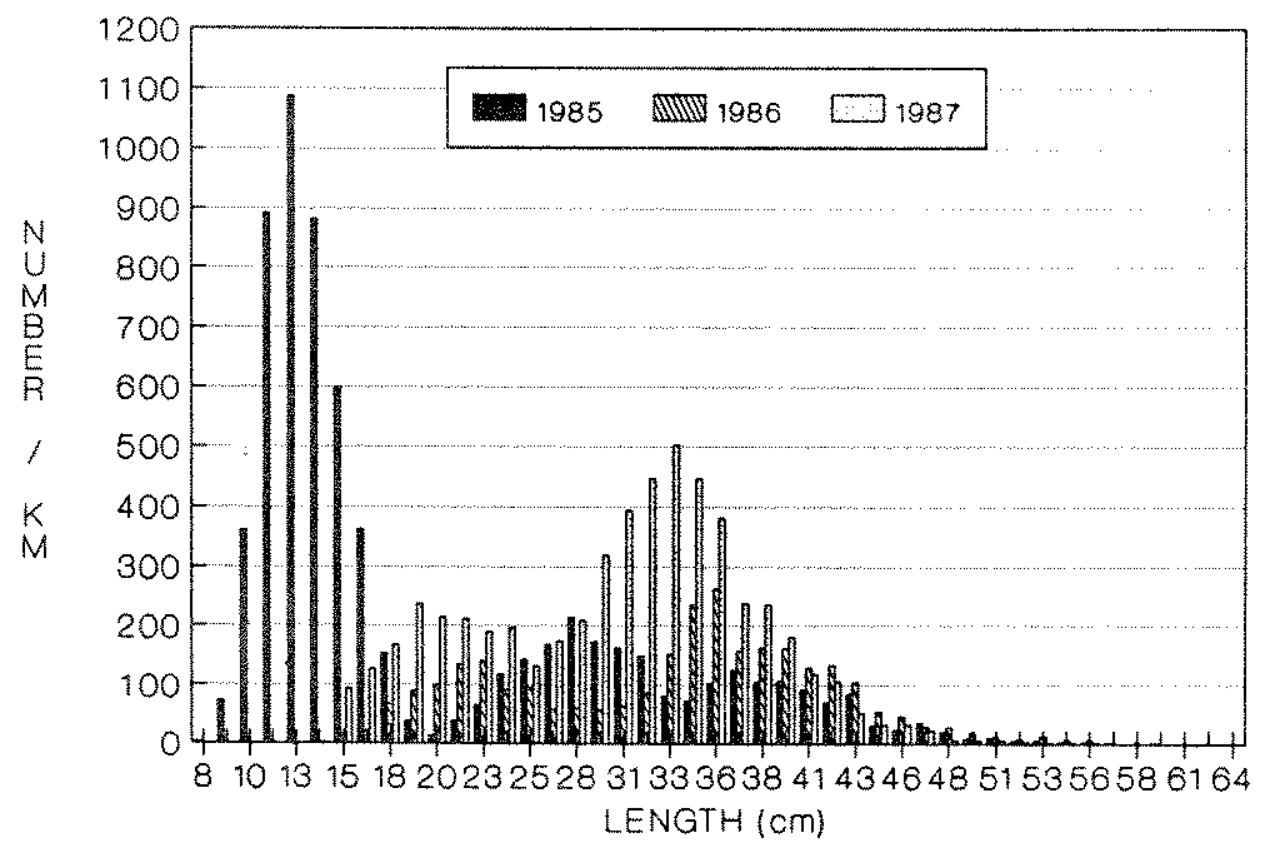

Figure 2.3.4. Spring brown trout population estimates by length group from $\mathrm{Rkm} 0-6.1$ (RM 0-3.8) below Afterbay Dam on the Bighorn River, 1985-87 (MCFRU and MDFWP data). 
Table 2.3.9. Fall brown trout population estimates by age group from Rkm 0-6.1 (RM 0-3.8) below Afterbay Dam on the Bighorn River during 1985-87 (MCFRU and MDFWP data).

Number / km (80\% Confidence intervals)

\begin{tabular}{llll}
\cline { 2 - 3 } Age & 1985 & 1986 & 1987
\end{tabular}

$\begin{array}{lccc}\text { I } & 5479( \pm 2067) & 3110( \pm 619) & - \\ \text { II } & 943( \pm 234) & 3560( \pm 278) & 2103( \pm 310) \\ \text { III } & 514( \pm 105) & 913( \pm 105) & 4118( \pm 399) \\ \text { IV } & 287( \pm 93) & 177( \pm 33) & 231( \pm 49) \\ \text { V } & 71( \pm 26) & 14( \pm 5) & 6( \pm 2)\end{array}$

Total:

Age II \& older $1815( \pm 274) \quad 4664( \pm 299)$

Age I \& older $7294( \pm 2085) \quad 7774( \pm 687)$

$6458( \pm 508)$

greater than spring or fall rainbow trout estimates in the same year. Growth of age II, III, and IV brown trout remained constant or decreased from 1985-1987 (Table 2.3.10). The mean length of age I fish varied from $188 \mathrm{~mm}$ in 1986 to $201 \mathrm{~mm}$ in 1987. The average size of age $\mathrm{V}$ brown trout increased each year. Except for age $V$ brown trout in 1987, the same age rainbow trout were always longer (Tables 2.3 .6 and 2.3.10). Fall population estimate data presented in length frequencies display relatively distinct peaks for age I fish; all other ages are integrated into the peaks to the right (Figure 2.3.5). Although fairly constant numbers of brown trout greater than $355 \mathrm{~mm}$ in 1985-86 represent age III and older, this difference was not seen in 1987.

Estimates of age I brown trout abundance were obtained in both spring and fall 1985 and fall 1986 (Tables 2.3.7 and 2.3.9). Smaller sizes, combined with schooling behavior and colder water temperature, resulted in inadequate samples for population estimation during spring 1986 and spring and fall 1987. 
Table 2.3.10. The average length (mm) and weight (g) at age of brown trout sampled during fall population estimates from $\mathrm{Rkm} \mathrm{0-}$ 6.1 (RM 0-3.8) below Afterbay Dam on the Bighorn River during 1985-87 (MCFRU and MDFWP data).

\begin{tabular}{|c|c|c|c|c|c|c|}
\hline \multirow[t]{2}{*}{ Age } & \multicolumn{2}{|c|}{1985} & \multicolumn{2}{|c|}{1986} & \multicolumn{2}{|c|}{1987} \\
\hline & Length & wt & Length & wt & Length & $w t$ \\
\hline$I$ & 198 & 113 & 188 & 95 & 201 & 141 \\
\hline II & 338 & 517 & 307 & 354 & 284 & 372 \\
\hline III & 414 & 830 & 414 & 685 & 366 & 572 \\
\hline IV & 480 & 1175 & 480 & 943 & 467 & 903 \\
\hline $\mathrm{V}$ & 511 & 1361 & 541 & 1270 & 551 & 1628 \\
\hline
\end{tabular}

FALL BROWN TROUT POPULATIONS - RKM 0-6.1

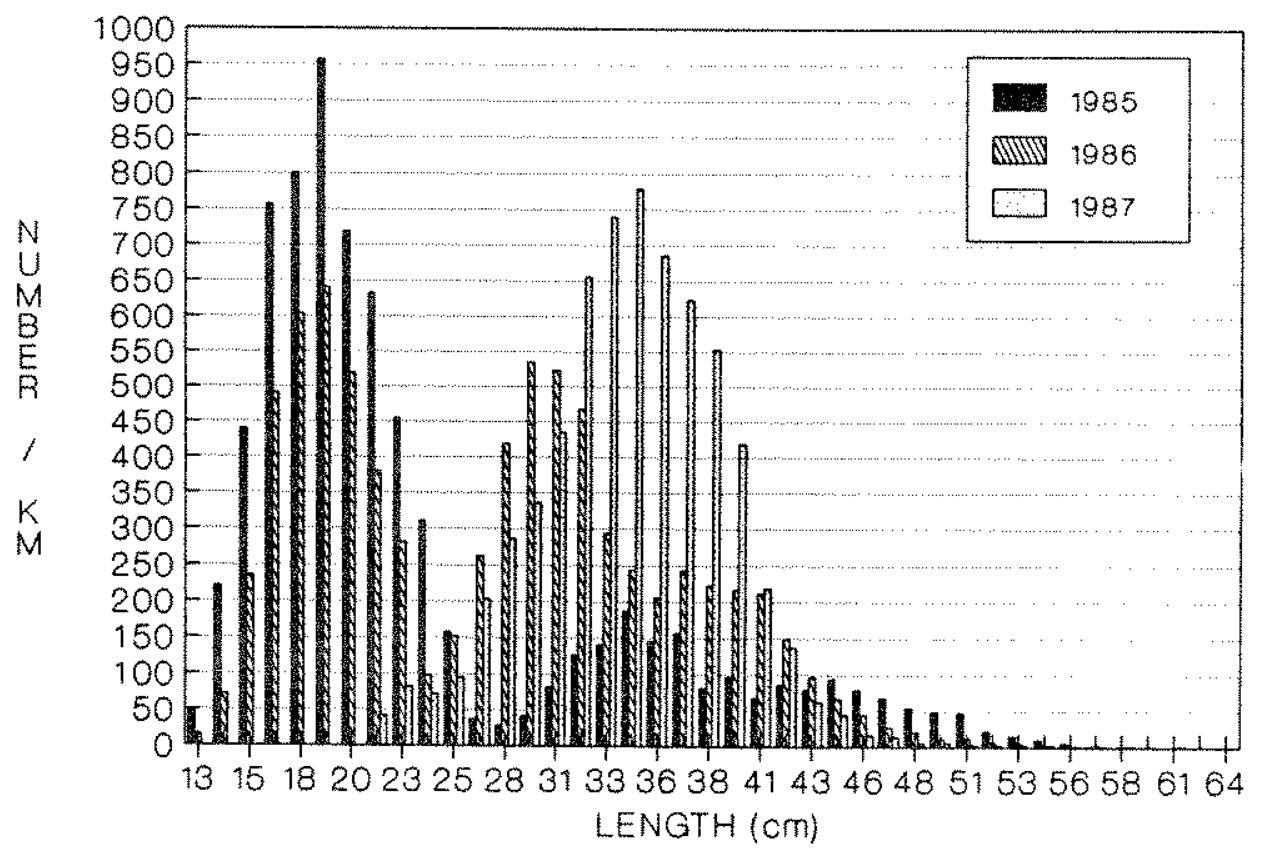

Figure 2.3.5. Fall brown trout population estimates by length group from Rkm 0-6.1 (RM 0-3.8) below Afterbay Dam on the Bighorn River, 1985-87 (MCFRU and MDFWP data). 
Mean brown trout condition factors calculated using metric measurements from spring population work in the Rkm 0-6.1 section were $0.98,1.04$, and 1.00 in 1985,1986 , and 1987, respectively. Condition factors increased over each growing season, with mean values of $1.29,1.16$, and 1.21 by fall 1985,1986 , and 1987 , respectively. In spring, mean condition factors for brown trout were always lower than for rainbow trout. However, condition factors of rainbow trout were less than brown trout in the falls of 1986 and 1987.

Although the estimated number of age II and older brown trout in the lower section (Rkm 3.9-15.4) during spring 1985 was greater than in the upper (Rkm 0-6.1) section, the $80 \%$ confidence intervals overlapped (Tables 2.3 .7 and 2.3 .11 ). In 1987 , numbers were substantially greater in the upper section. spring estimates of abundance of age II and older brown trout / $\mathrm{km}$ were $3083( \pm 464)$ in $1985,2329( \pm 234)$ in 1986 , and $2741( \pm 229)$ in 1987 (Table 2.3.11). The 1986 estimate was low since age II was underestimated. The 1985 and 1987 population estimates displayed by length showed initial peaks corresponding to age I fish in similar positions (Figure 2.3 .6 ). Other peaks, which represent older fish, were further to the right in 1985 than in the other 2 years (Figure 2.3.6). The average length at each age for age II-IV was greater in the Rkm 3.9-15.4 section than upstream in both 1985 and 1987 (Tables 2.3 .8 and 2.3.12). In 1986, the mean lengths for ages II-V were equal in the two sections. Mean condition factors for $\mathrm{Rkm} 3.9-15.4$ were $1.04,1.05$ and 0.92 during the springs of 1985, 1986, and 1987, respectively. Mean condition factors were slightly higher than in the upstream 
Table 2.3.11. Spring brown trout population estimates by age group from $\mathrm{Rkm} 3.9-15.4$ (RM 2.4-9.6) below Afterbay Dam on the Bighorn River during 1985-87 (MCFRU and MDFWP data).

Number / km ( $80 \%$ Confidence intervals)

Age

1985

1986

1987

$I$

II

III

IV

$\mathrm{V}$
$2966( \pm 756)$

$1428( \pm 394)$

$1053( \pm 224)$

$538( \pm 98)$

$64( \pm 12)$

-
$461( \pm 101)^{a}$
$1279( \pm 204)^{2}$
$519( \pm 54)$
$70( \pm 10)$

Age II \& older $3083( \pm 464) \quad 2329( \pm 234)^{\mathrm{a}} \quad 2741( \pm 229)$

$1258( \pm 322)^{b}$

$856( \pm 157)$

$1247( \pm 152)$

$563( \pm 66)$

$75( \pm 13)$

Total: Age I \& older $6049( \pm 887)$ $3999( \pm 395)$

anderestimate

b Age 1 estimate based on data collected from Rkm 6.1-15.4 only.

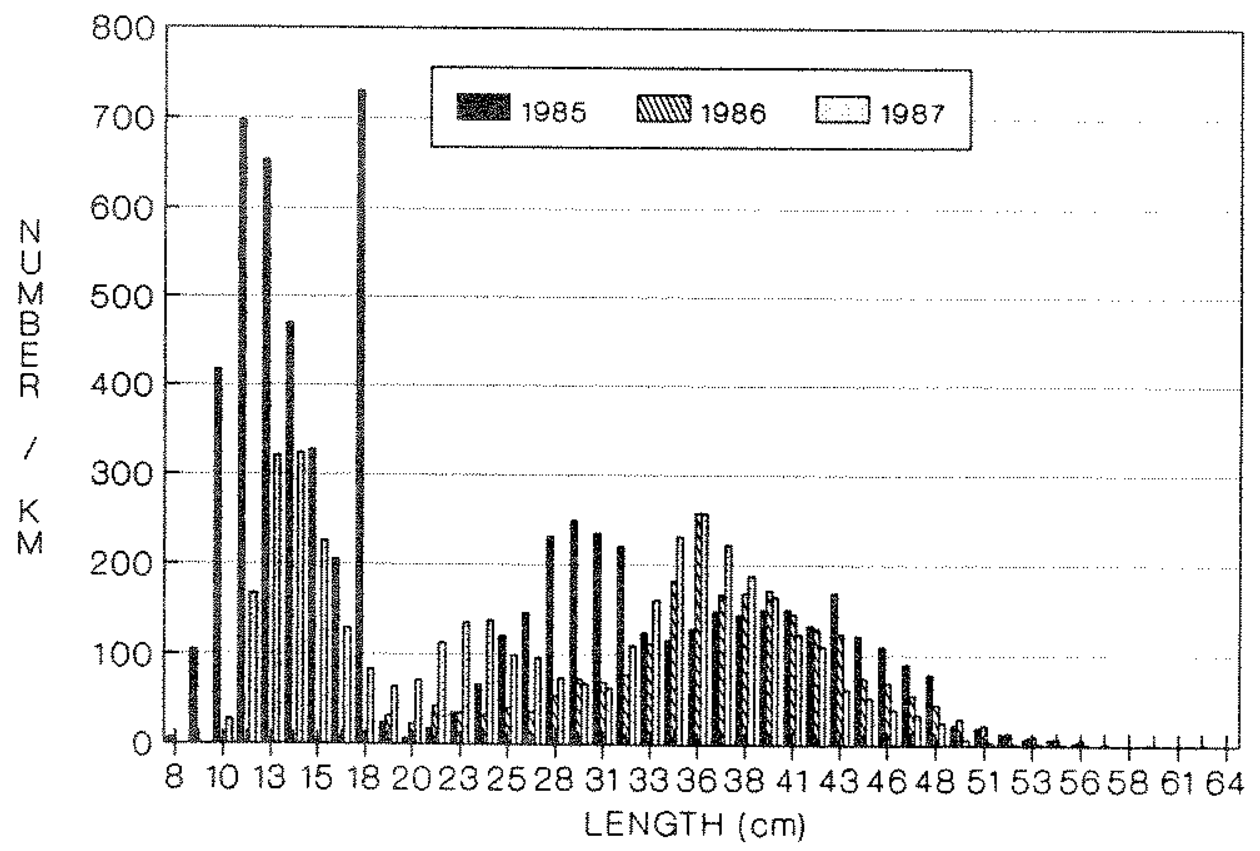

Figure 2.3.6. Spring brown trout population estimates by length group from Rkm 3.9-15.4 (RM 2.4-9.6) below Afterbay Dam on the Bighorn River, 1985-87 (MCFRU and MDFWP data). 
Table 2.3.12. The average length $(\mathrm{mm})$ and weight ( $\mathrm{g}$ ) at age of brown trout sampled during spring population estimates from $\mathrm{kmm}$ 3.9-15.4 (RM 2.4-9.6) below Afterbay Dam on the Bighorn River during 1985-87 (MCFRU and MDFWP data).

\begin{tabular}{|c|c|c|c|c|c|c|}
\hline \multirow[t]{2}{*}{ Age } & \multicolumn{2}{|c|}{1985} & \multicolumn{2}{|c|}{1986} & \multicolumn{2}{|c|}{1987} \\
\hline & Length & wt & Length & wt & Length & wt \\
\hline I & 132 & 27 & - & - & 1.42 & 27 \\
\hline II & 292 & 272 & 241 & 222 & 241 & 150 \\
\hline III & 391 & 626 & 371 & 590 & 351 & 435 \\
\hline IV & 455 & 885 & 467 & 857 & 439 & 621 \\
\hline $\mathrm{V}$ & 505 & 1143 & 531 & 1166 & 508 & 821 \\
\hline
\end{tabular}

section during 1985 and 1986, but considerably less in 1987.

Fall estimates of age II and older brown trout were similar between sections in 1985, but considerably smaller downstream in 1986 and 1987 (Tables 2.3 .9 and 2.3.13). There were always more age I brown trout in the upper section. Although the number per length group varied between years, the pattern was similar except for the shift of the first peak in 1987 (Figure 2.3.7). This change was probably due to an increase in the average length of age I brown trout, a decrease in the mean length of age II fish (Table 2.3.14), and an increase in the number of age II fish (Table 2.3.13). The mean length of brown trout at each age in the downstream (Rkm 3.9-15.4) section was greater than near Afterbay Dam except for ages III, IV and V in 1985 and age V in 1986. Mean condition factors were 1.29, 1.13, and 1.19 in 1985, 1986, and 1987, respectively; these were substantially higher than during spring and equal or slightly lower than in the Rkm $0-6.1$ section in fall.

We obtained two population estimates of mountain whitefish 
Table 2.3.13. Fall brown trout population estimates by age group from Rkm 3.9-15.4 (RM 2.4-9.6) below Afterbay Dam on the Bighorn (MCFRU and MDFWP data).

\begin{tabular}{lccc} 
& & Number $/ \mathrm{km}$ & $(80 \%$ Confidence intervals $)$ \\
\cline { 2 - 4 } Age & 1985 & 1986 & 1987 \\
\hline I & $1436( \pm 375)$ & $1745( \pm 202)$ & $962( \pm 294)$ \\
II III & $1011( \pm 179)$ & $1846( \pm 158)$ & $2203( \pm 327)$ \\
IV V & $569( \pm 94)$ & $648( \pm 77)$ & $2015( \pm 247)$ \\
Votal: & $227( \pm 37)$ & $138( \pm 28)$ & $111( \pm 18)$ \\
Age II \& older & $1848( \pm 206)$ & $2657( \pm 178)$ & $4( \pm 1)$ \\
Age I \& older & $3284( \pm 428)$ & $4402( \pm 269)$ & $4333( \pm 410)$ \\
& & $25( \pm 5)$ & $5295( \pm 505)$ \\
\hline
\end{tabular}

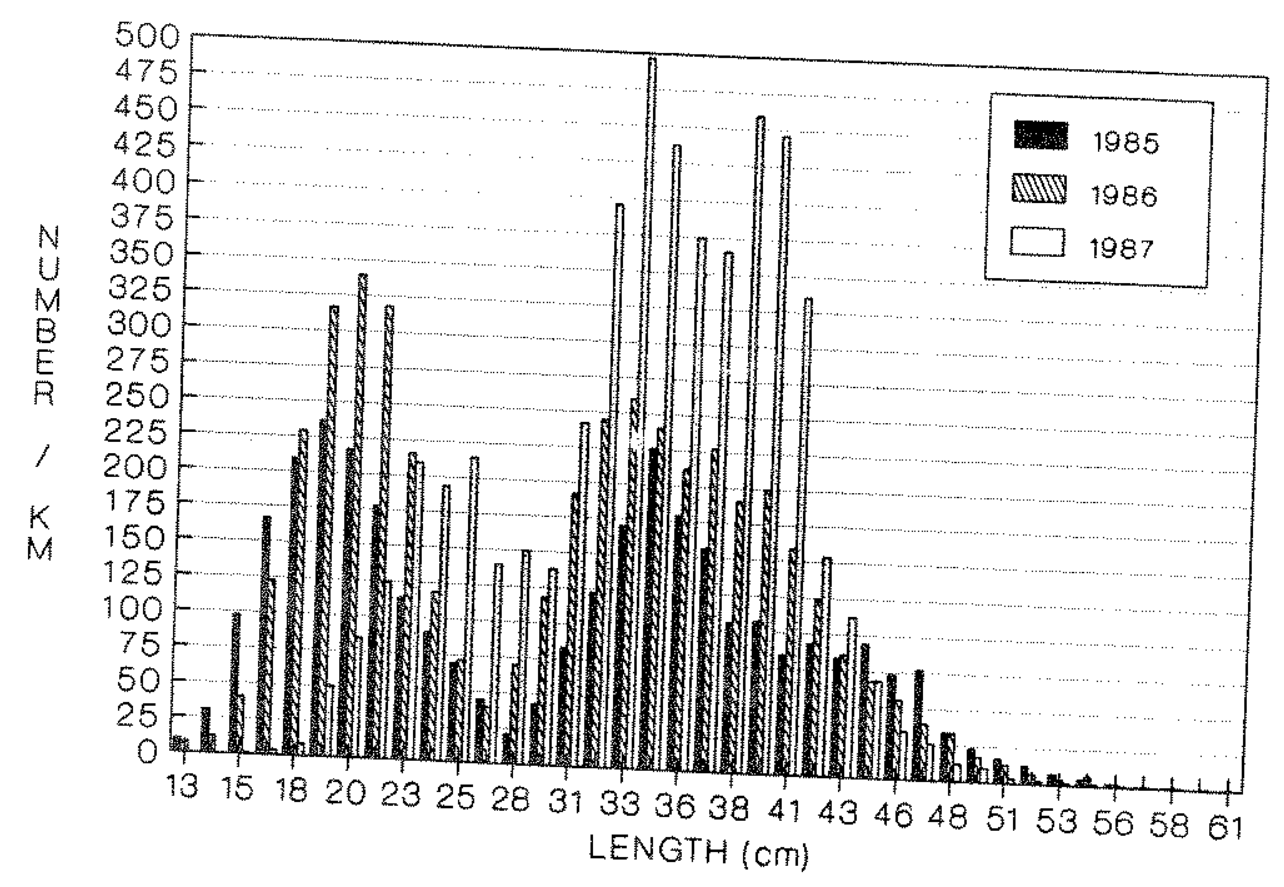

Figure 2.3.7. Fall brown trout population estimates by length
group from Rkm $3.9-15.4$ (RM 2.4-9.6) below Afterbay Dam on the
Bighorn River. $1985-87$ (MCFRU and MDFW data).
\[ 174 \] 
Table 2.3.14. The average length ( $\mathrm{mm}$ ) and weight ( $\mathrm{g}$ ) of brown trout sampled during fall population estimates from Rkm 3.9-15.4 (RM 2.4-9.6) below Afterbay Dam on the Bighorn River during 1985-87 (MCFRU and MDFWP data).

\begin{tabular}{|c|c|c|c|c|c|c|}
\hline \multirow[t]{2}{*}{ Age } & \multicolumn{2}{|c|}{1985} & \multicolumn{2}{|c|}{1986} & \multicolumn{2}{|c|}{1987} \\
\hline & Length & wt & Length & wt & Length & $w t$ \\
\hline$I$ & 203 & 127 & 203 & 109 & 226 & 177 \\
\hline II & 343 & 549 & 330 & 449 & 320 & 463 \\
\hline III & 411 & 830 & 427 & 771 & 404 & 708 \\
\hline IV & 472 & 1093 & 493 & 998 & 480 & 1052 \\
\hline $\mathrm{V}$ & 505 & 1247 & 536 & 1261 & 566 & 1547 \\
\hline
\end{tabular}

(Prosopium williamsoni) during the study (Table 2.3.15). The first was during March 1986, when mountain whitefish numbers were estimated to be $145( \pm 38) / \mathrm{km}$ from $\mathrm{Rkm} 0-15.4$ for fish $330-544 \mathrm{~mm}$. The following spring we obtained the second estimate, which encompassed only the upper $6.1 \mathrm{~km}$. The estimate also included a slightly larger range of lengths (279-546 mm). The density estimate increased to $309( \pm 95) / \mathrm{km}$. Although we attempted another estimate in fall 1987, insufficient numbers of mountain whitefish were captured.

Table 2.3.15. Mountain whitefish population estimates from the upper Bighorn River below Afterbay Dam during March 1986 and April 1987 (MCFRU and MDFWP data).

\begin{tabular}{lcccc}
\hline Year & $\begin{array}{c}\text { Section } \\
\mathrm{Rkm}\end{array}$ & $\begin{array}{c}\text { Length interval } \\
(\mathrm{mm})\end{array}$ & Number/km & $\begin{array}{c}80 \% \text { Confidence } \\
\text { intervals }\end{array}$ \\
\hline 1986 & $0.0-15.4$ & $330-544$ (Age 3+) & 145 & $107-183$ \\
1987 & $0.0-6.1$ & $279-546$ & 309 & $214-404$ \\
\hline
\end{tabular}




\subsection{Dissolved Gas Levels, Water Temperature and GBT}

\subsubsection{Methods}

We manually measured hyperbaric pressures two to three times per week at Rkm 0.6 (gagehouse), Rkm 4.8 (RM 3.0), and at each early life history study site (Figure 2.3.1). Long term monitoring began at early 1 ife history study sites on 16 and 17 May 1985, at Rkm 0.6 on 3 october 1985, and at Rkm 4.8 on 12 April 1986. Additional monitoring at Bighorn Access (Rkm 19.3) occurred from 18 December 1987 - 3 May 1988. Parameters measured included delta $P$ (the difference between total gas pressure in water and in air), absolute barometric pressure, water temperature and dissolved oxygen concentration. Delta $\mathrm{P}$ was determined using a Bouck gasometer (Bouck 1982), operated for 30 min before each measurement so the instrument could reach equilibrium with the water pressure. We measured barometric pressure at each field site with a Thommen 22000 pocket altimeter-barometer and in the office, two to three times daily, with a Princo Nova, fortin type mercury barometer. Beginning 14 March 1986, automatic collection of barometric pressures occurred every $30 \mathrm{~min}, 24 \mathrm{~h}$ per day from a model TBO-F Common sensing tensionometer at $\mathrm{Rkm} 0.6$. Water temperature was measured with a mercury thermometer. The azide modification of the winkler method (APHA 1976) using Iiquid reagents provided dissolved oxygen concentrations. Formulas used to calculate gas levels are discussed by colt (1984) and include:

Total Saturation $=\left[\left(\mathrm{BP}+{ }^{\wedge} \mathrm{P}\right) / \mathrm{BP}\right] * 100$

$\div \mathrm{O}_{2}$ saturation $\quad=\left(\left[\mathrm{O}_{2}\right] / \mathrm{B}-\mathrm{O}_{2} * 0.5318\right) /\left(\left(\mathrm{BP}-\mathrm{P}-\mathrm{H}_{2} \mathrm{O}\right) *\right.$ $0.20946)) * 100$ 


$$
\begin{aligned}
& \% \mathrm{~N}_{2}+\mathrm{Ar} \text { Saturation }=\left[\left(\mathrm{BP}+{ }^{A} \mathrm{P}-\left(\left[\mathrm{O}_{2}\right] / \mathrm{B}-\mathrm{O}_{2}\right) * 0.5318\right)\right. \\
& \left.-\mathrm{P}-\mathrm{H}_{2} \mathrm{O} /\left(\mathrm{BP}-\mathrm{P}-\mathrm{H}_{2} \mathrm{O}\right) * 0.7902\right] * 100 \\
& { }^{\wedge} \mathrm{P}=\mathrm{TGP}-\mathrm{BP} \\
& { }^{\wedge} \mathrm{P}-\mathrm{O}_{2}(\mathrm{mmHg})=\left(\left[\mathrm{O}_{2}\right] / \mathrm{B}-\mathrm{O}_{2} * 0.5318\right)-0.20946 *\left(\mathrm{BP}-\mathrm{P}-\mathrm{H}_{2} \mathrm{O}\right) \\
& { }^{\wedge} \mathrm{P}-\mathrm{N}_{2}+\mathrm{Ar}(\mathrm{mmHg})=\mathrm{BP}+{ }^{\wedge} \mathrm{P}-\left(\left[\mathrm{O}_{2}\right] / \mathrm{B}-\mathrm{O}_{2} * 0.5318\right)-\mathrm{P}-\mathrm{H}_{2} \mathrm{O} \\
& -0.7905 *\left(\mathrm{BP}-\mathrm{P}-\mathrm{H}_{2} \mathrm{O}\right) \\
& \mathrm{TGP}=\mathrm{BP}+{ }^{\wedge} \mathrm{P} \\
& \mathrm{P}-\mathrm{O}_{2}(\mathrm{mmHg})=\left[\mathrm{O}_{2}\right] / \mathrm{B}-\mathrm{O}_{2} * 0.5318 \\
& \mathrm{P}-\mathrm{N}_{2}+\mathrm{Ar}(\mathrm{mmHg})=\mathrm{BP}+{ }^{a} \mathrm{P}-\left[\mathrm{O}_{2}\right]-\mathrm{P}-\mathrm{H}_{2} \mathrm{O} \\
& \mathrm{B}-\mathrm{O}_{2}=\mathrm{e}^{\left[\mathrm{A} 1+\mathrm{A} 2 *(100 / \mathrm{T})+\mathrm{A} 3 * \log _{\mathrm{e}}(\mathrm{T} / 100)\right]} \\
& \mathrm{P}-\mathrm{H}_{2} \mathrm{O}=760 \mathrm{e}^{[24.4543-67.4509 *(100 / \mathrm{T})}-4.8489 * \log _{\mathrm{e}} \\
& (T / 100)]
\end{aligned}
$$$$
\text { Where: } \mathrm{BP}=\text { Barometric pressure }(\mathrm{mmHg})
$$$$
{ }^{*} \mathrm{P}=\text { Delta } \mathrm{P} \text { (mmHg) }
$$$$
\left[\mathrm{O}_{2}\right]=\text { Dissolved oxygen concentration (mg/L) }
$$$$
\left.\mathrm{B}-\mathrm{O}_{2}=\text { Bunsen coefficient of oxygen ( } \mathrm{L} /\left[\begin{array}{lll}
\mathrm{L} & \mathrm{x} & \mathrm{atm}
\end{array}\right]\right)
$$$$
\mathrm{P}-\mathrm{H}_{2} \mathrm{O}=\text { Vapor pressure of water }(\mathrm{mmHg})
$$$$
\text { TGP = Total gas pressure (mmHg) }
$$$$
{ }^{\wedge} \mathrm{P}-\mathrm{O}_{2}=\text { oxygen Component of " } \mathrm{P} \text { (mmHg) }
$$$$
{ }^{\wedge} \mathrm{P}-\mathrm{N}_{2}+\mathrm{Ar}=\text { Nitrogen and Argon Component of }{ }^{\wedge} \mathrm{P} \text { (mmHg) }
$$$$
\mathrm{P}-\mathrm{O}_{2}=\text { oxygen Pressure ( } \mathrm{mmHg} \text { ) }
$$$$
\mathrm{P}-\mathrm{N}_{2}+\mathrm{Ar}=\mathrm{Nitrogen} \text { and Argon Pressure (mmHg) }
$$$$
\mathrm{T}=\text { Absolute water temperature }\left({ }^{\circ} \mathrm{C}+273.15\right)
$$$$
A 1=-58.3877
$$$$
A 2=85.8079
$$$$
\mathrm{A} 3=23.8439
$$

Continuous water temperature monitoring occurred at five locations in the study area. A Taylor 30-d thermograph was maintained at the Saint Xavier gagehouse below Afterbay Dam (Rkm 0.6). Additional continuous recording temperature equipment was installed by the Bureau of Reclamation at Rkm 0.6 and at $\mathrm{Rkm} 4.8$. Maximum/minimum thermometers were located at $\mathrm{Rkm} 2.4,8.0$, and 14.5. Water temperature monitoring in the forebay of Bighorn Lake started on 15 April 1987 using the Hydromet system. 


\section{$2.3 .4 b$ Results}

Delta p's measured along the right bank at the gagehouse (Rkm $0.6)$ generally increased during the first 6 months of 1986 and 1987 (Figure 2.3.8a), with highest levels in 1986. An increase was not apparent in 1988 because of higher initial levels and a marked decrease in the latter part of June. During the second 6 months, delta $\mathrm{P}^{\prime} \mathrm{s}$ were substantially different between years (Figure $2.3 .8 \mathrm{~b}$ ). Periods of relatively low gas levels occurred from January to mid-February and mid-July to late August in 1986 and 1987. Although summer 1986 levels dropped below 1987 levels, in late August gas levels increased and remained high through November. Except for two short term increases, delta P in 1987 remained low until early october. Delta p's measured from midJuly to mid-August in 1988 varied more than in previous years, then leveled off and remained low during all other measurements. Gas levels at Rkm 0.6 in 1985 were usually below 1986 and 1987 levels. Hyperbaric pressures fluctuated substantially in fall and early winter 1985-1987.

The mean and range of delta $P^{\prime} \mathrm{s}$ at $\mathrm{Rkm} 0.6$ were similar for the 2 years (1986 and 1987) with year-long data collection (Table 2.3.16). The overall mean delta $\mathrm{P}$ from 357 Bouck measurements made at $\mathrm{Rkm} 0.6$ during the study was $111 \mathrm{mmHg}$. The maximum delta $P$ measured (153 mmHg) occurred on 15 June 1987 and the minimum ( $35 \mathrm{mmHg}$ ) on 2 December 1985. Several measurements in 1986 approached the 1987 maximum, but minimum readings during other years were substantially higher than the 1985 low.

Lower initial hyperbaric pressures at Rkm 2.4 than upstream 


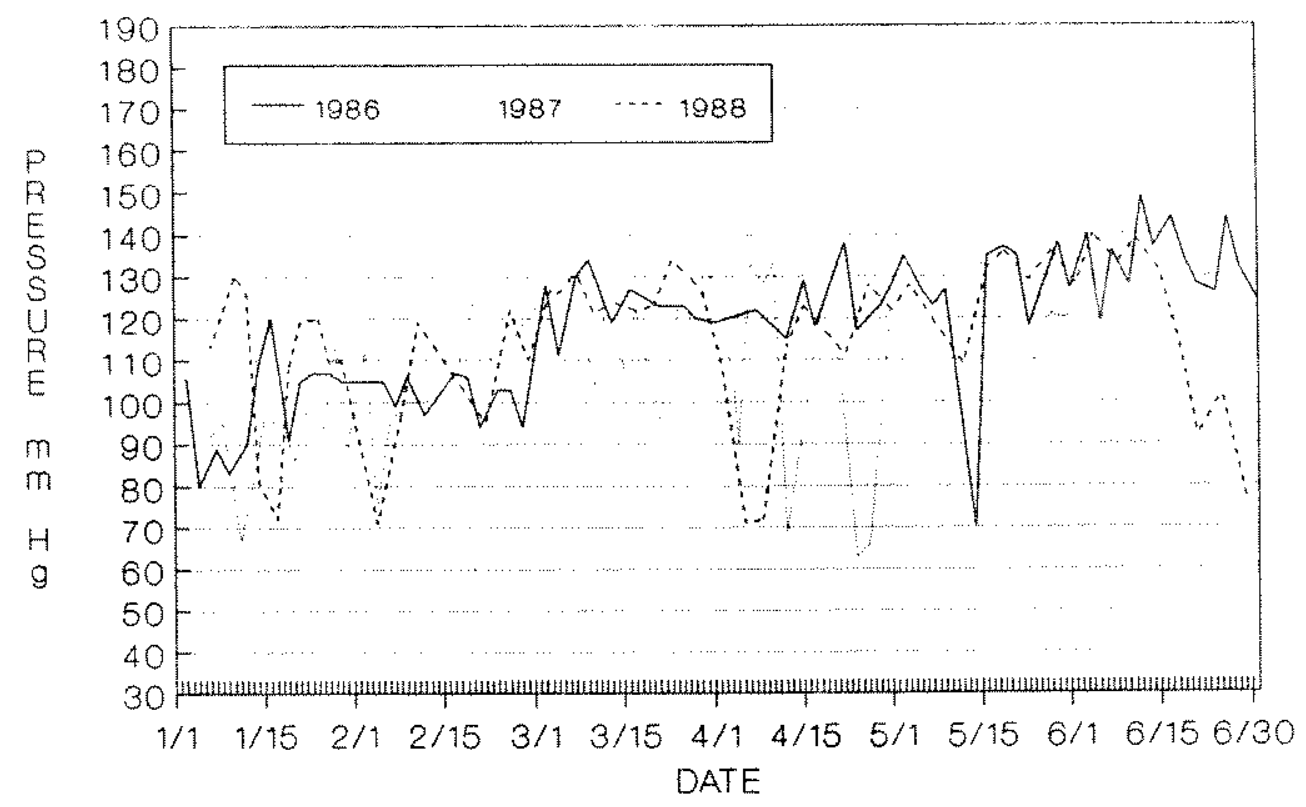

A).

RKm 0.6 - BOUCK DELTA.P

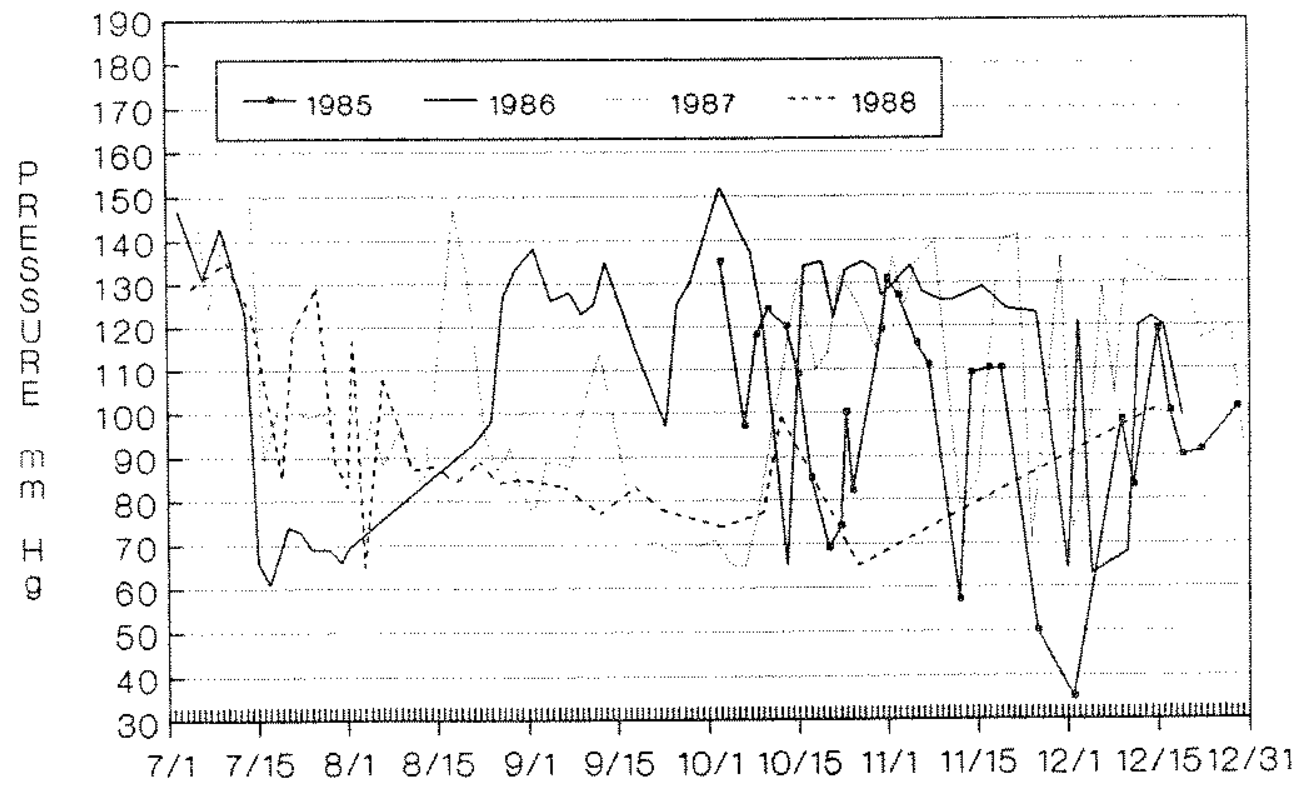

B).

Figure 2.3.8. Delta $\mathrm{P}$ (mmHig) measured by Bouck gasometers near the right bank at $\mathrm{Rkm} 0.6$ (gagehouse) between A) January - June and B) July - December from 1985-1988 on the Bighorn River, Montana. 
Table 2.3.16. Mean and range of hyperbaric pressure (mmHg) at gas monitoring sites on the Bighorn River, Montana.

\begin{tabular}{|c|c|c|c|c|c|}
\hline \multirow{2}{*}{$\begin{array}{c}\text { Location } \\
\text { (Rkm) }\end{array}$} & \multicolumn{5}{|c|}{ Year } \\
\hline & $1985^{\mathrm{a}}$ & 1986 & 1987 & $1988^{b}$ & $1985-88$ \\
\hline 0.6 & $\begin{array}{c}99 \\
(35-135)\end{array}$ & $\begin{array}{c}115 \\
(61-152)\end{array}$ & $\begin{array}{c}111 \\
(63-153)\end{array}$ & $\begin{array}{c}108 \\
(65-140)\end{array}$ & $\begin{array}{c}111 \\
(35-153)\end{array}$ \\
\hline 2.4 & $\begin{array}{c}104 \\
(37-152)\end{array}$ & $\begin{array}{c}106 \\
(46-143)\end{array}$ & $\begin{array}{c}109 \\
(58-154)\end{array}$ & $\begin{array}{c}119 \\
(74-155)\end{array}$ & $\begin{array}{c}109 \\
(37-155)\end{array}$ \\
\hline 4.8 & --- & $\begin{array}{c}100 \\
(48-140)\end{array}$ & $\begin{array}{c}107 \\
(46-188)\end{array}$ & $\begin{array}{c}122 \\
(63-169)\end{array}$ & $\begin{array}{c}108 \\
(46-188)\end{array}$ \\
\hline 8.0 & $\begin{array}{c}97 \\
(26-153)\end{array}$ & $\begin{array}{c}93 \\
(38-147)\end{array}$ & $\begin{array}{c}102 \\
(43-177)\end{array}$ & $\begin{array}{c}120 \\
(64-168)\end{array}$ & $\begin{array}{c}101 \\
(26-177)\end{array}$ \\
\hline 14.5 & $\begin{array}{c}90 \\
(23-164)\end{array}$ & $\begin{array}{c}83 \\
(16-159)\end{array}$ & $\begin{array}{c}100 \\
(20-191)\end{array}$ & $\begin{array}{c}115 \\
(54-175)\end{array}$ & $\begin{array}{c}94 \\
(16-191)\end{array}$ \\
\hline
\end{tabular}

a Data limited to October 1 - December 31
b Data limited to January 1 - December 15

resulted in a more pronounced increase during the first 6 months of 1986, 1987, and 1988 (Figure 2.3.9a), but

fluctuations were smaller than at Rkm 0.6. High gas pressures prevailed at $\mathrm{Rkm} 2.4$ from July through August in 1985 and 1987 and during the short period of data collection in July 1988 (Figure $2.3 .9 \mathrm{~b}$ ). Delta $\mathrm{P}^{\prime} \mathrm{s}$ at $\mathrm{Rkm} 2.4$ during each fall followed the same pattern as seen upstream at Rkm 0.6 .

Mean annual delta $\mathrm{P}^{\prime} \mathrm{s}$ remained relatively constant at $\mathrm{Rkm}$ 2.4 during the first 3 years of the study, increasing from 104 mmHg in 1985 to 106 and $109 \mathrm{mmHg}$ in 1986 and 1987, respectively. The 1988 mean delta $P$, calculated from measurements made between $15 \mathrm{January}$ and $26 \mathrm{July}$, was $119 \mathrm{mmHg}$. The highest minimum and maximum delta $\mathrm{P}^{\prime} \mathrm{s}$ of 74 and $155 \mathrm{mmHg}$, respectively, occurred in 1988. 


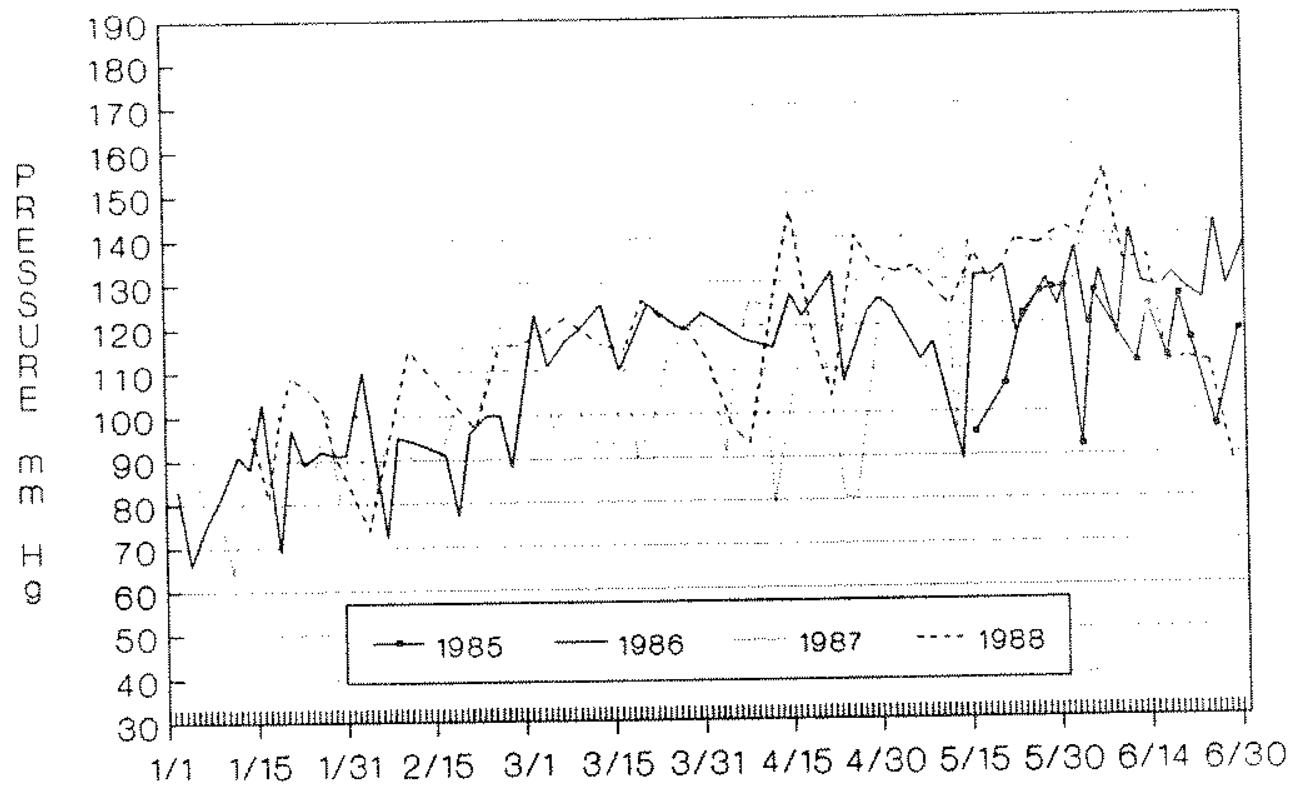

Rkm 2.4 - BOUCK DELTAP

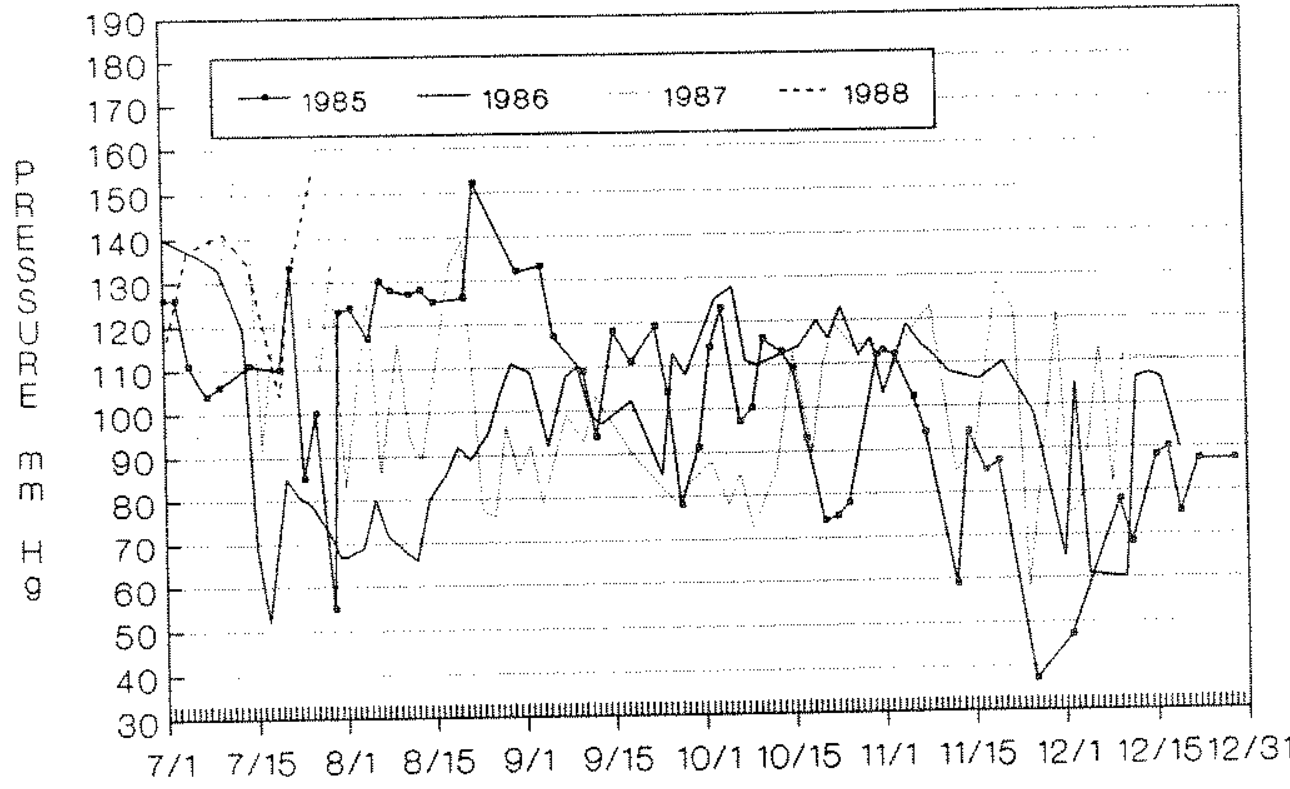

B).

Figure 2.3.9. Delta $\mathrm{P}$ (mmHg) measured by Bouck gasometers near the right bank at Rkm 2.4 (RM 1.5) between A) January - June and B) July - December from 1985-1988 on the Bighorn River, Montana. 
During the first 3 months in 1987 and 1988, Rkm 4.8 delta $\mathrm{P}^{\mathrm{s}} \mathrm{s}$ increased at similar rates (Figure 2.3.10a). From April to June, hyperbaric pressures were highest in 1988, intermediate in 1987, and 1owest in 1986. Delta $P$ in 1986 dropped

substantialiy in July and remained low except for large rises in early and late october (Figure 2.3.10b). In 1987 and 1988, gas pressure remained high through July and August. Maximum delta $\mathrm{P}^{\prime S}$ at $\mathrm{Rkm} 4.8$ in 1987 and 1988 were 188 and $169 \mathrm{mmHg}$, respectively, $14-35 \mathrm{mmHg}$ greater than at upstream sites (Table $2.3 .16)$. In 1986, however, the maximum delta $\mathrm{P}$ remained below maximums at the upstream monitoring sites. Mean delta P's increased from $100 \mathrm{mmHg}$ in 1986, to $107 \mathrm{mmHg}$ in 1987 and peaked at $122 \mathrm{mmHg}$ in 1988. The mean hyperbaric pressure determined from all Bouck measurements at Rkm 4.8 was $108 \mathrm{mmHg}$ and ranged from $46-188 \mathrm{mmHg}$.

Hyperbaric pressures at $\mathrm{Rkm} 8.0$ displayed the same pattern as at Rkm 4.8 (Figure 2.3.11a,b). However, the mean annual delta $P^{i} \mathrm{~s}$ and the highest pressure measured, except in 1987, declined from levels observed at Rkm 4.8. Delta $P^{\prime} \mathrm{s}$ in 1985 remained higher through september than in 1986 and 1987. The maximum delta $P$ we measured at $R k m$. 8 was $177 \mathrm{mmHg}, 11 \mathrm{mmHg}$ lower than at $\mathrm{Rkm} 4.8$ (Table 2.3 .16 ). The mean delta $\mathrm{P}$ at $\mathrm{Rkm}$ 8.0 over the entire study was $101 \mathrm{mmHg}$.

The most downstream monitoring site ( $\mathrm{km}$ 14.5) had variable gas pressures ranging from 16-191 $\mathrm{mmHg}$, with the lowest mean delta $P$ (94 mmg ) of stations monitored (Figure 2.3.12). Distinct differences in delta $p$ levels during the first quarter of each year were only apparent at this site. Also in contrast 
RKM 4.8 - BOUCK DELTAP

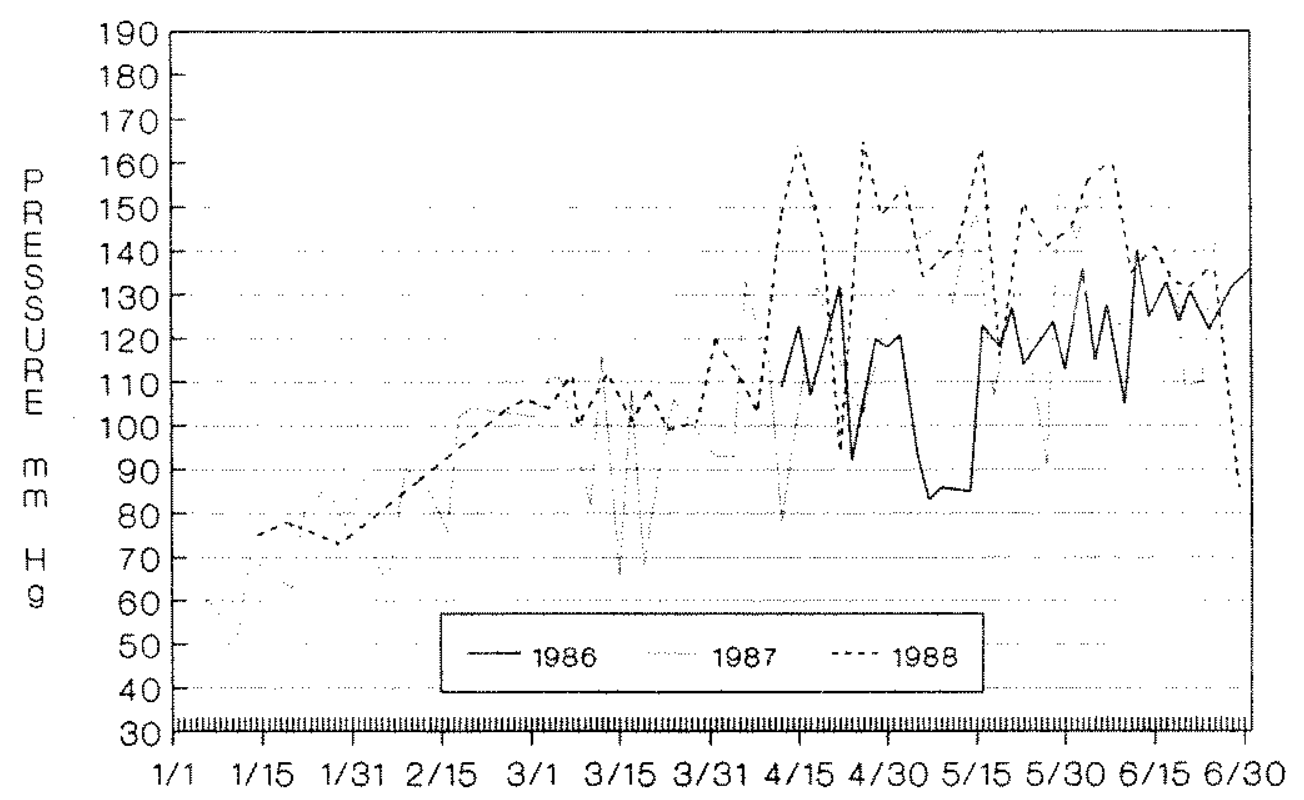

A).

Rkm 4.8 - BOUCK DELTAP

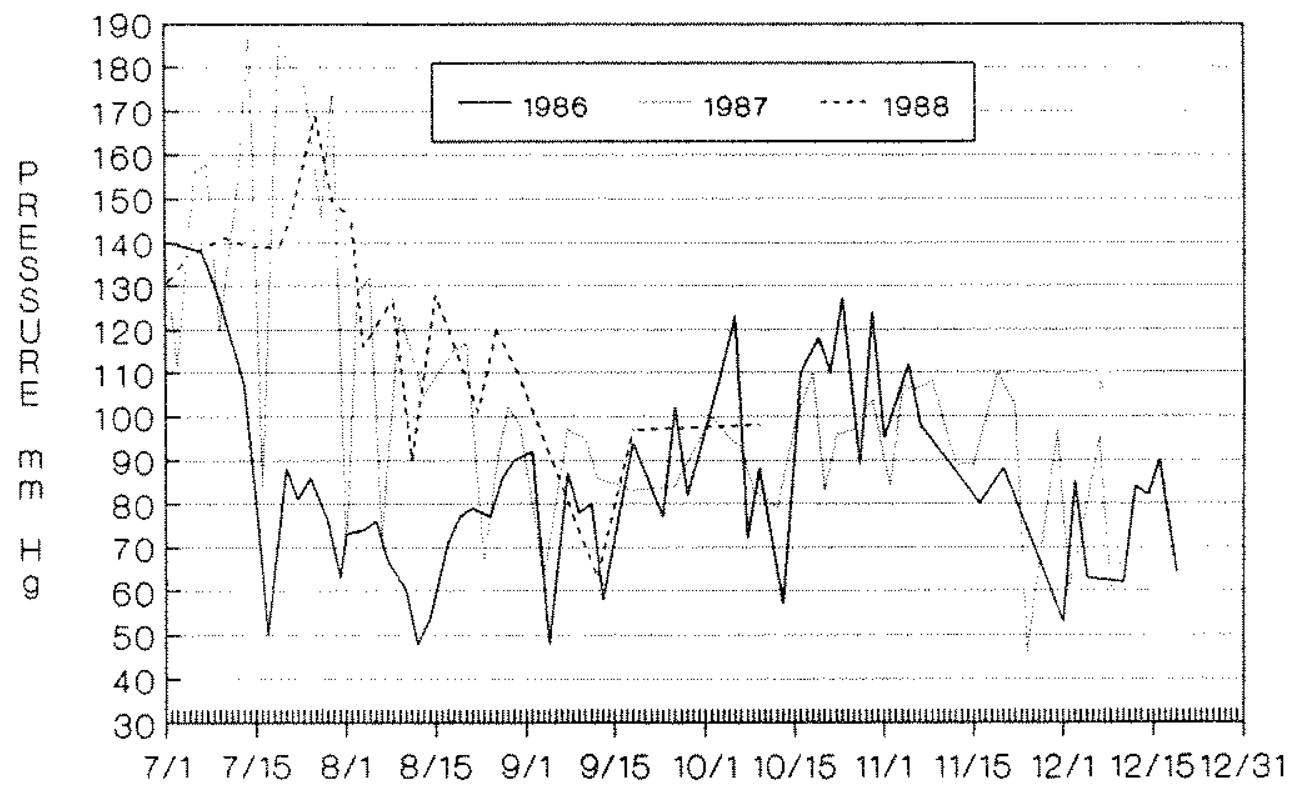

B).

Figure 2.3.10. Delta $\mathrm{P}$ (mmig) measured by Bouck gasometers near the right bank at $\mathrm{Rkm} 4.8$ (RM 3.0) between A) January June and B) July - December from 1986-1988 on the Bighorn River, Montana. 
RKM 8.0 - BOUCK DELTAP

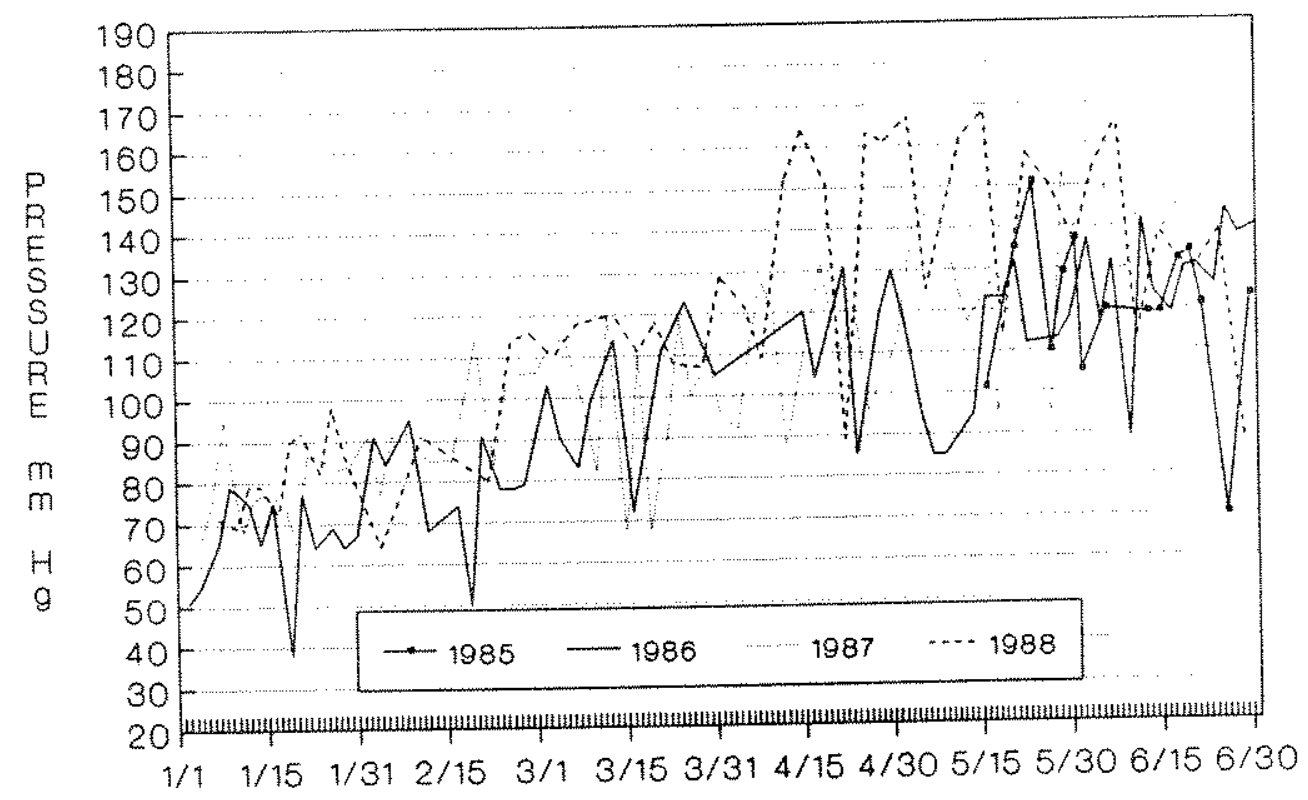

A).

RKM 8.0 - BOUCK DELTA P

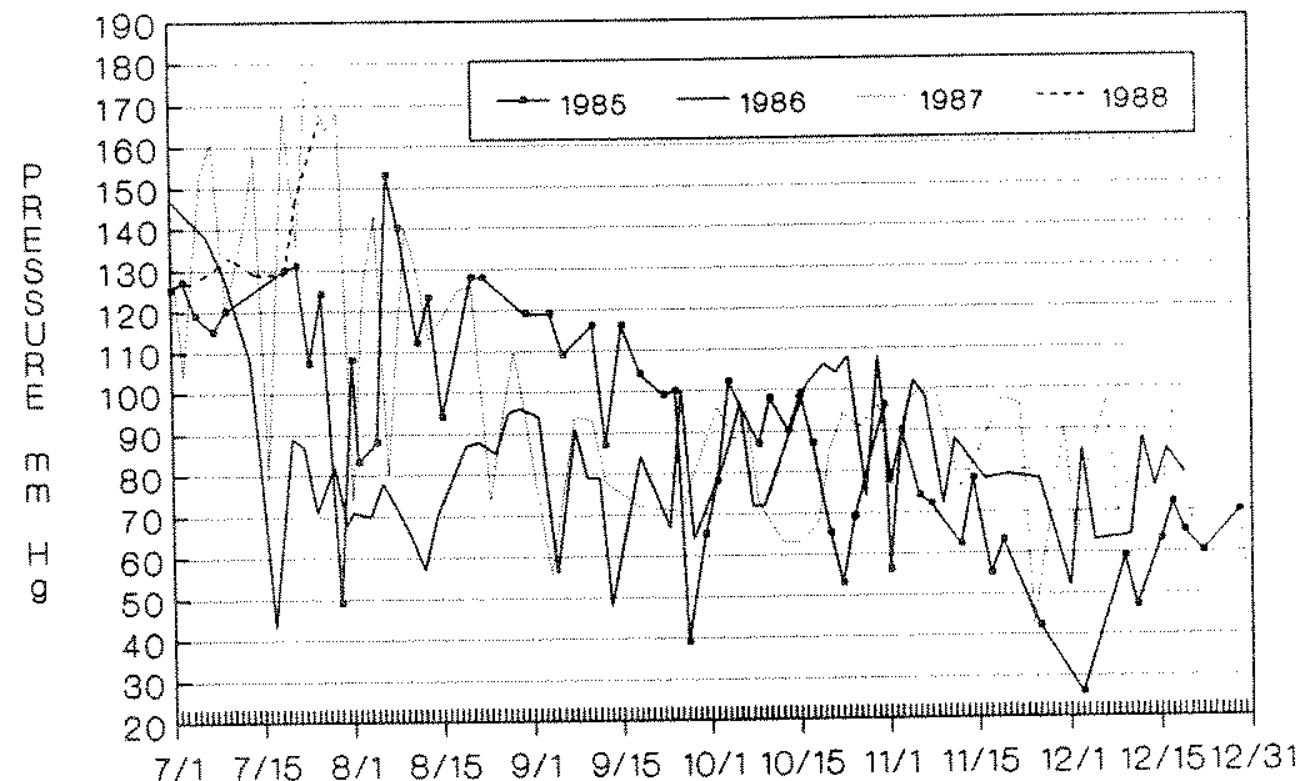

B).

Figure 2.3.11. Delta $\mathrm{p}$ (mmHg) measured by Bouck gasometers near the right bank at Rkm 8.0 (RM 5.0) between A) January June and B) July - December from 1985-1988 on the Bighorn River, Montana. 


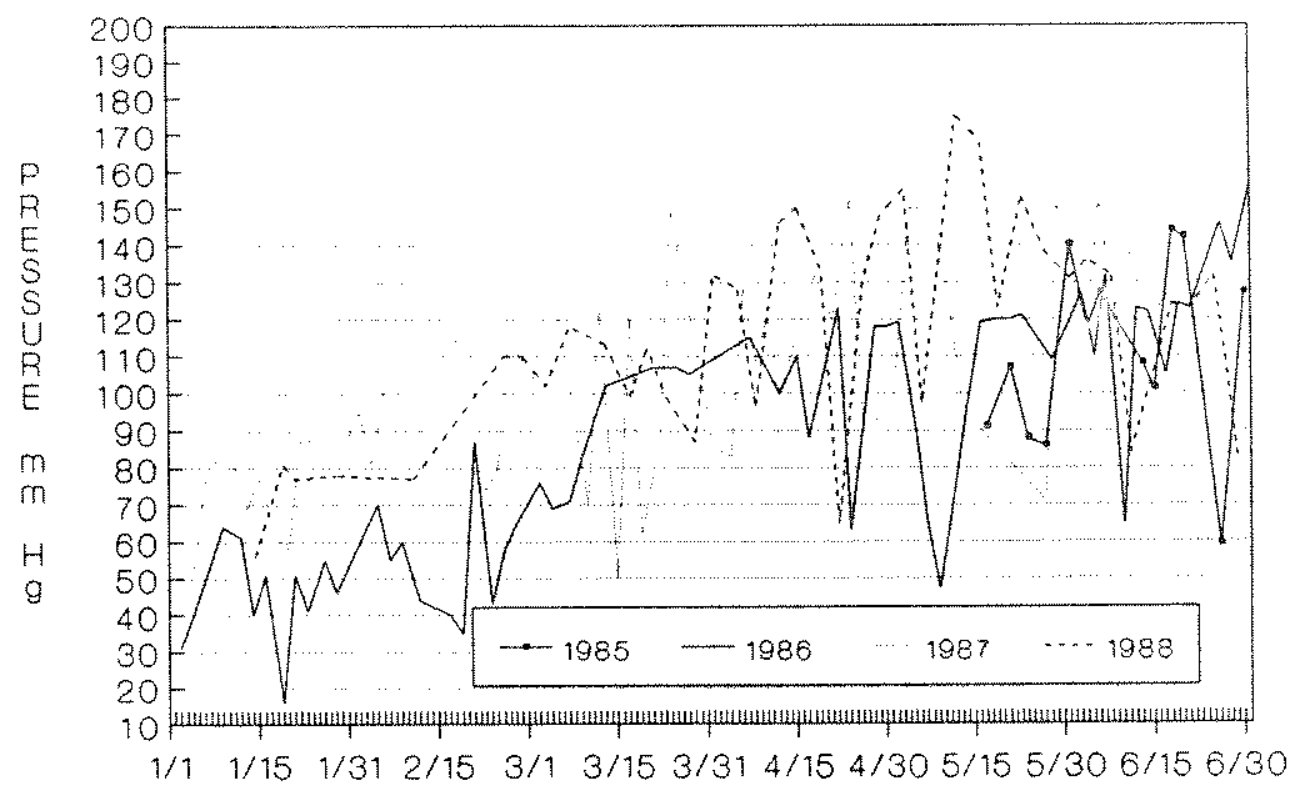

A).

Rkm 14.5 - BOUCK DELTAP

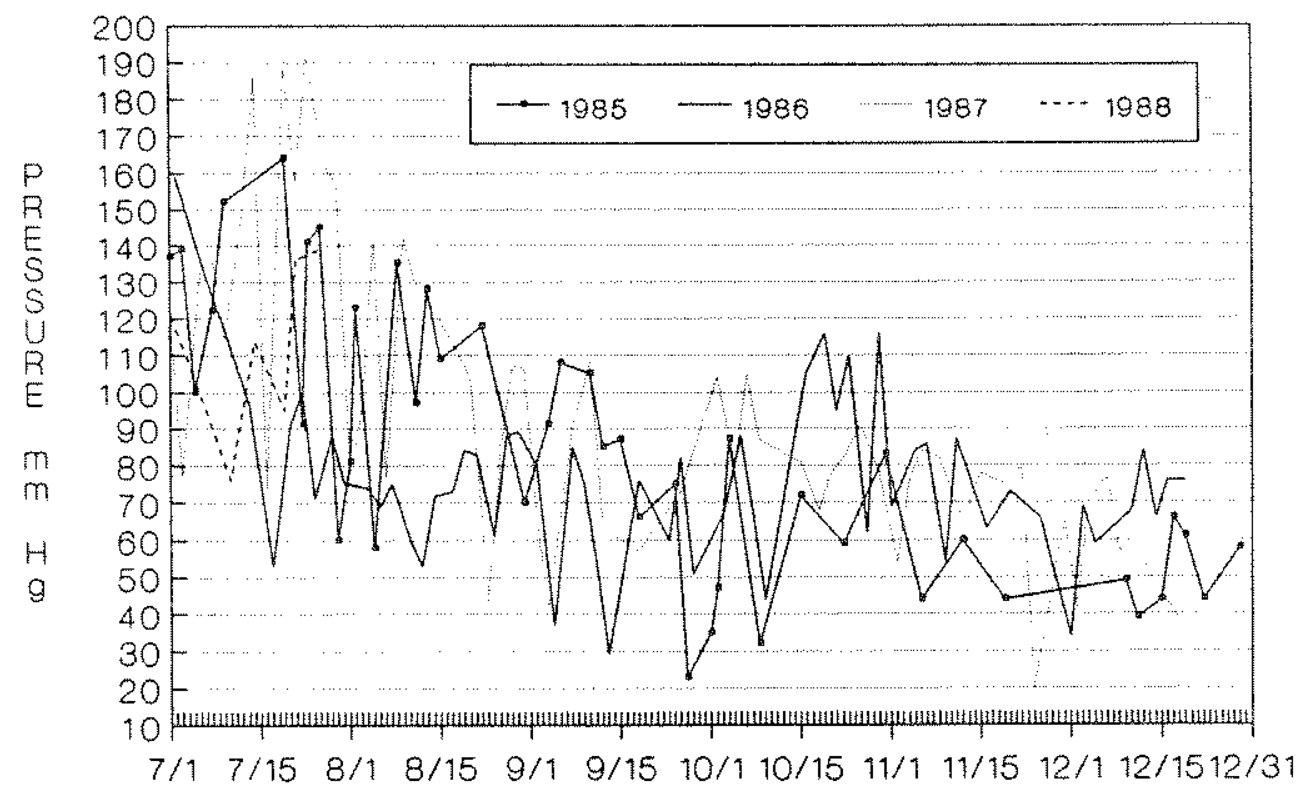

Figure 2.3.12. Delta $P$ (mnHg) measured by Bouck gasometers near the right bank at Rkm 14.5 (RM 9.0) between A) January June and B) July - December from 1985-1988 on the Bighorn River, Montana. 
with upstream sites, hyperbaric pressures dropped below $80 \mathrm{mmHg}$ during most of November and December in all years of data collection. All annual delta $\mathrm{P}^{\prime}$ s were lowest at Rkm 14.5 except in 1988 when the average hyperbaric pressure was $7 \mathrm{mmH}$ Lower at Rkm 0.6 .

Gas pressures were also monitored at Rkm 19.3 (Bighorn Fishing Access site) during a 4.5 month period from December 1987 - May 1988. Although low delta $P^{\prime}$ s characterized most of the monitoring period, hyperbaric pressures equaled or exceeded 130 mmHg on two occasions (Figure 2.3.13). The mean delta $P$ during this period was $74 \mathrm{mmHg}$ and individual measurements varied from $32-133 \mathrm{mmHg}$.

Mean weekly barometric pressure at $\mathrm{Rkm} 0.6$ or in Fort smith varied over a range of $19 \mathrm{mmHg}$ during the study (Figure 2.3.14). The substantial rise in March 1986 when instrumentation was changed suggests that the Princo Nova readings were lower than actual values even when differences in elevation are taken into account. Because of changes in elevation and barometric pressure during a monitoring run, we used a hand-held barometer for on site measurements. Barometric pressures measured at the study site increased slightly at each downstream site (Table 2.3.17). Individual measurements varied $30-39 \mathrm{mmHg}$.

oxygen pressure increased and become more variable downstream (Figures $2.3 .15-19$ ). The Rkm 0.6 mean weekly oxygen pressures remained essentially constant through the first 6 months of each year (Figure 2.3.15). oxygen pressures varied primarily between $150-160 \mathrm{mmHg}$ during this time in 1986 and 


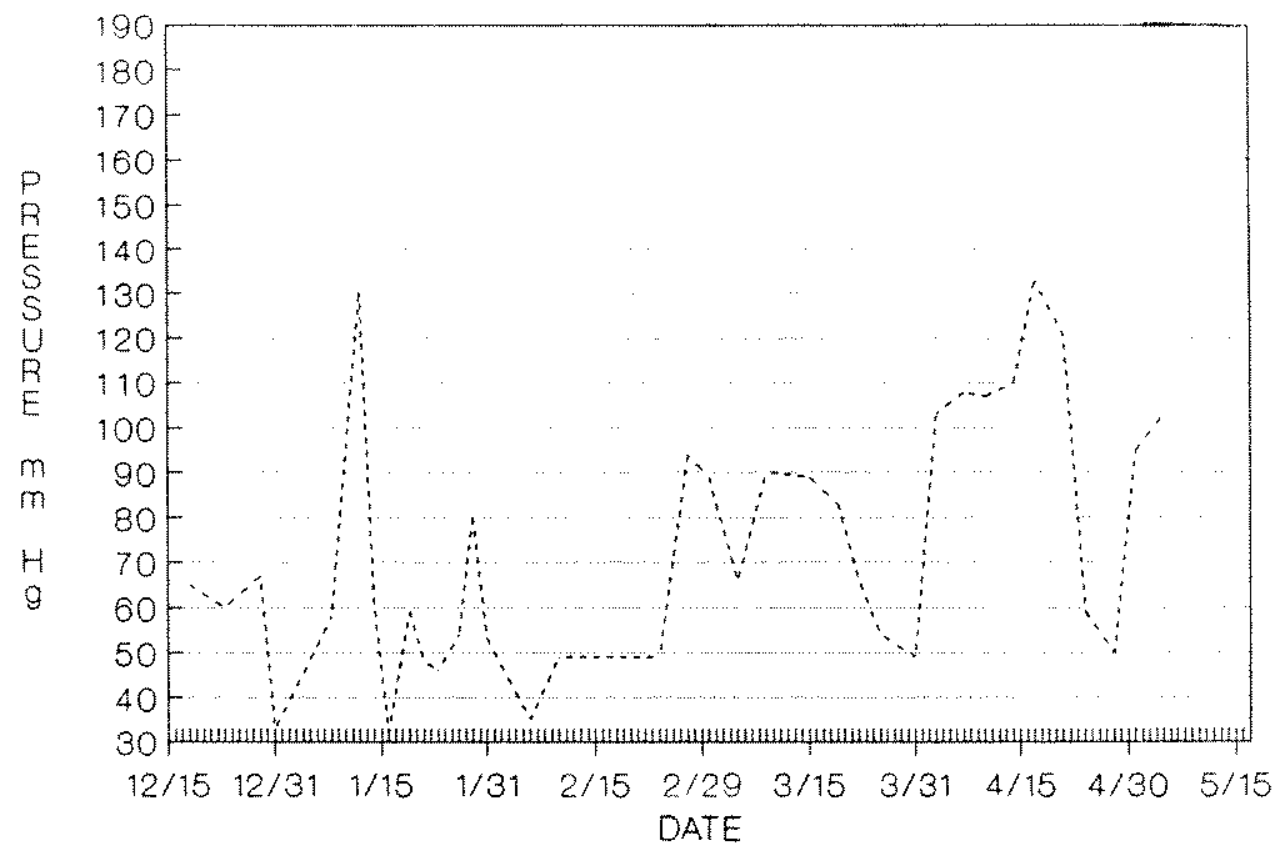

Figure 2.3.13. Delta $\mathrm{P}$ (mmHg) measured by Bouck gasometers near the right bank at Rkm 19.3 (RM 12.0) from 18 December, 1987 - 3 May, 1988 on the Bighorn River, Montana.

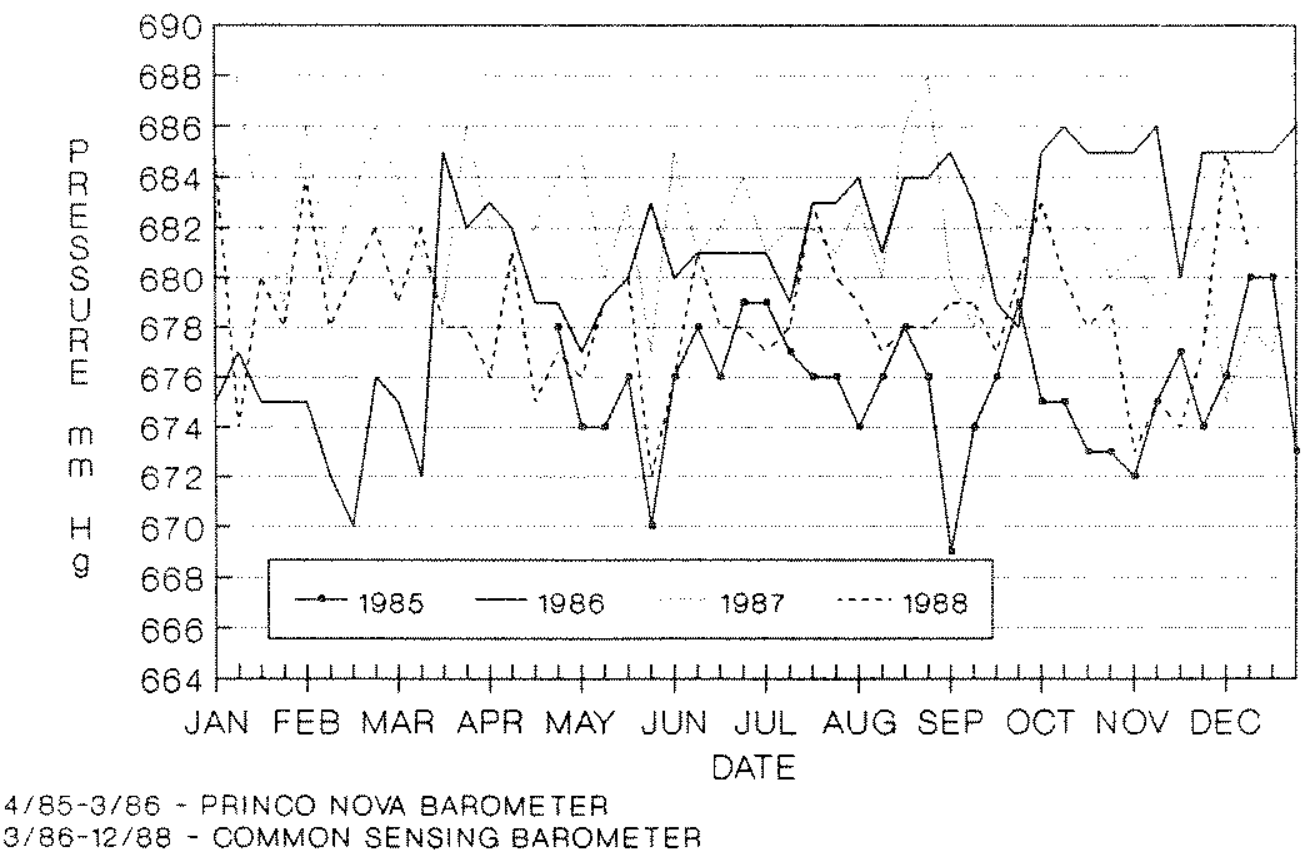

Figure 2.3.14. Mean weekly absolute barometric pressure measured at Fort Smith, Montana (elevation about $1001 \mathrm{~m}$ ) from April, 1985 - March, 1986 and at Rkm 0.6 (elevation about 965 m) from March 1986 - December, 1988 on the Bighorn River, Montana. 
Table 2.3.17. Mean and range of barometric pressure (mmH) at gas monitoring sites on the Bighorn River, Montana.

\begin{tabular}{ccc}
\hline $\begin{array}{c}\text { Location } \\
(\text { Rkm })\end{array}$ & Date & $\begin{array}{c}\text { Barometric } \\
\text { pressure } \\
(\mathrm{mmHg})\end{array}$ \\
\hline 0.6 & $10 / 3 / 85-10 / 26 / 88$ & 678.9 \\
& $5 / 16 / 85-7 / 26 / 88$ & $(658-697)$ \\
2.4 & $4 / 12 / 86-10 / 10 / 88$ & 679.0 \\
4.8 & & $(662-697)$ \\
& & 679.1 \\
8.0 & $5 / 16 / 85-7 / 26 / 88$ & $(661-691)$ \\
& & 679.2 \\
14.5 & $5 / 17 / 85-7 / 26 / 88$ & $(661-697)$ \\
& & 679.8 \\
& & $(663-697)$ \\
\hline
\end{tabular}

1987, but increased in 1988. Oxygen levels declined during summer months in all 3 years, and the drop occurred later each year. Oxygen pressure at $\mathrm{Rkm} 0.6$ generally reached a low point in september, then gradually increased over the remainder of the year. Measurements made in late 1985 showed more variation and lower pressures than other years (Table 2.3.18). Mean annual oxygen pressures in 1986 and 1987 were near $150 \mathrm{mmHg}$. The low 1985 mean oxygen pressure resulted from limited measurements made late in the year. Mean oxygen pressures measured at Rkm 0.6 varied $16 \mathrm{mmHg}$. Individual measurements ranged from a minimum of $120 \mathrm{mmHg}$ in 1985 to a maximum of $169 \mathrm{mmHg}$ in 1988 . The 1988 mean oxygen pressure at Rkm 0.6 and all other sites was greater than calculated for any other year. The higher 1988 oxygen pressures resulted from both higher levels measured and the discontinuation of monitoring later in the year when oxygen pressure tends to be lower: 


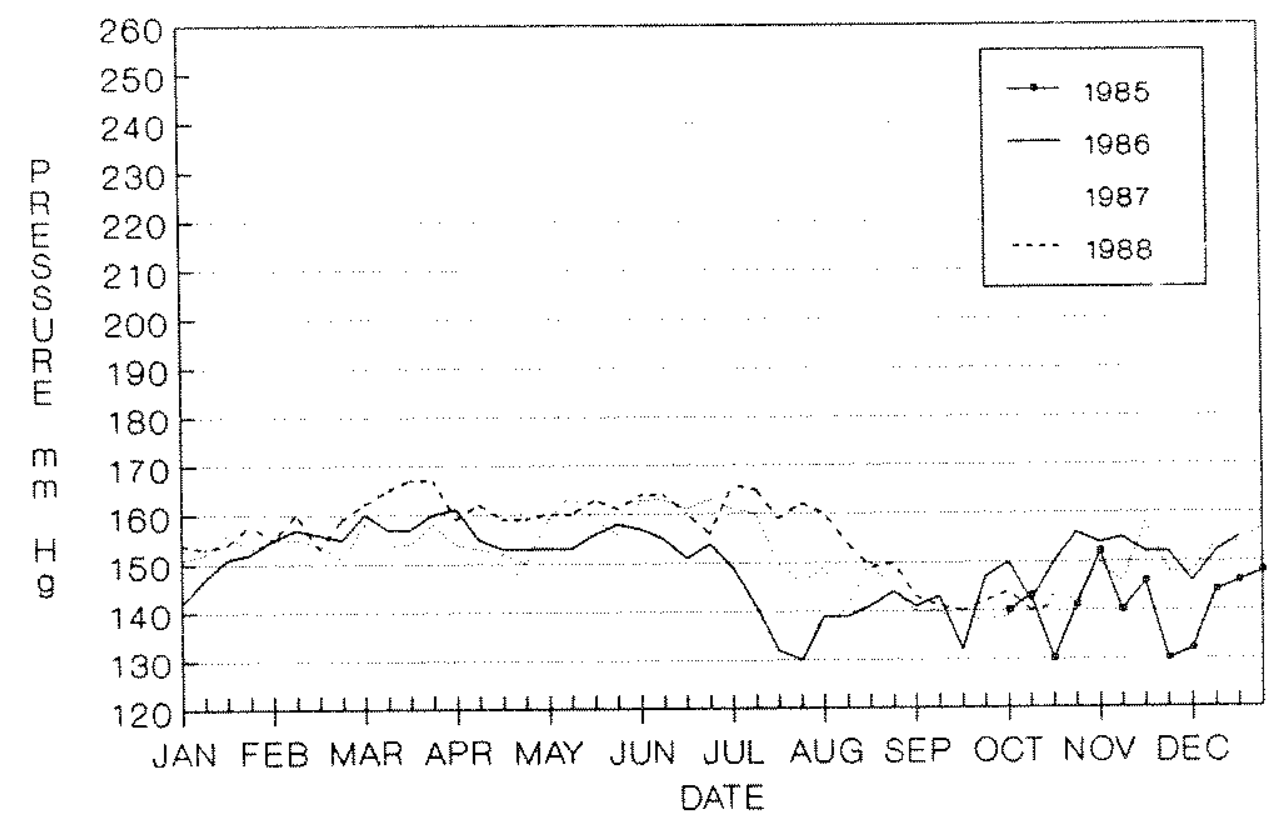

Figure 2.3.15. Mean weekly oxygen pressure (mmHg) calculated from modified winkler samples collected between 1985 and 1988 at $\mathrm{Rkm} 0.6$ (gagehouse), Bighorn River, Montana.

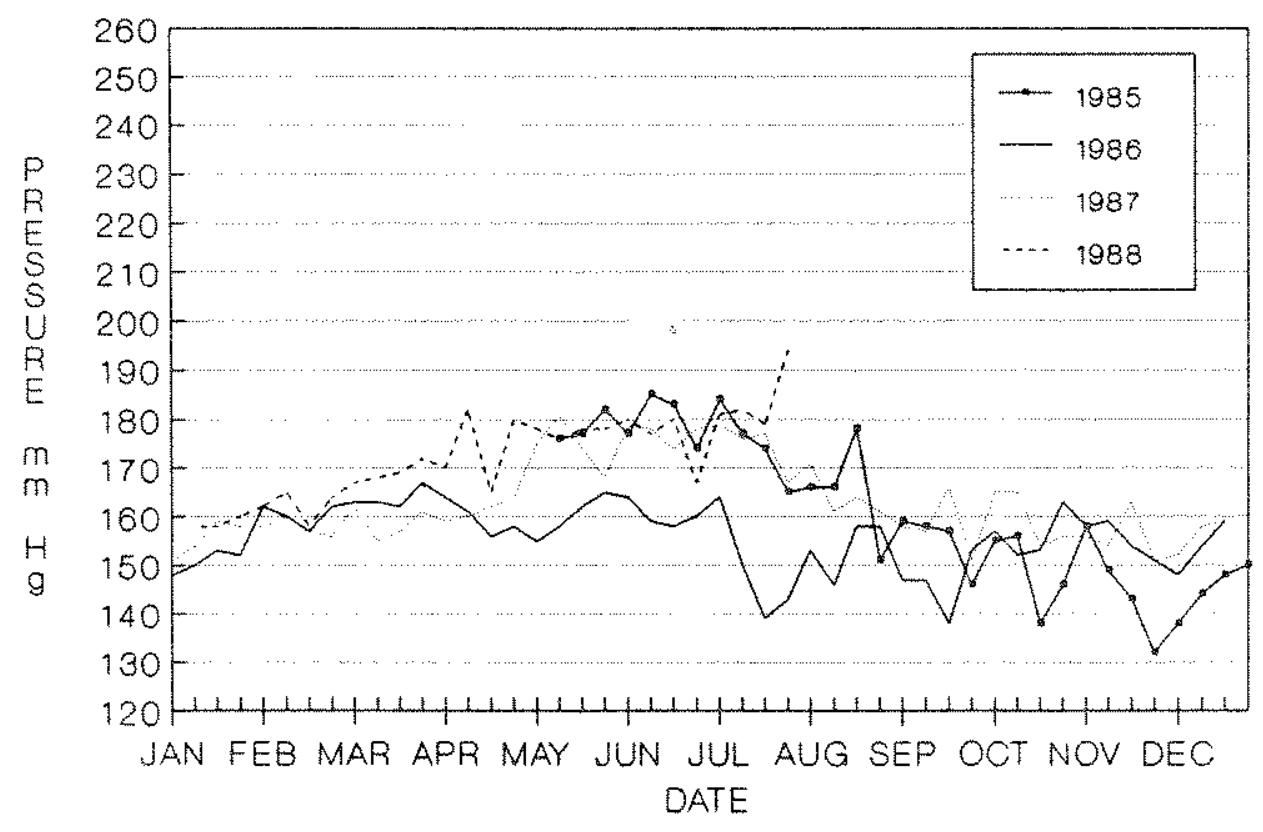

Figure 2.3.16. Mean weekly oxygen pressure (mmHg) calculated from modified winklex samples collected between 1985 and 1988 at $\mathrm{Rkm} 2.4$ (RM 1.5), Bighorn River, Montana. 


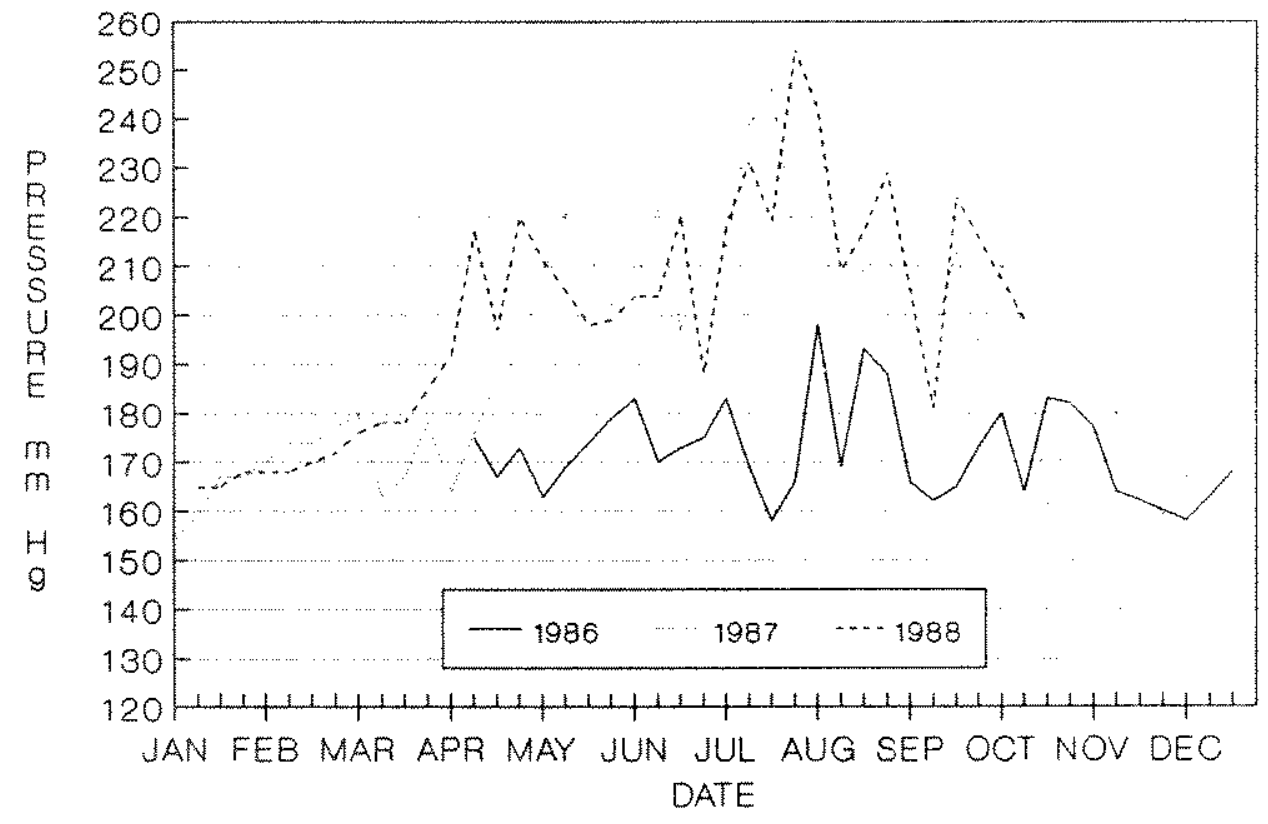

Figure 2.3.17. Mean weekly oxygen pressure (mmHg) calculated from modified winkler samples collected between 1985 and 1988 at Rkm 4.8 (RM 3.0), Bighorn River, Montana.

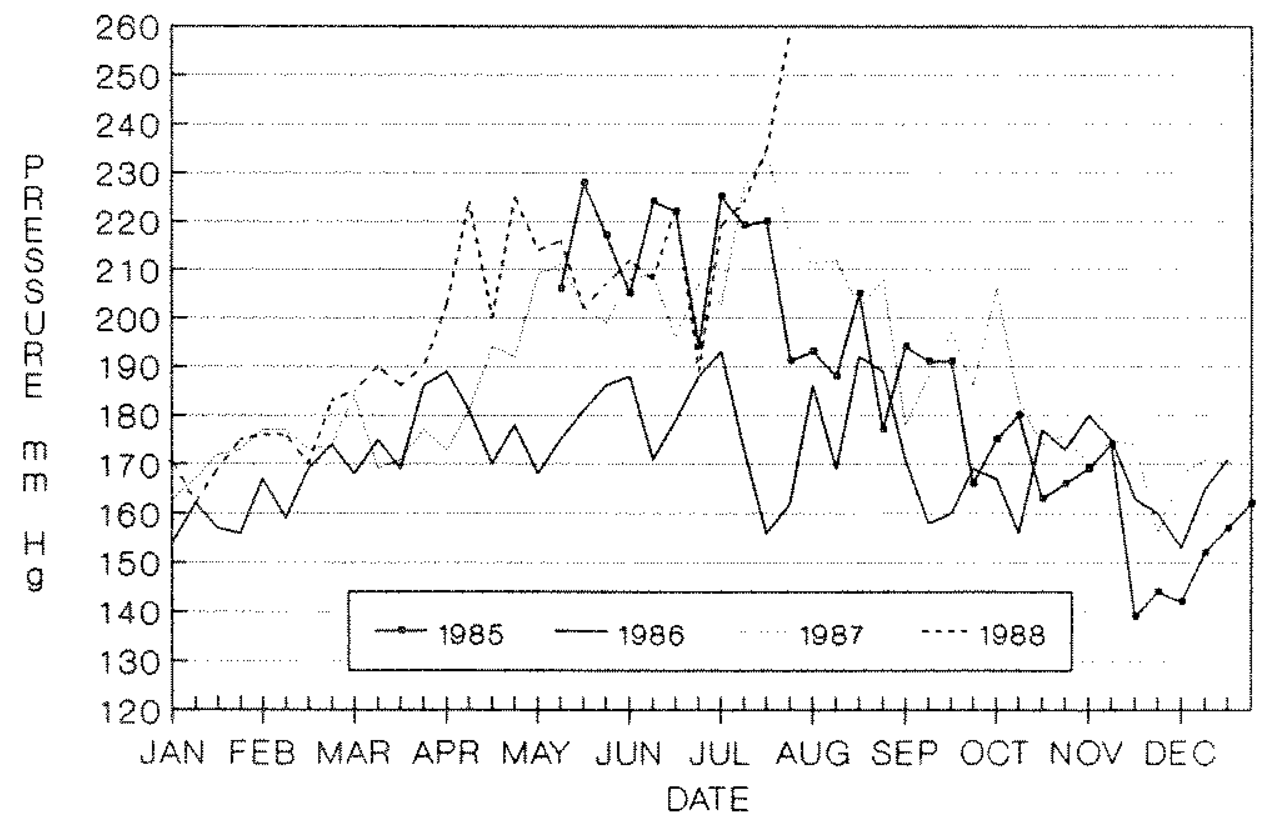

Figure 2.3.18. Mean weekly oxygen pressure (mmH) calculated from modified winkler samples collected between 1985 and 1988 at Rkm 8.0 (RM 5.0), Bighorn River, Montana. 


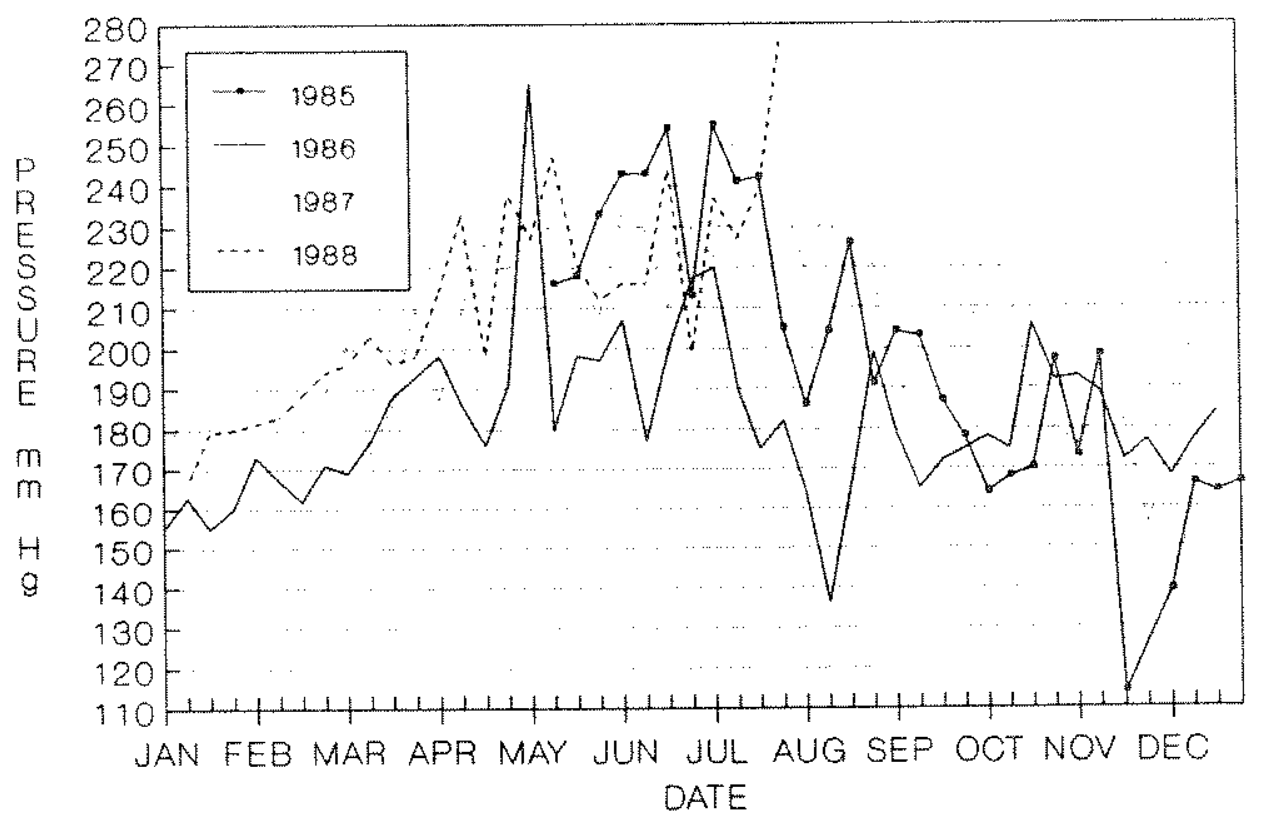

Figure 2.3.19. Mean weekly oxygen pressure (mmHg) calculated from modified Winkler samples collected between 1985 and 1988 at $\mathrm{Rkm} 14.5$ (RM 9.0), Bighorn River, Montana.

Table 2.3.18. Mean and range of oxygen pressure (mmitg) at gas monitoring sites on the Bighorn River, Montana.

\begin{tabular}{cccccc}
\hline \multirow{2}{*}{$\begin{array}{c}\text { Location } \\
(\mathrm{Rkm})\end{array}$} & 1985 & 1986 & 1987 & 1988 & $1985-88$ \\
\cline { 2 - 6 } & 142 & 150 & 152 & 158 & 152 \\
0.6 & $(120-154)$ & $(124-163)$ & $(134-167)$ & $(139-169)$ & $(120-169)$ \\
& 163 & 156 & 164 & 172 & 162 \\
2.4 & $(126-188)$ & $(132-179)$ & $(143-191)$ & $(156-194)$ & $(126-194)$ \\
4.8 & --0 & 172 & 193 & 203 & 189 \\
8.0 & 188 & $(139-203)$ & $(145-277)$ & $(163-257)$ & $(139-277)$ \\
& $(107-233)$ & $(136-198)$ & $(144-263)$ & $(157-259)$ & $(107-263)$ \\
14.5 & 204 & 181 & 205 & 216 & 199 \\
& $(114-281)$ & $(139-235)$ & $(142-312)$ & $(166-277)$ & $(114-312)$ \\
\hline
\end{tabular}


Seasonal changes in oxygen pressure at $\mathrm{Rkm} 2.4$ were similar during 1985, 1987, and 1988 (Figure 2.3.16). In 1986, however, oxygen levels from late spring through August were substantially lower than during the other 3 years (Figure 2.3.16). During the study, individual measurements at Rkm 2.4 ranged as high as $194 \mathrm{mmHg}$ and dropped as low as $126 \mathrm{mmHg}$. As at all downstream monitoring sites, the 1986 mean oxygen pressure was lower than other years. The mean oxygen pressure calculated from all measurements between 1985 and 1988 was 162 $\mathrm{mmHg}$ at $\mathrm{Rkm} 2.4$, an increase of $10 \mathrm{mmHg}$ from $\mathrm{Rkm} 0.6$.

Oxygen pressures at Rkm 4.8 in 1987 and 1988 increased substantially over 1986 levels. This increase, which began in April, was not apparent upstream. Peak values were $277 \mathrm{mmHg}$ or 197\% saturation in 1987 and $257 \mathrm{mmHg}$ or $184 \%$ saturation in 1988. Although 1986 oxygen pressures did not show the large rise, the $64 \mathrm{mmHg}$ variation in individual measurements was greater than in any year at the upstream stations. Mean annual oxygen pressure at Rkm 4.8 increased each year (Table 2.3.18); the overall mean was $189 \mathrm{mmHg}$.

Oxygen pressures at Rkm 8.0 showed similar trends to Rkm 4.8, but levels were slightly lower (Table 2.3.18). As at Rkm 2.4, 1985 oxygen pressures at $\mathrm{Rkm} 8.0$ followed the pattern observed during 1987 and 1988 rather than the 1986 trend (Figure 2.3.18). The peak oxygen pressure measured during 1987 at $\mathrm{Rkm} 8.0$ (263 $\mathrm{mmHg}, 187 \%$ saturation) was $14 \mathrm{mmHg}$ lower and in 1988 the maximum pressure (259 $\mathrm{mmHg}$, 185\% saturation) was 2 mmHg higher than at Rkm 4.8 .

Although the Rkm 14.5 oxygen pressures were generally lower 
in 1986 than all other years, they followed the same general pattern (Figure 2.3.19). Mean annual oxygen pressures were always highest at this site and spanned a $198 \mathrm{~mm}$ g range (Table 2.3.18). The maximum oxygen pressure measured on the Bighorn River during the study was $312 \mathrm{mmHg}$ or $220 \%$ saturation and occurred at this station in 1987 (Table 2.3.18). $14 \mathrm{~km} \mathrm{14.5,}$ the peak oxygen pressures in 1985, 1986, and 1988 reached 281 mmHg (202\% saturation), $235 \mathrm{mmHg}$ (168\% saturation), and 277 mmHg (199\% saturation), respectively. The mean annual oxygen pressure was over $200 \mathrm{mmHg}$ in all years except 1986, and the overall mean was $199 \mathrm{mmHg}$ (Table 2.3 .18 ).

During each year, the pattern of weekly mean water temperature was similar at $\mathrm{Rkm} 0.6,2.4,8.0$, and 14.5 (Figure $2.3 .20 a-d)$. In 1985, mean water temperatures were usually lower at all four sites compared to other years of the study. Maximum temperatures occurred in 1986, the only year with elevated spring flows that allowed a density gradient to establish in Bighorn Lake (Figure 2.3.20a-d). Water temperatures reached maximum levels in August 1986 compared to mid- to late september during other years (Figure 2.3.20a-d). Water temperature at $\mathrm{Rkm} 0.6$ was recorded throughout most of 1988. Data suggests maximum temperatures were intermediate of 1985 and 1987 values (Figure 2.3.20a).

Although annual patterns of water temperature were similar between years. During the period of measurement, water temperatures were generally lowest during 1985 and highest during 1986 (Table 2.3.19). In 1985 temperatures varied from $7.6-8.6^{\circ} \mathrm{C}$ between $\mathrm{Rkm} 0.6$ and 8.0 . In 1986 , mean annual water 


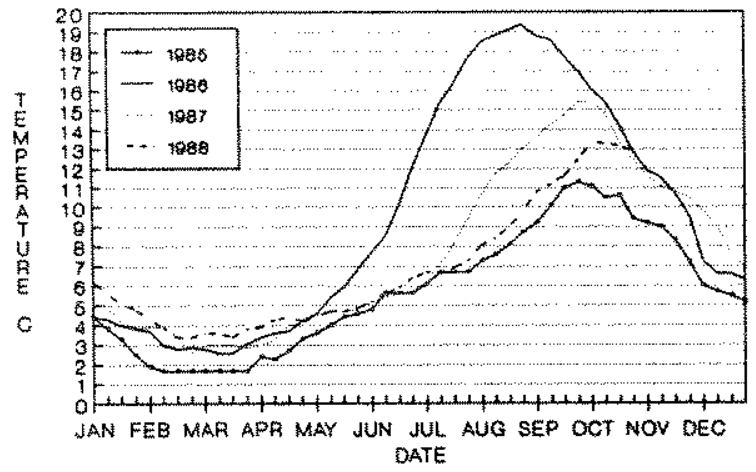

A).

Rkm 2.4

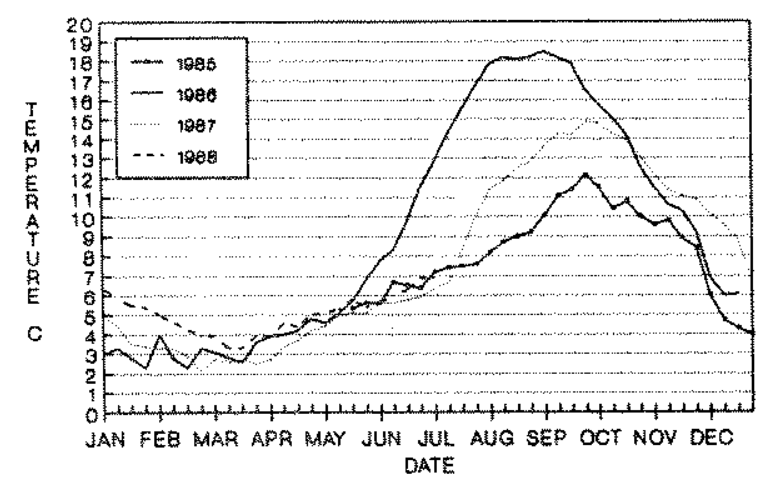

B).

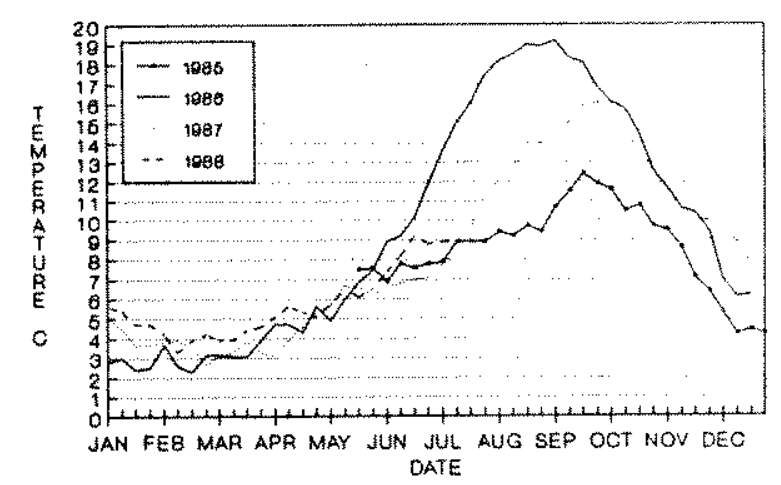

c).

Rkm 14.6

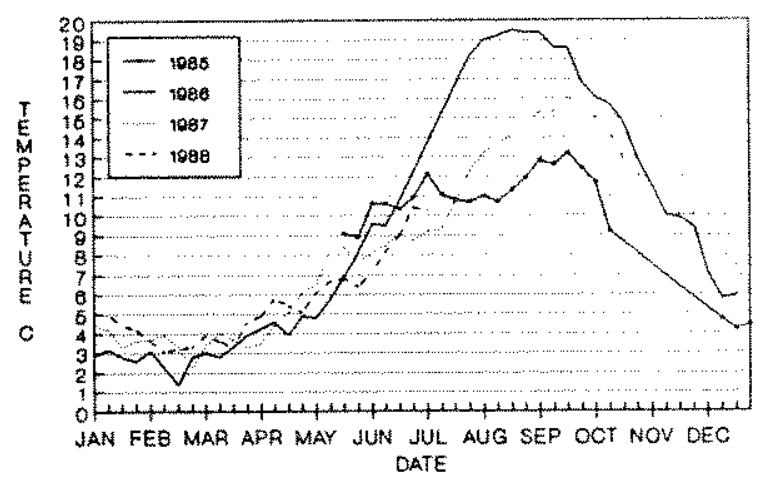

D).

Figure 2.3.20. Mean weekly water temperature of the Bighorn River, Montana from $1985-1988$ at A) Rkm 0.6 (gagehouse), B) Rkm 2.4 , C) $\mathrm{Rkm} 8.0$, and D) $\mathrm{Rkm} 14.5$.

temperatures varied only $0.4^{\circ} \mathrm{C}$, from $9.2-9.6^{\circ} \mathrm{C}$ between sites. The 1986 maximum mean weekly temperatures ranged from 18.5$19.5^{\circ} \mathrm{C}$ at Rkm 2.4 and 14.5 , respectively. Although several of the mean temperatures at each site were lower in 1987 than in 1985, they represent a year of warmer water temperatures than in the first year of the study, since the data include the 
Table 2.3.19. Mean and range of weekly water temperatures at gas monitoring sites on the Bighorn River, Montana.

\begin{tabular}{|c|c|c|c|c|c|}
\hline \multirow{2}{*}{$\begin{array}{l}\text { Location } \\
(\mathrm{Rkm})\end{array}$} & \multicolumn{5}{|c|}{ Year } \\
\hline & $1985^{\mathrm{a}}$ & 1986 & 1987 & $1988^{C}$ & $1985-88$ \\
\hline 0.6 & $\begin{array}{c}7.6 \\
(4.4-11.3)\end{array}$ & $\begin{array}{c}9.5 \\
(2.6-19.4)\end{array}$ & $\begin{array}{c}7.7 \\
(2.6-15.4)\end{array}$ & $\begin{array}{c}4.6 \\
(3.4-6.7)\end{array}$ & $\begin{array}{c}7.8 \\
(2.6-19.4)\end{array}$ \\
\hline 2.4 & $\begin{array}{c}8.1 \\
(4.1-12.1)\end{array}$ & $\begin{array}{c}9.2 \\
(2.3-18.5)\end{array}$ & $\begin{array}{c}7.8 \\
(2.1-15.1)\end{array}$ & $\begin{array}{c}5.1 \\
(3.3-6.9)\end{array}$ & $\begin{array}{c}7.9 \\
(2.1-18.5)\end{array}$ \\
\hline 8.0 & $\begin{array}{c}8.6 \\
(4.2-12.4)\end{array}$ & $\begin{array}{c}9.6 \\
(2.3-19.2)\end{array}$ & $\begin{array}{c}8.4 \\
(2.7-16.3)\end{array}$ & $\begin{array}{c}5.5 \\
(3.3-9.2)\end{array}$ & $\begin{array}{c}8.4 \\
(2.3-19.2)\end{array}$ \\
\hline 14.5 & $\begin{array}{c}10.2^{b} \\
(4.2-13.2)\end{array}$ & $\begin{array}{c}9.5 \\
(1.4-19.5)\end{array}$ & $\begin{array}{c}8.6 \\
(2.3-16.0)\end{array}$ & $\begin{array}{c}5.8 \\
(3.1-10.4)\end{array}$ & $\begin{array}{c}8.7 \\
(1.4-19.5)\end{array}$ \\
\hline $\begin{array}{l}\text { a data c } \\
\text { b dos does n } \\
\text { c data c }\end{array}$ & $\begin{array}{l}\text { hot include } \\
\text { collected }\end{array}$ & $\begin{array}{l}\text { ata from } 1 \\
\text { m January }\end{array}$ & $\begin{array}{l}2 \mathrm{~km} 2.4,8 \\
\text { october - } \\
\text { irough June }\end{array}$ & $\begin{array}{l}\text { and } 14.5 \\
\text { December } 1 \\
988 .\end{array}$ & $\begin{array}{l}\text { on } 20 \text { May } \\
1985 .\end{array}$ \\
\hline
\end{tabular}

months from January-May, a time of minimum water temperatures. Also in 1987, maximum values were higher and minimum values lower than in 1985. The low mean values from 1988 resulted from monitoring Iimited to January through June (Table 2.3.19). Both maximum and mean values in 1988 increased downstream. Minimum values show that water temperatures remained higher in 1988 than in 1986 or 1987.

In 1987, additional water temperature data were continuously collected every $0.5 \mathrm{~h}$ at $\mathrm{Rkm} 0.6$ (gagehouse) and Rkm 4.8 (RM 3.0) by common sensing tensionometers and from the epilimnion of Bighorn Lake at $15 \mathrm{~min}$ intervals by a Sutron-YSI thermistor (Figure 2.3.21). Water temperatures at the gagehouse, as determined by the tensionometer, followed the same pattern as shown by the thermograph (Figure 2.3.20a), but were slightly warmer. The mean weekly water temperature 
measured by the tensionometer from mid-February - December, 1987 was $8.5^{\circ} \mathrm{C}\left(47^{\circ} \mathrm{F}\right)$, while thermograph readings produced a mean weekly value $0.2^{\circ} \mathrm{C}$ lower. Water temperature at Rkm 4.8 remained higher than the two upstream sites through midoctober. It also tended to be warmer than the downstream locations from late July - October.

All river sites had a very different thermal regime than the forebay epilimion of Bighorn Lake (Figure 2.3.21) and showed the effect of the deep-water release from Yellowtail Dam. Water temperature in the forebay was substantially warmer than the Bighorn River through the summer and fall. We measured a maximum weekly mean temperature of $22.7^{\circ} \mathrm{C}\left(73^{\circ} \mathrm{F}\right)$, but higher temperatures during August may have occurred when the thermistor was inoperative.

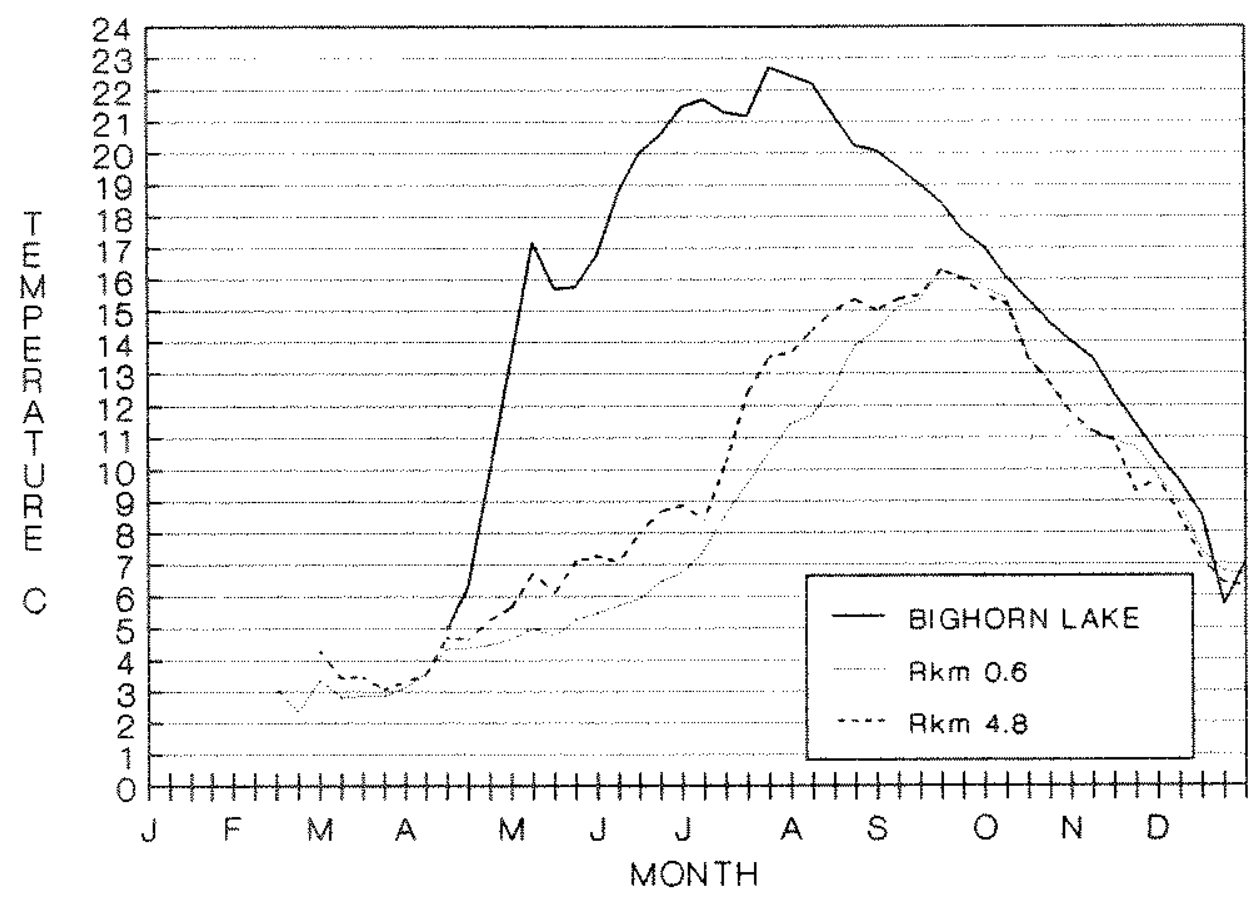

Figure 2.3.21. Mean weekly water temperatures of the forebay epilimion of Bighorn Lake, the Bighorn River at Rkm 0.6 (gagehouse), and Rkm 4.8 in 1987 reported via the Hydromet system. 


\subsubsection{Symptoms of GBT in Relation to Dissolved Gas Levels \\ 2.3.5.1 Embryo Incubation Tests}

\section{$2 \cdot 3 \cdot 5 \cdot 1 \mathrm{a}$ Methods}

Brown and rainbow trout embryo survival from egg

fertilization through hatching was tested. Eggs and sperm from several individuals were pooled to minimize handline or parental effects on egg survival between treatments. Eggs were incubated at high ( $R k m$ 2.4; RM 1.5), medium (Rkm 8.0; RM 5.0), and low (Rkm 14.5; RM 9.0) gas saturation areas in the Bighorn River, and in Afterbay Reservoir which served as a control (Figure 2.3.1).

Except for rainbow trout embryo tests in 1985, fertilized trout eggs were planted at each incubation site, including Afterbay Reservoir (control), in incubation boxes (subsequently referred to as wedge boxes) containing 200 eggs each. In addition, each year at least 1000 eggs were placed at each river site on astroturf substrate in a modified Porter (1973) fry holding box to provide fry for survival tests. In 1985, 100 eggs were placed in each of eight fiberglass bags and eight emergence traps at each site.

Egg bags and emergence traps were completely enclosed, except during rainbow trout tests in 1985 , and presifted $1.3-2.5$ $\mathrm{cm}(0.5-1 \mathrm{in})$ gravel was utilized. Wedge boxes simulated "natural" conditions and eliminated some ambient variables that could influence egg mortality. The wedge-shaped plywood box rested on top of the river bottom and effectively shed drifting aquatic vegetation; its rectangular main compartment measured $25.4 \times 20.3 \mathrm{~cm}$ on each side. Five of the sides had $15.2 \times 15.2$ $\mathrm{cm}$ screen-covered holes to provide intragravel flow. The 
presifted $1.3-2.5$ cm gravel in the egg compartment eliminated substrate as a variable and enclosing the eggs prevented mortality due to disturbance by spawning fish or fishermen. Loss due to lateral movement of fry was also prevented. Two fiberglass bags containing 100 eggs each were placed in each wedge box. Eggs were enumerated using individual counts, a paddle counter, or a von Bayer trough (Piper et al. 1982). The first two methods were exact while the von Bayer troughs were slightly less accurate. Two subsamples of 10 troughs each showed the mean number (standard deviation in parentheses) of eggs to be $50( \pm 0.67)$ and $50( \pm 0.0)$

\section{$2 \cdot 3 \cdot 5 \cdot 1 \mathrm{~b}$ Results}

Brown Trout: Mean survival among eyed brown trout embryos planted in egg bags during 1985 varied $21.4 \%$ between sites (Figure 2.3.22a.); significant differences (ANOVA, LSD; $\mathrm{P}<$ $0.05)$ in survival were detected only between Rkm 2.4 and 8.0 . Fry survival at hatching in egg bags was low. Survival through hatching in egg bags decreased downstream, but differences were not significant.

Variation in embryo and fry survival in 1985-1986 at each site was lower between wedge boxes than egg bags. Wedge box survival rates through eye-up and hatching in the river decreased in a downstream direction (Figure 2.3.22a.). Survival at eye-up was similar between the control sample in Afterbay Reservoir and wedge boxes at Rkm 2.4. However, hatching success was substantially lower in the control sample than at Rkm 2.4. Unintentional movement of the control wedge boxes between 
hardening and eye-up may have caused some of the control mortality.

Mean survival of brown trout embryos planted in wedge boxes on 20 November, 1986 varied 6.2 and $7.0 \%$ between sites from fertilization through eye-up and hatching, respectively (Figure $2.3 .22 b)$. Among wedge boxes, survival at hatching was $9.1-11.2 \%$ lower than at eye-up; no significant difference in embryo survival was detected between sites (ANOVA, $\mathrm{P}<0.05$ ). In Porter boxes, eyed embryo survival at $\mathrm{Rkm} 2.4$ and 8.0 was high, and varied only $3.2 \%$ between sites. Survival of eyed embryos dropped to $57.0 \%$ at $\mathrm{Rkm}$ 14.5. Unlike trends observed at eye-up, hatching success in Porter boxes was higher at Rkm 8.0 than at Rkm 2.4 . However, $235 \mathrm{fry}$ were not accounted for at hatching from the Porter box at Rkm 2.4. We assume that if these fry survived, hatching success would be $80.3 \%$

Eye-up survival was similar at both $\mathrm{Rkm} 2.4$ and 8.0 for brown trout embryos planted 19 November, 1987 in wedge boxes (Figure $2.3 .22 \mathrm{C})$, and approximated survival rates observed at these sites the previous year (Figure $2 \cdot 3 \cdot 22 b$ ). Hatching survival at the two sites remained high. Unlike 1986-87, survival in the Afterbay control sample and at $\mathrm{Rkm} 14.5$ was lower than the other sites. Analysis of variance and comparison of sample means (LSD; $P<$ $0.05)$ indicated survival at eye-up was significantly higher at both Rkm 2.4 and 8.0 than at Rkm 14.5 or in Afterbay Reservoir. Brown trout hatching success in Afterbay Reservoir wedge boxes was greater than survival at eye-up. Consequently, only hatching success at Rkm 14.5 was significantly lower than at Rkm 2.4 and 8.0 (ANOVA, LSD; $\mathrm{P}<0.05$ ). Survival at eye-up and hatching in 
Porter and wedge boxes was similar at Rkm 2.4. Embryo survival in Porter boxes at Rkm 8.0 and 14.5 was lower than in the respective wedge boxes.

Hyperbaric pressures during the first two brown trout embryo incubation experiments gradually rose from fertilization to hatching (Figure $2.3 .22 \mathrm{~d}$, e). This increase was not as apparent during the 1987-88 experiments (Figure 2.3.22f). In most instances delta $p^{\prime}$ s decreased in the downstream direction. An exception was the later part of incubation in 1986-87 when delta $P^{\prime} \mathrm{s}$ fluctuated at Rkm 14.5 (Figure 2.3.22e). Mean delta $\mathrm{P}^{\prime} \mathrm{s}$ ranged from 58-99 $\mathrm{mmHg}$ during brown trout embryo incubation at the three early life history study sites. The lowest hyperbaric pressure measured was $16 \mathrm{mmHg}$ while maximums were $128 \mathrm{mmHg}$ and $149 \mathrm{mmHg}$ through eye-up and hatching, respectively.

Rainbow Trout: In egg bags, rainbow trout embryo survival at eye-up increased at each downstream site in 1985 (Figure

2.3.23a). Survival remained constant between eye-up and hatching at Rkm 2.4 and 8.0 , but a substantial decrease occurred at Rkm 14.5. Egg bags at Rkm 2.4 may have been disturbed by spawning rainbow trout and those planted at $\mathrm{Rkm} 14.5$ were exposed to turbid irrigation return water. However, differences between embryo survival at each site were not significant (ANovA, $\mathrm{P}<$ $0.05)$.

In Porter boxes, eyed embryo survival was higher than in egg bags at each site. Survival in porter boxes at eye-up remained constant at $\mathrm{Rkm} 2.4$ and 8.0 and increased $11-12 \%$ at $\mathrm{Rkm} 14.5$ (Figure 2.3.23). At hatching, survival in Porter boxes remained 


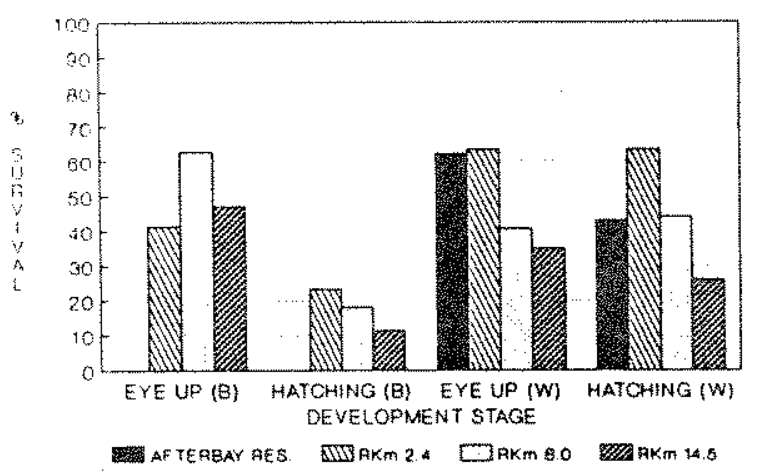

A).

(B) - BAGS, $(W)$ - WEOGE BOX

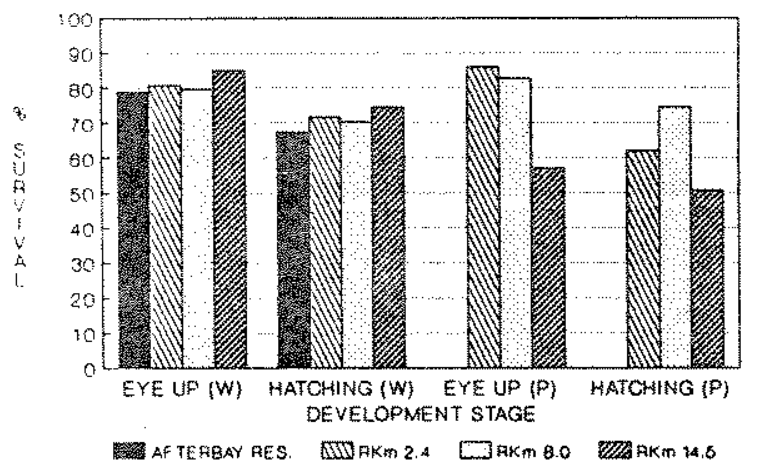

B).

$(W)$ - WEDGE BOX, $\{$ P - PORTEA BOX

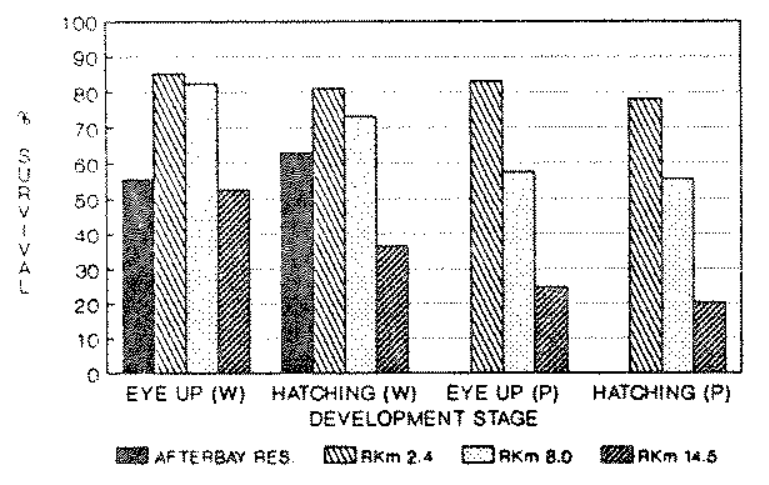

C).

$\left(W_{1}\right.$ \& WEDQE BOX, (P) + POATEG BOX

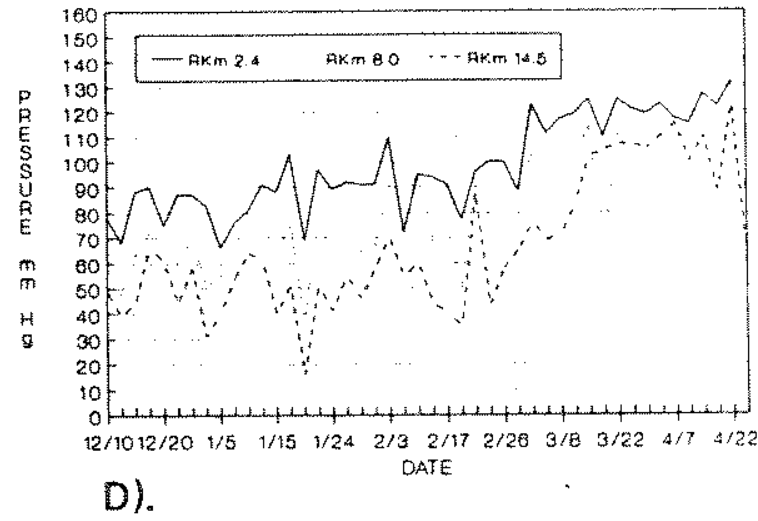

BROWN TROUT EMBRYO INCUBATION EYE UP - $11 / 20 / 8 \theta-1 / 27 / 87$ HATCHHNG + 11/2O/B6-4/6/8?

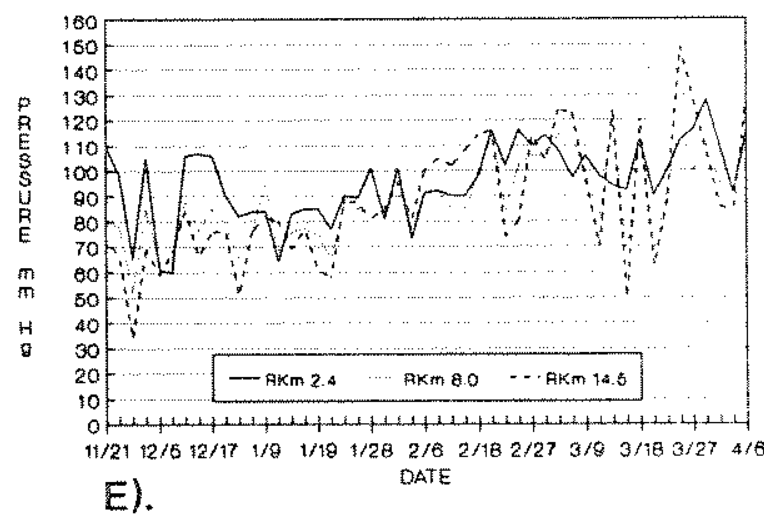

BROWN TROUT EMBRYO INCUBATION EYE UP 11/19/87-3/1/88 HATCHING 11/19/87-3/1/ES

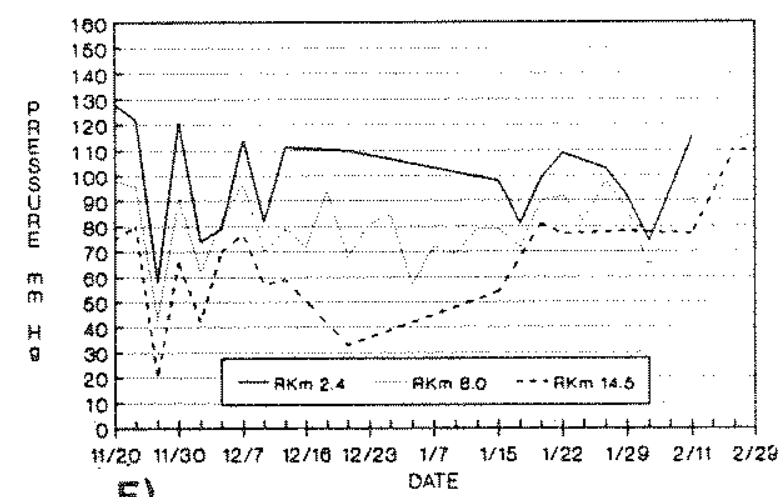

F).

Figure 2.3.22. Brown trout embryo survival in A) 1985, B) 1986 , and c) 1987 associated with Bouck delta P's (mmHg) during embryo incubation tests in D) 1985-1986, E) 1986-1987, and F) 1987-1988 at early life history sites on the Bighorn River and in Afterbay Reservoir, Montana. 
high at all sites and varied from 62-64\%. Survival was $95.1 \%$ among a control sample transported to laboratory facilities at MSU。

Different water temperature regimes at the study sites appeared to be the principal factor affecting the rate of embryo development. In 1985, eggs incubated at Rkm 14.5 needed more Daily Temperature Units (DTU) but fewer days to attain a particular developmental stage (Appendix D).

High flows interferred with the successful completion of rainbow trout embryo incubation experiments conducted in 1986 . None of these data are presented.

In wedge boxes, mean survival of rainbow trout embryos at eye-up during 1987 (Figure 2.3.23b) was substantially lower than in 1985. Survival was similar at $\mathrm{Rkm} 2.4$ and 8.0 and also between the control sample in Afterbay Reservoir and Rkm 14.5. Eye-up survival was significantly higher at Rkm 2.4 and 8.0 than at Rkm 14.5 and in Afterbay Reservoir (ANOVA $\mathrm{P}<0.05$ ). However, the low survival at all sites downplays the biological significance of the observation.

Mean survival during 1987 of rainbow trout embryos in porter boxes was greater than in wedge boxes (Figure 2.3.23b). Survival at eye-up in porter boxes varied from $42.4-53.5 \%$ at $\mathrm{Rkm} 8.0$ and 14.5, respectively. Survival to hatching in porter boxes dropped $6.6,11.4$, and $23.9 \%$ below eye-up survival at $\mathrm{Rkm} 2.4,8.0$, and 14.5, respectively.

Rainbow trout embryo survival at $\mathrm{Rkm} 8.0$ was consistently higher than at the other sites in 1988 (Figure $2.3 .23 \mathrm{C}$ ). Wedge box embryo survival in Afterbay Reservoir and at Rkm 14.5 was $20 \%$ 
or less at both developmental stages. Embryo survival in Rkm 2.4 wedge boxes at eye-up was intermediate of survival at Rkm 8.0 and 14.5. At hatching, however, survival rates at $\mathrm{Rkm} 2.4$ and $\mathrm{Rkm}$ 14.5 were similar. Statistical analysis (ANOVA and LSD) indicated eye-up survival at $\mathrm{Rkm} 8.0$ was significantly higher than at Rkm 14.5 and Afterbay Reservoir; also, survival at Rkm 2.4 was significantly greater than in Afterbay Reservoir.

Hatching survival at Rkm 8.0 was significantly greater (ANovA and LSD) than at any other location.

Porter box embryo survival at eye-up and hatching followed the same pattern we observed in wedge boxes. However, survival was greater in porter than in wedge boxes at Rkm 2.4 and 14.5 , but lower at Rkm 14.5 .

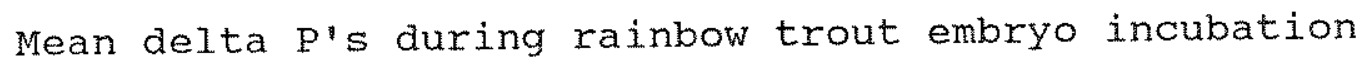
experiments showed a substantial elevation over levels encountered when brown trout embryos were developing. Mean delta P's ranged from 116-139 and 109-137 mmHg through eye-up and hatching, respectively. This is a 29-59 mmHg increase over values present during brown trout incubation. The minimum and maximum hyperbaric pressures measured were 59 and $175 \mathrm{mmHg}$, respectively. We obtained both values at $\mathrm{Rkm} 14.5$. Hyperbaric pressures during 1985 rainbow trout embryo incubation experiments showed no discernible increase from fertilization to hatching (Figure 2.3.23d). Also, no consistent trend was observed in pressures between sites. Delta P's measured during 1987 increased slightly between fertilization and hatching (Figure $2.3 .23 e)$. During most of the 1987 rainbow trout test, delta p's at Rkm 2.4 were greater than at downstream sites. In addition, 
hyperbaric pressures in 1987 usually decreased at downstream sites. Delta $P$ :s measured at $\mathrm{Rkm} 8.0$ and 14.5 just after fertilization in 1988 were the highest encountered during any embryo incubation tests (Figure 2.3.23f). In 1988, hyperbaric pressures at Rkm 8.0 and 14.5 gradually dropped during incubation. A similar decrease was not as apparent at Rkm 2.4.

\section{$2 \cdot 3 \cdot 5.10$ Discussion}

Brown and rainbow trout embryo survival data through eye-up and hatching showed no indication that hyperbaric pressure differences between sites affected mortality. Supporting evidence includes: 1) similar brown trout embryo survival at two sites even when gas pressures at one site were usually greater than the water bubble growth threshold and below the threshold at the other site; 2) high brown trout embryo survival at Rkm 2.4 where gas levels were highest; 3) high rainbow trout embryo survival at Rkm 8.0, where gas pressures were often the highest; 4) higher rainbow trout survival (1987) at Rkm 2.4 and 8.0 than at $R$ km 14.5 even though delta $P^{\prime} \mathrm{s}$ were greater at the upper two sites.

other investigators report that embryos appeared tolerant of supersaturated conditions (Weitkamp and Katz 1980), presumably because of hydrostatic and infracapsular pressures. Fertilized eggs located in the substrate at the bottom of the water column experience maximum hydrostatic pressure for that location. At water temperatures occurring during incubation, hydrostatic head/m of water provides $73.4-73.5 \mathrm{mmHg}$ compensation 


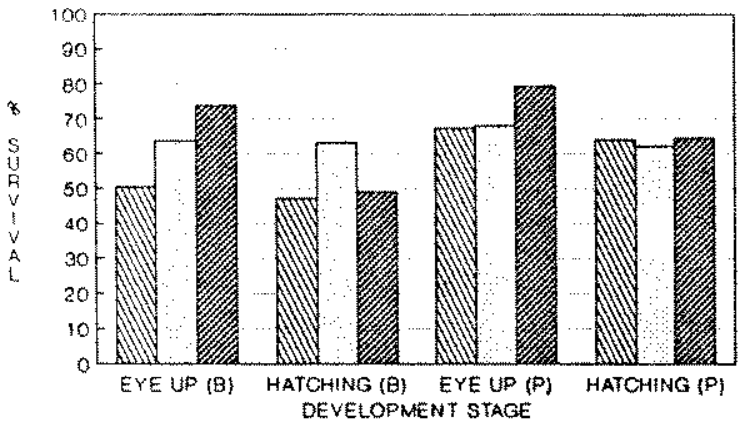

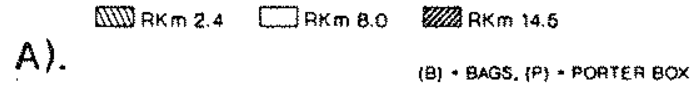

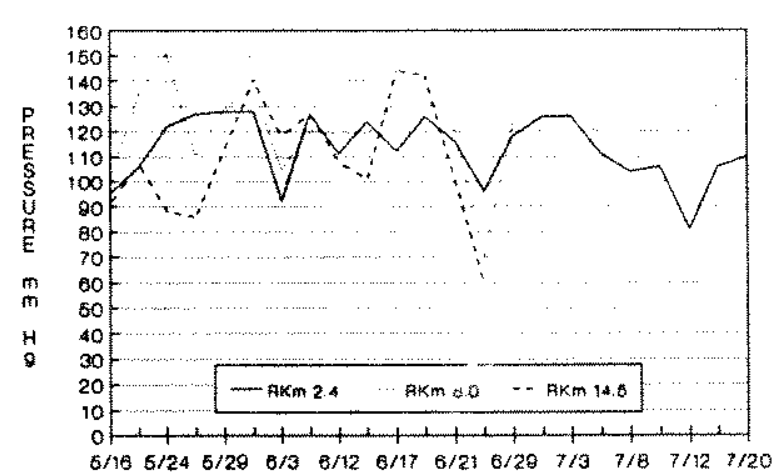

D).
RAINBOW TROUT EMBRYO INCUBATION EYE UP $5 / 6-6 / 25 / 87$
HATCHING $5 / 6-7 / 21 / 87$

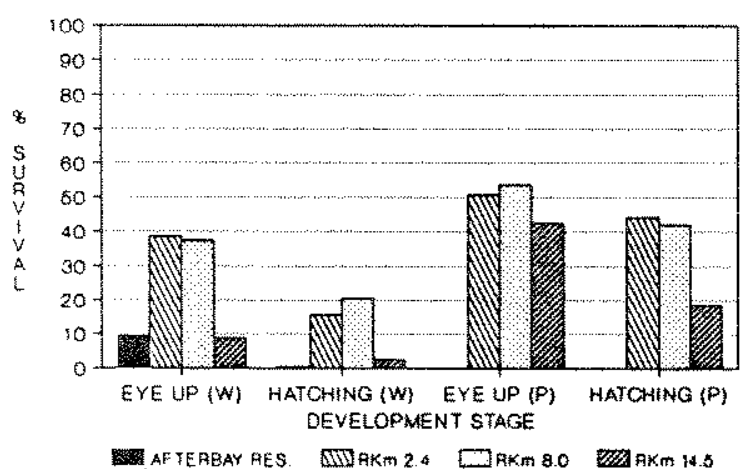
B).

$[W]$ - WEDGE BOK, $(F)$ - POATER BOK

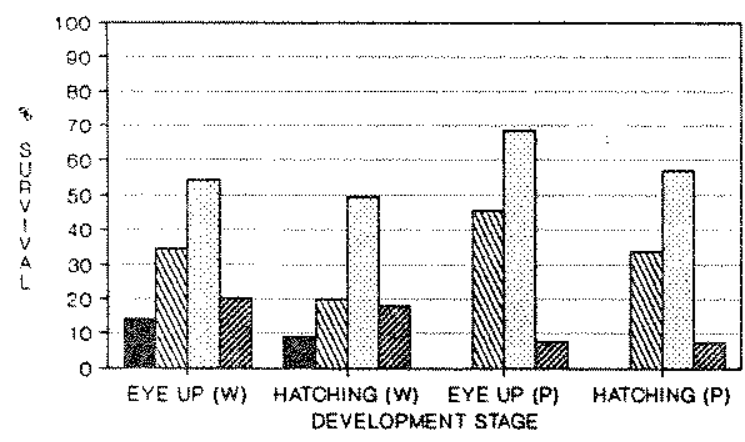

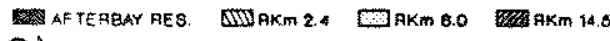
C).

[W] - WEDOE BOX, $\{$ P] * POATEA BOX

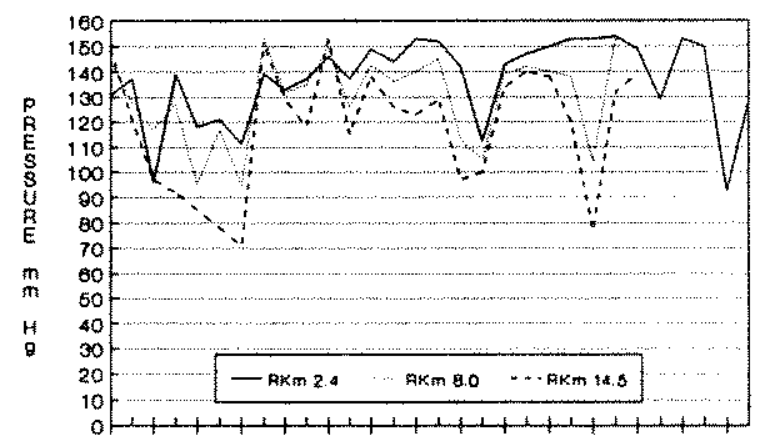

$0 / 8$ 0/30 8/185/27 0/1 0/5 0/100/45 0/10 6/240/20 7/3 7/8 7/13/7/7

E).
RAINBOW TROUT EMBRYO INCUBATION EYE UP $5 / 11-7 / 12 / 88$ HATCHING $5 / 11-7 / 14 / 88$

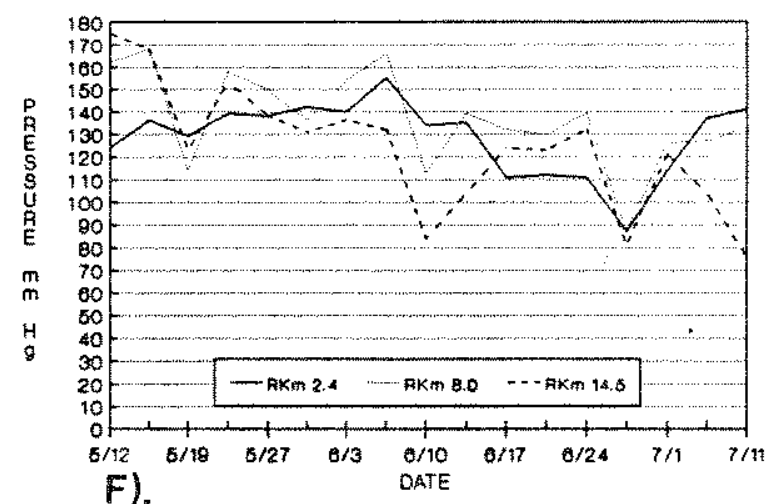

Figure 2.3.23. Rainbow trout embryo survival made in A) 1985 , B) 1987, and C) 1988 associated with Bouck delta P's (mmH) during embryo incubation tests in D) 1985, E) 1987 , and F) 1988 at early life history sites on the Bighorn River and in Afterbay Reservoir, Montana. 
(Colt 1984). Based on spawning depths used by 85 rainbow trout in the Bighorn River $(\bar{x}=40.9 \pm 15.5 \mathrm{~cm}$, range $=12.2-97.5 \mathrm{~cm}$ [Sanborn 1990]), mean hydrostatic compensation pressure during incubation is $30 \mathrm{mmHg}$ (range $9-72 \mathrm{mmHg}$ ). Internal pressures of fertilized salmonid eggs provide additional compensation, varying from 15-40 mmHg at 2-8 h after fertilization to 50-90 mmg near hatching (Alderice et al. 1984; Alderice and Jensen 1985). Thus compensating pressures vary from $24 \mathrm{mmHg}$ in a shallow water redd shortly after fertilization to $162 \mathrm{mmHg}$ near hatching in a deep water redd. An embryo near hatching in an average rainbow trout redd would be protected by approximately $80-120 \mathrm{mmHg}$ of compensating pressure. This would lower the maximum uncompensated delta $\mathrm{P}$ observed during rainbow embryo incubation to 34-70 mmHg in 1987 and 55-95 mming in 1988. After hatching and swim-up, however, the compensating effects of turgor pressure in the egg capsule and hydrostatic pressure would be lost, respectively. Nebeker et al. (1978) found that steelhead trout eggs, embryos, and newly hatched fry were more resistant to supersaturated waters before they attained the swim-up stage.

During embryo incubation experiments, we monitored gas pressures and determined the general range of compensating pressures. Utilizing these data and theoretical bubble growth threshold equations, predictions of when mortality will occur can be made. The threshold equation for bubble growth in environmental water (and the buccal cavity) is most applicable during incubation and after hatching (equation 3, section 2.1.1). Delta $\mathrm{P}^{\prime} \mathrm{s}$ exceeded the threshold at all locations during each brom trout embryo incubation experiment (Figure $2.3 .24 \mathrm{a}-\mathrm{c}$ ). We 
observed in 1985-86 and 1986-87 that pressures above the threshold increased in magnitude as brown trout embryo development proceeded. The increase in gas levels later in the development period would be beneficial to brown trout embryos since it corresponds to infracapsular pressure increases which to some extent counterbalance the rise. Delta $\mathrm{P}^{\prime} \mathrm{s}$ were low enough that brown trout embryos were easily protected by compensating factors; mean delta $\mathrm{P}^{\prime} \mathrm{s}$ were never more than 16 and $2 \mathrm{mmHg}$ greater than the environmental water threshold at $\mathrm{Rkm} 2.4$ and 8.0, respectively. The mean delta $P$ at $\mathrm{Rkm} 14.5$ was $13 \mathrm{mmHg}$ less than the environmental water threshold. Maximum hyperbaric pressures observed during all three experiments at eye-up and hatching were 45,40 , and $27 \mathrm{mmHg}$ and 49,48 , and $66 \mathrm{mmHg}$ greater than the threshold at $\mathrm{Rkm} 2.4,8.0$, and 14.5 , repectively. The maximum values represent levels below or at the minimum compensation pressure and suggest adequate protection.

Rainbow trout embryos develop when hyperbaric pressure is substantially higher than during brown trout incubation and consistently greater than or equal to threshold for bubble growth in water (Figure $2.3 .24 \mathrm{~d}-\mathrm{f}$ ). The largest mean delta $\mathrm{P}^{\prime} \mathrm{s}$ during rainbow trout experiments were 54,56 , and $42 \mathrm{mmHg}$ greater than the threshold at $\mathrm{Rkm} 2.4,8.0$, and 14.5 , respectively. The greatest maximums were 72,85 , and $92 \mathrm{mmHg}$ and increased downstream. Although no pressures measured were above the maximum compensation pressures expected near hatching for an average redd, at least one mean from each study site was greater than the minimum compensation pressure for redds just after fertilization. Also, maximum delta $P^{\prime} s$ measured at all locations 
BROWN TROUT EMPPYO INCUBATHON - 1966-80 DELTA P E.W. THRESHOLD

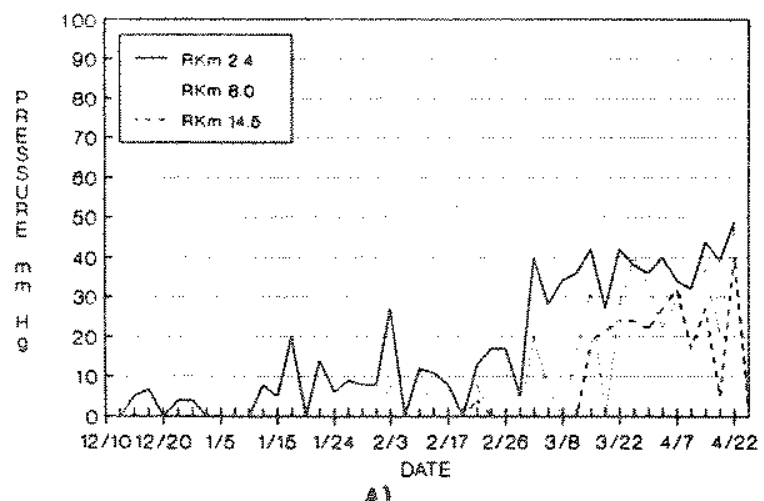

A).

BROWN TAOUT EMBAYO INCUBATION - 1080-87 DELTA P : E. TH. THESHOLD

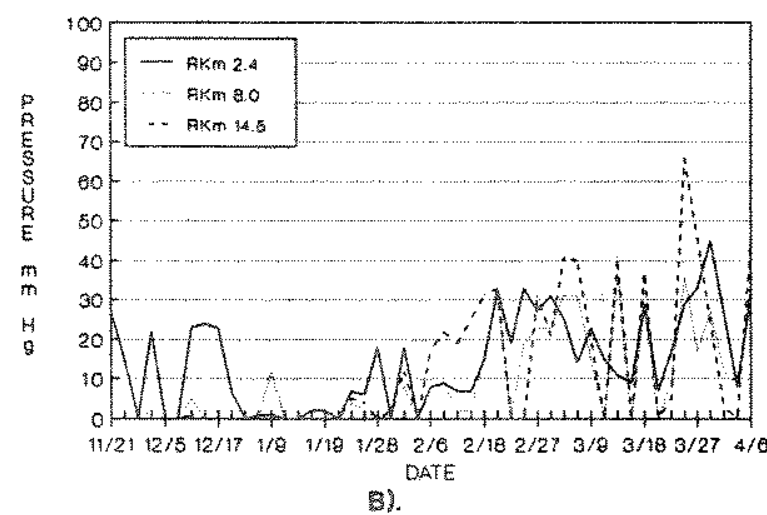

a).

QROWN ThOUT EMBRY INCURATION - 1987-80 DELTA $P>E$.W. FHRESHOLD

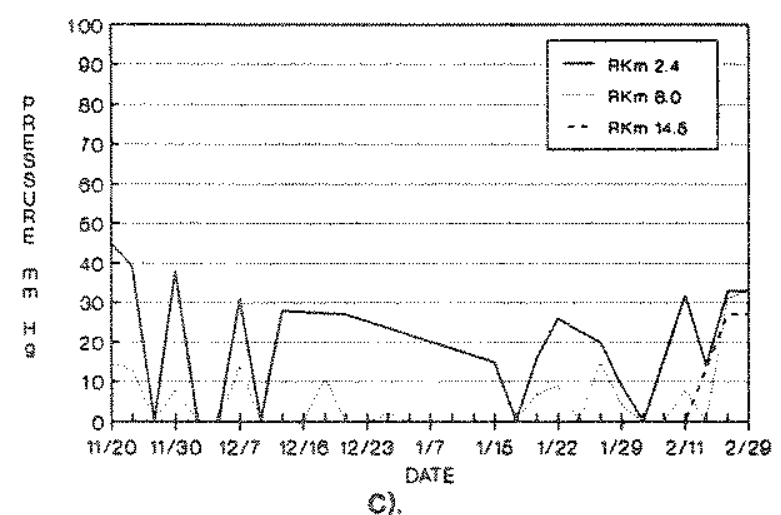

RAINBOW TROUT EMBRYO INCUBATION - 1985 DELTAP,$E$ W. THRESHOLD

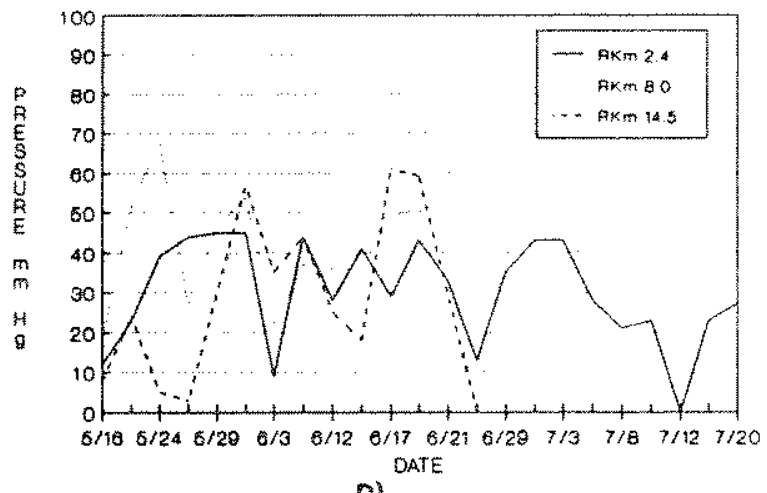

D).

RANEOW TROUT EMBRYO INCUEATION - 1987 DELTA P, EW. THRESHOLO

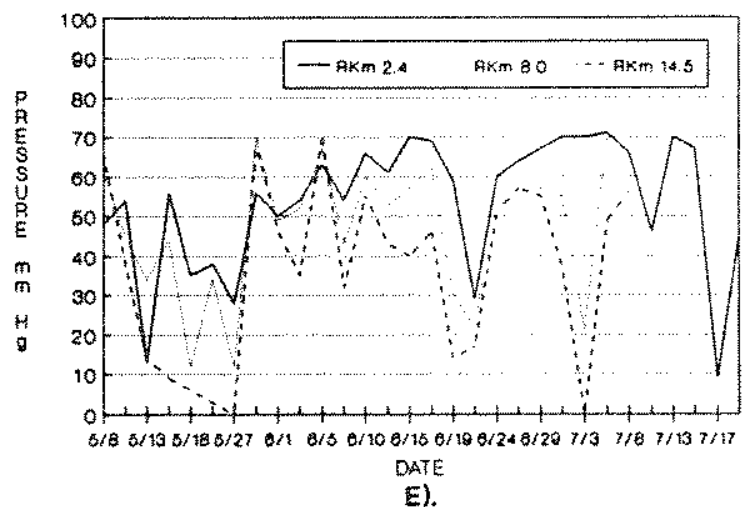

RAINBOW TROUT EMBRYO INCUBATION - 1989 DELTA P, EW. THRESHOLD

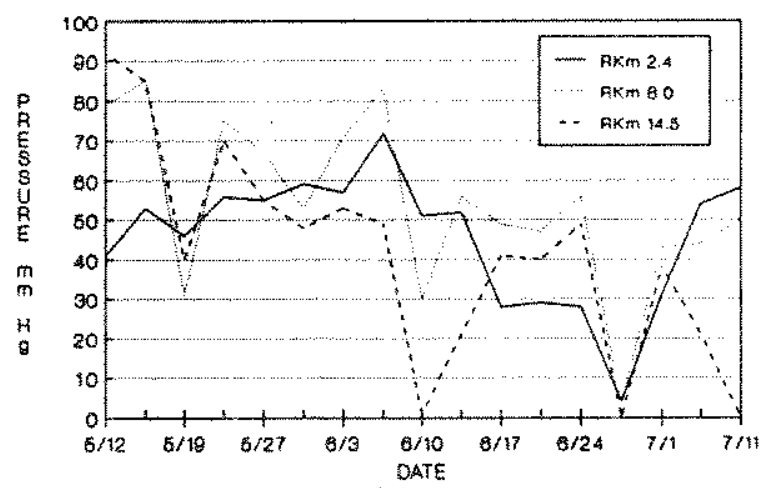

F).

Figure 2.3.24. Hyperbaric pressure (mmHg) greater than the threshold for bubble growth in environmental water during embryo incubation experiments for brown trout in A) 1985-86, B) 1986-87, and C) 1987-88 and for rainbow trout in D) 1985, E) 1987, and F) 1988 at early life history sites on the Bighorn River and in Afterbay Reservoir, Montana. 
in 1987 and 1988 approached or exceeded the minimum level of compensation near hatching. These data suggest that rainbow trout embryos in an average redd were not subjected to delta $\mathrm{P}^{\prime} \mathrm{s}$ high enough to result in substantial mortality. However, any increase in gas pressures due to dam operational changes or photosynthesis could potentially result in mortality to embryos.

An assessment of rainbow trout year class strength by the Montana Department of Fish, Wildlife, and Parks (MDFWP) indicated that reproduction in 1983 and 1984 was much lower than in 1985 and 1986 (Fredenberg 1987). If differences in year class strength are being influenced by dissolved gas levels, the embryo incubation field tests as well as Nebeker's et al. (1978) work imply the controlling mechanism operates after hatching. We noted a substantial difference in 1984 and 1986 gas levels at a time when swim-up fry might be vulnerable to GBT. In 1983, the first rainbow trout fry were located on $20 \mathrm{July}$ (Sanborn 1990). Each of our egg plants occurred near the end of the rainbow trout spawning season and suggest substantial fry emergence by late July. Weiss saturometer measurements made by MDFWP personnel from 13 July - 30 August 1984 showed a mean delta $p$ of 145 ( \pm 5 ) mmig (MDFWP, unpublished data), while valid Bouck gasometer measurements from the same period in 1986 yielded a mean hyperbaric pressure of $76( \pm 19) \mathrm{mmHg}$. Low gas levels in 1986 were in large part due to the shutdown of the sluiceway from 15 July - 21 August. The 1984 measurements suggest the sluiceway was in operation most of this time. Unfortunately, during this time in 1983 and 1985, we are not aware of any gas measurements made at $\mathrm{Rkm} 0.6$. However, 1985 Afterbay Dam operation records 
show the sluiceway was not in operation from 23-30 July. In addition, relatively low sluicegate openings from 13-23 July and 30 July - 6 August, 1985 suggest somewhat reduced delta $\mathrm{p}$ levels. Although 1983 Afterbay Dam operation records are not as extensive as from 1985-86, information should be adequate to estimate an approximate range of gas levels. If these records suggest high delta $\mathrm{P}^{\mathrm{S}} \mathrm{s}$, an experimental program should be developed where sluicegate openings are reduced to minimal levels and all scheduled shutdowns for sluiceway maintenance occur during the period when rainbow trout fry emergence. Continued monitoring of rainbow trout fry or yearlings would also be necessary to determine if any modification in dam operation produced tangible results. 


\subsubsection{Fry Survival Tests \\ $2 \cdot 3 \cdot 5 \cdot 2$ a Methods}

Artificially spawned and incubated Bighorn River brown and rainbow trout fry were held at various locations in the study area where gas saturation was high (Rkm 0.6, gagehouse; and Rkm 2.4; RM 1.5), medium ( $R \mathrm{~km} \mathrm{4.8;} \mathrm{RM} \mathrm{3.0,} \mathrm{and} R \mathrm{~km} \mathrm{8.0;RM} \mathrm{5.0),} \mathrm{and}$ low (Rkm 14.5; RM 9.0). Fry and fingerlings were held at each site in a $30.5 \times 22.9 \times 33 \mathrm{~cm}$ wooden box. Each box had three $24.1 \times 12.7 \mathrm{~cm}$ and two $17.8 \times 12.7 \mathrm{~cm}$ openings covered with 0.32 cm mesh screen. Fish were fed five or six times daily by a Sweeney model AF6 vibrator fish feeder equipped with an automatic timer powered by a 12 volt motorcycle battery. The feeder and holding box were enclosed in a larger wooden box attached to a steel post driven into the river bottom.

\section{$2 \cdot 3 \cdot 5 \cdot 2 \mathrm{~b}$ Results and Discussion}

Similar patterns of rainbow trout fry mortality were observed at all three early life history study sites in 1985 (Figure $2.3 .25)$. Mortality of rainbow trout fry hatched and reared at Rkm 2.4 was low until day 50, then high from day 50-60 and essentially zero after day 80 . Cumulative mortality surpassed $50 \%$ between days 50 and 60 and approached $80 \%$ when the experiment was terminated. At Rkm 8.0, mortality was highest between days

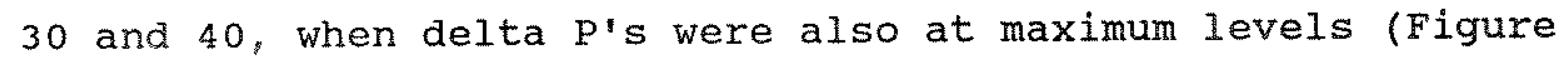
2.3.26). Cumulative mortality at Rkm 8.0 exceeded $50 \%$ between days 40 and 50. Mortality occurred earlier at Rkm 14.5, resulting in cumulative mortality surpassing $50 \%$ between days 20 


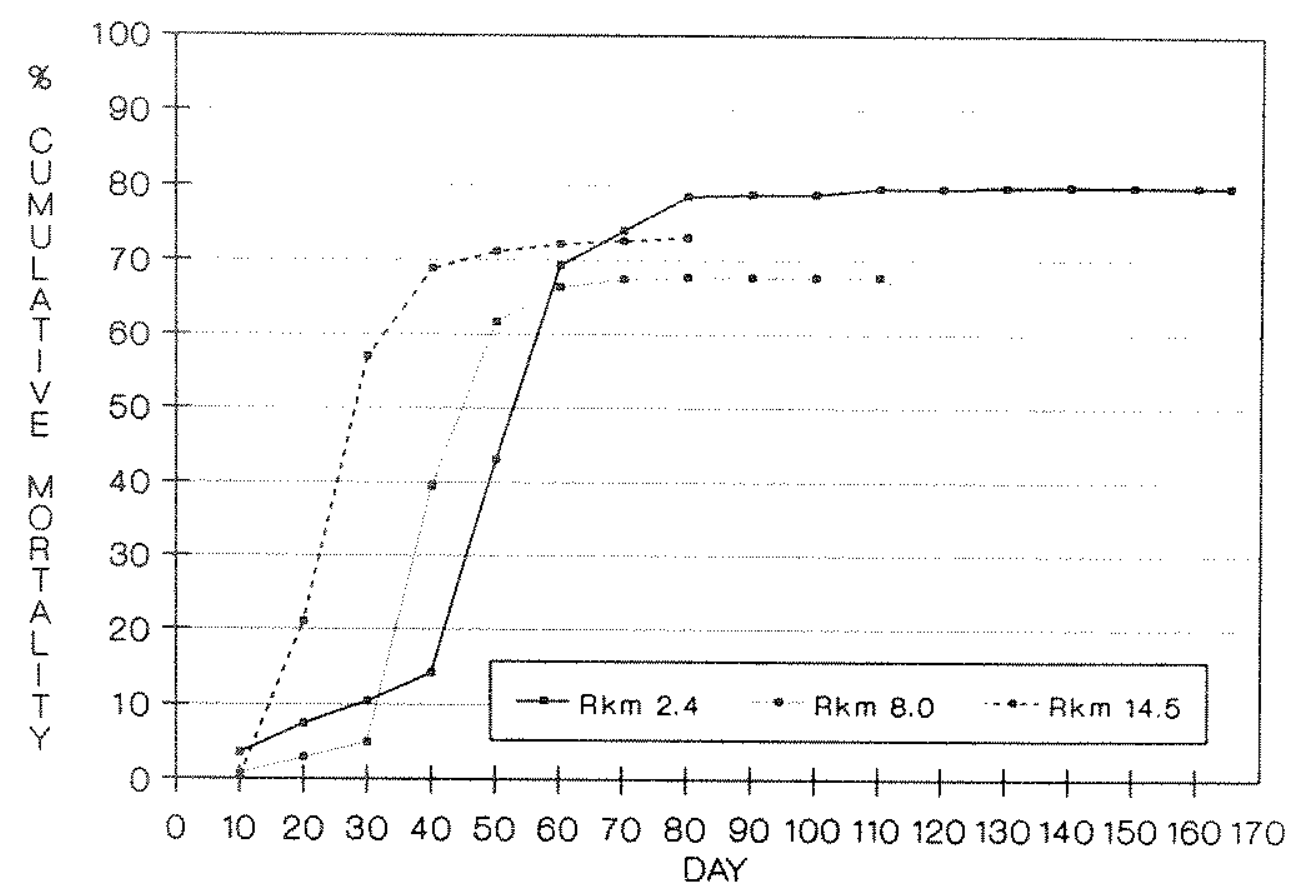

Figure 2.3 .25 . Cumulative mortality by day among rainbow trout fry held at $2.4,8.0$, and $14.5 \mathrm{~km}$ below Afterbay Dam on the Bighorn River, July 1985 - January 1986.

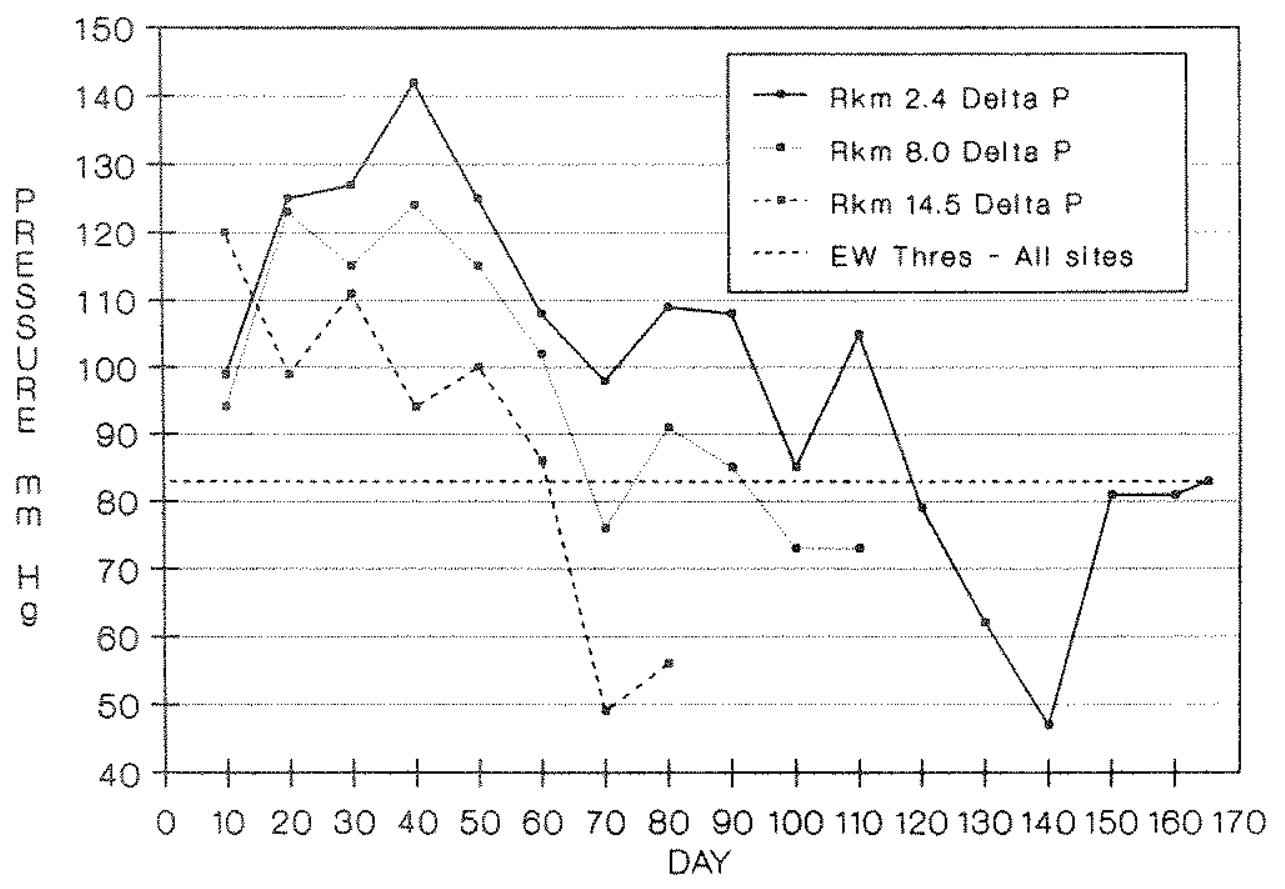

Figure 2.3.26. The environmental water (EW) threshold for bubble growth and mean delta $P^{\prime} \mathrm{s}$ (mnitg) at $2.4,8.0$, and $14.5 \mathrm{~km}$ downstream from Afterbay Dam during rainbow trout survival tests, July 1985 - January 1986. 
and 30 .

Hyperbaric pressures tended to decrease downriver and through time. Delta P's at $\mathrm{Rkm} 2.4$ were highest on day 40 immediately preceding peak fry mortality, then decreased during the remainder of the test (Figure 2.3.26). Although the delta $\mathrm{P}$ at Rkm 2.4 decreased, mean values remained above the threshold for bubble growth in environmental water (assuming no hydrostatic compensating pressure) until almost day 120, long after the mortality rate had declined to near zero. Delta $\mathrm{P}^{\mathrm{S}} \mathrm{s}$ at $\mathrm{Rkm} 8.0$ and 14.5 dropped below the environmental water threshold between days 60 and 70 . The threshold for bubble growth in environmental water probably represents the point above which hyperbaric pressures would have affected fry. Measurements were not made in 1985 to determine the maximum amount of hydrostatic pressure available to the fry for compensation. However, if the holding box at Rkm 2.4 was at its maximum depth in the water column and the fry utilized all the hydrostatic compensation, the threshold for bubble growth in environmental water would have been close to the gas pressures we observed on day 60 when mortality began to level off. Although some mortality may have been caused by excessive dissolved gases, we attributed most mortality to bacterial gill disease caused by crowding and accumulation of metabolic wastes. Treatment with Hyamine on day 60 at Rkm 2.4, and day 50 at $\mathrm{Rkm} 8.0$, substantially reduced mortalities. Bacterial gill disease appeared to have run its course before Hyamine application on day 50 at $\mathrm{Rkm} 14.5$.

Delta $P^{i} s$ at $\mathrm{Rkm} 8.0$ and 14.5 never reached the minimum threshold for bubble growth in the cardiovascular system. At Rkm 
2.4, delta $\mathrm{P}^{\prime}$ s remained above the cardiovascular system threshold from day 20 to 50 .

In early october 1985,241 rainbow trout reared at $\mathrm{Rkm} 14.5$, Where mean delta $P$ was $102 \mathrm{mmHg}$, were transported upstream to Rkm 0.6. Mortality rose to more than 50\% by day 10 , then remained near zero until after day 26 (Figure 2.3.27). From day 26 until the experiment ended on day 33, mortality increased slightly. Fifty-six percent of the dead fish had external symptoms of GBT. on day 12, $9 \%$ of 100 live fish examined showed signs of GBT; at the conclusion of the experiment, $21 \%$ of the survivors had external signs of GBT. The mean delta $\mathrm{p}$ during the $33-\mathrm{d}$ experiment was $106 \mathrm{mmHg}$, remaining greater than the environmental threshold for bubble growth during the entire test except on day 19. Hyperbaric pressures were greater than the threshold for bubble growth in the cardiovascular system from the beginning of the test through day 10 and from day 26 to the end of the test, which corresponds to the times when almost all mortality was observed.

After completion of the October 1985 test, one-half of the rainbow trout fry from Rkm 8.0 were moved up to Rkm 0.6 and half were retained at $\mathrm{Rkm} 8.0$ as a control. Mortality was negligible at the control site (Figure 2.3.28). However, at Rkm 0.6 cumulative mortality reached $50 \%$ between days 12 and 15 and was nearly $75 \%$ by day 40. Seventy-four percent of the fish that died had external symptoms of GBT: no symptoms were observed at Rkm 8.0 .

Test fish were reared at a mean delta $\mathrm{P}$ of $106 \mathrm{mmHg}$. During the test the mean delta $P$ at $R \mathrm{~km} 0.6$ was $91 \mathrm{mmHg}$, and $58 \mathrm{mmHg}$ at Rkm 8.0. The delta P's measured at $\mathrm{Rkm} 8.0$ never rose above 


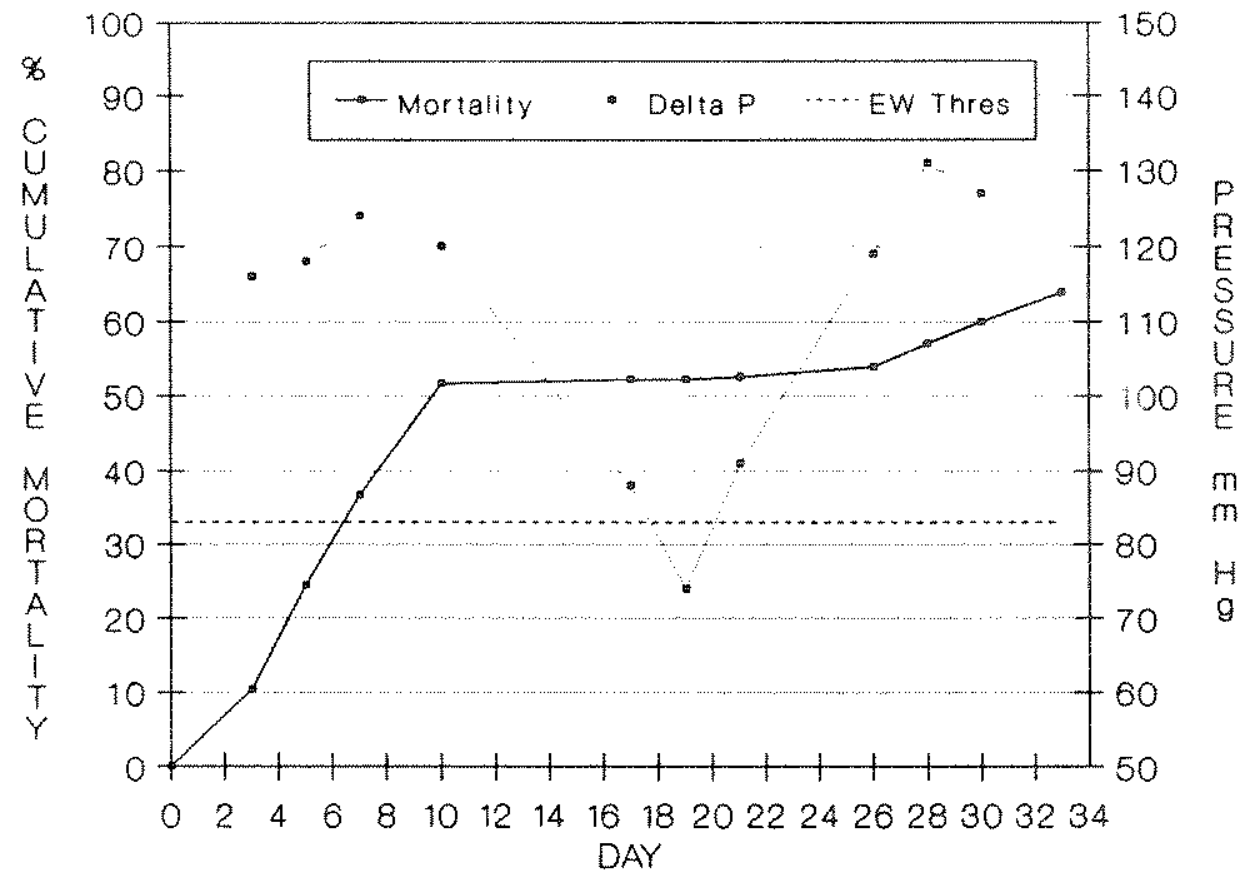

Figure 2.3.27. The environmental water (EW) threshold for bubble growth and delta $P$ ( $\mathrm{mmHg}$ ) at $R \mathrm{~km} 0.6$, and cumulative percent mortality among rainbow trout fry reared at $R \mathrm{~km} 14.5$ and noved to $0.6 \mathrm{~km}$ below Afterbay Dam on the Bighorn River, October November 1985.

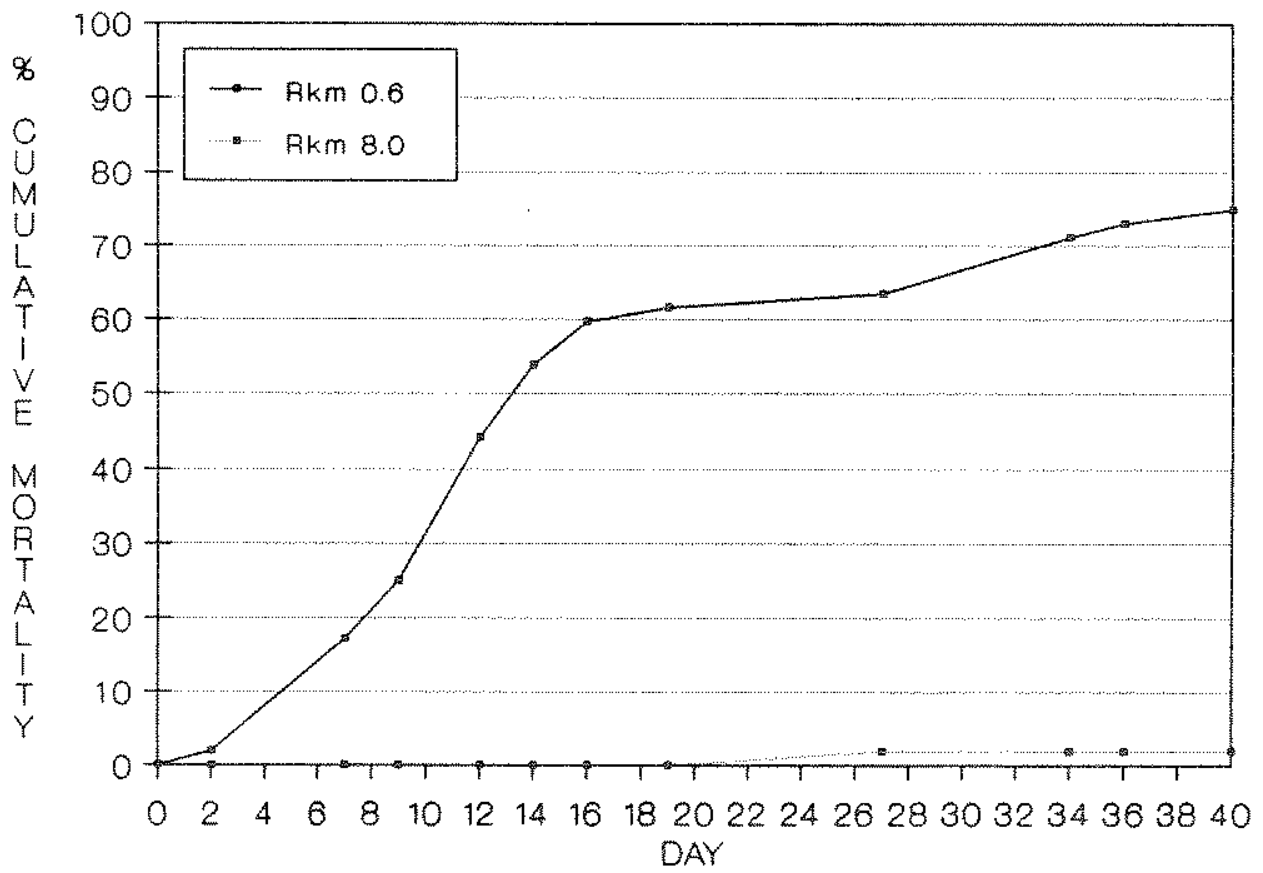

Figure 2.3.28. Percent cumulative mortality by day among rainbow trout fry reared at $\mathrm{Rkm} 8.0$ and held at Rkm 0.6 and 8.0 on the Bighorn River below Afterbay Dam, November - December 1985. 
the environmental water threshold for bubble growth (Figure 2.3.29), so we would not expect mortality from GBT at the site. At $\mathrm{Rkm} 0.6$, however, delta $\mathrm{P}^{\prime} \mathrm{s}$ were greater than the envirommental threshold for bubble growth during most of the test except from about day 16-32. As in the previous test, periods when gas pressures dropped below the environmental water threshold corresponded to a substantial decline in daily mortality. Delta $\mathrm{P}^{\mathrm{s}}$ measured at $\mathrm{Rkm} 0.6$ during the test were greater than the threshold for bubble growth in the cardiovascular system on only two occasions, days 0 and 40. Cumulative mortality at Rkm 0.6 was greater during this test than among fish reared at $\mathrm{Rkm} 14.5$ and moved to $\mathrm{Rkm} 0.6$, even though hyperbaric pressures were lower. Since mean delta P's measured during rainbow trout fry rearing at the two downstream sites were similar, previous exposure to elevated gas levels and any accompanying changes in susceptibility should not have caused the observed mortality. Compensating pressures, not measured during these tests, may have influenced the results. Nevertheless, this experiment demonstrates that substantial fry mortality can occur while gas pressures are below the threshold for bubble growth in the cardiovascular system.

On 3 January 1986 one-half of the rainbow trout fry at $\mathrm{Rkm}$ 2.4 were transported upstream to $\mathrm{Rkm} 0.6$. The mean delta $\mathrm{P}$ during rearing at Rkm 2.4 was $104 \mathrm{mmHg}$. The mean delta $\mathrm{P}^{\prime} \mathrm{s}$ during the tests at $\mathrm{Rkm} 0.6$ and 2.4 were 100 and $88 \mathrm{mmHg}$, respectively. Mortality remained low at Rkm 0.6 until increasing sharply after day 13 (Figure 2.3.30). The largest increase in mortality at $\mathrm{R} k \mathrm{~m} 2.4$ coincided with the period of maximum gas pressure 


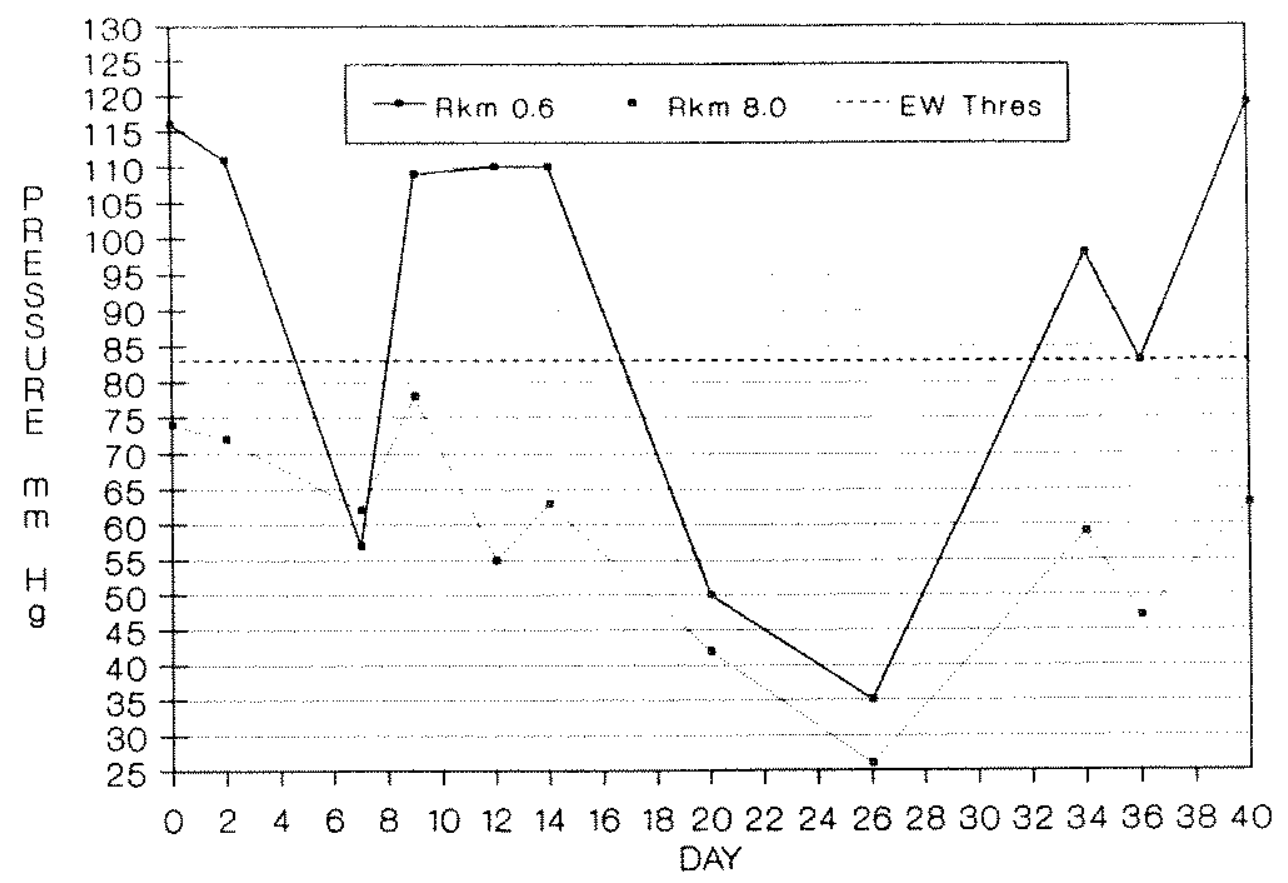

Figure 2.3.29. The environmental water threshold for bubble growth and delta P's (mmHg) measured at Rkm 0.6 and 8.0 below Afterbay Dam on the Bighorn River, Montana, during rainbow trout survival tests, November - December 1985.

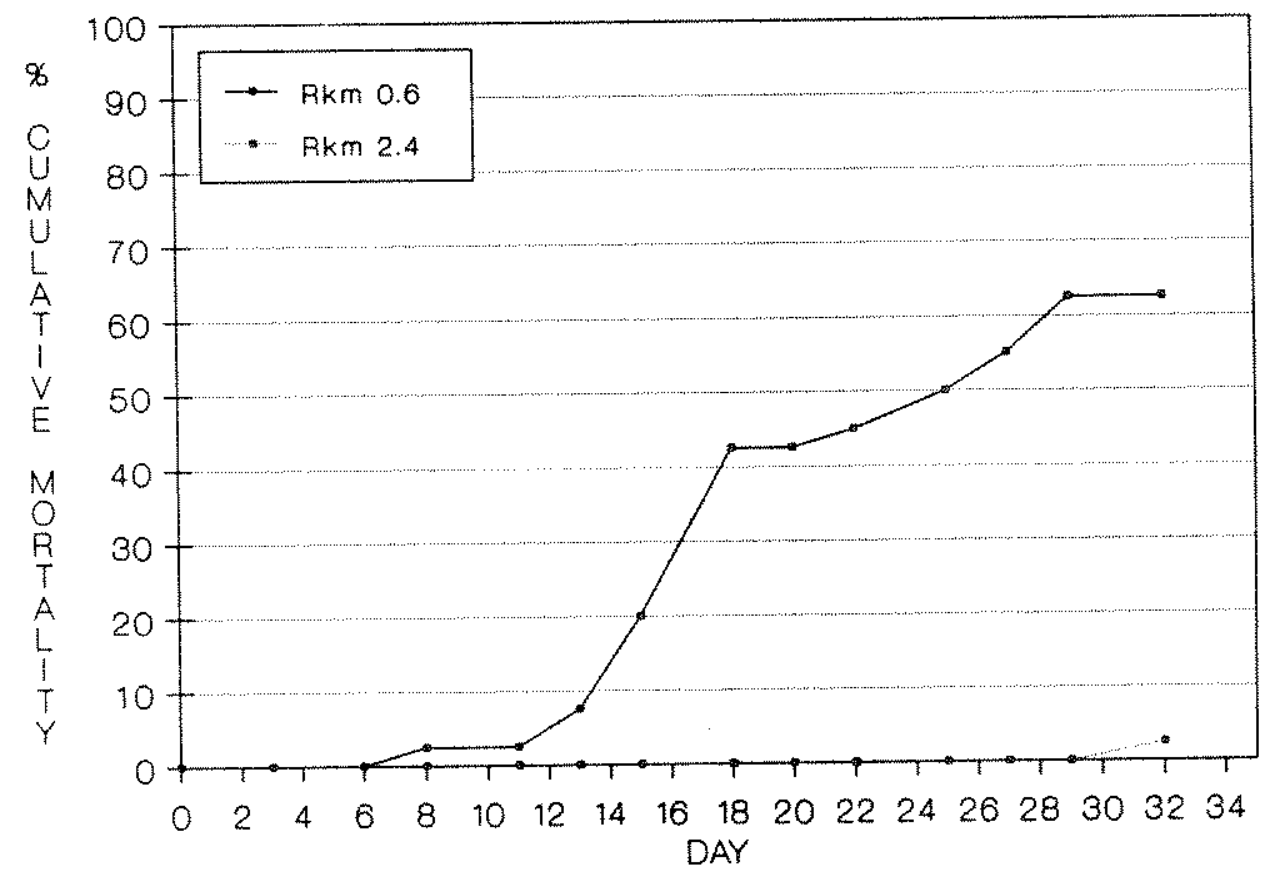

Figure 2.3.30. Percent cumulative mortality by day among rainbow trout fry reared at $R \mathrm{~km} 2.4$ and held at $R \mathrm{~km} 0.6$ and 2.4 on the Bighorn River below Afterbay Dam, January - February 1986. 
(Figure 2.3.31). Mortality of control fish at Rkm 2.4 was negligible and no external GBT symptoms were visible. Seventytwo percent of the fish that died at Rkm 0.6 exhibited external symptoms of GBT. Cumulative mortality of fish at this site reached $50 \%$ on day 25. Mean length of fry from both sites was similar at the end of the experiment, ranging from $71-73 \mathrm{~mm}$.

Although hyperbaric pressures were greater at $\mathrm{Rkm} 0.6$ than at Rkm 2.4, both sites had gas levels above the threshold for bubble growth in environmental water during much of the test. only gas levels at Rkm 0.6 exceeded the threshold level allowing bubble growth in the cardiovascular system and this occurred only once, on day 15.

Gas saturation at $\operatorname{Rkm} 0.6$ was sufficient to cause substantial mortality among rainbow trout fry. The bioassay using rish from Rkm 8.0 showed mortality was about $73 \%$ higher at Rkm 0.6 than at Rkm 8.0 when the mean delta $p$ was $33 \mathrm{mmHg}$ greater at the upstream site. However, comparison of the tests using rainbow trout fry incubated and reared at $\mathrm{Rkm} 2.4$ and 8.0 produced unexpected results. Fry moved upstream from Rkm 2.4 had a Lower cumulative mortality (62\%) than those moved from Rkm 8.0 (74\%). Hydrostatic pressure, not measured in 1985, may have contributed to the differences in survival. Also previous exposure to high delta p's may have altered one or both groups susceptability to elevated gas pressures. However, mean delta $\mathrm{P}^{\mathrm{S}}(104$ at $\mathrm{Rkm} 2.4$ and 106 at $\mathrm{Rkm} 8.0)$ during rearing at the two sites indicate little difference in prior exposure. To better relate mortality to gas pressures and to determine if the predictions of bubble threshold equations were consistent with 


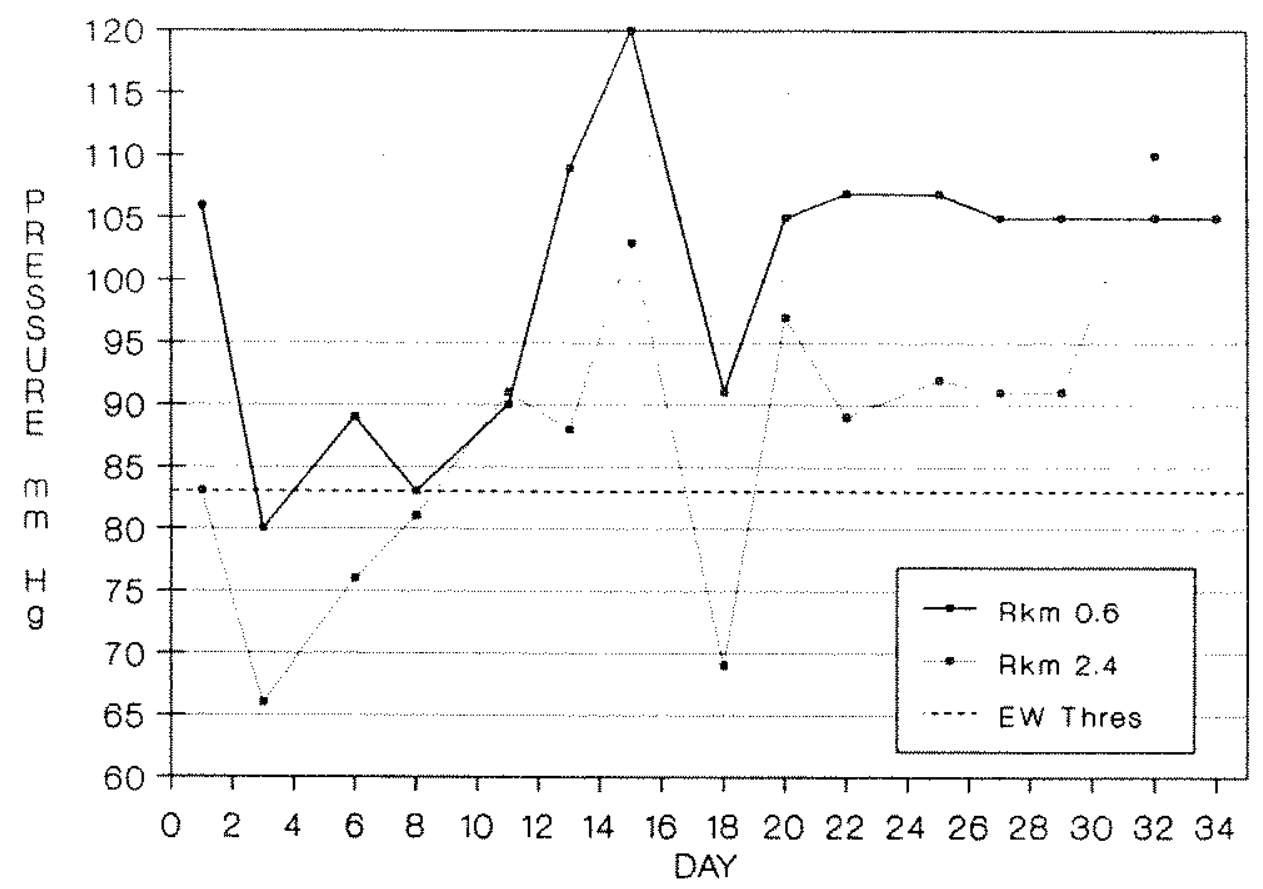

Figure 2.3.31. The environmental water threshold for bubble growth and delta P's (mmHg) measured at Rkm 0.6 and 2.4 below Afterbay Dam on the Bighorn River, Montana, during rainbow trout survival tests, January - February 1986.

observed mortality, we collected additional information in 1987 and 1988 .

In 1987, a control group of brown trout fry was incubated and reared in non-supersaturated water. On $3 \mathrm{March}$, one-half of the fry were transported to Rkm 0.6 on the Bighorn River. Fry ranged in size from 20-26 mm TI. Mortality in both groups remained low throughout the 59-a test (Figure 2.3.32). No mortality attributable to GBT was observed in the control group. Mortality first occurred among fry at Rkm 0.6 on day 18 and reached $5.9 \%$ by the end of the experiment. Maximum water depth was monitored in the holding box at Rkm 0.6 beginning on day 17 , which allowed calculation of bubble growth thresholds (Figure 2.3.33). The mean delta $\mathrm{P}$ at $\mathrm{Rkm} 0.6$ during the test was $111 \mathrm{mmHg}$, and gas 


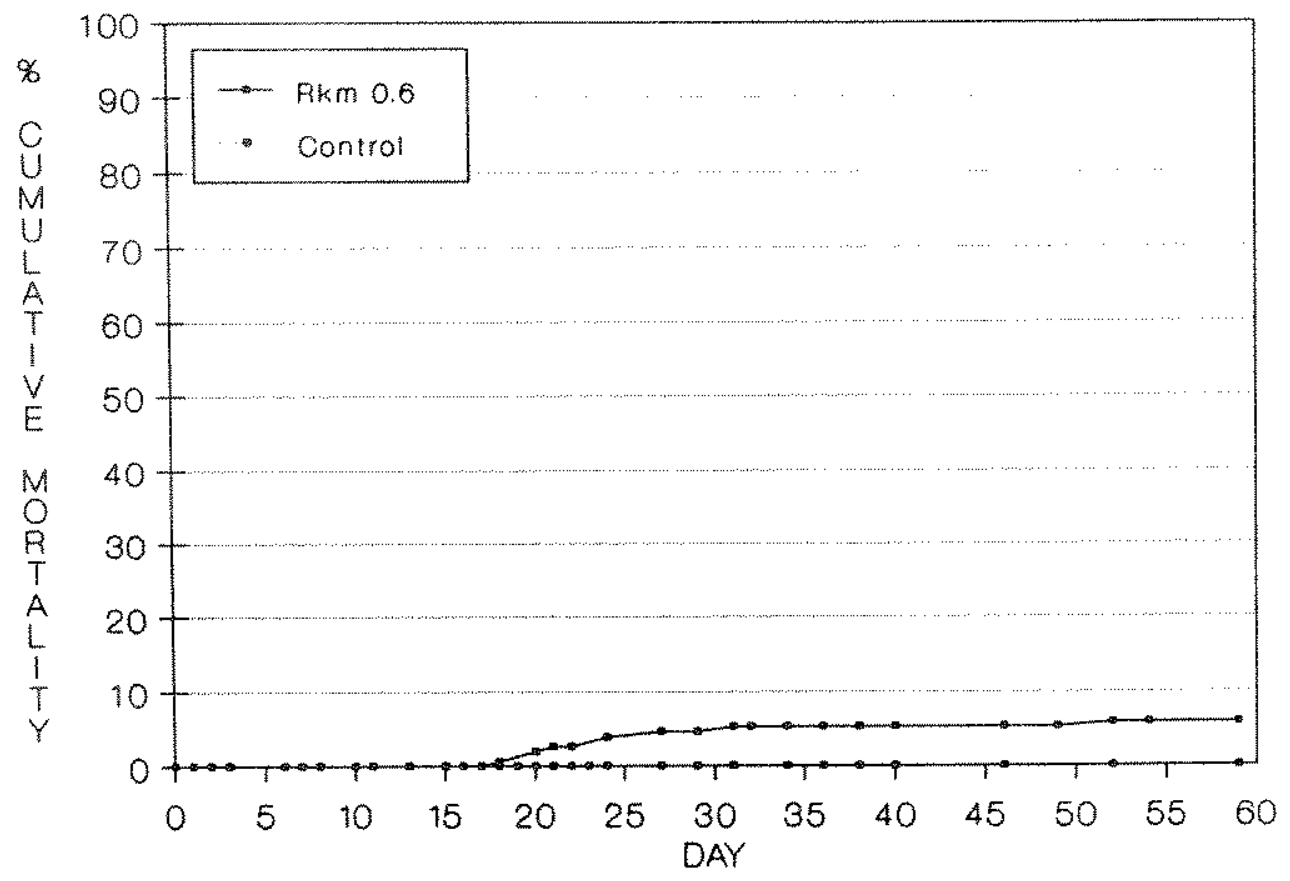

Figure 2.3.32. Percent cumulative mortality by day of brown trout fry reared in non-supersaturated water (control) and those held at Rkm 0.6 (test) on the Bighorn River below Afterbay Dam, March - April 1987.

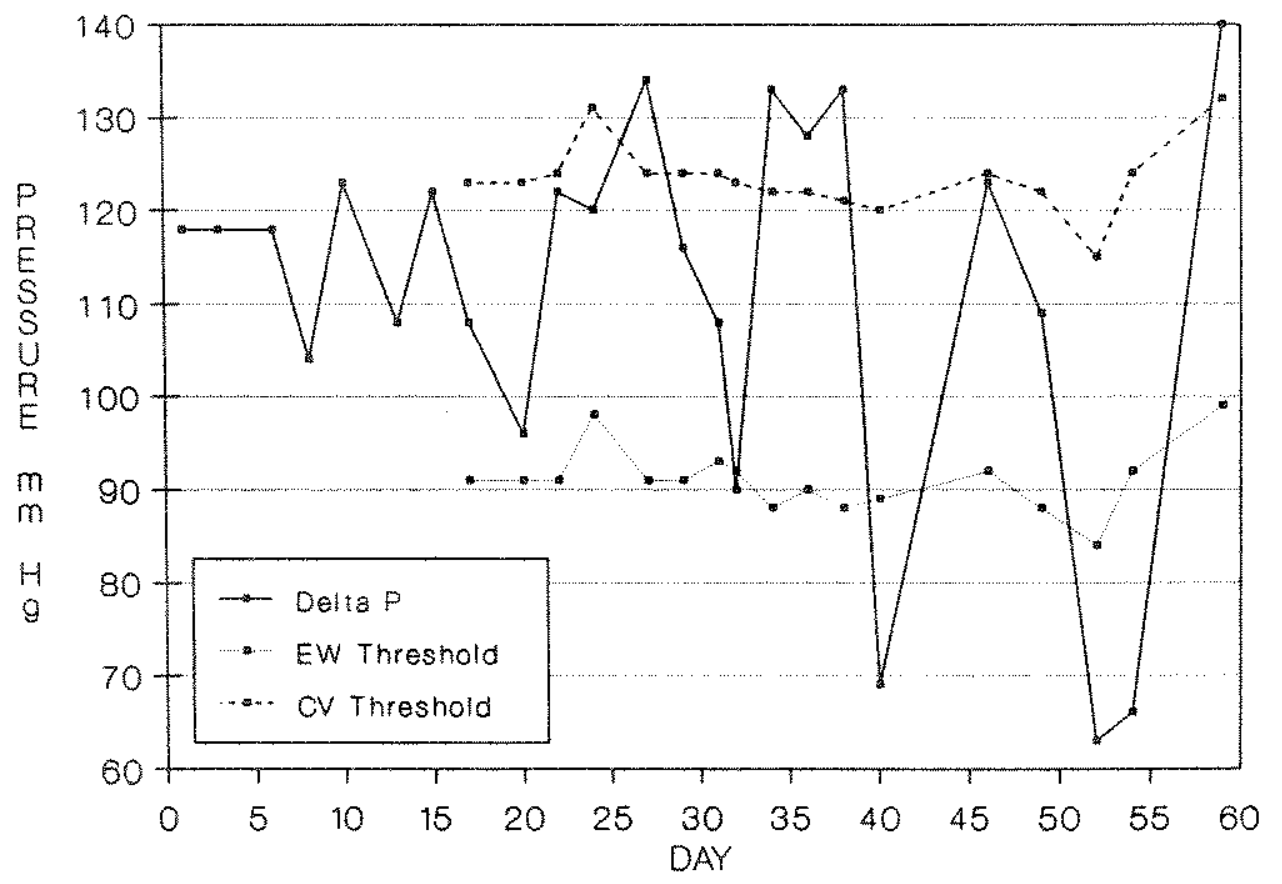

Figure 2.3.33. Environmental water (EW) and cardiovascular (CV) thresholds for bubble growth and delta pis (mmHg) measured at Rkm 0.6 below Afterbay Dam on the Bighorn River, Montana, during brown trout fry survival tests, March - April 1987. 
pressures were above the threshold for bubble growth in environmental water except on $4 \mathrm{~d}$. Delta $\mathrm{P}^{\prime} \mathrm{s}$ at $\mathrm{Rkm} 0.6$ exceeded the threshold for bubble growth in the cardiovascular system on days 27, 34-38, and 59. External GBT symptoms observed on mortalities included bubbles in the buccal cavity and popeye (exophthalmia).

In late March we moved 309 (32.8\%) brown trout fry hatched at $\mathrm{Rkm} 2.4$ to $\mathrm{Rkm} 0.6$. Mortality at both sites remained low throughout most of the 80-d bioassay (Figure 2.3.34). Cumulative mortality at $\mathrm{Rkm} 2.4$ and $\mathrm{Rkm} 0.6$ reached $7.6 \%$ and $36.2 \%$, respectively. During the test, mean delta $\mathrm{P}$ at $\mathrm{Rkm} 2.4$ (120 $\mathrm{mmHg}$ ) was slightly higher than at Rkm 0.6 (119 $\mathrm{mmHg}$ ). However, because of deeper water, the mean thresholds for bubble growth in environmental water and the cardiovascular system at Rkm 2.4 were higher (Figure 2.3.35). Eighty-six and $46 \%$ of the delta $p$ measurements at $\mathrm{Rkm} 2.4$ were greater than the environmental water and cardiovascular thresholds, respectively. Delta $\mathrm{P}$ at Rkm 0.6 was above the environmental water and cardiovascular thresholds $87 \%$ and $57 \%$ of the time, respectively (Figure 2.3.35). Most mortality occurred during the last $30 \mathrm{~d}$ of the test when gas pressures were always above the cardiovascular threshold and high, relatively constant delta $P^{\prime}$ s occurred (Figure 2.3.35). External symptoms of GBT were similar between sites, as was average size at death $(24 \mathrm{~mm})$. The most common GBT symptom was a ventral surface bubble which occurred on 54 and $58 \%$ of the mortalities at $\mathrm{Rkm} 2.4$ and 0.6 , respectively. At these same field sites, respectively, other symptoms included bubbles in the buccal cavity (26 and $30.6 \%)$, popeye (18 and $10.8 \%$ ), and external 


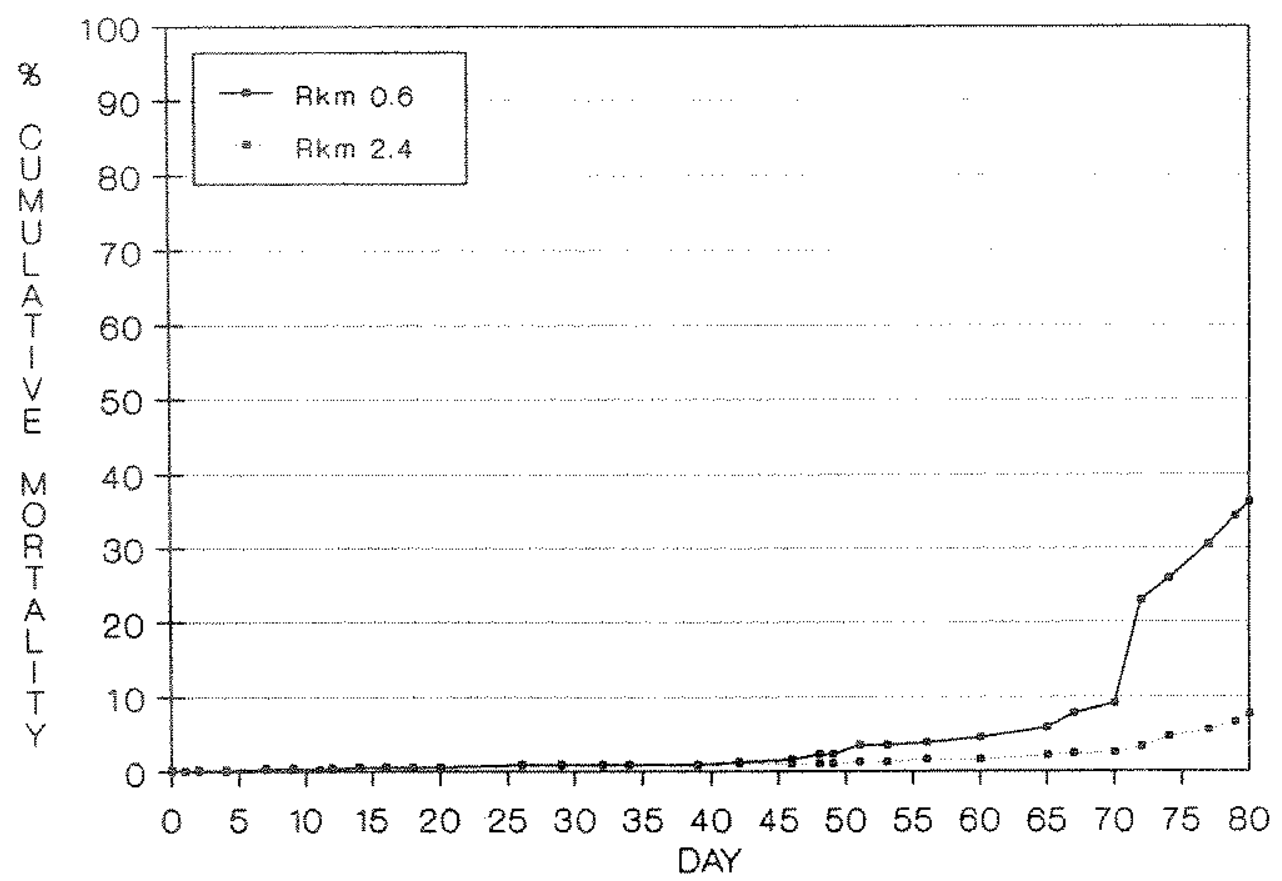

Figure 2.3.34. Percent cumulative mortality by day of brown trout fry reared at Rkm 2.4 and held at $\mathrm{Rkm} 0.6$ and 2.4 on the Bighorn River below Afterbay Dam, March - June, 1987.

bubbles $(2.0$ and $0.65 \%)$.

A second field bioassay (47 d) was conducted at Rkm 0.6 and 2. 4 during June and July using brown trout fry reared at Rkm 2.4. Mortality rates were much higher at both sites (Figure 2.3.36). Mortality at Rkm 0.6 rose to $72 \%$ by day 17 and then leveled off at about $80 \%$ by day 30 . Total fry mortality (30\%), as well as mortality rate, was much less at Rkm 2.4. Examination of hyperbaric pressures provides a reasonable explanation for the higher mortality during this test (Figure 2.3.37) but not the large difference in mortality between sites (Figure 2.3.36). Delta $p$ remained above the threshold for bubble growth in the cardiovascular system at both locations for all but one of the first 35 d. Delta p remained above the threshold for bubble growth in environmental water at both locations throughout the 

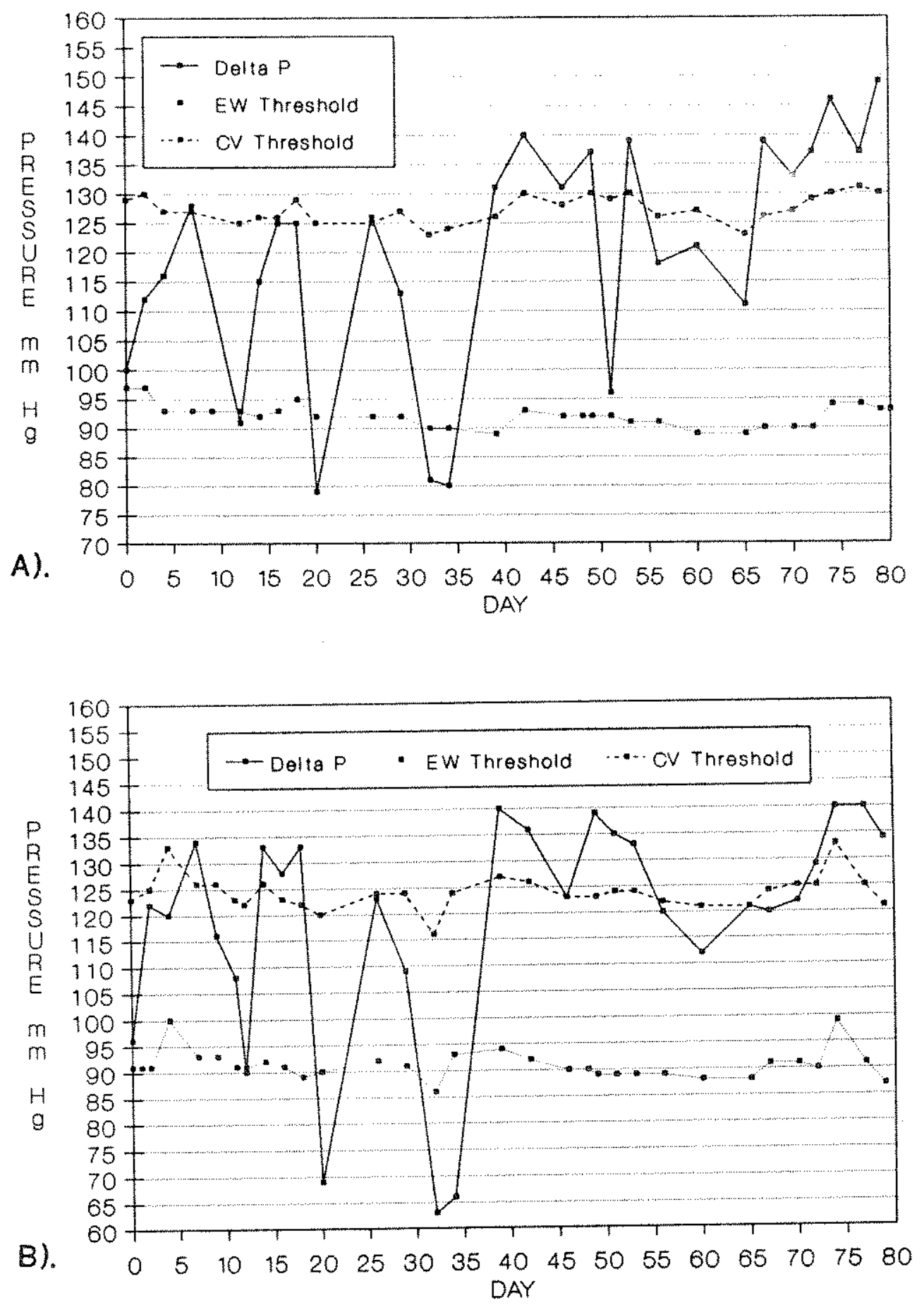

Figure 2.3.35. Environmental water (EW) and cardiovascular (CV) thresholds for bubble growth and delta $\mathrm{P}^{\prime \prime} \mathrm{s}$ (mmHg) measured at Rkm 2.4 (A) and 0.6 (B) below Afterbay Dam on the Bighorn River, Montana, during brown trout fry survival tests, March - June 1987. 


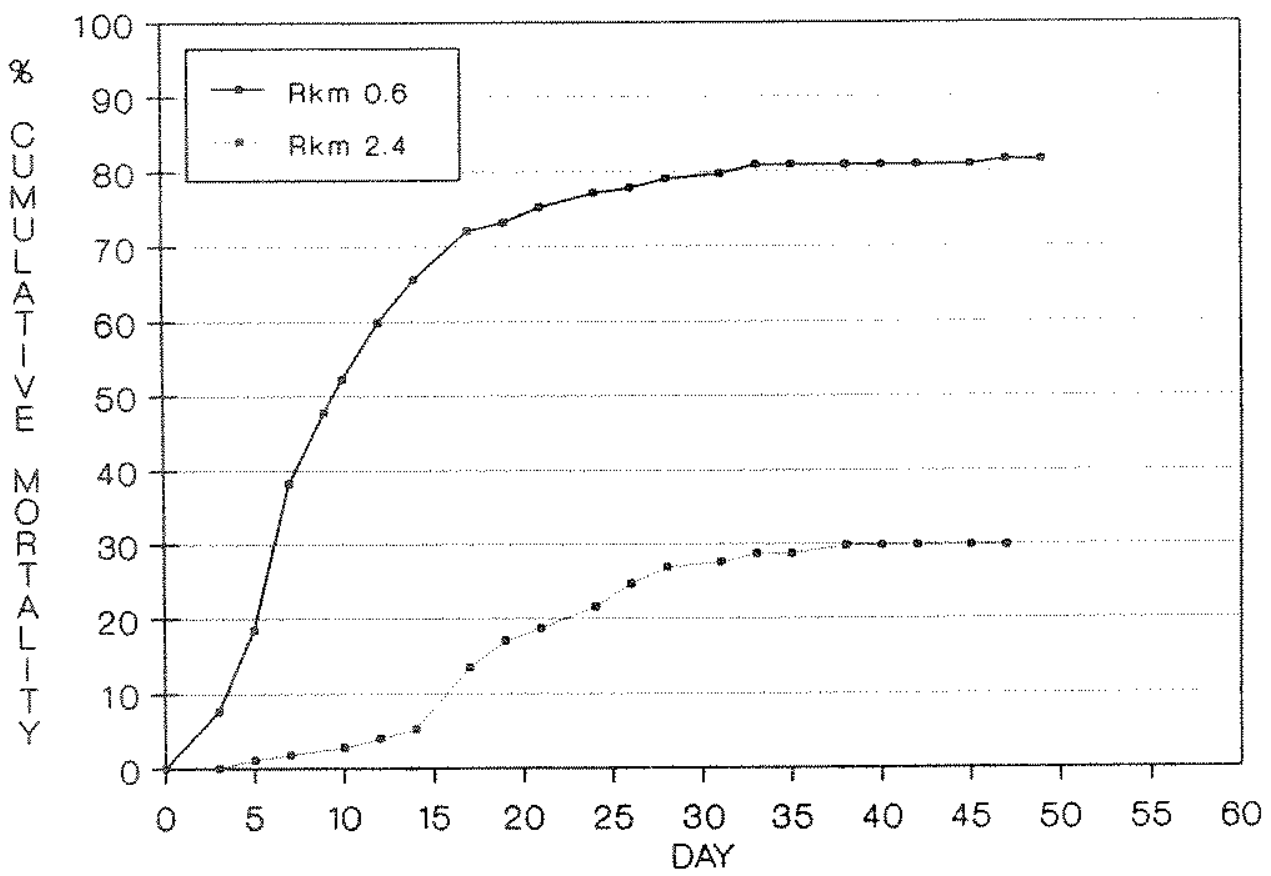

Figure $2.3,36$. percent cumulative mortality by day of brown trout fry reared at $\mathrm{Rkm} 2.4$ and held at $\mathrm{Rkm} 0.6$ and 2.4 on the Bighorn River below Afterbay Dam, June - July 1987.

entire experiment except for day 35 (Figure 2.3.37). The mean delta $\mathrm{P}^{\mathrm{s}}$ during the experiment were 138 and $130 \mathrm{~mm} g$, and mean cardiovascular thresholds were 132 and $124 \mathrm{mmHg}$ at Rkm 2.4 and 0.6, respectively.

More than four times more GBT symptoms (269) were observed among fxy mortalities at Rkm 0.6 than at Rkm 2.4 (64). Although a ventral bubble was still the most common symptom at both sites, other symptons such as popeye and buccal cavity bubbles were more prevalent than in the earlier test. Bubbles on the ventral sureace and in the buccal cavity, and popeye were found on 37.6 , 28.6, and 33.1\%, respectively, of the mortalities at Rkm 0.6. These same symptoms were seen on $54.7,12.5$, and $31.2 \%$ of the mortalities at Rkm 2.4. Also, internal hemorrhaging was observed on one pry. 

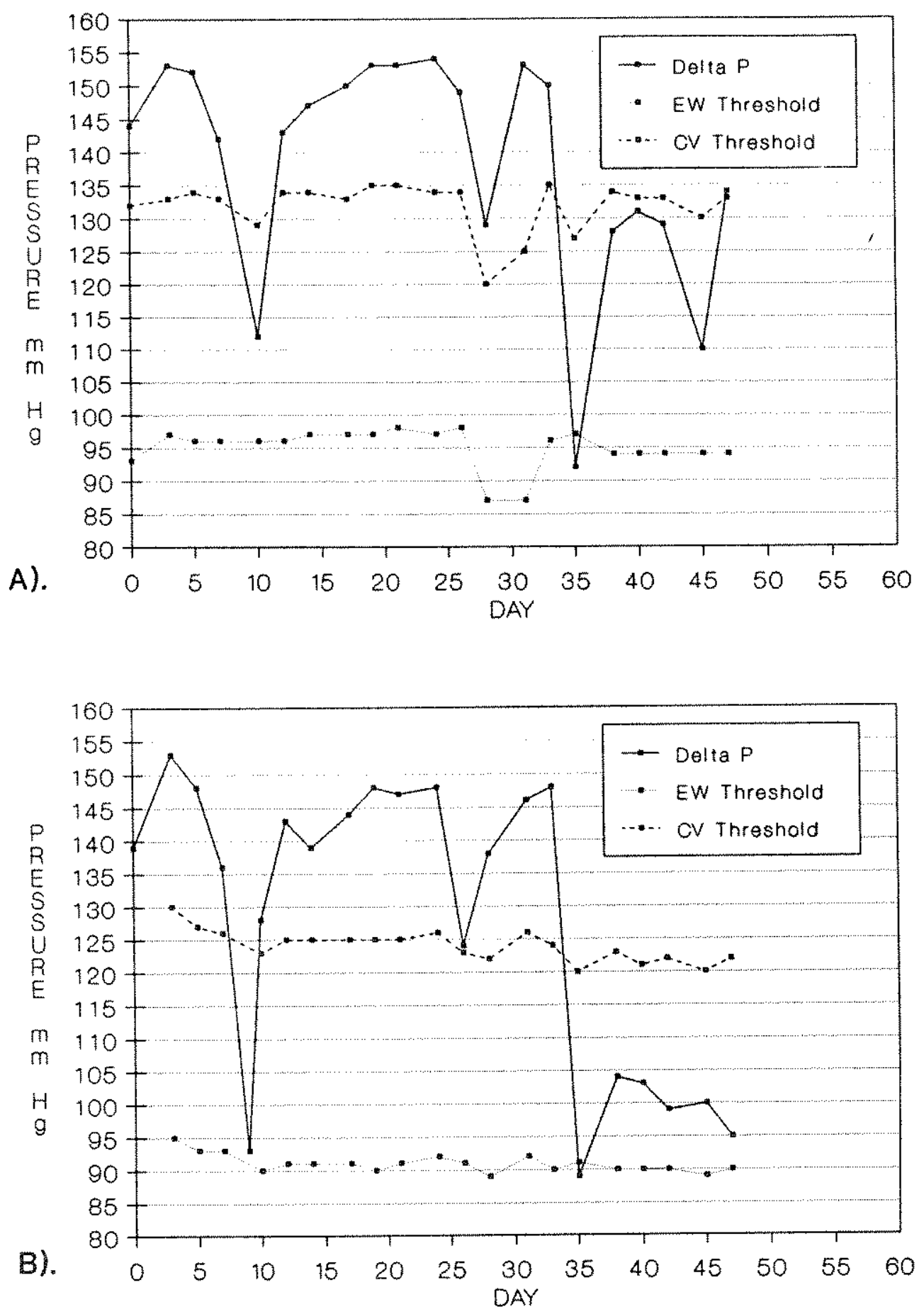

Figure 2.3.37. Environmental water (EW) and cardiovascular (CV) thresholds for bubble growth and delta $\mathrm{P}^{\prime} \mathrm{s}$ (mmHg) measured at Rkm 2.4 (A) and 0.6 (B) below Afterbay Dam on the Bighorn River, Montana during brown trout fry tests, June - July 1987. 
Simultaneous with survival tests using fry incubated at Rkm 2.4 , brown trout fry incubated at $\mathrm{Rkm} 8.0$ were moved to $\mathrm{Rkm} 0.6$. Mortality patterns at Rkm 0.6 and 8.0 from March to June were similar to that observed for the Rkm 2.4 fry (Figures 2.3.34 and 2.3.38). Mortality began to slowly increase after day 45; the largest increases at both $\mathrm{Rkm} 8.0$ and 0.6 occurred after day 65 . Although the mean delta $P$ during the experiment at both Rkm 8.0 and 0.6 was $119 \mathrm{mmHg}$, the greater mortality at Rkm 0.6 appears due to lower threshold levels. The mean threshold for bubble growth in environmental water and the cardiovascular system was 93 and $134 \mathrm{mmHg}$, respectively, at $\mathrm{Rkm} 8.0$, while at $\mathrm{Rkm} 0.6$ the respective thresholds were 91 and $124 \mathrm{mmHg}$. Delta $\mathrm{P}$ was greater than the environmental water threshold during 93 and $86 \%$ of the measurements at Rkm 8.0 and Rkm 0.6 , respectively (Figure 2.3.39). However, the situation was reversed for the threshold for bubble growth in the cardiovascular system. Delta p's were above the cardiovascular threshold 18 and $48 \%$ of the time at Rkm 8.0 and Rkm 0.6 , respectively. Also, when the cardiovascular threshold was exceeded at $\mathrm{kkm} 0.6$, it was exceeded for longer periods than at Rkm 8.0 (Figure 2.3.39). At both sites, the mean length of fry that died was $23 \mathrm{~mm}$. Ventral bubbles were the most common symptom of GBT, followed by buccal cavity bubbles and popeye.

The second experiment in 1987 involving brown trout fry hatched and reared at $\mathrm{Rkm} 8.0$ and moved to $\mathrm{kkm} 0.6$ was conducted from June - August. Results were substantially different than observed during the corresponding Rkm 2.4 test (Figure 2.3.40). Mortality and mortality pattern were nearly identical at Rkm 8.0 


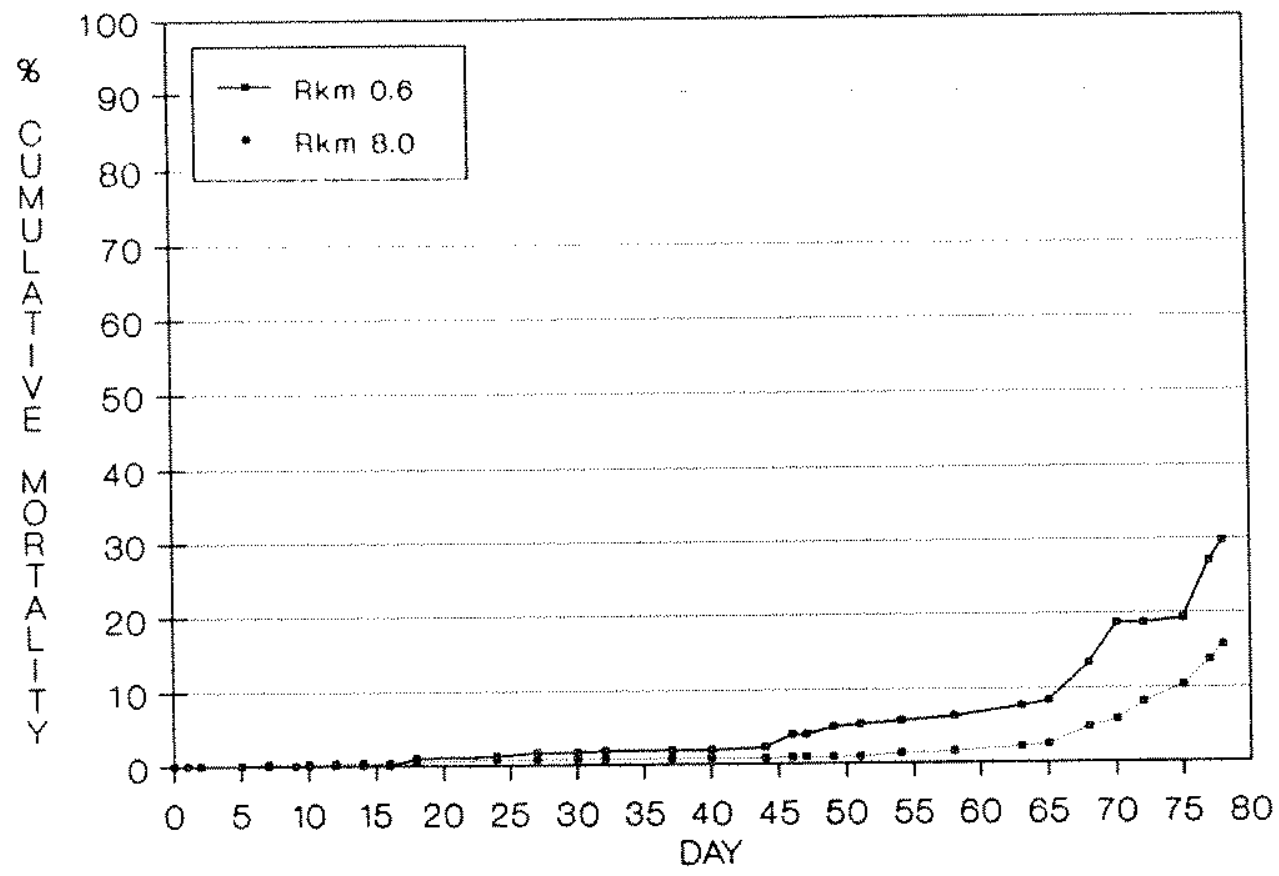

Figure 2.3.38. Percent cumulative mortality by day of brown trout fry reared at $\mathrm{Rkm} 8.0$ and held at $\mathrm{Rkm} 0.6$ and 8.0 on the Bighorn River below Afterbay Dam, March - June 1987.

and 0.6 . Mortality climbed quickly, leveling off at approximately $50 \%$ between days 25 and 30 . The fry test at Rkm 8.0 was discontinued on day 48 , while the Rkm 0.6 test was continued through day 80 . Cumulative mortality at $\mathrm{Rkm} 0.6$ remained at $51.4 \%$ from day 31 to 63 , then increased to $61.5 \%$ by day 80 . Unlike mortality rates, hyperbaric pressures at Rkm 8.0 and 0.6 were quite different (Figure 2.3 .41 ). The mean delta $\mathrm{P}$ measured at $\mathrm{R} \mathrm{km}$ 8.0 and $\mathrm{Rkm} 0.6$ was $138 \mathrm{mmHg}$ (range $=79-177$ ) and $129 \mathrm{mmHg}$ (range =89-153), respectively. The mean threshold for bubble growth in environmental water at these respective sites was 95 and $92 \mathrm{~mm} H$. Delta $P$ at both sites was above the environmental water threshold 95: of the time from day 0 to 48. The mean cardiovascular threshold level at $\mathrm{Rkm} 8.0$ (141 $\mathrm{mmHg}$ ) was also higher than at Rkm 

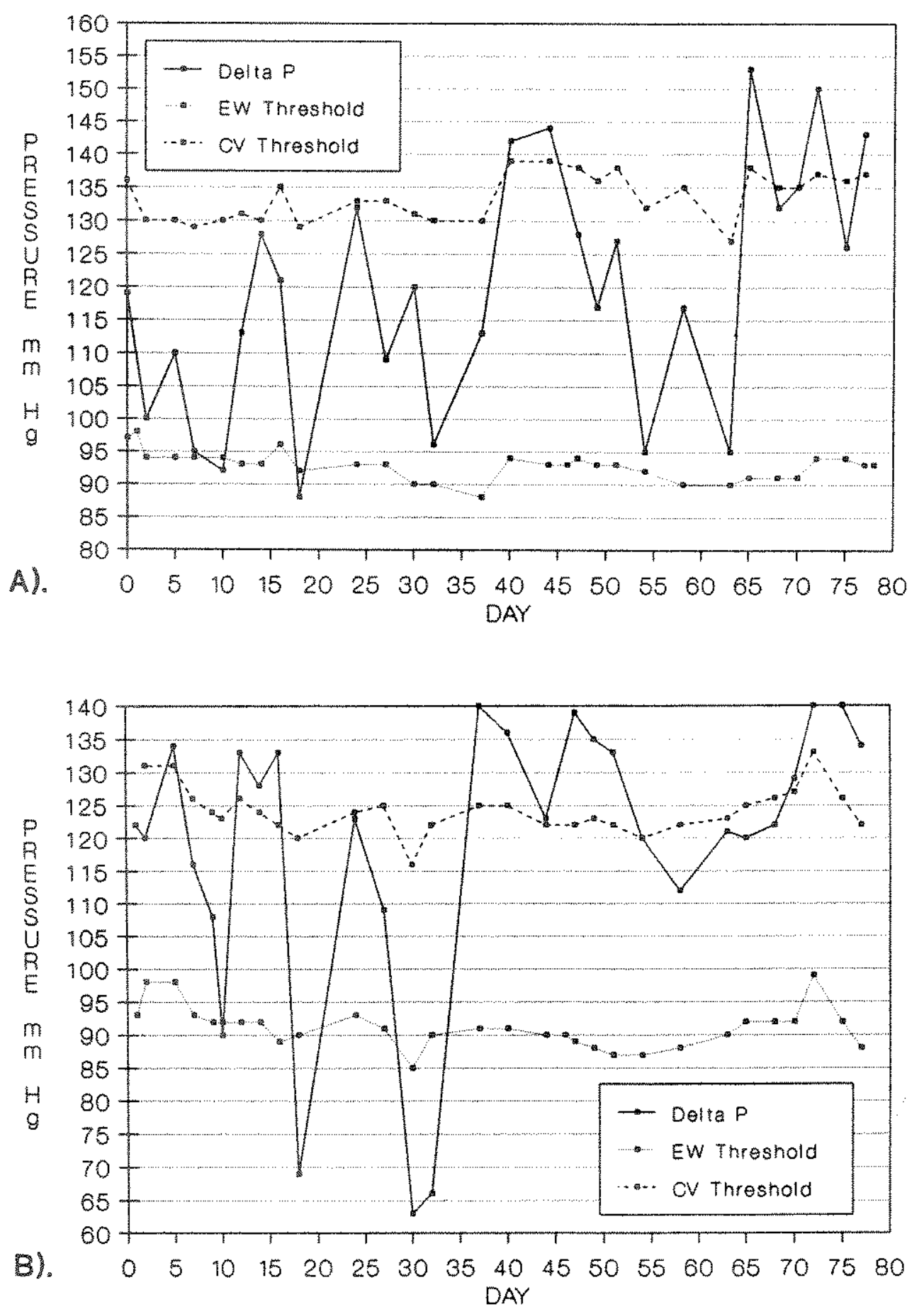

Figure 2.3.39. Environmental water (EW) and cardiovascular (CV) thresholds for bubble growth and delta P's (mmHg) measured at Rkm 8.0 (A) and 0.6 (B) below Afterbay Dam on the Bighorn River, Montana, during brown trout fry survival tests, March - June 1987. 


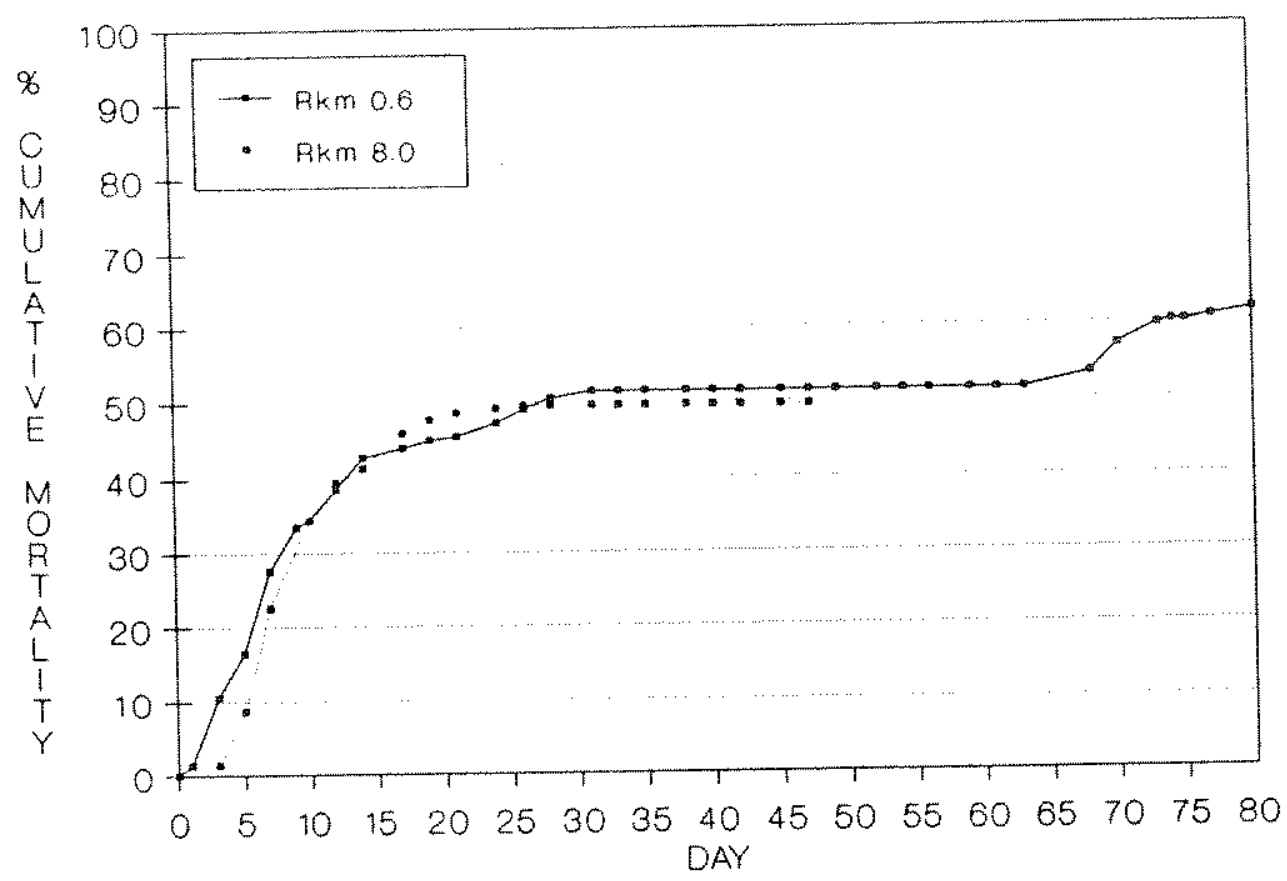

Figure 2.3.40. Percent cumulative mortality by day of brown trout fry reared at $\mathrm{Rkm} 8.0$ and held at $\mathrm{Rkm} 0.6$ and 8.0 on the Bighorn River below Afterbay Dam, June - August 1987.

0.6 (125 $\mathrm{mmHg})$. However, individual delta $p$ measurements were greater than the cardiovascular system threshold $55 \%$ of the time at $\mathrm{Rkm} 8.0$ compared to $67 \%$ at $\mathrm{Rkm} 0.6$. Delta $\mathrm{P}$ at $\mathrm{Rkm} 8.0$ fluctuated widely but did not rise much above the cardiovascular threshold until near the end of the bioassay. In contrast, delta $P$ at $R k m 0.6$ remained substantially higher than the cardiovascular threshold until day 35 (Figure 2.3.41). Some unmeasured factor apparently affected mortality at Rkm 8.0 since mortality was larger than would be expected based on threshold equations. The proportions of externally visible GBT symptoms were similar to those observed in other bioassays.

Brown trout fry mortality at $\mathrm{Rkm} 0.6$ after day 48 was low until day 68 (Figure 2.3 .40 ) then rose to $61.5 \%$. Increased mortality corresponded to a large increase in hyperbaric 

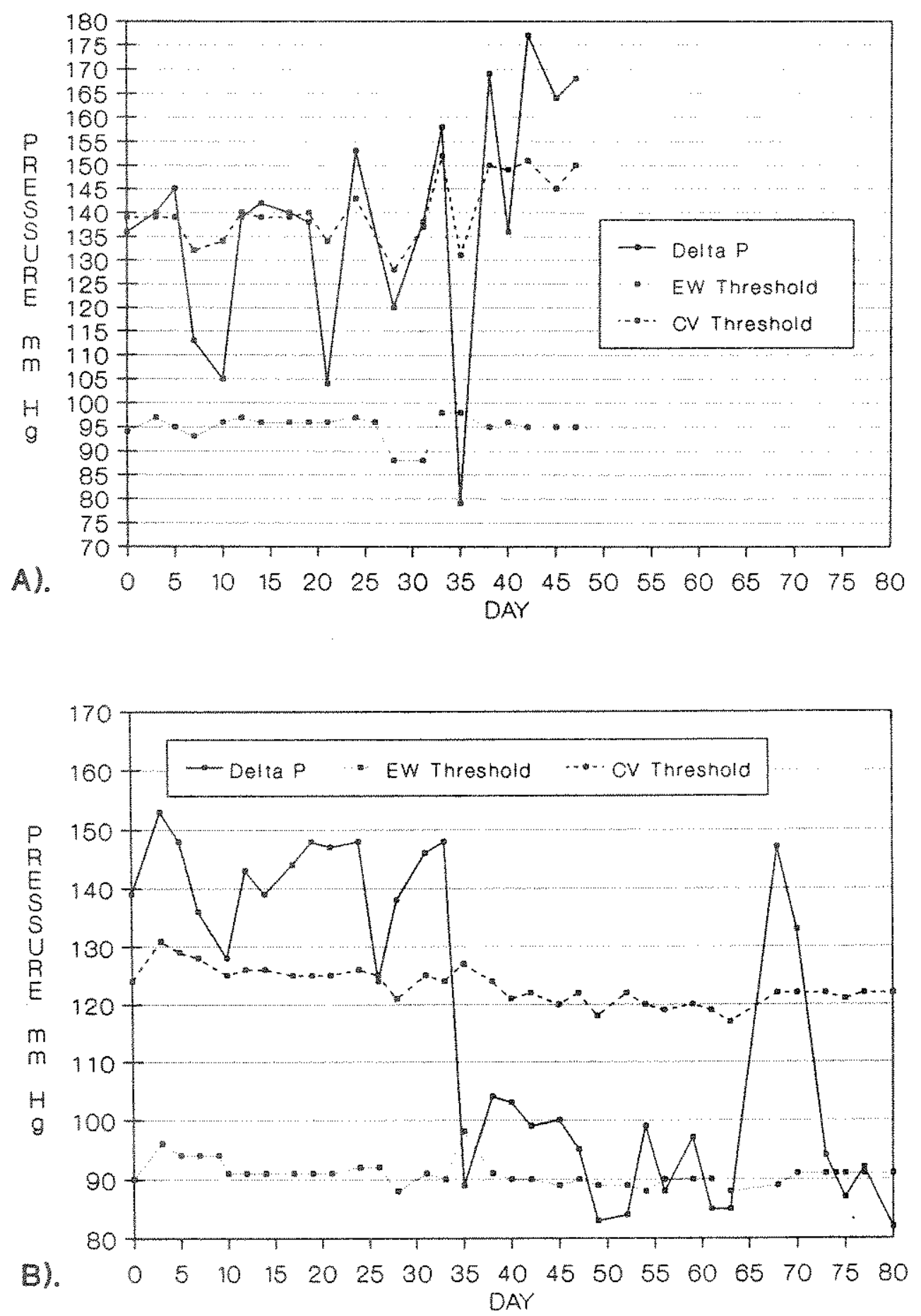

Figure 2.3.41. Environmental water (EW) and cardiovascular (CV) thresholds for bubble growth and delta $\mathrm{p}^{\prime} \mathrm{s}$ (mmHg) measured at Rkm 8.0 (A) and 0.6 (B) below Afterbay Dam on the Bighorn River, Montana, during brown trout fry survival tests, June - August 1987. 
pressures on day 68 (Figure 2.3 .41 ).

Another two bioassays were conducted during spring and summer 1987 using brown trout fry reared at Rkm 14.5 and tested there and at Rkm 0.6. The first experiment began on 10 April. Little mortality occurred until after day 35 at both $\mathrm{Rkm} 14.5$ and 0.6 (Figure 2.3.42). Brown trout fry were held at Rkm 14.5 through day 67 ; cumulative mortality at $\mathrm{Rkm} 14.5$ and 0.6 was equal on day 63 (Figure 2.3.42). Mortality at $\mathrm{Rkm} 0.6$ had reached $66.7 \%$ when the bioassay was terminated on day 74 . The increase in mortality at $\mathrm{Rkm} 14.5$ on day 48 corresponded to the greatest change in delta $\mathrm{P}$ observed at the site during the test. However, gas pressures were above the cardiovascular system threshold on three previous occasions (Figure 2.3.43). Substantial mortality unrelated to GBT occurred at $\mathrm{Rkm} 14.5$, making interpretation difficult. Mortality from other causes did not occur in brown trout fry at Rkm 0.6. However, mortality of the Rkm 14.5 fry moved to $\mathrm{Rkm} 0.6$ did not occur as early as would have been predicted based on gas pressures and threshold levels (Figure $2.3 .43)$. Symptoms of GBT at Rkm 0.6 were similar to those in previously described experiments; at Rkm 14.5, however, popeye was the most prevalent symptom.

Another bioassay using Rkm 14.5-reared brown trout fry was conducted June-october 1987. Less mortality occurred at both sites (Rkm 0.6 and $\mathrm{Rkm}$ 14.5) than during comparable tests with fry incubated upstream (Figures $2.3 .36,2.3 .40$ and 2.3.44). Fry were held at $\mathrm{Rkm} 14.5$ for $42 \mathrm{~d}$; at $\mathrm{Rkm} 0.6$ the test spanned 110 d. Fry mortality was nearly identical at the two sites and remained near $10 \%$ until the conclusion of the bioassay at Rkm 


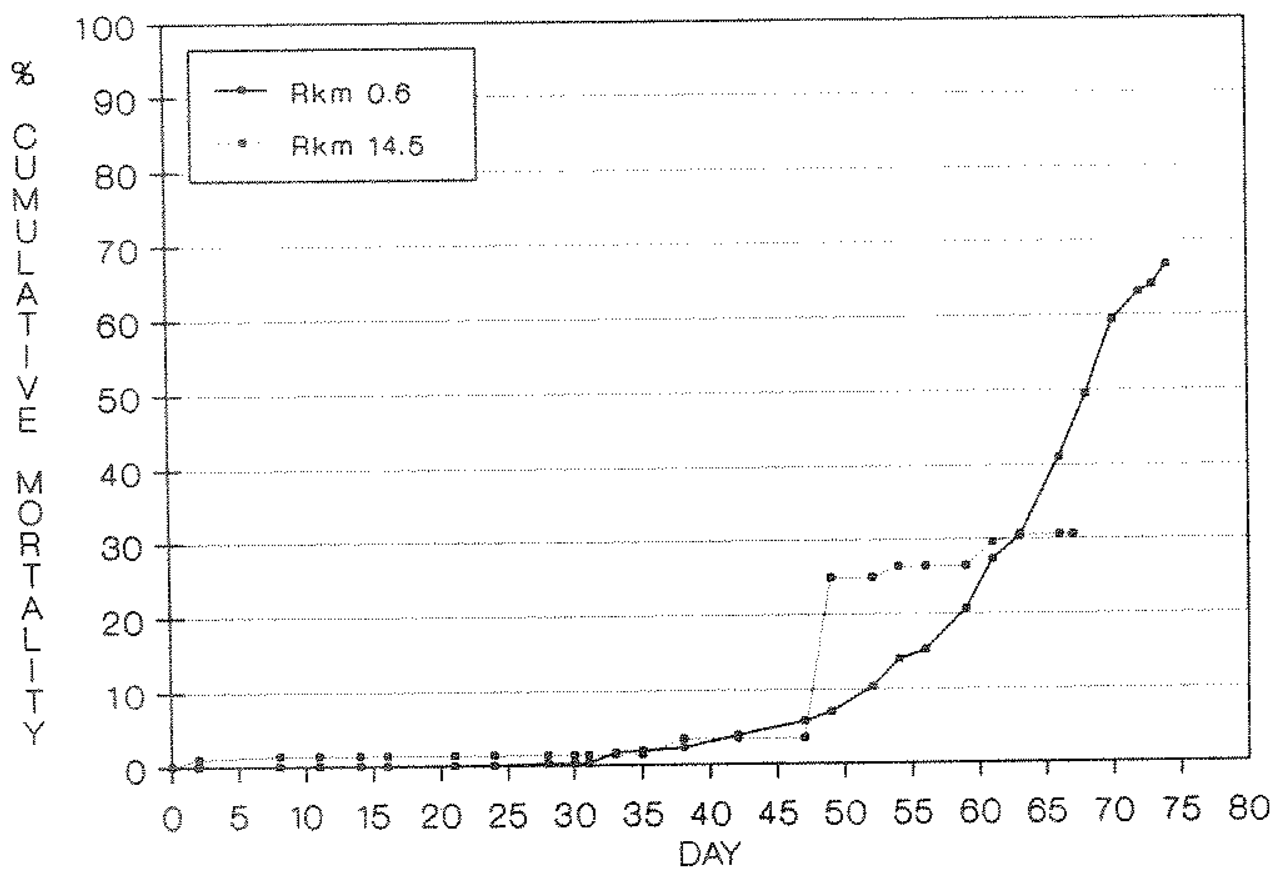

Figure 2.3.42. Percent cumulative mortality by day among brown trout fry reaxed at RMm 14.5 and held at Rkm 0.6 and 14.5 on the Bighorn River below Afterbay Dam, April - June 1987.

14.5. Mortality at Rm 0.6 increased to $35.3 \%$ between days 50 and 61 and remained chere through the end of the test.

Hyperbaric pressures were below the threshold for bubble growth in the cardiovascular system at Rkm 14.5 (days 1-8) when mortality occurred and were above the threshold during the period of no mortality (Figure 2.3 .44 and 2.3 .45 ). The large 1uctuations in gas pressures at $\mathrm{kmm} 14.5$ were not incorporated into the threshold graph and may have influenced survival. The only increase in delta $P$ at either site that coincided with an Increase in mortality was between days 51 and 56 at Rkm 0.6 (Figure 2.3 .45 ). Mean delta $p$ at both locations was lower than the mean cardiovascular threshold.

A comparison of cumulative brown trout fry mortality in the control sample, as well as among those moved upstream to Rkm 0.6 

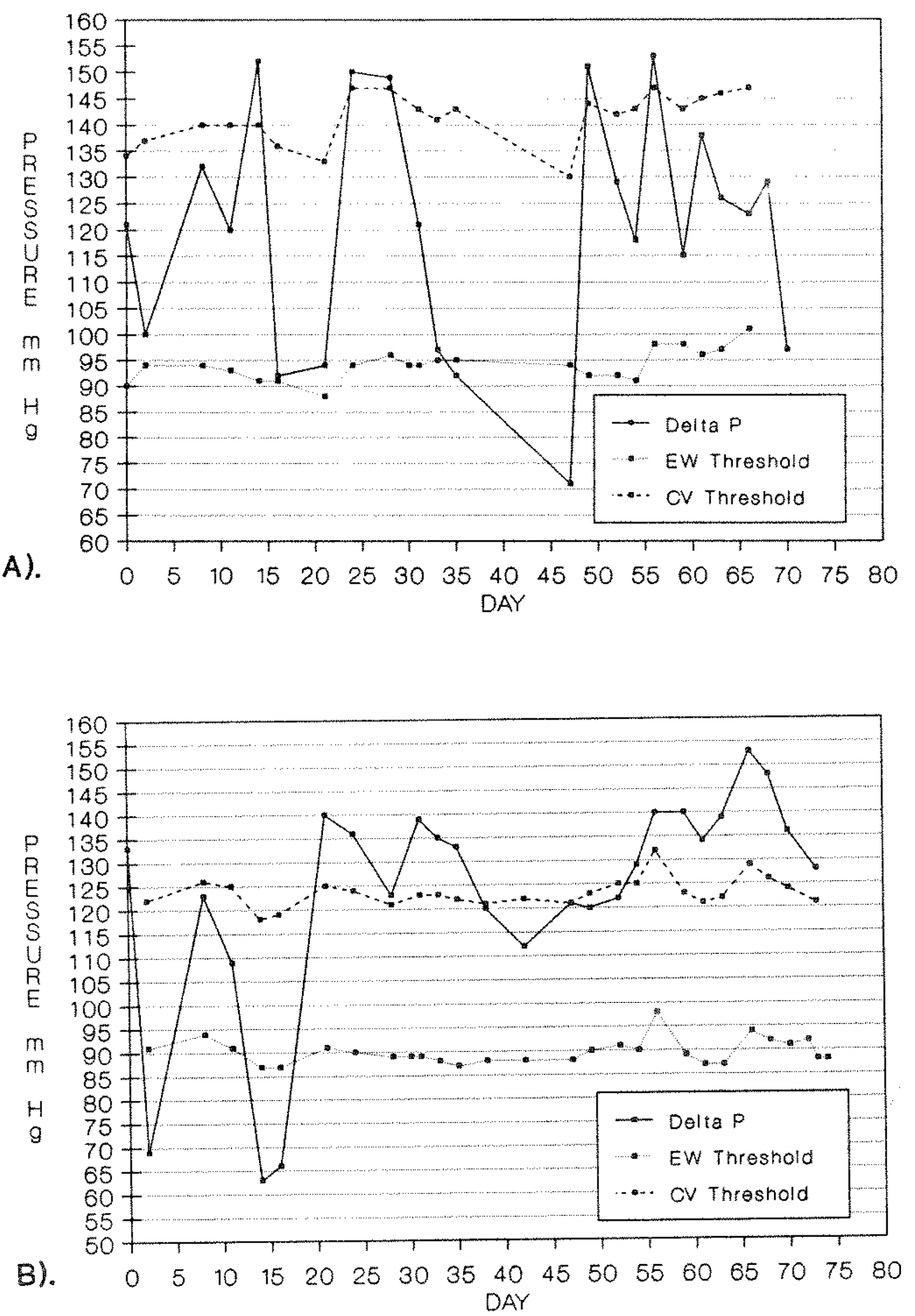

Figure 2.3.43. Environmental water (EW) and cardiovascular (CV) thresholds for bubble growth and delta P's (mmig) measured at Rkm 14.5 (A) and 0.6 (B) below Afterbay Dam on the Bighorn River, Montana, during brown trout fry survival tests, April - June 1987. 


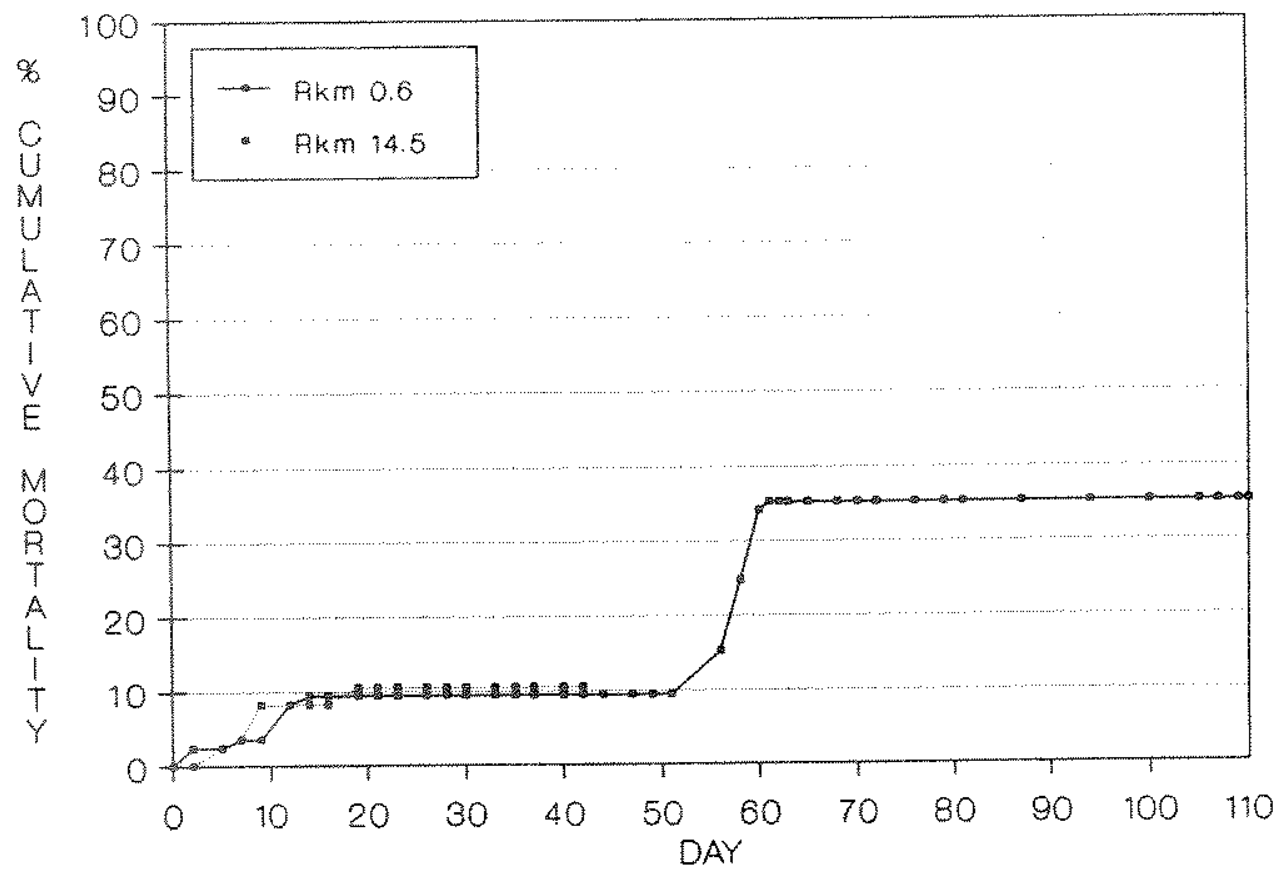

Figure 2.3.4.4. Percent cumulative mortality by day of brown trout fry reared at $\mathrm{Rkm} 14.5$ and held at $\mathrm{Rkm} 0.6$ and 14.5 on the Bighorn River below Afterbay Dam, June - October 1987.

from $R \mathrm{~km} 2.4,8.0$ and 14.5 , suggested that prior exposure to any particular gas pressures was independent of the effect of delta $P$ at Rkm 0.6 on a particular day. Mortality increased among all groups at Rkm 0.6 between $5 / 27$ and $6 / 1 / 87$. Cumulative mortality in two of the tests was similar among all four groups and varied from 27.3 to $36.2 \%$ even though individual groups had been held from 61 to 99 d. The second set of brown trout bioassays, conducted principally from June - August 1987, coincided with elevated gas pressures often greater than the cardiovascular system threshold. This period in 1987 was a critical time when hyperbaric pressures and GBT had a potentially profound effect on brown trout year class strength in at least the upper $8.0 \mathrm{Rkm}$ of the study area.

During the 1987 brown trout bioassays, we documented 

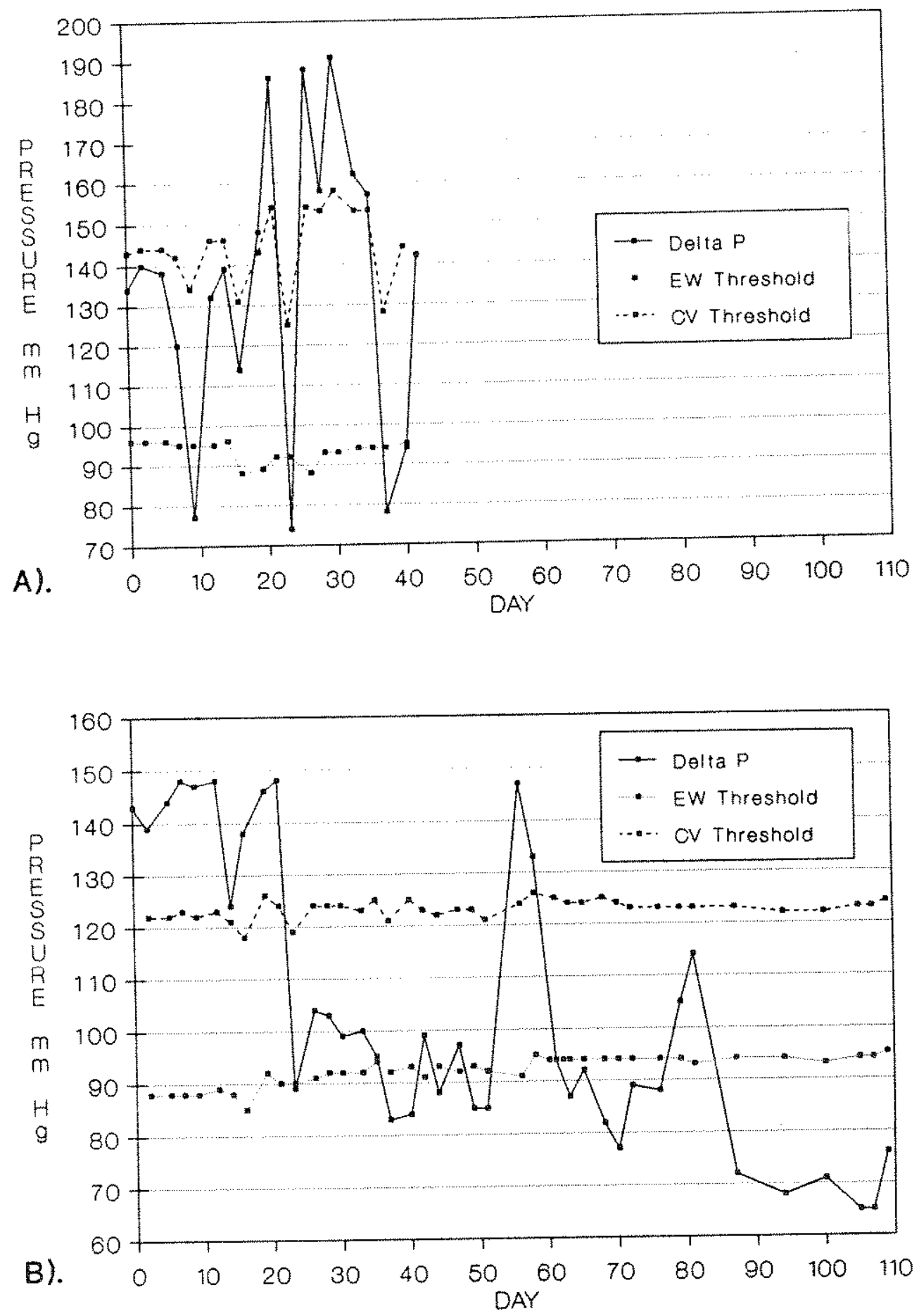

Figure 2.3.45. Environmental water (EW) and cardiovascular (CV) thresholds for bubble growth and delta $P^{\prime} s$ (mmHg) measured at $\mathrm{Rkm}$ 14.5 (A) and 0.6 (B) below Afterbay Dam on the Bighorn River, Montana, during brown trout fry survival tests, June - October 1987. 
sublethal effects of hyperbaric pressure that produced significant challenges to long-term fry survival. Examination of live brown trout fry held during May and June 1987 showed substantial numbers maintaining abnormal swimming positions due to the presence of bubbles (Table 2.3 .20 ). Overal1, $31.6 \%$ of the brown trout fry held at Rkm 0.6 in late May had to compensate for excess buoyancy: this increased to $49.7 \%$ on 11 June. Further downstream, the percentage of fry with buoyancy problems varied from 41.9-45.9\% during June, except for one occasion at Rkm 14.5 when almost no affected fish were observed. Free-ranging fish with similar equilibrium problems would have greatly reduced chances for long-term survival.

The first 1987 rainbow trout fry bioassay at Rkm 2.4 did not include a sample moved to $\mathrm{Rkm} 0.6$. Mortality of fry remained low until after day 20 then rose, reaching $47 \%$ on day 40 (Figure 2.3.46). Although hyperbaric pressures at $\mathrm{kkm} 2.4$ approached the cardiovascular system threshold several times early in the bioassay, the threshold was not exceeded until day 28 (Figure 2.3 .47 ), which corresponds closely to the onset of mortality. The elevated delta $P$ corresponds to an operational change at Afterbay Dam where all water was released from the sluicegates. Rainbow trout fry mortality continued through the end of the experiment even after delta $\mathrm{P}$ dropped below the lower environmental water threshold (Figure 2.3.46). A time lag may have been responsible for the continued mortality. Popeye accounted for $93.1 \%$ of the GBT symptoms, ventral bubbles comprised 5.3\%, buccal cavity bubbles made up $1.1 \%$, and a single hemorrhage on the ventral surface constituted $0.5 \%$ of the 
Table 2.3.20. Proportions of live brown trout fry with obvious buoyancy which affected swimming ability during May and June 1987 on the Bighorn River.

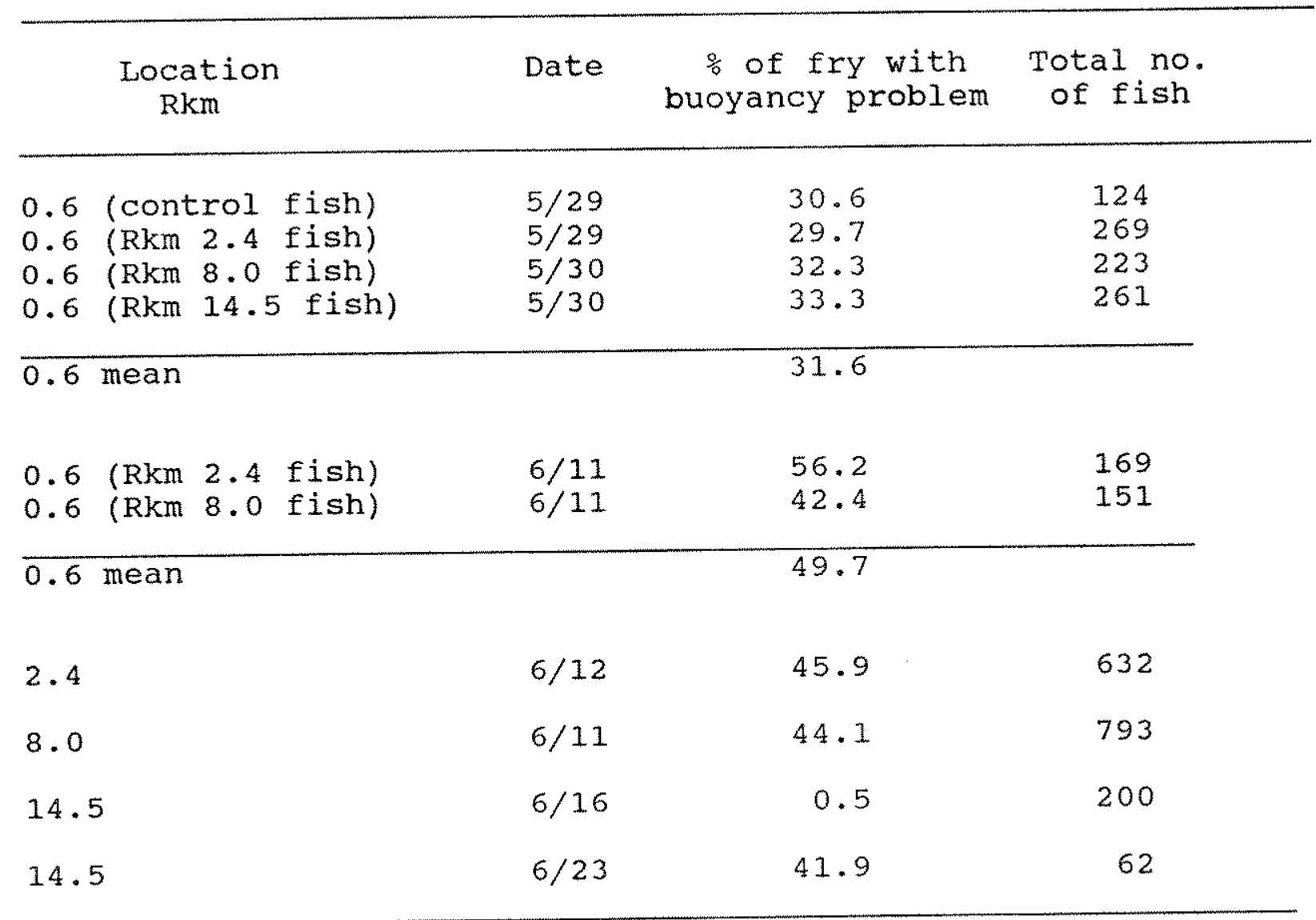

symptoms. The mean length of mortalities during this experiment was $24 \mathrm{~mm}$.

The next rainbow trout fry bioassay was conducted at $\mathrm{Rkm} 2.4$ and $\mathrm{Rkm} 0.6$ from 31 August - 19 october 1987. Mortality was low, never rising above $2.3 \%$ and $19.1 \%$ at $\mathrm{Rkm} 2.4$ and $\mathrm{Rkm} 0.6$, respectively (Figure 2.3 .48 ). Low mortality was a response to delta $\mathrm{P}$ levels lower than the threshold for bubble growth in environmental water at both sites during much of the time (Figure 2.3.49). The mean delta $P$ at $R \mathrm{~km} 2.4$ was $89 \mathrm{mmHg}$, or equal to the threshold for bubble growth in environmental water, and $34 \mathrm{mmHg}$ lower than the cardiovascular threshold. The mean delta $P$ at Rkm 0.6 was lower $(88 \mathrm{mmHg})$ than at $\mathrm{Rkm} 2.4$ and 


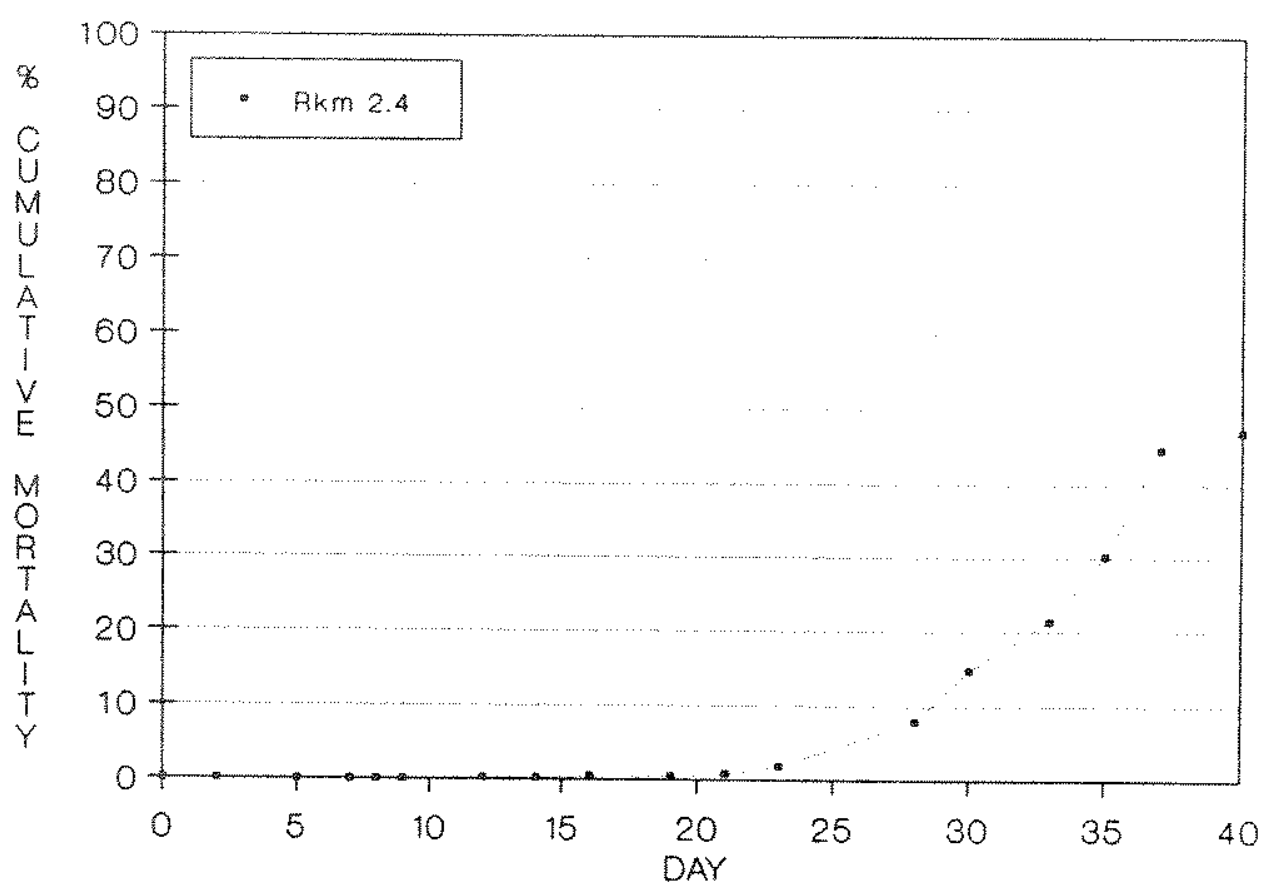

Figure 2.3.46. Percent cumulative mortality by day among rainbow trout fry reared and held at Rkm 2.4 on the Bighorn River below Afterbay Dam, July - August 1987.

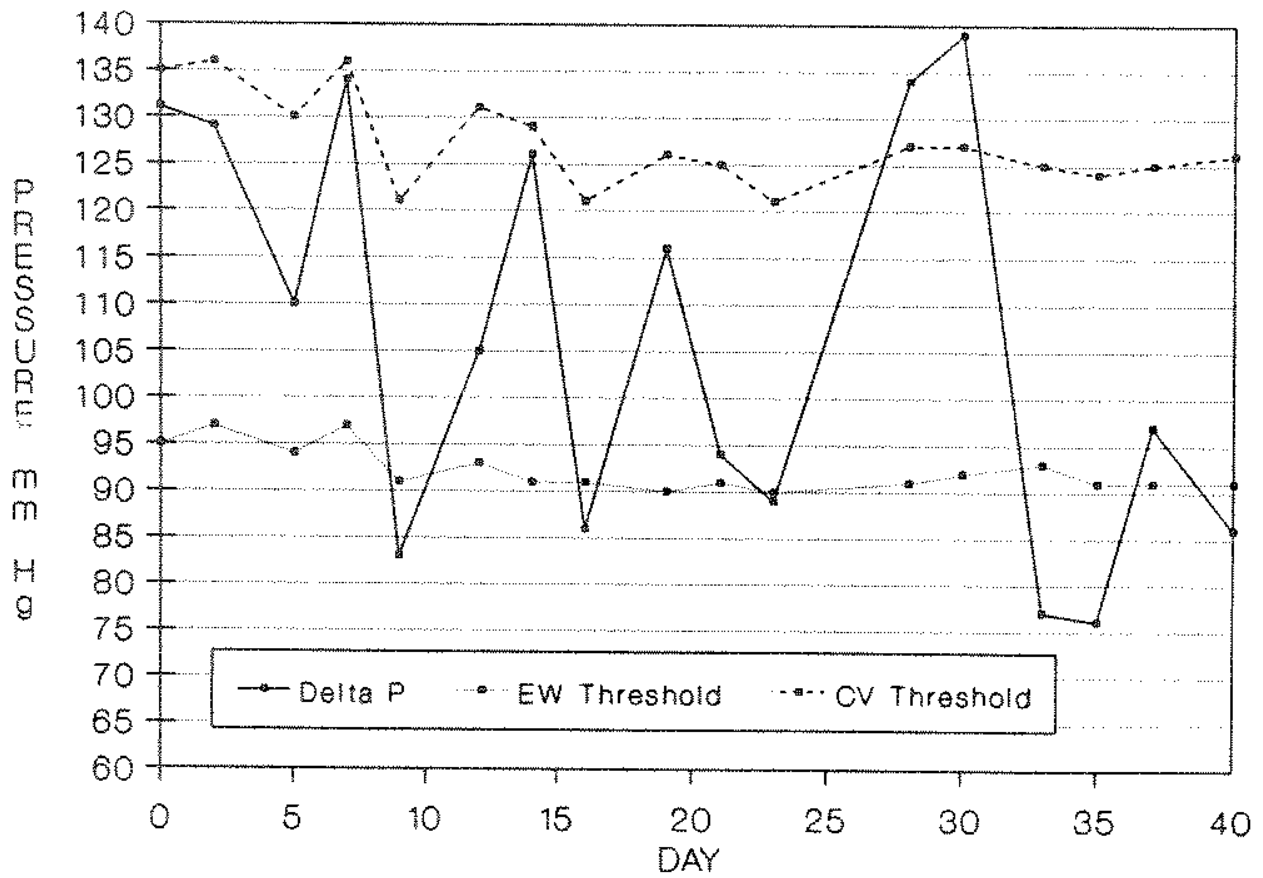

Figure 2.3.47. Environmental water (EW) and cardiovascular (CV) thresholds for bubble growth and delta $\mathrm{P}^{\prime} \mathrm{s}$ (mmHg) measured at Rkm 2.4 below Afterbay Dam on the Bighorn River, Montana, during rainbow trout fry survival tests, July - August 1987. 


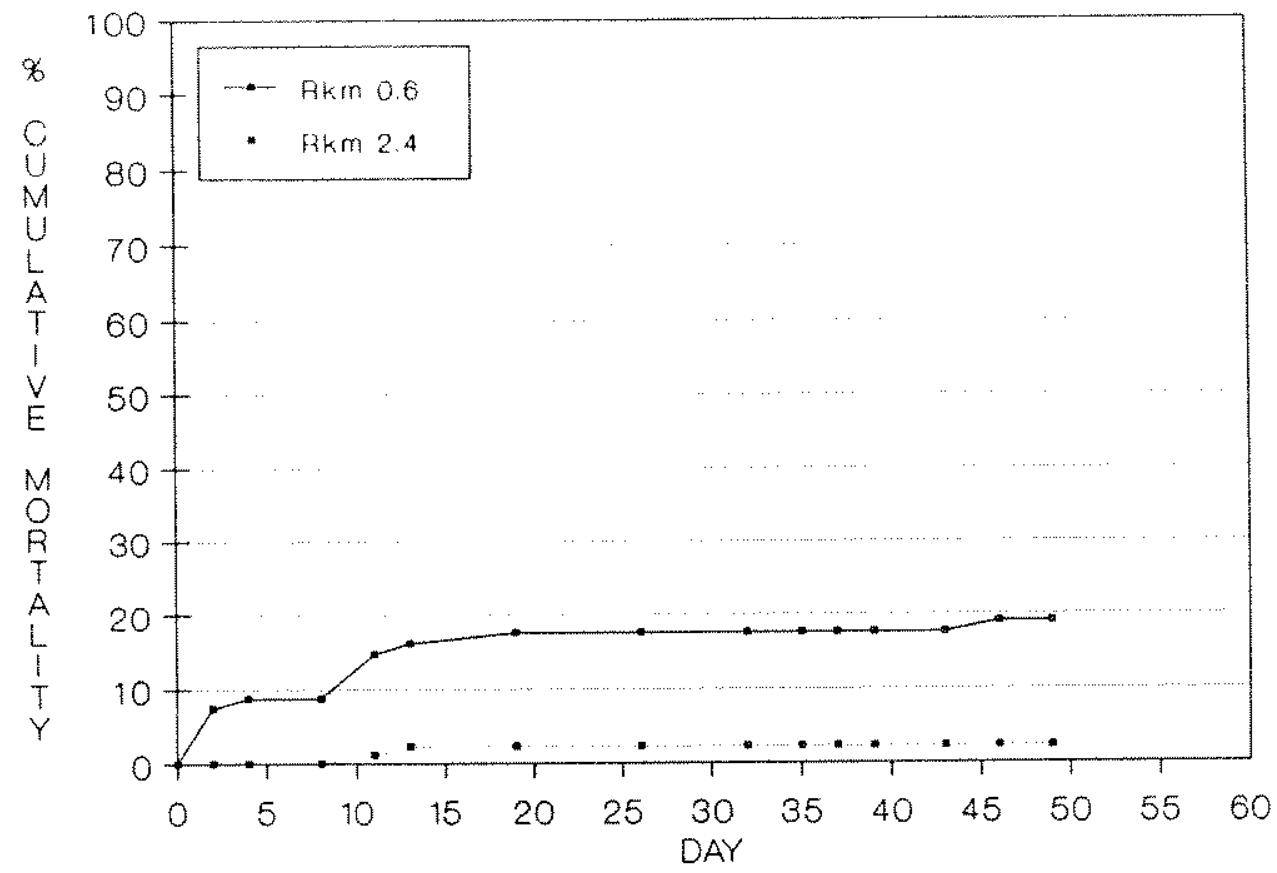

Figure 2.3.48. Percent cumulative mortality by day among rainbow trout fry reared at $\mathrm{Rkm} 2.4$ and held at $\mathrm{Rkm} 0.6$ and 2.4 on the Bighorn River below Afterbay Dam, August - october 1987.

was 4 and $33 \mathrm{mmHg}$ lower than the mean thresholds for bubble growth in environmental water and the cardiovascular system, respectively. The hyperbaric pressure at Rkm 0.6 did exceed the cardiovascular system threshold for bubble growth once, on day 46 (Figure 2.3.49). Gas bubble trauma symptoms at $\mathrm{Rkm} 0.6$ included popeye (60\%), buccal cavity bubbles $(26.6 \%)$, and external and ventral bubbles $(6.7 \%$ each). Two symptoms of GBT were observed on the two mortalities at $\mathrm{Rkm} 2.4$; one fry had a popeye while the other had a buccal cavity bubble. The mean length of fry that died during this experiment was $35 \mathrm{~mm}$ at $\mathrm{Rkm} 2.4$ and $32 \mathrm{~mm}$ at $\mathrm{Rkm}$ 0.6 .

The last field bioassay on rainbow trout fry hatched and reared in 1987 at $\mathrm{Rkm} \mathrm{2.4}$ continued for 91 d from 21 october 1987 - 20 January 1988. Major differences in mortality were observed 

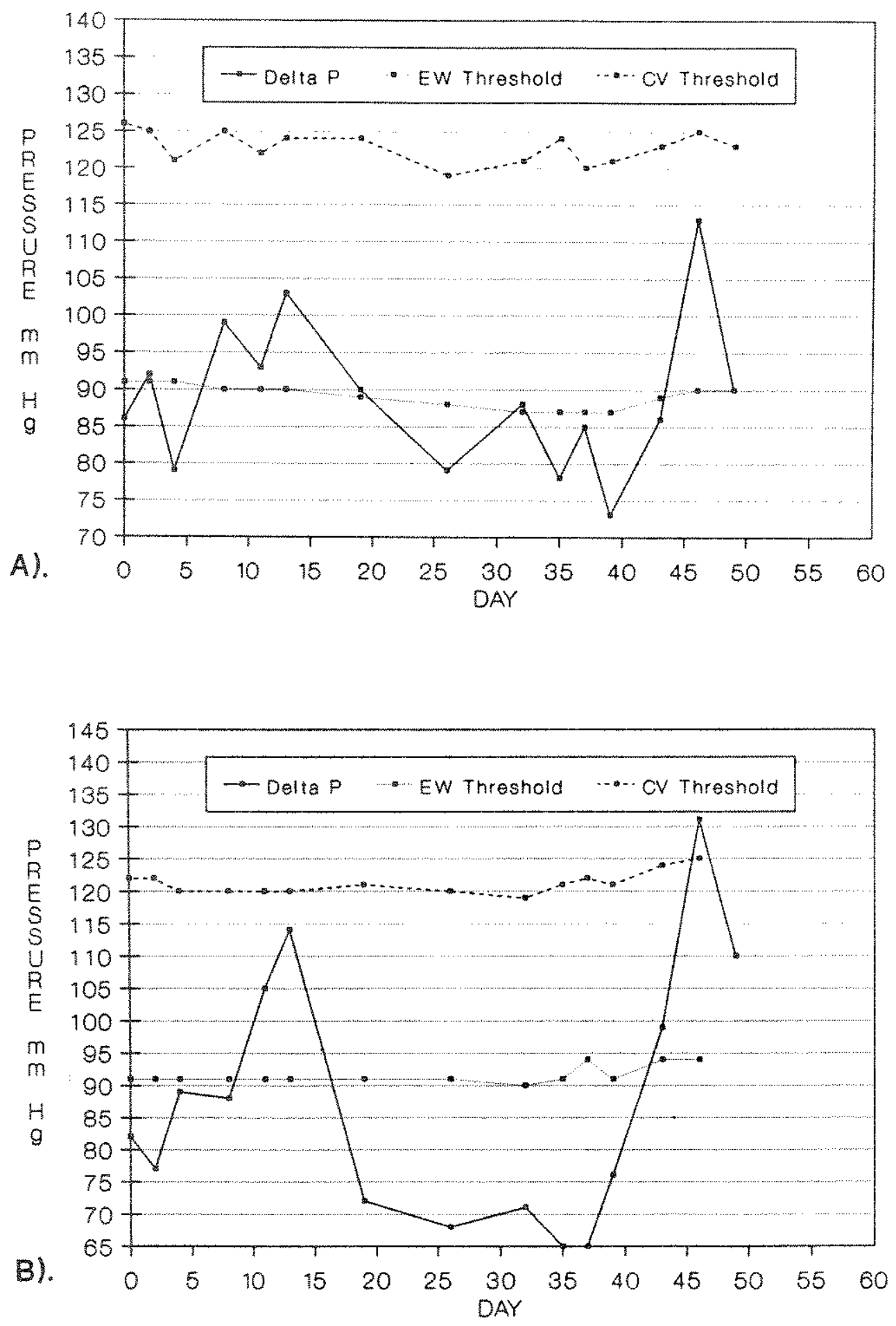

Figure 2.3.49. Environmental water (EW) and cardiovascular (CV) thresholds for bubble growth and delta $\mathrm{P}^{\prime} \mathrm{s}$ (mmHg) measured at Rkm 2.4 (A) and 0.6 (B) below Afterbay Dam on the Bighorn River, Montana, during rainbow trout fry survival tests, August october 1987. 
between the groups at $\mathrm{Rkm} 2.4$ and 0.6 (Figure 2.3.50). No mortality occurred at Rkm 2.4 until day 19; the maximum cumulative mortality reached was $13.2 \%$. In contrast, mortality rapidly increased at $\mathrm{Rkm} 0.6$ and reached $93.0 \%$ by the conclusion of the bioassay. Mean delta $\mathrm{P}$ was $103 \mathrm{mmHg}$ at $\mathrm{Rkm} 2.4$ and 114 $\mathrm{mmHg}$, or $11 \mathrm{mmHg}$ higher, at $\mathrm{Rkm} 0.6$. The mean threshold for bubble growth in environmental water during the bioassay was 92 mmHg at both sites; the mean cardiovascular system thresholds were 125 and $123 \mathrm{mmHg}$ at $\mathrm{Rkm} 2.4$ and 0.6 , respectively. The cause of the large difference in mortality between sites is not apparent from mean hyperbaric pressures. Although pressure was greater at Rkm 0.6, mean delta $\mathrm{P}$ at both sites was below the cardiovascular threshold level. Daily delta P, however, fluctuated much more at $\mathrm{Rkm} 0.6$ and was greater than the cardiovascular threshold $48 \%$ of the time (Figure 2.3.51).

Mean length of fry that died during the bioassay was $58 \mathrm{~mm}$ at each site. As the fish increased in size, GBT symptoms also changed. External bubbles represented 66.7 and $45.3 \%$ of the symptoms at $\mathrm{Rkm} 2.4$ and 0.6 , respectively. Popeye comprised the remaining $33.3 \%$ of the symptoms at $\mathrm{Rkm} 2.4$ and was present in $15.6 \%$ of the mortalities at $\mathrm{Rkm} 0.6$. Buccal cavity bubbles made up the remaining $39.1 \%$ of the GBT symptoms at Rkm 2.4. The external bubbles were classified using the system described for adult fish. Each fry with external bubbles averaged almost two affected body parts at both sites. At Rkm 0.6 the highest frequency of GBT incidence was found on the right operculum, left operculum, and the caudal fin. overall, symptoms were observed on 14 body parts. 


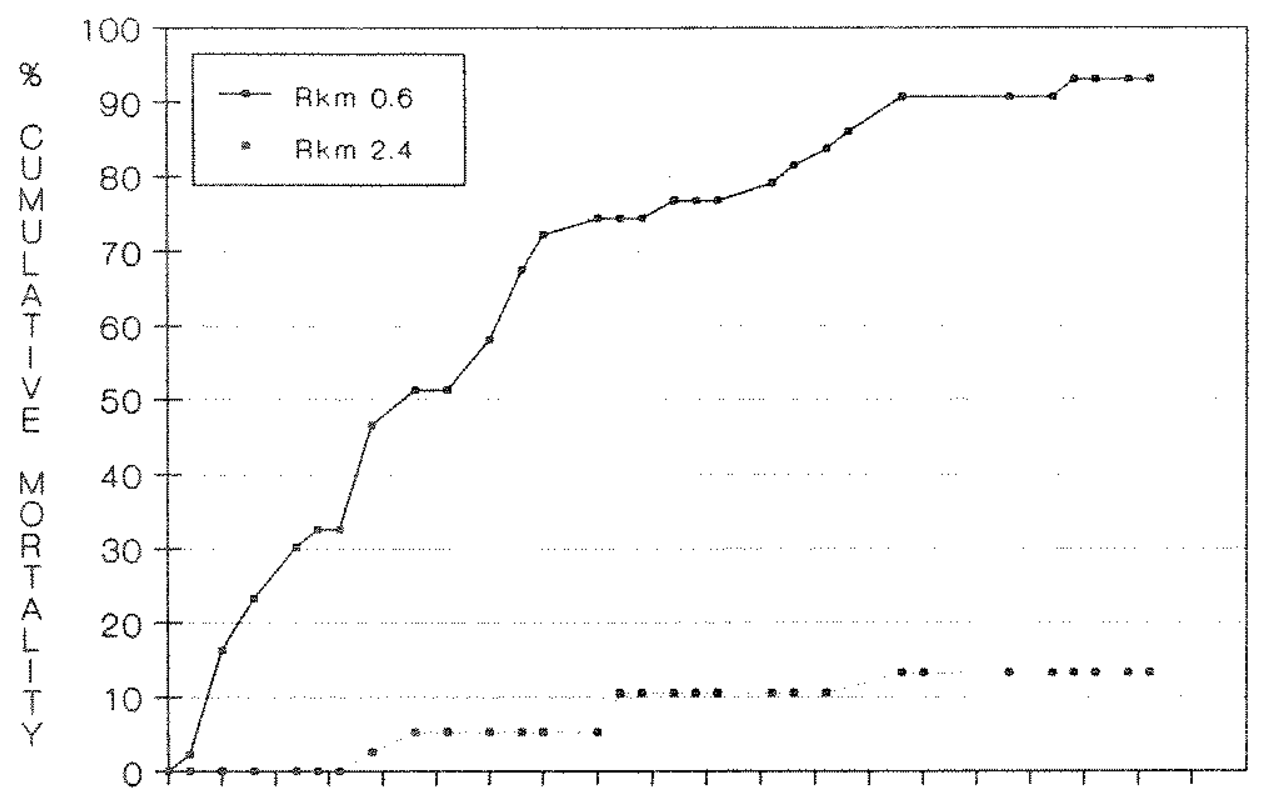

05101520253035404550556065707580859095100

DAY

Figure 2.3.50. Percent cumulative mortality by day among rainbow trout $\mathrm{pry}$ reared at Rkm 2.4 and held at $R \mathrm{~km} 0.6$ and 2.4 on the Bighorn River below Afterbay Dam, October 1987 - January 1988.

Rainbow trout fry incubated at Rkm 8.0 were monitored for 56 d before ary were moved upstream. No mortality occurred until day 21 (sigure 2.3.52). After day 30, mortality increased dramatically, reaching $72 \%$ by day 56. Mean delta $P$ was $124 \mathrm{mmHg}$, which 29 and 16 ming greater than the threshold for bubble growth in environmental water and the cardiovascuiar system, respectively. Delta $P$ was above the cardiovascular system threshold during 23 of the measurements, all of which occurred before day 21 (Figure 2.3 .53 ) when mortality was first observed. The time lag between the period of elevated gas pressures and onset of mortality may have resulted from cumulative effects of sublechal axposure.

The lower reaches of the study area, including $\mathrm{kkm} 8.0$, were characterized by substantial diurnal fluctuations in gas 

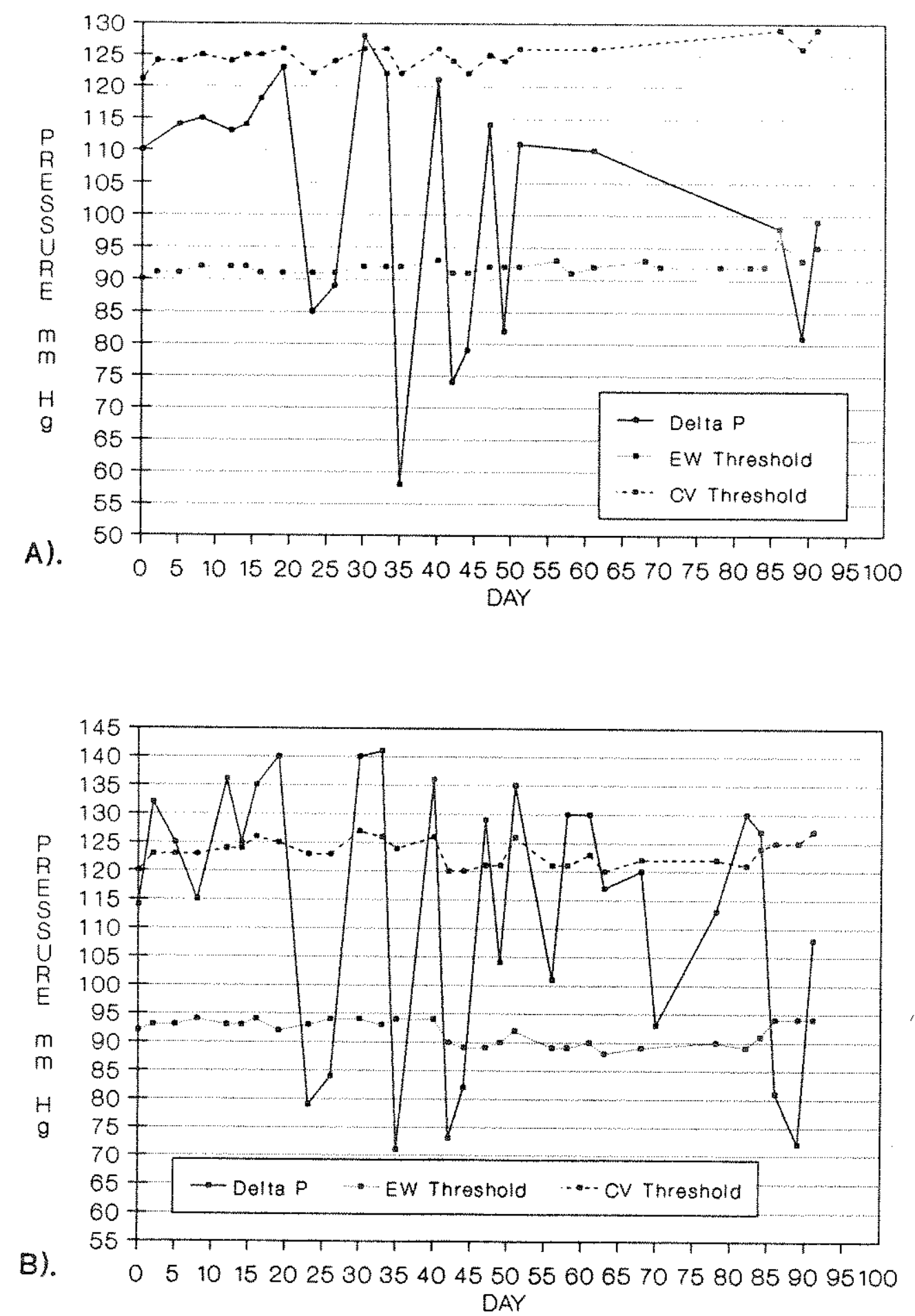

Figure 2.3.51. Environmental water (EW) and cardiovascular (CV) thresholds for bubble growth and delta $\mathrm{P}^{\prime} \mathrm{s}$ (mmig) measured at Rkm 2.4 (A) and 0.6 (B) below Afterbay Dam on the Bighorn River, Montana, during rainbow trout fry survival tests, October 1987 January 1988. 


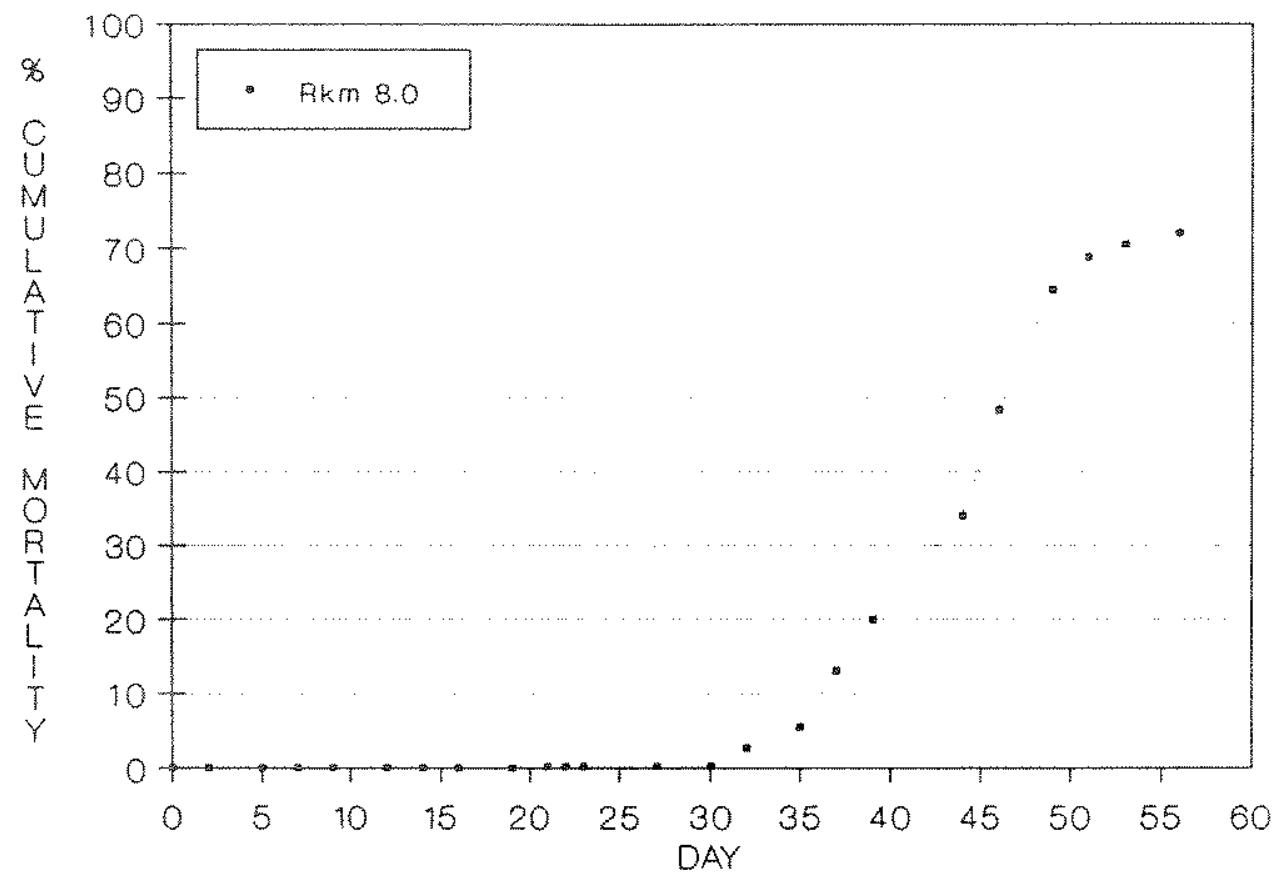

Figure 2.3.52. Percent cumulative mortality by day among rainbow trout fry reared and held at $\mathrm{Rkm} 8.0$ on the Bighorn River below Afterbay Dam, July - August 1987.

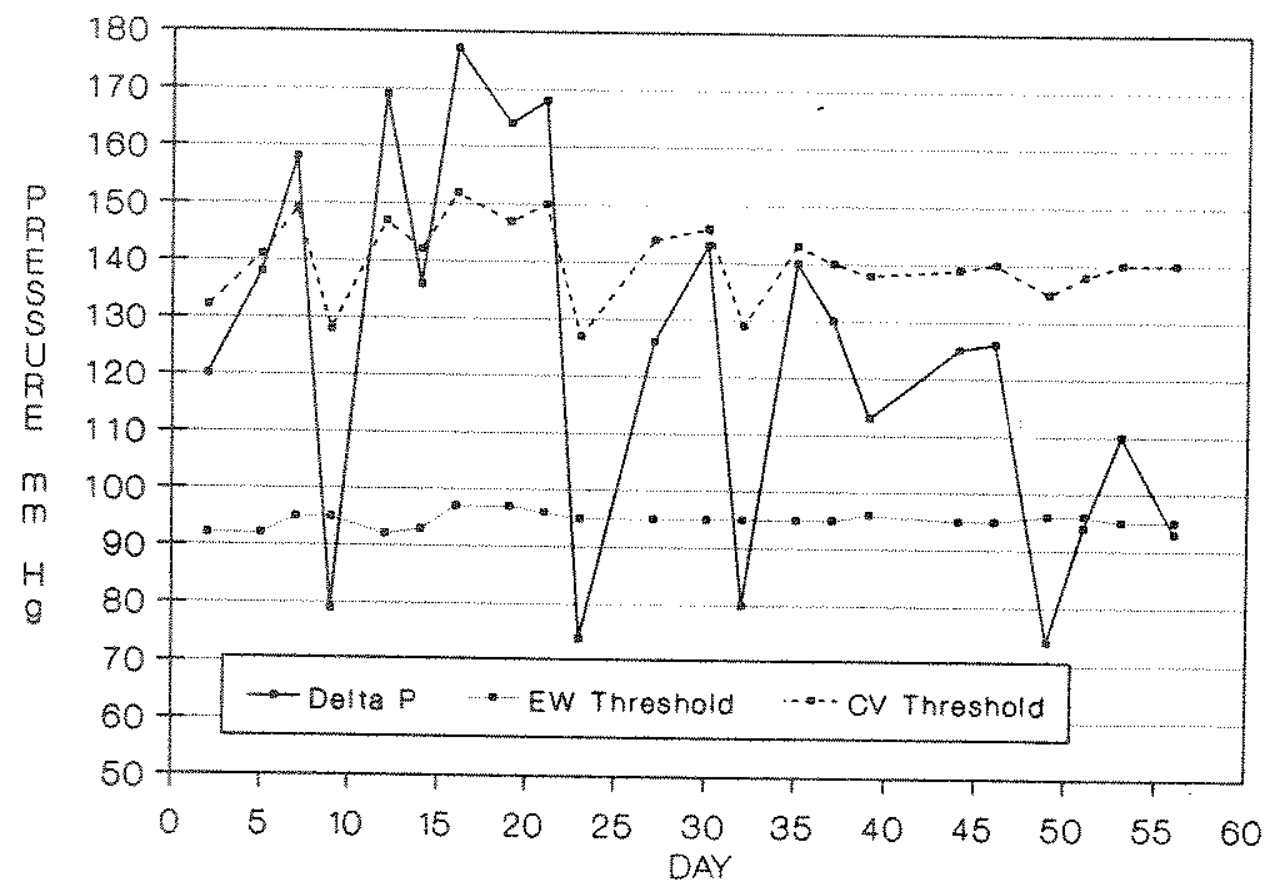

Figure 2.3.53. Environmental water (EW) and cardiovascular (CV) thresholds for bubble growth and delta $\mathrm{P}$ 's (mmHg) measured at $\mathrm{Rkm}$ 8.0 below Afterbay Dam on the Bighorn River, Montana, during rainbow trout fry survival tests, July - August 1987. 
pressures. These fluctuations, in combination with non-uniform sampling times $(10: 33-17: 14 \mathrm{~h})$, resulted in data which were inadequate to evaluate cause and effect between gas levels and mortality. The drop in delta $\mathrm{p}$ to $79 \mathrm{mmHg}$ on day 9 demonstrates the effect of sampling time. On day 9, gas levels were measured approximately $4.2 \mathrm{~h}$ earlier than day 8 and day 10 (Iigure $2.3 .53)$.

Popeye made up 99\% of the GBT symptoms observed on mortalities; the other $1 \%$ consisted of bubbles on the ventral surface of the fry. The mean length of rainbow trout fry that died was $26 \mathrm{~mm}$.

During the second 1987 rainbow trout field bioassay using fry from Rkm 8.0, a portion of the fry were moved to Rkm 0.6 . Mortality among both groups remained low throughout the 49-d monitoring period (Figure 2.3.54). No mortality occurred at Rkm 0.6 until day 49 , and all mortality (5.4\%) at $\mathrm{Rkm} 8.0$ occurred during the first 4 d. Although gas pressures at both locations were hyperbaric during the bioassay, delta $P$ was usually below the threshold for bubble growth in environmental water at Rkm 8.0 and exceeded the cardiovascular system threshold only once at Rkm 0.6 (Figure 2.3.55). Mean gas pressures and threshold values also show why little mortality was expected during this experiment. The mean delta $\mathrm{P}$ over the $49 \mathrm{~d}$ experiment was 78 $\mathrm{mmHg}$ at $\mathrm{Rkm} 8.0$ and $88 \mathrm{mmHg}$ at $\mathrm{Rkm} 0.6$. Mean delta $\mathrm{P}$ never approached the mean cardiovascular system thresholds, which were 132 and $122 \mathrm{mmHg}$ at $\mathrm{Rkm} 8.0$ and 0.6 , respectively, and were even lower than the thresholds for bubble growth in environmental water, which were 92 and $93 \mathrm{mmHg}$ at $\mathrm{Rkm} 8.0$ and 0.6 , 


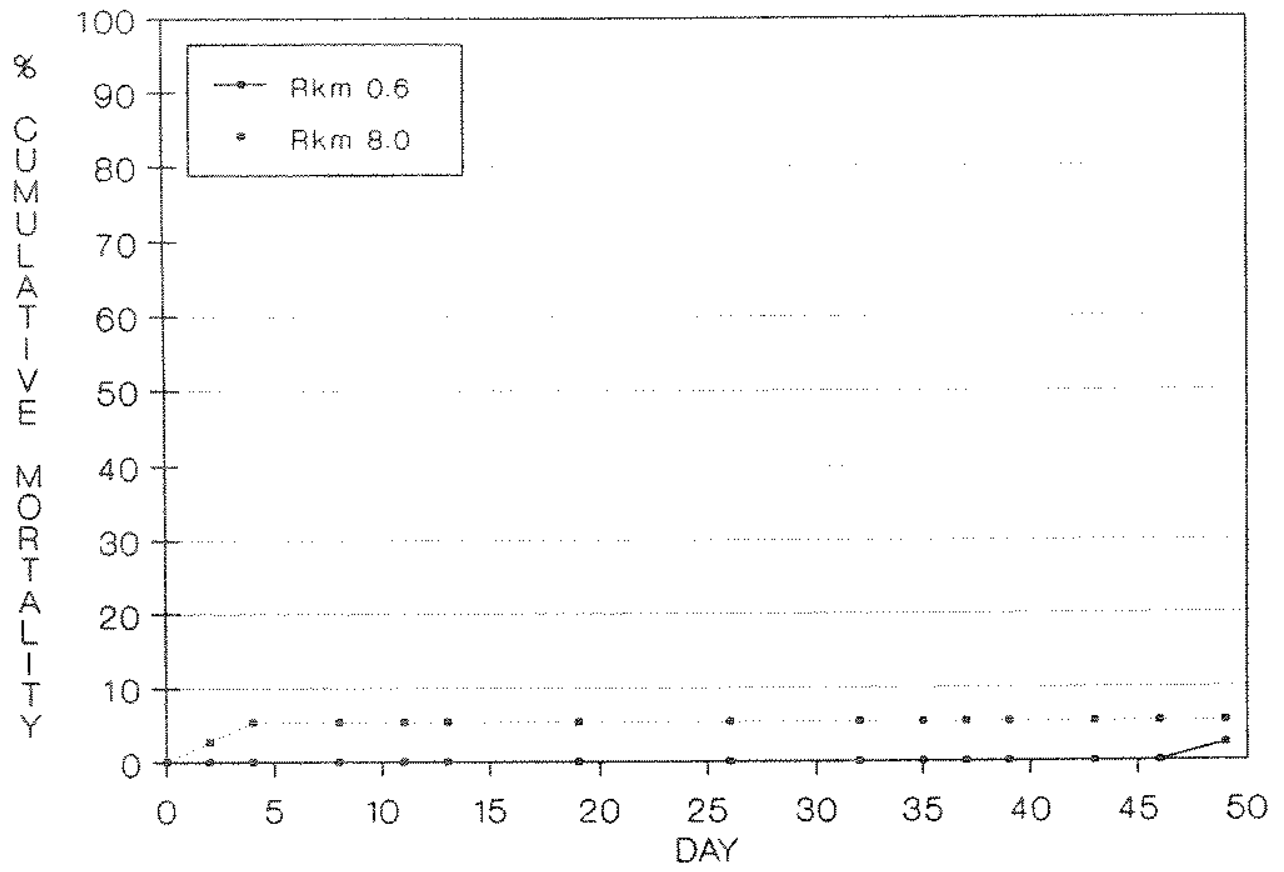

Igure 2.3.5. Percent cumulative mortality by day among rainbow trout fry reaxed at Rkm 8.0 and held at Rkm 0.6 and 8.0 on the Bighorn River below Afterbay Dam, August - october 1987.

respectively.

The last bloassay performed with rainbow trout from Rkm 8.0 resulted in large differences in survival between the rearing site and kkn 0.6 (Figure 2.3.56). No mortality from GBT occurred at $\mathrm{Rm}$ 8.0. By day 2, however, $6.3 \%$ cumulative mortality had occurred at Rkm 0.6 , increasing to $96.9 \%$ by day 30 where it remained for the duration of the $91-d$ test. Gas pressures during the experiment were low at $\mathrm{Rkm} 8.0$; delta $\mathrm{P}$ 'S never approached the threshold for bubble growth in the cardiovascular system and only 25.85 of the measurements exceeded the environmental water threshold (Figure 2.3 .57 ). The mean delta $p$ was $82 \mathrm{~mm} g$, which was 12 and $A B$ milg below the mean thresholds for bubble growth in environmental water and the cardiovascular system, respectively. At Rkm 0.6, hyperbaric pressures during the experiment were quite 

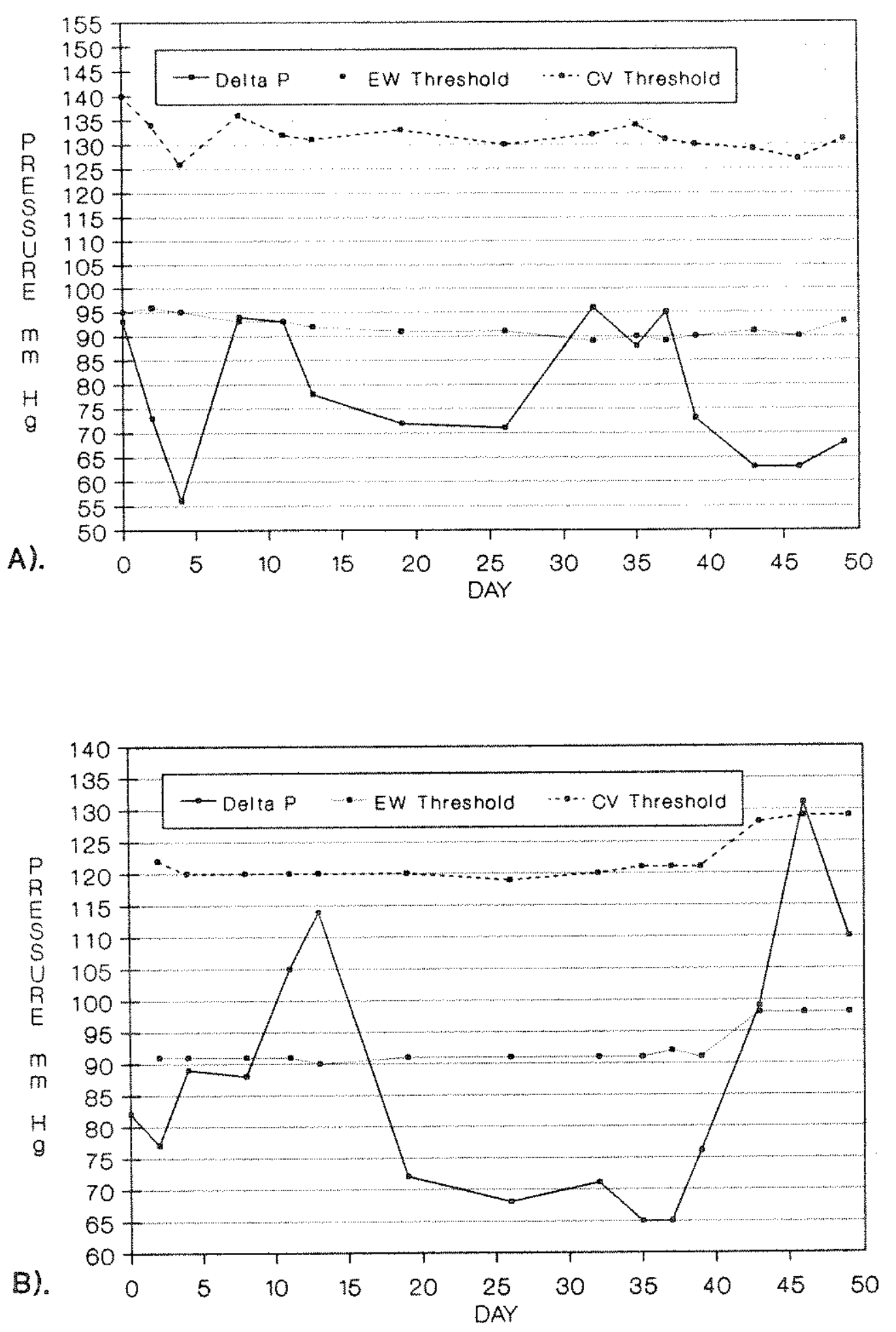

Figure 2.3.55. Environmental water (EW) and cardiovascular (CV) thresholds for bubble growth and delta $\mathrm{p}^{i} \mathrm{~s}$ (mmig) measured at Rkm 8.0 (A) and 0.6 (B) below Afterbay Dam on the Bighorn River, Montana, during rainbow trout fry survival tests, August october 1987. 


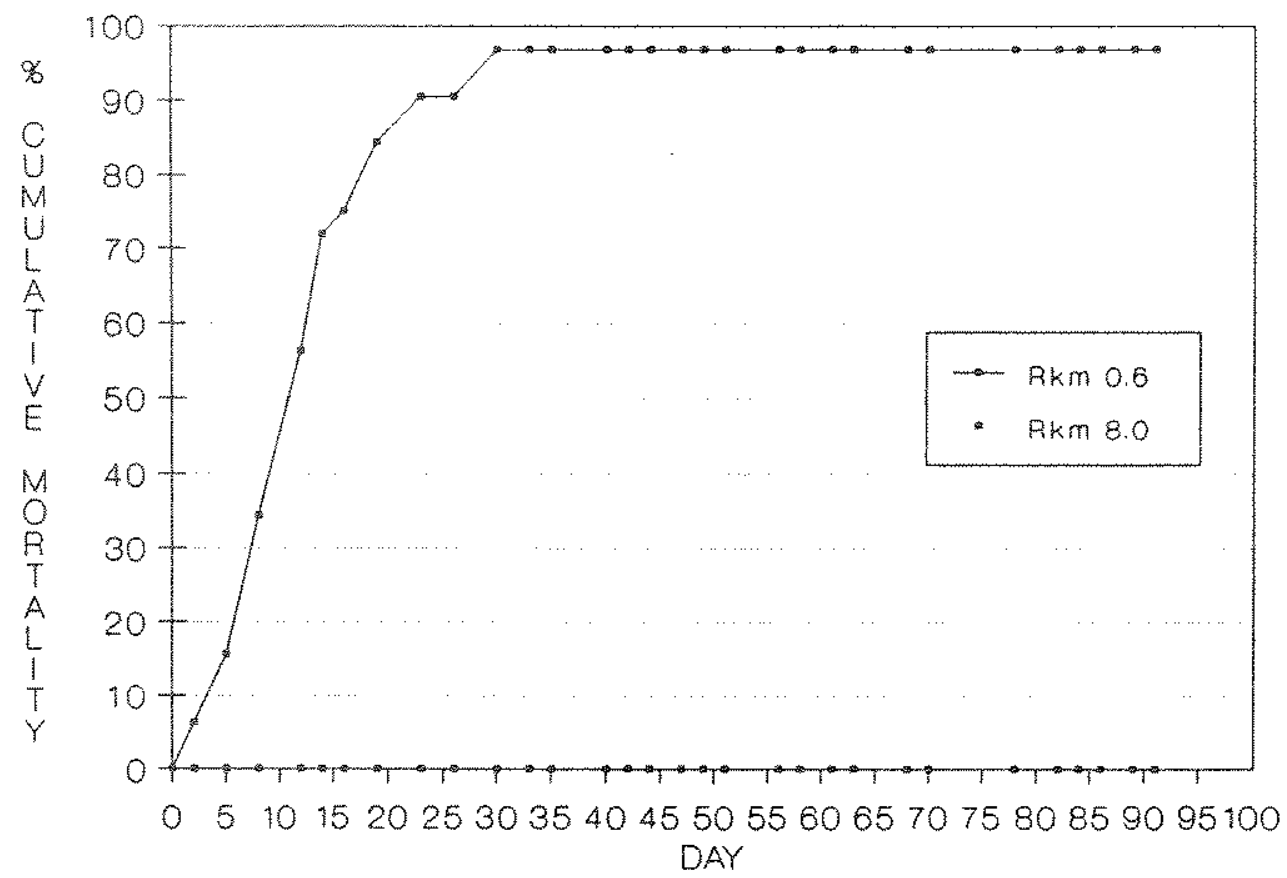

Figure 2.3.56. Percent cumulative mortality by day among rainbow trout fry reared at $R \mathrm{~km} 8.0$ and held at $\mathrm{Rkm} 0.6$ and 8.0 on the Bighorn River below Afterbay Dam, October 1987 - January 1988.

variable and substantially higher, explaining the high mortality; delta $\mathbb{P}$ was above the cardiovascular threshold almost half (48.4\%) of the time (Figure 2.3 .57 ). The mean of the delta $P$ measurements at $\mathrm{Rkm} 0.6$ was $114 \mathrm{mmHg}$, which was $32 \mathrm{mmHg}$ greater than at Rkm 8.0 and $23 \mathrm{mmHg}$ above the maximum calculated threshold for bubble growth in environmental water at Rkm 0.6 . Mean delta $P$, however, was $8 \mathrm{mmHg}$ below the mean maximum cardiovascular system threshold of $122 \mathrm{mmHg}$. Mean length of rainbow trout fry that died at $\mathrm{Rkm} 0.6$ was $61 \mathrm{~mm}$. The most prevalent GBT symptoms were external bubbles (52.5\%), followed by buccal cavity bubbles $(35.08)$ and popeye $(12.5 \%)$. External bubbles were found on 12 body parts, with the highest incidence on the dorsal fin followed by equal incidence on the caudal fin and right operculum. 

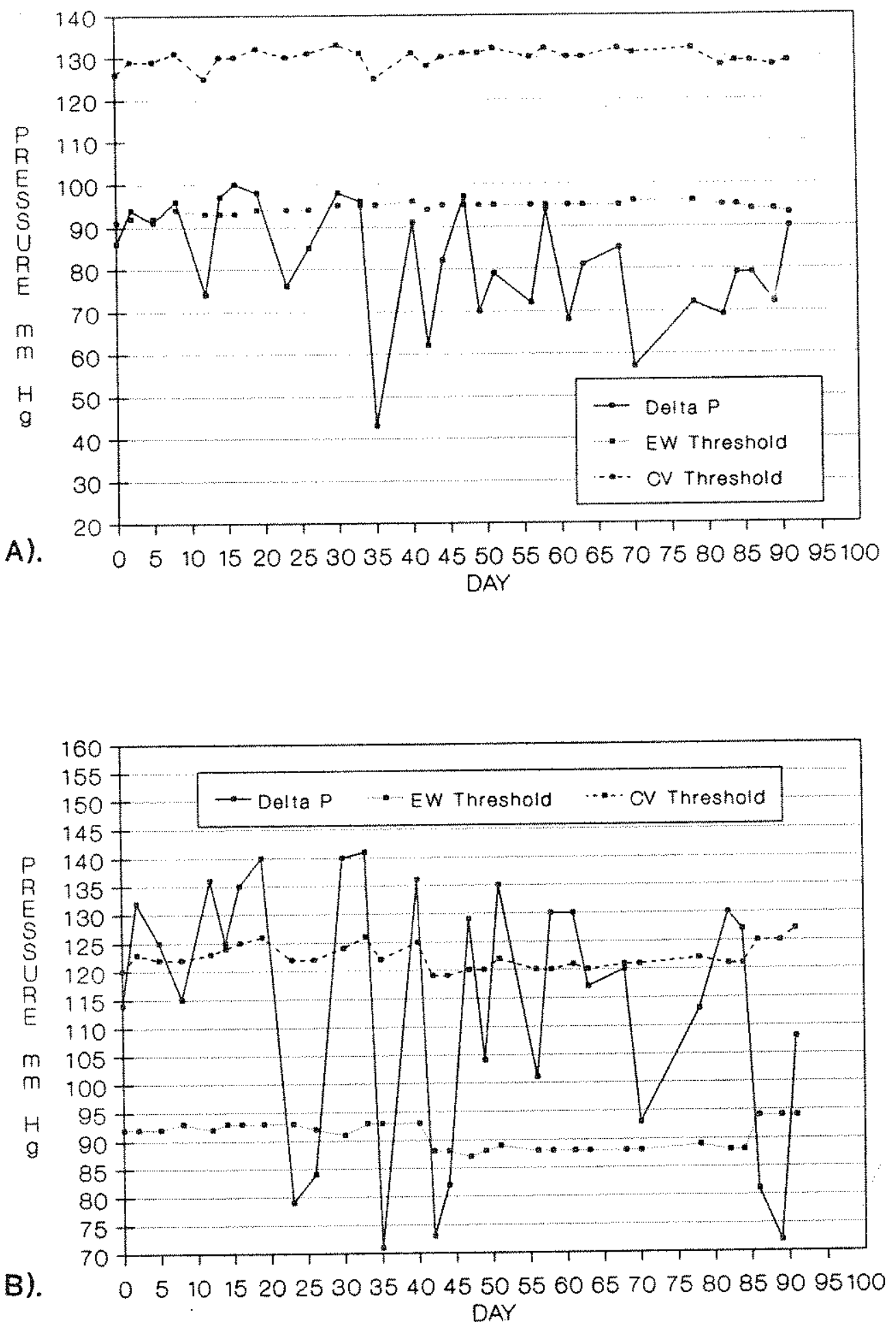

Figure 2.3.57. Environmental water (EW) and cardiovascular (CV) thresholds for bubble growth and delta P's (mmHg) measured at Rkm 8.0 (A) and 0.6 (B) below Afterbay Dam on the Bighorn River, Montana, during rainbow trout fry survival tests, October 1987 January 1988. 
only one experiment was conducted with rainbow trout fry reared at Rkm 14.5 in 1987, and no fry were transferred upstream. During the 93-a bioassay (July-october), fry mortality remained relatively low until day 28 (Figure 2.3.58). Mortality then increased rapidIy to $74 \%$ by day 47 , after which no mortality occurred. Delta $p$ was below the cardiovascular system threshold during the period of highest mortality (Figure 2.3.59). After day 37 , delta $P$ only intermittently rose slightly above the environmental water threshold. High mortality is thought to be a delayed response to approximately 2 weeks of delta $\mathrm{P}$ levels above bubble growth thresholds. The mean delta $P$ during the test was $110 \mathrm{mmHg}$, which was substantially above the threshold for bubble growth in environmental water (94 $\mathrm{mmHg})$ but lower than the mean cardiovascular system threshold of $142 \mathrm{mmHg}$. Fry monitored in this experiment were small (mean $=26 \mathrm{~mm}$ ). Popeye represented 89.8\% of the observed GBT symptoms. Buccal cavity bubbles comprised the other $10.2 \%$

In 1988, two series of brown trout fry field experiments were conducted. The first extended from 25 January - 29 July. Eggs were incubated and fry were reared and monitored at Rkm 2.4, 8.0 and 14.5. A control group, hatched in Afterbay Reservoir, was relocated to Rkm 19.3 to serve as a river control. Mortality at al1 locations remained low until about day 60 (Figure 2.3.60). Cumulative mortality among fry in the control group was $5.4 \%$ during $101 \mathrm{~d}$ of monitoring at Rkm 19.3. Mortality at Rkm 14.5 remained very similar to the control group until day 91 , then sceadily increased to $21.7 \%$ when monitoring was discontinued on day 151. Cumulative mortality at the conclusion of the bioassay 


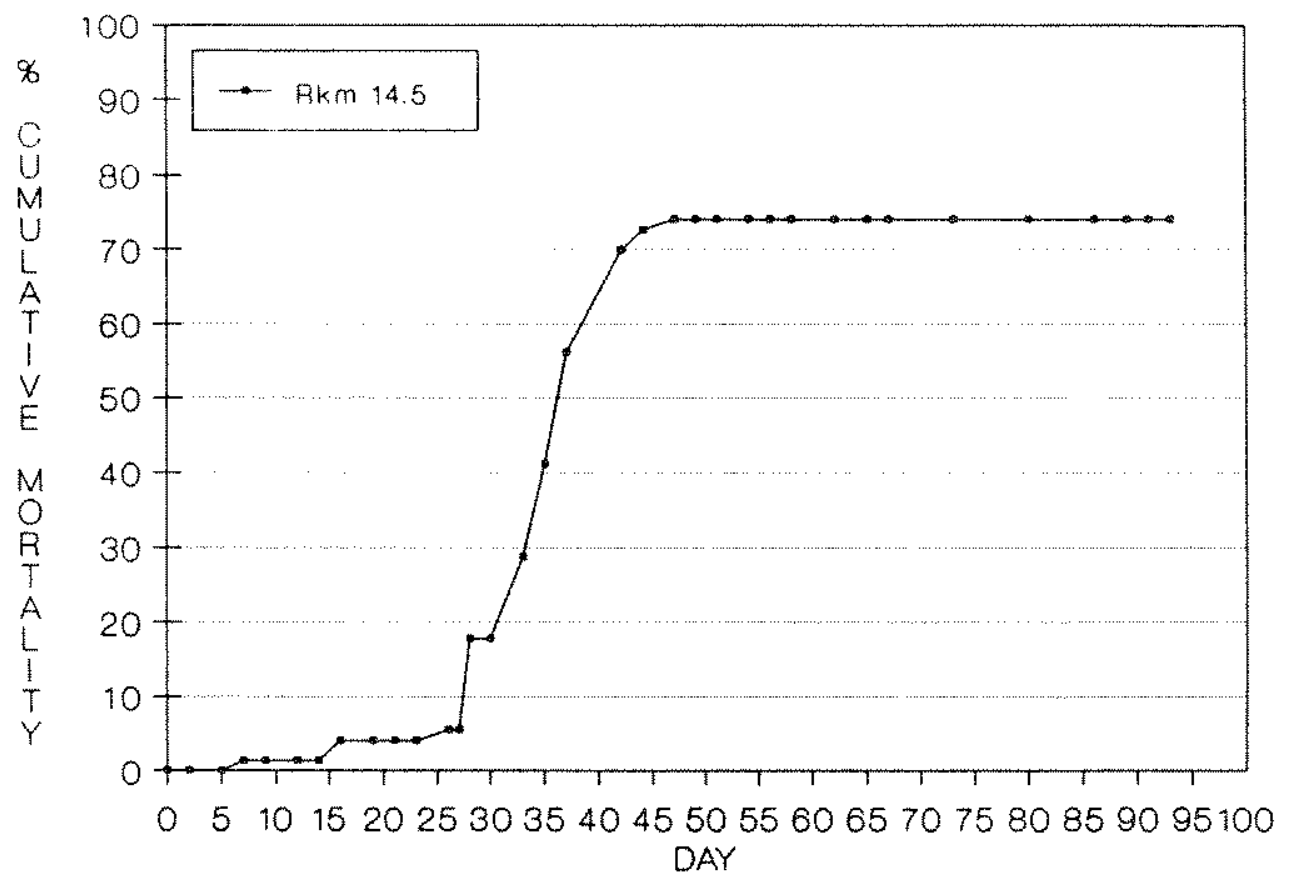

Figure 2.3.58. Percent cumulative mortality by day among rainbow trout fry reared and held at $\mathrm{Rkm} 14.5$ on the Bighorn River below Afterbay Dam, July - October 1987.

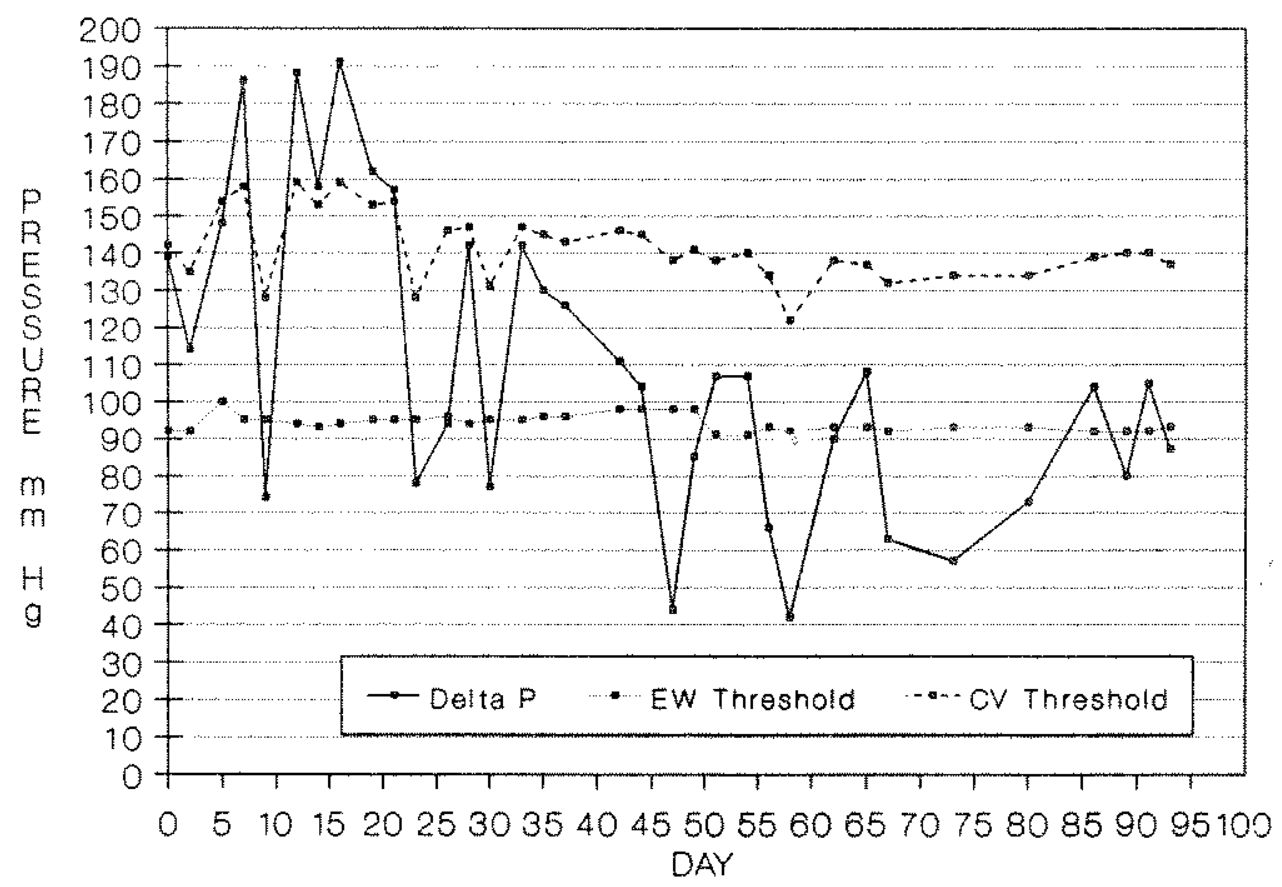

Figure 2.3.59. Environmental water (EW) and cardiovascular (CV) thresholds for bubble growth and delta $\mathrm{P}^{\prime} \mathrm{s}$ (mmig) measured at Rkm 14.5 below Afterbay Dam on the Bighorn River, Montana, during rainbow trout fry survival tests, July - October 1987. 


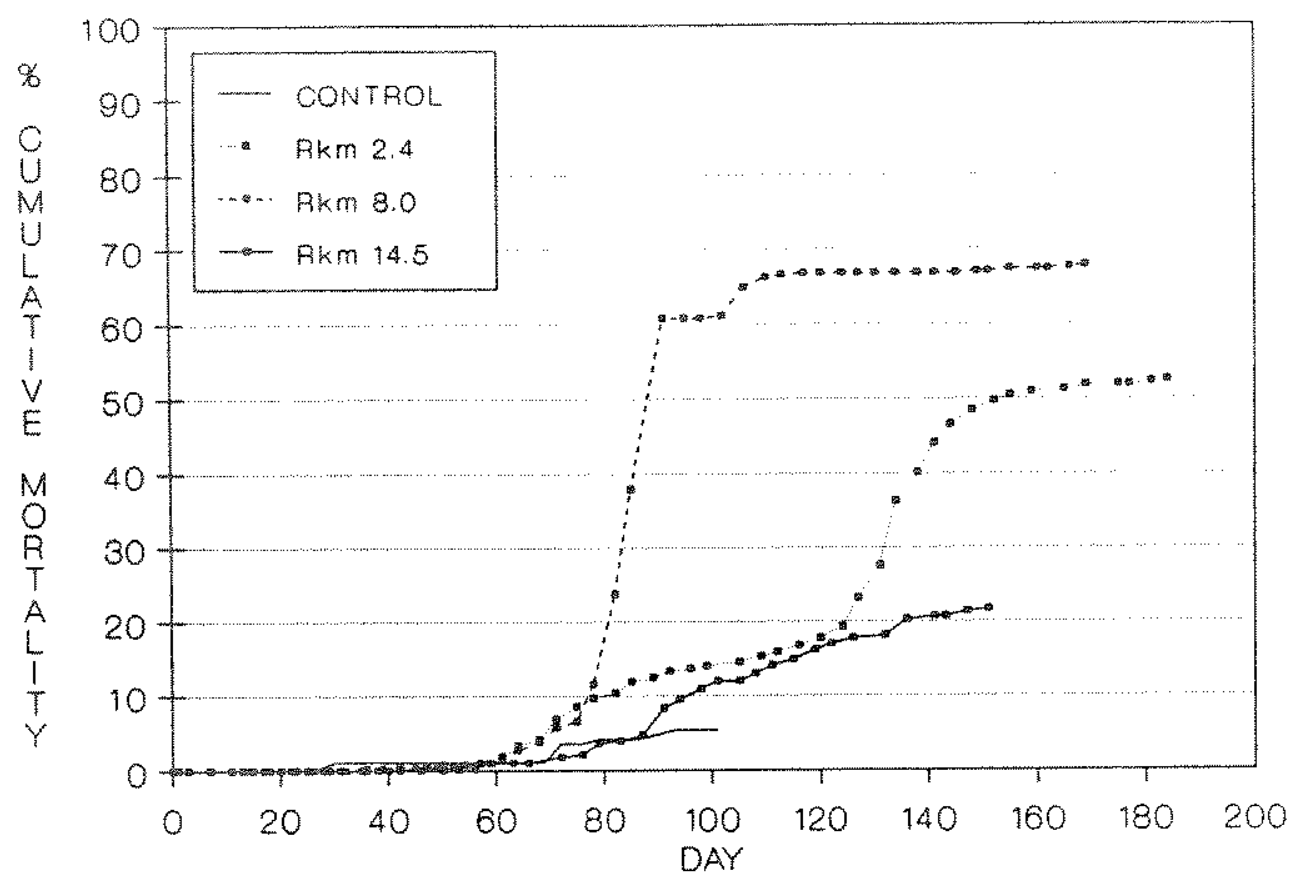

Figure 2.3.60. percent cumulative mortality by day among brown trout fry reared and held at Rkm 2.4, 8.0 , and 14.5 and a control group hela at Rkm 19.3 on the Bighorn River below Afterbay Dam, January $\rightarrow$ July 1988.

was substantialy higher at Rkm 8.0 and 2.4 than at the domstrean sites (Figure 2.3.60). Mortality at Rkm 8.0 rose emponentally at one point, increasing from $11.6 \%$ on day 78 to $60.8 \%$ on day 91 : cumulative mortality at the end of the bioassay was $67.3 \%$. Cumulative mortality on day 184 at Rkm 2.4 was $52.5 \%$, 14.88 lower than at Rkm 8.0. Mortality at Rkm 2.4 increased steadily from about day 60 until day 125 , then increased rapidly to approxinately $50 \%$ on day 152 , where it remained until the bioassay was concluded.

Low mortality in the control group (Rkm 19.3) was consistent With expectations based on gas pressure measurements (Figure 2.3.61). No delta greater than the threshold for bubble growth in the caxdiovasculax system was measured, and hyperbaric 


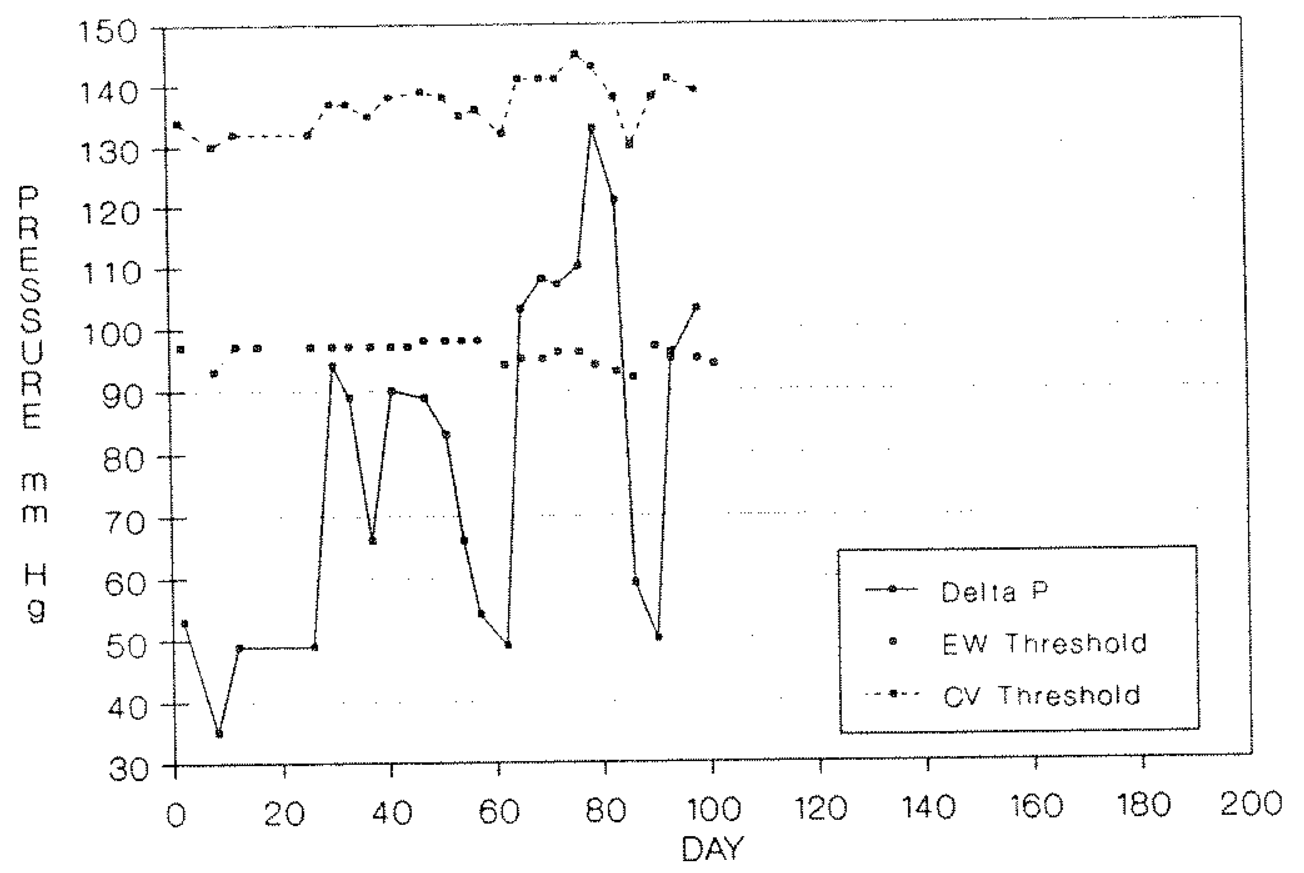

Figure 2.3.61. Environmental water (EW) and cardiovascular (CV) thresholds for bubble growth and delta $p^{\prime} \mathrm{s}$ (mmig) measured for the control group (Rkm 19.3) during brown trout fry survival tests, January - May 1988 on the Bighorn River, Montana.

pressure exceeded the environmental water bubble threshold only $26 \%$ of the time (Figure 2.3.61). Mean delta $P$ was $81 \mathrm{mmHg}$, which was lower than both the environmental water and cardiovascular system threshold and lower than the mean delta $P$ at any other site (Figure 2.3.62). At $\mathrm{Rkm} 2.4$, the most rapid increase in mortality occurred after day 125 and corresponded to the end of the longest period of hyperbaric pressures exceeding the cardiovascular system threshold (Figure 2.3.63). Delta $p$ at Rkm 2. A was above the threshold for bubble growth in environmental water $93.3 \%$ of the time and above the cardiovascular system threshold more than at any other site (35.6\%). However, the mean delta $\mathrm{P}$ (122 muHg) at Rkm 2.4 was 9 mmig lower than at Rkm 8.0 (Figure 2.3.62). The higher mortality at Rkm 8.0 apparently resulted from the higher hyperbaric pressures, which reached a 


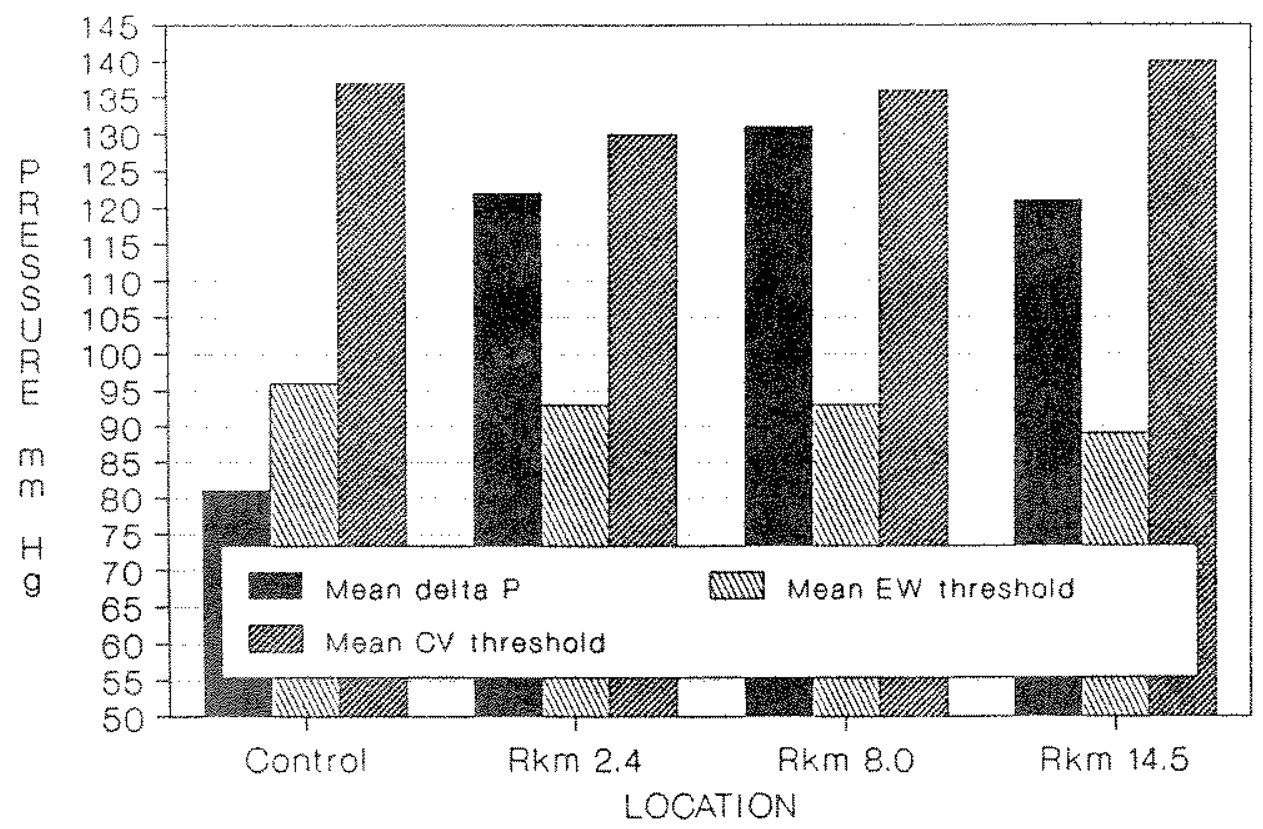

Figure 2.3.62. Mean delta $P$, mean environmental water (EW) and cardiovascular (CV) thresholds for bubble growth (mmHg) measured for the control group and $R \mathrm{~km} 2.4,8.0$, and 14.5 study sites during brown trout fry survival tests, January - May 1988, on the Bighorn River, Montana.

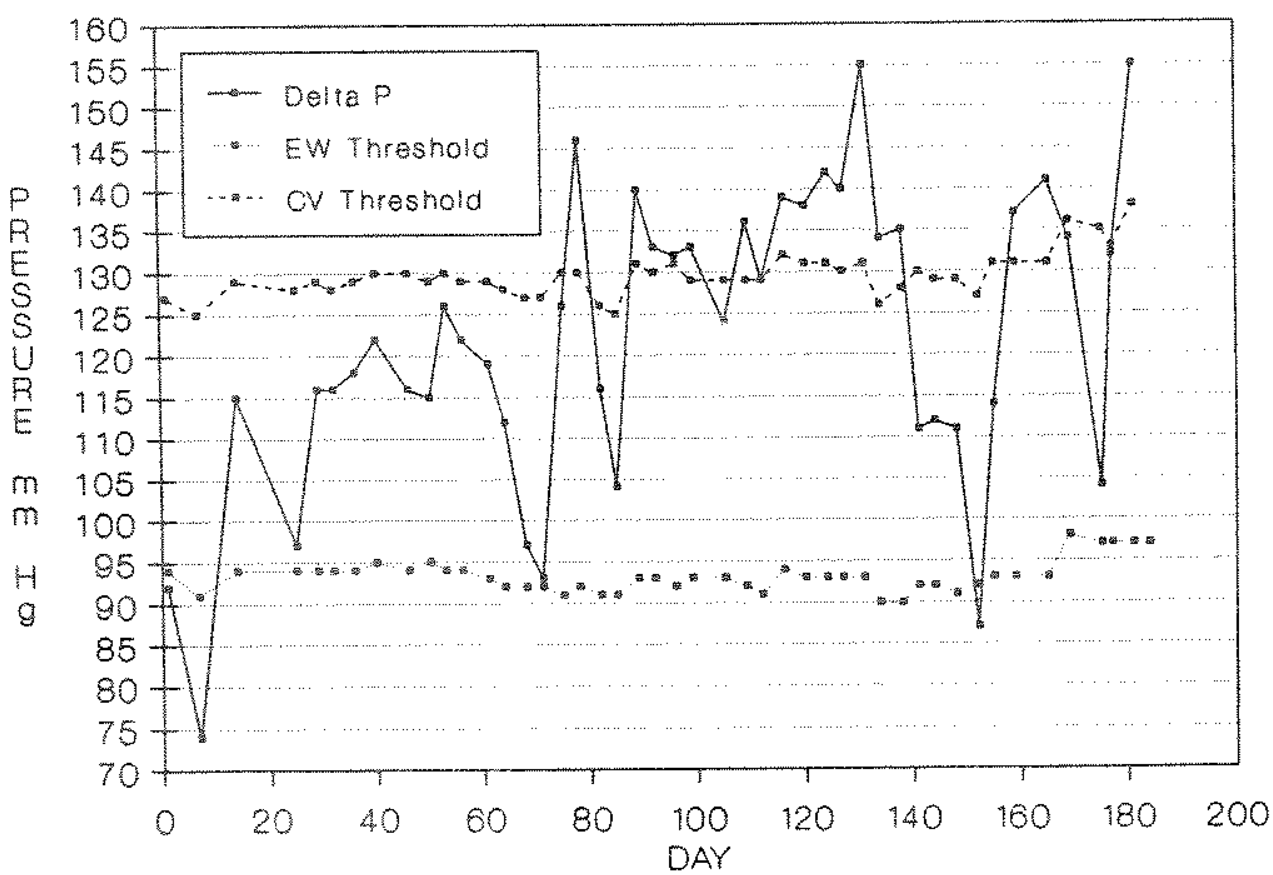

Figure 2.3.63. Environmental water (EW) and cardiovascular (CV) thresholds for bubble growth and delta $\mathrm{Pis}^{\mathrm{s}}$ (mmHg) measured at Rkm 2.4 below Afterbay Dam on the Bighorn River, Montana during brown trout fry survival tests, January - July 1988. 
maximum of $168 \mathrm{mmHg}$ (Figure 2.3.64). Gas levels at Rkm 14.5 peaked soon after mortality commenced (Figures 2.3 .61 and 2.3.65). Although the mean delta $\mathrm{P}$ at $\mathrm{Rkm} 14.5$ was $121 \mathrm{mmHg}, 1$ mmHg lower than at $\operatorname{Rkm} 2.4$, individual measurements were above the threshold for bubble growth in environmental water and the cardiovascular system on $87.2 \%$ and $18.4 \%$ of the dates, respectively.

Popeye was the principal external GBT symptom in all groups $(>60 \%)$ except the control where internal bubbles in the yolk sac comprised just over half of the GBT symptoms, with the remainder being popeye. Lower incidence of buccal cavity and ventral bubbles occurred in all groups, compared to previous tests. Mean size of fry that died ranged from $22 \mathrm{~mm}$ in the control group to $26 \mathrm{~mm}$ at $\mathrm{Rkm} 14.5$.

The second set of brown trout bioassays in 1988 involved the transfer of all fry from the early life history study sites (Rkm $2.4,8.0$ and 14.5 ) to $\operatorname{Rkm} 4.8$ where the fish were held $32-55 \mathrm{~d}$. Cumulative mortality remained low $(1-6 \%)$ throughout the experiment (Figure 2.3.66). Delta $P$, measured with a Bouck gasometer, approached the cardiovascular system threshold early in the experiment, then generally declined through the rest of the 52-d period (Figure 2.3.67). Mean delta $P$ at Rkm 4.8 (120 mmg) was mid-way between the threshold for bubble growth in environmental water $(97 \mathrm{mmHg})$ and the cardiovascular system (144 mmHg). Mean delta $P$ during the bioassay was similar to gas levels fry had been exposed to at Rkm 2.4 and 14.5 before being moved. Fry from Rkm 8.0, however, were reared at somewhat higher mean hyperbaric pressure $(131 \mathrm{mmHg})$. Popeye was the most common 


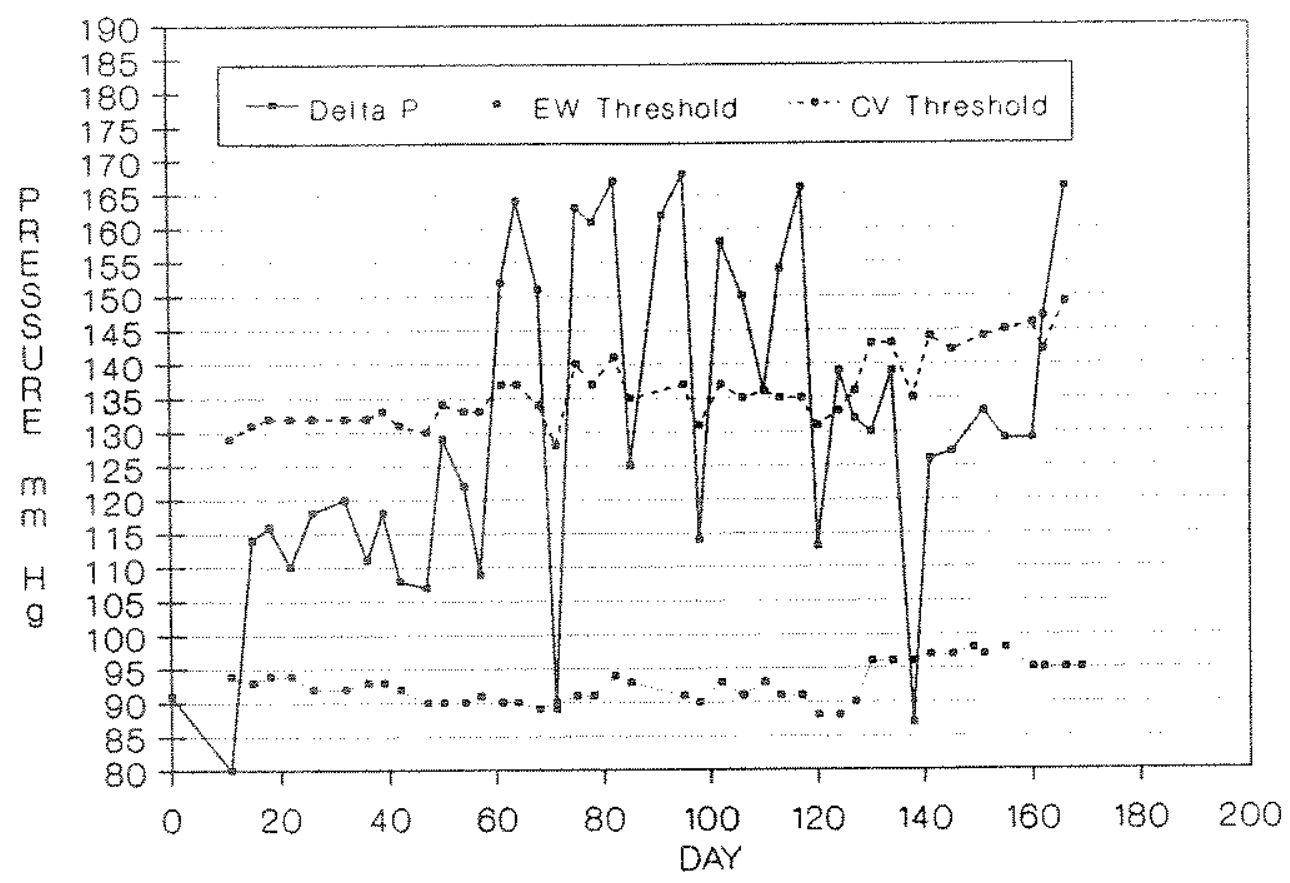

Figure 2.3.64. Snvixomental water (EW) and cardiovascular (CV) thresholds for bubble growth and delta $\mathrm{P}^{\prime} \mathrm{s}$ (mmlg) measured at Rkm 8.0 below Afterbay Dam on the Bighorn River, Montana, during brown trout fry survival tests, February - July 1988.

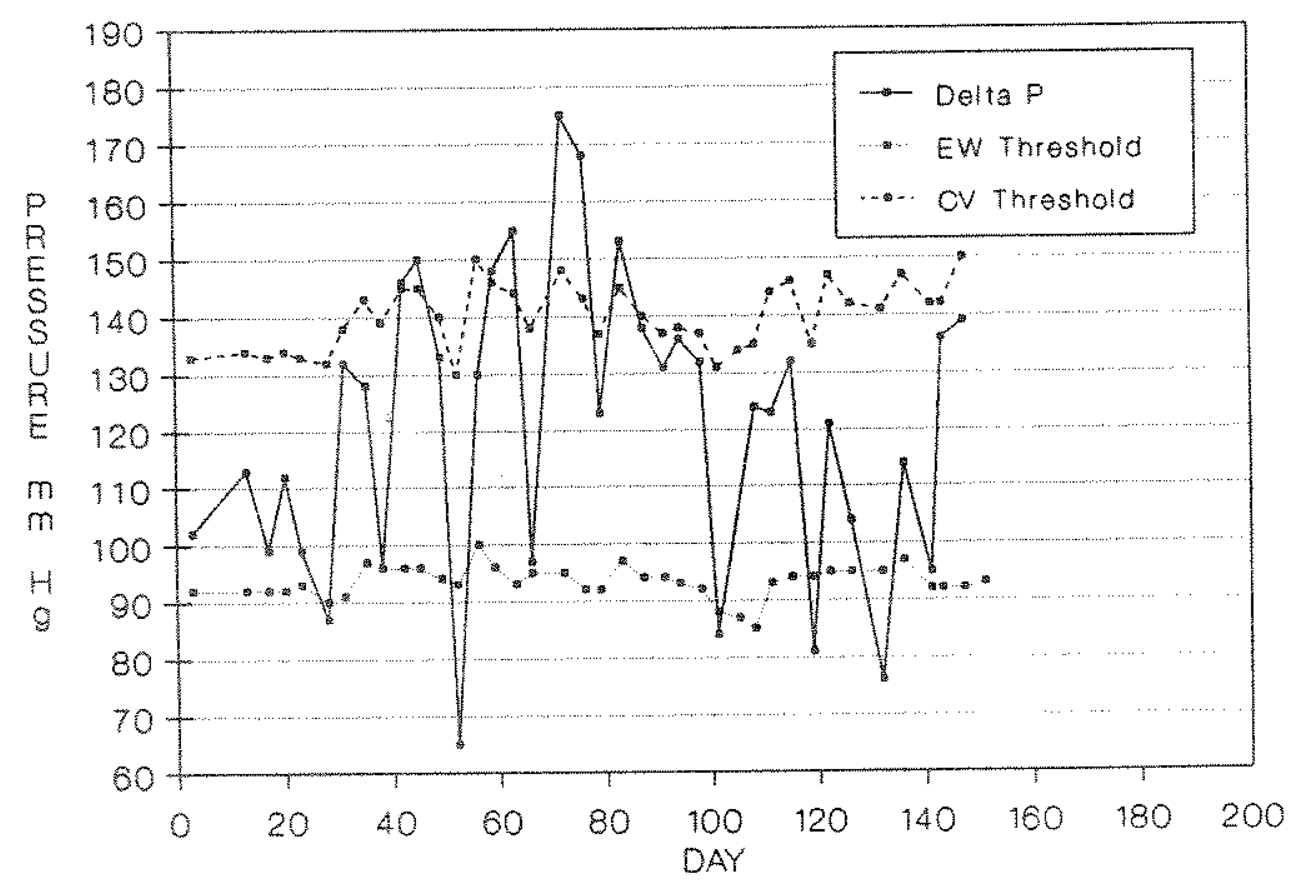

Figure 2.3.65. Environmental water (EW) and cardiovascular (CV) thresholds for bubble growth and delta $\mathrm{P}^{\prime} \mathrm{s}$ (mmH) measured at Rkm 14.5 (D) below Afterbay Dam on the Bighorn River, Montana, during brom trout Ery survival tests, March - July 1988. 


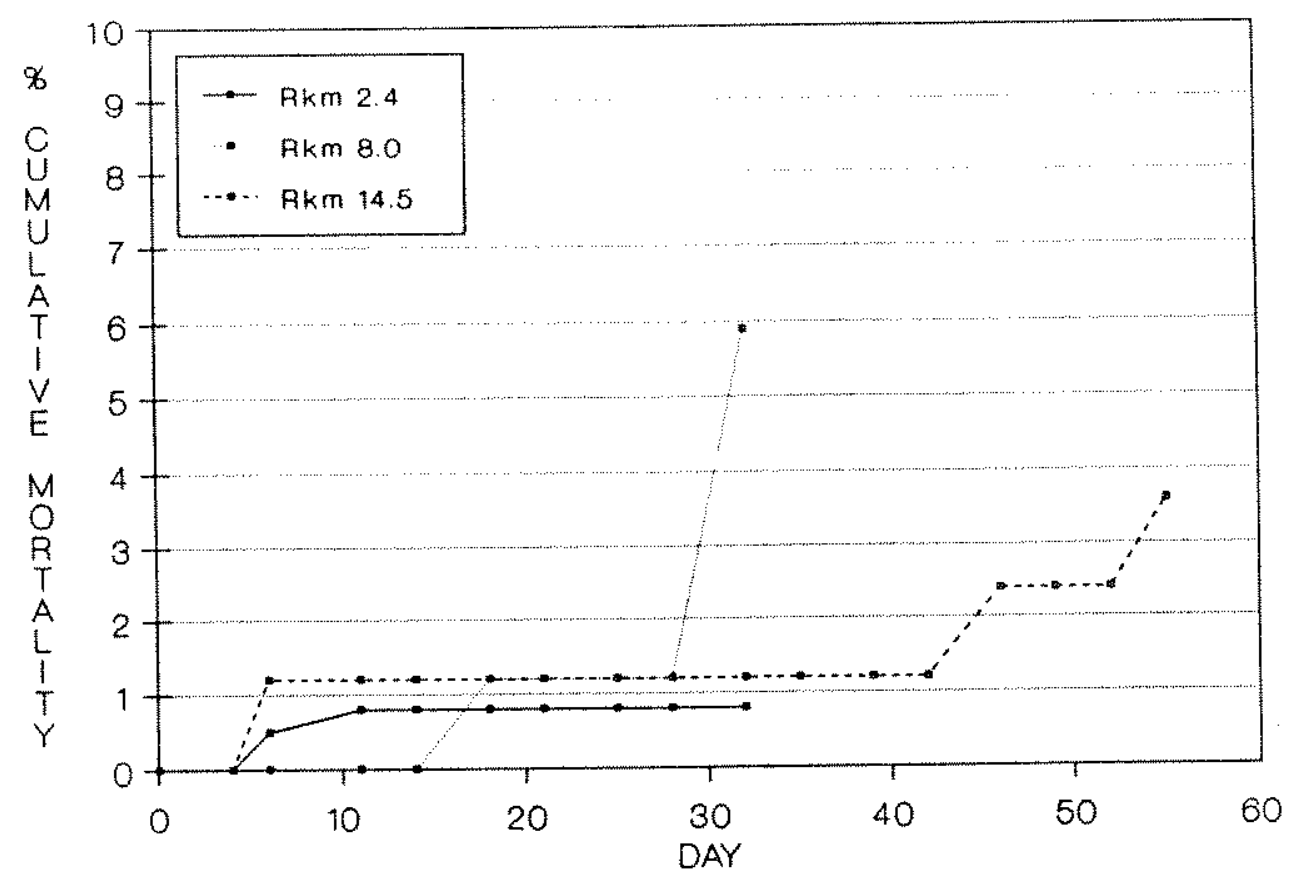

Figure 2.3.66. Percent cumulative mortality by day among brown trout fry reared at $\mathrm{Rkm} 2.4,8.0$, and 14.5 and held at $\mathrm{Rkm} 4.8$ on the Bighorn River below Afterbay Dam, July - September 1988.

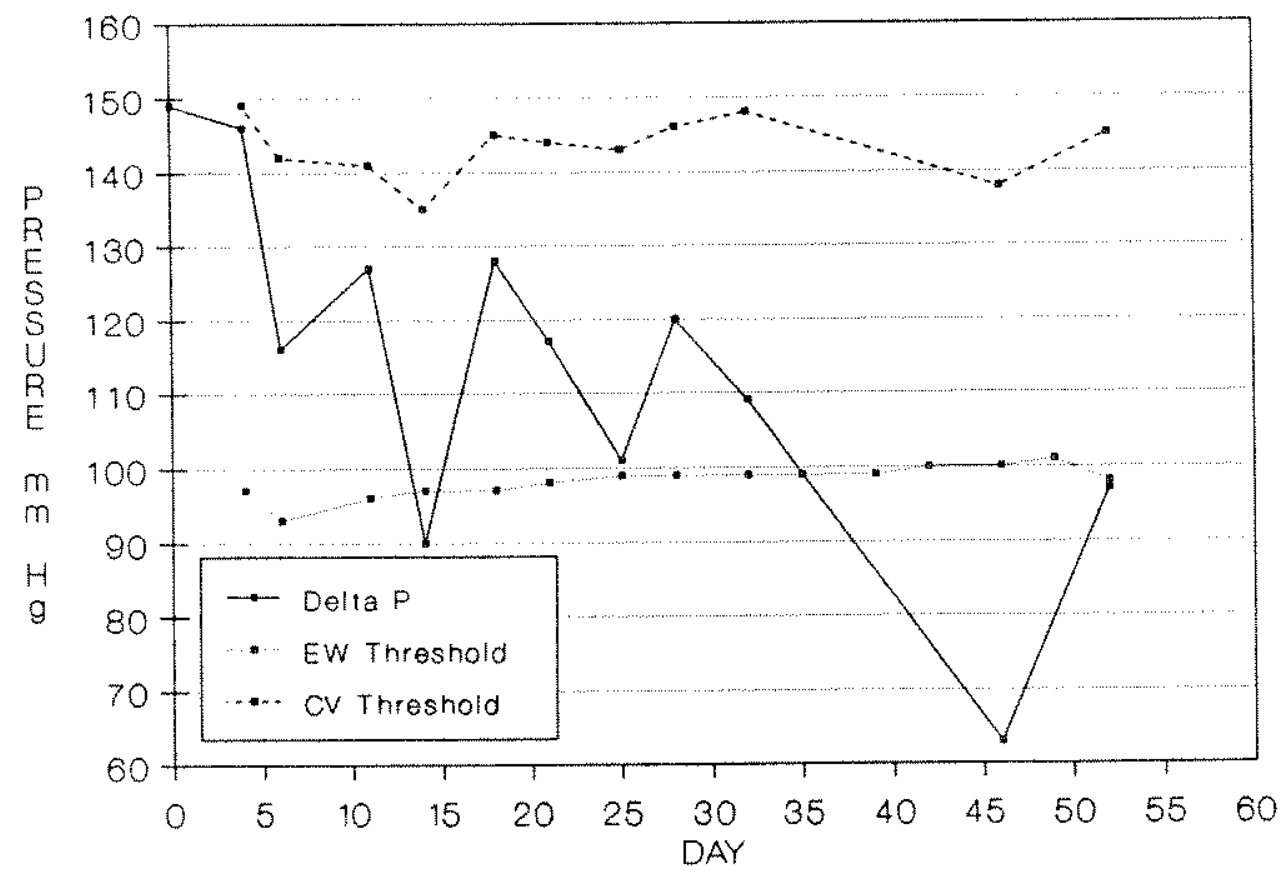

Figure 2.3.67. Environmental water (EW) and cardiovascular (CV) thresholds for bubble growth and delta p's (mmHg) measured while test groups from Rkm 2.4, Rkm 8.0, and $\mathrm{Rkm} 14.5$ were held $4.8 \mathrm{Rkm}$ below Afterbay Dam on the Bighorn River, Montana, during brown trout fry survival tests, July - september 1988. 
external GBT symptom. Fry with bubbles along the ventral surface were also observed among $\mathrm{Rkm} 2.4$ and $8.0 \mathrm{fish}$. The range in mean length of fish that died was highly variable between groups; mean length was 27,32 , and $53 \mathrm{~mm}$ for fry from Rkm 2.4, 8.0, and 14.5, respectively. This primarily resulted from time of mortality. For example, the comparatively small size of Rkm 2.4 fish that died was due to mortality occurring during the first 5 d of the test.

Rainbow trout fry transferred from rearing sites to Rkm 4.8 for the July - September 1988 bioassay sustained substantial mortality $(75.4,54.8$, and $57.5 \%$ of fry incubated at Rkm 2.4, 8.0, and 14.5, respectively) (Figure 2.3.68). Comparison of hyperbaric pressure measurements with predicted thresholds for bubble growth at Rkm 4.8 do not provide an adequate explanation for the high mortality (Figure 2.3.69). Delta P's measured never exceeded the cardiovascular system threshold and were lower than the environmental water/bubble growth threshold on two occasions. Mean delta $\mathrm{P}$ was $114 \mathrm{mmHg}, 6 \mathrm{mmHg}$ lower than the concurrent brown trout bioassays which resulted in low mortality. The mean environmental water threshold was $98 \mathrm{mmHg}$, substantially lower than the delta $P$, but the mean cardiovascular system threshold was much higher at $144 \mathrm{mmHg}$.

Manual gas level monitoring during this bioassay was inadequate to properly describe gas level exposure of the fish. Uncorrected data from continuous monitoring equipment (Figure $2.3 .70)$ showed daily peaks often exceeded the cardiovascular system threshold (Figure 2.3.69) during the first $10 \mathrm{~d}$ of the experiment. The substantial drop of delta $\mathrm{P}^{\prime} \mathrm{s}$ to approximately 


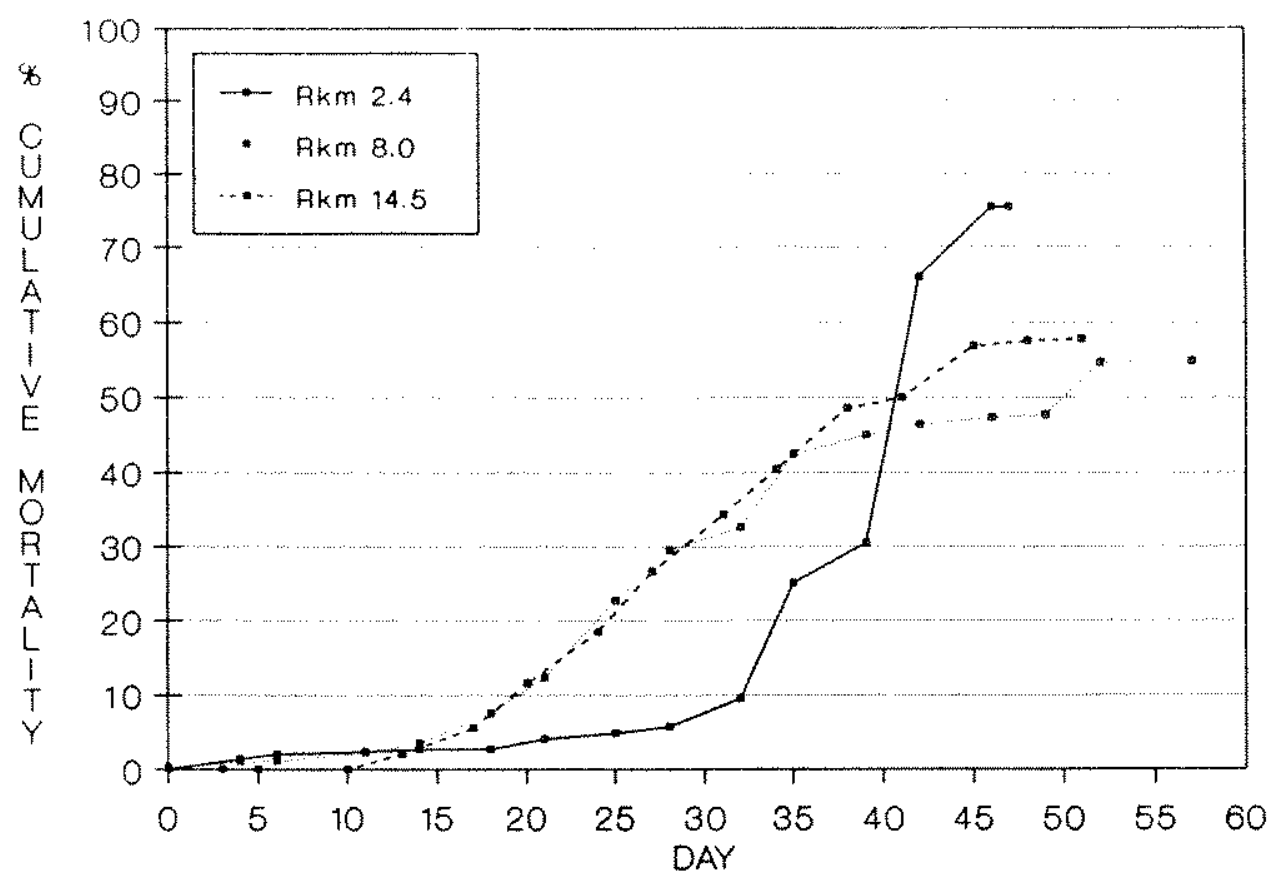

Figure 2.3.68. Percent cumulative mortality by day among rainbow trout fry reared at $\mathrm{Rkm} 2.4,8.0$, and 14.5 and held at $\mathrm{Rkm} 4.8$ on the Bighorn River below Afterbay Dam, July - September 1988.

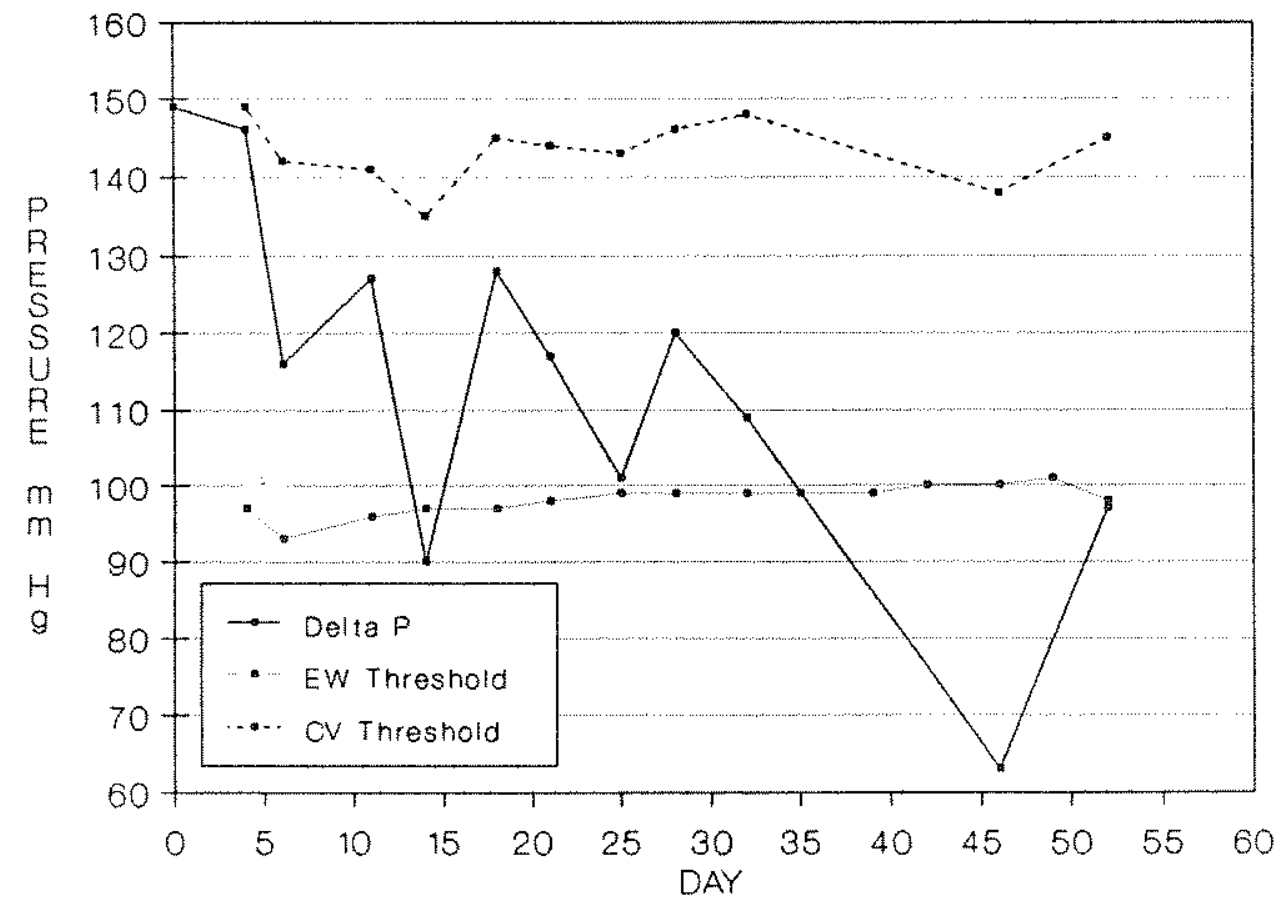

Figure 2.3.69. Environmental water (EW) and cardiovascular (CV) thresholds for bubble growth and delta p's (mmHg) measured while test groups from $\mathrm{Rkm} 2.4$, $\mathrm{Rkm} 8.0$, and $\mathrm{Rkm} 14.5$ were held $4.8 \mathrm{Rkm}$ below Afterbay Dam on the Bighorn River, Montana, during rainbow trout fry survival tests, July - september 1988. 


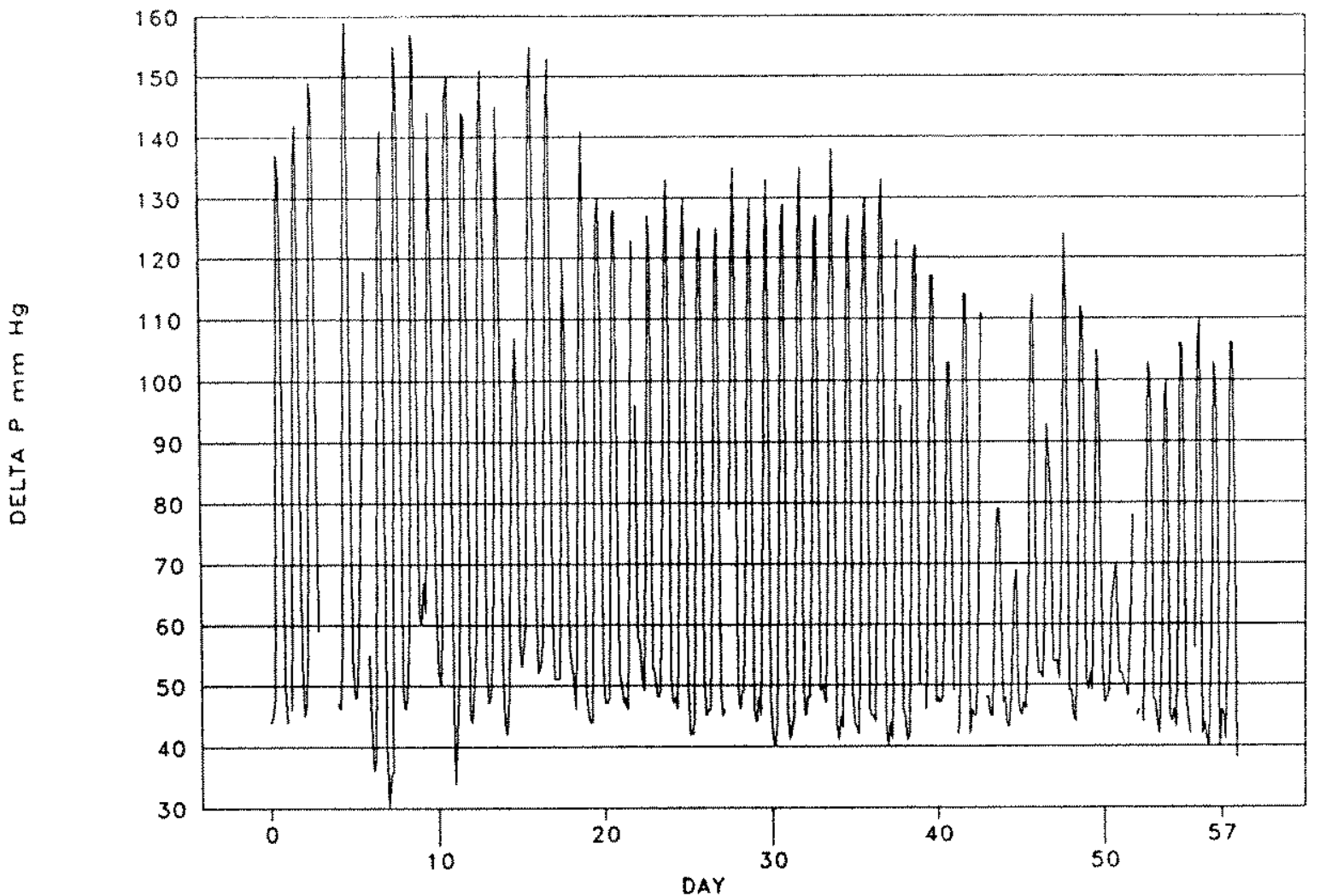

Figure 2.3.70. Uncorrected delta $\mathrm{P}$ (mmHg) measured at Rkm 4.8 by a Common sensing tensionometer during rainbow trout bioassays below Afterbay Dam on the Bighorn River, Montana, July September 1988 .

30-50 mmHg on a diurnal basis may have prevented immediate morta11ty.

More than $97 \%$ of all visible symptoms of GBT in this rainbow trout fry bioassay was popeye. Other GBT symptoms included ventral surface, buccal cavity, and external bubbles.

One difference between the brown and rainbow trout bioassays at $\mathrm{Rkm} 4.8$, July - September 1988, was that rainbow trout were 2-28 $\mathrm{mm}$ smaller. Mean size of mortalities was $25 \mathrm{~mm}$ for rainbow fry from both $\mathrm{Rkm} 2.4$ and 8.0 , and $27 \mathrm{~mm}$ for those from $\mathrm{Rkm} 14.5$. This does not, however, explain the higher mortality of rainbow trout fry since laboratory bioassays (Figures 2.1 .52 and 2.1.53) showed that mortality of fry of both species increased with size. A more plausible explanation relates to the brown trout fry 
having been exposed to ambient gas levels at the three rearing sites for 6 months before being moved to Rkm 4.8. During this period, GBT-related mortality ranged from 22-67\% (Figures 2.3.60 and 2.3 .66$)$. Surviving brown trout fry used in the July September bioassays may have been more resistant to high dissolved gas levels compared to the rainbow trout fry which had been exposed to no previous selective pressure.

\section{Summary}

Although results of field bioassays on trout fry were somewhat inconsistent and difficult to interpret, they demonstrated the effects of high dissolved gas supersaturation levels during different times of the year and provide a general range of delta $\mathrm{P}^{\prime} \mathrm{s}$ which cause substantial mortality. Several problems affected results. Depending on environmental conditions and dam operational patterns, daily Bouck gasometer measurements were often inadequate to describe hyperbaric conditions fry were exposed to. Large diurnal fluctuations in gas levels occurred at all locations at some time during the study. Continuous monitoring of gas levels at Rkm 0.6 and $\mathrm{Rkm} 4.8$ showed how non-uniform sampling times for point measurements would influence interpretation (Figure 2.3.71). Also point measurements may not reflect maximum hyperbaric pressures fry are exposed to. During some experiments, particularly in 1985, accumulation of metabolic wastes, bacterial gill disease, or cannibalism influenced survival. Also, fry did not always use the maximum compensating pressure available in holding boxes. Utilization of all or no hydrostatic pressure could change threshold levels as much as $18 \mathrm{mmHg}$ and account for 


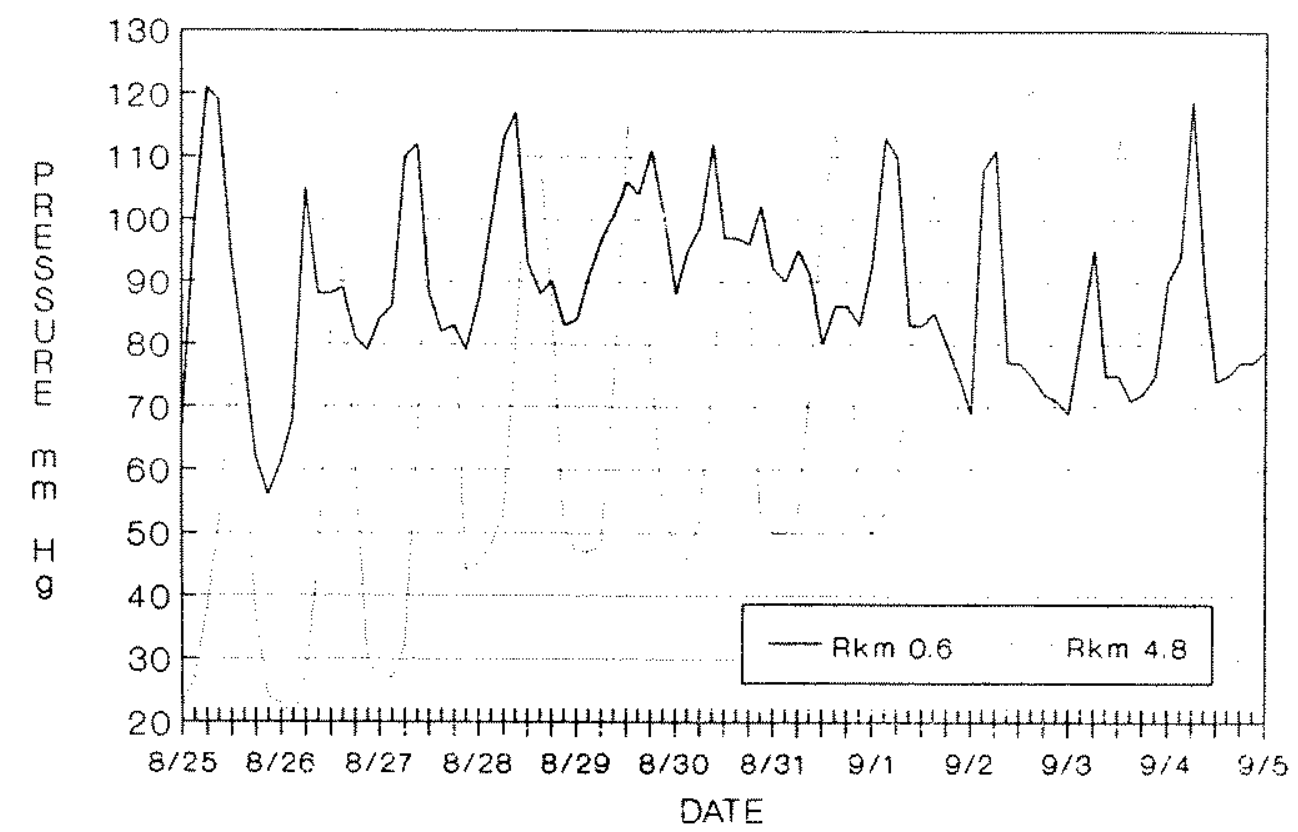

Figure 2.3.71. Corrected delta $\mathrm{P}(\mathrm{mmHg})$ measured by a Common Sensing tensionometer at Rkm 0.6 and 4.8 below Afterbay Dam on the Bighorn River, Montana, from July - September 1987.

most of the variation observed in some experiments.

Rainbow trout fry mortality was low in 1985 bioassays when gas levels were below or slightly above the threshold for bubble growth in environmental water. However, substantial gas-related fry mortality occurred in one group when gas levels measured were below the cardiovascular system threshold. Another group showed a direct response in mortality when gas pressure rose to 120 mmHg. Relatively high mortality rate occurred among fry from all three early life history sites when moved upstream to Rkm 0.6 , suggesting that level of prior exposure had no observable effect on susceptibility to GBT.

The bioassays from 1987 provided general guidelines on safe delta $P$ levels for fry. Relatively high delta P's, about 120 mmHg during the first set of 1987 brown trout fry bioassays, 
produced moderate mortality. The one exception (Figures 2.3 .32 and 2.3 .33 ) had low mortality and corresponded to the lowest mean delta $P, 111 \mathrm{mmHg}$. If all hydrostatic pressure was utilized by the fry, the delta $P$ could have dropped to $95 \mathrm{mmHg}$. The Rkm 14.5 group transferred to $\mathrm{Rkm} 0.6$ had the highest cumulative mortality and mean delta $\mathrm{P}, 122 \mathrm{mmHg}$ (Figures 2.3 .42 and 2.3.43). The mean hydrostatic pressure for the group was $11 \mathrm{mmHg}$, thus the mean delta $\mathrm{p}$ fry were exposed to could have been as low as $111 \mathrm{mmHg}$. During the second set of experiments in 1987, mean delta P's for fish hatched at $\mathrm{Rkm} 2.4$ and 8.0 increased 10 to $19 \mathrm{mmHg}$, which resulted in most hyperbaric pressures approaching or exceeding $130 \mathrm{mmHg}$ and associated high fry mortality. The groups with high

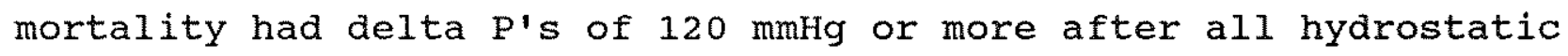
pressure was used for compensation. This group of tests also showed that, in general, the longer delta $\mathrm{P}$ was above the cardiovascular system threshold, the higher the cumulative mortality.

The first set of rainbow trout fry bioassays in 1987 utilized fry that were approximately $25 \mathrm{~mm} \mathrm{TL}$. Mortality of fry held at the two lower study sites ( $\mathrm{Rkm} 8.0$ and 14.5 ) was delayed until after gas levels permanently dropped below the cardiovascular system threshold. Mortality at Rkm 2.4, however, corresponded to a period when delta $\mathrm{p}$ rose above the cardiovascular system threshold. The delayed mortality at the lower sites may have been related to the greater oxygen component of TGP at these sites. Although cumulative mortality at both Rkm 8.0 and 14.5 was over $70 \%$, mean delta $\mathrm{P}^{\prime} \mathrm{s}$ were quite different, 124 and $110 \mathrm{mmHg}$, respectively. The mean delta $\mathrm{P}$ at Rkm 2.4 was 
only $3 \mathrm{mmHg}$ lower than at Rkm 14.5. GBT symptoms were almost exclusively popeye at all three sites.

The second set of rainbow trout bioassays resulted in relatively low mortality from $\mathrm{GBT}$ at $\mathrm{Rkm} 0.6,2.4$, and 8.0 . Mean delta P's varied from 78-89 mmig and were equal to or below the mean threshold for bubble growth at all locations. This experiment suggests that hyperbaric pressures that approximate or are below the environmental water threshold pose a minimal risk to rainbow trout fry.

Larger fry $(60 \mathrm{~mm})$ were used in the third rainbow trout bioassay in 1987. When gas pressure remained at approximately the threshold for bubble growth in environmental water and the mean delta $\mathrm{P}$ was $82 \mathrm{mmHg}$, mortality was low. Mortality increased slightly when delta $\mathrm{P}$ was greater than the environmental water threshold but below the cardiovascular system threshold. This corresponded to a mean delta $\mathrm{P}$ of $103 \mathrm{mmHg}$. High mortality was encountered when the mean delta $\mathrm{P}$ reached $114 \mathrm{mmHg}$ and individual measurements were regularly above the cardiovascular system threshold. As size increased, predominant GBT symptoms changed from popeye to external bubbles on body parts.

Results of the first brown trout bioassay in 1988 reinforced the previous year's findings. Mean delta $\mathrm{p}$ of more than $120 \mathrm{mmHg}$ resulted in substantial mortality at $\mathrm{Rkm} 2.4$ and 8.0 , but lower mortality at Rkm 14.5. This experiment also suggested the magnitude that delta $\mathrm{P}$ rose above the cardiovascular system threshold was a controlling factor of mortality just like the amount of time gas levels were above the threshold. Popeye remained the principal GBT symptom in 1988 among the fry when 
mean lengths were $22-26 \mathrm{~mm}$. Yolk sacs with internal bubbles were observed in large numbers among 1988 test fish. The low mortality among brown trout fry in the second 1988 experiment (compared to rainbow trout fry) may have resulted from using individuals which had survived 6 months of exposure to ambient gas levels at $\operatorname{Rkm} 2.4,8.0$ and 14.5 and thus were more tolerant to high dissolved gas pressures.

Field bioassays showed that swimming or feeding ability of fry can be affected by sub-lethal levels of hyperbaric pressure. Excess buoyancy in as many as $50 \%$ of the fry at Rkm 0.6 was documented. Free-ranging fry with equilibrium problems would have greatly reduced probability of long-term survival.

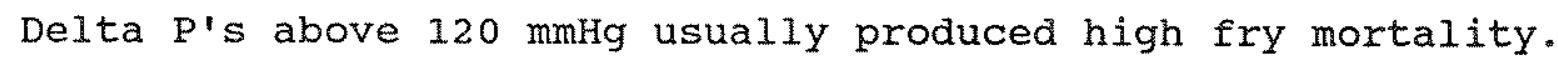
Mortality often occurred at mean delta $P^{\prime} \mathrm{s}$ above $110 \mathrm{mmHg}$. In one instance, high rainbow trout fry mortality was associated with a mean delta $\mathrm{P}$ of $114 \mathrm{mmHg}$. Our results demonstrate that the longer hyperbaric pressure is above the cardiovascular system threshold, the greater the cumulative mortality. Fry closer to Afterbay Dam are at the greatest risk, regardless of size or species. Delayed mortality may be a more common phenomena among younger, shorter fry. Delta $\mathrm{P}^{\prime} \mathrm{s}$ of 80-90 mmHg, and possibly up to $95 \mathrm{mmHg}$, pose minimal threat to fry. External GBT symptoms changed from mostly popeye (exopthalmia) on small fry to external bubbles on larger fry (> $35 \mathrm{~mm}$ TL). During 1987, fry were at risk primarily during June, July, and August, and again in November and December. Since brown trout fry emerge from the gravel earlier, they are larger than rainbow trout fry by June, when hydrostatic pressures increase in the Bighorn River. This 
larger size may improve survival of brown trout fry compared to rainbow trout fry since it allows them to occupy deeper water where compensating pressures are greater. Despite the inconsistencies of field bioassay results, high dissolved gas pressure appears to be the most important single factor affecting fry survival. 


\subsubsection{Adult symptoms, Frequency and severity Indices}

\subsubsection{3a Methods}

Gas pressures were measured as described in section 2.3.4. Incidence of gas bubble trauma and progression and duration of symptoms were monitored using a boom-mounted electrofishing system equipped with either a 110 or 220 volt rectifying unit. Electrofishing was conducted at night if capture efficiencies were low during daylight hours or if recreational fishing pressure was high. Approximately 150 brown trout and as many rainbow trout as possible were examined biweekly or monthly for external symptoms of gas bubble trauma. Lengths, weights, sex, missing fins and the presence of hooking scars were recorded. A GBT rating system was used to categorize fish into one of four groups:

category

0 - No visible external symptoms.

1 - Minor symptoms: symptoms normally restricted to a few bubbles on fins or a bubble in one eye. Fish in this category do not appear seriously stressed.

2 - Serious symptoms: Numerous bubbles present in fins; bubbles may also be found in the skin, inside the the mouth, on the opercle, or in one eye. Fish in this category appear stressed.

3 - Severe symptoms: Large numbers of gas bubbles are present; hemorrhaging and/or fungal growth may also occur and one or both eyes may be blind. These fish are severely debilitated, lethargic and have a poor condition factor. Chances for survival appear questionable.

A rating system was also developed to describe the severity of gas bubble trauma on each fin or body part. Parts exhibiting GBT symptoms were assigned one of the following codes: 


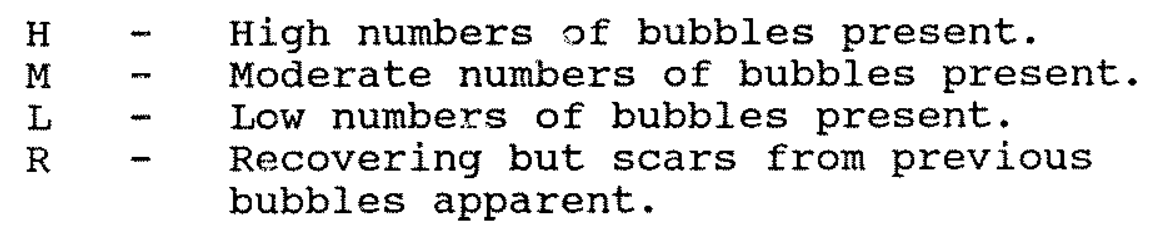

A total GBT rating that numerically summarized the severity and proportion of GBT on affected external surfaces of individual fish was developed. Fins or body parts with high, moderate, low and no visible GBT symptoms were assigned numerical values of 3 , 2,1, and 0 , respectively. Eighteen body parts were included in the total GBT rating, resulting in potential severity values ranging from 0 to 54 .

Two thousand and four trout in the upper $6.1 \mathrm{Rkm}(3.8 \mathrm{miles})$ of the Bighorn River were marked with numbered Floy t-tags that were treated with algacide. subsequent recaptures of tagged fish allowed us to evaluate progression or remission of GBT symptoms.

\subsubsection{2b Results and Discussion}

Brown trout made up $81 \%$ of the 25,420 trout sampled during gas bubble trauma (GBT) incidence surveys, 1985-1988 (Appendix E). Annually, brown trout comprised $70-87 \%$ of the fish sampled. Forty-six percent of the brown trout were $>356 \mathrm{~mm}$ in length compared to $59 \%$ of the rainbow trout.

Overall, 18,7 and $3 \%$ of the brown trout and 7,7 and $1 \%$ of the rainbow trout sampled in sections 1,2 and 3 , respectively, had external symptoms of GBT. Incidence among large brown trout in these sections was 25,13 and $4 \%$, compared to 15,19 and $2 \%$ for large rainbow trout (Appendix E, Tables 22-32 and 34-39). Numbers of mountain whitefish collected during incidence 
surveys (April 1986 - November 1987) were always small, ranging from 0 to 133 on any one sampling date. of 322 whitefish examined in 1986, only four had GBT symptoms; 2 of 60 in section 1, 2 of 183 in section 2 and none of 79 in section 3 (Appendix $E$, Table 33). Three of the four fish with symptoms were collected 1-3 April; and the remaining fish was collected on June. only two of 733 whitefish examined in 1987 had symptoms of GBT, one in each of sections 2 and 3 (Appendix E, Table 40). From these data, it could be concluded that mountain whitefish are less susceptible to gas supersaturation than trout. However, observations during snorkel surveys indicate that whitefish use deeper water and thus reduce their exposure to excessive gas pressures. Fickeisen and Montgomery (1975) found mountain whitefish to be less tolerant of gas supersaturation than other species tested, including trout. The whitefish population in the Bighorn River may, in fact, be limited by high gas levels.

Gas bubble trauma incidence was not uniformally distributed in space or time (Figures $2 \cdot 3 \cdot 72-2 \cdot 3 \cdot 75$ ). Incidence of GBT in the brown trout population was always largest in the $1.9 \mathrm{~km}$ (section 1) immediately below Afterbay Dam (Figure 2.3.72 and $2.3 .73)$. Maximum incidence ranged from $28 \%$ in 1985 to $65 \%$ in 1986. Although GBT incidence diminished with distance downstream, the pattern of occurrence through time was similar in each section. The magnitude of incidence among all brown trout varied by more than two-fold in the upper two sections between years, with percent GBT incidence among large trout (> $356 \mathrm{~mm}$ ) almost always greater than for all size groups combined. Maximum incidence among large brown trout in section 1 ranged from $68 \%$ in 

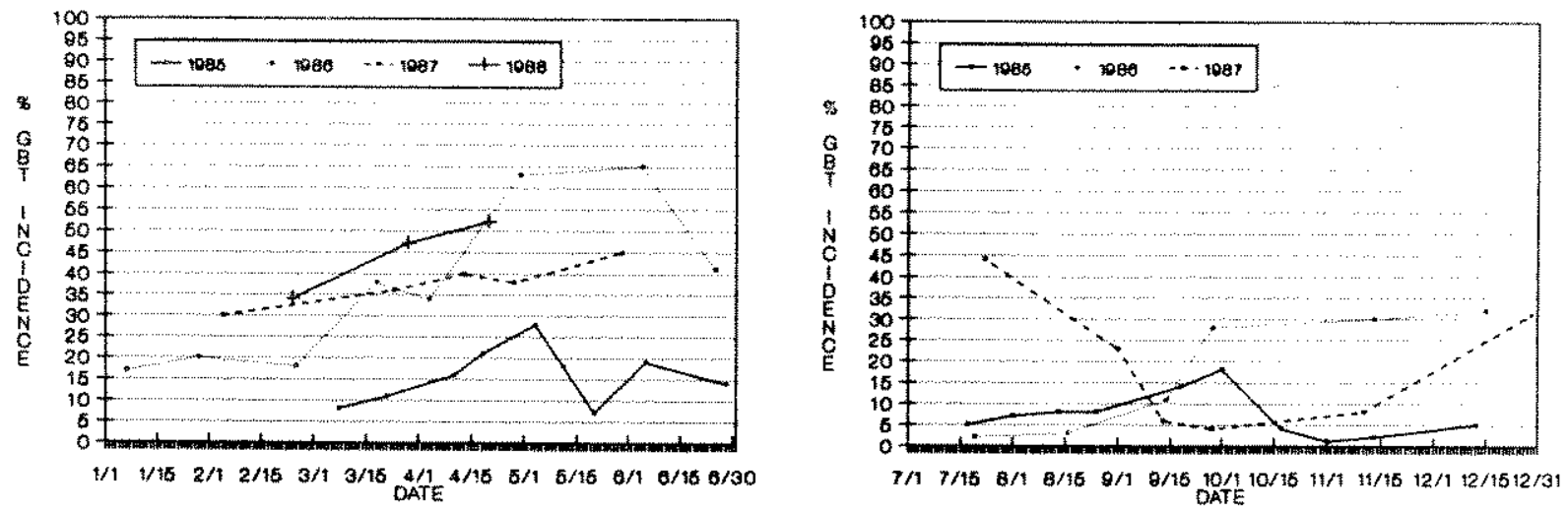

BECTION 1
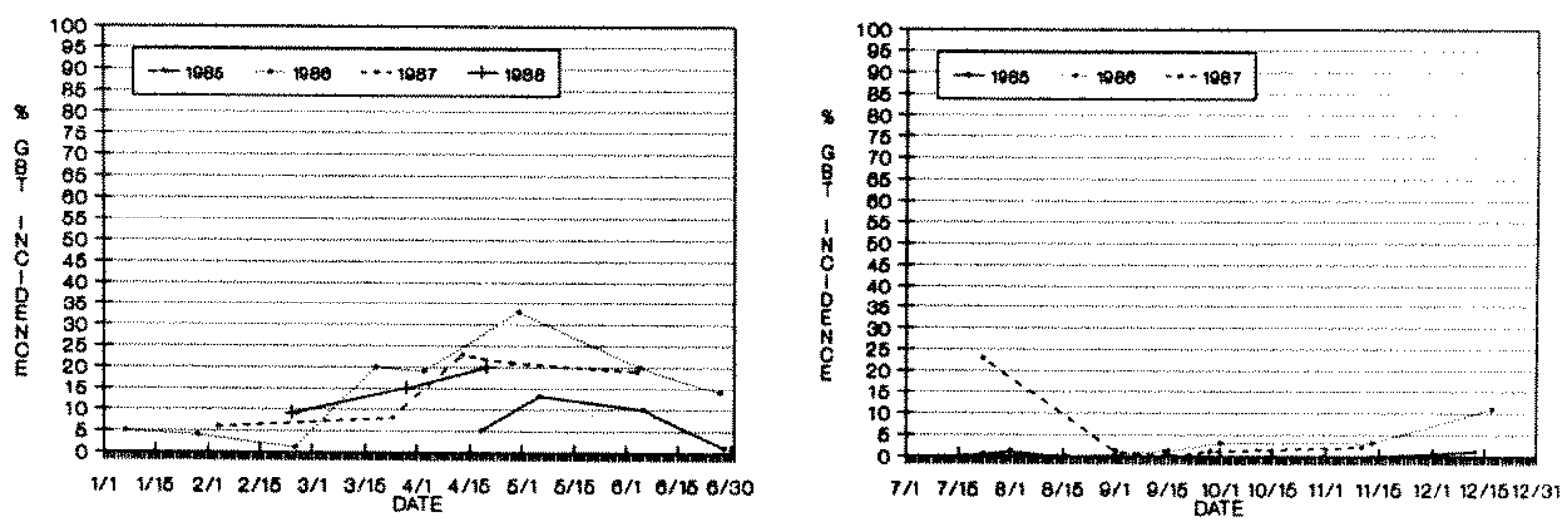

8ECTION 2
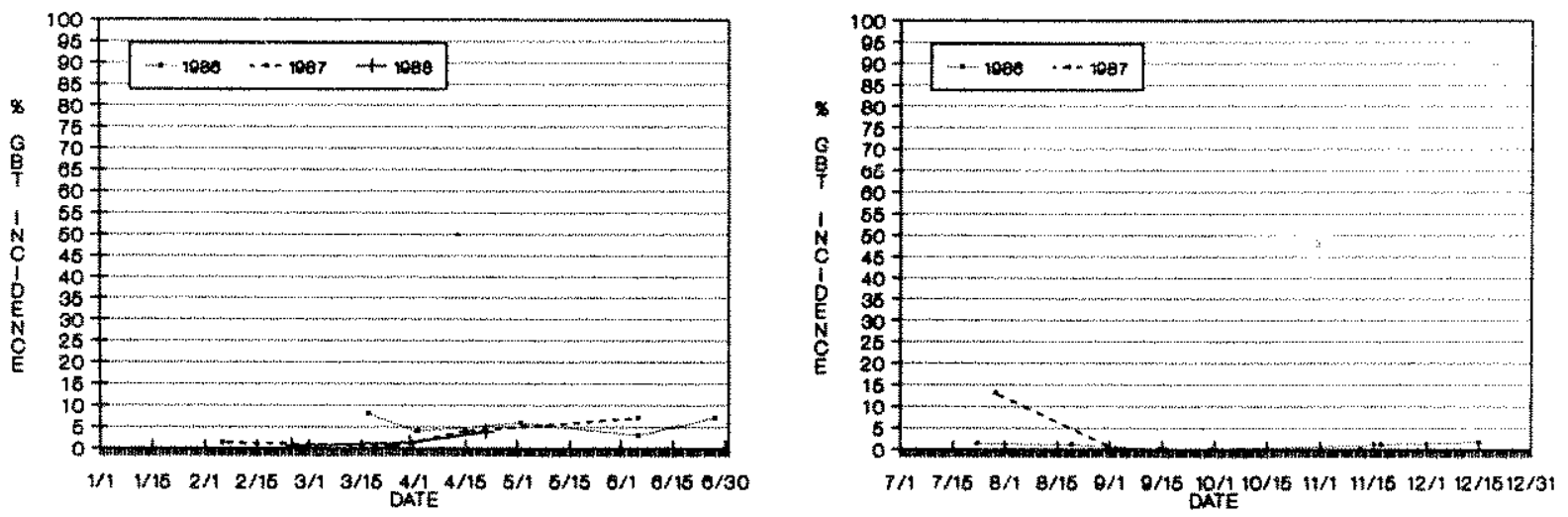

SECTION 3

Figure 2.3.72. Percent incidence in gas bubble trauma (GBT) among brown trout in sections 1,2 and 3, Bighorn River, March 1985 April 1987. 

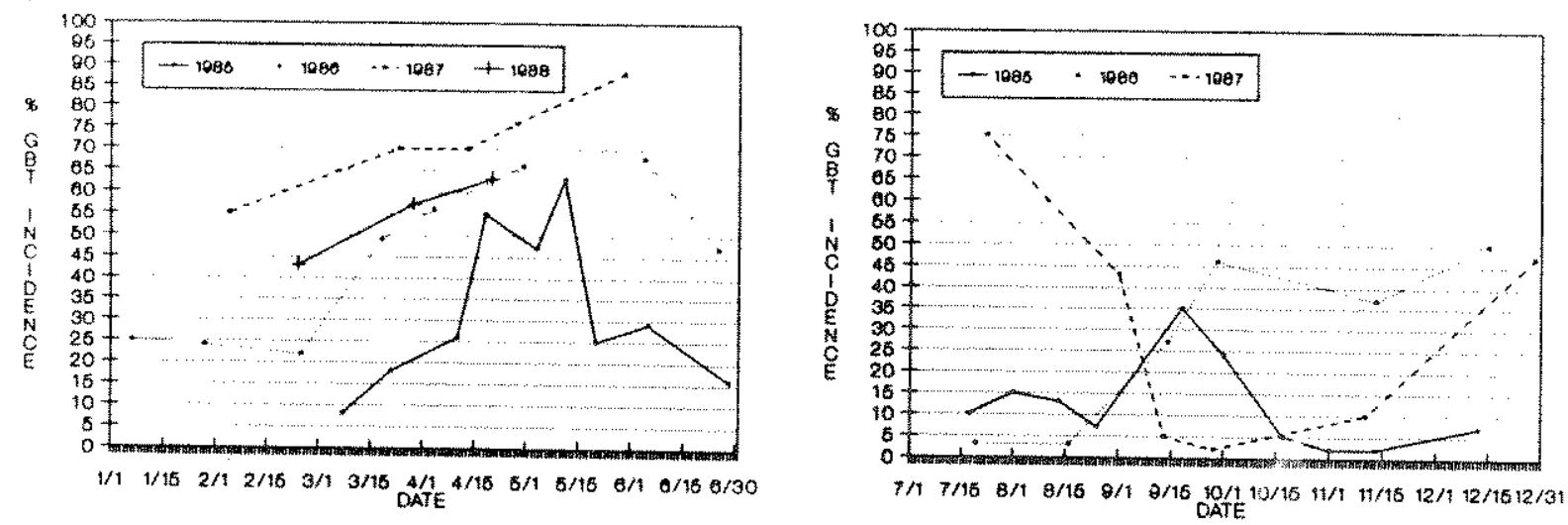

8ECTION 1
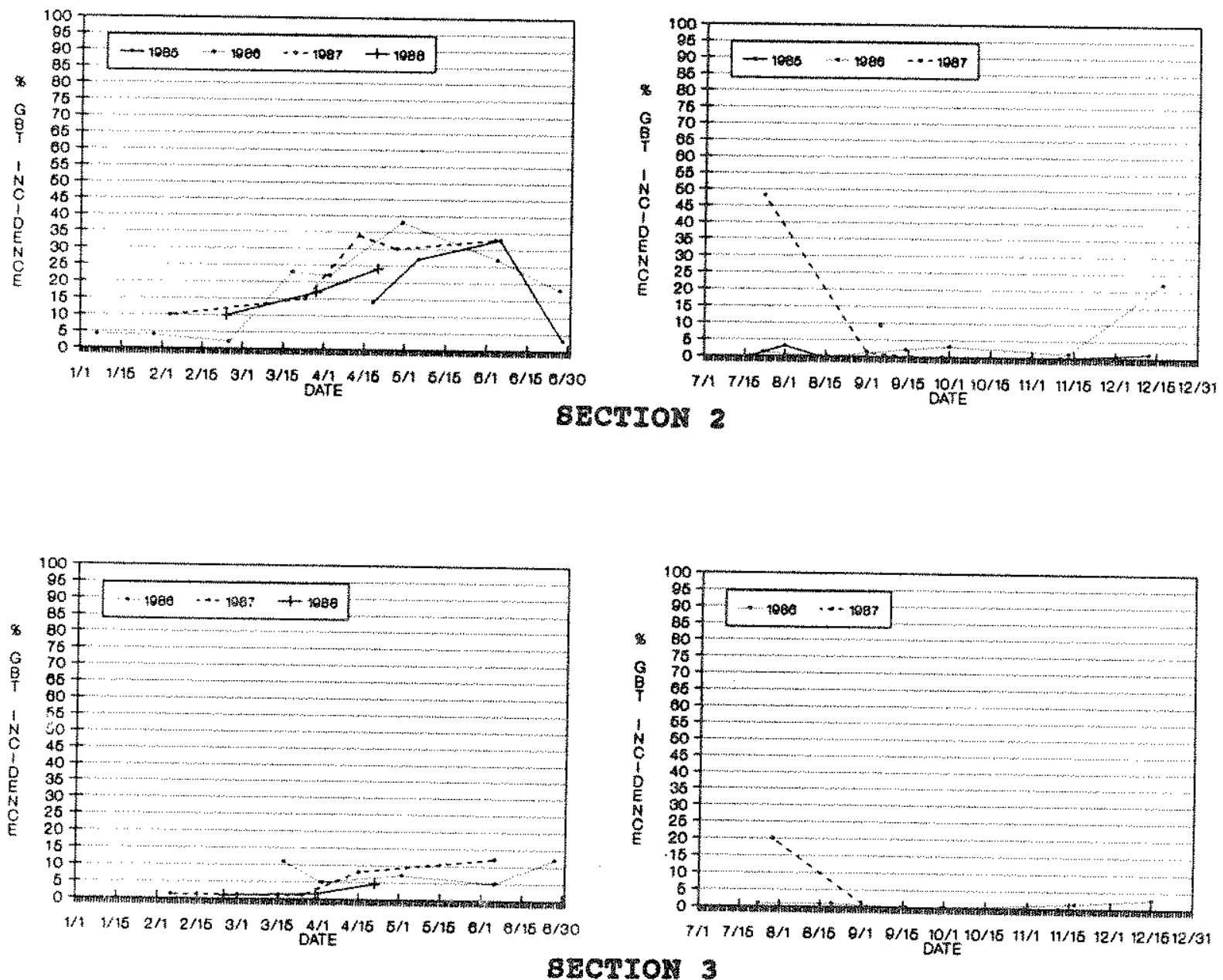

Figure 2.3.73. Percent incidence of gas bubble trauma (GBT) among brown trout $\geq 356 \mathrm{~mm}$ in total length in sections 1,2 and 3. Bighorn River, March 1985 - Apri1 1987. 

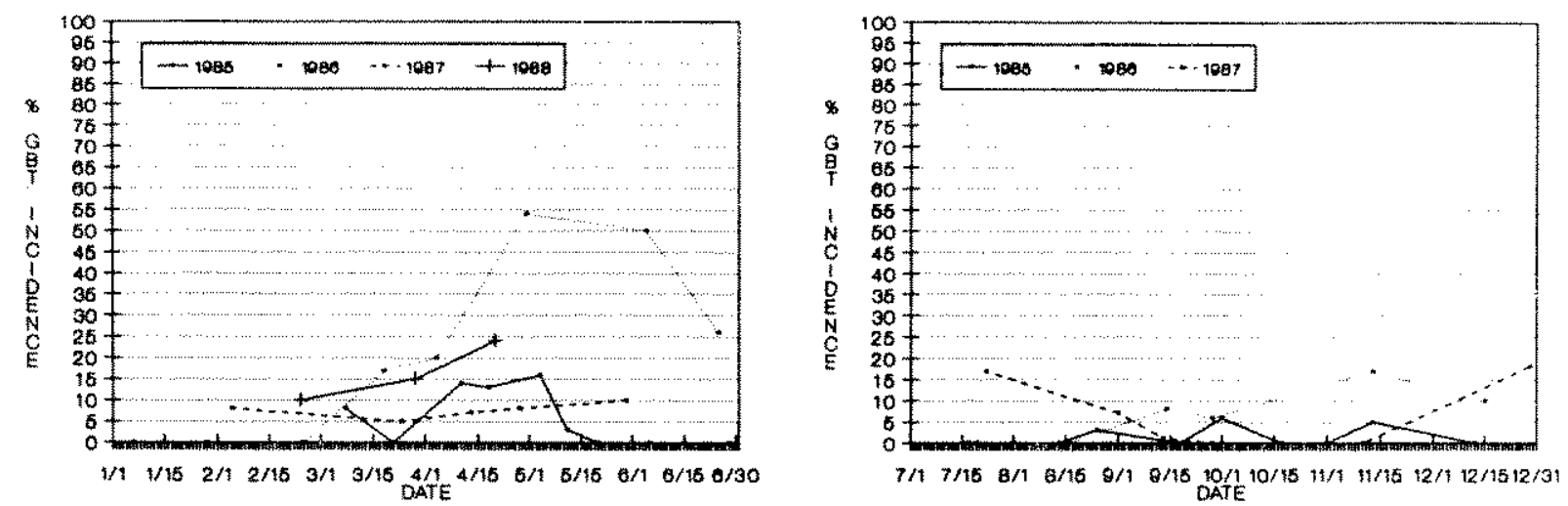

\section{BECTION 1}
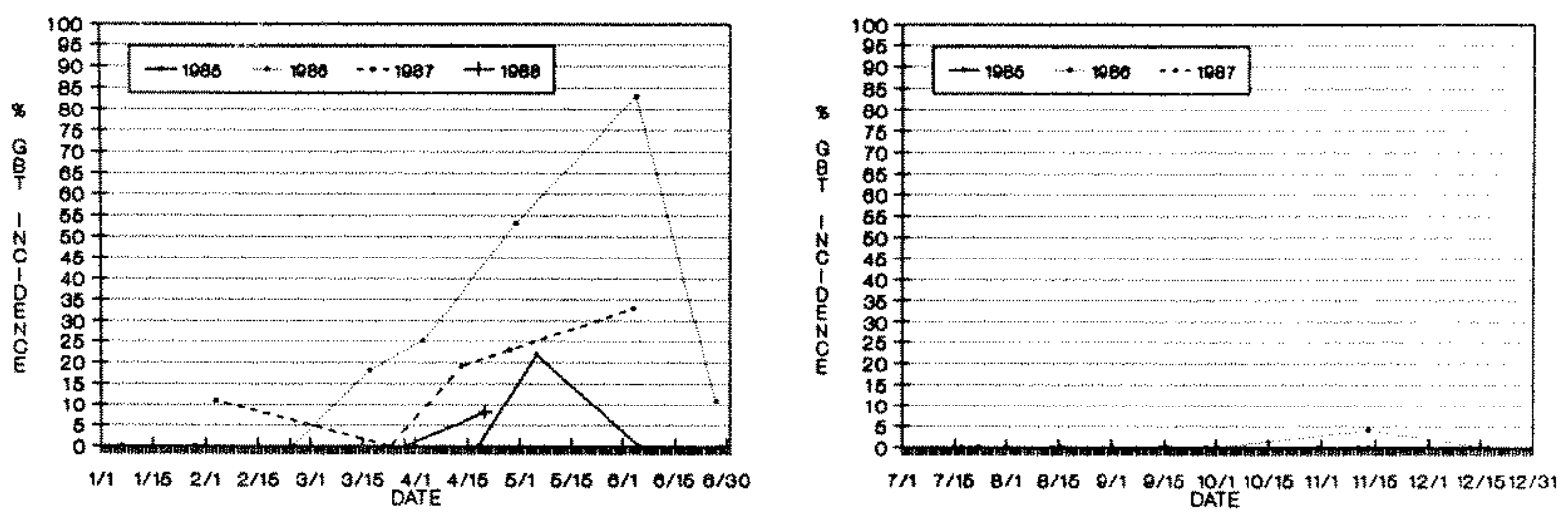

\section{SECTION 2}
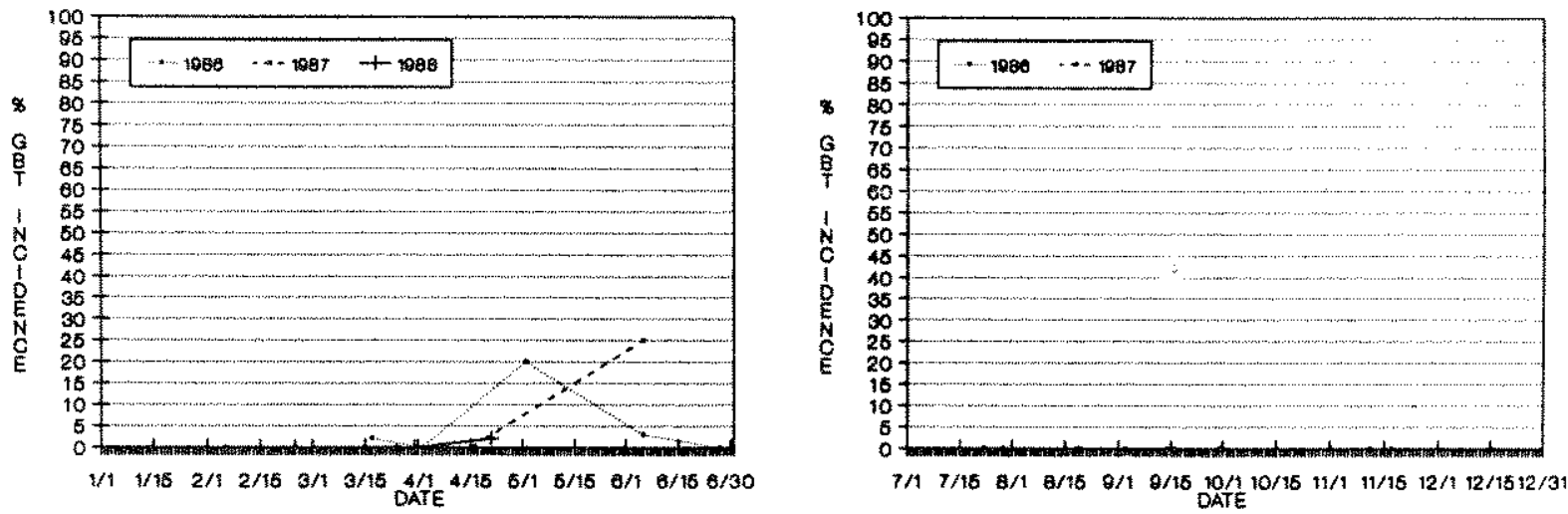

BECTION 3

Figure 2.3 .74 . Percent incidence of gas bubble trauma (GBT) among rainbow trout in sections 1, 2 and 3, Bighorn River, March 1985 - April 1987. 

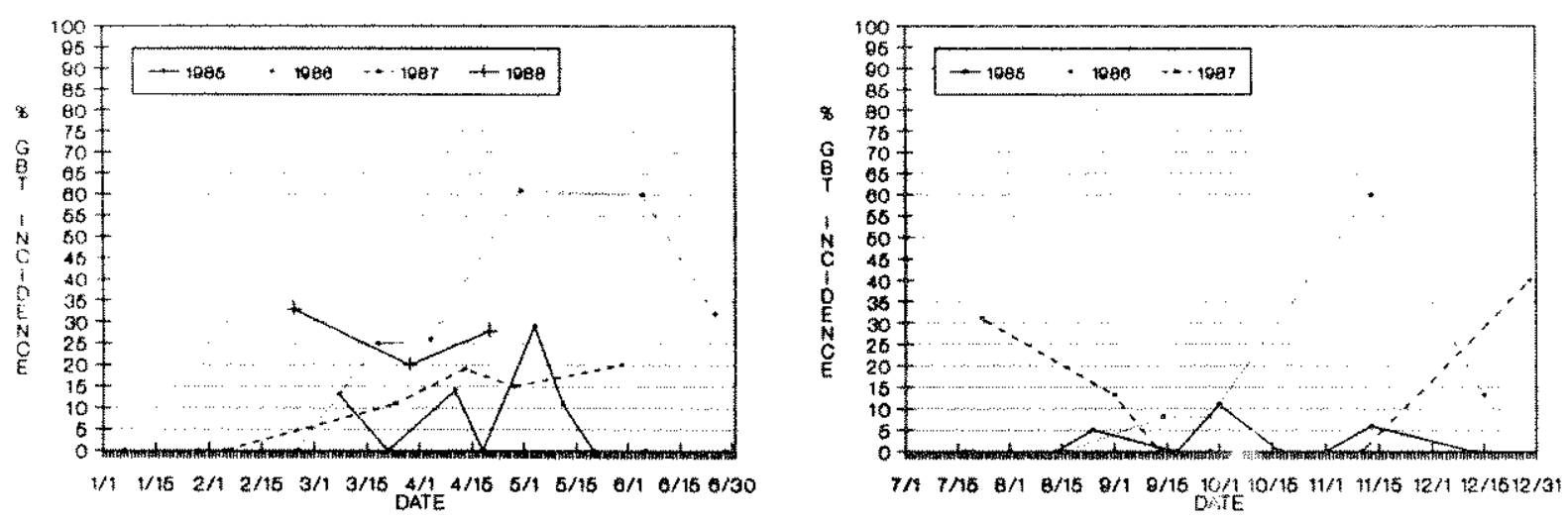

SECTION 1
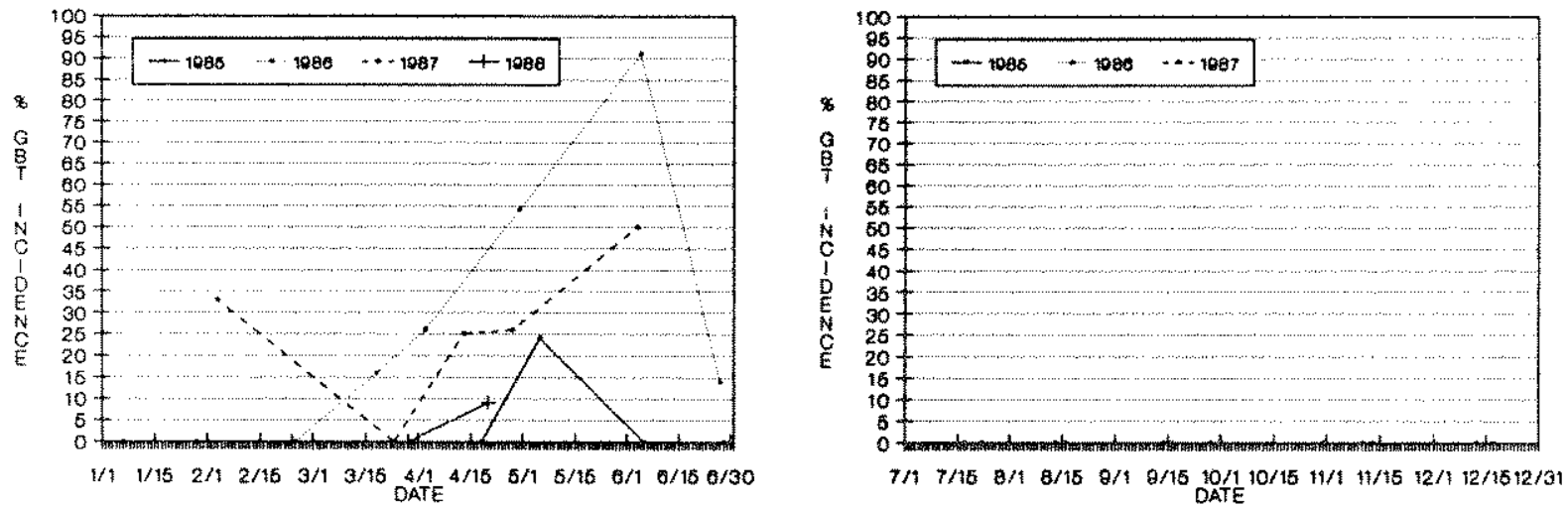

SECTION 2
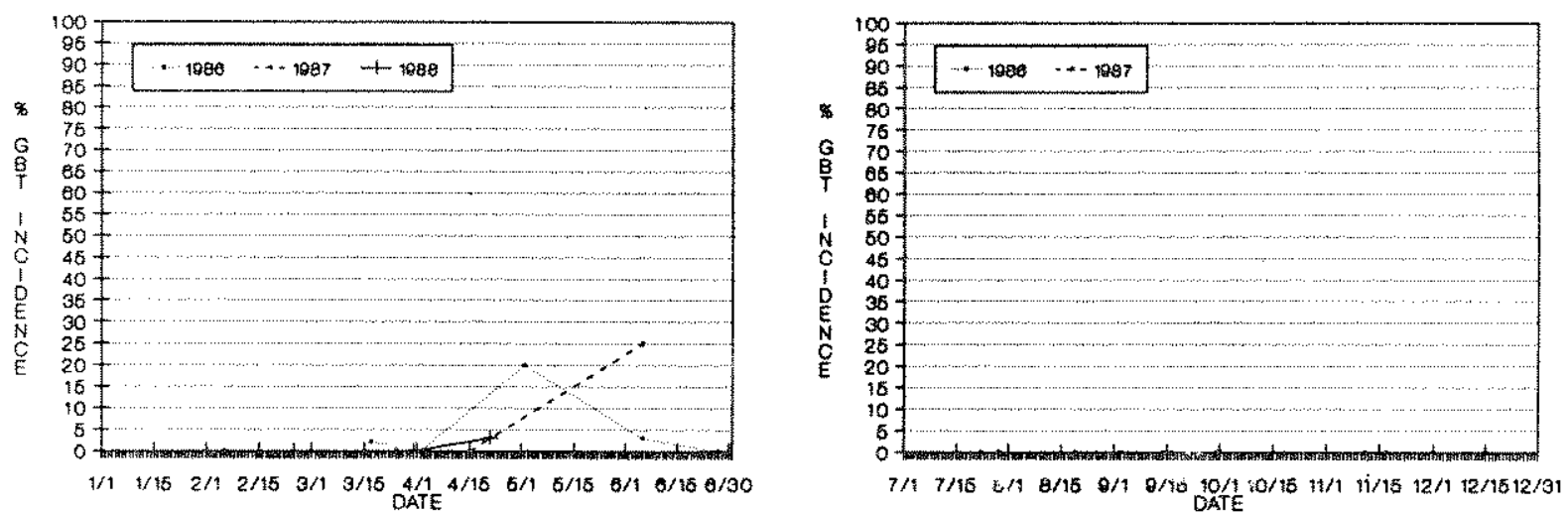

SECION 3

Figure 2.3.75. Percent incidence in gas bubble trauma (GBT) among rainbow trout $\geq 356 \mathrm{~mm}$ in total length in sections 1,2 and 3, Bighorn River, March 1985 - April 1987. 
1986 to $88 \%$ in 1987.

peak incidence of GBT in brown trout usually occurred between mid-April and early June. The highest incidence occurred in 1986, which was aiso the highest flow year (Figures 2.3.72 and 2.3.73). Although incidence usually increased as flow increased, incidence pattern was not directly related to discharge. For example, when flows were about 1,000 cfs lower in March and April 1988 (when GBT monitoring was discontinued), GBT incidence was approaching 1986 levels (Figure 2.3.72 and 2.3.76). In 1986, incidence increased with flow during the March - May period. In June, however, incidence declined dramatically when discharge increased more than $60 \%$.

A second, smaller peak in GBT incidence among brown trout occurred in late september or early october in 1985 and 1986 when flow increased and at the end of December, 1987 (Figures 2.3.72 and 2.3.73). Only in 1986 was the late-year increase seen in the two lower sections. The increase in GBT incidence during both spring and fall was apparent in all size groups. However, incidence among the larger size groups (> $356 \mathrm{~mm}$ ) always increased more quickly.

Highest GBT incidence and pattern of incidence among rainbow trout occurred at about the same time as in brown trout, but usually in a smaller percentage of the population (Figures 2.3.74 and 2.3 .75$)$. The exception was in spring in sections 2 and 3 where incidence among rainbow trout usually exceeded that of brown trout. In section 2, spring 1986 incidence among rainbow trout was more than $60 \%$ larger than among brown trout. A similar pattern was apparent when comparing large (>356 mm) rainbow and 


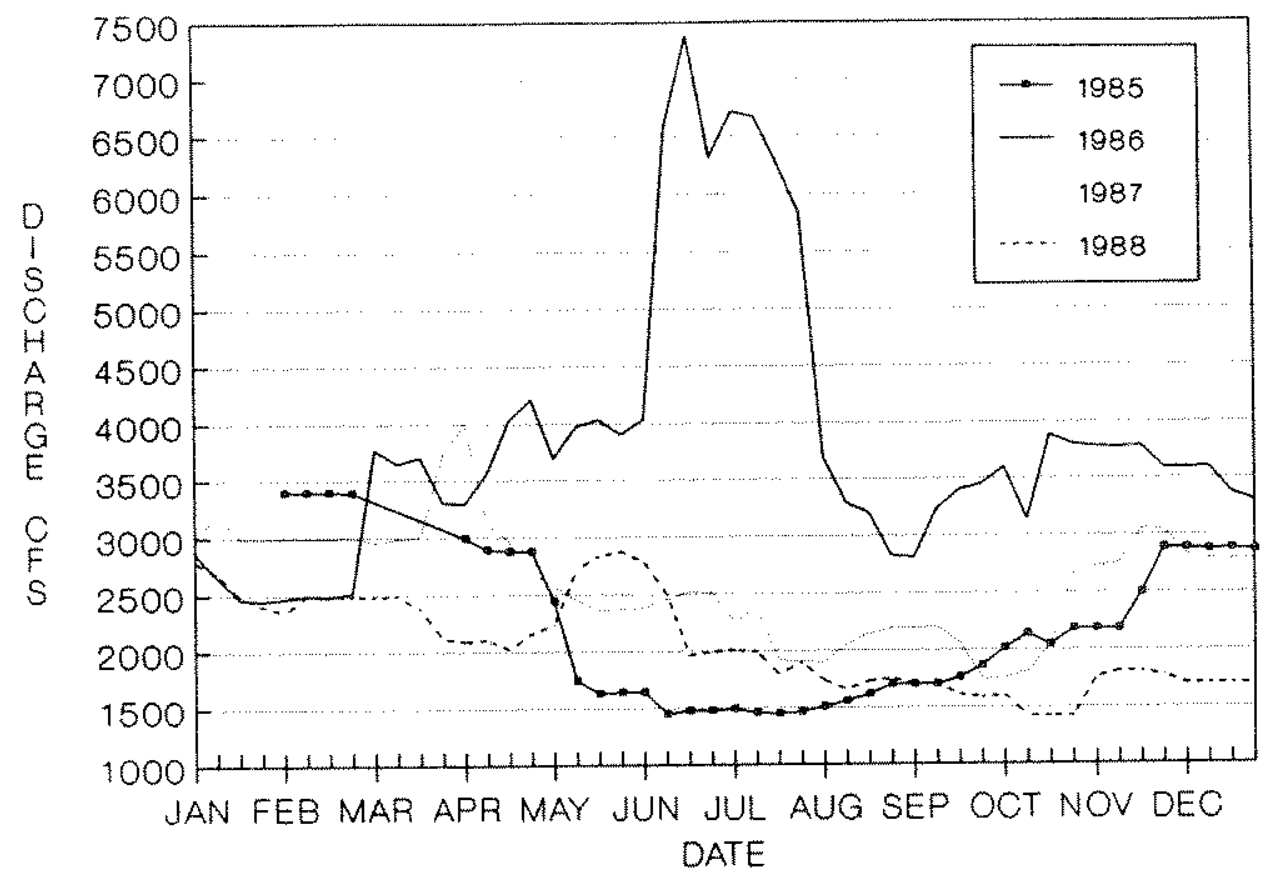

Figure 2.3.76. Mean weekly discharge (cfs) of the Bighorn River below Afterbay Dam measured at the $S t$. Xavier gagehouse from 1985-88.

brown trout. Also, incidence among large rainbow trout exceeded that of large brown trout by about $20 \%$ in mid-November of that year (Figures 2.3 .73 and 2.3 .75 ). Maximum incidence among large rainbow trout in section 2 ranged from $25 \%$ (1985) to 90\% (1986). Highest incidence of GBT among rainbow trout in all sections coincided with spawning periods. Spawning begins in early April and continues until about the third weex of June (Sanborn 1990). In 1986, peak incidence among rainbow trout in section 1 occurred before major increases in discharge or total gas pressure and was probably related to movement into shallow spawning areas.

Incidence in the middie reach followed a pattern similar to brown trout in sections 1 and 2 , but peak incidence was always higher. This higher GBr incidence was probably related to 
rainbow trout moving into two high-use spawning areas located in shallow side-channels. Sanborn (1990) reported that mean depth of trout redds in the Bighorn River was about $41 \mathrm{~cm}$. Use of this depth would increase vulnerability of rainbow trout to GBT because of reduced hydrostatic pressure.

In late spring and early fall, GBT incidence among brown trout along the river's right bank in section 1 was consistently higher than along the left bank. For example, no incidence was detected for brown trout on 17 september 1985 along the left bank, whereas $35 \%$ of brown trout $>356 \mathrm{~mm}$ in length captured along the right bank (18 and 20 september) had GBT symptoms. Also, brown trout with serious and severe symptoms were collected almost exclusively along the right bank (Appendix F). Percent GBT incidence among rainbow trout was similar between banks. The difference in incidence between species is probably, in part, related to the greater affinity of brown trout to bank-associated habitats and to higher dissolved gas levels along the right bank in section 1 .

Gas bubble trauma symptom severity was most acute in the upstream section, diminishing downstream. severity of GBr symptoms among affected rainbow trout was less than for brown trout (Table 2.3 .21 ). No rainbow trout examined during the 3 year study had severe symptoms of GBT. This compares to severe symptoms in $4 \%$ of all brown trout and $6 \%$ of those $>356 \mathrm{~mm}$. Most rainbow $(82-100 \%)$ and brown trout $(72-100 \%)$ with GBT had minor symptoms. As many as $24 \%$ of the brown trout had serious symptoms compared to a maximum of $13 \%$ of the rainbow trout. Severity of symptoms increased with increased duration of exposure to high 


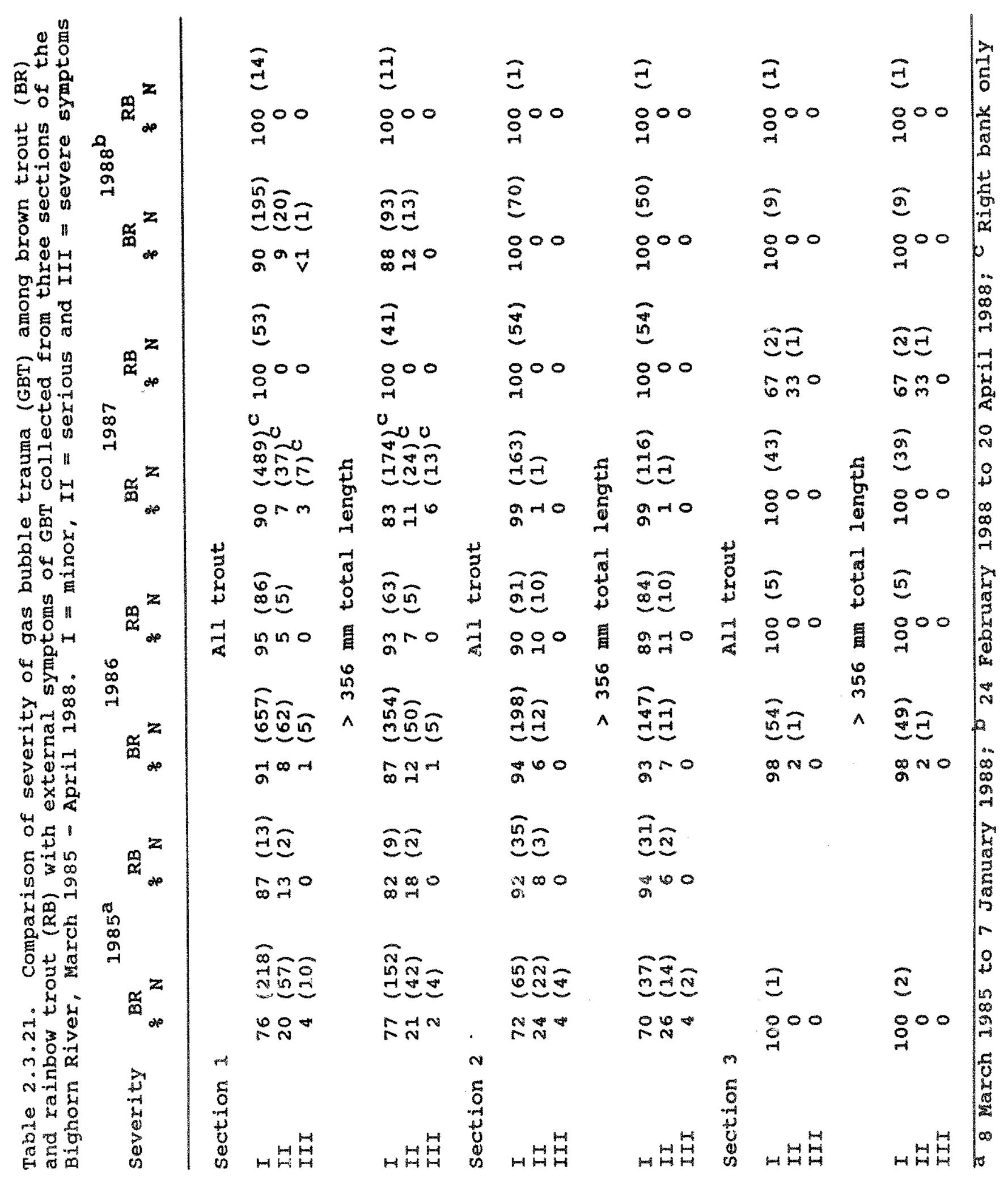


total gas pressure. Almost no rainbow trout and relatively few brown trout with GBT symptoms were found below Rkm 9.3. This corresponds with the area where percent nicrogen saturation dropped off during gas dissipation tests.

Gas bubble trauma incidence among trout in sections 1 and 2 followed the same general pattern as delta $P$ (Bouck gasometer measurements) (Figures 2.3 .77 and 2.3.78) until late spring of each year and then decreased, even though delta $\mathrm{p}$ remained high or increased (Figures 2.3.72-2.3.75). Incidence increased at a faster rate after delta $\mathrm{P}$ rose above the cardiovascular threshold for bubble growth (Figures $2 \cdot 3 \cdot 72-2 \cdot 3 \cdot 75$ ). Peak GBT usually occurred during periods of peak delta $\mathrm{P}$ with the exception of rainbow trout during the spawning period. The largest percent incidence occurred when delta $\mathrm{P}$ was above $120 \mathrm{mmHg}$.

The cardiovascular threshold represents the pressure at which many external symptoms of GBT begin to form. As delta $P$ decreased to near threshold levels, incidence dropped or leveled off. During prolonged periods when delta $\mathrm{P}$ remained below the cardiovascular threshold, symptoms of GBT were rare.

Although delta $P$ was often as high or higher in sections 2 and 3 compared to section 1 (Figures $2.3 .77-2.3 .79$ ), GBT incidence decreased downstream. For example, in 1987 delta P reached $190 \mathrm{mmHg}$ on July 15 in sections 2 and 3 compared to about $145 \mathrm{mmHg}$ in section 1. At the same time, incidence of GBT among brown trout was 45,28 and $14 \%$ in sections 1,2 and 3, respectively (Figure 2.3 .72 ). For rainbow trout, incidence was $17 \%$ in section 1 and no incidence was observed in sections 2 and 3 (Figure 2.3.74). This downstream decrease in GBT associated 

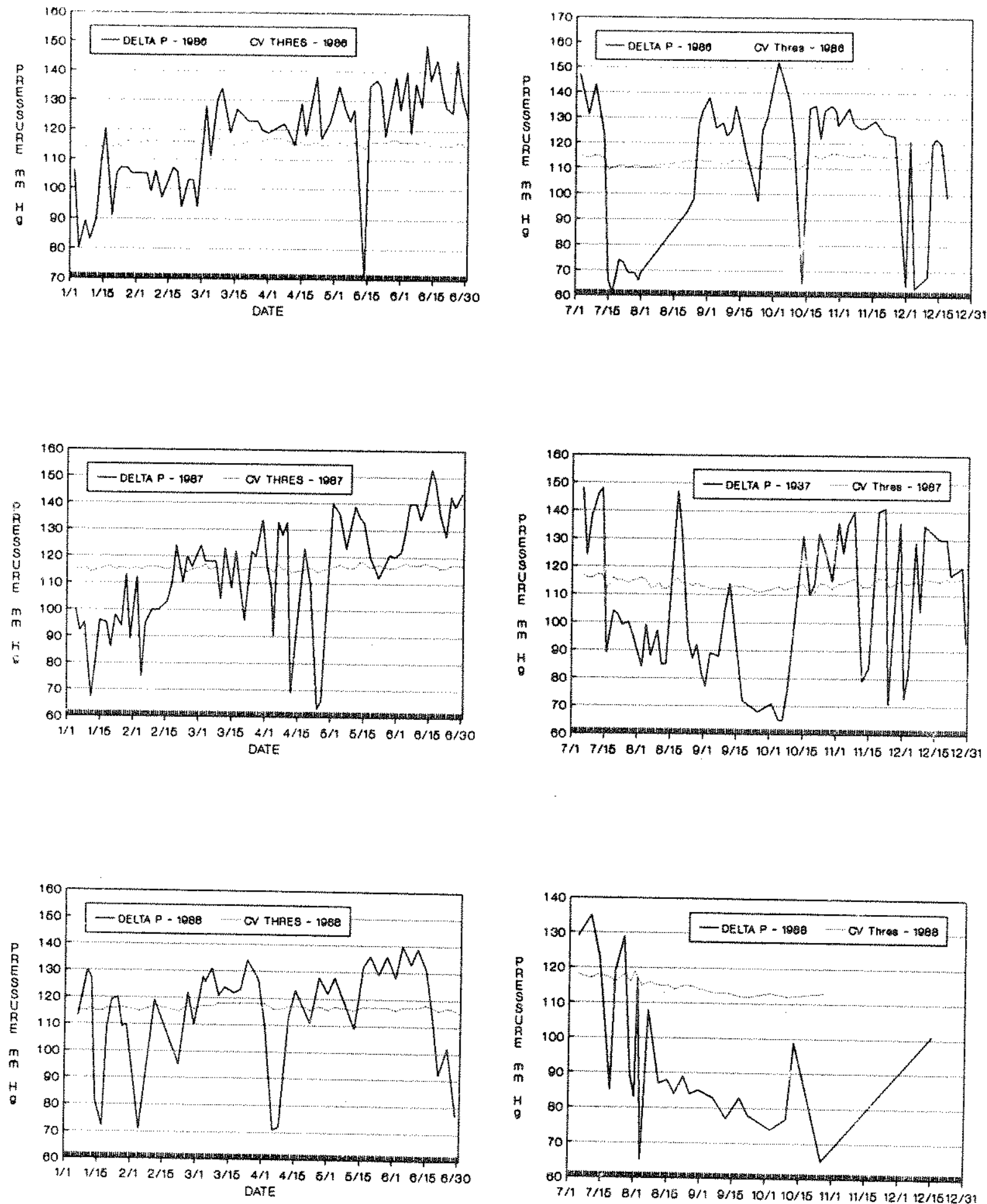

Figure 2.3.77. Bouck gasometer measurements of delta $p$ and the trout cardiovascular threshold for gas bubble trauma in section 1. Bighorn River, October 1985 - December 1988. 


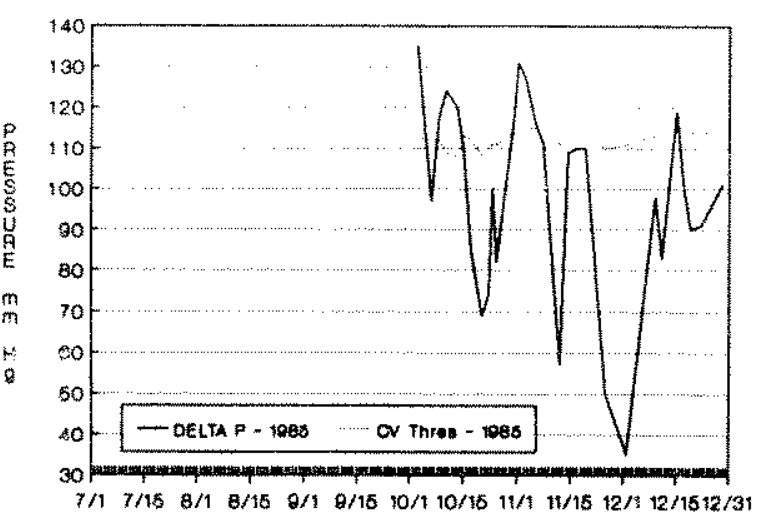

Figure 2.3.77. (Continued)

with equal or larger delta $P$ is due to increased threshold levels resulting from increasing oxygen partial pressure downstream due to increased abundance of aquatic macrophytes and associated photosynthesis. For example, in section 3, even though gas pressures were often higher than in section 1, they were usually below the cardiovascular threshold. Trout $0.5 \mathrm{~m}$ below the water surface in section 3 would not form bubbles in the vascular system during most periods in 1987, and those that remained in $1.0 \mathrm{~m}$ water would never suffer from any form of GBT (Figure 2.3.80). The only time external symptoms of GBT were observed on rainbow trout in section 3 was on 4 June (Figure 2.3.74), which also corresponded to peak rainbow incidence in section 2 . The three rainbow trout captured on 4 June that displayed signs of GBT represented $13 \%$ of all the rainbow and $25 \%$ of those $\geq 356 \mathrm{~mm}$. one had serious symptoms while the others had minor symptoms.

Large differences in GBT incidence occurred between years, even when delta $P$ and incidence patterns were similar. For example, while delta $\mathrm{P}$ pattern and levels were similar during the 

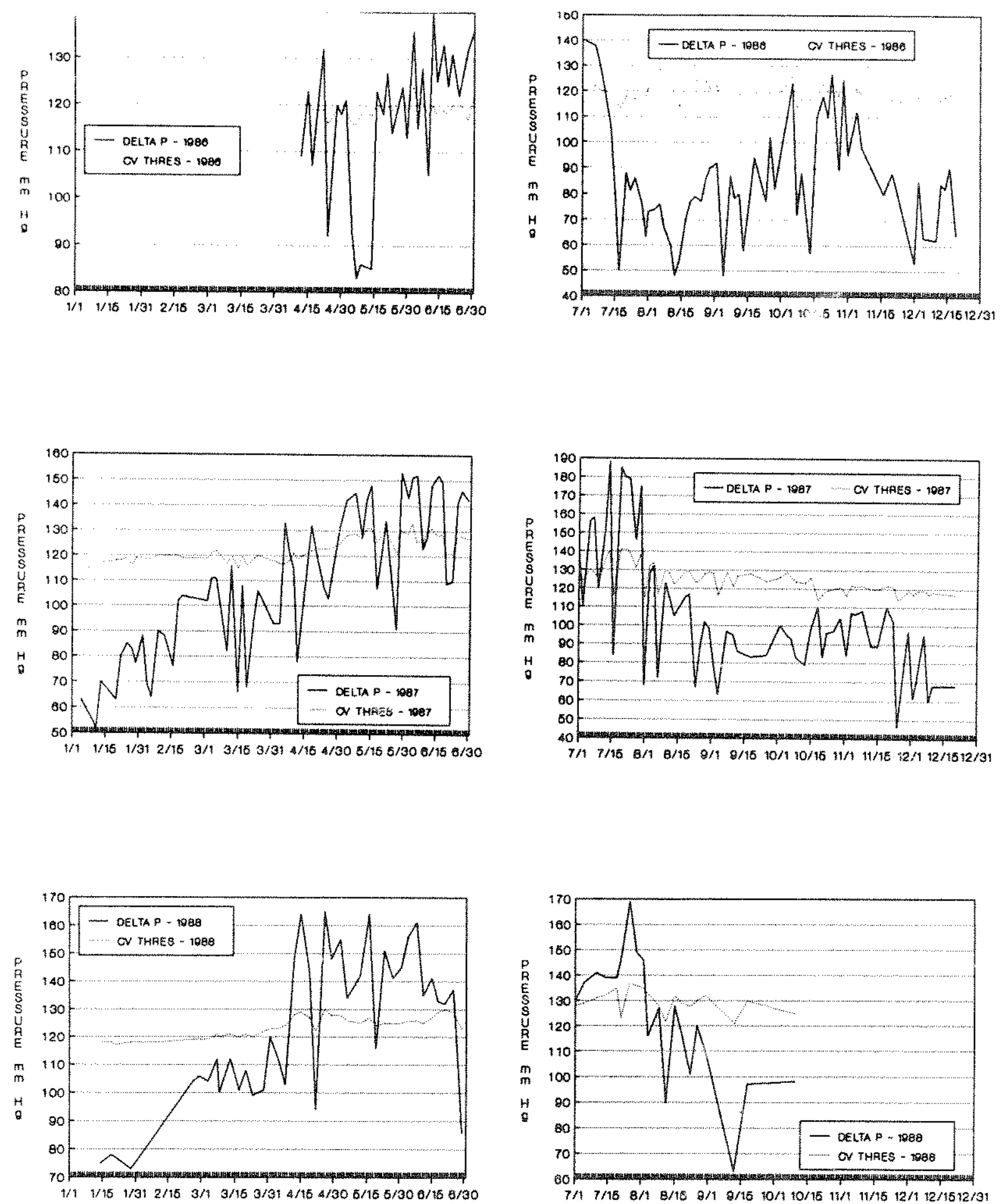

Figure 2.3.78. Bouck gasometer measurements of delta $P$ and the trout cardiovascular threshold for gas bubble trauma in section 2. Bighorn River, October 1985 - December 1988. 

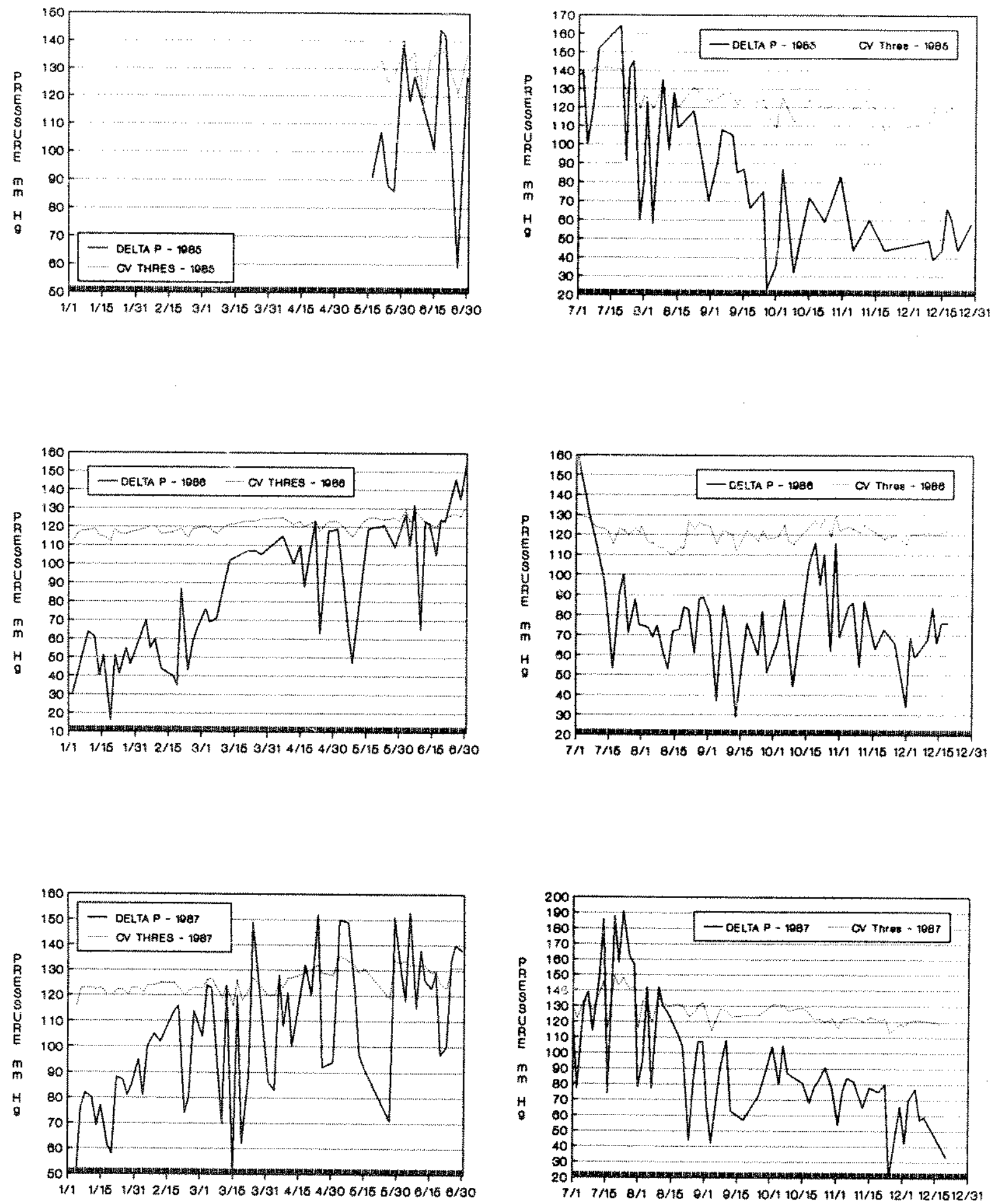

Figure 2.3.79. Bouck gasometer measurements of delta $p$ and the trout cardiovascular threshold for gas bubble trauma in section 3. Bighorn River, October 1985 - December 1988. 

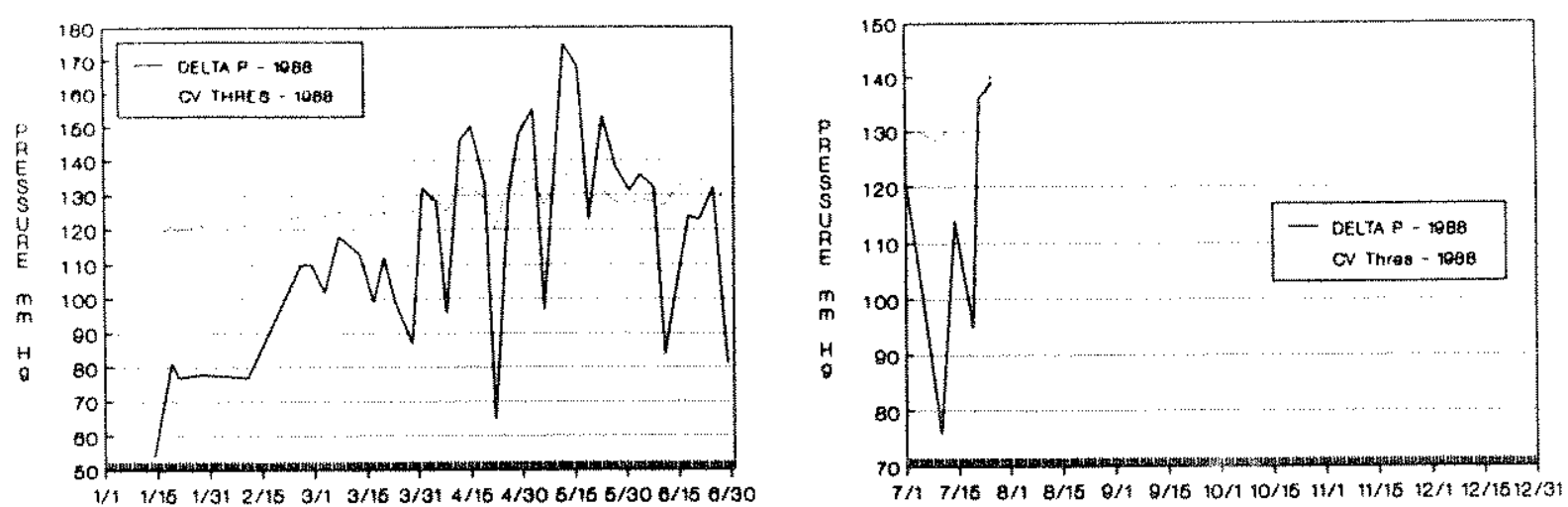

Figure 2.3.79. (Continued)

first half of 1986 and 1987, GBT incidence in the rainbow trout population in section 1 was as much as $45 \%$ greater in 1986 (Figures 2.3 .74 and 2.3 .76 ). Thus, other variables must influence susceptibility to GBT.

Two of the most important variables not accounted for are discharge patterns from Afterbay Dam and water stage (discharge). Discharge patterns and their effects are discussed in a later section. River stage is important because increased depth associated with increased discharge provides opportunity for hydrostatic compensation.

symptoms of GBT can occur only when total dissolved gas pressures (TGP) are greater than compensating pressures (PCOMP). Increased hydrostatic pressures (PH) that accompany higher flows may help fish compensate for increased gas levels. For example, the mean daily stage at the gagehouse (Rkm 0.6) for the period 29 Apri1 - 20 July 1986 increased from a low of about $963.4 \mathrm{~m}$ $(3160.89 \mathrm{ft})$ on 3 June to $964.0 \mathrm{~m}(3162.85 \mathrm{ft})$ on 18 June, and the mean barometric pressure for June was $681 \mathrm{~mm}$. This increased 


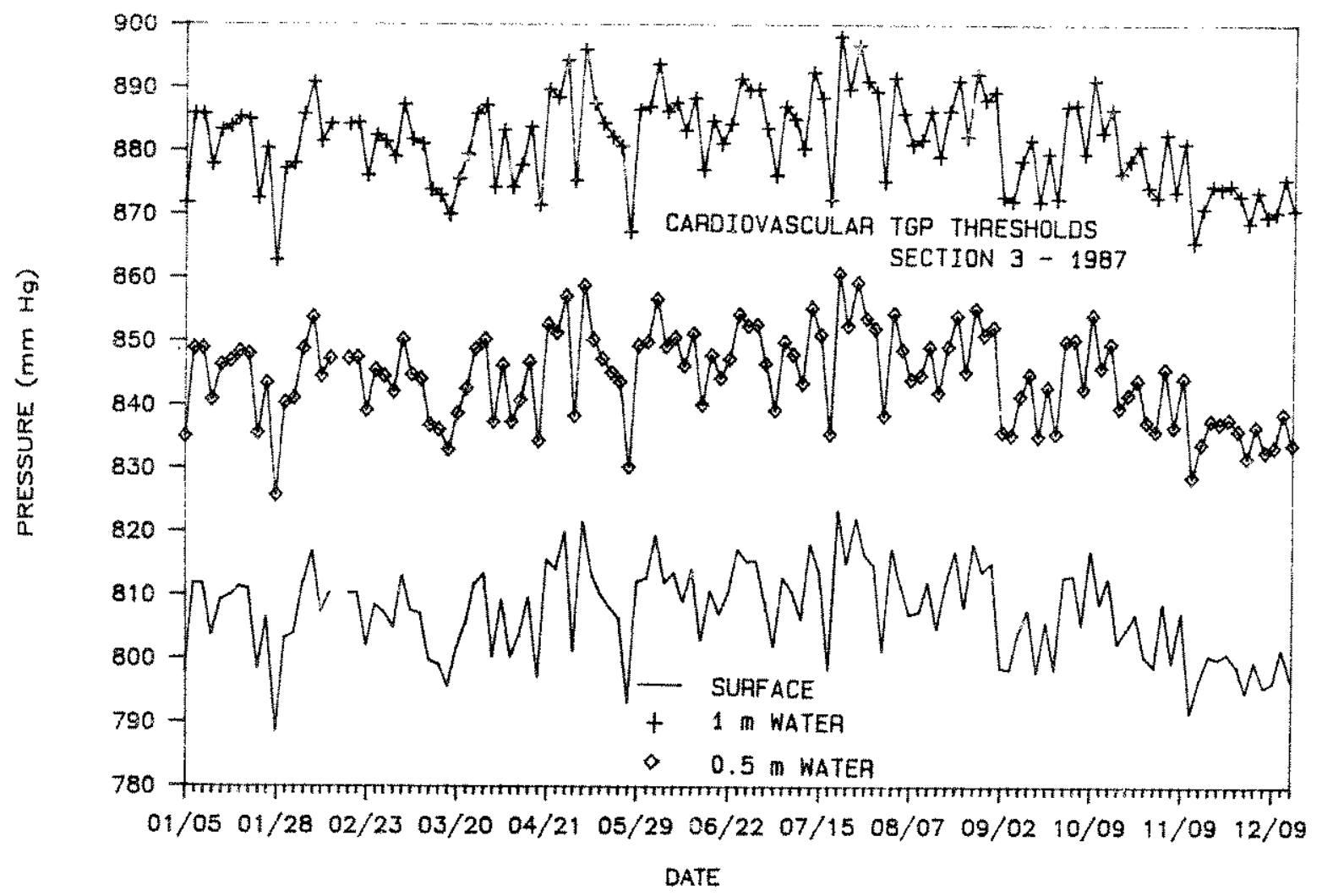

Figure 2.3.80. Threshold TGP for bubble formation in the cardiovascular system for trout in section 3 at the water surface, $0.5 \mathrm{~m}$, and $1.0 \mathrm{~m}$ below the surface during 1987.

height of the river provided an additional $41 \mathrm{mmHg}$ of hydrostatic pressure for compensation. Mean daily delta $P$ values at the gagehouse (representative of actual gas levels on GBT incidence sampling dates) rose from $126 \mathrm{mmHg}$ on 4 June to $138 \mathrm{mmHg}$ on 25 June. A fish on 4 June in $1 \mathrm{~m}$ of water would have been subjected to a ${ }^{a} P=$ "PSURFACE - $P H=126 \mathrm{mmHg}-73.5 \mathrm{mmHg}=52.5 \mathrm{mmHg}$. Assuming that fish took advantage of the additional depth, on 25 June exposure would have decreased to a ${ }^{a} P=138 \mathrm{mmHg}-73.5 \mathrm{mmHg}$ - $41 \mathrm{mmHg}=23.5 \mathrm{mmHg}$, or $23.5 \mathrm{mmHg}$ less than the hyperbaric pressure on 4 June.

Because Bouck gasometer measurements were manually taken biweekly, they do not reflect diurnal or other changes in delta $P$ 
between measurements. Thus, we re-examined 1987 results using gas measurements collected by the common sensing instrumentation. 


\subsubsection{Relationship Between GBT Thresholds and GBT Incidence on the Bighorn Fiver.}

Although GBT of adult fish was monitored throughout the study, we could not determine the extent of mortality because of the difficulty in recovering carcasses. Consequently, it was not possible to establish a correlation between adult trout mortality and the threshold for caräiovascular system bubble growth. Other complications in making correlations for water bubble growth (bubble growth between gij.l lamellae) and skin blister symptoms are the uncertainty in the data from the common sensing instrumentation prior to 1987. Since Bouck gasometer measurements do not reflect the major diurnal sweeps in delta $\mathrm{P}$, it was not possible to use these data to determine if reductions in delta $\mathrm{P}$ at night offered short-term relief from symptoms and thereby clouded the threshold effect. Thus, we evaluated the relationship between GBT thresholds and GBT incidence in trout using gas pressures collected by the common sensing instrumentation.

\section{$2.3 .5 .4 a$ Methods}

Dissolved gas data used in examining threshold relationships were collected during 1987 and May 1988 at Rkm 0.6 by Common Sensing instruments. Delta $\mathrm{P}$ and $\mathrm{po}_{2}$ data for this period were corrected based on the Bouck and Winkler measurements as described earlier. The corrected values were then used to calculate the three threshold conditions described by equations $5^{\prime}, 6^{\circ}$, and $7^{\prime}$ (see page 138). Monthly comparisons were made between delta $P, \mathrm{pO}_{2}$, percent of fish affected by GBT and 
threshold conditions. The GBT data are for river section 1 . The GBT rating described earlier was also used in evaluating the mean severity of symptoms. Instrumentation problems left gaps in some of the January and February data of 1987.

\section{$2 \cdot 3 \cdot 5 \cdot 4 \mathrm{~b}$ Results and Discussion}

January 1987: Delta $\mathrm{P}$ and $\mathrm{pO}_{2}$ were relatively constant at $\mathrm{Rkm} 0.6$ over most of January, with levels generally right at the threshold for bubble growth in the water and for the formation of external skin blisters (Figure 2.3.81). No GBT surveys were conducted during this period.

February 1987: During the first half of February, gas pressures were similar to January levels. Then delta p rose from the threshold level for water bubbles and skin blisters to the threshold level for vascular system bubble growth (Figure $2.3 .82)$

A survey of adult fish just before delta $\mathrm{P}$ increased in midFebruary showed $30 \%$ of all brown trout and $55 \%$ of brown trout over $356 \mathrm{~mm}$ in length were affected by GBT (Figure 2.3.82). The mean GBT rating for these fish was 6.85 . However, at the same time, only $8 \%$ of all rainbow trout were affected and nore over $356 \mathrm{~mm}$ in length had GBT symptoms. The mean GBT rating for all rainbow trout was 1.5 . These data show distinct differences between the two species in GBT susceptibility. They also illustrate the dramatic swings in delta $\mathrm{P}$ that occur between daylight and nighttime periods.

March 1987: During March 1987 deita $P$ remained fairly steady, oscillating between the water bubble-skin blister 


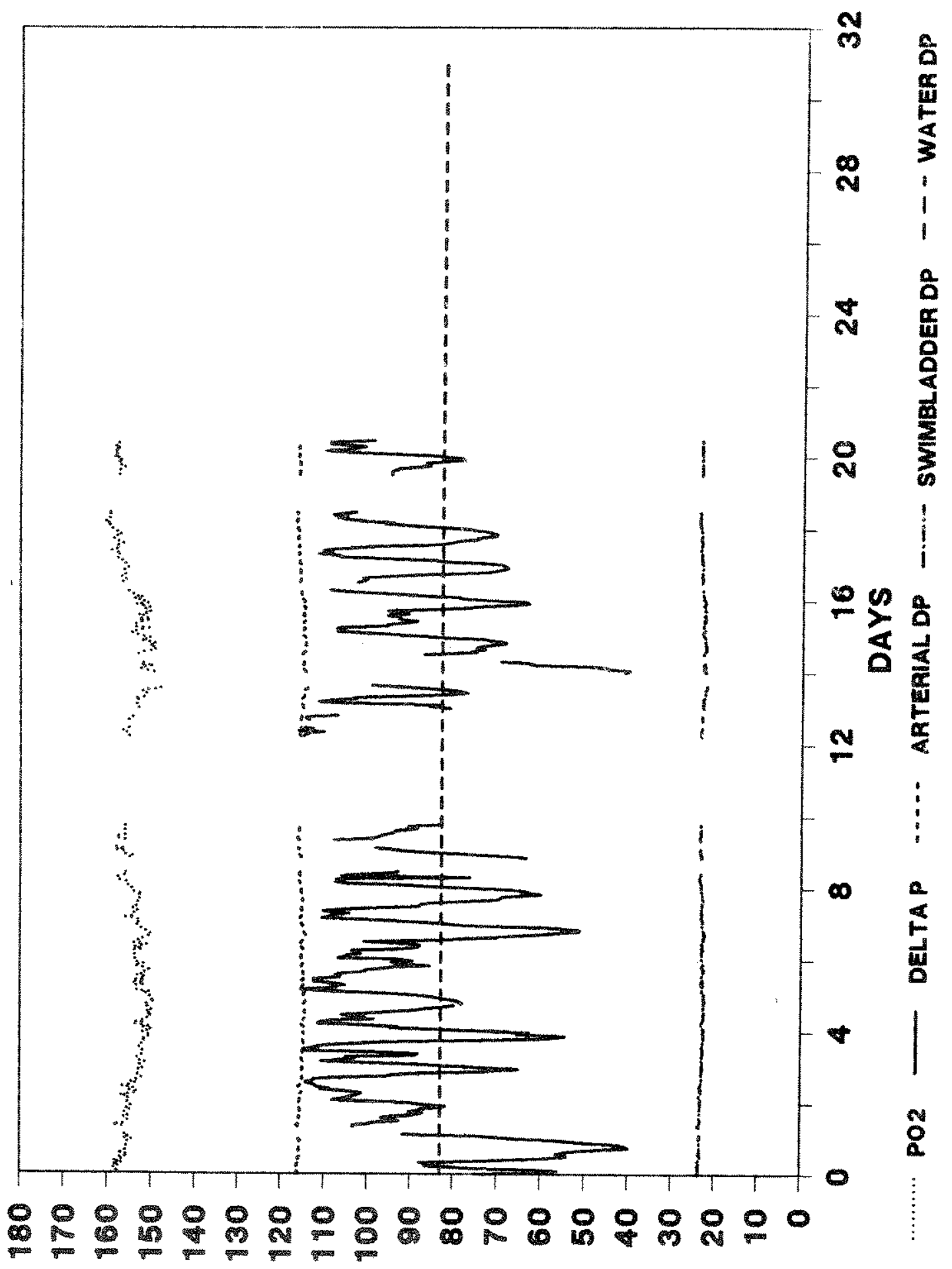

告
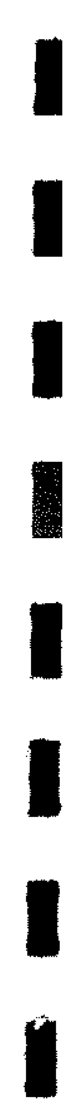

o.

H.

象

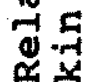

ज

i

$\infty$ 然

लं

. $\rightarrow-1$

N

0 车

40

क्ष

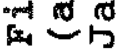

64uน - 3unsS3yd 


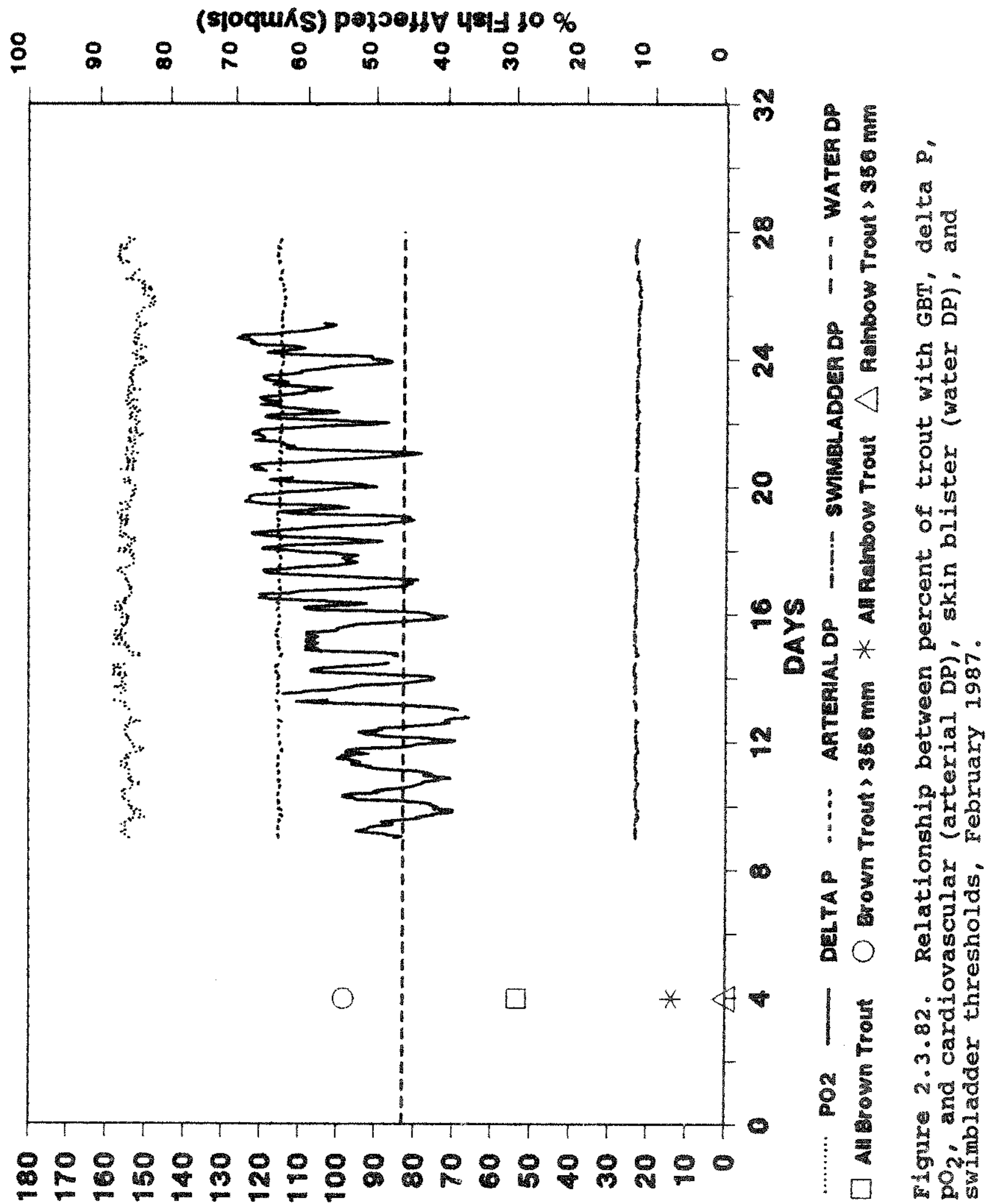

BHuru - 3unsS38d 
threshold and the cardiovascular bubble growth threshold (Figure 2.3.83). Diurnal swings in delta $\mathrm{P}$ were as much as $50 \mathrm{mmHg}$.

A symptom survey on adult fish taken on March 24 revealed very high percentages of brown trout affected by GBT. Thirty-six percent of all brown trout and $70 \%$ of brown trout over $356 \mathrm{~mm}$ in Iength were affected. Rainbow trout were far less affected with only 5\% of all fish and $11 \%$ of all these $>356 \mathrm{~mm}$ in length affected. Mean GBT rating for brown trout was 5.81 compared to 3.33 for rainbow trout.

April 1987: Delta $P$ levels of late March continued into the first week of April (Figure 2.3.84). Between that time and the beginning of the last week of April, average delta $\mathrm{P}$ decreased to near the water bubble-skin blister threshold; however, diurnal. fluctuations were larger. For a 4-d period during the last week of April, delta $\mathrm{P}$ fell below the water bubble-skin blister threshold but then rose sharply to levels above the threshold for cardiovascular system bubble growth and remained there for the rest of the month. There was little diurnal variation in delta $P$ during the last few days of April.

Symptom surveys in adult fish taken on April 13 again showed high numbers of brown trout affected by GBT. Forty percent of al1 fish were affected, with $70 \%$ of brown trout $>356 \mathrm{~mm}$ affected. The percent of rainbow trout affected rose slightly to $7 \%$, with $19 \%$ of all rainbow $\geq 356 \mathrm{~mm}$ showing symptoms. The mean GBT rating was 7.42 for brown trout and 2.92 for rainbow trout. on April 27, GBT symptoms among rainbow trout $\geq 356 \mathrm{~mm}$ had declined slightly to $15 \%$ while large brown trout with symptoms had increased from 70 to $76 \%$. The mean GBT rating was 7.67 for 


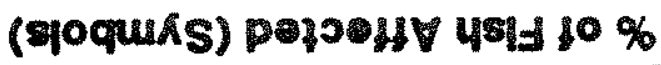

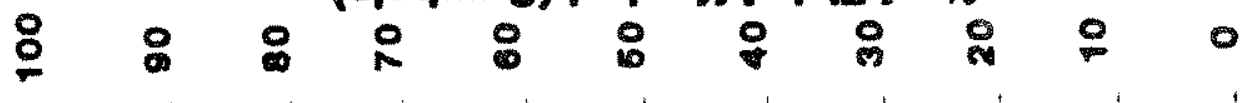

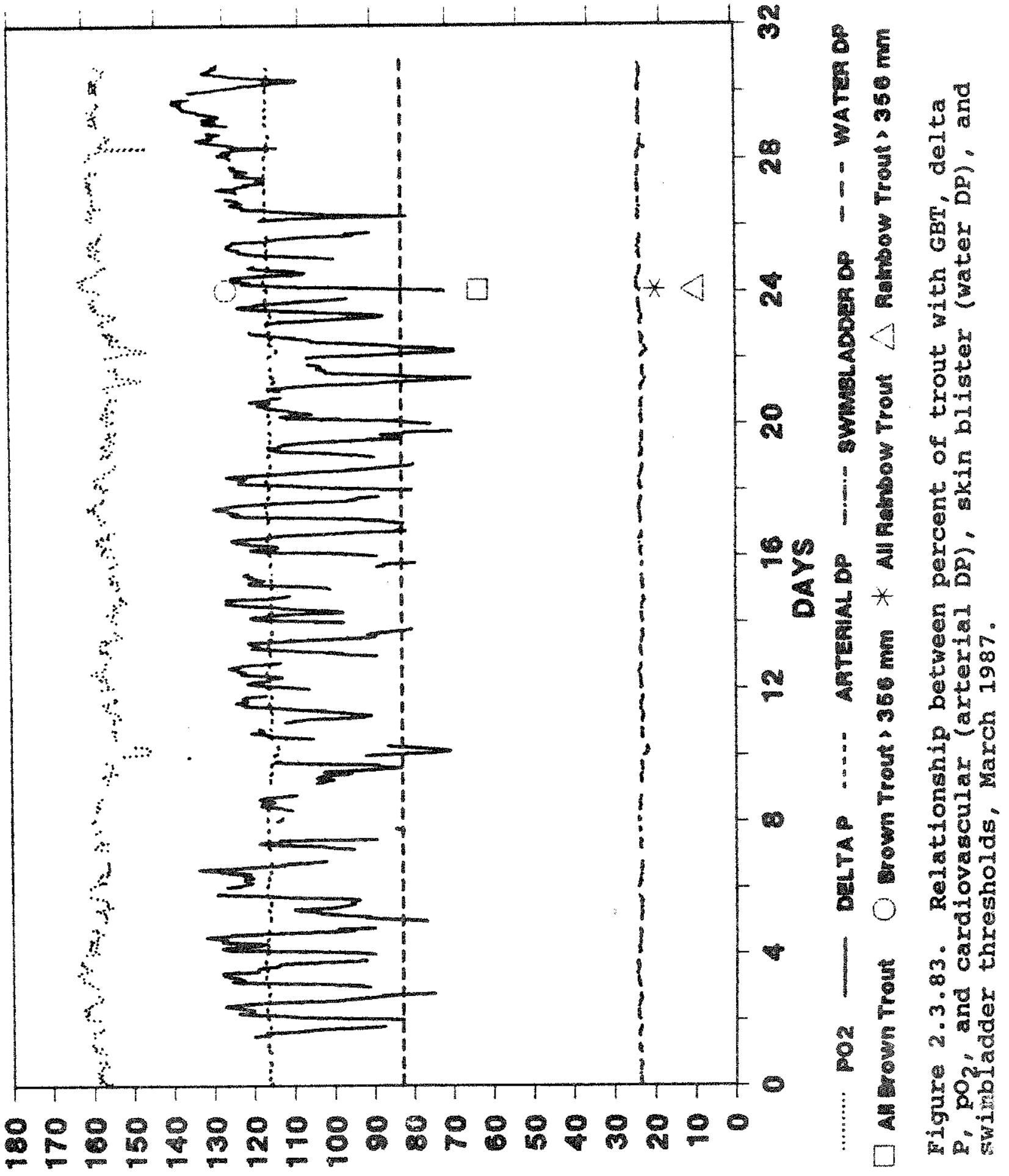

64uน - 34nss34d 
(\$оqun

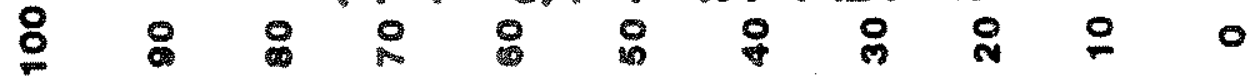

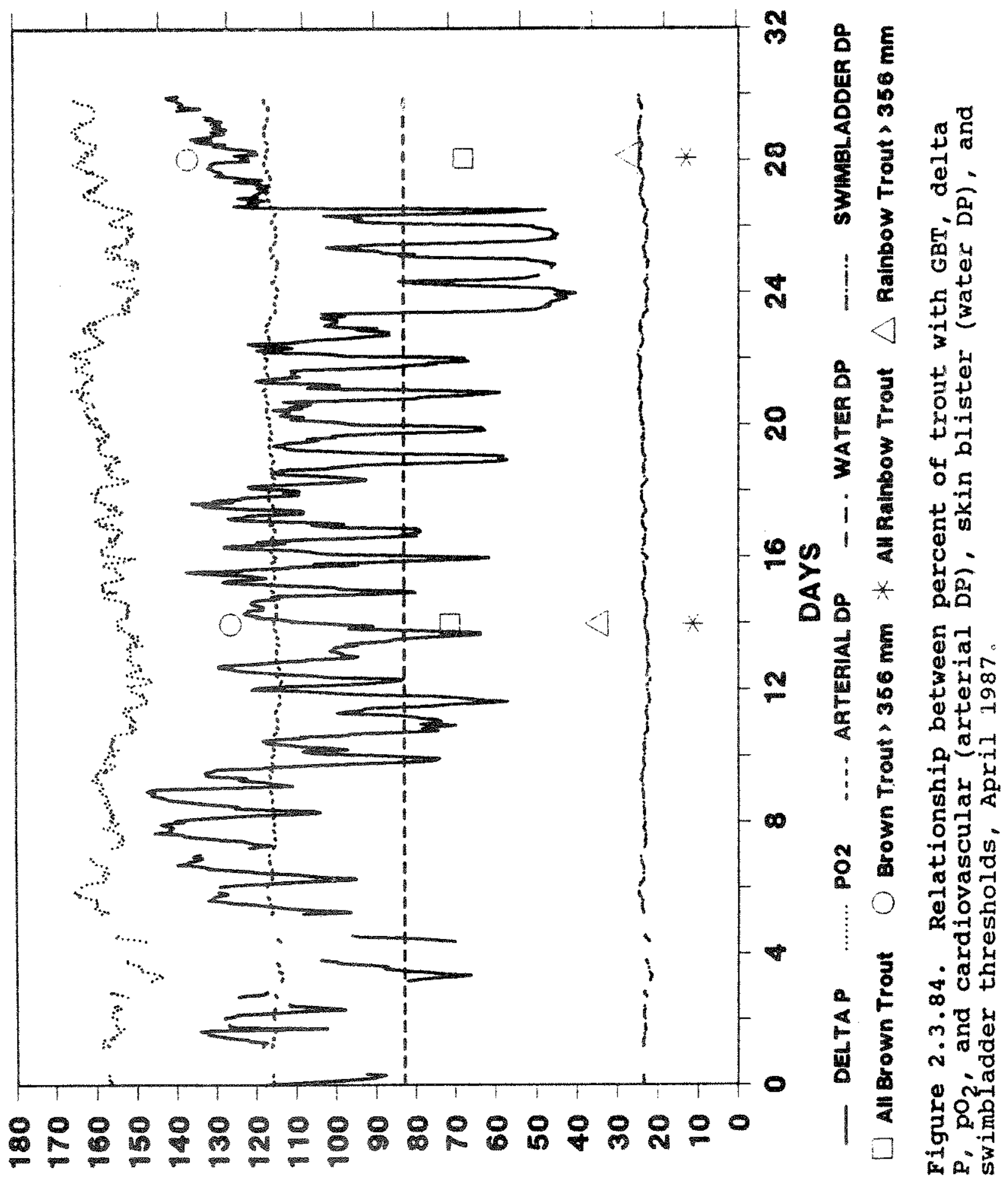

-44uน - 34nss3yd 
brown trout and 4 for rainbow trout.

May 1987: During May, delta $\mathrm{p}$ levels remained above the threshold for cardiovascular system bubble growth until the last week of the month when they decreased to the threshold level (Figure 2.3.85).

Brown trout GBT levels during the last week of ray were the highest observed during 1987 and early 1988. Forty-five percent of all brown trout and $88 \%$ of brown trout $\geq 356 \mathrm{~mm}$ were affected. Among rainbow trout, $10 \%$ of all fish and $20 \%$ for fish $\geq 356 \mathrm{~mm}$ were affected. The mean GBT rating for brown trout was 8.75 compared to 7.32 for rainbow trout. It is somewhat surprising that the mean GBT rating was similar for the two species when the GBT incidence was so dramatically different.

June 1987: In early June delta $\mathrm{p}$ levels again exceeded the cardiovascular system bubble growth threshold (Figure 2.3.86), and remained there for the rest of the month (Figure 2.3 .87 ). No GBT surveys were conducted during June 1987.

July 1987: The high late June levels of TGP persisted until mid-July then dropped to a level between the cardiovascular bubble growth threshold and the water bubble-skin blister threshold (Figure 2.3.87).

Surprisingly, the percent of affected brown trout sampled on July 23 had dropped slightly while the percent of affected rainbow trout had increased. The percent of all brown trout affected was $75 \%$, while $44 \%$ of those over $356 \mathrm{~mm}$ in length had symptoms. Rainbow trout GBT incidence rose to $17 \%$ for all fish and $31 \%$ for those $\geq 356 \mathrm{~mm}$. Also, the mean GBT rating for rainbow trout was slightly higher (8.75) than that for brown 


\section{(}

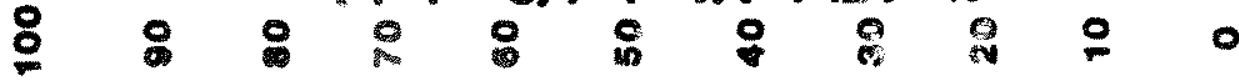

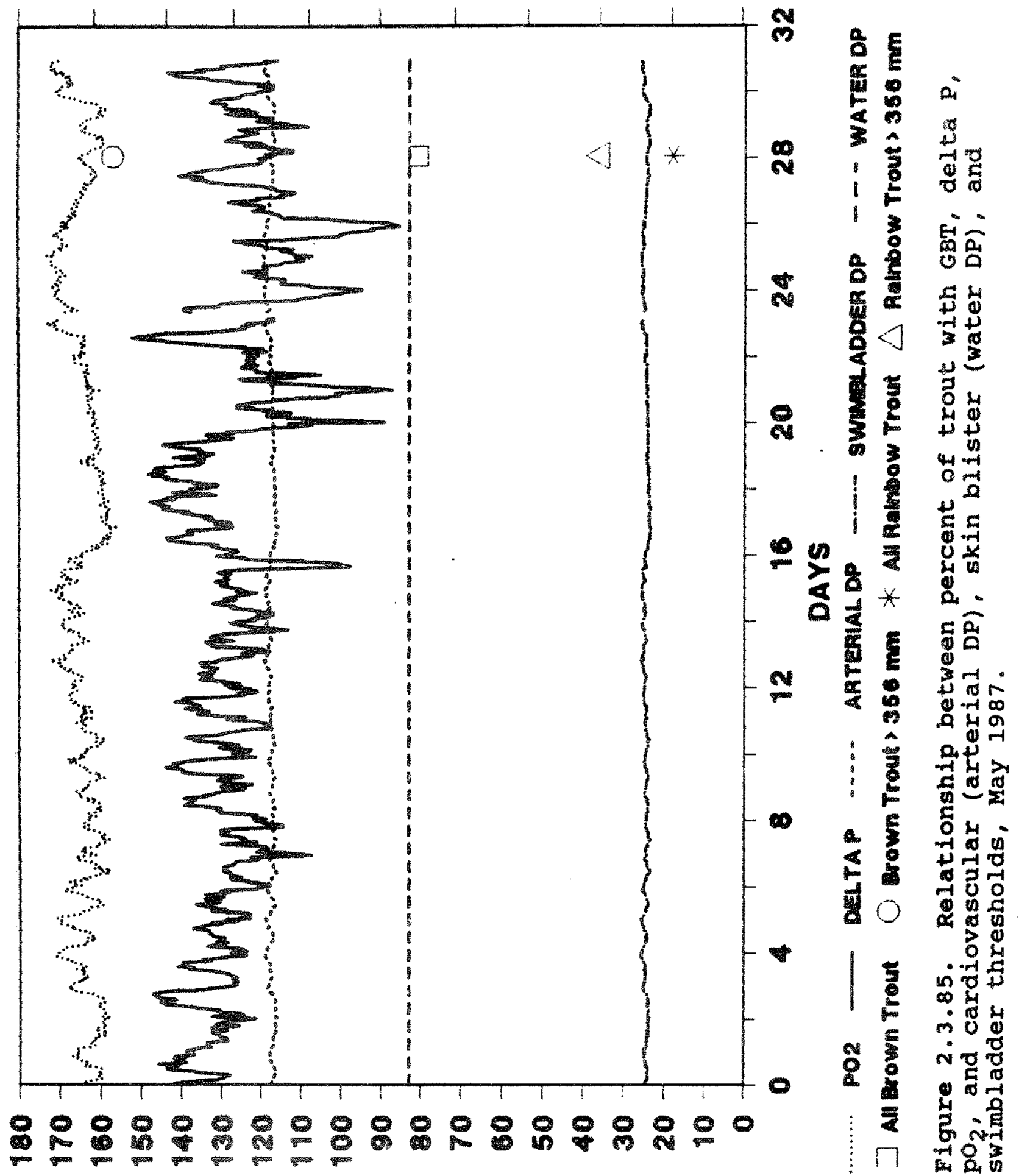

4. Buru - 3unss34d 


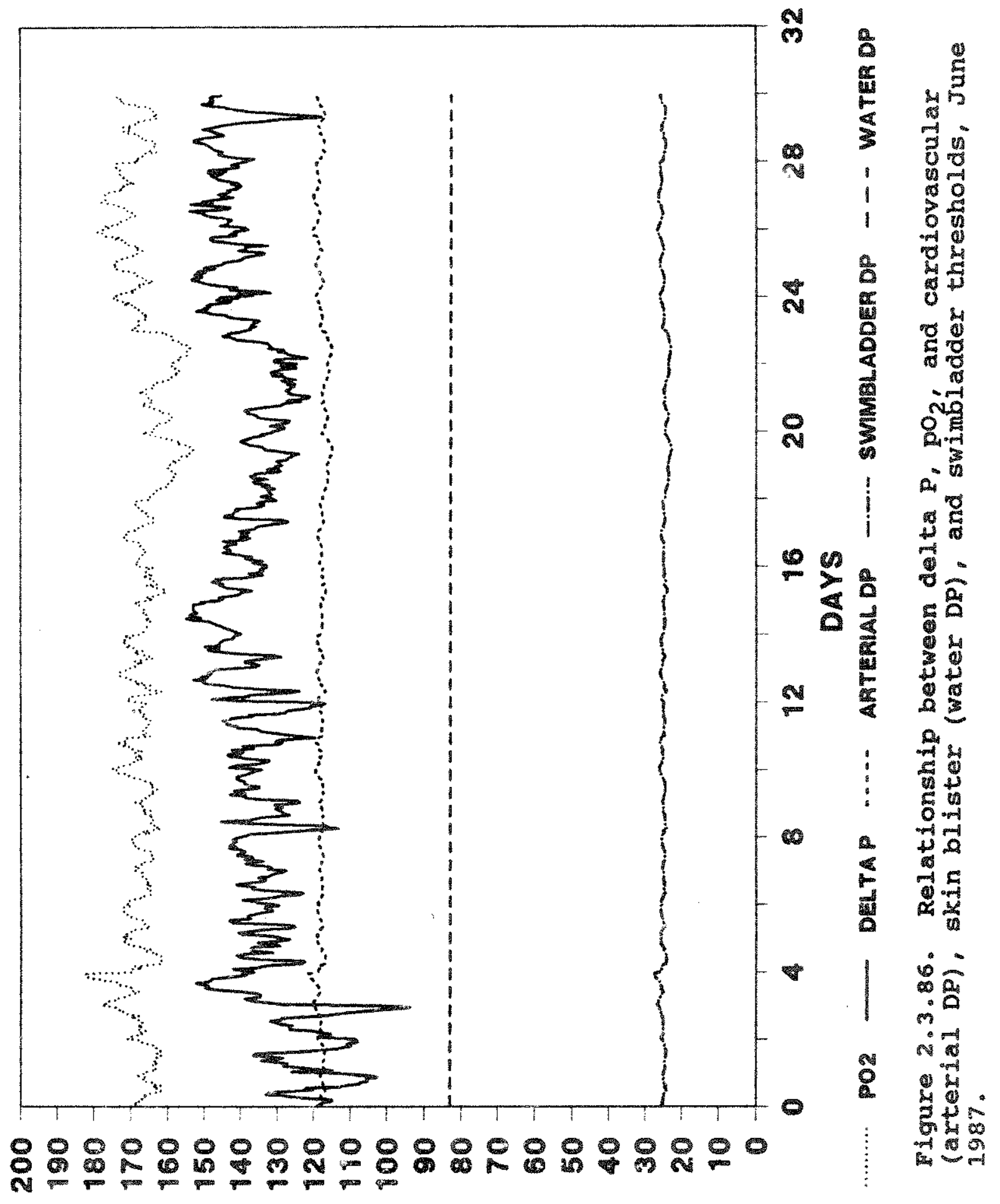

- Внuน - 34nSS34d 


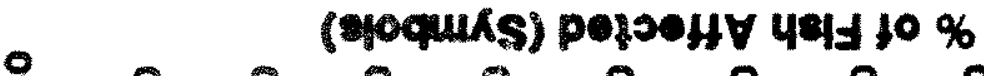

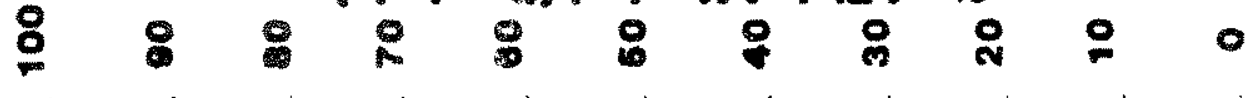

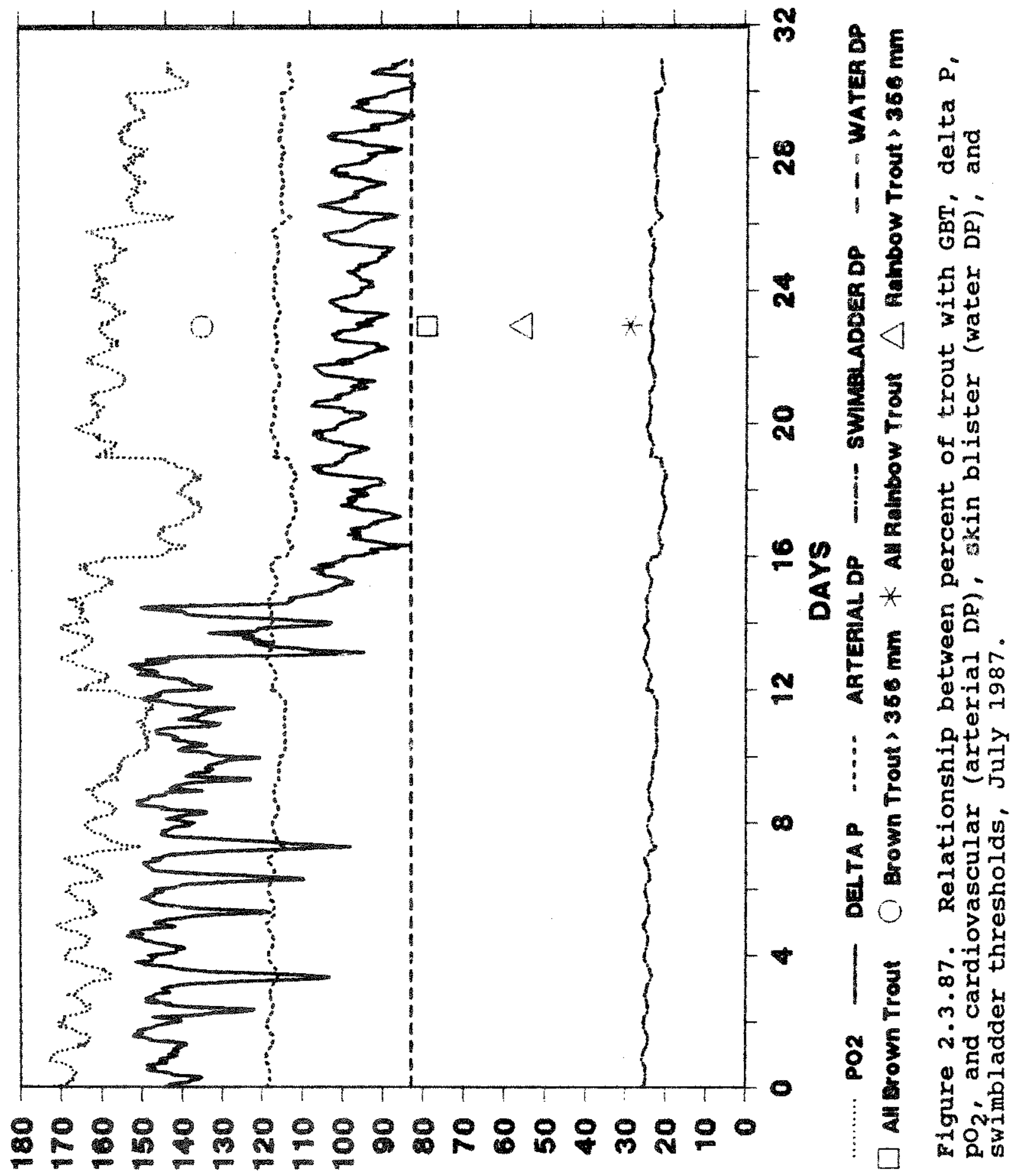

-4uนи - 3unss34d 
trout ( 8.57$)$. It is not clear whether this reversal from

previous trends is due to the more severely affected brown trout dying and being removed from the population or brown trout recovering from symptoms more rapidly than rainbow trout. If the latter is correct, there may be an inverse relationship between resistance to dissolved gas supersaturation and recovery.

August 1987: From the last few days of July until mid August, delta $\mathrm{P}$ levels dropped to the water bubble-skin blister threshold (Figure 2.3.88); nighttime variations in delta $\mathrm{P}$ were below this threshold. Near the middle of August, delta $P$ levels rose sharply, exceeding the cardiovascular system bubble growth threshold. About a week later, delta P dropped back to the water bubble-skin blister threshold. No adult fish surveys were conducted during this period.

september 1987: From the last week of August until the midale of september, delta $P$ levels were just at the water bubble-skin blister threshold (Figure 2.3.89). The low levels of delta $P$ during this period and up until mid-october were attributed to the sluiceway gates on the Afterbay Dam being operational.

Fish sampled on september 1 showed the percent of affected fish down to about one half of July levels. Mean GBT ratings were 4.64 for brown trout and 3.5 for rainbow trout. on September 14 the percent of affected fish rad declined to less than $6 \%$ for brown trout and near $0 \%$ for rainbow trout. Mean GBT ratings were 2.5 for brown trout and 0.0 for rainbow trout. This trend was also present in fish sampled on september 28 where only a small percent of both species showed symotoms with rainbow 


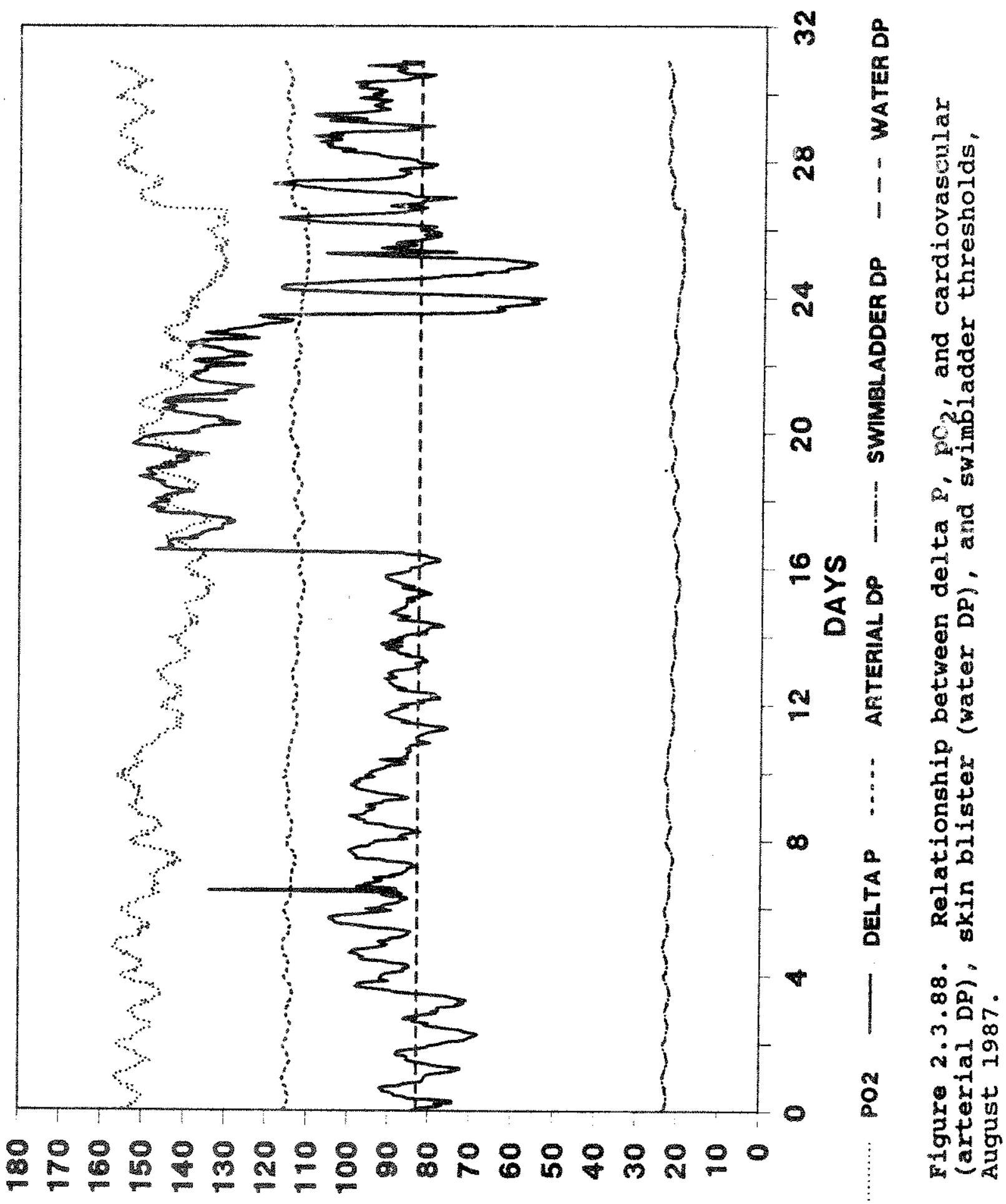

- ในuน - 3unss34d 


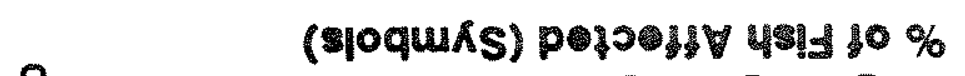

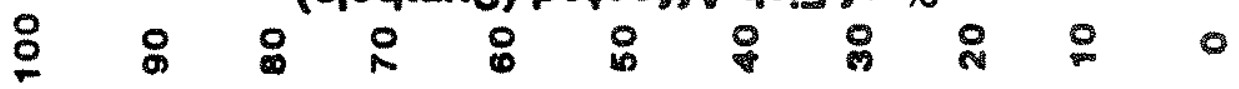

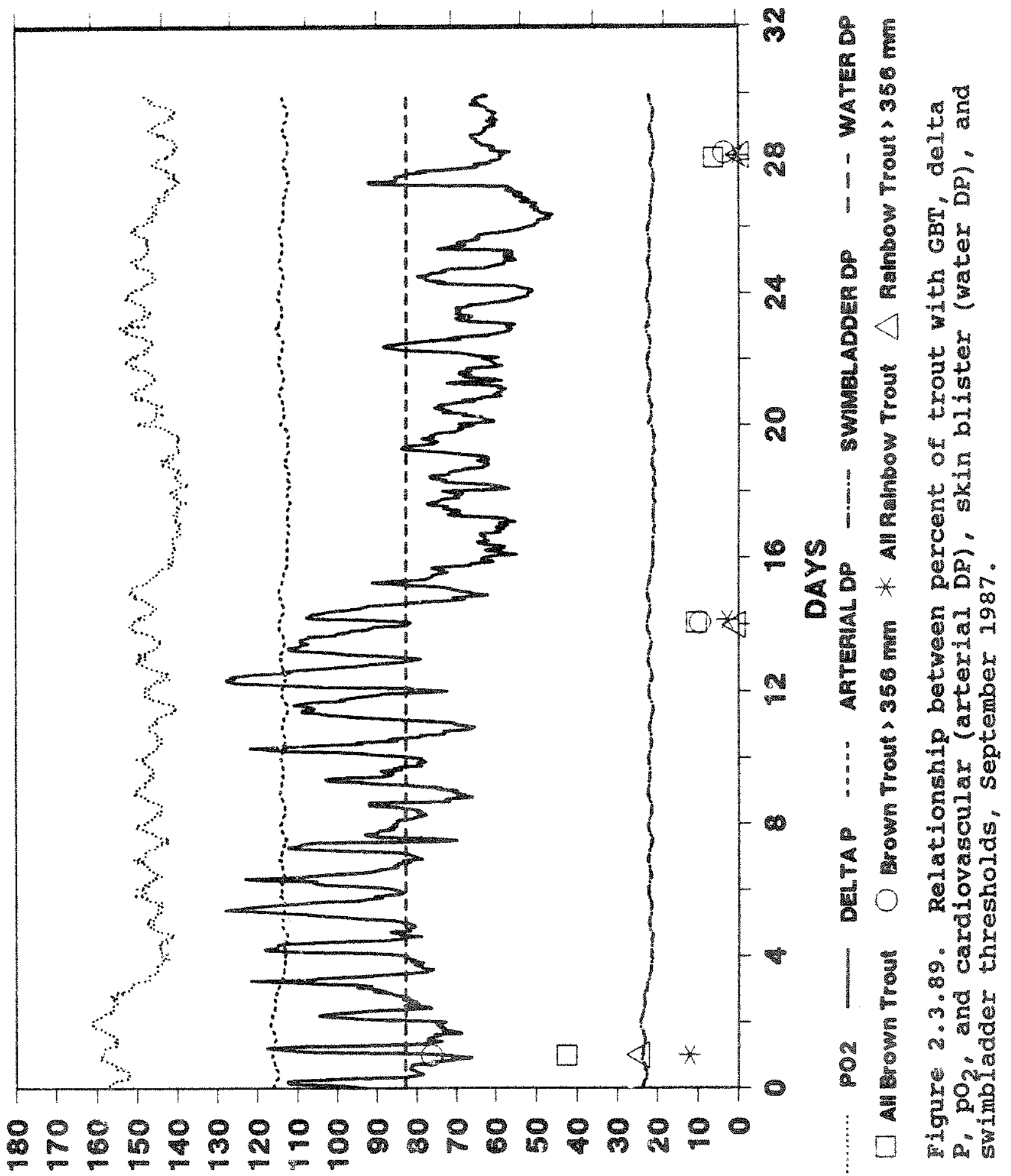

-8นuน - 3อกรS34d 
trout showing less than brown trout. This period was characterized by very large swings in daytime to nighttime delta $P$ levels. In comparing mean delta $P$ ievels for the first half of september with those for March when they were only slightly higher, there is a dramatic difference between the percent of fish affected on March 24 (Figure 2.3.83) and september 1 and 14 (Figure 2.3.89). There is a corresponding marked difference between the mean GBT ratings between these periods. This supports the existence of, and validates the level of, the water bubble-skin blister threshold.

october 1987: The low levels of TGP in late september continued until just before the middle of october (Figure 2.3.90). Starting at about october 12 , there was a graduai rise in TGP, and by october 16 it was above the cardiovascular system threshold. Except for a short drop around is October, TGP stayed above the cardiovascular system threshold well into November, 1987 (Figure 2.3.91). No adult fish symptom surveys were made during october.

November 1987: Most of November was characterized by levels of TGP above the cardiovascular system threshold (Figure 2.3.91). sharp drops in TGP to just below the water bubble-skin blister threshold occurred from November 11-15 and November 23-24.

percent of affected brown (8\% for all fish and $10 \%$ for fish $\geq 356 \mathrm{~mm}$ ) and rainbow trout ( $0 \%$ for all fish) was low on November 11. These levels were considerably lower than expected, considering that TGP levels were above the cardiovascular bubble growth threshold for nearly a month. It is unknown why the fish 


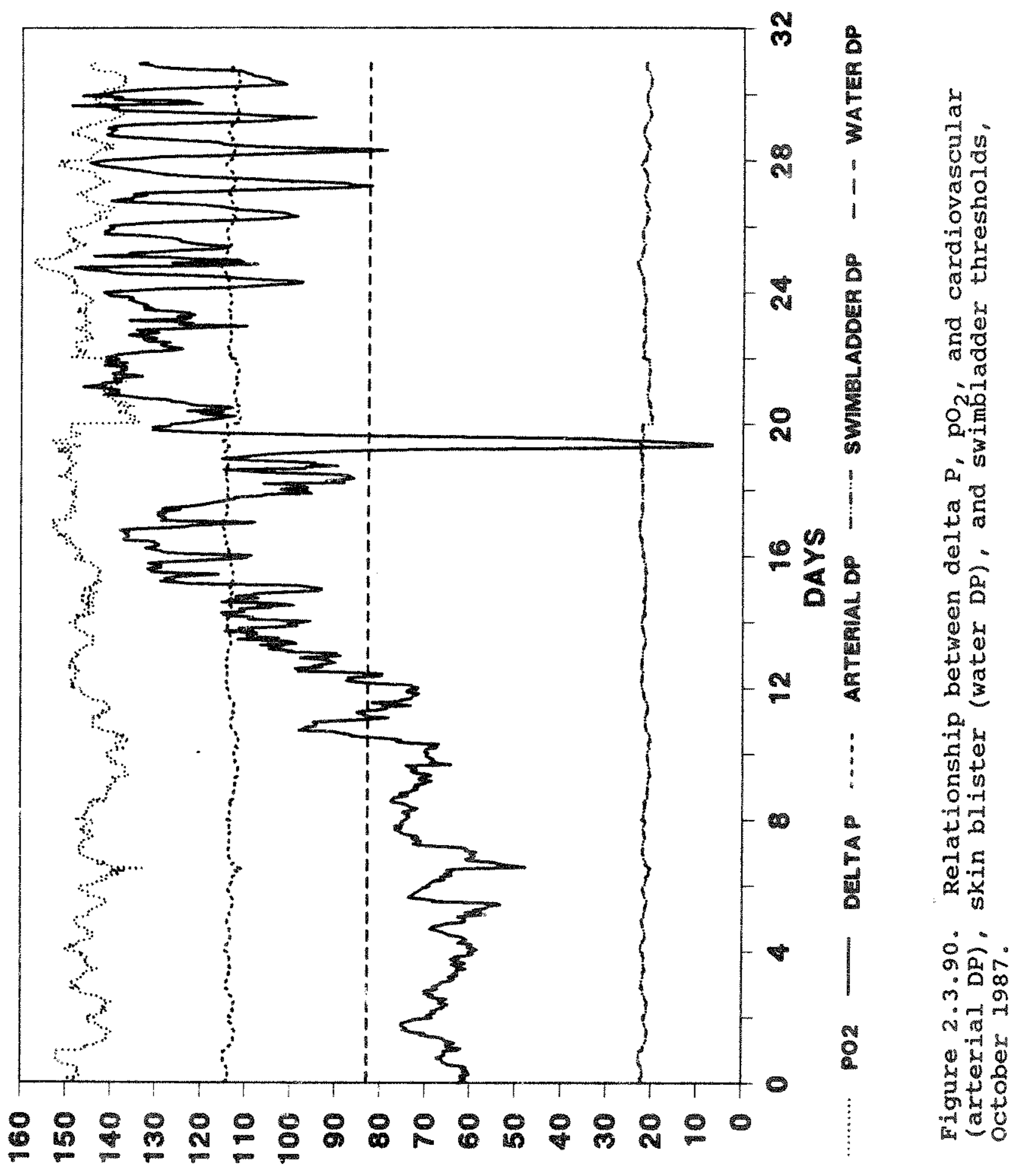

- 


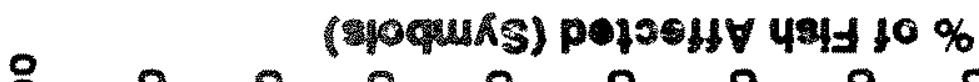

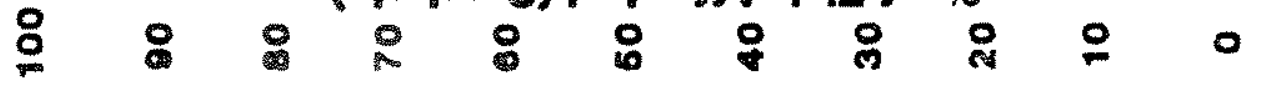

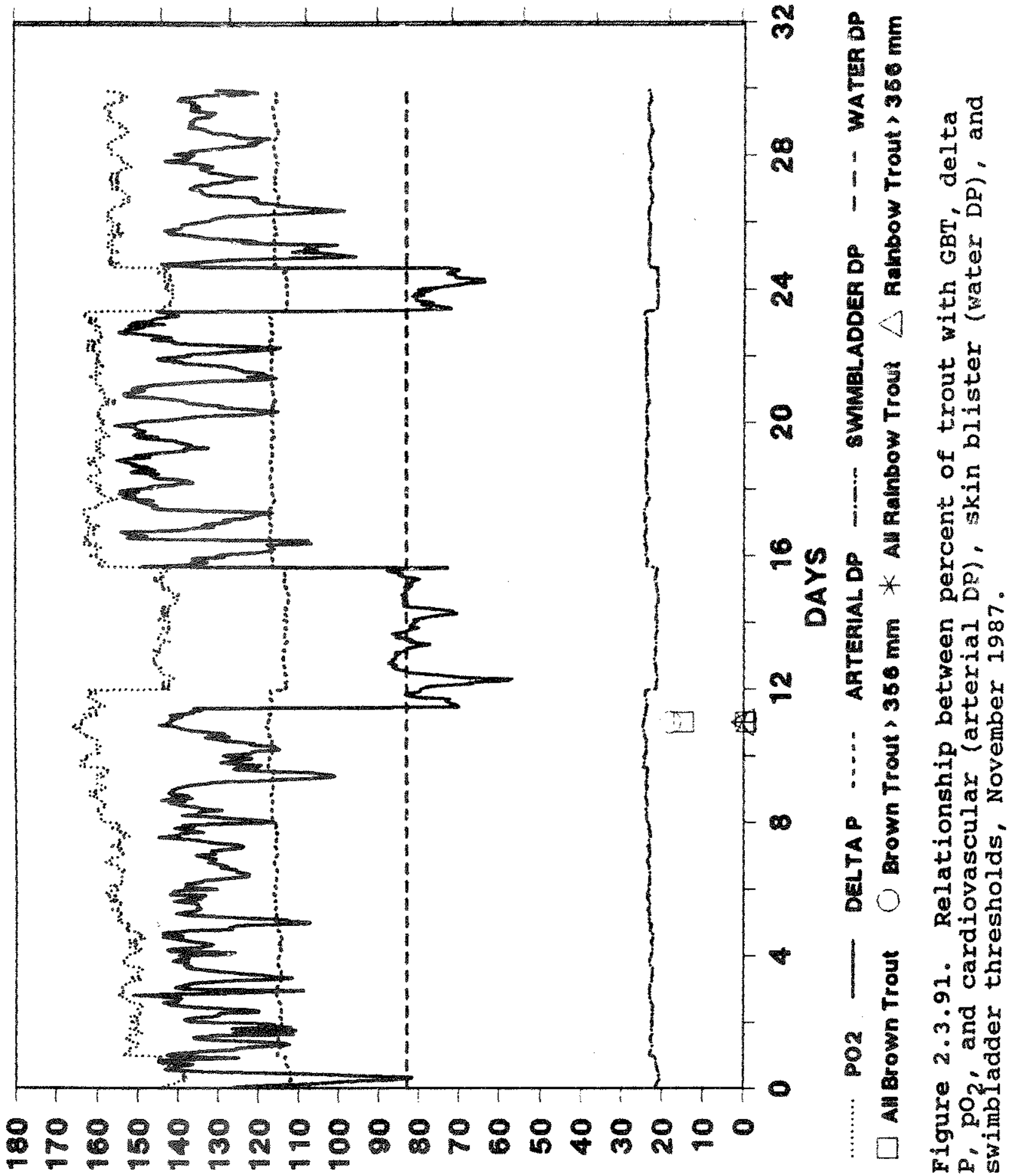

- 6uxu - 3unss3dd 
were not affected more severely. Even the mean GBT rating during this survey was low 2.5 for brown trout and 0.0 for rainbow trout). Based on laboratory experiments, data from the literature, and earlier episodes of high dissolved gas on the Bighorn River, there was more than enough time for vascular system bubble growth and skin blisters to occur. Furthermore, earlier bouts of similar high dissolved gas pressure (for example during June and July, 1987) left very high percentages of both species affected. It would be highly unlikely that some form of resistance to high dissolved gas levels could develop in such a short period. One possible explanation is that the earlier high dissolved gas levels may have reduced the adult trout population to only the more resistant individuals.

December 1987: Except for the first 3 d of December, when delta $P$ levels fell to the water bubble-skin blister threshold (Figure 2.3.92), delta p oscillated at or slightly below the cardiovascular bubble growth threshold. Some of the diurnal oscillations were as much as $50 \mathrm{mmHg}$.

Percent of affected adult fish on December 29 had risen to levels that might be expected for this range and duration of delta $P$. Thirty-one percent of all brown trout were affected and $47 \%$ of brown trout over $356 \mathrm{~mm}$ in length were affected. In rainbow trout, $18 \%$ of all cish were affected and $40 \%$ of all fish over $356 \mathrm{~mm}$ in length were affected. The percent of larger rainbow trout affected was nearly as large as the percent of larger brown trout affected. However, the mean GBT rating for rainbow trout was small $(0.136)$ compared to that of brown trout (5.023). Of the rainbow trout sampled, only one had external 


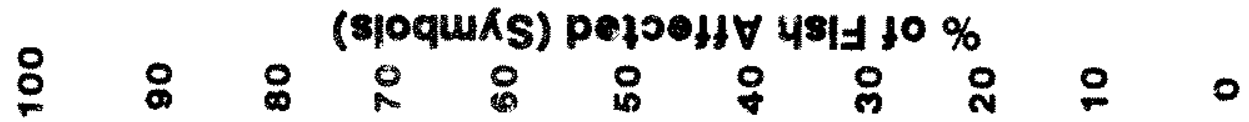

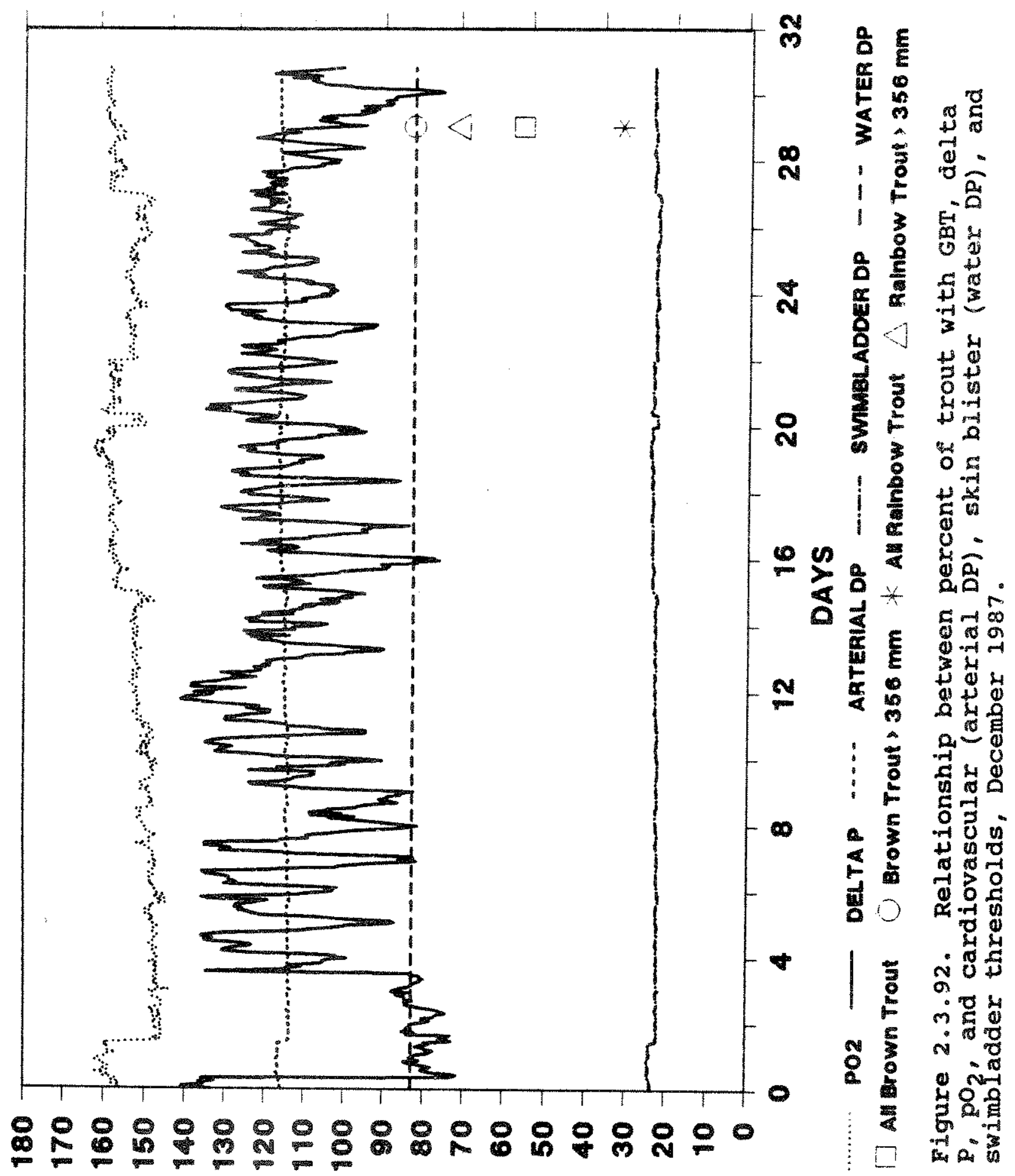

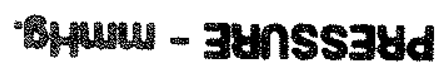


skin blisters. The other affected rainbow trout had various degrees of exothalmia.

January 1988: Up to 13 January 1989, water delta P stayed at or slightly below the cardiovascular bubble growth threshold (Figure 2.3.93). Between January 13 and January 18 it dropped to or slightly below the water bubble-skin blister threshold. Except for the last 2 d of the month, delta $\mathrm{p}$ oscillated between the cardiovascular bubble growth and the water bubble-skin blister thresholds. On the last 2 d of January, delta $p$ dropped to below the water bubble-skin blister threshold. No adult fish samples were collected during January 1988.

February 1988: During the first few days of February 1988, delta $\mathrm{p}$ rose to the water bubble-skin blister threshold, stayed there for $3 \mathrm{~d}$ and then rose to and remained near the cardiovascular bubble growth threshold for the rest of the month (Figure 2.3.94). Instrumentation problems near the end of the month prevented the collection of dissolved gas data at the gagehouse location.

on February $24,10 \%$ of all rainbow trout and $33 \%$ of those over $356 \mathrm{~mm}$ in length were affected by GBT. The mean CBT rating for rainbow trout was 4.0. For brown trout, $34 \%$ of all fish sampled and $43 \%$ of those over $356 \mathrm{~mm}$ in length had GBT symptoms: mean GBT rating was 5.22 .

March 1988: During March 1988 aissolved gas levels were high most of the nonth with delta $\mathrm{p}$ levels near or slightly above the cardiovascular bubble growth thresholds (Figure 2.3.95).

A large percentage of adult brown trout sampled March 28 was affected by GET; $46 \%$ of all and 55\% of those over $355 \mathrm{~mm}$. The 

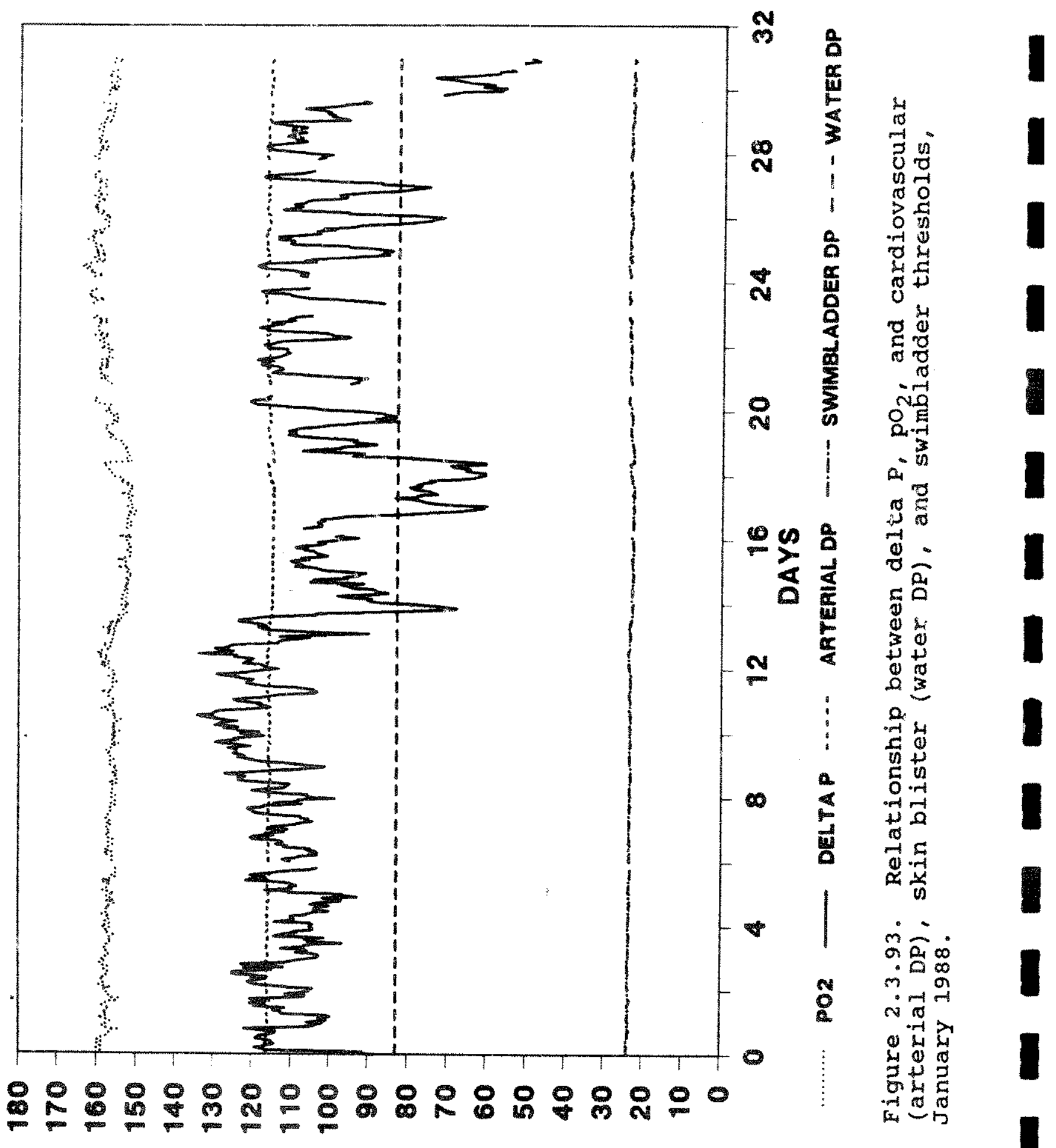

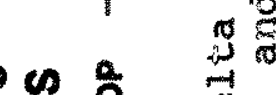

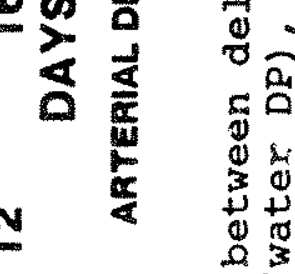

:

of 4

运哇

a. $\quad$ o.

$\alpha \quad 0 .-1$

$=$ i

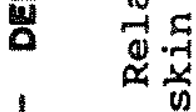

$\dot{m}=$

居我

N ? न्न

\& $\quad \dot{\pi} \pi$

a) 步 मे मै

कृष

.

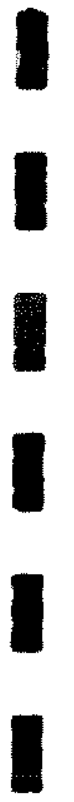

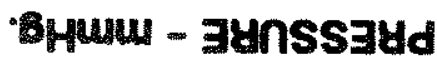



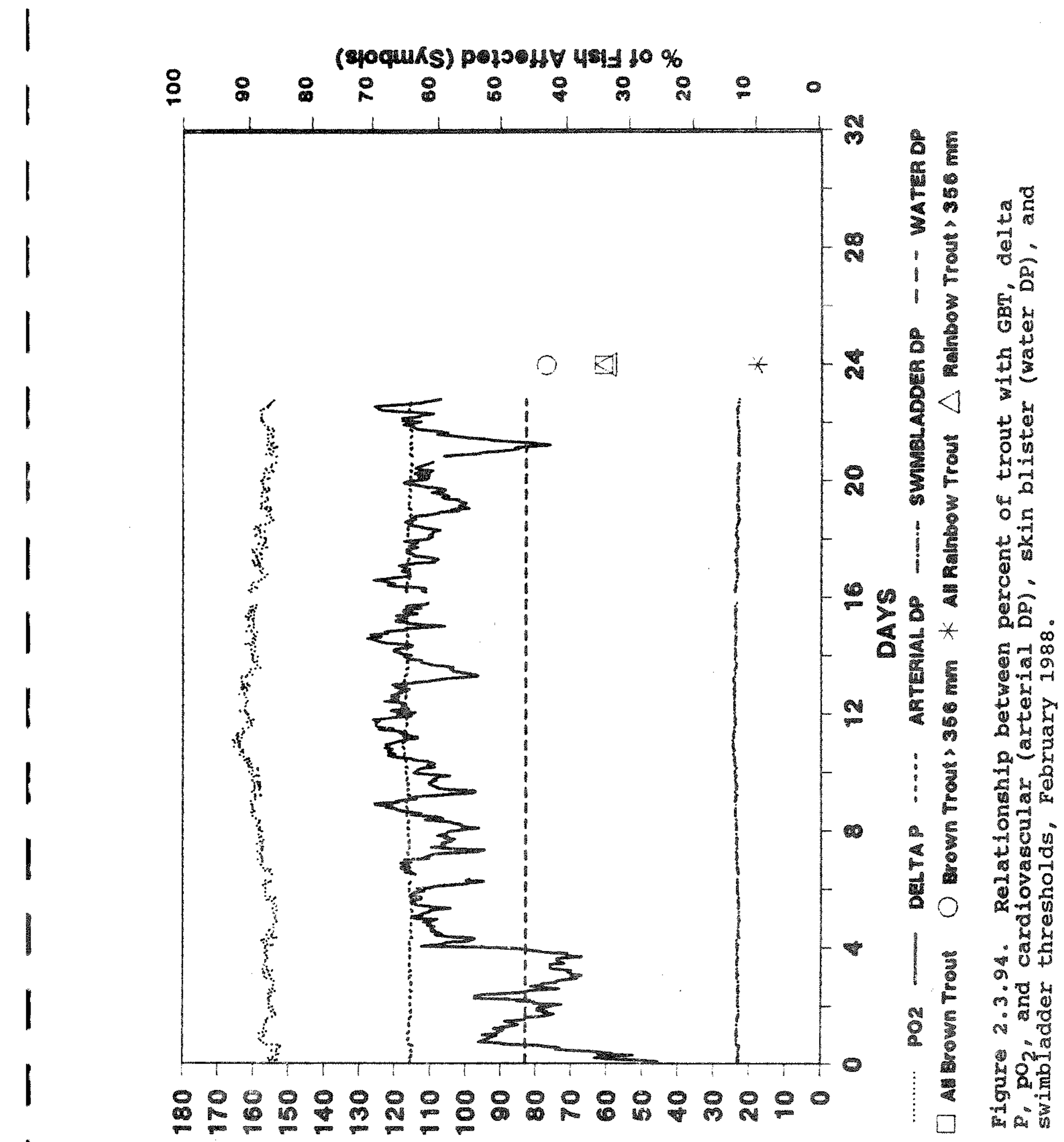

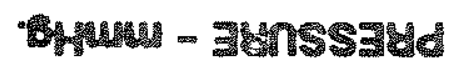




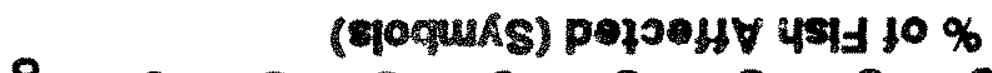

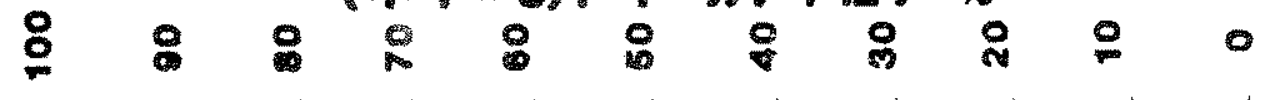

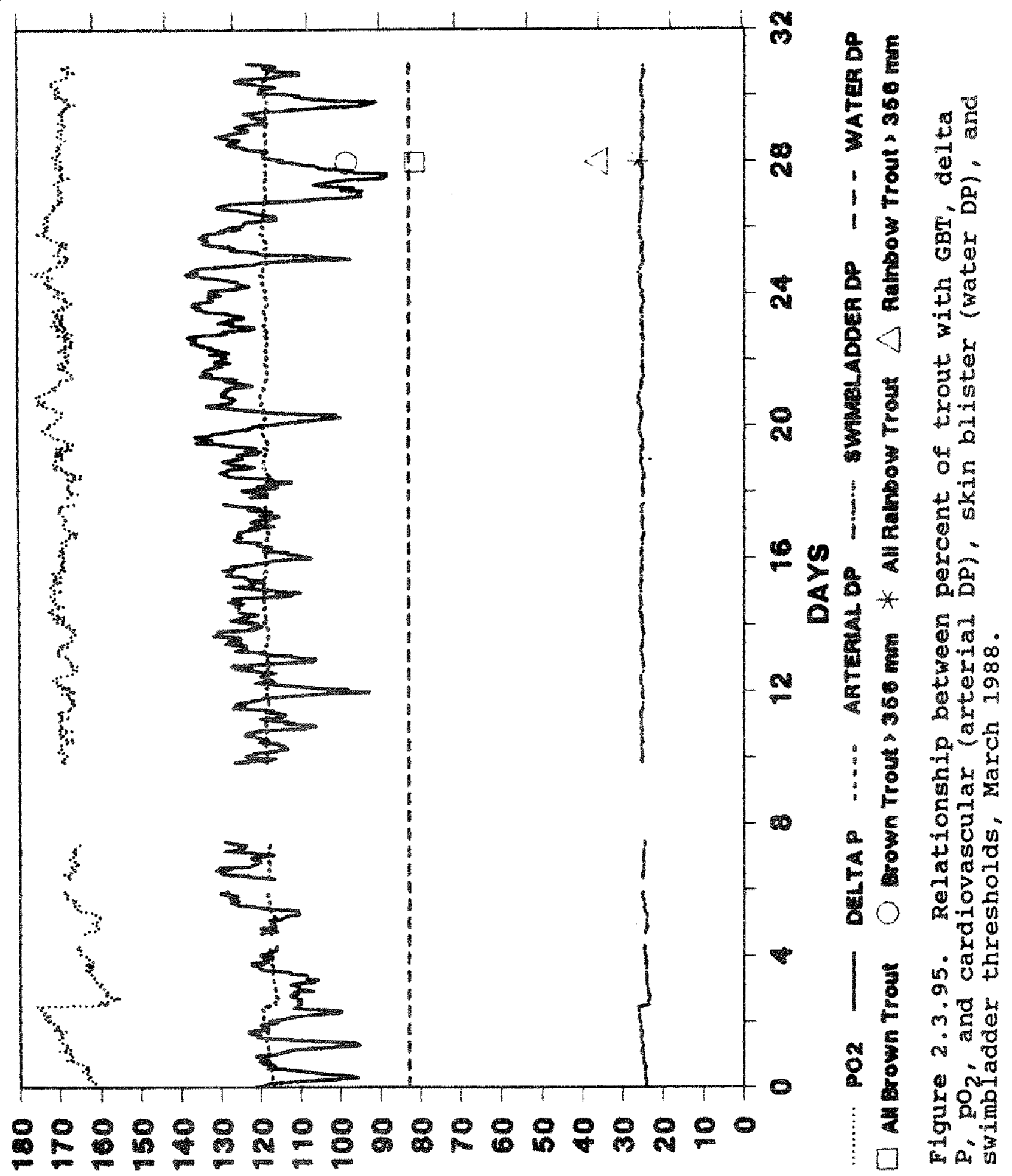

4mus - zunsszud 
mean GBT rating for brown trout was high at 6.67 . Incidence among rainbow trout was $15 \%$ of all fish and $20 \%$ of all fish over $356 \mathrm{~mm}$. The mean blister rating for rainbow trout was 1.5 .

April 1988: During the first few days of April, water delta $\mathrm{p}$ remained at the cardiovascular bubble growth threshold as it had most of March. However, on 4 April it dropped lo just below the water bubble-skin blister threshold (Figure 2.3.96). It remained there until 8 April when it rose sharply to and oscillated between the water bubble-skin blister threshold and the cardiovascular bubble threshold. These large oscillations (30- $40 \mathrm{mmHg})$ persisted until 22 April when the oscillations subsided somewhat and the mean delta $P$ was at the cardiovascular bubble growth threshold. This pattern remained until the end of the month.

On April 20 adult trout GBT incidence was higher for both species than in March. Brown trout had a $48 \%$ incidence in all fish and a $63 \%$ incidence in all fish over $356 \mathrm{~mm}$ in length. The mean GBT rating was 6.85 . Rainbow trout exhibited a $2.1 \%$ incidence in all fish and a $22 \%$ incidence in all fish over 356 mi mean GBT rating was 2.66 .

Bouck and Winkler Data: As mentioned earlier, delta $\mathrm{P}$ and $\mathrm{pO}_{2}$ data from the Bouck and Winkler measurements do not give a clear picture of the actual dissolved gas levels to which fish are exposed. As was evident in the common sensing data just described, diurnal oscillations often provided periods of relief from various threshold levels of delta $P$. To illustrate the difference, the Bouck and winkler data were used to calculate the various threshold conditions for the period January 1, 1987, 


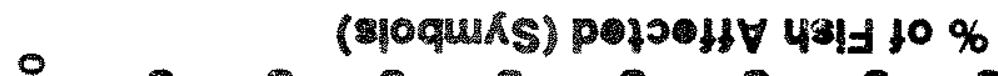

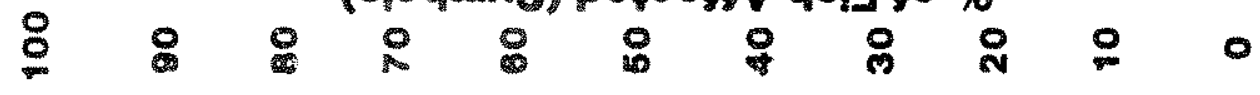

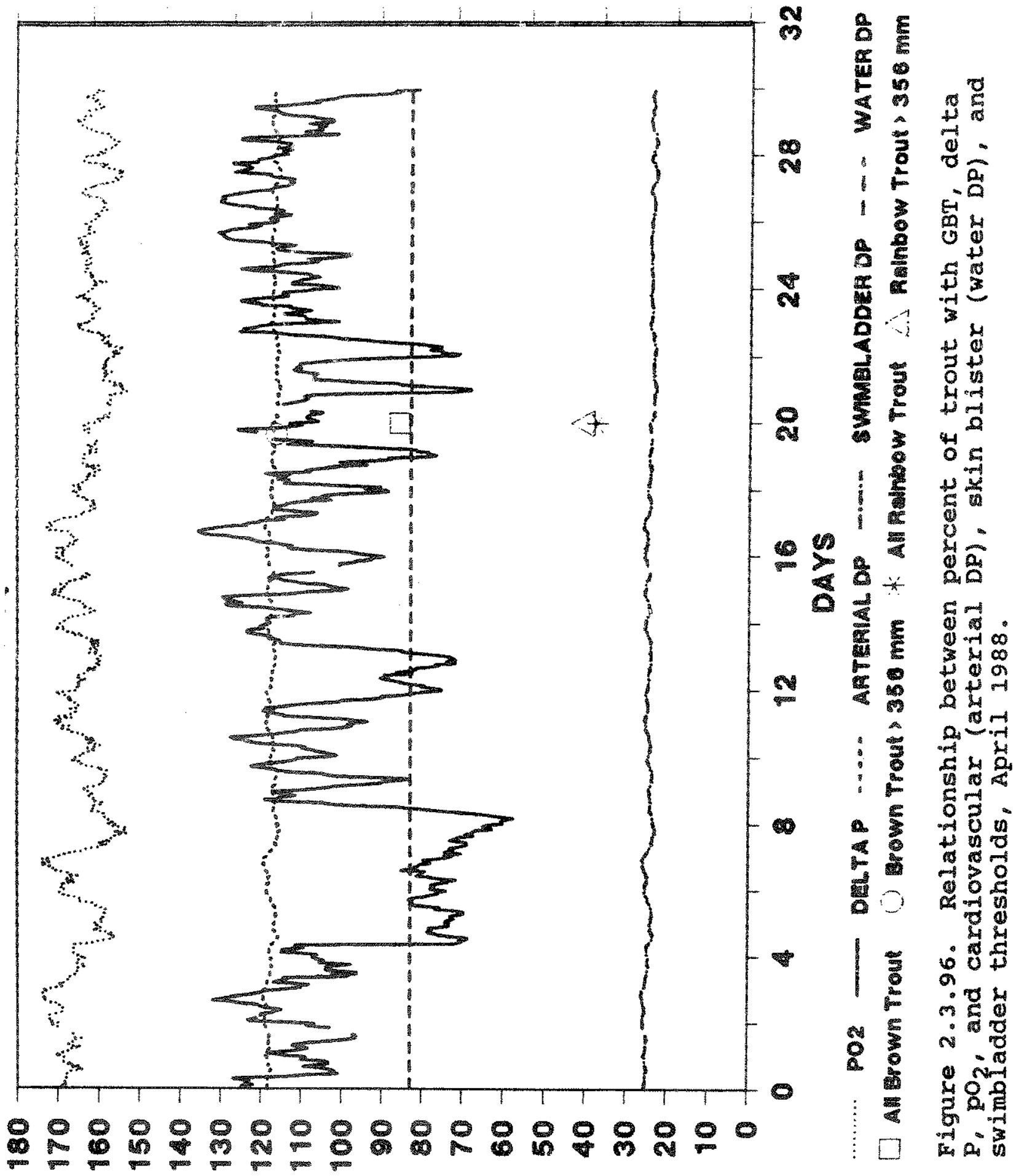

ชิน4น - zunss34d 
through April 30, 1988 (Figure 2.3.97). These data were replotted in terms of the excess delta $p$ above threshold levels, including the percent of all affected rainbow and brown trout and their mean GBT rating (Figure 2.3 .98 and 2.3 .99 ). An excess delta $\mathrm{P}$ of 0.0 corresponds to the particular threshold condition. In Figure 2.3.98, at approximately 240 to $252 \mathrm{~d}$, which

corresponds to about september 1 to 12,1987 , both the percent of affected fish and the mean GBT rating dropped to near zero. However, the delta $\mathrm{P}$ data show a significant spike that exceeds the water bubble-skin blister threshold during this period. Not apparent in the figure are the large diurnal oscillations in delta $P$ that are occurring during this period. During the nighttime hours, the delta $P$ dropped below both the water bubble-skin blister threshold and the cardiovascular bubble growth threshold. The same situation applies to Figure 2.3 .99 where the brown trout data are plotted.

Another interesting feature of Figures 2.3 .98 and 2.3 .99 is that excess delta $P$ levels do not exceed $73.5 \mathrm{mmHg}$, which corresponds to the hydrostatic pressure at $1 \mathrm{~m}$ of water depth. Thus, if 1 m of water was avaliable to fish during this 1987 and 1988 period, and the fish chose to position themselves at or below that depth, they would avoid all symptoms of GBT. The data of Figures 2.3 .98 and 2.3 .99 can be used to calculate the required compensation depth (in meters) for the cardiovascular or water bubble-skin blister thresholds by dividing the excess delta $\mathrm{P}$ by 73.5 . This is shown in Figure 2.3 .100 . 


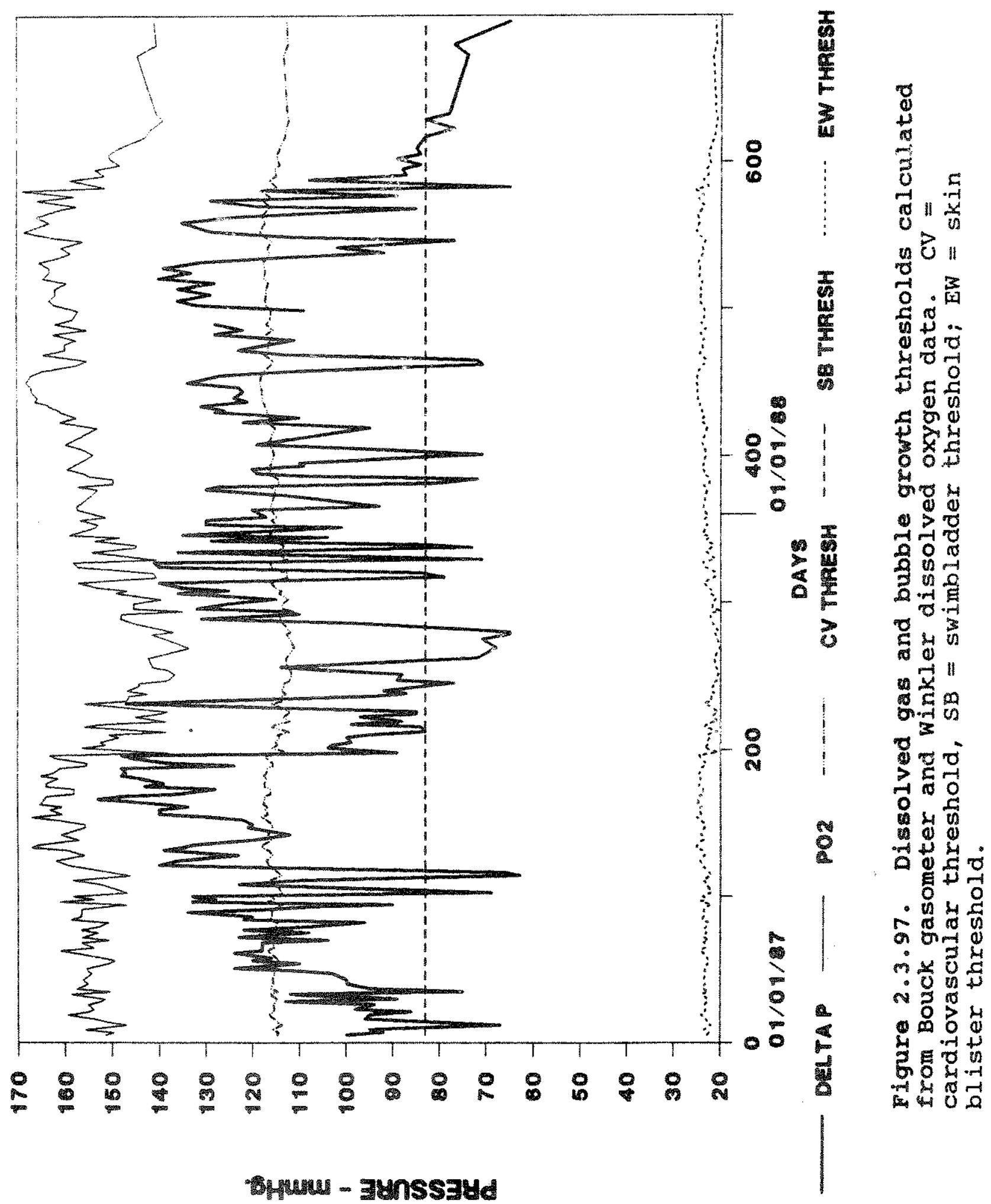


SNIIVY WOLINAS NYJW

HSIJ $03103 \mathrm{~A}$ \%

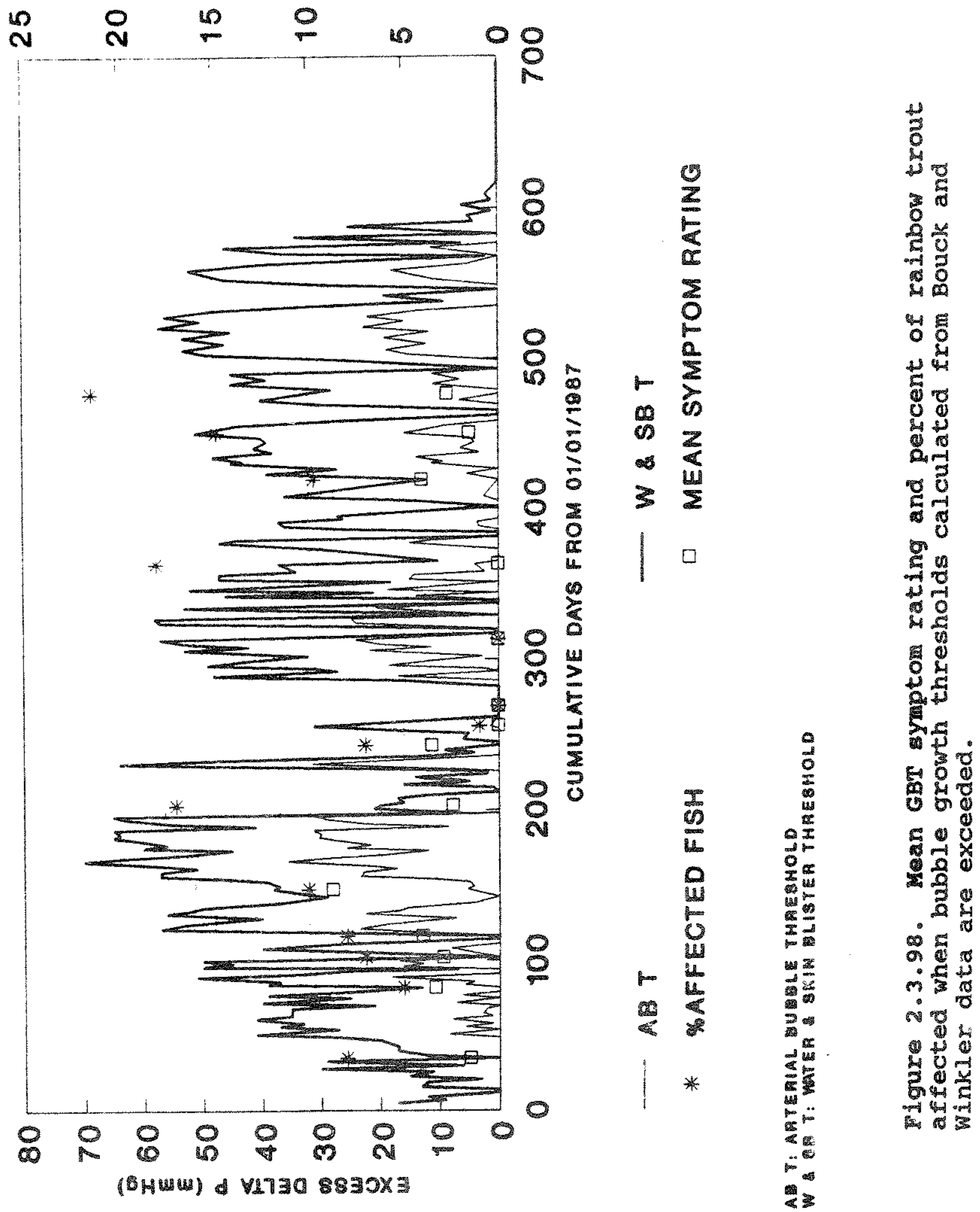




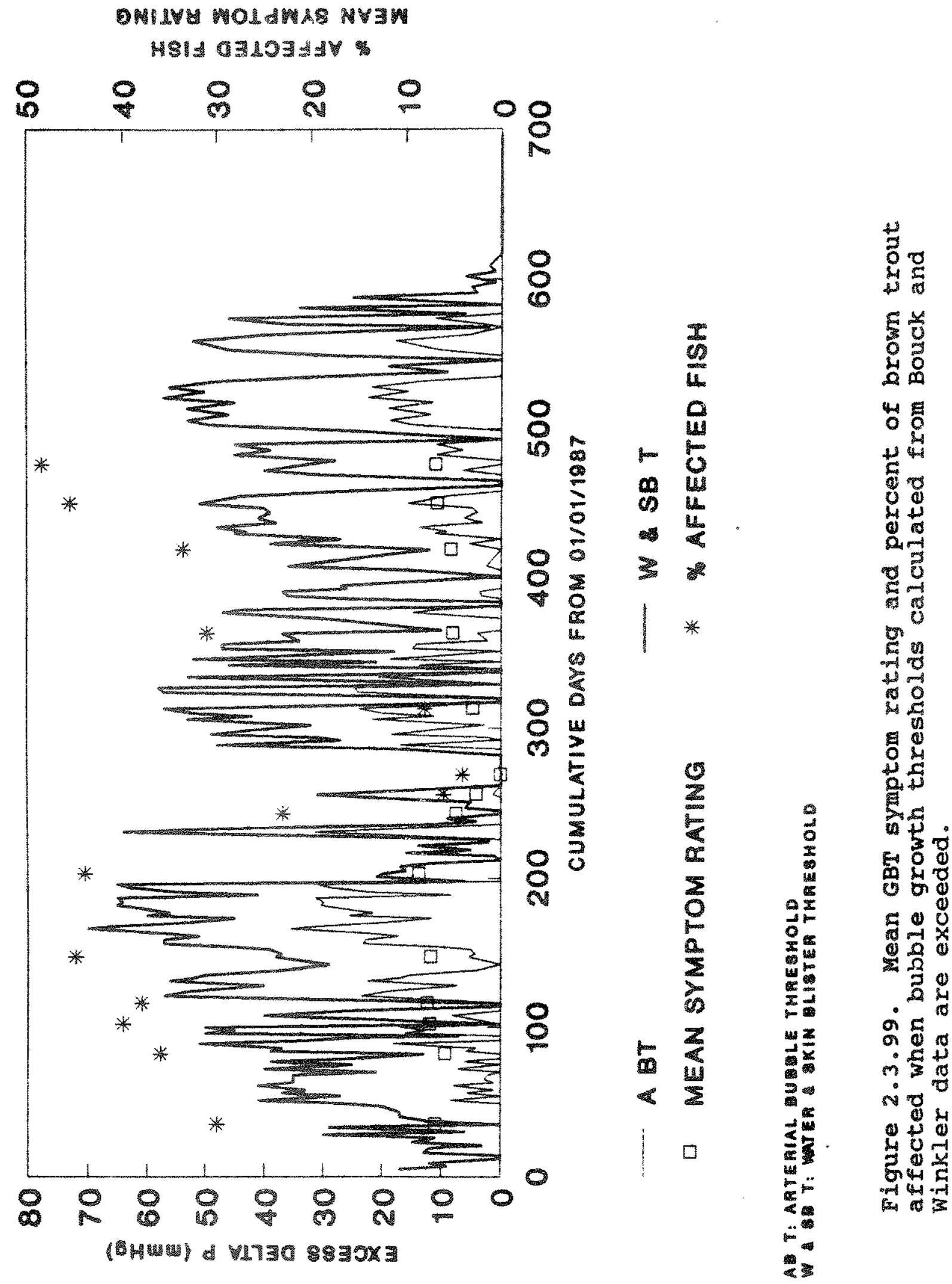




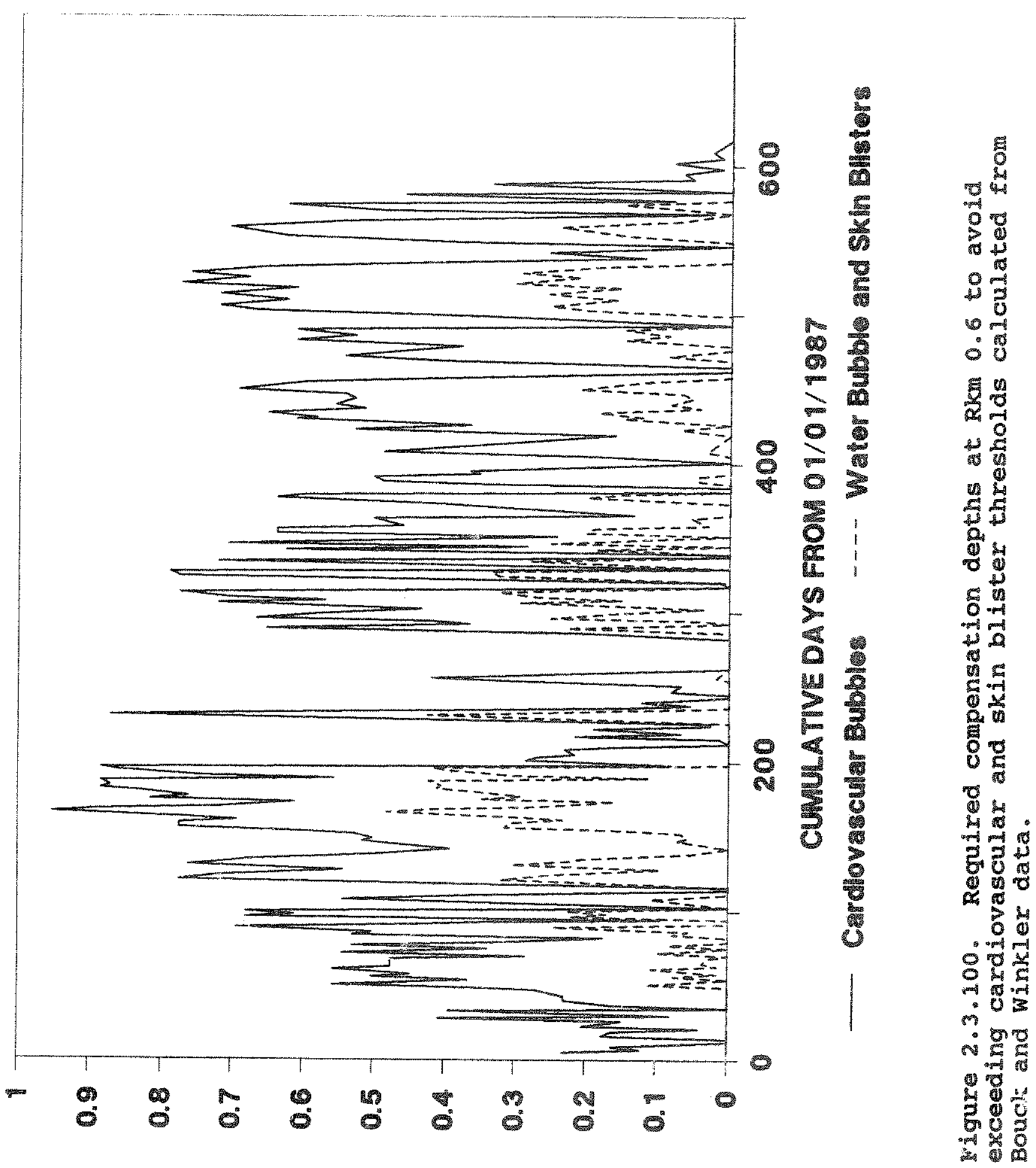

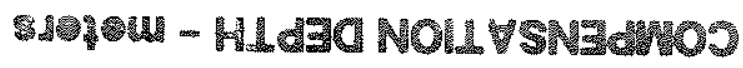


2.3.5.5 Changes in GBT symptoms Through Time Among Tagged Trout

\subsubsection{5a Methods}

We marked 1,837 trout with numbered Floy t-tags treated with algacide. During 1985, 900 tags were randomly placed in fish without regard to the presence of GBT. Beginning in 1986 most fish tagged had excernal signs of $\mathrm{CBT}$. We monitored the progression or remission of symptoms among recaptures. We applied the same rating system described in section 2.3 .5 .3 to categorize trout into four groups $(0-3)$.

In addition, we used a rating system that described the severity of GBT on each fin or body part ( 18 in a11) as having high, moderate, or low numbers of bubbles and assigned numerical values of 3,2, and 1 , respectively; if no bubbles were present, the rating was 0 . We also noted if recovery had occurred and if scars from previous bubbles were visible. The body part ratings were used to obtain a total GBT rating that numerically summarized the severity and proportion of GBT on all affected external surfaces of an individual fish.

\section{$2.3 .5 .5 \mathrm{~b}$ Results and Discussion}

of the 1,837 trout tagged, $87.6 \%$ were brown trout and $12.4 \%$ were rainbow trout. We recaptured tagged brown and rainbow trout 651 and 54 times, respectively. Most of the brown trout tagged $(90.9 \%)$, recaptured $(88.0 \%)$, and caught by anglers $(67.9 \%)$ were in the upper $1.9 \mathrm{RKm}$ (1.2 RM) (GBT monitoring section 1). Most other brown trout monitored were in GBT section 2, Rkm 3.9-6.1 (RM 2.4-3.8). The mark and recapture of rainbow trout was more equally distributed in the upper portion of the river: $57.5 \%$ and 
$38.1 \%$ were tagged and $51.9 \%$ and $38.9 \%$ were recaptured in GBT sections 1 and 2 , respectively. Almost $75 \%$ of tagged fish were captured along the right bank where monitoring was more frequent because of higher GBT incidence rates there. Approximately $7.6 \%$ of the rainbow trout and $4.2 \%$ of the brown trout tagged in the upper $1.9 \mathrm{Rkm}$ (1.2 RM) were subsequently recaptured downstream, suggesting greater movements among rainbow trout.

Severety of GBT symptoms remained unchanged in the majority of recaptured trout (Table 2.3 .22 ); most of these fish had no symptoms. The majority of recaptures with GBT had minor symptoms. GBT symptoms increased in $16 \%$ of the brown trout while they decreased in $22.2 \%$ (Table 2.3 .22 ). Only $6.8 \%$ of recaptured rainbow trout had more severe GBT, and $28.8 \%$ had less severe symptoms (Table 2.3.22). Although we observed the increase and remission of serious and severe GBT symptoms more among brown trout, the much smaller sample size for rainbow trout probably influenced results. Our data, however, demonstrate that some fish with serious and severe symptoms recover under conditions in the Bighorn River.

The average condition factor of tagged brown trout generally decreased as total fish length increased (Table 2.3.23). However, the same trend was not as apparent for rainbow trout (Table 2.3.24). For each size group of brown trout $\geq 275 \mathrm{~mm}$ (10.8 in), as well as the means for all tagged brown trout, condition decreased as GBT symptoms developed and became more severe (Table 2.3 .23 ). Overall, brown trout mean condition factors for fish with GBT codes of 1,2 , and 3 were 96.1, 87.3, and $76.5 \%$, respectively, of fish with no GBT symptoms. 
Table 2.3.22. Progression of GBT among recaptured brown and rainbow trout below Afterbay Dam in the Bighorn River 1985-1988.

Gas code change Brown trout Rainbow trout

No change

$\begin{array}{lrr}0 \text { to } 0 & 254 & 24 \\ 1 \text { to } 1 & 183 & 14 \\ 2 \text { to } 2 & 12 & - \\ & & 38\end{array}$

Increase

0 to 1

0 to 2

1 to 2

1 to 3

2 to 3

Total

2 to 0

2 to 1

3 to 1

3 to 2

Total

$\begin{array}{r}73 \\ 6 \\ 29 \\ 5 \\ 3 \\ \hline 116\end{array}$

Decrease
129

18

10

2

2

161
3

$-$

1

$-$

-

$\frac{4}{4}$

16

1

-

$-$

-

17

In the Beaverhead River, brown trout with GBT had condition factors about $10 \%$ lower when compared to the total sample (Oswald 1986). However, mean condition factors for brown trout $<275 \mathrm{~mm}$ either remained stable or increased as GBT severity increased. This may be due to small sample sizes or GBT having a greater effect on older fish that have been exposed to elevated gas pressures in previous years. 
Table 2.3.23. Mean condition factors (K) of brown trout tagged in the Bighorn River below Afterbay Dam for each size group and each GBT code category. GBT code: $0=$ no external symptoms, $1=$ minor, 2 = serious and 3 = severe symptoms. $N$ = sample size.

\section{GBT Code}

\begin{tabular}{ccccccccccccccc}
$\begin{array}{c}\text { Length } \\
(\mathrm{mm})\end{array}$ & $\mathrm{K}$ & $\mathrm{N}$ & $\mathrm{K}$ & & $\mathrm{N}$ & $\mathrm{K}$ & $\mathrm{N}$ & $\mathrm{K}^{3}$ & $\mathrm{~N}$ & $\mathrm{~K}$ & & $\mathrm{~N}$ \\
\hline
\end{tabular}

$\begin{array}{rrrrrrrrrrr}175-199 & - & (0) & 1.20 & (8) & 1.20 & (2) & - & (0) & 1.20 & (10) \\ 200-224 & 1.09 & (2) & 1.14 & (16) & - & (0) & - & (0) & 1.13 & (18) \\ 225-249 & 1.01 & (7) & 1.09 & (54) & 1.18 & (1) & - & (0) & 1.08 & (62) \\ 250-274 & 1.04 & (67) & 1.05 & (76) & - & (0) & - & (0) & 1.04 & (143) \\ 275-299 & 1.06 & (84) & 1.00 & (95) & 0.96 & (3) & 0.80 & (1) & 1.03 & (183) \\ 300-324 & 1.09 & (114) & 1.01 & (122) & 0.90 & (6) & 0.92 & (10) & 1.05 & (243) \\ 325-349 & 1.06 & (115) & 1.02 & (122) & 0.92 & (10) & 0.81 & (1) & 1.03 & (248) \\ 350-374 & 1.06 & (136) & 1.00 & (119) & 0.86 & (9) & 0.88 & (3) & 1.02 & (267) \\ 375-399 & 1.02 & (188) & 0.98 & (129) & 0.88 & (11) & 0.71 & (4) & 0.99 & (332) \\ 400-424 & 0.96 & (148) & 0.92 & (116) & 0.88 & (29) & 0.68 & (3) & 0.93 & (296) \\ 425-449 & 0.97 & (83) & 0.88 & (94) & 0.83 & (15) & 0.82 & (4) & 0.92 & (196) \\ 450-474 & 0.95 & (38) & 0.87 & (55) & 0.84 & (9) & 0.78 & (3) & 0.90 & (105) \\ 475-499 & 0.96 & (23) & 0.92 & (42) & 0.91 & (9) & - & (0) & 0.93 & (74) \\ 500-524 & 1.01 & (11) & 0.90 & (18) & 0.86 & (4) & - & (0) & 0.93 & (33) \\ 525-549 & 0.86 & (5) & 0.85 & (11) & 0.94 & (2) & - & (0) & 0.86 & (18) \\ 550-574 & 0.77 & (4) & 0.77 & (4) & 0.78 & (1) & - & (0) & 0.77 & (6) \\ 575-599 & 1.17 & (1) & - & (0) & - & (0) & - & (0) & 1.17 & (1) \\ \text { ALL } & 1.02 & (1025) & 0.98 & (1082) & 0.89 & (111) & 0.78 & (20) & 0.99 & (2238)\end{array}$

Mean condition of rainbow trout $\geq 275 \mathrm{~mm}$ always decreased as the GBT code increased from 0 to 1 (Table 2.3.24). However, except for one size group, as GBT codes increased from 1 to 2 , condition factors stabilized or increased. This may be due to small sample sizes or the large number of spawning fish with serious GBT symptoms due to use of shallow water spawning sites. The mean condition factors for rainbow trout with GBT ratings of 1 and 2 were $89.2 \%$ and $90.0 \%$, respectively, of the condition factor for fish with no external symptoms. 
Table 2.3.24. Mean condition factors $(K)$ of rainbow trout tagged in the Bighorn River: below Afterbay Dam for each size group and each gas code category. GBT code: $0=$ no external symptoms, $1=$ minor, 2 = serious and $3=$ severe symptoms. $N=$ sample size.

\begin{tabular}{|c|c|c|c|c|c|c|c|c|c|c|}
\hline \multirow{3}{*}{$\begin{array}{c}\text { Lengts } \\
\text { (mm) }\end{array}$} & \multicolumn{10}{|c|}{ GBT code } \\
\hline & \multicolumn{2}{|c|}{0} & \multicolumn{2}{|c|}{1} & \multicolumn{2}{|r|}{2} & \multicolumn{2}{|c|}{3} & \multicolumn{2}{|c|}{4} \\
\hline & $\mathrm{K}$ & $\mathbb{N}$ & $\mathrm{K}$ & $\mathrm{N}$ & $\mathrm{K}$ & $\mathrm{N}$ & $\mathrm{K}$ & $\mathbb{N}$ & $\mathbf{K}$ & $\mathrm{N}$ \\
\hline $200-224$ & - & $(0)$ & 1.35 & (1) & - & $(0)$ & - & (0) & 1.35 & (1) \\
\hline $225-249$ & - & (0) & 0.97 & (1) & - & (0) & - & (0) & 0.97 & (1) \\
\hline $250-274$ & 1.20 & $(7)$ & 1.22 & (2) & - & $(0)$ & - & $(0)$ & 1.20 & (9) \\
\hline $275-299$ & 1.27 & $(6)$ & 1.25 & (2) & - & $(0)$ & - & $(0)$ & 1.26 & (8) \\
\hline $300-321$ & 1.12 & (4) & 1.10 & (4) & - & $(0)$ & - & $(0)$ & 1.11 & (8) \\
\hline $325-349$ & 1.17 & (8) & 1.06 & (5) & - & $(0)$ & - & $(0)$ & 1.13 & (14) \\
\hline $350-374$ & 1.19 & $(13)$ & 1.12 & $(10)$ & - & $(0)$ & - & $(0)$ & 1.16 & (23) \\
\hline $375-399$ & 1.19 & (21) & 1.07 & $(16)$ & 1.11 & (1) & - & $(0)$ & 1.14 & (38) \\
\hline $400-424$ & 1.27 & $(20)$ & 1.07 & $(-8)$ & - & (0) & - & (0) & 1.17 & (38) \\
\hline $425-449$ & 1.28 & $(10)$ & 1.06 & $(25)$ & 1.06 & (3) & - & $(0)$ & 1.12 & (38) \\
\hline $450-474$ & 1.11 & (13) & 1.08 & $(32)$ & 1.08 & (4) & - & (0) & 1.09 & (49) \\
\hline $475-499$ & 1.24 & (7) & 1.03 & (13) & 1.17 & (2) & - & (0) & 1.11 & (22) \\
\hline $500-524$ & 1.19 & $(6)$ & 0.99 & $(6)$ & 1.12 & (1) & - & $(0)$ & 1.09 & (13) \\
\hline $525-549$ & 1.12 & (5) & 0.71 & (1) & - & (0) & - & $(0)$ & 1.05 & (6) \\
\hline $550-574$ & 1.17 & (1) & 0.94 & $(2)$ & 0.83 & (1) & - & (0) & 0.97 & (4) \\
\hline $575-599$ & 1.22 & (2) & 1.02 & (1) & - & $(0)$ & - & (0) & 1.15 & (3) \\
\hline $600-624$ & 1.57 & (1) & 0.86 & (2) & - & (0) & - & (0) & 1.10 & (3) \\
\hline ALL & 1.20 & $(124)$ & 1.07 & $(142)$ & 1.08 & (12) & - & (0) & 1.13 & $(278)$ \\
\hline
\end{tabular}

Anglers reported catching 81 tagged brown trout and 11 rainbow trout, which represents 5.0 and $4.8 \%$, respectively, of the total number originally tagged. Anglers made no mention of visible GBT symptoms on any rainbow caught, but three tagged brown trout caught by study employees had symptoms present: two with minor symptoms and one with serious symptoms.

GBT symptoms were most commonly observed on the caudal and dorsal fins of tagged brown trout (Table 2.3.25). Other fins affected in 20-25\% of tagged brown trout with GBT were the anal, both pectorals, and the left pelvic. Less than $1 \%$ of tagged brown trout had GBT symptoms on the adipose fin, in the buccal 
Table 2.3.25. The percentages of affected body parts on tagged brown trout exhibiting GBT when captured in the Bighorn River below Afterbay Dam, 1985-1988. GBT code: $0=$ no symptoms, $1=$ minor, 2 = serious, and 3 = severe symptoms.

\begin{tabular}{|c|c|c|c|c|c|}
\hline \multirow[b]{2}{*}{$\begin{array}{l}\text { Body } \\
\text { part }\end{array}$} & \multirow[b]{2}{*}{$\begin{array}{c}\text { severity } \\
\text { of GBT }\end{array}$} & \multicolumn{3}{|c|}{ GBT code } & \multirow{2}{*}{$\begin{array}{l}\text { \% Total } \\
\text { of all fish } \\
\text { with GBT }\end{array}$} \\
\hline & & $\begin{array}{l}1 \\
\%\end{array}$ & $\begin{array}{l}2 \\
\%\end{array}$ & $\begin{array}{l}3 \\
\%\end{array}$ & \\
\hline Adipose & $\begin{array}{l}\text { Low } \\
\text { Medium } \\
\text { High } \\
\text { All }\end{array}$ & $\begin{array}{l}0.1 \\
0.3 \\
0.0 \\
0.4\end{array}$ & $\begin{array}{l}0.0 \\
0.0 \\
1.8 \\
1.8\end{array}$ & $\begin{array}{l}0.0 \\
0.0 \\
0.0 \\
0.0\end{array}$ & $\begin{array}{l}0.1 \\
0.2 \\
0.2 \\
0.5\end{array}$ \\
\hline Anal & $\begin{array}{l}\text { Low } \\
\text { Medium } \\
\text { High } \\
\text { All }\end{array}$ & $\begin{array}{r}11.8 \\
4.0 \\
3.4 \\
19.3\end{array}$ & $\begin{array}{l}12.5 \\
12.5 \\
35.7 \\
60.7\end{array}$ & $\begin{array}{r}5.0 \\
20.0 \\
55.0 \\
80.0\end{array}$ & $\begin{array}{r}11.8 \\
5.1 \\
7.2 \\
24.1\end{array}$ \\
\hline Buccal cavity & $\begin{array}{l}\text { Medium } \\
\text { A11 }\end{array}$ & $\begin{array}{l}0.2 \\
0.2\end{array}$ & $\begin{array}{l}0.9 \\
0.9\end{array}$ & $\begin{array}{l}0.0 \\
0.0\end{array}$ & $\begin{array}{l}0.2 \\
0.2\end{array}$ \\
\hline Caudal & $\begin{array}{l}\text { Low } \\
\text { Medium } \\
\text { High } \\
\text { All }\end{array}$ & $\begin{array}{l}23.5 \\
13.0 \\
18.9 \\
55.5\end{array}$ & $\begin{array}{l}11.6 \\
17.9 \\
63.4 \\
92.9\end{array}$ & $\begin{array}{r}5.0 \\
10.0 \\
80.0 \\
95.0\end{array}$ & $\begin{array}{l}22.1 \\
13.4 \\
24.0 \\
59.5\end{array}$ \\
\hline Dorsal & $\begin{array}{l}\text { Low } \\
\text { Medium } \\
\text { High } \\
\text { All }\end{array}$ & $\begin{array}{l}18.2 \\
15.5 \\
21.5 \\
55.2\end{array}$ & $\begin{array}{r}7.1 \\
13.4 \\
64.3 \\
84.8\end{array}$ & $\begin{array}{r}5.0 \\
5.0 \\
80.0 \\
90.0\end{array}$ & $\begin{array}{l}17.0 \\
15.2 \\
26.4 \\
58.5\end{array}$ \\
\hline Head & $\begin{array}{l}\text { Low } \\
\text { Medium } \\
\text { High } \\
\text { All }\end{array}$ & $\begin{array}{l}0.9 \\
0.5 \\
0.8 \\
2.2\end{array}$ & $\begin{array}{r}0.0 \\
0.9 \\
13.4 \\
14.3\end{array}$ & $\begin{array}{r}0.0 \\
0.0 \\
65.0 \\
65.0\end{array}$ & $\begin{array}{l}0.8 \\
0.5 \\
3.0 \\
4.3\end{array}$ \\
\hline Left eye & $\begin{array}{l}\text { Low } \\
\text { Medium } \\
\text { High } \\
\text { All }\end{array}$ & $\begin{array}{l}1.1 \\
2.8 \\
0.8 \\
4.7\end{array}$ & $\begin{array}{l}0.9 \\
1.8 \\
0.9 \\
3.6\end{array}$ & $\begin{array}{r}0.0 \\
5.0 \\
5.0 \\
10.0\end{array}$ & $\begin{array}{l}1.1 \\
2.7 \\
0.9 \\
4.7\end{array}$ \\
\hline Right eye & $\begin{array}{l}\text { Low } \\
\text { Medium } \\
\text { High } \\
\text { All }\end{array}$ & $\begin{array}{l}2.3 \\
2.6 \\
1.3 \\
6.2\end{array}$ & $\begin{array}{l}1.8 \\
0.9 \\
3.6 \\
6.3\end{array}$ & $\begin{array}{r}5.0 \\
0.0 \\
5.0 \\
20.0\end{array}$ & $\begin{array}{l}2.3 \\
2.4 \\
1.6 \\
6.2\end{array}$ \\
\hline Left gill & $\begin{array}{l}\text { Low } \\
\text { Medium } \\
\text { Al1 }\end{array}$ & $\begin{array}{l}0.3 \\
0.2 \\
0.5\end{array}$ & $\begin{array}{l}0.0 \\
1.8 \\
1.8\end{array}$ & $\begin{array}{l}0.0 \\
0.0 \\
0.0\end{array}$ & $\begin{array}{l}0.2 \\
0.3 \\
0.6\end{array}$ \\
\hline Right gill & $\begin{array}{c}\text { Low } \\
\text { Medium } \\
\text { A11 }\end{array}$ & $\begin{array}{l}0.3 \\
0.2 \\
0.5\end{array}$ & $\begin{array}{l}0.0 \\
1.8 \\
1.8\end{array}$ & $\begin{array}{l}0.0 \\
0.0 \\
0.0\end{array}$ & $\begin{array}{l}0.2 \\
0.3 \\
0.6\end{array}$ \\
\hline
\end{tabular}


Table 2.3 .25$. (continued)...

\begin{tabular}{|c|c|c|c|c|c|}
\hline \multirow[b]{2}{*}{$\begin{array}{l}\text { Body } \\
\text { part }\end{array}$} & \multirow[b]{2}{*}{$\begin{array}{c}\text { Severity } \\
\text { of GBT }\end{array}$} & \multicolumn{3}{|c|}{ GBT code } & \multirow{2}{*}{$\begin{array}{l}\text { \%otal } \\
\text { of all fish } \\
\text { with GBT }\end{array}$} \\
\hline & & $\begin{array}{l}1 \\
\%\end{array}$ & $\begin{array}{l}2 \\
\frac{9}{0}\end{array}$ & $\begin{array}{l}3 \\
\%\end{array}$ & \\
\hline Left operculum & $\begin{array}{l}\text { Low } \\
\text { Medium } \\
\text { High } \\
\text { All }\end{array}$ & $\begin{array}{l}2.8 \\
3.5 \\
2.3 \\
8.5\end{array}$ & $\begin{array}{r}4.5 \\
13.4 \\
35.7 \\
53.6\end{array}$ & $\begin{array}{r}10.0 \\
5.0 \\
80.0 \\
95.0\end{array}$ & $\begin{array}{r}3.0 \\
4.4 \\
6.6 \\
14.1\end{array}$ \\
\hline Right operculum & $\begin{array}{l}\text { Low } \\
\text { Medium } \\
\text { High } \\
\text { All }\end{array}$ & $\begin{array}{l}3.7 \\
3.3 \\
2.3 \\
9.3\end{array}$ & $\begin{array}{r}3.6 \\
14.3 \\
33.9 \\
51.8\end{array}$ & $\begin{array}{r}5.0 \\
10.0 \\
70.0 \\
85.0\end{array}$ & $\begin{array}{r}3.7 \\
4.4 \\
6.3 \\
14.4\end{array}$ \\
\hline Left Pectoral & $\begin{array}{l}\text { Low } \\
\text { Medium } \\
\text { High } \\
\text { All }\end{array}$ & $\begin{array}{r}7.3 \\
4.3 \\
3.8 \\
15.4\end{array}$ & $\begin{array}{r}9.8 \\
8.9 \\
40.2 \\
58.9\end{array}$ & $\begin{array}{r}5.0 \\
15.0 \\
55.0 \\
75.0\end{array}$ & $\begin{array}{r}7.5 \\
4.9 \\
7.9 \\
20.4\end{array}$ \\
\hline Right Pectoral & $\begin{array}{l}\text { Low } \\
\text { Medium } \\
\text { High } \\
\text { All }\end{array}$ & $\begin{array}{r}9.4 \\
5.0 \\
3.6 \\
17.9\end{array}$ & $\begin{array}{l}12.5 \\
10.7 \\
38.4 \\
61.6\end{array}$ & $\begin{array}{r}5.0 \\
10.0 \\
65.0 \\
80.0\end{array}$ & $\begin{array}{r}9.6 \\
5.6 \\
7.8 \\
22.9\end{array}$ \\
\hline Left Pelvic & $\begin{array}{l}\text { Low } \\
\text { Medium } \\
\text { High } \\
\text { A11 }\end{array}$ & $\begin{array}{r}8.8 \\
4.8 \\
2.1 \\
15.7\end{array}$ & $\begin{array}{l}13.4 \\
12.5 \\
28.6 \\
54.5\end{array}$ & $\begin{array}{l}10.0 \\
15.0 \\
65.0 \\
90.0\end{array}$ & $\begin{array}{r}9.3 \\
5.7 \\
5.6 \\
20.5\end{array}$ \\
\hline Right pelvic & $\begin{array}{l}\text { Low } \\
\text { Medium } \\
\text { High } \\
\text { All }\end{array}$ & $\begin{array}{r}7.1 \\
5.4 \\
2.0 \\
14.5\end{array}$ & $\begin{array}{l}10.7 \\
15.2 \\
22.3 \\
48.2\end{array}$ & $\begin{array}{r}0.0 \\
15.0 \\
70.0 \\
85.0\end{array}$ & $\begin{array}{r}7.3 \\
6.5 \\
5.0 \\
18.8\end{array}$ \\
\hline Left popeye & $\begin{array}{l}\text { Low } \\
\text { Medium } \\
\text { High } \\
\text { Al1 }\end{array}$ & $\begin{array}{l}1.0 \\
0.8 \\
0.1 \\
1.9\end{array}$ & $\begin{array}{l}0.9 \\
0.9 \\
1.8 \\
3.6\end{array}$ & $\begin{array}{l}0.0 \\
0.0 \\
5.0 \\
5.0\end{array}$ & $\begin{array}{l}1.0 \\
0.8 \\
0.3 \\
2.1\end{array}$ \\
\hline Right popeye & $\begin{array}{l}\text { Low } \\
\text { Medium } \\
\text { High } \\
\text { A11 }\end{array}$ & $\begin{array}{l}0.8 \\
0.8 \\
0.7 \\
2.4\end{array}$ & $\begin{array}{l}0.0 \\
0.9 \\
1.8 \\
2.7\end{array}$ & $\begin{array}{l}0.0 \\
0.0 \\
0.0 \\
0.0\end{array}$ & $\begin{array}{l}0.7 \\
0.8 \\
0.8 \\
2.4\end{array}$ \\
\hline Mouth & $\begin{array}{l}\text { Low } \\
\text { Medium } \\
\text { High } \\
\text { सil }\end{array}$ & $\begin{array}{r}4.3 \\
6.8 \\
2.4 \\
13.5\end{array}$ & $\begin{array}{r}8.0 \\
21.4 \\
33.0 \\
62.5\end{array}$ & $\begin{array}{r}5.0 \\
10.0 \\
75.0 \\
90.0\end{array}$ & $\begin{array}{r}4.7 \\
8.2 \\
6.4 \\
19.2\end{array}$ \\
\hline skin & $\begin{array}{c}\text { Low } \\
\text { Medium } \\
\text { High } \\
\text { AII }\end{array}$ & $\begin{array}{l}3.1 \\
0.1 \\
0.0 \\
3.2\end{array}$ & $\begin{array}{r}10.7 \\
8.0 \\
4.5 \\
23.2\end{array}$ & $\begin{array}{r}10.0 \\
10.0 \\
0.0 \\
20.0\end{array}$ & $\begin{array}{l}3.9 \\
1.0 \\
0.4 \\
5.3\end{array}$ \\
\hline
\end{tabular}


cavity, or on the gills. Brown trout recovery from GBT symptoms was most evident in the mouth and on the caudal fin.

Most $(80.6 \%)$ tagged brown trout had total GBT rating's of $0-$ $10 ; 13.4 \%$ and $4.5 \%$ had ratings of $11-20$ and $21-30$, respectively, and $1.5 \%$ had severe GBT ratings ranging from $31-40$ out of a possible 54. The highest GBT total rating found arrong brown trout was 39. Mean GBT ratings increased from 4.5 to 17.0 to 26.6 for brown trout assigned to GBT codes 1, 2, and 3, respectively.

Although tagged rainbow trout with minor symptoms had a slightly lower mean GBT total rating than brown trout $(4.3 \mathrm{vs}$. 4.5), those with serious symptoms had a substantially higher rating (19.1). The mean GBT rating for all rainbow trout with symptoms was 5.4, which was slightly lower than the mean GBT rating of 6.0 for brown trout.

Most $(82.3 \%)$ rainbow trout had total GBT ratings ranging from $0-10$. Another $12.6 \%$ had ratings ranging from $11-20$, and the remaining $5.1 \%$ rated $20-30$. The maximum GBT total rating among tagged rainbow trout was 28 .

The dorsal fin $(62.4 \%)$, followed by the caudal fin (44.6\%), were the body parts most commonly afflicted with GBr among all tagged rainbow trout (Table 2.3 .26 ). Other body parts frequently affected included the skin $(41.4 \%)$, the aral fin (28.0\%), the mouth (22.3\%), and the right pectoral (21.\%). We observed no GBT symptoms on rainbow trout gills and no exophthalmia (popeye) in the left eye.

All rainbow trout with serious symptoms had bubbles in the dorsal fin (Table 2.3.26). Eighty-three percent had bubbles 
Table 2.3.26. The percentages of affected body parts on tagged rainbow trout exhibiting $G B T$ when captured in the Bighorn River below Afterbay Dam, 1985-1988. GBT code: $0=$ no symptoms, $1=$ minor, 2 = serious.

\begin{tabular}{|c|c|c|c|c|}
\hline \multirow[b]{2}{*}{$\begin{array}{l}\text { Bocy } \\
\text { part }\end{array}$} & \multirow[b]{2}{*}{$\begin{array}{l}\text { Severity } \\
\text { of GBT }\end{array}$} & \multicolumn{2}{|c|}{ GBT code } & \multirow{2}{*}{$\begin{array}{l}\text { \% Total } \\
\text { of all fish } \\
\text { with GBT }\end{array}$} \\
\hline & & $\begin{array}{l}1 \\
\frac{8}{0}\end{array}$ & $\begin{array}{l}2 \\
\%\end{array}$ & \\
\hline Adipose & High & 0.0 & 8.3 & 0.6 \\
\hline Anal & $\begin{array}{l}\text { Low } \\
\text { Medium } \\
\text { High } \\
\text { A11 }\end{array}$ & $\begin{array}{r}15.2 \\
4.8 \\
4.8 \\
24.8\end{array}$ & $\begin{array}{l}25.0 \\
25.0 \\
16.7 \\
66.7\end{array}$ & $\begin{array}{r}15.9 \\
6.4 \\
5.7 \\
28.0\end{array}$ \\
\hline Buccal cavity & A11 & 0.0 & 0.0 & 0.0 \\
\hline Caudal & $\begin{array}{l}\text { Low } \\
\text { Medium } \\
\text { High } \\
\text { All }\end{array}$ & $\begin{array}{r}32.4 \\
9.0 \\
2.1 \\
43.4\end{array}$ & $\begin{array}{r}8.3 \\
41.7 \\
8.3 \\
58.3\end{array}$ & $\begin{array}{r}30.6 \\
11.5 \\
2.5 \\
44.6\end{array}$ \\
\hline Dorsa 1 & $\begin{array}{l}\text { Low } \\
\text { Medium } \\
\text { High } \\
\text { AII }\end{array}$ & $\begin{array}{l}22.8 \\
22.1 \\
14.5 \\
59.3\end{array}$ & $\begin{array}{r}0.0 \\
25.0 \\
75.0 \\
100.0\end{array}$ & $\begin{array}{l}21.0 \\
22.3 \\
19.1 \\
62.4\end{array}$ \\
\hline Head & $\begin{array}{l}\text { Medium } \\
\text { A11 }\end{array}$ & $\begin{array}{l}0.7 \\
0.7\end{array}$ & $\begin{array}{l}8.3 \\
8.3\end{array}$ & $\begin{array}{l}1.3 \\
1.3\end{array}$ \\
\hline Left eye & $\begin{array}{l}\text { Low } \\
\text { Medium } \\
\text { A11 }\end{array}$ & $\begin{array}{l}0.7 \\
2.1 \\
2.8\end{array}$ & $\begin{array}{l}0.0 \\
0.0 \\
0.0\end{array}$ & $\begin{array}{l}0.6 \\
1.9 \\
2.5\end{array}$ \\
\hline Right eye & $\begin{array}{l}\text { Low } \\
\text { Medium } \\
\text { High } \\
\text { All }\end{array}$ & $\begin{array}{l}1.4 \\
1.4 \\
0.7 \\
3.4\end{array}$ & $\begin{array}{l}0.0 \\
8.3 \\
0.0 \\
8.3\end{array}$ & $\begin{array}{l}1.3 \\
1.9 \\
0.6 \\
3.8\end{array}$ \\
\hline Left gill & Al1 & 0.0 & 0.0 & 0.0 \\
\hline Right gill & A.1 & 0.0 & 0.0 & 0.0 \\
\hline Left operculum & $\begin{array}{l}\text { Low } \\
\text { Medium } \\
\text { High } \\
\text { All }\end{array}$ & $\begin{array}{r}2.8 \\
6.2 \\
2.1 \\
11.0\end{array}$ & $\begin{array}{r}8.3 \\
16.7 \\
58.3 \\
83.3\end{array}$ & $\begin{array}{r}3.2 \\
7.0 \\
6.4 \\
16.6\end{array}$ \\
\hline Right operculum & $\begin{array}{l}\text { Low } \\
\text { Medium } \\
\text { High } \\
\text { Al1 }\end{array}$ & $\begin{array}{r}4.8 \\
8.3 \\
2.1 \\
15.2\end{array}$ & $\begin{array}{r}8.3 \\
16.7 \\
33.3 \\
58.3\end{array}$ & $\begin{array}{r}5.1 \\
8.9 \\
4.5 \\
18.5\end{array}$ \\
\hline
\end{tabular}


Table 2.3.26. (continued).

\begin{tabular}{|c|c|c|c|c|}
\hline \multirow[b]{2}{*}{$\begin{array}{l}\text { Body } \\
\text { part }\end{array}$} & \multirow[b]{2}{*}{$\begin{array}{c}\text { Severity } \\
\text { of GBT }\end{array}$} & \multicolumn{2}{|c|}{ GBT code } & \multirow{2}{*}{$\begin{array}{l}\% \text { Total } \\
\text { of all fish } \\
\text { with GBT }\end{array}$} \\
\hline & & $\begin{array}{l}1 \\
\%\end{array}$ & $\begin{array}{l}2 \\
\%\end{array}$ & \\
\hline Left Pectoral & $\begin{array}{l}\text { Low } \\
\text { Medium } \\
\text { High } \\
\text { All }\end{array}$ & $\begin{array}{r}9.0 \\
2.1 \\
2.1 \\
13.1\end{array}$ & $\begin{array}{r}0.0 \\
25.0 \\
33.3 \\
58.3\end{array}$ & $\begin{array}{r}8.3 \\
3.8 \\
4.5 \\
10.2\end{array}$ \\
\hline Right Pectoral & $\begin{array}{l}\text { Low } \\
\text { Medium } \\
\text { High } \\
\text { All }\end{array}$ & $\begin{array}{r}12.4 \\
6.2 \\
0.7 \\
19.3\end{array}$ & $\begin{array}{r}0.0 \\
16.7 \\
25.0 \\
41.7\end{array}$ & $\begin{array}{r}11.5 \\
7.0 \\
2.5 \\
21.0\end{array}$ \\
\hline Left pelvic & $\begin{array}{l}\text { Low } \\
\text { Medium } \\
\text { High } \\
\text { A11 }\end{array}$ & $\begin{array}{r}8.3 \\
2.8 \\
2.1 \\
13.1\end{array}$ & $\begin{array}{r}0.0 \\
16.7 \\
41.7 \\
58.3\end{array}$ & $\begin{array}{r}7.6 \\
3.8 \\
5.1 \\
16.6\end{array}$ \\
\hline Right pelvic & $\begin{array}{l}\text { Low } \\
\text { Medium } \\
\text { High } \\
\text { All }\end{array}$ & $\begin{array}{r}6.2 \\
4.8 \\
1.4 \\
12.4\end{array}$ & $\begin{array}{r}8.3 \\
16.7 \\
25.0 \\
50.0\end{array}$ & $\begin{array}{r}6.4 \\
5.7 \\
3.2 \\
15.3\end{array}$ \\
\hline Left popeye & A11 & 0.0 & 0.0 & 0.0 \\
\hline Right popeye & $\begin{array}{l}\text { Low } \\
\text { Medium } \\
\text { A11 }\end{array}$ & $\begin{array}{l}1.4 \\
0.7 \\
2.1\end{array}$ & $\begin{array}{l}0.0 \\
0.0 \\
0.0\end{array}$ & $\begin{array}{l}1.3 \\
0.6 \\
1.9\end{array}$ \\
\hline Mouth & $\begin{array}{l}\text { Low } \\
\text { Medium } \\
\text { High } \\
\text { A11 }\end{array}$ & $\begin{array}{r}9.7 \\
7.6 \\
0.0 \\
17.2\end{array}$ & $\begin{array}{r}8.3 \\
25.0 \\
50.0 \\
83.3\end{array}$ & $\begin{array}{r}9.6 \\
8.9 \\
3.8 \\
22.3\end{array}$ \\
\hline skin & $\begin{array}{l}\text { Low } \\
\text { Medium } \\
\text { High } \\
\text { All }\end{array}$ & $\begin{array}{r}29.0 \\
8.3 \\
0.7 \\
37.9\end{array}$ & $\begin{array}{l}16.7 \\
16.7 \\
50.0 \\
83.3\end{array}$ & $\begin{array}{r}28.0 \\
8.9 \\
4.5 \\
41.4\end{array}$ \\
\hline
\end{tabular}

in the left operculum, skin and mouth. Signs of recovery from GBT were observed in the mouth and left operculum as well as on the caudal, dorsal, and right pectoral fins.

Nearly $10 \%$ of tagged brown trout and $6.6 \%$ of tagged rainbow trout were blind in at least one eye. only $0.2-0.3 \%$ of the trout 
were blind in both eyes.

Anglers reported releasing $17.3 \%$ of the tagged brown trout and $27.3 \%$ of the rainbow trout caught. The percentage of both species we could assume or know to have been creeled ranged from 63-68\%, which accounts for $85-91 \%$ of all tagged trout reported caught. We observed hooking scars on 12.9 and $21.3 \%$ of the tagged brown and rainbow trout, respectively. Frequencies of hooking scars on the right and left side of tagged fish were similar for both species and ranged from $40.0-45.4 \%$. About $8 \%$ of the tagged fish had hooking scars in the middle of the jaw while $4 \%$ of the brown trout and $6.7 \%$ of the rainbow trout had hooking scars on both sides.

Almost $5 \%$ of the tagged brown trout and $2.8 \%$ of the rainbow trout sampled were classified as humpies. Humpy was defined as any fish we could identify as having several adjacent, compressed vertebrae resulting in a convex posture.

Although only $2.6 \%$ of the brown trout tagged were in spawning condition, $50.7 \%$ of the rainbow trout were spawning. Sex ratios were $1: 1$ and $1: 53$ males: females for brown and rainbow trout, respectively. 


\section{Behavior and Habitat selection}

\subsubsection{Predation susceptibility}

\subsection{1a Methods}

Tank Tests: Sublethal effects of GBT on juvenile brown and rainbow trout were evaluated during predation susceptibility tests similar to those described by Bams (1967). the experimental procedure (Figure 2.4.1) was repeated 14 times.

One hour prior to each test, eight Arlee hatchery rainbow trout ranging in size from $200-270 \mathrm{~mm}$ (four during the first four tests) were transferred from a $1.8 \mathrm{~m}$ circular fiberglass rearing tank (80 cm deep) to three replicate $1.2 \mathrm{~m}$ tanks $(65 \mathrm{~cm}$ deep). Each tank was supplied with a constant flow of water which exited via a single stand-pipe. Different prey fish were used during

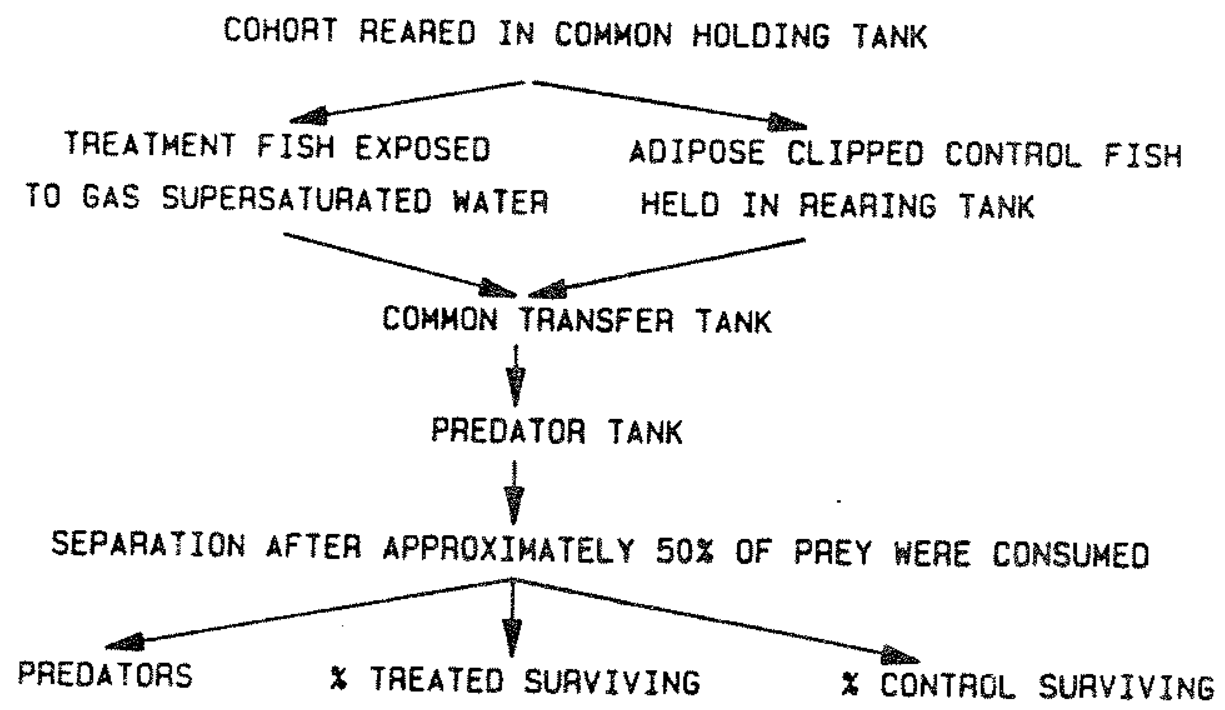

Figure 2.4.1. Flow diagram for tests to determine relative vulnerability to predation between fish exposed to gas supersaturated water and unexposed fish. 
each test. Juvenile rainbow (mean length $=46 \pm 8 \mathrm{~mm}$ )

and brown trout (mean length $=42 \pm 4 \mathrm{~mm}$ ) that had survived exposure to $124 \%$ supersaturated water for 30 d were used during the first four tests. Larger rainbow (mean length $=77 \pm 6 \mathrm{~mm}$ ) and brown trout (mean length $=56 \pm 7 \mathrm{~mm}$ ) that had been exposed for $30 \mathrm{~d}$ to $112 \%$ supersaturated water were used for the second four tests. Brown trout exposed for 30 d to $110 \%$ were used for two tests. Brown trout exposed $13 \mathrm{~h}$ to $130 \%$ supersaturated water were used during the remaining four tests.

Three days prior to predator avoidance tests, numbers of control fish equal to numbers of experimental fish were adipose clipped. Bams (1967) found that fin clipping control fish created no bias in testing. Control fish were from the same cohort, fed the same food, and reared at the same water temperature, but had not been exposed to supersaturated water.

prey ( 25 control and 25 test) were placed in a 4 L plastic bowl and gently stirred into the predator occupied water. When predators had ingested approximately $50 \%$ of the prey, they were removed from the tanks. Test results were discarded if more than 35 or less than 15 prey remained; five tests were invalidated. Surviving prey were counted, measured, and sorted according to experimental history. Instantaneous mortality ratios (Bams 1967) were calculated using the following equation:

$$
d p=\frac{-\log _{e}(i 1)}{-\log _{e}(i 2)}
$$

where:

$\mathrm{dp}=$ instantaneous mortality ratio

$i 1$ = surviving treatment fish/starting \# of treatment fish

12 = surviving control fish/ starting $\|_{\|}$of control fish 
When the instantaneous mortality rate of fish exposed to gas supersaturated water is greater than the instantaneous mortality rate of control fish, dp will be greater than 1 . When the predation ratio is equal, $d p=1$. The chi-square correction for continuity (Zar 1984) was used to test for statistical significance.

Experimental stream Tests: Seven predation tests were conducted in an experimental stream to determine if juvenile rainbow and brown trout exposed to high gas supersaturation levels are more susceptible to predation than non-exposed fish. The rectangular study section within an oval experimental stream channel was $5.1 \mathrm{~m}$ long, $0.6 \mathrm{~m}$ wide, $0.4 \mathrm{~m}$ deep, with a water depth of $0.39 \mathrm{~m}$ (Figure 2.4.2). Plastic-mesh screening formed upstream and downstream boundaries and metal screening placed on top of the channel kept fish from jumping out. Gravel (mean diameter $=1.9 \mathrm{~cm}$ ) was used to simulate natural conditions. The stream channel was provided with $8 \mathrm{~L} / \mathrm{min}$ of $16.6{ }^{\circ} \mathrm{C}$ water containing $6.2 \mathrm{ppm}$ DO. Water depth was regulated at $39.0 \mathrm{~cm}$ by a single stand pipe. A sears electric outboard motor (with 30 pounds thrust) created a mean stream velocity of $7 \mathrm{~cm} / \mathrm{sec}$. The thalweg of the section was partially directed using a $42.0 \mathrm{~cm}$ long, $10.0 \mathrm{~cm}$ wide, and $2.0 \mathrm{~cm}$ deep wing-deflector located at the upstream end of the channel. Three types of escape cover were provided for the prey (Figure 2.4.2). Two trapezoidal wooden slabs $(30.0 \mathrm{~cm} \times 21.0 \mathrm{~cm} \times 4.0 \mathrm{~cm} \times 10.5 \mathrm{~cm}$ and $33.5 \mathrm{~cm} \times 25.0 \mathrm{~cm}$ $\times 4.0 \mathrm{~cm} \times 9.0 \mathrm{~cm}$ ) furnished overhead cover, while a straight white plastic cylinder $(37.5 \mathrm{~cm}$ long, $6.0 \mathrm{~cm}$ diameter) and an 

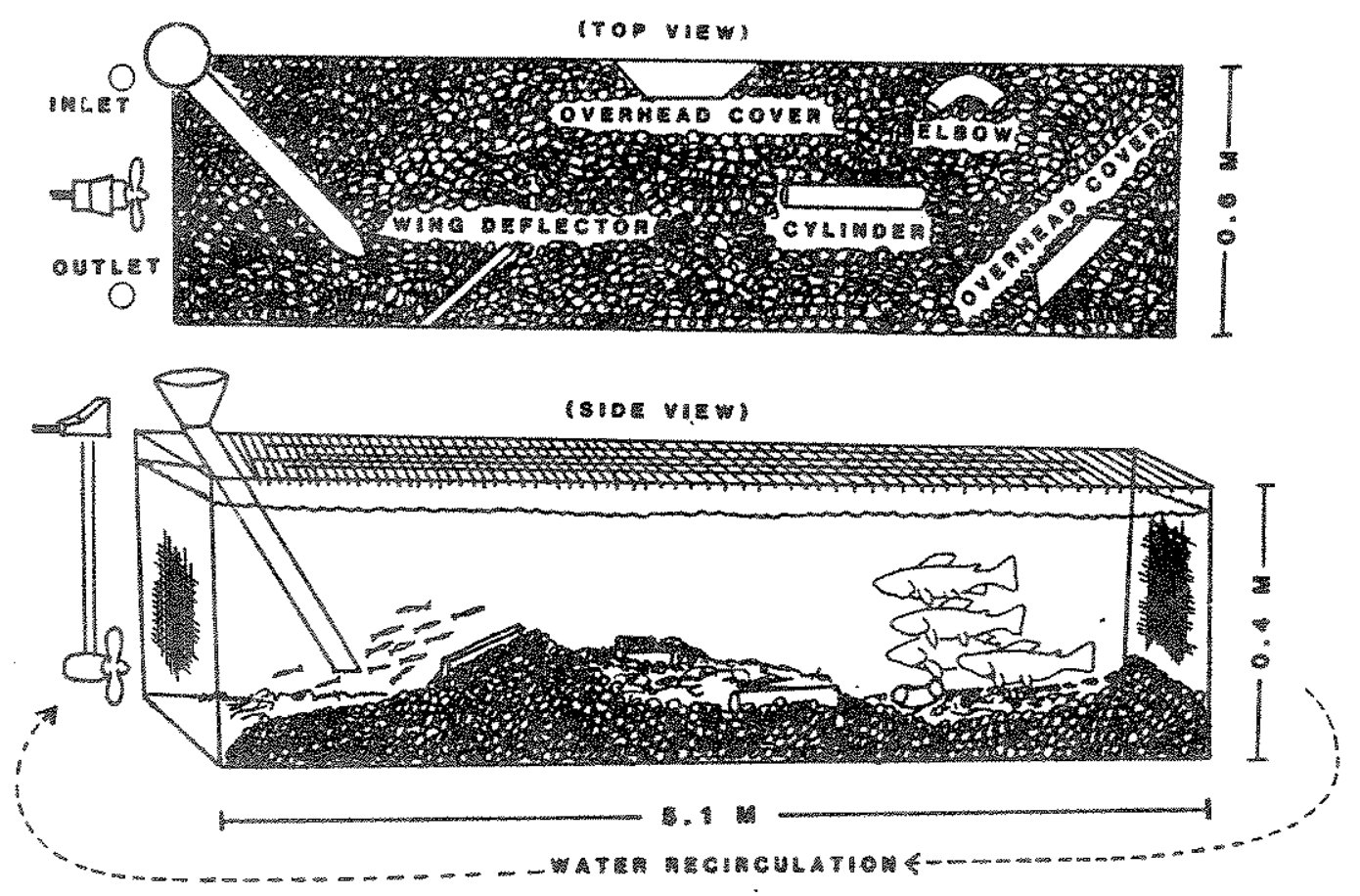

Igure 2.4.2. Axtificial study section used in the second series of predation tests May $1-21,1987$.

elbowed white plastic cylinder $(22.0 \mathrm{~cm}$ long, 6.5 cm diameter) provided tunnel-type cover.

The experimental procedure for the seven experimental stream tests was sinilax to that of tan tests except treacment fish With external symptoms of GBT were selected over those without external symptoms and predators vere maintained in the channel between tests. The predators (four Arlee hatchery rainbow trout) ranged in size from $270-286 \mathrm{~mm}$ total length. Different prey fish were used during each test. An equal number of creatment prey and control prey lexcept during rainbow test 2 when 14 treatment and 15 control fish were used) were placed in a 10 t bucket. The electric outboard motor was turned on, and prey were introduced into the channel through a funel located in the upstrean portion 
of study section (Figure 2.4.2). An observer watched the predators consume prey from behind a black curtain until approximately one-half the prey were consumed. This time ranged from $34 \mathrm{~min} 47 \mathrm{sec}$ to $88 \mathrm{~min} 54 \mathrm{sec}$. The predators were then removed and surviving prey were captured, separated into treatment and control groups, and measured. Because stream tests were not repeated, Fisher's exact test (Hintze 1987) was used to test for significant difference in predation on treatment and control fish.

Juvenile brown trout (mean total length $=46 \pm 3 \mathrm{~mm}$ ) were used as prey fish in the first five tests. Gas bubble trauma symptoms were induced in prey fish by exposure to $118 \%$ gas supersaturated water for $4-7$ d; control prey were not exposed to gas supersaturated conditions. Control fish were marked with an adipose clip $1 \mathrm{~h}$ prior to testing. Fish showing external symptoms of GBT were intentionally selected. All fish captured (up to five fish per dip including fish without external signs of GBT) were placed in a container with control fish until 25 treatment prey were obtained.

Two tests were conducted using rainbow trout (mean total length $60 \pm 6 \mathrm{~mm})$ as prey. One-half of the treatment rainbow trout prey in the first test had external symptoms of GBT induced by exposure to $118 \%$ gas supersaturation and the remaining 12 fish were randomly sampled from the exposure tank. During the second test the water level in exposure tank 1 was held at $10 \mathrm{~cm}$ and the water level in exposure tank 2 was raised to $45 \mathrm{~cm}$ (Figure 2.4.3). For a period of $4 \mathrm{~d}$, all fish in tank 1 were examined for 


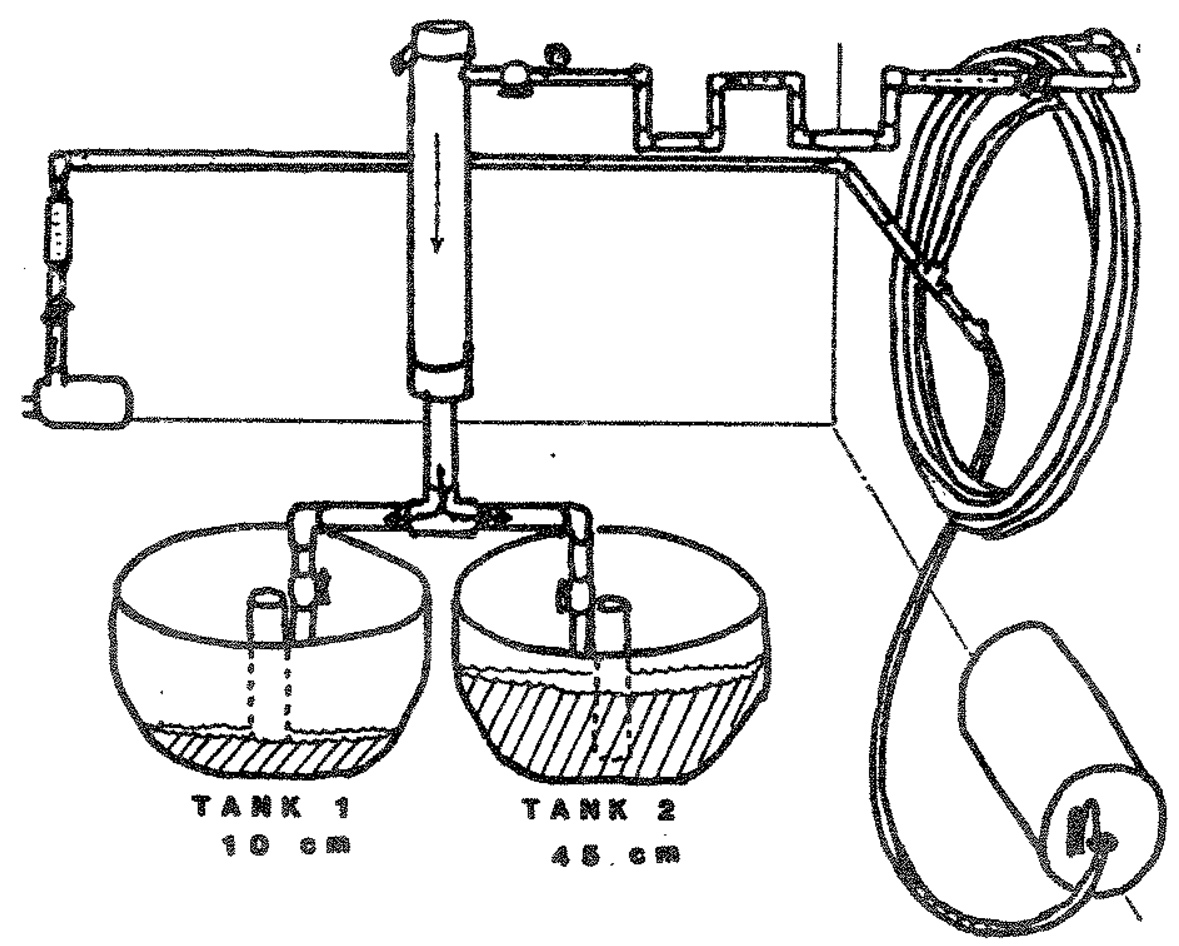

Figure 2.4.3. Apparatus and water level system used to induce external symptoms of GBT in rainbow trout for predation tests May $16-21,1987$.

external signs of GBT. Those with external signs were transfexred to tank 2 to allow for compensation, but not recovery. Fourteen rainbow trout prey with external signs of GBT were obtained in this mannex.

\subsection{1.b Results Discussion}

Tank Tests: Tests run in circular tanks using the procedure of Bams (1967) showed no difference in predation vulnerability between juvenile brown and rainbow trout exposed to gas supersaturated water and non-exposed fish (Tables $2.4 .1,2.4 .2$, $2.4 .3,2.4 .4$ and 2.4 .5$)$. Problems with the large size of rainbow trout prey used in the $112 \% / 30-d$ tests made it difficult for predators to consume a suitable number of prey before prey recovery from GBT (Table 2.4.1). Predator density was increased during the remaining tests (Tables $2.4 .2,2.4 .3,2.4 .4$, and 
Table 2.4.1. Number of survivors (beginning number $=25$ ), ratios of instantaneous predation rates (dp), and chi-square statistic corrected for continuity $\left(x^{2} c\right)$ for predation tests with juvenile rainbow trout prey $(77 \pm 5 \mathrm{~mm})$ after exposure for $30 \mathrm{~d}$ to $112 \%$ gas supersaturated water.

Test \begin{tabular}{cccc} 
Number surviving & Chi-square \\
\cline { 2 - 4 } & Treatment & $\begin{array}{c}\text { Control } \\
\text { (expected) }\end{array}$ & (expected)
\end{tabular}$\quad$ dp

\begin{tabular}{ccccc}
\hline 1 & 21 & 14 & 1.4 & 0.301 \\
2 & $(17.5)$ & $(17.5)$ & & \\
& 14 & 14 & 0.0 & 1.000 \\
\hline
\end{tabular}

Total of

chi-squares (2df)

1.4

Chi-square of totals (i.e, pooled chi-square 1 df)

35
$(31.5$

28

$(31.5)$

0.777

Heterogeneity

chi-square ( $1 \mathrm{df}$ )

$$
\begin{aligned}
& 0.623 \\
& 0.75>p>0.50 \\
& x^{2} c=\frac{([35-28]-1)^{2}}{73}=0.493 \\
& 0.50>p>0.25
\end{aligned}
$$

Chi-square correction for continuity 
Table 2.4.2. Number of survivors (beginning number $=25$ ), ratios of instantaneous predation rates (dp), and chi-square statistic corrected for continuity $\left(\mathrm{X}^{2} \mathrm{c}\right)$ for predation tests with juvenile rainbow trout prey $(45 \mathrm{~mm})$ after exposure for $30 \mathrm{~d}$ to $124 \% \mathrm{gas}$ supersaturated water.

\begin{tabular}{|c|c|c|c|c|}
\hline \multirow{2}{*}{ Test } & \multicolumn{2}{|c|}{ Number surviving } & \multirow{2}{*}{$\begin{array}{c}\text { Chi-square } \\
(1 \mathrm{df})\end{array}$} & \multirow{2}{*}{$\mathrm{dp}$} \\
\hline & $\begin{array}{l}\text { Treatment } \\
\text { (expected) }\end{array}$ & $\begin{array}{l}\text { Control } \\
\text { (expected) }\end{array}$ & & \\
\hline 1 & $\begin{array}{l}10 \\
(13.5)\end{array}$ & $\begin{array}{l}17 \\
(13.5)\end{array}$ & 1.815 & 2.376 \\
\hline 2 & $\begin{array}{l}16 \\
(14.5)\end{array}$ & $\begin{array}{c}13 \\
(14.5)\end{array}$ & 0.301 & 0.682 \\
\hline 3 & 12 & 5 & 2.882 & 0.456 \\
\hline
\end{tabular}

Total of

chi-squares (2df)

5.007

Chi-square of

totals (i.e,

pooled chi-square

$1 \mathrm{df})$

$\begin{array}{lll}38 & 35 & \\ (31.5) & (31.5) & 0.123\end{array}$

Heterogeneity

chi-square ( $I d f$ )

$$
0.10>p>0.05
$$

Chi-square correction

for continuity

$$
\begin{aligned}
& x^{2} c=\frac{([38-35]-1)^{2}}{63}=0.0541 \\
& 0.90>p>0.75
\end{aligned}
$$


Table 2.4.3. Number of survivors (beginning number $=25$ ), ratios of instantaneous predation rates (dp), and chi-square statistic corrected for continuity $\left(\mathrm{x}^{2} \mathrm{c}\right)$ for predation tests with juvenile brown trout prey $(69 \pm 7 \mathrm{~mm})$ after exposure for $30 \mathrm{~d}$ to $110 \% \mathrm{gas}$ supersaturated water.

\begin{tabular}{|c|c|c|c|c|}
\hline \multirow{2}{*}{ Test } & \multicolumn{2}{|c|}{ Number surviving } & \multirow{2}{*}{$\begin{array}{c}\text { Chi-square } \\
(1 \mathrm{df})\end{array}$} & \multirow{2}{*}{$d p$} \\
\hline & $\begin{array}{l}\text { Treatment } \\
\text { (expected) }\end{array}$ & $\begin{array}{l}\text { Control } \\
\text { (expected) }\end{array}$ & & \\
\hline 1 & $\begin{array}{c}15 \\
(17)\end{array}$ & $\begin{array}{c}23 \\
(17)\end{array}$ & 1.684 & 6.126 \\
\hline 2 & $\begin{array}{l}10 \\
(14.5)\end{array}$ & $\begin{array}{l}18 \\
(14.5)\end{array}$ & 2.285 & 2.789 \\
\hline
\end{tabular}

Total of

chi-squares ( $2 \mathrm{df}$ )

3.969

Chi-square of

totals (i.e,

pooled chi-square

1 df)

25

(34.5)

41

(34.5)

2.249

Heterogeneity

chi-square (Idf)

$0.25>\mathrm{p}>0.10$

Chi-square correction

for continuity

$$
\begin{aligned}
x^{2} c=\frac{([25-41]-1)^{2}}{69} & =3.260 \\
0.10 & >p>0.05
\end{aligned}
$$


Table 2.4.4. Number of survivors (beginning number $=25$ ), ratios of instantaneous predation rates $(\mathrm{dp})$, and chi-square statistic corrected for continuity $\left(\mathrm{X}^{2} \mathrm{c}\right)$ for predation tests with juvenile brown trout prey $(56 \pm 7 \mathrm{~mm})$ after exposure for 30 d to $112 \%$ gas supersaturated water.

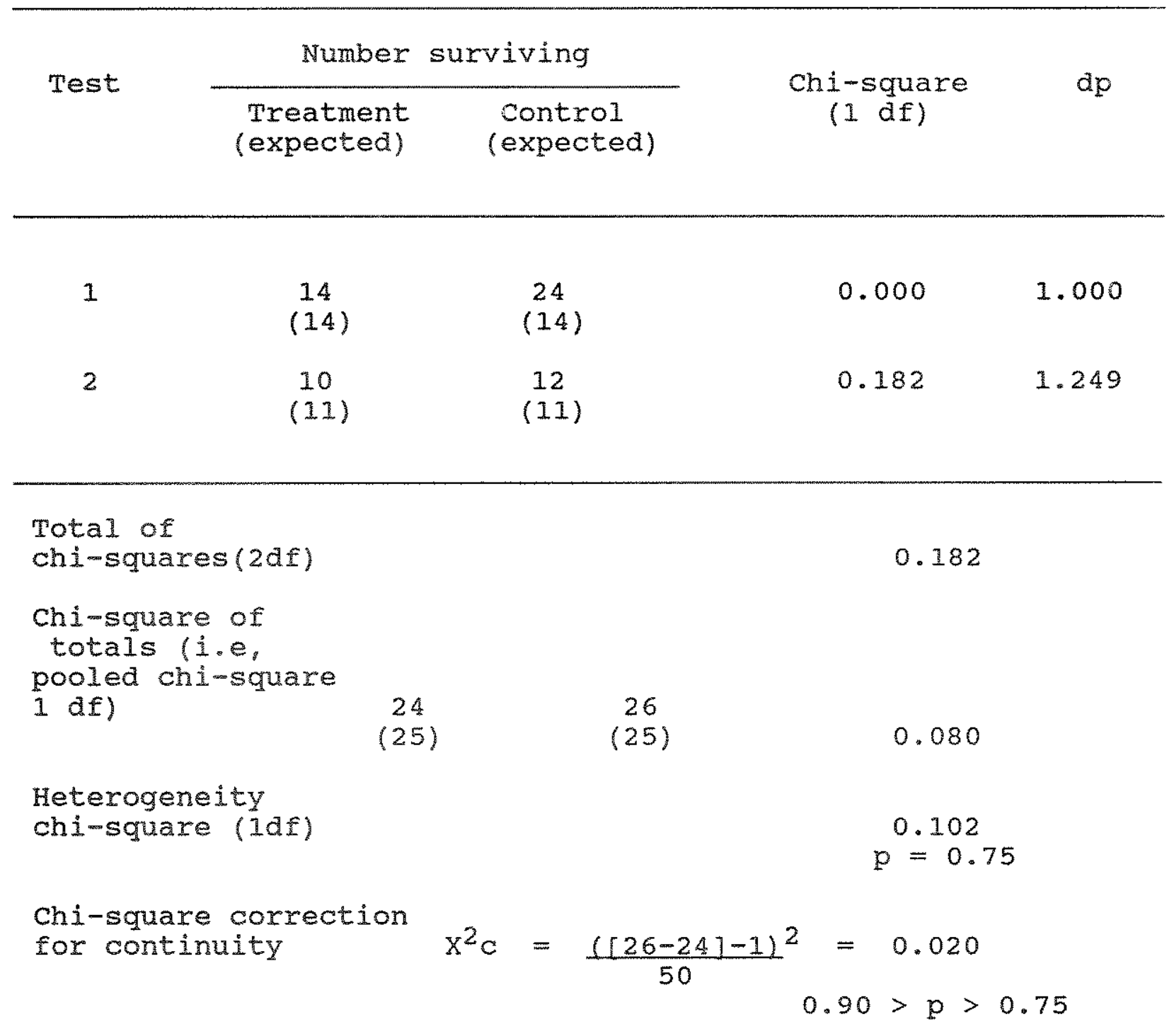


Table 2.4.5. Number of survivors (beginning number $=25$ ), ratios of instantaneous predation rates (dp), and chi-square statistic corrected for continuity $\left(\mathrm{x}^{2} \mathrm{c}\right)$ for predation tests with juvenile brown trout prey $(56 \pm 5 \mathrm{~mm})$ after exposure for $13 \mathrm{~h}$ to $130 \%$ gas supersaturated water.

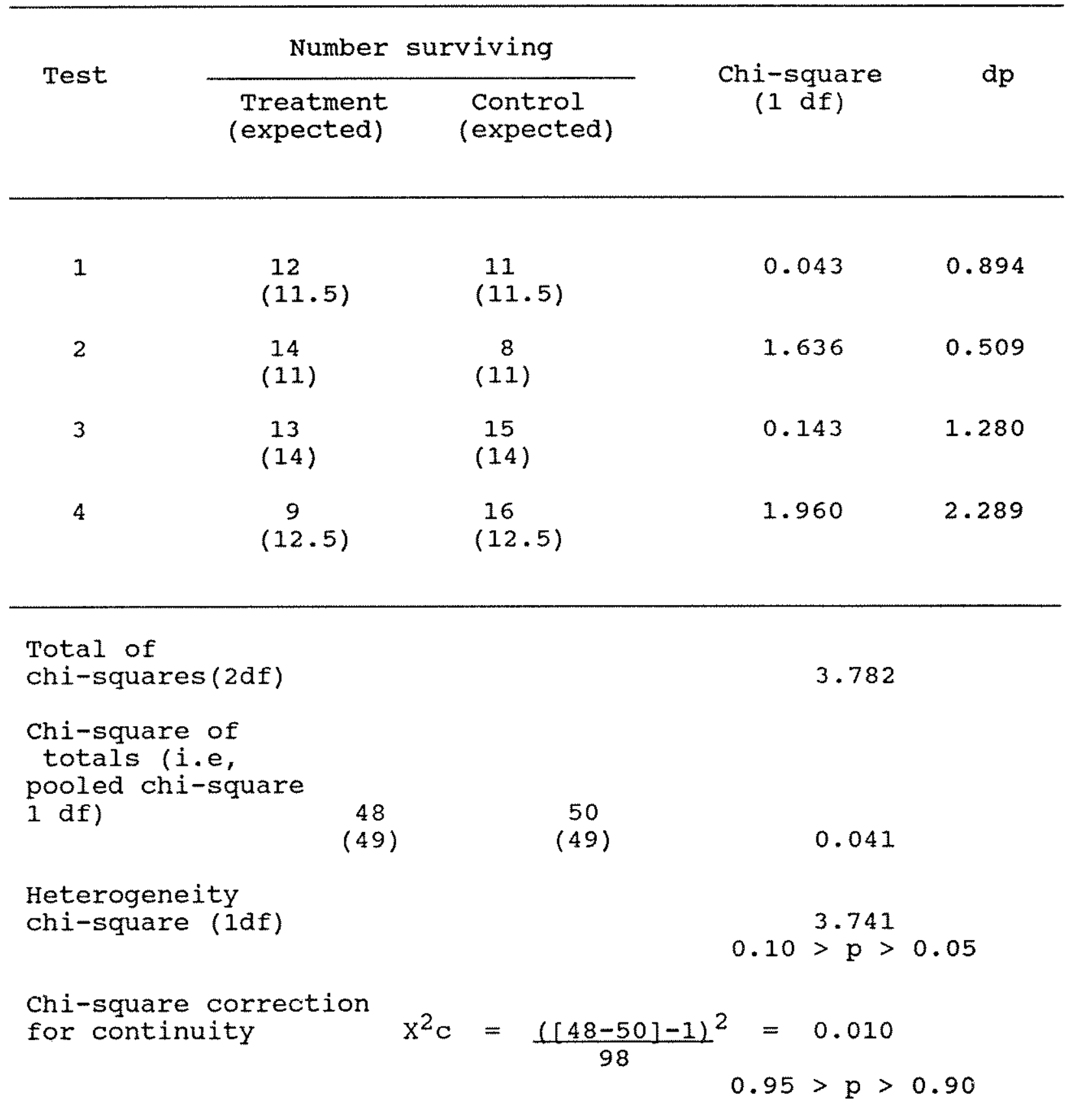


2.4.5) to avoid this problem.

Reduced fitness of prey can increase their vulnerability to predation. Kania and o'Hara (1974) found that the ability of misquito fish (Gambusia affinia) to avoid predation by largemouth bass was impaired after exposure to sublethal concentrations of mercury. Rainbow trout, chinook salmon, and sockeye salmon experienced elevated predation vulnerability after exposure to high water temperature (Coutant 1973, sylvester 1972). Brook trout consumed more atlantic salmon that had been exposed to insecticide than unexposed fish (Hatfield and Anderson 1972). Hertig and witt (1967) concluded that bluegill and largemouth bass suffering from columnaris disease, parasitism, or starvation were more vulnerable to predation than healthy fish. Predators selected impaired prey because they were slow swimming and easy to catch. Hatchery reared sockeye salmon fry did not swim as well as wild fry, leading to increased vulnerability to predation (Bams 1967).

Gas Bubble Trauma affects fish swimming performance and vision. These impairments could make it easier for predators to discover and capture GBT inflicted fish. However, under laboratory conditions I did not observe increased vulnerabilty to predation.

Experintal stream Tests: Predation tests in the experimental stream showed that brown trout exposed to $118 \%$ gas supersaturated water for 5 d experienced significantly increased vulnerability to predation (Table 2.4 .6$)$. There was no statistically significant selective predation on brown trout exposed for 4, 6, or 7 d (Table 2.4.6), nor on rainbow trout 
Table 2.4.6. Ratio of instantaneous predation rates (dp) of juvenile brown trout exposed to $118 \%$ gas supersaturated water and non-exposed fish.

\begin{tabular}{cccccc}
\hline $\begin{array}{c}\text { Duration } \\
\text { of } \\
\begin{array}{c}\text { exposure } \\
\text { (days) }\end{array}\end{array}$ & $\begin{array}{c}\text { Mean fish } \\
\text { length } \\
(\mathrm{mm} \pm \mathrm{SD})\end{array}$ & $\begin{array}{c}\text { Number } \\
\text { of } \\
\text { replicates }\end{array}$ & $\begin{array}{c}\text { Number } \\
\text { surviving }\end{array}$ & treatment control & dp \\
\hline 4 & $46 \pm 3$ & 1 & 14 & 19 & 2.113 \\
5 & $47 \pm 4$ & 1 & 13 & 21 & $3.751 *$ \\
6 & $45 \pm 3$ & 1 & 13 & 15 & 1.280 \\
7 & $46 \pm 4$ & 1 & 15 & 13 & 0.781
\end{tabular}

* Significantly different using Fisher's exact test at alpha = 0.05

exposed for 11 to $12 \mathrm{~d}$ (Table 2.4.7).

Juvenile brown trout exposed to $118 \%$ gas supersaturation for 5 d may have been most susceptible to predation because of the nature of fish response to supersaturated water. Some fish die quickly, some acquire severe symptoms of GBT and die slowly and some are never affected. I observed that the number of brown trout with external symptoms of GBT peaked on day 5 of exposure. Fish removed from the exposure system for a given test were the first to display symptoms. Hence, remaining fish after each successive test were those which were most tolerant of supersaturation. The trend in instantaneous mortality rates supports this hypothesis (Figure 2.4.4) and may explain why predation was greatest on fish exposed to gas supersaturation for 5 d.

The ability of rainbow trout to rapidly recover from overinflation of the swimbladder may decrease their 
Table 2.4.7. Ratio of instantaneous predation rates (dp) of juvenile rainbow trout exposed to $118 \%$ gas supersaturated water and non-exposed fish.

\begin{tabular}{|c|c|c|c|c|c|}
\hline \multirow{2}{*}{$\begin{array}{c}\text { Duration } \\
\text { of } \\
\text { exposure } \\
\text { (days) }\end{array}$} & \multirow{2}{*}{$\begin{array}{l}\text { Mean Eish } \\
\text { length } \\
\text { (mm I SD) }\end{array}$} & \multirow{2}{*}{$\begin{array}{c}\text { Number } \\
\text { of } \\
\text { replicates }\end{array}$} & \multicolumn{2}{|c|}{$\begin{array}{l}\text { Number } \\
\text { surviving }\end{array}$} & \multirow[b]{2}{*}{$d p$} \\
\hline & & & treatment & control & \\
\hline 11 & $58 \pm 7$ & 1 & 12 & 8 & 0.644 \\
\hline 12 & $61 \pm 5$ & 1 & 7 & 6 & 0.892 \\
\hline
\end{tabular}

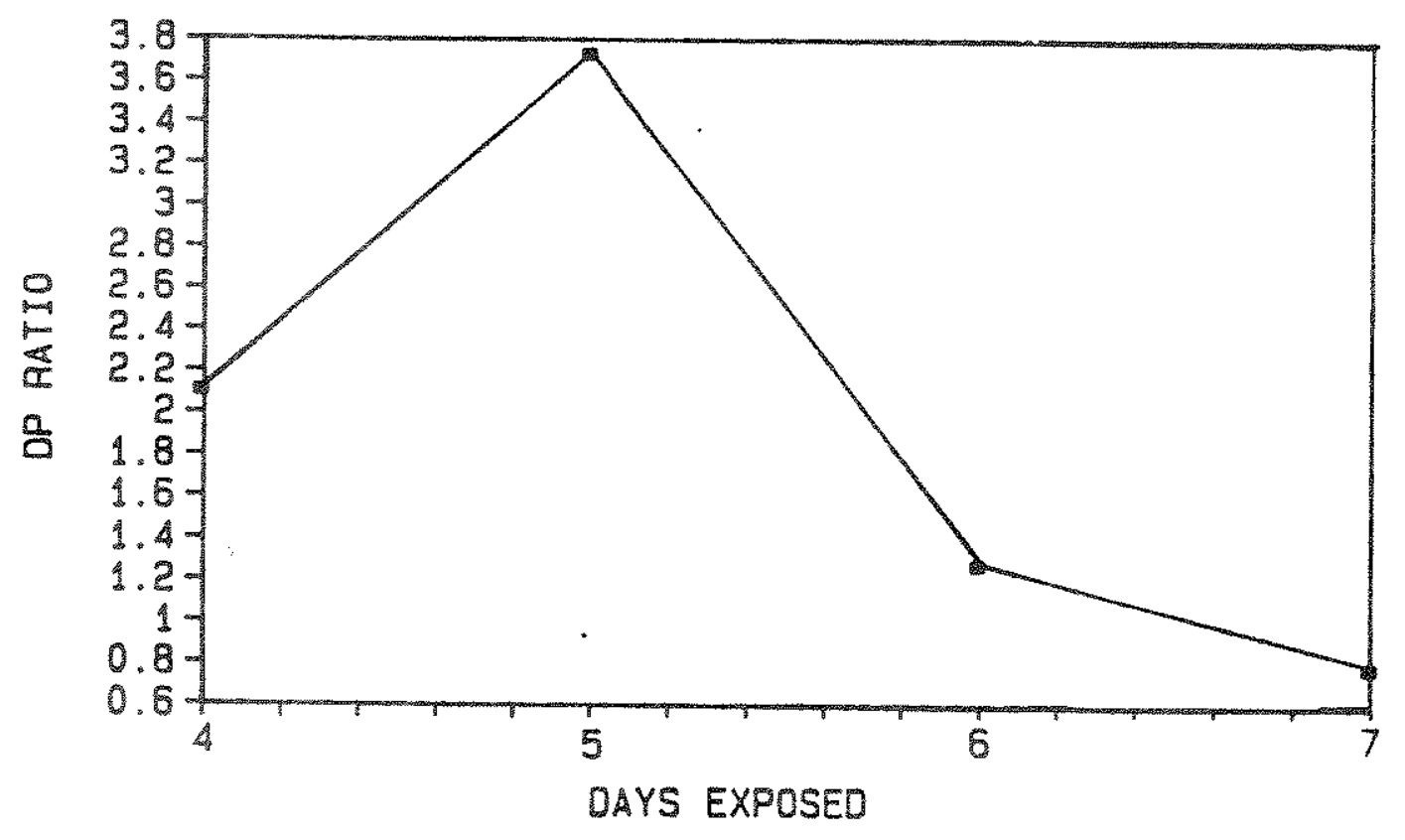

Figure 2.4.4. Ratio of instantaneous predation rates (dp) by rainbow trout predators on juvenile brown trout exposed $118 \%$ gas supersaturated water for $4-7$ and control fish (dp $>1$ signifies greater predation on brown trout exposed to gas supersaturation). 
susceptibility to increased predation from exposure to gas supersaturated water. Schiewe (1974) reported that swimming impairment of juvenile chinook salmon caused by overinflation of the swimbladder was reduced within $2 \mathrm{~h}$ of removal from $120 \%$ gas supersaturated water to equilibrated water. During my second series of rainbow trout tests, overinflation of the swimbladder was the only symptom in 18 of the 26 affected prey fish.

Tests to evaluate recovery time showed that rainbow trout released in a non-supersaturated pond lost all signs of excess buoyancy in 1-3 min. More than $3 \mathrm{~min}$ were required for the predators to discover the prey during stream predation tests. Rainbow trout prey may have expelled excess gas via the pneumatic duct, thereby regaining equilibrium prior to predator feeding. predators were not replaced between tests which also may have biased the study. Coutant (1973) suggested that reusing predator fish did not bias predator-prey study results. However, with time, predators may become more efficient at capturing prey. The four predators in the experimental stream were trained to feed on prey three to four times prior to testing and were used six times during testing. Werner et al. (1981) found that bluegill foraging efficiency increased four-fold over the course of six to eight foraging opportunities and ware (1971) reported that rainbow trout increased foraging efficiency six-fold in $8 \mathrm{~d}$. My rainbow trout predators may have become too efficient at consuming prey, thereby biasing results. 


\section{4 .2 Hablet vat ve}

Reasons for the much larger incidence of GBT in brown trout compared to rainbow trout in the Bighorn River are not known but could be related to differences in habitat selection between species and among different size or age classes. Differences in habitat selection that expose a particular species or age class to high gas supersaturation may also influence relative abundance of these species by causing a differential mortality.

Detection and avoidance of supersaturated water could influence survival of fish in waters that contain high levels of dissolved gases (Jensen et al. 1986). Avoidance can occur by fish sounding or moving away from supersaturated water (stevens et a1. 1980). Change in hydrostatic pressure associated with an increase in water depth of $1 \mathrm{~m}$ reduces gas saturation by approximately 10\% (Gray and Haynes 1977). Most studies of detection, avoldance, and tolerance of supersaturated waters by salmonids have been conducted with juvenile fish.

It is generally accepted that fish are not able to detect supersaturated water and therefore do not avoid it (Weitkamp and Katz 1980), although there appear to be exceptions. Lund and Heggberget (1985) found that 2-year-old rainbow trout (oncorhynchus mykiss) did not avoid 115 to $125 \%$ supersaturated water in tank experiments. An apparent avoidance of high dissolved gas levels by squawfish (Ptychocheilus oregonenis) below Little Goose Dam on the Snake River was described by Bentley et al. (1976). They collected fewer squawish during periods of high supersaturation than during periods of lower supersaturation. Stickney (1968) found that Atlantic herring 
(Clupea harengus harengus) avoided gas supersaturated water when dissolved gas levels were high enough to produce GBT (122\% TGP) . Dawley et al. (1976) reported that juvenile chinook salmon (Oncorhynchus tschawytscha) and steelhead trout (oncorhynchus mykiss) apparently avoided high dissolved gas levels by using deeper water that provided hydrostatic compensation. Both species were found at deeper average depths as gas concentrations increased in deep $(2.4 \mathrm{~m})$ tank experiments. Shrimpton et al. (1989) showed that small rainbow trout in supersaturated water seek depth to overcome excess buoyancy induced by swimbladder overinflation. Stevens et al. (1980) found that rainbow trout (30 to $45 \mathrm{~g}$ ) moved laterally to avoid supersaturated water in tank experiments. Heggberget (1984) found that brown trout (Salmo trutta) were less tolerant to supersaturated water than were perch (Perca fluviatilis) and eel (Anguilla anguilla) in river cage studies.

The objectives of this part of the study were to conduct in situ observations of trout behavioral distribution in the Bighorn River to determine: 1) whether differences in incidence of GBT between brown trout and rainbow trout can be explained by habitat use, 2) whether habitat used by different length groups of trout results in differences in exposure to GBT between length groups, and 3) whether brown trout and rainbow trout avoid high dissolved gas levels. Field observations were conducted between June 1986 and December 1988 .

\subsubsection{Macrohabitat}

This research was conducted in the upper $4.9 \mathrm{~km}$ of the study area (Figure 2.3.1). River width was approximately $80 \mathrm{~m}$ with a 
midchannel water depth of about $1.8 \mathrm{~m}$. Channel substrate was homogeneous, dominated by large (256 mm - $128 \mathrm{~mm}$ ) and small cobble $(128 \mathrm{~mm}-64 \mathrm{~mm})$. Areas with low velocities were characterized by gravel less than $20 \mathrm{~mm}$ in diameter.

Two 400-m long sections were established to observe trout macro- and microhabitat use in relation to stream habitat characteristics such as water depth, focal point velocity, distance to bank, side of river, substrate, depth of vegetation, discharge, cover, dissolved gases, and water temperature.

Locations were designated in relation to distance downstream from the Afterbay Dam. Section 1 extended from Rkm 0.5 to 0.9 . High nitrogen saturation and high delta $P$ levels were typical for this area. A slight gradient of decreasing gas levels from the right to left bank occurred due to operation of the sluiceway, located at the right side of Afterbay Dam. Section 2, located at Rkm 4.5 to 4.9 , had lower nitrogen levels, and higher oxygen levels than section 1. Delta $\mathrm{P}$ was lower in 1987 and higher in 1988 in section 2. Incidence of GBT in brown trout was highest in section 1 while incidence of GBT in rainbow trout was higher in section 2. Aquatic vegetation, dominated by horned pond weed (Zannichellia palustris) and a moss (Fontinalis hypnoides) was dense from late spring through early fall.

\subsubsection{Methods}

To contrast fish distribution in shallow near-bank areas with deeper midchannel areas, three 400-m long snorkel lanes (left, middle, and right) were monitored from late May to october in 1987 and January to November in 1988 in both study sections. 
Snorkel lanes along the left and right banks were surveyed while maintaining a distance of approximately $9 \mathrm{~m}$ from shore and counting trout between the diver and the bank. Middle lane surveys were conducted while maintaining a midchannel position and counting trout visible in a forward direction. Middle lane water depths were greater than $1.75 \mathrm{~m}$. Water velocities $0.5 \mathrm{~m}$ above the stream bottom in the middle lanes during periods without aquatic vegetation where approximately 2.2 to $2.6 \mathrm{~m} / \mathrm{s}$. Lanes were swum moving downstream counting the total numbers of brown trout and rainbow trout and the numbers of each species $>36$ cm total length (TL). Data were recorded on underwater slates. All counts were made during daylight hours between 0900 and 1700 (MDT). Above-water light levels were measured using a Li-Cor integrating quantum/radiometer/photometer.

Correlations of trout number and day number (day of the year) were used to examine seasonal changes in fish distribution. A non-parametric sign test was used on the correlation results (Sprent 1981). Testing the null hypotheses that correlations have a binomial distribution ( $p=0.5)$, the non-parametric sign test determined the probability of getting the observed correlations by chance. To ensure independence of trout counts (the number of trout in middle lane is not independent to the number of fish in near bank lanes) for the sign test, only one lane count per size group and species was used. Lack of significance of correlations does not affect the sign test.

\section{$2.4 .2 .1 \mathrm{~b}$ Results}

Correlation among snorkel lane counts and day number indicated seasonal changes in trout distribution in 1987 . Trout 
numbers generally decreased along banks and increased in middle lanes from 26 May to 17 October (Figures 2.4.5 and 2.4.6). Trout abundance was negatively correlated with day number for all near bank lanes (Table 2.4 .8 ). The probability of getting all eight 1987 left or right lane counts to correlate the same as was observed was 0.004 . Positive correlations with day number were observed in all middle lane counts in 1987 with the exception of section 1 , rainbow trout $>36 \mathrm{~cm}$ TL, which was negatively correlated with day number $(x=-0.19)$. The probability of one or less of the eight correlations being negative due to chance alone was 0.035 (sign test) for 1987 middle lane counts. Twelve of the 24 correlations were significant $(P<0.05)$.

Correlations among snorkel lane counts and day number in 1988 were not similar to 1987 results. Trout numbers did not show sinilar decreases along banks and increases in the middle lanes as in 1987 (Figures 2.4 .7 and 2.4 .8 ). Only 1 of 24 correlations was significant (Table 2.4.9). Sample sizes for 1988 were smaller than in 1987 (1987, $N=27$ for both sections: 1988, $N=7$ for section $1, N=6$ for section 2$)$. Six of the eight middle lane counts were positively correlated with day number, and three of eight left lane counts had positive correlation with day number. Assuming binomial distribution, the probability of two or less of eight middle lane counts being negative was 0.145 . The probability of three or fewer left lane counts being positive, as was observed, was 0.363 .

The percentage of total trout counted in the middle snorkel lanes during 1987 had significant, positive correlation with day 


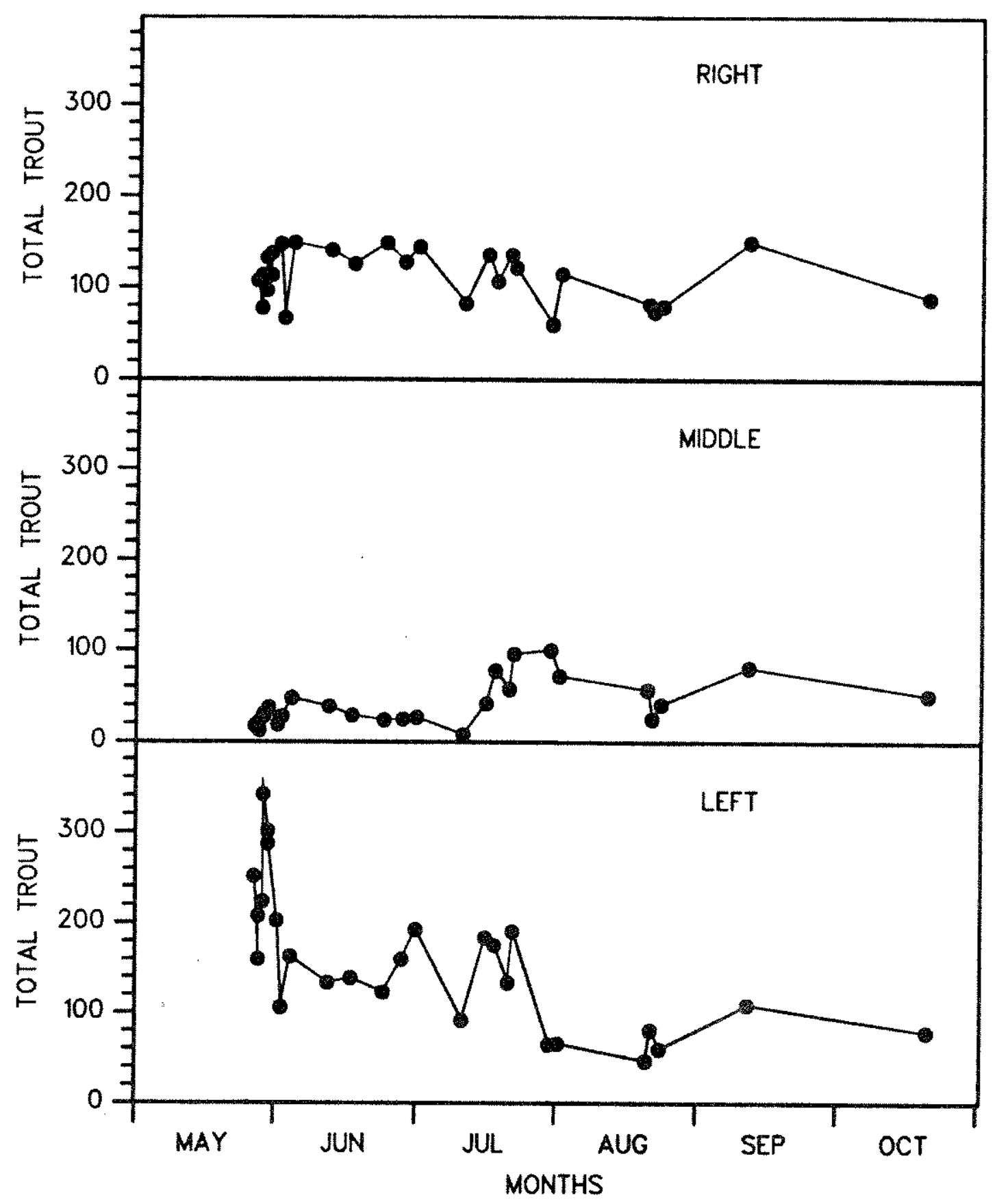

Figure 2.4.5. Number of rainbow trout and brown trout counted in section 1 snorkel lanes, 26 May - 17 October 1987, Bighorn River, Montana. 


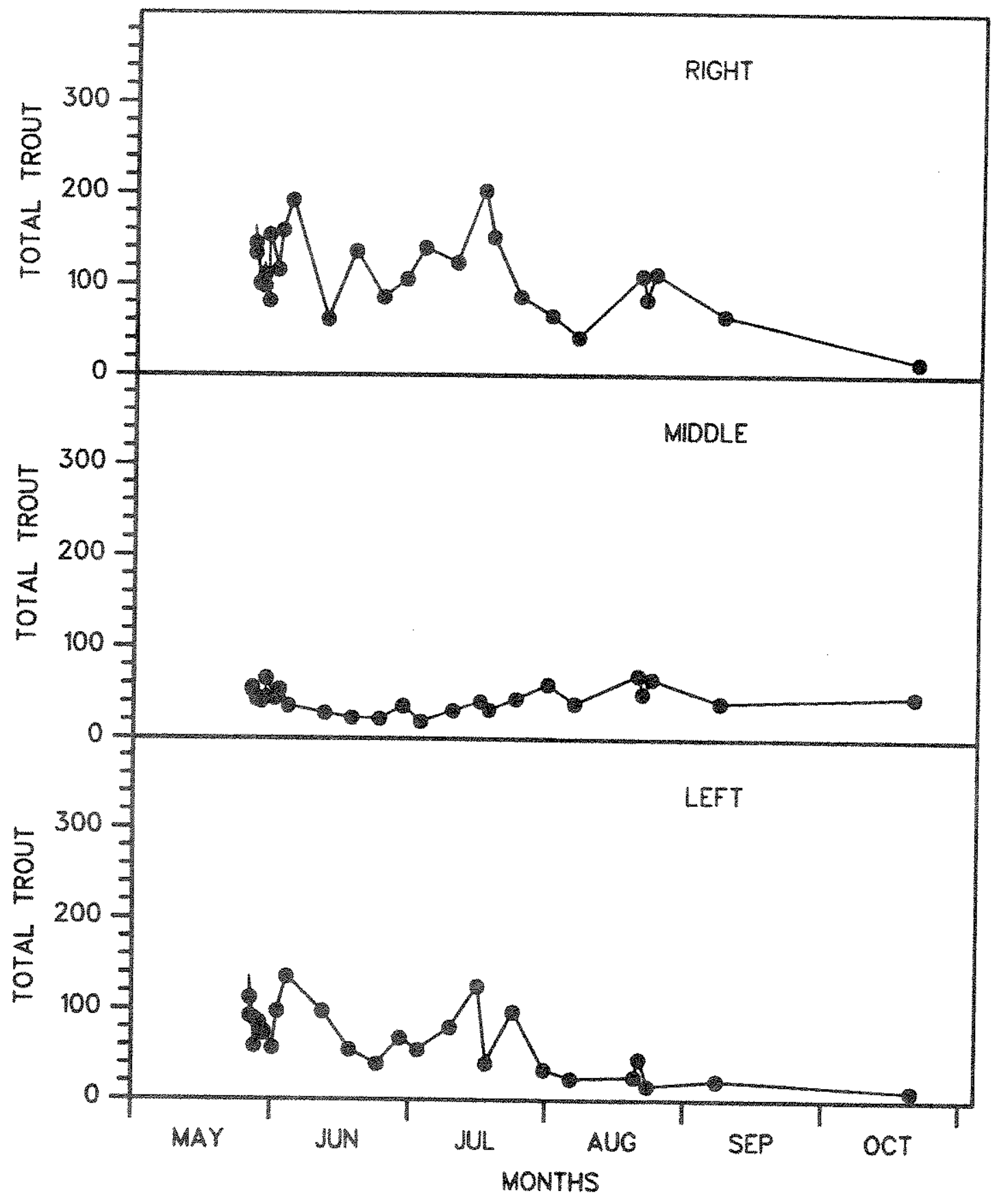

Figure 2.6.6. Number of rainbow trout and brown trout counted in section 2 snorikel lanes, 26 May - 17 october 1987, Bighorn River, Montana. 
Table 2.4.8. Correlation among trout abundance in sections 1 and 2 snorkel lane counts and day number (26 May - 17 october) 1987, Bighorn River, Montana ( $N=27$ for both sections).

Section species size Bank $^{a} \quad \begin{aligned} & \text { Correlation significance } \\ & \text { coefficient }\end{aligned}$

(r)

\begin{tabular}{|c|c|c|c|c|c|c|}
\hline 1 & Brown & $<36$ & $\mathrm{~cm}$ & LB & -0.52 & $* *$ \\
\hline 1 & Brown & $>36$ & $\mathrm{~cm}$ & LB & -0.64 & ** \\
\hline 1 & Brown & $<36$ & $\mathrm{~cm}$ & $\mathrm{M}$ & 0.47 & $\star$ \\
\hline 1 & Brown & $>36$ & $\mathrm{~cm}$ & $\mathrm{M}$ & 0.34 & NS \\
\hline 1 & Brown & $<36$ & $\mathrm{~cm}$ & $\mathrm{RB}$ & -0.20 & NS \\
\hline 1 & Brown & $>36$ & $\mathrm{~cm}$ & $\mathrm{RB}$ & -0.14 & NS \\
\hline 1 & Rainbow & $<36$ & $\mathrm{~cm}$ & LB & -0.79 & ** \\
\hline 1 & Rainbow & $>36$ & $\mathrm{~cm}$ & LB & -0.55 & ** \\
\hline 1 & Rainbow & $<36$ & $\mathrm{~cm}$ & $\mathbf{M}$ & 0.63 & ** \\
\hline 1 & Rainbow & $>36$ & $\mathrm{~cm}$ & $\mathbf{M}$ & -0.19 & NS \\
\hline 1 & Rainbow & $<36$ & $\mathrm{~cm}$ & $\mathrm{RB}$ & -0.09 & NS \\
\hline 1 & Rainbow & $>36$ & $\mathrm{~cm}$ & $\mathrm{RB}$ & -0.35 & NS \\
\hline 2 & Brown & $<36$ & $\mathrm{~cm}$ & LB & -0.60 & ** \\
\hline 2 & Brown & $>36$ & $\mathrm{~cm}$ & LB & -0.72 & $\star *$ \\
\hline 2 & Brown & $<36$ & $\mathrm{~cm}$ & $\mathrm{M}$ & 0.09 & NS \\
\hline 2 & Brown & $>36$ & $\mathrm{~cm}$ & $\mathrm{M}$ & 0.04 & NS \\
\hline 2 & Brown & $<36$ & $\mathrm{~cm}$ & $\mathrm{RB}$ & -0.41 & $*$ \\
\hline 2 & Brown & $>36$ & $\mathrm{~cm}$ & $\mathrm{RB}$ & -0.51 & ** \\
\hline 2 & Rainbow & $<36$ & $\mathrm{~cm}$ & LB & -0.35 & NS \\
\hline 2 & Rainbow & $>36$ & $\mathrm{~cm}$ & LB & -0.30 & NS \\
\hline 2 & Rainbow & $<36$ & $\mathrm{~cm}$ & $\mathrm{M}$ & 0.46 & $*$ \\
\hline 2 & Rainbow & $>36$ & $\mathrm{~cm}$ & M & 0.16 & NS \\
\hline 2 & Rainbow & $<36$ & $\mathrm{~cm}$ & $\mathrm{RB}$ & -0.22 & NS \\
\hline 2 & Rainbow & $>36$ & $\mathrm{~cm}$ & $\mathrm{RB}$ & -0.50 & ** \\
\hline
\end{tabular}

a LB - left bank, M - middle, RB - right bank

$\mathrm{b}$ NS $\mathrm{P}>0.05, * \mathrm{P}<0.05, * * \mathrm{P}<0.01$

number (26 May - 17 october) also indicating seasonal movement away from near bank areas (Table 2.4.10, Figure 2.4.9). A less pronounced increase in the percentages of total trout in middle lanes was observed in 1988 (Table 2.4.10, Figure 2.4.10). Neither 1988 correlations are significant $(P<0.05)$. Correlations between total gas pressure (TGP) and trout number in snorkel lanes were observed only with middle lane 


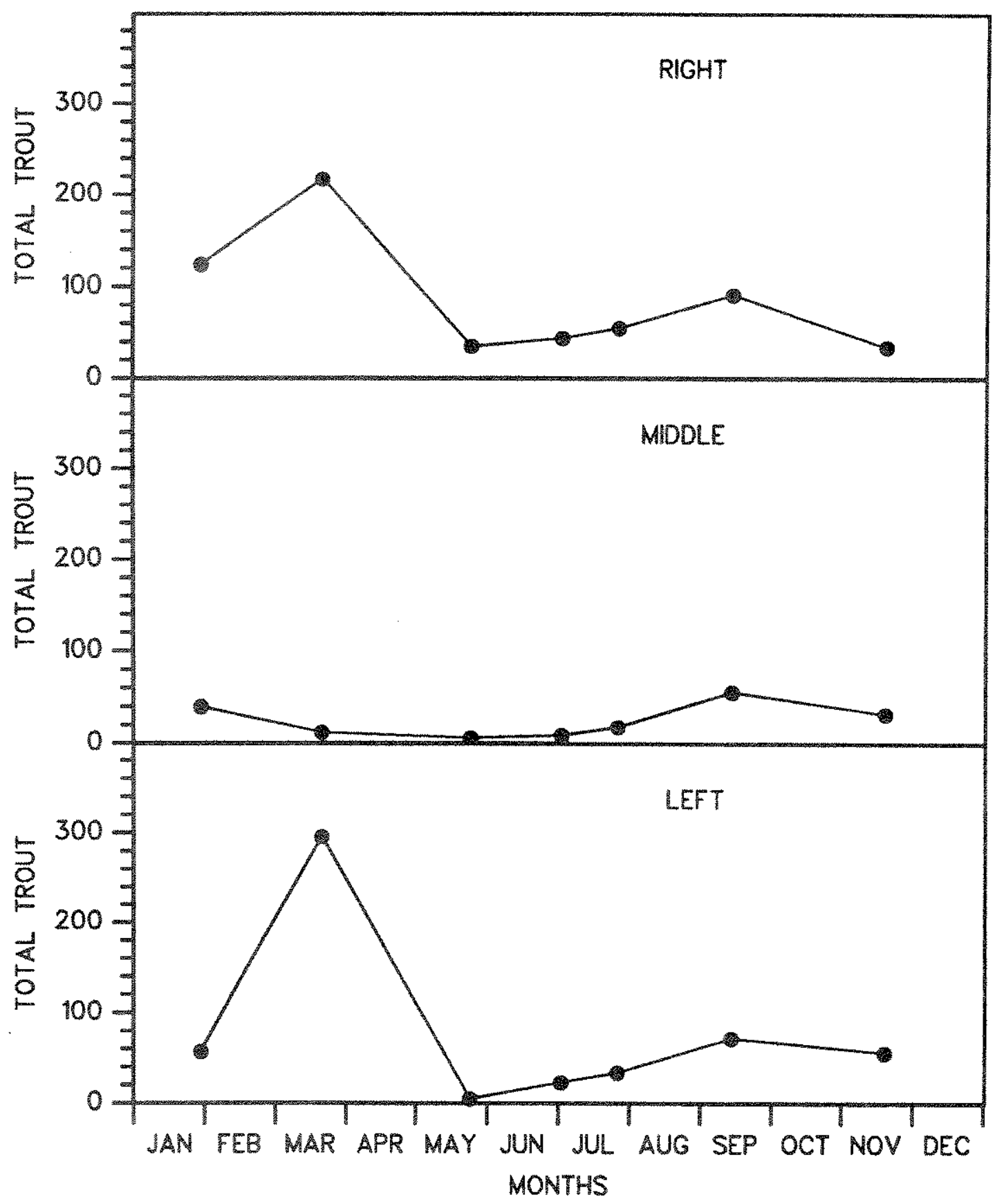

Figure 2.4.7. Number of rainbow trout and brown trout in section 1 snorkel lanes, 30 January - 23 November 1988 , Bighorn River. Montana. 


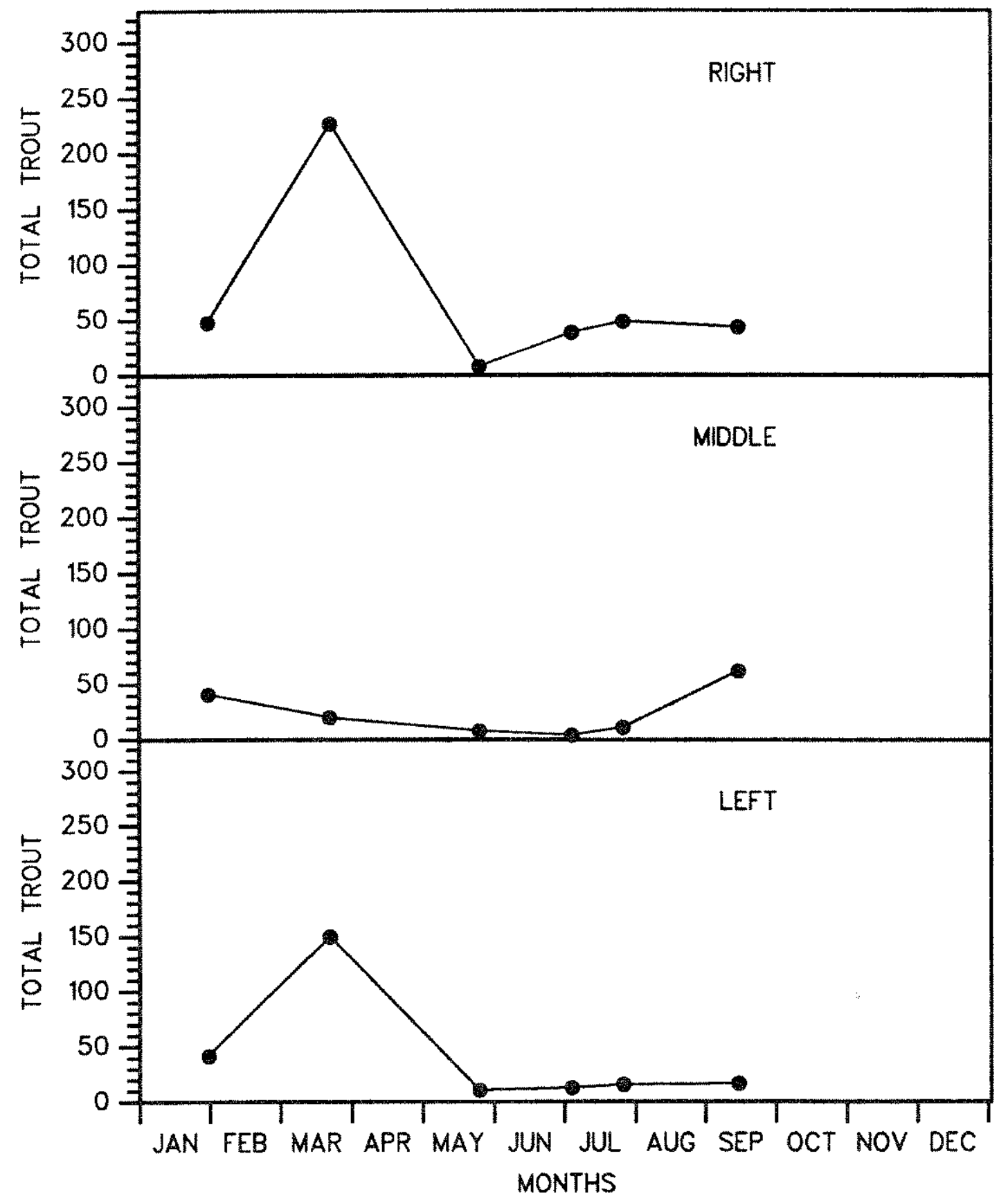

Figure 2.4.8. Number of rainbow trout and brown trout counted in section 2 snorkel lanes, 30 January - 18 September 1988, Bighorn River, Montana. 
Table 2.4.9. Correlation among trout abundance in sections 1 and 2 snorkel lane counts and day number (30 January - 23 November) 1988, Bighorn River, Montana ( $N=7$ for section $1, N=6$ for section 2).

Section species size Bank $\begin{aligned} & \text { Correlation significance } \\ & \text { coefficient }\end{aligned}$

(x)

\begin{tabular}{|c|c|c|c|c|c|c|}
\hline 1 & Brown & $<36$ & $\mathrm{~cm}$ & LB & -0.31 & NS \\
\hline 1 & Brown & $>36$ & $\mathrm{~cm}$ & $L B$ & 0.20 & NS \\
\hline 1 & Brown & $<36$ & $\mathrm{~cm}$ & $M$ & 0.12 & NS \\
\hline 1 & Brown & $>36$ & $\mathrm{~cm}$ & $M$ & 0.48 & NS \\
\hline 1 & Brown & $<36$ & $\mathrm{~cm}$ & $\mathrm{RB}$ & -0.47 & NS \\
\hline 1 & Brown & $>36$ & $\mathrm{~cm}$ & $\mathrm{RB}$ & -0.23 & NS \\
\hline 1 & Rainbow & $<36$ & $\mathrm{~cm}$ & LB & -0.22 & NS \\
\hline 1 & Rainbow & $>36$ & $\mathrm{~cm}$ & $\mathrm{LB}$ & 0.14 & NS \\
\hline 1 & Rainbow & $<36$ & $\mathrm{~cm}$ & $M$ & 0.08 & NS \\
\hline 1 & Rainbow & $>36$ & $\mathrm{~cm}$ & $\mathrm{M}$ & 0.56 & NS \\
\hline 1 & Rainbow & $<36$ & $\mathrm{~cm}$ & $\mathrm{RB}$ & -0.68 & NS \\
\hline 1. & Rainbow & $>36$ & $\mathrm{~cm}$ & $\mathrm{RB}$ & -0.10 & $\mathbb{N S}$ \\
\hline 2 & Brown & $<36$ & $\mathrm{~cm}$ & $L B$ & -0.40 & NS \\
\hline 2 & Brown & $>36$ & $\mathrm{~cm}$ & $\mathrm{LB}$ & -0.47 & NS \\
\hline 2 & Brown & $<36$ & $\mathrm{~cm}$ & $M$ & -0.38 & NS \\
\hline 2 & Brown & $>36$ & $\mathrm{~cm}$ & $M$ & 0.71 & NS \\
\hline 2 & Brown & $<36$ & $\mathrm{~cm}$ & $\mathrm{RB}$ & -0.05 & NS \\
\hline 2 & Brown & $>36$ & $\mathrm{~cm}$ & $\mathrm{RB}$ & -0.36 & NS \\
\hline 2 & Rainbow & $<36$ & $\mathrm{~cm}$ & LB & -0.41 & NS \\
\hline 2 & Rainbow & $>36$ & $\mathrm{~cm}$ & LB & 0.30 & NS \\
\hline 2 & Rainbow & $<36$ & $\mathrm{~cm}$ & $M$ & -0.24 & NS \\
\hline 2 & Rainbow & $>36$ & $\mathrm{crn}$ & $\mathrm{M}$ & 0.97 & $* *$ \\
\hline 2 & Rainbow & $<36$ & $\mathrm{~cm}$ & $\mathrm{RB}$ & 0.00 & NS \\
\hline 2 & Rainbow & $>36$ & $\mathrm{~cm}$ & $\mathrm{RB}$ & 0.50 & NS \\
\hline
\end{tabular}

a LB - left bank, M- middle, RB - right bank

b NS $\mathrm{P}>0.05, * \mathrm{P}<0.05, * * \mathrm{P}<0.01$

counts (Tables 2.4 .11 and 2.4 .12 ). Trout numbers in the midale lane of section 1 were the only counts significantly correlated with TGP in 1987. Trout numbers in the middle lane of sections 1 and 2 were significantly correlated with TGP in 1988.

Correlations between water temperature and discharge followed seasonal trends. Water temperature increased from 5 to $15^{\circ} \mathrm{C}$ (section 1, 1987, correlation between water temperature and day 
Table 2.4.10. Correlation among percentage of total trout in the middle snorkel lane in sections 1 and 2 and day number, 1987 and 1988, Bighorn River, Montana ( $N=27$ for $1987 ; \mathrm{N}=7$ for section 1 , $\mathrm{N}=6$ for section 2 for 1988).

$\begin{array}{ccc}\text { Section } & \begin{array}{c}\text { Mean percentage of } \\ \text { trout in middle lane } \\ \text { (range) }\end{array} & \begin{array}{c}\text { Correlation } \\ \text { coefficient }\end{array} \\ & (r) & \text { Significance }\end{array}$

1987

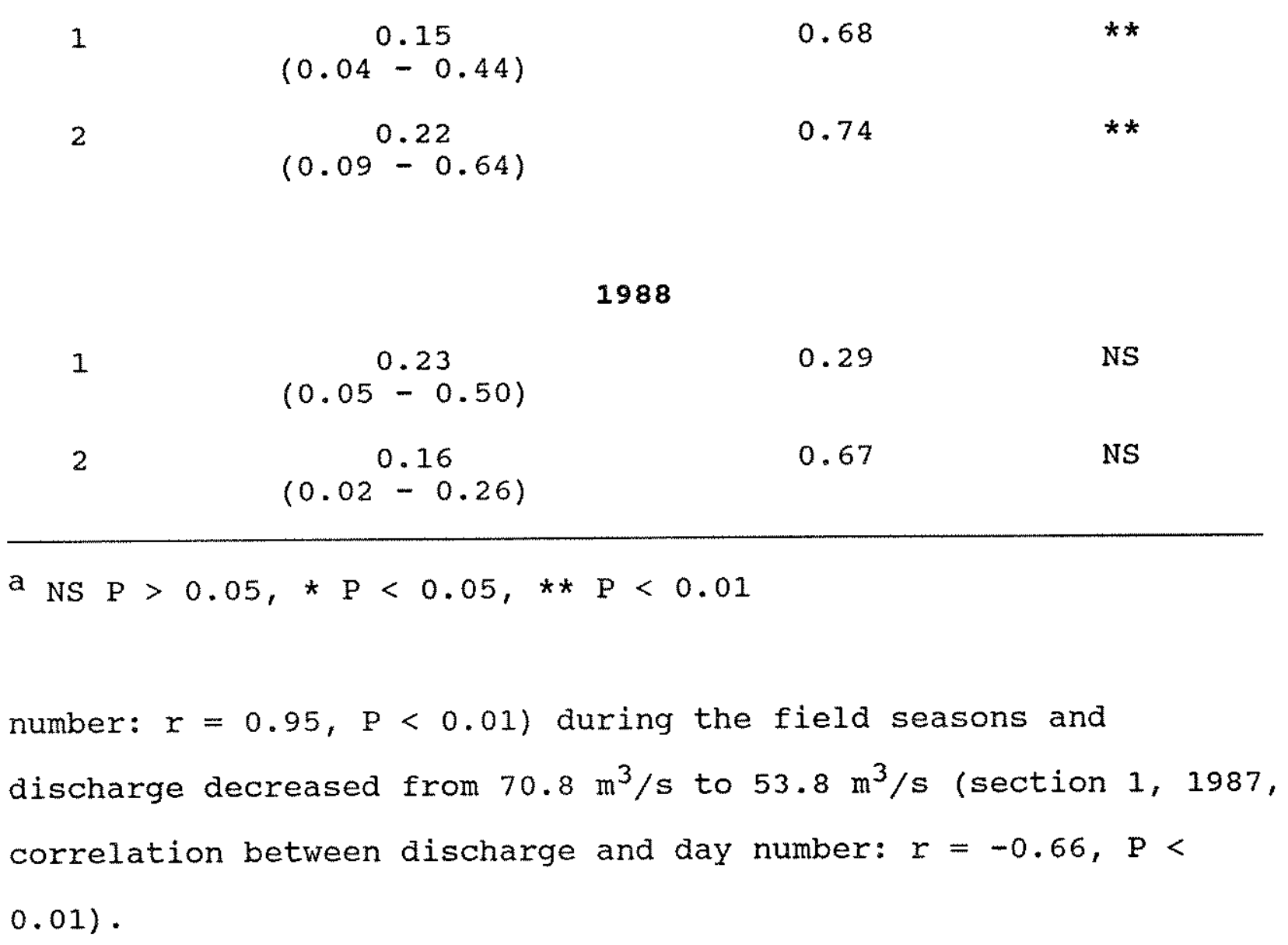

\section{4 .2 .2 Microhabitat}

\section{$2.4 .2 .2 \mathrm{a}$ Methods}

To examine possible differences between brown trout and rainbow trout habitat use in relation to physical habitat and water quality conditions, individual trout were located while snorkeling, and microhabitat variables were recorded. All 


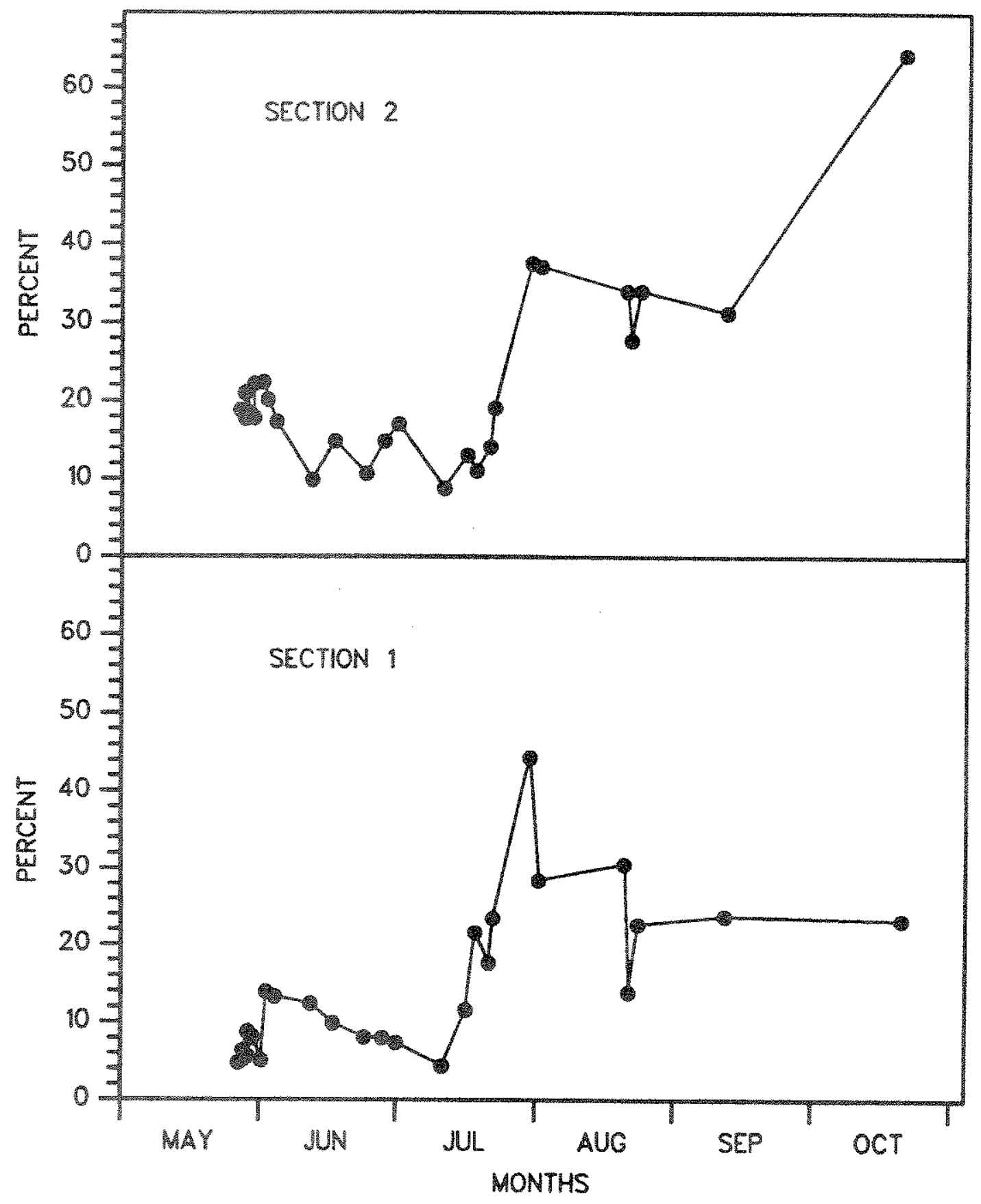

Figure 2.4.9. Percentage of total rainbow trout and brown trout counted in middie lane of snorkel sections 1 and 2,1987 , Bighorn River, Montana. 


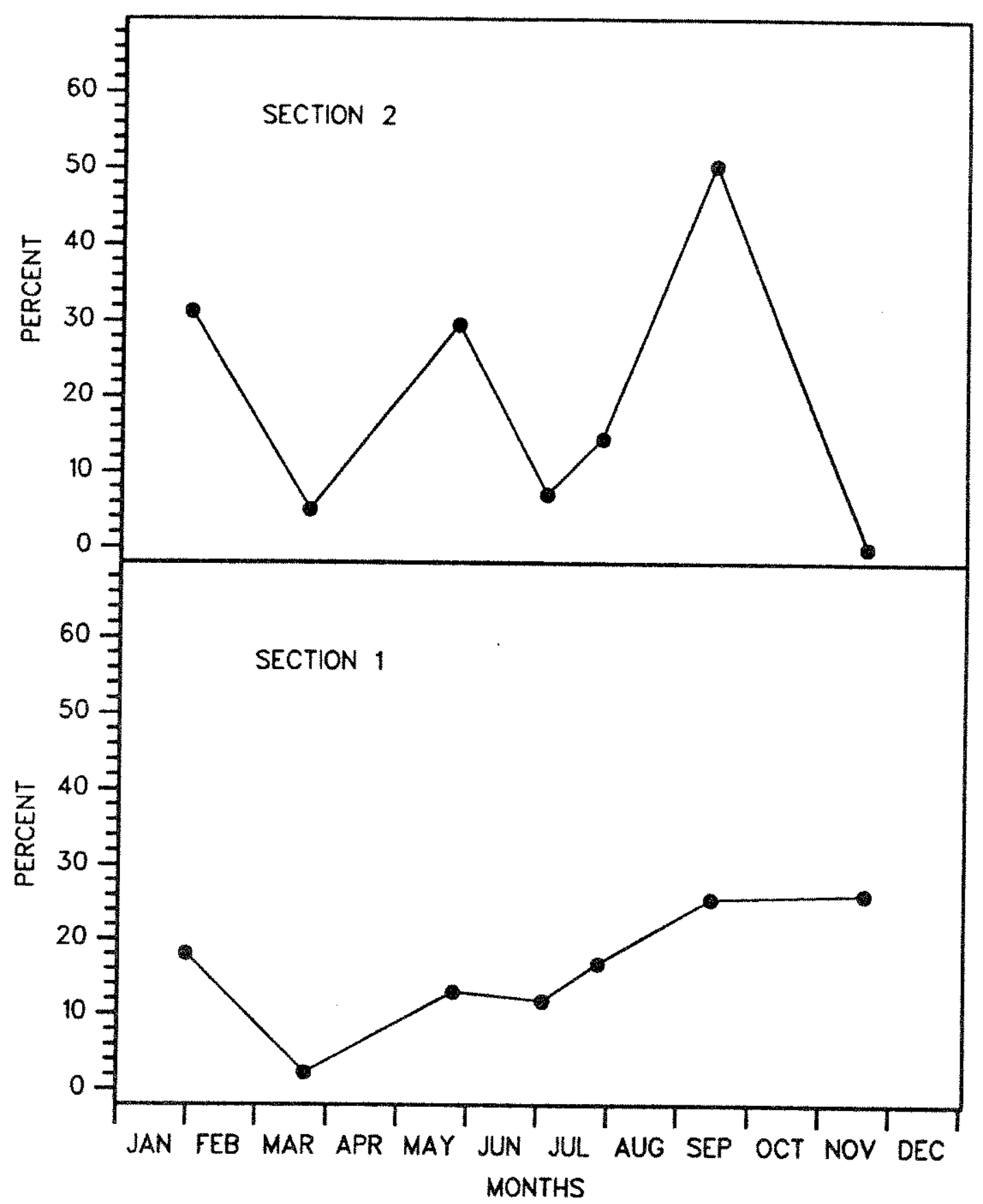

Figure 2.4.10. Percentage of total rainbow trout and brown trout counted in middle lane of snorkel sections 1 and 2 , 1988, Bighorn River, Montana.

355 
Table 2.4.11. Correlations among trout number in snorkel lanes and discharge, visibility, water temperature, above water light level, and total gas pressure, sections 1 and 2, 1987, Bighorn River, Montana $(\mathrm{N}=27)$. The upper triangular matrix indicates simple significance levelsa.

1 Trout, left lane

2 Trout, middle lane

3 Trout, right lane

4 Discharge

5 visibility

6 Light

7 Water temperature

8 TGP
1 Trout, left lane

2 Trout, middle lane

3 Trout, right bank

4 Discharge

5 visibility

6 Light

7 Water temperature

8 TGP

Section 1

$\begin{array}{cccccccc}1 & 2 & 3 & 4 & 5 & 6 & 7 & 8 \\ -- & \text { NS } & * & \text { NS } & * * & \text { NS } & * * & \text { NS } \\ -.29 & -- & \text { NS } & * * & \text { NS } & \text { NS } & * & * * \\ .44 & -.03 & -- & \text { NS } & \text { NS } & \text { NS } & * & \text { NS } \\ .38 & -.69 & .39 & -- & \text { NS } & \text { NS } & * * & * \\ .75 & -.21 & .32 & .38 & -- & \text { NS } & * * & \text { NS } \\ .07 & .02 & .28 & .17 & .25 & -- & \text { NS } & \text { NS } \\ -.67 & .05 & -.40 & -.75 & -.79 & -.06 & -- & \text { NS } \\ -.06 & -.54 & -.02 & .40 & -.02 & .24 & -.07 & --\end{array}$

\section{section 2}

$\begin{array}{cccccccc}1 & 2 & 3 & 4 & 5 & 6 & 7 & 8 \\ -- & \text { NS } & * * & \text { NS } & * * & * * & \text { NS } & \text { NS } \\ -.12 & -- & \text { NS } & * * & \text { NS } & \text { NS } & \text { NS } & \text { NS } \\ .62 & .00 & -- & \text { NS } & * & \text { NS } & \text { NS } & \text { NS } \\ .32 & -.64 & .27 & -- & * * & * * & * * & \text { NS } \\ .66 & -.17 & .42 & .64 & -- & * * & * & \text { NS } \\ -.18 & .15 & .01 & -.55 & -.41 & -- & * * & \text { NS } \\ -.58 & .33 & -.36 & -.81 & -.86 & .64 & -- & \text { NS } \\ .17 & -.20 & .27 & -.13 & -.13 & .37 & .26 & --\end{array}$

a NS $\mathrm{P}>0.05, * \mathrm{P}<0.05, * \mathrm{P}<0.01$

microhabitat data were collected within the two snorkel sections used for macrohabitat studies. Microhabitat use was analyzed for three time periods (June, July, september - October) to determine If time-related changes in habitat use existed.

Locations of individual trout observed using mask and snorkel were marked with a weight attached to a line and float. The section was entered at the upstream end, and the diver floated downstream until a trout was located. Since the diver was drifting with the current, trout position had to be determined rapidly before trout moved. only trout that were 
Table 2.4.12. Correlations among trout number in snorkel lanes and discharge, visibility, water temperature, above water light level, and total gas pressure, sections 1 and 2,1988, Bighorn River, Montana ( $N=7$ for section $1, N=6$ for section 2). The upper triangular matrix indicates simple significance levels ${ }^{\mathrm{a}}$.

\section{Section 1}

1 Trout, left lane

2 Trout, middle lane

3 Trout, right lane

4 Discharge

5 Visibility

6 Light

7 Water temperature

8 TGP

$\begin{array}{rrrrrrrc}1 & 2 & 3 & 4 & 5 & 6 & 7 & 8 \\ -- & \text { NS } & * * & \text { NS } & \text { NS } & \text { NS } & \text { NS } & \text { NS } \\ -.08 & -- & \text { NS } & \text { NS } & * & \text { NS } & \text { NS } & * \\ .92 & .07 & -- & \text { NS } & \text { NS } & \text { NS } & \text { NS } & \text { NS } \\ .04 & -.65 & -.01 & -- & \text { NS } & \text { NS } & \text { NS } & * \\ .42 & -.76 & .37 & .28 & -- & \text { NS } & \text { NS } & \text { NS } \\ -.62 & -.64 & -.67 & .32 & .37 & -- & \text { NS } & \text { NS } \\ -.43 & .56 & -.48 & -.75 & -.48 & .13 & -- & \text { NS } \\ .12 & -.82 & -.07 & .85 & .61 & .51 & -.55 & --\end{array}$

\section{section 2}

1 Trout, left lane

2 Trout, middle lane

3 Trout, right bank

4 Discharge

5 Visibility

6 Light

7 Water temperature

8 TGP

$\begin{array}{cccccccc}1 & 2 & 3 & 4 & 5 & 6 & 7 & 8 \\ -- & \text { NS } & * * & \text { NS } & \text { NS } & \text { NS } & \text { NS } & \text { NS } \\ .01 & -- & \text { NS } & \text { NS } & \text { NS } & \text { NS } & \text { NS } & * \\ .98 & -.01 & -- & \text { NS } & \text { NS } & \text { NS } & \text { NS } & \text { NS } \\ .18 & -.53 & .04 & -- & \text { NS } & \text { NS } & \text { NS } & \text { NS } \\ .56 & -.56 & .53 & .49 & -- & \text { NS } & \text { NS } & \text { NS } \\ -.53 & -.76 & -.45 & .11 & -.01 & -- & \text { NS } & * \\ -.60 & .22 & -.45 & -.60 & -.56 & .41 & -- & \text { NS } \\ -.19 & -.90 & -.12 & .34 & .40 & .89 & .20 & --\end{array}$

$\mathrm{a}$ NS $\mathrm{P}>0.05, * \mathrm{P}<0.05, * * \mathrm{P}<0.01$

initially observed maintaining a relatively constant position were used. The weight was placed directly below the fish position (the location trout was first observed was marked with a weight attached to a line and float) and the float number was recorded. Several trout positions were marked before taking measurements. Habitat measurements were recorded on an underwater slate. Fish habitat parameters included: 1) water depth, 2) fish depth (estimated distance from fish to substrate), 3) velocity at focal point of fish, 4) estimated size of fish, 5) species, 6) signs of GBT, 7) estimated distance to bank (0-3 $\mathrm{m}$, 3-9 $\mathrm{m}, 9+\mathrm{m}), 8$ ) side of river, 9) substrate (fines $<0.2 \mathrm{~cm}$, 
gravel $0.2-6.4 \mathrm{~cm}$, cobble $6.42-24.99 \mathrm{~cm}$, boulder $>24.99 \mathrm{~cm}), 10$ ) height of vegetation at the location of the marking weight, 11) surface light, 12) cover type within $0.5 \mathrm{~m}$ from the focal point (substrate, vegetation, and bank associated, i.e. overhanging vegetation, fences, undercut banks). Water depth, fish depth, and vegetation height were measured to the nearest $0.03 \mathrm{~m}$ with a metal measuring rod. Focal point velocity was measured at the estimated fish depth with an electromagnetic current meter (Marsh/McBirney model 201). Discharge, dissolved gases, and water temperature data were obtained from satellite stations. Air temperature was measured with a mercury thermometer.

Plywood cutouts of various sizes in the shape of trout were used to practice underwater estimation of trout size. Accuracy of length estimates was typically within $2.54 \mathrm{~cm}$ of actual length. Trout observed for microhabitat measurements were estimated to the nearest $2.54 \mathrm{~cm}$ and divided into three size groups (<15 cm, $15-40 \mathrm{~cm}$, and $>40 \mathrm{~cm}$ TL) for data analysis.

Discriminant analysis was used to determine if brown trout and rainbow trout were selecting different habitat locations. Fisherian discriminant analysis (Mardia et al. 1979) attempts to construct a rule which best allocates an individual fish to either brown trout or rainbow trout on the basis of the measured variables for that observation. This analysis finds sets of axes where the ratio of between group variance to within group variance is maximized and is unaffected by scaling of variables. A jackknife validation procedure was used on discriminant analysis results to prevent over-fitting the data. over-fitting occurs when the number of variables is large relative to the 
number of observations. The jackknife validation estimates the true predictive ability of the discriminant function. A jackknife validation repeats the original analysis for the entire data set after removing one observation at a time. Each time the jackknife validation is done, its performance is evaluated using the excluded observation. Analysis of variance was used to examine differences within species.

Microhabitat data were also examined using principal component analysis (PCA) (Mardia et al. 1979). The use of PCA assumed no group membership and therefore is not biased for finding groups. Principal component analysis maximally separated the fish on axes which can facilitate comparison between groups. plots of principal components were examined to identify groups, if any. A correlation matrix was used for PCA.

Monte Carlo simulations were used to repeat PCA many times using randomly constructed data. Data were constructed to represent the null hypotheses of random correlation. Monte Carlo simulations assess the probability of obtaining an observed statistical result, given the null hypotheses is true.

\section{$2 \cdot 4 \cdot 2 \cdot 2 b$ Results}

Significant differences in microhabitat use (ANOVA) between the three size groups of rainbow trout were found for water depth, fish depth, substrate, velocity, and cover (Table 2.4.13). Small rainbow trout $(<15 \mathrm{~cm} T L)$ were significantly $(P<0.05)$ different from larger rainbow trout in microhabitat use for the variables measured. Rainbow trout $15-40 \mathrm{~cm} \mathrm{TL}$ and $>40 \mathrm{~cm} \mathrm{TL}$ used similar microhabitat with only slight differences in 
Table 2.4.13. Comparison of microhabitat use between rainbow trout size groups using analysis of variance, 1987, Bighorn River, Montana ( $1=$ rainbow trout less than $15 \mathrm{~cm}, N=23 ; 2=r a$ inbow trout $15-40 \mathrm{~cm}, \mathrm{~N}=65 ; 3=$ rainbow trout greater than $40 \mathrm{~cm}, \mathrm{~N}=27$; $* \mathrm{p}<0.05, * * \mathrm{p}<0.01)$.

\begin{tabular}{|c|c|c|c|c|}
\hline \multirow[b]{2}{*}{ Variable } & \multicolumn{3}{|c|}{ Size group } & \multirow[b]{2}{*}{$F_{2,112}$} \\
\hline & $\begin{array}{c}1 \\
\text { Mean } \\
\text { (SD) }\end{array}$ & $\begin{array}{c}2 \\
\text { Mean } \\
(S D)\end{array}$ & $\begin{array}{c}3 \\
\text { Mean } \\
(\mathrm{SD})\end{array}$ & \\
\hline Water depth (m) & $\begin{array}{c}0.56 \\
(0.42)\end{array}$ & $\begin{array}{l}1.14 \\
(0.28)\end{array}$ & $\begin{array}{c}1.22 \\
(0.38)\end{array}$ & $31.07 * *$ \\
\hline Fish depth (m) & $\begin{array}{c}0.45 \\
(0.33)\end{array}$ & $\begin{array}{c}0.81 \\
(0.34)\end{array}$ & $\begin{array}{c}1.02 \\
(0.40)\end{array}$ & $16.93 \%$ \\
\hline Substrate & $\begin{array}{c}1.39 \\
(0.50)\end{array}$ & $\begin{array}{c}1.86 \\
(0.39)\end{array}$ & $\begin{array}{c}1.82 \\
(0.17)\end{array}$ & $11.24 * *$ \\
\hline $\begin{array}{l}\text { Vegetation } \\
\text { height (cm) }\end{array}$ & $\begin{array}{c}17.67 \\
(13.56)\end{array}$ & $\begin{array}{c}16.57 \\
(14.67)\end{array}$ & $\begin{array}{l}12.98 \\
(15.36)\end{array}$ & 0.77 \\
\hline Velocity $(\mathrm{cm} / \mathrm{s})$ & $\begin{array}{c}11.13 \\
(15.96)\end{array}$ & $\begin{array}{c}32.03 \\
(13.16)\end{array}$ & $\begin{array}{c}18.37 \\
(18.37)\end{array}$ & $16.62 * *$ \\
\hline Cover & $\begin{array}{l}1.04 \\
(0.64)\end{array}$ & $\begin{array}{c}1.80 \\
(0.72)\end{array}$ & $\begin{array}{c}1.70 \\
(0.67)\end{array}$ & $10.50 * *$ \\
\hline
\end{tabular}

vegetation height and velocity. The mean vegetation height used by rainbow trout 15 - $40 \mathrm{~cm}$ TL was greater than that used by rainbow trout $>40 \mathrm{~cm}$ TL. The mean focal point velocity was greater for rainbow trout $15-40 \mathrm{~cm}$ TL than for the larger rainbow trout.

Significant differences $(P<0.05)$ in the use of water depth, fish depth, substrate, vegetation height, velocity, and cover were observed between brown trout of different size groups (ANOVA, Table 2.4.14). Brown trout $<15 \mathrm{~cm}$ TL were found in shallower water and lower velocities than brown trout $>15 \mathrm{~cm}$ TL. Brown trout $>15 \mathrm{~cm}$ TL were found using similar water depth, fish 
Table 2.4.14. Comparison of microhabitat use between brown trout size groups using analysis of variance, 1987, Bighorn River, Montana ( 1 =brown trout less than $15 \mathrm{~cm}, \mathrm{~N}=33 ; 2=\mathrm{brown}$ trout 15 $40 \mathrm{~cm}, \mathrm{~N}=141 ; 3=$ brown trout greater than $40 \mathrm{~cm}, \mathrm{~N}=44 ; * \mathrm{P}<0.05$, $* * \mathrm{P}<0.01)$.

\begin{tabular}{|c|c|c|c|c|}
\hline \multirow[b]{2}{*}{ Variable } & \multicolumn{3}{|c|}{ Size group } & \multirow[b]{2}{*}{$\mathrm{F}_{2,215}$} \\
\hline & $\begin{array}{c}1 \\
\text { Mean } \\
\text { (SD) }\end{array}$ & $\begin{array}{c}2 \\
\text { Mean } \\
(\mathrm{SD})\end{array}$ & $\begin{array}{c}3 \\
\text { Mean } \\
(S D)\end{array}$ & \\
\hline Water depth (m) & $\begin{array}{c}0.57 \\
(0.38)\end{array}$ & $\begin{array}{c}1.08 \\
(0.29)\end{array}$ & $\begin{array}{c}1.03 \\
(0.23)\end{array}$ & $42.18 * *$ \\
\hline Fish depth (m) & $\begin{array}{c}0.49 \\
(0.33)\end{array}$ & $\begin{array}{c}0.91 \\
(0.30)\end{array}$ & $\begin{array}{c}0.90 \\
(0.23)\end{array}$ & $28.98 * *$ \\
\hline Substrate & $\begin{array}{c}1.24 \\
(0.61)\end{array}$ & $\begin{array}{c}1.85 \\
(0.41)\end{array}$ & $\begin{array}{l}1.73 \\
0.54)\end{array}$ & $21.89 * *$ \\
\hline $\begin{array}{l}\text { Vegetation } \\
\text { height }(\mathrm{cm})\end{array}$ & $\begin{array}{l}13.16 \\
(16.48)\end{array}$ & $\begin{array}{c}12.16 \\
(11.53)\end{array}$ & $\begin{array}{c}11.49 \\
(10.31)\end{array}$ & 0.18 \\
\hline Velocity $(\mathrm{cm} / \mathrm{s})$ & $\begin{array}{c}8.22 \\
(9.86)\end{array}$ & $\begin{array}{c}24.69 \\
(12.74)\end{array}$ & $\begin{array}{l}17.39 \\
(12.41)\end{array}$ & $26.05 * *$ \\
\hline Cover & $\begin{array}{c}1.27 \\
(1.35)\end{array}$ & $\begin{array}{c}1.83 \\
(0.78)\end{array}$ & $\begin{array}{c}1.68 \\
(1.01)\end{array}$ & $4.78 * *$ \\
\hline
\end{tabular}

depth, substrate, vegetation height, and cover. Brown trout 15 $40 \mathrm{~cm}$ TL had higher focal point velocities than brown trout $>40$ cm TL (Table 2.4.14).

Discriminant analysis of microhabitat use (1987) by small brown trout and rainbow trout $(<15 \mathrm{~cm} \mathrm{TL}, \mathrm{N}=56)$ resulted in a jackknife misclassification of $52 \%$ (Table 2.4.15). Fish depth had the largest weighting (absolute value), -0.95 , followed by substrate and water depth with values of 0.22 and 0.20 , respectively. Seasonal differences could not be determined due to differences in time of fry emergence.

Discrimination between rainbow trout and brown trout $>15 \mathrm{~cm}$ 
Table 2.4.15. Discriminant analysis of use of selected microhabitat variables by brown trout and rainbow trout $<15 \mathrm{~cm}$ TL, June - October 1987, Bighorn River, Montana (brown trout $N=33$, rainbow trout $N=23 ; J K=j a c k k n i f e$ validation).

\begin{tabular}{|c|c|c|c|c|}
\hline Variable & $\begin{array}{l}\text { Discriminant } \\
\text { eigen vector }\end{array}$ & $\begin{array}{l}\text { Eigen } \\
\text { value }\end{array}$ & $\begin{array}{c}\text { Number } \\
\text { misclassified }\end{array}$ & $\begin{array}{c}\text { Number } \\
\text { misclassified } \\
\text { (JK) }\end{array}$ \\
\hline
\end{tabular}

$\begin{array}{lr}\text { Water depth } & 0.20 \\ \text { Fish depth } & -0.95 \\ \text { Substrate } & 0.22 \\ \text { Vegetation } & \\ \quad \text { height } & 0.01 \\ \text { Velocity } & 0.01 \\ \text { Cover } & -0.08\end{array}$

TL $(N=104)$ based on microhabitat use in June 1987 had a jackknife misclassification of $46 \%$ using the same variables analyzed for size group 1 (Table 2.4.16). Fish depth and water depth were the largest weighted variables. No significant differences $(P<$ $0.05)$ were found in microhabitat use between brown trout and rainbow trout for variables measured (t test, Table 2.4.17), although rainbow trout used slightly higher water velocities $(P=0.069,26.93 \mathrm{~cm} / \mathrm{s}$ Vs. $32.70 \mathrm{~cm} / \mathrm{s})$

Misclassification of brown trout and rainbow trout $>15 \mathrm{~cm}$ TL using discriminant analysis decreased to $36 \%$ in July 1987 (Table 2.4.16). Water depth and fish depth again received the largest weighting. The eigen value increased from 0.108 in June to 0.154 in July, an increase in the ratio of between-group variance to within-group variance, indicating an increase in the separation of species. Significant differences in water depth $(P=0.009)$ and velocity $(P=0.000)$ were observed (t test, Table $2 \cdot 4 \cdot 18)=$ 
Table 2.4.16. Discriminant analysis of use of selected

microhabitat variables by brown trout and rainbow trout $>15 \mathrm{~cm}$ TL, June - October 1987, Bighorn River, Montana (June: brown trout $\mathrm{N}=71$, rainbow trout $\mathrm{N}=33$; July: brown trout $\mathrm{N}=92$, rainbow trout $\mathrm{N}=41$; September/October: brown trout $\mathrm{N}=22$, rainbow trout $\mathrm{N}=18$; JK=jackknife validation).

Variable

Discriminant Eigen

Number

Number

eigen vector value misclassified

misclassified

(JK)

\section{June}

$\begin{array}{lr}\text { Water depth } & 0.67 \\ \text { Fish depth } & -0.72 \\ \text { Substrate } & 0.18 \\ \text { Vegetation } & \\ \quad \text { height } & 0.00 \\ \text { Velocity } & 0.00 \\ \text { Cover } & 0.00\end{array}$

$0.108 \quad 42(40 \%)$

$48(46 \%)$

July

$\begin{array}{lr}\text { Water depth } & 0.78 \\ \text { Fish depth } & -0.61 \\ \text { Substrate } & -0.15 \\ \text { Vegetation } & \\ \quad \text { height } & 0.00 \\ \text { Velocity } & 0.01 \\ \text { Cover } & -0.07\end{array}$

$0.154 \quad 44 \quad(33 \%)$

49 (36\%)

\section{september - October}

Water depth

0.76

Fish depth

$-0.65$

Substrate

0.03

Vegetation

height

0.00

velocity

Cover

0.01

$-0.01$

$$
1.046
$$

$5(13 \%)$

$8(20 \%)$

Discrimination between brown trout and rainbow trout $>15 \mathrm{~cm}$ TL $(\mathrm{N}=40)$ in september - October 1987 was improved over other 
Table 2.4.17. Comparisons between brown trout and rainbow trout $>15 \mathrm{~cm}$ TL use of selected microhabitat variables, t test for equality of means, June 1987, Bighorn River, Montana (brown trout $\mathrm{N}=71$, rainbow trout $\mathrm{N}=33$ ).

\begin{tabular}{|c|c|c|c|c|}
\hline Variable & $\begin{array}{l}\text { Brown trout } \\
\text { Mean } \\
\text { (SD) }\end{array}$ & $\begin{array}{l}\text { Rainbow trout } \\
\text { Mean } \\
\text { (SD) }\end{array}$ & $t$ & P value \\
\hline Water depth (m) & $\begin{array}{c}1.09 \\
(0.26)\end{array}$ & $\begin{array}{c}1.12 \\
(0.31)\end{array}$ & -0.39 & 0.700 \\
\hline Fish depth (m) & $\begin{array}{c}0.92 \\
(0.29)\end{array}$ & $\begin{array}{c}0.87 \\
(0.33)\end{array}$ & 0.79 & 0.433 \\
\hline Substrate & $\begin{array}{c}1.83 \\
(0.48)\end{array}$ & $\begin{array}{c}1.97 \\
(0.17)\end{array}$ & -1.62 & 0.109 \\
\hline $\begin{array}{l}\text { Vegetation heig } \\
(\mathrm{cm})\end{array}$ & $\begin{array}{l}11.20 \\
(8.03)\end{array}$ & $\begin{array}{l}11.47 \\
(5.72)\end{array}$ & -0.17 & 0.862 \\
\hline Velocity $(\mathrm{cm} / \mathrm{s})$ & $\begin{array}{c}26.92 \\
(15.02)\end{array}$ & $\begin{array}{c}32.70 \\
(14.80)\end{array}$ & -1.83 & 0.069 \\
\hline Cover & $\begin{array}{c}1.92 \\
(0.69)\end{array}$ & $\begin{array}{c}2.06 \\
(0.35)\end{array}$ & -1.14 & 0.258 \\
\hline
\end{tabular}

time periods with a misclassification of $20 \%$ (Table 2.4 .16 ). As in June and July, water depth and fish depth had the largest weighting, 0.76 and -0.65 , respectively. Significant differences were found in use of depth $(P=0.001)$ and velocity $(P=0.000)$ by brown trout and rainbow trout (t test, Table 2.4.19). Brown trout were closer to the river bottom (deeper in the water column, mean fish depth) and in slower water than rainbow trout. Rainbow trout were found in deeper water (mean water depth 1.14 m) but closer to the surface (mean fish depth $0.59 \mathrm{~m}$ ), than brown trout.

Principal component analysis of microhabitat use by all trout from all periods produced no detectable differences between 
Table 2.4.18. Comparisons between brown trout and rainbow trout $>15 \mathrm{~cm}$ TL use of selected microhabitat variables, $t$ test for equality of means, July 1987, Bighorn River, Montana (brown trout $\mathrm{N}=92$, rainbow trout $\mathrm{N}=41$ ).

\begin{tabular}{|c|c|c|c|c|}
\hline Variable & $\begin{array}{l}\text { Brown trout } \\
\text { Mean } \\
\text { (SD) }\end{array}$ & $\begin{array}{l}\text { Rainbow trout } \\
\text { Mean } \\
\text { (SD) }\end{array}$ & $t$ & p value \\
\hline Water depth (m) & $\begin{array}{c}1.06 \\
(0.29)\end{array}$ & $\begin{array}{c}1.21 \\
(0.36)\end{array}$ & -2.63 & 0.009 \\
\hline Fish depth (m) & $\begin{array}{c}0.91 \\
(0.29)\end{array}$ & $\begin{array}{c}1.01 \\
(0.38)\end{array}$ & -1.51 & 0.132 \\
\hline Substrate & $\begin{array}{c}1.89 \\
(0.38)\end{array}$ & $\begin{array}{c}1.88 \\
(0.40)\end{array}$ & 0.18 & 0.854 \\
\hline $\begin{array}{l}\text { Vegetation height } \\
(\mathrm{cm})\end{array}$ & $\begin{array}{c}8.70 \\
(5.00)\end{array}$ & $\begin{array}{c}9.97 \\
(5.43)\end{array}$ & $-1 \cdot 33$ & 0.187 \\
\hline Velocity $(\mathrm{cm} / \mathrm{s})$ & $\begin{array}{c}21.63 \\
(11.28)\end{array}$ & $\begin{array}{l}30.70 \\
(15.23)\end{array}$ & -3.83 & 0.000 \\
\hline Cover & $\begin{array}{c}1.84 \\
(0.89)\end{array}$ & $\begin{array}{l}1.66 \\
(0.73)\end{array}$ & 1.12 & 0.263 \\
\hline
\end{tabular}

brown trout and rainbow trout (Figure 2.4.11). Principal component (PC) I was heavily weighted (absolute value) by water depth, fish depth, velocity, and substrate (Table 2.4.20). The explained variances of all principal components except PC I was expected, indicating that PC I was capturing a distinct feature of the data not due to chance (Appendix G: Table 50). Plotting PC I versus PC II indicates a clustering of size groups (Figure 2.4.12). Size group 1 was scored lower on PC I than size groups 2 and 3. No separation between groups 2 and 3 was detected. Microhabitat use by all trout $<15 \mathrm{~cm}$ TL observed in 1987 produced no detectable separation of brown trout and rainbow trout using principal component analysis (Figure 2.4.13). Principal 
Table 2.4.19. Comparisons between brown trout and rainbow trout $>15 \mathrm{~cm}$ TL use of selected microhabitat variables, $t$ test for equality of means, September - October 1987, Bighorn River, Montana (brown trout $\mathrm{N}=22$, rainbow trout $\mathrm{N}=18$ ).

\begin{tabular}{|c|c|c|c|c|}
\hline Variable & $\begin{array}{l}\text { Brown trout } \\
\text { Mean } \\
\text { (SD) }\end{array}$ & $\begin{array}{l}\text { Rainbow trout } \\
\text { Mean } \\
\text { (SD) }\end{array}$ & $t$ & P value \\
\hline Water depth (m) & $\begin{array}{c}1.05 \\
(0.25)\end{array}$ & $\begin{array}{c}1.14 \\
(0.14)\end{array}$ & -1.40 & 0.168 \\
\hline Fish depth (m) & $\begin{array}{c}0.86 \\
(0.28)\end{array}$ & $\begin{array}{c}0.59 \\
(0.22)\end{array}$ & 3.49 & 0.001 \\
\hline Substrate & $\begin{array}{l}1.50 \\
(0.51)\end{array}$ & $\begin{array}{l}1.56 \\
(0.51)\end{array}$ & -0.34 & 0.734 \\
\hline $\begin{array}{l}\text { Vegetation heig } \\
(\mathrm{cm})\end{array}$ & $\begin{array}{c}28.40 \\
(21.20)\end{array}$ & $\begin{array}{c}35.56 \\
(22.87)\end{array}$ & -1.02 & 0.312 \\
\hline Velocity $(\mathrm{cm} / \mathrm{s})$ & $\begin{array}{l}15.66 \\
(7.89)\end{array}$ & $\begin{array}{l}29.46 \\
(14.52)\end{array}$ & -3.83 & 0.000 \\
\hline Cover & $\begin{array}{c}1.23 \\
(0.87)\end{array}$ & $\begin{array}{c}1.50 \\
(0.92)\end{array}$ & -0.96 & 0.343 \\
\hline
\end{tabular}

component I explained $52 \%$ of the variance and had an eigen value of 3.13 (Table 2.4.21). Monte Carlo simulations indicated that a variance of $20-31 \%$ was expected and there was less than a 1 in 1000 chance of obtaining the observed value of $52 \%$ (Appendix G: Table 51).

Principal component analysis of microhabitat use of all trout $>15 \mathrm{~cm}$ TL from June 1987 resulted in a first principal component that explained $40 \%$ of the variance (Table 2.4.22). All weightings of variables were positive, with water depth, fish depth, and velocity having the largest values. Monte carlo simulations indicated that PC I was capturing a unique feature in the data (Appendix G: Table 52). Principal component II captured 


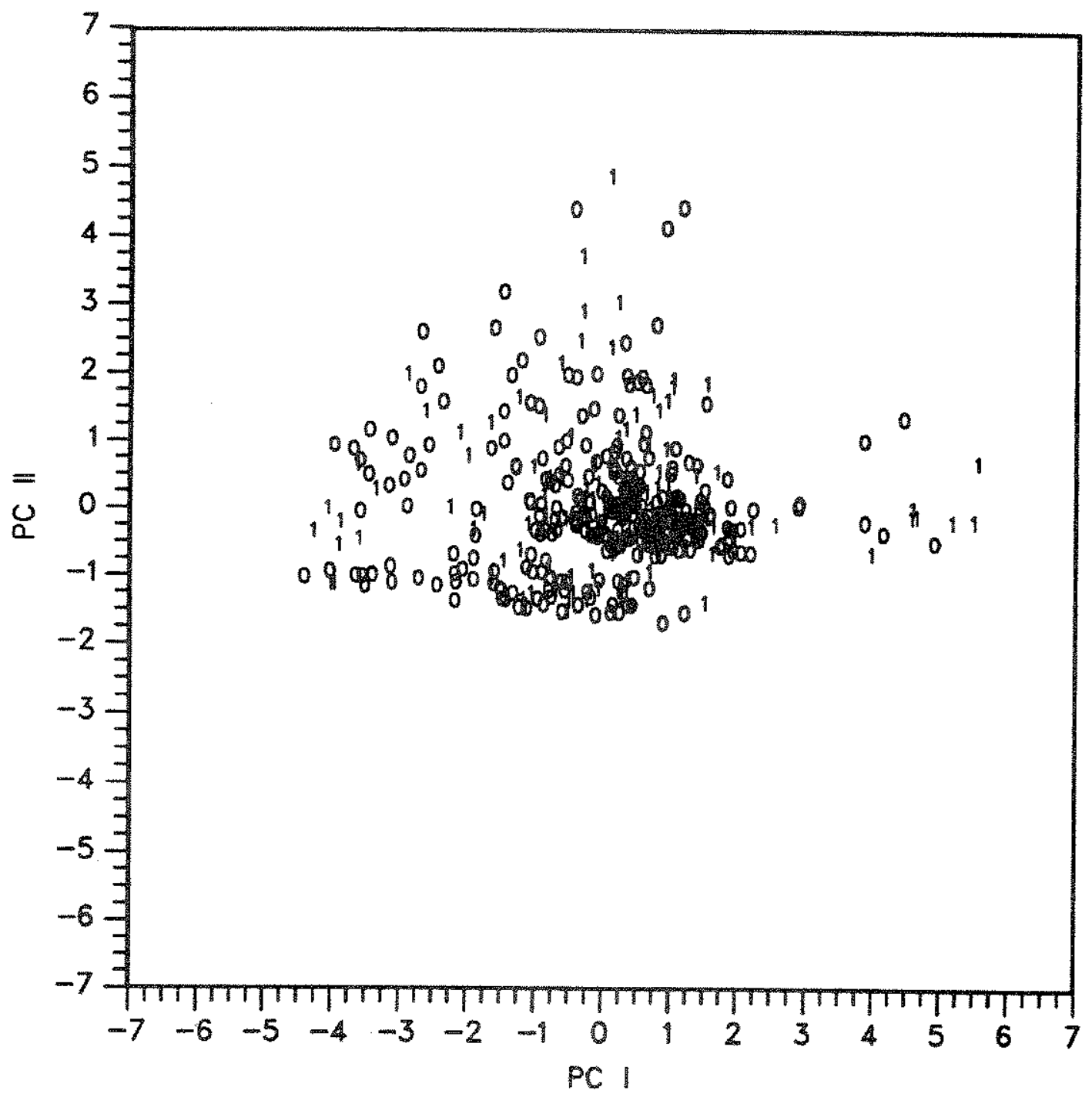

Figure 2.4.11. Principal component I versus principal component II for brown trout and rainbow trout of all size groups using the variables water depth, fish depth, substrate, vegetation height, velocity, and cover, June - october 1987, Bighorn River, Montana ( 0 =brown trout, 1=rainbow trout). 
Table 2.4.20. Principal component analysis for all brown trout and rainbow trout using selected habitat variables, June october 1987. Bighorn River, Montana.

\begin{tabular}{|c|c|c|c|c|c|c|}
\hline \multirow[t]{2}{*}{ Variable } & \multicolumn{6}{|c|}{ Principal component } \\
\hline & $I$ & II & III & IV & $\mathrm{V}$ & VI \\
\hline Water depth & 0.58 & 0.05 & -0.01 & -0.34 & 0.00 & -0.74 \\
\hline Fish depth & 0.53 & -0.14 & 0.03 & -0.47 & -0.29 & 0.63 \\
\hline Substrate & 0.38 & -0.07 & -0.32 & 0.70 & -0.51 & -0.03 \\
\hline Vegetation depth & 0.05 & 0.91 & -0.37 & -0.07 & 0.04 & 0.14 \\
\hline Velocity & 0.44 & -0.12 & -0.12 & 0.29 & 0.81 & 0.20 \\
\hline Cover & 0.21 & 0.36 & 0.86 & 0.28 & -0.05 & 0.05 \\
\hline Eigen value & 2.58 & 1.03 & 0.93 & 0.76 & 0.63 & 0.07 \\
\hline Percent of variance & 42.93 & 17.20 & 15.54 & 12.70 & 10.44 & 1.19 \\
\hline Cumulative percent & 42.93 & 60.13 & 75.67 & 88.37 & 98.81 & 100.00 \\
\hline
\end{tabular}

a structural aspect of the data with the heaviest weighting being cover and substrate. Water depth and fish depth switched from positive weighting in PC I to negative weighting in PC II. The structural aspect of the data was also captured by PC III with the largest weighting being vegetation height, substrate, and cover. Brown trout tended to receive lowex scores in PC I than rainbow trout although considerable overlap still occurred between species (Figure 2.4 .14 ).

Water depth and fish depth had the largest weighting of the six variables used in PC I for trout $>15 \mathrm{~cm}$ TL observed in July (Table 2.4.23). Unlike PC I from June observations, a negative weighting was contained in PC I of July data. A value of -0.03 for cover differed from the June weighting of 0.09 . In PC II vegetation height had the largest weighting and velocity the smallest. As in the June data, brown trout generally had lower scores than rainbow trout in PC I (Figure 2.4.15).

Principal component I obtained from microhabitat of trout 


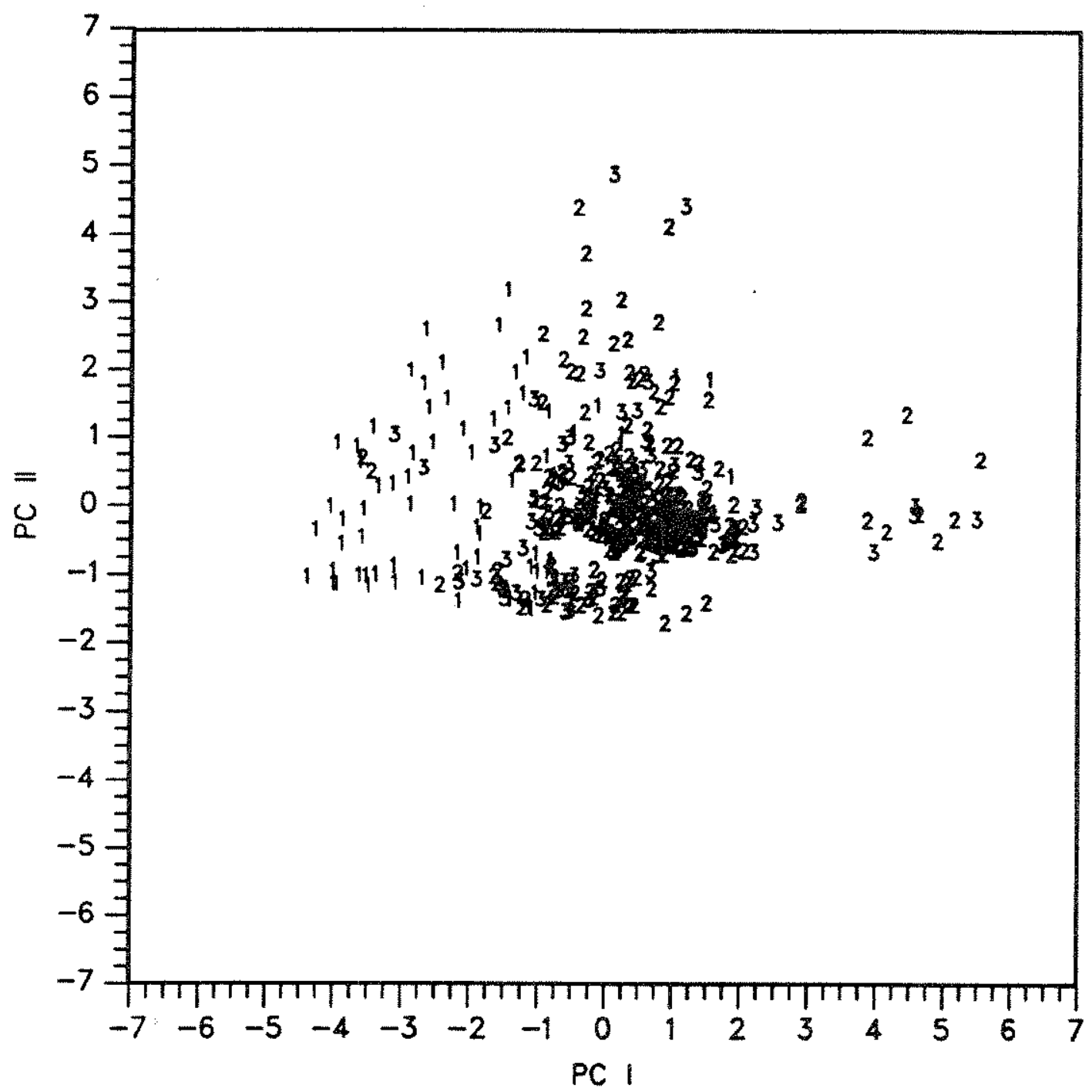

Figure 2.4.12. Principal component I versus principal component II for brown trout and rainbow trout of all size groups using the variables water depth, fish depth, substrate, vegetation height, velocity, and cover, June - october 1987, Bighorn River, Montana (1=size group 1, <15 cm TL; $2=s i z e$ group 2, 15-40 cm TL; 3=size group $3,>40 \mathrm{~cm}$ TL). 


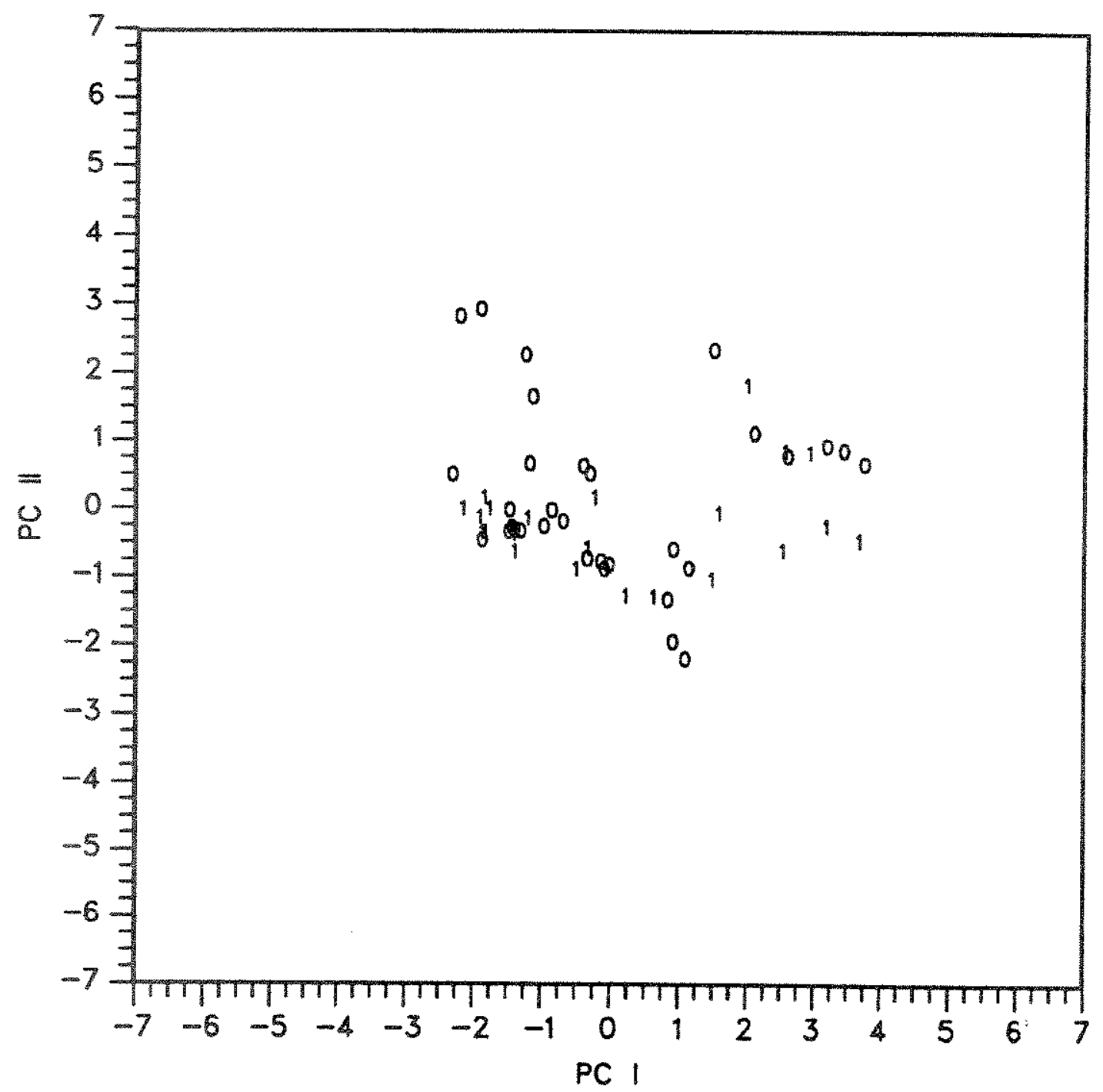

Figure 2.4.13. Principal component I versus principal component II for brown trout and rainbow trout $<15 \mathrm{~cm} \mathrm{TL}$ using the variables water depth, fish depth, substrate, vegetation height, velocity, and cover, June - October 1987, Bighorn River, Montana ( $0=$ brown trout, $1=$ rainbow trout). 
Table 2.4.21. Principal component analysis for all brown trout and rainbow trout $<15 \mathrm{~cm} \mathrm{TL}$ using selected habitat variables, June - October 1987, Bighorn River, Montana.

\begin{tabular}{|c|c|c|c|c|c|c|}
\hline \multirow[t]{2}{*}{ Variable } & \multicolumn{3}{|c|}{ Principal } & \multicolumn{2}{|c|}{ component } & \multirow[b]{2}{*}{ VI } \\
\hline & $I$ & II & III & IV & $\mathrm{V}$ & \\
\hline Water depth & 0.54 & 0.15 & -0.10 & -0.18 & -0.26 & -0.76 \\
\hline Fish depth & 0.51 & 0.09 & -0.13 & -0.52 & -0.27 & 0.61 \\
\hline Substrate & 0.43 & -0.44 & -0.01 & -0.08 & 0.78 & -0.02 \\
\hline Vegetation depth & 0.22 & -0.49 & 0.73 & 0.19 & -0.37 & 0.05 \\
\hline velocity & 0.44 & 0.18 & -0.27 & 0.81 & -0.06 & 0.21 \\
\hline Cover & 0.13 & 0.71 & 0.61 & -0.01 & 0.33 & 0.05 \\
\hline Eigen value & 3.13 & 1.10 & 0.98 & 0.45 & 0.33 & 0.02 \\
\hline Percent of variance & 52.09 & $18 \cdot 34$ & 16.24 & 7.54 & 5.42 & 0.36 \\
\hline Cumulative percent & 52.09 & 70.43 & 86.68 & 94.22 & 99.64 & 100.00 \\
\hline
\end{tabular}

Table 2.4.22. Principal component analysis for brown trout and rainbow trout $>15 \mathrm{~cm}$ TL using selected habitat variables, June 1987, Bighorn River, Montana.

Variable

$$
\text { I II III IV IV V }
$$

VI

$\begin{array}{lrrrrrr}\text { Water depth } & 0.58 & -0.25 & 0.10 & -0.27 & 0.16 & -0.70 \\ \text { Fish depth } & 0.54 & -0.43 & 0.01 & -0.25 & -0.16 & 0.66 \\ \text { Substrate } & 0.34 & 0.43 & -0.53 & 0.08 & -0.63 & -0.12 \\ \text { Vegetation depth } & 0.27 & 0.05 & 0.65 & 0.66 & -0.28 & -0.02 \\ \text { Velocity } & 0.41 & 0.37 & -0.24 & 0.35 & 0.69 & 0.20 \\ \text { Cover } & 0.09 & 0.66 & 0.48 & -0.56 & 0.00 & 0.12 \\ & & & & & & \\ \quad \text { Eigen value } & 2.39 & 1.11 & 0.99 & 0.86 & 0.58 & 0.07 \\ \quad \text { Percent of variance } & 39.90 & 18.50 & 16.51 & 14.35 & 9.66 & 1.09 \\ \quad \text { Cumulative percent } & 39.90 & 58.40 & 74.90 & 89.25 & 98.91100 .00\end{array}$

$>15 \mathrm{~cm}$ TL during september - October 1987 differed considerably

from earlier months. Water depth had the largest value, followed by vegetation height and cover (Table 2.4.24). Fish depth received a low weighting of -0.03 in $P C I$. Monte Carlo simulations indicated that the percent of the variance explained 


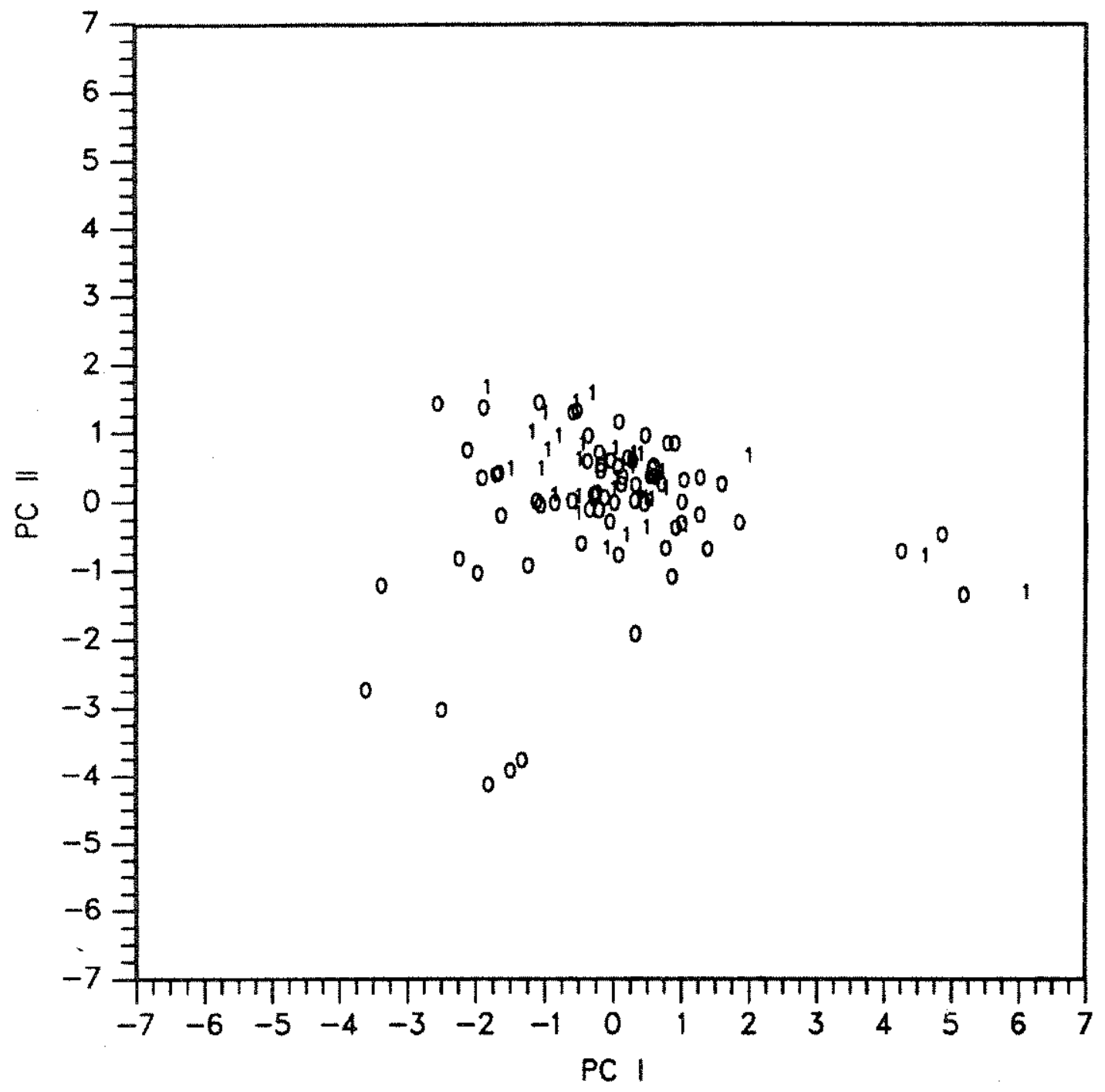

Figure 2.4.14. Principal component I versus principal component II for brown trout and rainbow trout $>15 \mathrm{~cm} \mathrm{TL}$ using the variables water depth, fish depth, substrate, vegetation height, velocity, and cover, June 1987, Bighorn River, Montana (0=brown trout, $1=$ rainbow trout). 
Table 2.4.23. Principal component analysis for brown trout and rainbow trout $>15 \mathrm{~cm}$ TL using selected habitat variables, July 1987, Bighorn River, Montana.

\begin{tabular}{|c|c|c|c|c|c|c|}
\hline \multirow{2}{*}{ Variable } & \multicolumn{6}{|c|}{ Principal component } \\
\hline & $I$ & II & III & IV & $\mathrm{V}$ & VI \\
\hline water depth & 0.57 & -0.26 & 0.23 & 0.10 & -0.07 & -0.74 \\
\hline Fish depth & 0.52 & -0.37 & 0.22 & 0.33 & -0.11 & 0.65 \\
\hline Substrate & 0.28 & 0.36 & -0.59 & 0.57 & 0.35 & -0.05 \\
\hline Vegetation depth & 0.35 & 0.63 & -0.04 & -0.19 & -0.66 & 0.08 \\
\hline velocity & 0.46 & 0.07 & -0.09 & -0.69 & 0.53 & 0.15 \\
\hline Cover & -0.03 & 0.52 & 0.74 & 0.22 & 0.37 & 0.02 \\
\hline Eigen value & 2.57 & 1.19 & 1.07 & 0.68 & 0.44 & 0.05 \\
\hline Percent of variance & 42.86 & 19.85 & 17.90 & 11.36 & 7.27 & 0.77 \\
\hline Cumulative percent & 42.86 & 62.70 & 80.60 & 91.96 & 99.23 & 100.00 \\
\hline
\end{tabular}

by all principal components was within expected ranges (Appendix G: Table 54). Principal component II had its largest weighting from the variables fish depth and velocity. Brown trout received lower scores than rainbow trout producing more distinct differences between species than that found in June and July data when plotted (Figure 2.4.16).

\section{4 .2 .3 Radio Telemetry}

\section{4 .2 .3 a Methods}

Pressure-sensitive radio transmitters (PSRTs) were implanted in four brown trout and four rainbow trout in 1987 and in two brown trout and one rainbow trout in 1988. Six brown trout and four rainbow trout were implanted with location transmitters in 1987. Transmitters were designed and built by Custom Telemetry and Consulting (CTC), Athens, Georgia. Pressure-sensitive transmitters were temperature compensating. Transmitter frequencies were individually identifiable and were in the $30 \mathrm{MHz}$ 


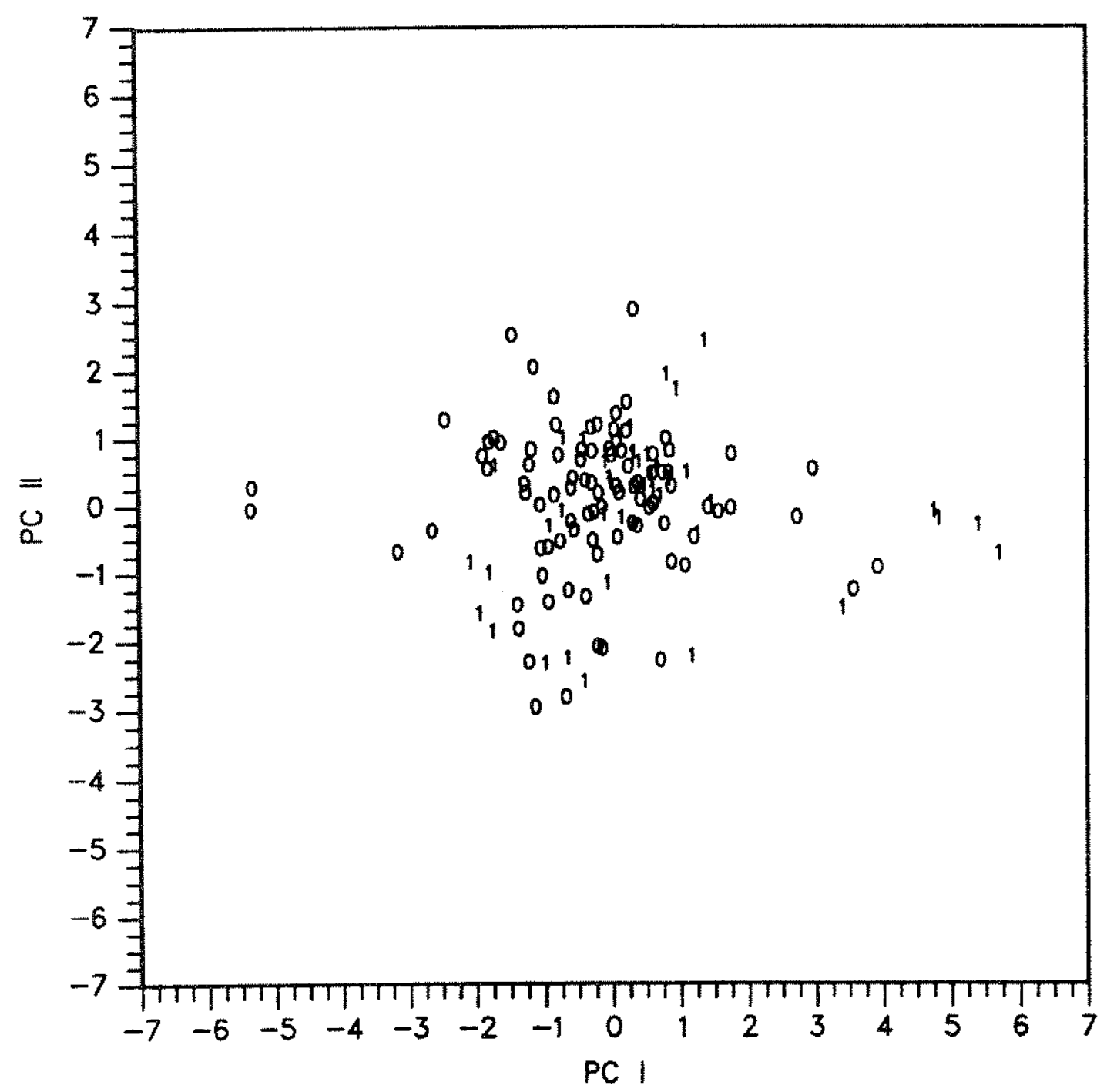

Figure 2.4.15. Principal component I versus principal component II for brown trout and rainbow trout $>15 \mathrm{~cm}$ TL using the variables water depth, fish depth, substrate, vegetation height, velocity, and cover, July 1987, Bighorn River, Montana (0=brown trout, 1 =rainbow trout). 
Table 2.4.24. Principal component analysis for brown trout and rainbow trout $>15 \mathrm{~cm}$ TL using selected habitat variables, September - October 1987, Bighorn River, Montana.

\begin{tabular}{|c|c|c|c|c|c|c|}
\hline \multirow[t]{2}{*}{ Variable } & \multicolumn{6}{|c|}{ Principal compon } \\
\hline & I & II & III & IV & $\mathrm{V}$ & VI \\
\hline Water depth & 0.53 & 0.38 & 0.03 & -0.44 & -0.29 & -0.55 \\
\hline Fish depth & -0.03 & 0.63 & 0.56 & 0.00 & -0.15 & 0.51 \\
\hline Substrate & 0.41 & -0.61 & 0.03 & 0.80 & -0.43 & -0.06 \\
\hline Vegetation depth & 0.52 & 0.17 & -0.60 & -0.08 & 0.21 & 0.54 \\
\hline velocity & 0.23 & -0.61 & 0.24 & -0.40 & -0.47 & 0.37 \\
\hline Cover & 0.48 & -0.22 & 0.52 & 0.07 & 0.67 & -0.06 \\
\hline Eigen value & 1.84 & 1.53 & 1.07 & 0.94 & 0.46 & 0.15 \\
\hline Percent of variance & 30.71 & 25.52 & 17.89 & 15.60 & 7.72 & 2.56 \\
\hline Cumulative percent & 30.71 & 56.23 & 74.12 & 89.72 & 97.44 & 100.00 \\
\hline
\end{tabular}

range in order to operate in the highly conductive (mean: 737 micromhos/cm) Bighorn River water. The eight PSRTs used in 1987 were cylindrical in shape with a mean length of $70.9 \mathrm{~mm}$, a mean diameter of $20.3 \mathrm{~mm}$, and an average weight of $9.6 \mathrm{~g}$ in water (Table 2.4.25). The three PSRTs used in 1988 were similar in shape to those used in 1987, but mean diameter was $14.0 \mathrm{~mm}$ and average weight was $6.3 \mathrm{~g}$ in water (Table 2.4.26). Lithium batteries used in the 1987 PSRTs were replaced by silver batteries in the 1988 PSRTs. Mean size of 10 location transmitters used in 1987 was $29 \mathrm{~mm}$ long by $16 \mathrm{~mm}$ diameter, and average weight in water was $2.5 \mathrm{~g}$ (Table 2.4 .27 ).

Prior to implantation, pressure sensitive transmitters were operated for 3 a to prevent false calibration due to electronic drift from battery power drain (Haynes 1978). Calibration tests were conducted in Afterbay Reservoir. where water quality and temperature were the same as in the Bighorn River (Appendix H). Each transmitter was suspended on a metered line at depths of 0 , 


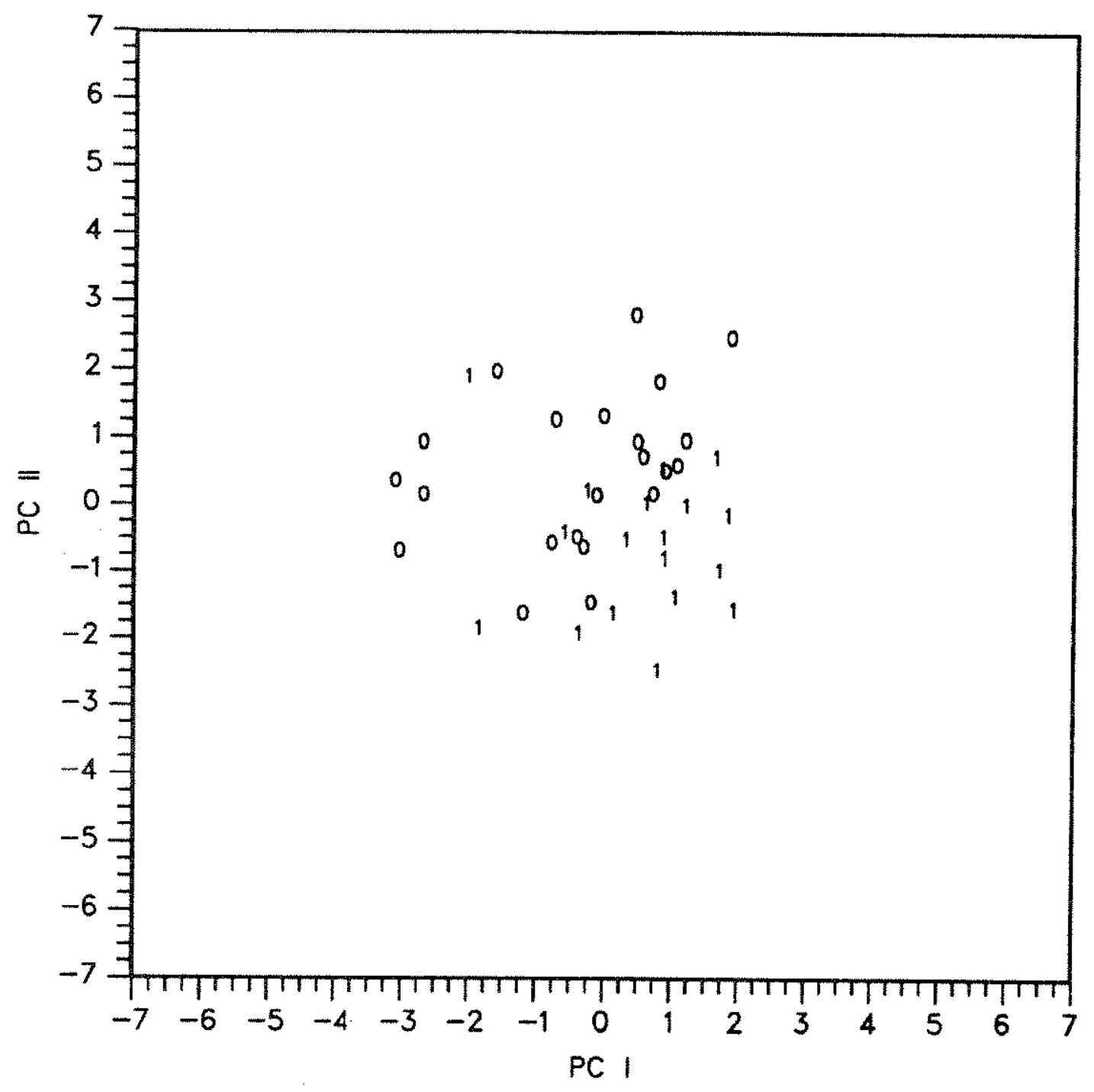

Figure 2.4.16. Principal component I versus principal component II for brown trout and rainbow trout $>15 \mathrm{~cm}$ TL using the variables water depth, fish depth, substrate, vegetation height, velocity, and cover, september - October 1987, Bighorn River, Montana ( $0=$ brown trout, $1=$ rainbow trout). 
Table 2.4.25. Specifications of eight pressure-sensitive radio transmitters implanted in brown trout and rainbow trout, 1987, Bighorn River, Montana.

$\begin{array}{ll}\text { Length (mean) } & 70.9 \mathrm{~mm} \\ \text { Diameter (mean) } & 20.3 \mathrm{~mm} \\ \text { Weight in air } & 33.6-35.0 \mathrm{~g} \\ \text { Weight in water } & 9.3-10.0 \mathrm{~g} \\ \text { Battery type } & 3.5 \mathrm{v} \text { lithium } \\ \text { Sensor } & \begin{array}{l}\text { Cantlever silicon, } \\ \text { with pleated stainless } \\ \text { steel diaphragm }\end{array} \\ \text { Frequency } & 30.071-30.276 \mathrm{MHz} \\ \text { Theoretical life } & 28-35 \mathrm{~d}\end{array}$

Table 2.4.26. Specifications of three pressure-sensitive radio transmitters implanted in brown trout and rainbow trout, 1988, Bighorn River, Montana.

$\begin{array}{ll}\text { Length (mean) } & 72.3 \mathrm{~mm} \\ \text { Diameter (mean) } & 14.0 \mathrm{~mm} \\ \text { Weight in air } & 17.0-17.5 \mathrm{~g} \\ \text { Weight in water } & 6.0-6.5 \mathrm{~g} \\ \text { Battery type } & \text { silver } \\ \text { Sensor } & \text { Cantlever silicon, } \\ & \text { with pleated stainless } \\ & \text { steel diaphragm } \\ \text { Frequency } & 30.255-30.279 \mathrm{MHz} \\ \text { Theoretical life } & 18-22 \mathrm{~d}\end{array}$


Table 2.4.27. Specifications of ten location radio transmitters implanted in brown trout and rainbow trout, 1987, Bighorn River, Montana.
Dimension (mean)
$29 \times 16 \mathrm{~mm}$
Weight in air
$5.4-6.49$
Weight in water
$1.7-3.19$
Battery type
silver
Frequency
$30.043-30.237 \mathrm{MHz}$
Theoretical life
$90 \mathrm{~d}$

1, 2 , and $3 \mathrm{~m}$ and pulse repetitions were measured. Pulse repetition was measured from the leading edge of one pulse to the leading edge of the succeeding pulse as determined from pulse counters. Pulse repetition time decreased with increases in hydrostatic pressure (depth).

Transmitters were surgically implanted in the abdomens of brown trout and rainbow trout ranging in weight from $0.29 \mathrm{~kg}$ to $1.83 \mathrm{~kg}$ and total length from $29.2 \mathrm{~cm}$ to $53.1 \mathrm{~cm}$ (Tables 2.4 .28 and 2.4 .29 ). The general procedures of Hart and summerfelt (1975) were followed. Trout were collected by electrofishing within the upper $4 \mathrm{~km}$ of the river. They were anesthetized (MS222) and were inverted for surgery so that gills were submerged in anesthetic solution. Fish were tagged with a bright orange anchor tag (Floy Tag Company) behind the dorsal fin for identification. Radio-tagged trout were held for less than $20 \mathrm{~h}$ before release at the location of capture in 1987 . In 1988 trout were released as soon as they recovered from anesthesia. A curved cutting needle with 2-0 synthetic absorbable suture (Ethicon J-4534) was used to close incisions. Two to three 


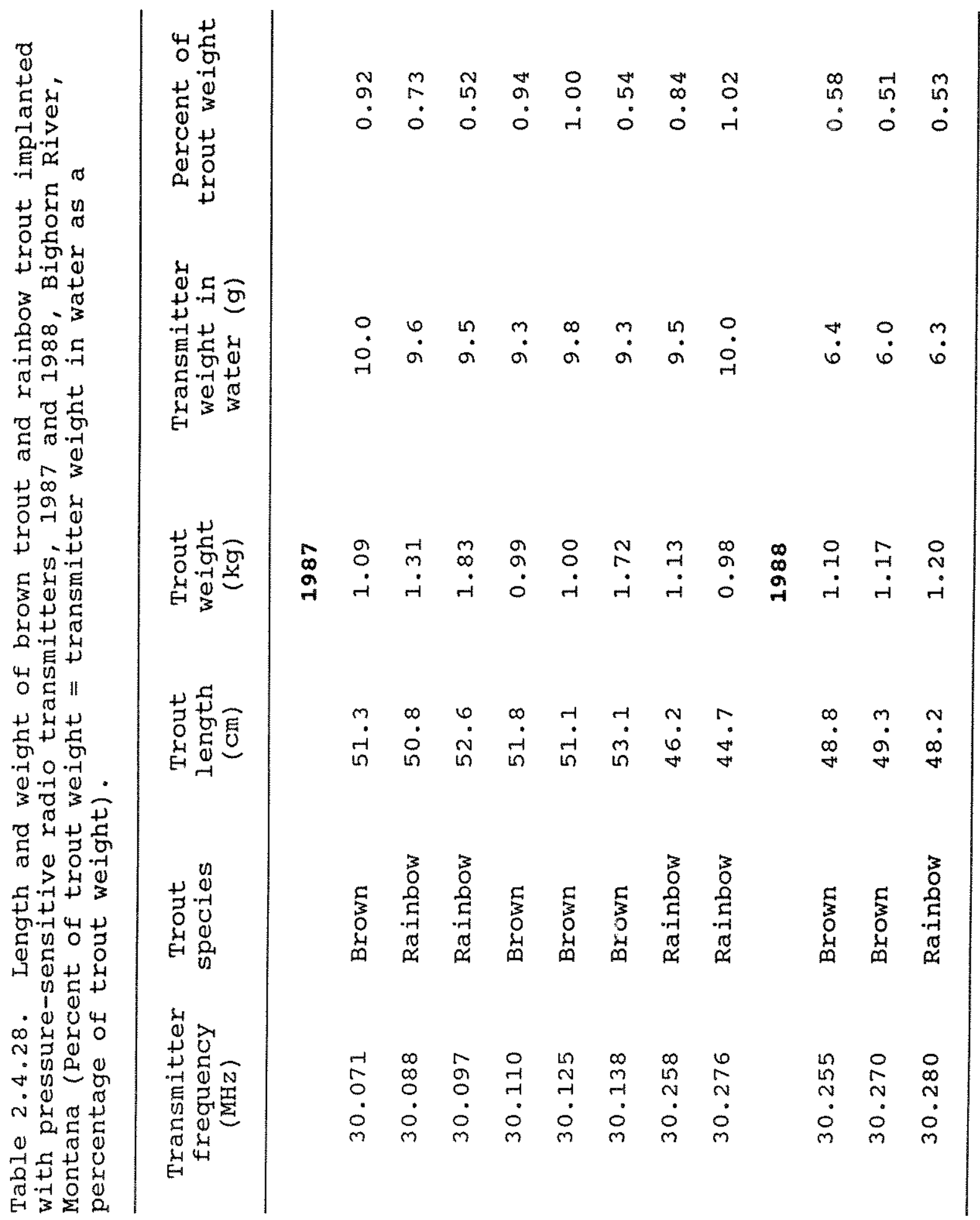




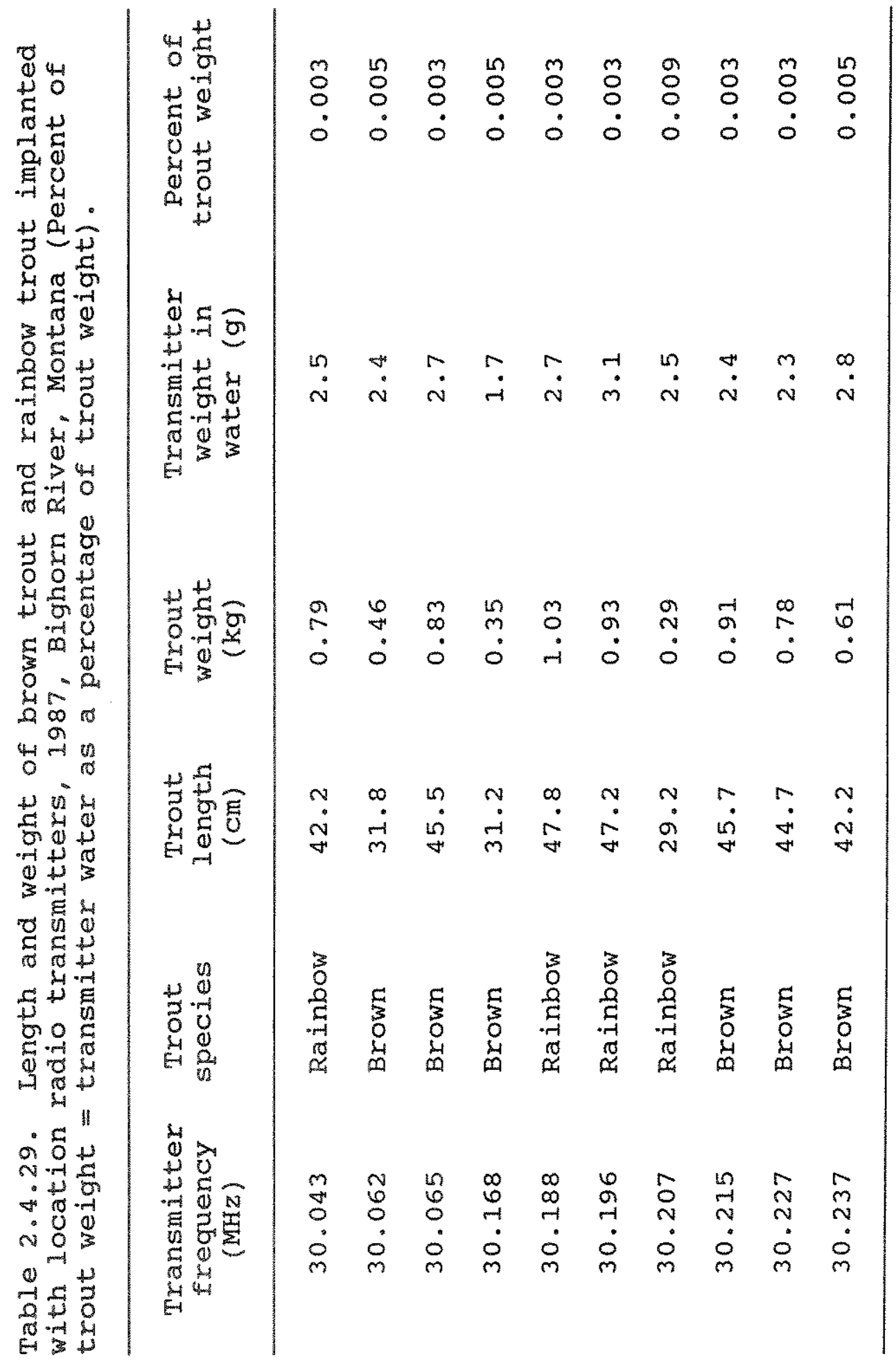


subcutaneous stitches and four to six exterior stitches were used. Erythromycin (Erythro-200, $25 \mathrm{mg} / 1 \mathrm{~kg}$ ) was injected into the dorsal sinus and tetracycline (Polyotic) was applied to the incision to reduce possible infection. Transmitter weight in water did not exceed $1.25 \%$ of trout weight out of water as recommended by winter et al. (1978).

After release, radio tagged trout were located and monitored using a $124 \mathrm{~cm}^{2}$ "loop" antenna and one of two different receivers. A programmable scanning-receiver and pulse counter (Model 2000 Challenger Programmable Scanner and Pulse Decoder) manufactured by Advanced Telemetry systems, Incorporated (ATS), Isanti, Minnesota, and a second receiver and pulse counter, manufactured by CTC (Model CTC-AR-12 and Pulse Counter), were used to locate radio-tagged trout and record pulse repetition. Transmitter range was approximately $300 \mathrm{~m}$ depending on transmitter depth and orientation to the antenna. During 1987 an attempt was made to locate each fish daily, and $24 \mathrm{~h}$ monitoring of PSRTs was done on two occasions on two different transmitters (radio-tagged trout) to assess diurnal trout movements. Fish were also located at night to evaluate night time habitat use. Monitoring of radio-tagged trout in 1988 was done $24 \mathrm{~h} /$ day. Fish locations were determined by triangulation and by signal strength. Fish locations were plotted on maps that were overlaid with a grid so that XY coordinates could be determined for each fish location. A computer program designed by Dan Gustafson (Montana State University) was used to analyze the data and produce location graphics.

Four of the eight PSRTs implanted into trout in 1987 were 
recovered after 21 d and were recalibrated to determine if electronic drift had occurred. All three trout radio tagged in 1988 were recovered after $10 \mathrm{~d}$. The approach used for preimplant calibration was repeated.

Flow pattem through Afterbay Dam was modified to manipulate gas levels and examine behavior of trout when exposed to various levels of supersaturated water (Table 2.4.30). Radio-tagged trout were exposed to three different gas regimes in 1987 (Figure 2.4.17). From 9 August (release date) until 16 August, trout were exposed to moderate delta $\mathrm{P}$ levels with low variation (77 mmig - $103 \mathrm{mmHg}$ ). High delta $\mathrm{p}$ levels with low variation (123 mmHg - 152 mmig) were present from 16 August to 23 August. After 23 August, radio-tagged trout were exposed to moderate delta P levels with high variation (57 mmig - $125 \mathrm{mmHg})$. Total gas pressure and nitrogen pressure followed the pattern of delta $P$ levels (Appendix I: Figures 4-7). Oxygen pressure ranged from $133 \mathrm{mmHg}$ to $158 \mathrm{mmHg}$. Three levels of discharge ranging from $52.5 \mathrm{~m}^{3} / \mathrm{s}$ to $66.0 \mathrm{~m}^{3} / \mathrm{s}$ were encountered over the period (Figure $2.4 .18)$

Five periods of dissolved gas levels were created by changes in Afterbay Dam sluice gate/radial gate configuration during the 1988 radio telemetry study (Figure 2.4.19). From 19 to 20 July and from 27 to 29 July trout were exposed to moderate delta $P$ levels with high variation $(63 \mathrm{mmHg}-104 \mathrm{mmHg})$. High delta $P$ levels with low variation were present for two periods, 20 to 22 July and 25 to $27 \mathrm{July}(104 \mathrm{mmHg}-123 \mathrm{mmHg})$. From 22 to $25 \mathrm{July}$ moderate delta $\mathrm{P}$ levels with low variation were created (72 $\mathrm{mmH}$ - $90 \mathrm{~mm} g)$. Discharge was relatively constant during the 10-d 


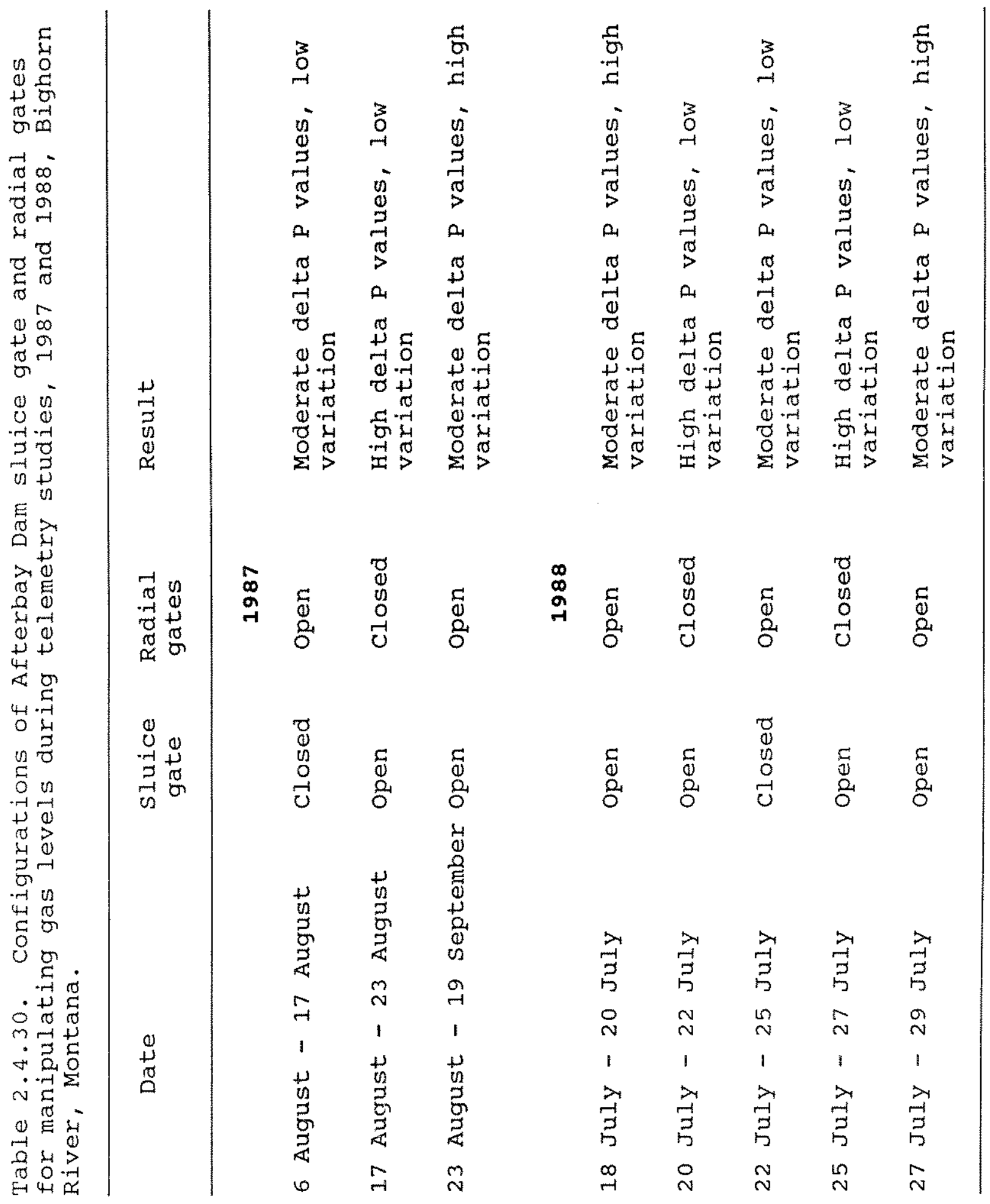




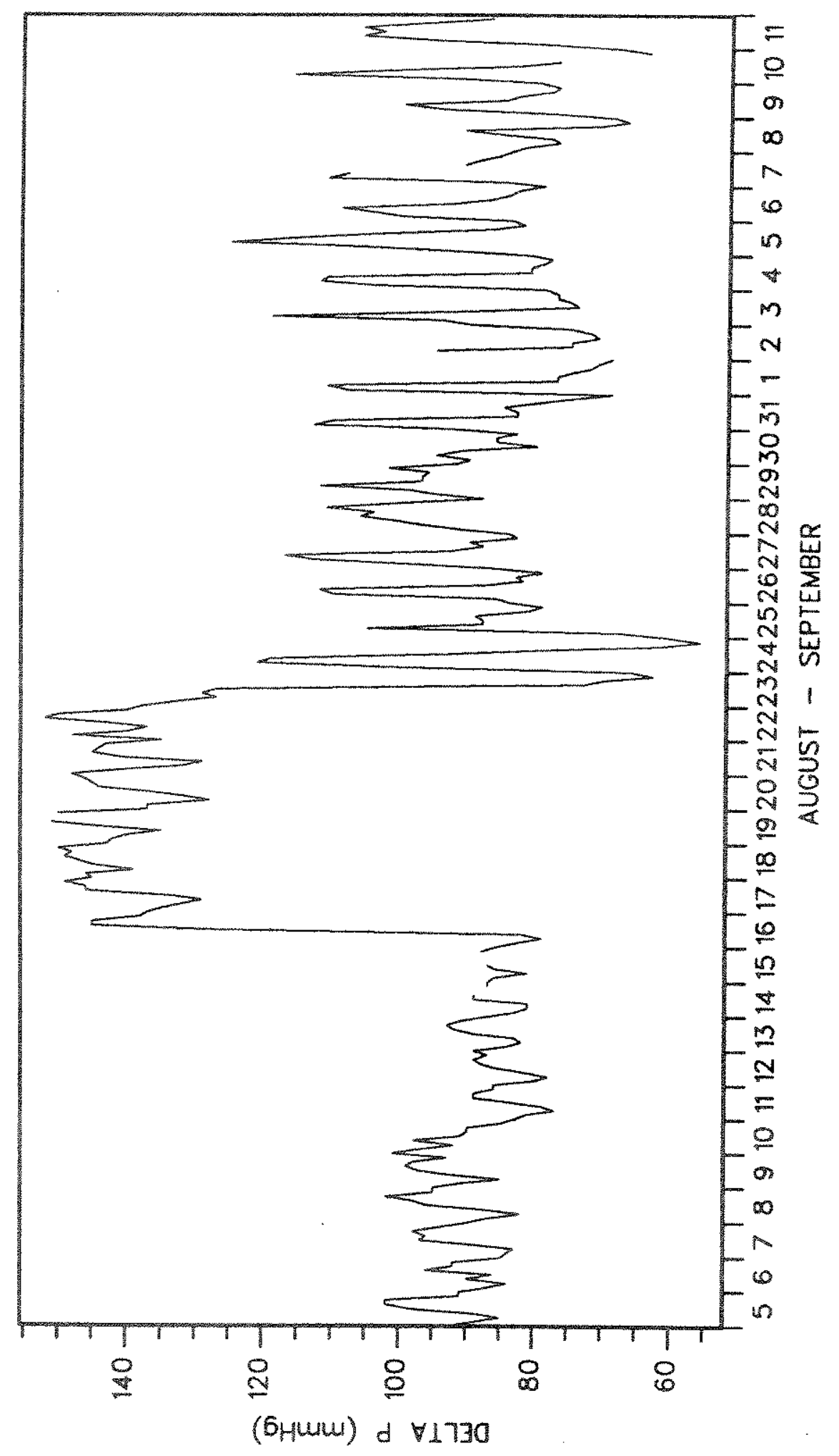

0

至

㐘

t.

40

का

04

计

대요

-1

$+y 0$

os

a

+ $\frac{1}{0}$

$\rightarrow 0$

영

5 .

जै

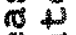

00

$\exists 0$

0

का

w

色

E.

oㄹ

n

4

叫

$\rightarrow$ on

$a_{4}+{ }_{-1}$

ช

is

$\rightarrow 0$

का क

क्षै

엉

$\therefore$

fy

-

小

ง

(c) 8

4.

3

-

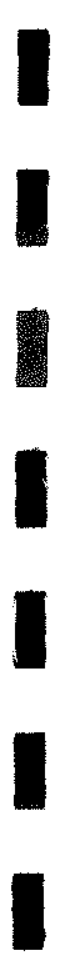

s n
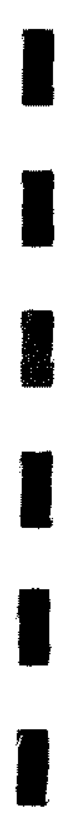

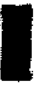

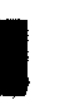




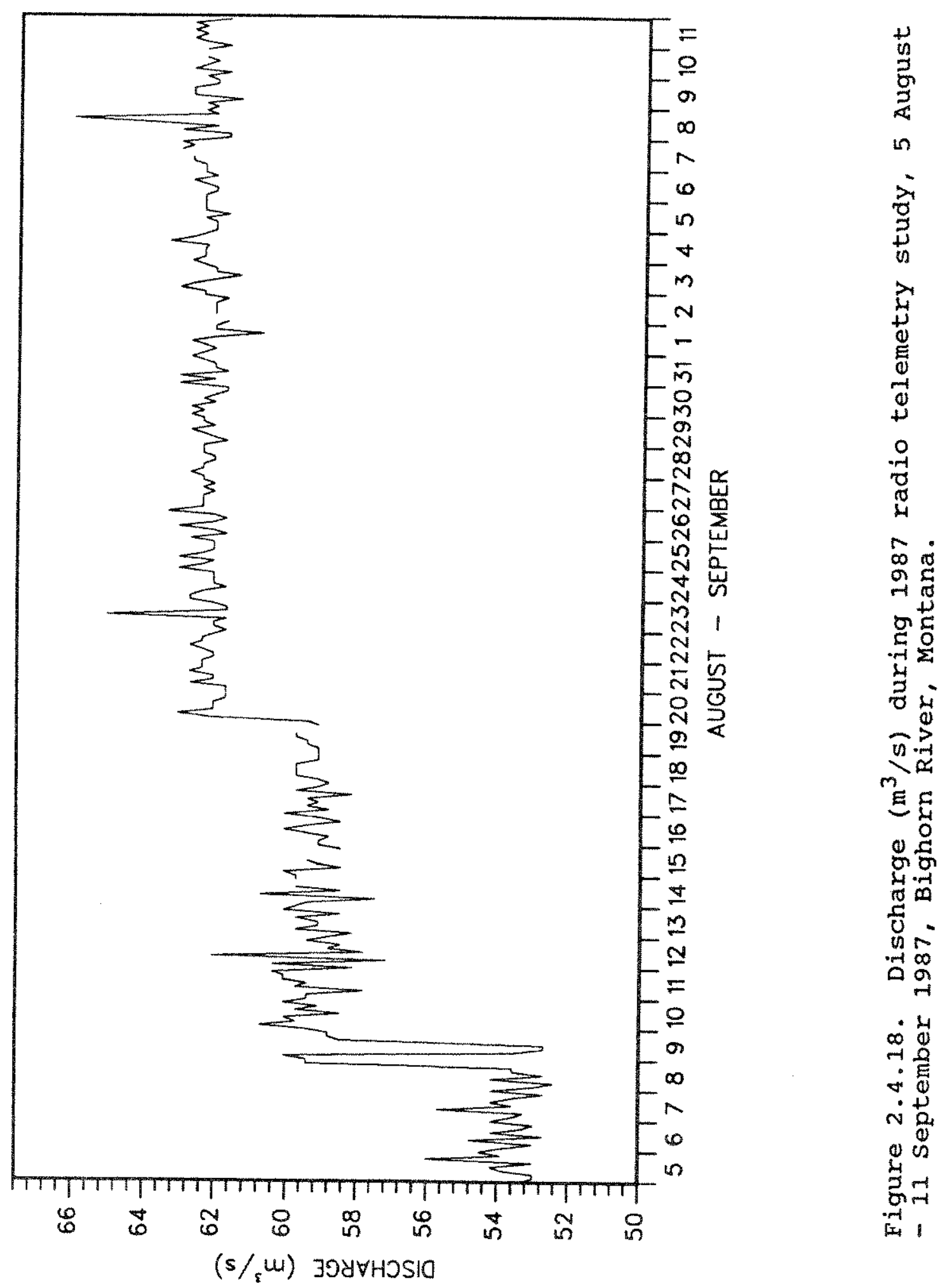



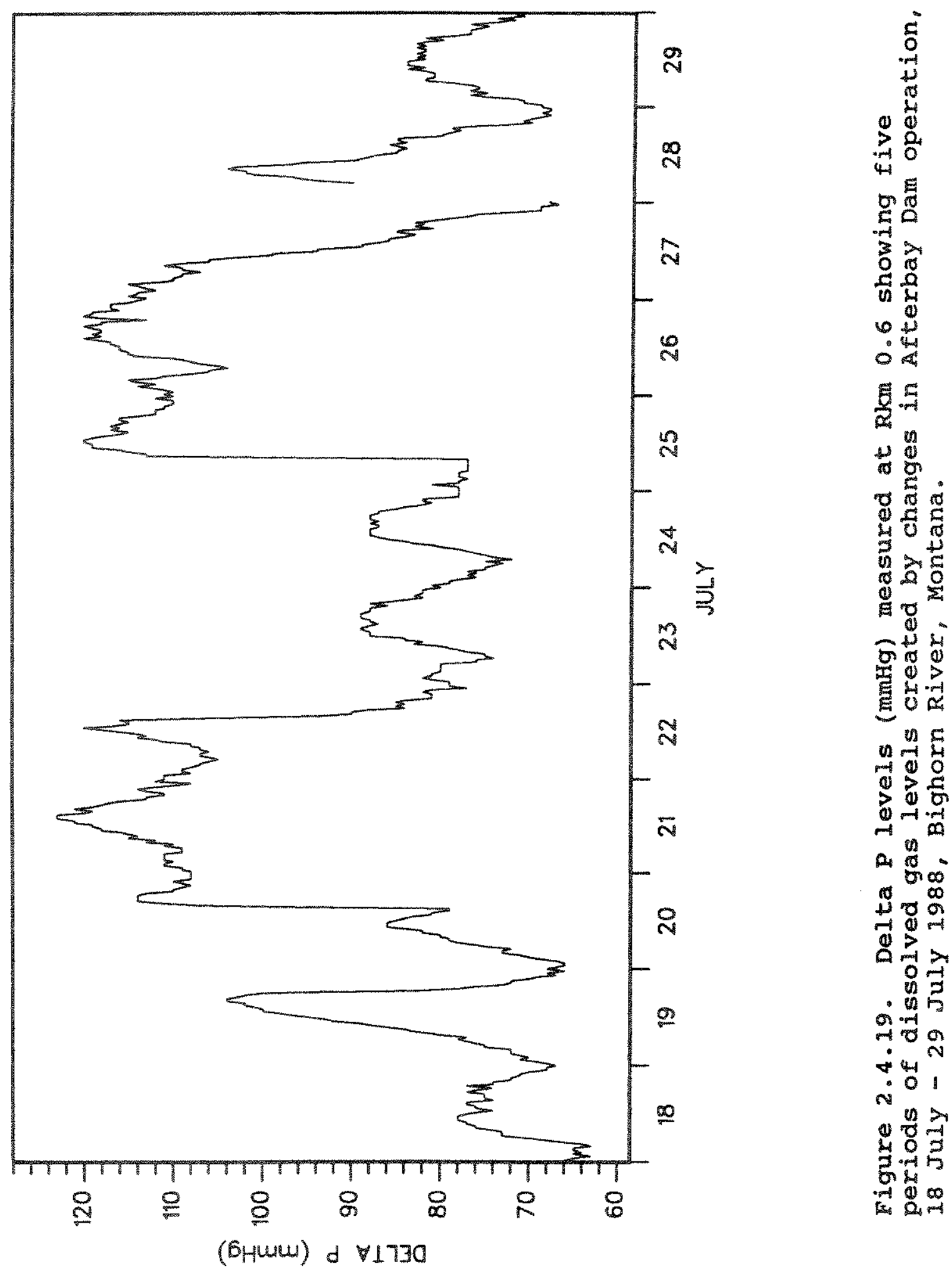
period (Figure 2.4.20). A large increase in discharge $\left(54 \mathrm{~m}^{3} / \mathrm{s}-\right.$ $74 \mathrm{~m}^{3} / \mathrm{s}$ ) occurred for $3 \mathrm{~h}$ on 22 July due to problems with Afterbay Dam operation.

A linear regression was used to determine the relationship between pulse repetition and depth for each transmitter. To determine depth resolution, unlimited simultaneous discrimination intervals were used (Lieberman et al. 1967). This method accounts for both the uncertainty in the observed pulse rate and the uncertainty in the relationship between pulse repetition and depth. Methods which do not take both uncertainties into account are not appropriate for measurements from precalibrated instruments. Transmitter calibration was done by regressing pulse rate on known depths. Field pulse rates were then converted to confidence intervals for depth using this regression equation, along with estimates of both types of uncertainty (Appendix H). A computer program designed by Milo Adkison and Dan Gustafson (Montana State University) performed the regression analysis.

\section{$2.4 .2 .3 b$ Results}

Pressure-sensitive radio transmitters in two brown trout and one rainbow trout were monitored from 19 July to 28 July 1988 (Tables 2.4.31, 2.4.32, and 2.4.33). The rainbow trout (Fish 280) occupied a deeper position following surgery and release than at other times during the observation period (Figure 2.4.21). After the initial location, this fish maintained a relatively constant depth. None of the fish appeared to change depth in response to changes in dissolved gas levels (Figures 2.4 .22 and 2.4 .23$)$. Depths occupied by the two brown trout 

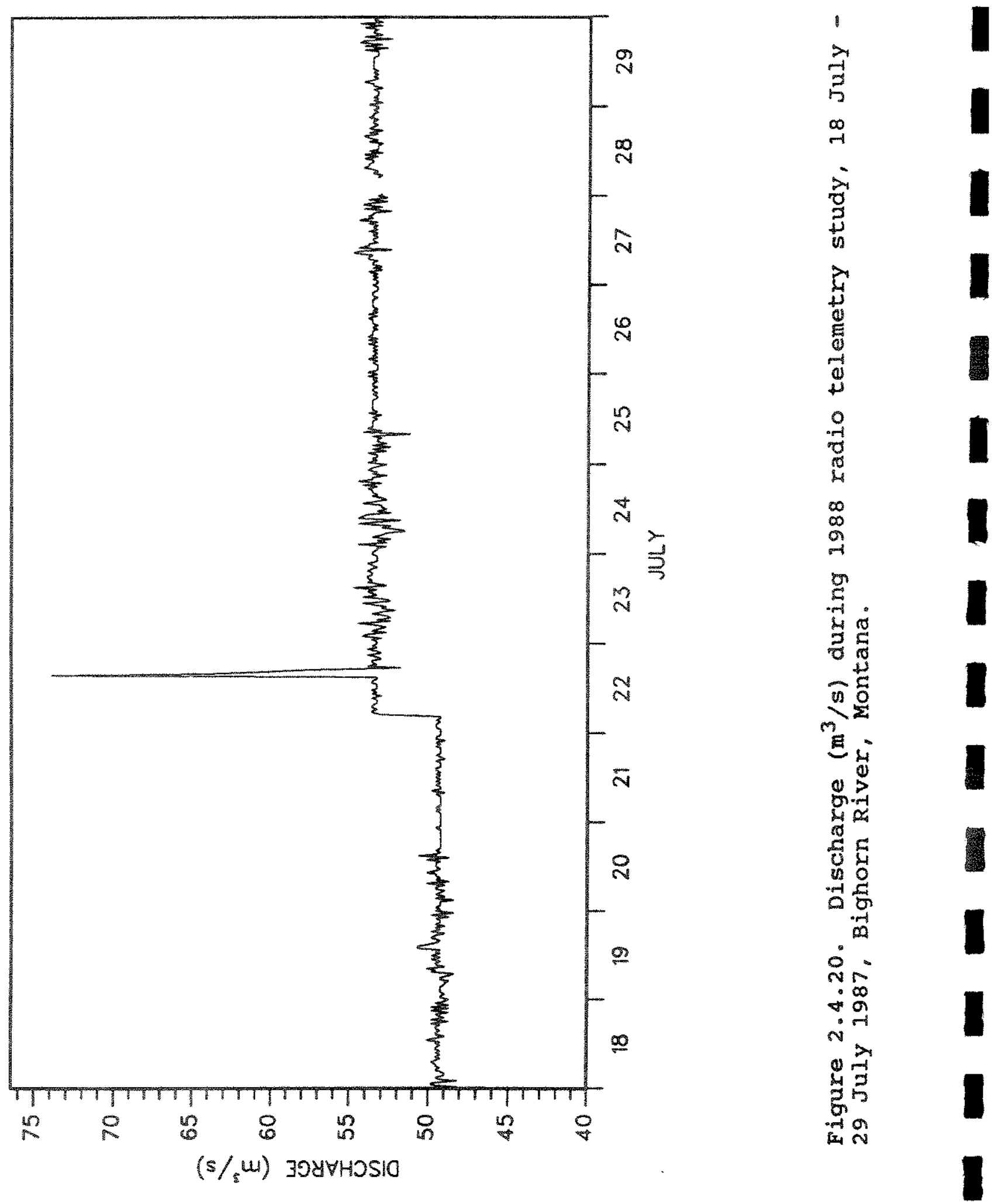
Table 2.4.31. Number of locations per day and daily range of fish 255 (brown trout) implanted with pressure-sensitive radio transmitter, 19 July - 28 July 1988, Bighorn River, Montana.

\begin{tabular}{ccc} 
July & Number & $\begin{array}{c}\text { Daily range } \\
(\mathrm{m})\end{array}$ \\
& & 0.00 \\
19 & 3 & 10.06 \\
20 & 14 & 17.83 \\
21 & 15 & 17.83 \\
22 & 19 & 29.34 \\
23 & 22 & 19.96 \\
24 & 19 & 21.70 \\
25 & 17 & 26.26 \\
26 & 12 & 67.34 \\
27 & 7 & - \\
28 & 1 & Mean \\
& 129 & 23.38 \\
\hline
\end{tabular}

Table 2.4.32. Number of locations per day and daily range of fish 270 (brown trout) implanted with pressure-sensitive radio transmitter, 19 July - 28 July 1988, Bighorn River, Montana.

\begin{tabular}{|c|c|c|}
\hline July & Number & $\begin{array}{c}\text { Daily range } \\
(\mathrm{m})\end{array}$ \\
\hline 19 & 2 & 0.00 \\
\hline 20 & 14 & 0.00 \\
\hline 21 & 12 & 59.86 \\
\hline 22 & 17 & 27.02 \\
\hline 23 & 19 & 28.57 \\
\hline 24 & 15 & 32.57 \\
\hline 25 & 23 & 51.38 \\
\hline 26 & 19 & 65.94 \\
\hline 27 & 19 & 45.94 \\
\hline \multirow[t]{2}{*}{28} & 1 & - \\
\hline & Total & 34.59 \\
\hline
\end{tabular}


Table 2.4.33. Number of locations per day and daily range of fish 280 (rainbow trout) implanted with pressure-sensitive radio transmitter, 19 July - 28 July 1988, Bighorn River, Montana.

\begin{tabular}{|c|c|c|}
\hline JuIy & Number & $\begin{array}{c}\text { Daily range } \\
(\mathrm{m})\end{array}$ \\
\hline 19 & 3 & 0.00 \\
\hline 20 & 21 & 8.46 \\
\hline 21 & 10 & 0.00 \\
\hline 22 & 19 & 2.64 \\
\hline 23 & 10 & 0.00 \\
\hline 24 & 17 & 0.00 \\
\hline 25 & 10 & 0.00 \\
\hline 26 & 8 & 4.18 \\
\hline 27 & 5 & 0.00 \\
\hline \multirow[t]{2}{*}{28} & 1 & 0.00 \\
\hline & Total & Mean \\
\hline
\end{tabular}

(Fish 255 and Fish 270) varied considerably throughout the 10-d monitoring period.

Light levels (Figure 2.4.24) or time of day (Figure 2.4.25) had no apparent influence on fish depth, although one brown trout (fish 270) was often located at shallow depths during periods when field notes indicated high levels of trout surface feeding activity and insect emergence $(0600-1100$ and $1400-1600)$. The other brown trout (Fish 255) also was frequently located in shallow water during the early morning when high levels of feeding activity were noted (0500 - 0900). The large increase in discharge on 22 July did not appear to influence fish depth (Figure 2.4.21).

Fourteen of 18 brown trout and rainbow trout implanted with radio tags in 1987 were subsequently located for up to $35 \mathrm{~d}$ (Table 2.4.34). Four of the radio-tagged trout were not located after release. Most movement of the 14 radio-tagged trout 


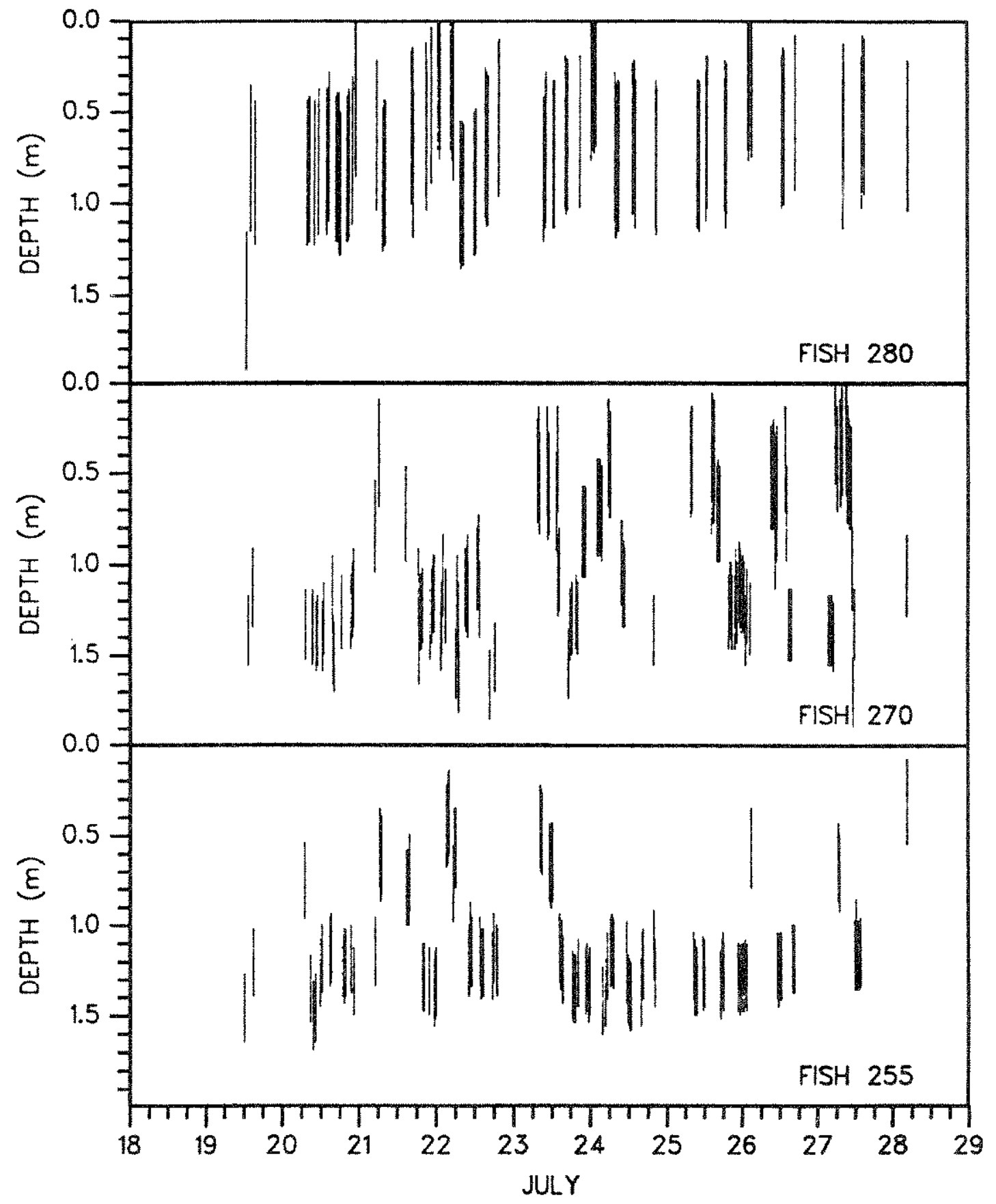

Figure 2.4.21. Fish depth determined from pressure-sensitive radio transmitters, 19 July - 28 July 1988, Bighorn River, Montana (bars represent confidence interval for depth from measured pulse repetition). Fish 280 , rainbow trout: fish 270 and 255 , brown trout. 


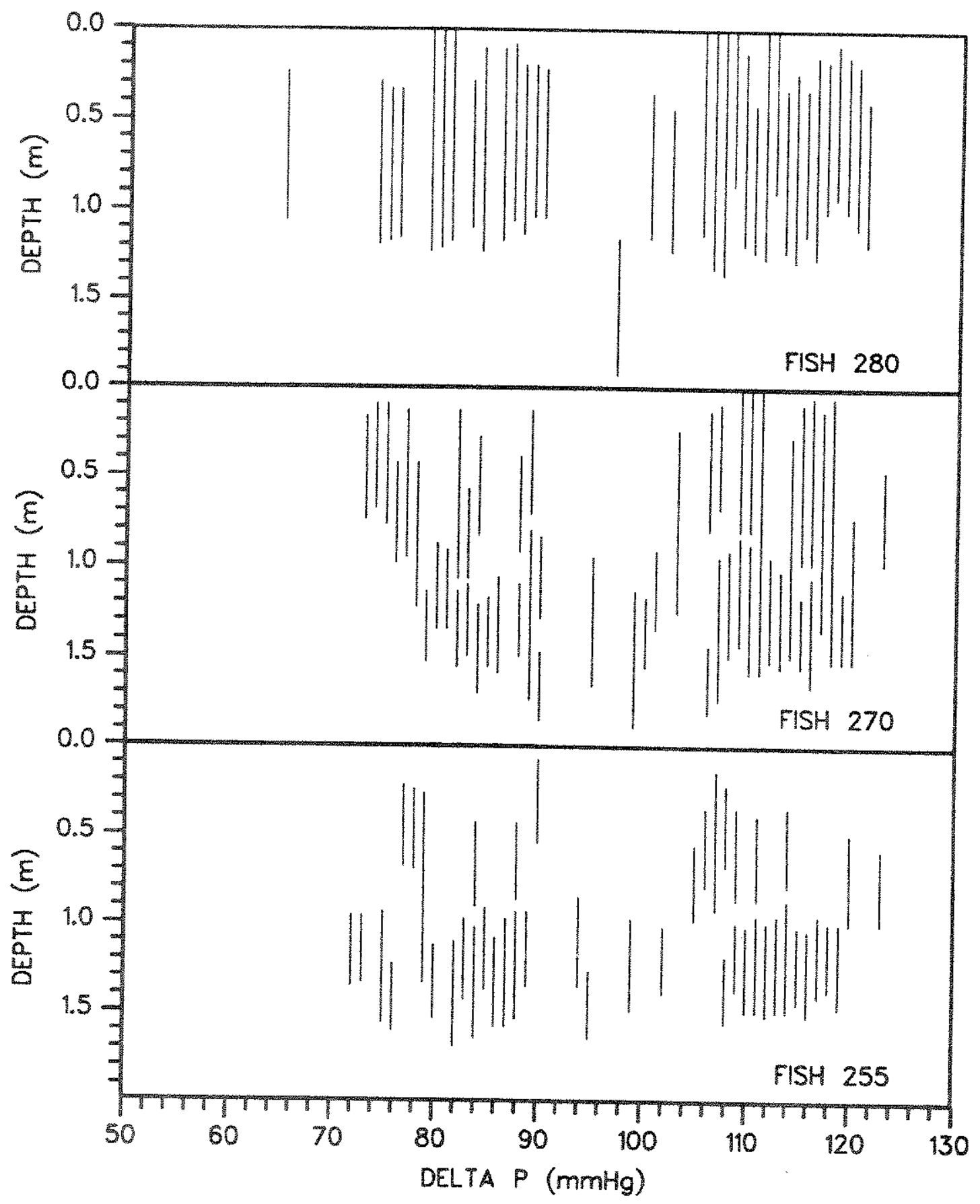

Figure 2.4.22. Wish depth determined from pressure-sensitive radio transmitters at delta $p$ levels encountered during monitoring geriod, 19 July - 28 July 1988, Bighorn River, Montana (baxs represent confidence interval for depth from measured pulse repetition). Fish 280 , rainbow trout: fish 270 and 255 , brown trout. 


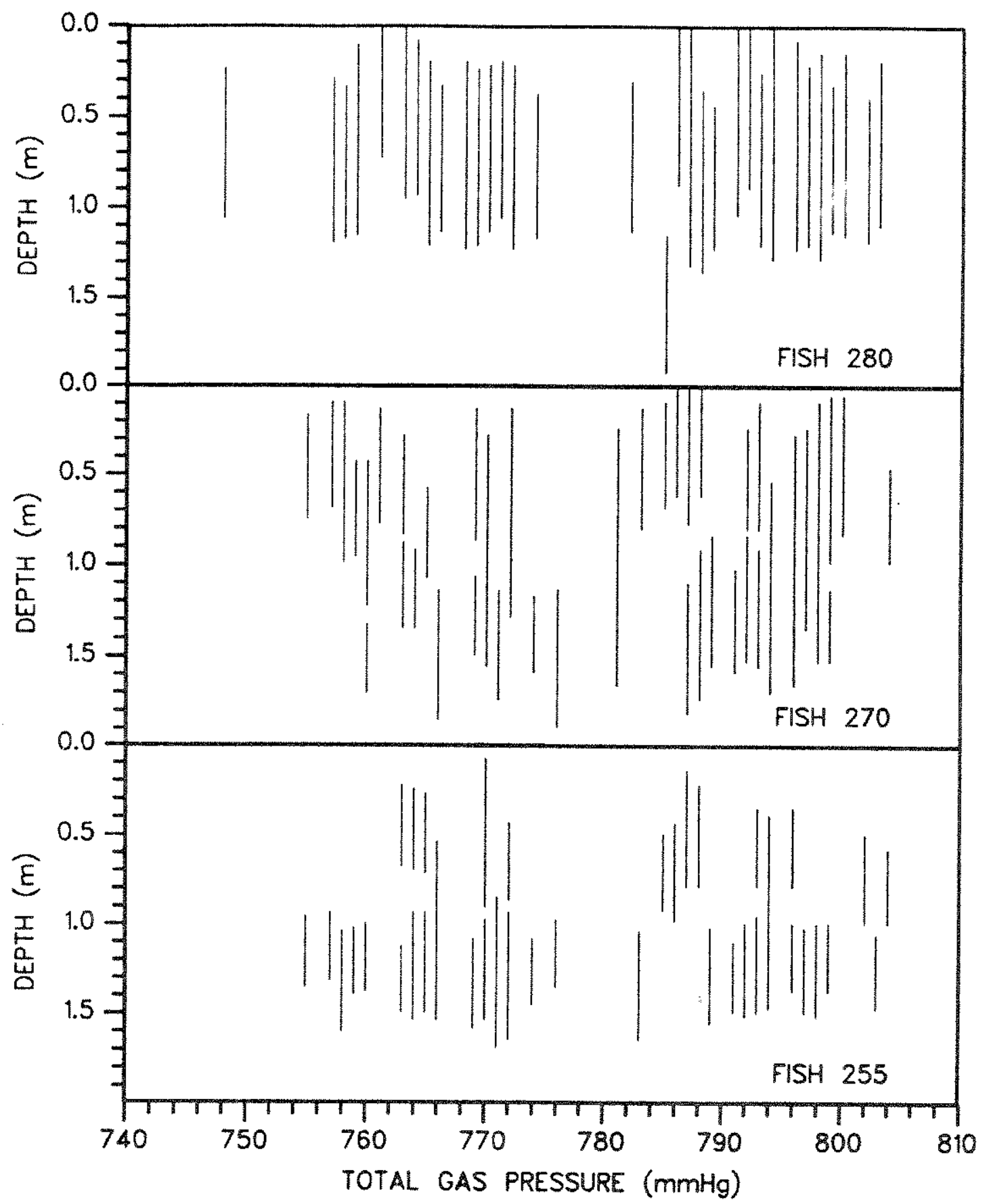

Figure 2.4.23. Fish depth determined from pressure-sensitive radio transmitters at total gas pressures encountered during monitoring period, 19 July - 28 July 1988, Bighorn River, Montana (bars represent confidence interval for depth from measured pulse repetition). Fish 280, rainbow trout: fish 270 and 255 , brown trout. 


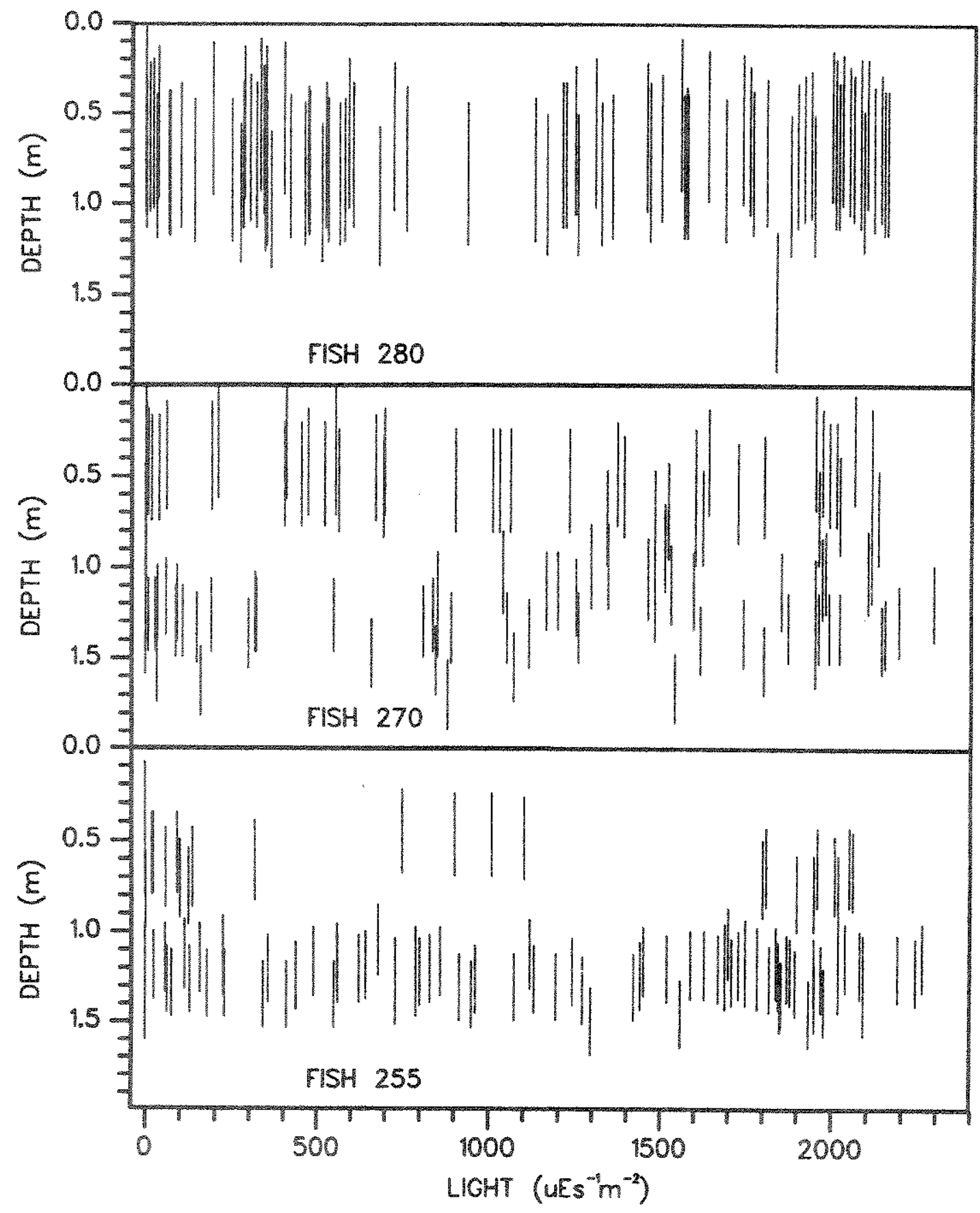

Figure 2.4.24. Fish depth detemined from pressure-sensitive radio transmitters at light levels recorded at time of observation, 19 July -28 July 1988 , Bighorn River, Montana (bars represent confidence interval for depth from measured pulse repetition). Fish 280, rainbow trout; fish 270 and 255 , brown trout. 


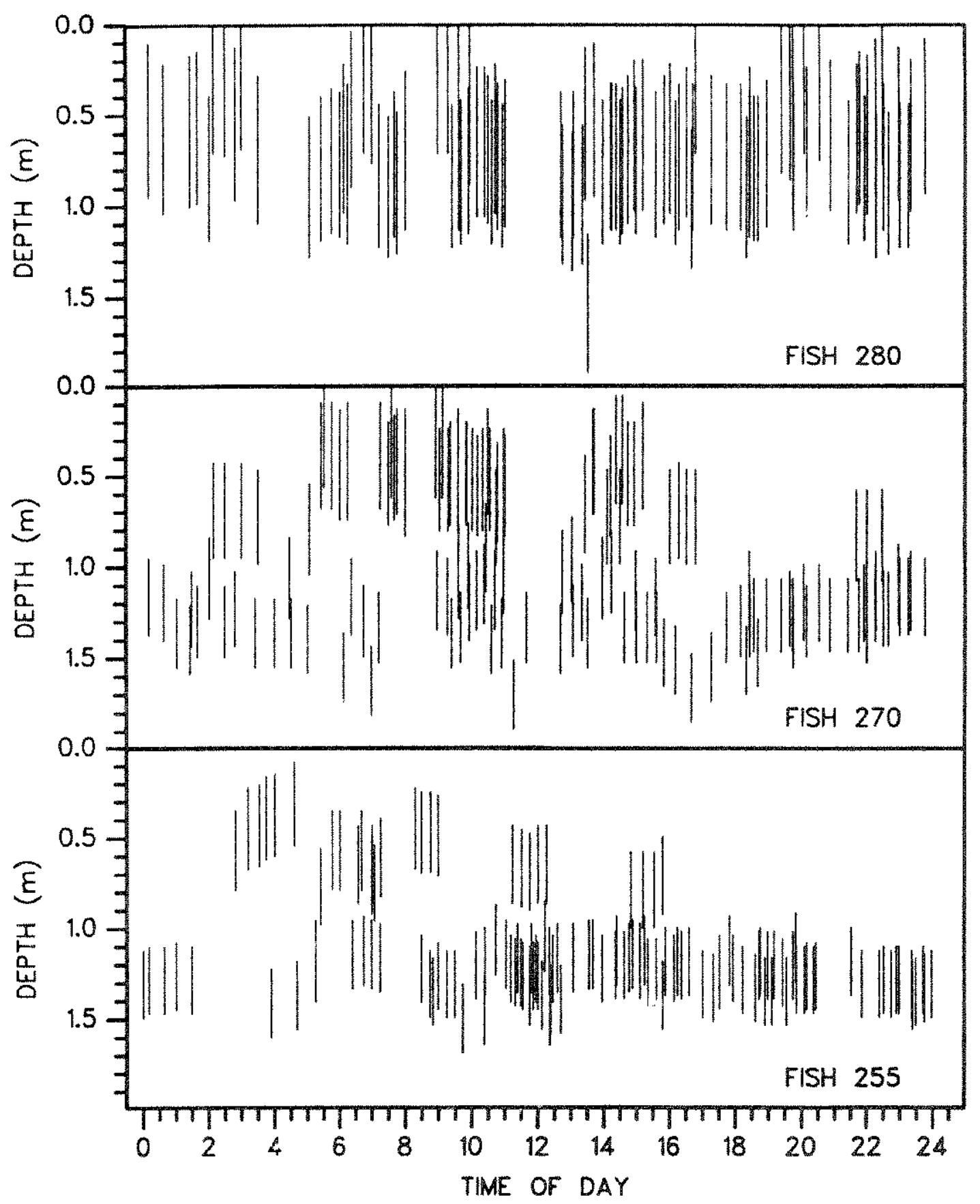

Figure 2.4.25. Fish depth determined from pressure-sensitive radio transmitters at various times of day, data combined from 19 July - 28 July 1988, Bighorn River, Montana (bars represent confidence interval for depth from measured pulse repetition). Fish 280, rainbow trout; fish 270 and 255 , brown trout. 
Table 2.4.34. Number of locations and duration of monitoring (days) of trout implanted with radio transmitters, 8 August - 11 September 1987, Bighorn River, Montana.

\begin{tabular}{|c|c|c|c|}
\hline $\begin{array}{l}\text { Fish } \\
\text { number }\end{array}$ & Species & $\begin{array}{l}\text { Number of } \\
\text { locations }\end{array}$ & $\begin{array}{l}\text { Duration of } \\
\text { monitoring (days) }\end{array}$ \\
\hline 43 & Rainbow & 31 & 35 \\
\hline 62 & Brotn & 31 & 35 \\
\hline 65 & Brown & 36 & 35 \\
\hline 71 & Brown & 21 & 20 \\
\hline 88 & Rainbow & 21 & 20 \\
\hline 97 & Rainbow & 23 & 20 \\
\hline 110 & Brown & 12 & 11 \\
\hline 125 & Brown & 21 & 20 \\
\hline $138^{a}$ & Brown & & \\
\hline 168 & Brown & 35 & 35 \\
\hline 188 & Rainbow & 34 & 35 \\
\hline $196^{a}$ & Rainbow & & \\
\hline $207^{a}$ & Rainbow & & \\
\hline 215 & Brown & 21 & 35 \\
\hline 227 & Brom & 27 & 35 \\
\hline 237 & Brown & 34 & 35 \\
\hline $258^{a}$ & Rainbow & & \\
\hline 276 & Rainbow & 16 & 20 \\
\hline
\end{tabular}

a Trout not located after release.

occurred during the first 5 d following release (Figure 2.4.26). During the period, little upstream/downstream movement occurred. Average daily distance traveled for all radio-tagged trout was $-0.82 \mathrm{~m}$ (Appendix J: Figures 12-25). Changes in dissolved gas levels did not have a detectable influence on daily distance traveled.

Location data from 1988 radio-tagged trout indicated that two trout (Fish 270 brown and 280 rainbow) increased their daily range during periods of high dissolved gas levels (104 mmH - 123 mHig delta p) on 20 July to 22 July and 25 July to 27 July (Figures 2.4 .27 and 2.4 .28 ). Mean daily range for the $10-\mathrm{d}$ period was largest for the two brown trout (Tables 2.4 .31 , 


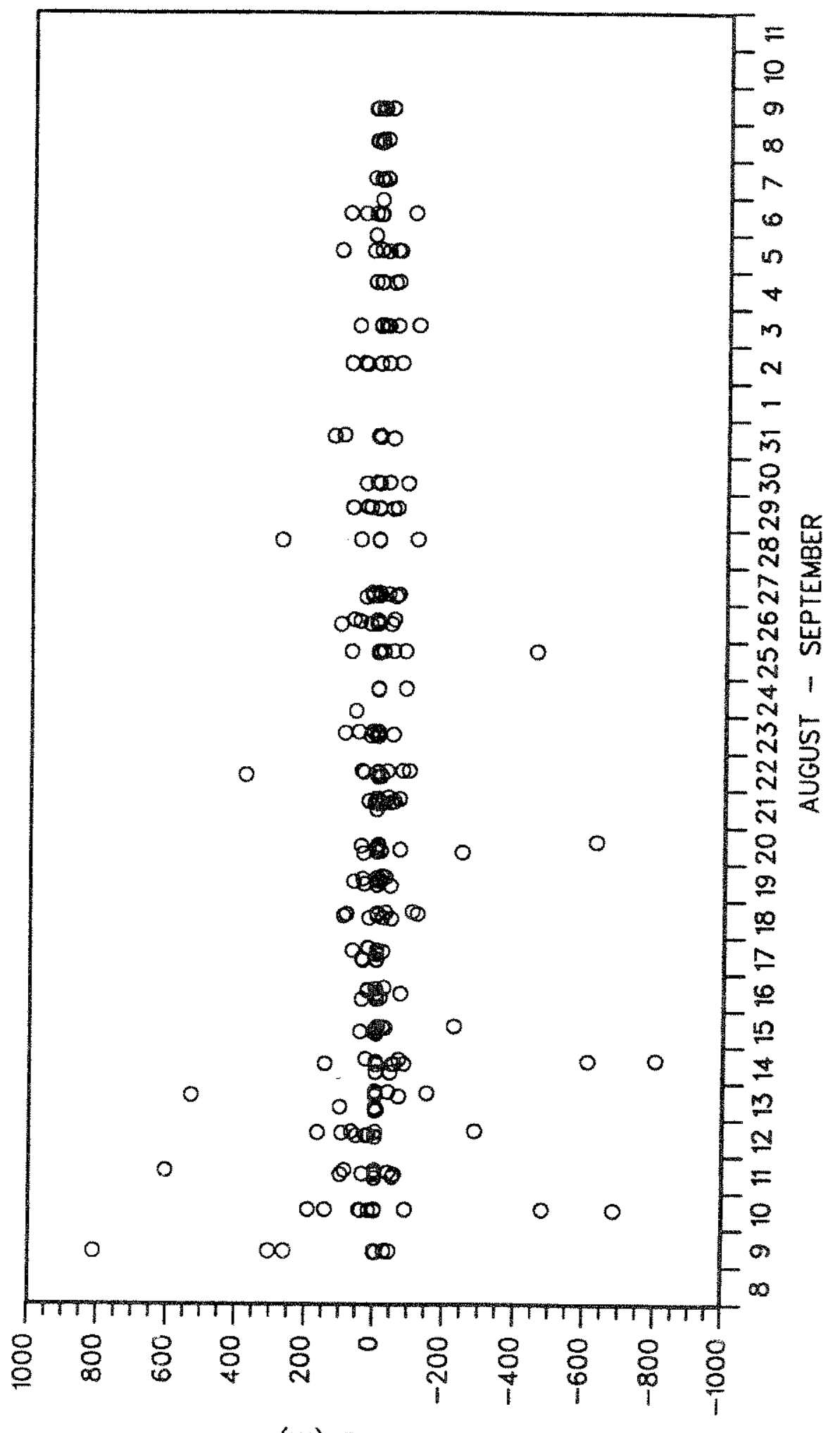

1 造 $+40$ $y+y$ 당 4 व $\infty \cdot-1$ $>0$

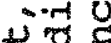
उ O

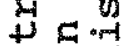
\% (8) o.ris क्षे फ 5 1 . 용 ठ당 क्ष 200 20 4 $>\pi 0$ ชั $+$ 노요 Q o 0 영 $0>$ a 的 (4) $+55$ o) 0 둥 क क्ष कि 约的 Nㅜㅇㅇㅇ $\infty 04$ or 5 $\therefore$ $\circlearrowleft$ t

त. * ब ซ

N@

(1) O. 0

\& 4 \&

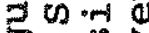
का की

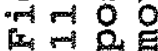

(w) $30 N \forall I S I O$ 


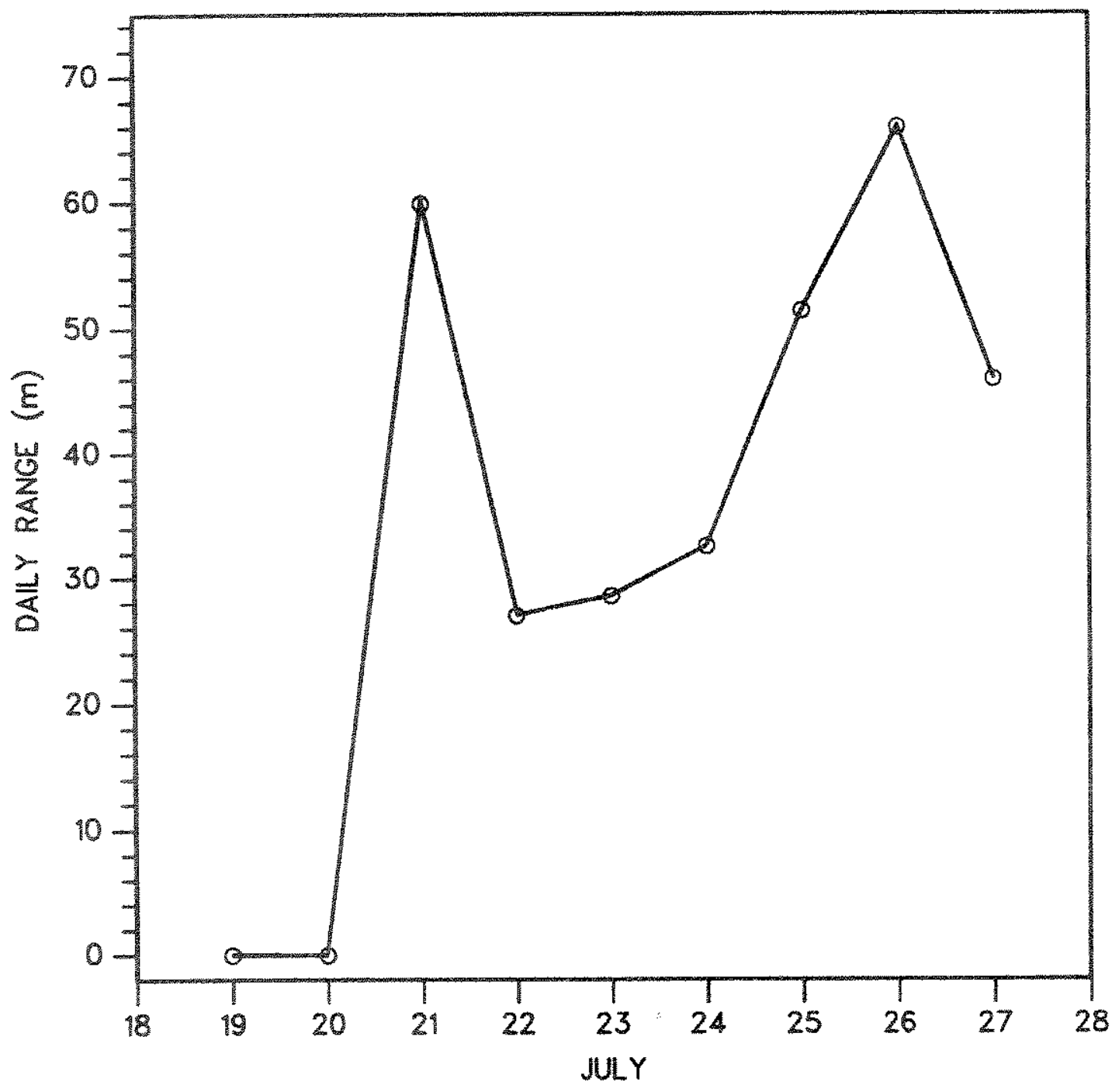

Figure 2.4.27. Daily range (m) of fish 270 (brown trout). 19 July - 28 July 1988, Bighorn River, Montana. 


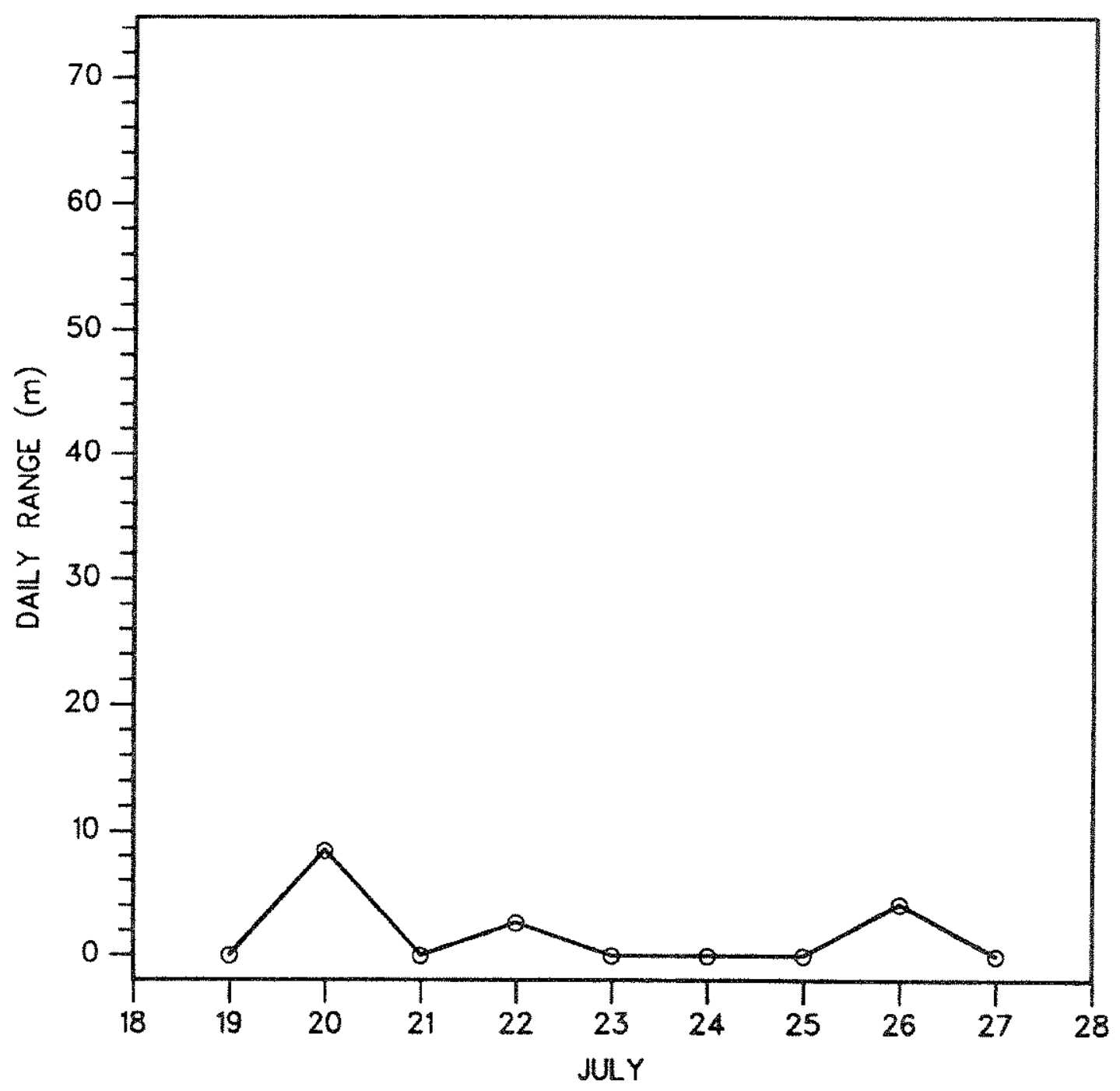

Figure 2.4.28. Daily range (m) of fish 280 (rainbow trout), 19 July - 28 July 1988, Bighorn River, Montana. 
$2.4 .32,2.4 .33$; Appendix J: Figures 26-28). One brown trout increased its daily range throughout the 10-d period; largest movements were on days with moderate gas levels (Figure 2.4.29).

\subsubsection{Discussion}

Differences in habitat use between brown trout and rainbow trout did not account for the higher incidence of GBT observed in the adult brown trout population and the large difference in relative abundance between the two species. Habitat use varied seasonally as well as between size groups with few interspecific differences observed. Brown trout larger than $15 \mathrm{~cm}$ maintained positions relatively close to the substrate whereas rainbow trout larger than $15 \mathrm{~cm}$ were found at shallower average depth (fish depth). Rainbow trout, however, used a wider range of depths within the water column. Hearn and Kynard (1986) reported similar use of pool habitat by rainbow trout determined from electrofishing habitat use surveys in tributaries of the white River, vermont. In stream channel experiments they found that rainbow trout cended to occupy positions off the substrate. The maximum difference in mean fish depth between brown trout and rainbow trout for all time periods studied in the Bighorn River was 27 cm in september - october, with brown trout occupying deeper water. For depth compensation to account for lower GBT incidence in rainbow trout, the opposite pattern of habitat use would have been expected.

Although fish depth aid not differ greatly between species, rainbow trout were usually associated with slightly deeper water areas than were brown trout $(1.16 \mathrm{~m}$ versus $1.07 \mathrm{~m})$. If rainbow 


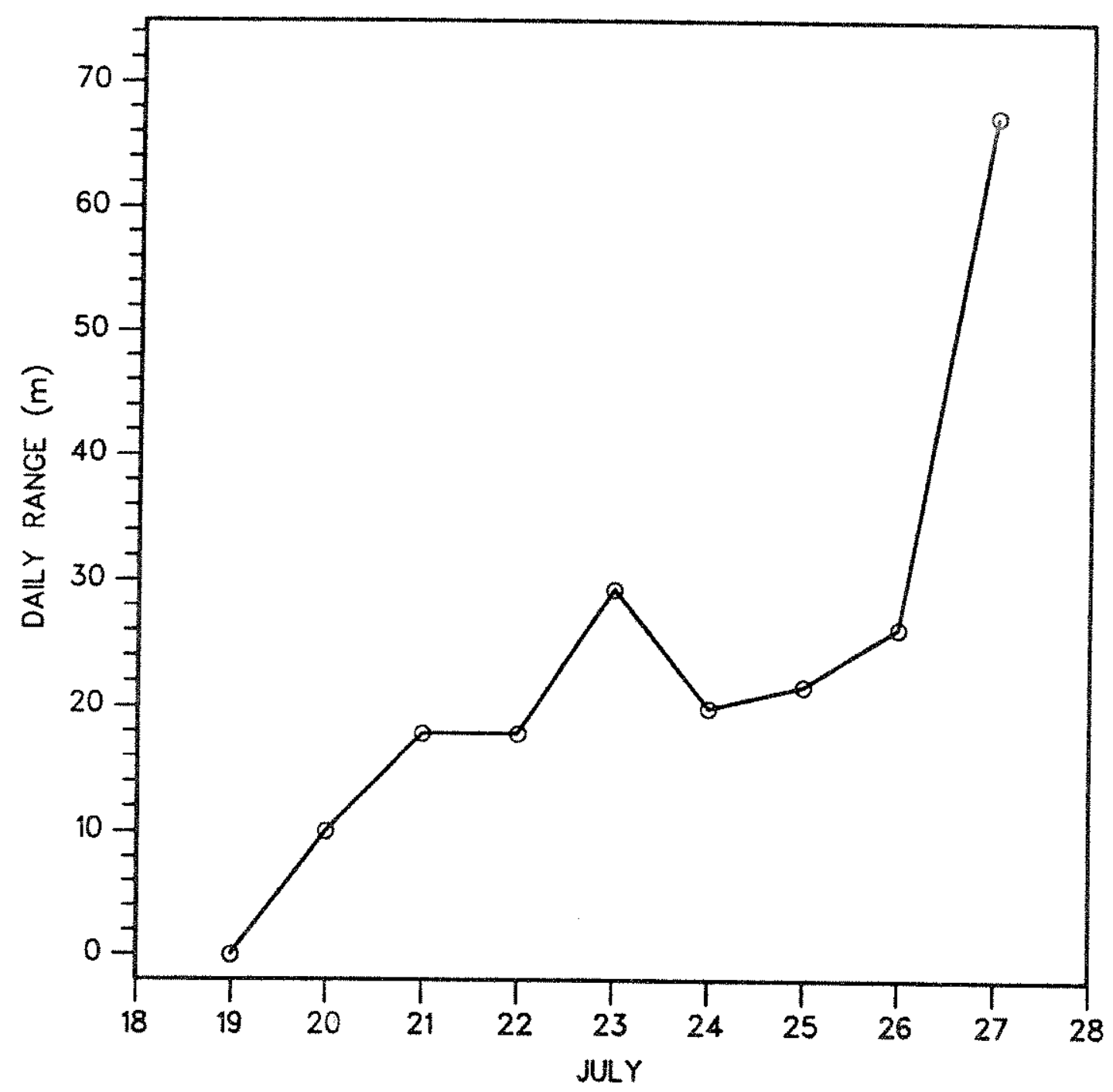

Figure 2.4.29. Daily range (m) of fish 255 (brown trout), 19 July - 28 July 1988, Bighorn River, Montana. 
trout were to use the complete water column in these areas, a small amount 10 cm would provide a $1 \%$ decrease in gas supersaturation) of hydrostatic compensation would be provided. It is conceivable that rainbow trout occupied deeper water at night, decreasing their exposure to gas supersaturation, and that brown trout moved into shallower water at night, increasing their exposure. However, no diurnal movements of this type were detected using radio telemetry.

Differences in habitat use between brown trout and rainbow trout varied seasonally, with the most significant differences being fish depth and velocity. Brown trout occupied significantly deeper habitat in only one time period, septemberoctober, whereas rainbow trout used significantly higher velocity water during all three time periods. The most critical variable in habitat selection by fluvial trout is generally thought to be water velocity (Jenkins 1969; Bachman 1984; Fausch 1984; Gatz et al. 1987), followed by cover (Lewis 1969; Gatz et al. 1987). Similar water velocities $(26.7 \mathrm{~cm} / \mathrm{s}$ versus $22.95 \mathrm{~cm} / \mathrm{s}$ in this study) and slightly shallower depths $(0.65 \mathrm{~m}$ versus $0.91 \mathrm{~m}$ ) were found for adult brown trout in this study compared to those reported by Shrivell and Dungey (1983). Velocities and depths found in this study were similar to those reported for brown trout and rainbow trout in sympatry by Gatz et al. (1987). velocity and depth used by adult brown trout and rainbow trout in the Bighorn River fell within the optimal conditions described by the suitability index (SI) curves developed for instream flow analysis borown trout adult velocity: $S I=1.0$ at $15.24 \mathrm{~cm} / \mathrm{s}$ : depth: SI=1.0 at $0.79 \mathrm{~m}$ (Raleigh et al. 1986): rainbow trout 
adult velocity: $S I=1.0$ at $15.24-60.96 \mathrm{~cm} / \mathrm{s}$; depth: $S I=1.0$ at $>0.45 \mathrm{~m}$ (Raleigh et al. 1984)].

Observed differences in habitat use by adult trout of both species did not result in differences in exposure to high dissolved gas levels between length groups. Thus, size specific mortality of trout $>15 \mathrm{~cm}$ was unlikely in the Bighorn River and does not account for the large difference in relative abundance between the two species.

Use of shallow water habitat by brown trout (mean water depth $=0.49 \mathrm{~m}$ ) and rainbow trout (mean water depth $=0.45 \mathrm{~m}$ ) $<15$ $\mathrm{cm}$ exposed this size group to a greater potential of developing GBT than that encountered by larger trout. Juvenile brown trout and rainbow trout are typically found at shallower depths and lower velocities than adults (Raleigh et al. 1984; Raleigh et al. 1986). Gatz et al. (1987) found allopatric age 0 rainbow trout and brown trout occupied similar water depth (25 to $35 \mathrm{~cm}$ ), but age 0 brown trout displaced age 0 rainbow trout from shallow water in sympatric situations. Exclusion of juvenile rainbow trout from shallow water was not observed in the Bighorn River. Differences in emergence time between the two species may reduce overlap of habitat use by trout <15 cm TL. Habitat occupied by trout $<15 \mathrm{~cm}$ TL provided less than a $0.5 \%$ reduction in hydrostatic pressure and thus exposure to gas saturation was highest in these size groups. White et al. (1988) found that 32 to $50 \%$ of brown trout fry held in cages in the Bighorn River exhibited excessive buoyancy. connor (1988) found that daily mortality of juvenile brown trout exposed to $125 \%$ gas saturation was always greater than for rainbow trout of similar size. He 
found no difference in vulnerability to predation between juvenile brown trout and rainbow trout exposed to high dissolved gas levels in circular tanks compared to controls, but this may have been an artifact of the experimental design. Mortality indirectly related to high dissolved gas levels of juvenile trout with excessive buoyancy might be expected in the Bighorn River due to increased vulnerability to predation, to decreased feeding efficiency, and/or to increased energy demand related to maintaining their position in the water column. If differences in species specific mortality of juvenile trout related to dissolved gas levels occur in the Bighorn River, they are not due to differences in habitat use.

Few studies have examined sympatric populations of brown trout and rainbow trout. of the studies examining sympatric populations of the two species, similar habitat use was found (Lewis 1969: Jenkins 1969; Bachman 1984; Baltz and Moyle 1984; Carty 1985). Gatz et al. (1987) reported evidence of asymetrical interspecific competition. Habitat use varied significantly between allopatric and sympatric rainbow trout in their comparison. They found the water velocity the fish faced, followed by cover, to be the most critical habitat variables in the interaction between the two species.

contrary to other studies that have found slight differences in habitat use (Raleigh et al. 1984, Raleigh et al. 1986), simialr-sized brown trout and rainbow trout in the Bighorn River occupied similar habitat with respect to water depth, fish depth (except september-october), substrate, vegetation height, and cover. Several possible conditions in the study area may account 
for this similarity of habitat use: 1) lack of forage fish, 2) food limitation, 3) evolutionary isolation, and 4) homogeneous habitat.

Brown trout and rainbow trout have similar diets except that brown trout $>30 \mathrm{~cm}$ are predominantly piscivorous (Kaeding and Kaya 1978; scott and Crossman 1973). Few forage fish were present within the study area, thus large brown trout were relegated to feed primarily on aquatic invertebrates. If food was limited in the Bighorn River, brown trout and rainbow trout could be forced to use similar habitat due to the premium it would put on food availability and on associated bioenergetically suitable feeding locations (Fausch 1984). This does not appear to be a likely explanation since aquatic invertebrates were much more abundant than reported for most Montana streams (Jim Brammer, personal communication). However, since trout densities in the Bighorn River are higher than trout densities in most other Montana streams, the possibility exists that food may have been limiting.

Brown trout and rainbow trout evolved in geographic isolation, thus overlap in habitat use is not unexpected. Nikolskii (1963) maintained that species with similar geographic origins have evolved mechanisms allowing coexistence. Where brown trout have been introduced into streams with other salmonids, a decline in the other salmonid populations often occurs (Nilsson 1967).

The most likely explanation for use of similar habitat by brown trout and rainbow trout is the homogeneous nature of the Bighorn River channel. Lack of habitat diversity appears to 
force the species into similar patterns of habitat use. Interspecific competition (interactive segregation: Nilsson 1967) cannot be assumed simply because brown trout and rainbow trout overlapped in their use of habitat variables examined. For competition to occur the demand for the resource must exceed resource avaliability at any particular point in time (Birch 1957). Since allopatric conditions were not available, we could not determine if brown trout were being forced to occupy feeding locations similar to rainbow trout or if differences in water velocity use were due to species interactions. The latter appears unlikely since rainbow trout have been reported to use higher velocities, even in allopatric situations (Raleigh et al. 1984). The possibility exits that similarities in habitat use by the two species resulted from observing them at feeding sites, not refuge sites. Jenkins (1969) reported that brown trout and rainbow trout used similar feeding sites but different refuge sites. Since this study was designed to determine differences in habitat use between the two species, questions concerning interactive segregation cannot be addressed.

Seasonal shifts of trout distribution in the Bighorn River appeared to be related to seasonal changes in availability of suitable habitat created by aquatic vegetation. Trout were concentrated along river banks during winter and spring and used bank and midchannel areas during summer and fall. We hypothesize that increased abundance of trout in midchannel areas during late spring and summer was associated with habitat refuges provided by aquatic vegetation growth. As water temperature and photo-period increased in summer and fall, aquatic vegetation became dense. 
Because of uniform stream channel shape and the scarcity of low velocity microhabitat locations in the midchannel areas during winter and early spring, we believe trout concentrated along the banks to take advantage of lower river velocities. Maximum velocity theoretically occurs at the surface in the centerline of the channel (Leopold et al. 1964). Near-bank areas in shallow water provide large quantities of substrate area per volume of water (compared to the volume of water over an equal size area in deeper midchannel area) creating microhabitat locations energetically suitable for trout. As the season progressed, growth of aquatic vegetation may have provided reduced velocity areas in midchannel, making this habitat more available to trout. Movement of trout into midchannel areas and the decrease of external symptoms of GBT coincided with seasonal increases in aquatic vegetation. The Bighorn River was typically free of dense aquatic vegetation in the winter and spring. The seasonal shift in distribution of trout was more apparent in 1987 than in 1988, perhaps due to small sample size in 1988 and/or reduced amounts of aquatic vegetation that year. Water temperature was cooler in 1988 than in 1987 (maximum in section 1 in 1987: $15.0^{\circ} \mathrm{C}$, in $1988: 11.3^{\circ} \mathrm{C}$ ) and remained relatively constant from spring through fall, probably explaining the reduced growth of aquatic vegetation. A seasonal shift in trout distribution was detected even with similar spring, summer, and fall water temperatures in 1988 suggesting that even small amounts of cover created by aquatic vegetation influenced trout distribution. No correlations among snorkel lane counts and dissolved gas level, visibility, light, and discharge were detected; some snorkel lane 
counts were correlated with water temperature. Dissolved gas levels remained relatively high throughout the year (Tables 2-5), and the percent of contribution of oxygen and nitrogen remained relatively constant at various levels of TGP (White et al. 1988).

The importance of aquatic vegetation as cover for trout has been reported by several authors. Gosse and Helm (1979) found that aquatic macrophytes were very important to brown trout as cover in the Provo River, Utah. The aquatic macrophytes produced areas of reduced velocity near the stream bottom. Brown trout used this cover to a larger extent than available bankside cover. Boussu (1954) also found that aquatic vegetation provided cover for trout in Trout creek, Gallatin County, Montana. In a study to determine relationships between a trout population and cover, he found a $71 \%$ reduction in total trout biomass after aquatic vegetation was removed. In a study to determine if microhabitat use changed temporally or spatially, Ross et al. (1987) reported findings (discriminant function analysis) that suggest that seasonal changes in aquatic vegetation may result in seasonal shifts in habitat use of fish in Black Creek, Mississippi.

Bighorn River trout appear to show seasonal variation in microhabitat use, changing their distribution in response to changes in habitat availability. Felley and Felley (1987) found habitat choice to be a dynamic process. Using factor analysis they found that different species select habltat according to different environmental parameters and seasonally change their habitat use. of the species studied, most selected habitats based on current speed, amount of debris (leaves and sticks on bottom: presence or absence), and cover (defined as structure or 
vegetation in which fish might hide: presence or absence). Grossman et al. (1987a and $1987 \mathrm{~b})$ found seasonal variation in cyprinid (Barbus haasi, Barbus graellsii, and Chondrostoma toxostoma) microhabitat use was strongly correlated with seasonal changes in microhabitat availability.

The availability of midchannel habitat may have provided hydrostatic compensation for trout moving in and out of deeper midchannel areas. An increase in hydrostatic pressure associated with an increase in water depth of $1 \mathrm{~m}$ would decrease gas saturation by approximately $10 \%$ (Gray and Haynes 1977). If dissolved gas levels were near thresholds for bubble growth in salmonids (see section 2.1), the compensation provided by midchannel depths (approximately $1.8 \mathrm{~m}$ ) would provide a refuge from gas supersaturation. Critical thresholds for bubble growth vary, ranging from swimbladder overinflation, which is the lowest, to the threshold for bubble formation in the cardiovascular system. Estimates of threshold levels for bubble formation (swimbladder and cardiovascular) range from $110-117 \%$ TGP saturation. Total gas pressure averaged $116.3 \%$ saturation (range: $109.2-122.7$ ) at $\mathrm{Rkm} 0.6$ and averaged $115.5 \%$ saturation (range: $106.8-127.9$ ) at $\mathrm{Rkm} 4.8$ in 1987. Threshold criteria apply to fish at the water surface. Hydrostatic compensation may be important in minimizing mortality. This may explain the decreased incidence of GBT in trout during late spring and early summer though dissolved gas levels remained high and relatively constant. When sufficient vegetation was present, trout used deeper water areas that may have compensated for high gas levels. Shallow depths provided little hydrostatic compensation when 
trout were concentrated along the banks. In section 1 during 1987, cardiovascular bubble growth would not have occurred in fish at a depth $>0.5 \mathrm{~m}$ (White et al. 1988). The only

alternative to depth compensation for avoiding high dissolved gas levels was for trout to move downstream into areas of lower dissolved gas pressure. Downstream movements would have had to be large due to the persistence of high dissolved gas levels throughout the length of the study area. Such large downstream movements were not detected among radio tagged brown trout and rainbow trout. Also, the number of trout counted in section 1 (highest dissolved gas levels) was always greater than in section 2 .

Radio-tagged trout did not appear to compensate for high dissolved gas levels. During tests in which river dissolved gas levels were modified, radio tagged trout generally did not shift location or depth. Diel shifts in fish depth were probably associated with insect emergence. Both brown trout tagged in 1988 used a wider range of depth than the rainbow trout and were often located in shallow riffle areas during periods of insect emergence. Movement of the rainbow trout was very localized. The only indication of a possible behavioral response was a slight increase in daily range (not sounding) in one brown trout and the rainbow trout during periods of high dissolved gas levels In 1988. The larger amount of local movement during the $6 \mathrm{~d}$ following release in 1987 was probably due to handling stress rather than dissolved gas levels since these trout were held for up to $20 \mathrm{~h}$ before release. This increased movement activity was not detected in 1988 when fish were released immediately after 
surgery.

Resident trout have been reported to remain in localized areas during most of the year. Using mark and recapture methods Harcup et al. (1984) found that movements $>50 \mathrm{~m}$ of brown trout (1+ years and older) were rare with the majority being $<15 \mathrm{~m}$. Hesthagen (1988) recaptured between $85-89 \%$ of resident brown trout (age $2+$ to $9+$ years) within $45 \mathrm{~m}$ of their release point throughout a 3-month period. Data collected in this study indicated a similar lack of movement. 


\subsection{Impacts of GBT on Trout catchability}

on 13 June 1987 we conducted a catchability study to determine if fish with GBI made up a smaller percentage of the catch than the actual proportion in the population. If this was the case, it would suggest that gas supersaturation may effectively remove a portion of the population from the sport fishery. Approximately 24 volunteer anglers assisted and 59 trout were caught, 45 brown trout and 14 rainbow trout. Anglers fished on the right bank between Afterbay Dam and Rkm 1.9 (RM 1.2) with most effort expended between $\mathrm{Rkm} 0.8$ and 1.2 (RM 0.5 $0.75)$

Approximately 78 and $93 \%$ of all brown trout caught and those over $356 \mathrm{~mm}$ (14 in), respectively, had external GBT symptoms. Eighty-three percent of all brown trout caught had minor symptoms of $\mathrm{GBI}, 14 \%$ had serious symptoms, and $3 \%$ had severe symptoms. We determined that $61 \%$ of the larger brown trout had minor symptoms, $31 \%$ serious symptoms, and about $8 \%$ had severe symptoms, which substantially reduced the chances for long-term survival. Rainbow trout showed a much lower GBT incidence rate - $21 \%$ for al1 rainbow trout and $29 \%$ for $f$ ish $356 \mathrm{~mm}$ (14 in) or greater: also, only minor symptoms were observed. Seventy-nine percent of the rainbow trout caught were of hatchery origin.

On the evening of 14 June 1987 we electrofished the same river reach. From Rkm 0.0-1.2 (RM 0.00-0.75), GBT incidence among all brom trout was $50 \%$, and for brown trout $356 \mathrm{~mm}$ (14 in) or greater we noted a $56 \%$ rate. Rainbow trout had an overall GBT incidence rate of $9 \%$, while for larger rainbow trout the rate was 17\%. These incidence rates are substantially lower than data 
obtained by angling. Since more than $96 \%$ of the brown trout and $100 \%$ of the rainbow trout were caught by anglers between $\mathrm{Rkm}$ 0.8-1.2 (RM 0.5-0.75), we examined the data for this area to see if incidence was higher there than in other reaches. of the 122 brown trout and 21 rainbow trout captured, GBT incidence for brown trout was $65 \%$ in both categories, and $10 \%$ for all rainbow trout; rainbow trout over $356 \mathrm{~mm}$ (14 in) had a 15\% incidence rate. Most brown trout had minor symptoms, $77 \%$ for all brown trout and 65\% for fish $356 \mathrm{~mm}$ (14 in) or larger, while serious symptoms were present on 22 and $29 \%$ of all brown trout and the larger brown trout, respectively. Severe symptoms were displayed by $1 \%$ of all brown trout and $6 \%$ of the larger brown trout. As with the angler-caught rainbow trout, only minor symptoms were observed among the rainbow captured during electrofishing.

Although GBT incidence from Rkm 0.8-1.2 (RM $0.5-0.75$ ) was higher than in the entire stretch we electrofished, it was still lower than the incidence rate determined from angling. This suggests that minor GBT symptoms do not remove brown and rainbow trout from the fishery. However, most trout severely affected by GBT are probably unavailable to anglers. As found in other parts of this study, the proportion of the population affected and the severity of symptoms was greater among brown trout than rainbow trout. 


\subsection{Impacts on Invertebrate and Forage Fis Communties}

\subsubsection{Aguatic Invertebrates}

Information concerning potential effects of hyperbaric pressure on aquatic invertebrates is Iimited, although occurrence of GBT in these organisms is well documented (Marsh and Gorham 1905; Evans and Walder 1969; Malouff et al. 1972). Fickeisen and Montgomery (1975) conducted bioassays on effects of gas supersaturated water on six families (representing four orders) of aquatic insects and reported that pteronarcys californica acquired GBT when exposed to $132 \%$ and $140 \%$ supersaturation for 10 d. Nebeker (1976) noted body distension and gas bubbles throughout the body fluids of stoneflies at $125 \%$ saturation, but no mortality occurred.

An understanding of gas supersaturation influences on aquatic invertebrates is needed to determine potential impacts to food resources of Bighorn River fishes. This portion of the study was designed to:

1) Determine invertebrate abundance and community composition in areas of the Bighorn River where high and low incidence of GBT occur in fish.

2) Define gas supersaturation levels harmful to different invertebrate taxa.

3) Relate findings to gas saturation levels in the river.

\subsubsection{Methods}

site selection: The physical characteristics of four riffle areas were analyzed before selecting two of these as invertebrate sampling sites. Two riffles were near Afterbay Dam and represented areas where high levels of GBT occurred in fish. The remaining two were further downstream where incidence of GBT was 
lower. At each riffle, three transects (perpendicular to the current) were established. Water depth and velocity, and substrate size were measured along each transect. Depth and velocity were measured at $1.5 \mathrm{~m}$ intervals using a Marsh-McBirney current meter and a top setting rod, while substrate was measured by embedding a $0.085 \mathrm{~m}^{2}$ modified Hess invertebrate sampler (Waters and Knapp 1961) approximately 3-7 cm into the river bottom at $2 \mathrm{~m}$ intervals. All rocks within the sampler with a maximum diameter greater than $6 \mathrm{~cm}$ were measured.

Riffles 2.4 and 14.5 river kilometers ( $\mathrm{rkm}$ ) below Afterbay Dam were chosen as invertebrate collection sites due to their physical similarities and their proximity to monitoring stations where gas saturation levels and water temperatures were measured two or three times weekly (Figure 2.6.1).

Benthic Invertebrate sampling: Benthic samples were collected during three periods. Sampling dates were chosen to allow evaluation of invertebrate comminity structure before runoff and after run-off, the period of highest gas supersaturation and high GBT incidence in trout. In 1986, sampling was done in August (post-runoff). In 1987, samples were taken in April and september. Twenty-five samples per site were collected in August. This was reduced to 15 samples per site in April and September 1987.

Riffles used in benthic sampling (Figure 2.6.1) were divided into $1 \mathrm{~m}^{2}$ grids, and random numbers, generated by computer, were used to determine where samples would be collected within the riffle. Samples were collected by embedding the modified Hess sampler into the substrate to a depth of $5-10 \mathrm{~cm}$. All rocks 


\section{IMVETTESATE COLLECTION \\ O OAS MOMIORIMC STATION}

BIOHOR

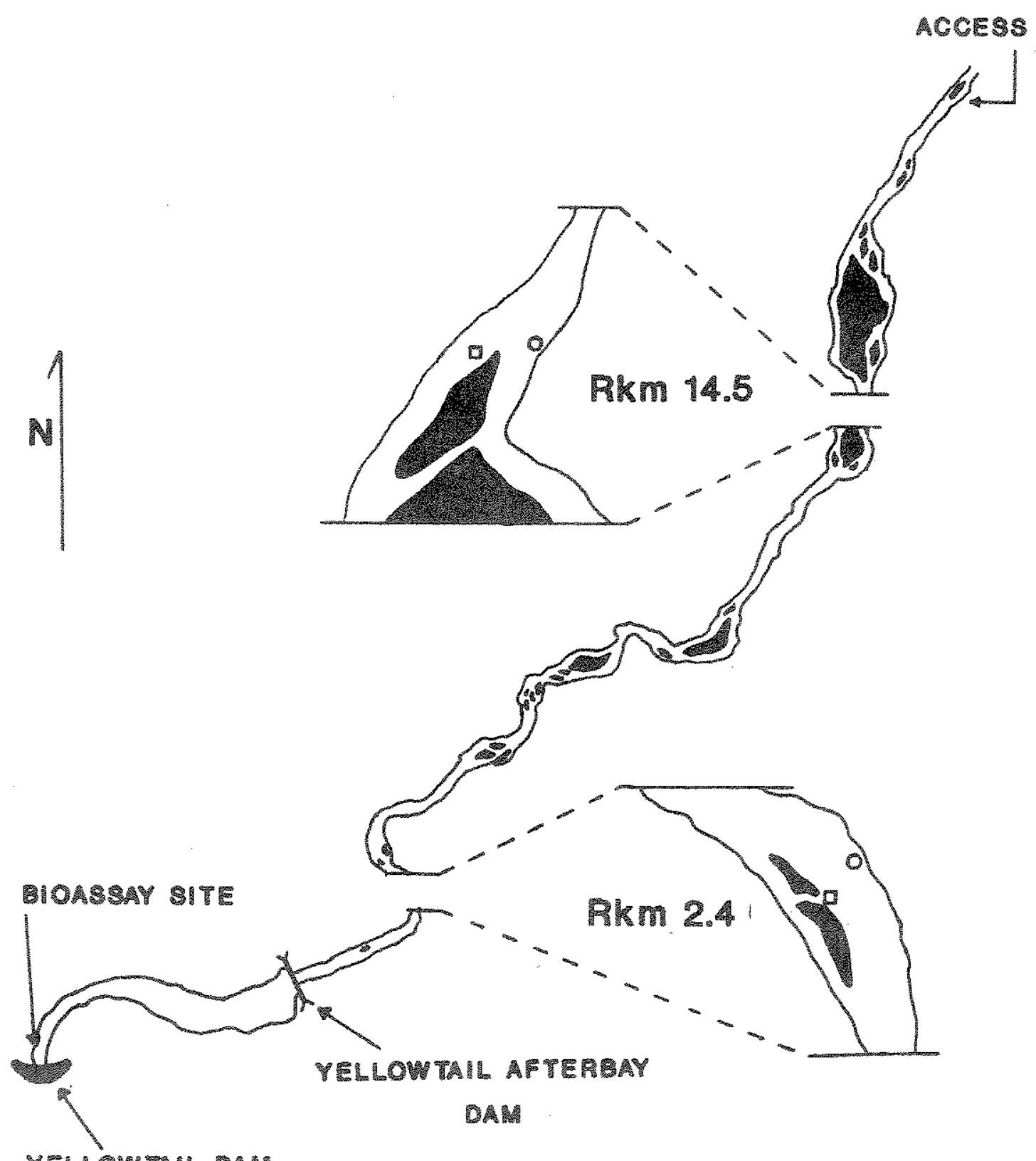

Y

Figure 2.6.1. Bighorn River study area, illustrating channel characteristics at invertebrate collection sites and gas monitoring stations. 
with periphytic algae were individually scrubbed with a soft bristled brush, examined to ensure no organisms remained, then removed from the sampler. Remaining substrate was agitated with a steel rod to a depth of $7-10 \mathrm{~cm}$, before removal of the sampler. At each sample location and prior to sampling, water velocities (0.6 depth and $5-7 \mathrm{~cm}$ above the substrate) and depth were measured using a Marsh-McBirney current meter, and a top-setting rod. Only water velocities at 0.6 depth were collected during the August 1986 sampling period. During all three collection periods, samples were gathered from each riffle over a 2 d interval. Samples were preserved in Kahle's solution (Pennak 1978) containing rose bengal and sorted in the lab at a later date.

Invertebrate sorting was facilitated by straining samples through a series of sieves $(8.0,1.0$, and $0.5 \mathrm{~mm}$ mesh size) before picking. Due to sample richness, the sieved material smaller than $1.0 \mathrm{~mm}$ in the 1987 samples was subsampled before sorting, using a device constructed to divide the material into quarters. Invertebrates were removed from one quarter of the material smaller than $1.0 \mathrm{~mm}$. After sorting, the organisms were identified to family or genus and counted.

Vegetative materials from the samples were dried in an oven at $105^{\circ} \mathrm{C}$ for $24 \mathrm{~h}$. The dry weight values provided a samplespecific measure as an index to compare vegetative standing crop.

Drift Sampling: Fickeisen and Montgomery (1975) noted that during gas supersaturation bioassays, several invertebrate taxa experienced buoyancy problems due to gas bubbles adhering to their bodies. Thus, drift samples were collected during the 
April and september sampling periods to evaluate drift rates of various taxa.

Allan and Russek (1985) found that fewer drift samples were required to quantify invertebrate abundance if replicate samples were collected during the period of peak drist, as opposed to other sampling designs. Thus, during the April and september 1987 sampling periods, four synchronized drift samples were taken at the lower end of the two sampling riffles. Nets were placed in the river shortly after dark, when drift is nomally greatest (Allen and Russek 1985) and removed $0.5 \mathrm{~h}$ later. The nets had $0.15 \mathrm{~m}^{2}$ openings with a mesh size of $0.5 \mathrm{~mm}$. Each net sampled the entire water column. Water velocities were measured 10.5 depth of the water column) at the mouth of each net at the beginning, middle and end of each sample. Samples were preserved, sieved, subsampled, sorted and counted the same as described for benthic samples.

Seriphyton collecton and Analysis: periphyton samples were taken during both 1987 sampling periods to provide another measure of site similarity. During April, the standing crop of periphyton appeared to vary substantially within collection sites. Thus, upstream and downstream riffles were partitioned into subsections based on visual differences in periphyton abundance, and mapped (Figure 2.6.2). Statistical analysis of data by subsection allowed determination of habitat differences within sites and between sites. In september 1987 , differences in periphyton abundance were not visually apparent within sites and riffles were not subsectioned.

Periphyton sampling sites were chosen by throwing a steel. 

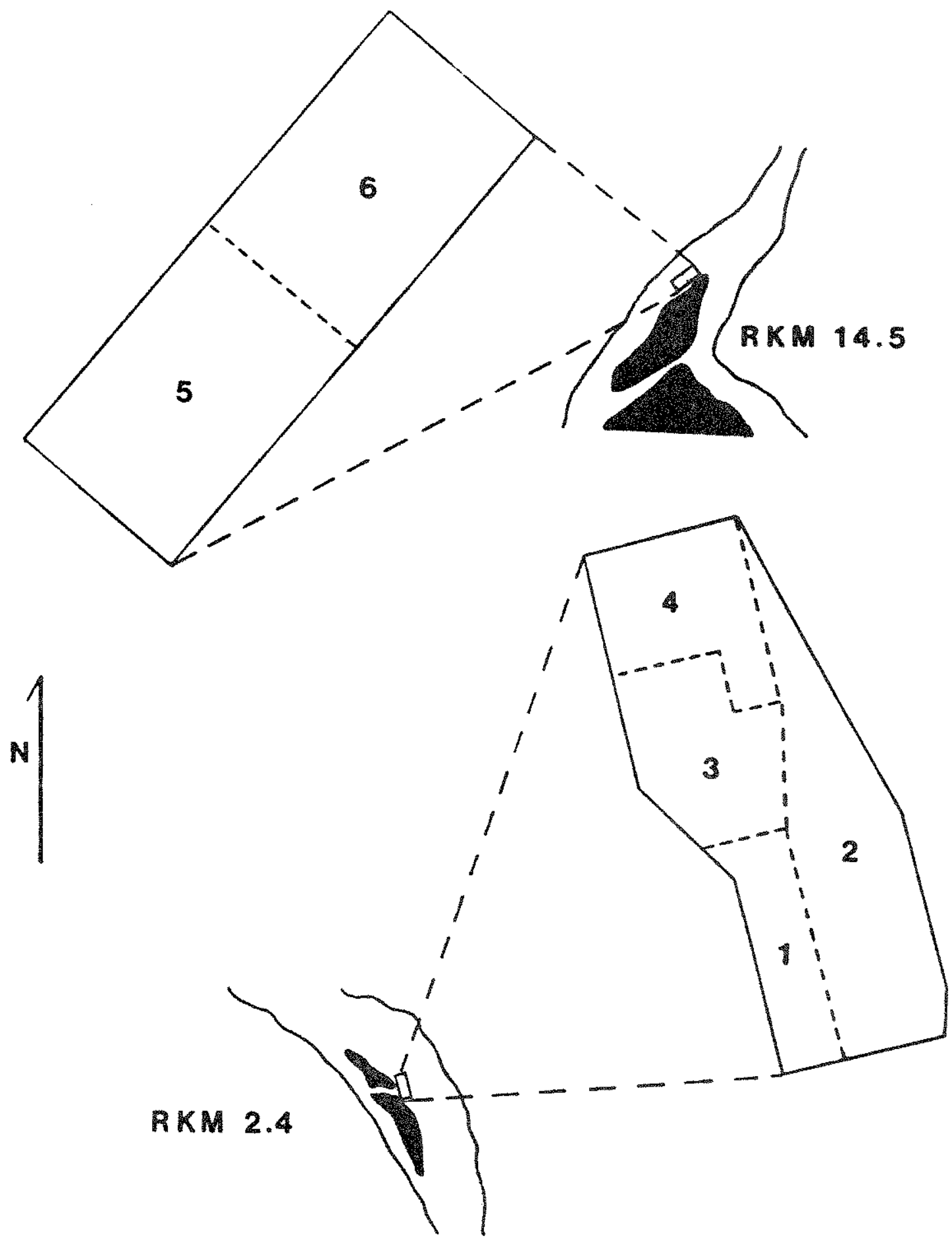

Figure 2.6.2. Invertebrate collection sites illustrating how riffles were divided into subsections in April 1987. 
rod, marked on one end, into the water, and collecting the rock nearest the marked end. Periphyton was scraped off the rock, placed in a labeled plastic bag, put on ice in a cooler, and later frozen for analysis in the lab. The periphytic attachment area was estimated by taking two measurements - essentially length and width - to allow comparison between samples. In April, 42 and 39 samples were collected from the upper and lower sites, respectively, and the number of periphyton samples were proportioned according to subsection area. In september, 40 samples were collected from each site.

Periphyton samples were analyzed for chlorophyll a concentration and ash-free dry weight. To do this each periphytic mass was thawed, blotted with paper towels, and weighed. Three subsamples of approximately $0.2 \mathrm{~g}$ were removed for chlorophyll extraction and analysis.

subsamples were placed in a centrifuge tube with $10 \mathrm{ml}$ of extractant (DMSO:90\% acetone), agitated, and placed in a dark freezer for $24 \mathrm{~h}$. Upon removal they were centrifuged for $10 \mathrm{~min}$. One milliliter of the chlorophyll solution was then diluted with an additional $5 \mathrm{ml}$ of extractant. Spectrophotometry was done using a spectronic 20 spectrophotometer and methods described by Wilkinson (1983) were used to correct for absorbance by phaeopigments. The following formula (modified from wilkinson 1983) was used to determine chlorophyll a concentration per unit weight of vegetation:

$$
\begin{gathered}
c=\left(E_{\mathrm{a}}-E_{\mathrm{b}}\right) \times 27.31 \times \mathrm{v} / \mathrm{m} \times 1 \\
c=\begin{array}{c}
\text { Chlorophyll } \\
\text { vegetation) }
\end{array}
\end{gathered}
$$




$$
\begin{aligned}
& \mathrm{E}_{\mathrm{a}}=\text { Extract absorbance at } 665 \mathrm{~nm} \text { minus } \\
& \text { absorbance at } 750 \mathrm{~nm} \text { before } \\
& \text { acidification } \\
& \mathrm{E}_{\mathrm{b}}=\text { Extract absorbance at } 665 \mathrm{~nm} \text { minus } \\
& \text { absorbance at } 750 \mathrm{~nm} \text { after } \\
& \text { acidification } \\
& 27.31=\text { constant used for extractant } \\
& \mathrm{v}=\text { Volume, in } \mathrm{ml} \text { of extractant solution } \\
& \text { used } \\
& \mathrm{m}=\text { Blotted wet weight, in grams, of } \\
& \text { vegetation } \\
& I=\text { Path length (usually } 1 \mathrm{~cm} \text { ) }
\end{aligned}
$$

The remaining vegetative mass was placed in a drying oven at $105^{\circ} \mathrm{C}$ for $24 \mathrm{~h}$, weighed and placed in a muffle furnace at $500^{\circ} \mathrm{C}$ for another $24 \mathrm{~h}$. A final weight was taken to determine ash-free dry weight.

Invertebrate Bioassays: A transportable gas supersaturation unit was constructed so tests could be conducted with water from the Bighorn River. The unit was installed approximately $400 \mathrm{~m}$ below the base of Yellowtail Dam (Figure 2.6.1) and housed in a $2.44 \times 3.05 \mathrm{~m}$ metal shed. Supersaturation was achieved by sprinkling water through a vertical, $2.13 \mathrm{~m}$ PVC column $(20.3 \mathrm{~cm}$ in diameter), pressurized by an air compressor. As water passed through the column, high pressure forced the gas into solution (Figure 2.6.3). Varying proportions of treated and untreated water were used to achieve mean gas saturation levels which progressively increased from tank 1 through tank 6. Tank 1 received only untreated water and was used as a control. Flows into all tanks $(37.9 \mathrm{~L}$ aquaria with standpipes maintaining volumes at $30.3 \mathrm{~L}$ ) were approximately $0.75 \mathrm{~L} / \mathrm{min}$ exposing 


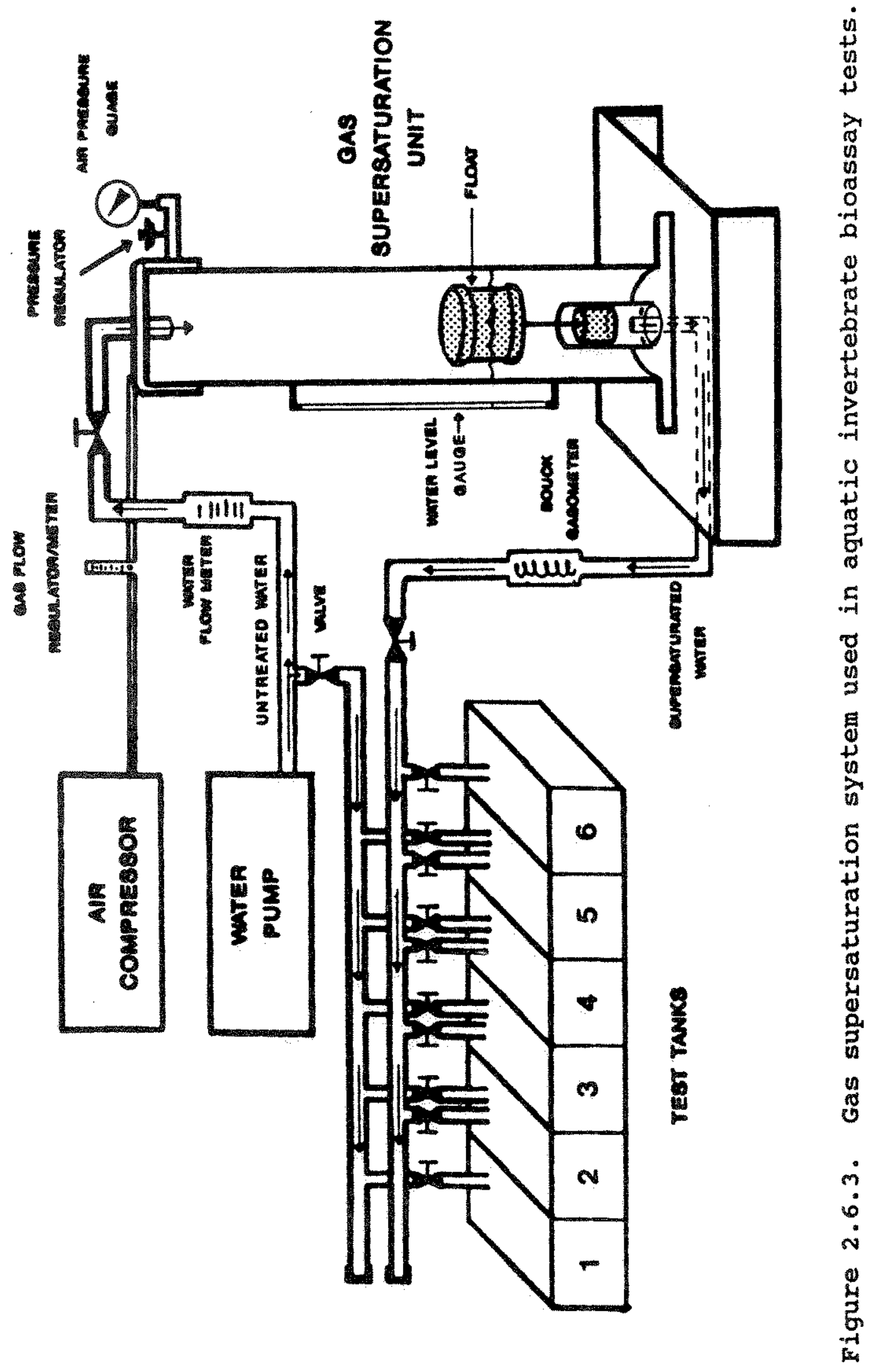


organisms to a water turnover time of about $40 \mathrm{~min}$.

Two 7-d (168 h) bioassays were conducted, each testing four invertebrate taxa. The first was run from August 2-9, 1988 and the second from September $8-15,1988$. Test organisms were collected at Bighorn Access (Figure 2.6.1), approximately 19.5 rkm below Afterbay Dam, taken to the bioassay site, and placed in the control tank for $24 \mathrm{~h}$. Twenty organisms of each taxon were then placed into each of the six tanks. Total gas pressures (TGP) were measured twice daily in all tanks using a Bouck gasometer (Bouck 1982). The following formula (Colt 1984) was used to calculate gas supersaturation levels referred to as delta P.

$$
\begin{aligned}
& { }^{\wedge} \mathrm{P}=\mathrm{TGP}-\mathrm{BP} \\
& \text { Where: }{ }^{\wedge} \mathrm{P}=\text { Delta } \mathrm{P}(\mathrm{mm} \mathrm{Hg}) \\
& \text { TGP }=\text { Total gas pressure }(\mathrm{mm} \mathrm{Hg}) \\
& \mathrm{BP}=\text { Barometric pressure }(\mathrm{mm} \mathrm{Hg})
\end{aligned}
$$

Liquid reagents and the azide modification of the winkler method (APHA 1976) were used once (first test) or twice (second test) daily to determine associated dissolved $\mathrm{O}_{2}$ levels in the tanks. Water temperatures were measured three or four times daily using a mercury thermometer. Barometric pressures were measured at least once daily using a Prince Nova, fortin type mercurial barometer or a Thommen 22000 pocket altimeterbarometer. The status of test organisms was assessed and recorded at $4 \mathrm{~h}$ intervals for the duration on of the bioassays. During both tests, organisms often attached themselves to the top and sides of the stand-pipe. To determine if this was behavioral, all organisms on stand-pipes were dislodged, 
encouraging them to swim to the bottom of the test tank. If unable to successfully swim to the bottom, organisms were classified as stranded. Buoyant animals were examined to determine whether external bubbles coalescing on the body were the cause. If none could be seen, buoyancy was assumed to be physiological and associated with GBT. Organisms were left in the test tanks until they became immobile and unresponsive when disturbed, or were dead. At this time they were recorded as "gas related mortalities", examined under a dissecting scope to determine if visible signs of GBT were present, and preserved. Nonbuoyant dead organisms found on the bottom of a test tank or on the stand-pipe, were classified as "non-gas related mortalities".

Statistical Analysis: Benthic invertebrate data were transformed $(1 n+1)$ for all analyses. All other data were left in raw form and parametric statistics were used, when variances were homogeneous $(p=0.05)$. Non-parametric methods were employed when this was not the case. 


\subsection{1b Results}

Taxanomic composition: Twenty-five genera of benthic invertebrates, representing 20 families and 10 orders, were collected from Bighorn River sampling sites. An additional five, three, and two taxa were identified to family, order and class, respectively (Table 2.6 .1 ).

Relative abundance in the Bighorn River was dominated by few taxa. Eighty-seven percent of all organisms collected in 107 benthic samples were members of five taxa (Table 2.6.2), and only Tricladida, Chironomidae, Baetis tricaudatis and Tricorythodes minutus were present in all samples. Isoperla quinquepunctata was the only stonefly that occurred commonly in Bighorn River benthic samples, and densities were low.

sample variation: Principal components (PC) analysis was performed on benthic data for the 14 taxa/groups exhibiting the greatest variance in abundance between samples (Table 2.6.3). Three vectors explained $73.5 \%$ of the total variance. When principal components were plotted (Figures 2.6.4 and 2.6.5), most variation in abundance of individual taxa occurred between samples collected during different periods and from different sites.

Principal component 1 explained $39 \%$ of the total variance in abundance for those taxa tested and had an eigen value of 14.84 , with the heaviest weightings given to Physella, Simulium, Hydropsychidae, and Serratella. This vector showed a separation between the three sampling periods, and between sites during August 1986 and september 1987 (Figure 2.6.4). 


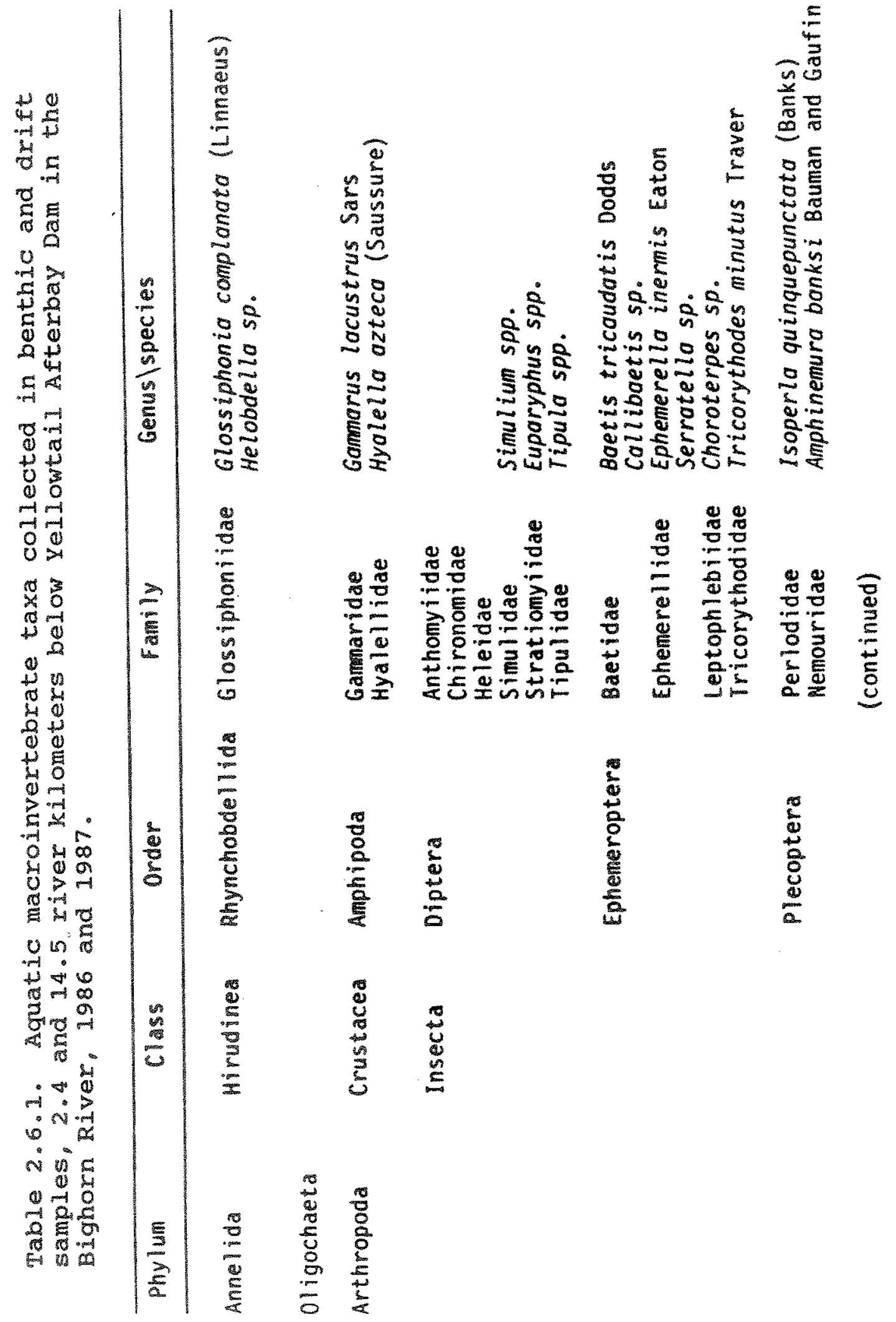




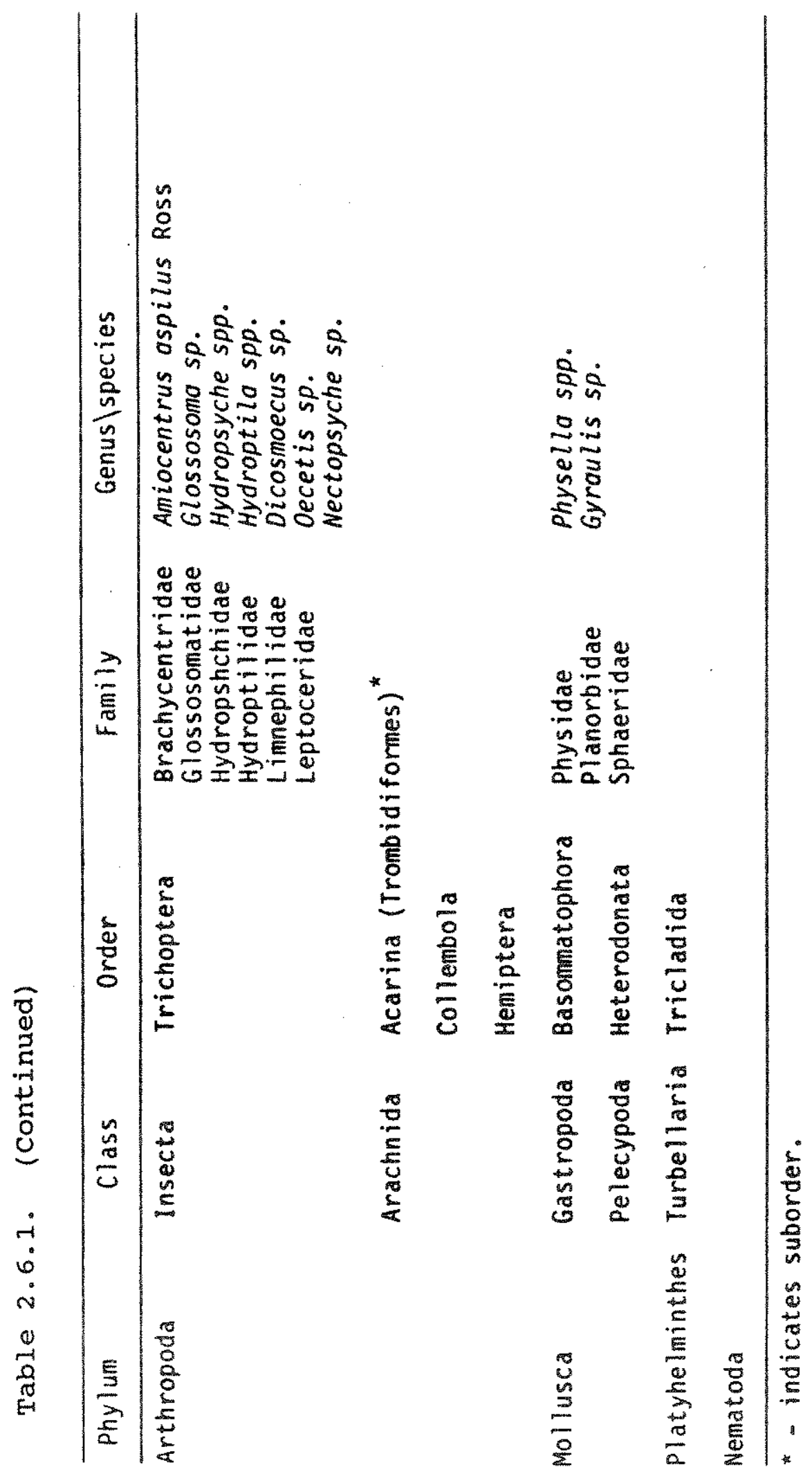




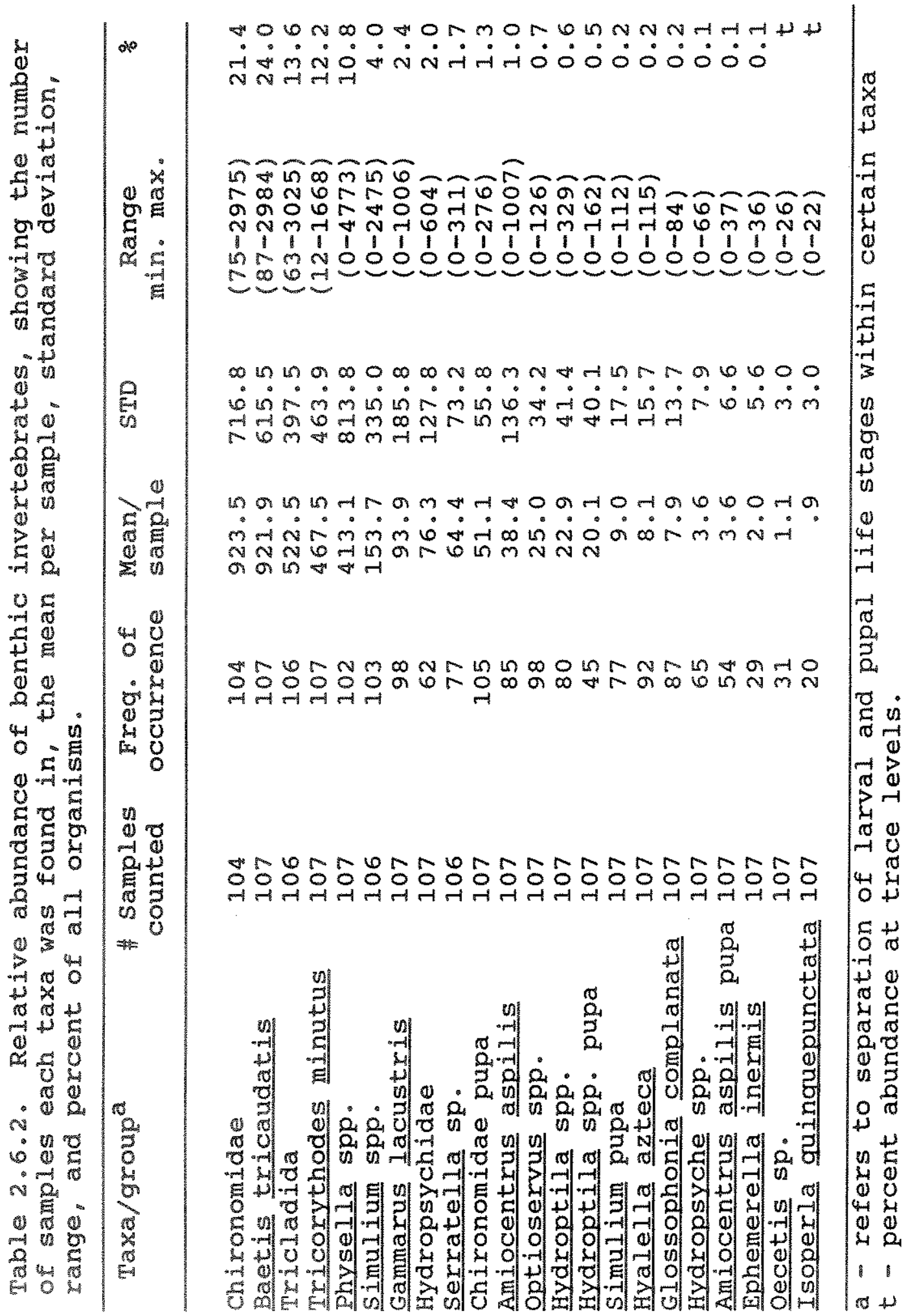


Table 2.6.3. Principal components analysis of the 14 "taxa/groups a which varied most in abundance among 107 benthic samples collected at Rkm 2.4 and Rkm 14.5, Bighorn River, 1986 and 1987.

\begin{tabular}{|c|c|c|c|}
\hline \multirow[t]{2}{*}{ Taxa/group ${ }^{a}$} & \multicolumn{3}{|c|}{$\begin{array}{c}\text { Principal components } \\
\text { vector }\end{array}$} \\
\hline & 1 & 2 & 3 \\
\hline Tricorythodes minutus & 0.15 & 0.11 & 0.33 \\
\hline Physella spp. & 0.41 & -0.44 & 0.34 \\
\hline Simulium spp. & 0.34 & 0.21 & -0.17 \\
\hline Simulium spp. pupa & 0.08 & 0.26 & -0.17 \\
\hline Gammarus lacustris & 0.24 & -0.49 & -0.32 \\
\hline Hydropsychidae & 0.50 & 0.25 & -0.29 \\
\hline Serratella sp. & 0.48 & -0.04 & -0.10 \\
\hline Amiocentrus aspilis & -0.08 & 0.16 & -0.29 \\
\hline Amiocentrus aspilis pupa & 0.16 & 0.15 & 0.15 \\
\hline optioservus spp. & -0.07 & -0.08 & 0.40 \\
\hline Hydroptila spp. & -0.14 & 0.33 & 0.33 \\
\hline Hydroptila spp. pupa & 0.27 & 0.44 & 0.23 \\
\hline Hyalella azteca & -0.12 & -0.02 & 0.01 \\
\hline Glossophonia complanata & 0.08 & -0.14 & 0.30 \\
\hline Eigen values & 14.84 & 7.90 & 5.14 \\
\hline Percent of variance & 39.17 & 20.84 & 13.57 \\
\hline Cumulative percent & 39.17 & 60.01 & 73.58 \\
\hline
\end{tabular}

Principal component 2 featured differences in individual taxa abundance between late summer-early fall samples and those collected in April. It also focused on differences between sites that were present in August 1986 (Figures 2.6 .4 and 2.6.5). Physella and Gammarus were given heavy negative weightings, while Hydroptila pupae had a large positive weighting. The amount of variance accounted for by PC 2 was $21 \%$, and its eigen value was 7.89.

Approximately $14 \%$ of the total variance in abundance of individual taxa was explained by PC 3 , with heavy weightings given to Physella, Tricorythodes, Gammarus, optioservus and 


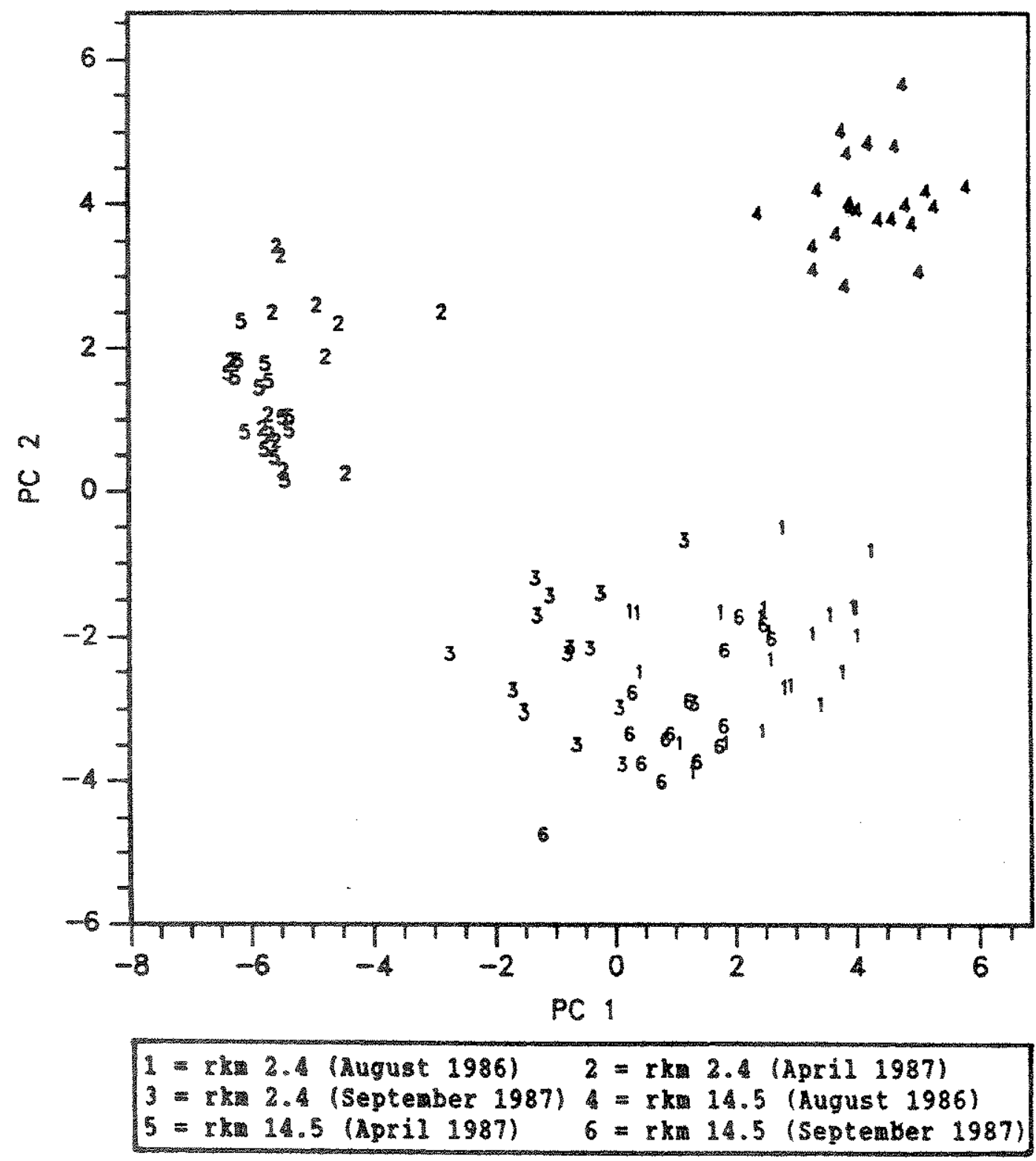

Figure 2.6.4. Principal components (PC) of vectors 1 and 2, generated from benthic samples collected at Rkm 2.4 and 14.5 during all sampling periods. 


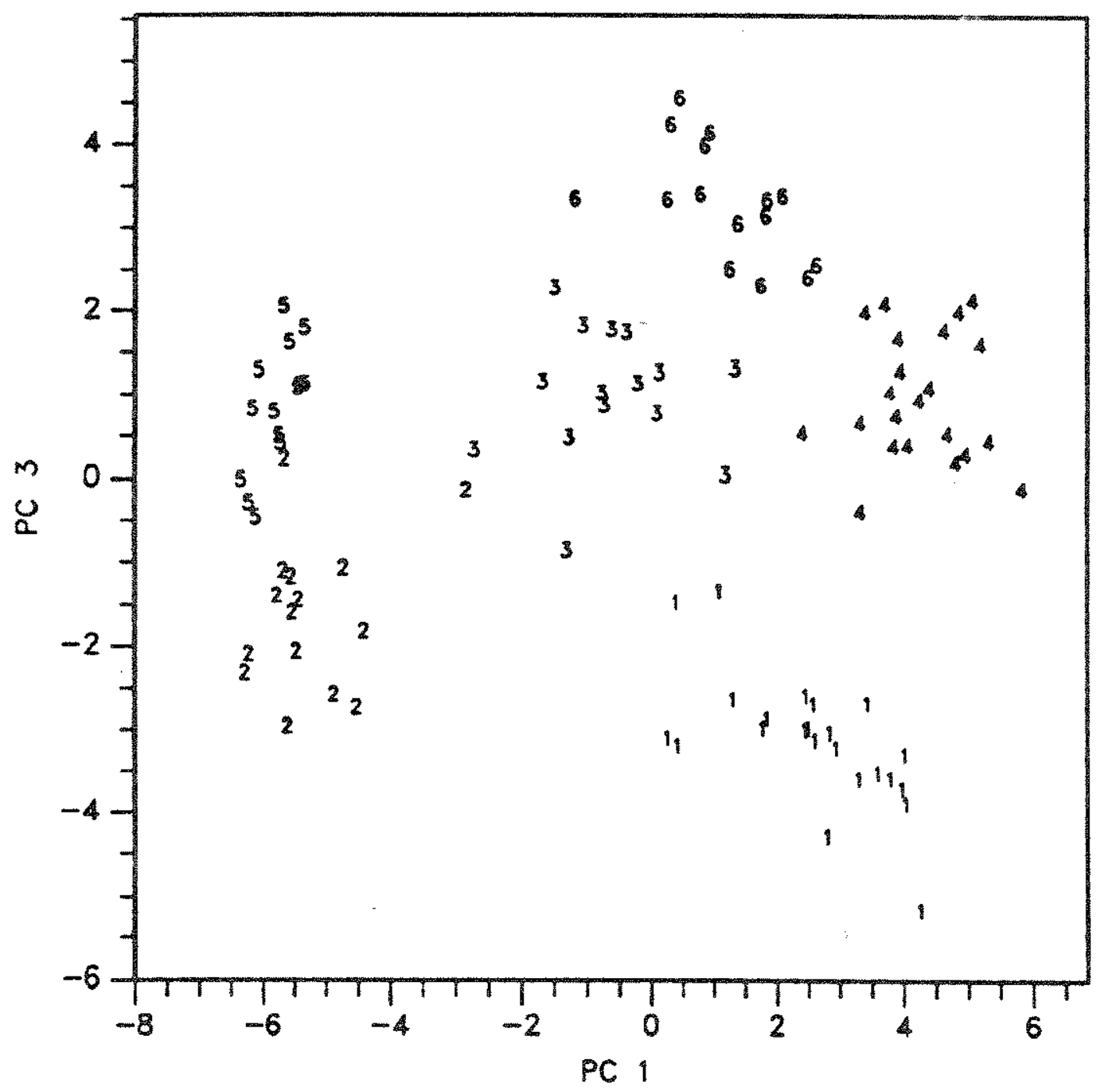

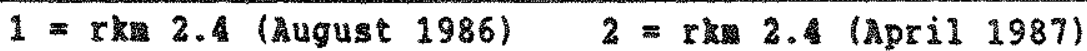

$$
\begin{aligned}
& 3=r k 2.4 \text { (September 1987) }=r \text { 14.5 (August 1986) } \\
& 5=r \text { 14.5 (April 1987) } 6=r \text { m } 14.5 \text { (September 1987) }
\end{aligned}
$$

Figure 2.6.5. Principal components (PC) of vectors 1 and 3 , generated from benthic samples collected at $\mathrm{Rkm} 2.4$ and 14.5 during all sampling periods. 
Hydroptila. This vector represents differences between upstream and downstream sites during all collection periods (Figure $2.6 .5)$

Benthic Abundance: Mean total invertebrate density was greater at $\mathrm{Rkm} 14.5$ during all sampling periods (Table 2.6.4). In August 1986 and september 1987, differences were significant with downstream densities being $22 \%(p<0.01)$ and $55 \%(p<0.01)$ greater than those upstream, respectively. In April, differences were not significant $(p=0.51)$ with 32,658 and 36,456 organisms per square meter at $\mathrm{Rkm} 2.4$ and 14.5 , respectively.

During August 1986, 16 taxa or groups showed significant differences in abundance between sites $(p \leq 0.05)$. Twelve of these were more common at Rkm 14.5 (Table 2.6.4). Eleven taxa differed significantly in september 1987; six of these were more common downstream. In April 1987, four of seven taxa which differed significantly between sites were more abundant downstream.

Most commonly occurxing taxa fell into three general categories with regard to density differences between sites: first, those taxa that were more abundant at $\mathrm{Rkm} 2.4$, when significant differences occurred (Group 1): second, those taxa found in significantly greater densities at one site during one period, but at the other site during a subsequent period (Group 2): and third, those taxa that were more abundant downstream, when significant differences occurred (Group 3).

Group 1. Densities of group 1 taxa were hypothesized to be least affected by gas supersaturation, assuming the pattern of influence on invertebrates is similar to that on trout (i.e. increased affiction near Afterbay Dam). However, data for taxa 
Table 2.6.4. Estimated density $\left(\# / \mathrm{m}^{2}\right)$ for taxa/groups ${ }^{\mathrm{a}}$ commonly occurring in benthic invertebrate samples. Results are provided by site for collection period 1 (August 23-24, 1986), period 2 (April 13-14, 1987) and period 3 (September 22-23, 1987), Bighorn River, MT.

\begin{tabular}{|c|c|c|c|c|c|c|}
\hline \multirow{2}{*}{ Taxa/groupa } & \multicolumn{2}{|c|}{$\begin{array}{l}\text { Period } 1 \\
\text { Rkm }\end{array}$} & \multicolumn{2}{|c|}{$\begin{array}{l}\text { Period } 2 \\
\text { Rkm }\end{array}$} & \multicolumn{2}{|c|}{$\begin{array}{l}\text { Period } 3 \\
\text { Rkm }\end{array}$} \\
\hline & 2.4 & 14.5 & 2.4 & 14.5 & 2.4 & 14.5 \\
\hline Amiocentrus aspilis ${ }^{2}$ & 249 & 64 & 2608 & 60 & 72 & 18 \\
\hline Amiocentrus pupa ${ }^{1}$ & 11 & 145 & 1 & 0 & 50 & 16 \\
\hline Baetis tricaudatis ${ }^{1} 3$ & 9603 & 12356 & 10354 & 12519 & 3061 & 16290 \\
\hline Chironomidae 3 & 9783 & 1664 & 12109 & 14511 & 21966 & 10117 \\
\hline Chironomidae pupa 13 & 690 & 77 & 1305 & 981 & 490 & 352 \\
\hline Ephemerella inermis ${ }^{2}$ & 0 & 0 & 138 & 24 & 0 & 0 \\
\hline Gammarus lacustris 3 & 4157 & 44 & 25 & 25 & 93 & 638 \\
\hline Glossophonia complanata ${ }^{1}$ & 30 & 56 & 12 & 17 & 194 & 309 \\
\hline Hyalella azteca ${ }^{3}$ & 40 & 26 & 321 & 83 & 145 & 31 \\
\hline Hydropsyche spp. ${ }^{1}$ & 3 & 14 & 156 & 52 & 28 & 46 \\
\hline Hydropsychidae & 1493 & 2393 & 7 & 0 & $<1$ & 142 \\
\hline Hydroptila spp. 123 & 11 & 275 & 352 & 982 & 3 & 224 \\
\hline Hydroptila pupa 1 & 1 & 1053 & 5 & $<1$ & 3 & 18 \\
\hline Isoperla quinquepunctata ${ }^{2}$ & 0 & 0 & 10 & 70 & 0 & 0 \\
\hline oecetis sp. 3 & 0 & 5 & 42 & 10 & 26 & 10 \\
\hline optioservus spp, ${ }^{1} 23$ & 51 & 80 & 163 & 574 & 181 & 1016 \\
\hline Physella spp. 1 & 2162 & 1387 & 138 & 31 & 3105 & 24529 \\
\hline Serratella sp. 1 & 619 & 2027 & 0 & 0 & 814 & 478 \\
\hline Simulium spp. 1 & 1686 & 6236 & 208 & 69 & 431 & 400 \\
\hline Simulium pupa ${ }^{12}$ & 83 & 276 & 156 & 26 & 43 & 10 \\
\hline Tricladida & 6001 & 5036 & 2756 & 3575 & 8924 & 11483 \\
\hline Tricorythodes minutus ${ }^{1} 2$ & 31506 & 13358 & 1792 & 2847 & 2132 & 9578 \\
\hline TOTAL NUMBER ${ }^{1} 3$ & $\begin{array}{c}38179 \\
N=23\end{array}$ & $\begin{array}{r}46572 \\
N=25\end{array}$ & $\begin{array}{r}32658 \\
N=14\end{array}$ & $\begin{array}{r}36456 \\
N=15\end{array}$ & $\begin{array}{r}41761 \\
N=15\end{array}$ & $\begin{array}{r}75670 \\
N=15\end{array}$ \\
\hline
\end{tabular}

a - refers to separation of larva and pupa within certain taxa 1,2 , and 3 - sampling period when abundance was significantly different between Rkm 2.4 and $14.5 \quad(p=0.050)$

included in this and the other two groups were sometimes confounded by asynchronous life cycles.

Hyalella azteca, oecetis, Amiocentrus aspilis larvae, and Ephemerella inermis were all more abundant at Rkm 2.4 when differences were significant (Table 2.6.4). Amiocentrus aspilis larvae were significantly more abundant at Rkm 2.4 during April $1987(\mathrm{p}<0.01)$. The pupae, however, were more abundant at Rkm 
14.5 in August $1986(p<0.01)$ and at Rkm 2.4 in September 1987 $(p=0.04)$. These differences might result from a lack of synchrony in . aspilis"s life cycle between sites, prompted by differences in water temperatures.

High densities at Rkm 2.4 in April 1987 resulted from presence of many newly hatched larvae. Early instar larvae were not present downstream. Similarly, the difference in August 1986 pupae abundance reflects earlier pupation and/or emergence at $\mathrm{Rkm}$ 14.5 than at Rkm 2.4. Four percent of $\mathrm{A}$. aspilis at the upstream site were in the pupal stage versus $69 \%$ downstream (Table 2.6.4). september 1987 differences were probably also influenced by asynchronous life cycles between sites, since this was the case during the two earliex sampling periods.

Eohemerella inermis was present only in the April 1987 samples (Table 2.6.4). Although its life cycle in the Bighorn River was not directly evaluated, late instar nymphs were collected at Bighorn Access (Rkm 19.5) the first week in August 1988. Thus, this species was probably present in the Bighorn River through most summer months, but had emerged prior to late August - early september sampling. Given this, a considerable portion of its nymph stage coincided with the periods of highest gas supersaturation levels in the river; any resulting density differences would have been missed.

Group 2. Taxa exhibiting greater abundance at Rkm 2.4 and 14.5 during different periods include: Physella spp., Simulium spp. pupae and Gammarus lacustris (Table 2.6.4). Multiple species of Physella and simulium are present in the Bighorn River. This, along with asynchronous life cycies, made 
interpretation of density differences difficult.

Physella were more abundant at Rkm 2.4 during August 1986 $(\mathrm{p}=0.01)$ and April 1987, however, differences were not significant during the latter period $(p=0.17)$. In september 1987, presence of newly hatched young resulted in snail densities being eight times greater at Rkm 14.5. Had the sites been compared at similar life cycle stages, results could have been different.

In April 1987, $43 \%$ of the Simulium were pupae at Rkm 2.4 versus $27 \%$ at $\mathrm{Rkm} 14.5$ (Table 2.6.4). This greater ratio of pupae to larvae contributed to the significant increase in upstream pupae abundance $(p=0.05)$, even though total blackfly densities were higher. During August 1986, pupae abundance at both sites corresponded closely with larvae densities.

Gammarus lacustris was $98.9 \%$ more abundant at $\mathrm{Rkm} 2.4$ than at Rkm 14.5 during August $1986(p<0.01$ ) (Table 2.6.4). Differences between sites were again significant during september 1987, although $\underline{G}$. lacustris was more common downstream.

Group 3. Baetis tricaudatis, Glossophonia complanata, Hydropsyche spp., Hydropsychidae, Hydroptila spp., Isoperla quinquepunctata, optioservus spp., serratella sp., and Tricorythodes minutus all occurred in greater densities at Rkm 14.5 when differences were significant (Table 2.6.4). Assuming aquatic invertebrates near the dam are more affected by the higher gas supersaturation there, these taxa are the ones most likely being impacted.

Baetis tricaudatis was more abundant downstream during all collections, but only significantly so during August 1986 
$(p=0.02)$ and september $1987(p<0.01)$ (Table 2.6.4). This taxa had two emergence periods in the Bighorn River. A spring emergence occurred from about mid-April through much of June, and a more limited fall emergence occurred from mid-october through mid-November (Gail Larr personal communication). It is unknown whether this results from two separate cohorts or from a single multivoltine population.

Glossophonia complanata was significantly more abundant at Rkm 14.5 in August $1986(\mathrm{p}<0.01)$. It also had greater downstream densities in April and September 1987, but differences were not significant (Table 4 ).

Hydropsyche spp. and Hydropsychidae were significantly more abundant at Rkm 14.5 only in August $1986(p=0.03$ and 0.01 , respectively). Early instar larvae were identified to family level only. All mid to late instar larvae collected in benthic samples, however, were Hydropsyche.

Hydroptila spp. occurred in significantly greater numbers at Rkm 14.5 during all sampling periods. Although we were not able to differentiate individual species and their life cycles, pupae to larvae ratios indicate that life cycles between sites were not synchronized. Even so, the magnitude of difference in Hydroptila densities during all periods indicates that this taxa is probably more common downstream.

optioservus spp. also occurred in significantly greater densities at Rkm 14.5 during all sampling periods. Most larvae collected are thought to be $\underline{0}$. divergens.

I. guinguepunctata had emerged prior to the late sumer/early fall sampling periods, thus were present only in the 
spring samples. April 1987 densities were seven times greater at Rkm 14.5 (p<0.01) (Table 2.6.4).

Serratella sp. was present only in late summer and fall collections. Densities were significantly different between sites only during August 1986 ( $p<0.01)$, with downstream abundance three times greater than upstream (Table 2.6.4). Although estimated densities were 1.7 times greater at $\mathrm{Rkm} 2.4$ in September 1987 , they were not significantly different from densities upstream. Nymphs probably appear in early August and emerge sometime prior to early May. Thus, they are not present in the river during the period of highest gas saturation levels. Tricorythodes minutus, was one of only three taxa that were significantly more abundant at Rkm 14.5 during all three collection periods. Adults emerge for approximately 1 month beginning about the third week in september (Gail Larr personal communication). Thus, nymphs are present during the period of highest gas saturation levels in the Bighorn River. Additionally, August and september sampling was likely done prior to the occurrence of any extensive emergence and probably provide reliable comparisons of $\underline{T}$. minutus abundance.

Community structure: Community structure differed markedly between sites during August and september, but was similar during Apri1. In August 1986, percentages of Chironomidae, Tricladida, and Gammarus lacustris in the community decreased from $26 \%, 16 \%$, and $11 \%$, respectively, at the upstream site to $4 \%, 11 \%$, and $0 \%$ at the downstream site (Figure 2.6 .6$)$. In contrast, Tricorythodes and Simulium gained in representation downstream from $4 \%$ each, to $29 \%$ and $13 \%$, respectively. 


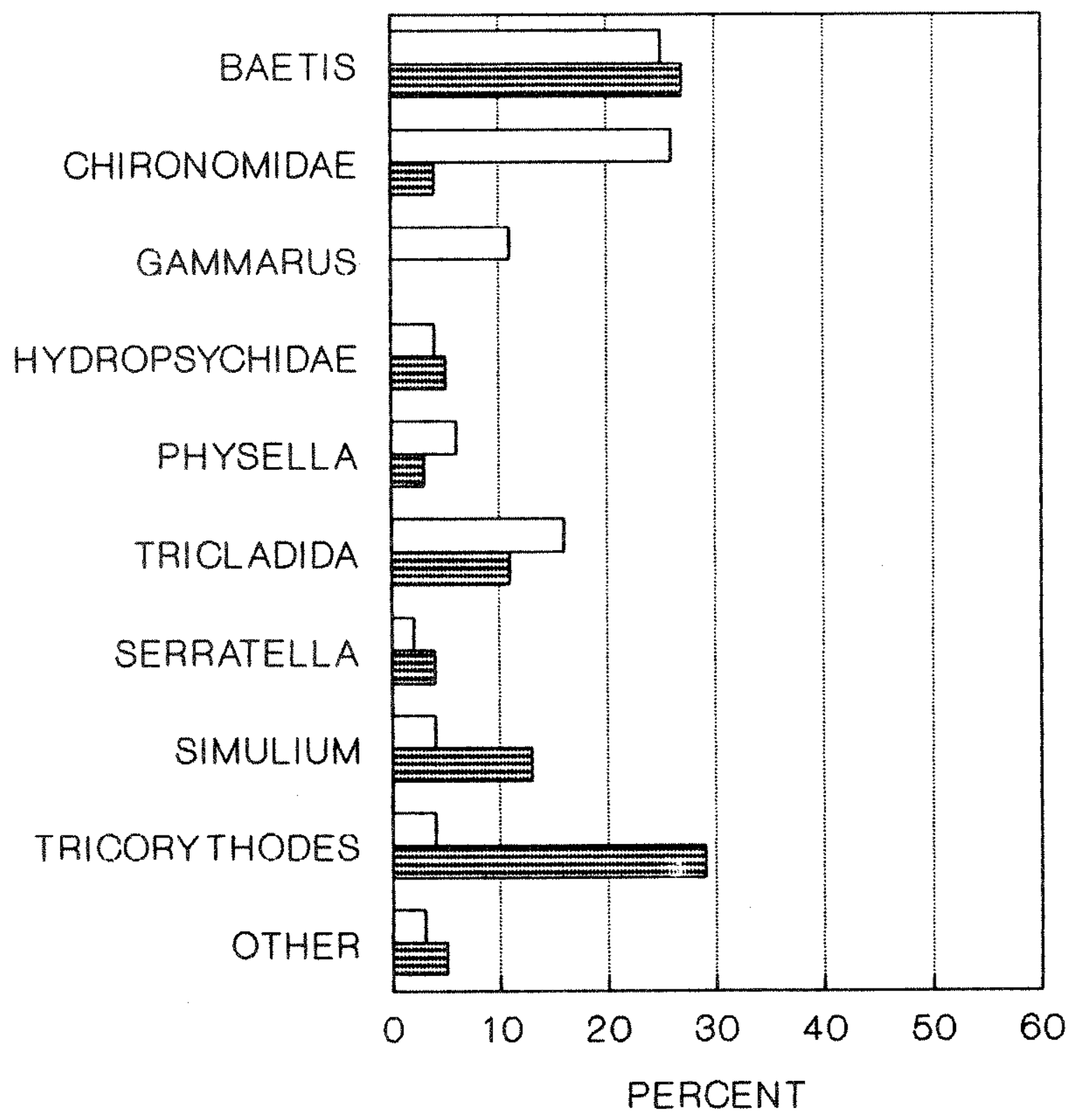

$\square$ RKM $2.4 \cong R K M 14.5$

Figure 2.6.6. Mean percent of total invertebrate abundance comprised by the nine most common taxa, at Rkm 2.4 and 14.5 , Bighorn River, August 1986. 
In september 1987, abundance of Chironomidae and Tricladida was lower downstream, while abundance of $\underline{B}$. tricaudatis, Physella sp., and Tricorythodes was higher (Figure 2.6.7). A large number of small individuals was responsible for the increase in Physella.

Community structure during April 1987, was remarkably similar between sites. A. aspilis, the only taxa exhibiting a major difference in representation, decreased from $8 \%$ (2608 organisms per $\mathrm{m}^{2}$ ) upstream to less than $1 \%$ (60 organisms per $\mathrm{m}^{2}$ ) downstream (Figure 2.6.8). Slightly greater percent representation of $\underline{B}$. tricaudatis, Chironomidae, Tricladida, and T. minutus at $\mathrm{Rkm} 14.5$ resulted from A. aspilis' decreased abundance.

Drift: Invertebrate drift was dominated by chironomids (pupae and larvae) and $\underline{B}$. tricaudatis during both collection periods. In April 1987, drift densities of five invertebrate groups were 0.1 to 12.2 organisms per cubic meter (Table 2.6.5). In september 1987,10 groups dominated the samples. The increase in drift densities coincided with fall increases in total benthic abundance. These increases were commonly due to recruitment of early instar organisms.

To determine whether differences in drift densities between Rkm 2.4 and 14.5 resulted from different benthic densities, drift rate at each site was compared to benthic abundance. In April 1987, Baetis, chironomid pupae, and Simulium exhibited proportionately greater drift rates at $\mathrm{Rkm} 14.5$, relative to their benthic densities (Figure 2.6.9). Although gas saturation levels were not measured when drift samples were collected (April 


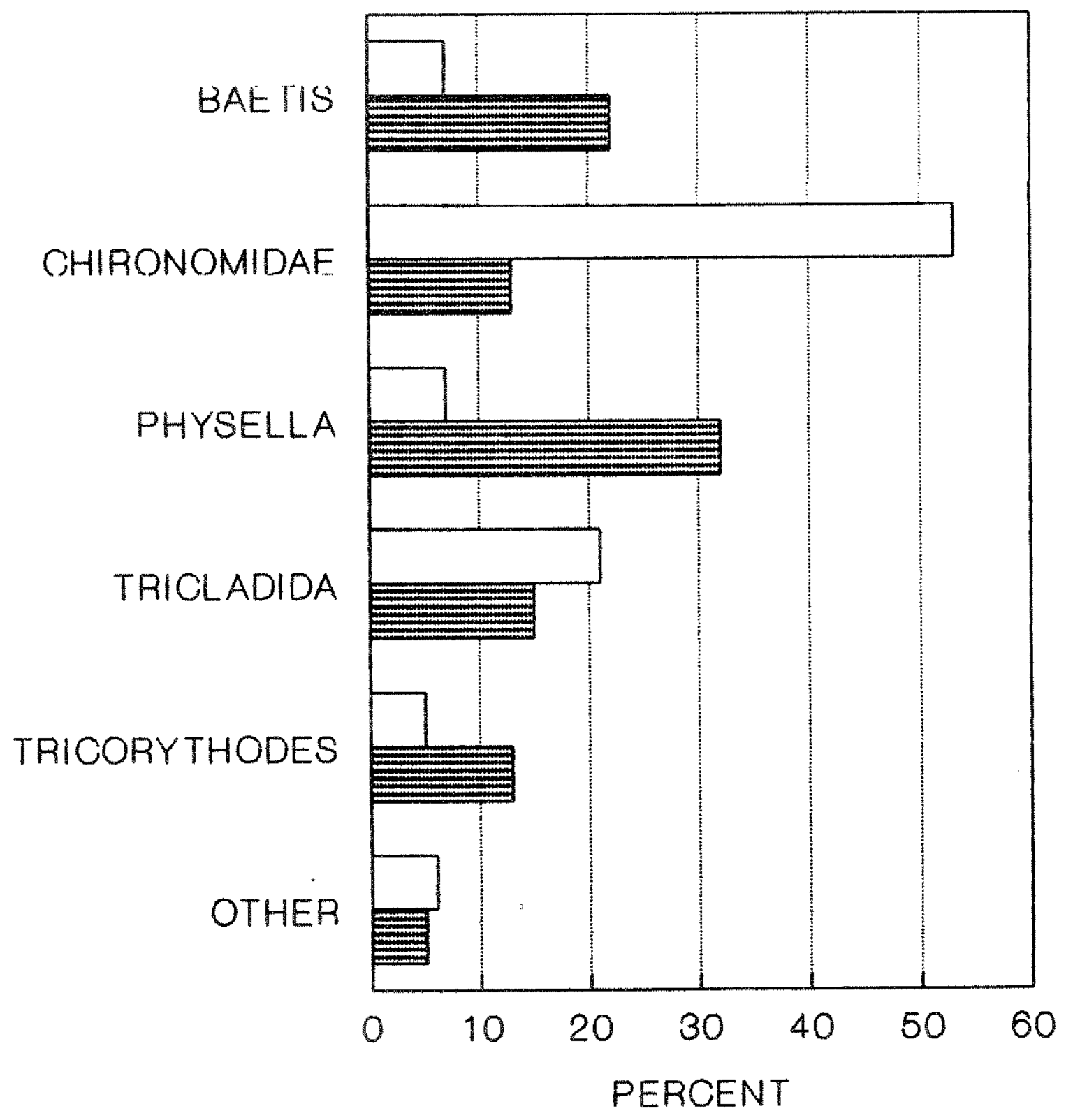

$\square R K M 2.4 \cong R K M 14.5$

Figure 2.6.7. Mean percent of total invertebrate abundance comprised by the five most common taxa, at Rkm 2.4 and 14.5 , Bighorn River, september 1987. 


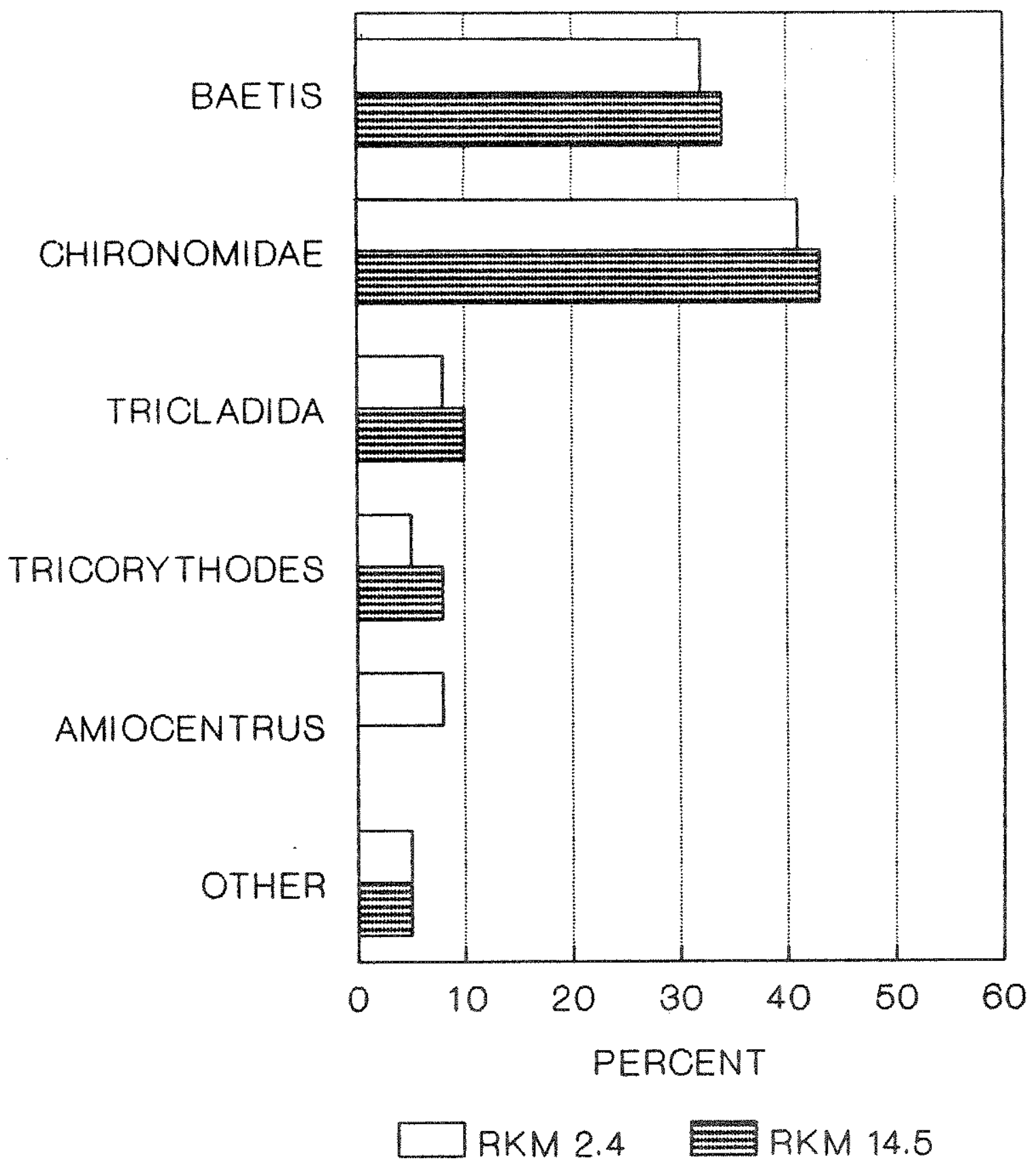

Figure 2.6.8. Mean percent of total invertebrate abundance comprised by the five most common taxa, at $\mathrm{Rkm} 2.4$ and 14.5 , Bighorn River, April 1987. 
Table 2.6.5. Mean drift densities (\#/ $\mathrm{m}^{3}$ ) and standard deviations at $\mathrm{Rkm} \mathrm{2.4}$ and 14.5 , for those taxa most commonly present in the drift during collection periods 2 and 3 , Bighorn River.

\begin{tabular}{|c|c|c|c|c|c|c|c|c|}
\hline \multirow{2}{*}{$\begin{array}{l}\text { Taxanomic } \\
\text { group }\end{array}$} & \multicolumn{2}{|c|}{ APRIL 15, } & \multicolumn{2}{|l|}{1987} & \multicolumn{2}{|c|}{ SEPTEMBER } & \multicolumn{2}{|c|}{24,1987} \\
\hline & $\frac{\mathrm{RKM}}{\overline{\mathrm{x}}}$ & $\begin{array}{l}2.4 \\
\text { S.D. }\end{array}$ & $\underset{\overline{\mathrm{x}}}{\mathrm{RKM}}$ & $\begin{array}{r}14.5 \\
\text { S.D. }\end{array}$ & $\underset{\overline{\mathbf{x}}}{\mathrm{RKM}}$ & $\begin{array}{l}2.4 \\
\text { S.D. }\end{array}$ & $\underset{\overline{\mathrm{x}}}{\mathrm{RKM}}$ & $\begin{array}{l}14.5 \\
\text { S.D. }\end{array}$ \\
\hline Baetis & 4.1 & 2.4 & 11.7 & 2.8 & 9.8 & 2.8 & 112.2 & 36.7 \\
\hline Tricorythodes & 0.1 & 0.0 & $\mathrm{p} /$ & -- & 2.3 & $1 \cdot 4$ & 2.2 & 1.1 \\
\hline serratella & a/ & -- & a/ & $-\infty$ & 1.6 & 0.4 & $2 \cdot 3$ & 1.0 \\
\hline Physel1a & a) & $-\infty$ & a/ & $-\infty$ & 2.7 & 1.1 & 12.6 & 10.2 \\
\hline Chironomidae (I) & 12.2 & 7.5 & 7.5 & 3.0 & 8.2 & 2.1 & 3.9 & 3.2 \\
\hline Chironomidae (P) & 4.6 & 1.9 & 5.8 & 1.4 & 7.8 & 1.8 & 5.4 & 1.6 \\
\hline Simulium (L) & 0.1 & 0.1 & 0.1 & 0.1 & 0.5 & 0.2 & 0.1 & 0.1 \\
\hline Gammarus & $\mathrm{p} /$ & --- & $\mathrm{p} /$ & --- & 0.4 & 0.3 & 0.5 & 0.2 \\
\hline Hyalella & $\mathrm{p} /$ & -- & $\mathrm{p} /$ & -- & 0.6 & 0.2 & 0.1 & 0.1 \\
\hline Tricladida & $\mathrm{p} /$ & --- & a/ & -- & 3.1 & 2.0 & 3.5 & 3.2 \\
\hline $\begin{array}{l}\text { a/ absent from dr } \\
\text { p/ present in sam } \\
\text { less than } 0.1 \\
\text { (L) larvae } \\
\text { (P) pupae }\end{array}$ & $t s$ & $\begin{array}{l}\text { mples } \\
\text { but }\end{array}$ & $n d r$ & ift & $i t$ & es were & much & \\
\hline
\end{tabular}

13 and 14), delta $\mathrm{p}$ trends indicate that gas levels were greater at this site (Appendix K: Figure 29).

In september, drift densities of $\underline{B}$. tricaudatis, chironomid larvae and pupa, Physella, Hyalella and triclads were proportionately close to benthic densities at Rkm 14.5 (Figure 2.6.10). This suggests that drift rate differences between sites, for these taxa, are associated with differences in benthic abundance.

Tricorythodes, Simulium, and Gammarus had lower drift rates downstream relative to their benthic abundance, while serratella 


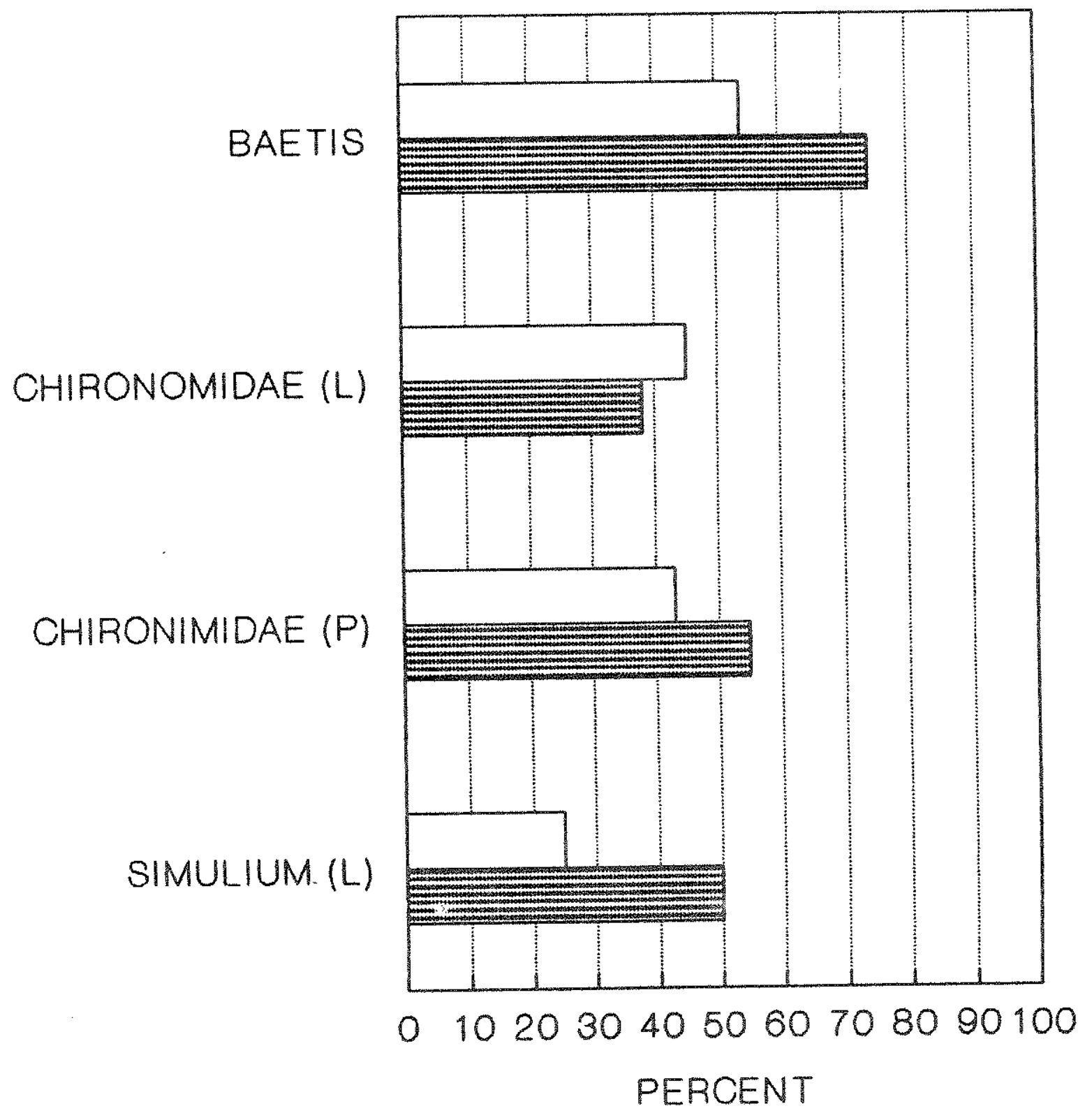

$\square$ BENTHIC $\cong D R I F T$

Figure 2.6.9. Mean percent of Baetis tricaudatis, Chironomidae larvae and pupae, and Simulium larvae collected at $\mathrm{Rkm} 14.5$, in benthic and drift samples, Bighorn River, April 1987. 


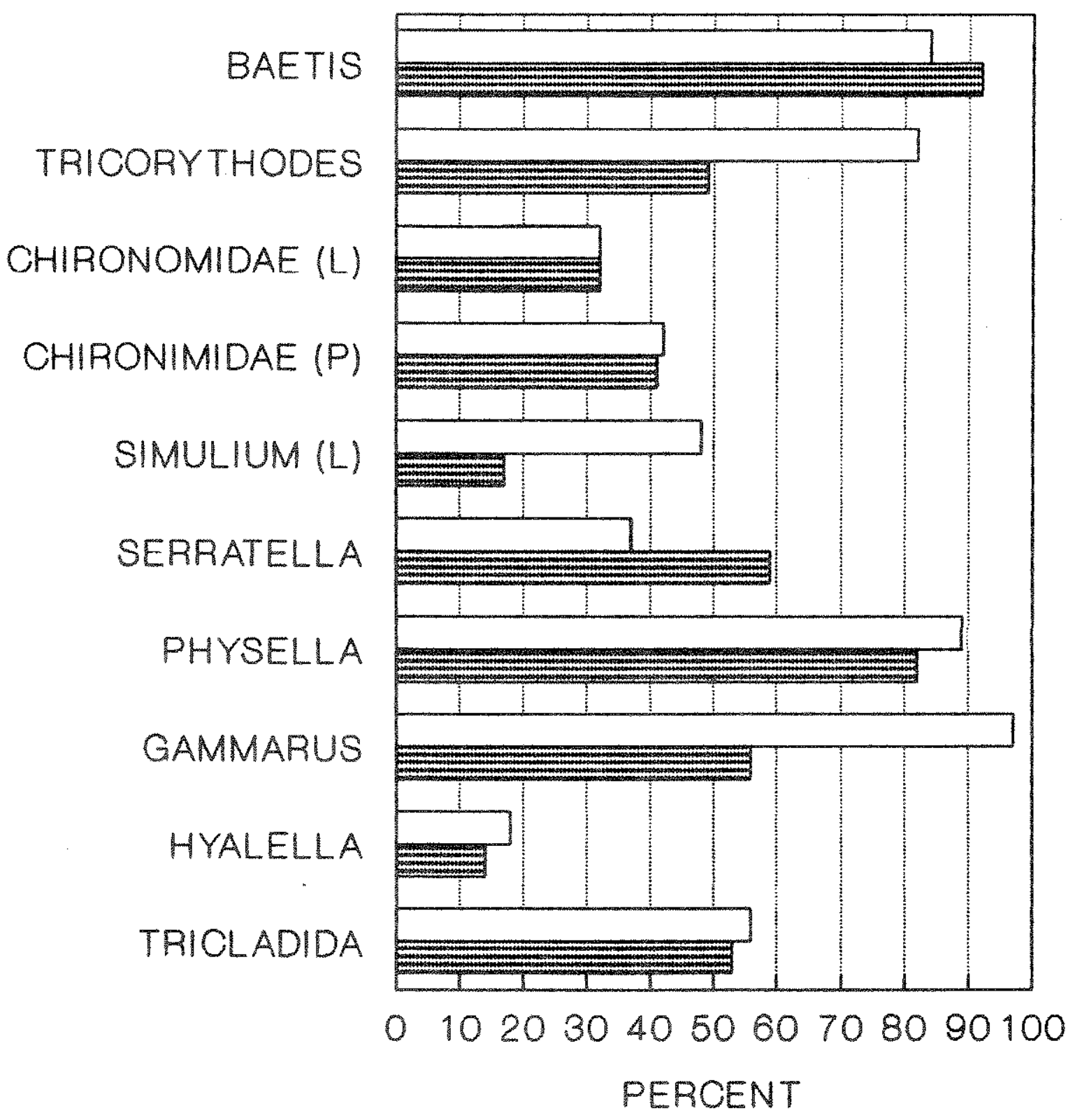

$\square$ BENTHIC DDIFT

Figure 2.6.10. Mean percent of nine taxa collected at Rkm 14.5 in benthic and dxift samples, Bighorn River, september 1987. 
drift rates were higher. The delta $p$ trend during the sampling period (september 22 and 23) indicates that gas saturation levels were higher at the upstream site (Appendix K: Figure 30).

site characteristics: Mean daily discharges were assumed to be the same at both sites during each sampling period.

Discharges near Afterbay Dam remained at $85 \mathrm{~m}^{3} / \mathrm{s}$ during sampling in August 1986 and at $99 \mathrm{~m}^{3} / \mathrm{s}$ in september 1987. In April 1987, discharges decreased from $56 \mathrm{~m}^{3} / \mathrm{s}$ on the first sampling day to 50 $\mathrm{m}^{3} / \mathrm{s}$ the following day.

other site specific characteristics are addressed in four parts: 1) "Associated Habitat Characteristics" - includes water velocities, water depths, and dry weights of vegetation (DWV) collected in each benthic sample. 2) Periphyton - includes periphytic ash-free dry weights (AFDW), and chlorophyll a concentrations. Ash-free dry weights were used to quantify standing crops of aquatic plants at sampling riffles, and to determine if DWV accurately depicted vegetation abundance; 3) Temperature; and 4) Gas saturation.

Associated Habitat characteristics. The greatest variation in water depth, water velocity, and DWV (Table 2.6.6) occurred between sampling periods, although some differences between sites during a single sampling period were significant. of the habitat characteristics measured during August 1986, only water depth varied significantly between sites $(p=0.05)$, averaging $0.1 \mathrm{~m}$ deeper at $\mathrm{Rkm} 2.4$. Water velocity differences at 0.6 depth approached significance $(p=0.08)$ with the faster water upstream, while differences in DWV were insignificant $(p=0.72)$.

In April 1987, differences in water depth neared 
Table 2.6.6. Mean water depths, water velocities $(M=0.6$ depth: $B=3$ to $5 \mathrm{~cm}$ above the substrate), and dry weight of vegetation (DWV), measured for each benthic sample, according to site and collection period. Ranges are in parentheses.

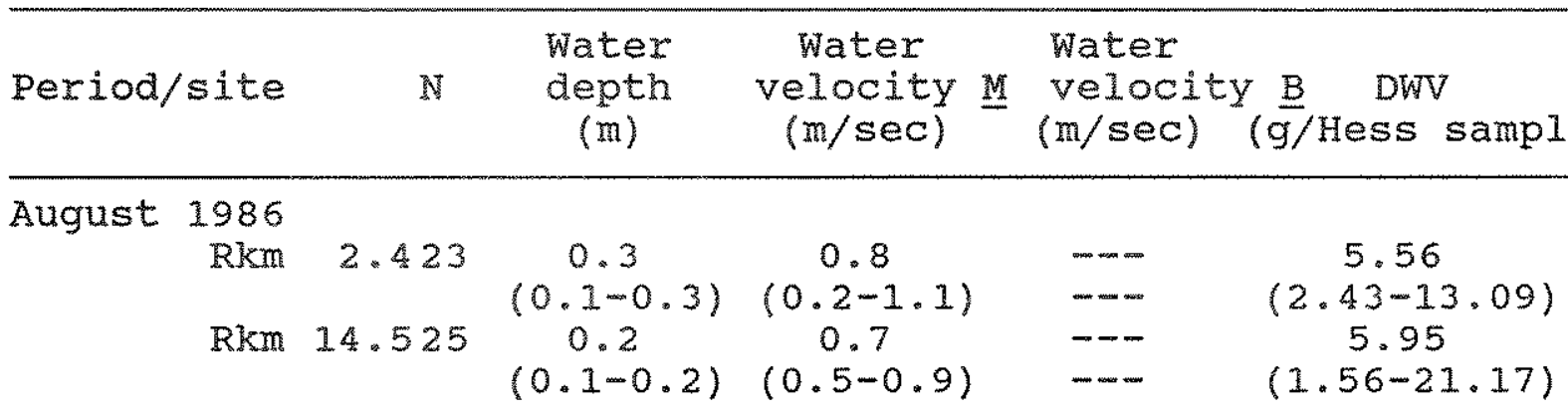

April 1987

$\begin{array}{lccccc}\text { Rkm } & 2.415 & 0.3 & 0.4 & 0.1 & 12.29 \\ \text { Rkm } 14.514 & (0.1-0.4) & (0.1-0.9) & (0.0-0.3) & (2.21-26.56) \\ & 0.2 & 0.5 & 0.2 & 10.91 \\ & & (0.2-0.3) & (0.2-0.7) & (0.0-0.5) & (2.44-23.52)\end{array}$

September 1987
Rkm 2.415
0.2
0.3
0.1
6.62
Rkm 14.515
$(0.1-0.2)$
0.2
$(0.2-0.5)$
0.6
$(0.0-0.2)$
0.2
$(2.34-15.94)$
12.98
$(0.1-0.2)$
$(0.2-0.9)$
$(0.0-0.3)$
$(7.56-24.35)$

significance $(p=0.07)$, with greater depths occurring at $\mathrm{Rkm} 2.4$ (Table 2.6.6). Mean water velocities at 0.6 depth were greater, although not significantly different at the downstream site.

Velocities near the substrate, however, were significantly different $(p=0.02)$, averaging $0.1 \mathrm{~m} / \mathrm{s}$ faster at $\mathrm{Rkm} 14.5$. Means for DWV were similar, indicating comparable vegetative standing crops between riffles. Correlations between water velocity and individual taxa in the Bighorn River, however, were poor. oddly, invertebrate covariance correlations were commonly higher with velocities at 0.6 depth than with velocities at the substrate. This may result from the lack of an effective means of measuring substrate velocities. 
The largest differences in physical characteristics (Table $2.6 .6)$ between sites occurred during september 1987. Average water velocity $(0.6$ depth) and vegetation abundance (DWV) were approximately $100 \%$ greater at the downstream site $(p<0.01)$. Correlations between invertebrate numbers and DWV were not strong, possibly indicating that the specific type of vegetation, not overall abundance, was important for individual taxa.

Sampling sites were subdivided in April 1987 (Figure 2.6.2) to determine if certain subsections from each site were more comparable than others. Some differences between subsections (Table 2.6.7) were present for each characteristic measured. However, comparable subsections changed with the physical parameter used to evaluate them. Thus, no unique combination of subsections could be used to compare upstream and downstream invertebrate communities. These results did show that some parameters differed significantly within sites but not between sites when the entire riffles were compared.

Periphyton. Ash-free dry weight (AFDW) (Table 2.6.8) showed trends similar to those from dry weights of vegetation (DWV) (Table 2.6.7). Some differences were found among subsections in April 1987 (Tables 7 and 8), probably because of smaller sample size. Both AFDW and DWV indicated that sampling sites had similar vegetation abundance in April 1987, but variance was large. In september 1987 a significantly greater standing crop of vegetation was present at $\mathrm{Rkm} 14.5 \quad(\mathrm{p}=0.01)$.

In April 1987, chlorophyl1 a concentrations were similar between sites, but differences were present between subsections within the upstream site. Mean concentrations in subsections 4 
Table 2.6.7. Mean water depths, water velocities (M $=0.6$ depth: $\underline{B}=3$ to $5 \mathrm{~cm}$ above the substrate), and dry weight of vegetation (DWV) associated with benthic samples collected in April 1987 in each riffle subsection. Ranges are in parentheses.

\begin{tabular}{|c|c|c|c|c|}
\hline $\begin{array}{l}\text { Site/ } \\
\text { subsection }\end{array}$ & $\mathrm{N}$ & $\begin{array}{l}\text { Watex } \\
\text { depth } \\
(m)\end{array}$ & $\begin{array}{l}\text { Water } \\
\text { velocity M } \\
(\mathrm{m} / \mathrm{sec})\end{array}$ & $\begin{array}{l}\text { Water } \\
\text { velocity B DWV } \\
\text { (m/sec) (g/Hess sample) }\end{array}$ \\
\hline
\end{tabular}

Rkm 2.4

$\begin{array}{ccccccc}1 & 3 & 0.2 & 0.2 & 0.1 & 5.30 \\ 2 & & 5 & (0.2-0.3) & (0.1-0.3) & (0.0-0.1) & (4.98-5.87) \\ 3 & & & 0.4 & 0.5 & 0.1 & 15.64 \\ 3 & 4 & (0.3-0.4) & (0.2-0.9) & (0.0-0.2) & (11.79-17.97) \\ 4 & & & (0.2 & 0.6 & 0.1 & 13.43 \\ 4 & 3 & 0.2 & 0.3) & (0.6-0.7) & (0.0-0.3) & (2.21-26.56) \\ & & & (0.1-0.2) & (0.1-0.4) & (0.0-0.2) & (3.80-18.82)\end{array}$

Rkm 1.4.5

$\begin{array}{llccccc}5 & 7 & 0.2 & 0.5 & 0.2 & 5.52 \\ & & (0.2-0.3) & (0.2-0.6) & (0.0-0.5) & (2.44-10.22) \\ 6 & 7 & 0.2 & 0.5 & 0.2 & 16.30 \\ & & (0.2-0.2) & (0.4-0.7) & (0.1-0.5) & (9.91-23.52)\end{array}$

and 5 were $60 \%$ and $71 \%$ of those in subsection 6 (Table 2.6.8) $(\mathrm{p}<0.01)$. Chlorophyll a data were not collected in september 1987.

Water Temperature. Watex temperatures at $\mathrm{Rkm} 14.5$ began to exceed those at Rkm 2.4 in early spring during both years of the study. These differences persisted until late fall (Figures 2.6.11 and 2.6.12). Temperature disparity between sites was probably the primary cause of asynchronous life cycles in many of the taxa collected.

In 1986 the highest and lowest mean weekly water temperatures occurred at Rkm 14.5 (Figure 2.6.11). Temperatures were similar at both sites in January, but were cooler downstream during Feoruary. Water temperatures were wamer at Rkm 14.5 from 
Table 2.6.8. Mean and standard deviation of periphytic AFDW $\left(9 / \mathrm{cm}^{2} \cdot 10^{-3}\right)$ and Chlorophyll a concentration ( $\mu \mathrm{g} / \mathrm{g}$ of blotted wet weight) at $\mathrm{Rkm} 2.4$ and 14.5 for April and september 1987 sampling periods. Data are also presented by subsection for April 1987.

\begin{tabular}{|c|c|c|c|c|c|c|c|}
\hline \multirow{2}{*}{\multicolumn{2}{|c|}{$\begin{array}{l}\text { Period/site/ } \\
\text { subsection }\end{array}$}} & \multicolumn{3}{|c|}{$\begin{array}{c}\text { Ash-free dry } \\
\text { weight }\end{array}$} & \multicolumn{3}{|c|}{$\begin{array}{c}\text { Chlorophyll } \\
\text { a }\end{array}$} \\
\hline & & mean & S.D. & $\mathrm{N}$ & mean & S.D. & $\mathrm{N}$ \\
\hline \multicolumn{8}{|c|}{ April 1987} \\
\hline $\mathrm{Rkm}$ & $\begin{array}{l}2.4 \\
1 \\
2 \\
3 \\
4\end{array}$ & $\begin{array}{r}6.34 \\
38.52 \\
13.47 \\
17.75\end{array}$ & $\begin{array}{r}3.40 \\
50.62 \\
8.27 \\
27.61\end{array}$ & $\begin{array}{r}6 \\
15 \\
5 \\
7\end{array}$ & $\begin{array}{l}683.0 \\
659.9 \\
697.4 \\
428.3\end{array}$ & $\begin{array}{r}324.8 \\
172.2 \\
104.3 \\
94.7\end{array}$ & $\begin{array}{r}6 \\
18 \\
5 \\
6\end{array}$ \\
\hline \multicolumn{2}{|l|}{ SITE } & 24.47 & 38.17 & 33 & 628.1 & 199.3 & 35 \\
\hline Rkm & $\begin{array}{c}14.5 \\
5 \\
6\end{array}$ & $\begin{array}{l}11.64 \\
65.61\end{array}$ & $\begin{array}{r}6.79 \\
60.30\end{array}$ & $\begin{array}{l}20 \\
13\end{array}$ & $\begin{array}{l}509.0 \\
718.8\end{array}$ & $\begin{array}{l}155.1 \\
196.1\end{array}$ & $\begin{array}{l}20 \\
11\end{array}$ \\
\hline SITE & & 32.90 & 45.91 & 33 & 583.5 & 196.1 & 31 \\
\hline \multicolumn{8}{|c|}{ sept. 1987} \\
\hline $\mathrm{Rkm}$ & 2.4 & 11.34 & 10.90 & 34 &.--- &.--- & -- \\
\hline Rkm & 14.5 & 17.92 & 10.11 & 37 &.--- &.--- & -- \\
\hline
\end{tabular}

the second week in March through the third week of October. Temperatures were warmer downstream by the third week in January 1987 (Figure 2.6.12). This difference persisted until the third week in october. The minimum average weekly temperature was similar between sites, while the maximum was approximately $1^{\circ} \mathrm{C}$ warmer at $\mathrm{Rkm} 14.5$ (Figure 2.6.12).

Temperature differences between sites were substantially larger in 1987, likely associated with lower flows during the second year of the study.

Gas Saturation. Gas saturation levels were generally higher at $\mathrm{Rkm} 2.4$ than at $\mathrm{Rkm} 14.5$ during 1986, except for a few periods 


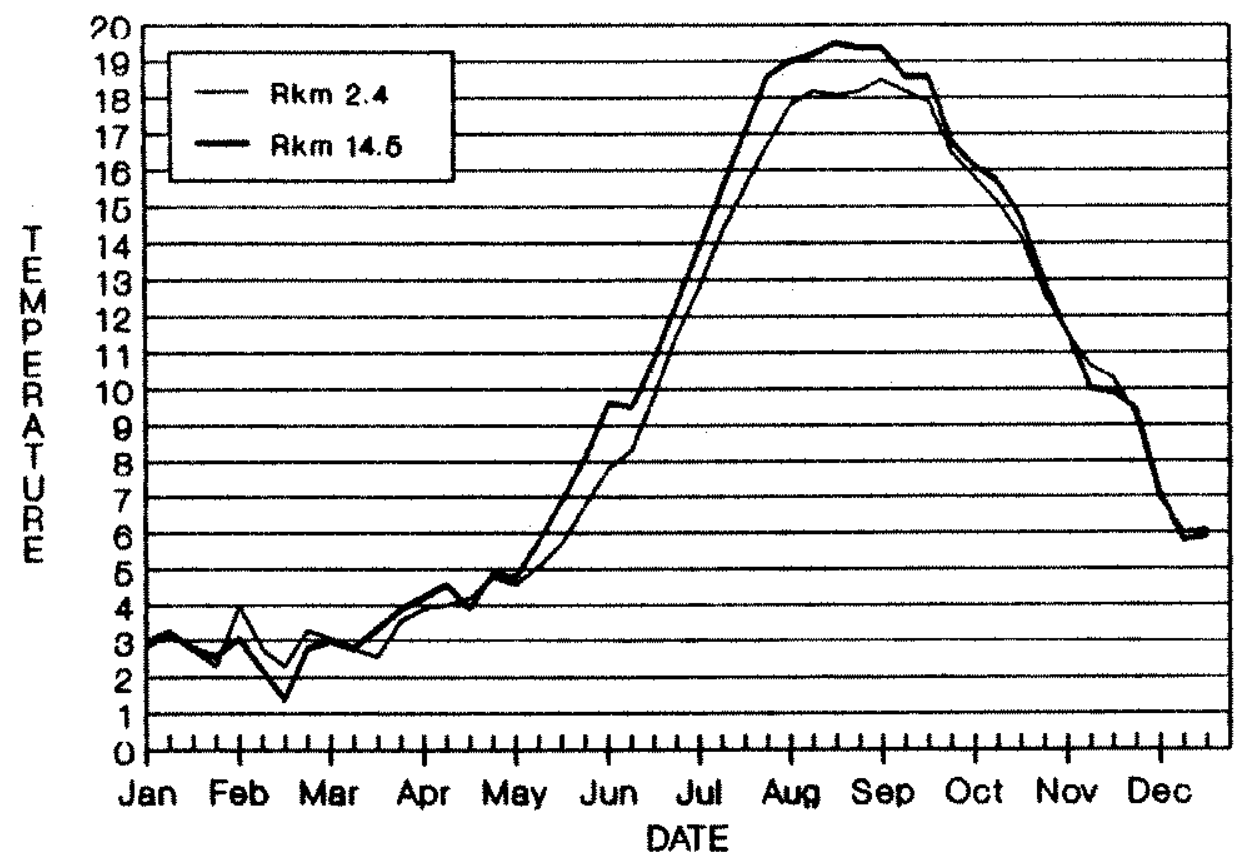

Figure 2.6.11. Mean weekly, Bighorn River water temperatures ('C) at $\mathrm{Rkm} \mathrm{2.4}$ and $14.5,1986$.

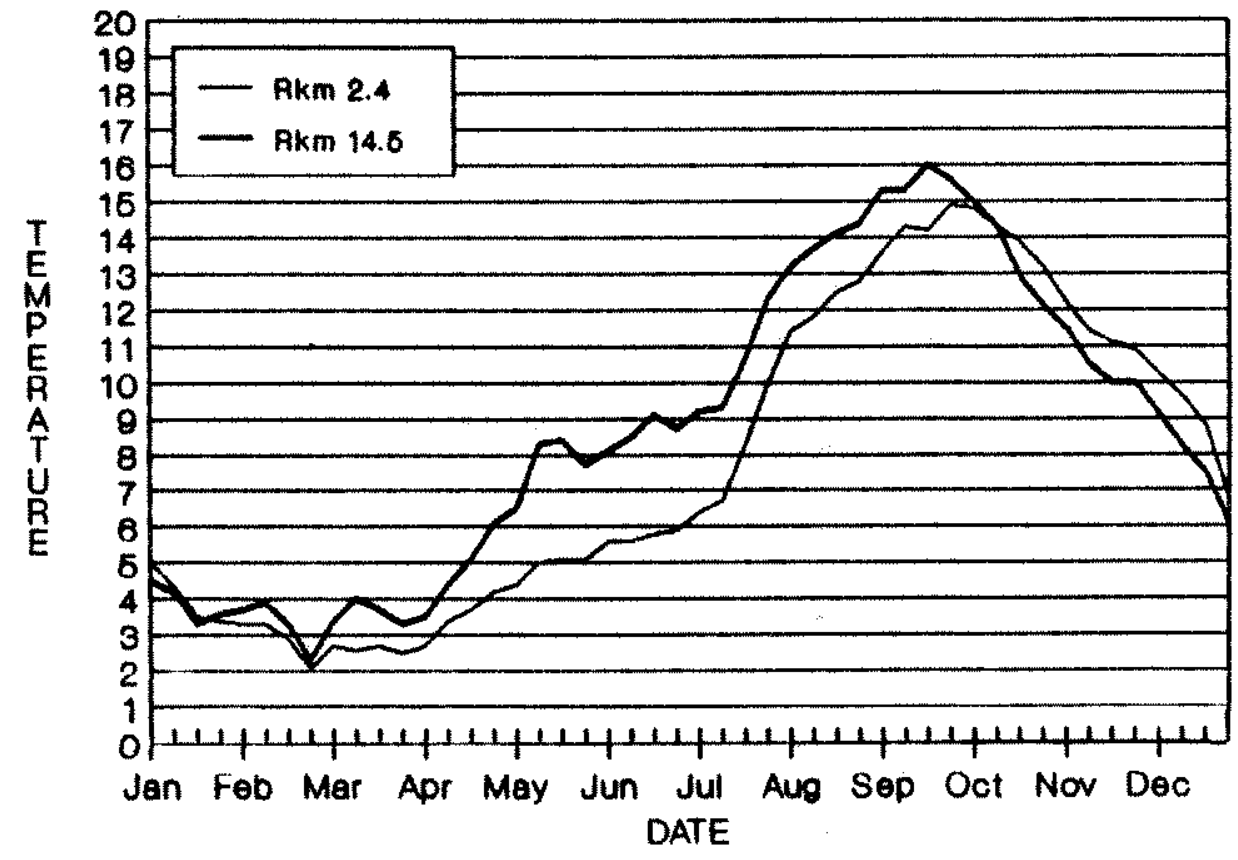

Figure 2.6.12. Mean weekly Bighorn River water temperatures $\left({ }^{\circ} \mathrm{C}\right)$ at $\mathrm{Rkm} 2.4$ and $14.5,1987$. 
between late June and mid-August (Figure 2.6.13). The highest gas saturation levels occurred at Rkm 14.5 on June 30 and July 2

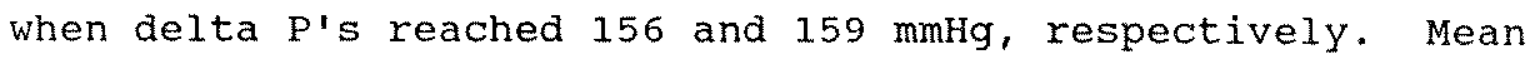
monthly gas levels were greater upstream throughout the year, except in July when Rkm 14.5 delta $P^{\prime} \mathrm{s}$ averaged $2.1 \mathrm{mmHg}$ higher (Figure 2.6.14).

Gas saturation levels during 1987 at Rkm 14.5 exceeded those at $\mathrm{Rkm} 2.4$ much more frequently than the previous year (Figure 2.6.15). Higher downstream delta P's were observed on at least one occasion in each of the first 10 months of the year. As in 1986, the highest delta $\mathrm{P}$ levels were recorded at Rkm 14.5, with gas pressures reaching $191 \mathrm{mmHg}$. The highest delta $\mathrm{P}$ recorded at the upstream site was $154 \mathrm{mmHg}$.

Mean monthly gas saturation levels were generally higher in 1987 than for the same months in 1986, with less disparity between sites. Downstream average delta $\mathrm{P}^{\prime} \mathrm{s}$ exceeded those at Rkm 2.4 during April, July, and August (Figure 2.6.16).

The composition of supersaturated gases varied between sites, with oxygen $\left(\mathrm{O}_{2}\right)$ partial pressures always influencing delta $P^{\prime}$ s more at Rkm 14.5 than at $\mathrm{Rkm} 2.4$. In 1986, the percentage of total delta $\mathrm{P}$ due to $\mathrm{O}_{2}$ at $\mathrm{Rkm} 14.5$ ranged from $36 \%$ in January to $76 \%$ in August. Values for the same months at Rkm 2.4 were $11 \%$ and $17 \%$, respectively (Figure 2.6.17). This trend was repeated in 1987 . In 5 of 12 months, the contribution of $\mathrm{O}_{2}$ to total delta $\mathrm{P}^{\prime}$ s averaged $70 \%$ or more downstream, but averaged no higher than $28 \%$ in any month at Rkm 2.4 (Figure 2.6.18).

Invertebrate Bioassays: Eight macroinvertebrate taxa were tested under controlled bioassay conditions. Taxa were selected 

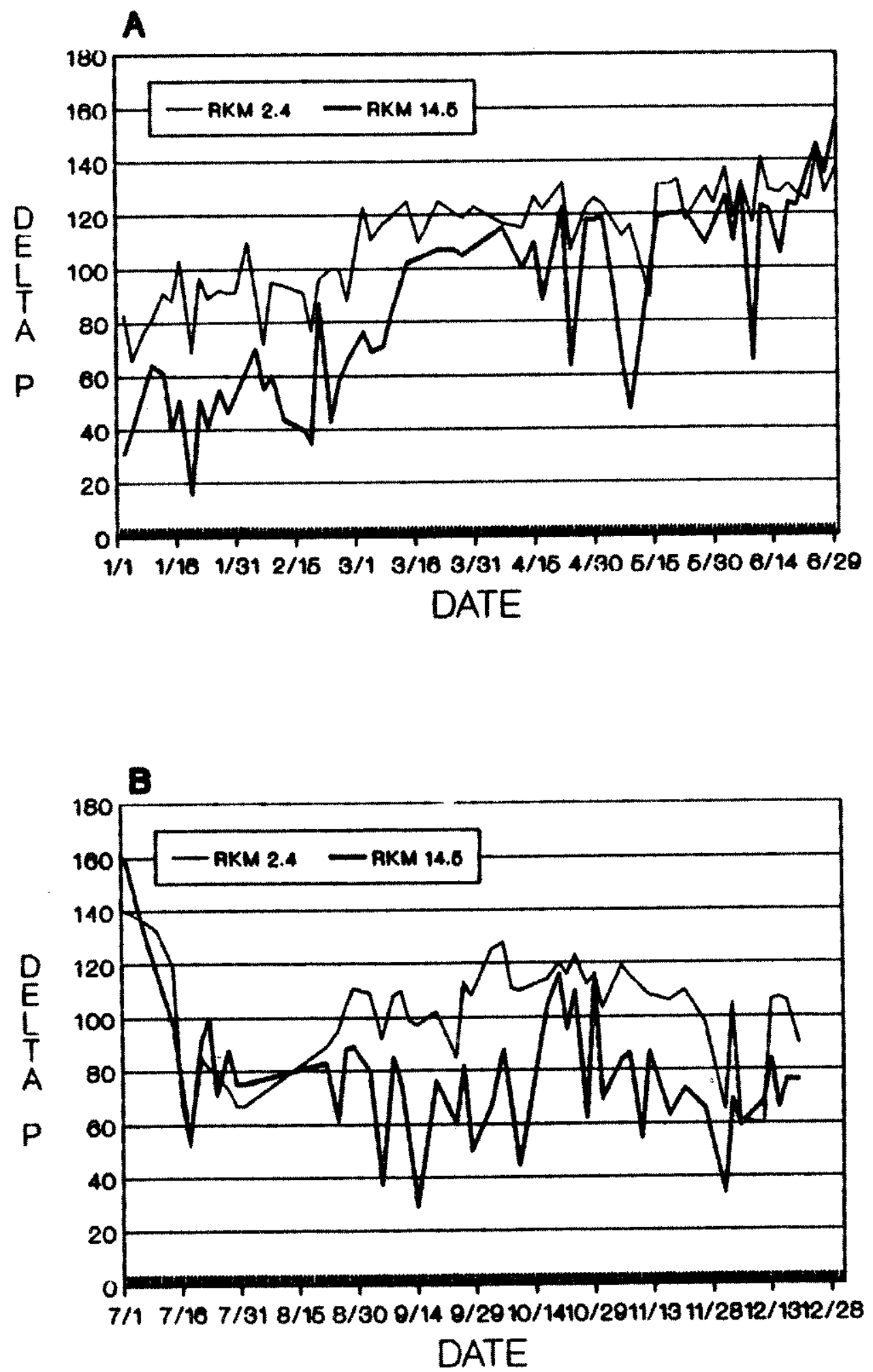

Figure 2.6.13. Delta $p$ levels (mmHg) measured two or three times weekly on the Bighorn River, at Rkm 2.4 and 14.5 , from January 1 through June 30 (A) and from July 1 through December 31 (B) 1986 . 


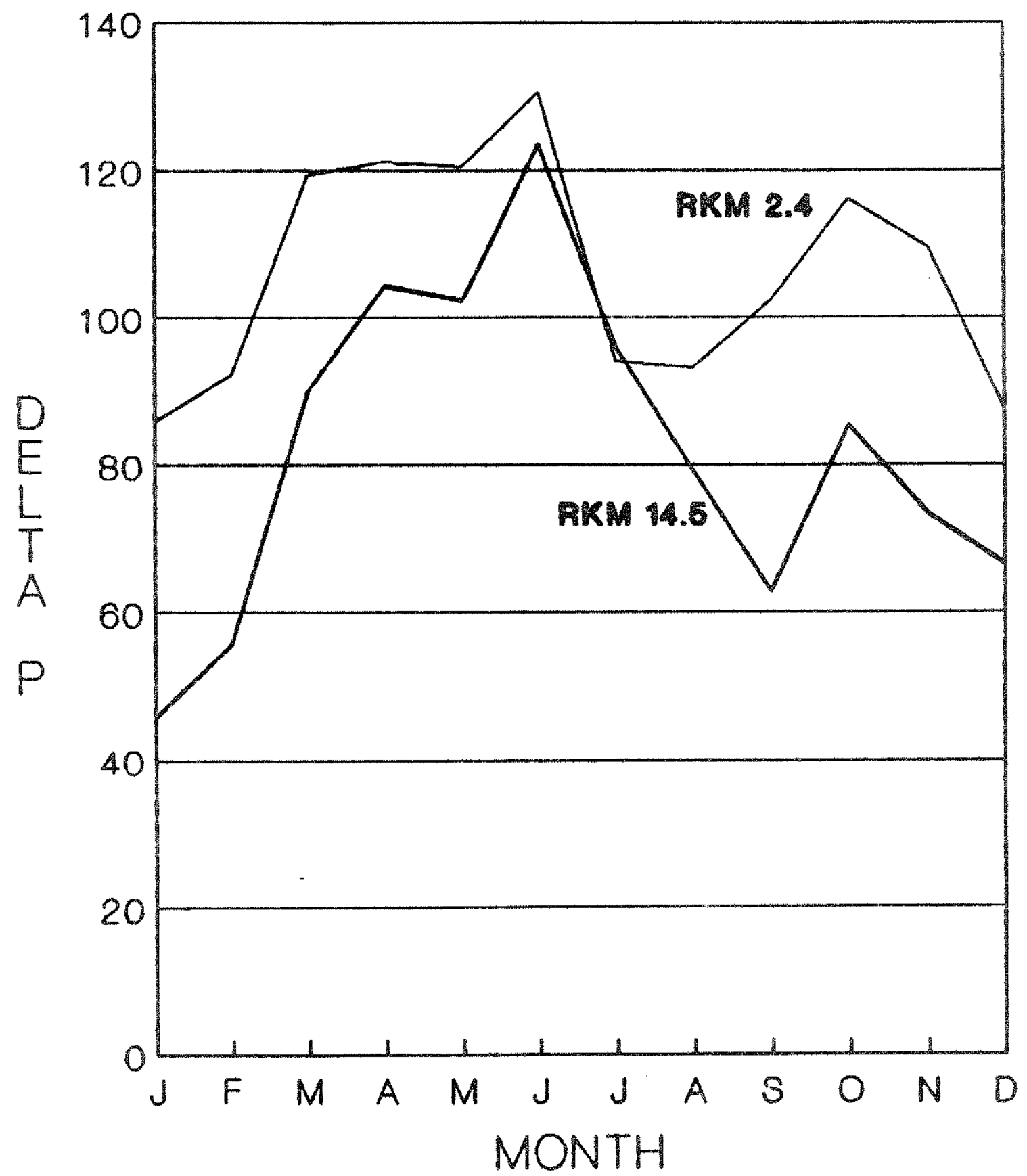

Figure 2.6.14. Mean monthly delta $p$ levels (mmHg) at Rkm 2.4 and 14.5, Bighorn River, 1986. 


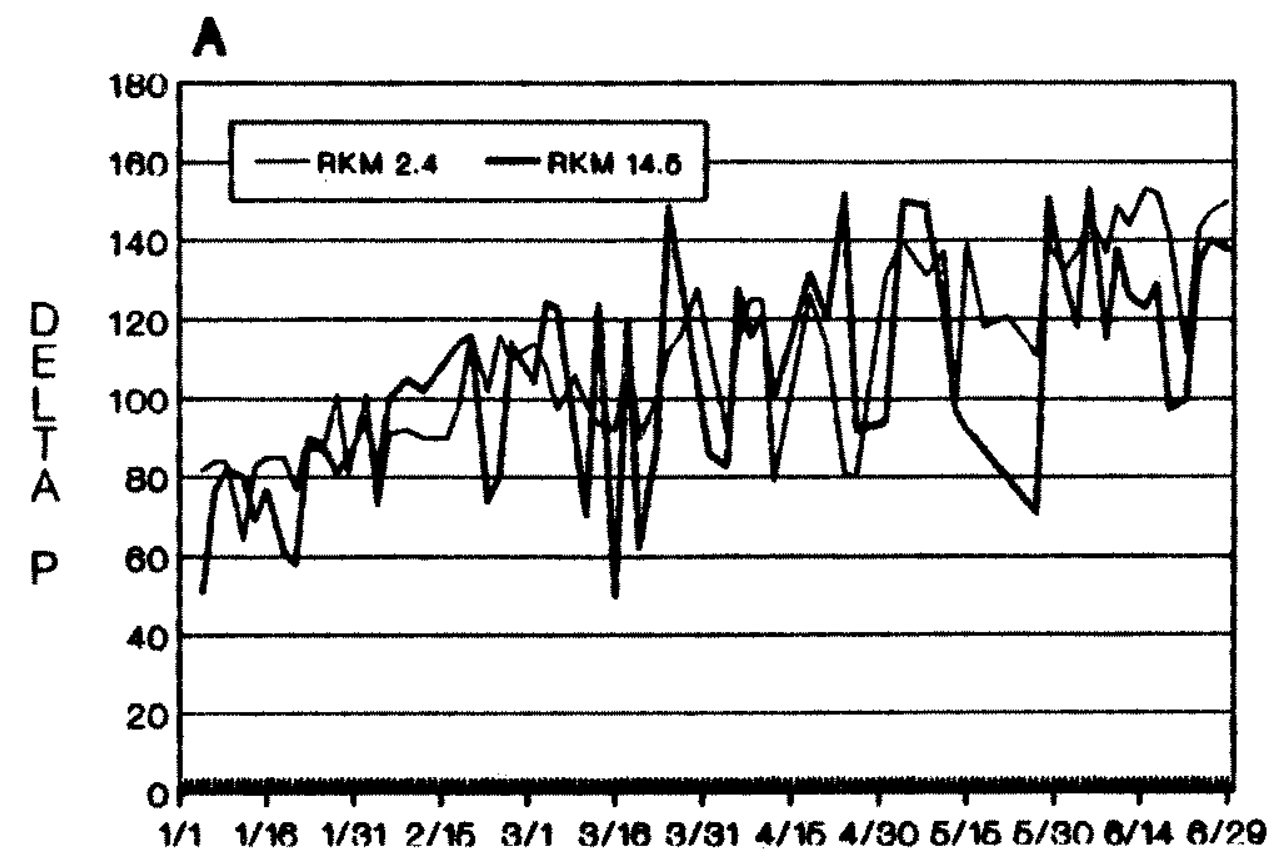

DATE

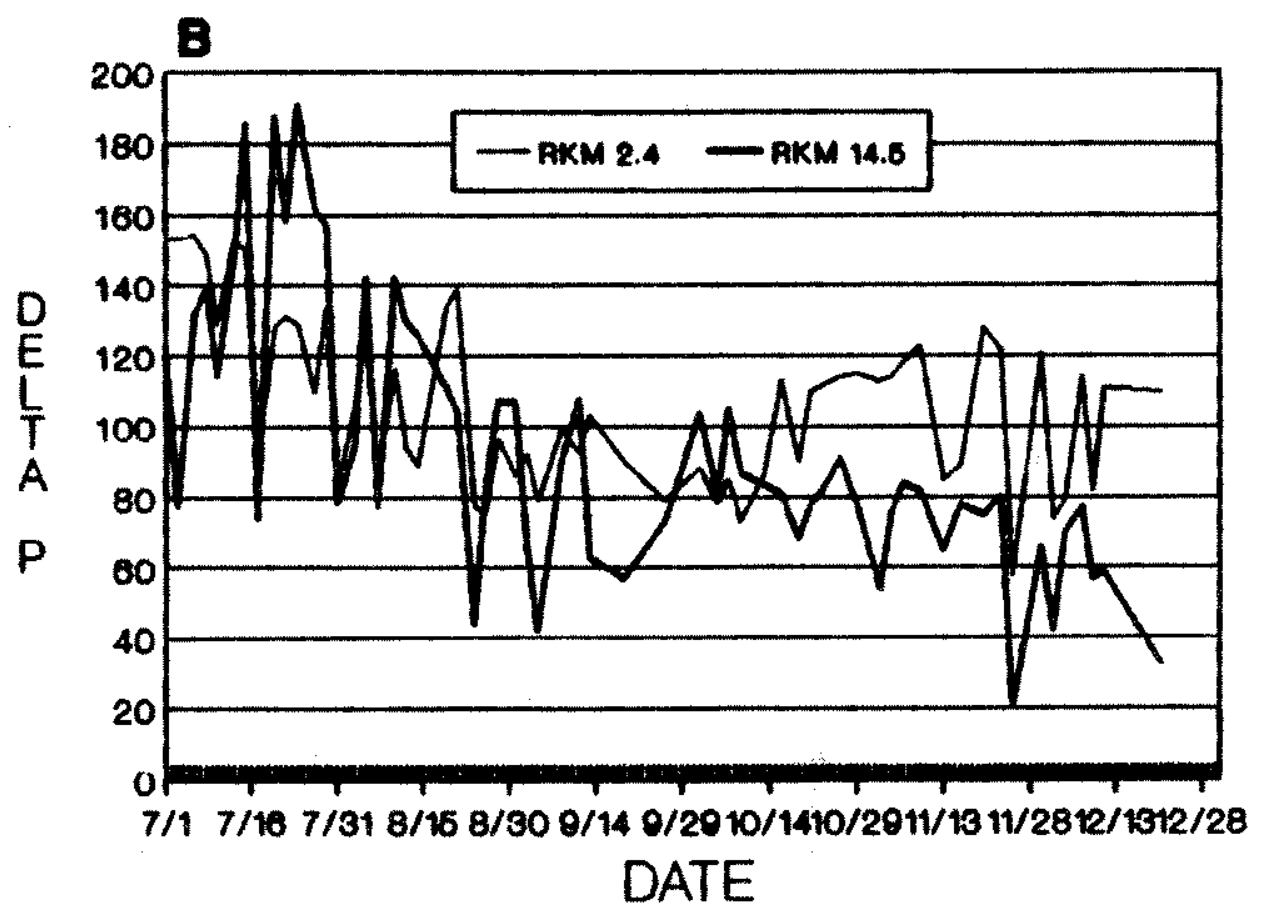

Figure 2.6.15. Delta $P$ levels (mmHg) measured two or three times weekly on the Bighorn river, at $R \mathrm{~km} 2.4$ and 14.5 , from January 1 through June 30 (A) and from July 1 through December 31 (B), 1987. 


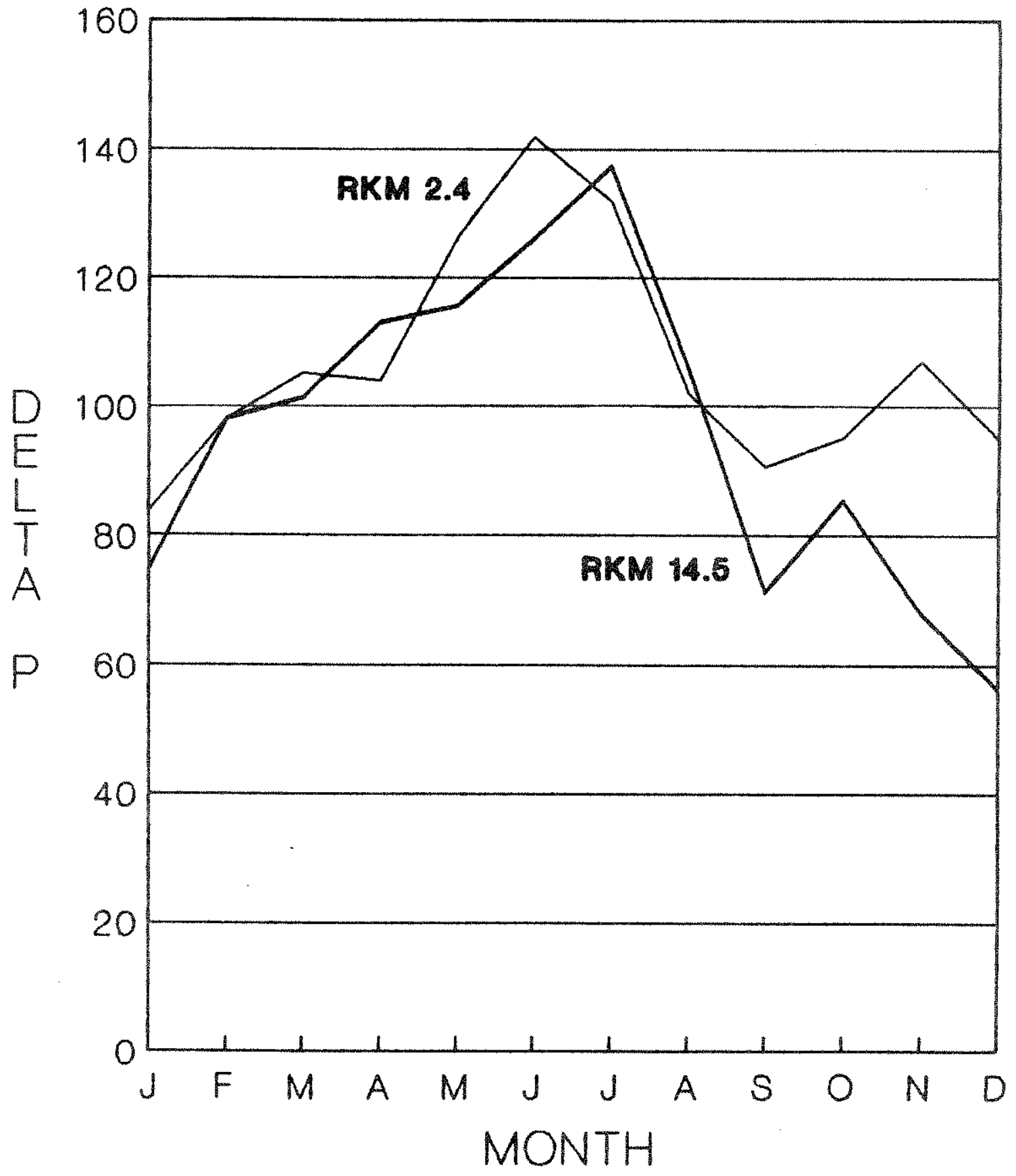

Figure 2.6.16. Mean monthly delta $p$ levels (mmHg) at Rkm 2.4 and 14.5, Bighorn River, 1987. 


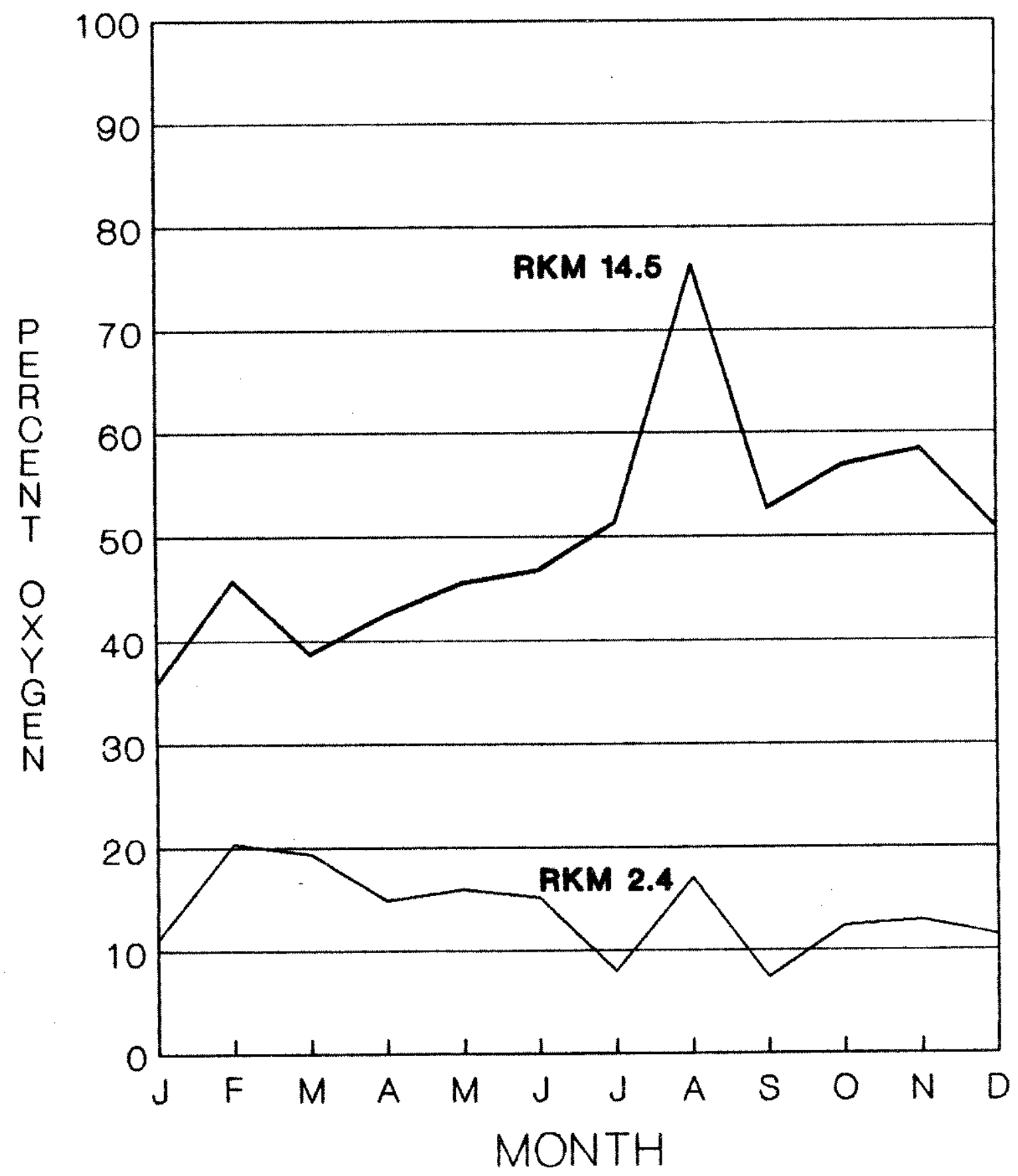

Figure 2.6.17. Percent of mean monthly delta $P$ levels comprised of oxygen at Rkm 2.4 and 14.5, Bighorn River, 1986. 


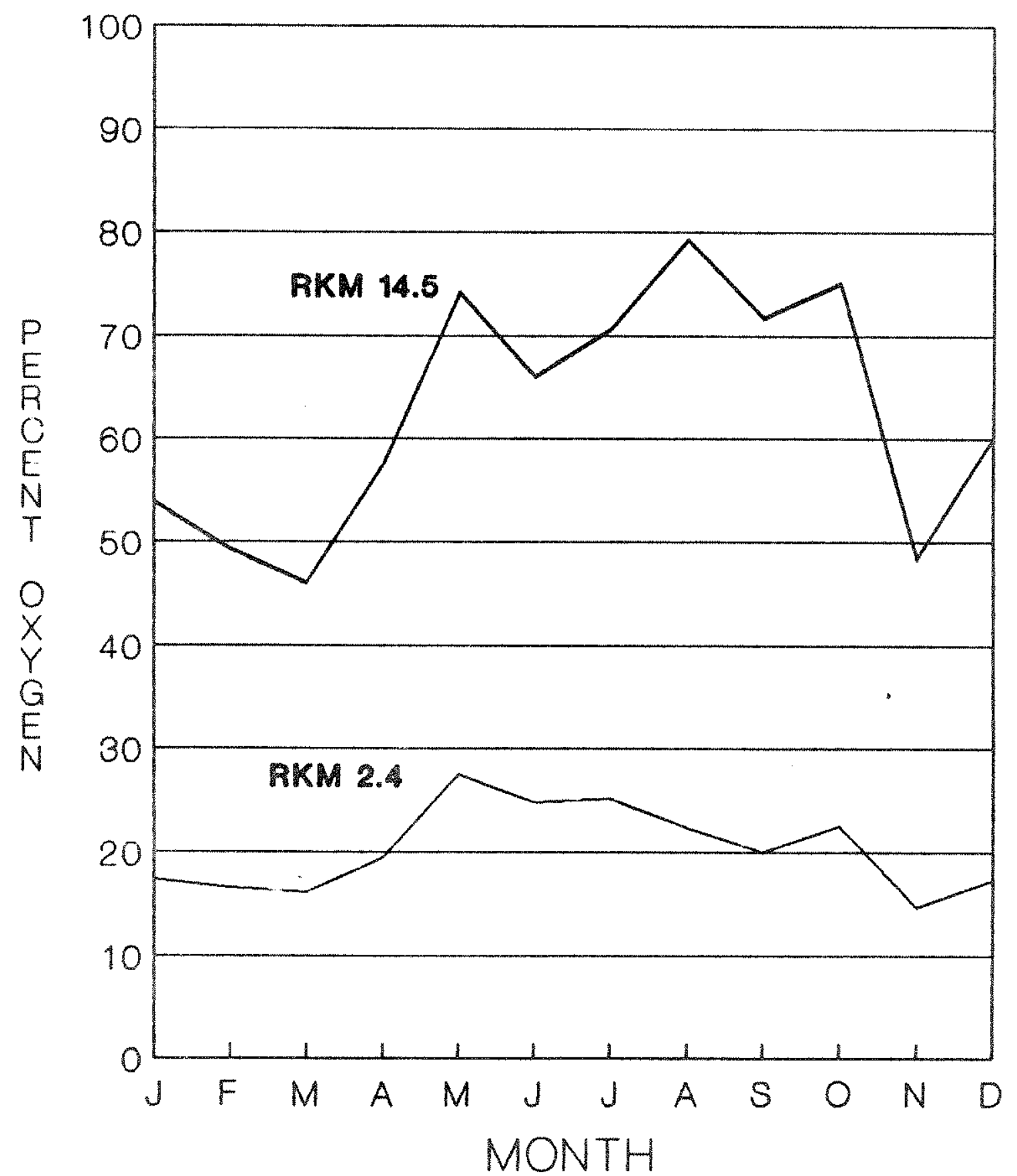

Figure 2.6.18. Percent of mean monthly delta $P$ levels comprised of oxygen at Rkm 2.4 and 14.5, Bighorn River, 1987. 
to include representatives from groups 1,2 and 3 . Amiocentrus aspilis and Ephemerella inermis were significantly more abundant at Rkm 2.4 when differences occurred (group 1). Physella and Gammarus lacustris had significantly greater densities at one site during one period, but at the other site during a subsequent period (group 2). Baetis tricaudatis, Isoperla quinquipunctata, Hydropsyche spp. and Tricorythodes minutus were all more abundant downstream when differences were significant (group 3).

The initial effect on all taxa exposed to high levels of gas supersaturated water was buoyancy which resulted in organisms becoming stranded at the water surface. Buoyancy was caused by gas bubbles forming and clinging to the body externally or forming internally within the organism. stranding from external bubbles was temporary, lasting until the organism floated to the surface where the bubbles would eventually burst. This was most prevalent during the first $24 \mathrm{~h}$.

Buoyancy from internal gas accumulation (gas bubble trauma or GBT) was more permanent than externally caused buoyancy. Recovery from GBT was not observed to occur within the 168 -h test periods. Gas bubbles were observed at various locations within appendages and body cavities of afflicted organisms (Appendix L). Severity increased with increasing delta $\mathrm{P}$ levels and with time of exposure. Advanced GBT symptoms included protraction of the head from the thorax, separation of abdominal segments (i.e. distension), and loss of torsal mobility. Death was common in organisms exhibiting these symptoms.

Mean delta $P$ levels were lower in all tanks during bioassay \#2 (Figure 2.6.19). This made comparing susceptibility of taxa 
tested in different bioassays difficult.

Bioassay \#1. Although delta $\mathrm{P}$ levels fluctuated during tests, the overlap between tanks was small except between the two highest gas levels tested (Table 2.6.9). This overlap resulted from one measurement of $270 \mathrm{mmHg}$ in tank 5. Excluding this, mean delta $P$, standard deviation, and range would be $221.6,9.9$, and 204-237, respectively, in tank 5. With this exception, delta $P$ varied more at low supersaturation levels, probably reflecting varying gas pressures in the untreated water. Delta $P$ fluctuations at high saturation levels were attributed to gases coming out of solution and impeding water flow through the valves (i.e. vapor lock), rather than to variations in gas levels produced by the column. This problem was never rectified, but was partially overcome by periodically "bleeding" the water line. Water temperatures also varied between tanks (Table 2.6.9). Average temperatures increased with the proportion of treated water used, but differences were small and the ranges similar. Water chemistry (Table 2.6.10) was similar to that reported for the Bighorn River (White et al. 1986).

I. quinquepunctata, A. aspilis, E. inermis, and Hydropsyche spp. were tested during bioassay \#1. Individuals of three of the four taxa suffered GBT induced stranding (Figure 2.6.20) and death (Figure 2.6.21) at delta $\mathrm{P}^{\prime} \mathrm{s}$ of $181 \mathrm{mmHg}$ and greater, but none were negatively influenced by delta P's of 75 or less.

Isoperla guinquepunctata. The lowest susceptibility to GBTinduced stranding during bioassay \#I occurred in $I$. quinquepunctata. Of the 20 nymphs tested, three (15\%), four $(20 \%)$ and six $(30 \%)$ became permanently stranded at delta P's of 


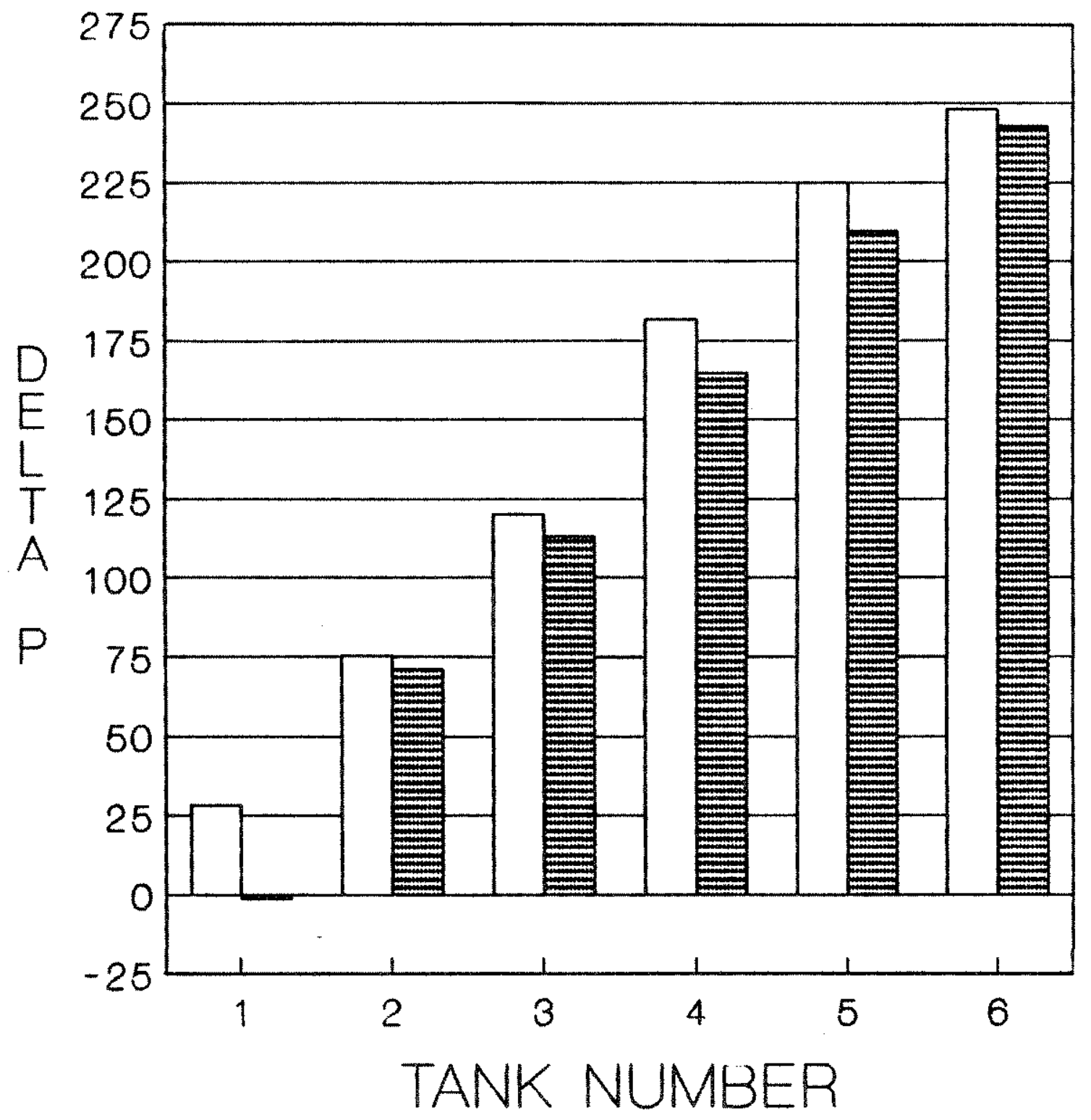

$\square$ BIOASSAY \#1

Figure 2.6.19. Comparison of mean delta $P$ levels (mmHg) in all test tanks during bioassays 1 and 2 . 
Table 2.6.9. Means, standard deviations (S.D.), and ranges of Delta $\mathrm{P}$ ( $\mathrm{mmHg})$, dissolved oxygen (mg/L), and temperature $\left({ }^{\circ} \mathrm{C}\right)$ during bioassay $\# 1$, August 2 to 9, 1988.

\begin{tabular}{|c|c|c|c|c|c|c|c|c|c|}
\hline Tank & mean & $\begin{array}{c}\text { DeIta } \\
\text { S.D. }\end{array}$ & $\begin{array}{l}\mathrm{P} \\
\text { range }\end{array}$ & \multicolumn{2}{|c|}{$\begin{array}{l}\text { Dissolved } \\
\text { mean S.D. }\end{array}$} & $\begin{array}{l}\text { d oxygen } \\
\text { range }\end{array}$ & \multicolumn{3}{|c|}{$\begin{array}{c}\text { Temperature } \\
\text { mean S.D. range }\end{array}$} \\
\hline 1 & 28.4 & 13.6 & $14-57$ & 8.0 & 0.29 & 9.4 & 8.5 & 0.6 & $7.8-8.9$ \\
\hline 2 & 75.5 & 11.8 & $54-92$ & 9.8 & 0 . & 10.0 & 8.7 & 0.6 & 7. \\
\hline 3 & 120.3 & 8.1 & 104 & 10.8 & 0.34 & 10.2 & 8.7 & 0.6 & $7.8-10.0$ \\
\hline 4 & 181.6 & 6.9 & $170-193$ & 11.9 & 0.24 & 11.3 & 8.9 & 0.6 & $7.8-10.6$ \\
\hline 5 & 225.0 & 16.0 & $204-270$ & 13.3 & 0.48 & $12 \cdot 2-13 \cdot 8$ & 9.0 & 0.7 & $7.8-10.6$ \\
\hline 6 & 248.2 & 4.3 & $244-258$ & 14.7 & 0.29 & $14.1-15.0$ & 9.2 & 0.8 & $8 \cdot 3-11 \cdot 1$ \\
\hline
\end{tabular}

Table 2.6.10. Water chemistry measurements taken the day following gas supersaturation bioassays, Bighorn River, Montana 1988.

\begin{tabular}{lcc}
\hline \multicolumn{1}{c}{ Parameter } & Bioassay \#1 & Bioassay \#2 \\
\hline Total hardness $\left(\mathrm{CaCo}_{3}\right)$ & $332 \mathrm{mg} / \mathrm{L}$ & $309 \mathrm{mg} / \mathrm{L}$ \\
Ca hardness & $205 \mathrm{mg} / \mathrm{L}$ & $200 \mathrm{mg} / \mathrm{L}$ \\
$\mathrm{pH}$ & 8.3 & 8.3 \\
Total alkalinity & $1.78 \mathrm{mg} / \mathrm{L}$ & $216 \mathrm{mg} / \mathrm{L}$ \\
\hline
\end{tabular}

181, 225, and $248 \mathrm{mmHg}$, respectively (Figure 2.6.20). A similar trend in mortality occurred (Figure 2.6.21). The threshold for GBT induced stranding and death in this taxa appears to be between a delta $\mathrm{P}$ of 181 and $120 \mathrm{mmHg}$.

Ejght I. guinguepunctata emerged during the test. Emergence was observed from tanks $1,2,3$ and 6 . Only one stonefly (tank 3) died during emergence. During molts prior to the final molt for emergence, one Isoperla died, and another became buoyant due 


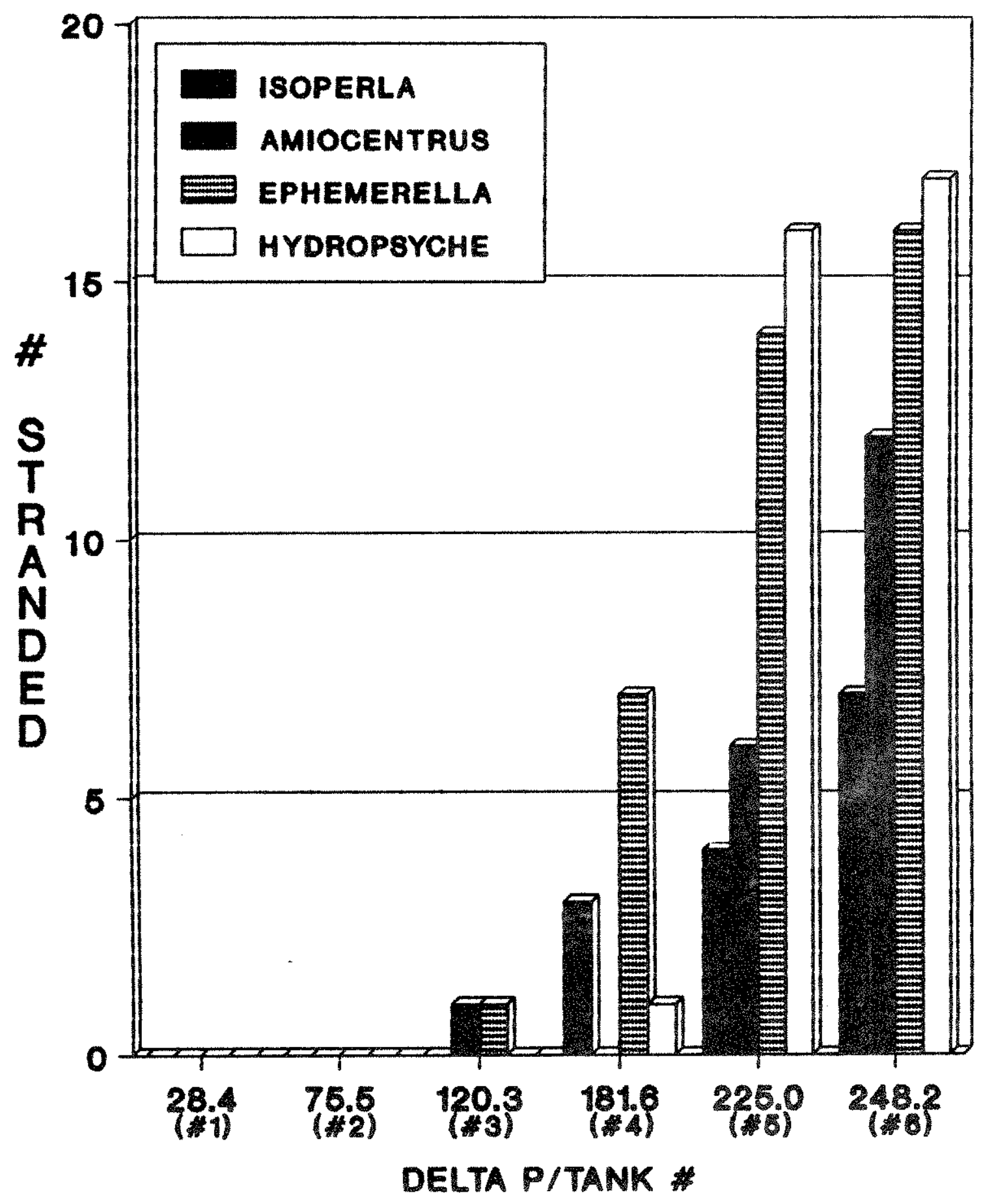

Figure 2.6.20. Susceptibility of four taxa to stranding at six delta $\mathrm{P}$ levels used in bioassay $\# 1 . \quad \mathrm{N}=20$. 


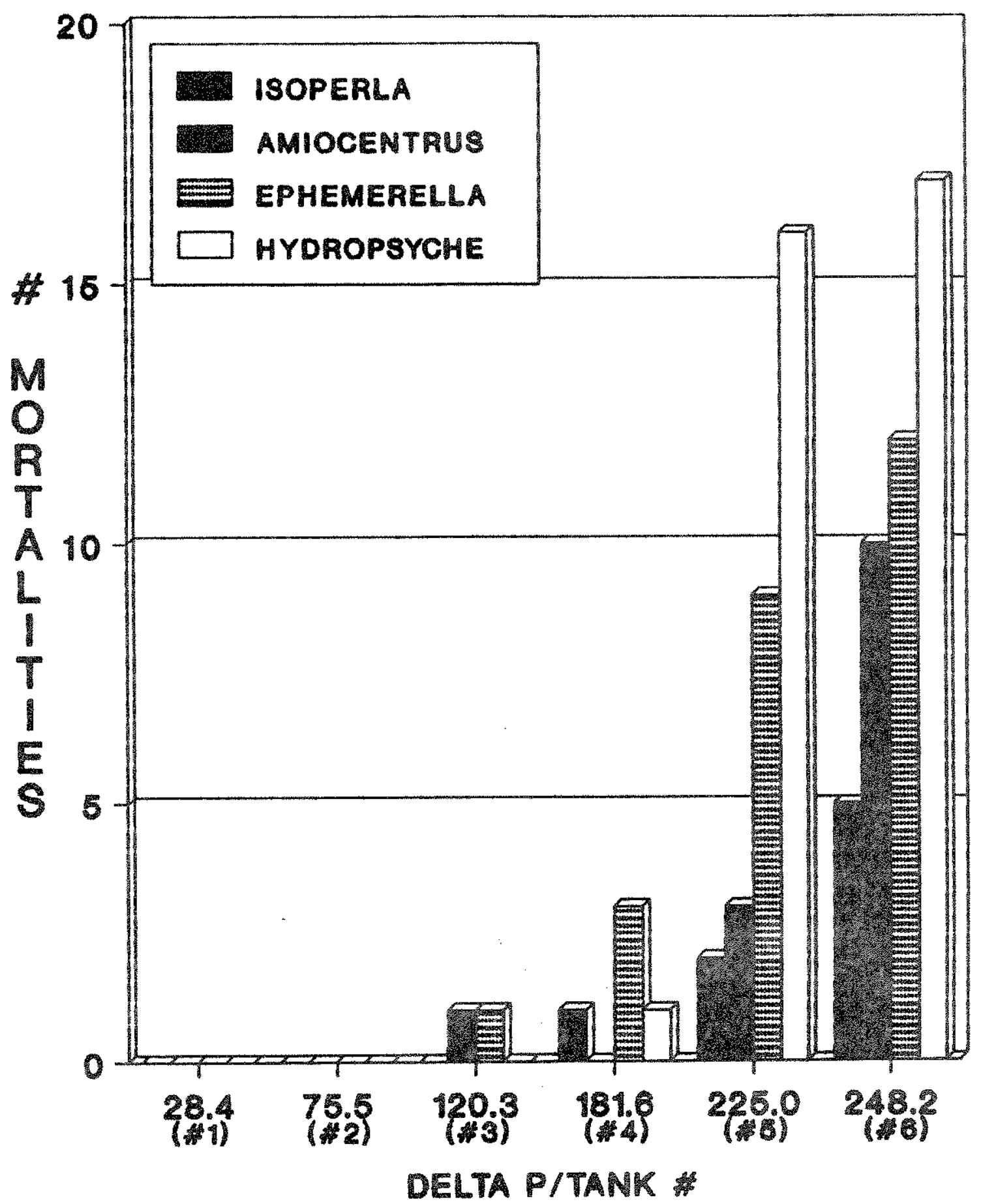

Figure 2.6.21. Gas-related mortalities for four taxa cested at six delta $P$ levels during bioassay $1 . \quad N=20$. 
to external bubbles.

Amiocentrus aspilis. The case of $\AA$. aspilis inhibited detection of the onset of GBT and may have resulted in some GBT caused mortalities not being observed. When gas accumulation became severe, however, symptoms were readily visible (Appendix L).

Nineteen $\underline{A}$. aspilis were stranded during the test; 18 were in tanks 5 and 6 (Figure 2.6.20). The single stranded larvae in tank 3 that subsequently died suffered from external gas bubbles and not GBT. Death resulted from stranding above the water surface due to water level fluctuations. Total GBT-related mortality was 13 for the test, with 3 (15\%) and 10 (50\%) occurring in tanks 5 and 6 , respectively (Figure 2.6.21).

The threshold for GBT-induced stranding and death appeared to be above a mean delta $\mathrm{p}$ of $181 \mathrm{mmHg}$. Pupation decreased at higher gas saturation levels. Nine, 8, and 2 Amiocentrus larvae pupated in tanks 1,2 and 5 , respectively.

Ephemerella inermis. E. inermis nymphs were more susceptible to stranding during bioassay \#1 than any other taxa. one $(5 \%), 7(35 \%), 14(70 \%)$ and $16(80 \%)$ nymphs became stranded in tanks 3,4,5, and 6, respectively (Figure 2.6.20). Total E. inermis mortality caused by GBT was 25 (Figure 2.6.21). Non-gasrelated mortality ranged from three (15\%) in tanks 3 and 5 to four (20\%) in tanks 1 and 2 and was not correlated with mean gas supersaturation levels.

The threshold for GBT-induced stranding appeared to be near a delta $\mathrm{p}$ of $120 \mathrm{mmHg}$. Longer exposure at lower gas levels, however, could affect this species. 
Extremely high delta $P$ levels could also affect this species' ability to mature and/or emerge. Successful emergence was seen in tanks 1 and 2 , while observed attempts to emerge from tanks 5 and 6 were unsuccessful. Also, two nymphs died while trying to molt in tank 6 .

Hydropsyche spp. Hydropsyche was the taxon most susceptible to GBT at the highest gas saturation levels. Stranding occurred almost entirely in tanks 5 and 6 (delta $P=225$ and 248.6) (Figure 2.6.20). By the end of the test, only five larvae remained in these two tanks. A single larvae developed GBT in tank 4, indicating that Hydropsyche's threshold is probably around $181 \mathrm{mmHg}$, and that it is less susceptible to GBT than Ephemerella inermis at lower delta $\mathrm{P}^{\prime} \mathrm{s}$.

Incidence of mortality (Figure 2.6.21) and of stranding (Figure 2.6.20) were identical. This occurred because larvae remained in their nets near the tank bottom until they became quite distended. By the time larvae were noted at the water surface, GBT had been severe for some time and they were near death. Other taxa became stranded when GBT was less severe and remained on the water surface longer before death.

No larvae attempted to pupate during the test. Thus, potential gas supersaturation effects on pupation are unknown.

Bioassay \#2: Physella spp., Gammarus lacustris, Tricorythodes minutus, and Baetis tricaudatis were tested during bioassay \#2. Al1 taxa had individuals that became permanently stranded during the test. Physella was least susceptible to GBT-induced stranding, while B. tricaudatis was most susceptible. water chemistry was similar to that observed after bioassay 
\#1 (Table 2.6.9). Total hardness was $23 \mathrm{mg} / \mathrm{L}$ less, while total alkalinity had increased from $178 \mathrm{mg} / \mathrm{L}$ to $216 \mathrm{mg} / \mathrm{L} \mathrm{CaCO}_{3}$.

Large variations in delta $\mathrm{p}$ (Table 2.6.11) occurred during bioassay \#2. Temperature ranges were large, but mean temperatures were more similar between tanks than during bioassay \#1. Tanks 1 through 5 averaged $11.2^{\circ} \mathrm{C}$, while tank 6 averaged $11.3^{\circ} \mathrm{C}$

Physella sp. Physella was the least affected of the organisms tested in bioassays \#1 and \#2. Four were stranded at the end of the test (Figure 2.6.22). These data, however, are somewhat misleading. Although snails were commonly seen stranded during the test, none remained that way.

The randomness exhibited by the number of buoyant gastropods from observation to observation (Appendix $M$ : Figure 45) suggested that Physella may have been able to alter its buoyancy. This in turn suggests that criteria used for determining gas supersaturation effects might have been unreliable for this taxon.

Physella commonly clung to the tank walls and were less frequently associated with the bottom than other taxa tested. Those in the upper one-half of the tanks were dislodged during each observation ( 4 - h intervals) to determine if they would float or sink. Thus, the status of most gastropods was regularly checked. Although some individuals became stranded in tanks 1 , 2 , and 3, most stranding occurred in tanks 4,5 and 6 (Figure 2.6.22). Thus, while stranding was temporary, incidence increased at delta $\mathrm{P}^{\prime} \mathrm{s}$ of $164 \mathrm{mmHg}$ and greater. 
Table 2.6.11. Means, standard deviations (S.D.), and ranges for Delta $\mathrm{P}$ (mmHg), dissolved oxygen (mg/L), and temperature $\left({ }^{\circ} \mathrm{C}\right)$ during bioassay \#2, september 8 to 15 , 1988.

\begin{tabular}{|c|c|c|c|c|c|c|c|c|c|}
\hline & mean & $\begin{array}{l}\text { Delt } \\
\text { S.D. }\end{array}$ & $\begin{array}{l}\mathrm{p} \\
\text { range }\end{array}$ & $\begin{array}{c}\mathrm{Di} \\
\text { mean }\end{array}$ & $\begin{array}{l}\text { ssolv } \\
\text { S.D. }\end{array}$ & $\begin{array}{c}\text { oxygen } \\
\text { range }\end{array}$ & mean & $\begin{array}{l}\text { Tempe } \\
\text { S.D. }\end{array}$ & $\begin{array}{l}\text { rature } \\
\text { range }\end{array}$ \\
\hline 1 & -1.5 & 4.7 & $-8-12$ & 7.2 & 0.24 & $6.7-7.4$ & 11.2 & 0.4 & $10.6-11$. \\
\hline 2 & 71.3 & 9.9 & $59-88$ & 8.6 & 0.12 & $8.5-8.7$ & 11.2 & 0.4 & $10.6-11$ \\
\hline 3 & 113.1 & 9.2 & $94-135$ & 9.4 & 0.15 & $9.2-9.6$ & 11.2 & 0.4 & $10 \cdot 6-11$. \\
\hline 4 & 164.7 & 12.7 & $141-189$ & 10.6 & 0.25 & $10.2-10.9$ & 11.2 & 0.5 & $10.0-11.7$ \\
\hline 5 & 209.6 & 9.0 & $199-222$ & 11.7 & 0.32 & $11 \cdot 1-12 \cdot 1$ & 11.2 & 0.5 & $10.0-12$ \\
\hline 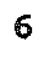 & 242.6 & 7.3 & $222-254$ & 13.9 & 0.24 & $13 \cdot 6-14 \cdot 2$ & 11.3 & 0.5 & $10.0-12$. \\
\hline
\end{tabular}

One gas-caused mortality occurred in tank 6 (Figure 2.6.23). It appeared that gas accumulated between the shell and the mantle, forcing the organism out of the shell.

Gammarus lacustris. Relatively few Gammarus became permanently stranded during the test. Only one (5\%), two (10\%), and four $(20 \%)$ of the amphipods were restricted to the water surface at delta $\mathrm{P}^{\prime} \mathrm{S}$ of 164,209 , and $242 \mathrm{mmHg}$, respectively (Figure 2.6 .22 ). Internal bubbles were not observed in individuals exposed to gas saturation levels of $113 \mathrm{mmHg}$ or less. Substantial buoyancy was necessary to strand Gammarus at the surface. Because of their strong swimming ability, some individuals with internal bubbles were able to return to the tank botton. Thus, GBT was often severe before the organism became helpless, leaving little time between stranding and death. For this reason, GBT-induced mortality (Figure 2.6.23) was identical to stranding results (Figure 2.6 .22 ). 


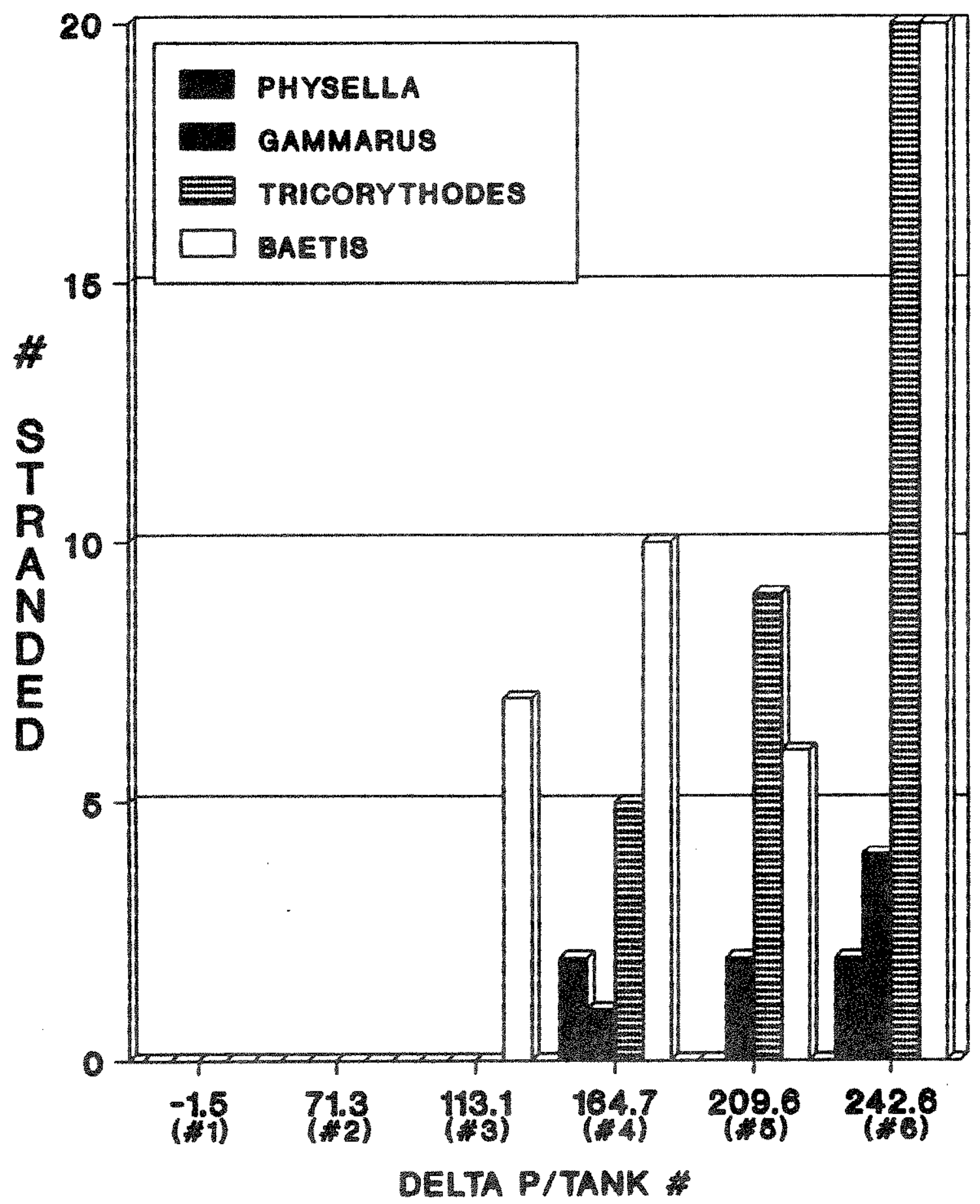

Figure 2.6 .22 . Susceptibility of four tasa to stranding at six delta $P$ levels used in bioassay $2 . \quad N=20$. 


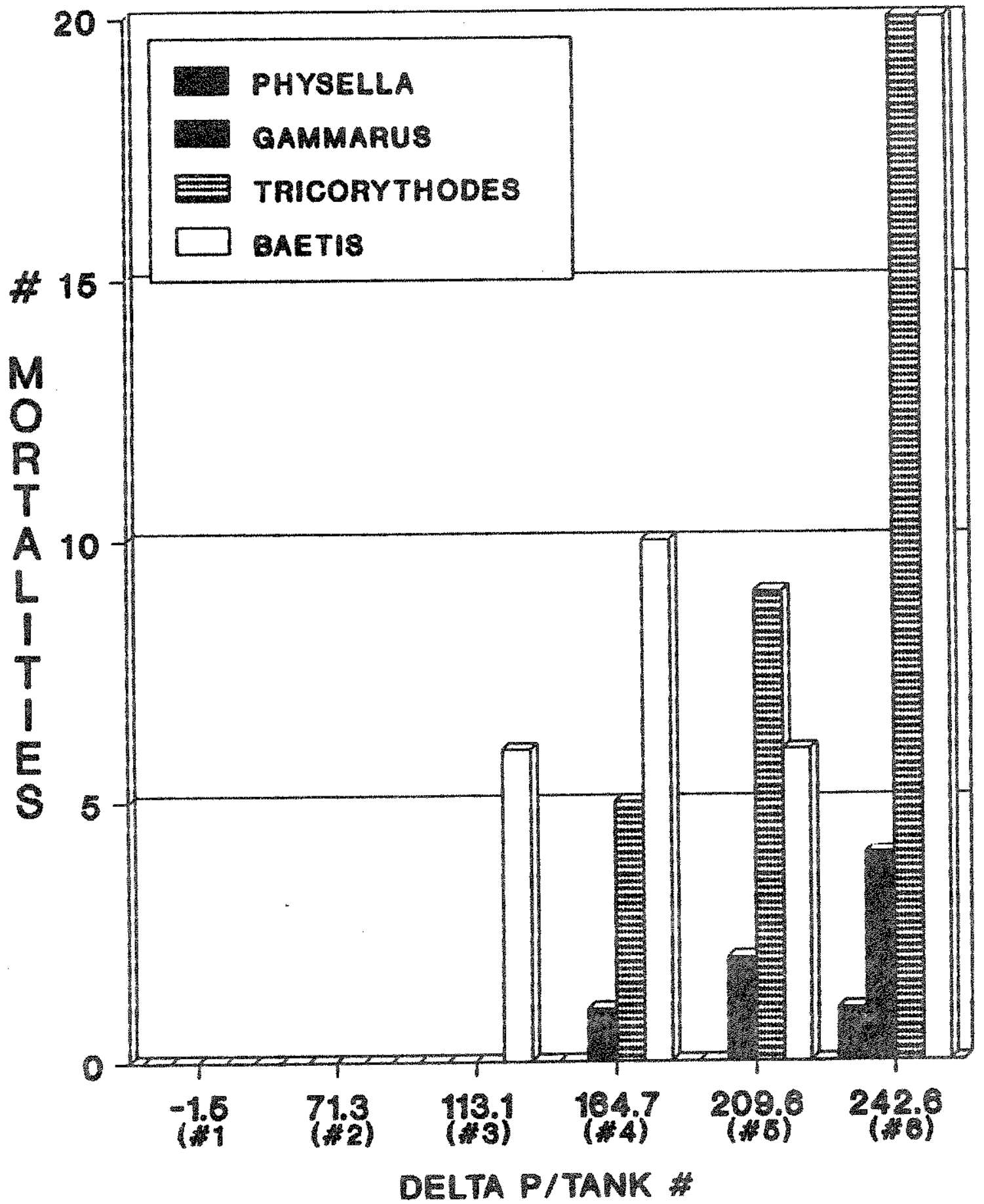

Figure 2.6.23. Gas-related mortalities for four taxa tested at six delta $P$ levels during bioassay \#2. $\mathbb{N}=20$. 
As with Physella, applicability of criteria used to determine gas supersaturation effects on Gammarus was also suspect. While stranding was not common, Gammarus appeared to more commonly occupy the upper portion of tanks 4,5 and 6 , compared to tanks with lower delta Pis.

To determine if differences in their positions were related to increasing delta $P^{\prime}$, the number of individuals in the upper one-fourth of each tank was noted in all observations after hour $84(N=20)$. The mean number (per observation) of amphipods occupying the top one-fourth of the tanks was $0.63,0.21,0.16$, $2.95,4.79$, and 5.95 for tanks 1 through 6 , respectively.

These results indicate that gas supersaturation levels in tank 4 affected Gammarus more than indicated by the numbers stranded (Figure 2.6 .22 ). These data also suggest that the threshold for behavioral response of Gammarus to gas supersaturation levels occurs somewhere between a mean delta $P$ of 113 and $164 \mathrm{mmHg}$.

Tricorythodes minutus. Susceptibility of Tricorythodes to GBT was high at mean delta $P$ levels of $164.7 \mathrm{mmHg}$ or greater. Stranded individuals comprised 20,45 , and $100 \%$ of all T. minutus in tanks 4, 5, and 6, respectively (Figure 2.6.22). Mortality and stranding results were identical (Figure 2.6 .23 ). No stranding or mortality from GBT occurred at delta $P$ levels of 113 mmH or less. Thus, the threshold appears to lie between delta P's of 113 and 165 . No nymphs atcempted to emerge during the test.

I. minutus nymphs were much less mobile than E. inermis, I. guinquepunctata and B. tricaudatis. Thus, their ability to 
overcome initial buoyancy was inferior to other, more mobile, invertebrate taxa tested.

Gammarus was observed feeding on $\underline{T}$. minutus nymphs once in tank 5 and three times in tank 6 . Only one of the nymphs was alive at the time of observation: all nymphs being fed upon were buoyant. Most GBT-afflicted I. minutus were removed and classified as mortalities when body movement and response to disturbance was nearly absent, but before death. Thus, predation rather than scavenging probably occurred. Predation likely occurred in other tanks as wel1. Only 15, 14, and 17 Tricorythodes were accounted for in tanks 1, 2, and 4, respectively.

Baetis tricaudatis. of the taxa tested, B. tricaudatis were the most sensitive to gas supersaturation. One (5\%), six $(30 \%)$, seven $(35 \%)$, and $14(70 \%)$ of the individuals in tanks 3 through 6 were classified as "stranded" by hour 8 of the test (Appendix M: Figure 43). At the end of the test, 7 (35\%), 10 $(50 \%), 6(30 \%)$, and $20(100 \%)$ were stranded in these same tanks (Figure 2.6.22). Mortalities from gas supersaturation ranged from six in tank 3 to 20 in tank 6 (Figure 2.6.23).

Disappearance of nymphs from tanks 1 through 5 confounded the results. At the end of the test, the number of Baetis accounted for was $5,8,14,12,8$, and 20 in tanks 1 through 6 , respectively. Most of the depletion occurred early in the test. By hour 80 few Baetis nymphs remained in the tanks.

Emergence and predation were the probable cause of $B$. tricaudatis's depletion. Eight nymphs are known to have successfully emerged; four from tank 2, and two from each of 
tanks 3 and 4. Since most nymphs were not late instar, predation appears more important than emergence in explaining disappearance from the tanks.

Baetis nymphs were strongly affected by GBT early in the bioassay and exhibited little time between initial buoyancy and loss of mobility. Stranded individuals became easy prey for an agile predator. Gammarus were observed eating nymphs and were likely the main predator. Seven of the nine partially eaten nymphs recovered, were buoyant.

Early depletion in tank 5 appeared to cause the decrease in stranded nymphs from $35 \%$ at $h 8$ to $30 \%$ at $h 168$, the end of the test (Appendix M: Figure 43). A11 organisms in tank 6 were accounted for. This likely resulted from the short time required for stranding and death (Appendix M: Figures 43 and 44). Also, delta $\mathrm{P}^{\prime} \mathrm{s}$ averaging $242 \mathrm{mmHg}$ may have decreased Gammarus's predatory behavior.

The pattern of gas-related mortality for $\underline{B}$. tricaudatis (Appendix M: Figure 44) was similar to the number stranded in that mortality in tank 4 surpassed that in tank 5 by $h 32$. This was probably due to predation. Total GBT-associated mortality was 42 and would have probably been higher had depletion of Baetis not occurred.

Baetis $^{\prime} s$ threshold for GBT-induced stranding appears closer to a delta $P$ of 71 than $113 \mathrm{mmHg}$. One nymph in tank 2 was strongly buoyant at $\mathrm{h} 80$ (Appendix $\mathrm{M}:$ Figure 43 ) but not in subsequent observations, possibly because it was preyed upon. The short time required for depletion of Baetis may have prevented other individuals from becoming stranded in tank 2 . 


\section{$2.6 .1 \mathrm{c}$ Discussion}

Community composition: Community composition in the Bighorn River below Yellowtail Afterbay Dam was typical of other tailwater communities. In a survey of regulated streams in six major drainage basins in the Rocky Mountains of Colorado, Zimmerman and ward (1984) found that Tricorythodes ninutus, Baetis spp. Ephemerella infrequens and chironomids were often present in high numbers below deep-release impoundments. Isoperla quinquepunctata was one of two plecopterans that were commonly found. Other common groups included amphipods, snails, oligochaetes, turbellarians, sphaeriid clams, and water mites. The Bighorn River invertebrate community was dominated by five taxa: Chironomidae, Baetis tricaudatis, Tricladida sp., Tricorythodes minutus, and Physella spp. Community dominance by few taxa has been documented below other deep-release impoundments (Spence and Hynes 1971; Ward and Short 1978; Ward and stanford 1979). Ward (1976) found that mayflies, Baetis sp. and Ephemere1la inermis, along with dipterans, Cardiocladius sp. and simulium arcticum, accounted for $87 \%$ of the organisms in a study section on the south Platte River below Cheesman Lake in colorado. He suggested that diurnal and seasonal temperature constancy, summer cold and winter warm conditions, and delayed seasonal temperature maximums are primarily responsible for the limited success of some species and the elimination of others below deep-release dams.

In addition to temperature patterns, the Bighorn River's abundant aquatic vegetation is important in determining invertebrate community composition and abundance. Total 
invertebrate densities increase (Hynes 1970), while species diversity tends to decrease (Yount 1956) with increased vegetative productivity.

The Bighorn River invertebrate fauna is comprised of taxa typically associated with aquatic vegetation. Most taxa are considered "collectors" which depend primarily on fine particle detritus for food. Abundant vegetation benefits members of this trophic group by filtering particulate organic matter from the water (Armitage 1984) and providing a source of recruitable food. other commonly occurring Bighorn River taxa have differing trophic habits but still tend to rely on aquatic vegetation. Physella spp., Hyalella azteca, and Gammarus lacustris are considered omnivores but may depend heavily on detritus or live plants (Pennak 1978). Hydroptila is considered a herbivore along with certain species of oecetis, while Isoperla quinquepunctata and Glossophonia are predators and probably associated with vegetation indirectly through their prey.

Invertebrate Densities and Community structure: Certain invertebrate community similarities between upstream (Rkm 2.4) and downstream (Rkm 14.5) sites in spring were absent during late summer-early fall in the Bighorn River. Total benthic densities were not statistically different between sites during April 1987, but were significantly greater downstream during August 1986 and September 1987. Likewise, invertebrate community structure differed considerably between sites during late summer/fall sampling periods, but not in the spring (Figures $2.6 .6,2.6 .7$, and 2.6 .8$)$.

Principal components analysis showed that a major portion of 
the variation in individual taxa abundance resulted from samples being collected at different times and from different sites. physical and chemical habitat factors control invertebrate distribution and abundance (Cummins 1975). Thus, the loss of invertebrate community similarities between sites in the fall undoubtedly resulted from habitat changes during simmer.

Attempts to link site differences (other than gas

supersaturation) to changes in invertebrate communities, however, were not completely successful. correlations between individual taxa and water depth, water velocity or vegetation abundance were invariably poor and inconsistent. Because a complex set of conditions influences invertebrate densities, it is understandable why individual habitat parameters inadequately explained all differences that occurred. In spite of this, habitat data provided some description of the physical variability between sites and, when related to the ecology of individual taxa, allowed some inference into potential effects on the invertebrate community.

\section{Habitat Comparisons Between sites: During each sampling} period, at least one of the habitat parameters measured differed significantly between sites. In general, however, both sites were similar with regard to variability and range of conditions provided. Comparisons between sites in August 1986 indicated that only water depth was significantly different. Hynes (1970) states that current speed, temperature, and substratum are the most important factors regulating aistribution of aquatic invertebrates. Microhabitat distribution is primarily determined by food availability, nature of the sediments, and current 
velocity (Cummins 1975). It seems unlikely, then, that a slight difference in depths would cause substantial variation in invertebrate abundance. The influence of depth is probably more indirect than direct, first affecting aquatic vegetation through light availability, which in turn directly influences invertebrates. With the high water clarity of the Bighorn River, small changes in depth would have little influence on light availability.

Mean water velocity near the substrate (Table 2.6.6) was significantly different between sites in April $1987(0.1 \mathrm{~m} / \mathrm{s}$ faster at $\mathrm{Rkm} 14.5)$; at 0.6 depth it was significantly different between sites in september $1987(0.3 \mathrm{~m} / \mathrm{s}$ faster at Rkm 14.5). Changes in water velocity can alter invertebrate communities by influencing the occurrence and densities of species (Hynes 1970). Specific responses of invertebrate communities to differing water velocities at Bighorn River sampling sites, however, were difficult to determine. Correlations with densities of individual taxa were always poor, although they were commonly better with velocities at 0.6 depth than those near the substrate. poor correlations suggest that factors other than water velocity were more important in decermining distribution. Since water velocities approach zero near the substrate, velocities near mid-depth may better reflect velocities associated with food resources and oxygen.

In summary, it is unknown whether velocity differences present between sampling sites were sufficient to significantly alter the invertebrate communities. The data only indicate that differences existed, and thus the potential to influence the 
invertebrate community was present.

Greater abundance of downstream vegetation in september 1987 was likely responsible for some of the increase in invertebrate density. Increases in algae and macrophytes alter the character of the substrate, providing increased surface area for invertebrate colonization and retention of particulatice organic materials through the filtering action of the plants (Armitage 1984). The trophic habits of most taxa common to the Bighorn River indicate that increases in benthic vegetation are beneficial. Correlations between the taxa that increased at Rkm 14.5 and dry weights of vegetation, however, were not strong, suggesting that specific types of vegetation may be more important than overall abundance.

In April 1987, total invertebrate abundance and invertebrate community structure were similar between sites. Vegetative standing crop was also comparable between sites. A variety of periphytic plants existed on sampling riffles, but different types were not quantified. Analysis showed that chlorophyll a concentrations significantly differed within both sites but not between them. Concentrations of chlorophyll a differ among species of periphytic plants (Wilkinson 1983). Thus, a patchy distribution of different algae and macrophytes might explain differences in chlorophyll a concentrations within riffles. Alternately, the lack of difference between sites could suggest a similaxity in vegetative types and distributions at Rkm 2.4 and Rkm 14.5.

Temperature is often used by aquatic invertebrates as an environmental cue to synchronize life cycles. Thus, a single 
species occurring at sites having different temperature regimes can exhibit considerable life history differences (Merritt and Cummins 1984). Different aspects of aquatic invertebrate life histories that can be affected include the rhythm of life cycles, emergence times (Hynes 1970), and the length of the emergence period (Ide 1935).

Temperature differences between invertebrate sampling sites were largest from early spring through late fall, with temperatures increasing downstream. These differences were undoubtedly responsible for asynchronous life cycles exhibited by Amiocentrus aspilis, Physella spp., Simulium spp., and Hydroptila sp. during late summer and early fall sampling periods. This made interpretation of benthic data difficult. Abundance differences could exist between sites at any one time, while densities at similar stages of the life cycle could be similar. Had densities been compared at a similar time in the life cycles of these taxa, results may have differed. other species likely had asynchronous life cycles that were not detected at the level of sampling in this study.

Temperature can also affect distribution of species (Merritt and Cummins 1984; Hynes 1970). Plecopterans are often negatively affected by altered temperature regimes downstream of deeprelease dams (Zimmerman and Ward 1984: Spence and Hynes, 1971). Isoperla guinquepunctata was the only stonefly that occurred commonly in Bighorn River benthic samples. Densities were low at both sites but greater downstream. It seems likely that the more nomal downstream diurnal and seasonal temperature patterns are favorable for this species. 
optioservus divergens was significantly more abundant at Rkm 14.5 during all sampling periods. This species is a habitat generalist, becoming very abundant under the right conditions (Dan Gustafson, personal communication). In Limekiln creek, a tributary of Afterbay Reservoir, densities were considerably greater than those in the Bighorn River (Dan Gustafson, personal communication). Limekiln Creek exhibits temperature patterns typical of prairie streams. From this, it seems likely that downstream temperatures are more suitable for this species than those near Afterbay Dam.

potentially Affected Taxa: Assuming that invertebrates are most susceptible to gas bubble trauma (GBT) near Afterbay Dam where nitrogen saturation is highest, taxa that were more abundant downstream are candidates for being negatively impacted by gas supersaturation. Significantly greater densities at Rkm 14.5 occurred with Tricorythodes minutus, Hydroptila spp. optioservus divergens, Hydropsyche spp. Isoperla quinquepunctata, Baetis tricaudatis, and Glossophonia complanata.

Increased abundance of $\underline{O}$. divergens and $I$. quinguepunctata at $\mathrm{Rkm} 14.5$ is 1 ikely related to temperature rather than gas supersaturation, for reasons discussed earlier. Multiple species of Hydroptila and Hydropsyche occur in the Bighorn River, confounding interpretation. Hydroptila, however, was one of only three taxa (along with Tricorythodes minutus and optioservus divergens) that was significantly more abundant downstream during all sampling periods, indicating that all Bighorn River species within this genera could be affected by high gas saturation levels. 
Ephemerella inemis was only present in the April 1987 benthic samples, occurring in significantly greater densities at Rkm 2.4. It had emerged prior to the late summer/early fall sampling periods, but was present in the river during the period of highest gas saturation levels. Because abundance differences caused by gas supersaturation would have been missed, this taxon also might be affected.

Invertebrate Bioassays: Eight potentially affected taxa were tested during bioassays. These included: Amiocentrus aspilis, Ephemerella inemis, Physella, Gammarus lacustris, Isoperla guinguepunctata, Hydropsyche, Iricorythodes minutus, and Baetis tricaudatis.

Externa11y Induced Buoyancy. Invertebrates tested varied in tolerance to elevated delta $\mathrm{P}^{\prime} \mathrm{s}$, but at some level tested, all experienced externally and/or internally induced buoyancy. Fickeisen and Montgomery (1975) found that supersaturated water from 107 to $123 \%$, or water with a high concentration of entrained gas bubbles, induced invertebrate buoyancy through bubbles adhering to the body surface. In our study, externally induced buoyancy seemed to result from gas bubbles coming out of solution and forming on test organisms rather than the adherence of entrained bubbles. If entrained gas bubbles cause invertebrates to become buoyant in the Bighom River, those in the stilling basin and immediately downstream would be most affected. Gas in solution, however, potentially chreatens invertebrates for miles downstream of Afterbay Dam. Setae and/or short spines common on many aquatic insects, as well as silt or organic matter adhering to the body, may provide nucleation sites for bubble formation 
directly on organisms.

All taxa tested experienced some buoyancy due to external gas bubbles, which generally occurred at delta $\mathrm{p}^{\prime} \mathrm{s} \geq 120 \mathrm{mmHg}$. A. aspilis, I. quinquepunctata, and B. tricaudatis were most affected. Bubbles occurred on the terminal end of $A$. aspilis's case and often persisted until larvae floated to tre surface since there was no way for the larvae to dislodge them. Usually stranding occurred only during the first $12 \mathrm{~h}$ of the bioassay (Appendix M: Figure 37), although bubbles were observed throughout the test. After a short time, most larvae had secured themselves to the tank walls or bottom.

Species behavior and location of bubble formation may determine the extent to which externally induced buoyancy influences invertebrates of the Bighorn River. Bubbles primarily occurred on the wings and abdomen of Isoperla and Baetis nymphs. After test organisms became familiar with their new surroundings, they became more stationary and the incidence of stranding decreased. It is possible that abdominal movements used for ventilation (i.e. gill beating in Ephemeroptera) or during swimming could encourage formation of external bubbles. Ephemerella was also affected by external buoyancy but did not seem to be as susceptible as Baetis, even though it uses similar ventilation and swimming movements.

In contrast, mobility associated with an organism's strength as a swimner may help moderate the incidence of permanent stranding. Isoperla was sometimes able to dislodge external bubbles while swimming. Fickeisen and Montgomery (1976) observed this in Cleon. I. quinquepunctata and $G$. lacustris were the 
strongest swimmers tested and were able to overcome slight

positive buoyancy and swim to the tank bottom. Swimming strength alone could not compensate for extreme buoyancy caused by supersaturated water, but it may allow certain taxa to do better than others.

It is conceivable that buoyant organisms modify their behavior to reduce the chance of involuntary drift. If so, this is probably the most important factor in determining what effects externally induced buoyancy will have on invertebrates. In bioassays, all taxa seemed to better cope with externally induced buoyancy after $24 \mathrm{~h}$ by becoming more stationary. Movements after this time did not appear sluggish, and organisms were assumed to be healthy. E. inermis and I. guinquepunctata nymphs were also observed attempting to dislodge bubbles while clinging to the bottom of the tank. Under natural conditions, these types of behavior would minimize the occurrence of externally induced stranding when gas saturation levels in the river exceed the thresholds required for bubble formation.

Tolerance to external bubbles 1 ikely depends on the location of bubble formation. Fickeisen and Montgomery (1975) report that bubbles were located around the mouth of cleon, which may have inhibited their feeding. This would likely cause increased activity due to discomfort. None of the taxa that I tested, however, exhibited this symptom.

Isoperla, Baetis and probably other invertebrates could be affected by externally induced stranding in the Bighorn River. The potential for impacts are likely greater at the upstream site since delta $P^{\prime}$ s exceeded $120 \mathrm{mmHg}$ more frequently than downstream 
(Appendix N: Tables 57 and 58). However, the most extreme gas

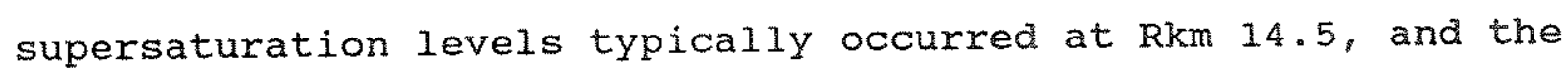
composition of gases differs there. It is difficult to know how these factors would affect the incidence of externally induced stranding downstream. Bioassay results indicated, however, that invertebrates minimize stranding by becoming more sitationary. Thus, while some involuntary drift due to external buoyancy probably occurs, we do not believe this significantly reduces invertebrate densities in the Bighorn River.

Internally Induced Buoyancy. In bioassays, buoyancy of organisms without external gas bubbles resulted from gas accumulation in the body cavity. Gas bubble trauma was more permanent and seemed less tolerable than external buoyancy. Problems were first expressed by the organism becoming stranded on the water surface. Advanced GBT was evident by anatomical distensions and loss of abdominal mobility.

The mode in which internal gas accumulation occurs is not totally understood. Most hemi- and holometabolous immature aquatic insects have tracheal gills for oxygen uptake and a network of trachea for transporting gases to various parts of the body. In addition, respiration may occur through the body surface. Cuticles of immature aquatic insects are thinner than in most adults and are underlain by a rich network of tracheae (Pennak 1978).

In terrestrial insects, tracheae originate at spiracles. Spiracle valves open and close the passageway for oxygen uptake. A pressure gradient, created when oxygen diffuses through tracheal walls and is taken up by cells, results in aix uptake 
and expulsion of carbon dioxide during a series of valve openings and closures (Hainsworth 1981). In immature aquatic insects, reduced pressure in the tracheal system likely occurs as oxygen is utilized. No valve is present, however, to isolate the tracheal system from the aquatic environment. As partial pressures of gases in solution increase, pressures in the tracheal system must climb until equilibrium is reached. If pressures become great enough to cause gases to diffuse through tracheal walls faster than cells can utilize them, or the tracheal walls rupture, gas accumulation in the body cavity of the organism would result. Pressures in the body cavity then increase until equilibrium, or near equilibrium with the partial pressures of the gases in solution, is achieved.

While the manner in which organisms are affected is different between external and internal gas bubbles, both result in buoyancy and potential downstream displacement. In April, Baetis drift rates were greater at Rkm 14.5 in proportion to benthic densities than at Rkm 2.4. Downstream delta $\mathrm{P}^{\prime} \mathrm{s}$ ranged from 100 to $127 \mathrm{mmHg}$ during a $10-\mathrm{d}$ period that included the days that drift was collected (Appendix K: Figure 29). These gas levels are in a range that may exceed Baetis's GBT threshold (between 71 and $113 \mathrm{mmHg}$ ), leaving gas supersaturation as a possible cause. Because of limited sampling, other potential causes for increased drift can not be addressed.

In september 1987, Tricorythodes and Gammarus exhibited disproportionately higher drift rates at Rkm 2.4. Gas saturation levels ranged between a delta $P$ of 79 and $90 \mathrm{mmHg}$ (Appendix $\mathrm{K}$ : Figure 30), well below the GBT thresholds determined for these 
two taxa during bioassays. Thus, other factors were Iikely responsible for their drift patterns.

Although drift sampling results did not conclusively show that drift rates increase because of high delta p's, combined results from bioassays, benthic sampling and river gas saturation data suggest that $\underline{B}$. tricaudatis, $\underline{E}$. inermis and $\underline{\underline{T}}$. ninutus were the most likely affected. The GBT threshold for Baetis, determined from bioassay \#2, was near a delta $\mathrm{P}$ of $71 \mathrm{mmHg}$. From February through October 1987 , gas saturation levels at Rkm 2.4 always exceeded this level. This was also true at Rkm 14.5 from April through July (Appendix N: Tables 59 and 60). E. inermis's GBT threshold was near a delta $\mathrm{p}$ of $120 \mathrm{mmHg}$ (Figure 2.6.22). $\mathrm{T}$. minutus's GBT threshold was between 113 and $164 \mathrm{~mm}$; however, the trend established by the numbers stranded indicated that it was probably closer to a delta $\mathrm{p}$ of $113 \mathrm{mmHg}$ (Figure 2.6.22). Gas saturation levels equal to or greater than this were also common at both sites in 1986 and 1987 .

T. minutus was significantly more abundant at $\mathrm{Rkm} 14.5$ during all three sampling periods. Densities were $58 \%$ greater downstream in April 1987 (prior to extended periods of high gas saturation levels) increasing to $350 \%$ greater in september. Similarly, Baetis densities at $\mathrm{Rkm} 14.5$ were $21 \%$ greater than those upstream in April 1987 and 430\% greater in september. It seems likely that a similar trend would have been documented with E. inermis if late summer sampling had occurred prior to their emergence.

Although observed thresholds were exceeded at both $\operatorname{Rkm} 2.4$ and 14.5 , they were exceeded over longer periods of time at the 
upstream site. During bioassays some organisms became affected within 1 or $2 \mathrm{~h}$. Others, however, did not become noticeably affected for days, especially at gas saturation levels near their thresholds. Thus, increased densities at the downstream site would be consistent with these observations.

Benthic abundance patterns in Gammarus, Physella and Amiocentrus suggested that the distributions of these taxa are little affected by high gas levels in the Bighorn River. Bioassay results supported this, as GBT thresholds for these taxa were rarely or never exceeded in the River (Appendix N: Tables $57,58,59$, and 60$)$. Also, Isoperla's relatively low susceptibility to GBT seems to support the assumption that temperature, rather than gas supersaturation, is determining its aistribution.

Delta $\mathrm{P}$ fluctuation patterns and the partial pressures of individual supersaturated gases must be considered when assessing "in stream" impacts on the invertebrate community. We attempted to maintain constant delta $\mathrm{P}$ levels during bioassays. Gas saturation levels in the Bighorn River, however, exhibited large fluctuations over relatively short periods (Figures 2.6.8, 2.6.9, 2.6.11, and 2.6.12). Nebeker et al. (1976) noted that stoneflies exposed to gas supersaturation levels of $135 \%$ for 7 d acquired internal gas bubbles. After $4 \mathrm{~h}$ at a lower gas level, however, symptoms disappeared. Thus, invertebrates in the river might tolerate exposure to delta $\mathrm{P}^{\prime} \mathrm{s}$ above their susceptibility threshold when periods of lower delta $\mathrm{P}^{\prime} \mathrm{s}$ commonly occur.

The composition of supersaturated gases in water may also determine the extent of influence on aquatic invertebrates. 
During bioassays, the ratio of dissolved oxygen to nitrogen was similar to that in supersaturated water immediately below Afterbay Dam. Downstream, however, the oxygen component of total dissolved gases increased. In fish, nitrogen is more important in causing GBT than oxygen (Marsh and Gorham 1905). Thus, while total gas saturation levels were sometimes higher downstream, the incidence of GBT in trout was lower (White et al. 1987). This may or may not be the case with invertebrates. How supersaturated levels of oxygen and nitrogen separately affect aquatic invertebrates must be answered before specific "in river" effects can be understood.

\subsection{1d Conclusions}

Total invertebrate densities and invertebrate community structure were similar between sites in the spring but were quite different by the end of summer. Temperature and certain physical habitat differences were responsible for the density patterns of most taxa. However, gas saturation levels in the Bighorn River frequently exceed thresholds for formation of external gas bubbles and for acquiring gas bubble trauma in Baetis tricaudatis, Ephemerella inermis, and Tricorythodes minutus. Significantly greater downstream abundances of Baetis and Iricorythodes are probably related to lower gas saturation levels at that site. These taxa were also the most affected by bioassay gas levels which commonly occur in the Bighorn River. Late summer distribution patterns in Ephemerella could not be determined because nymphs had emerged prior to sampling, but they were likely similar to Baetis and Iricorythodes. 
After being exposed to gas supersaturated water for less than 1 d, most test organisms exhibited behavior that minimized the occurrence of involuntary drift (stranding in the bioassay tanks) due to external gas bubbles. Invertebrates in the river might also adjust their behavior when afflicted with externally induced buoyancy, thus limiting displacement.

Most taxa tested did not acquire gas bubble trauma until gas saturation levels were much higher than normally occur in the Bighorn River. Once afflicted, however, internally induced buoyancy was physically more harmful to test organisms than external gas bubbles, causing death in some.

From a management perspective, the primary concern is the well being of the trout populations. Benthic invertebrate densities were very high at both sampling sites, and invertebrate community composition was comparable to other rivers downstream of deep-release impoundments. If downstream displacement occurs as a result of high gas saturation levels, it appears that upstream migration by adults before ovipositing compensates for losses that occur. Increased invertebrate drift caused by gas supersaturation might be beneficial in terms of food availability for trout so long as invertebrate populations are not depleted. 


\subsubsection{Forage Fish}

\subsubsection{Dace}

\section{$2 \cdot 6.2 .1 \mathrm{a}$ Methods}

The transportable gas supersaturation unit described in the invertebrate bioassay section was used to examine effects of gas supersaturation on forage fish. The proportions of supersaturated water and unaltered water from Afterbay Reservoir added to four aquaria were varied to achieve various gas saturation levels. One tank received only untreated water and served as a control. Flows into all tanks (10 gal aquaria with standpipes maintaining volumes at 8 gal) were approximately 0.75 L/min which provided a turnover time of about $40 \mathrm{~min}$.

Delta P's (mmHg) were measured daily in all tanks using a Bouck gasometer (Bouck 1982). Oxygen pressure was determined with a Yellow Springs Instrument Model 54 oxygen meter or the azide modification of the winkler method (APHA 1976) using Iiquid reagents. Water temperatures were measured with a mercury thermometer. Barometric pressures were measured using a Thommen 22000 pocket altimeter-barometer.

Longnose dace (Rhinichthys cataractae) bioassays were conducted for $120 \mathrm{~h}$ (5 d) and involved four test groups of 25 fish each. Group 1 was the control. We designed the experiment so group 2 was exposed to medium levels of hyperbaric pressures while both groups 3 and 4 were subjected to high pressures. The mean total length of fish was $90.3( \pm 17.9), 95.7( \pm 16.6)$, $81.6( \pm 15.2)$, and $84.6( \pm 17.3) \mathrm{mm}$ for groups $1,2,3$, and 4, respectively. Fish were selected at random and measured and weighed at the end of the test or at the time of mortality. This 
resulted in significantly greater average length of longnose dace in group 2 than in groups 3 and 4 (Analysis of variance; LSD). At the time of mortality or at the conclusion of the experiment, Fish were examined for external signs of GBT and assigned GBT ratings using the system described for adult trout.

\section{$2.6 .2 .1 . b$ Results and Discussion}

No mortality was observed in control and medium treatment (groups 1 and 2) bioassay. Mortality first occurred in high treatment group 3 on day 3 (Figure 2.6.24). Cumulative mortality rose to $40 \%$ on day 4 and was $48 \%$ when the bioassay ended on day 5. We observed mortality on day 1 in high treatment group 4; cumulative mortality quickly increased to $72 \%$ after $48 \mathrm{~h}(2 \mathrm{~d})$ and on day 4 reached $100 \%$ (Figure 2.6 .24 ).

Gas pressures in the control tank (group 1) remained stable and hypobaric (Figure 2.6.25); mean delta $\mathrm{P}$ was $-33 \mathrm{mmH}$ with a range of -30 to $-38 \mathrm{mmHg}$. In other tanks, we were unable to maintain constant gas pressures. The mean and range of delta p's increased in each successive group; the mean and range (in parentheses) in delta $P$ for groups 2, 3, and 4 were 104 (86120) $\mathrm{mmHg}, 163(132-206) \mathrm{mmHg}$, and 204 (144-240) $\mathrm{mmHg}$, respectively. Although oxygen pressure also increased in each succeeding group, variation within groups 1 and 4 remained low (Figure 2.6.26).

Mean oxygen pressures (range in parentheses) were $66(61-70), 118$ $(105-136), 155(133-182)$, and $174(176-169) \mathrm{mmHg}$ for groups 1, 2, 3, and 4, respectively. Nitrogen and argon pressure remained stable throughout the tests for groups 1 and 2 but varied substantially in the other groups (Figure 2.6.27). Nitrogen and 


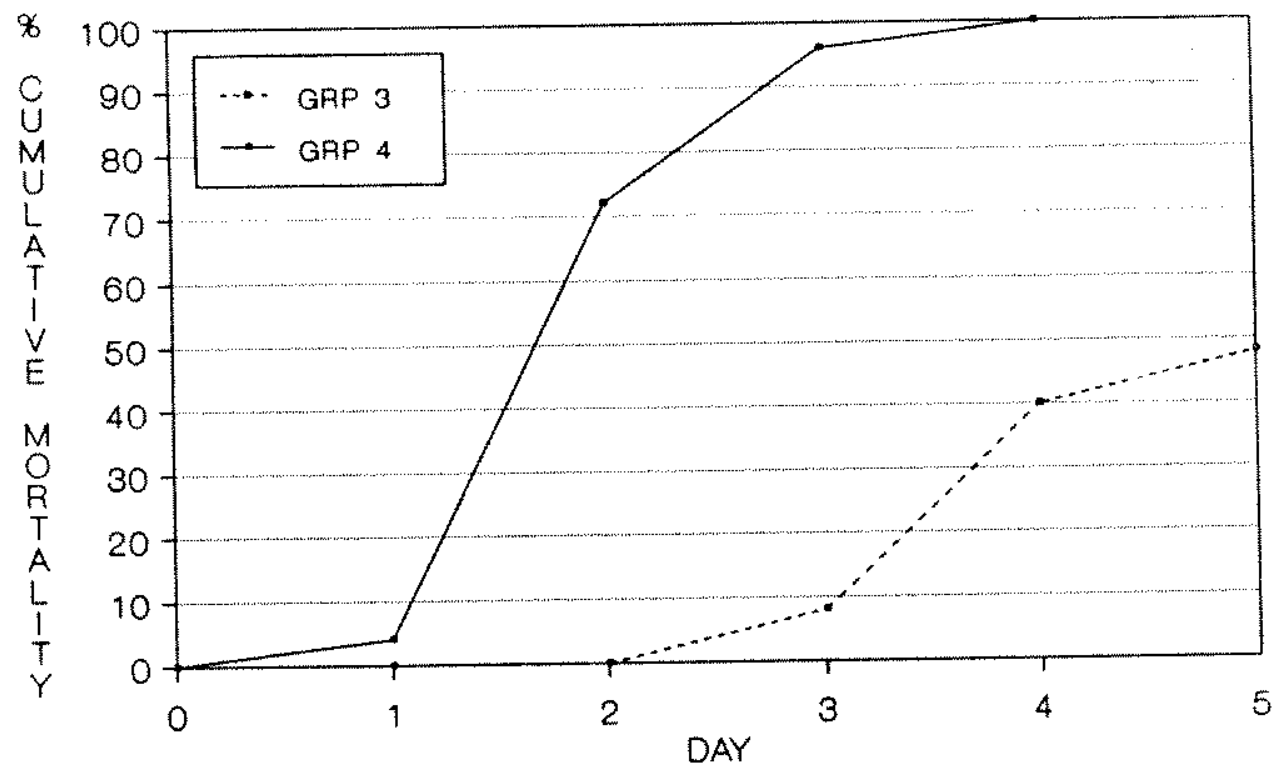

Figure 2.6.24. Cumulative mortality by day among four groups of longnose dace during bioassays at Fort Smith, Montana in 1988. No mortality occurred in groups 1 and 2 . Mean delta $P$ for groups $1,2,3$ and 4 were $-33,104,163$, and $204 \mathrm{mmHg}$, respectively.

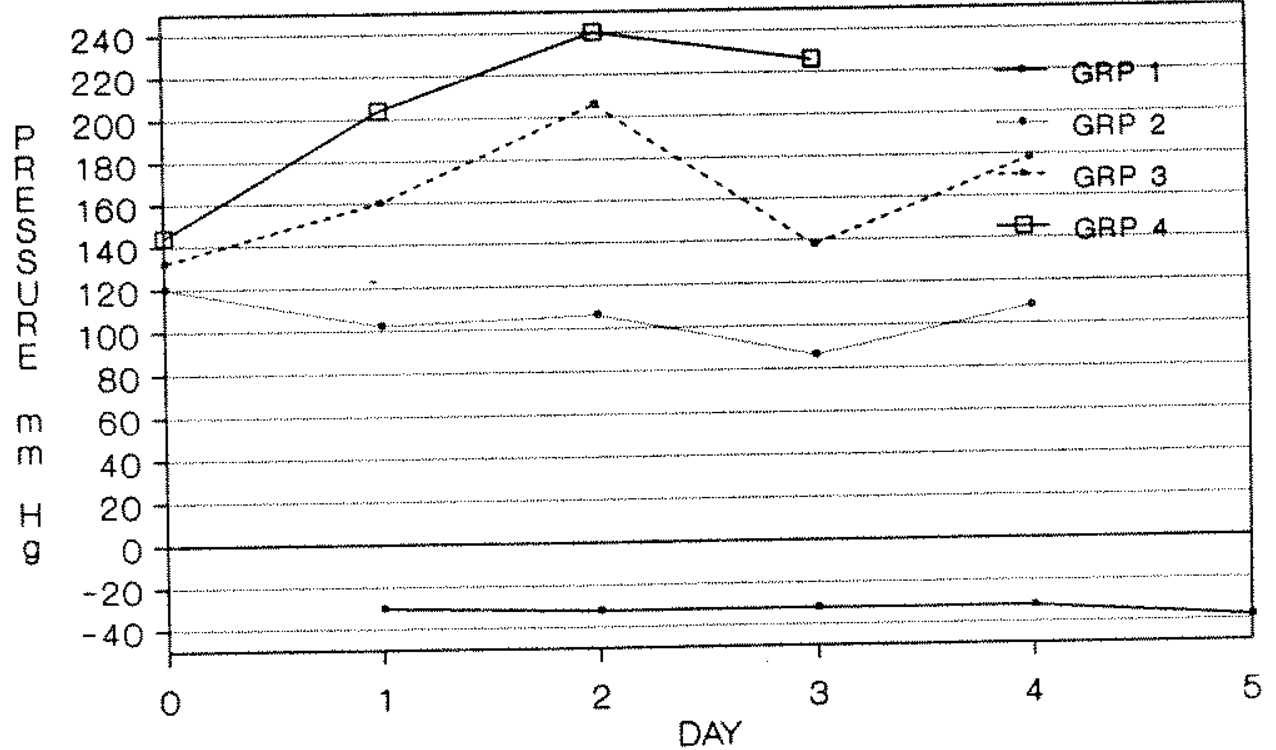

Figure 2.6.25. Hyperbaric gas pressures (mmHg) which each group of longnose dace were exposed to during bioassays at fort smith, Montana, september and october 1988. 


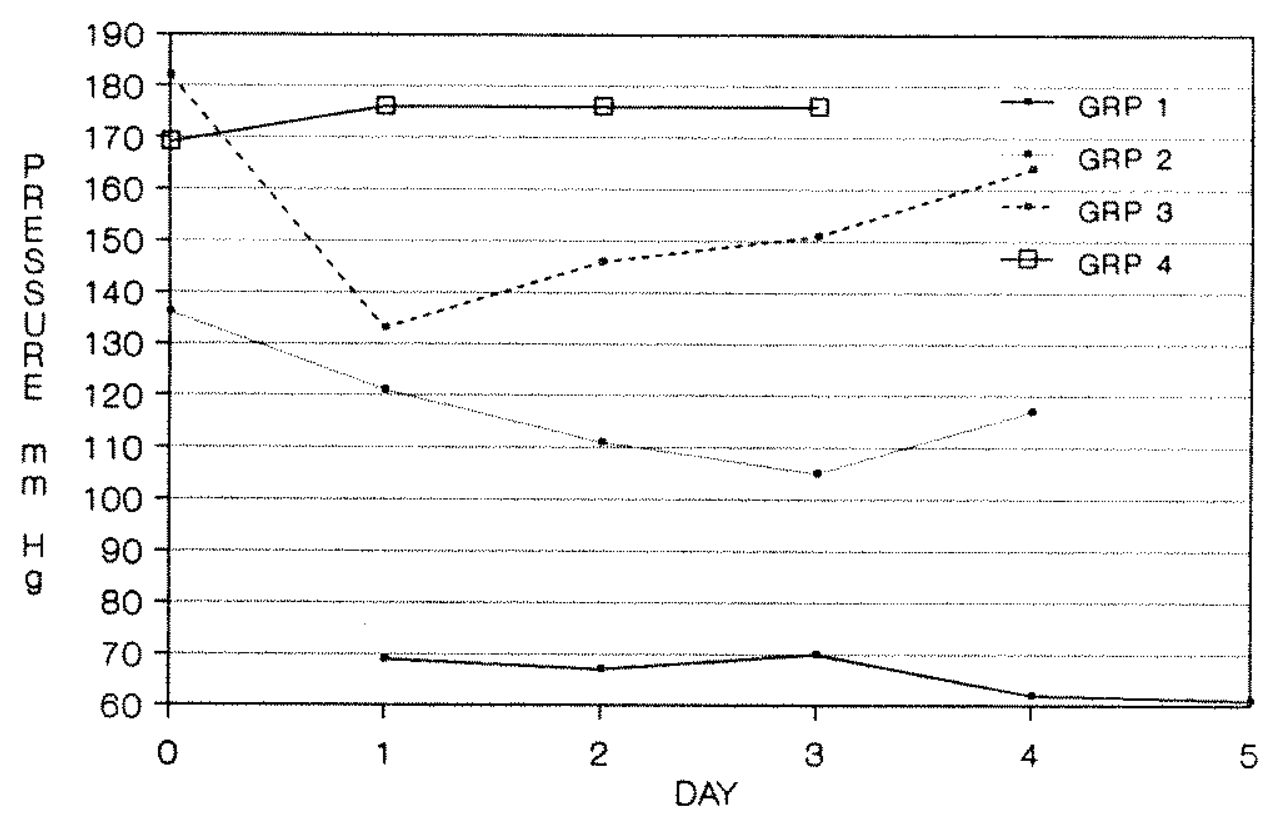

Figure 2.6.26. Oxygen pressures (mmH) which each group of longnose dace was exposed to during bioassays at Fort Smith, Montana, september and october 1988 .

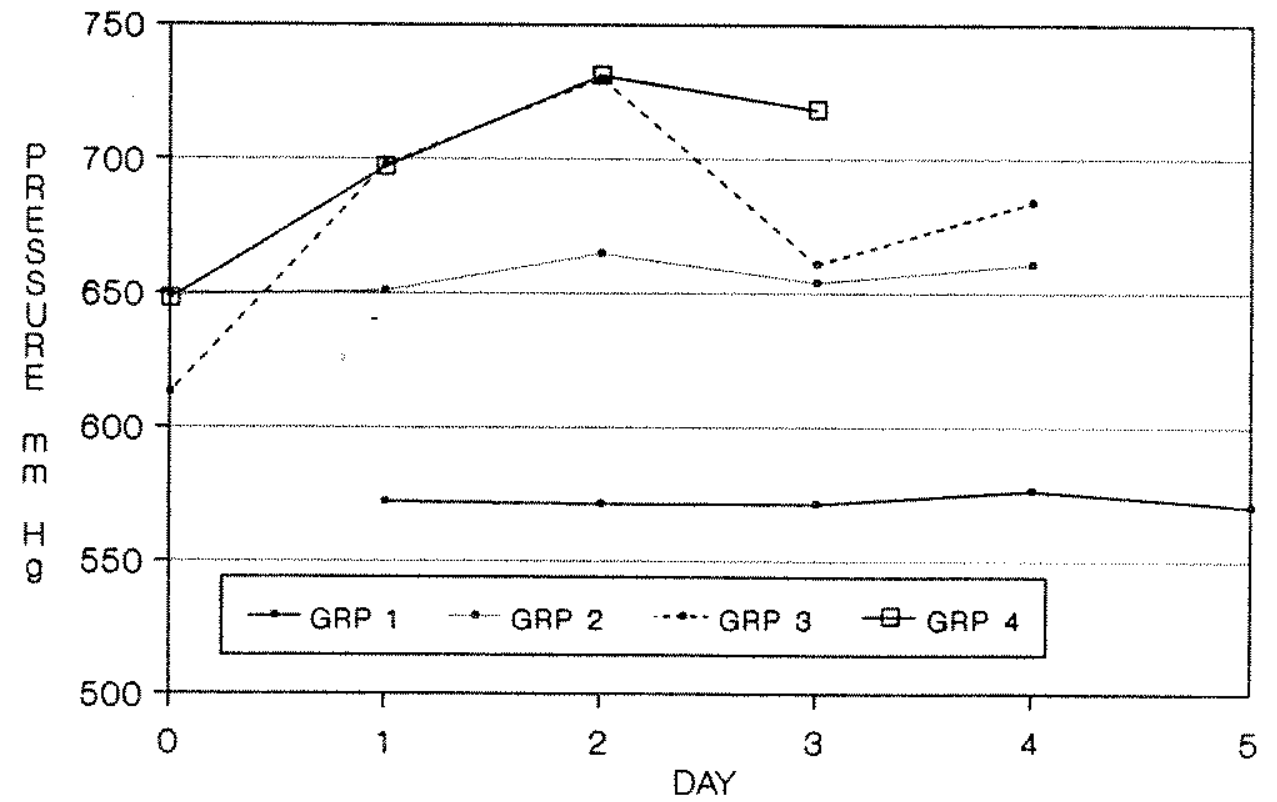

Figure 2.6.27. Nitrogen and argon gas pressures (mmHg) which each group of longnose dace was exposed to during bioassays at Fort Smith, Montana, September and October 1988. 
argon pressure measured on days 1 and 2 were almost identical for groups 3 and 4 .

The rainbow trout threshold equation for bubble growth in the cardiovascular system was applied to longnose dace bioassay results. We did not expect mortality from GBT among the control group (\#1) since pressures remained hypobaric and never approached the cardiovascular system (CV) threshold (Figure 2.6.28). Although group 2 was initially exposed to delta p's exceeding the threshold, time was insufficient for mortality to occur and/or the longnose dace utilized depth to compensate for delta $\mathrm{P}$ levels above the minimum threshold (assuming similar threshold). The maximum hydrostatic compensation at the bottom of the tank was about $15 \mathrm{mmHg}$ which, if utilized by the fish, resulted in increased cardiovascular threshold levels substantially greater than any gas level group 2 was exposed to. The delta $P$ for both groups 3 and 4 remained above cardiovascular system thresholds throughout the experiment (Figure 2.6.29). However, observed mortality differed between groups (Figure 2.6.24). Group 4 was exposed to delta p's of 204 and $240 \mathrm{mmHg}$ during the first $2 \mathrm{~d}$. These were 34 and $44 \mathrm{mmHg}$ greater than those of group 3 and resulted in immediate mortality. Mortality in group 3 was not immediate and lagged at least $1 \mathrm{~d}$ behind increases in delta $P$ (Figure 2.6.24). In addition, the decrease in delta $\mathrm{p}$ on day 3 may have resulted in reduced mortality rate in group 3 between days 4 and 5 . Considering potential hydrostatic compensation, the delta $\mathrm{P}$ on day 3 decreased to a level only $8 \mathrm{mmHg}$ above the maximum cardiovascular threshold and was below the maximum threshold on day 0 . 


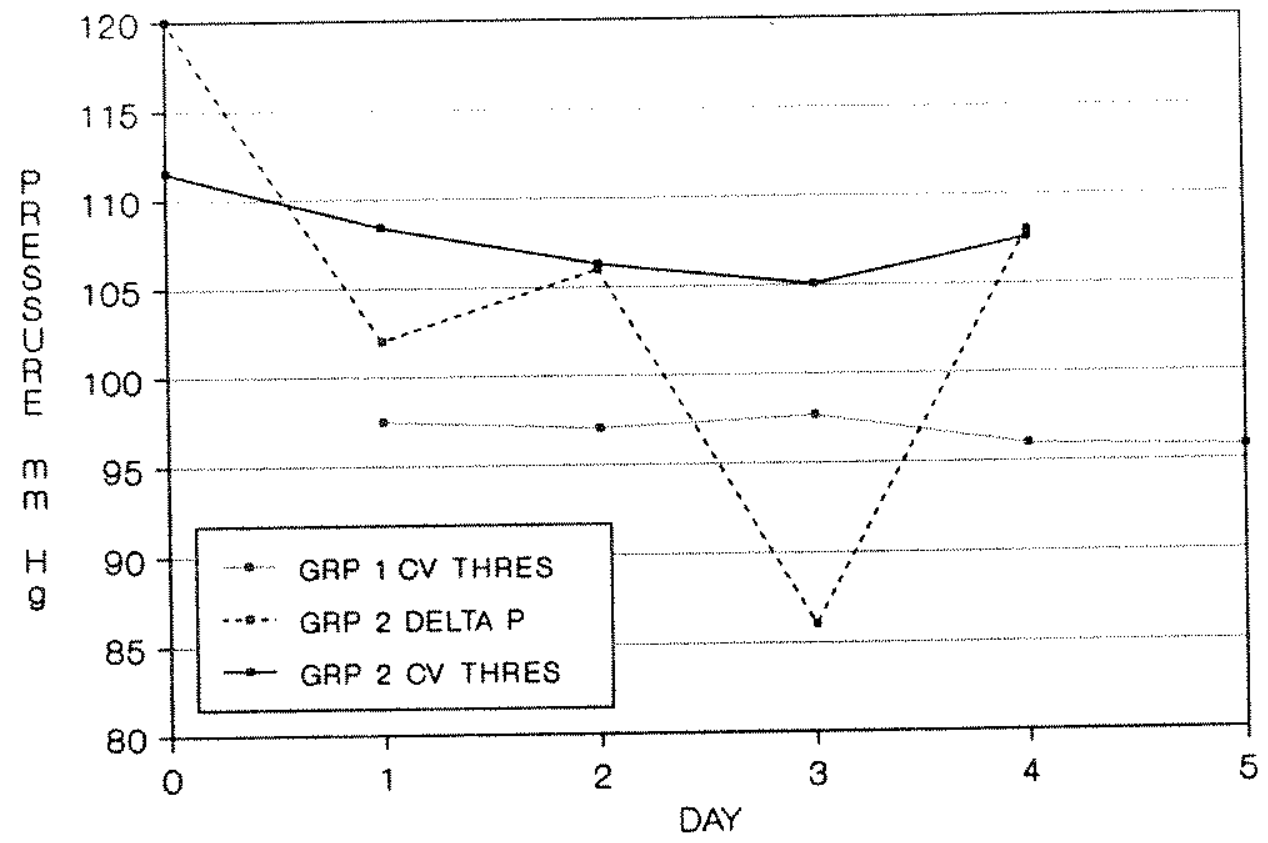

Figure 2.6.28. Trout cardiovascular (CV) system GBT threshold delta $\mathrm{P}$ applied to longnose dace groups 1 and 2 compared to the delta $P$ that group 2 was exposed to during bioassay work at Fort Smith, Montana, september and October 1988. Group 1 delta P remained hypobaric. (The threshold assumes no hydrostatic compensation).

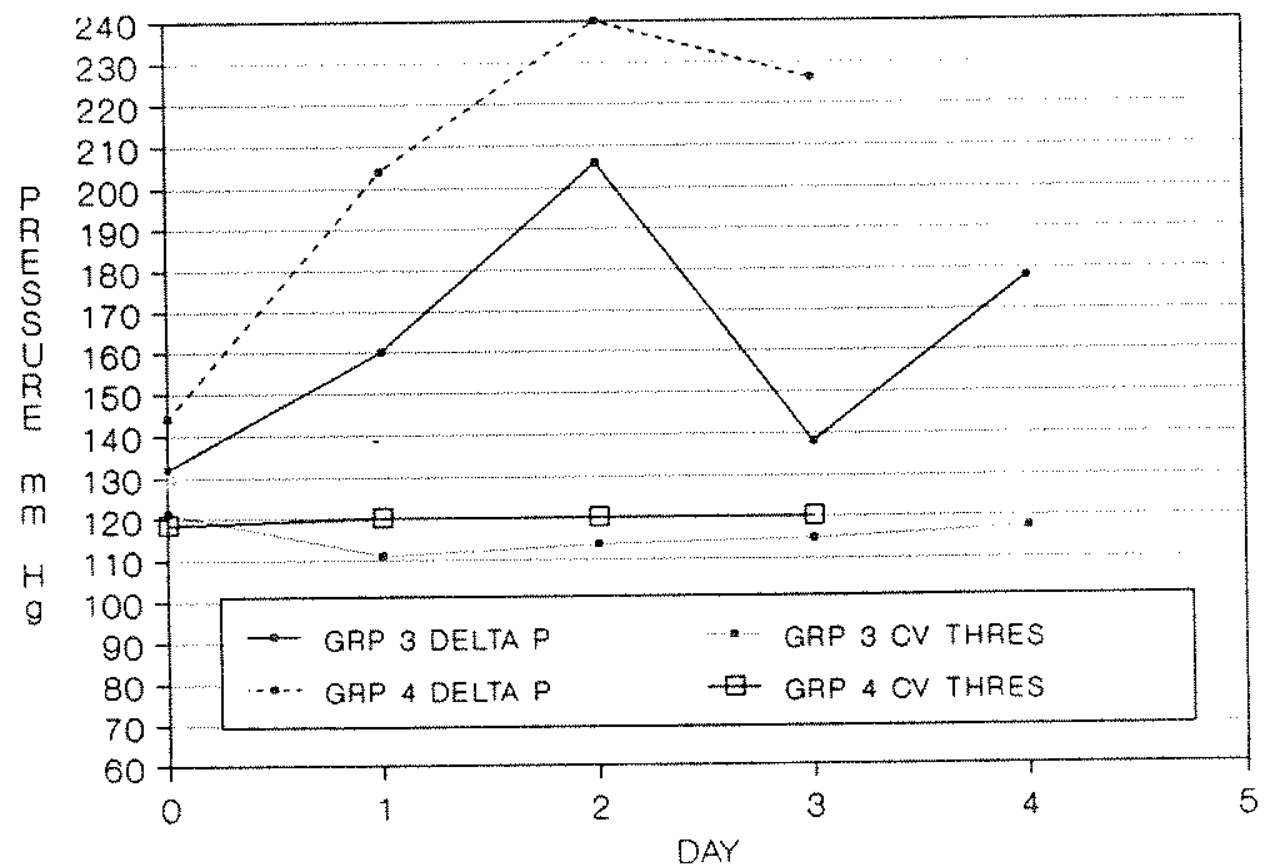

Figure 2.6.29. Trout cardiovascular (CV) system GBT threshold delta $P$ applied to longnose dace groups 3 and 4 compared to the delta $\mathrm{p}^{\prime} \mathrm{s}$ measured during bioassay work at Fort Smith, Montana, september and October 1988. (The threshold assumes no hydrostatic compensation). 
We observed external symptoms of GBT on $75 \%$ and $64 \%$ of the mortalities in groups 3 and 4 , respectively. At the conclusion of the experiment, $29 \%$ of surviving longnose dace displayed external GBT. Among group 3 mortalities, external GBT symptoms were most numerous on the right pectoral fin, but also present on the anal, caudal, right pectoral, right pelvic, and left pectoral fins as well as the right and left lateral lines and the right pectoral insertion. We classified the external symptoms as $28.6 \%$ minor, $42.8 \%$ serious, and $28.6 \%$ severe. In group 4 , external GBT symptoms were most numerous on the anal and right pectoral fins, closely followed by the dorsal, left pectoral, and caudal fins. In addition, GBT symptoms were also noted on the top of the head and snout as well as the premaxillary of some group $4 \mathrm{fish}$. Group 4 had more severe symptoms of GBT with $15.6 \%$ classified as minor, $40.6 \%$ serious, and $43.8 \%$ severe.

Bioassay results suggest that cardiovascular system threshold equation for bubble growth in trout adequately predicts the gas levels where longnose dace mortality would occur. Since accurate hydrostatic pressures were not accounted for in the calculations and in the graphs, we believe the actual thresholds would be lower or more conservative than the hyperbaric pressures at which mortality occurred during the bioassay. The hyperbaric pressures that group 3 were exposed to best represent the maximum gas levels expected in the Bighorn River downstream of Afterbay Dam.

\section{$2 \cdot 6.2 .2$ Mountain Whitefish}

only six mountain whitefish (Prosopium williamsoni) of suitable size were available for bioassays. All were tested 
simultaneously with longnose dace group 3 (Figures 2.6.25). Mean total length of whitefish was $147( \pm 10) \mathrm{mm}$. Mountain whitefish were much less tolerant of the hyperbaric pressures than longnose dace. Whitefish mortality first occurred on day 1 when two fish died. Mortality increased to $50 \%$ on day 2 , and by day 3 mortality was $100 \%$. only two fish displayed external GBT symptoms. One mortality on day 3 had hemmorhaged from the lower jaw, the left pelvic fin, and the bases of the right and left pectoral fins. Another mortality on day 3 had minor GBT symptoms on the left pelvic fin.

These limited data suggests that mountain whitefish are less tolerant of high hyperbaric pressures compared to Iongnose dace and rainbow trout. The cardiovascular system threshold for bubble growth in rainbow trout would not be a suitable method to predict critical dissolved gas levels for mountain whitefish. This supports the findings of other research which found mountain whitefish less tolerant to gas supersaturation than cutthroat trout (Oncorhynchus clarki lewisi), largescale sucker (Catostomus machrocheilus), and torrent sculpin (Cottus rhotheus) (Fickeisen and Montgomery 1975).

\section{$2 \cdot 6 \cdot 2 \cdot 3$ sculpin}

\section{$2.6 .2 .3 \mathrm{a}$ Methods}

Mottled sculpin (Cottus bairdi) obtained from Rock Creek were placed at Rkm 0.6 (gagehouse) and Rkm 19.3 (Bighorn Access) from February - May 1988. Two replicates of 65 or 66 fish each were placed at each site. Rkm 0.6 was representative of high gas saturation levels while Rkm 19.3 (RM 12.0) represented low gas 
levels and served as a control. Test fish were held in wooden boxes which were $30.5 \times 22.9 \times 33 \mathrm{~cm}$. Each box had three $24.1 \times$ $12.7 \mathrm{~cm}$ and two $17.8 \times 12.7 \mathrm{~cm}$ openings covered with $0.32 \mathrm{~cm}$ mesh screen to allow water flow. The holding box was enclosed in a larger wooden box attached to a steel post secured in the river bottom. Each time the site was visited, average water depth in the holding box was measured to allow calculation of hydrostatic pressure.

Delta P's were measured at both sites with a Bouck gasometer, oxygen pressures determined by the azide modification of the winkler method (APHA 1976), and water temperatures obtained with a mercury thermometer. Gas pressures were also continuously monitored at $\mathrm{Rkm} 0.6$ by the common sensing tensionometer and a Hydrolab 8000 interfaced to the Sutron-Hydromet system.

Lengths and weights of mottled sculpin were measured at the beginning and end of the bioassay. Also, we measured length of each fish and categorized all external GBT symptoms twice during the experiment. All mortalities were removed as soon as possible, measured, and examined for GBT. The sculpins were not artificially fed during the experiment.

\section{$2.6 .2 .3 b$ Results}

No mortality occurred among sculpins in groups 1 and 2 at Rkm 0.6 until day 28 (Figure 2.6.30). Mortality rose slowly and temporarily leveled off in both groups around day 40 . Mortality increased sharply in group 2 on day 49 and continued to rise, reaching $25 \%$ at the end of the experiment. Group 1 mortality rose at a much slower rate than that in group 2 after day 49 


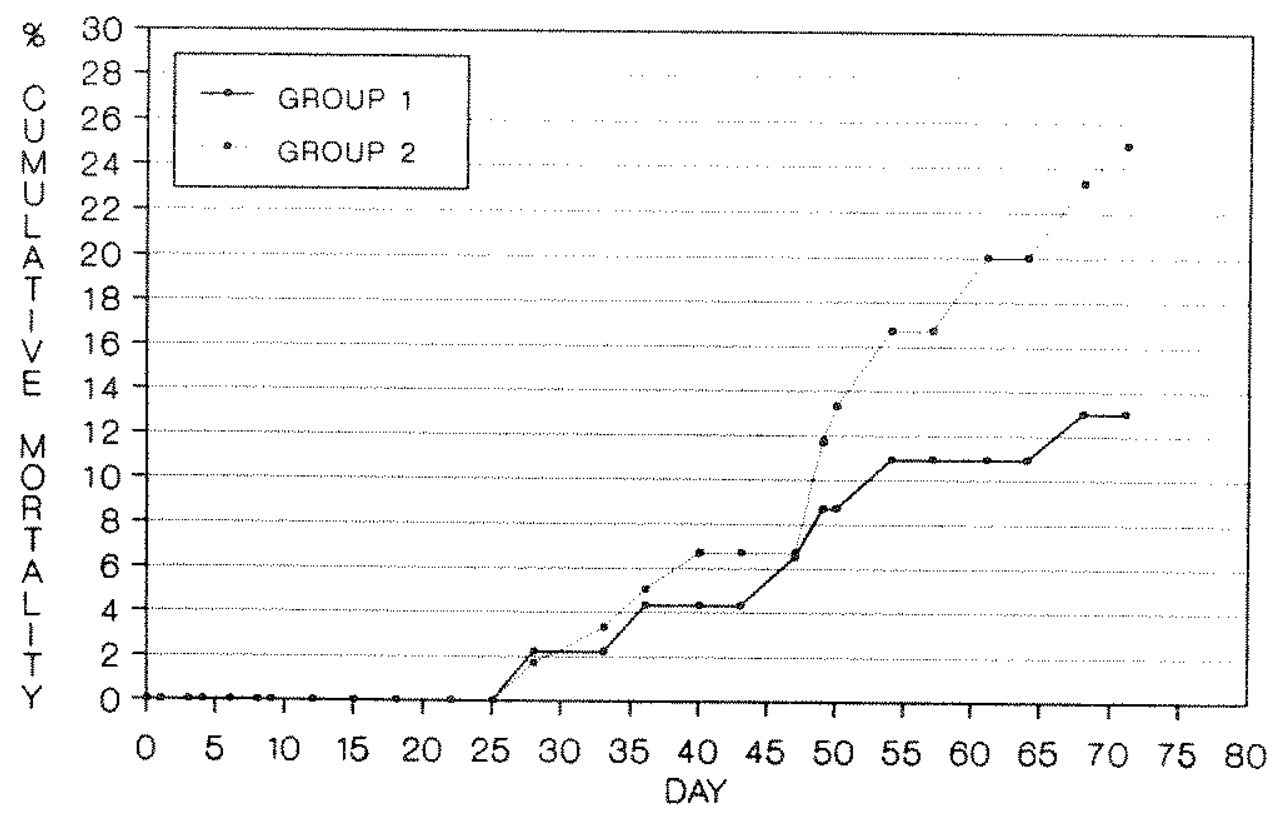

Figure 2.6.30. Cumulative daily mortality in two groups of mottled sculpin held near the right bank at Rkm 0.6 (gagehouse) on the Bighorn River, Montana, February - May 1988.

(Figure 2.6 .30 ). Cumulative mortality in group 1 reached $13 \%$.

Delta P's at the gagehouse (Rkm 0.6) remained above the maximum threshold for bubble growth in environmental water much of the time (Figures $2.6 .31-2.6 .33$ ). During days 40-44 and 47-49 hyperbaric pressure remained below the environmental water threshold (Figure 2.6.32). Diurnal fluctuations dropped delta $P$ below the environmental water threshold on day 2 , on days $66-68$, and on three occasions between days 54-58. Both groups were exposed to delta $\mathrm{P}^{\prime} \mathrm{s}$ exceeding the cardiovascular system thresholds for bubble growth on days $24,26,27,28,29,33$, and 52. In addition, delta $\mathrm{P}$ exceeded the cardiovascular threshold for group 2 on day 50 and equaled the threshold on days 55,61 , and 62. The environmental water and cardiovascular system 


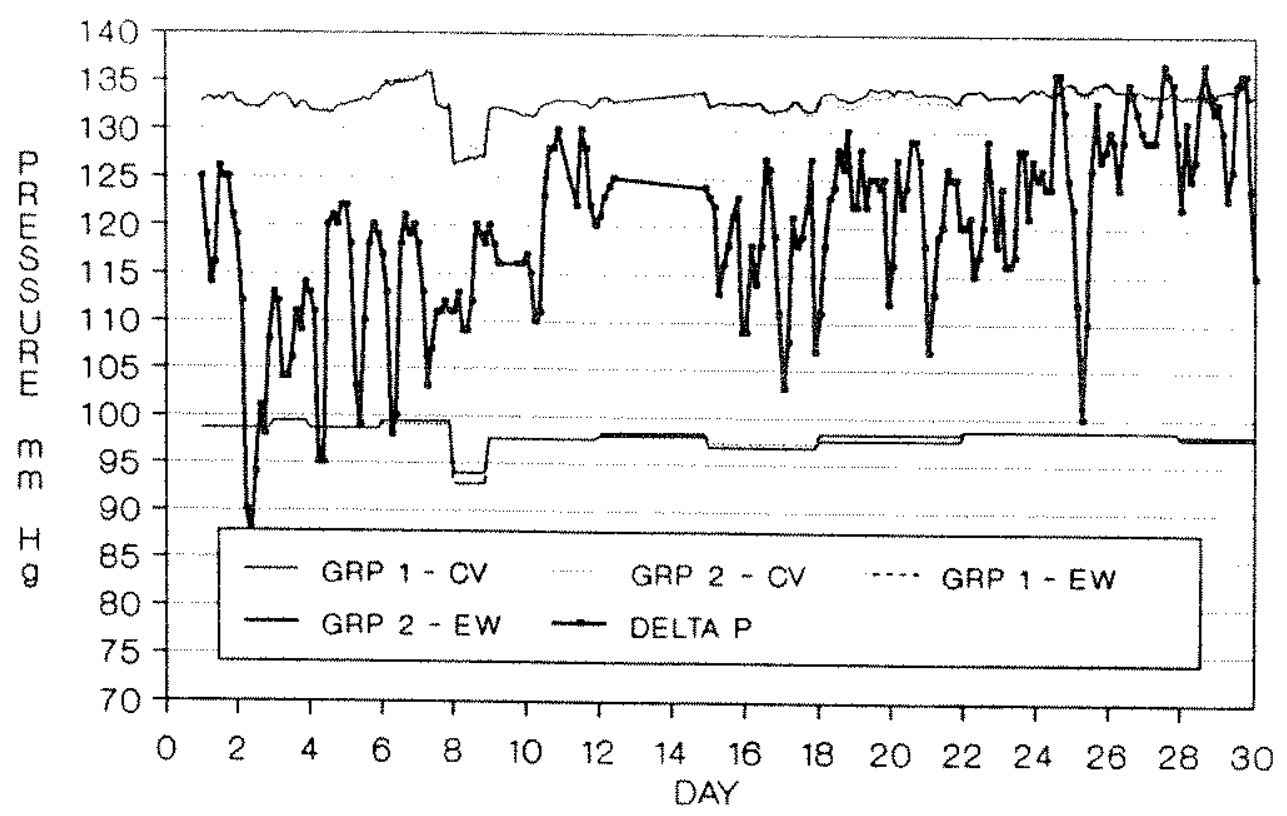

Figure 2.6.31. The maximum cardiovascular system (CV) (rainbow trout) and environmental water thresholds (EW) for bubble growth and delta P's (common sensing) at Rkm 0.6 on the Bighorn River in 1988 for days $0-30$ (thresholds incorporate hydrostatic compensation).

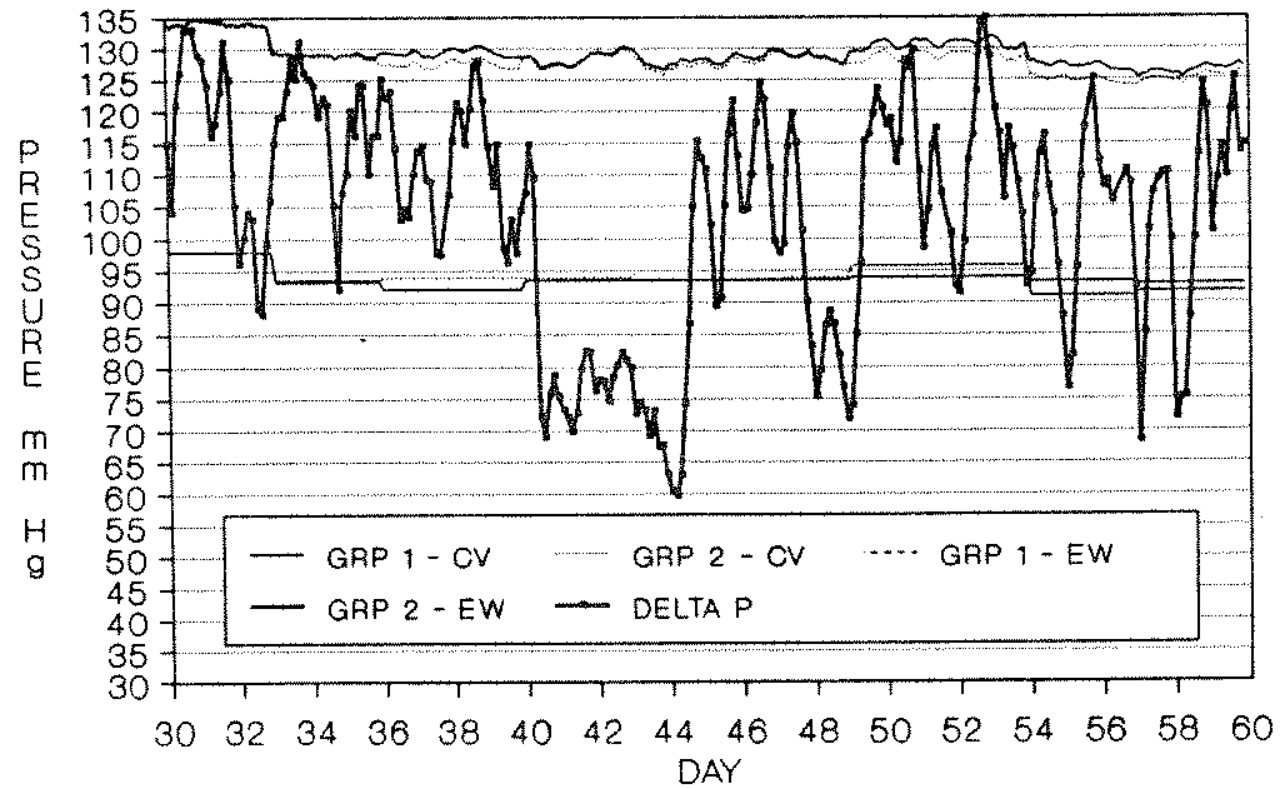

Figure 2.6.32. The maximum cardiovascular system (CV) (rainbow trout) and environmental water thresholds (EW) for bubble growth and delta p's (common sensing) at $\mathrm{Rkm} 0.6$ on the Bighorn River in 1988 for days 30-60 (thresholds incorporate hydrostatic compensation). 


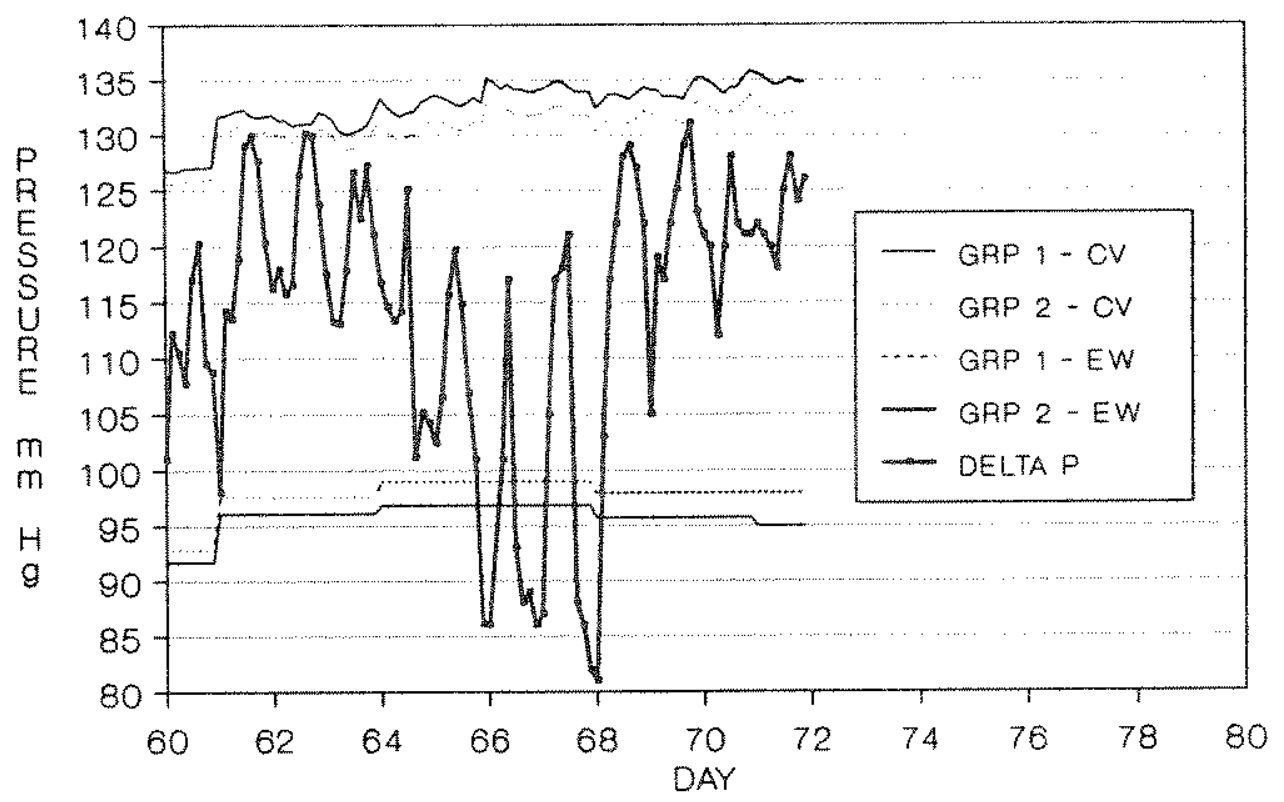

Figure 2.6.33. The maximum cardiovascular system (CV) (rainbow trout) and environmental water thresholds (EW) for bubble growth and the delta $\mathrm{p}^{\prime} \mathrm{s}$ (common sensing) at Rkm 0.6 on the Bighorn River in 1988 for days 60-72 (thresholds incorporate hydrostatic compensation).

thresholds for groups 1 and 2 were nearly identical throughout the bioassay. The largest difference occurred from day 60 to the conclusion of the test, when group 2 thresholds were lower.

Initial sculpin mortality in groups 1 and 2 (Figure 2.6 .30 ) corresponded closely to the first time delta $\mathrm{P}^{\prime} \mathrm{s}$ exceeded the cardiovascular system thresholds (Figure 2.6.31). However, mortality in both groups remained low. No mortality occurred between days 40 and 44 when hyperbaric pressures were below the cardiovascular threshold (Figure 2.6.32). Although increased mortality on day 49 parallels a sharp rise in delta $P$, cardiovascular thresholds were not exceeded. The higher mortality rate in group 2 after day 47 (Figure 2.6 .30 ) may be related to a slight reduction in hydrostatic pressures which 
effectively lowered the bubble growth thresholds so delta $P$ was greater than the thresholds on day 50, equaled them on days 55, 61, and 62, and was $1 \mathrm{mmHg}$ below on day 69.

During the bioassay, 14 sculpin in group 1 and 5 in group 2 were lost to cannibalism. We observed cannibalism on several occasions. Also, the mean length of fish in group $1(71.7 \pm 19.5$ $\mathrm{mm})$, was significantly less than group $2(83.2 \pm 18.1 \mathrm{~mm})$ as well as both $\mathrm{Rkm} 19.3$ groups which had mean lengths of $78.5 \pm 17.9 \mathrm{~mm}$ and $82.3 \pm 15.6 \mathrm{~mm}$ (analysis of variance; LSD). The larger size of sculpin in group 2 may have resulted in higher mortality due to greater sensitivity of larger fish to GBT. The mean length of fish that died was 11.8 and $9.2 \mathrm{~mm}$ greater than for all $\mathrm{fish}$ in groups 1 and 2, respectively. However, the lower bubble growth threshold levels associated with group 2 provide an adequate explanation for the observed differences in mortality.

All sculpin mortalities had external symptoms of GBT with severe symptoms more prevalent in group 2 . Severe symptoms were observed on $73.3 \%$ of group 2 mortalities; $20 \%$ had serious symptoms, and $6.7 \%$ had minor symptoms. This compares to $50 \%$ severe, $16.7 \%$ serious, and 33.3\% minor among group 1 mortalities. Sculpin mortalities had GBT symptoms on an average of 6.8 body parts, with the skin, head, opercules, dorsal fin, and jaws having the highest frequency of symptoms. We also noted symptoms on the pectoral, pelvic, caudal, and anal fins, as well as the gills and lateral lines.

Live fish in groups 1 and 2 were examined for signs of GBT on days 20,49 , and at the conclusion of the bioassays (Table $2.6 .12)$. On day $20,67.4 \%$ of the fish in group 1 and $43.3 \%$ of 
Table 2.6.12. The percentages of mottled sculpin in groups 1 and 2 with external GBT symptoms and the severity of the symptoms on days 20,49 , and 72 of the bioassay near the right bank at $\mathrm{Rkm}$ 0.6 (gagehouse) on the Bighorn River, Montana, February - May 1988 .

\begin{tabular}{rcccc}
\hline & \multicolumn{2}{c}{ Severity GBT symptoms } & Fish with \\
Day & Minor & Serious & Severe & symptoms (\%) \\
\hline Group 1 & 67.4 & 6.5 & 11.5 & 67.4 \\
20 & 76.9 & 11.5 & 11.5 & 61.9 \\
72 & 64.0 & 16.0 & 20.0 & 62.5 \\
Group 2 & & & \\
20 & 43.3 & 19.2 & 0.0 & 43.3 \\
79 & 75.0 & 15.6 & 9.4 & 60.1 \\
72 & 51.9 & 18.5 & 29.6 & 60.0 \\
\hline
\end{tabular}

the fish in group 2 displayed GBT symptoms. on day 49, GBT was similar among groups 1 and 2 with $61.9 \%$ and $60.1 \%$, respectively. At the end of the experiment, $62.5 \%$ of the remaining fish in group 1 and $60.0 \%$ in group 2 had GBT symptoms. The percentage with minor symptoms was always largest. only on day 20 did no fish have severe symptoms. In general the severity of symptoms increased with time.

On 30 May 1988, we examined 73 sculpins that had been transferred from Rkm 0.6 to $\mathrm{Rkm} 19.3$ on 13 May. Unexpectedly, after 17 d at $\operatorname{Rkm} 19.3,32.9 \%$ still had external GBT symptoms. Perhaps delta p's at Rkm 19.3 were high enough to allow maintenance of external symptoms.

No mortality attributed to GBT occurred in the two groups of 
sculpin tested at the lower site, Rkm 19.3. The original sample size in each group was 65 and 66 . However, at the conclusion of the experiments, 56 and 55, respectively, remained in each box. The loss of $\mathrm{fish}$ in both groups was attributed to cannibalism, which was supported by observations. only one sculpin at Rkm 19.3 had external symptoms of GBT. During the bioassay, the mean delta $P$ measured was $88 \mathrm{mmHg}$ with values ranging from 49-133 mmHg. Oxygen pressures averaged $202 \mathrm{mmHg}$ and ranged from 176-234 mmHg. Based on gas measurements made, hyperbaric pressures remained $10.4 \mathrm{mmHg}$ or more below the maximum cardiovascular system. Delta P's averaged $49 \mathrm{mmHg}$ lower than the cardiovascular threshold with the range of differences varying from 10.4 to 87 mmHg

Bioassay results suggest the trout cardiovascular threshold equation can be used to predict safe levels of delta $\mathrm{p}$ for mottled sculpin. We also may speculate that the thresholds are conservative for mottled sculpins. Although delta $\mathrm{P}^{\prime}$ s exceeded the cardiovascular thresholds on several days, total mortality did not increase above $13 \%$ and $25 \%$ for groups 1 and 2 . Also, even though hyperbaric pressures remained above the environmental water threshold most of the time, the incidence of externally visible GBT symptoms seemed to stabilize near $60 \%$

Fickeisen and Montgomery (1975) found the mortality curves for torrent sculpin were much flatter than for other species, and mortality was not observed at $116 \%$ TGP. However, sculpins developed large external bubbles behind the pectoral fin insertion at lower TGP's, which caused a loss of equilibrium and increased buoyancy. Fickeisen and Montgomery (1975) also 
observed a substantial lag between the loss of equilibrium and death. Similar bubble growth and loss of equilibrium was observed on mottled sculpins at Rkm 0.6 on the Bighorn River. Even so, sculpins appear to be more tolerant than trout or dace. This suggests that sculpin transplants, if deemed desirable, have potential for success in the Bighorn River provided that suitable habitats exist. 


\subsection{Dam Operational Characteristics and River Dissolved Gas Lovels}

\subsubsection{River Discharge}

Discharge in 1985 reached maximum levels near 3400 cfs 196.29 $\mathrm{m}^{3} / \mathrm{s}$ ) early in the year, then dropped and remained slightly less than 3000 cfs $\left(84.96 \mathrm{~m}^{3} / \mathrm{s}\right)$ through April (Figure 2.7.1). During May, low inflows forced a further reduction in releases from Bighorn Lake. Summer discharge was the lowest during 1985, reaching a weekly low of $1445 \mathrm{cfs}\left(40.92 \mathrm{~m}^{3} / \mathrm{s}\right)$ the first week of June. Discharge increased from mid-August to early November, then rose and remained constant through the end of the year. Discharge during most of 1986 was substantially greater than in other years (Figure 2.7.1); mean daily discharge from Afterbay Dam was 3820 cfs $\left(108.18 \mathrm{~m}^{3} / \mathrm{s}\right)$. Discharge aropped to near 2500 cfs $\left(70.80 \mathrm{~m}^{3} / \mathrm{s}\right)$ in mid-January and remained constant through February. Flows frequently fluctuated from March through early June; mean daily discharge commonly oscillated over $1000 \mathrm{cfs}$ $\left(28.32 \mathrm{~m}^{3} / \mathrm{s}\right)$. Flows peaked near $7400 \mathrm{cfs}\left(209.57 \mathrm{~m}^{3} / \mathrm{s}\right)$ in June and remained high through early August, then aropped to between 2700-3700 cfs for the remainder of the year.

Discharge from Afterbay Dam in 1987 contrasts sharply with 1986 since no high-water period occurred. From 1 January - 26 March, mean daily discharge remained near 3000 cfs $\left(84.96 \mathrm{~m}^{3} / \mathrm{s}\right)$. Flows rose to near 4000 cfs $\left(113.28 \mathrm{~m}^{3} / \mathrm{s}\right)$ in late March and gradually decreased thereafter, reaching a low in late october. The mean daily discharge from Afterbay Dam in 1987 was 2612 cfs $\left(73.97 \mathrm{~m}^{3} / \mathrm{s}\right)$

Discharge in 1988 generally decreased as the year progressed 


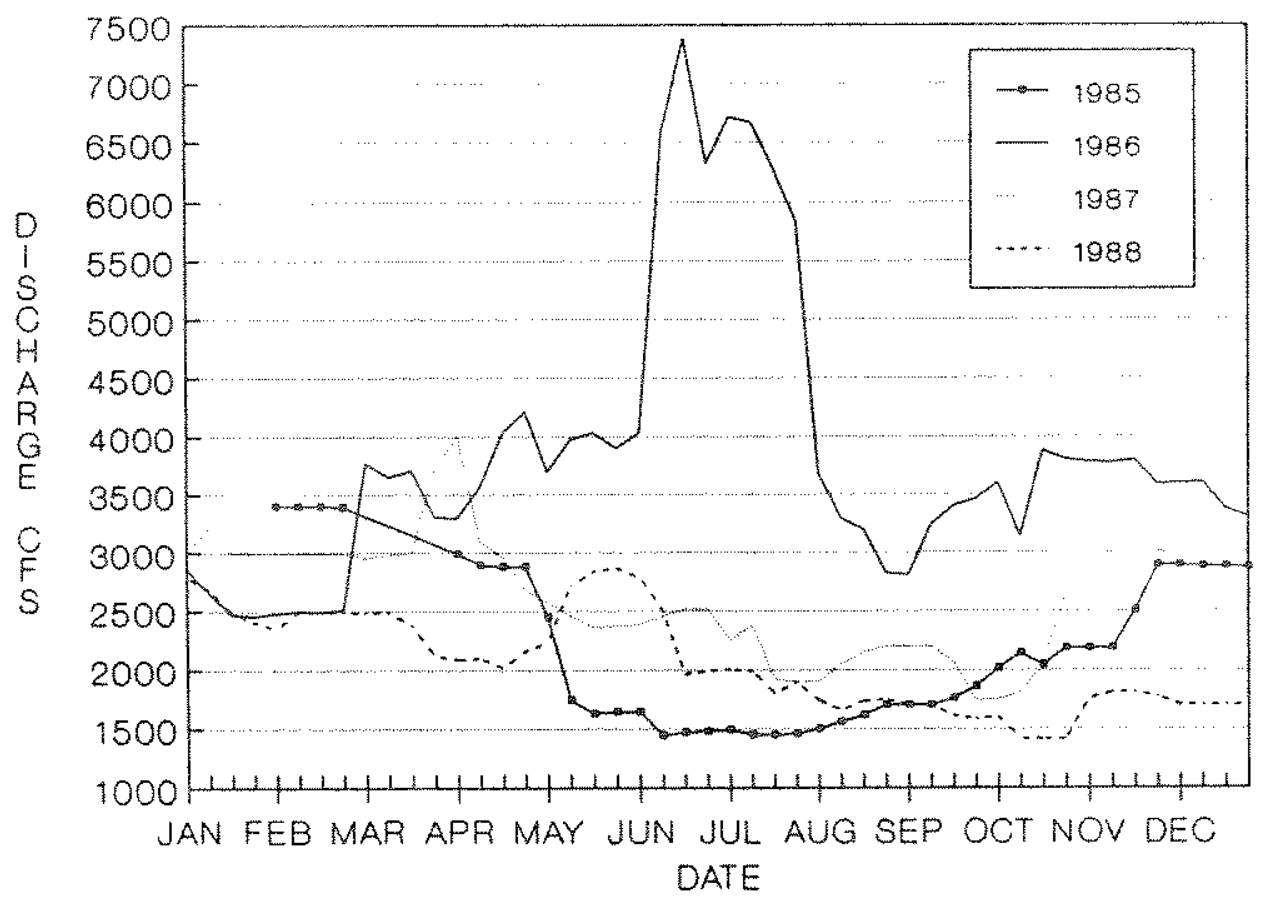

Figure 2.7.1. Mean weekly discharge (cfs) of the Bighorn River below fterbay Dam measured at the $s t$. Xavier gagehouse from 1985-88.

(Figure 2.7.1) with the exception of a modest peak during May (2868 cfs: $\left.81.22 \mathrm{~m}^{3} / \mathrm{s}\right)$. Discharge dropped below 2000 cfs $(56.64$ $\mathrm{m}^{3} / \mathrm{s}$ ) in June and gradually declined, reaching a minimum in October. The lowest mean weekly discharge was 1415 cfs $(40.07$ $\left.\mathrm{m}^{3} / \mathrm{s}\right)$; mean daily discharge was $2060 \mathrm{cfs}\left(58.34 \mathrm{~m}^{3} / \mathrm{s}\right)$.

\section{7 .2 Afterbay Reservoir Gas honitoring}

We measured dissolved gas levels in the irrigation canal 0.6 $\mathrm{km}$ downstream from Afterbay Dam on six occasions from 20 June 10 July, 1986. All delta $\mathrm{p}^{\prime} \mathrm{s}$ were negative $(\overrightarrow{\mathrm{x}}=-14 ;$ range $=$ -4 to $-24 \mathrm{mmHg})$, i.e. total gas saturation was below $100 \%$ ( $\bar{x}=$ 98.08 : range $=96.5-98.48$ ). Oxygen pressures varied from 93-109 mmHg and averaged $103 \mathrm{rmHg}$-corresponding to mean oxygen saturation of $73.7 \%$ (range $=66.4-77.88$ ) and dissolved oxygen 
concentration of $7.0 \mathrm{mg} / \mathrm{L}$ (range $=5.9-7.7 \mathrm{mg} / \mathrm{L}$ ). Nitrogen and argon pressures $\left(\mathrm{N}_{2}+\mathrm{Ar}\right)$ averaged $552 \mathrm{mmHg}$ and ranged from 548$561 \mathrm{mmHg}$; the $\mathrm{N}_{2}+$ Ar pressures were slightly above saturation $(\bar{x}=104.4 \%$, range $=103.8-105.4 \%)$ and lower than expected; total gas saturation was $21.4 \%$ lower in the canal than in the river (Rkm 0.6) during 1986 monitoring.

To examine causes of low oxygen and total gas levels in the canal, we made nine measurements in Afterbay Reservoir 3 September 1986. Little difference between gas pressures and temperature occurred between sites (Table 2.7.1). Nitrogen + Ar pressure and temperature were similar throughout its length. Delta $P$ and oxygen pressure increased an average of 17 and 15 mmHg, respectively, from 3.3 to $0.05 \mathrm{~km}$ upstream of Afterbay Dam. However, delta $\mathrm{P}^{\prime} \mathrm{s}$ in the canal and Afterbay Reservoir in September were much more hypobaric than levels measured in June and July 1986.

Gas tension monitoring in Afterbay Reservoir continued during 1987 using a Common sensing tensionometer at the boat ramp from 8 May - 30 July (Table 2.7.2). The average delta $\mathrm{p}$ from the 10 measurements was $28 \pm 12 \mathrm{mmHg}$ with values ranging from $3-47 \mathrm{mmHg}$; all delta P's were positive. The average oxygen pressure was 134 mmHg (range $=119-145)$, and the mean calculated $\mathrm{N}_{2}+$ Ar pressure was $565 \mathrm{mmHg}$ (range $=543-580$ ). Oxygen saturation on all but two occasions remained just below equilibrium, while nitrogen plus argon saturation was always slightly above equilibrium. oxygen remained substantially higher than 1986 levels measured in both the irrigation canal and Afterbay Reservoir. 
Table 2.7.1. Gas pressures (mmHg) measured at selected sites on Afterbay Reservoix and the irrigation canal below Afterbay Dam, 3 September, 1986.

\begin{tabular}{|c|c|c|c|c|c|c|c|c|}
\hline \multirow[t]{2}{*}{$\mathrm{Rkm}^{\mathrm{a}}$} & \multirow[t]{2}{*}{ Bank $k^{b}$} & \multirow[t]{2}{*}{ Time } & \multicolumn{5}{|c|}{ Pressure (mmig) } & \multirow{2}{*}{$\begin{array}{l}\text { Temp } \\
\text { (C) }\end{array}$} \\
\hline & & & Barometric & ${ }^{\wedge} \mathrm{P}$ & $\mathrm{O}_{2}$ & $\mathrm{~N}_{2}+\mathrm{A} \mathrm{r}^{\mathrm{C}}$ & Water vapor & \\
\hline 3.3 & $\mathrm{R}$ & $16: 18$ & 682 & -65 & 73 & 528 & 16.4 & 18.9 \\
\hline 2.5 & $\mathrm{R}$ & $16: 43$ & 682 & -64 & 74 & 528 & 16.3 & 18.8 \\
\hline$n$ & L & $17: 00$ & 682 & -65 & 76 & 525 & 16.3 & 18.8 \\
\hline 1.5 & $\mathrm{R}$ & $17: 20$ & 682 & -57 & 84 & 525 & 16.5 & 19.0 \\
\hline " & L & $17: 34$ & 682 & -59 & 79 & 528 & 16.3 & 18.8 \\
\hline 0.4 & $\mathrm{R}$ & $17: 52$ & 682 & -29 & 116 & 520 & 16.6 & 19.1 \\
\hline$n$ & I & $18: 10$ & 683 & -44 & 87 & 536 & 16.4 & 18.9 \\
\hline 0.05 & $\mathrm{R}$ & $18: 27$ & 683 & -42 & 94 & 531 & 16.5 & 19.0 \\
\hline$n$ & L & $18: 43$ & 683 & -54 & 82 & 531 & 16.5 & 19.0 \\
\hline-0.6 & Canal & $19: 25$ & 683 & -54 & 83 & 530 & 16.4 & 18.9 \\
\hline
\end{tabular}

a distance upstream from Afterbay Dam.

b $R=$ right bank, $L=$ left bank when viewed facing downstream.

c calculated values

Gas levels in Afterbay Reservoir fluctuate greatly and probably depend upon conditions in Bighorn Lake and the river upstream. A permanent solution to the air entrainment problem below Afterbay Reservoir, such as construction of a powerhouse and bypass, could at times cause a substantial change in the oxygen regime in the upper $2.4 \mathrm{~km}$ of the river. oxygen concentrations in Afterbay Reservoir on 3 September 1986 were below Montana water-quality criteria $(7.0 \mathrm{mg} / \mathrm{L})$ for cold water aquatic 1ife (DHES 1986), trout production could be reduced 
Table 2.7.2. Gas tensions (mmHg) measured at the boat ramp on Afterbay Reservoir from 8 May - $30 \mathrm{July}, 1987$.

\begin{tabular}{|c|c|c|c|c|c|c|c|}
\hline \multirow[t]{2}{*}{ Date } & \multirow[t]{2}{*}{ Time } & \multicolumn{5}{|c|}{ Pressure (mmHg) } & \multirow{2}{*}{$\begin{array}{l}\text { Temperature } \\
\text { (C) }\end{array}$} \\
\hline & & B.P. & ${ }^{*} \mathrm{P}$ & $\mathrm{O}_{2}$ & $\mathrm{~N}_{2}+\mathrm{Ar}$ & Water Vapor & \\
\hline $5 / 8$ & $14: 56$ & 675 & 23 & 134 & 557 & 7.1 & 6.1 \\
\hline $5 / 22$ & $14: 39$ & 683 & 39 & 136 & 580 & 6.8 & 5.6 \\
\hline $5 / 29$ & $16: 38$ & 676 & 28 & 138 & 558 & 7.1 & 6.1 \\
\hline $6 / 5$ & $14: 20$ & 678 & 25 & 134 & 562 & 6.8 & 5.6 \\
\hline $6 / 17$ & $17: 07$ & 675 & 47 & 142 & 572 & 7.8 & 7.5 \\
\hline $6 / 29$ & $11: 25$ & 684 & 3 & 137 & 543 & 7.1 & 6.1 \\
\hline $7 / 3$ & $10: 06$ & 677 & 33 & 145 & 558 & 7.0 & 5.9 \\
\hline $7 / 9$ & $16: 50$ & 676 & 35 & 127 & 577 & 7.6 & 7.2 \\
\hline $7 / 17$ & $13: 42$ & 677 & 23 & 119 & 573 & 8.3 & 8.4 \\
\hline $7 / 30$ & $19: 11$ & 676 & 25 & 125 & 568 & 8.7 & 9.2 \\
\hline Mean & & 678 & 28 & 134 & 565 & 7.4 & 6.8 \\
\hline std dev. & & 3 & 12 & 8 & 11 & 0.7 & 1.3 \\
\hline
\end{tabular}

a calculated values

when compared to the high oxygen levels now present (Davis et al.1979). Also, angler-induced stress in combination with lower oxygen concentrations may increase fishing mortality in the upper reaches at certain times of the year. These potential effects would not extend far downriver because of the high input of oxygen by photosynthesis as well as reaeration. Any detrimental effects in the first $2.4 \mathrm{~km}$ would be outweighed by an increase in overall fish health, particularly in larger fish, resulting from lowering TGP and $\mathrm{N}_{2}+\mathrm{Ar}$ levels. 


\subsubsection{Afterbay operation Monitoring \\ 2.7 .3 .2 low Tests}

On 17 October 1985 the Bureau of Reclamation reduced the discharge from Afterbay Dam to $11.3 \mathrm{~m}^{3} / \mathrm{s}$ (400 cfs), and then increased water elevations $3.1-15.2 \mathrm{~cm} / \mathrm{h}(0.1-0.5 \mathrm{ft} / \mathrm{h})$ until normal operational flows were attained: only the sluice gates were used. As discharge and water levels rose, we measured delta $\mathrm{p}$ every $15 \mathrm{~min}$ (Figure 2.7 .2 ); delta $\mathrm{p}$ continued to increase from 22-104 migg as sluiceway discharge rose from $11.16-43.75 \mathrm{~m}^{3} / \mathrm{s}$ (394-1545 cfs). A regression equation was developed to describe the relationship over this range of sluiceway flows with water temperatures at $11.6 \mathrm{C}(52.8 \mathrm{~F})$ :

$$
{ }^{A} \mathrm{P}=2.542(\mathrm{Q})-4.27 \quad \mathrm{I}=0.99
$$

where: ${ }^{\wedge} \mathrm{P}=$ delta $\mathrm{p}$ (mmg)

$Q=$ discharge $\left(\mathrm{m}^{3} / \mathrm{sec}\right)$

oxygen pressure also increased from about $130 \mathrm{mmHg}$ to 139 mmHg throughout the last half of monitoring. computation of the proportion of the total discharge routed through the sluicegates was necessary to establish a relationship between hyperbaric pressure in Bighorn River water and the operation of Afterbay Dam. Since sluicegate openings vary automatically throughout the day and correspond to frequent flow changes, we developed equations of sluiceway discharge based on Afterbay elevations between 3176.00 and 3189.00 ft $(968.04-972.01 \mathrm{~m}$ ) (Table 2.7.3); flows calculated deviated $0.01-2.96 \%$ from flows determined from Bureau of Reclamation sluiceway discharge curves. Equations in Table 2.7 .3 are applicable only if the gates are open exactly to the nearest foot. Additional equations were developed to 

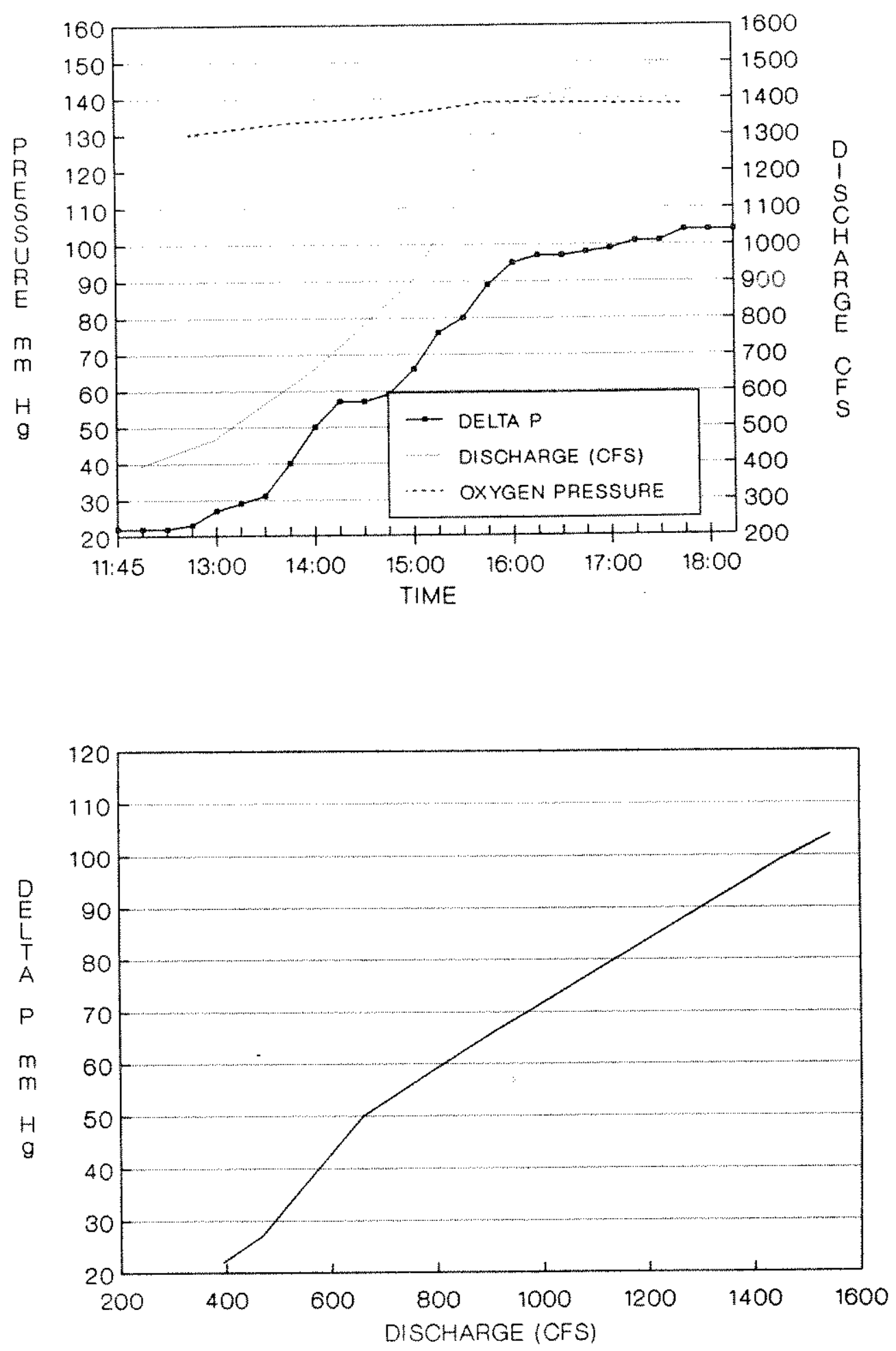

Figure 2.7.2. Delta $P$, oxygen pressure (mmHg), and sluiceway discharge (cfs) measured on the right bank (Rkm 0.6 ) on 17 october 1985 (top) and the relationship between delta $P$ and discharge from the sluiceway (bottom). 
Table 2.7.3. Discharge equations for the sluiceway gates of Afterbay Dam on the Bighorn River.

Gate opening (feet)

Equation

$\begin{array}{ll}1.0 & Q=\left(-0.91(E)^{2}+171.3(E)-7137.6\right) / 3 \\ 2.0 & Q=\left(-1.43(E)^{2}+290.1(E)-12794.5\right) / 3 \\ 3.0 & Q=\left(-3.12(E)^{2}+594.9(E)-25631.7\right) / 3 \\ 4.0 & Q=\left(-2.86(E)^{2}+579.8(E)-25448.1\right) / 3 \\ 5.0 & Q=\left(-2.15(E)^{2}+464.8(E)-19812.1\right) / 3 \\ 6.0 & Q=\left(-2.54(E)^{2}+546.3(E)-22953.1\right) / 3 \\ 7.0 & Q=\left(-2.99(E)^{2}+645.3(E)-27046.1\right) / 3\end{array}$

where: $Q=$ discharge from one $10 \mathrm{ft} \times 8 \mathrm{ft}$ sluiceway gate (cfs)

$\mathrm{E}=$ Afterbay elevation $-3100.00 \mathrm{ft}$

calculate discharge for any gate opening.

During a flow reduction, water $(14.3 \mathrm{C} ; 57.7 \mathrm{~F})$ was discharged only from the sluiceway beginning at $12: 41$ on 15 October 1986. Sluiceway discharge varied from 3123-1500 cfs $\left(88.4-42.5 \mathrm{~m}^{3} / \mathrm{s}\right)$ as determined from the U.S.G.S. stage-discharge rating curve (Figure 2.7.3). A substantial discrepancy occurred between the observed (rating curve) discharge and the discharge calculated from the sluiceway discharge equations (Figure 2.7.3); calculated sluiceway discharge was $720-845$ cfs $\left(20.39-23.93 \mathrm{~m}^{3} / \mathrm{s}\right)$ greater than observed. This large difference between methods of determining discharge resulted in data analysis problems. Conclusions and recommendations based on discharge would be 


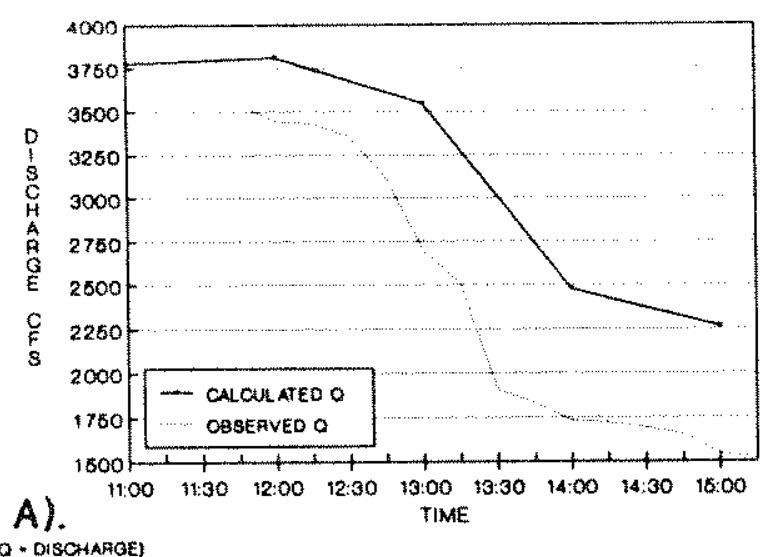

(O. - DISCHAFGE)

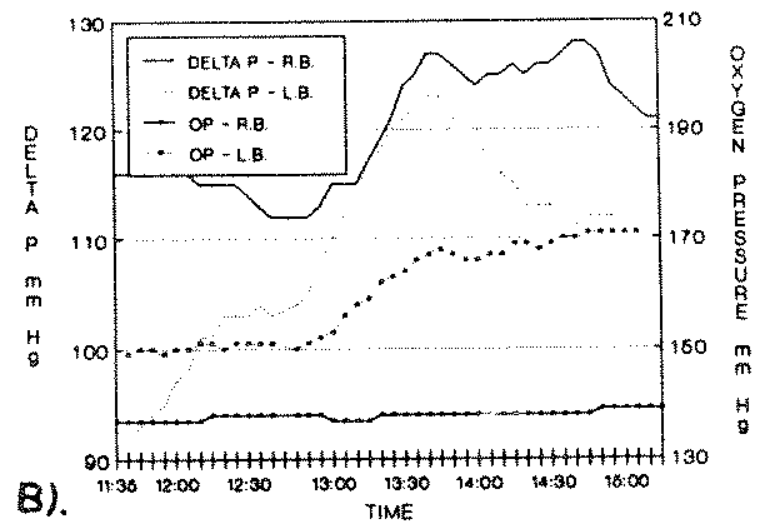

IOP * COY YOEN PAESSUAE)

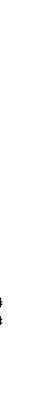

OAAWDOWN - 10/15/86

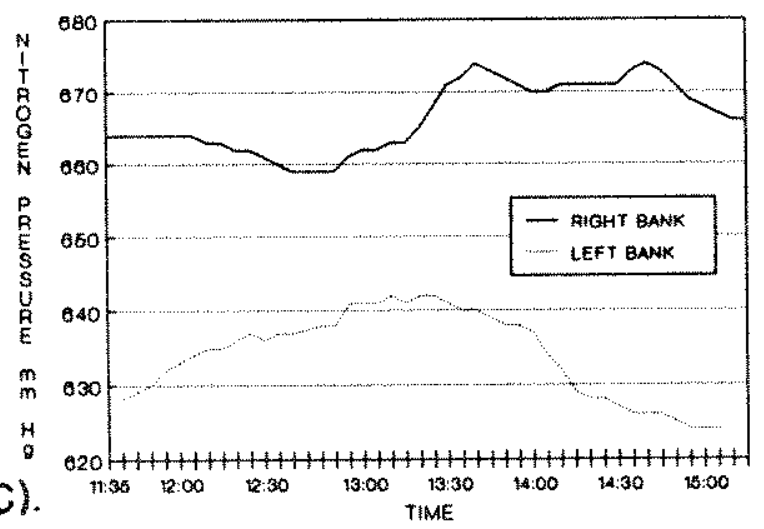

DRANDOWH - 10/15/86

OBSERVED O (HYDROMET) CALCULATED O

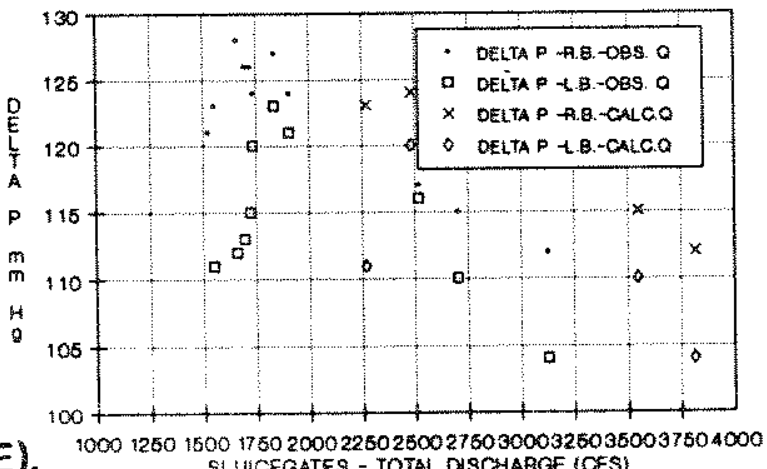

E). SL UICEGATES - TOTAL DISCAAPGE (CFS)
DRAWDOWN - $10 / 16 / B 0$

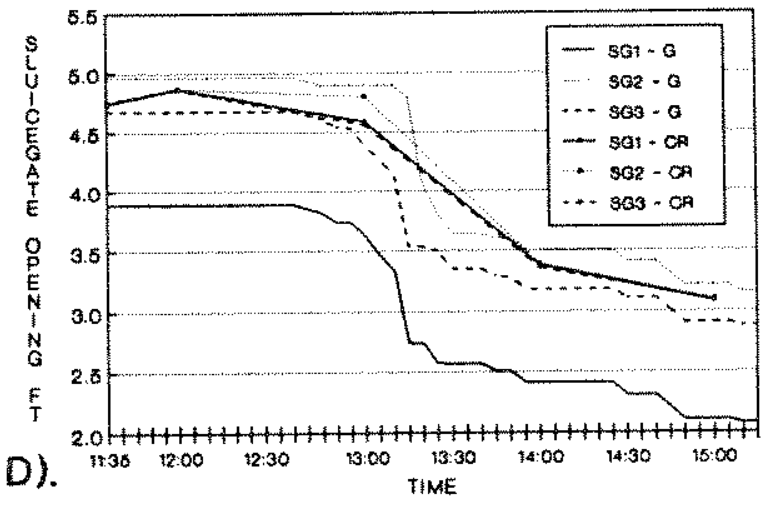

(BOE- SLULOCATE NUMBEA)

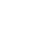


considerably different depending on the technique used.

Gas monitoring began at $11: 35$, and all flow was routed through the sluiceway by $12: 41$. Initial hyperbaric pressures were much higher on the right than the left bank (Figure 2.7.3). Delta $\mathrm{p}$ along the left bank increased as discharge was diverted to the sluiceway. We observed a first peak in delta $P$ at about $13: 45$ on both the right and left banks 1127 and $123 \mathrm{mmHg}$, respectively), which corresponds to an observed flow of 1831 cfs $\left(51.85 \mathrm{~m}^{3} / \mathrm{s}\right)$. This general pattern is comparable to that found on 19 October 1973 when water temperatures were 13.9 C (57 F) (Bureau of Reclamation 1973). As flows decreased below 1800 cfs $\left(50.98 \mathrm{~m}^{3} / \mathrm{s}\right)$, gas levels on the left bank dropped until stabilizing at 111-112 mmg--still higher than when monitoring began (Figure 2.7.3). Pressures remained high on the right bank and delta $\mathrm{p}$ reached a second peak at about 14:45 when observed discharge was about 1654 cfs $\left(46.84 \mathrm{~m}^{3} / \mathrm{s}\right)$. Minimum delta $\mathrm{P}$ occurred when the sluiceway discharge was above 2700 cfs (76.5 $\left.\mathrm{m}^{3} / \mathrm{s}\right)$.

oxygen pressure on the right bank remained nearly constant (137-139 mmg) throughout the flow test (Figure 2.7.3). On the left bank, oxygen pressure remained near $150 \mathrm{mmHg}$ until after rerouting of all Afterbay Dam discharge to the sluiceway. Unlike delta $\mathrm{P}$, oxygen on the left bank rose after the routing change and peaked at $171 \mathrm{mmHg}$ near the end of monitoring at 14:45. The constancy of oxygen pressure on the right bank was not expected and may be a result of a malfunctioning oxygen sensor.

Nitrogen and Ar pressure followed the same general pattern as delta $P$ (Figure 2.7.3). We expected this along the right bank 
because under normal conditions, when oxygen and barometric pressures were constant, any substantial change in hyperbaric pressure would result from changes in $\mathrm{N}_{2}+$ Ar tensions. Maximum $\mathrm{N}_{2}+$ Ar levels corresponded to peak delta $P$ levels. Nitrogen + Ar tension measured along the left bank was always lower than near the right bank and, in contrast to the right bank, declined to a lower level than we observed at the beginning of the test. During the flow test, sluicegate openings were recorded hourly in the powerplant control room and every $5 \mathrm{~min}$ at the st. Xavier gagehouse (Figure 2.7.3). Initially, all gate openings were between 4.6 and 4.9 ft (except sluicegate number 1 which was partially closed). Delta p's rose above $120 \mathrm{mmHg}$ on both banks when the opening of sluicegate 2 dropped below $4.05 \mathrm{ft}$ and when the opening of sluicegate 3 was less than $3.54 \mathrm{ft}$. Gagehouse readings of sluicegates 2 and 3 were $3.60-3.64$ ft and $3.29-3.35$ ft, respectively, at the first peak in delta $P$ and $3.31-3.41$ and 3.01-3.11 ft, respectively, during the second peak.

Discrepancy between observed discharge and discharge calculated from sluiceway discharge equations (Figure 2.7.3) results in considerably different conclusions concerning what sluiceway flows should be avoided (Figure 2.7.3). If we use observed flows, all right bank delta P's measured when discharge was 1500-1900 cfs $\left(42.48-53.81 \mathrm{~m}^{3} / \mathrm{s}\right)$ were greater than $120 \mathrm{mmHg}$. Delta $\mathrm{P}^{\prime} \mathrm{s}$ on the left bank climbed above $120 \mathrm{mmHg}$ between $1730-$ $1900 \mathrm{cfs}\left(48.99-53.81 \mathrm{~m}^{3} / \mathrm{s}\right)$. Right and left bank delta $\mathrm{P}^{\prime} \mathrm{s}$ at approximately 2500,2700 and $3125 \mathrm{cfs}(70.8,76.46$, and 88.5 $\mathrm{m}^{3} / \mathrm{s}$ ) displayed a gradual decline to safer levels as discharge increased. Using the same gas data but with sluiceway calculated 
discharges and a reduced data set, we found peak gas production between 2250 and $2500 \mathrm{cfs}\left(63.72-70.80 \mathrm{~m}^{3} / \mathrm{s}\right.$ ) (Figure 2.7.3). Although the actual discharge values vary between methods, the mean sluicegate openings used to calculate flows that were recorded in the control room ( 3.09 and $3.38 \mathrm{ft}$ ) during peak delta P's coincided with the openings of sluicegates 2 and 3 we recorded in the gagehouse. Since we encountered problems with the sluicegate rating curves, we will use gate openings and observed flows in data analysis and recommend these methods in any future work.

During a flow test on 9 July 1986, we intended to maintain total discharge at approximately 6350 cfs $\left(179.83 \mathrm{~m}^{3} / \mathrm{s}\right)$ while routing 55-70\% of the discharge through the sluiceway. We also changed discharge patterns from the spillway to establish if utilizing a certain group of gates would effectively lower hyperbaric pressures. Interpretation of results proved difficult, however, since discharge varied from 6167-7026 cfs $\left(174.65-198.98 \mathrm{~m}^{3} / \mathrm{s}\right)$ and the proportion routed through the sluiceway ranged from only 56-65\%. Moreover, the adjustment was not 1 inear. Delta $\mathrm{p}$ on the right bank at the gagehouse as measured by both a Bouck gasometer and a common sensing tensionometer (model TBO) remained higher and relatively constant when compared to measurements obtained from similar equipment on the left bank (Figure 2.7.4). Right bank delta P's never decreased below $111 \mathrm{mmHg}$, and although pressures rose as high as $127 \mathrm{mmHg}$, most were under $120 \mathrm{mmHg}$. The lower delta $\mathrm{P}^{\prime} \mathrm{s}$ measured on the left bank with a Bouck gasometer and a common Sensing model TGO-F tensionometer, fluctuated over a $44 \mathrm{mmHg}$ range but 
DELTA P - RKM 0.0 - 7/9/00

TEMPEAATURE $-16.20180 \mathrm{~F})$

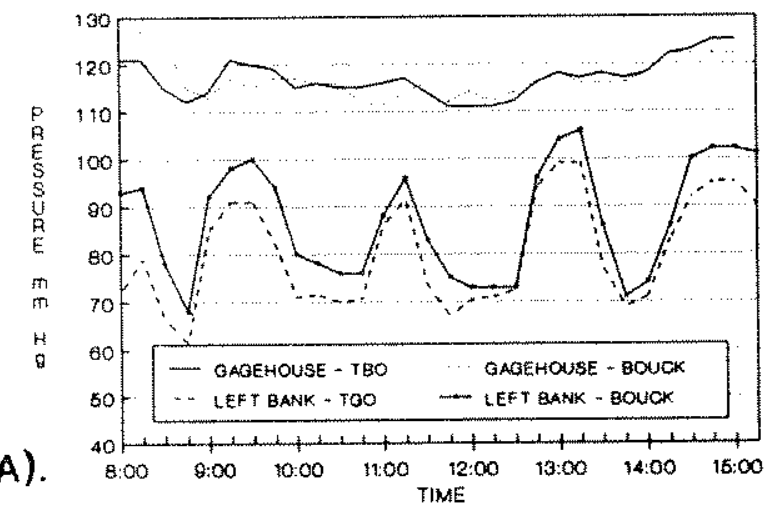

NITROGEN PRESSURE - RKM O.O - T/8/80 TEMPERATURE - $15.2 \mathrm{C}(50 \mathrm{~F})$

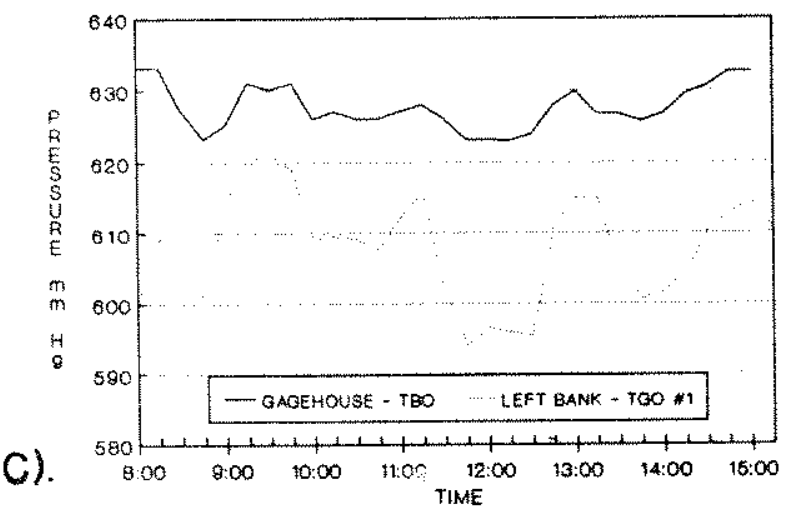

OXYGEN PRESSURE - GKM $0.0-7 / 9 / 88$

TEMPEAATURE $=18.2 \mathrm{C}(50 \mathrm{~F})$

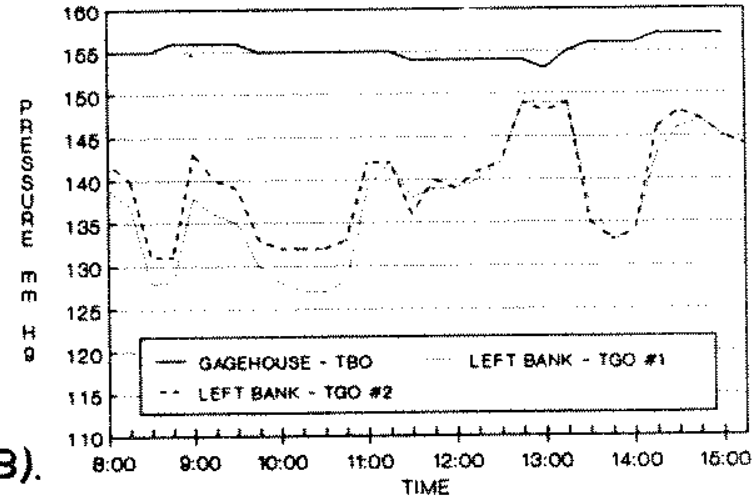

RKM $0.6-7 / 8 / 86$

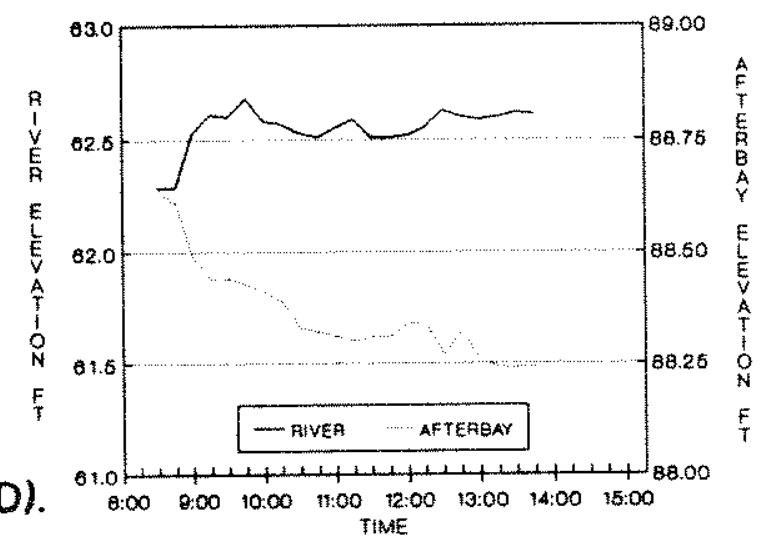

Figure 2.7.4. Delta $P$ (A), oxygen pressure (mmHg) (B), $\mathrm{N}_{2}+\mathrm{Ar}$ pressure (mmH) (C), and river-Afterbay Reservoir water elevations measured at $\mathrm{Rkm} 0.6$ using models TBO and TGO Common sensing tensionometers on the Bighorn River, 9 July 1986. 
did not rise above $106 \mathrm{mmHg}$. All individual gas pressures(except oxygen on the right bank) changed as delta $\mathrm{p}$ varied (Figure

2.7.4). Mean barometric pressure during the test was 679 (range $=678-680) \mathrm{mmHg}$.

The fluctuations in river discharge and elevations in addition to the drafting of Afterbay Reservoir (Figure 2.7.4) make it nearly impossible to isolate the results of gate changes. However, delta $\mathrm{P}^{\mathrm{s}} \mathrm{s}$ along the right bank remained higher than expected for sluiceway discharges from $3850-4300$ cfs (109.03$\left.121.78 \mathrm{~m}^{3} / \mathrm{s}\right)$ and sluicegate openings from $4.45-4.81$ ft when compared to data collected on 19 october 1973 (Bureau of Reclamation 1973). Other data, not directly comparable, suggest the results are valid. Although the cycling of delta p's on the left bank suggests a response due to changes in spillway radial. gate openings, no clear relationship was observed under this flow regime and gas level.

In contrast to the 15 october 1986 flow test, sluicegate openings recorded during the July flow test were constant at both the st. Xavier gagehouse and the control room. However, inconsistencies were noted between remote and local radial gate readings.

Three operational patterns observed during August and early September 1987 produced substantial changes in delta P's, oxygen and nitrogen pressures at both Rkm 0.6 and 4.8 (Figure 2.7.5). From August 6 to 16 the sluiceway was closed. Rkm 0.6 delta $P^{\prime} \mathrm{s}$ remained relatively low (mean $=89 \mathrm{~mm}$ ) and stable, ranging from 77-102 $\mathrm{mmHg}$. At Rkm 4.8, however, large diurnal fluctuations (34-135 mmg) increased the mean delta $P$ to $67 \mathrm{mmHg}$. During this 

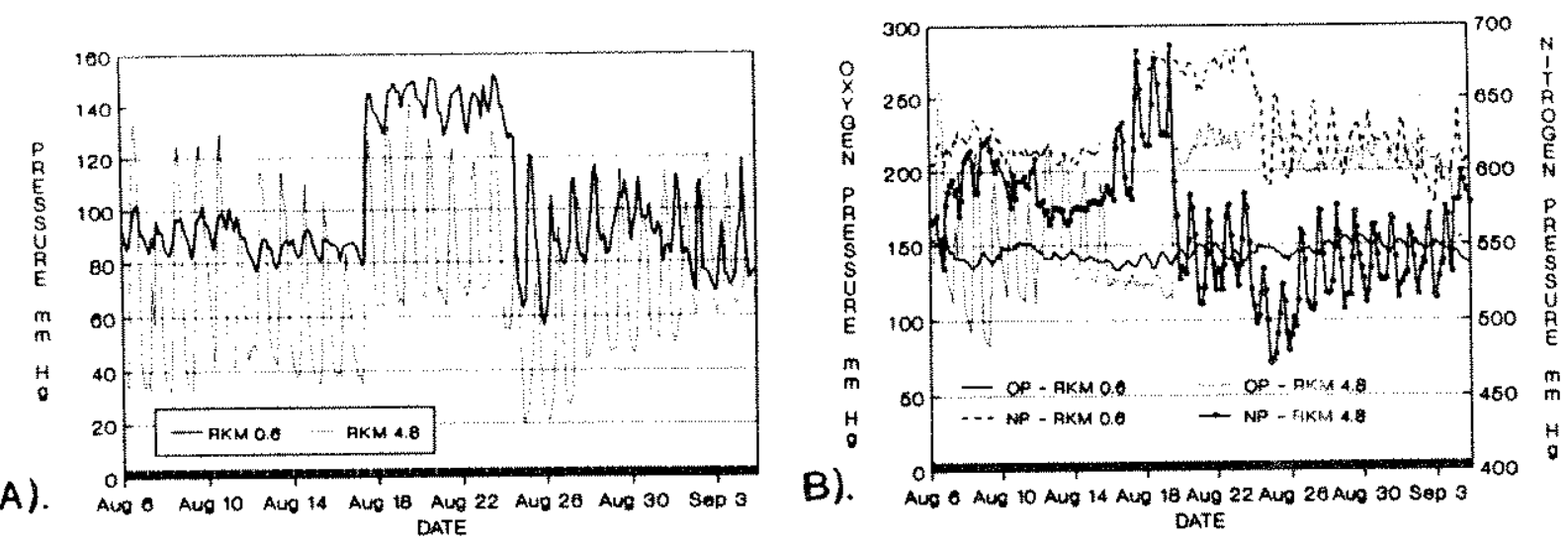

C).
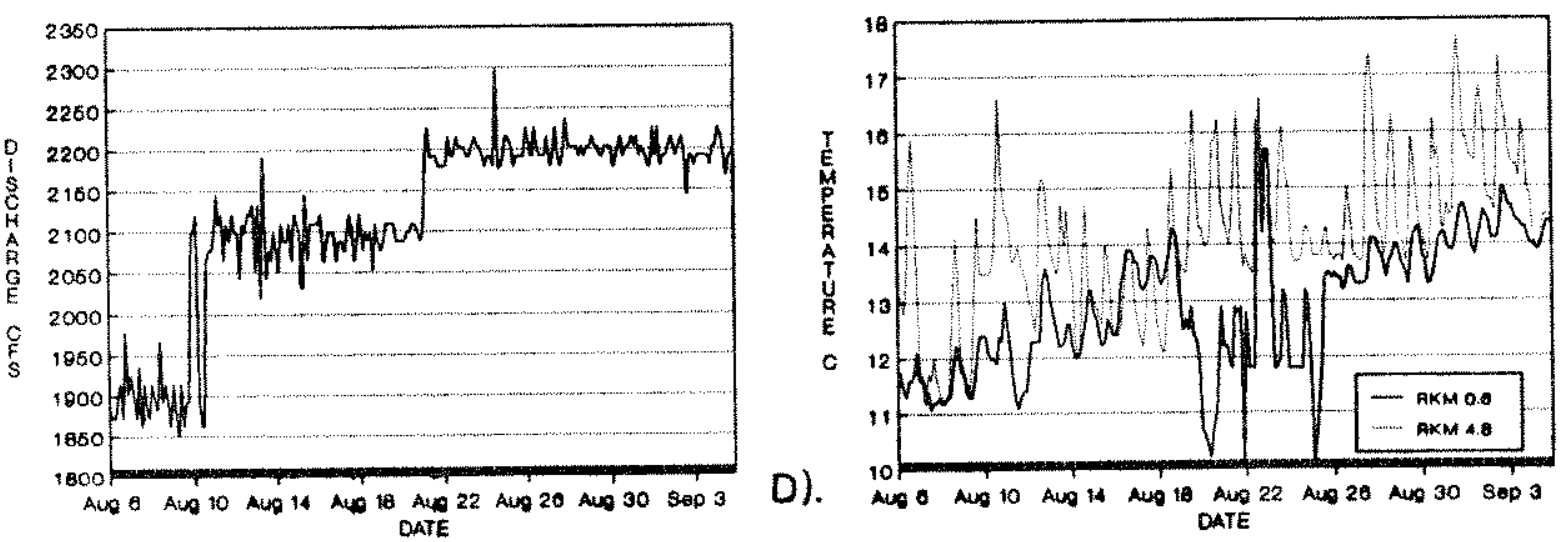

MEAN DALLY AFTERBAY GATE OPENINGS
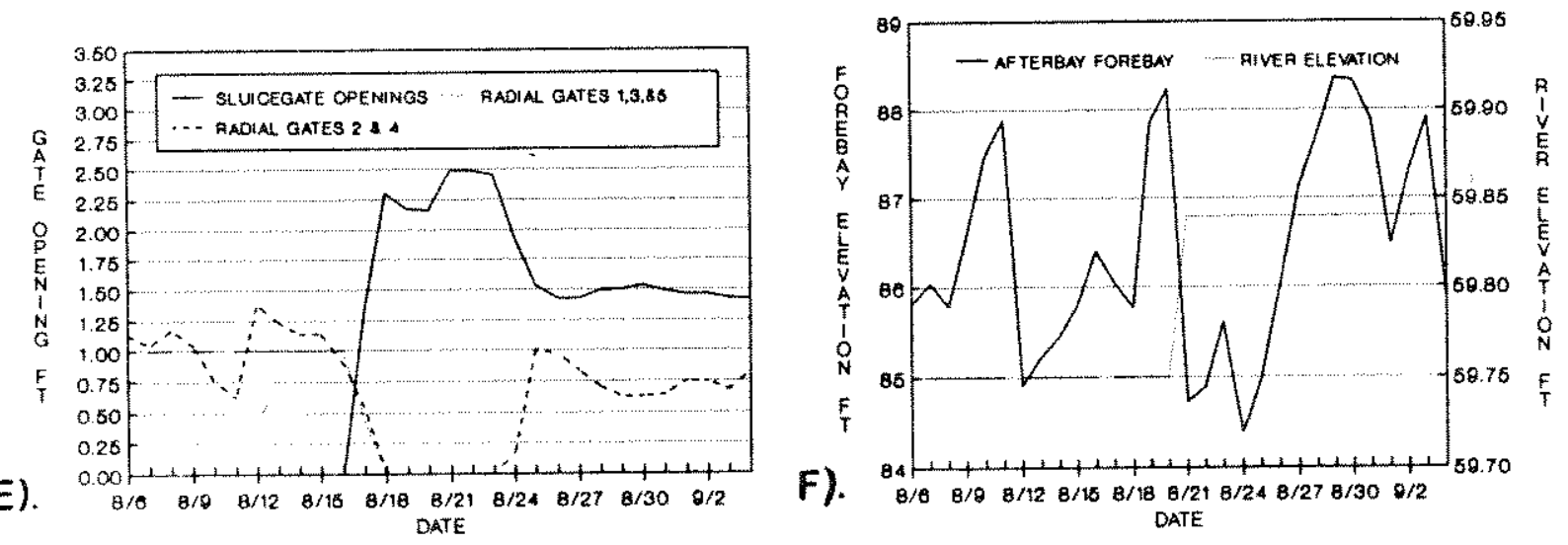

Figure 2.7.5. Delta $P$ (mmHg) (A), oxygen (OP) and $N_{2}+A r$ (NP) pressure (B) at $\mathrm{Rkm} 0.6$ and 4.8 , river discharge (C), water temperature (D), mean daily Afterbay Dam gate openings (E), and Afterbay Reservoir - river water elevations (F) on the Bighorn River from 6 August - 4 September 1987. 
time, radial gates 1,3 , and 5 were open $1.0 \mathrm{ft}$, and gates 2 and 4 varied from $0.63-1.14 \mathrm{ft}$.

The second operational pattern routed all water through the sluiceway and demonstrates the resulting rise in hyperbaric pressures (Figure 2.7.5). This operational regime was initiated at approximately 11:00 A.M. on 17 August 1987 and remained in effect until 11:00 A.M., 24 August 1987, when radial gates 2 \& 4 were opened. As in early August, Rkm 0.6 delta $P^{\prime} s$ varied 25 mmHg from 127-152 mmHg (Figure 2.7.5). The Rkm 0.6 mean delta $P$ rose $52 \mathrm{mmHg}$ to $141 \mathrm{mmHg}$. Downstream at Rkm 4.8 we also observed an increase in delta $\mathrm{P}$ (Figure 2.7 .5 ). The mean delta $\mathrm{P}$ (88 $\mathrm{mmHg})$, however, remained nearly constant. Mean daily sluicegate openings varied from $2.13-2.53$ on days when no other gates discharged water.

The third pattern simulated a typical Afterbay Dam operational mode utilized by the Bureau of Reclamation where both the sluiceway and spillway discharged water. In contrast to the other discharge patterns, $\mathrm{Rkm} 0.6$ delta $\mathrm{P}^{\prime} \mathrm{s}$ were highly variable, ranging from 56-127 $\mathrm{mmHg}$ (Figure 2.7.5). Although variation increased almost threefold, the mean $\mathrm{Rkm} 0.6$ delta $P$ remained 89 mmH--the same as measured using only the spillway radial gates. Similarly, at Rkm 4.8 the mean delta $p$ was the same (67 mmHg) as during the first period when only the spillway was used. The minimum and maximum values, 20 and $121 \mathrm{mmHg}$, respectively, were slightly lower than in the first period, but the range was similar. Mean daily sluicegate openings varied from $1.32-1.65$ ft, and mean daily openings in radial gates 2 and 4 ranged from $0.63-1.00 \mathrm{ft}$ (Figure 2.7 .5 ). All other radial gates were closed. 
oxygen pressure remained quite constant at $\mathrm{Rkm} 0.6$ during all discharge patterns tested (Figure 2.7.5); mean values for each period varied from 143-148 mmg. In contrast to oxygen, $\mathrm{N}_{2}$ + Ar pressure at $\mathrm{Rkm} 0.6$ responded with changes in TGP and delta $\mathrm{p}$ (Figure 2.7.5). Mean levels rose from $615 \mathrm{mmHg}$ during the first period to $673 \mathrm{mmHg}$ during the second and then dropped to $614 \mathrm{mmHg}$ during the last.

No rise in oxygen pressures was measured during the second test period, probably because of malfunctioning of the oxygen censor. Oxygen data from $\mathrm{Rkm} 4.8$ also appeared questionable even though we had replaced the instrument and probe. The mean oxygen pressure increased from 151 to $191 \mathrm{mmHg}$ between the first and second test periods, then rose to $205 \mathrm{mmHg}$ during the third. A major change in oxygen pressure is evident on 19 August when gas tensions almost doubled; this change occurred the same day a calibration adjustment of $12 \mathrm{mmHg}$ was made. In addition, $3 \mathrm{~d}$ earlier the typically large diurnal fluctuations stopped and were not evident the remainder of the test. As we would anticipate from instrumentation problems when TGP values remain correct but the oxygen sensor malfunctions, mean $\mathrm{N}_{2}+$ Ar pressures varied in an inverse relationship to oxygen pressures at $\mathrm{Rkm} 4.8$ and followed delta $P$ changes in a manner similar to what we would expect from oxygen. The $\mathrm{N}_{2}+\mathrm{Ar}$ pressure during the first period was $586 \mathrm{mmHg}$ and dropped to 571 and $535 \mathrm{mmHg}$ in the second and third periods, respectively.

River discharge was altered during August 1987 (Figure $2.7 .5)$. Discharge fluctuated near $1900 \mathrm{cfs}\left(53.81 \mathrm{~m}^{3} / \mathrm{s}\right)$ until 11 August when it increased to approximately 2100 cfs $\left(59.47 \mathrm{~m}^{3} / \mathrm{s}\right)$, 
which is also roughly the average flow $\left(2118 \mathrm{Cfs}, 59.98 \mathrm{~m}^{3} / \mathrm{s}\right)$. Another flow increase to $2200 \mathrm{cfs}\left(62.30 \mathrm{~m}^{3} / \mathrm{s}\right)$ took place on 21 August. Although discharge increased on 11 August, the river water elevation did not fluctuate (Figure 2.7.5). This was due to the U.S.G.S. recalibrating the stage-discharge rating curve. Water temperature continued to increase at both sites through August and early september (Figure 2.7.5). Mean water temperatures were $12.9 \mathrm{C}(55 \mathrm{~F})$ and $14.2 \mathrm{C}(57.6 \mathrm{~F})$ at $\mathrm{Rkm} 0.6$ and 4.8 , respectively. The large rise in water temperature at $\mathrm{Rkm} 0.6$ on 22-23 August was a result of instrumentation problems. Forebay elevations of Afterbay reservoir varied throughout the test period (Figure 2.7.5). However, the fluctuation was only 3.96 ft $(3184.39-3188.35 \mathrm{ft})$ which is small: levels always remained above the spillway crest elevation of $3179.5 \mathrm{ft}$ (969.11 m).

This test demonstrated that the risk of GBT in fish can be reduced if only radial gates are utilized to discharge water from Afterbay Reservoir. During period 1, hyperbaric pressures at Rkm 0.6 were substantially below the threshold for bubble growth in the cardiovascular system; and during the latter part of the period, levels were at times below the environmental water threshold even assuming no hydrostatic pressure provided compensation (Figure 2.3.88). Tests also demonstrate that sole use of slulceway gates in the $2 \cdot 10-2.5$ ft range should be avoided. The mean delta $P$ at $R \mathrm{~km} 0.6$ during period 2 was almost $30 \mathrm{mmHg}$ above the cardiovascular system threshold: even minimum values remained greater than the threshold. The risk at Rkm 4.8 was much lower since only a few of the peaks, all of which 
occurred during the second period, rose above the vascular system threshold. Although the operational mode which utilized both the spillway and sluiceway had maximum daily pressures substantially above those in the first period and included levels above the cardiovascular threshold, many days also had the lowest gas levels observed. If peak levels are of short duration and not substantially above the bubble growth threshold and pressures drop below the threshold for a long enough period of time, high delta P's could occur without severely affecting trout. close monitoring is recommended during periods when delta $\mathrm{P}^{\prime} \mathrm{s}$ are high and frequently exceed $115-120 \mathrm{mmHg}$. Minimum delta $\mathrm{P}^{\prime} \mathrm{s}$ during the last operational period appeared to correspond with sluicegate openings of approximately $1.25 \mathrm{ft}$ or less.

This flow test also showed the value of continuous monitoring in that peaks in delta $\mathrm{P}$ above GBT thresholds were documented. If we had examined only the mean delta $P^{\prime} s$ for the first and third periods, no difference would have been observed since means were identical.

We monitored another series of discharge patterns from Afterbay Reservoir in July 1988 (Figure 2.7.6). Discharge from both the sluiceway and the spillway occurred from 16-20 and 27-31 July. On 20-22 and 25-27 July, all discharge was routed through the sluiceway. Only radial gates were used to discharge water from 22-25 July.

The results reinforced what we observed in August 1987. Although maximum hyperbaric pressures were lower than during the 1987 monitoring, the two periods where only the sluiceway was in operation produced the highest gas regime. Mean delta p's were 

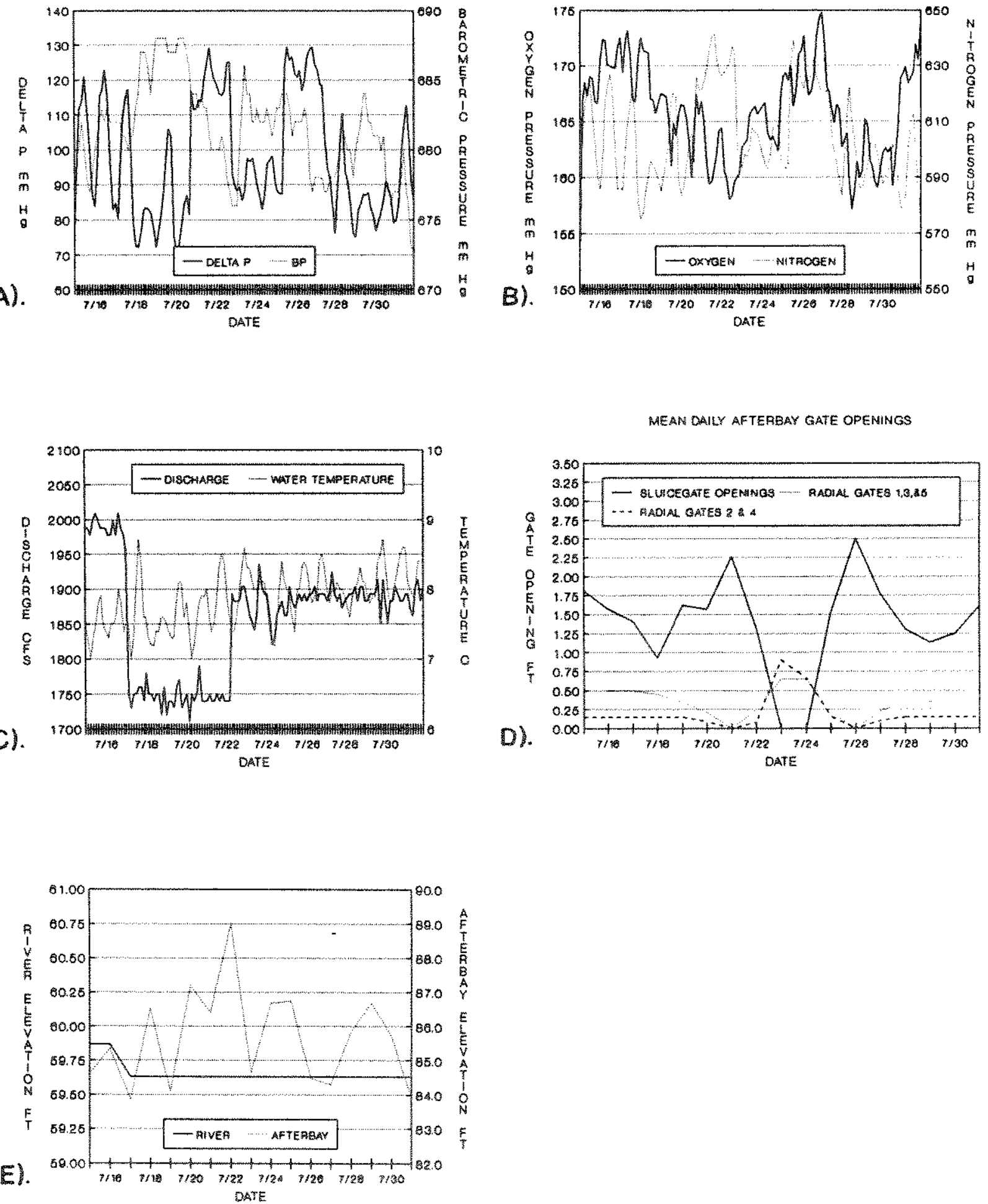

Figure 2.7.6. Delta $P$ and barometric pressure (mmig) (A), oxygen and $\mathrm{N}_{2}+\mathrm{Ar}$ pressure (mHH) (B), river discharge and water temperature (C), mean daily Afterbay Dam gate openings (ft) (D), and Afterbay Reservoir - river water elevations (ft) (E) at Rkm 0.6 on the Bighorn River from 15-31 July 1988. 
119 (range $=111-129)$ and 124 (range $=117-130)$ mmig during the first and second sluiceway-only periods, respectively. other periods had mean delta $P^{\prime}$ s about $30 \mathrm{mmHg}$ lower, varying from 90$92 \mathrm{mmHg}$. When both sluice and radial gates were in use, delta p's ranged from $71-123 \mathrm{mmHg}$; when only radial gates were used, less variability in pressures occurred (83-98 $\mathrm{mmHg}$ ).

oxygen pressure did not show the consistent response to changes in TGP and delta P (Figure 2.7.6). Nitrogen + Ar pressure appeared to closely follow the fluctuations in hyperbaric pressure. Barometric pressure varied from 673-688 mmHg and averaged $682 \mathrm{mmHg}$.

Discharge also changed during the test period (Figure $2.7 .6)$. The first 2 -d discharge remained near $2000 \mathrm{cfs}$ (56.64 $\left.\mathrm{m}^{3} / \mathrm{s}\right)$ then dropped to approximately 1750 cfs $\left(49.56 \mathrm{~m}^{3} / \mathrm{s}\right)$ on 17 July, remaining at that level until 22 July when flows increased to about $1900 \mathrm{cfs}\left(53.81 \mathrm{~m}^{3} / \mathrm{s}\right)$. River elevations also changed on 17 July but remained constant through the rest of July (Figure 2.7.6). Mean daily Afterbay elevations fluctuated $5.15 \mathrm{ft}$ (1.57 m) from $3183.86-3189.01$ ft $(971.35-972.01 \mathrm{~m})$; hourly readings remained above the spillway crest except for $2 \mathrm{~h}$ on $27 \mathrm{July}$.

water temperature gradually increased through the monitoring period and fluctuated diurnally (Figure 2.7.6). Mean water temperature from $15-31$ July was $7.9 \mathrm{C}(46 \mathrm{~F})$.

\subsection{3b Dye Tests}

on 28 March 1985 we determined mixing patterns of water released from different portions of the dam as well as the rate of water movement downstream. Aerial observations of rhodamine 
dye added to the sluiceway water indicated complete lateral mixing occurred near Lind Access, about $5.8 \mathrm{~km}(3.6 \mathrm{mi})$ below Afterbay Dam. The leading edge of the dye plume progressed 8.4 $\mathrm{km}(5.2 \mathrm{mi})$ downstream after $136 \mathrm{~min}$, indicating a downstream movement of approximately $3.7 \mathrm{~km} / \mathrm{h}(2.3 \mathrm{mi} / \mathrm{h})$. The central portion of the dye plume moved about $7.4 \mathrm{~km}(4.6 \mathrm{mi})$ downstream in the same period, a rate of $3.3 \mathrm{~km} / \mathrm{h}(2.0 \mathrm{mi} / \mathrm{h})$.

\subsection{3.c Downstream Gas Measurements}

In conjunction with the 1985 dye test, we first measured the rate of downstream gas dissipation on 29 March 1985. Seven locations $0.6-24.6 \mathrm{Rkm}$ below the Afterbay Dam were sampled to obtain dissolved gas levels and oxygen concentrations (Table 2.7.4). Dissolved $\mathrm{N}_{2}+$ Ar levels were substantially higher on the right bank (sluiceway side) than on the left bank at the gage house (Rkm 0.6); this difference had disappeared at Rkm 3.2 and 6.1. Nitrogen levels dropped sharply between 6.1 and $10.5 \mathrm{~km}$ downstream but stayed relatively constant below Rkm 10.5 . External evidence of gas bubble trauma in trout is confined primarily to the first $8.0 \mathrm{~km}$ below the Afterbay Dam (Porter and Viel 1980); this is approximately where $\mathrm{N}_{2}+$ Ar saturation dissipates. Delta $P$ and total gas saturation on the right bank remained high until dropping substantially at Rkm 10.5 to $85 \mathrm{mmHg}$ or 112\%. Delta $P$ increased downstream of Rkm 10.5. Although hyperbaric pressure was highest along the right bank at Rkm 0.6 , it was higher near the left bank at both $\operatorname{Rkm} 3.2$ and 6.1 . Oxygen pressure was always higher along the left bank than on the right bank and increased downstream with the exception of measurements 
Table 2.7.4. Results of downriver gas dissipation test conducted on the Bighorn River downstream from the Afterbay Dam on 29 March 1985.

\begin{tabular}{|c|c|c|c|c|c|c|}
\hline \multirow{2}{*}{$\begin{array}{c}\text { Location }{ }^{\mathrm{a}} \\
(\mathrm{Rkm})\end{array}$} & \multirow{2}{*}{$\begin{array}{l}\text { River } \\
\text { bank }\end{array}$} & \multirow{2}{*}{$\begin{array}{l}\text { starting } \\
\text { time }\end{array}$} & \multicolumn{4}{|c|}{ Pressure (mmHg) } \\
\hline & & & Delta & $\mathrm{P} \quad \mathrm{N}_{2}+\mathrm{Ar}$ & $\mathrm{O}_{2}$ & $\mathrm{BP}$ \\
\hline 0.6 & right & $9: 30 \mathrm{~A}$ & 125 & 646 & 154 & 681 \\
\hline 0.6 & left & $"$ & 103 & 619 & 159 & 681 \\
\hline 3.2 & right & $10: 48 \mathrm{~A}$ & 107 & 620 & 163 & 682 \\
\hline 3.2 & left & $"$ & 125 & 624 & 177 & 682 \\
\hline 6.1 & right & $12: 01 \mathrm{P}$ & 125 & 625 & 176 & 682 \\
\hline 6.1 & left & $"$ & 135 & 632 & 179 & 682 \\
\hline 10.5 & right & $9: 45 A$ & 85 & 596 & 166 & 683 \\
\hline 14.5 & right & $10: 40 \mathrm{~A}$ & 92 & 588 & 181 & 683 \\
\hline 19.3 & right & $11: 40 \mathrm{~A}$ & 99 & 596 & 180 & 683 \\
\hline 24.6 & right & $12: 25 \mathrm{P}$ & 112 & 597 & 193 & 684 \\
\hline
\end{tabular}

a Locations are river kilometers below Afterbay Dam.

at $\mathrm{Rkm}$ 10.5. This may have been related to the time of day of day measurements were made. Downstream from $\mathrm{Rkm} 10.5$ oxygen pressure remained relatively constant or increased.

on 18 July 1986 we conducted another downstream survey; we determined gas levels on the right bank with a Bouck gasometer at the usual monitoring stations and on the left bank every $0.8 \mathrm{~km}$ $(0.5 \mathrm{mi})$ with two tensionometers (Figure 2.7.7). During this test, sluiceway gates were not in use, resulting in low hyperbaric pressures. Delta $\mathrm{P}$ values ranged from $21 \mathrm{~mm}$ H on the right bank at Rkm 19.3 (Bighorn Access) to $77 \mathrm{mmHg}$ on the left bank at $\mathrm{Rkm} 4.8$ (opposite Sutron satellite station near Lind 
DONNAIVER GAS DISSIPATION TEST

t8 JULY $: 080$

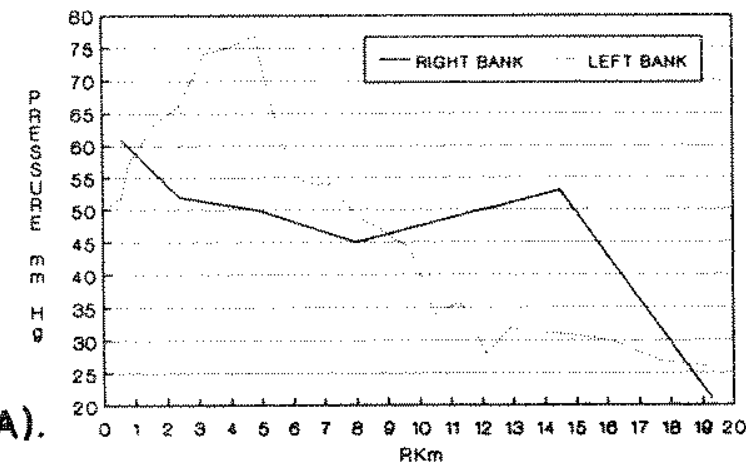

DOWNAIVEA OAS DISSIPATION TEST OXYGEN IOPI \& NITROOEN (NP) PAESSUAE 18 JULY 1986

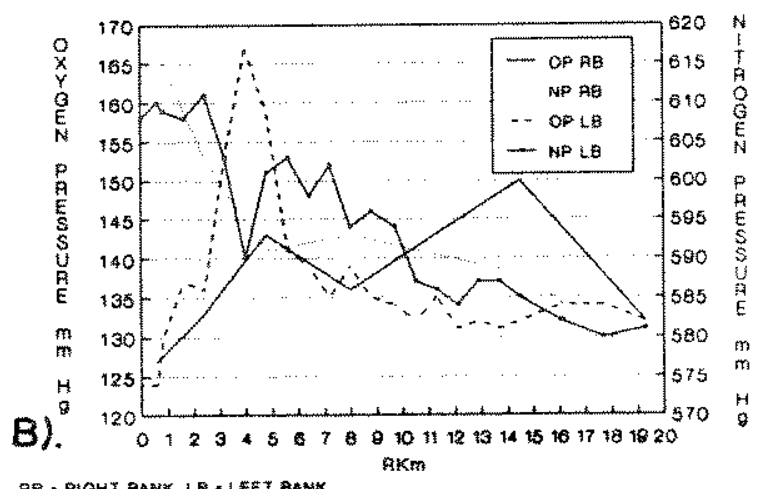

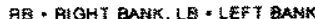

Figure 2.7.7. Downxiver survey of delta $\mathrm{P}$ (A) and oxygen and nitrogen-argon pressures (B) on the Bighorn River downstream from Afterbay Dam, $18 \mathrm{JuIy} 1986 . \quad(Q=6262$ cfs: slulceway gates closed, opening of radial gates $1,3,+5=3.0 \mathrm{ft}$, mean opening of radial gates $2+4=2.44 \mathrm{ft}$. , day $=$ primaxily overcast).

Access) (Plgure 2.7.7). Delta $\mathrm{P}^{\mathrm{i}} \mathrm{s}$ on the left bank increased rapidLy until Rkm 4.8 and then quickly dropped. Below Rkm 7.2 values along the left bank continued to decline downstream but at a much slower rate. Right bank measurements remained more constant than left bank delta $\mathrm{P}^{\prime}$ s over the upper $14.5 \mathrm{Rkm}$ of the study area. Delta $\mathrm{P}^{\prime} \mathrm{s}$ were higher on the right bank at Rkm 0.6 (gagehouse) and Rkm 14.5 (RM 9.0), while at Rkm 2.4, 4.8, 8.0 and 19.3 they were lower.

We compared readings from the Bouck gasometer, the permanent1y installed tensionometer, and the two tensionometers used on the left bank at $\mathrm{Rkm} 4.8$ to ensure validity of measurements. The comparison verified that gas pressures were Lower on the right bank, an unlikely event had the sluiceway been operating. We anticipate that all delta $P^{\prime} s$ measured during this survey would have little or no impact on the fisheries in the 
Bighorn River.

Oxygen pressure along the left bank displayed the same peak at Rkm 4.0 as delta $\mathrm{P}$ (Figure 2.7 .7 ); this, along with a corresponding lower oxygen regime on the right bank, suggests the peak was caused by biological oxygen production. oxygen pressures measured on the right bank tended to increase in a downstream direction. Nitrogen and argon $\left(\mathrm{N}_{2}+\mathrm{Ar}\right)$ pressures were highest at $\mathrm{Rkm} 0.6(617 \mathrm{mmHg})$ and decreased with distance downstream.

Temperature also peaked at $\mathrm{Rkm} 4.0(17.6 \mathrm{C} ; 63.7 \mathrm{~F})$ and then dropped to approximately $16.6 \mathrm{C}(61.9 \mathrm{~F})$ and remained there through Rkm 19.3. Essentially no difference was observed in water temperatures between banks; the overall mean water temperature during the downriver run was $16.6 \mathrm{C}(61.9 \mathrm{~F})$.

During August 1987 gas tensions were measured throughout the study area on both sides of the river while Afterbay Dam was operated under two spill patterns (Figure 2.7.8). On 16 August pressures were measured between Afterbay Dam and Rkm 12.9. Only radial gates discharged water, resulting in relatively low hyperbaric pressures; all five radial gates were open $0.94-1.0$ ft. Mean discharge was $59.44 \mathrm{~m}^{3} / \mathrm{s}$ (2099 cfs), average water temperature was $13.4 \mathrm{C}(56 \mathrm{~F})$, and mean barometric pressure was $680 \mathrm{mmHg}$ with a range from $679-685 \mathrm{mmHg}$. Delta $\mathrm{p}$ was similar near both banks at each site and increased downstream (Figure 2.7.8). Mean delta $P$ was $92 \mathrm{mmHg}$ along the left bank compared to $89 \mathrm{mmHg}$ along the right bank. Minimum and maximum delta p's on both banks were measured at $\mathrm{Rkm} 3.2$ and 11.3 , respectively, and pressures varied over a range of $36 \mathrm{mmHg}$ near the right bank and 
DOWNAEEA GAS DESSIPATION TEST

DELTA F

10 ALCUST 1987

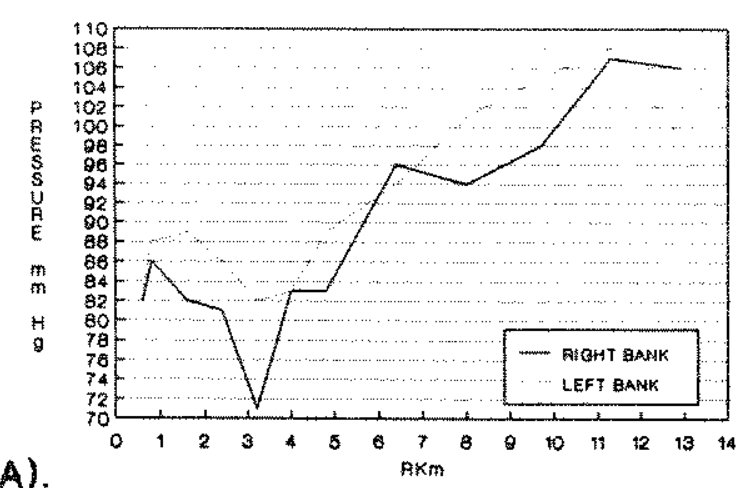

A).

DOWNAIVEA OAS DISSIPATION TEST OXYGEN IOPI AITPOOEN (AP) PRESSUAES

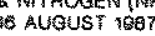

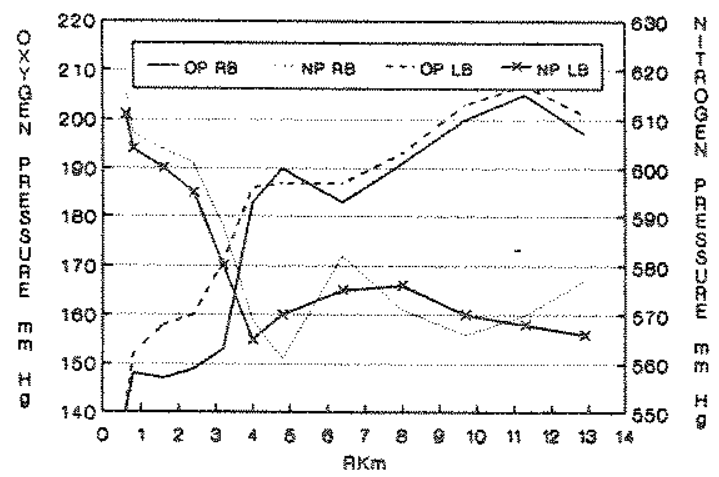

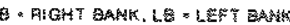

c).
DOWNFIVEA GAS DISSIPATION TEST

DELTAP

22 AUUUST 1887

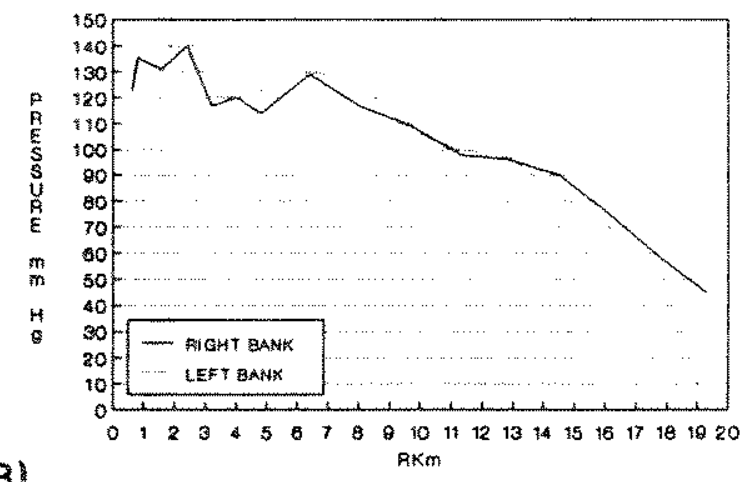

B).

COWNAIVEA GAS DISSIPATION TEST OXYOEN (OP) \& NITROGEN (NP) PRESSUAES 22 AUGUST 1087

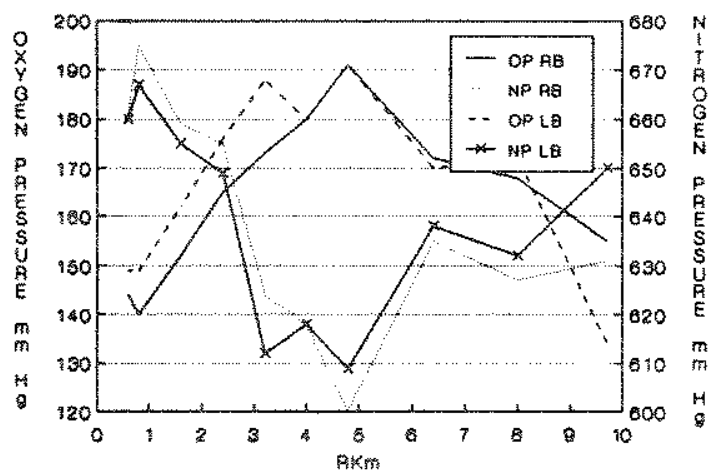

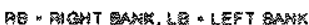

D).

Figure 2.7.8. Downriver survey of delta $p$ (milg) on 16 August (A), and 22 August 1987 (B), and the corresponding oxygen and $\mathrm{N}_{2}$ + Ar pressure (mmig) (C\& D) in the Bighorn River downstream from Afterbay Dam in 1987. 
$26 \mathrm{mmHg}$ near the left bank. The maximum TGP of $788 \mathrm{mmHg}$ represented a delta $\mathrm{p}$ of $108 \mathrm{mmHg}$ and was about $20 \mathrm{mmHg}$ less than the cardiovascular system threshold for bubble formation.

Oxygen pressures on 16 August increased along both banks about $65 \mathrm{mmHg}$ from a minimum near $140 \mathrm{mmHg}$ at the gagehouse to peak levels at Rkm 11.3 (Figure 2.7.8). Calculated $\mathrm{N}_{2}+\mathrm{Ar}$ pressure was highest in the uppermost reach of the river (Figure $2.7 .8)$. Water temperature warmed approximately $2.5 \mathrm{C}$ along both banks between $\mathrm{Rkm} 0.6$ and $\mathrm{Rkm} 12.9$. Gas pressures were relatively low and constant both across and downriver when only the radial gates were used to discharge $59.44 \mathrm{~m}^{3} / \mathrm{s}$ (2099 cfs). Similar sampling on 18 July 1986 , when only radial gates were passing water and discharge was $177.34 \mathrm{~m}^{3} / \mathrm{s}(6262 \mathrm{cfs})$, showed larger variation in gas pressures between banks and through the study area. Although hyperbaric pressures were not critically high during either test, this demonstrates that gas tension changes occur due to variations of discharge, river elevation, water temperature, and weather conditions, even during the same operational mode.

on 22 August 1987 , while all of the $62.16 \mathrm{~m}^{3} / \mathrm{s}$ (2195 cfs) discharge was released through the sluiceway, downriver gas pressures and temperatures were measured. The mean delta $\mathrm{P}$ increased 17 and $20 \mathrm{mmHg}$ over levels measured $6 \mathrm{~d}$ before, to 106 and $112 \mathrm{mmHg}$ near the right and left banks, respectively. Maximum delta p's were 140 and $145 \mathrm{mmHg}$ along the right and left banks at Rkm 2.4, respectively (Figure 2.7.8). This corresponds to 22 and $18 \mathrm{mmHg}$ above cardiovascular bubble formation. As on 16 August, each parameter exhibited the same general pattern on 
both banks. The pattern of delta $P$, oxygen, and $\mathrm{N}_{2}+\mathrm{Ar}$ pressure, however, was different between tests. Gas entrainment by the sluiceway gates produced higher delta $\mathrm{P}^{\prime} \mathrm{s}$ in the upper portion of the study area with pressures decreasing substantially downstream. Nitrogen + Ar pressures showed a greater tendency to rise in the lower part of the study area on 22 August than on 16 August.

Failure of oxygen probes on both tensionometers prevented the determination of oxygen and $\mathrm{N}_{2}+\mathrm{Ar}$ pressures downstream from Rkm 9.7. Upriver of this, oxygen pressure achieved maximum levels at Rkm 4.8 and then declined. Maximum levels were not as high as on 16 August, probably due to differences in solar insolation and the time of measurement. The average barometric pressure on 22 August was $691 \mathrm{mmHg}$, with individual measurements varying from $688-693 \mathrm{mmHg}$. As on 16 August, watex temperature increased in a downstream direction and was nearly the same on both sides of the river; mean water temperature was $1.8 \mathrm{C}$ higher on 22 August than on 16 August.

\subsection{3.d Cross River Gas Measurements}

on 31 July, 4 september, and 14 october 1986, gas pressures were measured across the river every $3.1 \mathrm{~m}$ (10 ft) along the UsGS cableway at Rkm 0.6 (Figure 2.7.9). Mean flows were 5766 cfs $\left(163.29 \mathrm{~m}^{3} / \mathrm{s}\right)($ range $=5710-5936), 2796 \mathrm{cfs}\left(79.18 \mathrm{~m}^{3} / \mathrm{s}\right)$, and $3606 \mathrm{cfs}\left(102.12 \mathrm{~m}^{3} / \mathrm{s}\right)$ on these dates, respectively. The sluiceway gates were closed during the 31 July and 14 october sampling (Table 2.7 .5 ), resulting in substantially lower gas levels. 


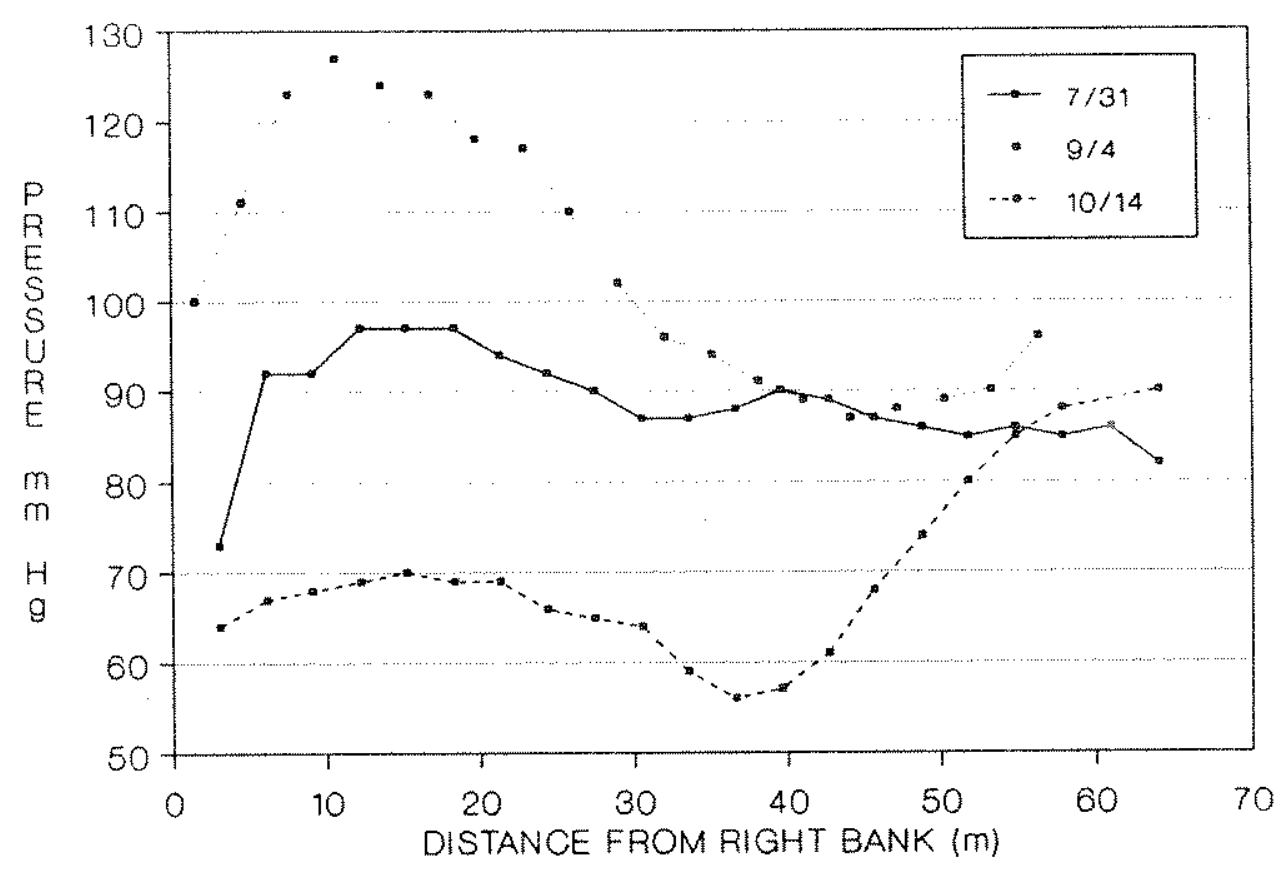

Figure 2.7.9. Cross-river measurments of delta $P$ at $\mathrm{Rkm} 0.6$ on 31 July, 4 September, and 14 October 1986.

Table 2.7.5. Mean barometric pressure (B.P.) and Afterbay Dam gate openings (in feet) during cross-river transect measurements in 1986.

\begin{tabular}{|c|c|c|c|c|c|c|}
\hline \multirow{2}{*}{ Date } & \multirow{2}{*}{ B. P. } & \multicolumn{3}{|c|}{ sluicegate number } & \multicolumn{2}{|c|}{ Radial gate no. } \\
\hline & & 1 & 2 & 3 & $1,3 \& 5$ & $2 \& 4$ \\
\hline $7 / 31$ & 684 & 0 & 0 & 0 & $3.0-2.25$ & $\begin{array}{c}1.64 \\
(1.58-1.70)\end{array}$ \\
\hline $9 / 4$ & 683 & $\begin{array}{c}1.79 \\
(1.46-2.10)\end{array}$ & $\begin{array}{c}1.89 \\
(1.61-2.19)\end{array}$ & $\begin{array}{c}2.18 \\
(1.91-2.40)\end{array}$ & 0.75 & 0.25 \\
\hline $10 / 14$ & 685 & 0 & 0 & 0 & 1.50 & $\begin{array}{c}2.79 \\
(2.78-2.80)\end{array}$ \\
\hline
\end{tabular}

on 31 July, radial gates 1,3 , and 5 were partially closed to prepare for lower flows. This change increased gas levels by about $15 \mathrm{mmHg}$. The situation persisted during all subsequent 
measurements. Delta $P$ varied $24 \mathrm{mmHg}$, and both the maximum and minimum values occurred near the right bank. Mean delta $P$ from al1 transect measurements was $89 \pm 6 \mathrm{mmHg}$. Gas Pressures were intermediate of the other two tests; this test was conducted when discharge and Afterbay elevation was greatest and radial gates 1 , 3 , and 5 released most of the water. Oxygen and $\mathrm{N}_{2}+\mathrm{Ar}$ pressures were relatively constant across the entire transect. We measured the lowest delta $\mathrm{P} 3.0 \mathrm{~m}$ from the right bank and attributed the low value to the measurement being taken shortly after the Afterbay Dam operational change occurred but before gas levels increased. Temperature was very constant, ranging from $18.5 \mathrm{C}(65.3 \mathrm{~F})$ to $18.7 \mathrm{C}(65.7 \mathrm{~F})$.

on 14 october (sluiceway gates closed), discharge was lower (3606 cfs) and the gas pattern was somewhat different (Figure 2.7.9). Like data from July, peak delta $P$ along the right bank was at $15.2 \mathrm{~m}$ and then decreased $14 \mathrm{mmHg}$ to the minimum at $36.6 \mathrm{~m}$ from the right bank. The maximum delta $P$ measured near the right bank was $27 \mathrm{mmHg}$ lower in October than in July. This difference appears representative of gas levels in general since the mean delta $P$ over the course of the transect was $69 \pm 10 \mathrm{mmHg}$, or 20 mmig lower. Beyond $36.6 \mathrm{~m}$, delta $\mathrm{P}$, oxygen, and $\mathrm{N}_{2}+\mathrm{Ar}$ pressures tended to increase. The total variation in delta $P$ was $34 \mathrm{mmHg}$ with the maximum delta $\mathrm{p}$ and oxygen pressure found at $61.0 \mathrm{~m}$, adjacent to the left bank.

oxygen pressure on 14 October showed the same pattern across the river as delta $P$. Oxygen levels remained above $130 \mathrm{~mm} H \mathrm{~g}$ except for three measurements $33.5-39.6 \mathrm{~m}$ from the right bank. The lowest oxygen pressure observed was $127 \mathrm{mmg}$ at $36.6 \mathrm{~m}$ : 
pressure increased near the left bank where the last measurement made was also the maximum (163 $\mathrm{mmHg})$. Mean oxygen pressure was $136 \pm 8 \mathrm{mmHg}$. Nitrogen and Ar pressure remained more constant than oxygen, ranging $18 \mathrm{mmHg}$ from 609-627 $\mathrm{mmHg}$. The mean $\mathrm{N}_{2}+\mathrm{Ar}$ was $618 \pm 5 \mathrm{mmHg}$. Water temperature again showed little variation during the cross-river survey with a mean value of 13.8 $\pm 0.1 \mathrm{C}(56.8 \mathrm{~F})$.

In contrast to the other two sampling dates, substantial differences in gas pressures were noted on 4 september, when approximately $57 \%$ of the discharge was routed through the sluiceway (Figure 2.7.9). The maximum delta $\mathrm{P}\left(127 \mathrm{mmH}\right.$ ) and $\mathrm{N}_{2}$ + Ar (663 mmHg) pressures were found at $10.7 \mathrm{~m}$ from the right bank. Nitrogen + Ar continually decreased across the channel, dropping $39 \mathrm{mmHg}$ between 10.7 and $53.3 \mathrm{~m}$ from $663-624 \mathrm{mmHg}$. The minimum delta $P$, found at $44.2 \mathrm{~m}$, was $40 \mathrm{mmHg}$ lower than the highest level. The sluiceway discharge of approximately $1595 \mathrm{cfs}$ $\left(45.17 \mathrm{~m}^{3} / \mathrm{s}\right)$ produced the highest mean and range in delta $\mathrm{P}(\overline{\mathrm{x}}=$ 104, range $=87-127 \mathrm{mmHg}$ ) observed in 1986 tests. Oxygen

pressure was essentially constant across the river (mean $147 \pm 7$ ) with the exception of measurements adjacent to the banks. Water temperature was nearly constant with a mean of $18.9 \mathrm{C}$. These data demonstrate that when the sluiceway is used, considerable variation in gas levels occur laterally. Total gas pressure dropped 5.3\% from 118 to $111.8 \%$ and $\mathrm{N}_{2}+\operatorname{Ar}$ dropped $6.7 \%$ from 122.3 to $114.1 \%$ between 7.6 and $44.2 \mathrm{~m}$. During sluiceway use, fish may be able to reduce exposure to high gas levels by moving short distances laterally in the river in addition to sounding and taking advantage of the hydrostatic pressure if they can 
detect or differentiate pressure differences.

During August 1987, we examined hyperbaric gradients across the river under the different operational modes at three of the long-term gas monitoring sites, Rkm 0.6, 4.8, and 14.5. Along with the 1986 transects at Rkm 0.6, these data provide a framework from which to predict gradients as affected by both discharge and operating regime. Mean barometric pressures from each transect varied from 675-686 (Table 2.7.6).

Hyperbaric pressures at Rkm 0.6 on 12 August 1987 when the sluiceway gates were closed and mean discharge was $60.0 \mathrm{~m}^{3} / \mathrm{s}$ (2119 cfs) showed relatively little change across the river (Figure 2.7.10). Mean delta $P$ was $82 \mathrm{mmHg}$, and excluding the 3.0 m reading, varied only $8 \mathrm{mmHg}$. Nitrogen + Ar pressure fluctuated slightly, while oxygen tensions increased $14 \mathrm{mmHg}$ from $6.1-51.8 \mathrm{~m}$ across the transect (Figure 2.7.10). Further downstream, delta $P$ was higher and more variable (Figure 2.7.10). At Rkm 4.8, mean delta $P$ was $104 \mathrm{mmHg}$ and ranged from $91-113 \mathrm{mmHg}$ on 13 August 1987. Nitrogen + Ar pressure was highest in mid-channel; oxygen pressure was greatest near the left bank (Figure 2.7.10). The mean delta $\mathrm{P}$ at $\mathrm{Rkm} 14.9$ on 14 August was $96 \mathrm{mmHg}$ and values varied over a $70 \mathrm{mmHg}$ range (Figure 2.7 .10 ). The cross-river transect at the lower station was located at Rkm 14.9. The low mean delta $\mathrm{P}$ and sudden decline at about $50 \mathrm{~m}$ from the right bank was probably related to a severe thunderstorm that reduced photosynthesis and probably increased reaeration. If measurements made after the storm (1ater than 17:14) are excluded, the mean delta $P$ increased to $114 \mathrm{mmHg}$ and the range decreased to $33 \mathrm{mmHg}$. Oxygen pressure before the storm tended to 
Table 2.7.6. Mean barometric pressure (mmHg) during measurements of cross-river transects in 1987 on the Bighorn River, Montana below Afterbay Dam.

\begin{tabular}{|c|c|c|c|c|c|c|}
\hline \multirow[b]{2}{*}{ Rkm } & \multicolumn{2}{|c|}{ Ist transect } & \multicolumn{2}{|c|}{ 2nd transect } & \multicolumn{2}{|c|}{$3 r d$ transect } \\
\hline & Date & $\begin{array}{c}\text { B.P. } \\
(\mathrm{mmHg})\end{array}$ & Date & $\underset{(\mathrm{mmHg})}{\mathrm{B} . \mathrm{P} .}$ & Date & $\begin{array}{c}\mathrm{B} \cdot \mathrm{P} . \\
(\mathrm{mmHg})\end{array}$ \\
\hline 0.6 & $8 / 12$ & 680 & $8 / 18$ & 686 & $8 / 28$ & 683 \\
\hline 4.8 & $8 / 13$ & 675 & $8 / 19$ & 683 & -- & -- \\
\hline 14.9 & $8 / 14$ & 675 & $8 / 20$ & 677 & -- & -- \\
\hline
\end{tabular}

be greater than $200 \mathrm{mmHg}$ and unlike all upstream transects, $\mathrm{N}_{2}+$ Ar pressures were less than $600 \mathrm{mmHg}$.

Although maximum delta $P$ and TGP increased at each downstream transect, no maximum delta $\mathrm{P}$ or TGP was higher than the threshold for bubble growth in the cardiovascular system of fish at the water surface. However, the difference between the maximum delta $P$ and the threshold decreased from $28 \mathrm{mmHg}$ at $\mathrm{Rkm}$ 0.6 to $9 \mathrm{mmHg}$ at $\mathrm{Rkm} 4.8$ and only $1-2 \mathrm{mmHg}$ at Rkm 14.9 .

The mean delta $P$ of $82 \mathrm{mmHg}$ at Rkm 0.6 measured on 12 August was intermediate of the mean delta $\mathrm{P}$ levels determined from two 1986 profiles when the sluicegates were also closed ( 31 July and 14 october). Based on the cross-river transect data collected when water temperatures ranged from about $12-19^{\circ} \mathrm{C}\left(53.6-66.2^{\circ} \mathrm{F}\right)$ and discharges ranged from $59.47-163.29 \mathrm{~m}^{3} / \mathrm{s}(2100-5766 \mathrm{cfs})$, maximum delta $\mathrm{P}$ would be less than $100 \mathrm{mmHg}$ under similar conditions. Mean delta $\mathrm{P}$ levels would range from approximately 70-90 $\mathrm{mmHg}$ when water is discharged only over the radial gates of Afterbay Dam. other data, however, suggest we could expect this range to extend up to approximately 110-112 $\mathrm{mmHg}$. 
CROSS RIVER TAANSECT - 1987

DELTA P \{Rkm O.e

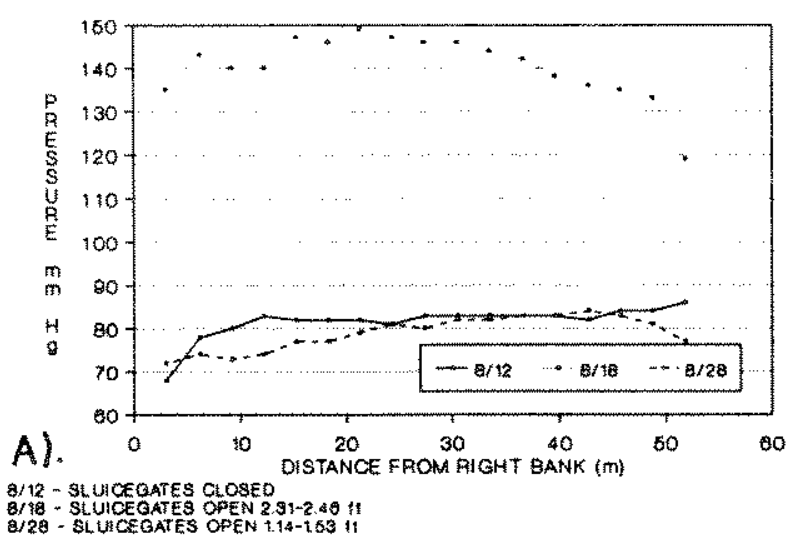

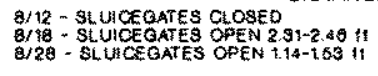

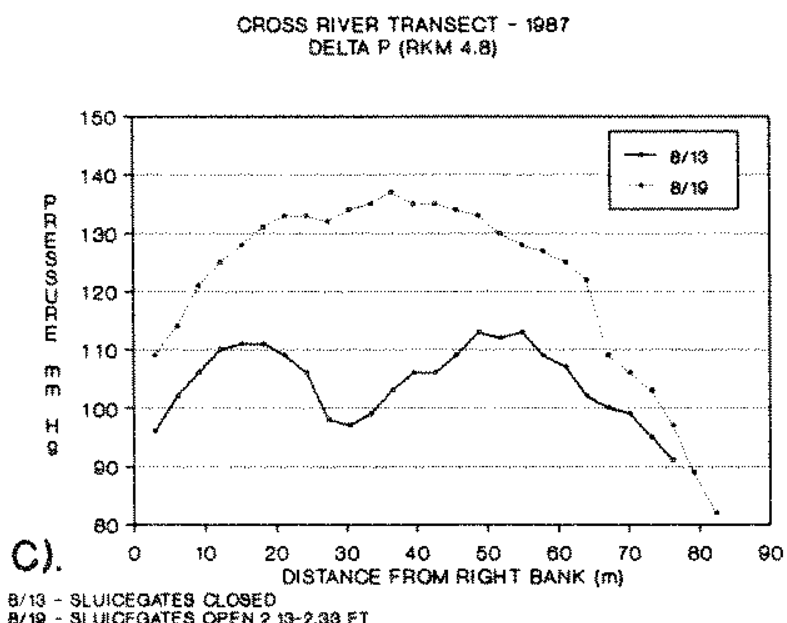

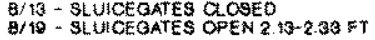

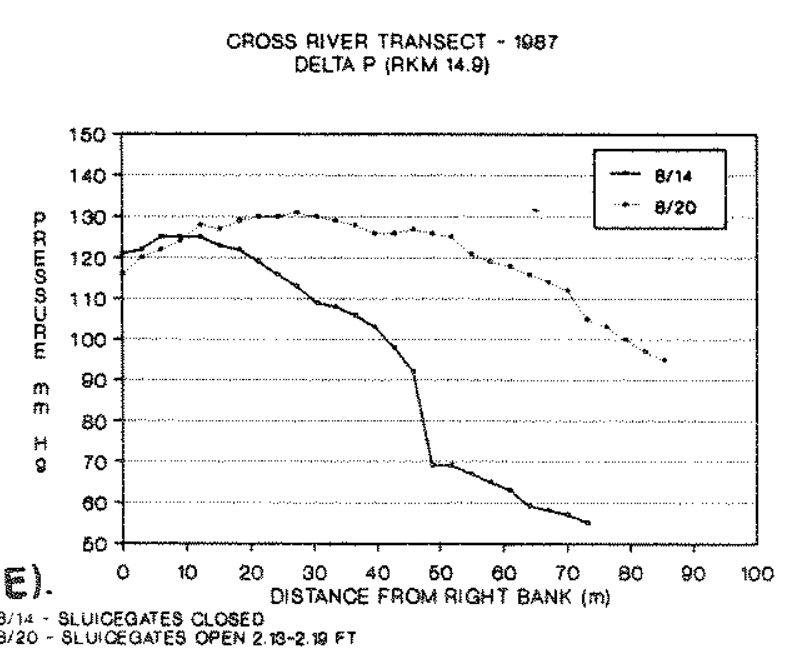
CROSS AIVER TRANSECT - 1987 - AKM O.6
OXYGEN (OP) AND NITROGEN (NP) PRESSURE

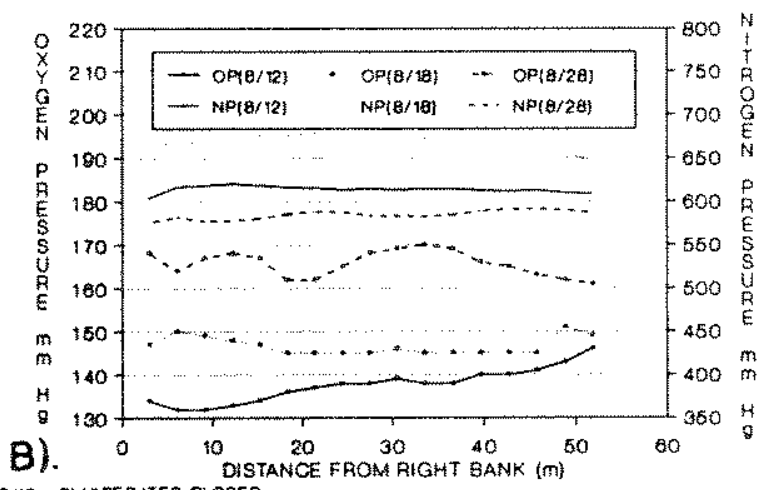

9/12 - \$LLICEGATES CLOBEO

8/18 - SLUICEGATES OFEN 2.31-2.40

CROSS RIVEA TRANSECT - 1987 - RKM 4.8 OXYGEN (OP) AND NITROGEN (NP) PRESSURE

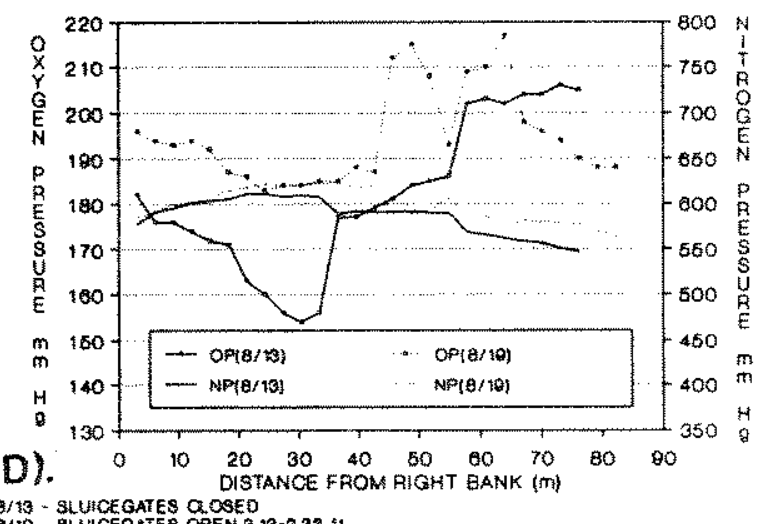

\$/13 - SLULCEATES Q.OSED
B/19 - SLUICEQATES OPEN $2.13-2.33$ H1

CROSS RIVER TRANSEC - 1987 - RKM 14.8 OXYGEN (OP) AND NITROGEN (NP) PAESSURE

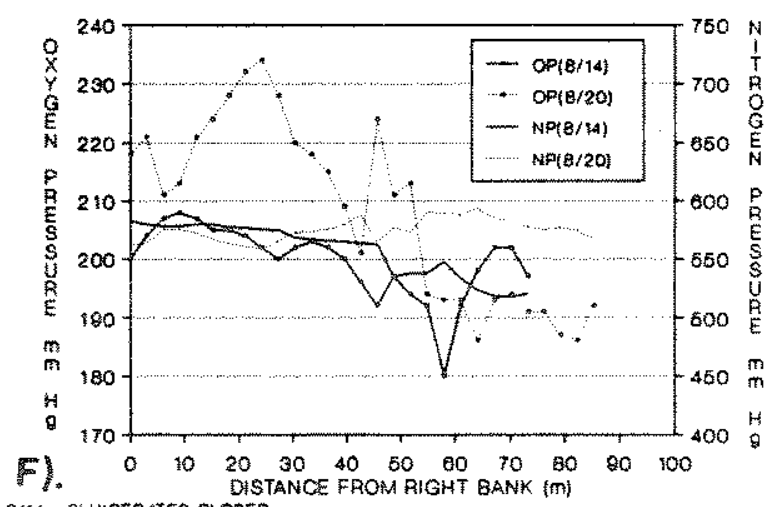

8/14 - SLUHCEQATS CLOSED
B/20 - SLUICEATES OPEN 2.13-2.

Figure 2.7.10. Cross-river transects of delta $P$ (mmHg) at Rkm 0.6 (A), Rkm 4.8 (C), and Rkm 14.9 (E), and oxygen and $\mathrm{N}_{2}+\mathrm{Ar}$ pressures (mmHg) at Rkm 0.6 (B), Rkm 4.8 (D), and Rkm 14.9 (F). 
The same transects were remeasured 6 d later when only the sluiceway gates were used to release water from Afterbay

Reservoir (Figure 2.7.10). Although discharge remained near $59.47 \mathrm{~m}^{3} / \mathrm{s}$ (2100 cfs), major increases in hyperbaric pressures occurred. The largest increase in the mean and maximum delta $P$ was 58 and $63 \mathrm{mmHg}$, respectively, at $\mathrm{Rkm} 0.6$. The $\mathrm{rise}$ of 18 and $24 \mathrm{mmHg}$ in mean delta $\mathrm{P}$ values at $\mathrm{Rkm} 4.8$ and 14.9 , respectively, was substantially lower than immediately below Afterbay Dam but still pose possible negative impacts for trout. Maximum delta p's at each site were equal to or greater than all threshold criteria for bubble formation. Maximum delta P's were 36 and 15 mmHg above the vascular system threshold at $\mathrm{Rkm} 0.6$, and 4.8 , respectively, and equal to the threshold at $\mathrm{Rkm} 14.9$.

At $\mathrm{Rkm} 0.6$, we also observed increases in component gases when discharge was rerouted from the spillway to the sluiceway. Mean $\mathrm{N}_{2}+$ Ar gas tension increased $55 \mathrm{mmHg}$ and mean oxygen tension rose $9 \mathrm{mmHg}$. Individual gases remained fairly constant across the river channel (Figure 2.7.10).

Although hyperbaric pressures changed, the difference between low and high values across the river at both Rkm 0.6 and 14.9 remained similar, irrespective of how water was released from Afterbay Dam (with the exceptions of the TGP measurement at $51.8 \mathrm{~m}$ on 18 August at Rkm 0.6 , and TGP determined after the thunderstorm at $\mathrm{Rkm} 14.9$ on 14 August). At Rkm 4.8, however, the cross-river transect showed a considerably larger range in delta $\mathrm{P}$ on 19 August when water was passed through the sluicegates. Difference between low and high values increased from 22 to 55 mmHg. Almost one-half of the increase may have been due to 
extending the transect an additional $6 \mathrm{~m}$ into slow-moving water near the left bank. Even so, the range would have still increased $40 \mathrm{mmHg}$.

on 28 August, another cross-channel profile of gas pressures was measured at $\mathrm{Rkm} 0.6$ (Figure 2.7 .10 ). At this time, both the spillway radial gates and the sluiceway were used to release the $62.1 \mathrm{~m}^{3} / \mathrm{s}(2193 \mathrm{cfs})$ flow from Afterbay Reservoir. The mean hyperbaric pressure was lower and the range was less than in either of the previous profiles. The mean delta $P$ was $79 \mathrm{mmHg}$ and ranged from $72-84 \mathrm{mmHg}$ (Figure 2.7 .10 ). The lack of a gradient across the river was unexpected but probably resulted from the small sluicegate openings which ranged from $1.14-1.53$ ft. Usually, delta $P$ decreases when sluicegates are open either a small or large amount and increases when openings are in a mid-range. Maximum delta $P$ measured on this transect was $34 \mathrm{mmHg}$ below the cardiovascular bubble growth threshold, but 1 and 59 mmHg greater than the environmental water and swim bladder thresholds, respectively. No other profiles were measured downstream under this flow regime because of the lack of gradient in hyperbaric pressure at $\mathrm{Rkm} 0.6$.

The Rkm 0.6 cross-channel gas tensions on 28 August 1987 were markedly different than on 4 september 1986. In 1986, mean discharge was $17.08 \mathrm{~m}^{3} / \mathrm{s}(603 \mathrm{cfs})$ greater, sluicegates were open about one-half way, all radial gates were open, and water temperature was higher. Mean delta $P$ in 1986 was about $25 \mathrm{mmHg}$ higher and the $40 \mathrm{mmHg}$ gradient was much larger than the $12 \mathrm{mmHg}$ gradient on 28 August 1987.

The downiver and cross-river gas measurements illustrated 
that if all flow is passed through either the sluicegates or radial gates, pressure gradients did not develop. Data collected in 1986, however, demonstrated that under certain conditions, discharge split between the spillway and sluiceway may establish a gradient and reduce gas levels near the left bank, benefiting fish there. However, splitting the discharge may maximize production of hyperbaric pressures on the right bank. The last profile performed at $\mathrm{Rkm} 0.6$ in 1987 showed that excessive gas entrainment does not always occur when the sluiceway is in use, i.e., when sluicegate openings are relatively small. Attainment of these conditions should be possible at times even while operating under the constraints imposed by irrigation demand, power production, or recreational concerns.

\subsection{3e Target Gas Level Recommendations}

Potential operational changes at Afterbay Reservoir will not result in total dissolved gas pressures dropping below the Environmental Protection Agency (EPA) criterion of $110 \%$ saturation (EPA 1976) or the corresponding average delta $\mathrm{P}$ for this site, $68 \mathrm{mmHg}$. The maximum gas pressure we recommend at $\mathrm{Rkm}$ 0.6 (gagehouse) to protect fry is a delta $\mathrm{p}$ of $83 \mathrm{mmHg}$ or $112.2 \%$ saturation TGP, regardless of oxygen or $\mathrm{N}_{2}+$ Ar pressures. These values correspond to the threshold for external bubble growth and assume no compensation by hydrostatic pressure. Because fry use shallow, stream margin areas, almost no compensation is available when they are most vulnerable. This target gas level, however, is also unattainable given the current design of Afterbay Dam and the stilling basin. Although recruitment of brown trout is 
sufficient under existing conditions, this is not true for rainbow trout.

The goal of modifying Afterbay Dam operation should be to decrease mean gas levels below bubble growth thresholds for the cardiovascular system, which vary with oxygen pressure. We calculated an average threshold delta $P$ of $115 \mathrm{mmHg}$ (116.9\% TGP) for $\mathrm{Rkm} 0.6$ based on oxygen data collected at the gagehouse from 1985-1988. The expected variation in the threshold would be roughly 108-118 $\mathrm{mmHg}$, which corresponds to the thresholds calculated from Hydromet data as shown in section 2.3. Changes in Afterbay Dam operation which would decrease gas entrainment could also decrease the mean threshold by as much as $3 \mathrm{mmHg}$, which represents a delta $\mathrm{P}$ of $112 \mathrm{mmHg}$ or $116.5 \% \mathrm{TGP}$, if oxygen tension reached atmospheric equilibrium.

Altering Afterbay Dam operations to decrease mean gas levels below the cardiovascular system bubble growth threshold would provide more flexibility to dam operators since it would allow hyperbaric pressure to exceed the threshold for short time periods. However, the risk to fish is increased substantially if gas tensions are maintained at high levels for extended periods. operational changes could realistically achieve these target gas levels. However, it is beyond the scope of this study to determine if those operational changes would conflict with other uses of the project.

\section{7 .31 operation options}

Large variations in Afterbay Dam gate openings, water elevations, and gas levels occurred during this study (Figures $2.7 .11-2.7 .14)$. The general level of each factor was predictable 

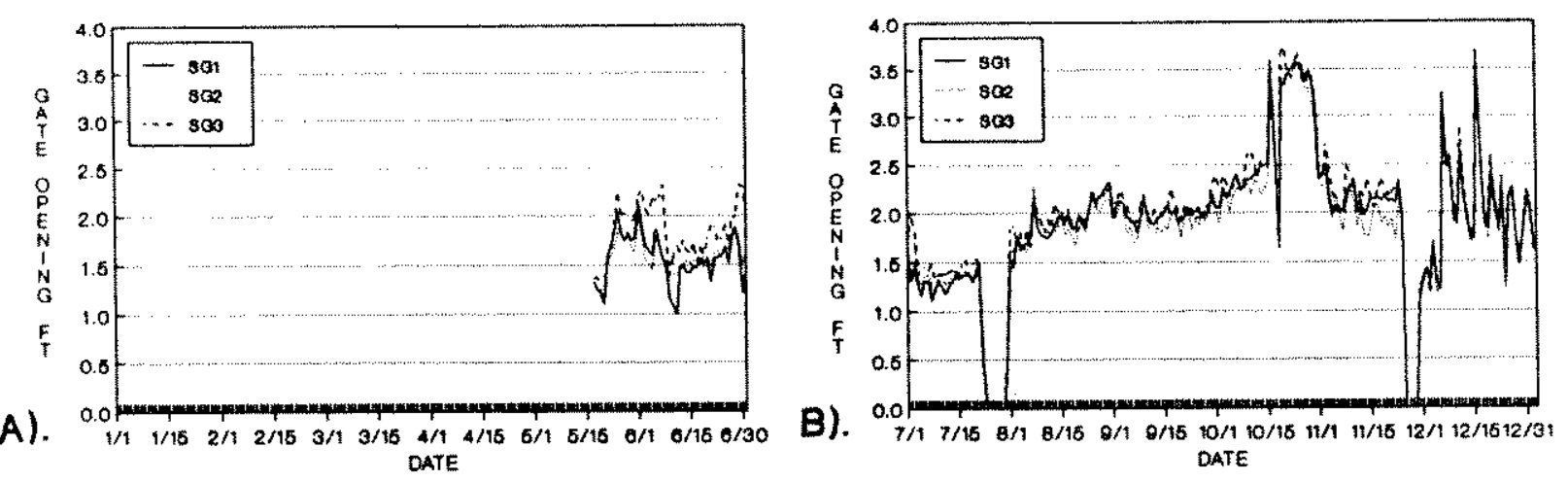

RADIAL GATES - 19BS
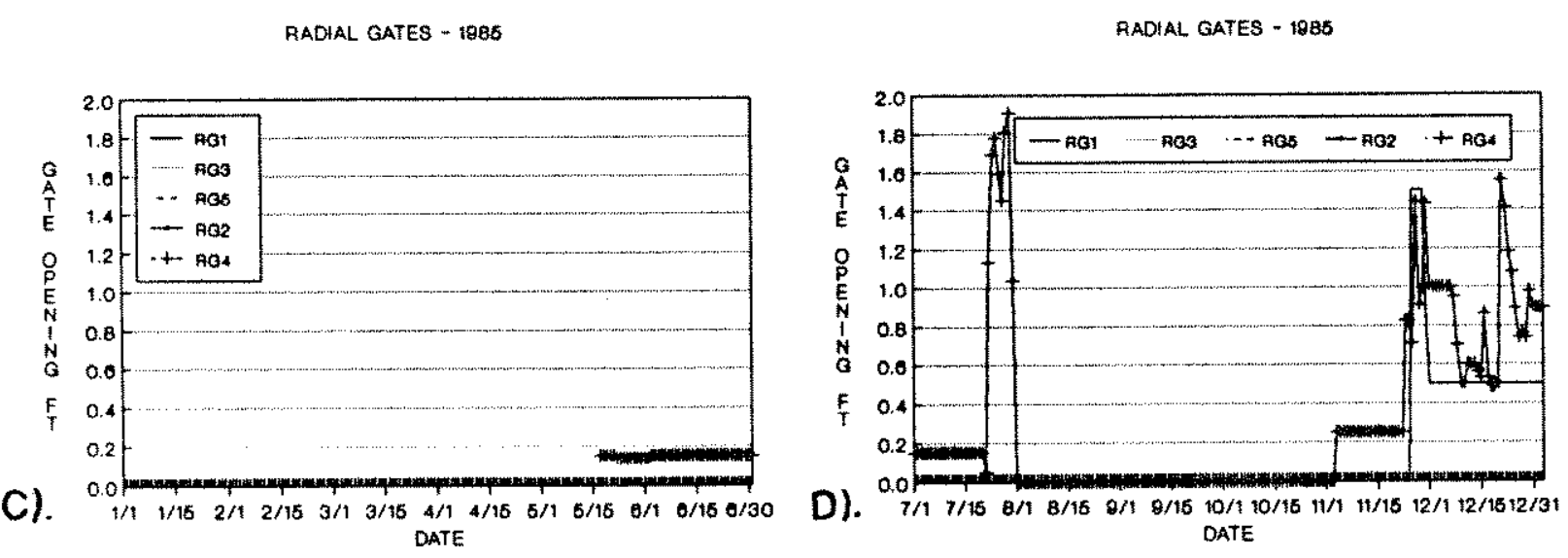

$1285-101$ HALF
(ELEVATION $+3100 \mathrm{FT}$ )

$1860-2$ 2nd HALF
(ELEVATION $+3100 \mathrm{FT})$
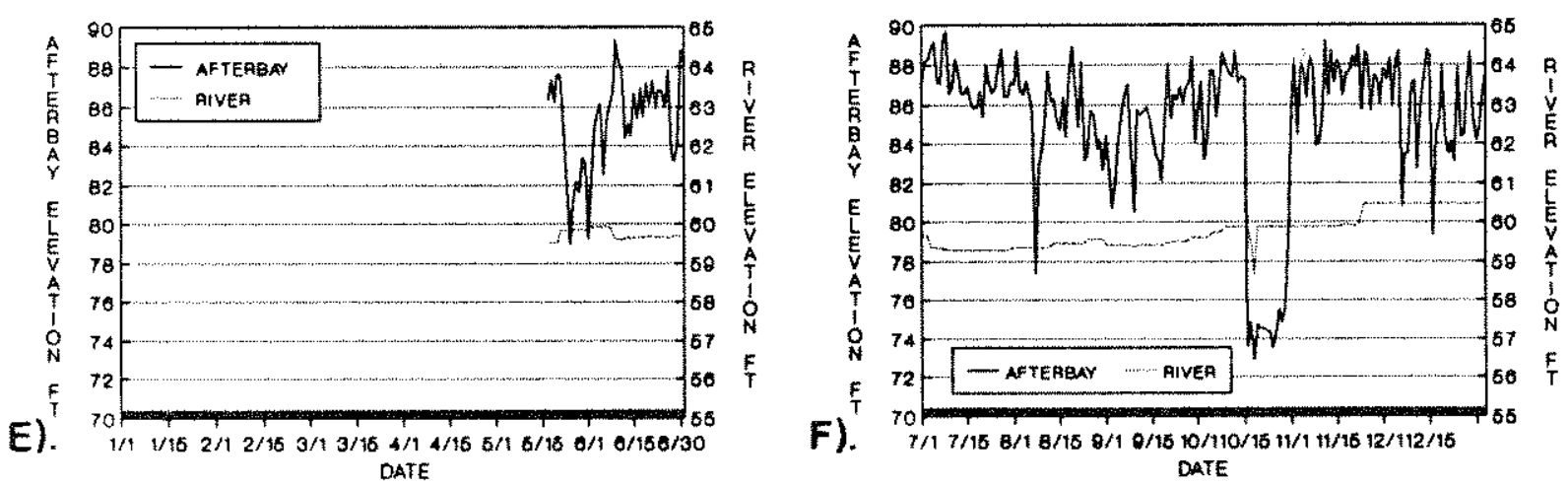

Figure 2.7.11. Mean daily opening of sluiceway gates (A \& B), spillway radial gates (C\&D) on Afterbay Dam, and Afterbay Reservolr and river elevations (ft) (E\& F) during 1985. 

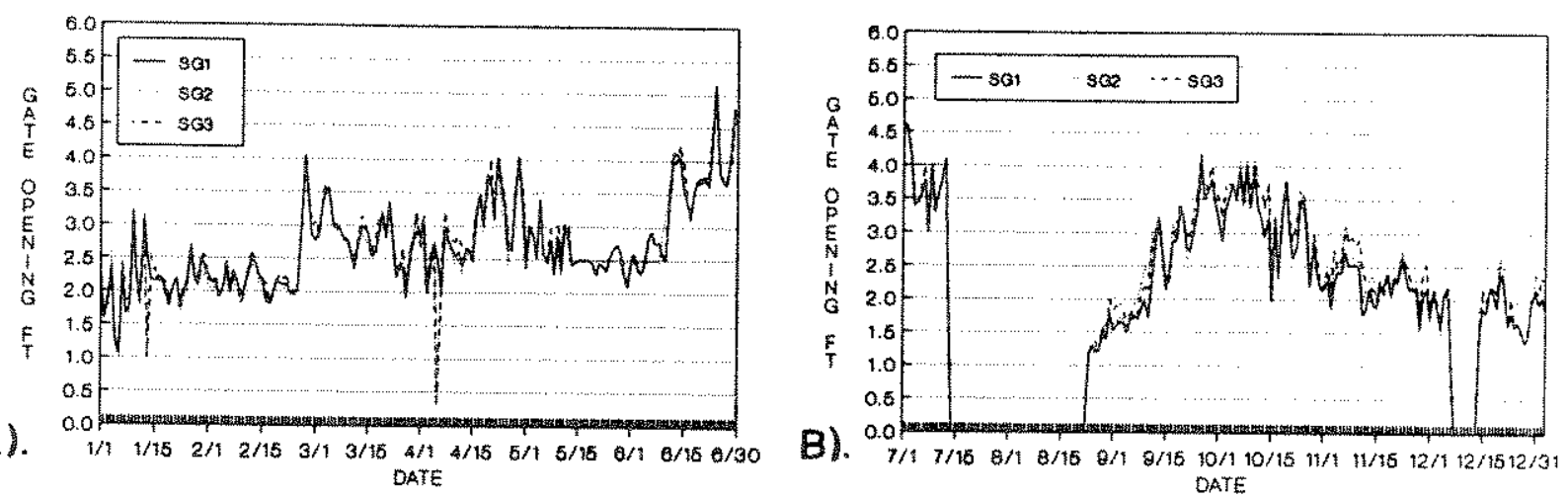

RAOIAL GATES - 1988
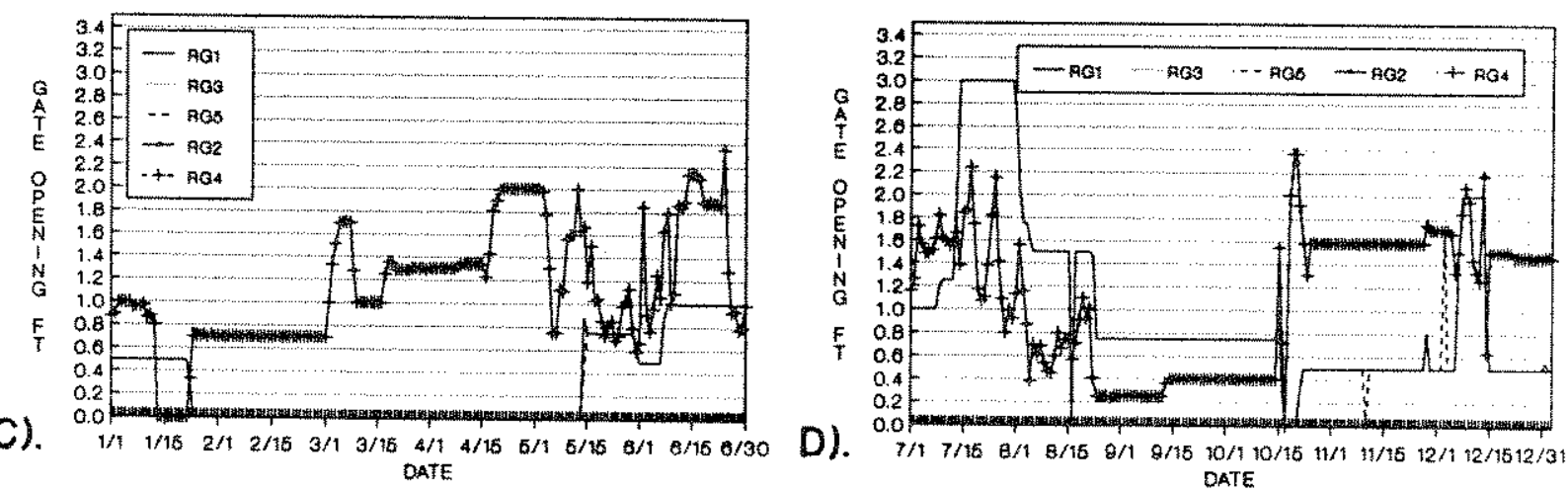

1880 - 18T HALF

(ELEVATION $+3100 \mathrm{FT}$ )

$1080-$ 2nd HALF

(ELEVATION + 3100 FT)
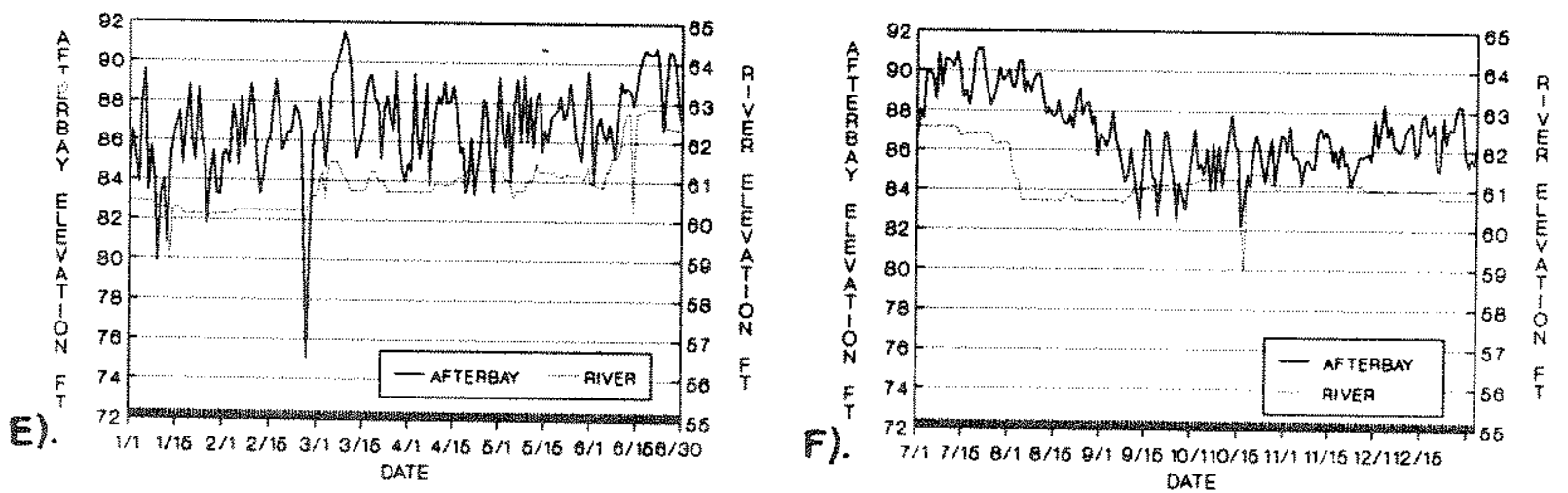

Figure 2.7.12. Mean daily opening of sluiceway gates (A B), spillway radial gates (C D) on Afterbay Dam, and Afterbay Reservoir and river elevations (ft) $(E \& F)$ during 1986. 

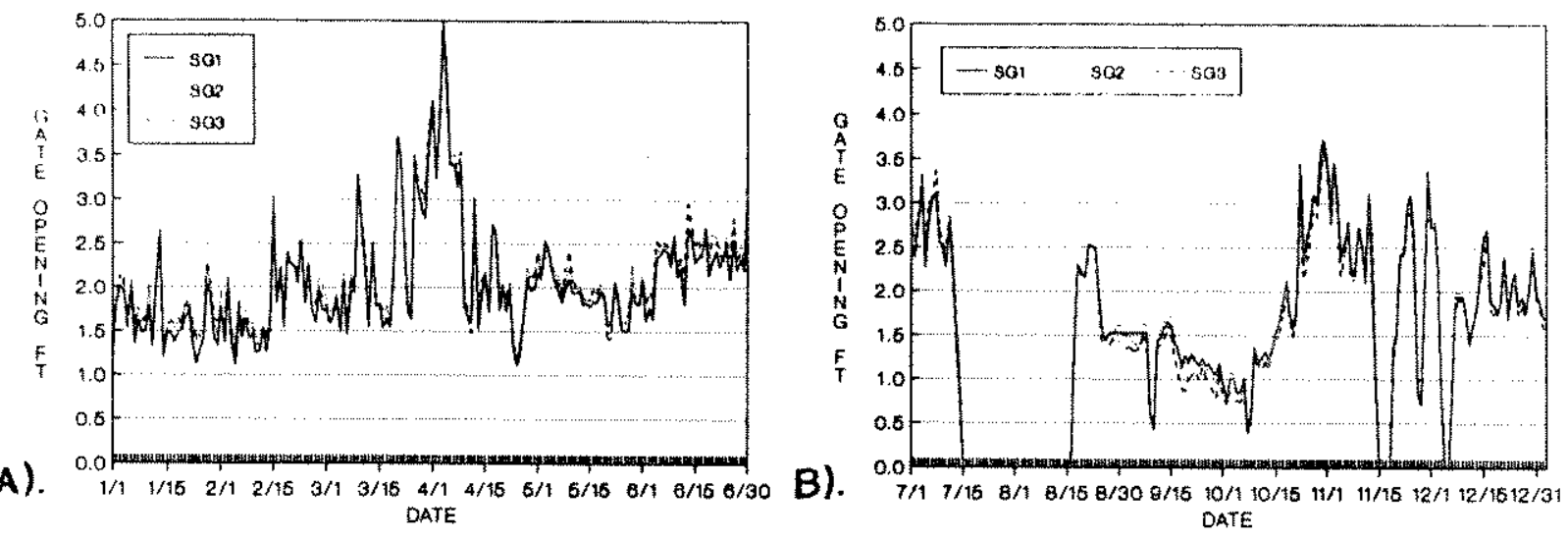

AADIAL GATES - 1887

RADIAL GATES - 1987
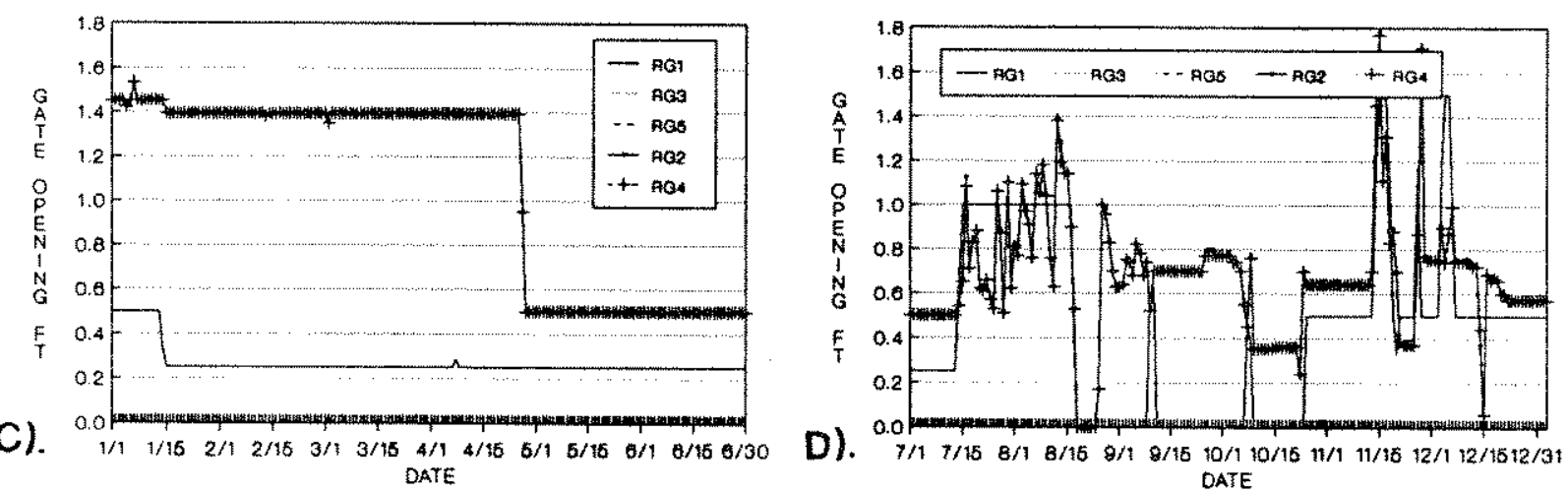

1987 - ISt HALF (ELEVATION + 3100 FT)

$18 a 7$ - Ist HALF

\{ELEVATION $+3100 \mathrm{FT}$ \}
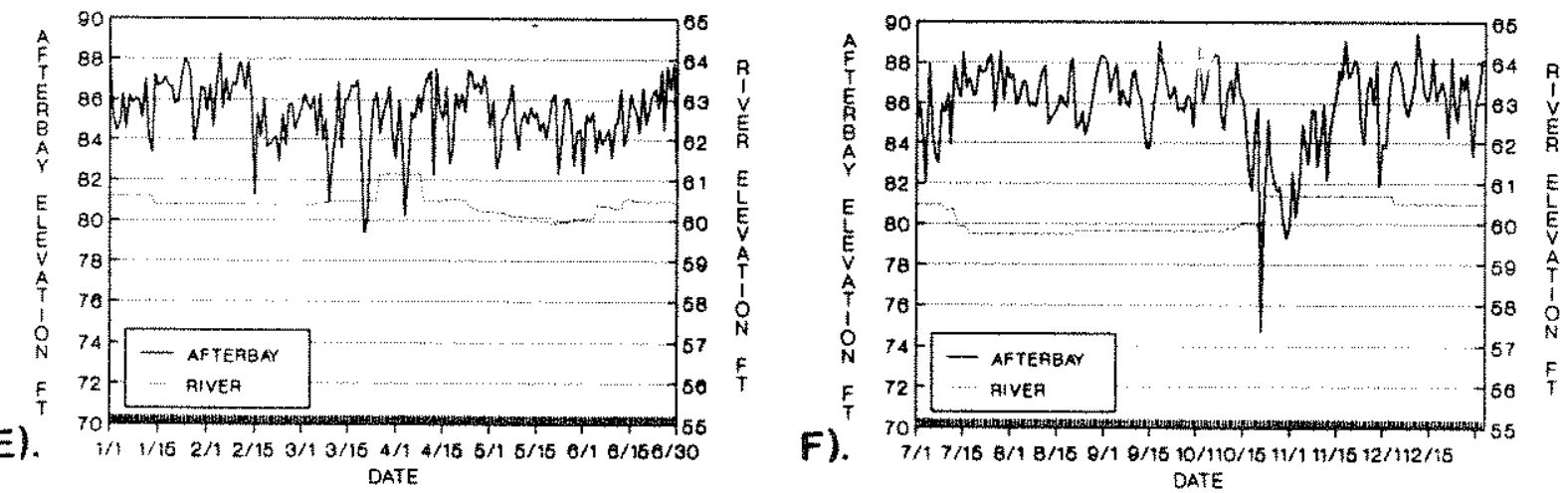

Figure 2.7.13. Mean daily opening of sluiceway gates (A \& B), spillway radial gates (C \& D) on Afterbay Dam, and Afterbay Reservoir and river elevations (ft) (E\& F) during 1987. 


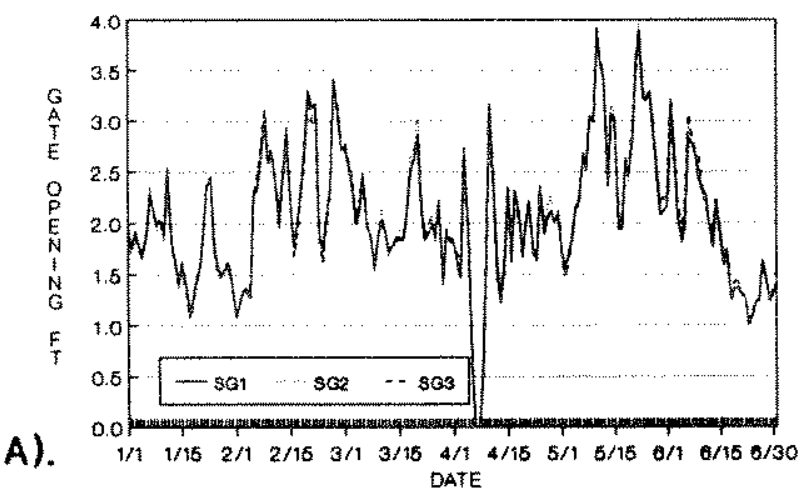

RADIAL GATES - 1998

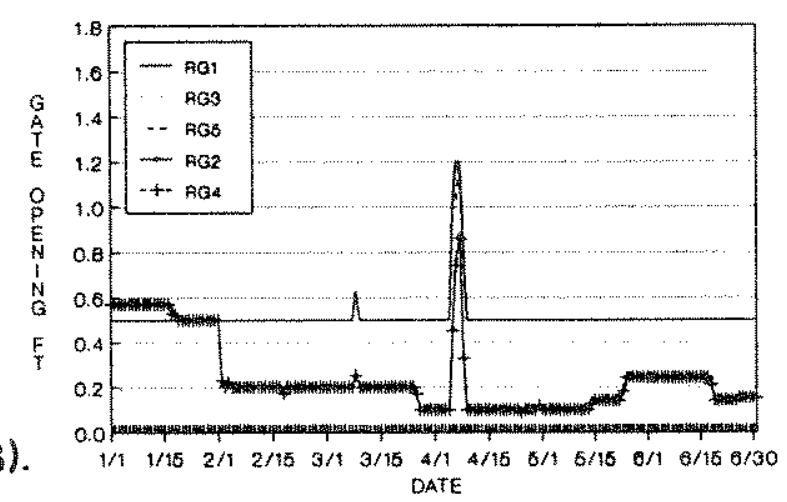

108B - 19: HALF (ELEVATION + 3100 FT)

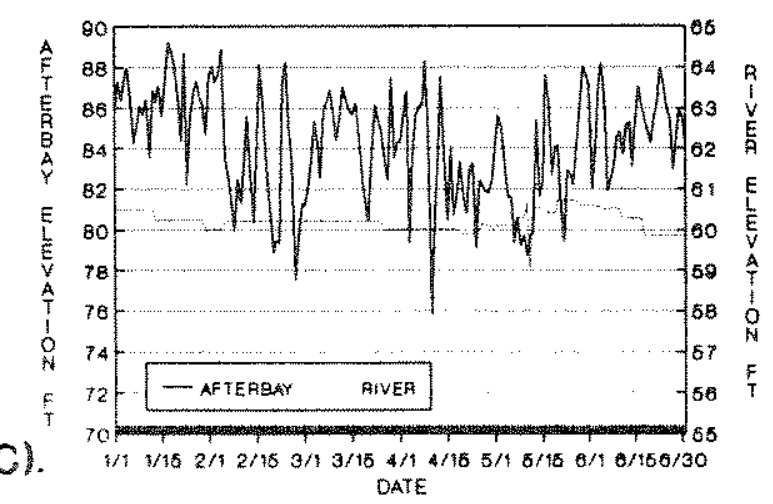

Figure 2.7.14. Mean daily opening of sluiceway gates (A), spillway radial gates (B) on Afterbay Dam, and Afterbay Reservoir and river elevations ( $f t$ ) (C) during the irst half of 1988 . 
depending on the Afterbay operational mode and total discharge. In 1985, mean sluicegate openings were $1.84,1.81$, and $1.95 \mathrm{ft}$ for sluicegates 1,2 , and 3, respectively (Figure 2.7.11). The maximum mean daily sluicegate opening was $3.74 \mathrm{ft}$. All sluice and radial gates were closed sometime during each year except for the radial gates in 1988 . Radial gates 1,3 , and 5 each had an annual mean and maximum gate opening in 1985 of $0.10 \mathrm{ft}$ and 1.5 ft, respectively. Radial gates 2 and 4 averaged $0.27 \mathrm{ft}$ and were opened as much as $1.91 \mathrm{ft}$. Lowest river elevations occurred in 1985 (mean $59.75 \mathrm{ft}$ ). Afterbay Reservoir elevations varied from a low of 72.88 ft up to $89.74 \mathrm{ft}$.

In 1986, mean daily sluicegate openings varied from 2.29-2.35 ft and maximum mean daily gate openings ranged from 4.15-5.14 ft, reflecting higher flow conditions (Figure 2.7.12). Radial gates 1,3 , and 5 mean daily openings were $0.65-0.66 \mathrm{ft}$ with a maximum of $3.00 \mathrm{ft}$ while radial gates 2 and 4 had mean openings of 1.12 ft with mean daily maximums of only 2.35 and $2.37 \mathrm{ft}$, respectively. Mean river elevation rose to $61.09 \mathrm{ft}$ in 1986 and Afterbay elevations remained higher, varying from 75.06-91.57 ft.

The mean daily sluicegate openings were less in 1987 than 1986, ranging from 1.77-1.81 ft with high daily maximums ranging from 4.89-5.01 ft (2.7.13). The mean and maximum settings of radial gates 1,3 , and 5 were 0.36 and $1.5 \mathrm{ft}$, respectively. Radial gates 2 and 4 had a mean opening of $0.87 \mathrm{ft}$ and maximum mean daily openings of $1.77-1.78 \mathrm{ft}$. Mean river elevation in 1987 was intermediate between 1985 and 1986 levels, at $60.29 \mathrm{ft}$. Mean daily Afterbay Reservoir elevations varied $14.77 \mathrm{ft}$ in 1987 from $74.7-89.47 \mathrm{ft}$. 
Mean daily sluicegate openings during the first half of 1988 increased from 1987 and ranged from 2.06-2.08 ft (Figure 2.7.14). Maximum daily sluicegate openings remained slightly higher than in 1985 but lower than all other years, varying from 3.89-4.00 ft. The mean, minimum, and maximum settings of radial gates 1 , 3 , and 5 were $0.51,0.50$, and $1.2 \mathrm{ft}$, respectively. Radial gates 2 and 4 had a relatively low mean opening of $0.24 \mathrm{ft}$, maximum mean daily openings of $0.86-0.87 \mathrm{ft}$, and minimum mean daily openings from $0.86-0.87 \mathrm{ft}$. The mean river elevation in 1988 was $60.22 \mathrm{ft}$. Mean daily Afterbay Reservoir elevations varied from $75.83-89.22 \mathrm{ft}$.

All Afterbay Dam operations data analyzed during the study demonstrated that regulation of the sluiceway gates had the greatest effect on delta $P$ or TGP measured at Rkm 0.6 (White et al. 1986, 1987, 1988). This was demonstrated by Pearson correlation coefficients which described the linear relationship between TGP or delta $P$ and individual sluicegate openings. The correlation coefficients varied from 0.45 to 0.53 in 1985 and increased to 0.76-0.77 during 1986 (White et al. 1986, 1987). The increase was due at least partially to more accurate data collection in 1986. Sluice gates were usually operated identically (Figures 2.7.11-14).

Mean daily openings of the spillway radial gates did not correspond with gas levels or sluice gate openings. The openings of radial gates 1,3, and 5 , and of 2 and 4 were generally adjusted simultaneously. The relationship between openings in the radial gates and delta $\mathrm{p}$ was negative in all years of the study. During periods when the sluiceway was not in service, 
radial gates 2 and 4 maintained a uniform river discharge, which resulted in frequent and/or large fluctuation in gate openings. Radial gates were usually opened to a specific height, however, and maintained there for a long period of time (Figures 2.7.1114). Even though the gates remained open the same amount, fluctuations in Afterbay Reservoir elevations altered their discharge. Discharge through the radial gates ceases when the Afterbay Reservoir drops below the spillway crest elevation of $3179.50 \mathrm{ft}(969.11 \mathrm{~m})$.

our data and those collected in 1973 (BOR 1973) suggest that maximum TGP production may be reduced by developing guidelines for regulating spill pattern through sluiceway gates. Guidelines would be adjusted seasonally to account for changes in discharge and water temperature, necessitating monitoring of water temperatures when they are low such as in the spring and early summer and avoid use of the sluicegates in the mid-range when possible since this operating regime often produces the highest hyperbaric pressures. Sluiceway discharges from 1500-2000 cfs should be avoided whenever possible, with discharges of $1000 \mathrm{cfs}$ or less most ideal. Achievement of the following recommendations would minimize the probability of adult trout mortality.

Minimizing use of sluiceway gate openings between 25 to $75 \%$ would not eliminate hyperbaric pressures above trout cardiovascular threshold levels; it would reduce the length of exposure and could be accomplished during parts of the year. In 1987 we observed elevated gas pressures when sluicegates were open halfway. Mean daily delta $\mathrm{P}$ reached $120 \mathrm{mmHg}$ and above (Figure 2.7 .15 ) at daily mean sluicegate openings of 1.75 to 3.5 
DELTAP $(\mathrm{mm} \mathrm{Hg})$

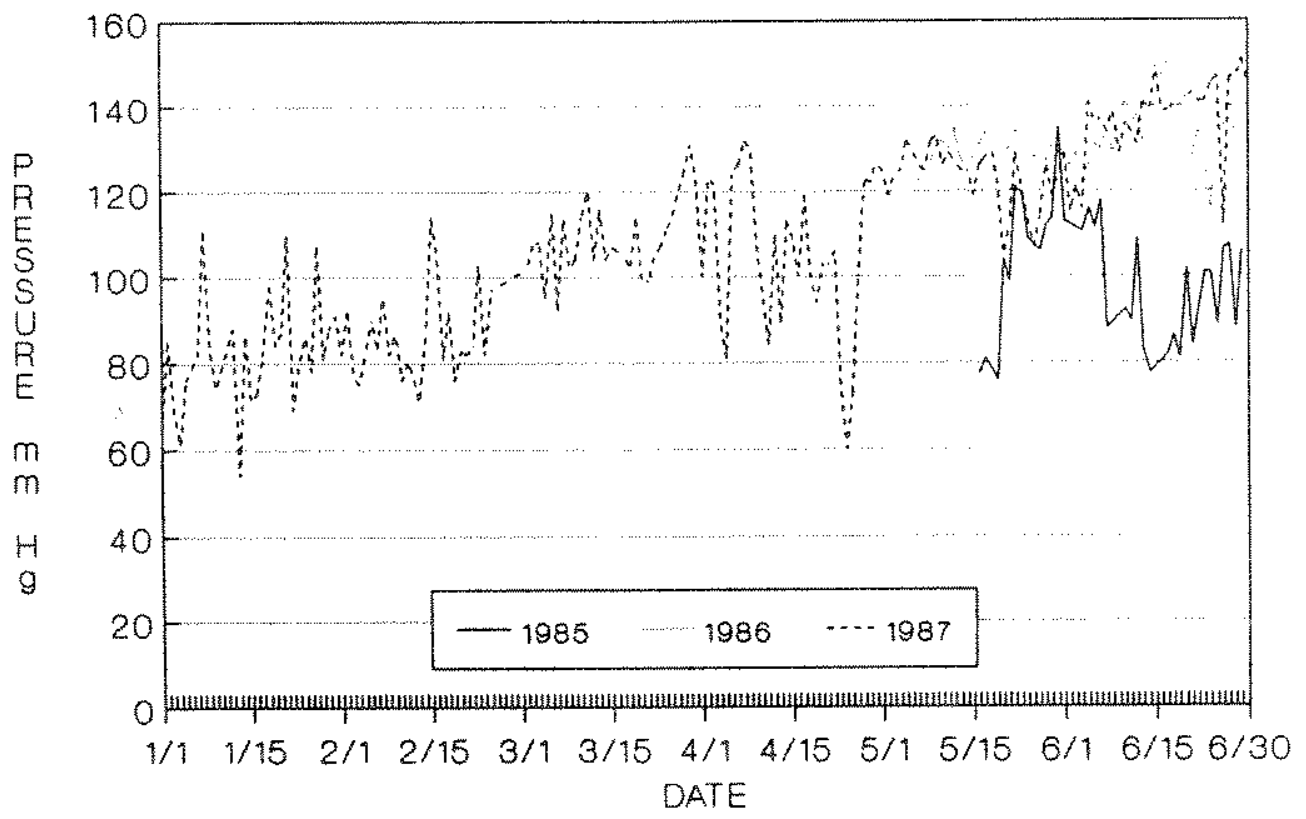

DELTAP

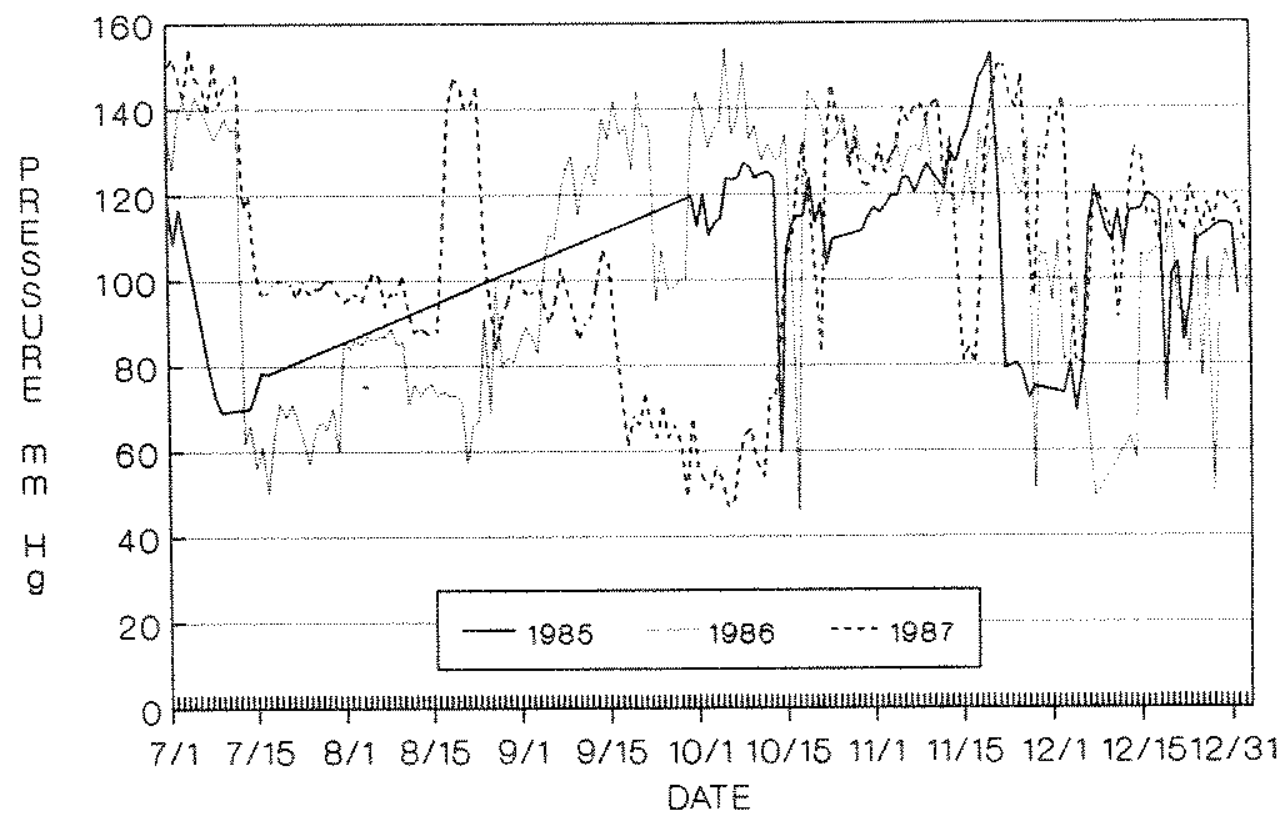

Figure 2.7.15. Uncorrected mean daily delta $p$ (mmig) measured at Rkm 0.6 (gagehouse) by the common sensing tensionometer from 1985-1987 (Hydromet archive files data). 
ft (mean opening $2.44 \pm 0.52 \mathrm{ft}$ ). These delta $P$ levels were above the Rkm 0.6 mean cardiovascular system threshold level of $119 \mathrm{mmHg}$. However, both larger and smaller sluicegate openings coincided with lower hyperbaric pressures. The mean opening of sluice gate 1 was $1.42 \pm 0.84 \mathrm{ft}$ for mean daily delta $P$ levels less than $120 \mathrm{mmHg}$. As would be expected, the range of openings for the lower gas pressures was larger, varying from $0-5.1 \mathrm{ft}$ compared to $0.91-3.87 \mathrm{ft}$ for those above the mean cardiovascular system threshold. So, under some conditions, altering the percentage of total river flow through the sluiceway from onehalf to either one-fourth or three-fourths would reduce TGP since the equal split between the sluice and spillway results in discharges that at times create maximum delta p's.

Maximizing discharge through the radial gates would benefit the fishery and would not necessitate frequent large adjustments of the sluice gates under some operational modes. The daily minimum Afterbay water elevation dropped below the spillway's crest elevation for $28,8,45$, and $73 \mathrm{~d}$ in 1985, 1986, 1987, and 1988, respectively. These usually occurred in groups of consecutive days. The 1985 and 1988 data did not include the entire year. Although there was substantial variation between years, during $88.0 \%$ of the $1280 \mathrm{~d}$ monitored, the Afterbay Reservoir minimum pool elevation was greater than the spillway radial gate crest elevation $(3179.5 \mathrm{ft} ; 969.11 \mathrm{~m})$ that would allow continuous spillway discharge (Figure 2.7.16). Most minimum elevations were substantially greater than the spillway crest elevation which would enable the radial gates to discharge much of the water from Afterbay Dam. As an example, even if 


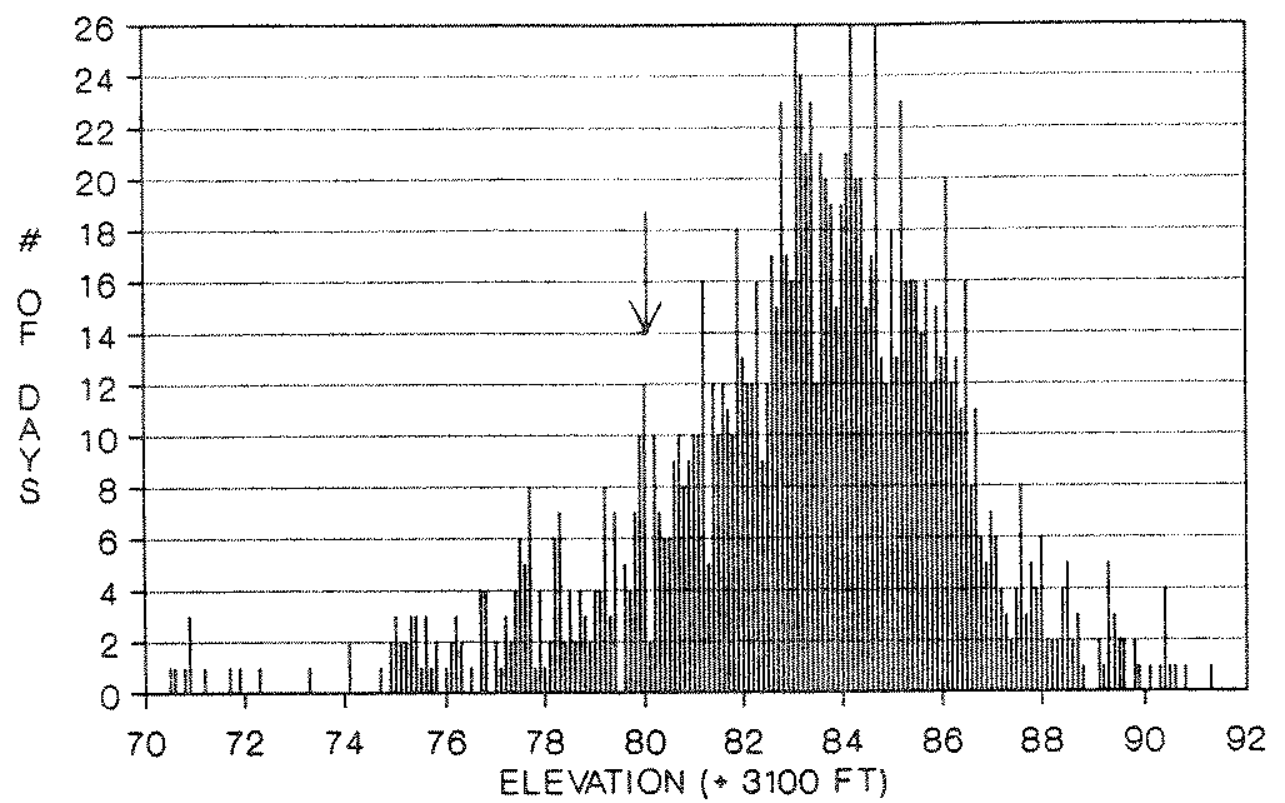

DOESN'T INCLUDE 5 DAYS WITH ELEV, 70 FT

Figure 2.7.16. The frequency of occurrence of Afterbay Reservoir minimum elevations from 30 March 1985 - 5 October 1988. Arrow designates elevation below which splilway discharge is not possible.

minimum daily elevation dropped to $3182.00 \mathrm{ft}$ spillway discharge, according to BOR rating curves, could still be maintained at 1950 cfs $\left(55.21 \mathrm{~m}^{3} / \mathrm{s}\right)$ using all five radial gates or 790 cfs $\left(22.37 \mathrm{~m}^{3} / \mathrm{s}\right)$ using two radial gates.

Also, in periods of peak runoff, the Afterbay Reservoir water elevation may remain constant. We observed this in 1986 when the pool was maintained near 971.7 s (3188 ft). During these periods, almost all discharge could be routed through radial gates. Since gas levels near Afterbay Dam tend to increase as discharge increases, this mode of operation should reduce saturation levels during a critical time of the year. However, large changes in Afterbay Reservoir water elevation over the course of a day would require frequent manual gate changes 
and make this unfeasible. The average variation in daily Afterbay levels during the second half of 1987 was $5.76 \mathrm{ft}$. The minimum variation of $0.79 \mathrm{ft}$ would not pose operational problems but the maximum observed, which was 15.15 ft would cause logistical difficulties.

The magnitude of the hyperbaric pressure reduction resulting from maximizing spillway flow would depend primarily on the amount of sluiceway flow reduction and the nearness of the discharge to that which would produce maximum saturation for a given temperature. Although reductions in delta $P$ may be relatively small, they may provide significant benefit if levels drop below thresholds for bubble growth in trout. Also, reductions may minimize the potential for adult fish kills in the future.

Presently, only sluice gates are automated to respond to changes in river elevation and serve as the only means to discharge water when the Afterbay Reservoir is drafted below the spillway crest elevation $(969.11 \mathrm{~m} ; 3179.50 \mathrm{ft})$. Also, only the sluice gates and two radial gates $(2 \& 4)$ can be remotely adjusted from the Yellowtail project control room. The other radial gates are adjustable only from Afterbay Dam. The existing operating regime releases approximately half of the water through the radial gates and half through the sluiceway provided Afterbay Reservoir water elevations are above the spillway crest. Engineering constraints limit the maximum difference in openings between the radial gates to $0.76 \mathrm{~m}(2.5 \mathrm{ft})$, while sluicegate openings vary up to $30 \%$ of each other. Since the function of Afterbay Dam is to reregulate discharge in the river below 
Yellowtail Dam, daily reservoir fluctuations and associated changes in sluicegate openings are part of the usual operation. Avoiding mid-range sluicegate openings could result in additional wear and less flexibility for the sluicegates to respond to changes in the river stage height resulting in a corresponding fluctuation in river discharge.

Reducing gas tensions by operational changes would require a more consistent discharge via radial gates. Automation of radial gates 2 and 4 or all radial gates would allow shifts in flow pattern through Afterbay Dam when the Afterbay levels exceed $969.11 \mathrm{~m}$ (3179.5 ft) and would benefit the fishery. However, even with only radial gate discharge, hyperbaric pressures would still climb to $112 \mathrm{mmHg}$ at times. Although this would approach the cardiovascular threshold, it would be a substantial improvement over present gas levels.

Seasonal operational changes at Afterbay Dam could be implemented to decrease maximum gas pressures and reduce the potential of adult fish kills. The feasibility of increasing storage in Afterbay Reservoir, construction of a bypass penstock without electrical generation facilities, placement of a cascade downstream of the sluiceway stilling basin, use of the irrigation ditch and sandtrap as a bypass after renovation at the sandtrap headgates, or construction of a powerhouse should be explored to ensure that TGP is reduced in the upper reaches of the river. 


\section{LITERATURE CITED}

APHA (American Public Health Association). 1976. Standard methods for the examination of water and wastewater, 14th edition. American Public Health Association, Washington, D.C., USA.

Adams, E. S. 1974. Use of a recompression chamber to alleviate gas bubble disease in coho sac-fry. Progressive Fish culturist $36(1): 41$.

Alderice, D. F., J. T. Jensen, and F. J. Velson. 1984. Measurement of hydrostatic pressure in salmonid eggs. Canadian Journal of Zoology $62: 1977-1987$.

Alderdice, D. F. and J. O. T. Jensen. 1985a. An explanation for the high resistance of incubating salmonid eggs to atmospheric gas supersaturation of water. Aquaculture $49: 85-88$.

Allan, J. D. and E. Russek. 1985. The quantification of stream drift. Canadian Journal of Fisheries and Aquatic Sciences $42: 210-215$.

Altman, P. L. and D. S. Dittmer. 1961. Blood and other body fluids. Federation of American Societies for Experimental Biology.

Armitage, P. D. 1984. Environmental changes induced by stream regulation and their effect on lotic macroinvertebrate communities. Pages 139-165 in A. Lillehammer and S. J. Saltveit, editors. Regulated rivers. Columbia University Press. New York. USA.

Bachman, R. A. 1984. Foraging behavior of free-ranging wild and hatchery brown trout in a stream. Transactions of the American Fisheries Society 113:1-32.

Baltz, D. M. and P. B. Moyle. 1984. Segregation by species and size class of rainbow trout (Salmo gairdneri) and Sacramento sucker (Catostomus occidentalis) in three California streams. Environmental Biology of Fishes 10:101-110.

Bams, R. A. 1967. Differences in performance of naturally and artificially propagated sockeye salmon migrant fry, as measured with swimming and predation tests. Journal of the Fisheries Research Board of Canada 24(5):1117-1153.

Beiningen, K. T. and W. J. Ebel. 1970. Effect of John Day Dam on dissloved nitrogen concentrations and salmon in the Columbia River. Transactions of the American Fisheries Society $99: 664-671$. 
Bentley, W. W., E. M. Dawley, and T. W. Newcomb. 1976. Some effects of excess dissolved gas on squawfish, ptychochelius oregonensis (Richardson). Pages 41-46 in D. H. Fickeisen and M. J. Schneider, editors. Gas bubble disease. Technical Information Center, office of Public Affairs, Energy Research and Development Administration.

Berg, A. 1984. Supersaturation of dissolved air in the waterways of hydroelectric power plants: causal relationships, detrimental effects, and preventative measures. Norwegian Hydrodynamics Laboratories.

Blahm, T. H., R. J. McConnel1, and G. R. Snyder. 1973. Effect of gas supersaturated Columbia River water on the survival of juvenile salmonids. Final Report Part 1. National Marine Fisheries Service. Environmental Field station, prescott, oregon.

Blahm, T. H., R. J. McConnell, and G. R. Snyder. 1975. Effect of gas supersaturated Columbia River water on the survival of juvenile chinook and coho salmon. NOAA Technical Report, National Marine Fisheries Service. SSRF 688.

Blahm, T. H., R. J. McConnell, and G. R. Snyder, 1976. Gas supersaturation research. National Marine Fisheries Service Prescott Facility. Pages 11-19 in D.H. Fickeisen and M.J. Schneider, editors. Gas bubble disease. Technical Information Center, office of Public Affairs, Energy Research and Development Administration.

Bouck, G. R., G. A. Chapman, P. W. Schneider Jx., and D. G. Stevens. 1970. Observations on gas bubble disease in adult Columbia River sockeye salmon. Pacific Northwest Laboratory, Federal Water Quality Administration, Corvallis, oregon.

Bouck, G. E., G. A. Chapman, W. Schneider, Jr., and D. G. Stevens. 1976. Observations on gas bubble disease among wild adult columbia River fishes. Transactions of the American Fisheries Society 105:114-115.

Bouck, G. R. 1980. Etiology of gas bubble disease. Transactions of the American Fisheries Society 109:703-707.

Bouck, G. R. 1982. Gasometer: an inexpensive device for continuous monitoring of dissolved gases and supersaturation. Transactions of the American Fisheries Society 111:505-516.

Bouck, G. R. and R. E. King. 1983. Tolerance to gas supersaturation in freshwater and sea water by steelhead trout (Salmo gairaneri). Journal of Fish Biology 23:293300 .

Boussu, M. F. 1954. Relationship between trout populations and cover on a small stream. Journal of Wildilfe Management $18: 229-239$. 
Bowser, P. R., R. Toal, H. R. Robinette, and M. W. Brunson. 1983. Coelomic distension in channel catfish fingerlings. Progressive Fish Culturist 45:208-209.

Boyer, P. B. 1974. Lower Columbia and lower snake Rivers nitrogen gas supersaturation and related data analysis and interpretations. U.S. Army Corps of Engineers, North Pacific Division, Portland, Oregon.

Bureau of Reclamation. 1965. Definite plan report on Yellowtail Unit, Montana-Wyoming lower Bighorn division, Missouri River Basin Project. USDI, Bureau of Reclamation, Billings, MT, USA.

Bureau of Reclamation. 1973. Total dissolved gas supersaturation evaluation - Bighorn River below Yellowtail Afterbay. USDI. Bureau of Reclamation, Billings, Montana, USA.

Carty, D. G. 1985. Potential impacts of altering discharge pattern from Hauser Dam, Missouri River, on young-of-theyear brown trout and rainbow trout. Master thesis. Montana State University, Bozeman, Montana, USA.

Colt, J. 1984. Computation of dissolved gas concentrations in water as functions of temperature, salinity, and pressure. Special publication 14. American Fisheries society, Bethesda, Maryland, USA.

Colt, J., K. Orwicz, and D. Brooks. 1985. Impact of gas supersaturation on the growth of juvenile channel catfish. Aquaculture 50:153-160.

Colt, J., G. Bouck, and L. Fidler. 1986. Review of current literature and research on gas supersaturation and gas bubble trauma. American Fisheries Society, Bioengineering section Special Publiation 1.

Connor, W. P. 1988. Effects of gas supersaturated water on juvenile brown and rainbow trout. Master thesis. Montana State University, Bozeman, Montana, USA.

Corbel, M. J. 1975. The immune response in fish: a review. Journal of Fish Biology 7:539-563.

Cornacchia, J.W. and J. E. Colt. 1984. The effects of dissolved gas supersaturation on larval striped bass, Morone saxatilis. Journal of Fish Diseases 7:15-27.

coutant, C. C. and R. B. Genoway. 1968. Final report on the exploratory study of interaction of increased temperature and nitrogen supersaturation on the mortality of adult salmonids. U.S. Bureau of Commercial Fisheries, Pacific Northwest Laboratory, Richland, Washington. 
Coutant, C. C. 1973. Effect of thermal shock on vulnerability of juvenile salmonids to predation. Journal of Fisheries

Research Board of Canada 30:965-973.

Cramer, S. P. and J. D. McIntyre. 1975. Heritable resistance to gas bubble disease in chinook salmon. Fishery Bulletin $73: 934-938$.

Cummins, K. W. 1975. Macroinvertebrates. Pages 170-198 in B. A. Whitton (editor). River ecology. Blackwell, England.

Curry, R. L. and K. B. Curry. 1981. Bighorn River nitrogen supersaturation study, final report, February-August 1981. USDI, Fish and Wildlife Service, Hardin, Montana, USA.

DHES (Department of Health and Environmental Sciences). 1986. Montana water quality, 1986. The 1986 Montana 305 (b) report. Department of Health and Environmental Sciences, Environmental Sciences Division, water quality Bureau, Helena, Montana, USA.

Davis, J. C. and J. N. Cameron. 1971. Water flow and gas exchange at the gills of rainbow trout (Salmo gairdneri). Journal of Experimental Biology 54:1-18.

Davis, J. C., G. I. Bresnick, P. Doudoroff, T. R. Doyle, A. J. Mearns, F. B. Pearce, J. J. Peterka, J. G. Robinson, and D. L. Swanson. 1979. Dissolved oxygen. Pages 169-174 in R. V. Thurston, R. C. Russo, C. M. Fetterolf, Jr., T. A. Edsal1, and $Y$. M. Barber, Jr., editors. A review of the EPA Red Book: quality criteria for water. Water quality Section, American Fisheries Society, Bethesda, Maryland, USA.

Dawley, E. M. and W. J. Ebel. 1975. Effects of various concentrations of dissolved gas on juvenile chinook salmon and steelhead trout. U.S. National Marine Fisheries Service Fishery Bulletin 73:787-796.

Dell, M. B. and M. W. Erho. 1975. Occurrence of gas bubble disease symptoms on fish in mid-Columbia reservoirs. Grant, Douglas, and Chelan County Public Utility Districts. Ephrata and Wenatchee, Washington.

Dixon, W. J., M. B. Brown, L. Engleman, J. W. Frane, M. A. Hill, R. I. Jénnich, J. D. Toporek. 1983. BMDP statistical software. University of California Press. Berkely California, USA.

EPA (Environmental Protection Agency). 1976. Quality criteria for water. U. S. Environmental Protection Agency, Washington, D.C., USA.

Ebel, W. J. 1969. Supersaturation of nitrogen in the Columbia River and its effect on salmon and steelhead trout. Fishery Bulletin $68(1): 1-10$. 
Ebel, W. J. 1971. Dissolved nitrogen concentrations in the Columbia and snake Rivers in 1970 and their effect on chinook salmon and steelhead trout. NOAA Technical Report, National Marine Fisheries Service. SSRF 646.

Ebel W. J., E. M. Dawley, and B. H. Monk. 1971. Thermal tolerance of juvenile pacific salmon and steelhead trout in relation to supersaturation of nitrogen gas. Fishery Bulletin $69(4): 833-843$.

Ebel, W. J., R. W. Kroma, and H. L. Raymond. 1973. Evaluation of fish protective facilities at Little Goose Dam and review of other studies relating to protection of juvenile salmonids in the Columbia and Snake Rivers, 1973. National Marine Fisheries Service. Northwest Fisheries center, seattle, Washington.

Ebel, W. J., H. L. Raymond, G. E. Monan, W. E. Farr, and G. K. Tanonaka. 1975. Effect of atmospheric gas supersaturation caused by dams on salmon and steelhead trout of the snake and Columbia Rivers. National Marine Fisheries Service. Northwest Fisheries Center, Seattle, Washington.

Ebel, W. J. 1979. Effects of atmospheric gas supersaturation on survival of fish and evaluation of proposed solutions. 5th Progress Report, U.S. Army Corps of Engineers.

Ebel, W. J., K. T. Beningen, G. R. Bouck, W. R. Penrose, and D. E. Weitkamp. 1979. Gases, total dissolved. Pages 113-118 in R. V. Thurston, R. C. Russo, C. M. Fetterolf, T. A. Edsail, and Y. M. Barber, editors. A review of the EPA red book: quality criteria for water. Water Quality section American Fisheries Society, Bethesda, Maryland.

Evans, A. and D. N. Walder. 1969. Significance of gas micronuclei in the aetiology of decompression sickness. Nature (London) 222:251-252.

Fausch, K. D. 1984. profitable stream positions for salmonids: relating specific growth rate to net energy gain. Canadian Journal of Zoology 62:441-451.

Felley, J. D. and S. M. Felley. 1987. Relationships between habitat selection by individuals of a species and patterns of habitat segregation among species: fishes of the calcasieu drainage. Pages 61-68 in Matthews and Heins, editors. Community and evolutionary ecology of North America stream fishes. University of oklahoma Press, Norman, oklahoma, USA.

Fickeisen, D. H. and J. C. Montgomery. 1975. Dissolved gas supersaturation: bioassays of Kootenai River organisms. Batelle Pacific Northwest Laboratories, Richland, Washington, USA. 
Fickeisen, D. H. and M. J. Schneider, editors. 1976. Gas bubble disease. Conf-741033, Technical Information Center, Energy Research and Development Administration, Oak Ridge, Tennessee, USA.

Fidler, L. E. 1983. Design and analysis methods for hatchery aerations systems. Report to the Department of Fisheries and Oceans, Canada. Salmon Enhancement Program.

Fidler, L. E. 1985. A study of biophysical phenomena associated with gas bubble trauma in fish. M. S. Thesis, University of British Columbia, Vancouver, B.C. Canada.

Fidler, L. E. 1988. Gas bubble trauma in fish. Doctoral dissertation. University of British Columbia, Vancouver, canada.

Fredenberg, W. 1985. South-central Montana fisheries investigations, Bighorn Lake and Bighorn River postimpoundment study. Job Progress Report, Montana Department of Fish, Wildilfe and Parks, project No. F-20-R-29, Job IVa, Helena, Montana, USA.

Fredenberg, W. 1987. South-central Montana fisheries, Bighorn Lake and Bighorn River post-impoundment study. Job Progress Report, Montana Department of Fish, Wildlife, and Parks, Project No. F-20-R-31, Job IV-a, Helena, Montana, USA.

Garton, R. R. and R. V. Nebeker. 1977. A multiple approach to solving the gas supersaturation problem. Pages 5-19 in R.A. Tubb, editor. Recent advances in fish toxicology.

Environmental protection Agency Ecological Research series EPA-600/3-77-085.

Garvey, S., N. E. Cremer, and D. H. Sussdorf. 1977. Methods in immunology. W. A. Benjamin, Incorporated. New York, New York.

Gatz, A. J., Jr., M. J. Sale, and J.M. Loar. 1987, Habitat shifts in rainbow trout: competitive influences of brown trout. oecologia $74: 7-19$.

Gorham, F. P. 1901. The gas bubble disease of fish and its causes. Bulletin of the United States Fish Commission (1899) $19: 33-37$.

Gosse, J. C. and W. T. Helm. 1979. Effects of flow alterations on brown trout microhabitat in the Provo River. United States Bureau of Reclamation. Provo, Utah. Contract Number 8-07-40-S0729.

Gray, R. H. and J. M. Haynes. 1977. Depth distribution of adult chinook salmon (oncorhynchus tshawytscha) in relation to season and gas-supersaturated water. Transactions of the American Fisheries Society 106:617-620. 
Gray, R. H., T. L. Page, M. G. Saro, and P. Bronzi. 1982. Comparative tolerance to gas supersaturated water of carp (Cyprinus carpio), and black bullhead (Ictalurus melas), from the U.S.A. and Italy. Journal of Fish Biology 20:223227 .

Gray, R. H., M. F. Saroglia, and G. Scarano. 1985. Comparative tolerance to gas supersaturated water of two marine fishes, Dicentrarchus labrax and Mugil cephalus. Aquaculture 48:8389.

Grossman, G. D., A. de Sostoa, M. C. Freeman, and J. LobonCervia. 1987a. Microhabitat use in a Mediterranean riverine fish assemblage: fishes of the lower Matarrana. oecologia $73: 490-500$.

Grossman, G. D., A. de Sostoa, M. C. Freeman, and J. LobonCervia. 1987b. Microhabitat use in a Mediterranean riverine fish assemblage: fishes of the upper Matarrana. oecologia 73:501-512.

Hainsworth, F. R. 1981. Air respiration: Discontinuous respiration in insect pupae. Pages 69-71 in F. R. Hainsworth, editor. Animal physiology - Adaptations in function. Addison-Wesley Publishing Company. Reading Massachusetts, Menlo Park, California, London, Amsterdam, Don Mills, Ontario, Sydney.

Harcup, M. F., R. Williams, and D. M. Ellis. 1984. Movements of brown trout, Salmo trutta L., in the River Gwyddon, South Wales. Journal of Fish Biology 24:415-426.

Hart, L. G. and R. C. Summerfelt. 1975. Surgical procedures for implanting ultrasonic transmitters into flathead catfish (Pylodictis olivaris). Transactions of the American Fisheries society 104:56-59.

Harvey, E. N., D. K. Barnes, W. D. McElroy, A. H. Whitely, D. C. Pease, and K. W. Cooper. 1944. Bubble formation in animals. Journal of Cellular and Comparative Physiology $24: 1-24$.

Harvey, E. N. 1951. Physical factors in bubble formation in $F$. J. Fulton, editor. Decompression sickness. Saunders, Philadelphia.

Hatfield, C. T. and J. M. Anderson. 1972. Effects of two insecticides on the vulnerability of Atlantic salmon (Salmo salar) parr to brook trout (Salvelinus fontinalis) predation. Journal of the Fisheries Research Board of Canada 29:27-29.

Haynes, J. M. 1978. Movement and habitat studies of chinook salmon and white sturgeon. Battele, Pacific Northwest Laboratory, Richland, Washington, USA. 
Hearn, W. E. and B. E. Kynard. 1986. Habitat utilization and behavioral interaction of juvenile Atlantic salmon (Salmo salar) and rainbow trout (S. gairaneri) in tributaries of the White River of Vermont. Canadian Journal of Fisheries and Aquatic Sciences 43:1988-1998.

Heggberget, T. G. 1984. Effect of supersaturated water on fish in the River Nidelva, southern Norway. Joumal of Fish Biology $24: 65-74$.

Heming, T. A. 1984. The role of fish erythrocytes in transport and excretion of carbon dioxide. Doctoral dissertation. University of British Columbia, Vancouver, Canada.

Hertig, G. E., and W. Witt Jr. 1967. The role of physical fitness of forage fishes in relation to their vulnerability to predation by bowfin (Amia calva). Transactions of the American Fisheries Society $96: 427-430$.

Hesthagen, T. 1988. Movements of brown trout, Salmo trutta, and juvenile Atlantic salmon, Salmo salar, in a coastal stream in northern Norway. Journal of Fish Biology 32:639-653.

Hills, B. A. 1977. Decompression sickness. John Wiley \& Sons, New York.

Hintze, J. I. 1987. NCSSplus. 865 East 400 North, Kaysville, Utah, USA 84037 .

Hoffert, R. J. and P. O. Fromm. 1965. Biomicroscopic, gross, and microscopic observations of corneal lesions in the lake trout (Salvelinius namaycush). Journal of the Fisheries Research Board of Canada 22(3) $761-766$.

Holeton, G. F. and D. J. Randa11. 1967a. The effect of hypoxia upon the partial pressure of gases in the blood and water afferent and efferent to the gills of rainbow trout. Journal of Experimental Biology 46:317-327.

Holeton, G. F. and D.J. Randall. 1967b. Changes in blood pressure in the rainbow trout during hypoxia. Journal of Experimental Biology 46:297-305.

Hynes, H. B. N. 1970. The ecology of running waters. University of Toronto Press. Toronto.

Ide, F. F. 1935. Life history notes on Eohoron, Potamanthus, Lepthophlebia and Blasturus with descriptions

(Ephemeroptera) Canadian Entomologist 67:113-125.

Jenkins, T. M. 1969. Social structure, position choice and microdistribution of two trout species (Salmo trutta and Salmo gairaneri) resident in mountain streams. Animal Behaviour Monographs $2: 57-123$. 
Jensen, J. O. T. 1980. Effect of TGP and total water hardness in steelhead eggs and alevins. Pages 15-22 in Proceedings of the Northwest conference of Fish Culturists. Courtney, B.C., Canada.

Jensen, J. O. T., A. N. Halley, and J. Schnute. 1985. Literature data on salmonid response to gas supersaturation and ancillary factors. Canadian Data Report of Fisheries and Aquatic Sciences No. 501.

Jensen, J. O. T, J. Schnute, and D. F. Alderice. 1986. Assessing juvenile salmonid response to gas supersaturation using a general multivariate dose - response model. Canadian Journal of Fisheries and Aquatic Sciences 43:16941709 .

Johnson, D. W and I. Katavic. 1984. Mortality, growth and swimbladder stress syndrome of sea bass larvae under varied environmental conditions. Aquaculture 38:67-78.

Johnson, R. C. and E. M. Dawley. 1974. The effect of spill way flow deflectors at Bonneville Dam on the total gas supersaturation and survival of juvenile salmon. National Marine Fisheries Service. Seattle, Washington, USA. Cited In Weitkamp and Katz (1980), page 673.

Kaeding, L. R. and C. M. Kaya. 1978. Growth and diets of trout from contrasting environments in a geothermaliy heated stream: the Firehole River of Yellowstone National Park. Transactions of the American Fisheries Society 107:432-438.

Kania, H. J. and J. O'Hara. 1974. Behavioral alterations in a simple predator-prey system due to sublethal exposure to mercury. Transactions of the American Fisheries Society $103: 134-136$.

Kirsch, R. and G. Nonnote. 1977. Cutaneous respiration in three freshwater teleosts. Respiratory Physiology 29:339-354.

Knittel, M. D., G. A. Chapman, and R. R. Garton. 1980. Effects of hydrostatic pressure on steelhead survival in air supersaturated water. Transactions of the American Fisheries Society 109:755-759.

Kolbeinshavn A., and J. C. Wallace. 1985. Observations on swimbladder stress syndrome in arctic charr (Salvelinius alpinus) induced by inadequate water depth. Aquaculture $6: 259-261$.

Kral, S. 1983. Results and hypothesis for the propagation of the grey mullet. Aquaculture 30:273-284.

Leopold, L. B., M. G. Wolman, and J. P. Miller. 1964. Fluvial processes in geomorphology. W. H. Freeman and Company, San Francisco, USA. 
Lewis, S. I. 1969. Physical factors influencing fish populations in pools of a trout stream. Transactions of the American Fisheries Society $98: 14-19$.

Lieberman, G. J., R. G. Millex, Jr., and M. A. Hamilton. 1967. Unlimited simultaneous discrimination intervals in regression. Biometrika 54:133-145.

Lund, M., and T. G. Heggberget. 1985. Avoldance response of two-year-old rainbow trout, salmo gairdneri Richardson, to air-supersaturated water: hydrostatic compensation. Journal of Fish Biology 26:193-200.

Machado, J. P., D. L. Garling Ir., N. R. Kevern, A. L. Trapp, and T. G. Bell. 1987. Gas bubble disease: a histopathologic study of the pathogenesis of embolism in rainbow trout. Canadian Journal of Fisheries and Aquatic Science 44:19851994.

Malouf, R., R. Keck, D. Maurer, and C. Epifanio. 1972. occurrence of gas-bubble disease in three species of bivalve molloscs. Journal of the Fisheries Research Board of Canada $29: 588-589$.

Mardia, K. V., J. T. Kent, and J. M. Bibby. 1979. Multivariate analysis. Academic Press, New York, New York, USA.

Marsh, M. C. and F. P. Gorham. 1905. The gas disease in fishes. Report of the United States Bureau of Fisheries 1904:343376.

Meekin, T. K. and B. K. Turner. 1974. Tolerance of salmonid eggs, juveniles and squawish to supersaturated nitrogen. Washington Depaxtment of Fisheries Technical Report 12:78126.

Meisel, S., A. Nir, and D. Kerem. 1981. Bubble dynamics in perfused tissue undergoing decompression. Respiratory Physiology 43:89-98.

Merritt, $R$. W. and $K$. W. Cummins. 1984. An introduction to the aquatic insects of North America. (2nd ed.). Kendall/Hunt Publishing Co. USA.

Mott, J. C. 1957. The cardiovascular system. Pages 81-108 in M. E. Brown, editor. The physiology of fishes. Academic Press, New York.

Nebeker, A. V. 1976. Survival of Daphnia, crayfish and stoneflies in air - supersaturated water. Journal of the Fisheries Research Board of Canada 33:1208-1212.

Nebeker, A. V., D. G. Stevens, and R. K. Stroud. 1976. Effects of air supersaturated water on adult sockeye salmon.

Journal of Fisheries Research Board of Canada $33: 2629-2633$. 
Nebeker, A. V. and J. R. Brett. 1976. Effects of gassupersaturated water on survival of Pacific salmon and steelhead smolts. Transactions of the American Fisheries Society 105:338-342.

Nebeker, A. V., J. D. Andros, J. K. McCrady, and D. G. Stevens. 1978. Survival of steelhead trout (Salmo gairdneri) eggs, embryos, and fry in air-supersaturated water. Journal of the Fisheries Research Board of Canada 35:261-264.

Nebeker, A. V., A. K. Hauck, and R. J. Baker. 1979. Temperature and oxygen-nitrogen gas ratios affecting fish survival in air supersaturated water. Water Resources 13:299-303.

Nebeker, A. V., A. K. Hauck, F. D. Baker, and S. L. Weitz. 1980. Comparative responses of speckled dace and cutthroat trout to air-supersaturated water. Transactions of the American Fisheries society 109:760-764.

Nikolskii, G. A. 1963. The ecology of fishes. Academic Press, New York, USA.

Nilsson, N. A. 1967. Interactive segregation between fish species. Pages 295 - 313 in S. D. Gerking, editor. The biological basis of freshwater fish production. Blackwell Scientific Publications, oxford, England.

Oswald, R. A. 1986. Southwestern Montana fisheries study, Investigation of the influence of Clark Canyon Reservoir on the stream fishery of the Beaverhead River. Job Progress Report, Montana Department of Fish, wildlife, and Parks, Project No. F-9-R-34, Job II-a, Helena, Montana, USA.

Pennak, R. W. 1978. Freshwater invertebrates of the United States (2nd ed.). John Wiley \& Sons, N.Y.

Piiper, J. and P. Scheid. 1984. Model analysis of gas transfer in fish gills. Pages 230-262 in W.S. Hoar and D.J. Randall, editors. Fish physiology, Volume X Part A. Academic Press, New York.

Piper, R. G., I. B. McElwain, L. E. Orme, J. P. Mccraren, L. G. Fowler and J. R. Leonard. 1982. Fish hatchery management. USDI, Fish and Wildlife Service, Washington, D.C., USA.

Porter, T. R. 1973. Fry emergence trap and holding box. Progressive Fish Culturist 35:104-106.

Porter, J. F. and T. J. Viel. 1980. Progress Report: Bighorn River nitrogen supersaturation study, July - September 1980. USDI, Fish and Wildlife Service, Hardin, Montana, USA.

Post, G. W. 1983. Text book of fish health. TFH Publications Incorporated, LTD. Neptune, New Jersey. 
Poston, H. A., D. L. Livingston, and D. G. Hedrick. 1973. Effect of method of introducing well water on the growth, body chemistry, and incidence of eye abnormalities of juvenile brown and rainbow trout. Progressive Fish culturist $35: 187-190$.

Raleigh, R. F., T. Hickman, R. C. Solomon, and P. C. Nelson. 1984. Habitat suitability index models and instream flow suitability curves: rainbow trout. United states Fish and Wildlife service. FWS/OBS $-82 / 10.60$.

Raleigh, R. F., L. D. Zuckerman, and P. C. Nelson. 1986. Habitat suitability index models and instream flow suitability curves: brown trout. United States Fish and Wildlife service. Biological Report 82(10.124).

Randall, D. J. 1970. Gas exchange in fish. Pages 253-292 in W.S. Hoar and D.J. Randall, editors. Fish physiology, volume IV. Academic Press, New York.

Randal1, D. J., and C. D. Daxboeck. 1984. Oxygen and carbon dioxide transfer across fish gills. Pages 263-314 in W.S. Hoar and D.J. Randall, editors. Fish Physiology, volume XA. Academic Press, New York.

Reid, R. C., J. M. Prausnitz, and T. K. Sherwood. 1977. The properties of gases and liquids, 3rd edition. McGraw Hill Company, New York.

Renfro, W. C. 1963. Gas bubble mortality of fishes in Galveston Bay, Texas. Transactions of the American Fisheries Society $92: 664-671$.

Ricker, W. E. 1975. Computation and interpretation of biological statistics of fish populations. Bulletin 191, Department of the Environment, Fisheries and Marine Service, ottawa, Canada.

Ross, S. T., J. A. Baker, and K. E. Clark. 1987. Microhabitat partitioning of southeastern stream fishes: temporal and spatial predictability. Pages 42-51 in Matthews and Heins, editors. Community and evolutionary ecology of North America stream fishes. University of oklahoma Press, Norman, Oklahoma, USA.

Rucker, R. R. and K. Hodgeboom. 1953. Observations on gas bubble disease of fish. Progressive Fish Culturist 15:2426.

Rucker, R. R. 1974. Effect of nitrogen supersaturated water on coho and chinook salmon. Progressive Fish Culturist 36:152159 . 
Rucker, R. R. 1975a. Gas bubble disease mortalities of coho salmon in water with constant total gas pressure and different oxygen-nitrogen ratios. U.S. National Marine Fisheries Service Fishery Bulletin 73:915-918.

Rucker, R. R. 1975b. Excess nitrogen gas in water not a cause of coagulated yolk disease in chinook salmon. Progressive Fish Culturist $37: 101-102$.

Rucker, R. R. 1976. Gas bubble disease of salmonids: variation in oxygen-nitrogen ratio with constant total gas pressure. Pages 85-89 in D.H. Fickeisen and M.J. Schneider, editors. Gas bubble disease. Technical Information center, office of Public Affairs, Energy Research and Development Administration.

Sanborn, B. W. 1990. The ecology of rainbow trout in the Bighorn River, Montana. M.S. Thesis, Montana State University, Bozeman.

Schiewe, M. H. 1974. Influence of dissolved atmospheric gas on swimming performance of juvenile chinook salmon.

Transactions of the American Fisheries Society 4:717-721.

Schnute, J. and M. McKinnell. 1984. A biological meaningful approach to response surface analysis. Canadian Journal of Fisheries and Aquatic Sciences 41:936-953.

Scott, W. B. and E. J. Crossman. 1973. Freshwater fishes of Canada. Fisheries Research Board of Canada. Bulletin 184.

Shirahata, S. 1966. Experiments on nitrogen gas disease with rainbow trout fry. Bulletin of the Freshwater Research Laboratory, Tokyo 15:197-211.

Shirvell, C. S. and R. G. Dungey. 1983. Microhabitats chosen by brown trout for feeding and spawning in rivers.

Transactions of the American Fisheries Society 112:355-367.

Shrimpton, M. J., D. J. Randall, and L. E. Fidler. (manuscript) Factors affecting swimbladder volume in rainbow trout (oncorhynchus mykiss) held in gas supersaturated water. University of British Columbia, Vancouver, Canada.

Shrimpton, M. J., D. J. Randall, and L. E. Fidler. (manuscript) Assessing the effects of positive buoyancy on rainbow trout (Oncorhynchus mykiss) held in gas supersaturated water. University of British Columbia, Vancouver, Canada.

Smith, C. G., W. M. Lewis, and H. M. Kaplan. 1952. Comparative morphologic and physiologic study of fish blood. Progressive Fish Culturist 14:169-172. 
Smith, C. E. 1988. Histopathology of gas bubble disease in juvenile rainbow trout. U.S. Fish and wildlife service. Fish Technology Center, Bozeman, Montana.

Snieszko, S. F. 1974. The effects of environmental stress on outbreaks of infectious diseases of fishes. Journal of Fish Biology $6: 197-208$.

Soltero, R. A. 1971. Limnological studies on Bighorn Lake (Yellowtail Dam) and its tributaries. Doctorate thesis, Montana State University. Bozeman, MT.

Sovio, A., K. Westerman and K. Nyholm. 1972. Improved methods of dorsal aorta catheterization: Haematological effects followed for three weeks in rainbow trout, salmo gairdneri. Finnish Fisheries Research 1:11-12.

Sovio, A, M. Nikinmaa, K. Nyholm, and K. Westman. 1981. The role of gills in the responses of (Salmo gairdneri) during moderate hypoxia. Comparative Biochemistry and Physiology 70A: 133-139.

Spence, J. A. and H. B. N. Hynes. 1971. Differences in benthos upstream and downstream of an impoundment. Journal of Fisheries Research Board Canada 28:35-43.

Sprent, P. 1981. Quick statistics: an introduction to nonparametric methods. Penguin Books, New York, New York, USA.

steen, J. B. 1970. The swim bladder as a hydrostatic organ. pages $414-443$ in W.S.Hoar and D.J. Randall, editors. Fish Physiology, volume IV. Academic Press, New York.

Stevens, D. G., A. V. Nebeker, and R. J. Baker. 1980. Avoidance responses of salmon and trout to air-supersaturated water. Transactions of the American Fisheries Society 109:751-754.

Stevenson, H. R. 1975. The trout fishery of the Bighorn River below Yellowtail dam, Montana. M.S. Thesis, Montana state University, Bozeman.

Stickney, A. P. 1968. Supersaturation of atmospheric gases in coastal waters of the Gulf of Maine. United States Fish and Wildlife Service Fishery Bulletin 67:117-123.

Stroud, R. K., G. R. Bouck, and A. V. Nebeker. 1975. Pathology of acute' and chronic exposure of salmonid fishes to supersaturated water. pages 435-489 in W.A. Adams, editor. Chemistry and physics of aqueous gas solutions. The Electrochemical Society, Princeton, New Jersey. 
Stroud, F. K. and A. V. Nebeker. 1976. A study of the pathogenesis of gas bubble disease in steelhead trout (Salmo gairdneri). Pages 66-71 in D.H. Fickeisen and M.J. Schneider, editors. Gas bubble disease. Technical Information Center, Office of Public Affairs, Energy Research and Development Administration.

Swedberg, S. 1973. South-central Montana fisheries study, Bighorn Lake, Bighorn River and Bighorn River postimpoundment study. Job Progress Report, Montana Department of Fish, Wildlife and Parks, Project F-20-R-18, Job IV-a, Helena, Montana, USA.

Sylvester, J.R. 1972. Effect of thermal stress on predator avoidance in sockeye salmon. Journal Fisheries Research Board of Canada 29:601-603.

Thomas, S. and G. M. Hughes. 1982. A study of the effects of hypoxia on acid-base status of rainbow trout blood using an extracorporeal blood circulation. Respiratory Physiology $49: 371-382$.

Thorn, W. C., C. Lessman, and R. Glazer. 1978. Some effects of controlied levels of dissolved gas supersaturation on selected salmonids and other fishes. Minnesota Department of Natural Resources, Division of Fish, wildlife, Section of Fisheries Investigational Report Number 307.

Thorpe, J. E. and R. J. Roberts. 1972. An aeromonad epidemic in the brown trout (Salmo trutta L.). Journal of Fish Biology $4: 441-451$.

Trust, T. J. and R. A. H sparrow. 1974. The bacterial flora in the alimentary tract of freshwater salmonid fishes. Canadian Journal of Microbiology 20:1219-1228.

Trust, T. J. 1986. Pathogenesis of infectious diseases of fish. Annual Review of Microbiology 40:479-502.

Vann, R. D. and H. G. Clark. 1975. Bubble growth and mechanical properties of tissue in decompression. Undersea Biomedical Research 2:185-194.

Vincent, E. R. 1971. River electrofishing and fish population estimates. Progressive Fish Culturist 33:163-167.

Vincent, E. R. 1974. Addendum to river electrofishing and fish population estimates. Progressive Fish Culturist $36: 182$.

WPRS. 1980. Yellowtail Afterbay powerplant appraisal report. USDI, Water and Power Resources Service, Upper Missouri Region, Billings, Montana, USA.

Ward, J. V. and R. A. Short. 1978. Macroinvertebrate community structure of four special lotic habitats in Colorado, U.S.A. verh. Int. Ver. Limnol. 20:1382-1387. 
Ward, J. V. 1976. Effects of thermal constancy and seasonal temperature displacement on community structure of stream macroinvertebrates. Pages 302-307 in G. W. Esch and R. W. Mcfarlane, editors. Thermal ecology II. ERDA Symposium Series (Conf. 750425).

Ward, J. V. and J. A. Stanford. 1979. Ecological factors controlling stream zoobenthos with emphasis on thermal modification of regulated streams. pages 35-55 in J.V. Ward and J. A. Stanford, editors. The ecology of regulated streams. Plenum Press, New York and London.

Ware, D. M. 1971. Predation by rainbow trout (Salmo gairdneri): the effect of experience. Journal of the Fisheries Research Board of Canada 28:1847-1852.

Waters, T. F. and R. J. Knapp. 1961. An improved stream bottom fauna sampler. Transactions of the American Fisheries society $90: 225-226$.

Weitkamp, D. E. 1974. Dissolved gas supersaturation in the Columbia River system: salmonid bioassay and depth distribution studies, 1973 and 1974. Parametrix, Inc. Report to Utility Cooperative, Idaho Power Company, Boise.

Weitkamp, D. E. 1976. Dissolved gas supersaturation Iive cage bioassays at Rock Island Dam, Washington. Pages 24-36 in D. H. Fickeisen and M. J. Schneider, editors. Gas bubble disease. Technical Information center, office of Public Affairs, Energy Research and Development Administration.

Weitkamp, D. E. and M. Katz. 1980. A review of dissolved gas supersaturation iterature. Transactions of the American Fisheries Society 109:659-702.

Werner, E. E., G. G. Mittlebach, and D. J. Hall. 1981. The role of foraging profitability and experience in habitat use by the bluegill sunfish. Ecology 62:116-125.

White, R. G., G. Phillips, G. Liknes, and S. Sanford. 1986. The effects of supersaturation of dissolved gases on the fishery of the Bighorn River downstream of the Yellowtail Afterbay Dam. Montana Cooperative Rishery Research Unit and Montana Department of Fish, Wildlife, and Parks, Bozeman, Montana.

White, R. G., Phillips, G., Liknes, G., C. Sprague, J. Brammer, W. Connor, L. Fidler, T. Williams, and W. P. Dwyer. 1987. The effects of supersaturation of dissolved gases on the fishery of Bighorn River downstream of the Yellowtail Afterbay Dam. Montana Cooperative Fishery Research Unit and Montana Department of Fish Wildife and Parks, Bozeman, Montana. 
White, R. G., G. Phillips, G. Liknes, C. Sprague, J. Brammer, W. Connor, L. Fidler, T. Williams, and W. P. Dwyer. 1988. The effects of supersaturation of dissolved gases on the fishery of the Bighorn river downstream of the Yellowtail Afterbay Dam. Montana Cooperative Rishery Research Unit and Montana Department of Fish, Wildlife, and Parks, Bozeman, Montana.

Wilkinson, V. 1983. Chlorophyll a analysis - guidelines and procedures for extraction, measurement and calculation of chlorophyll a concentration. Taupo Research Laboratory, Taupo, New Zealand.

Winter, J. D., V. B. Kuechle, D. B. Siniff, J. R. Tester. 1978. Equipment and methods for radio tracking freshwater fish. Agricultural Experiment Station, University of Minnesota, Miscellaneous Report 152-1978.

Wood, C. M. and E. B. Jackson. 1980. Blood acid base regulation during environmental hypoxia in the rainbow trout, (Salmo gairaneri). Respiratory Physiology 42:351-372.

Wood, C. M., M. G. Wheatly, and H. Hobe. 1984. The mechanisms of acid base and ion regulation in the freshwater rainbow trout during environmental hyperoxia and subsequent normoxia. III. Branchial exchanges. Respiratory Physiology $55: 175-192$.

Wright, J. C. and R. A. Soltero. 1973. Limnology of Yellowtail Reservoir and the Bighorn River. U.S. Environmental Protection Agency. Report NO. EPA-R3-73-002. Washington D.C., USA.

Yang, W. J. and C. Y. Liang. 1972. Dynamics of the dissolution of gas bubbles or pockets in tissue. Journal of Biomechanics $5: 331-332$.

Yount, J. L. 1956. Factors that control species numbers in Silver Springs, Florida. Limnology Oceanography 1:286-295.

Zar, J. H. 1984. Biostatistical analysis. Second edition. Prentice-Hall, Incorporated, Englewood Cliff, New Jersey, USA.

Zimmerman, H. J. and J. V. Ward. 1984. A survey of regulated streams in the Rocky Mountains of Colorado, U.S.A. Pages 251-262 in A. Lillehammer and S. J. Saltveit, editors. Regulated rivers. Columbia University Press, New York. USA. 
APPENDIX A

Afterbay Gates 

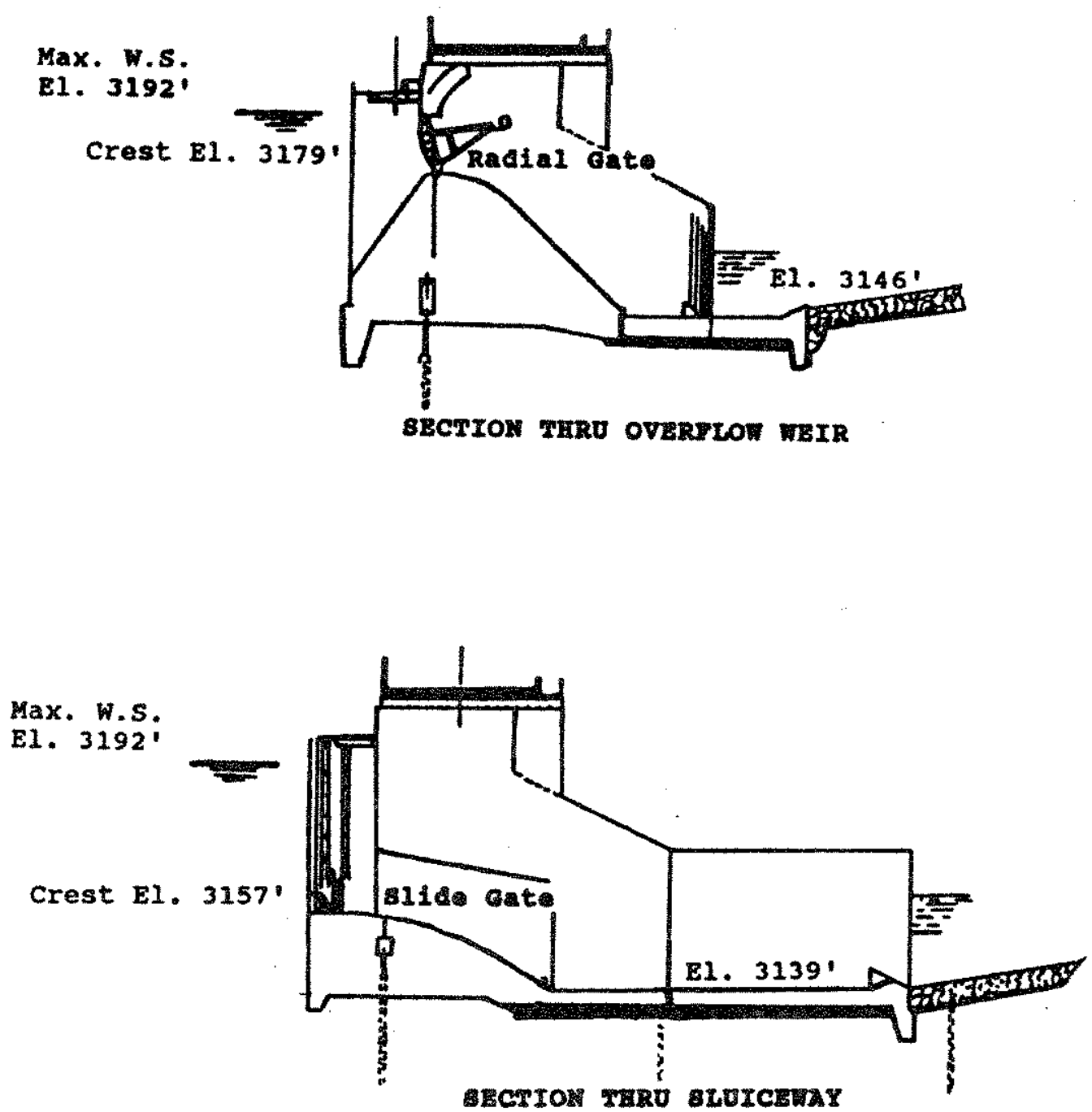

Figure 1. Cross-sectional schematic of overflow weir with radial gate position and sluiceway showing slide gates (BOR). 
APPENDIX B

Bighorn River water quality 
Table 1. Water quality parameters measured from each water sample 17 May 1985 - 1 June 1988 near the gagehouse (Rkm 0.6) below Afterbay Dam on the Bighorn River.

\begin{tabular}{|c|c|c|c|c|}
\hline $\begin{array}{l}\text { Date } \\
\text { sampled }\end{array}$ & $\mathrm{pH}$ & $\begin{array}{c}\text { Conductivity } \\
\text { (microomhos } / \mathrm{cm})\end{array}$ & $\begin{array}{l}\text { Total dissolved } \\
\text { solids }(\mathrm{mg} / \mathrm{l})\end{array}$ & $\begin{array}{l}\text { Sodium adsorption } \\
\text { ratio (SAR) }\end{array}$ \\
\hline $05 / 17 / 85$ & 8.2 & 867 & 608 & - \\
\hline $06 / 03 / 85$ & 7.7 & 944 & 590 & 1.96 \\
\hline $06 / 18 / 85$ & 8.0 & 622 & 436 & 1.91 \\
\hline $07 / 18 / 85$ & 7.5 & 755 & 534 & 1.75 \\
\hline $08 / 01 / 85$ & 7.8 & 866 & 544 & 1.81 \\
\hline $08 / 16 / 85$ & 8.2 & 810 & 570 & 1.96 \\
\hline $09 / 12 / 85$ & 7.6 & 743 & 488 & 1.90 \\
\hline $09 / 18 / 85$ & 6.7 & 759 & 544 & 1.81 \\
\hline $10 / 03 / 85$ & 7.7 & 890 & 642 & 2.31 \\
\hline $10 / 22 / 85$ & 7.7 & 966 & 690 & 2.29 \\
\hline $11 / 03 / 85$ & 7.9 & 1081 & 720 & 2.37 \\
\hline $12 / 02 / 85$ & 7.9 & 944 & 672 & 2.13 \\
\hline $01 / 02 / 86$ & 7.8 & 1040 & 784 & 2.30 \\
\hline $01 / 16 / 86$ & 7.7 & 1040 & 752 & 2.26 \\
\hline $02 / 02 / 86$ & 7.8 & 919 & 682 & 2.17 \\
\hline $02 / 26 / 86$ & 8.1 & 936 & 696 & 2.18 \\
\hline $04 / 05 / 86$ & 8.0 & 1020 & 756 & 2.02 \\
\hline $05 / 02 / 86$ & 8.0 & 1010 & 706 & 2.09 \\
\hline $06 / 04 / 86$ & 8.1 & 947 & 705 & 2.10 \\
\hline $07 / 07 / 86$ & 8.1 & 699 & 484 & 1.82 \\
\hline $08 / 01 / 86$ & 7.9 & 523 & 344 & 1.53 \\
\hline $09 / 03 / 86$ & 7.8 & 475 & 360 & 1.23 \\
\hline $10 / 01 / 86$ & 7.9 & 682 & 516 & 1.65 \\
\hline $11 / 06 / 86$ & 8.1 & 707 & 508 & 1.75 \\
\hline $12 / 01 / 86$ & 8.1 & 752 & 524 & 1.76 \\
\hline $01 / 05 / 87$ & 8.2 & 662 & 484 & 1.62 \\
\hline $02 / 02 / 87$ & 8.2 & 617 & 472 & 1.64 \\
\hline $03 / 02 / 87$ & 8.2 & 769 & 528 & 1.57 \\
\hline $04 / 02 / 87$ & 8.1 & 641 & 544 & 1.77 \\
\hline $05 / 01 / 87$ & 8.1 & 786 & 556 & 1.90 \\
\hline $06 / 01 / 87$ & 8.1 & 769 & 544 & 1.86 \\
\hline $07 / 01 / 87$ & 7.9 & 649 & 452 & 1.90 \\
\hline $08 / 03 / 87$ & 8.0 & 590 & 644 & 1.94 \\
\hline $09 / 08 / 87$ & 8.0 & 661 & 524 & 1.91 \\
\hline $10 / 02 / 87$ & 8.0 & 866 & 700 & 2.25 \\
\hline $11 / 01 / 87$ & 8.3 & 895 & 512 & 1.94 \\
\hline $12 / 01 / 87$ & 7.9 & 937 & 624 & 2.17 \\
\hline $01 / 04 / 88$ & - & - & - & - \\
\hline $02 / 01 / 88$ & 8.4 & 930 & - & - \\
\hline $03 / 01 / 88$ & 8.2 & 945 & - & - \\
\hline $04 / 01 / 88$ & 8.3 & 977 & - & - \\
\hline $05 / 03 / 88$ & 8.3 & 400 & - & - \\
\hline $06 / 01 / 88$ & 8.1 & 998 & - & - \\
\hline
\end{tabular}


Table 1. (continued).

\begin{tabular}{|c|c|c|c|c|c|}
\hline $\begin{array}{l}\text { Date } \\
\text { sampled }\end{array}$ & $\begin{array}{l}\text { Total hardness } \\
\left(\mathrm{mg} / 1 \text { as } \mathrm{CaCO}_{3}\right)\end{array}$ & $\begin{array}{l}\text { Calcium } \\
(\mathrm{mg} / \mathrm{l})\end{array}$ & $\begin{array}{l}\text { Magnesium } \\
(\mathrm{mg} / \mathrm{l})\end{array}$ & $\begin{array}{l}\text { Sodium } \\
(\mathrm{mg} / \mathrm{l})\end{array}$ & $\begin{array}{c}\text { Potassium } \\
(\mathrm{mg} / \mathrm{l})\end{array}$ \\
\hline $05 / 17 / 85$ & 307 & 80 & 26 & 88 & 4 \\
\hline $06 / 03 / 85$ & 264 & 63 & 26 & 73 & $<5$ \\
\hline $06 / 18 / 85$ & 261 & 60 & 27 & 71 & $<5$ \\
\hline $07 / 18 / 85$ & 294 & 75 & 26 & 69 & $<5$ \\
\hline $08 / 01 / 85$ & 258 & 62 & 25 & 67 & 7 \\
\hline $08 / 16 / 85$ & 248 & 58 & 25 & 71 & $<1$ \\
\hline $09 / 12 / 85$ & 272 & 66 & 26 & 72 & $<5$ \\
\hline $09 / 18 / 85$ & 299 & 77 & 26 & 72 & $<5$ \\
\hline $10 / 03 / 85$ & 276 & 66 & 27 & 88 & 5 \\
\hline $10 / 22 / 85$ & 320 & 77 & 31 & 94 & 3 \\
\hline $11 / 03 / 85$ & 312 & 74 & 31 & 96 & 3 \\
\hline $12 / 02 / 85$ & 295 & 72 & 28 & 84 & 2 \\
\hline $01 / 02 / 86$ & 288 & 66 & 30 & 90 & 3 \\
\hline $01 / 16 / 86$ & 293 & 68 & 30 & 89 & 3 \\
\hline $02 / 02 / 86$ & 334 & 76 & 35 & 91 & 4 \\
\hline $02 / 26 / 86$ & 330 & 81 & 31 & 91 & 5 \\
\hline $04 / 05 / 86$ & 369 & 95 & 32 & 89 & 4 \\
\hline $05 / 02 / 86$ & 390 & 100 & 34 & 95 & 5 \\
\hline $06 / 04 / 86$ & 346 & 89 & 30 & 90 & 4 \\
\hline $07 / 07 / 86$ & 220 & 57 & 19 & 62 & 4 \\
\hline $08 / 01 / 86$ & 193 & 51 & 16 & 49 & 2 \\
\hline $09 / 03 / 86$ & 173 & 46 & 14 & 37 & 1 \\
\hline $10 / 01 / 86$ & 234 & 59 & 21 & 58 & 1 \\
\hline $11 / 06 / 86$ & 251 & 66 & 21 & 64 & 3 \\
\hline $12 / 01 / 86$ & 260 & 68 & 22 & 65 & 3 \\
\hline $01 / 05 / 87$ & 268 & 71 & 22 & 61 & 3 \\
\hline $02 / 02 / 87$ & 247 & 66 & 20 & 59 & 2 \\
\hline $03 / 02 / 87$ & 287 & 77 & 23 & 61 & 4 \\
\hline $04 / 02 / 87$ & 279 & 72 & 24 & 68 & 4 \\
\hline $05 / 01 / 87$ & 311 & 80 & 27 & 77 & 4 \\
\hline $06 / 01 / 87$ & 292 & 74 & 26 & 73 & 3 \\
\hline $07 / 01 / 87$ & 209 & 49 & 21 & 63 & 3 \\
\hline $08 / 03 / 87$ & 283 & 69 & 27 & 75 & 4 \\
\hline $09 / 08 / 87$ & 276 & 71 & 24 & 73 & 3 \\
\hline $10 / 02 / 87$ & 316 & 82 & 27 & 92 & 4 \\
\hline $11 / 01 / 87$ & 304 & 79 & 26 & 87 & 4 \\
\hline $12 / 01 / 87$ & 332 & 85 & 29 & 91 & 4 \\
\hline $01 / 04 / 88$ & 324 & 83 & 28 & 88 & 3 \\
\hline $02 / 01 / 88$ & 334 & 85 & 29 & 86 & 3 \\
\hline $03 / 01 / 88$ & 313 & 75 & 30 & 95 & 4 \\
\hline $04 / 01 / 88$ & 340 & 86 & 30 & 91 & 4 \\
\hline $05 / 03 / 88$ & 357 & 91 & 31 & 93 & 4 \\
\hline $06 / 01 / 88$ & 342 & 85 & 31 & 93 & 5 \\
\hline
\end{tabular}


Table 1. (continued).

\begin{tabular}{|c|c|c|c|c|}
\hline $\begin{array}{c}\text { Date } \\
\text { sampled }\end{array}$ & $\begin{array}{c}\text { Total } \\
\text { alkalinity } \\
\left(\mathrm{mg} / \mathrm{l} \text { as } \mathrm{CaCO}_{3}\right)\end{array}$ & $\begin{array}{c}\text { Bicarbonate } \\
\text { alkalinity } \\
\left(\mathrm{mg} / 1 \text { as } \mathrm{CaCO}_{3}\right)\end{array}$ & $\begin{array}{c}\text { Chloride } \\
(\mathrm{mg} / \mathrm{l})\end{array}$ & $\begin{array}{c}\text { Fluoride } \\
(\mathrm{mg} / 1)\end{array}$ \\
\hline $05 / 17 / 85$ & 170 & 208 & 12 & - \\
\hline $06 / 03 / 85$ & 122 & 149 & 12 & 0.5 \\
\hline $06 / 18 / 85$ & 124 & 151 & 11 & 0.5 \\
\hline $07 / 18 / 85$ & 140 & 171 & 14 & 0.5 \\
\hline $08 / 01 / 85$ & 136 & 166 & 9 & 0.5 \\
\hline $08 / 16 / 85$ & 126 & 154 & 10 & 0.5 \\
\hline $09 / 12 / 85$ & 136 & 166 & 19 & $<0.1$ \\
\hline $09 / 18 / 85$ & 159 & 194 & 22 & $<0.1$ \\
\hline $10 / 03 / 85$ & 136 & 166 & 8 & 0.4 \\
\hline $10 / 22 / 85$ & 150 & 183 & 9 & 0.4 \\
\hline $11 / 03 / 85$ & 145 & 177 & 9 & 0.4 \\
\hline $12 / 02 / 85$ & 154 & 188 & 9 & 0.4 \\
\hline $01 / 02 / 86$ & 146 & 178 & 13 & 0.57 \\
\hline $01 / 16 / 86$ & 157 & 192 & 13 & 0.54 \\
\hline $02 / 02 / 86$ & 147 & 179 & 12 & 0.6 \\
\hline $02 / 26 / 86$ & 176 & 215 & 13 & 0.51 \\
\hline $04 / 05 / 86$ & 195 & 238 & 14 & 0.48 \\
\hline $05 / 02 / 86$ & 194 & 237 & 14 & 0.55 \\
\hline $06 / 04 / 86$ & 198 & 242 & 12 & 0.58 \\
\hline $07 / 07 / 86$ & 135 & 165 & 9 & 0.27 \\
\hline $08 / 01 / 86$ & 114 & 139 & 8 & 0.3 \\
\hline $09 / 03 / 86$ & 141 & 172 & $<1$ & 0.32 \\
\hline $10 / 01 / 86$ & 146 & 178 & 6 & 0.36 \\
\hline $11 / 06 / 86$ & 146 & 178 & 7 & 0.4 \\
\hline $12 / 01 / 86$ & 147 & 179 & 7 & 0.4 \\
\hline $01 / 05 / 87$ & 147 & 179 & 9 & 0.35 \\
\hline $02 / 02 / 87$ & 147 & 179 & 7 & 0.45 \\
\hline $03 / 02 / 87$ & 154 & 188 & 8 & 0.36 \\
\hline $04 / 02 / 87$ & 164 & 200 & 10 & 0.45 \\
\hline $05 / 03 / 87$ & 165 & 201 & 11 & 0.58 \\
\hline $06 / 01 / 87$ & 167 & 204 & 13 & 0.54 \\
\hline $07 / 01 / 87$ & 80 & 98 & 12 & 0.46 \\
\hline $08 / 03 / 87$ & 155 & 189 & 10 & 0.4 \\
\hline $09 / 08 / 87$ & 148 & 181 & 8 & 0.3 \\
\hline $10 / 02 / 87$ & 161 & 196 & 11 & 0.45 \\
\hline $11 / 01 / 87$ & 172 & 210 & 12 & 0.41 \\
\hline $12 / 01 / 87$ & 172 & 210 & 13 & 0.5 \\
\hline $01 / 04 / 88$ & 174 & 212 & 13 & 0.45 \\
\hline $02 / 01 / 88$ & 196 & 225 & 14 & 0.47 \\
\hline $03 / 01 / 88$ & 188 & 229 & 11 & 0.47 \\
\hline $04 / 01 / 88$ & 191 & 233 & 12 & 0.51 \\
\hline $05 / 01 / 88$ & 197 & 241 & 14 & 0.49 \\
\hline $06 / 01 / 88$ & 205 & 251 & 14 & 0.45 \\
\hline
\end{tabular}


Table 1. (continued).

\begin{tabular}{|c|c|c|c|c|c|}
\hline $\begin{array}{c}\text { Date } \\
\text { sampled }\end{array}$ & $\begin{array}{l}\text { Nitrate \& } \\
\text { Nitrite } \\
(\mathrm{mg} / \mathrm{l})\end{array}$ & $\begin{array}{c}\text { Sulfate } \\
(\mathrm{mg} / 1)\end{array}$ & $\begin{array}{l}\text { Total } \\
\text { phosphorus } \\
\text { as } \mathrm{P}(\mathrm{mg} / \mathrm{l})\end{array}$ & $\begin{array}{c}\text { Ortho- } \\
\text { phosphate as } \\
\text { p (mg/l) }\end{array}$ & $\begin{array}{c}\text { Ammonia } \\
\text { Nitrogen as } \\
\mathrm{N}(\mathrm{mg} / \mathrm{l})\end{array}$ \\
\hline $05 / 17 / 85$ & - & 281 & - & - & - \\
\hline $06 / 03 / 85$ & 0.30 & 295 & 0.02 & 0.01 & $<0.2$ \\
\hline $06 / 18 / 85$ & 0.32 & 285 & 0.01 & $<0.01$ & $<0.2$ \\
\hline $07 / 18 / 85$ & 0.33 & 263 & 0.02 & $<0.01$ & $<0.2$ \\
\hline $08 / 01 / 85$ & $<0.05$ & 261 & 0.01 & $<0.01$ & $<0.2$ \\
\hline $08 / 16 / 85$ & 0.39 & 253 & 0.04 & 0.02 & $<0.2$ \\
\hline $09 / 12 / 85$ & $<0.05$ & 263 & 0.04 & 0.01 & $<0.2$ \\
\hline $09 / 18 / 85$ & 0.48 & 266 & 0.03 & 0.02 & $<0.2$ \\
\hline $10 / 03 / 85$ & 0.64 & 317 & 0.08 & 0.08 & $<0.2$ \\
\hline $10 / 22 / 85$ & 0.73 & 352 & 0.07 & 0.07 & $<0.2$ \\
\hline $11 / 03 / 85$ & 0.77 & 367 & 0.06 & 0.06 & 0.65 \\
\hline $12 / 02 / 85$ & 0.64 & 322 & 0.05 & 0.05 & $<0.2$ \\
\hline $01 / 02 / 86$ & 0.70 & 338 & 0.20 & 0.05 & $<0.2$ \\
\hline $01 / 16 / 86$ & 0.70 & 306 & 0.09 & 0.04 & $<0.2$ \\
\hline $02 / 02 / 86$ & 0.71 & 346 & 0.01 & $<0.01$ & 0.21 \\
\hline $02 / 26 / 86$ & 0.66 & 357 & 0.01 & $<0.01$ & $<0.2$ \\
\hline $04 / 05 / 86$ & 0.71 & 352 & 0.13 & 0.12 & $<0.2$ \\
\hline $05 / 02 / 86$ & 0.68 & 343 & 0.04 & 0.05 & 0.31 \\
\hline $06 / 04 / 86$ & 0.52 & 323 & 0.02 & 0.02 & $<0.2$ \\
\hline $07 / 07 / 86$ & 0.51 & 201 & 0.09 & 0.04 & $<0.2$ \\
\hline $08 / 01 / 86$ & 0.45 & 123 & 0.08 & 0.05 & $<0.2$ \\
\hline $09 / 03 / 86$ & 0.45 & 114 & 0.08 & 0.06 & $<0.2$ \\
\hline $10 / 01 / 86$ & 0.62 & 184 & 0.13 & 0.06 & $<0.2$ \\
\hline $11 / 06 / 86$ & 0.66 & 224 & 0.06 & 0.05 & 0.22 \\
\hline $12 / 01 / 86$ & 0.55 & 236 & 0.06 & 0.05 & 0.21 \\
\hline $01 / 05 / 87$ & 0.40 & 228 & 0.04 & 0.03 & $<0.2$ \\
\hline $02 / 02 / 87$ & 0.96 & 188 & 0.03 & 0.02 & $<0.2$ \\
\hline $03 / 02 / 87$ & 0.44 & 241 & 0.03 & 0.02 & $<0.2$ \\
\hline $04 / 02 / 87$ & 0.50 & 252 & 0.03 & 0.02 & $<0.2$ \\
\hline $05 / 01 / 87$ & 0.37 & 278 & 0.06 & 0.05 & 0.22 \\
\hline $06 / 01 / 87$ & 0.37 & 266 & 0.18 & 0.06 & $<0.2$ \\
\hline $07 / 01 / 87$ & 0.36 & 225 & $<0.02$ & $<0.02$ & $<0.2$ \\
\hline $08 / 03 / 87$ & 0.42 & 277 & 0.05 & 0.05 & $<0.2$ \\
\hline $09 / 08 / 87$ & 0.54 & 266 & 0.07 & 0.07 & $<0.2$ \\
\hline $10 / 02 / 87$ & 0.90 & 332 & 0.28 & 0.18 & $<0.2$ \\
\hline $11 / 01 / 87$ & 0.56 & 309 & 0.07 & 0.05 & $<0.2$ \\
\hline $12 / 01 / 87$ & 0.50 & 308 & 0.11 & 0.07 & 0.13 \\
\hline $01 / 04 / 88$ & 0.66 & 294 & - & $<0.01$ & $<0.1$ \\
\hline $02 / 01 / 88$ & 0.66 & 298 & 0.01 & $<0.01$ & $<0.1$ \\
\hline $03 / 01 / 88$ & 0.60 & 301 & 0.02 & $<0.01$ & $<0.1$ \\
\hline $04 / 01 / 88$ & 0.54 & 309 & 0.02 & $<0.01$ & $<0.1$ \\
\hline $05 / 03 / 88$ & 0.47 & 324 & 0.02 & 0.02 & $<0.1$ \\
\hline $06 / 01 / 88$ & 0.53 & 325 & 0.03 & $<0.01$ & $<0.1$ \\
\hline
\end{tabular}


Table 1. (continued).

\begin{tabular}{|c|c|}
\hline $\begin{array}{c}\text { Date } \\
\text { sampled }\end{array}$ & $\begin{array}{c}\text { Total kjeldahl } \\
\text { Nitrogen as } \\
\text { N (mg/1) }\end{array}$ \\
\hline $05 / 17 / 85$ & - \\
\hline $06 / 03 / 85$ & 0.68 \\
\hline $06 / 18 / 85$ & 0.95 \\
\hline $07 / 18 / 85$ & 0.2 \\
\hline $08 / 01 / 85$ & 0.88 \\
\hline $08 / 16 / 85$ & $<0.5$ \\
\hline $09 / 12 / 85$ & $<0.5$ \\
\hline $09 / 18 / 85$ & $<0.5$ \\
\hline $10 / 03 / 85$ & $<0.5$ \\
\hline $10 / 22 / 85$ & $<0.5$ \\
\hline $11 / 03 / 85$ & $<0.5$ \\
\hline $12 / 02 / 85$ & $<0.5$ \\
\hline $01 / 02 / 86$ & 0.6 \\
\hline $01 / 16 / 86$ & 0.8 \\
\hline $02 / 02 / 86$ & 0.35 \\
\hline $02 / 26 / 86$ & 0.34 \\
\hline $04 / 05 / 86$ & $<0.5$ \\
\hline $05 / 02 / 86$ & 0.62 \\
\hline $06 / 04 / 86$ & 0.52 \\
\hline $07 / 07 / 86$ & 0.43 \\
\hline $08 / 01 / 86$ & 0.28 \\
\hline $09 / 03 / 86$ & 0.21 \\
\hline $10 / 01 / 86$ & 0.38 \\
\hline $11 / 06 / 86$ & 0.41 \\
\hline $12 / 01 / 86$ & 0.34 \\
\hline $01 / 05 / 87$ & 0.39 \\
\hline $02 / 02 / 87$ & $<0.2$ \\
\hline $03 / 02 / 87$ & 0.35 \\
\hline $04 / 02 / 87$ & 0.57 \\
\hline $05 / 01 / 87$ & 0.22 \\
\hline $06 / 01 / 87$ & 0.48 \\
\hline $07 / 01 / 87$ & $<0.2$ \\
\hline $08 / 03 / 87$ & 0.26 \\
\hline $09 / 08 / 87$ & 0.48 \\
\hline $10 / 02 / 87$ & 1.52 \\
\hline $11 / 01 / 87$ & 0.49 \\
\hline $12 / 01 / 87$ & 0.82 \\
\hline $01 / 04 / 88$ & 0.7 \\
\hline $02 / 01 / 88$ & 0.3 \\
\hline $03 / 01 / 88$ & 0.2 \\
\hline $04 / 01 / 88$ & 0.3 \\
\hline $05 / 03 / 88$ & 0.4 \\
\hline $06 / 01 / 88$ & 0.2 \\
\hline
\end{tabular}


Table 2. Dissolved metal concentrations measured from each water sample collected near the gagehouse below Afterbay Dam on the Bighorn River from 18 June 1985 - 1 August 1986.

Dissolved metals (ug/1)

\begin{tabular}{llrrrr}
\cline { 3 - 4 } Date & Copper & Iron & Lead & Manganese & Zinc \\
\hline $6 / 18 / 85$ & $<20$ & 40 & $<20$ & 20 & $<20$ \\
$7 / 18 / 85$ & $<20$ & 40 & $<20$ & 20 & 40 \\
$8 / 16 / 85$ & $<20$ & $<50$ & $<20$ & $<20$ & $<20$ \\
$12 / 2 / 85$ & $<20$ & $<50$ & $<20$ & 20 & $<20$ \\
$4 / 5 / 86$ & $<20$ & 70 & $<20$ & 80 & $<20$ \\
$5 / 2 / 86$ & $<20$ & $<50$ & $<20$ & $<20$ & $<20$ \\
$8 / 1 / 86$ & $<20$ & $<70$ & $<20$ & $<20$ & $<20$ \\
\hline
\end{tabular}




\section{APPENDIX C \\ Trout Population statistics}


Table 3. Fall brown trout population estimates, biomass, and condition factors by length group from $\mathrm{Rkm} 0-6.1$ (RM 0-3.8) below Afterbay Dam on the Bighorn River in 1985 (Collection Code = 585009).

\begin{tabular}{|c|c|c|c|c|c|c|}
\hline \multicolumn{2}{|c|}{ Length } & $\begin{array}{l}\text { Total } \\
\text { number }\end{array}$ & $\begin{array}{l}\text { Total } \\
\text { weight }\end{array}$ & $\begin{array}{l}\text { Condition } \\
\text { Factor }\end{array}$ & $\begin{array}{c}\text { Number } \\
\text { per mile }\end{array}$ & $\begin{array}{l}\text { Number } \\
\text { per } \mathrm{km}\end{array}$ \\
\hline 127 & 5.0 & 292 & 29 & 66.94 & 76.8 & 47.9 \\
\hline 140 & 5.5 & 1343 & 129 & 50.47 & 353.4 & 220.2 \\
\hline 152 & 6.0 & 2686 & 322 & 49.43 & 706.8 & $\begin{array}{l}220.2 \\
440.3\end{array}$ \\
\hline 165 & 6.5 & 4613 & 680 & 48.76 & 1213.9 & $\begin{array}{l}440.3 \\
756.2\end{array}$ \\
\hline 178 & 7.0 & 4876 & 935 & 51.53 & 1283.2 & $\begin{array}{l}756.2 \\
799.3\end{array}$ \\
\hline 191 & 7.5 & 5839 & 1356 & 50.68 & 1536.6 & $\begin{array}{l}799.3 \\
957.2\end{array}$ \\
\hline 203 & 8.0 & 4379 & 1203 & 49.78 & 1152.4 & $\begin{array}{l}957.2 \\
717.9\end{array}$ \\
\hline 216 & 8.5 & 3854 & 1243 & 49.07 & 1014.2 & $\begin{array}{l}717.9 \\
631.8\end{array}$ \\
\hline 229 & 9.0 & 2774 & 1073 & 49.40 & 730.0 & $\begin{array}{l}631.8 \\
454.8\end{array}$ \\
\hline 241 & 9.5 & 1898 & 834 & 48.89 & 499.5 & $\begin{array}{l}454.8 \\
311.1\end{array}$ \\
\hline 254 & 10.0 & 963 & 497 & 48.87 & 253.4 & $\begin{array}{l}311.1 \\
157.9\end{array}$ \\
\hline 267 & 10.5 & 230 & 142 & 49.86 & 60.5 & $\begin{array}{r}157.9 \\
37.7\end{array}$ \\
\hline 279 & 11.0 & 175 & 128 & 51.91 & 46.1 & $\begin{array}{l}37.7 \\
28.7\end{array}$ \\
\hline 292 & 11.5 & 258 & 212 & 50.72 & 67.9 & $\begin{array}{l}28.7 \\
42.3\end{array}$ \\
\hline 305 & 12.0 & 506 & 458 & 49.84 & 133.2 & $\begin{array}{l}42.3 \\
83.0\end{array}$ \\
\hline 318 & 12.5 & 773 & 769 & 48.42 & 203.4 & \\
\hline 330 & 13.0 & 856 & 956 & 48.38 & 225.3 & $\begin{array}{l}126.7 \\
140.3\end{array}$ \\
\hline 343 & 13.5 & 1141 & 1373 & 46.75 & 300.3 & $\begin{array}{l}140.3 \\
187.0\end{array}$ \\
\hline 356 & 14.0 & 883 & 1143 & 45.07 & 232.4 & $\begin{array}{l}187.0 \\
144.8\end{array}$ \\
\hline 368 & 14.5 & 957 & 1354 & 44.62 & 251.8 & $\begin{array}{l}144.8 \\
156.9\end{array}$ \\
\hline 381 & 15.0 & 498 & 774 & 44.17 & 131.1 & $\begin{array}{r}156.9 \\
81.6\end{array}$ \\
\hline 394 & 15.5 & 586 & 991 & 43.7 & 154.2 & \\
\hline 406 & 16.0 & 411 & 742 & 42.51 & 108.2 & $\begin{array}{l}96.1 \\
67.4\end{array}$ \\
\hline 419 & 16.5 & 525 & 1019 & 41.62 & 138.2 & $\begin{array}{l}67.4 \\
86.1\end{array}$ \\
\hline 432 & 17.0 & 485 & 994 & 40.15 & 127.6 & $\begin{array}{l}86.1 \\
79.5\end{array}$ \\
\hline 445 & 17.5 & 575 & 1273 & 39.98 & 151.3 & $\begin{array}{l}79.5 \\
94.3\end{array}$ \\
\hline 457 & 18.0 & 483 & 1139 & 39.2 & 127.1 & $\begin{array}{l}94.3 \\
79.2\end{array}$ \\
\hline 470 & 18.5 & 408 & 1010 & 37.96 & 107.4 & \\
\hline 483 & 19.0 & 325 & 887 & 38.57 & 85.5 & $\begin{array}{l}66.9 \\
53.3\end{array}$ \\
\hline 495 & 19.5 & 292 & 810 & 36.19 & 76.8 & \\
\hline 508 & 20.0 & 283 & 830 & 35.36 & 74.5 & $\begin{array}{l}47.9 \\
46.4\end{array}$ \\
\hline 521 & 20.5 & 133 & 428 & 36.38 & 35.0 & $\begin{array}{l}46.4 \\
21.8\end{array}$ \\
\hline 533 & 21.0 & 92 & 312 & 35.44 & 24.2 & $\begin{array}{l}21.8 \\
15.1\end{array}$ \\
\hline 546 & 21.5 & 67 & 225 & 33.53 & 17.6 & $\begin{array}{l}15.1 \\
11.0\end{array}$ \\
\hline 559 & 22.0 & 33 & 119 & 33.06 & 8.7 & $\begin{array}{r}11.0 \\
5.4\end{array}$ \\
\hline 572 & 22.5 & 8 & 47 & 49.16 & 2.1 & $\begin{array}{l}5.4 \\
1.3\end{array}$ \\
\hline & & & & & & 1.3 \\
\hline Total & & 44500 & 26436 & 45.18 & 11710.5 & 7295.1 \\
\hline
\end{tabular}


Table 4. Fall brown trout population estimates, biomass, and condition factors by length group from Rkm 0-6.1 (RM 0-3.8) below Afterbay Dam on the Bighorn River in 1986 (Collection Code = 586014).

\begin{tabular}{|c|c|c|c|c|c|c|}
\hline \multicolumn{2}{|c|}{ Length } & $\begin{array}{l}\text { Total } \\
\text { number }\end{array}$ & $\begin{array}{l}\text { Total } \\
\text { weight }\end{array}$ & $\begin{array}{c}\text { Condition } \\
\text { Factor }\end{array}$ & $\begin{array}{c}\text { Number } \\
\text { per mile }\end{array}$ & $\begin{array}{l}\text { Number } \\
\text { per } \mathrm{km}\end{array}$ \\
\hline 127 & 5.0 & 94 & 8 & 57.77 & 24.7 & 15.4 \\
\hline 140 & 5.5 & 436 & 43 & 51.42 & 114.7 & 71.5 \\
\hline 152 & 6.0 & 1439 & 173 & 49.26 & 378.7 & 235.9 \\
\hline 165 & 6.5 & 2996 & 441 & 48.99 & 788.4 & 491.1 \\
\hline 178 & 7.0 & 3680 & 656 & 47.53 & 968.4 & 603.3 \\
\hline 191 & 7.5 & 3904 & 833 & 46.71 & 1027.4 & 640.0 \\
\hline 203 & 8.0 & 3161 & 818 & 47.05 & 831.8 & 518.2 \\
\hline 216 & 8.5 & 2323 & 702 & 46.3 & 611.3 & 380.8 \\
\hline 229 & 9.0 & 1722 & 601 & 44.88 & 453.2 & 282.3 \\
\hline 241 & 9.5 & 597 & 233 & 42.72 & 157.1 & 97.9 \\
\hline 254 & 10.0 & 924 & 427 & 43.21 & 243.2 & 151.5 \\
\hline 267 & 10.5 & 1601 & 814 & 41.01 & 421.3 & 262.5 \\
\hline 279 & 11.0 & 2559 & 1472 & 40.69 & 673.4 & 419.5 \\
\hline 292 & 11.5 & 3265 & 2118 & 40.48 & 859.2 & 535.2 \\
\hline 305 & 12.0 & 3190 & 2350 & 40.57 & 839.5 & 523.0 \\
\hline 318 & 12.5 & 2852 & 2321 & 39.81 & 750.5 & 467.5 \\
\hline 330 & 13.0 & 1790 & 1648 & 40.20 & 471.1 & 293.4 \\
\hline 343 & 13.5 & 1488 & 1569 & 41.10 & 391.6 & 243.9 \\
\hline 356 & 14.0 & 1256 & 1488 & 41.48 & 330.5 & 205.9 \\
\hline 368 & 14.5 & 1483 & 1924 & 40.73 & 390.3 & 243.1 \\
\hline 381 & 15.0 & 1350 & 1887 & 39.75 & 355.3 & 221.3 \\
\hline 394 & 15.5 & 1315 & 1991 & 39.19 & 346.1 & 215.6 \\
\hline 406 & 16.0 & 1288 & 2090 & 38.21 & 338.9 & 211.1 \\
\hline 419 & 16.5 & 906 & 1572 & 37.53 & 238.4 & 148.5 \\
\hline 432 & 17.0 & 585 & 1077 & 36.44 & 153.9 & 95.9 \\
\hline 445 & 17.5 & 405 & 792 & 35.35 & 106.6 & 66.4 \\
\hline 457 & 18.0 & 270 & 551 & 33.83 & 71.1 & 44.3 \\
\hline 470 & 18.5 & 163 & 353 & 33.21 & 42.9 & 26.7 \\
\hline 483 & 19.0 & 131 & 301 & 32.83 & 34.5 & 21.5 \\
\hline 495 & 19.5 & 73 & 176 & 31.37 & 19.2 & 12.0 \\
\hline 508 & 20.0 & 69 & 168 & 29.87 & 18.2 & 11.3 \\
\hline 521 & 20.5 & 33 & 92 & 32.11 & 8.7 & 5.4 \\
\hline 533 & 21.0 & 33 & 92 & 29.52 & 8.7 & 5.4 \\
\hline 546 & 21.5 & 20 & 66 & 32.33 & 5.3 & 3.3 \\
\hline 559 & 22.0 & 8 & 26 & 29.42 & 2.1 & 1.3 \\
\hline 572 & 22.5 & 12 & 45 & 31.01 & $\begin{array}{l}2.1 \\
3.2\end{array}$ & 2.0 \\
\hline 584 & 23.0 & 4 & 19 & 36.53 & 1.1 & 0.7 \\
\hline Total & & 47425 & 31937 & 39.74 & 12480.3 & 7774.6 \\
\hline
\end{tabular}


Table 5. Fall brown trout population estimates, biomass, and condition factors by length group from $\mathrm{Rkm} 0-6.1$ (RM 0-3.8) below Afterbay Dam on the Bighorn River in 1987 (Collection Code = $587073)$.

\begin{tabular}{|c|c|c|c|c|c|c|}
\hline \multicolumn{2}{|c|}{ Length } & \multirow{2}{*}{$\begin{array}{l}\text { Total } \\
\text { number }\end{array}$} & \multirow{2}{*}{$\begin{array}{l}\text { Total } \\
\text { weight }\end{array}$} & \multirow{2}{*}{$\begin{array}{l}\text { Condition } \\
\text { Factor }\end{array}$} & \multirow{2}{*}{$\begin{array}{l}\text { Number } \\
\text { per mile }\end{array}$} & \multirow{2}{*}{$\begin{array}{l}\text { Number } \\
\text { per km }\end{array}$} \\
\hline$\overline{(m m)}$ & (inches) & & & & & \\
\hline 127 & 5.0 & - & - & - & - & - \\
\hline 140 & 5.5 & - & - & - & $=$ & - \\
\hline 152 & 6.0 & - & - & 55.31 & - & - \\
\hline 165 & 6.5 & - & - & 44.53 & - & - \\
\hline 178 & 7.0 & - & - & 44.89 & - & - \\
\hline 191 & 7.5 & - & - & 51.5 & - & - \\
\hline 203 & 8.0 & - & - & 51.62 & - & - \\
\hline 216 & 8.5 & 255 & 85 & 50.11 & 67.1 & 41.8 \\
\hline 229 & 9.0 & 500 & 195 & 49.68 & 131.6 & 82.0 \\
\hline 241 & 9.5 & 439 & 205 & 50.5 & 115.5 & 72.0 \\
\hline 254 & 10.0 & 582 & 298 & 48.47 & 153.2 & 95.4 \\
\hline 267 & 10.5 & 1236 & 720 & 47.44 & 325.3 & 202.6 \\
\hline 279 & 11.0 & 1746 & 1146 & 46.51 & 459.5 & 286.2 \\
\hline 292 & 11.5 & 2053 & 1524 & 46.04 & 540.3 & 336.6 \\
\hline 305 & 12.0 & 2652 & 2196 & 45.37 & 697.9 & 434.8 \\
\hline 318 & 12.5 & 3998 & 3675 & 44.87 & 1052.1 & 655.4 \\
\hline 330 & 13.0 & 4511 & 4605 & 44.37 & 1187.1 & 739.5 \\
\hline 343 & 13.5 & 4752 & 5358 & 43.9 & 1250.5 & 779.0 \\
\hline 356 & 14.0 & 4184 & 5255 & 43.9 & 1101.1 & 685.9 \\
\hline 368 & 14.5 & 3803 & 5204 & 43.15 & 1000.8 & 623.4 \\
\hline 381 & 15.0 & 3375 & 4956 & 42.01 & 888.2 & 553.3 \\
\hline 394 & 15.5 & 2559 & 4043 & 41.00 & 673.4 & 419.5 \\
\hline 406 & 16.0 & 1331 & 2245 & 39.91 & 350.3 & 218.2 \\
\hline 419 & 16.5 & 837 & 1536 & 39.5 & 220.3 & 137.2 \\
\hline 432 & 17.0 & 378 & 698 & 36.35 & 99.5 & 62.0 \\
\hline 445 & 17.5 & 267 & 556 & 37.64 & 70.3 & 43.8 \\
\hline 457 & 18.0 & 100 & 223 & 36.84 & 26.3 & 16.4 \\
\hline 470 & 18.5 & 85 & 198 & 35.8 & 22.4 & 13.9 \\
\hline 483 & 19.0 & 37 & 96 & 36.67 & 9.7 & 6.1 \\
\hline 495 & 19.5 & 33 & 86 & 34.48 & 8.7 & 5.4 \\
\hline 508 & 20.0 & 15 & 45 & 37.44 & 3.9 & 2.5 \\
\hline 521 & 20.5 & 15 & 53 & 40.02 & 3.9 & 2.5 \\
\hline 533 & 21.0 & 11 & 35 & 32.81 & 2.9 & 1.8 \\
\hline 546 & 21.5 & 11 & 42 & 36.39 & 2.9 & 1.8 \\
\hline 559 & 22.0 & 4 & 16 & 41.79 & 1.1 & 0.7 \\
\hline 572 & 22.5 & 4 & 17 & 40.37 & 1.1 & 0.7 \\
\hline 584 & 23.0 & 4 & 17 & 36.83 & 1.1 & 0.7 \\
\hline 597 & 23.5 & 0 & 0 & - & 0.0 & 0.0 \\
\hline 610 & 24.0 & 4 & 20 & 39.79 & 1.1 & 0.7 \\
\hline Tota] & & 39781 & 45348 & 41.61 & 10468.7 & 6521.5 \\
\hline
\end{tabular}


Table 6. Fall brown trout population estimates, biomass, and condition factors by length group from Rkm 3.9-15.4 (RM 2.4-9.6) below Afterbay Dam on the Bighorn River in 1985 (Collection Code $=585010$ ).

\begin{tabular}{|c|c|c|c|c|c|c|}
\hline \multicolumn{2}{|c|}{ Length } & \multirow{2}{*}{$\begin{array}{l}\text { Total } \\
\text { number }\end{array}$} & \multirow{2}{*}{$\begin{array}{l}\text { Total } \\
\text { weight }\end{array}$} & \multirow{2}{*}{$\begin{array}{c}\text { Condition } \\
\text { Factor }\end{array}$} & \multirow{2}{*}{$\begin{array}{c}\text { Number } \\
\text { per mile }\end{array}$} & \multirow{2}{*}{$\begin{array}{l}\text { Number } \\
\text { per km }\end{array}$} \\
\hline (mm) & (inches) & & & & & \\
\hline 127 & 5.0 & 113 & 9 & 52.82 & 15.7 & 9.8 \\
\hline 140 & 5.5 & 355 & 34 & 51.93 & 49.3 & 30.9 \\
\hline 152 & 6.0 & 1121 & 141 & 52.01 & 155.7 & 97.5 \\
\hline 165 & 6.5 & 1915 & 294 & 50.32 & 266.0 & 166.5 \\
\hline 178 & 7.0 & 2412 & 474 & 52.26 & 335.0 & 209.7 \\
\hline 191 & 7.5 & 2724 & 645 & 51.65 & 378.3 & 236.9 \\
\hline 203 & 8.0 & 2497 & 718 & 52.18 & 346.8 & 217.1 \\
\hline 216 & 8.5 & 2043 & 689 & 51.29 & 283.8 & 177.7 \\
\hline 229 & 9.0 & 1305 & 517 & 51.10 & 181.3 & 113.5 \\
\hline 241 & 9.5 & 1036 & 477 & 50.66 & 143.9 & 90.1 \\
\hline 254 & 10.0 & 794 & 438 & 51.41 & 110.3 & 69.0 \\
\hline 267 & 10.5 & 511 & 326 & 51.86 & 71.0 & 44.4 \\
\hline 279 & 11.0 & 243 & 188 & 55.00 & 33.8 & 21.1 \\
\hline 292 & 11.5 & 495 & 449 & 56.32 & 68.8 & 43.0 \\
\hline 305 & 12.0 & 952 & 915 & 52.62 & 132.2 & 82.8 \\
\hline 318 & 12.5 & 1410 & 1471 & 50.69 & 195.8 & 122.6 \\
\hline 330 & 13.0 & 1961 & 2231 & 49.17 & 272.4 & 170.5 \\
\hline 343 & 13.5 & 2605 & 3156 & 47.08 & 361.8 & 226.5 \\
\hline 356 & 14.0 & 2064 & 2765 & 46.70 & 286.7 & 179.5 \\
\hline 368 & 14.5 & 1802 & 2658 & 46.45 & 250.3 & 156.7 \\
\hline 381 & 15.0 & 1211 & 1960 & 46.00 & 168.2 & 105.3 \\
\hline 394 & 15.5 & 1235 & 2145 & 44.75 & 171.5 & 107.4 \\
\hline 406 & 16.0 & 966 & 1801 & 43.88 & 134.2 & 84.0 \\
\hline 419 & 16.5 & 1068 & 2090 & 41.89 & 148.3 & 92.9 \\
\hline 432 & 17.0 & 958 & 1963 & 40.21 & 133.1 & 83.3 \\
\hline 445 & 17.5 & 1084 & 2317 & 38.51 & 150.6 & 94.3 \\
\hline 457 & 18.0 & 839 & 1971 & 39.00 & 116.5 & 73.0 \\
\hline 470 & 18.5 & 886 & 2194 & 37.86 & 123.1 & 77.0 \\
\hline 483 & 19.0 & 389 & 1008 & 36.45 & 54.0 & 33.8 \\
\hline 495 & 19.5 & 267 & 714 & 35.05 & 37.1 & 23.2 \\
\hline 508 & 20.0 & 192 & 541 & 34.22 & 26.7 & 16.7 \\
\hline 521 & 20.5 & 145 & 438 & 34.12 & 20.1 & 12.6 \\
\hline 533 & 21.0 & 84 & 259 & 32.11 & 11.7 & 7.3 \\
\hline 546 & 21.5 & 52 & 170 & 32.66 & 7.2 & 4.5 \\
\hline 559 & 22.0 & 28 & 88 & 28.78 & 3.9 & $2 \cdot 4$ \\
\hline 572 & 22.5 & 5 & 17 & 31.96 & 0.7 & 0.4 \\
\hline 584 & 23.0 & 5 & 17 & 27.71 & 0.7 & 0.4 \\
\hline Total & & 37772 & 38288 & 44.56 & 5246.1 & 3284.5 \\
\hline
\end{tabular}


Table 7. Fall brown trout population estimates, biomass, and condition factors by length group from Rkm 3.9-15.4 (RM 2.4-9.6) below Afterbay Dam on the Bighorn River in 1986 (Collection Code $=586015$ ).

\begin{tabular}{|c|c|c|c|c|c|c|}
\hline \multicolumn{2}{|c|}{ Length } & \multirow{2}{*}{$\begin{array}{l}\text { Total } \\
\text { number }\end{array}$} & \multirow{2}{*}{$\begin{array}{l}\text { Total } \\
\text { weight }\end{array}$} & \multirow{2}{*}{$\begin{array}{c}\text { Condition } \\
\text { Factor }\end{array}$} & \multirow{2}{*}{$\begin{array}{l}\text { Number } \\
\text { per mile }\end{array}$} & \multirow{2}{*}{$\begin{array}{l}\text { Number } \\
\text { per } \mathrm{km}\end{array}$} \\
\hline$(\mathrm{mm})$ & (inches) & & & & & \\
\hline 127 & 5.0 & 88 & 6 & 48.13 & 12.2 & 7.7 \\
\hline 140 & 5.5 & 139 & 13 & 48.73 & 19.3 & 12.1 \\
\hline 152 & 6.0 & 469 & 50 & 44.15 & 65.1 & 40.8 \\
\hline 165 & 6.5 & 1407 & 197 & 46.29 & 195.4 & 122.3 \\
\hline 178 & 7.0 & 2638 & 432 & 43.42 & 366.4 & 229.4 \\
\hline 191 & 7.5 & 3634 & 716 & 42.93 & 504.7 & 316.0 \\
\hline 203 & 8.0 & 3905 & 921 & 42.83 & 542.4 & 339.6 \\
\hline 216 & 8.5 & 3656 & 1020 & 42.58 & 507.8 & 317.9 \\
\hline 229 & 9.0 & 2484 & 797 & 41.63 & 345.0 & 216.0 \\
\hline 241 & 9.5 & 1363 & 503 & 40.76 & 189.3 & 118.5 \\
\hline 254 & 10.0 & 821 & 354 & 41.19 & 114.0 & 71.4 \\
\hline 267 & 10.5 & 452 & 231 & 41.69 & 62.8 & 39.3 \\
\hline 279 & 11.0 & 801 & 482 & 42.25 & 111.3 & 69.7 \\
\hline 292 & 11.5 & 1355 & 900 & 41.34 & 188.2 & 117.8 \\
\hline 305 & 12.0 & 2211 & 1640 & 40.77 & 307.1 & 192.3 \\
\hline 318 & 12.5 & 2819 & 2322 & 40.10 & 391.5 & 245.1 \\
\hline 330 & 13.0 & 2988 & 2724 & 39.68 & 415.0 & 259.8 \\
\hline 343 & 13.5 & 2765 & 2822 & 39.87 & 384.0 & 240.4 \\
\hline 356 & 14.0 & 2445 & 2807 & 40.18 & 339.6 & 212.6 \\
\hline 368 & 14.5 & 2614 & 3345 & 40.29 & 363.1 & 227.3 \\
\hline 381 & 15.0 & 2193 & 3115 & 40.50 & 304.6 & 190.7 \\
\hline 394 & 15.5 & 2295 & 3574 & 40.18 & 318.8 & 199.6 \\
\hline 406 & 16.0 & 1830 & 3062 & 39.45 & 254.2 & 159.1 \\
\hline 419 & 16.5 & 1420 & 2566 & 38.95 & 197.2 & 123.5 \\
\hline 432 & 17.0 & 986 & 1883 & 37.63 & 136.9 & 85.7 \\
\hline 445 & 1.7 .5 & 780 & 1570 & 36.30 & 108.3 & 67.8 \\
\hline 457 & 18.0 & 642 & 1353 & 35.06 & 89.2 & 55.8 \\
\hline 470 & 18.5 & 453 & 1014 & 34.23 & 62.9 & 39.4 \\
\hline 483 & 19.0 & 390 & 913 & 33.20 & 54.2 & 33.9 \\
\hline 495 & 19.5 & 201 & 497 & 32.55 & 27.9 & 17.5 \\
\hline 508 & 20.0 & 149 & 385 & 31.58 & 20.7 & 13.0 \\
\hline 521 & 20.5 & 80 & 220 & 30.92 & 11.1 & 7.0 \\
\hline 533 & 21.0 & 46 & 137 & 31.19 & 6.4 & 4.0 \\
\hline 546 & 21.5 & 75 & 230 & 30.30 & 10.4 & 6.5 \\
\hline 559 & 22.0 & 17 & 49 & 26.59 & 2.4 & 1.5 \\
\hline 572 & 22.5 & 6 & 21 & 31.19 & 0.8 & 0.5 \\
\hline 584 & 23.0 & 11 & 53 & 36.76 & 1.5 & 1.0 \\
\hline Tota] & & 50628 & 42924 & 38.79 & 7031.7 & 4402.4 \\
\hline
\end{tabular}


Table 8. Fall brown trout population estimates, biomass, and condition factors by length group from Rkm 3.9-15.4 (RM 2.4-9.6) below Afterbay Dam on the Bighorn River in 1987 (Collection Code $=587074$ ).

\begin{tabular}{|c|c|c|c|c|c|c|}
\hline \multicolumn{2}{|c|}{ Length } & \multirow{2}{*}{$\begin{array}{l}\text { Total } \\
\text { number }\end{array}$} & \multirow{2}{*}{$\begin{array}{l}\text { Total } \\
\text { weight }\end{array}$} & \multirow{2}{*}{$\begin{array}{c}\text { Condition } \\
\text { Factor }\end{array}$} & \multirow{2}{*}{$\begin{array}{l}\text { Number } \\
\text { per mile }\end{array}$} & \multirow{2}{*}{$\begin{array}{l}\text { Number } \\
\text { per } \mathrm{km}\end{array}$} \\
\hline$(\mathrm{mm})$ & (inches) & & & & & \\
\hline 127 & 5.0 & - & - & - & - & - \\
\hline 140 & 5.5 & - & - & - & - & - \\
\hline 152 & 6.0 & 14 & 1 & 31.99 & 1.9 & 1.2 \\
\hline 165 & 6.5 & 43 & 6 & 49.20 & 6.0 & 3.7 \\
\hline 178 & 7.0 & 100 & 20 & 50.07 & 13.9 & 8.7 \\
\hline 191 & 7.5 & 571 & 115 & 43.46 & 79.3 & 49.7 \\
\hline 203 & 8.0 & 970 & 247 & 45.58 & 134.7 & 84.3 \\
\hline 216 & 8.5 & 1427 & 417 & 44.42 & 198.2 & $124 \cdot 1$ \\
\hline 229 & 9.0 & 2411 & 863 & 45.94 & 334.9 & 209.7 \\
\hline 241 & 9.5 & 2226 & 955 & 46.81 & 309.2 & 193.6 \\
\hline 254 & 10.0 & 2468 & 1203 & 45.99 & 342.8 & 214.6 \\
\hline 267 & 10.5 & 1610 & 920 & 46.61 & 223.6 & 240.0 \\
\hline 279 & 11.0 & 1731 & 1157 & 47.40 & 240.4 & 150.5 \\
\hline 292 & 11.5 & 1586 & 1196 & 46.91 & 220.3 & 137.9 \\
\hline 305 & 12.0 & 2784 & 2330 & 45.77 & 386.7 & 242.1 \\
\hline 318 & 12.5 & 4552 & 4240 & 45.35 & 632.2 & 395.8 \\
\hline 330 & 13.0 & 5714 & 5855 & 44.44 & 793.6 & 496.9 \\
\hline 343 & 13.5 & 5028 & 5729 & 44.27 & 698.3 & 437.2 \\
\hline 356 & 14.0 & 4304 & 5390 & 43.64 & 597.8 & 374.3 \\
\hline 368 & 14.5 & 4202 & 5739 & 42.96 & 583.6 & 365.4 \\
\hline 381 & 15.0 & 5283 & 7890 & 42.52 & 733.8 & 459.4 \\
\hline 394 & 15.5 & 5130 & 8284 & 41.73 & 712.5 & 446.1 \\
\hline 406 & 16.0 & 3855 & 6766 & 41.28 & 535.4 & 335.2 \\
\hline 419 & 16.5 & 1767 & 3309 & 40.22 & 245.4 & 153.7 \\
\hline 432 & 17.0 & 1287 & 2553 & 38.98 & 178.8 & 111.9 \\
\hline 445 & 17.5 & 789 & 1637 & 37.49 & 109.6 & 68.6 \\
\hline 457 & 18.0 & 388 & 870 & 37.31 & 53.9 & 33.7 \\
\hline 470 & 18.5 & 293 & 678 & 35.45 & 40.7 & 25.5 \\
\hline 483 & 19.0 & 141 & 364 & 36.34 & 19.6 & 12.3 \\
\hline 495 & 19.5 & 116 & 299 & 34.04 & 16.1 & 10.1 \\
\hline 508 & 20.0 & 42 & 125 & 35.90 & 5.8 & 3.7 \\
\hline 521 & 20.5 & 28 & 80 & 31.86 & 3.9 & 2.4 \\
\hline 533 & 21.0 & 18 & 55 & 32.90 & 2.5 & 1.6 \\
\hline 546 & 21.5 & 14 & 46 & 32.17 & 1.9 & 1.2 \\
\hline 559 & 22.0 & 4 & 9 & 24.89 & 0.6 & 0.3 \\
\hline 572 & 22.5 & 4 & 13 & 32.05 & 0.6 & 0.3 \\
\hline 584 & 23.0 & 4 & 14 & 32.27 & 0.6 & 0.3 \\
\hline 597 & 23.5 & 4 & 18 & 39.06 & 0.6 & 0.3 \\
\hline Total & & 60908 & 69393 & 40.48 & 8459.4 & 5296.3 \\
\hline
\end{tabular}


Table 9. Spring brown trout population estimates, biomass, and condition factors by length group from Rkm $0-6.1$ (RM $0-3.8$ ) below Afterbay Dam on the Bighorn River in 1985 (Collection code = 585005).

\begin{tabular}{|c|c|c|c|c|c|c|}
\hline \multicolumn{2}{|c|}{ Length } & \multirow{2}{*}{$\begin{array}{l}\text { Total } \\
\text { number }\end{array}$} & \multirow{2}{*}{$\begin{array}{l}\text { Total } \\
\text { weight }\end{array}$} & \multirow{2}{*}{$\begin{array}{c}\text { Condition } \\
\text { Factor }\end{array}$} & \multirow{2}{*}{$\begin{array}{l}\text { Number } \\
\text { per mile }\end{array}$} & \multirow{2}{*}{$\begin{array}{l}\text { Number } \\
\text { per km }\end{array}$} \\
\hline$\overline{(\mathrm{mm})}$ & (inches) & & & & & \\
\hline 76 & 3.0 & 16 & - & & 4.2 & 2.6 \\
\hline 89 & 3.5 & 442 & 13 & 56.15 & 116.3 & 72.5 \\
\hline 102 & 4.0 & 2196 & 86 & 50.4 & 577.9 & 360.0 \\
\hline 114 & 4.5 & 5440 & 226 & 39.54 & 1431.6 & 891.8 \\
\hline 127 & 5.0 & 6637 & 323 & 34.5 & 1746.6 & 1088.0 \\
\hline 140 & 5.5 & 5375 & 302 & 30.58 & 1414.5 & 881.1 \\
\hline 152 & 6.0 & 3654 & 294 & 33.88 & 961.6 & 599.0 \\
\hline 165 & 6.5 & 2212 & 218 & 33.30 & 582.1 & 362.6 \\
\hline 178 & 7.0 & 934 & 118 & 34.33 & 245.8 & 153.1 \\
\hline 191 & 7.5 & 229 & 40 & 39.03 & 60.3 & 37.5 \\
\hline 203 & 8.0 & 85 & 20 & 44.01 & 22.4 & 13.9 \\
\hline 216 & 8.5 & 242 & 61 & 38.14 & 63.7 & 39.7 \\
\hline 229 & 9.0 & 399 & 117 & 37.11 & 105.0 & 65.4 \\
\hline 241 & 9.5 & 726 & 240 & 36.02 & 191.1 & 119.0 \\
\hline 254 & 10.0 & 871 & 341 & 36.24 & 229.2 & 142.8 \\
\hline 267 & 10.5 & 1028 & 453 & 35.88 & 270.5 & 168.5 \\
\hline 279 & 11.0 & 1307 & 649 & 35.37 & 343.9 & 214.3 \\
\hline 292 & 11.5 & 1053 & 596 & 35.20 & 277.1 & 172.6 \\
\hline 305 & 12.0 & 992 & 649 & 35.95 & 261.1 & 162.6 \\
\hline 318 & 12.5 & 907 & 664 & 35.83 & 238.7 & 148.7 \\
\hline 330 & 13.0 & 492 & 395 & 35.08 & 129.5 & 80.7 \\
\hline 343 & 13.5 & 446 & 402 & 35.06 & 117.4 & 73.1 \\
\hline 356 & 14.0 & 629 & 625 & 34.64 & 165.5 & 103.1 \\
\hline 368 & 14.5 & 765 & 859 & 35.19 & 201.3 & 125.4 \\
\hline 381 & 15.0 & 638 & 777 & 34.74 & 167.9 & 104.6 \\
\hline 394 & 15.5 & 656 & 836 & 32.91 & 172.6 & 107.5 \\
\hline 406 & 16.0 & 565 & 800 & 33.36 & 148.7 & 92.6 \\
\hline 419 & 16.5 & 428 & 665 & 33.33 & 112.6 & 70.2 \\
\hline 432 & 17.0 & 519 & 883 & 33.19 & 136.6 & 85.1 \\
\hline 445 & 17.5 & 186 & 341 & 33.14 & 48.9 & 30.5 \\
\hline 457 & 18.0 & 142 & 283 & 32.92 & 37.4 & 23.3 \\
\hline 470 & 18.5 & 211 & 455 & 33.04 & 55.5 & 34.6 \\
\hline 483 & 19.0 & 117 & 271 & 32.89 & 30.8 & 19.2 \\
\hline 495 & 19.5 & 49 & 119 & 31.97 & 12.9 & 8.0 \\
\hline 508 & 20.0 & 59 & 151 & 31.22 & 15.5 & 9.7 \\
\hline 521 & 20.5 & 26 & 68 & 29.66 & 6.8 & 4.3 \\
\hline 533 & 21.0 & 33 & 97 & 30.74 & 8.7 & 5.4 \\
\hline 546 & 21.5 & 21 & 68 & 32.07 & 5.5 & 3.4 \\
\hline 559 & 22.0 & 15 & 48 & 28.66 & 3.9 & 2.5 \\
\hline 572 & 22.5 & 8 & 26 & 28.38 & 2.1 & 1.3 \\
\hline 584 & 23.0 & 0 & 0 & - & 0 & 0 \\
\hline 597 & 23.5 & 5 & 20 & 29.59 & 1.3 & 0.8 \\
\hline Tota] & & 40755 & 13599 & 35.08 & 10725.0 & 6681.1 \\
\hline
\end{tabular}


Table 10. Spring brown trout population estimates, biomass, and condition factors by length group from Rkm 0-6.1 (RM 0-3.8) below Afterbay Dam on the Bighorn River in 1986 (Collection Code $=$ $586006)$.

\begin{tabular}{|c|c|c|c|c|c|c|}
\hline \multicolumn{2}{|c|}{ Length } & \multirow{2}{*}{$\begin{array}{l}\text { Total } \\
\text { number }\end{array}$} & \multirow{2}{*}{$\begin{array}{l}\text { Total } \\
\text { weight }\end{array}$} & \multirow{2}{*}{$\begin{array}{c}\text { Conaition } \\
\text { Factor }\end{array}$} & \multirow{2}{*}{$\begin{array}{c}\text { Number } \\
\text { per mile }\end{array}$} & \multirow{2}{*}{$\begin{array}{l}\text { Number } \\
\text { per } \mathrm{km}\end{array}$} \\
\hline$(\mathrm{mm})$ & (inches) & & & & & \\
\hline 152 & 6.0 & 82 & 9 & 45.79 & 21.6 & 13.4 \\
\hline 165 & 6.5 & 124 & 17 & 43.15 & 32.6 & 20.3 \\
\hline 178 & 7.0 & 412 & 60 & 38.47 & 108.4 & 67.5 \\
\hline 191 & 7.5 & 544 & 101 & 40.62 & 143.2 & 89.2 \\
\hline 203 & 8.0 & 601 & 130 & 38.83 & 158.2 & 98.5 \\
\hline 216 & 8.5 & 824 & 215 & 39.56 & 216.8 & 135.1 \\
\hline 229 & 9.0 & 857 & 260 & 38.99 & 225.5 & 140.5 \\
\hline 241 & 9.5 & 560 & 200 & 39.01 & 147.4 & 91.8 \\
\hline 254 & 10.0 & 585 & 234 & 37.64 & 153.9 & 95.9 \\
\hline 267 & 10.5 & 354 & 166 & 38.15 & 93.2 & 58.0 \\
\hline 279 & 11.0 & 363 & 202 & 39.46 & 95.5 & 59.5 \\
\hline 292 & 11.5 & 345 & 217 & 39.11 & 90.8 & 56.6 \\
\hline 305 & 12.0 & 390 & 288 & 40.54 & 102.6 & 63.9 \\
\hline 318 & 12.5 & 523 & 424 & 39.73 & 137.6 & 85.7 \\
\hline 330 & 13.0 & 924 & 867 & 40.73 & 243.2 & 151.5 \\
\hline 343 & 13.5 & 1437 & 1496 & 40.36 & 378.2 & 235.6 \\
\hline 356 & 14.0 & 1604 & 1825 & 39.63 & 422.1 & 263.0 \\
\hline 368 & 14.5 & 956 & 1140 & 37.73 & $251 \cdot 6$ & 156.7 \\
\hline 381 & 15.0 & 990 & 1312 & 37.99 & 260.5 & 162.3 \\
\hline 394 & 15.5 & 983 & 1417 & 37.28 & 258.7 & 161.1 \\
\hline 406 & 16.0 & 792 & 1218 & 36.16 & 208.4 & 129.8 \\
\hline 419 & 16.5 & 812 & 1336 & 35.24 & 213.7 & 133.1 \\
\hline 432 & 17.0 & 635 & 1109 & 34.42 & 167.1 & 104.1 \\
\hline 445 & 17.5 & 331 & 644 & 34.97 & 87.1 & 54.3 \\
\hline 457 & 18.0 & 280 & 575 & 34.12 & 73.7 & 45.9 \\
\hline 470 & 18.5 & 182 & 389 & 32.7 & 47.9 & 29.8 \\
\hline 483 & 19.0 & 168 & 384 & 32.52 & 44.2 & 27.5 \\
\hline 495 & 19.5 & 113 & 275 & 32.08 & 29.7 & 18.5 \\
\hline 508 & 20.0 & 69 & 178 & 31.23 & 18.2 & 11.3 \\
\hline 521 & 20.5 & 51 & 138 & 30.31 & 13.4 & 8.4 \\
\hline 533 & 21.0 & 80 & 230 & 30.33 & 21.1 & 13.1 \\
\hline 546 & 21.5 & 40 & 125 & 30.66 & 10.5 & 6.6 \\
\hline 559 & 22.0 & 36 & 118 & 29.41 & 9.5 & 5.9 \\
\hline 572 & 22.5 & 0 & 0 & - & 0 & 0 \\
\hline 584 & 23.0 & 7 & 30 & 33.48 & 1.8 & 1.1 \\
\hline Tota & & 17054 & 17329 & 36.78 & 4487.9 & 2795.7 \\
\hline
\end{tabular}


Table 11. Spring brown trout population estimates, biomass, and condition factors by length group from Rkm 0-6.1 (RM 0-3.8) below Afterbay Dam on the Bighorn River in 1987 (Collection Code = 587051).

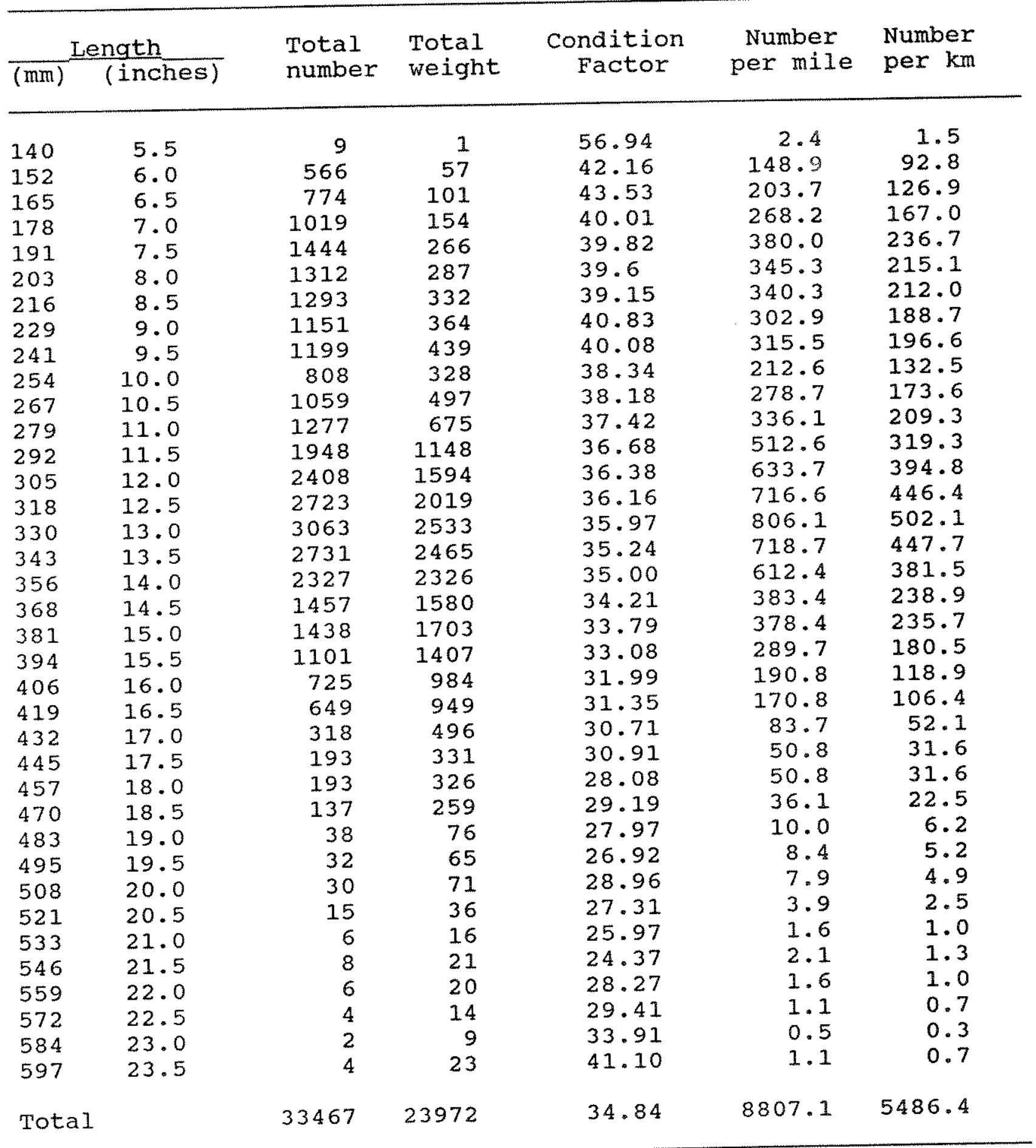


Table 12. Spring brown trout population estimates, biomass, and condition factors by length group from Rkm 3.9-15.4 (RM 2.4-9.6) below Afterbay Dam on the Bighorn River in 1985 (Collection Code $=585006$ ).

\begin{tabular}{|c|c|c|c|c|c|c|}
\hline \multicolumn{2}{|c|}{ Lenoth } & \multirow{2}{*}{$\begin{array}{l}\text { Total } \\
\text { number }\end{array}$} & \multirow{2}{*}{$\begin{array}{l}\text { Total } \\
\text { weight }\end{array}$} & \multirow{2}{*}{$\begin{array}{c}\text { Condition } \\
\text { Factor }\end{array}$} & \multirow{2}{*}{$\begin{array}{c}\text { Number } \\
\text { per mile }\end{array}$} & \multirow{2}{*}{$\begin{array}{l}\text { Number } \\
\text { per } \mathrm{km}\end{array}$} \\
\hline$(\mathrm{mm})$ & (inches) & & & & & \\
\hline 76 & 3.0 & 78 & 2 & - & 10.8 & 6 \\
\hline 89 & 3.5 & 1209 & 40 & 60.83 & 167.9 & 105 \\
\hline 102 & 4.0 & 4799 & 188 & 51.08 & 666.5 & 417 \\
\hline 114 & 4.5 & 8017 & 341 & 40.92 & 1113.5 & 697 \\
\hline 127 & 5.0 & 7510 & 371 & 35.26 & 1043.1 & 653 \\
\hline 140 & 5.5 & 5403 & 316 & 31.85 & 750.4 & 469 \\
\hline 152 & 6.0 & 3765 & 306 & 34.18 & 522.9 & 327 \\
\hline 165 & 6.5 & 2360 & 235 & 33.90 & 327.8 & 205 \\
\hline 178 & 7.0 & 8397 & 112 & 36.12 & 1166.3 & 730 \\
\hline 191 & 7.5 & 273 & 47 & 38.41 & 37.9 & 23 \\
\hline 203 & 8.0 & 79 & 19 & 42.74 & 11.0 & 6 \\
\hline 216 & 8.5 & 204 & 54 & 39.26 & 28.3 & 17 \\
\hline 229 & 9.0 & 409 & 131 & 40.28 & 56.8 & 35 \\
\hline 241 & 9.5 & 770 & 283 & 40.01 & 106.9 & 67 \\
\hline 254 & 10.0 & 1383 & 605 & 40.67 & 192.1 & 120 \\
\hline 267 & 10.5 & 1682 & 817 & 39.44 & 233.6 & 146 \\
\hline 279 & 11.0 & 2657 & 1420 & 37.93 & 369.0 & 231 \\
\hline 292 & 11.5 & 2861 & 1743 & 37.88 & 397.4 & 248 \\
\hline 305 & 12.0 & 2704 & 1877 & 38.11 & 375.6 & 235 \\
\hline 318 & 12.5 & 2531 & 1939 & 37.36 & 351.5 & 220 \\
\hline 330 & 13.0 & 1432 & 1272 & 38.64 & 198.9 & 124 \\
\hline 343 & 13.5 & 1339 & 1380 & 40.10 & 186.0 & 116 \\
\hline 356 & 14.0 & 1479 & 1648 & 38.81 & 205.4 & 128 \\
\hline 368 & 14.5 & 1712 & 2081 & 38.06 & 237.8 & 148 \\
\hline 381 & 15.0 & 1665 & 2231 & 38.19 & 231.3 & 144 \\
\hline 394 & 15.5 & 1735 & 2513 & 37.29 & 241.0 & 150 \\
\hline 406 & 16.0 & 1735 & 2709 & 36.67 & 241.0 & 150 \\
\hline 419 & 16.5 & 1525 & 2554 & 35.95 & 211.8 & 132 \\
\hline 432 & 17.0 & 1945 & 3410 & 34.44 & 270.1 & 169 \\
\hline 445 & 17.5 & 1399 & 2636 & 34.00 & 194.3 & 121 \\
\hline 457 & 18.0 & 1259 & 2517 & 33.21 & 174.9 & 109 \\
\hline 470 & 18.5 & 1037 & 2250 & 33.23 & 144.0 & 90 \\
\hline 483 & 19.0 & 905 & 2061 & 32.16 & 125.7 & 78 \\
\hline 495 & 19.5 & 256 & 628 & 32.18 & 35.6 & 22 \\
\hline 508 & 20.0 & 223 & 570 & 30.96 & 31.0 & 19 \\
\hline 521 & 20.5 & 148 & 404 & 30.97 & 20.6 & 12 \\
\hline 533 & 21.0 & 86 & 241 & 29.18 & 11.9 & 7 \\
\hline 546 & 21.5 & 83 & 257 & 30.29 & 11.5 & 7 \\
\hline 559 & 22.0 & 36 & 122 & 30.94 & 5.0 & 3 \\
\hline 572 & 22.5 & 4 & 13 & 30.09 & 0.6 & 0 \\
\hline 584 & 23.0 & 4 & 14 & 31.23 & 0.6 & 0 \\
\hline 597 & 23.5 & 7 & 27 & 27.78 & 1.0 & 0 \\
\hline 610 & 24.0 & 4 & 14 & 28.43 & 0.6 & 0 \\
\hline Total & & 77109 & 42398 & 36.1 & 10709.6 & 6705 \\
\hline
\end{tabular}


Table 13. Spring brown trout population estimates, biomass, and condition factors by length group from Rkm 3.9-15.4 (RM 2.4-9.6) below Afterbay Dam on the Bighorn River in 1986 (Collection Code $=586007$ ).

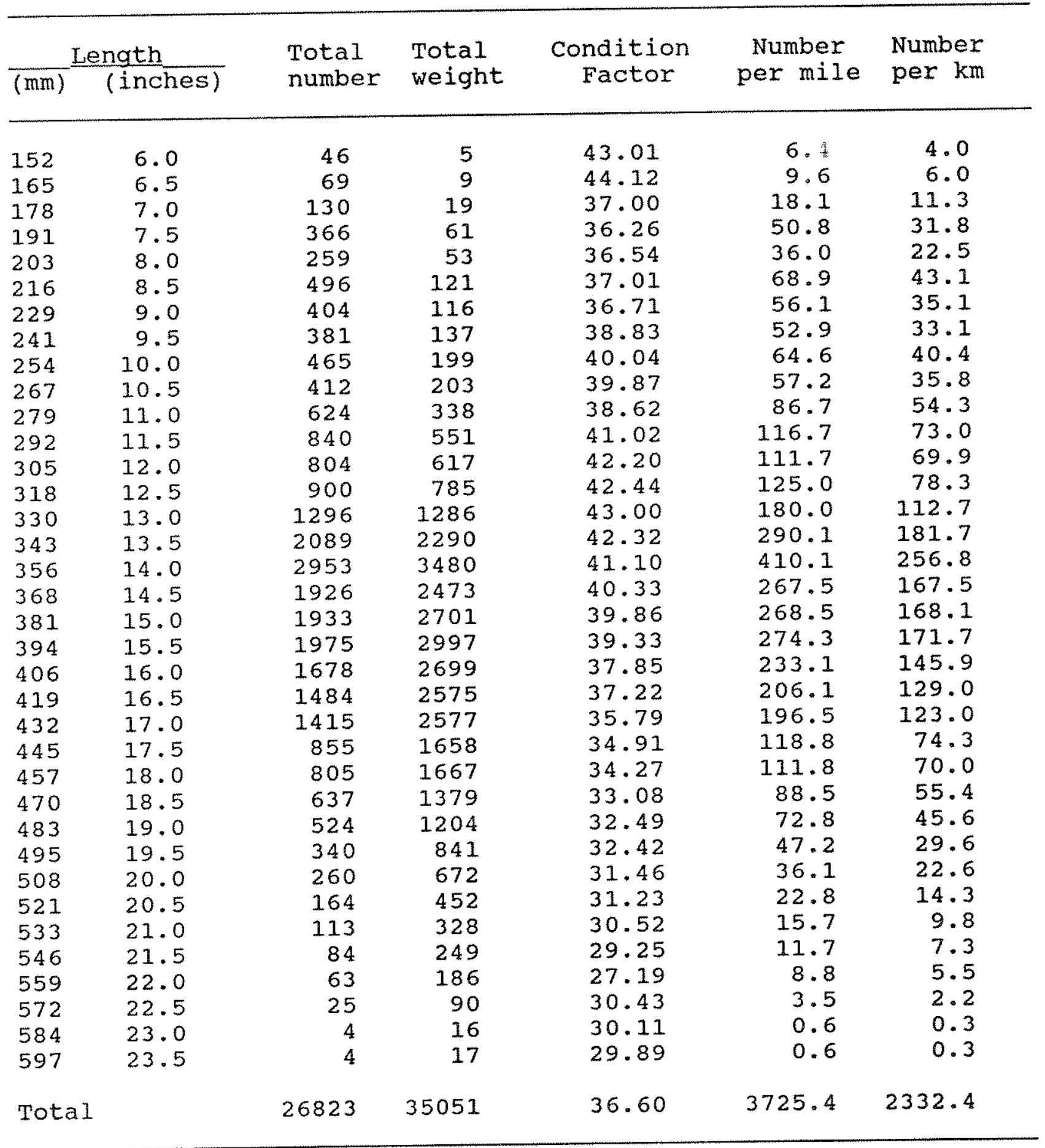


Table 14. Spring brown trout population estimates, biomass, and condition factors by length group from Rkm 3.9-15.4 (RM 2.4-9.6) below Afterbay Dam on the Bighorn River in 1987 (Collection Code $=587052$ ).

\begin{tabular}{|c|c|c|c|c|c|c|}
\hline \multicolumn{2}{|c|}{ Length } & \multirow{2}{*}{$\begin{array}{l}\text { Total } \\
\text { number }\end{array}$} & \multirow{2}{*}{$\begin{array}{l}\text { Total } \\
\text { weight }\end{array}$} & \multirow{2}{*}{$\begin{array}{c}\text { Condition } \\
\text { Factor }\end{array}$} & \multirow{2}{*}{$\begin{array}{l}\text { Number } \\
\text { per mile }\end{array}$} & \multirow{2}{*}{$\begin{array}{l}\text { Number } \\
\text { per } \mathrm{km}\end{array}$} \\
\hline (mm) & (inches) & & & & & \\
\hline 102 & 4.0 & 316 & 13 & 50.18 & 43.9 & 27.5 \\
\hline 114 & 4.5 & 1922 & 79 & 38.41 & 266.9 & 167.1 \\
\hline 127 & 5.0 & 3699 & 180 & 34.64 & 513.8 & 321.7 \\
\hline 140 & 5.5 & 3726 & 197 & 28.89 & 517.5 & 324.0 \\
\hline 152 & 6.0 & 2594 & 191 & 31.03 & 360.3 & 225.6 \\
\hline 165 & 6.5 & 1488 & 143 & 32.24 & 206.7 & 129.4 \\
\hline 178 & 7.0 & 961 & 125 & 34.64 & 133.5 & 83.6 \\
\hline 191 & 7.5 & 735 & 123 & 36.44 & 102.1 & 63.9 \\
\hline 203 & 8.0 & 821 & 158 & 35.01 & 114.0 & 71.4 \\
\hline 216 & 8.5 & 1298 & 293 & 34.30 & 180.3 & 112.9 \\
\hline 229 & 9.0 & 1556 & 418 & 34.37 & 216.1 & 135.3 \\
\hline 241 & 9.5 & 1594 & 515 & 35.30 & 221.4 & 138.6 \\
\hline 254 & 10.0 & 1138 & 421 & 34.72 & 158.1 & 99.0 \\
\hline 267 & 10.5 & 1113 & 478 & 35.15 & 154.6 & 96.8 \\
\hline 279 & 11.0 & 858 & 418 & 34.74 & 119.2 & 74.6 \\
\hline 292 & 11.5 & 781 & 439 & 35.05 & 108.5 & 67.9 \\
\hline 305 & 12.0 & 730 & 472 & 35.48 & 101.4 & 63.5 \\
\hline 318 & 12.5 & 1265 & 924 & 35.55 & 175.7 & 110.0 \\
\hline 330 & 13.0 & 1851 & 1499 & 35.00 & 257.1 & 161.0 \\
\hline 343 & 13.5 & 2658 & 2360 & 34.42 & 369.2 & 231.1 \\
\hline 356 & 14.0 & 2956 & 2875 & 34.00 & 410.6 & 257.0 \\
\hline 368 & 14.5 & 2551 & 2707 & 33.45 & 354.3 & 221.8 \\
\hline 381 & 15.0 & 2152 & 2483 & 32.90 & 298.9 & 187.1 \\
\hline 394 & 15.5 & 1891 & 2356 & 32.24 & 262.6 & 164.4 \\
\hline 406 & 16.0 & 1417 & 1905 & 31.69 & 196.8 & 123.2 \\
\hline 419 & 16.5 & 1258 & 1813 & 30.95 & 174.7 & 109.4 \\
\hline 432 & 17.0 & 714 & 1094 & 30.18 & 99.2 & 62.1 \\
\hline 445 & 17.5 & 609 & 1003 & 29.67 & 84.6 & 53.0 \\
\hline 457 & 18.0 & 454 & 794 & 28.92 & 63.1 & 39.5 \\
\hline 470 & 18.5 & 393 & 712 & 27.85 & 54.6 & 34.2 \\
\hline 483 & 19.0 & 293 & 567 & 27.29 & 40.7 & 25.5 \\
\hline 495 & 19.5 & 73 & 150 & 26.81 & 10.1 & 6.3 \\
\hline 508 & 20.0 & 45 & 105 & 28.25 & 6.3 & 3.9 \\
\hline 521 & 20.5 & 26 & 60 & 26.13 & 3.6 & 2.3 \\
\hline 533 & 21.0 & 19 & 50 & 26.80 & 2.6 & 1.7 \\
\hline 546 & 21.5 & 13 & 33 & 25.18 & 1.8 & 1.1 \\
\hline 559 & 22.0 & 11 & 34 & 28.66 & 1.5 & 1.0 \\
\hline 572 & 22.5 & 2 & 7 & 29.11 & 0.3 & 0.2 \\
\hline 584 & 23.0 & 0 & 0 & - & 0 & 0 \\
\hline 597 & 23.5 & 2 & 12 & 42.38 & 0.3 & 0.2 \\
\hline Total & & 45983 & 28206 & 32.77 & 6386.5 & 3998.5 \\
\hline
\end{tabular}


Table 15. Fall rainbow trout population estimates, biomass, and condition factors by length group from Rkm $0-15.4$ (RM $0-9.6$ ) below Afterbay Dam on the Bighorn River in 1985 (Collection code $=585011$ ).

\begin{tabular}{|c|c|c|c|c|c|c|}
\hline \multicolumn{2}{|c|}{ Length } & \multirow{2}{*}{$\begin{array}{l}\text { Total } \\
\text { number }\end{array}$} & \multirow{2}{*}{$\begin{array}{l}\text { Total } \\
\text { weight }\end{array}$} & \multirow{2}{*}{$\begin{array}{c}\text { Condition } \\
\text { Factor }\end{array}$} & \multirow{2}{*}{$\begin{array}{l}\text { Number } \\
\text { per mile }\end{array}$} & \multirow{2}{*}{$\begin{array}{l}\text { Number } \\
\text { per } \mathrm{km}\end{array}$} \\
\hline$(\mathrm{mm})$ & (inches) & & & & & \\
\hline 279 & 11.0 & 52 & 33 & 47.46 & 5.4 & 3.4 \\
\hline 292 & 11.5 & 52 & 42 & 49.36 & 5.4 & 3.4 \\
\hline 305 & 12.0 & 42 & 42 & 56.09 & 4.4 & 2.7 \\
\hline 318 & 12.5 & 125 & 120 & 46.66 & 13.0 & 8.1 \\
\hline 330 & 13.0 & 83 & 92 & 48.01 & 8.6 & 5.4 \\
\hline 343 & 13.5 & 83 & 96 & 45.16 & 8.6 & 5.4 \\
\hline 356 & 14.0 & 73 & 98 & 46.17 & 7.6 & 4.7 \\
\hline 368 & 14.5 & 136 & 188 & 43.69 & 14.2 & 8.8 \\
\hline 381 & 15.0 & 240 & 389 & 45.78 & 25.0 & 15.6 \\
\hline 394 & 15.5 & 354 & 621 & 45.31 & 36.9 & 23.0 \\
\hline 406 & 16.0 & 396 & 757 & 44.98 & 41.3 & 25.7 \\
\hline 419 & 16.5 & 354 & 768 & 46.58 & 36.9 & 23.0 \\
\hline 432 & 17.0 & 417 & 965 & 45.31 & 43.4 & 27.1 \\
\hline 445 & 17.5 & 313 & 764 & 44.14 & 32.6 & 20.3 \\
\hline 457 & 18.0 & 229 & 649 & 46.40 & 23.9 & 14.9 \\
\hline 470 & 18.5 & 292 & 836 & 43.60 & 30.4 & 19.0 \\
\hline 483 & 19.0 & 250 & 755 & 42.84 & 26.0 & 16.2 \\
\hline 495 & 19.5 & 136 & 415 & 40.06 & 14.2 & 8.8 \\
\hline 508 & 20.0 & 94 & 316 & 41.01 & 9.8 & 6.1 \\
\hline 521 & 20.5 & 94 & 344 & 41.76 & 9.8 & 6.1 \\
\hline 533 & 21.0 & 104 & 393 & 39.28 & 10.8 & 6.8 \\
\hline 546 & 21.5 & 63 & 239 & 37.62 & 6.6 & 4.1 \\
\hline 559 & 22.0 & 52 & 228 & 39.84 & 5.4 & 3.4 \\
\hline 572 & 22.5 & 21 & 88 & 36.42 & 2.2 & 1.4 \\
\hline 584 & 23.0 & 52 & 200 & 30.85 & 5.4 & 3.4 \\
\hline 597 & 23.5 & 21 & 105 & 38.23 & 2.2 & 1.4 \\
\hline 610 & 24.0 & 10 & 89 & & 1.0 & 0.6 \\
\hline Tota & & 4138 & 9632 & 43.56 & 431.0 & 268.7 \\
\hline
\end{tabular}


Table 16. Fall rainbow trout population estimates, biomass, and condition factors by length group from Rkm $0-15.4$ (RM 0-9.6) below Afterbay Dam on the Bighorn River in 1986 (Collection Code $=586094$ ).

\begin{tabular}{|c|c|c|c|c|c|c|}
\hline \multicolumn{2}{|c|}{ Length } & \multirow{2}{*}{$\begin{array}{l}\text { Total } \\
\text { number }\end{array}$} & \multirow{2}{*}{$\begin{array}{l}\text { Total } \\
\text { weight }\end{array}$} & \multirow{2}{*}{$\begin{array}{c}\text { Condition } \\
\text { Factor }\end{array}$} & \multirow{2}{*}{$\begin{array}{l}\text { Number } \\
\text { per mile }\end{array}$} & \multirow{2}{*}{$\begin{array}{l}\text { Number } \\
\text { per } \mathrm{km}\end{array}$} \\
\hline$(\mathrm{mm})$ & (inches) & & & & & \\
\hline 127 & 5.0 & 118 & 8 & 46.90 & 12.3 & 7.7 \\
\hline 140 & 5.5 & 105 & 9 & 46.91 & 10.9 & 6.8 \\
\hline 152 & 6.0 & 158 & 16 & 42.67 & 16.5 & 10.3 \\
\hline 165 & 6.5 & 204 & 28 & 44.77 & 21.3 & 13.2 \\
\hline 178 & 7.0 & 223 & 37 & 44.32 & 23.2 & 14.5 \\
\hline 191 & 7.5 & 375 & 77 & 44.42 & 39.1 & 24.4 \\
\hline 203 & 8.0 & 486 & 119 & 44.48 & 50.6 & 31.6 \\
\hline 216 & 8.5 & 539 & 154 & 43.59 & 56.1 & 35.0 \\
\hline 229 & 9.0 & 427 & 142 & 42.70 & 44.5 & 27.7 \\
\hline 241 & 9.5 & 289 & 110 & 41.81 & 30.1 & 18.8 \\
\hline 254 & 10.0 & 151 & 64 & 40.29 & 15.7 & 9.8 \\
\hline 267 & 10.5 & 72 & 37 & $42 \cdot 22$ & 7.5 & 4.7 \\
\hline 279 & 11.0 & 45 & 25 & 40.03 & 4.7 & 2.9 \\
\hline 292 & 11.5 & 72 & 49 & 41.91 & 7.5 & 4.7 \\
\hline 305 & 12.0 & 99 & 75 & 40.83 & 10.3 & 6.4 \\
\hline 318 & 12.5 & 162 & 132 & 39.90 & 16.9 & 10.5 \\
\hline 330 & 13.0 & 153 & 141 & 40.16 & 15.9 & 9.9 \\
\hline 343 & 13.5 & 189 & 193 & 39.46 & 19.7 & 12.3 \\
\hline 356 & 14.0 & 189 & 212 & 39.20 & 19.7 & 12.3 \\
\hline 368 & 14.5 & 256 & 323 & 39.80 & 26.7 & 16.6 \\
\hline 381 & 15.0 & 189 & 260 & 39.00 & 19.7 & 12.3 \\
\hline 394 & 15.5 & 300 & 445 & 38.38 & 31.3 & 19.5 \\
\hline 406 & 16.0 & 398 & 641 & 37.97 & 41.5 & 25.8 \\
\hline 419 & 16.5 & 398 & 722 & 38.89 & 41.5 & 25.8 \\
\hline 432 & 17.0 & 371 & 714 & 37.95 & 38.6 & 24.1 \\
\hline 445 & 17.5 & 453 & 940 & 37.38 & 47.2 & 29.4 \\
\hline 457 & 18.0 & 333 & 750 & 37.37 & 34.7 & 21.6 \\
\hline 470 & 18.5 & 289 & 692 & 36.79 & 30.1 & 18.8 \\
\hline 483 & 19.0 & 164 & 417 & 36.44 & 17.1 & 10.6 \\
\hline 495 & 19.5 & 147 & 389 & 34.79 & 15.3 & 9.5 \\
\hline 508 & 20.0 & 55 & 154 & 33.78 & 5.7 & 3.6 \\
\hline 521 & 20.5 & 55 & 167 & 34.69 & 5.7 & 3.6 \\
\hline 533 & 21.0 & 49 & 156 & 33.29 & 5.1 & 3.2 \\
\hline 546 & 21.5 & 65 & 228 & 34.30 & 6.8 & 4.2 \\
\hline 559 & 22.0 & 11 & 41 & 34.90 & 1.1 & 0.7 \\
\hline 572 & 22.5 & 27 & 107 & 33.75 & 2.8 & 1.8 \\
\hline 584 & 23.0 & 5 & 23 & 35.26 & 0.5 & 0.3 \\
\hline Tota. & & 7621 & 8797 & 39.49 & 793.9 & 494.9 \\
\hline
\end{tabular}


Table 17. Fall rainbow trout population estimates, biomass, and condition factors by length group from $\mathrm{Rkm} 0-15.4$ (RM $0-9.6$ ) below Afterbay Dam on the Bighorn River in 1987 (Collection code = 587099).

\begin{tabular}{|c|c|c|c|c|c|c|}
\hline \multicolumn{2}{|c|}{ Length } & \multirow{2}{*}{$\begin{array}{l}\text { Total } \\
\text { number }\end{array}$} & \multirow{2}{*}{$\begin{array}{l}\text { Total } \\
\text { weight }\end{array}$} & \multirow{2}{*}{$\begin{array}{l}\text { Condition } \\
\text { Factor }\end{array}$} & \multirow{2}{*}{$\begin{array}{c}\text { Number } \\
\text { per mile }\end{array}$} & \multirow{2}{*}{$\begin{array}{l}\text { Number } \\
\text { per km }\end{array}$} \\
\hline$(\mathrm{mm})$ & (inches) & & & & & \\
\hline 127 & 5.0 & 20 & 1 & 39.20 & 2.1 & 1.3 \\
\hline 140 & 5.5 & 20 & 2 & 44.73 & 2.1 & 1.3 \\
\hline 152 & 6.0 & 20 & 2 & 40.05 & 2.1 & 1.3 \\
\hline 165 & 6.5 & 10 & 1 & 33.25 & 1.0 & 0.6 \\
\hline 178 & 7.0 & 20 & 3 & 41.13 & 2.1 & 1.3 \\
\hline 191 & 7.5 & 70 & 15 & 48.38 & 7.3 & 4.5 \\
\hline 203 & 8.0 & 50 & 13 & 49.72 & 5.2 & 3.2 \\
\hline 216 & 8.5 & 110 & 37 & 49.91 & 11.5 & 7.1 \\
\hline 229 & 9.0 & 100 & 35 & 44.66 & 10.4 & 6.5 \\
\hline 241 & 9.5 & 190 & 82 & 46.91 & 19.8 & 12.3 \\
\hline 254 & 10.0 & 359 & 175 & 46.39 & 37.4 & 23.3 \\
\hline 267 & 10.5 & 469 & 265 & 45.75 & 48.9 & 30.5 \\
\hline 279 & 11.0 & 509 & 322 & 44.72 & 53.0 & 33.1 \\
\hline 292 & 11.5 & 569 & 410 & 44.76 & 59.3 & 36.9 \\
\hline 305 & 12.0 & 539 & 437 & 44.31 & 56.1 & 35.0 \\
\hline 318 & 12.5 & 759 & 688 & 44.30 & 79.1 & 49.3 \\
\hline 330 & 13.0 & 664 & 659 & 43.09 & 69.2 & 43.1 \\
\hline 343 & 13.5 & 655 & 735 & 43.70 & 68.2 & 42.5 \\
\hline 356 & 14.0 & 522 & 643 & 42.98 & 54.4 & 33.9 \\
\hline 368 & 14.5 & 655 & 886 & 42.32 & 68.2 & 42.5 \\
\hline 381 & 15.0 & 418 & 624 & 42.71 & 43.5 & 27.1 \\
\hline 394 & 15.5 & 541 & 851 & 40.53 & 56.4 & 35.1 \\
\hline 406 & 16.0 & 437 & 747 & 40.22 & 45.5 & 28.4 \\
\hline 419 & 16.5 & 456 & 883 & 41.50 & 47.5 & 29.6 \\
\hline 432 & 17.0 & 184 & 372 & 39.87 & 19.2 & 11.9 \\
\hline 445 & 17.5 & 267 & 578 & 39.20 & 27.8 & 17.3 \\
\hline 457 & 18.0 & 254 & 597 & 38.98 & 26.5 & 16.5 \\
\hline 470 & 18.5 & 263 & 664 & 38.60 & 27.4 & 17.1 \\
\hline 483 & 19.0 & 68 & 186 & 38.91 & 7.1 & 4.4 \\
\hline 495 & 19.5 & 68 & 196 & 37.97 & 7.1 & 4.4 \\
\hline 508 & 20.0 & 46 & 137 & 36.45 & 4.8 & 3.0 \\
\hline 521 & 20.5 & 29 & 95 & 36.95 & 3.0 & 1.9 \\
\hline 533 & 21.0 & 22 & 76 & 36.32 & 2.3 & 1.4 \\
\hline 546 & 21.5 & 10 & 43 & 43.10 & 1.0 & 0.6 \\
\hline 559 & 22.0 & 7 & 29 & 36.49 & 0.7 & 0.5 \\
\hline 572 & 22.5 & 0 & 0 & - & 0.0 & 0.0 \\
\hline 584 & 23.0 & 0 & 0 & - & 0.0 & 0.0 \\
\hline 597 & 23.5 & 2 & 11 & 33.60 & 0.2 & 0.1 \\
\hline Tota & & 9382 & 11500 & 41.71 & 977.3 & 609.2 \\
\hline
\end{tabular}


Table 18. Spring rainbow trout population estimates, biomass, and condition factors by length group from Rkm 0-15.4 (RM 0-9.6) below Afterbay Dam on the Bighorn River in 1985 (Collection Code $=585095$ ).

\begin{tabular}{|c|c|c|c|c|c|c|}
\hline \multicolumn{2}{|c|}{ Length } & \multirow{2}{*}{$\begin{array}{l}\text { Total } \\
\text { number }\end{array}$} & \multirow{2}{*}{$\begin{array}{l}\text { Total } \\
\text { weight }\end{array}$} & \multirow{2}{*}{$\begin{array}{c}\text { Condition } \\
\text { Factor }\end{array}$} & \multirow{2}{*}{$\begin{array}{c}\text { Number } \\
\text { per mile }\end{array}$} & \multirow{2}{*}{$\begin{array}{l}\text { Number } \\
\text { per km }\end{array}$} \\
\hline$(\mathrm{mm})$ & (inches) & & & & & \\
\hline 267 & 10.5 & 9 & 6 & 55.51 & 0.9 & 0.6 \\
\hline 279 & 11.0 & 90 & 54 & 43.05 & 9.4 & 5.8 \\
\hline 292 & 11.5 & 81 & 53 & 40.70 & 8.4 & 5.3 \\
\hline 305 & 12.0 & 179 & 134 & 41.38 & 18.6 & 11.6 \\
\hline 318 & 12.5 & 206 & 180 & 42.40 & 21.5 & 13.4 \\
\hline 330 & 13.0 & 431 & 426 & 42.97 & 44.9 & 28.0 \\
\hline 343 & 13.5 & 296 & 310 & 40.65 & 30.8 & 19.2 \\
\hline 356 & 14.0 & 269 & 319 & 41.56 & 28.0 & 17.5 \\
\hline 368 & 14.5 & 449 & 582 & 40.83 & 46.8 & 29.2 \\
\hline 381 & 15.0 & 296 & 439 & 42.26 & 30.8 & 19.2 \\
\hline 394 & 15.5 & 368 & 585 & 41.03 & 38.3 & 23.9 \\
\hline 406 & 16.0 & 431 & 735 & 40.04 & 44.9 & 28.0 \\
\hline 419 & 16.5 & 419 & 781 & 39.88 & 43.6 & 27.2 \\
\hline 432 & 17.0 & 312 & 647 & 40.75 & 32.5 & 20.3 \\
\hline 445 & $17 \cdot 5$ & 518 & 1183 & 41.18 & 54.0 & 33.6 \\
\hline 457 & 18.0 & 391 & 925 & 39.32 & 40.7 & 25.4 \\
\hline 470 & 18.5 & 447 & 1137 & 38.89 & 46.6 & 29.0 \\
\hline 483 & 19.0 & 305 & 838 & 38.79 & 31.8 & 19.8 \\
\hline 495 & 19.5 & 334 & 1002 & 39.05 & 34.8 & 21.7 \\
\hline 508 & 20.0 & 355 & 1169 & 39.90 & 37.0 & 23.1 \\
\hline 521 & 20.5 & 241 & 802 & 37.59 & 25.1 & 15.6 \\
\hline 533 & 21.0 & 220 & 795 & 37.98 & 22.9 & 14.3 \\
\hline 546 & 21.5 & 199 & 766 & 37.71 & 20.7 & 12.9 \\
\hline 559 & 22.0 & 142 & 564 & 36.51 & 14.8 & 9.2 \\
\hline 572 & 22.5 & 50 & 234 & 39.73 & 5.2 & 3.2 \\
\hline 584 & 23.0 & 57 & 260 & 36.56 & 5.9 & 3.7 \\
\hline 597 & 23.5 & 21 & 86 & 30.88 & 2.2 & 1.4 \\
\hline 610 & 24.0 & 28 & 138 & 34.53 & 2.9 & 1.8 \\
\hline 622 & 24.5 & 0 & 0 & - & 0.0 & 0.0 \\
\hline 635 & 25.0 & 7 & 32 & 28.80 & 0.7 & 0.5 \\
\hline Total & & 7151 & 15182 & 39.67 & 744.9 & 464.4 \\
\hline
\end{tabular}


Table 19. Spring rainbow trout population estimates, biomass, and condition factors by length group from $\mathrm{Rkm} 0-15.4$ (RM 0-9.6) below Afterbay Dam on the Bighorn River in 1986 (Collection Code $=586008$ ).

\begin{tabular}{|c|c|c|c|c|c|c|}
\hline \multicolumn{2}{|c|}{ Length } & \multirow{2}{*}{$\begin{array}{l}\text { Total } \\
\text { number }\end{array}$} & \multirow{2}{*}{$\begin{array}{l}\text { Total } \\
\text { weight }\end{array}$} & \multirow{2}{*}{$\begin{array}{c}\text { Condition } \\
\text { Factor }\end{array}$} & \multirow{2}{*}{$\begin{array}{c}\text { Number } \\
\text { per mile }\end{array}$} & \multirow{2}{*}{$\begin{array}{l}\text { Number } \\
\text { per km }\end{array}$} \\
\hline$\overline{(\mathrm{mm})}$ & (inches) & & & & & \\
\hline 305 & 12.0 & 33 & 25 & 41.94 & 3.4 & 2.1 \\
\hline 318 & 12.5 & 109 & 94 & 42.22 & 11.4 & 7.1 \\
\hline 330 & 13.0 & 38 & 36 & 40.99 & 4.0 & 2.5 \\
\hline 343 & 13.5 & 76 & 78 & 40.37 & 7.9 & 4.9 \\
\hline 356 & 14.0 & 141 & 177 & 43.57 & 14.7 & 9.2 \\
\hline 368 & 14.5 & 158 & 209 & 41.44 & 16.5 & 10.3 \\
\hline 381 & 15.0 & 212 & 323 & 43.15 & 22.1 & 13.8 \\
\hline 394 & 15.5 & 228 & 385 & 43.65 & 23.8 & 14.8 \\
\hline 406 & 16.0 & 266 & 493 & 43.32 & 27.7 & 17.3 \\
\hline 419 & 16.5 & 315 & 615 & 41.80 & 32.8 & 20.5 \\
\hline 432 & 17.0 & 402 & 863 & 42.18 & 41.9 & 26.1 \\
\hline 445 & 17.5 & 408 & 969 & 42.78 & 42.5 & 26.5 \\
\hline 457 & 18.0 & 386 & 969 & 41.68 & 40.2 & 25.1 \\
\hline 470 & 18.5 & 193 & 504 & 40.04 & 20.1 & 12.5 \\
\hline 483 & 19.0 & 176 & 506 & 40.59 & 18.3 & 11.4 \\
\hline 495 & 19.5 & 160 & 494 & 40.18 & 16.7 & 10.4 \\
\hline 508 & 20.0 & 98 & 319 & 39.25 & 10.2 & 6.4 \\
\hline 521 & 20.5 & 115 & 403 & 39.62 & 12.0 & 7.5 \\
\hline 533 & 21.0 & 66 & 238 & 37.77 & 6.9 & 4.3 \\
\hline 546 & 21.5 & 53 & 228 & 41.98 & 5.5 & 3.4 \\
\hline 559 & 22.0 & 62 & 264 & 39.14 & 6.5 & 4.0 \\
\hline 572 & 22.5 & 29 & 120 & 35.60 & 3.0 & 1.9 \\
\hline 584 & 23.0 & 16 & 75 & 36.28 & 1.7 & 1.0 \\
\hline 597 & 23.5 & 16 & 92 & 42.47 & 1.7 & 1.0 \\
\hline 610 & 24.0 & 8 & 38 & 33.38 & 0.8 & 0.5 \\
\hline \multicolumn{2}{|c|}{ Total } & 3764 & 8517 & 40.62 & 392.1 & 244.4 \\
\hline
\end{tabular}


Table 20. Spring rainbow trout population estimates, biomass, and condition factors by length group from $\mathrm{Rkm} 0-15.4$ (RM 0-9.6) below Afterbay Dam on the Bighorn River in 1987 (Collection Code $=587059$ ).

\begin{tabular}{|c|c|c|c|c|c|c|}
\hline \multicolumn{2}{|c|}{ Length } & \multirow{2}{*}{$\begin{array}{l}\text { Total } \\
\text { number }\end{array}$} & \multirow{2}{*}{$\begin{array}{l}\text { Total } \\
\text { weight }\end{array}$} & \multirow{2}{*}{$\begin{array}{c}\text { Condition } \\
\text { Factor }\end{array}$} & \multirow{2}{*}{$\begin{array}{c}\text { Number } \\
\text { per mile }\end{array}$} & \multirow{2}{*}{$\begin{array}{l}\text { Number } \\
\text { per } \mathrm{km}\end{array}$} \\
\hline$(\mathrm{mm})$ & (inches) & & & & & \\
\hline 89 & 3.5 & 33 & 1 & 61.62 & 3.4 & 2.1 \\
\hline 102 & 4.0 & 375 & 15 & 49.72 & 39.1 & 24.4 \\
\hline 114 & 4.5 & 739 & 31 & 39.90 & 77.0 & 48.0 \\
\hline 127 & 5.0 & 882 & 44 & 36.08 & 91.9 & 57.3 \\
\hline 140 & 5.5 & 1003 & 54 & 29.04 & 104.5 & 65.1 \\
\hline 152 & 6.0 & 1400 & 119 & 35.06 & 145.8 & 90.9 \\
\hline 165 & 6.5 & 1290 & 138 & 35.63 & 134.4 & 83.8 \\
\hline 178 & 7.0 & 1069 & 145 & 36.65 & 111.4 & 69.4 \\
\hline 191 & 7.5 & 661 & 111 & 36.67 & 68.9 & 42.9 \\
\hline 203 & 8.0 & 430 & 91 & 38.37 & 44.8 & 27.9 \\
\hline 216 & 8.5 & 408 & 108 & 40.34 & 42.5 & 26.5 \\
\hline 229 & 9.0 & 386 & 124 & 41.89 & 40.2 & 25.1 \\
\hline 241 & 9.5 & 195 & 71 & 39.96 & 20.3 & 12.7 \\
\hline 254 & 10.0 & 195 & 82 & 39.46 & 20.3 & 12.7 \\
\hline 267 & 10.5 & 159 & 77 & 39.73 & 16.6 & 10.3 \\
\hline 279 & 11.0 & 183 & 104 & 40.23 & 19.1 & 11.9 \\
\hline 292 & 11.5 & 208 & 130 & 39.27 & 21.7 & 13.5 \\
\hline 305 & 12.0 & 171 & 120 & 38.73 & 17.8 & 11.1 \\
\hline 318 & 12.5 & 128 & 103 & 39.31 & 13.3 & 8.3 \\
\hline 330 & 13.0 & 116 & 105 & 38.63 & 12.1 & 7.5 \\
\hline 343 & 13.5 & 102 & 105 & 39.75 & 10.6 & 6.6 \\
\hline 356 & 14.0 & 106 & 121 & 39.73 & 11.0 & 6.9 \\
\hline 368 & 14.5 & 177 & 216 & 38.01 & 18.4 & 11.5 \\
\hline 381 & 15.0 & 217 & 294 & 38.50 & 22.6 & 14.1 \\
\hline 394 & 15.5 & 186 & 282 & 39.20 & 19.4 & 12.1 \\
\hline 406 & 16.0 & 279 & 443 & 37.28 & 29.1 & 18.1 \\
\hline 419 & 16.5 & 310 & 541 & 37.26 & 32.3 & 20.1 \\
\hline 432 & 17.0 & 256 & 474 & 36.38 & 26.7 & 16.6 \\
\hline 445 & 17.5 & 301 & 596 & 35.67 & 31.4 & 19.5 \\
\hline 457 & 18.0 & 252 & 542 & 35.84 & 26.3 & 16.4 \\
\hline 470 & 18.5 & 270 & 630 & 35.75 & 28.1 & 17.5 \\
\hline 483 & 19.0 & 231 & 566 & 34.84 & 24.1 & 15.0 \\
\hline 495 & 19.5 & 119 & 312 & 34.55 & 12.4 & 7.7 \\
\hline 508 & 20.0 & 92 & 248 & 33.08 & 9.6 & 6.0 \\
\hline 521 & 20.5 & 37 & 115 & 34.96 & 3.9 & 2.4 \\
\hline 533 & 21.0 & 20 & 63 & 32.10 & 2.1 & 1.3 \\
\hline 546 & 21.5 & 31 & 104 & 33.35 & 3.2 & 2.0 \\
\hline 559 & 22.0 & 0 & 0 & - & 0 & 0 \\
\hline 572 & 22.5 & 7 & 28 & 35.46 & 0.7 & 0.5 \\
\hline 584 & 23.0 & 7 & 35 & 41.19 & 0.7 & 0.5 \\
\hline Total & & 13031 & 7488 & 38.18 & 1357.4 & 846.2 \\
\hline
\end{tabular}


APPENDIX D

Rainbow Trout Embryo Development Rates 
Table 21. Developmental rates in number of days and daily temperature units (DTU) of rainbow trout embryos planted 8 May 1985 at the three early life history sites on the Bighorn River, Montana.

\begin{tabular}{llccc}
\hline \multirow{2}{*}{$\begin{array}{c}\text { River } \\
\mathrm{km}\end{array}$} & \multicolumn{2}{c}{ Eyed stage } & & \multicolumn{2}{c}{ Hatching } \\
\cline { 2 - 3 } \cline { 5 - 5 } & Days & DTU & Days & DTU \\
\hline 2.4 & 45 & 470 & $58-68$ & $625-760$ \\
8.0 & 41 & 525 & $53-57$ & $700-740$ \\
14.5 & 31 & 525 & $44-48$ & $750-825$ \\
\hline
\end{tabular}


APPENDIX E

Incidence of Gas Bubble Trauma 
Table 22. Incidence of gas bubble trauma (GBT) in brown trout from Rkm 0-1.9 of the Bighorn River between 8 March $1985-7$ January 1986. (MDFWP and MCFRU data).

\begin{tabular}{|c|c|c|c|c|c|c|}
\hline \multirow[t]{2}{*}{ Date } & \multirow[t]{2}{*}{ Time } & \multicolumn{2}{|c|}{ All brown trout } & \multicolumn{3}{|c|}{ Brown trout $\geq 356 \mathrm{~mm}$} \\
\hline & & No. caught & $\% \mathrm{~W} / \mathrm{GBT}$ & No. caught & $q$ & $\mathrm{w} / \mathrm{GBT}$ \\
\hline $3 / 8$ & day & 123 & 8 & 62 & & 8 \\
\hline $3 / 22$ & $"$ & 62 & 11 & 40 & & 18 \\
\hline $4 / 10$ & $"$ & 44 & 16 & 19 & & 26 \\
\hline $4 / 18$ & $"$ & 62 & 21 & 22 & & 55 \\
\hline $5 / 3$ & $"$ & 143 & 28 & 47 & & 47 \\
\hline $5 / 11$ & $"$ & 92 & 18 & 19 & & 63 \\
\hline $5 / 20$ & $"$ & 102 & 7 & 24 & & 25 \\
\hline $6 / 4$ & $"$ & 75 & 19 & 28 & & 29 \\
\hline $6 / 27$ & night & 201 & 14 & 93 & & 16 \\
\hline $7 / 18$ & $"$ & 206 & 5 & 80 & & 10 \\
\hline $8 / 1$ & $n$ & 170 & 7 & 53 & & 15 \\
\hline $8 / 14$ & $"$ & 147 & 8 & 70 & & 13 \\
\hline $8 / 25$ & $"$ & 146 & 8 & 91 & & 7 \\
\hline $9 / 18 \& 20$ & day & 146 & 14 & 43 & & 35 \\
\hline $9 / 30-10 / 2$ & 21 & 177 & 18 & 87 & & 24 \\
\hline $10 / 18$ & night & 161 & 4 & 89 & & 5 \\
\hline $10 / 31$ & $"$ & 148 & 1 & 107 & & 2 \\
\hline $11 / 14$ & $"$ & 173 & 2 & 106 & & 2 \\
\hline $12 / 13$ & day & 147 & 5 & 107 & & 7 \\
\hline $1 / 7 / 86$ & $"$ & 156 & 17 & 95 & & 25 \\
\hline
\end{tabular}


Table 23. Incidence of gas bubble trauma (GBT) in rainbow trout from Rkm 0-1.9 of the Bighorn River from 8 March 1985 - 7 January 1986. (MDFWP and MCFRU data).

\begin{tabular}{|c|c|c|c|c|c|c|c|c|}
\hline \multirow[t]{2}{*}{ Date } & \multirow[t]{2}{*}{ Time } & \multicolumn{3}{|c|}{ A11 rainbow trout } & \multicolumn{4}{|c|}{ Rainbow trout $\geq 356 \mathrm{~mm}$} \\
\hline & & No. & caught & $\% \mathrm{w} / \mathrm{GBT}$ & No. & caught & $\%$ & $w / G B T$ \\
\hline $3 / 8$ & day & & 13 & 8 & & 8 & & 13 \\
\hline $3 / 22$ & $n$ & & 9 & 0 & & 4 & & 0 \\
\hline $4 / 10$ & $"$ & & 7 & 14 & & 4 & & 14 \\
\hline $4 / 18$ & 11 & & 15 & 13 & & 5 & & 0 \\
\hline $5 / 3$ & " & & 37 & 16 & & 14 & & 29 \\
\hline $5 / 11$ & $"$ & & 29 & 3 & & 9 & & 11 \\
\hline $5 / 20$ & $n$ & & 9 & 0 & & 4 & & 0 \\
\hline $6 / 4$ & n & & 7 & 0 & & 2 & & 0 \\
\hline $6 / 27$ & night & & 17 & 0 & & 8 & & 0 \\
\hline $7 / 18$ & $n$ & & 11 & 0 & & 9 & & 0 \\
\hline $8 / 1$ & "1 & & 13 & 0 & & 8 & & 0 \\
\hline $8 / 14$ & $n$ & & 23 & 0 & & 14 & & 0 \\
\hline $8 / 25$ & $n$ & & 29 & 3 & & 20 & & 5 \\
\hline $9 / 18 \& 20$ & day & & 18 & 0 & & 14 & & 0 \\
\hline $9 / 30-10 /$ & $12 "$ & & 31 & 6 & & 18 & & 11 \\
\hline $10 / 18$ & night & & 13 & 0 & & 9 & & 0 \\
\hline $10 / 31$ & n & & 33 & 0 & & 25 & & 0 \\
\hline $11 / 14$ & $"$ & & 20 & 5 & & 16 & & 6 \\
\hline $12 / 13$ & day & & 15 & 0 & & 10 & & 0 \\
\hline $1 / 7 / 86$ & १ & & 11 & 0 & & 10 & & 0 \\
\hline
\end{tabular}


Table 24. Incidence of gas bubble trauma (GBT) in brown trout from Rkm 3.9-6.1 on the Bighorn River, 18 April 1985 - 7 January 1986. (MDFWP and MCFRU data).

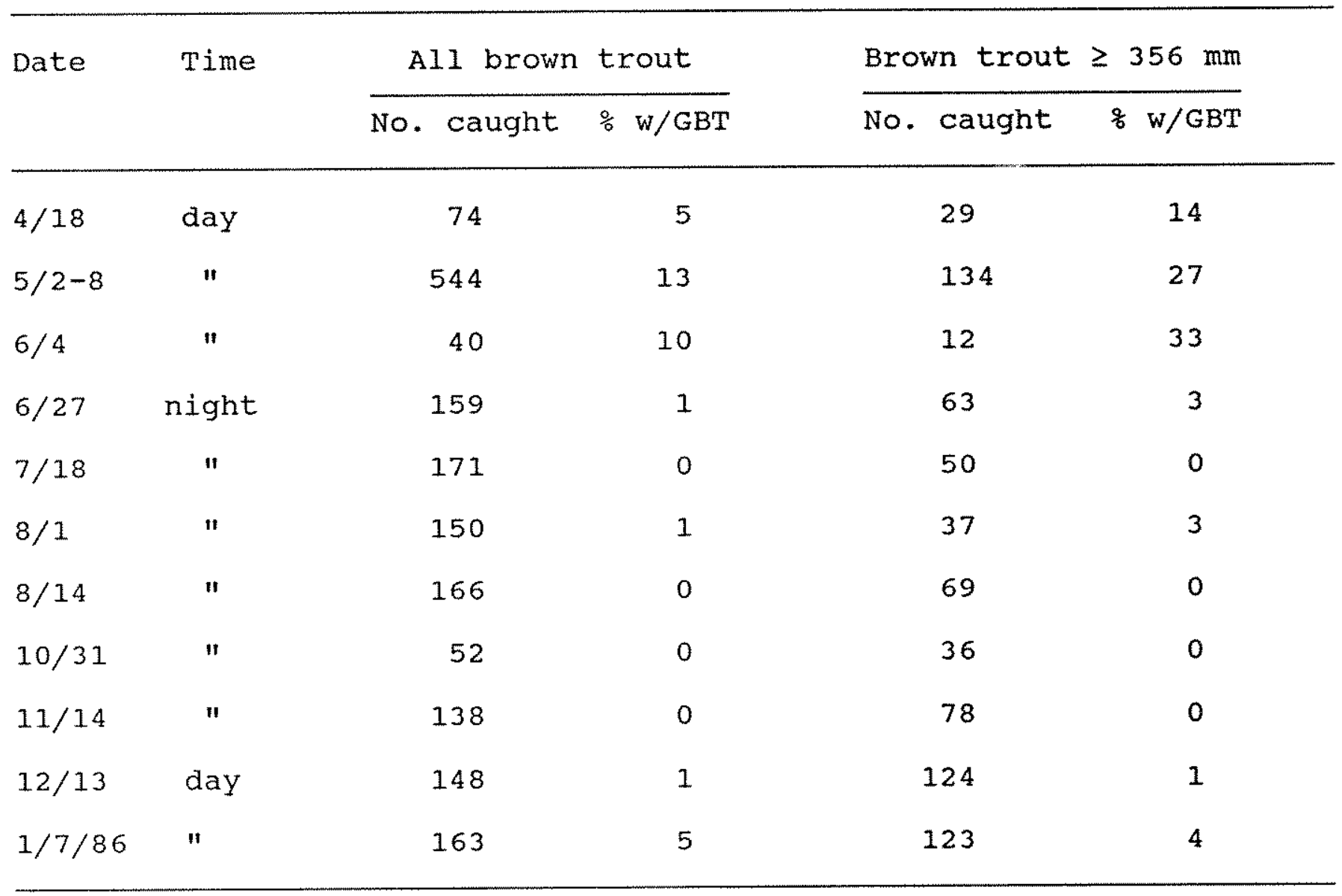


Table 25. Incidence of gas bubble trauma (GBT) in rainbow trout from Rkm 3.9-6.1 on the Bighorn River, 18 April 1985 - 7 January 1986. (MDFWP and MCFRU data).

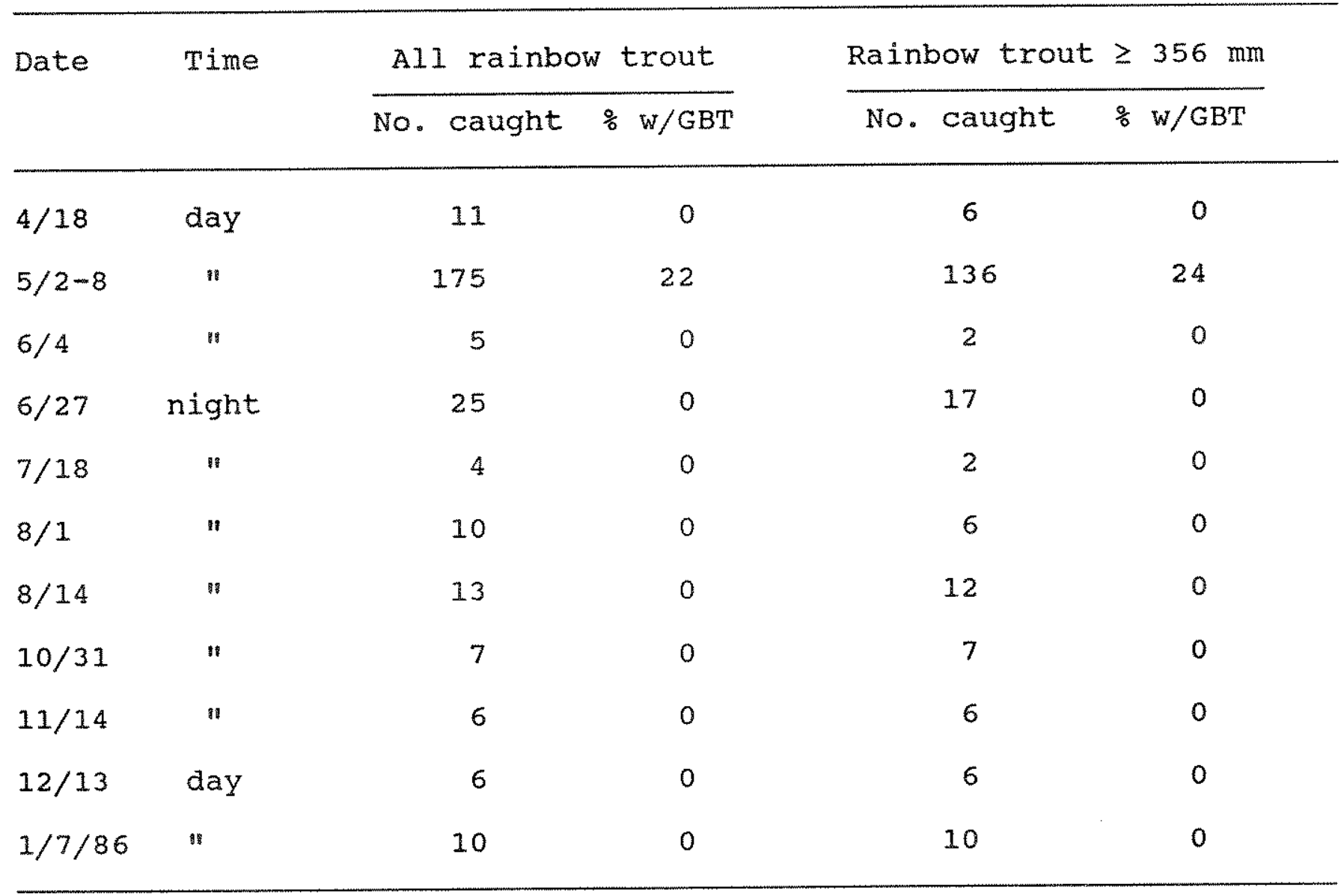


Table 26. Incidence of external symptoms of GBT in brown trout from subsections of the 15.4 river kilometers below Afterbay Dam on the Bighorn River, 2-3 May 1985.

\begin{tabular}{|c|c|c|c|c|c|c|}
\hline \multirow{2}{*}{$\begin{array}{c}\text { River } \\
\mathrm{km}\end{array}$} & \multicolumn{2}{|c|}{ All brown trout } & \multicolumn{4}{|c|}{ Brown trout $\geq 356 \mathrm{~mm}$} \\
\hline & No. caught & $\% \mathrm{w} / \mathrm{GBT}$ & No. & caught & 웅 & $\mathrm{w} / \mathrm{GBT}$ \\
\hline $0.0-1.8$ & 143 & 28 & & 47 & & 47 \\
\hline $3.9-6.1$ & 75 & 19 & & 22 & & 32 \\
\hline $6.1-9.3$ & 138 & 10 & & 74 & & 16 \\
\hline $9.3-11.3$ & 110 & 2 & & 65 & & 2 \\
\hline $11.3-15.4$ & 140 & 1 & & 85 & & 2 \\
\hline
\end{tabular}


Table 27. Incidence of gas bubble trauma (GBT) in brown trout from Rkm 0-1.9 (section 1 ) of the Bighorn River during 1986 (data are from right bank unless noted otherwise).

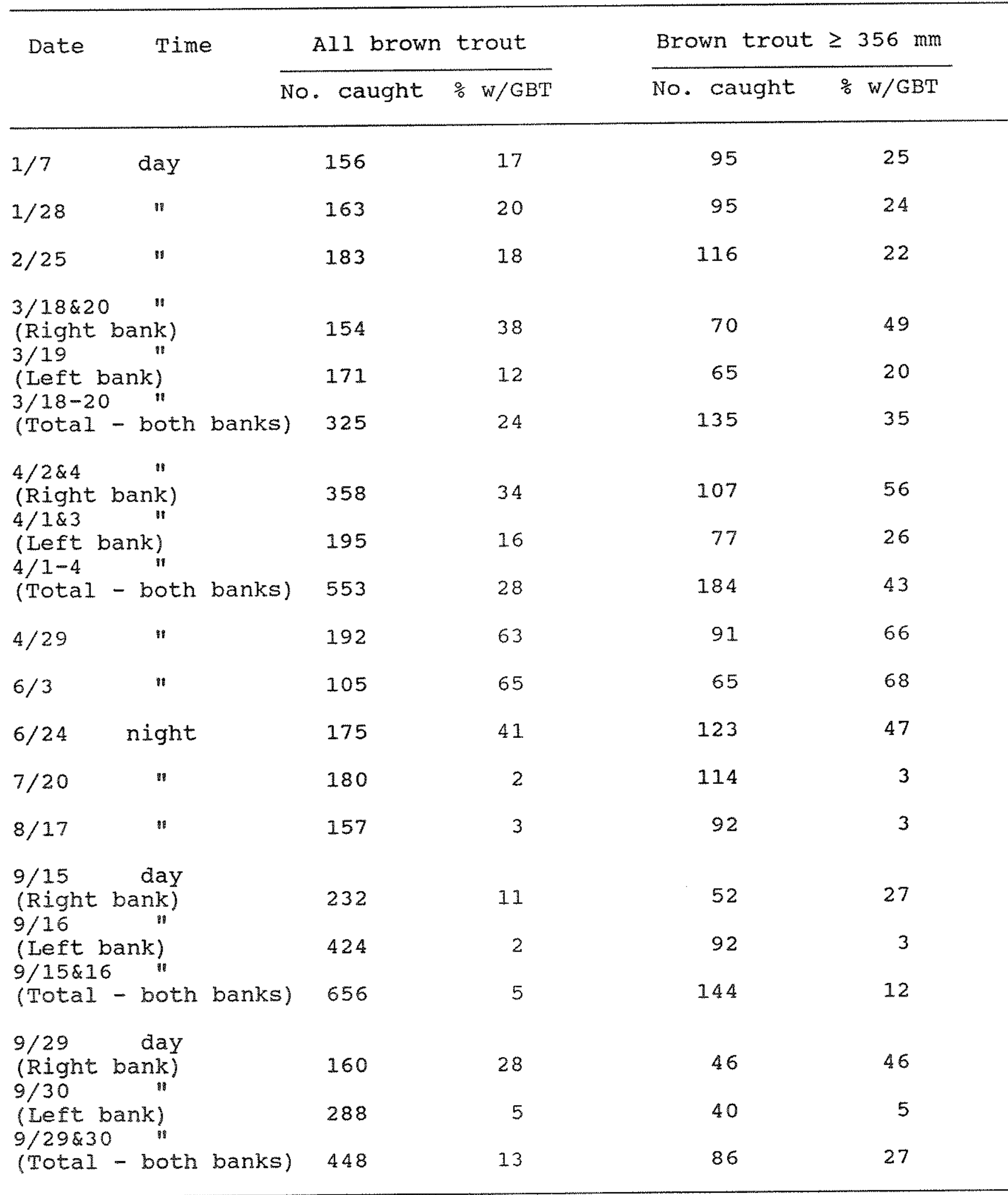


Table 27. (continued).

\begin{tabular}{lcccccc}
\hline \multirow{2}{*}{ Date } & Time & \multicolumn{2}{c}{ All brown trout } & & \multicolumn{2}{c}{ Brown trout $\geq 356 \mathrm{~mm}$} \\
\cline { 3 - 5 } & & No. caught $\%$ w/GBT & & No. caught $\%$ w/GBT \\
\hline $11 / 14$ & day & 174 & 30 & 57 & 36 & 50 \\
$12 / 16$ & " & 192 & 32 & 30 & 44 & 55 \\
$2 / 4 / 87$ & 168 & & &
\end{tabular}

1

1

$\mid$

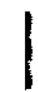

1

I

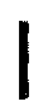


Table 28. Incidence of gas bubble trauma (GBT) in rainbow trout from $R \mathrm{~km}$ 0-1.9 (section 1 ) of the Bighorn River during 1986 (data are from right bank unless noted otherwise).

Date Time $\frac{A 11 \text { rainbow trout }}{\text { No. caught } \% \mathrm{w} / \mathrm{GBT}} \quad \frac{\text { Rainbow trout } \geq 356 \mathrm{~mm}}{\text { No. caught } \% \mathrm{w} / \mathrm{GBT}}$

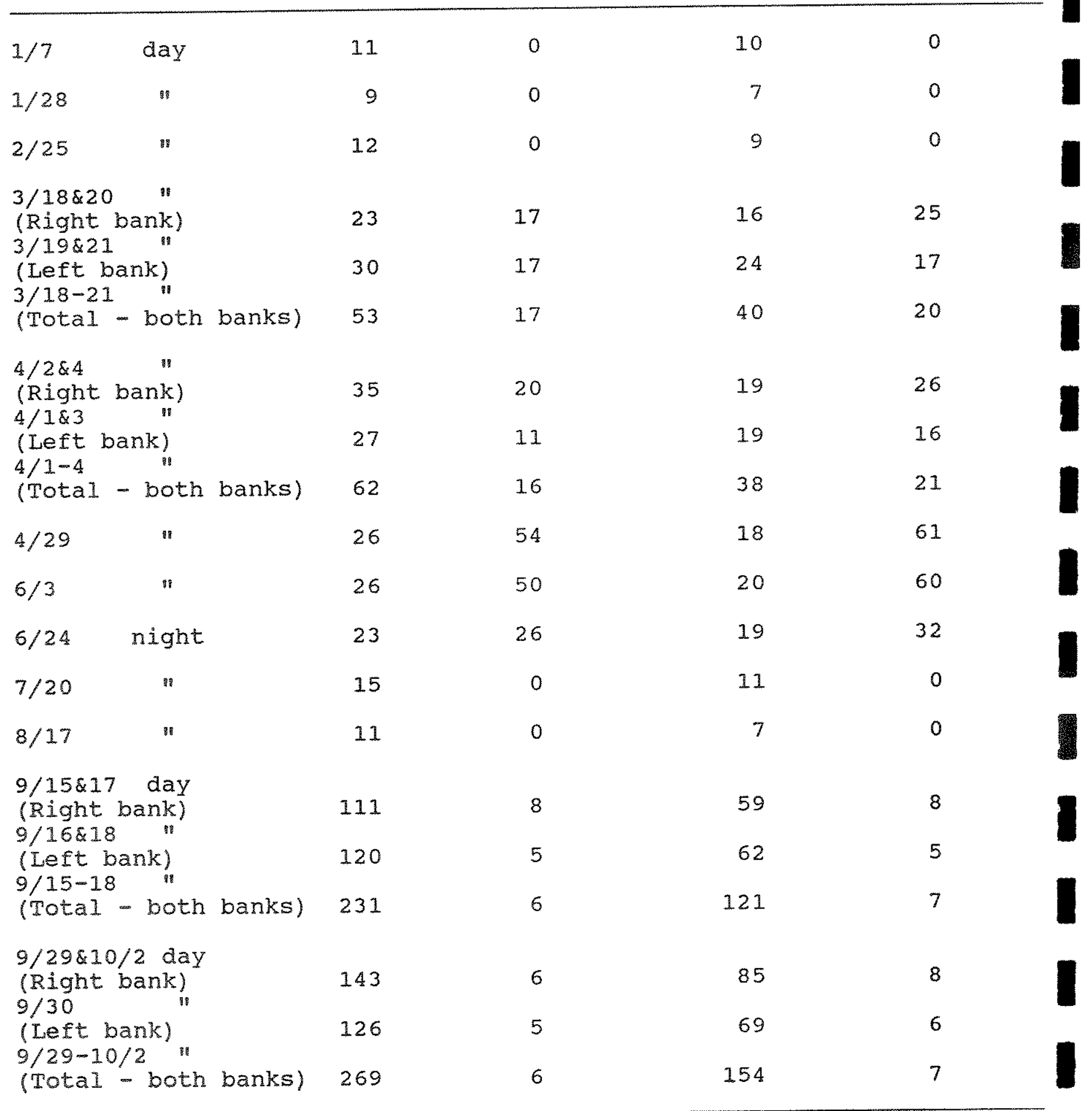


Table 28. (continued).

\begin{tabular}{lcccccc}
\hline Date & Time & \multicolumn{2}{c}{ All rainbow trout } & & \multicolumn{2}{c}{ Rainbow trout $\geq 356 \mathrm{~mm}$} \\
\cline { 5 - 6 } & & No. caught $\%$ w/GBT & & No. caught & $\%$ w/GBT \\
\hline $11 / 14$ & day & 18 & 17 & 5 & 60 \\
$12 / 16$ & " & 41 & 10 & 8 & 2 & 13 \\
$2 / 4 / 87$ & " & 26 & 8 & & 2 & 0 \\
\hline
\end{tabular}


Table 29. Incidence of gas bubble trauma (GBT) in brown trout from Rkm 3.9-6.1 (section 2) on the Bighorn River during 1986 (data are from both banks unless noted otherwise).

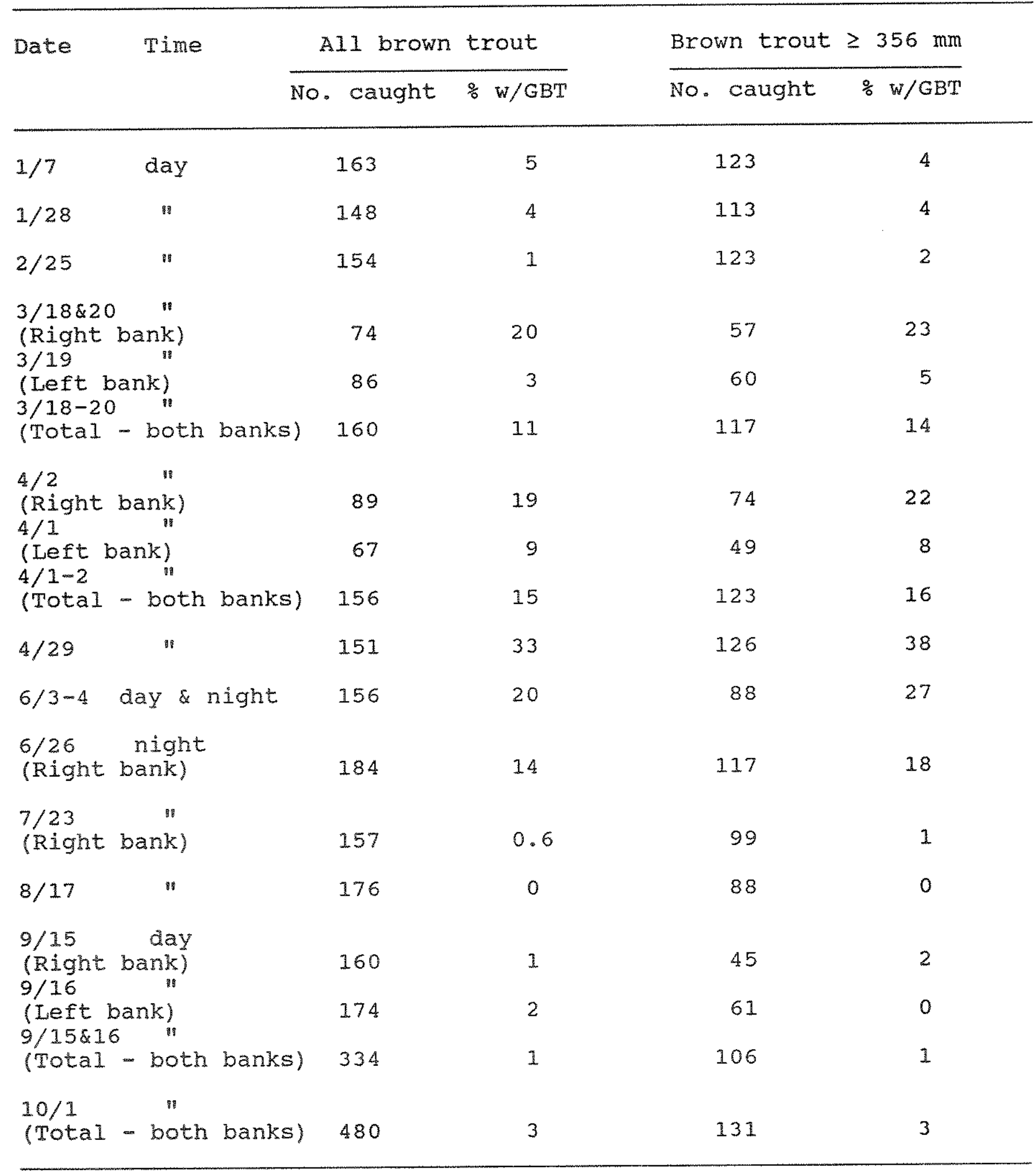


Table 29. (continued).

\begin{tabular}{|c|c|c|c|c|c|c|c|}
\hline \multirow[t]{2}{*}{ Date } & \multirow[t]{2}{*}{ Time } & \multicolumn{2}{|c|}{ All brown trout } & \multicolumn{4}{|c|}{ Brown trout $\geq 356 \mathrm{~mm}$} \\
\hline & & No. caught & $\% \mathrm{~W} / \mathrm{GBT}$ & No. & caught & 字 & $\mathrm{w} / \mathrm{GBT}$ \\
\hline $\begin{array}{l}11 / 14 \\
\text { (Right }\end{array}$ & $\begin{array}{r}\text { day } \\
\text { bank) }\end{array}$ & 160 & 3 & & 72 & & 1 \\
\hline $\begin{array}{l}12 / 18 \\
\text { (Right }\end{array}$ & $\begin{array}{r}\text { day } \\
\text { bank) }\end{array}$ & 207 & 11 & & 50 & & 22 \\
\hline $2 / 3 / 87$ & $n$ & 157 & 6 & & 52 & & 10 \\
\hline
\end{tabular}


Table 30. Incidence of gas bubble trauma (GBT) in rainbow trout from Rkm 3.9-6.1 (section 2) on the Bighorn River during 1986 (data are from both banks unless noted otherwise).

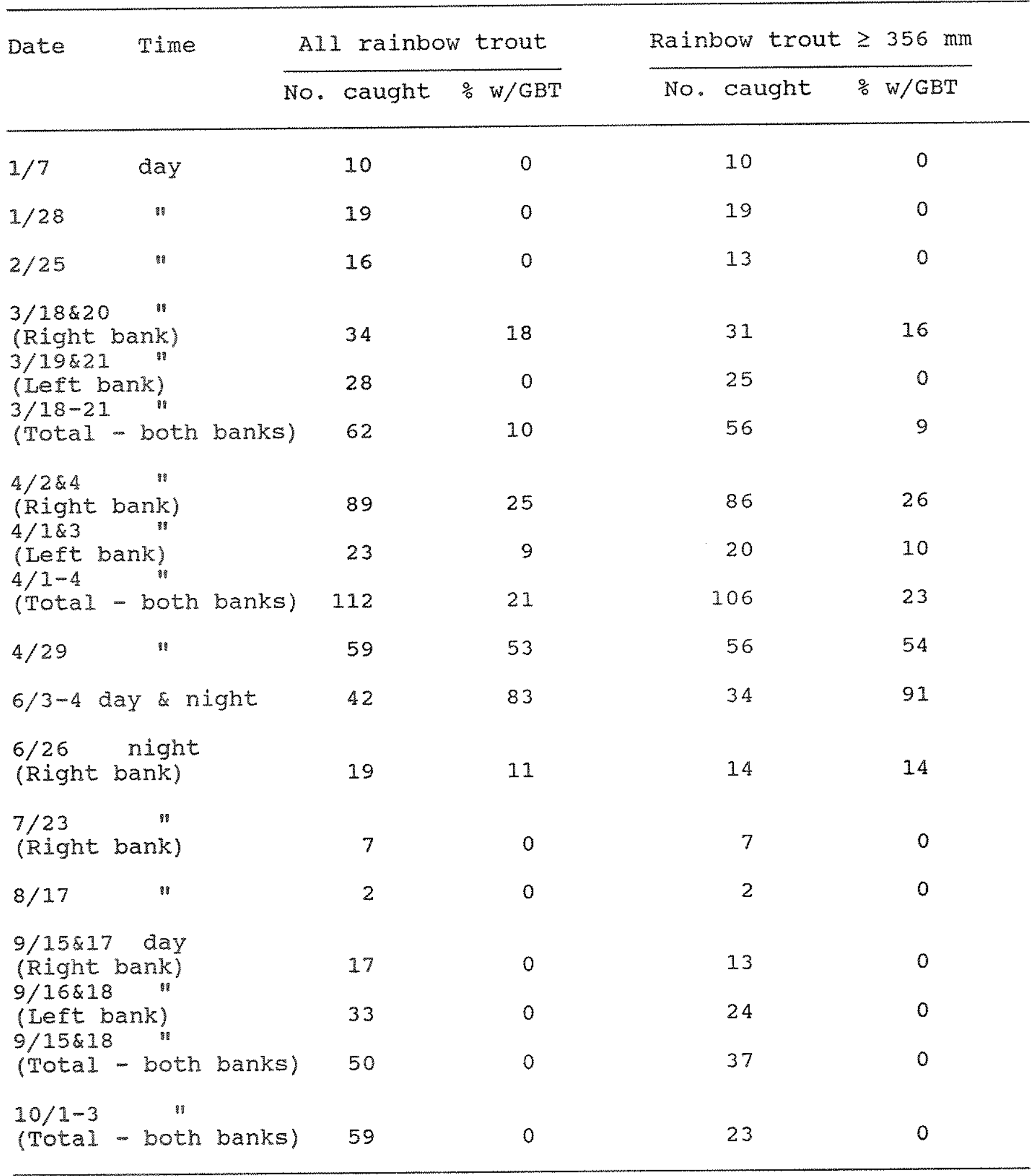


Table 30. (continued).

\begin{tabular}{|c|c|c|c|c|c|c|c|c|}
\hline \multirow[t]{2}{*}{ Date } & \multirow[t]{2}{*}{ Time } & \multicolumn{3}{|c|}{ All rainbow trout } & \multicolumn{4}{|c|}{ Rainbow trout $\geq 356 \mathrm{~mm}$} \\
\hline & & No. & caught $\%$ & $\mathrm{~W} / \mathrm{GBT}$ & No. & caught & $\%$ & $\mathrm{~W} / \mathrm{GBT}$ \\
\hline $\begin{array}{l}\text { 11/14 } \\
\text { (Right }\end{array}$ & $\begin{array}{r}\text { day } \\
\text { bank) }\end{array}$ & & 28 & 4 & & 13 & & 0 \\
\hline $\begin{array}{l}12 / 18 \\
\text { (Right }\end{array}$ & bank) & & 11 & 0 & & 4 & & 0 \\
\hline $\begin{array}{l}2 / 3 / 87 \\
\text { (Right }\end{array}$ & 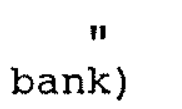 & & 18 & 11 & & 6 & & 33 \\
\hline
\end{tabular}


Table 31. Incidence of gas bubble trauma (GBT) in brown trout from Rkm 12.2-15.4 (section 3) on the Bighorn River during 1986 (data are from both banks unless noted otherwise).

\begin{tabular}{|c|c|c|c|c|c|}
\hline \multirow[t]{2}{*}{ Date } & \multirow[t]{2}{*}{ Time } & \multicolumn{2}{|c|}{ A11 brown trout } & \multicolumn{2}{|c|}{ Brown trout $\geq 356 \mathrm{~mm}$} \\
\hline & & No. caught & $\% \mathrm{w} / \mathrm{GBT}$ & No. caught & $\% \mathrm{~W} / \mathrm{GBT}$ \\
\hline $3 / 18$ & day & 129 & 8 & 95 & 11 \\
\hline $4 / 1 \& 2$ & $n$ & 231 & 4 & 169 & 5 \\
\hline $5 / 1$ & 19 & 166 & 6 & 137 & 7 \\
\hline $\begin{array}{l}6 / 4 \\
\text { (Right }\end{array}$ & $\begin{array}{l}\text { night } \\
\text { Bank) }\end{array}$ & 184 & 3 & 111 & 5 \\
\hline $\begin{array}{l}6 / 26 \\
\text { (Right }\end{array}$ & bank) & 166 & 7 & 90 & 12 \\
\hline $\begin{array}{l}7 / 23 \\
\text { (Right }\end{array}$ & bank) & 153 & 1.3 & 107 & 0.9 \\
\hline $\begin{array}{l}8 / 20 \\
\text { (Right }\end{array}$ & Bank) & 174 & 1.1 & 113 & 0.9 \\
\hline $\begin{array}{l}9 / 17 \\
\text { (Right }\end{array}$ & $\begin{array}{r}\text { day } \\
\text { bank) }\end{array}$ & 181 & 0 & 65 & 0 \\
\hline $10 / 1$ & day & 190 & 0 & 71 & 0 \\
\hline $\begin{array}{l}11 / 18 \\
\text { (Right }\end{array}$ & $\begin{array}{r}\text { day } \\
\text { bank) }\end{array}$ & 180 & 1.1 & 82 & 1.2 \\
\hline $\begin{array}{l}12 / 16 \\
\text { (Right }\end{array}$ & bank) & 180 & 1.7 & 73 & 2.7 \\
\hline $2 / 5 / 87$ & II & 165 & 1.2 & 94 & 1.1 \\
\hline
\end{tabular}


Table 32. Incidence of gas bubble trauma (GBT) in rainbow trout from Rkm 12.2-15.4 (section 3) on the Bighorn River during 1986 (data is from both banks unless noted otherwise).

\begin{tabular}{|c|c|c|c|c|c|c|c|c|}
\hline \multirow[t]{2}{*}{ Date } & \multirow[t]{2}{*}{ Time } & \multicolumn{3}{|c|}{ All rainbow trout } & \multicolumn{4}{|c|}{ Rainbow trout $\geq 356 \mathrm{~mm}$} \\
\hline & & No. & caught & $\% \mathrm{w} / \mathrm{GBT}$ & No. & caught & $\%$ & $\mathrm{w} / \mathrm{GBT}$ \\
\hline $3 / 18$ & day & & 27 & 0 & & 27 & & 0 \\
\hline $4 / 1 \& 2$ & $"$ & & 47 & 2 & & 43 & & 2 \\
\hline $5 / 1$ & $"$ & & 16 & 19 & & 15 & & 20 \\
\hline $\begin{array}{l}6 / 4 \\
\text { (Right }\end{array}$ & $\begin{array}{l}\text { night } \\
\text { bank) }\end{array}$ & & 31 & 3 & & 31 & & 3 \\
\hline $\begin{array}{l}6 / 26 \\
\text { (Right }\end{array}$ & bank) & & 24 & 0 & & 22 & & 0 \\
\hline $\begin{array}{l}7 / 23 \\
\text { (Right }\end{array}$ & bank) & & 13 & 0 & & 12 & & 0 \\
\hline $\begin{array}{l}8 / 20 \\
\text { (Right }\end{array}$ & "Bank) & & 15 & 0 & & 10 & & 0 \\
\hline $\begin{array}{l}9 / 17 \\
\text { (Right }\end{array}$ & $\begin{array}{r}\text { day } \\
\text { bank) }\end{array}$ & & 36 & 0 & & 23 & & 0 \\
\hline $10 / 1$ & day & & 42 & 0 & & 26 & & 0 \\
\hline $\begin{array}{l}11 / 18 \\
\text { (Right }\end{array}$ & $\begin{array}{r}\text { day } \\
\text { bank) }\end{array}$ & & 36 & 0 & & 21 & & 0 \\
\hline $\begin{array}{l}12 / 16 \\
\text { (Right }\end{array}$ & bank) & & 32 & 0 & & 9 & & 0 \\
\hline $2 / 5 / 87$ & " & & 34 & 0 & & 9 & & 0 \\
\hline
\end{tabular}


Table 33. Incidence of gas bubble trauma (GBT) in mountain whitefish from the Bighorn River during 1986 (data are from right bank unless noted otherwise).

Date Time $\frac{\text { All mtn whitefish }}{\text { No. caught } \% \mathrm{w} / \mathrm{GBT}} \quad \frac{\text { Mtn whitefish } \geq 356 \mathrm{~mm}}{\text { No. caught } \% \mathrm{w} / \mathrm{GBT}}$

$$
\text { Rkm } 0.0-1.9
$$

$4 / 183$ day

(Left bank)

41

5

39

5

$4 / 2 \& 4$

14

13

0

(Right bank)

55

(Total both banks)

55

4

52

4

$4 / 29$

$n$

2

0

1

0

$6 / 3$ day

2

0

1

0

$6 / 24$ night

0

$7 / 20$

"

0

$8 / 17$

$n$

1

$11 / 14$ day

0

$12 / 16$

$n$

$2 / 4$

ห

2

Rkm 3.9-6.1

$4 / 1 \& 3$ day

(Left bank)

$4 / 2 \& 4$

"

(Right bank)

4/1-4

กิ

(Total both banks)

30

3

22

5

$41 \quad 0$

20

71

1

42

2

\section{$4 / 29$}

(Both banks)

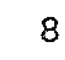

10

10

5

0

$6 / 3$

n

7

17

an

0

5

20

$6 / 26$ night

$7 / 23$

o

5

0

16

0 
Table 33. (continued)

Date Time $\frac{\text { All mtn whitefish }}{\text { No. caught } \% \text { w/GBT }} \quad \frac{\text { Mtn whitefish } \geq 356 \mathrm{~mm}}{\text { No. caught } 8 \mathrm{w} / \mathrm{GBT}}$

$$
\mathrm{Rkm} 3 \cdot 9-6 \cdot 1
$$

$\begin{array}{lccccr}8 / 17 & \text { " } & 14 & 0 & 6 & 0 \\ 11 / 14 & \text { day } & 21 & 0 & 8 & 0 \\ 12 / 18 & " & 35 & 0 & 21 & 0 \\ 2 / 3 & " & 12 & 8 & 8 & 13\end{array}$

$\mathrm{Rkm} 12 \cdot 2-15.4$

$\begin{array}{lccccc}5 / 1 & \text { day } & 3 & 0 & 3 & 0 \\ 6 / 4 & \text { night } & 18 & 0 & 15 & 0 \\ 6 / 26 & \text { " } & 9 & 0 & 5 & 0 \\ 7 / 23 & \text { " } & 13 & 0 & 7 & 0 \\ 8 / 20 & \text { " } & 0 & - & 0 & 0 \\ 11 / 18 & \text { day } & 18 & 0 & 3 & 0 \\ 12 / 16 & \text { " } & 29 & 0 & 8 & 0 \\ 2 / 5 & \text { " } & 6 & 0 & 4 & \end{array}$


Table 34. Incidence of gas bubble trauma (GBT) in brown trout from Rkm 0-1.9 (section 1) of the Bighorn River during 1987 (data are from right bank unless noted otherwise).

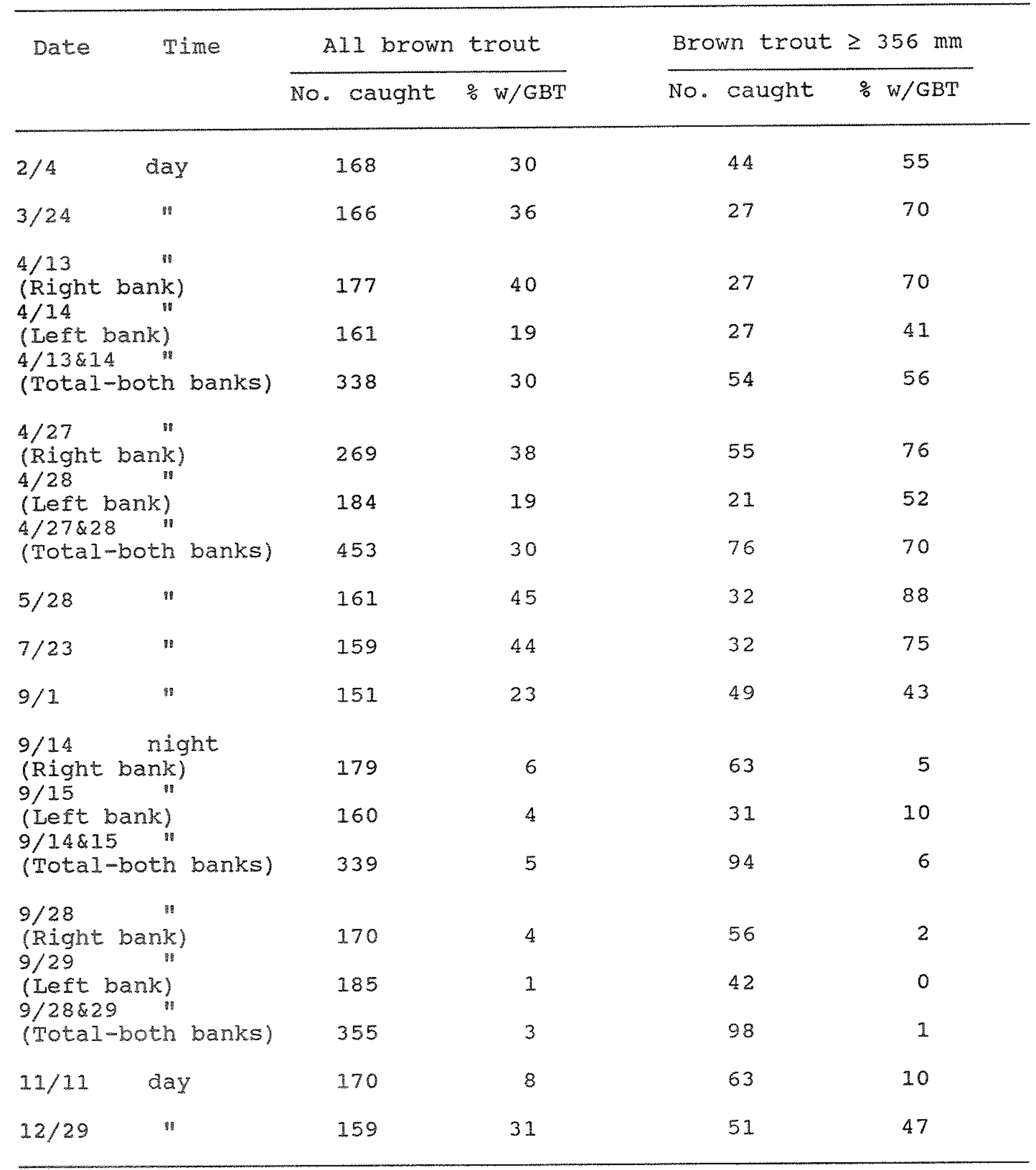


Table 35. Incidence of gas bubble trauma (GBT) in rainbow trout from Rkm 0-1.9 (section 1) of the Bighorn River during 1987 (data are from right bank unless noted otherwise).

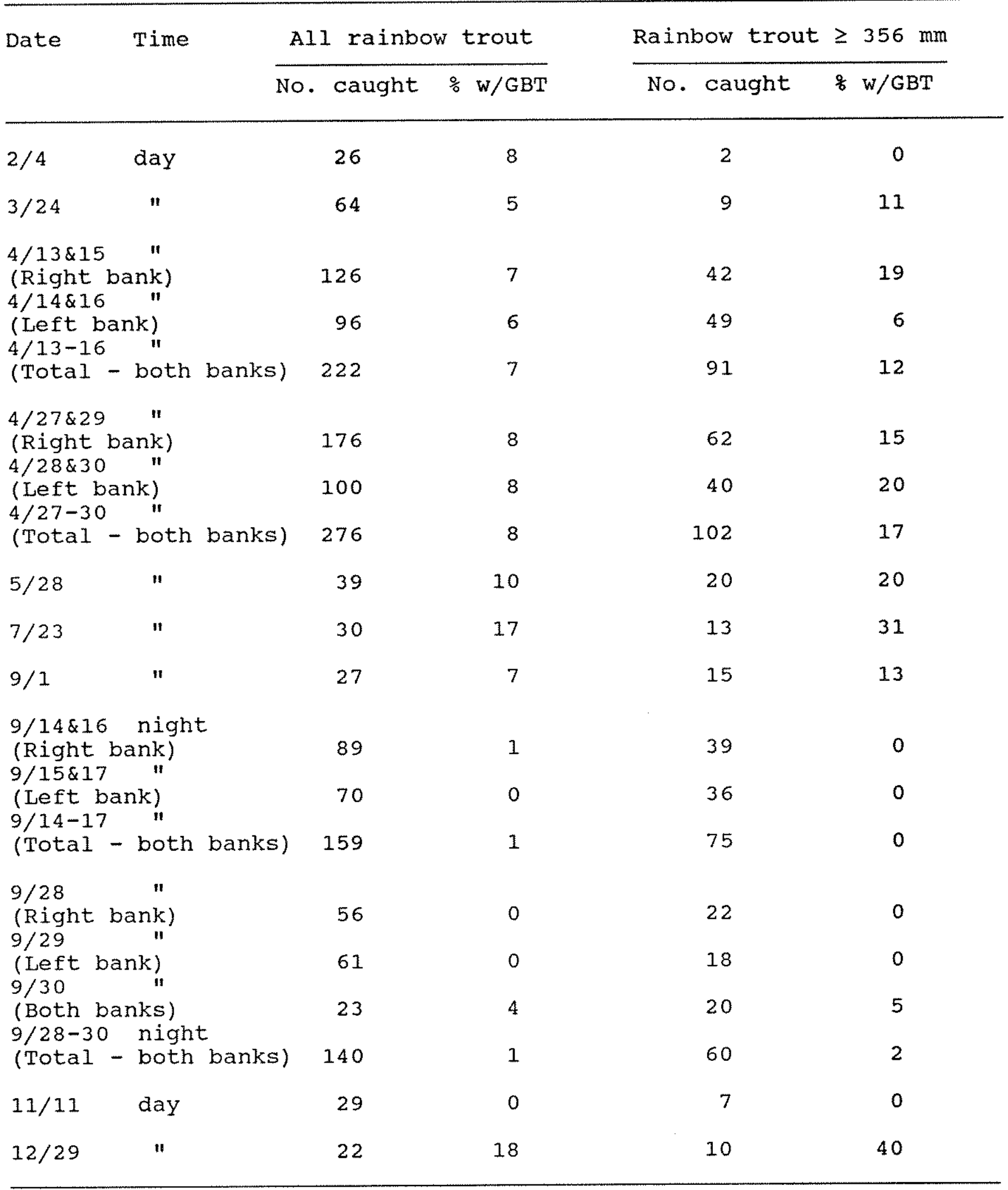


Table 36. Incidence of gas bubble trauma (GBT) in brown trout from RkM 3.9-6.1 (section 2) on the Bighorn River during 1987 (data are from both banks unless noted otherwise).

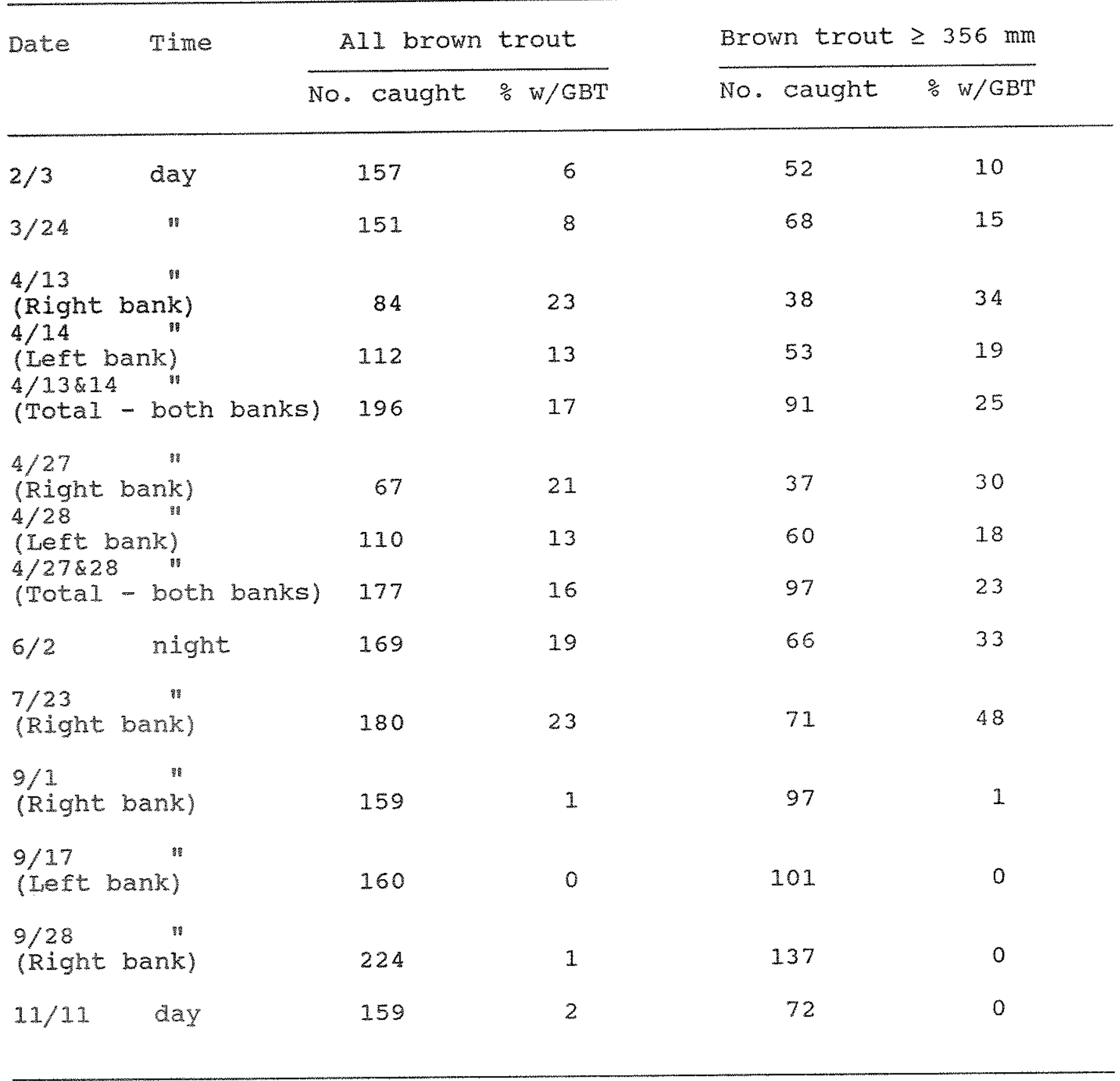


Table 37. Incidence of gas bubble trauma (GBT) in rainbow trout from Rkm 3.9-6.1 (section 2) on the Bighorn River during 1987

(data are from both banks unless noted otherwise).

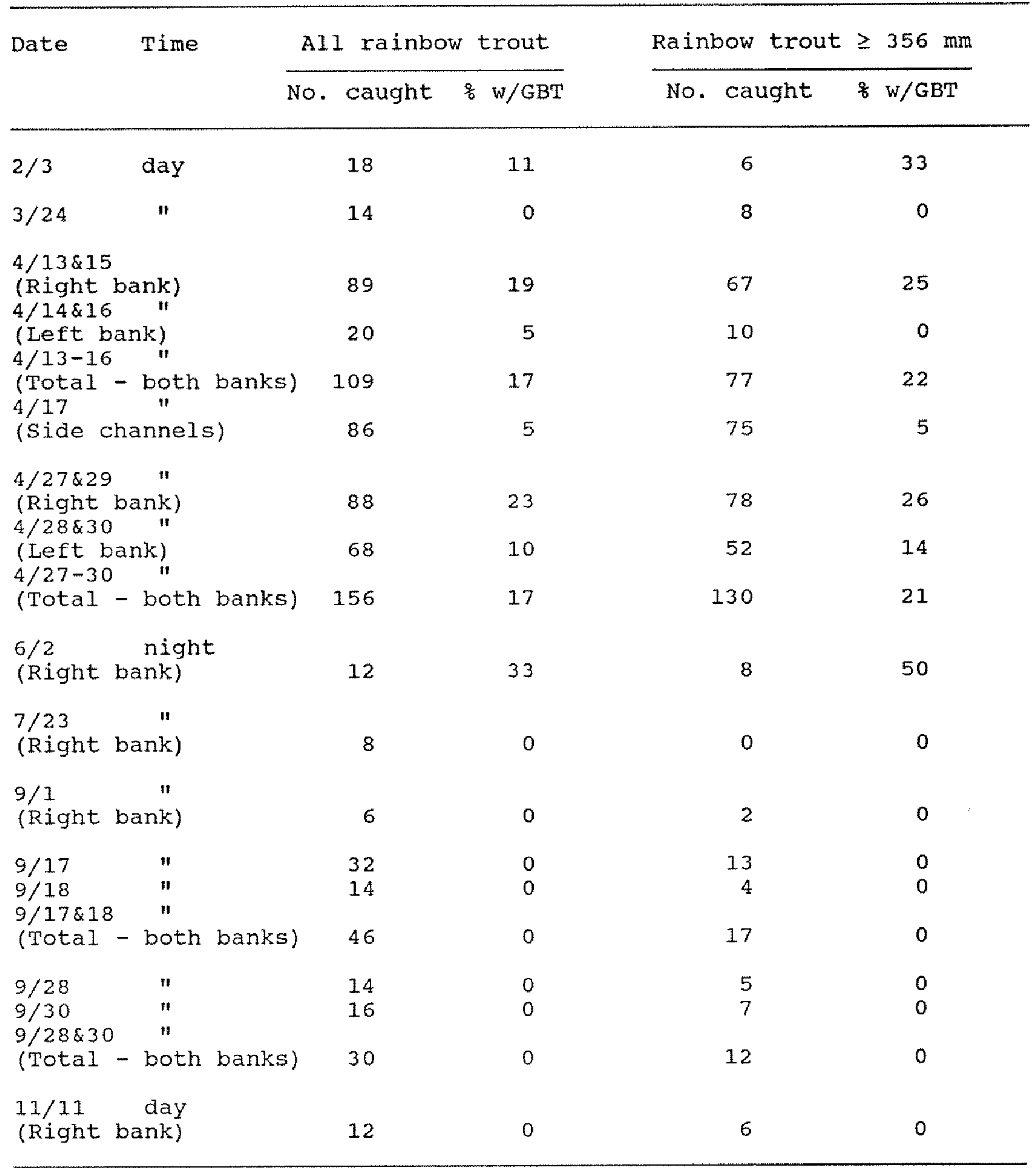


Table 38. Incidence of gas bubble trauma (GBT) in brown trout from Rkm 12.2-15.4 (section 3) on the Bighorn River during 1987 (data are from both banks unless noted otherwise).

\begin{tabular}{|c|c|c|c|c|c|c|}
\hline \multirow[t]{2}{*}{ Date } & \multirow[t]{2}{*}{ Time } & \multicolumn{2}{|c|}{ A11 brown trout } & \multicolumn{3}{|c|}{ Brown trout $\geq 356 \mathrm{~mm}$} \\
\hline & & No. caught & $\frac{\circ}{6} \mathrm{~W} / \mathrm{GBT}$ & No. caught & \% & $\mathrm{w} / \mathrm{GBT}$ \\
\hline $2 / 5$ & day & 165 & 1.2 & 94 & & 1.1 \\
\hline $3 / 25$ & 战 & 163 & 0.6 & 101 & & 1.0 \\
\hline $4 / 15$ & $n$ & 213 & 4 & 85 & & 8 \\
\hline $6 / 4$ & " & 151 & 7 & 82 & & 12 \\
\hline $7 / 28$ & & 167 & 13 & 99 & & 20 \\
\hline $9 / 3$ & n & 151 & 0 & 100 & & 0 \\
\hline $9 / 15$ & 政 & 207 & 0 & 117 & & 0 \\
\hline $9 / 30$ & " & 144 & 0 & 78 & & 0 \\
\hline $\begin{array}{l}11 / 12 \\
\text { (Right }\end{array}$ & $\begin{array}{c}\text { sank) } \\
\text { pank }\end{array}$ & 153 & 0 & 75 & & 0 \\
\hline
\end{tabular}


Table 39. Incidence of gas bubble trauma (GBT) in rainbow trout from Rkm 12.2-15.4 (section 3) on the Bighorn River during 1987 (data is from both banks unless noted otherwise).

\begin{tabular}{|c|c|c|c|c|c|c|c|c|}
\hline \multirow[t]{2}{*}{ Date } & \multirow[t]{2}{*}{ Time } & \multicolumn{3}{|c|}{ All rainbow trout } & \multicolumn{4}{|c|}{ Rainbow trout $\geq 356 \mathrm{~mm}$} \\
\hline & & No. & caught $\%$ & $\mathrm{~W} / \mathrm{GBT}$ & No. & caught & $\%$ & $\mathrm{w} / \mathrm{GBT}$ \\
\hline $2 / 5$ & day & & 34 & 0 & & 9 & & 0 \\
\hline $3 / 25$ & $"$ & & 36 & 0 & & 12 & & 0 \\
\hline $4 / 15$ & $"$ & & 72 & 0 & & 21 & & 0 \\
\hline $6 / 4$ & $"$ & & 23 & 13 & & 12 & & 25 \\
\hline $7 / 28$ & $"$ & & 20 & 0 & & 9 & & 0 \\
\hline $9 / 3$ & $"$ & & 22 & 0 & & 17 & & 0 \\
\hline $9 / 15$ & $"$ & & 47 & 0 & & 37 & & 0 \\
\hline $9 / 30$ & $"$ & & 39 & 0 & & 24 & & 0 \\
\hline $11 / 12$ & $"$ & & 27 & 0 & & 18 & & 0 \\
\hline
\end{tabular}


Table 40. Incidence of gas bubble trauma (GBT) in mountain Whitefish from the Bighorn River during 1987 (data are from right bank unless noted otherwise).

Date Time $\frac{\text { All mtn whitefish }}{\text { No. caught } \% \text { w/GBT }} \quad \frac{\text { Men whitefish } \geq 356 \mathrm{~mm}}{\text { No. caught } \% \mathrm{~W} / \mathrm{GBT}}$

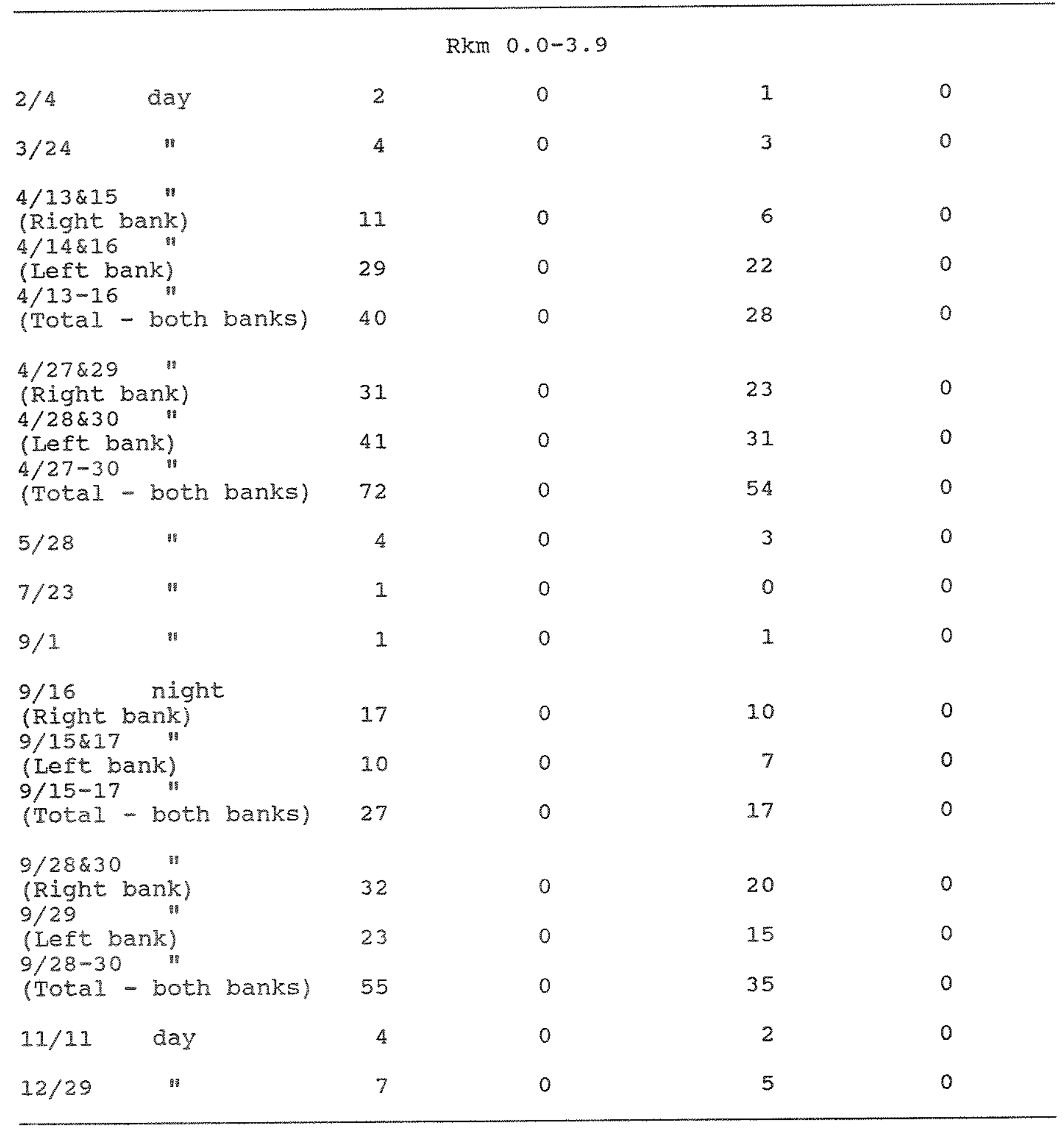


Table 40. (continued)

Date Time $\frac{\text { All mtn whitefish }}{\text { No. caught } \% \mathrm{w} / \mathrm{GBT}} \quad \frac{\text { Mtn whitefish } \geq 356 \mathrm{~mm}}{\text { No. caught } \% \mathrm{w} / \mathrm{GBT}}$

$\mathrm{Rkm} 3 \cdot 9-6 \cdot 1$

$2 / 3$ day

(Both banks)

12

8

8

13

$3 / 24$

18

0

14

0

4/13-17 "

(Total - both banks)

117

0

71

0

4/27-30 "

(Total - both banks)

133

0

87

0

$6 / 2$

night

13

0

10

0

$7 / 23$

"

21

0

14

0

$9 / 1$

$"$

21

0

13

0

$9 / 17 \& 18 \quad "$

(Total - both banks) 36

0

20

0

$9 / 28 \& 30 \quad "$

(Total - both banks) 76

$11 / 11$ day

30

0

38

0

1

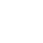

$2 / 5$
$3 / 25$
$6 / 4$
$7 / 28$
$9 / 3$

$11 / 12$

"

6

$\mathrm{Rkm} \mathrm{12.2-15.4}$

(1)

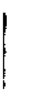


Table 41. Categorization of the severity of gas bubble trauma (GBT) in brown trout from Rkm 0-1.9 of the Bighorn River, 8 March 1985 - 7 January 1986. I = minor, $I I=$ serious and III = severe symptoms (MDFWP and MCFRU data).

\begin{tabular}{|c|c|c|c|c|c|c|c|}
\hline \multirow{3}{*}{ Date } & \multirow{3}{*}{ Time } & \multicolumn{3}{|c|}{ All brown trout } & \multirow{2}{*}{$\frac{\text { Brown }}{I}$} & \multicolumn{2}{|c|}{ trout $\geq 356 \mathrm{~mm}$} \\
\hline & & $I$ & II & III & & II & III \\
\hline & & No. $/ \%$ & No. $/ \%$ & No./\% & No. $/ \%$ & No./\% & No. $/ \%$ \\
\hline $3 / 8$ & day & $10 / 100$ & & & $5 / 100$ & & \\
\hline $3 / 22$ & $"$ & $3 / 43$ & $3 / 43$ & $1 / 14$ & $3 / 43$ & $3 / 43$ & $1 / 14$ \\
\hline $4 / 10$ & $"$ & $7 / 100$ & & & $5 / 100$ & & \\
\hline $4 / 18$ & $"$ & $4 / 31$ & $8 / 61$ & $1 / 8$ & $4 / 33$ & $8 / 67$ & \\
\hline $5 / 3$ & $"$ & $19 / 47$ & $17 / 43$ & $4 / 10$ & $10 / 45$ & $10 / 45$ & $2 / 10$ \\
\hline $5 / 11$ & $"$ & $15 / 88$ & $2 / 12$ & & $10 / 83$ & $2 / 17$ & \\
\hline $5 / 20$ & $"$ & $7 / 100$ & & & $6 / 100$ & & \\
\hline $6 / 4$ & $"$ & $12 / 86$ & $2 / 14$ & & $7 / 88$ & $1 / 12$ & \\
\hline $6 / 27$ & night & $25 / 86$ & $4 / 14$ & & $12 / 80$ & $3 / 20$ & \\
\hline $7 / 18$ & $"$ & $9 / 90$ & $1 / 10$ & & $7 / 88$ & $1 / 12$ & \\
\hline $8 / 1$ & $"$ & $9 / 82$ & $1 / 9$ & $1 / 9$ & $8 / 100$ & & \\
\hline $8 / 14$ & $"$ & $10 / 83$ & $2 / 17$ & & $7 / 78$ & $2 / 22$ & \\
\hline $8 / 25$ & " & $11 / 100$ & & & $6 / 100$ & & \\
\hline $9 / 18 \& 20$ & day & $13 / 62$ & $8 / 38$ & & $10 / 67$ & $5 / 33$ & \\
\hline $9 / 30-10 / 2$ & $2 "$ & $20 / 64$ & $8 / 26$ & $3 / 10$ & $14 / 67$ & $6 / 28$ & $1 / 5$ \\
\hline $10 / 18$ & night & $5 / 100$ & & & $4 / 100$ & & \\
\hline $10 / 31$ & $"$ & $2 / 100$ & & & $2 / 100$ & & \\
\hline $11 / 14$ & $n$ & $3 / 100$ & & & $2 / 100$ & & \\
\hline
\end{tabular}


Table 41. (continued).

\begin{tabular}{|c|c|c|c|c|c|c|c|}
\hline \multirow{3}{*}{ Date } & \multirow{3}{*}{ Time } & \multicolumn{3}{|c|}{ All brown trout } & \multicolumn{3}{|c|}{ Brown trout $\geq 356 \mathrm{~mm}$} \\
\hline & & $I$ & II & III & $I$ & II & III \\
\hline & & No. $/ \%$ & No. $/ \%$ & No. $/ \%$ & No. $1 \%$ & No. $/ \%$ & No. $1 \%$ \\
\hline $12 / 13$ & day & $8 / 100$ & & & $7 / 100$ & & \\
\hline $1 / 7 / 86$ & \% & $26 / 96$ & $1 / 4$ & & $23 / 96$ & $1 / 4$ & \\
\hline Total & & $218 / 76$ & $57 / 20$ & $10 / 4$ & $152 / 77$ & $42 / 21$ & $4 / 2$ \\
\hline
\end{tabular}


Table 42. Categorization of the severity of gas bubble trauma (GBT) in brown trout from $\mathrm{kkm} 3.9-6.1$ on the Bighorn River, 18 April 1985 - 7 January 1986. I = minor, $I I=$ serious and III = severe symptoms. (MDFWP and MCFRU data).

\begin{tabular}{|c|c|c|c|c|c|c|c|}
\hline \multirow{3}{*}{ Date } & \multirow{3}{*}{ Time } & \multicolumn{3}{|c|}{ Al1 brown trout } & \multicolumn{3}{|c|}{ Brown trout $\geq 356 \mathrm{~mm}$} \\
\hline & & $I$ & II & III & $I$ & II & III \\
\hline & & No. $/ \%$ & No. $/ \%$ & No. $/ \%$ & No. $/ 8$ & No. $/ 8$ & No. $/ \%$ \\
\hline $4 / 18$ & day & $4 / 1.00$ & & & $4 / 100$ & & \\
\hline $5 / 2-8$ & $"$ & $46 / 65$ & $21 / 30$ & $4 / 5$ & $21 / 58$ & $13 / 36$ & $2 / 6$ \\
\hline $6 / 4$ & $"$ & $3 / 75$ & $1 / 25$ & & $3 / 75$ & $1 / 25$ & \\
\hline $6 / 27$ & night & $2 / 100$ & & & $2 / 100$ & & \\
\hline $7 / 18$ & $"$ & - & & & - & & \\
\hline $8 / 1$ & $"$ & $1 / 100$ & & & $1 / 100$ & & \\
\hline $8 / 14$ & $"$ & - & & & - & & \\
\hline $10 / 31$ & $"$ & - & & & - & & \\
\hline $11 / 14$ & $"$ & - & & & - & & \\
\hline $12 / 13$ & day & $1 / 100$ & & & $1 / 100$ & & \\
\hline $1 / 7 / 86$ & $"$ & $8 / 100$ & & & $5 / 100$ & & \\
\hline Total & & $65 / 72$ & $22 / 24$ & $4 / 4$ & $37 / 70$ & $14 / 26$ & $2 / 4$ \\
\hline
\end{tabular}


Table 43. Categorization of the severity of gas bubble trauma (GBT) in brown trout from Rkm 0-1.9 (section 1) of the Bighorn River during 1986. $I=$ minor, $I I=$ serious and $I I I=$ severe symptoms (MDFWP and MCFRU data).

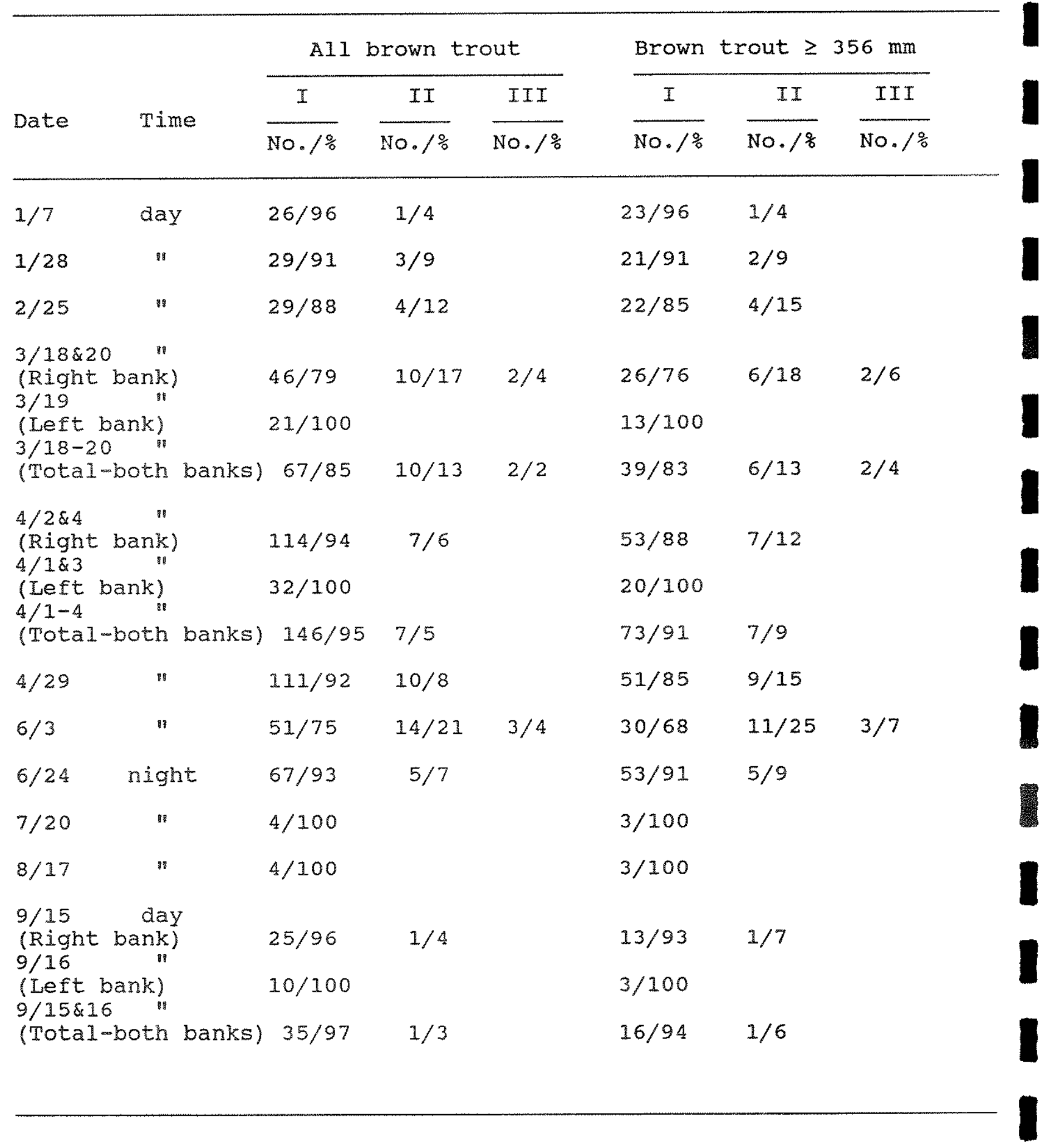


Table 43. (continued).

\begin{tabular}{|c|c|c|c|c|c|c|c|}
\hline \multirow{3}{*}{ Date } & \multirow{3}{*}{ Time } & \multicolumn{3}{|c|}{ All brown trout } & \multicolumn{3}{|c|}{ Brown trout $\geq 356 \mathrm{~mm}$} \\
\hline & & \multirow{2}{*}{$\frac{\mathrm{I}}{\mathrm{NO} \cdot / \%}$} & \multirow{2}{*}{$\frac{\mathrm{II}}{\mathrm{NO} / \mathrm{\%}}$} & \multirow{2}{*}{$\frac{\text { III }}{\text { No. } / \%}$} & \multirow{2}{*}{$\frac{I}{N O \cdot / \%}$} & \multirow{2}{*}{$\frac{\mathrm{II}}{\mathrm{No} \cdot / q}$} & \multirow{2}{*}{$\frac{\mathrm{III}}{\mathrm{NO} \cdot / \mathrm{g}}$} \\
\hline & & & & & & & \\
\hline \multirow{5}{*}{\multicolumn{2}{|c|}{$\begin{array}{l}9 / 29 \text { day } \\
\text { (Right bank) } \\
9 / 30 \text { " } \\
\text { (Left bank) } \\
9 / 29 \& 30 \text { " } \\
\text { (Total-both banks) }\end{array}$}} & & & & & & \\
\hline & & $41 / 93$ & $3 / 7$ & & $20 / 95$ & $1 / 5$ & \\
\hline & & $15 / 100$ & & & $2 / 100$ & & \\
\hline & & 10100 & & & $27+00$ & & \\
\hline & & $56 / 95$ & $3 / 5$ & & $22 / 96$ & $1 / 4$ & \\
\hline $11 / 14$ & daỳ & $49 / 92$ & $4 / 8$ & & $18 / 86$ & $3 / 14$ & \\
\hline $12 / 16$ & $"$ & $61 / 100$ & & & $18 / 100$ & & \\
\hline $2 / 4 / 87$ & $"$ & $43 / 84$ & $4 / 8$ & $4 / 8$ & $18 / 75$ & $2 / 8$ & $4 / 17$ \\
\hline $\begin{array}{r}1986 \mathrm{TO} \\
\text { (R. Ba }\end{array}$ & f) & $7 / 91$ & $62 / 8$ & $5 / 1$ & $354 / 87$ & $50 / 12$ & $5 / 1$ \\
\hline
\end{tabular}


Table 44. Categorization of the severity of gas bubble trauma (GBT) in brown trout from Rkm 3.9-6.1 (section 2) of the Bighorn River during 1986. $I=$ minor, $I I=$ serious and $I I I=$ severe symptoms (MDFWP and MCFRU data).

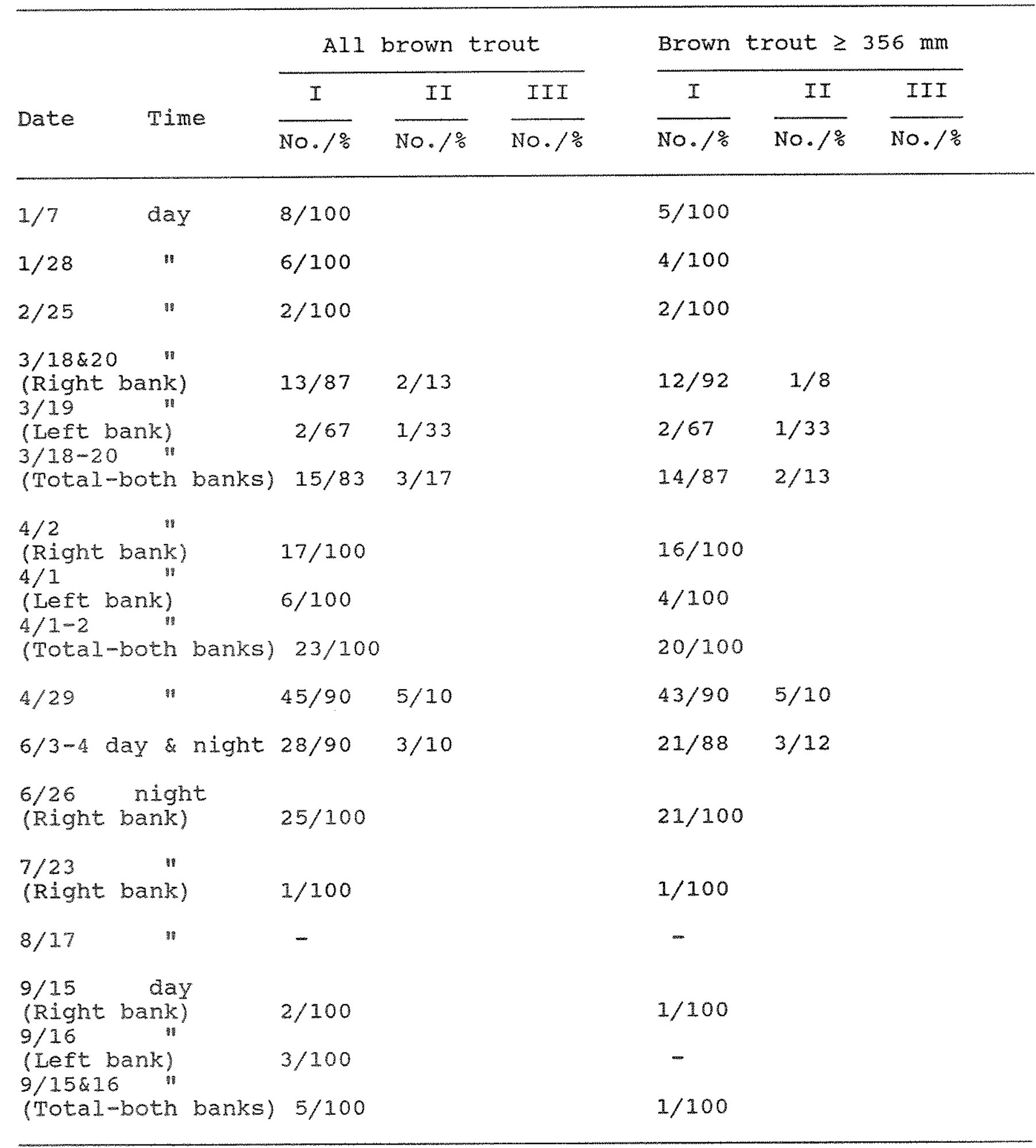


Table 44. (continued).

\begin{tabular}{|c|c|c|c|c|c|c|}
\hline \multirow{3}{*}{ Date } & \multicolumn{3}{|c|}{ Al1 brown trout } & \multicolumn{3}{|c|}{ Brown trout $\geq 356 \mathrm{~mm}$} \\
\hline & $I$ & II & III & $I$ & II & III \\
\hline & $\overline{\mathrm{No} . / \%}$ & $\overline{\text { No. } / \%}$ & $\overline{\mathrm{NO} . / \%}$ & $\overline{\mathrm{No} . / \mathrm{q}}$ & $\overline{\mathrm{No} .1 \%}$ & No. $/ \%$ \\
\hline $\begin{array}{l}10 / 1 \\
\text { (Total-both banks) }\end{array}$ & $14 / 93$ & $1 / 7$ & & $3 / 75$ & $1 / 25$ & \\
\hline $\begin{array}{l}11 / 14 \text { day } \\
\text { (Right bank) }\end{array}$ & $4 / 100$ & & & $1 / 100$ & & \\
\hline $\begin{array}{l}\text { 12/18 day } \\
\text { (Right bank) }\end{array}$ & $22 / 100$ & & & $11 / 100$ & & \\
\hline $2 / 3 / 87$ & $10 / 100$ & & & $5 / 100$ & & \\
\hline 1986 TOTAL & $198 / 94$ & $12 / 6$ & & $147 / 93$ & $11 / 7$ & \\
\hline
\end{tabular}


Table 45. Categorization of the severity of gas bubble trauma (GBT) in brown trout from Rkm 12.2-15.4 (section 3 ) of the Bighorn River during 1986. $I=$ minor, $I I=$ serious and $I I I=$ severe symptoms (MDFWP and MCFRU data).

\begin{tabular}{|c|c|c|c|c|c|c|}
\hline \multirow{3}{*}{ Date } & \multicolumn{3}{|c|}{ A11 brown trout } & \multicolumn{3}{|c|}{ Brown trout $\geq 356 \mathrm{~mm}$} \\
\hline & $I$ & II & III & $I$ & II & III \\
\hline & No. $/ \%$ & No. $/ \%$ & $\mathrm{NO} . / \%$ & No. $/ \%$ & No. $/ \%$ & No. $/ \%$ \\
\hline $3 / 18$ & $10 / 100$ & & & $10 / 100$ & & \\
\hline $4 / 1 \& 2$ & $7 / 87$ & $1 / 13$ & & $7 / 87$ & $1 / 13$ & \\
\hline $5 / 1$ & $10 / 100$ & & & $10 / 100$ & & \\
\hline $\begin{array}{l}\text { 6/4 night } \\
\text { (Right Bank) }\end{array}$ & $6 / 100$ & & & $6 / 100$ & & \\
\hline $\begin{array}{l}6 / 26 \\
\text { (Right bank) }\end{array}$ & $12 / 100$ & & & $11 / 100$ & & \\
\hline $\begin{array}{l}7 / 23 \\
\text { (Right bank) }\end{array}$ & $2 / 100$ & & & $1 / 100$ & & \\
\hline $\begin{array}{l}8 / 20 \\
\text { (Right Bank) }\end{array}$ & $2 / 100$ & & & $1 / 100$ & & \\
\hline $\begin{array}{l}9 / 17 \text { day } \\
\text { (Right bank) }\end{array}$ & - & & & - & & \\
\hline $10 / 1$ & - & & & - & & \\
\hline $\begin{array}{l}11 / 18 \text { day } \\
\text { (Right bank) }\end{array}$ & $2 / 100$ & & & $1 / 100$ & & \\
\hline $\begin{array}{l}12 / 16 \\
\text { (Right bank) }\end{array}$ & $3 / 100$ & & & $2 / 100$ & & \\
\hline $2 / 5 / 87$ & $2 / 100$ & & & $1 / 100$ & & \\
\hline 1986 TOTAL & $54 / 98$ & $1 / 2$ & & $49 / 98$ & $1 / 2$ & \\
\hline
\end{tabular}


Table 46. Categorization of the severity of gas bubble trauma (GBT) in brown trout from Rkm 0-1.9 (section 1) of the Bighorn River during 1987. I $=$ minor, $I I=$ serious and $I I I=$ severe symptoms (MDFWP and MCFRU data).

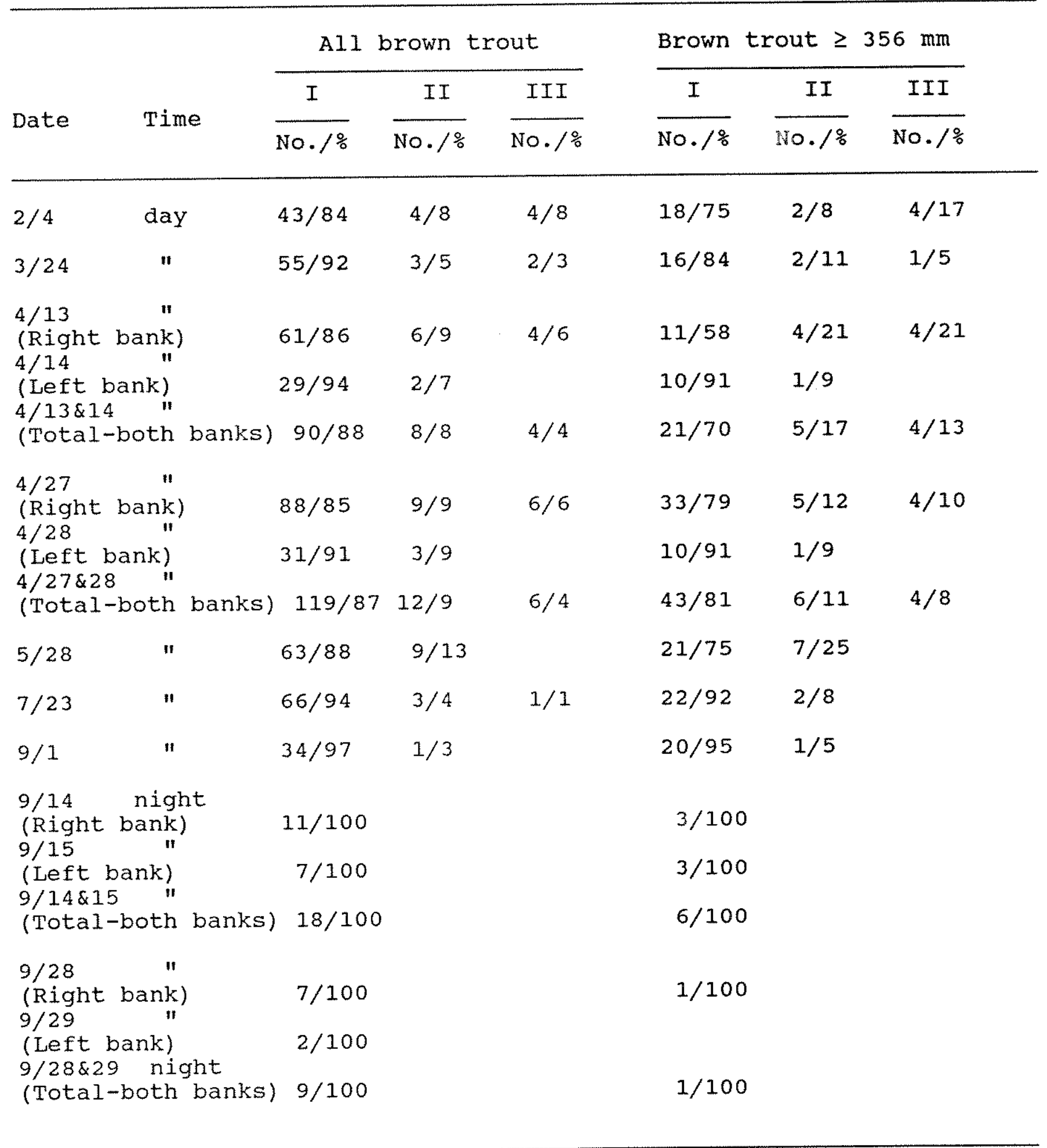


Table 46. (continued).

\begin{tabular}{|c|c|c|c|c|c|c|c|}
\hline \multirow{3}{*}{ Date } & \multirow{3}{*}{ Time } & \multicolumn{3}{|c|}{ All brown trout } & \multicolumn{3}{|c|}{ Brown trout $\geq 356 \mathrm{~mm}$} \\
\hline & & I & II & III & $I$ & II & III \\
\hline & & No. $/ \%$ & No. $/ \%$ & No. $/ \%$ & No. $/ \%$ & No./\% & No. $/ \%$ \\
\hline $11 / 11$ & day & $14 / 100$ & & & $6 / 100$ & & \\
\hline $12 / 29$ & 的 & $47 / 96$ & $2 / 4$ & & $23 / 96$ & $1 / 4$ & \\
\hline \multicolumn{2}{|c|}{$\begin{array}{l}1987 \text { TOTAL } \\
\text { (Right bank) }\end{array}$} & $489 / 90$ & $37 / 7$ & $17 / 3$ & $174 / 83$ & $24 / 11$ & $13 / 6$ \\
\hline
\end{tabular}


Table 47. Categorization of the severity of gas bubble trauma (GBT) in brown trout from Rkm 3.9-6.1 (section 2) of the Bighorn River during 1987. $I=$ minor, $I I=$ serious and $I I I=$ severe symptoms (MDFWP and MCFRU data).

\begin{tabular}{|c|c|c|c|c|c|c|}
\hline \multirow{3}{*}{ Date } & \multicolumn{3}{|c|}{ All brown trout } & \multicolumn{3}{|c|}{ Brown trout $\geq 356 \mathrm{~mm}$} \\
\hline & $I$ & II & III & $I$ & II & III \\
\hline & $\overline{\mathrm{NO} .1 \%}$ & No. $1 \%$ & $\mathrm{No} . / \%$ & No. $/ \%$ & $\sqrt{N 0.1 \%}$ & $\overline{\text { No. } / \%}$ \\
\hline day & $10 / 100$ & & & $5 / 100$ & & \\
\hline $3 / 24$ & $12 / 100$ & & & $10 / 100$ & & \\
\hline $\begin{array}{l}4 / 13 \text { " } \\
\text { (Right bank) } \\
4 / 14 \text { " } \\
\text { (Left bank) } \\
4 / 13 \& 14 \text { " } \\
\text { (Total-both banks) }\end{array}$ & $\begin{array}{l}19 / 100 \\
14 / 100 \\
33 / 100\end{array}$ & & & $\begin{array}{l}13 / 100 \\
10 / 100 \\
23 / 100\end{array}$ & & \\
\hline $\begin{array}{l}4 / 27 \text { " } \\
\text { (Right bank) } \\
4 / 28 \text { " } \\
\text { (Left bank) } \\
4 / 27 \& 28 \text { " } \\
\text { (Total-both banks) }\end{array}$ & $\begin{array}{l}14 / 100 \\
14 / 100 \\
28 / 100\end{array}$ & & & $\begin{array}{l}11 / 100 \\
11 / 100 \\
22 / 100\end{array}$ & & \\
\hline $\begin{array}{l}6 / 2 \quad \text { night } \\
\text { (Right bank) }\end{array}$ & $32 / 100$ & & & $22 / 100$ & & \\
\hline $\begin{array}{l}7 / 23 \quad " \\
\text { (Right bank) }\end{array}$ & $40 / 98$ & $1 / 2$ & & $33 / 97$ & $1 / 3$ & \\
\hline $9 / 1$ & $2 / 100$ & & & $1 / 100$ & & \\
\hline $9 / 17$ (Left bank) & $0 / 0$ & & & $0 / 0$ & & \\
\hline $\begin{array}{l}9 / 28 \text { " } \\
\text { (Right bank) }\end{array}$ & $3 / 100$ & & & $0 / 0$ & & \\
\hline $\begin{array}{l}\text { 11/11 day } \\
\text { (Right bank) }\end{array}$ & $3 / 100$ & & & $0 / 0$ & & \\
\hline 1987 TOTAL & $163 / 99$ & $1 / 1$ & & $116 / 99$ & $1 / 1$ & \\
\hline
\end{tabular}


Table 48. Categorization of the severity of gas bubble trauma (GBT) in brown trout from Rkm 12.2-15.4 (section 3) of the Bighorn River during 1987. I = minor, $I I=$ serious and $I I I=$ severe symptoms (MDFWP and MCFRU data).

\begin{tabular}{|c|c|c|c|c|c|c|c|}
\hline \multirow{3}{*}{ Date } & \multirow{3}{*}{ Time } & \multicolumn{3}{|c|}{ Al1 brown trout } & \multicolumn{3}{|c|}{ Brown trout $\geq 356 \mathrm{~mm}$} \\
\hline & & I & II & III & I & II & III \\
\hline & & $\overline{N O} / \%$ & No. $/ \%$ & No. $/ \%$ & No. $/ \%$ & No. $/ \%$ & No. $/ \%$ \\
\hline $2 / 5$ & day & $2 / 100$ & & & $1 / 100$ & & \\
\hline $3 / 25$ & " & $1 / 100$ & & & $1 / 100$ & & \\
\hline $4 / 15$ & " & $8 / 100$ & & & $7 / 100$ & & \\
\hline $\begin{array}{l}6 / 4 \\
\text { (Right }\end{array}$ & Bank) & $10 / 100$ & & & $10 / 100$ & & \\
\hline $7 / 28$ & 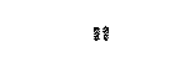 & $22 / 100$ & & & $20 / 100$ & & \\
\hline $9 / 3$ & 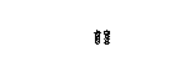 & - & & & - & & \\
\hline $9 / 15$ & 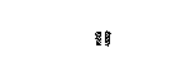 & - & & & - & & \\
\hline $9 / 30$ & $n$ & - & & & - & & \\
\hline $11 / 12$ & 的 & - & & & - & & \\
\hline $1987 \mathrm{TO}$ & OTAL & $43 / 100$ & & & $39 / 100$ & & \\
\hline
\end{tabular}


Table 49. Severity of gas bubble trauma (GBT) in brown and rainbow trout sampled during February - April 1988 in the Bighorn River. No GBT symptoms were observed on mountain whitefish. $I=$ minor; $I I=$ serious; $I I I=$ severe.

\begin{tabular}{|c|c|c|c|c|c|c|}
\hline \multirow{2}{*}{ Date } & $I$ & II & III & $I$ & II & III \\
\hline & No. $/ \%$ & No. $/ \%$ & No. $/ \%$ & No. $/ \%$ & No. $/ \%$ & No. $/ \%$ \\
\hline
\end{tabular}

$2 / 24$

$3 / 28$

$4 / 20$

$2 / 24$

$3 / 28$

$4 / 20$

$2 / 25$

$3 / 30$

$4 / 21$

$2 / 24$

$3 / 28$

$4 / 20$

$2 / 24$

$3 / 28$

$4 / 20$

$2 / 25$

$3 / 30$

$4 / 21$
All brown trout

$\mathrm{Rkm} 0.0-3.9$

$65 / 88$

$75 / 92$

$9 / 2$

$7 / 8$

$1 / 2$
0
0

$\mathrm{Rkm} 3.9-6.1$

$15 / 100$

$23 / 100$

$32 / 100$

$$
\begin{aligned}
& 0 \\
& 0 \\
& 0
\end{aligned}
$$

0
0
0

$\mathrm{Rkm} 12.2-15.4$

$1 / 100 \quad 0$

$2 / 100$

$6 / 100$

0

0

0
0
0

A11 rainbow trout

$\mathrm{Rkm} \quad 0.0-3.9$

$3 / 100$

$4 / 100$

$7 / 100$
Brown trout $\geq 356 \mathrm{~mm}$

$\begin{array}{lll}29 / 91 & 3 / 9 & 0 \\ 36 / 84 & 7 / 16 & 0 \\ 28 / 90 & 3 / 10 & 0\end{array}$

0
0
0

$\begin{array}{lll}11 / 100 & 0 & 0 \\ 21 / 100 & 0 & 0 \\ 27 / 100 & 0 & 0\end{array}$

$\begin{array}{lll}1 / 100 & 0 & 0 \\ 2 / 100 & 0 & 0 \\ 6 / 100 & 0 & 0\end{array}$

Rainbow trout $\geq 356 \mathrm{~mm}$

$\begin{array}{lll}3 / 100 & 0 & 0 \\ 3 / 100 & 0 & 0 \\ 5 / 100 & 0 & 0\end{array}$

$\mathrm{Rkm} 3.9-6.1$

$\begin{array}{cccccc}0 & 0 & 0 & 0 & 0 & 0 \\ 0 & 0 & 0 & 0 & 0 & 0 \\ 1 / 100 & 0 & 0 & 1 / 100 & 0 & 0\end{array}$

Rkm 12.2-15.4

$\begin{array}{cccccc}0 & 0 & 0 & 0 & 0 & 0 \\ 0 & 0 & 0 & 0 & 0 & 0 \\ 1 / 100 & 0 & 0 & 1 / 100 & 0 & 0\end{array}$




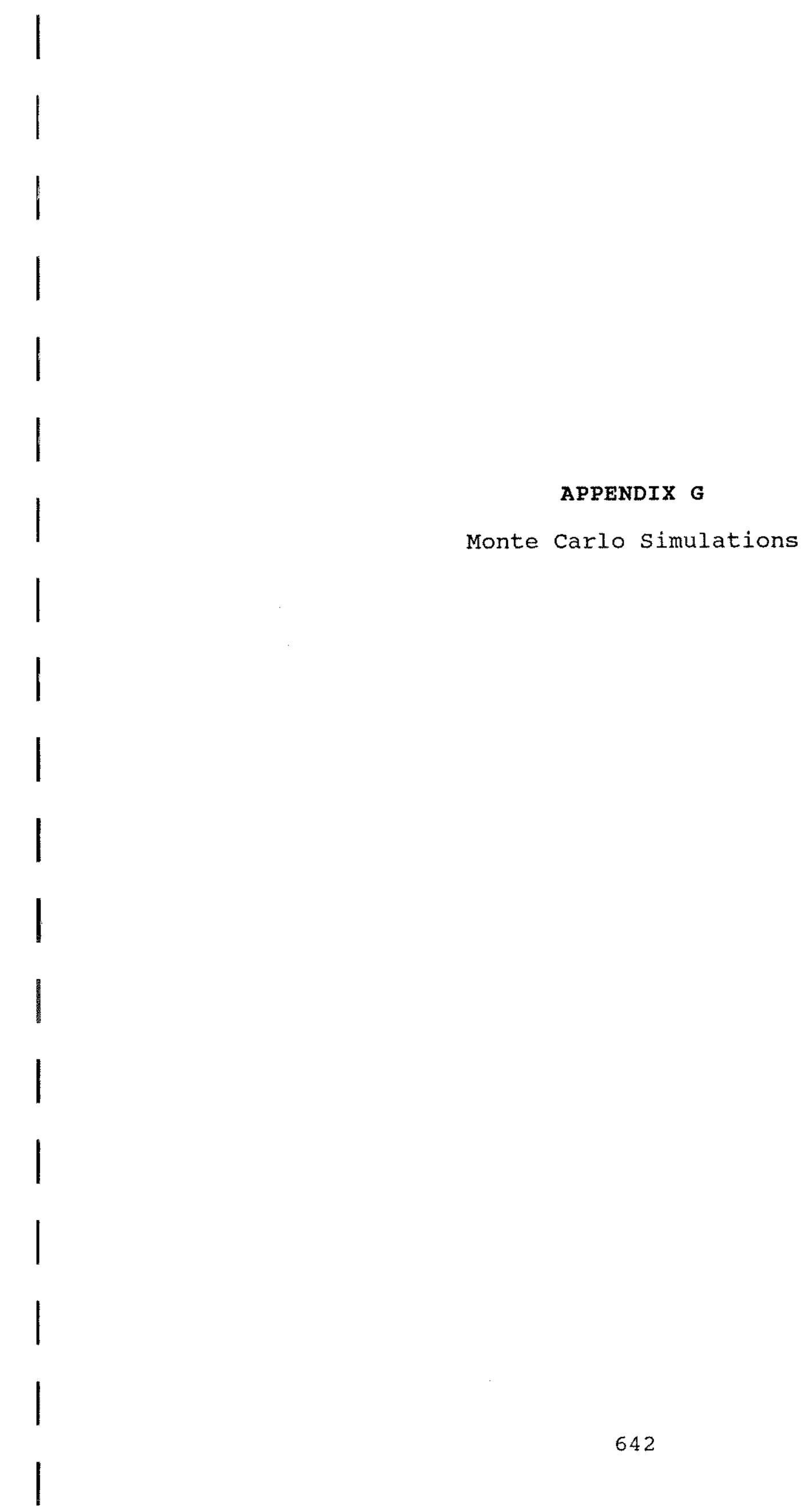


Table 50. Monte carlo simulations to access the probability of obtaining the observed PCA result, all brown trout and rainbow trout, percent of variance explained by eigen vector, June october 1987, Bighorn River, Montana.

\begin{tabular}{llllll}
\hline Principal component & $N$ & Mean & SD & Minimum & Maximum \\
\hline I & 1000 & 19.32 & 0.61 & 17.84 & 21.44 \\
II & 1000 & 18.07 & 0.42 & 17.01 & 19.69 \\
III & 1000 & 17.10 & 0.36 & 15.91 & 18.32 \\
IV & 1000 & 16.18 & 0.37 & 14.98 & 17.75 \\
V & 1000 & 15.24 & 0.42 & 13.87 & 16.32 \\
VI & 1000 & 14.09 & 0.55 & 11.88 & 15.43 \\
\hline
\end{tabular}


Table 51. Monte carlo simulations to access the probability of obtaining the observed PCA result, brown trout and rainbow trout $<15 \mathrm{~cm} \mathrm{TL}$, percent of variance explained by eigen vector, June october 1987, Bighorn River, Montana.

\begin{tabular}{cccccc}
\hline Principal component & $\mathrm{N}$ & Mean & SD & Minimum & Maximum \\
\hline I & 1000 & 24.48 & 1.86 & 19.70 & 30.77 \\
II & 1000 & 20.45 & 1.26 & 16.89 & 25.02 \\
III & 1000 & 17.59 & 0.98 & 14.65 & 20.88 \\
IV & 1000 & 15.06 & 1.03 & 11.86 & 17.88 \\
V & 1000 & 12.62 & 1.07 & 8.90 & 15.91 \\
VI & 1000 & 9.81 & 1.30 & 4.83 & 13.83 \\
\hline
\end{tabular}


Table 52. Monte Carlo simulations to access the probability of obtaining the observed PCA result, brown trout and rainbow trout $>15 \mathrm{~cm} \mathrm{TL}$, percent of variance explained by eigen vector, June 1987, Bighorn River, Montana.

\begin{tabular}{llllll}
\hline Principal component & $\mathrm{N}$ & Mean & SD & Minimum & Maximum \\
\hline I & 1000 & 22.31 & 1.34 & 19.32 & 27.71 \\
II & 1000 & 19.49 & 0.89 & 17.41 & 22.30 \\
III & 1000 & 17.39 & 0.71 & 15.28 & 19.67 \\
IV & 1000 & 15.57 & 0.76 & 12.86 & 17.57 \\
V & 1000 & 13.72 & 0.80 & 11.10 & 15.97 \\
VI & 1000 & 11.52 & 1.04 & 8.12 & 14.60
\end{tabular}


Table 53. Monte Carlo simulations to access the probability of obtaining the observed PCA result, brown trout and rainbow trout $>15$ cm TL, percent of eigen vector explained by eigen vector, July 1987, Bighorn River, Montana.

\begin{tabular}{cccccc}
\hline Principal component & $\mathrm{N}$ & Mean & SD & Minimum & Maximum \\
\hline I & 1000 & 21.61 & 1.17 & 18.64 & 26.66 \\
II & 1000 & 19.18 & 0.83 & 16.91 & 22.12 \\
III & 1000 & 17.31 & 0.62 & 15.52 & 19.30 \\
IV & 1000 & 15.70 & 0.64 & 13.41 & 17.50 \\
V & 1000 & 14.08 & 0.75 & 11.54 & 16.19 \\
VI & 1000 & 12.13 & 0.94 & 9.18 & 14.60 \\
\hline
\end{tabular}


Table 54. Monte Carlo simulations to access the probability of obtaining the observed PCA result, brown trout and rainbow trout $>15 \mathrm{~cm}$ TL, percent of eigen vector explained by eigen vector, September and October 1987, Bighorn River, Montana.

\begin{tabular}{cccccc}
\hline Principal component & $\mathrm{N}$ & Mean & SD & Minimum & Maximum \\
\hline I & 1000 & 26.08 & 2.34 & 20.11 & 34.83 \\
II & 1000 & 21.16 & 1.53 & 17.57 & 26.60 \\
III & 1000 & 17.63 & 1.20 & 13.48 & 21.15 \\
IV & 1000 & 14.68 & 1.22 & 10.68 & 17.73 \\
V & 1000 & 11.78 & 1.32 & 7.52 & 15.88 \\
VI & 1000 & 8.67 & 1.44 & 4.70 & 12.73 \\
\hline
\end{tabular}




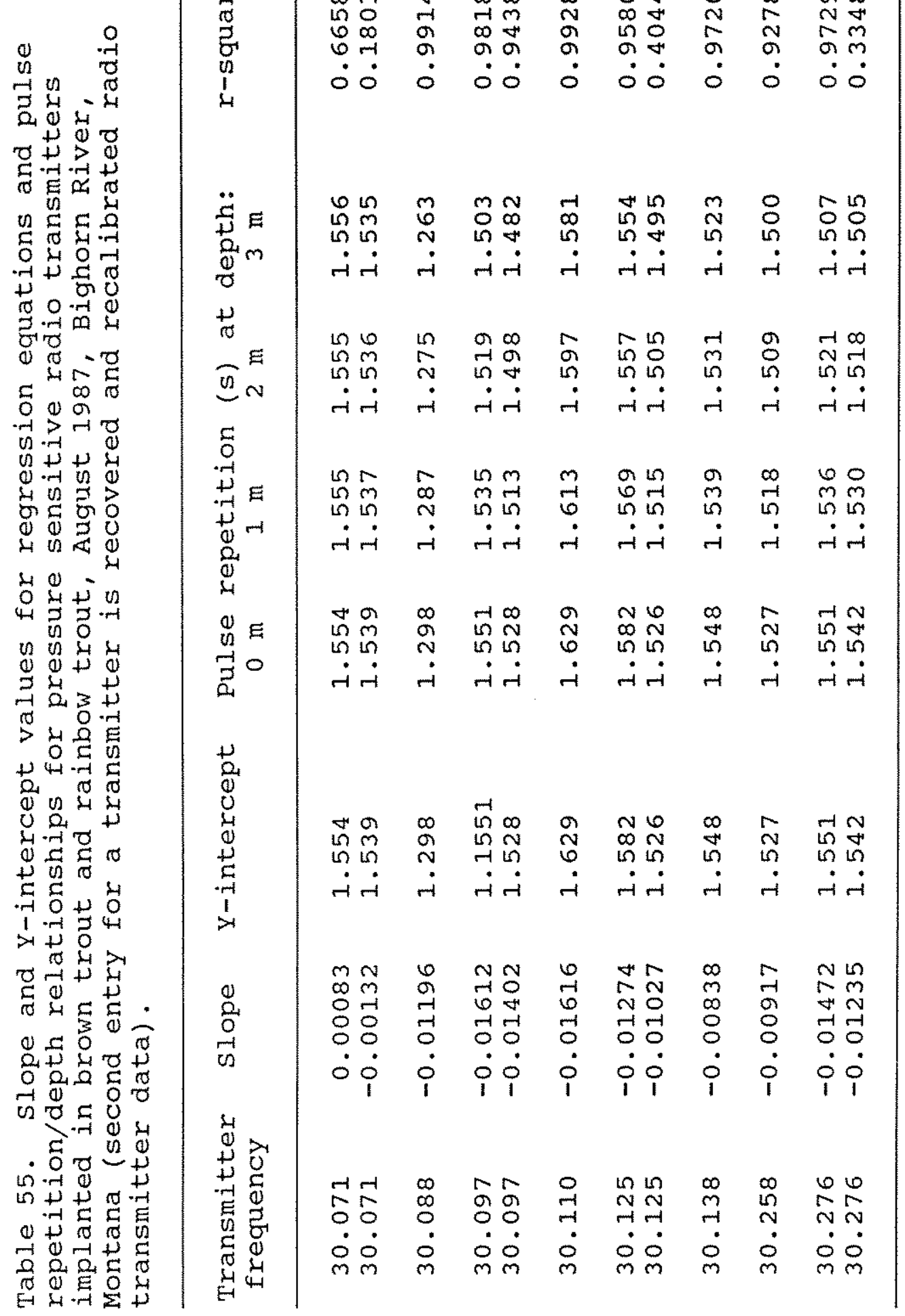




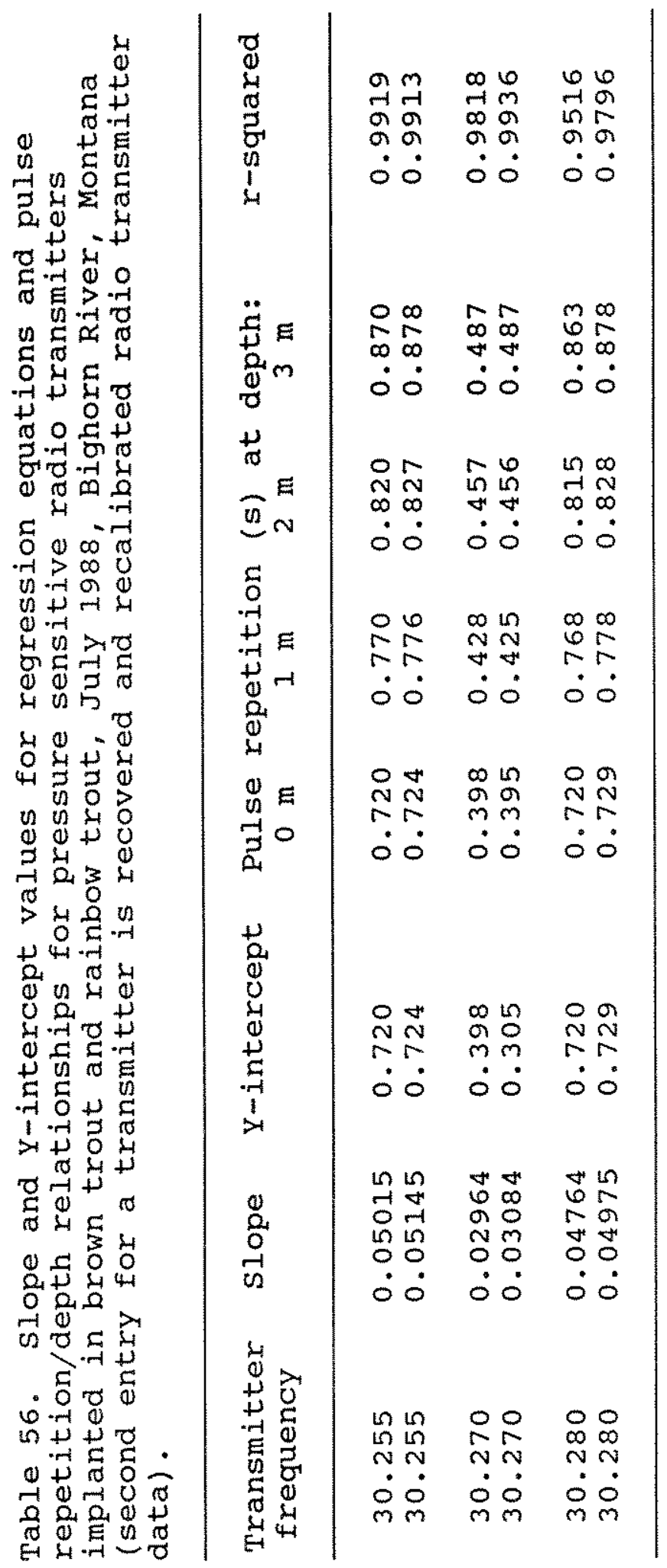



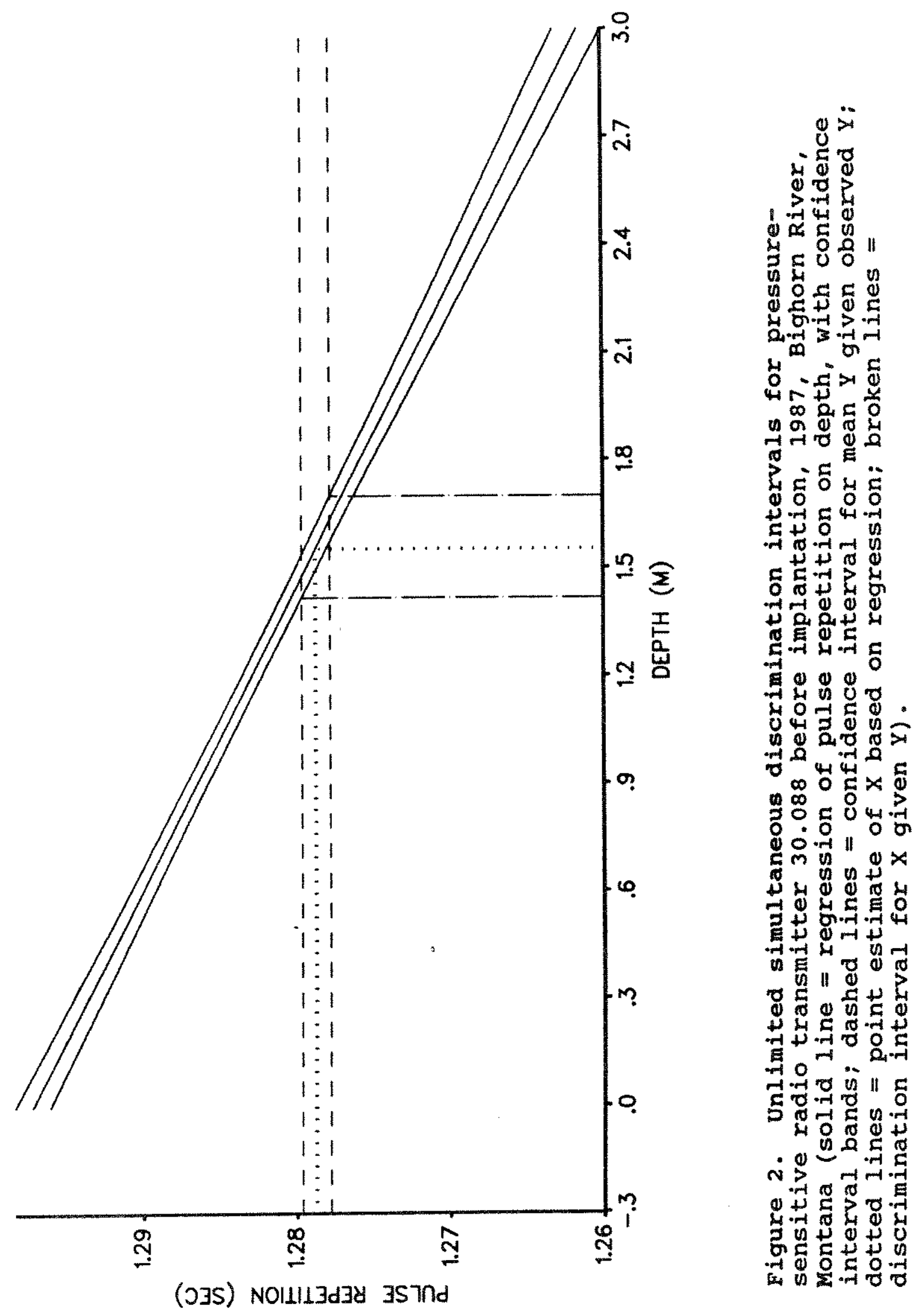

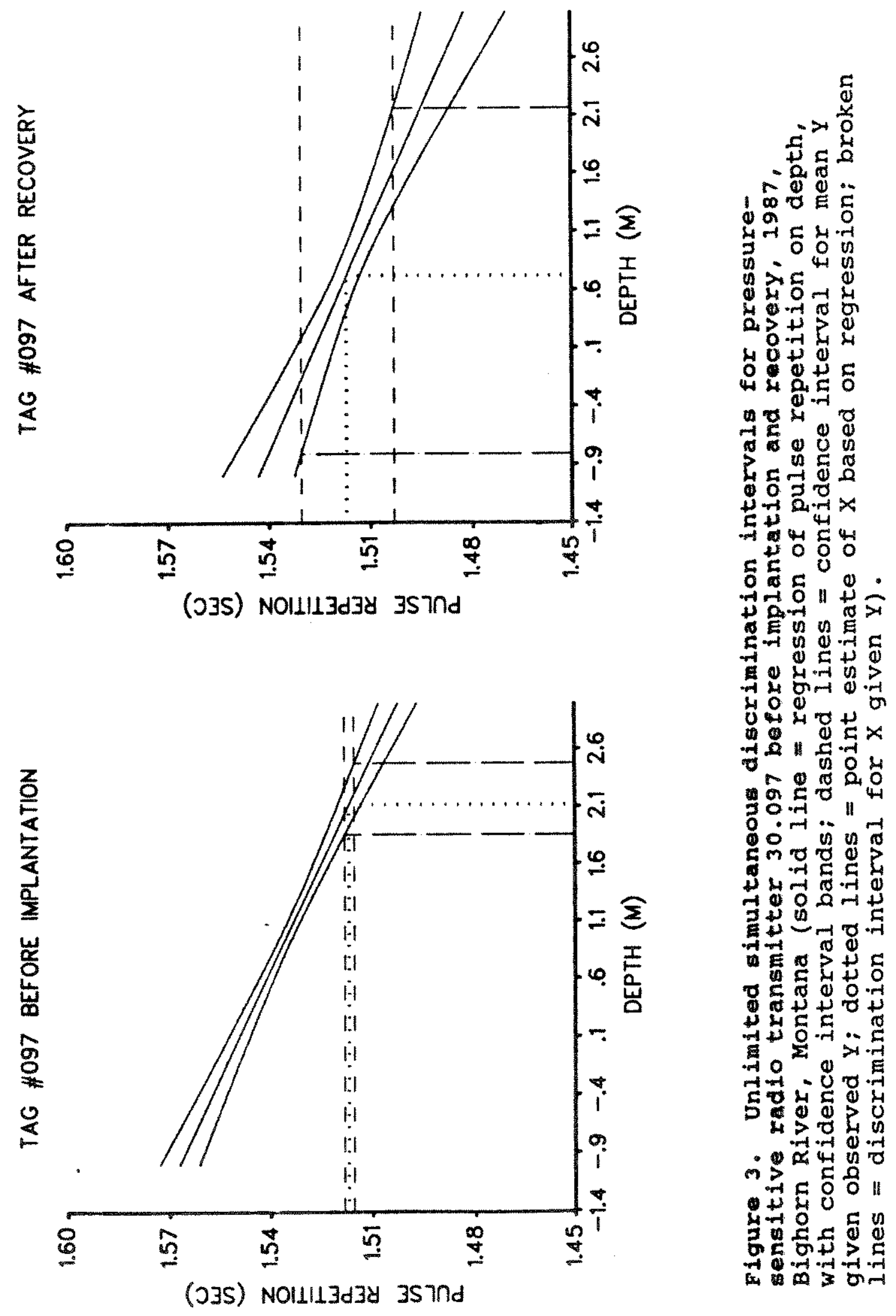
APPENDIX I

Gas Levels 


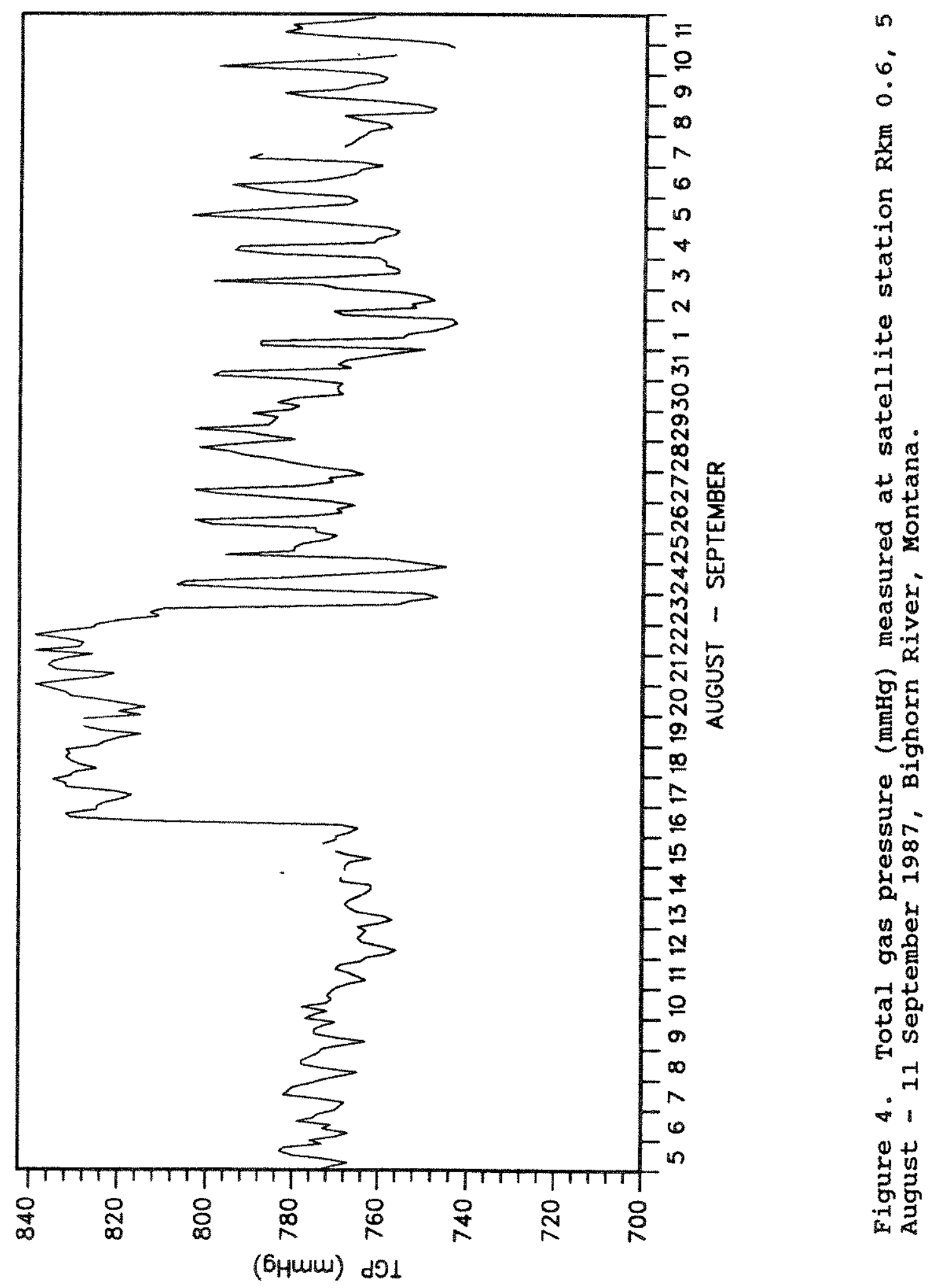




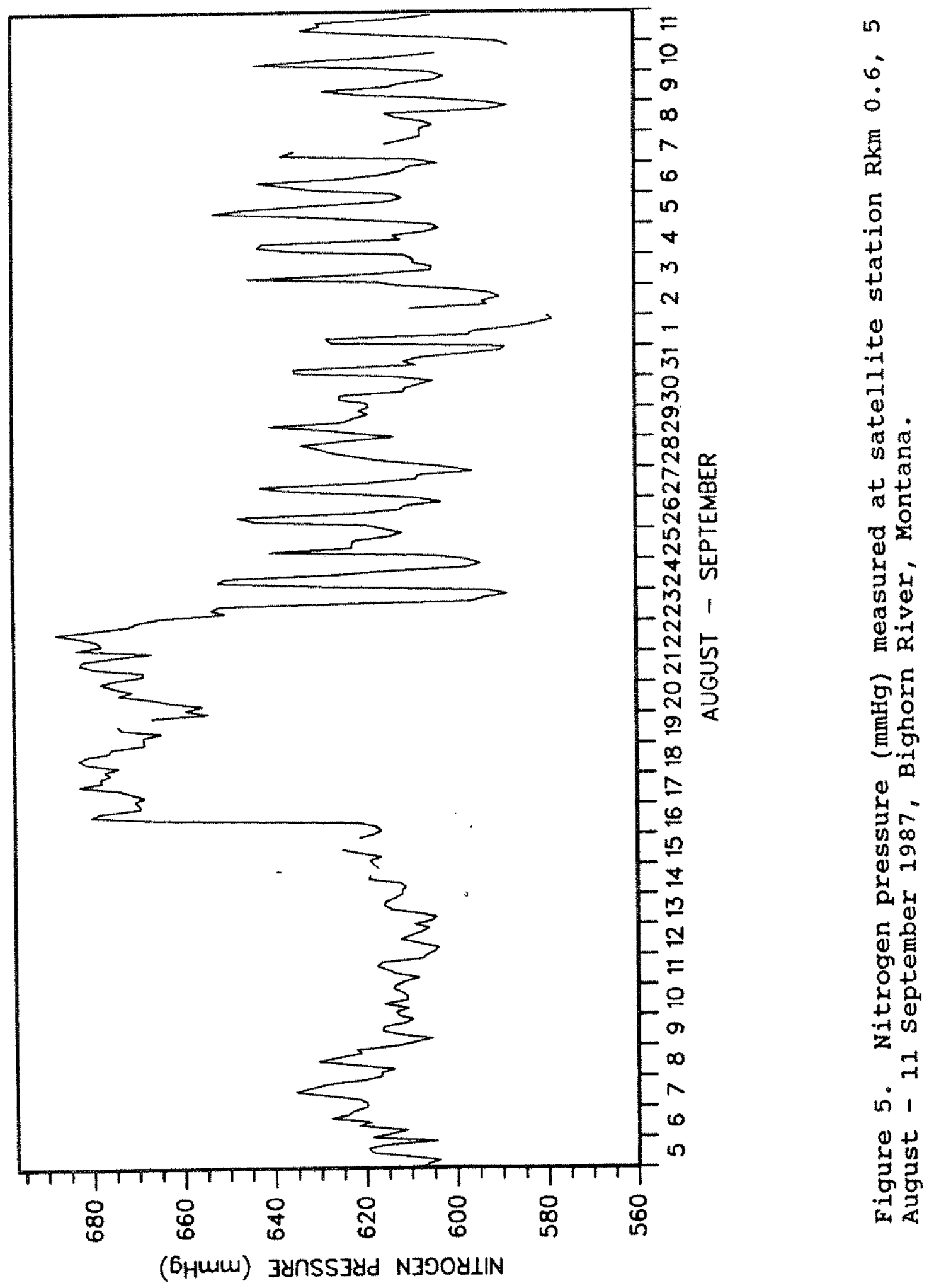




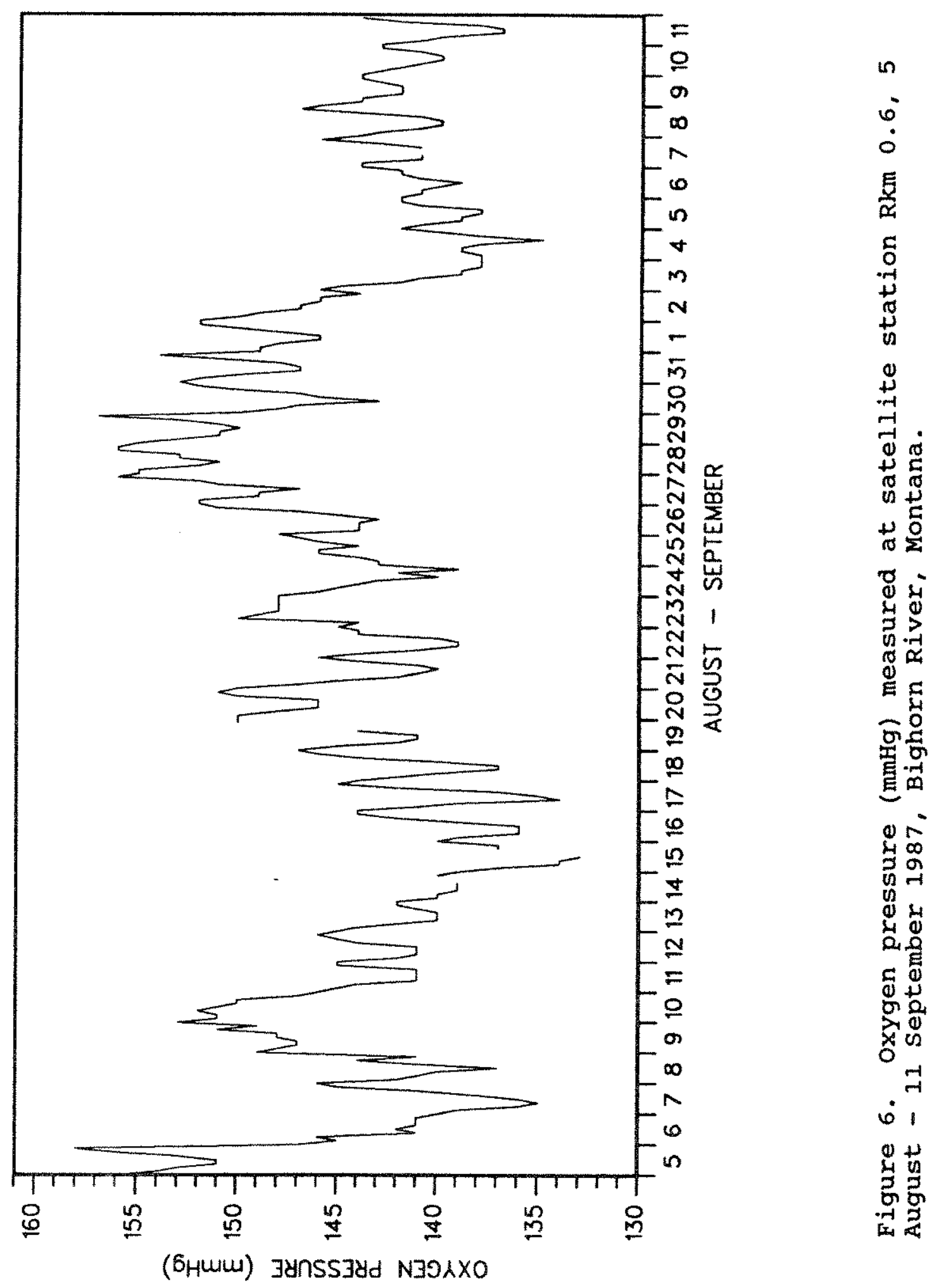




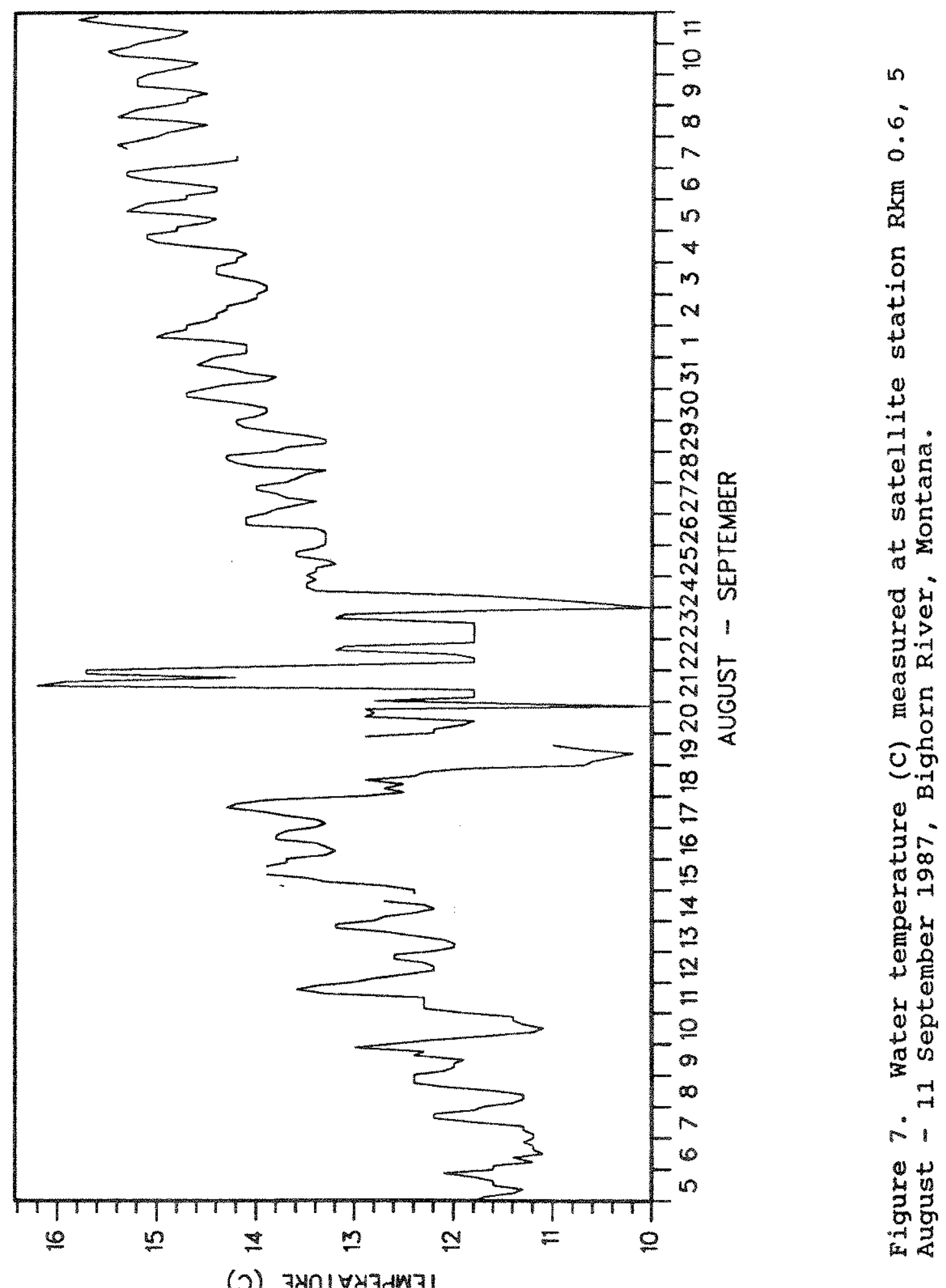

(0) $38 n 1 \forall y 3 d w] 1$ 


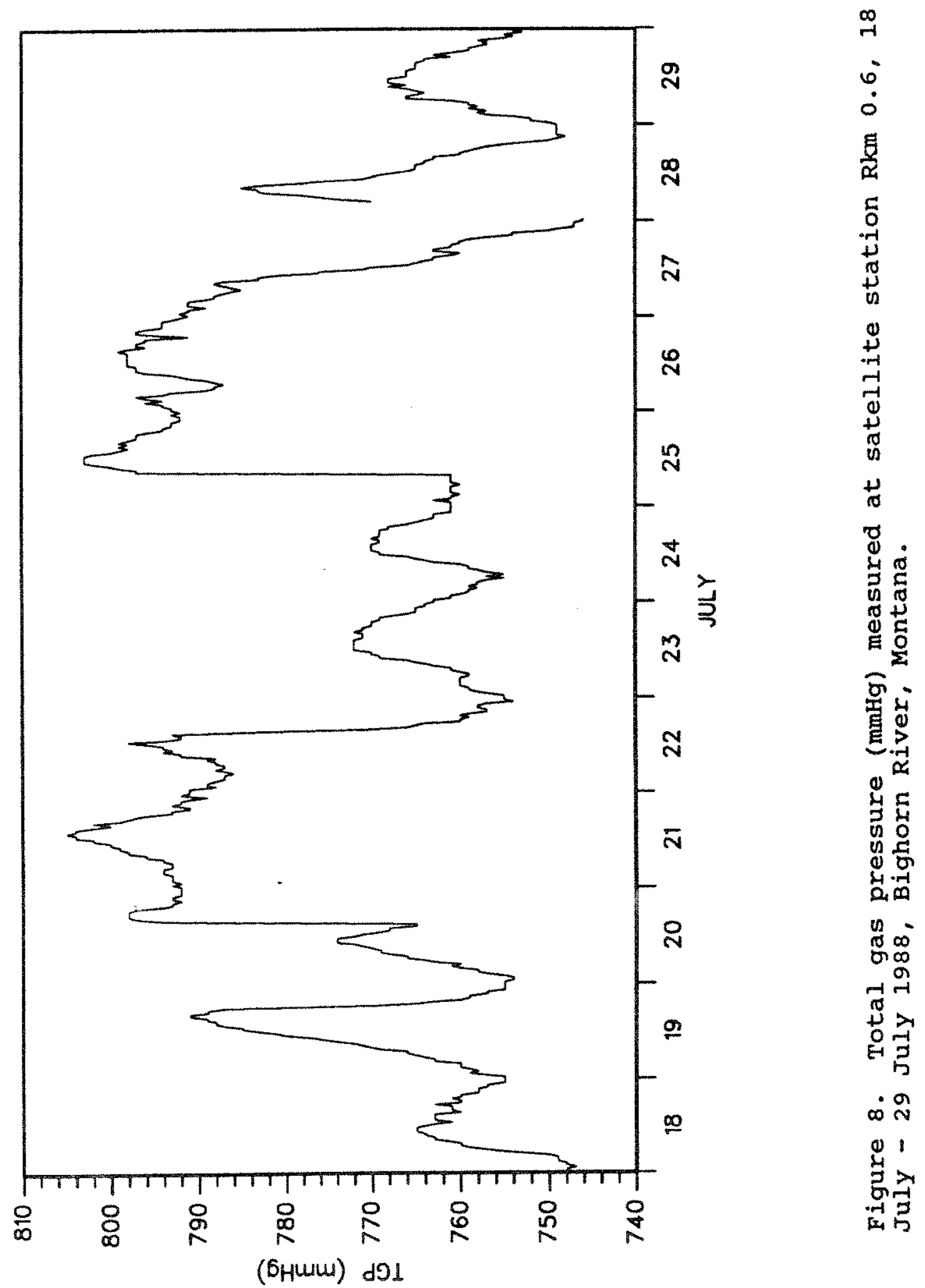



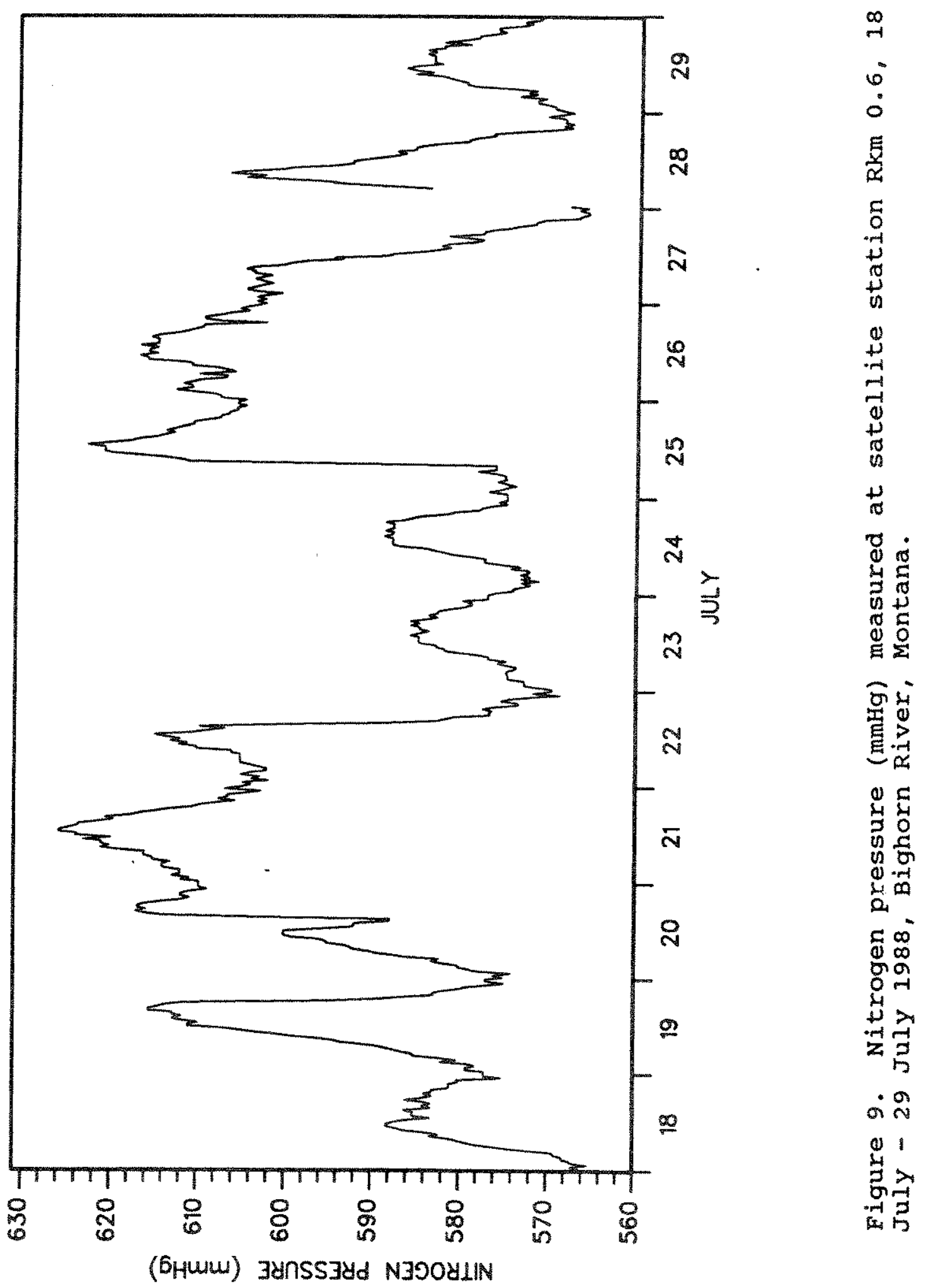

659 


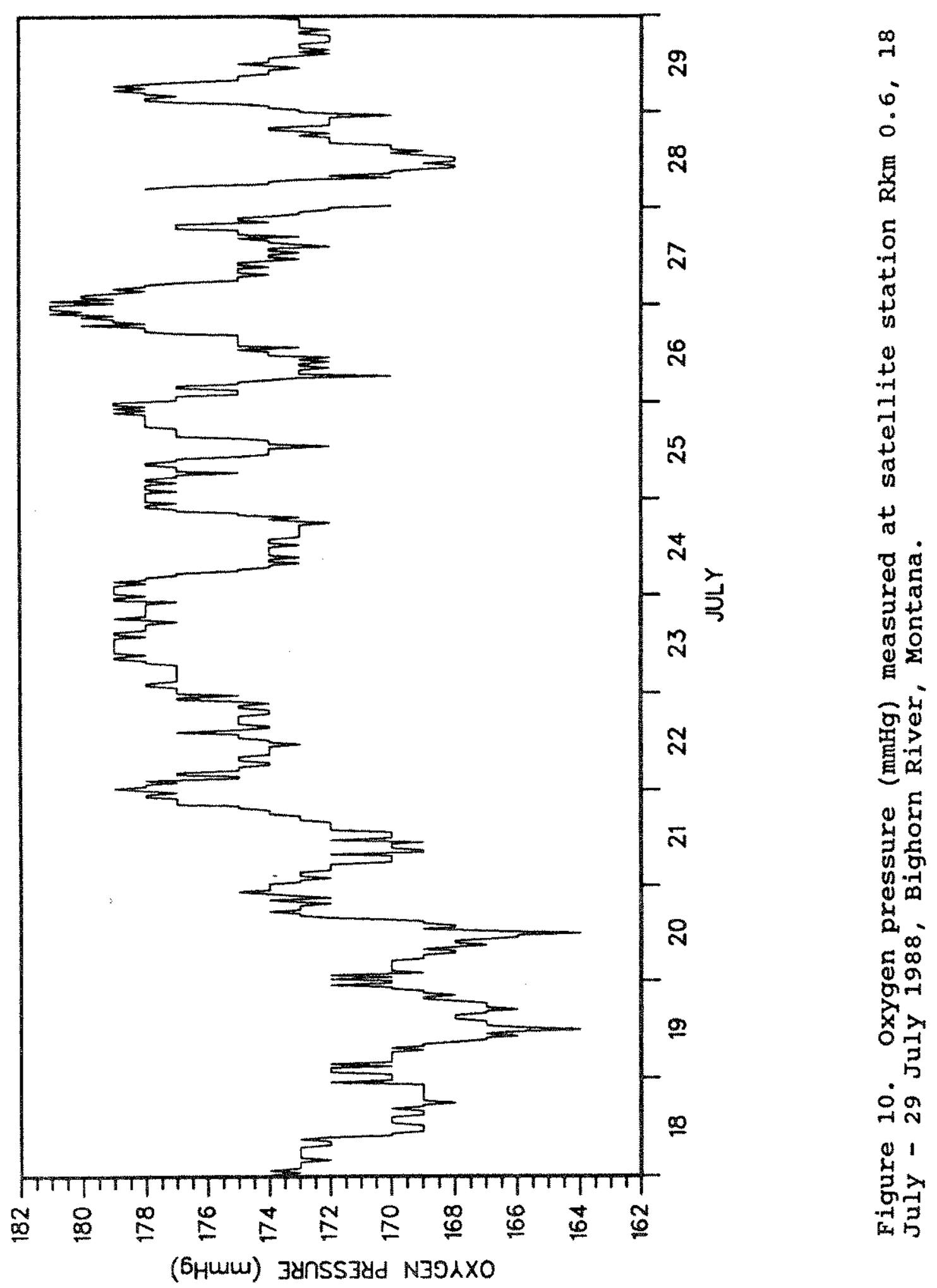




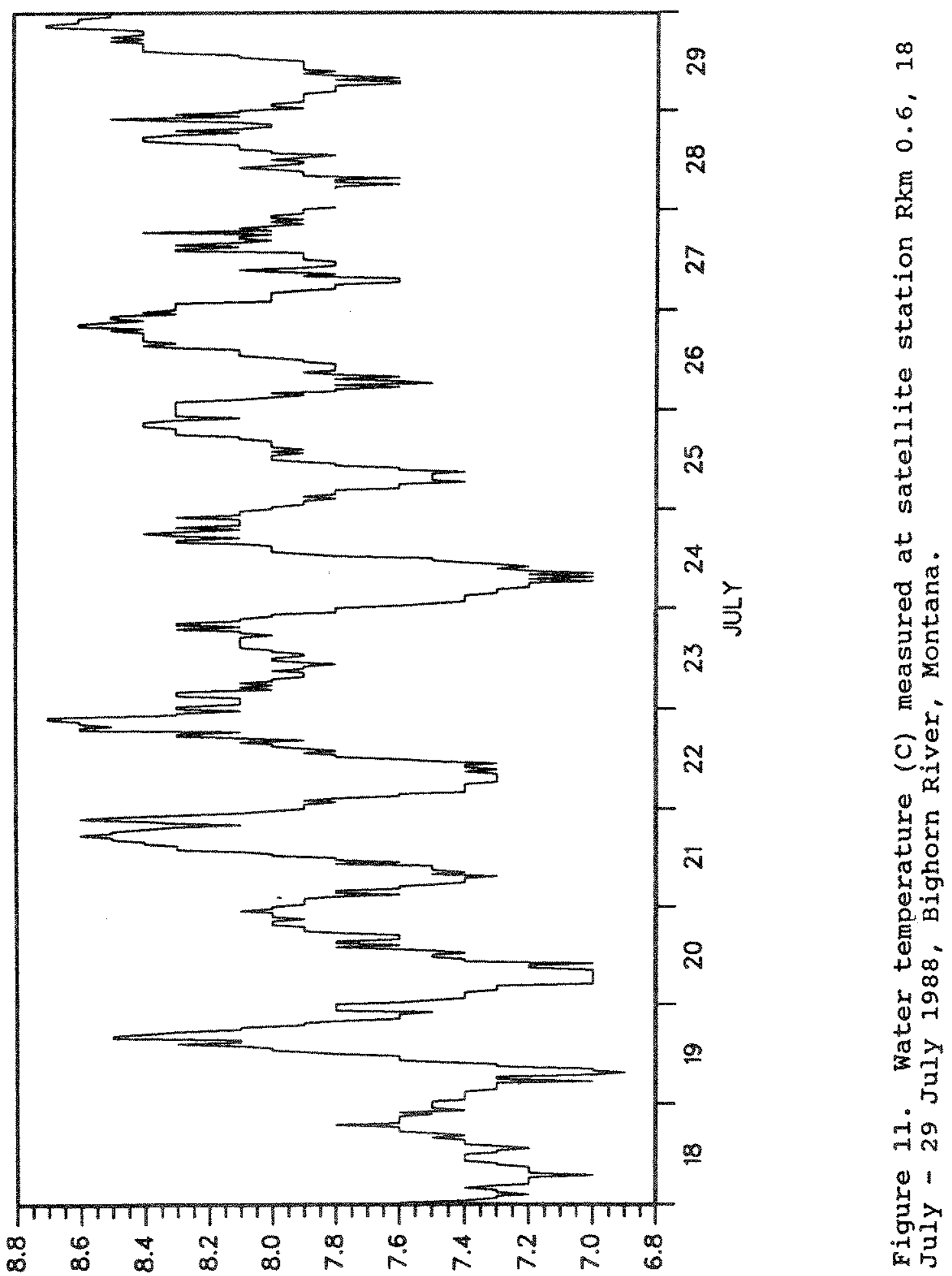

(0) $38 n 1783 d w 31$ 


\section{APPENDIX J}

\section{Fish Movement Maps}

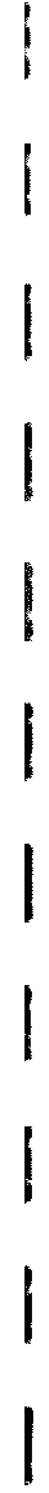




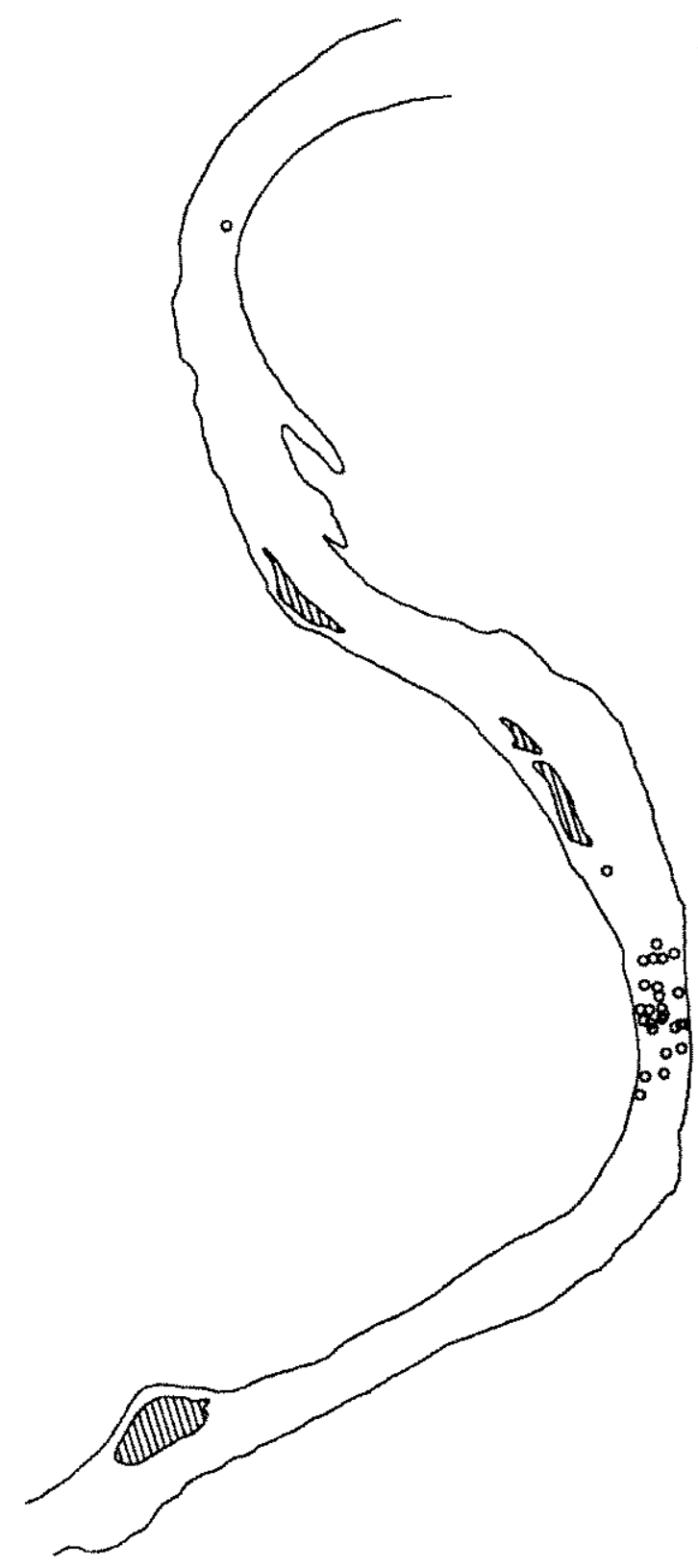

Figure 12. Movement of radio-tagged trout $43(30.043 \mathrm{MHz}), 1987$, Bighorn River, Montana. 


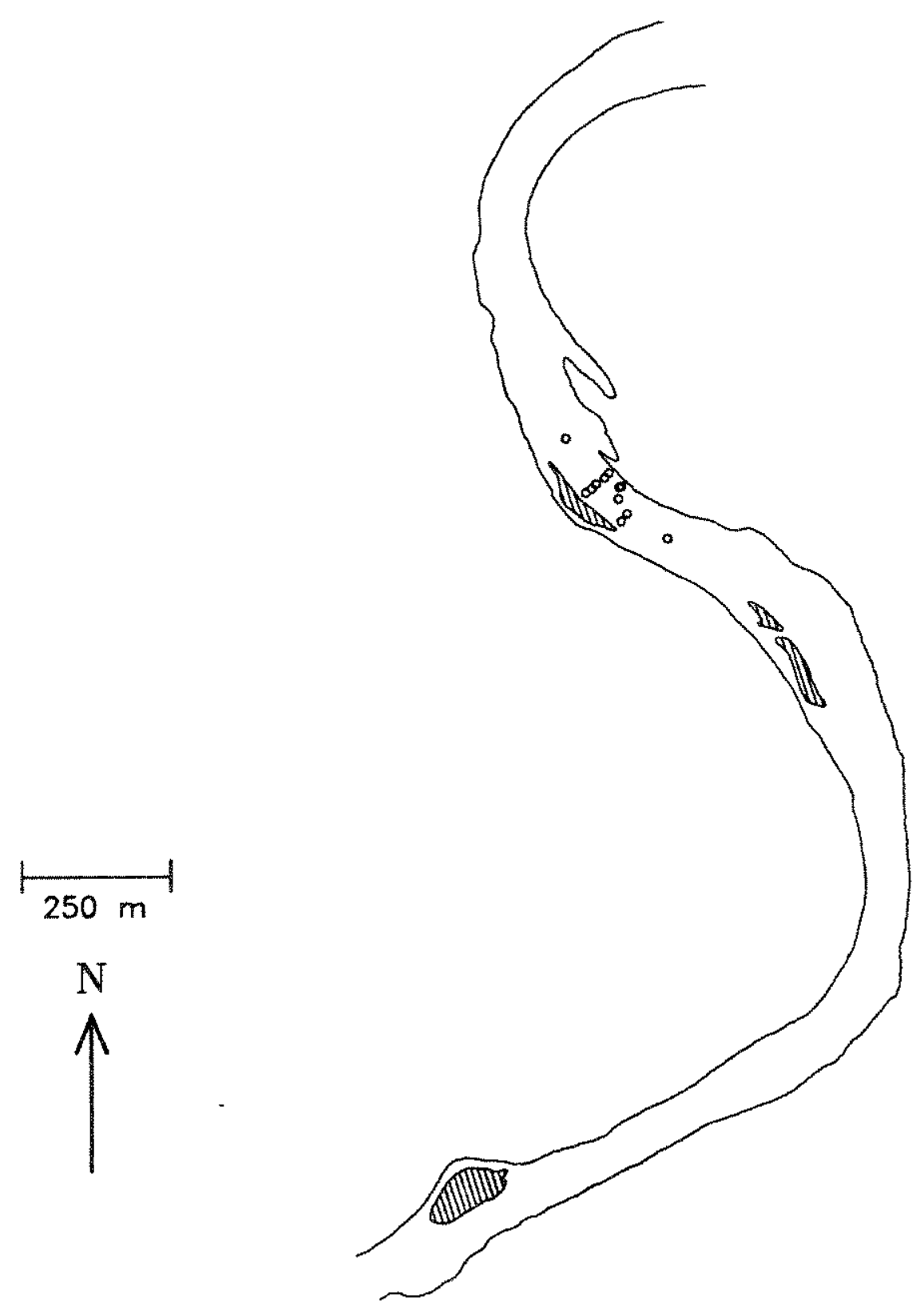

Figure 13. Movement of radio-tagged trout $62(30.062 \mathrm{MHz}), 1987$, Bighorn River, Montana. 

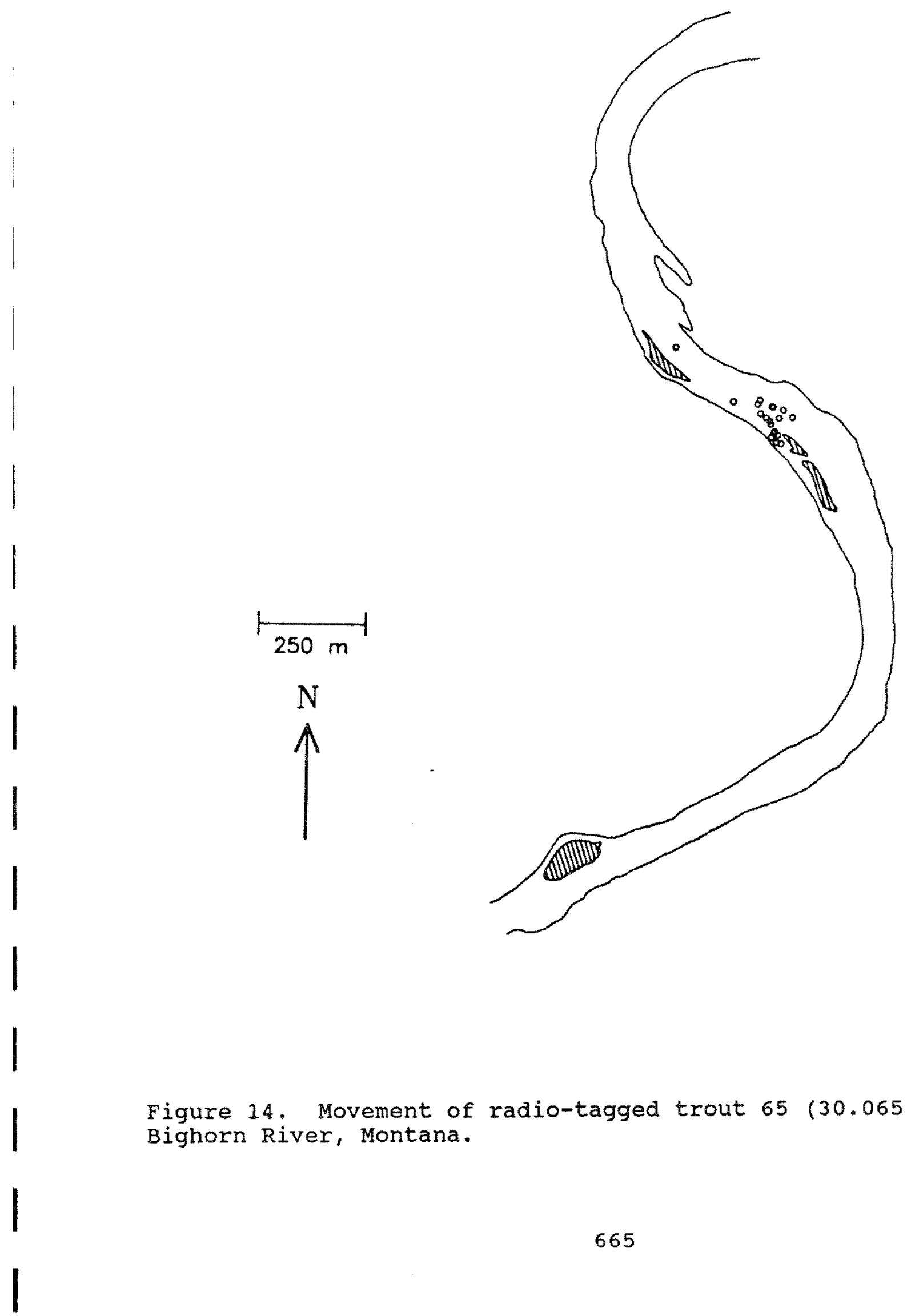

Figure 14. Movement of radio-tagged trout $65(30.065 \mathrm{MHz}), 1987$, Bighorn River, Montana. 


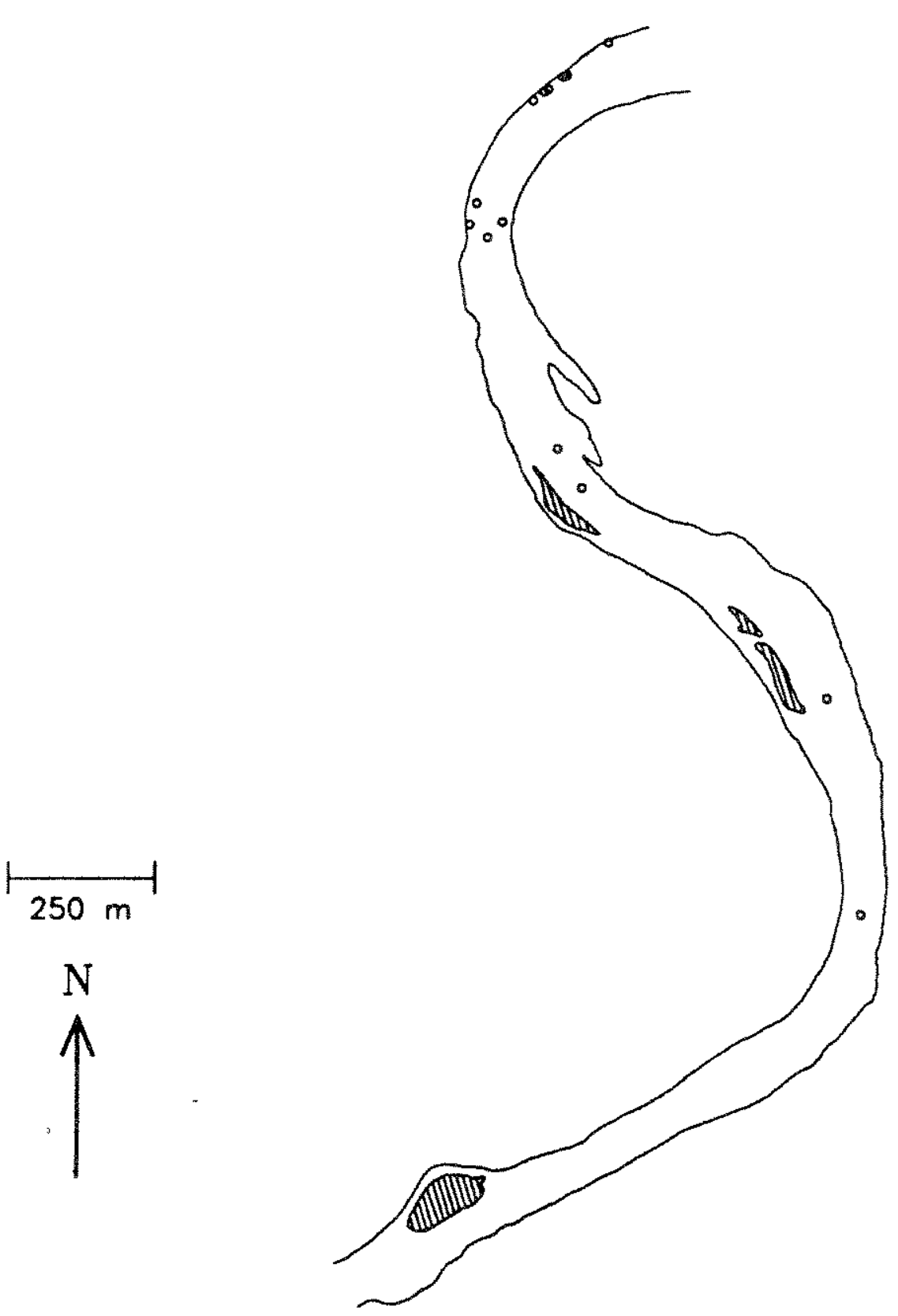

Figure 15. Movement of radio-tagged trout $71(30.071 \mathrm{MHz}), 1987$, Bighorn River, Montana. 


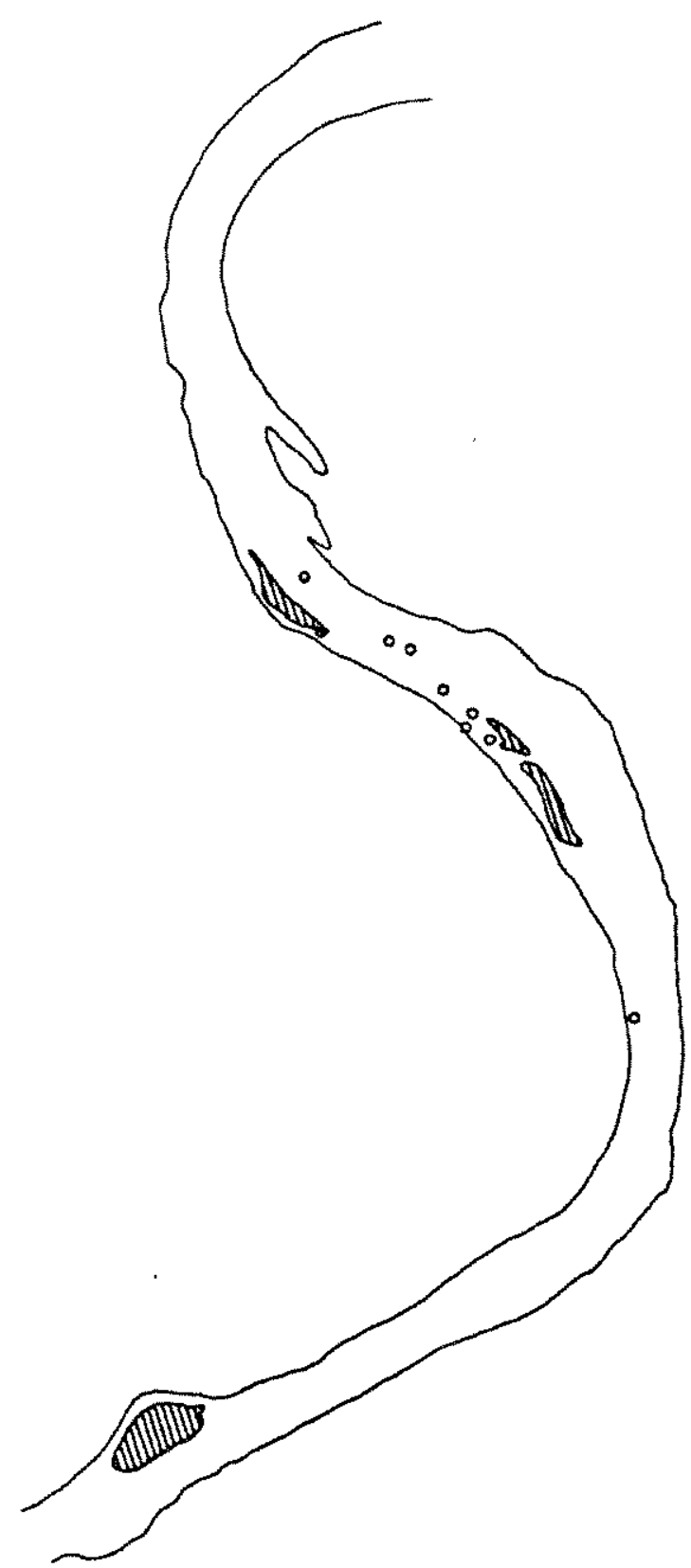

Figure 16. Movement of radio-tagged trout $88(30.088 \mathrm{MHz}), 1987$, Bighorn River, Montana. 


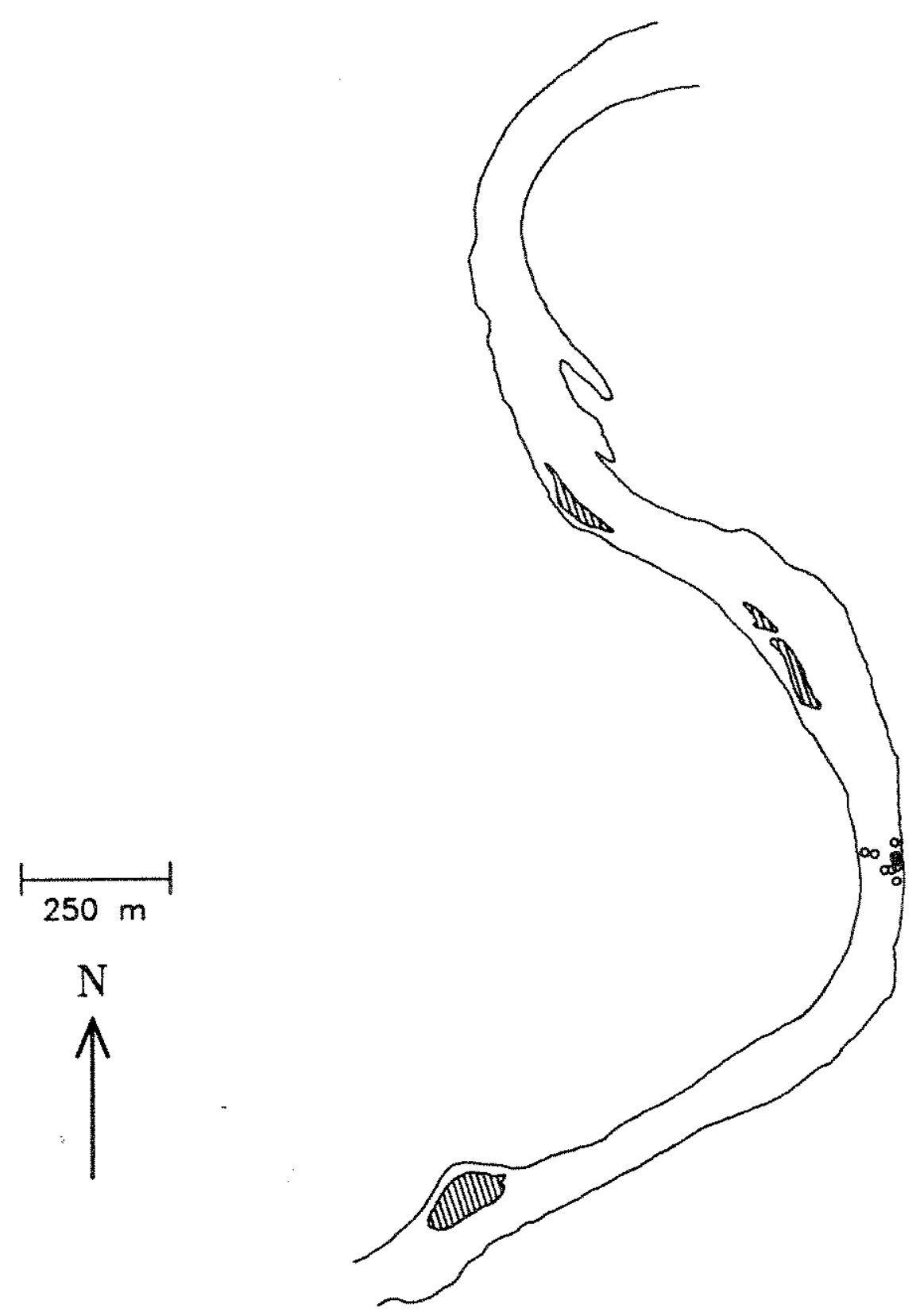

Figure 17. Movement of radio-tagged trout 97 (30.097 MHz), 1987, Bighorn River, Montana. 

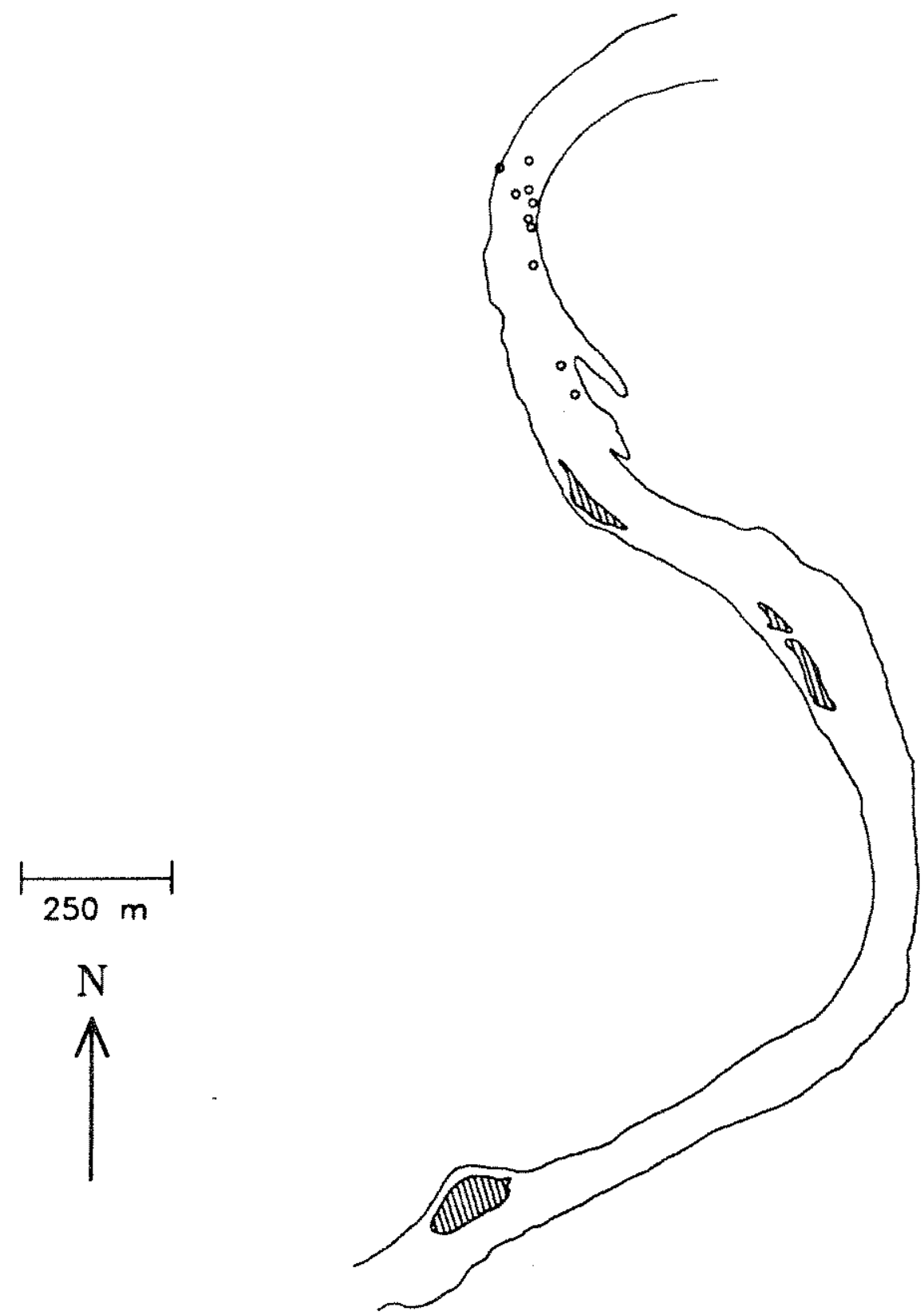

Figure 18. Movement of radio-tagged trout $110(30.110 \mathrm{MHz})$, 1987, Bighorn River, Montana. 


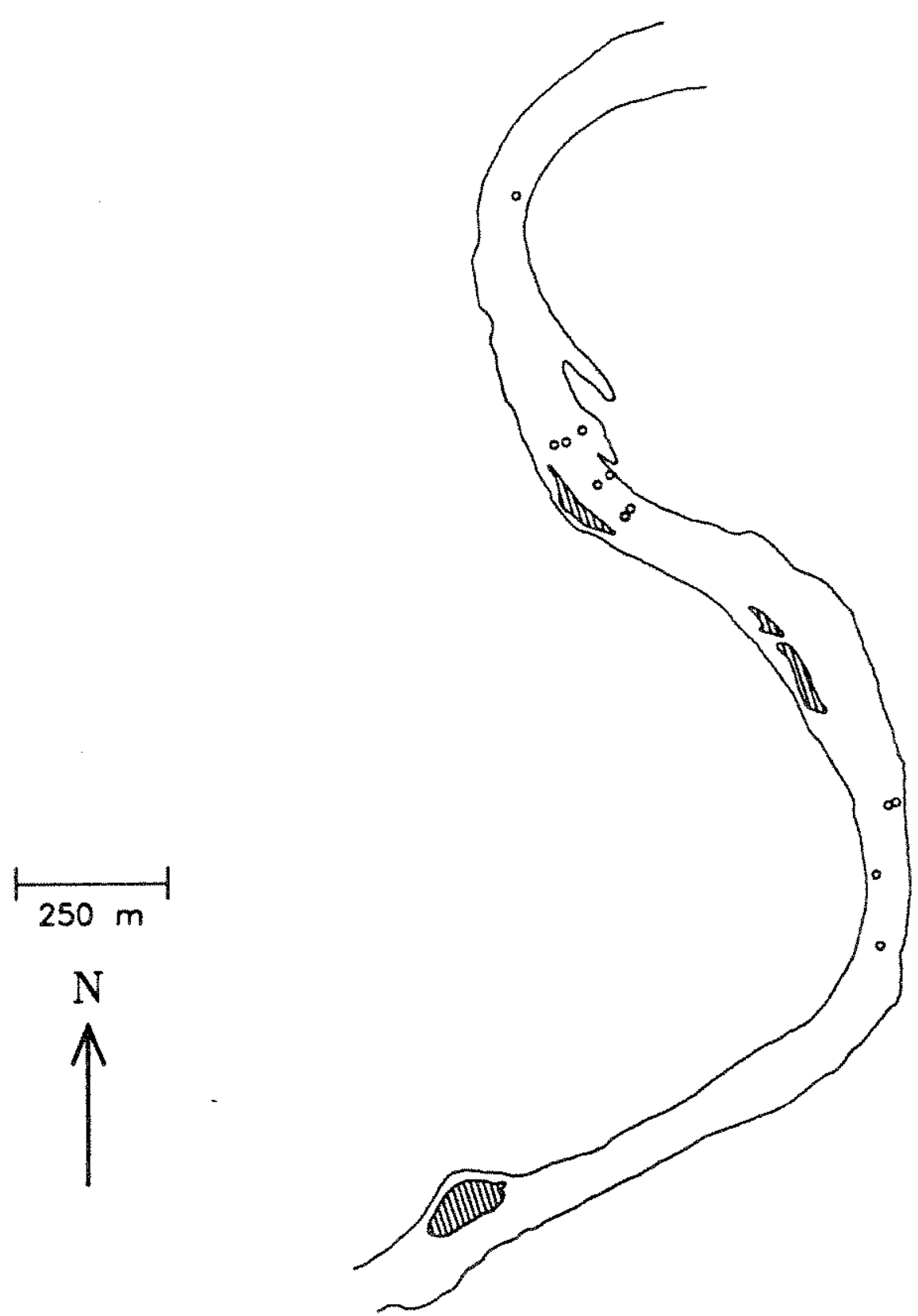

Figure 19. Movement of radio-tagged trout 125 (30.125 MHz), 1987, Bighorn River, Montana. 


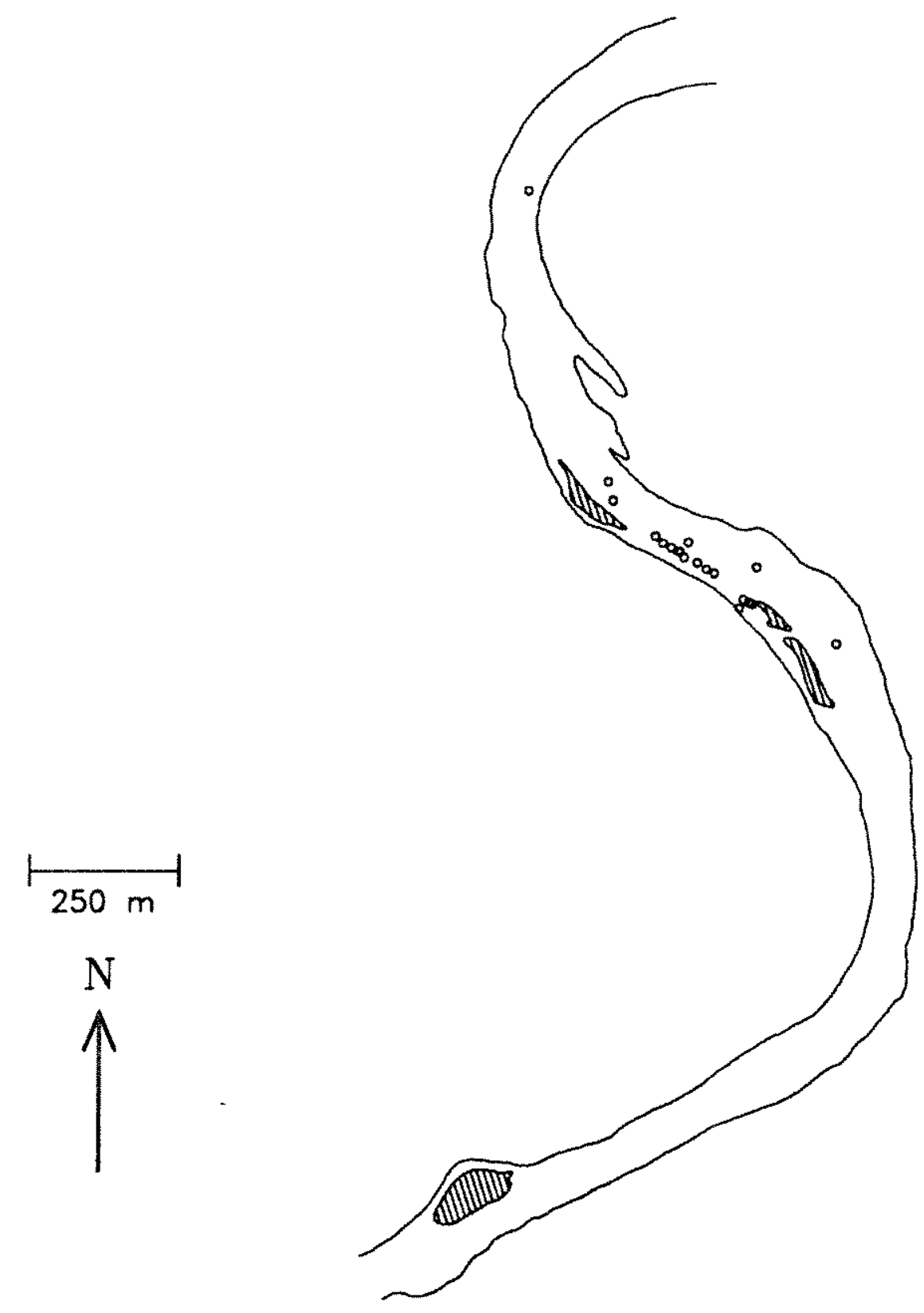

Figure 20. Movement of radio-tagged trout 168 (30.168 MHz), 1987, Bighorn River, Montana. 


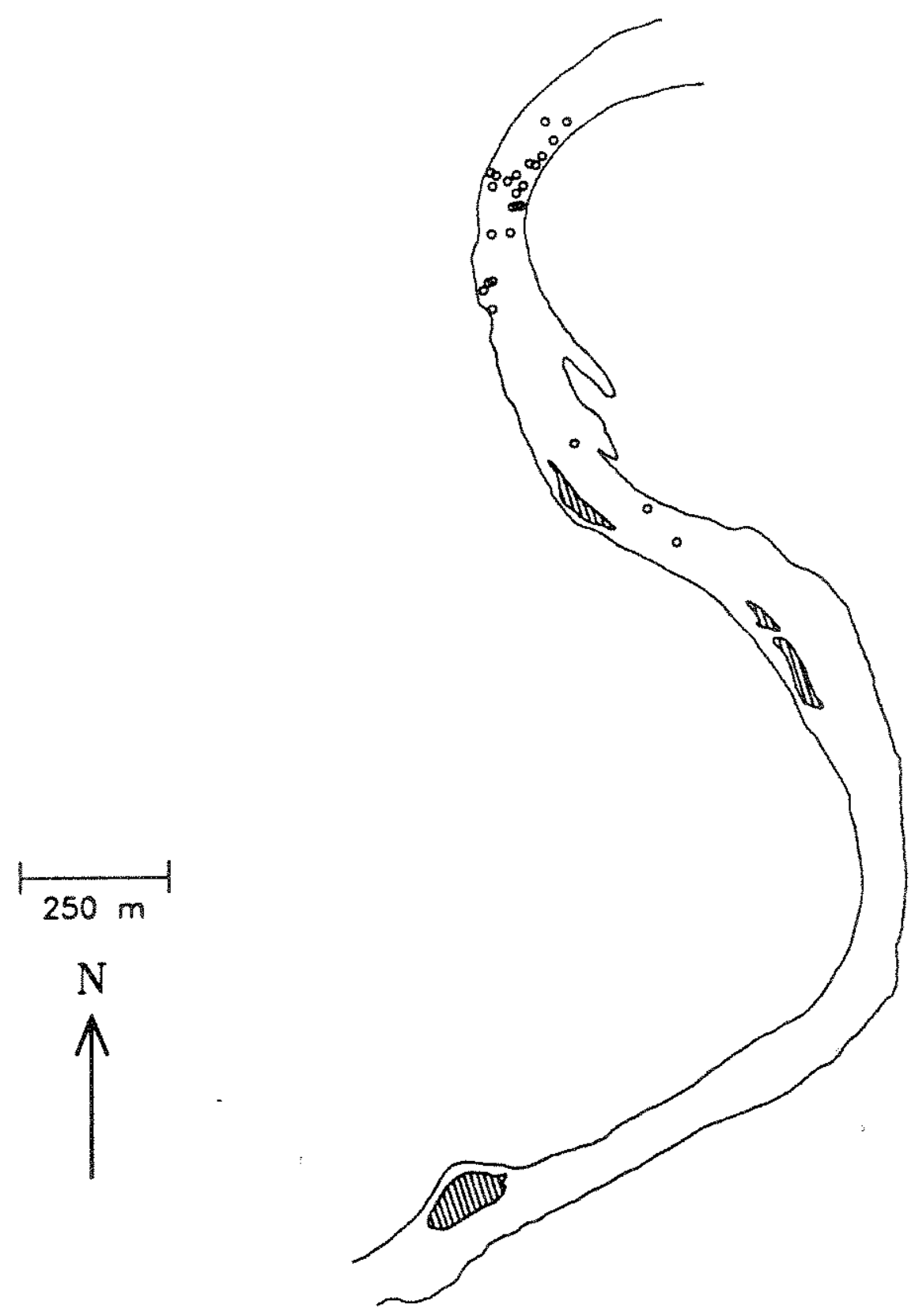

Figure 21. Movement of radio-tagged trout $188(30.188 \mathrm{MHz})$, 1987, Bighorn River, Montana. 


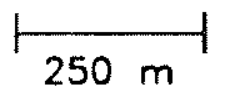

$\mathrm{N}$
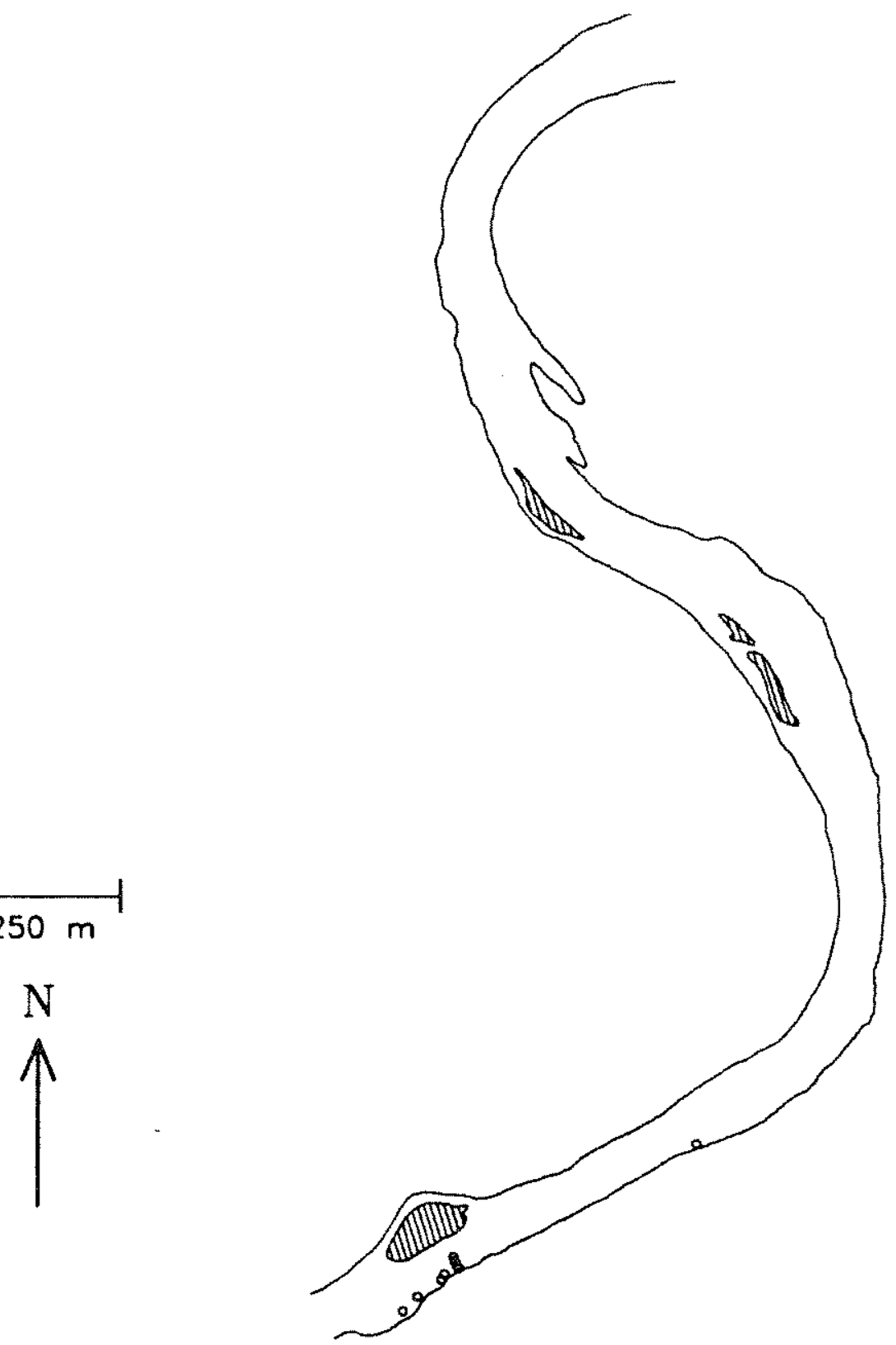

Figure 22. Movement of radio-tagged trout $215(30.215 \mathrm{MHz})$, 1987, Bighorn River, Montana. 


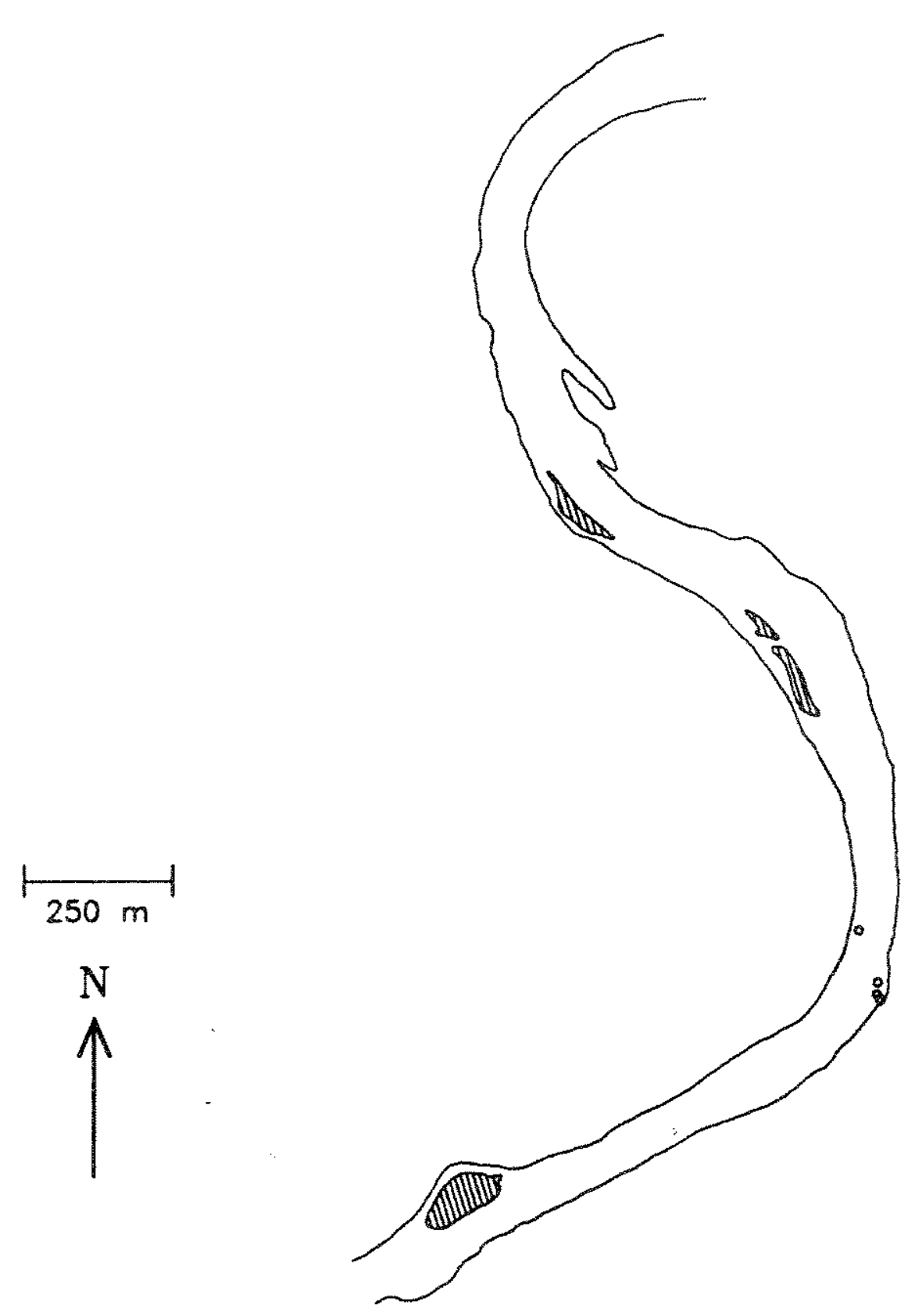

Figure 23. Movement of radio-tagged trout 227 (30.227 MHz), 1987, Bighorn River, Montana. 


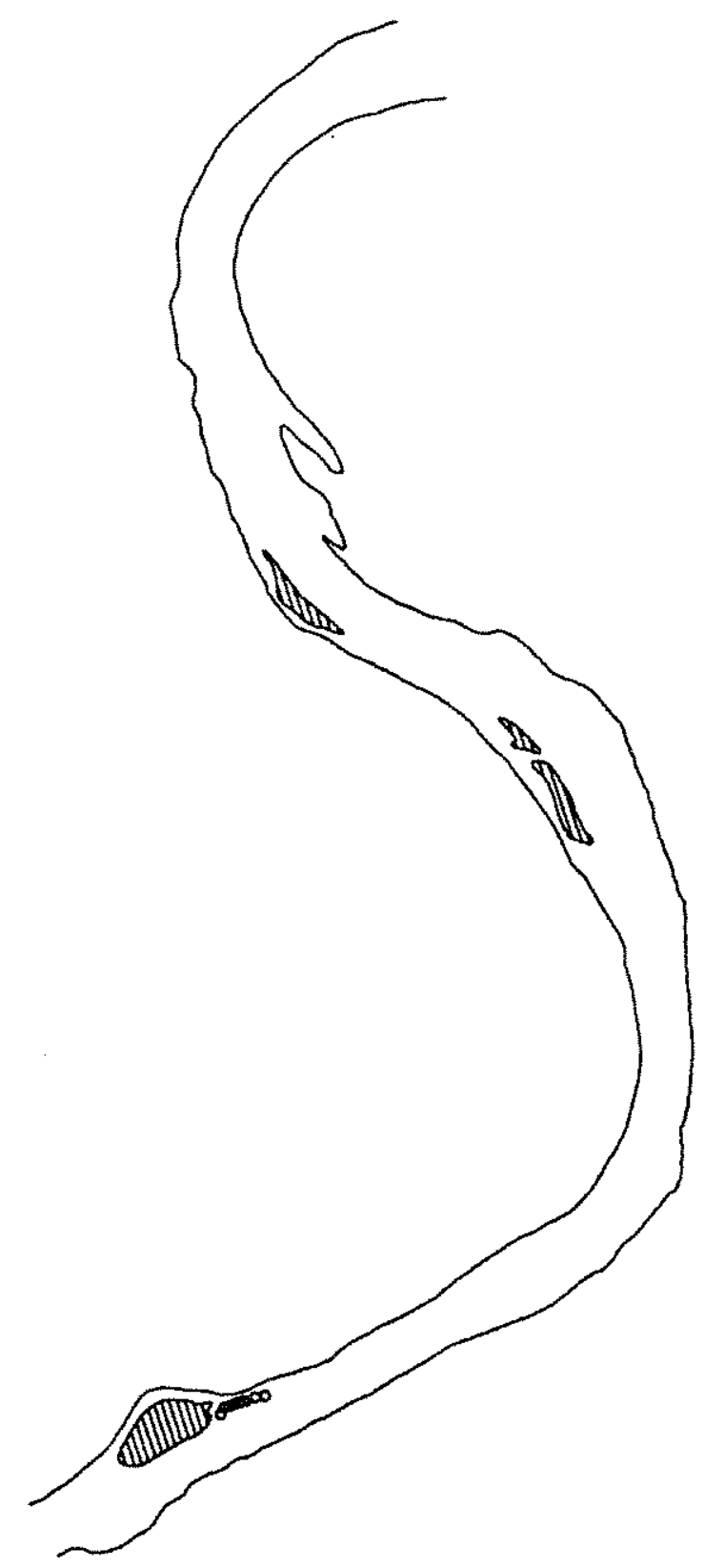

Figure 24. Movement of radio-tagged trout $237(30.237 \mathrm{MHz})$, 1987, Bighorn River, Montana. 

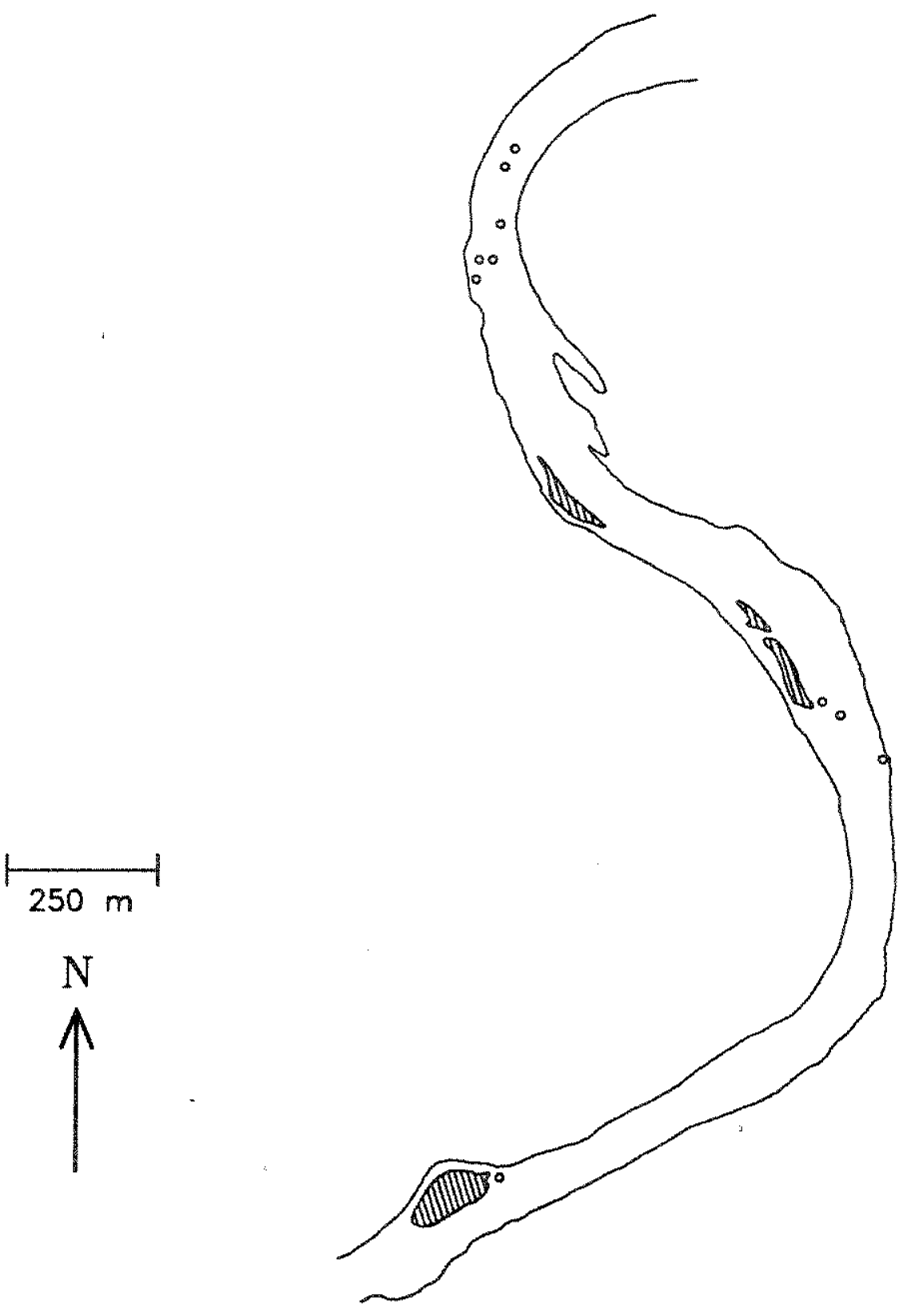

Figure 25. Movement of radio-tagged trout $276(30.276 \mathrm{MHz})$, 1987, Bighorn River, Montana. 


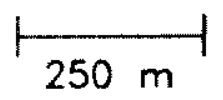

$\mathrm{N}$

$\uparrow$

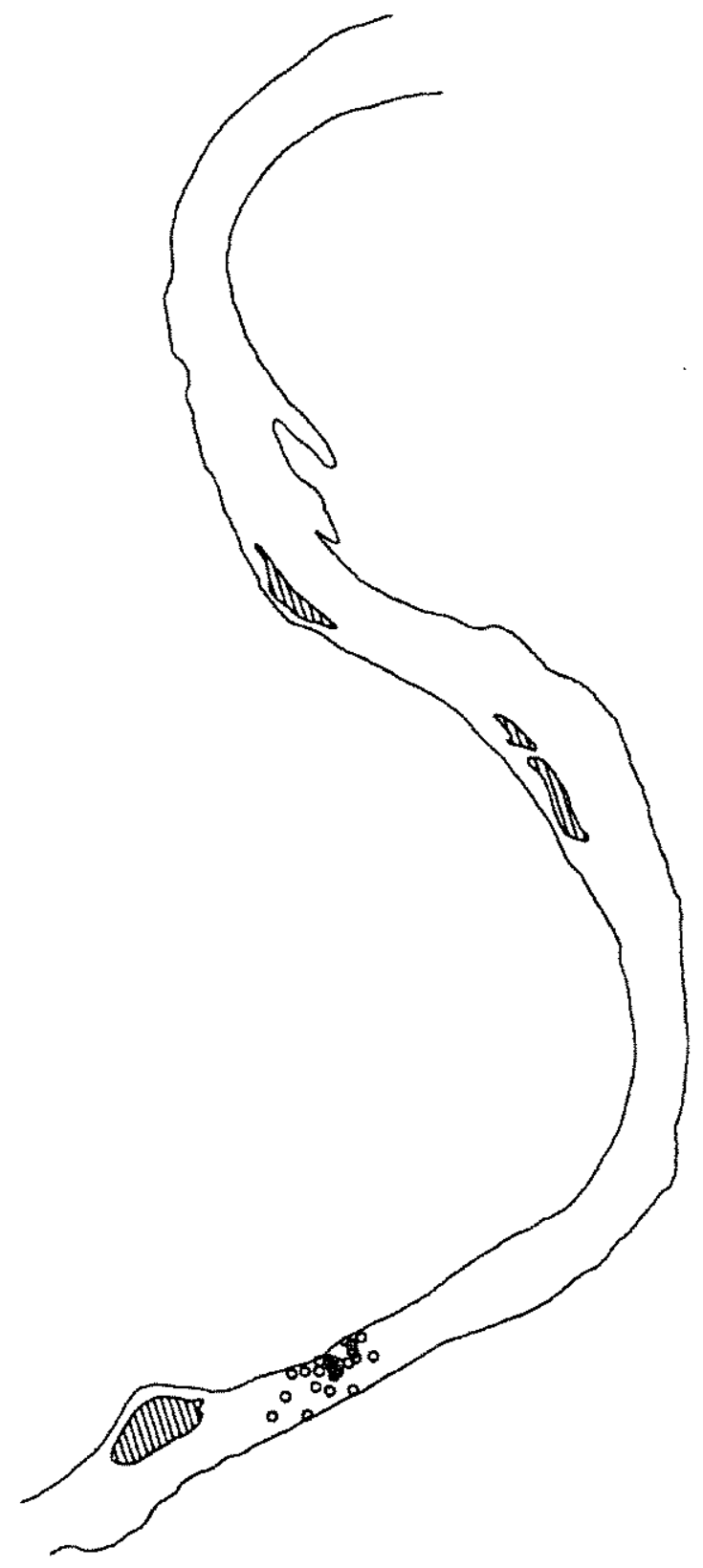

Figure 26. Movement of radio-tagged trout 255 (30.255 MHz), 1988, Bighorn River, Montana. 

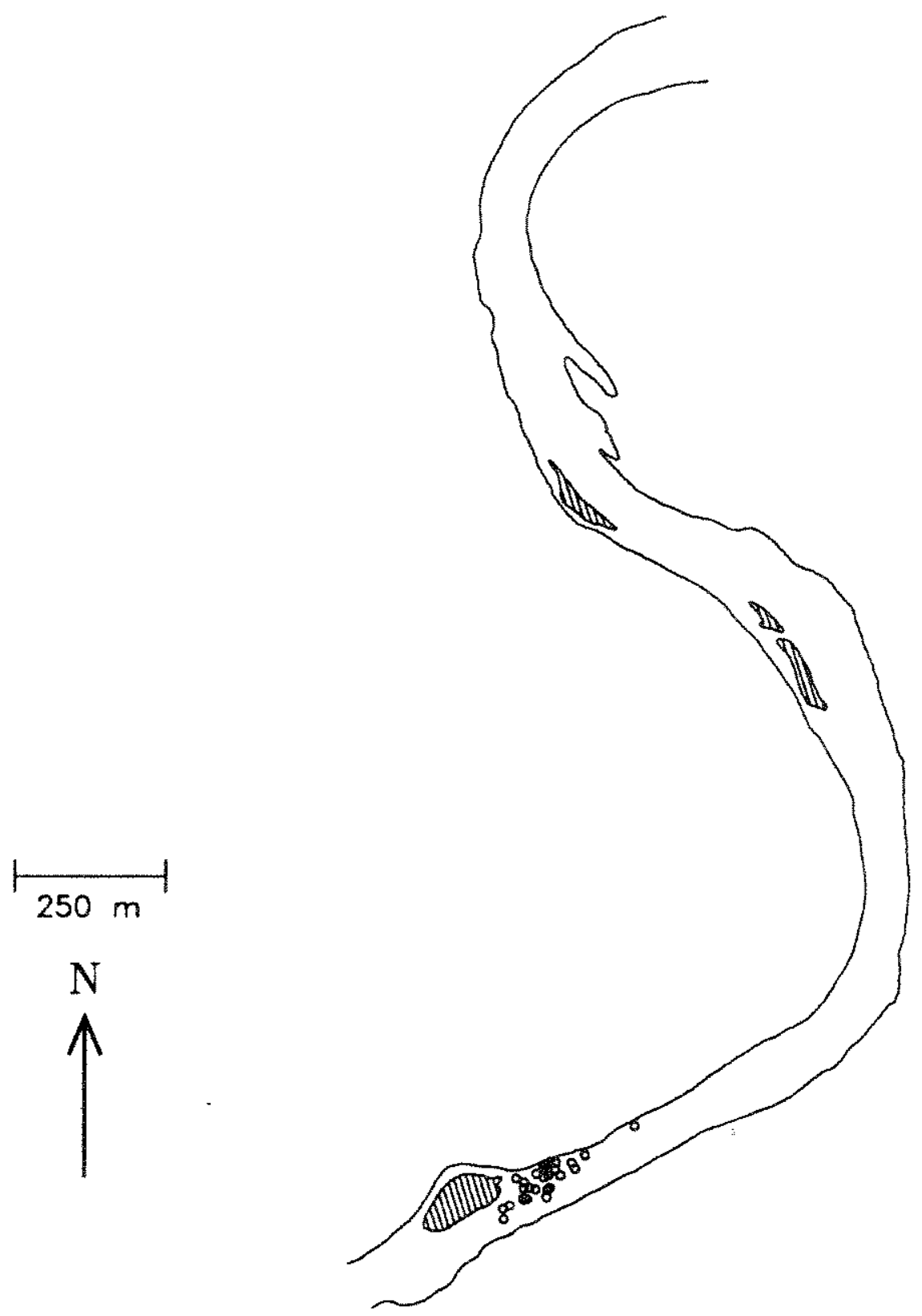

Figure 27. Movement of radio-tagged trout 270 (30.270 $\mathrm{MHz}$ ), 1988, Bighorn River, Montana. 


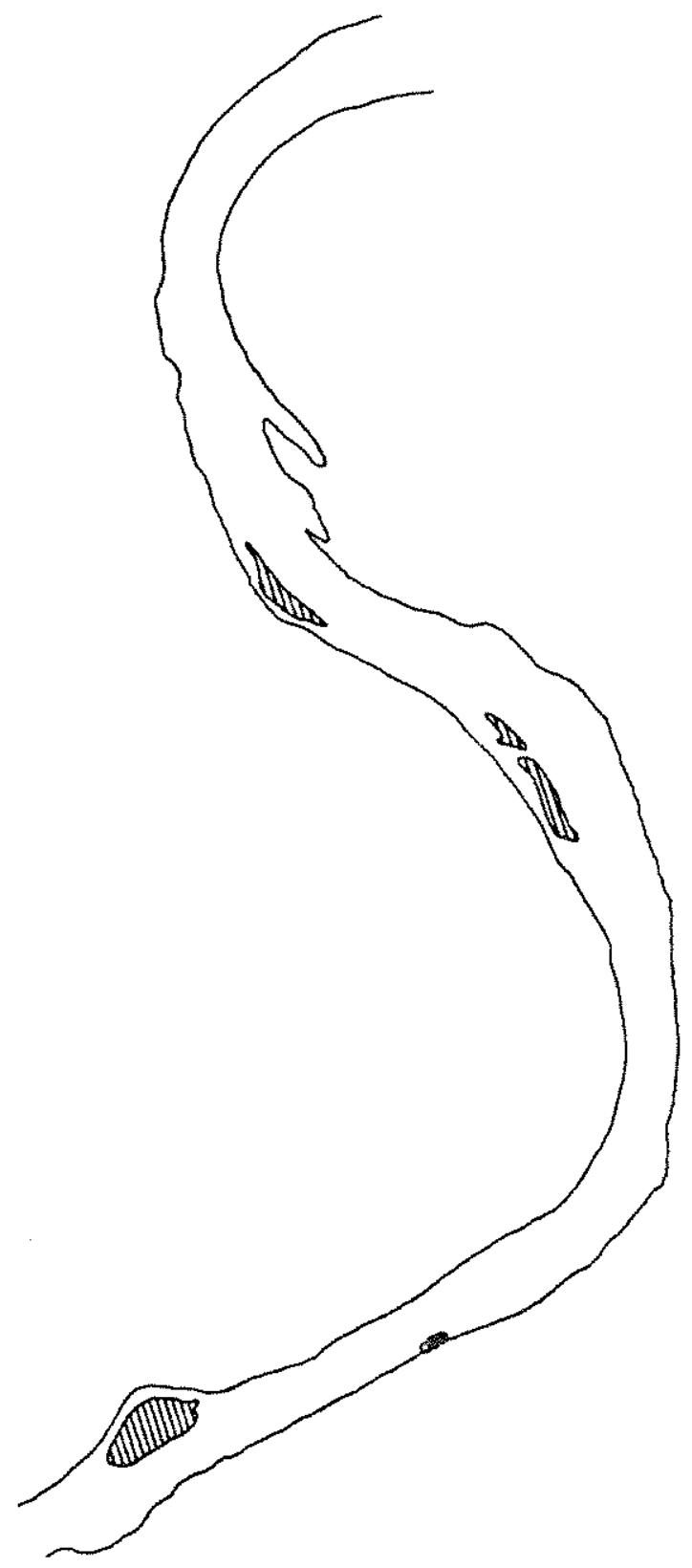

Figure 28. Movement of radio-tagged trout $280(30.280 \mathrm{MHz})$, 1988, Bighorn River, Montana. 
APPENDIX K

Gas Saturation Trends During Sampling Periods 


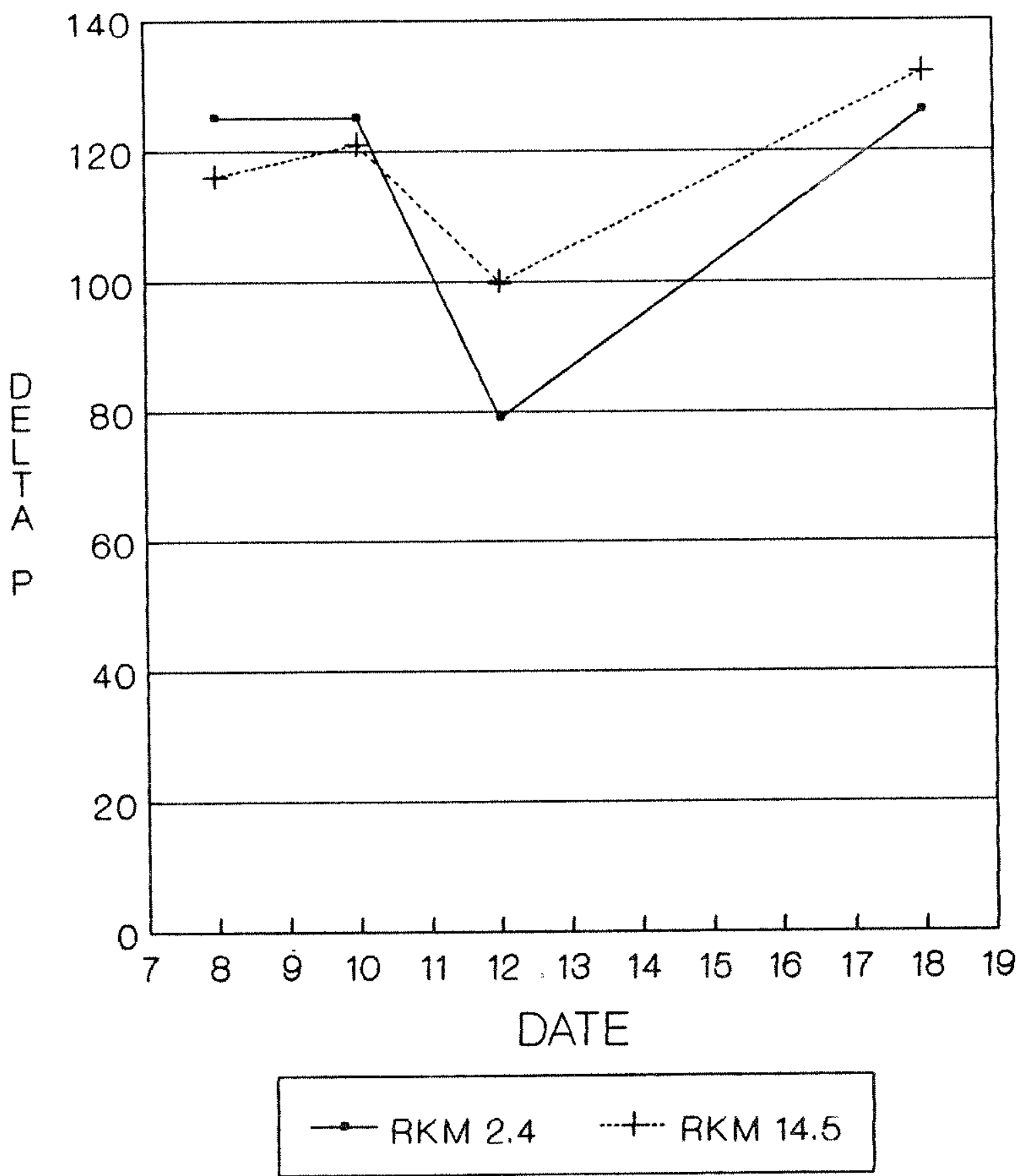

Figure 29. Comparison of Bighorn River delta P's measured at Rkm 2.4 and 14.5 from April 8 to April 19, 1987. 


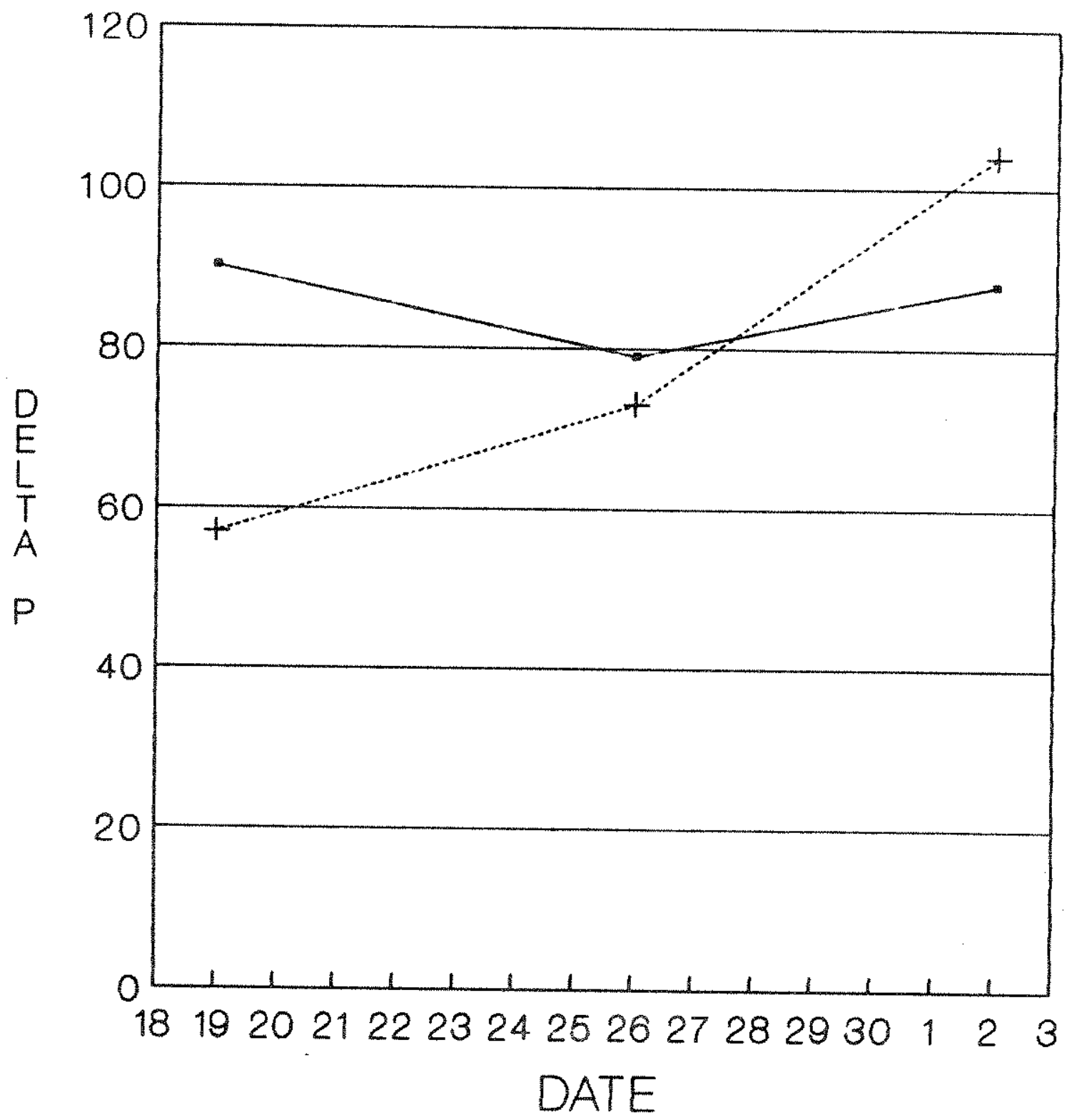

$\rightarrow$ RKM $2.4 \quad \cdots+$ RKM 14.5

Figure 30. Comparison of Bighorn River delta $\mathrm{p}^{\mathrm{i}} \mathrm{s}$ measured at Rkm 2. 4 and 14.5 from September 19 to November $2,1987$. 
APPENDIX I

Invertebrate Gas Bubble Trauma symptoms 


\section{Bioassay \#1}

\section{Ephemerella inermis}

E. inermis afflicted with GBT usually floated on the water surface, ventral side up, suggesting initial gas accumulation occurred ventrally within the nymph. Under magnification, bubbles could occasionally be seen in the ventral thorax, abdomen and appendages. During early stages of GBT, organisms readily responded to disturbance. Gills appeared to function properly and nymphs swam, albeit upside down, with the undulatory abdominal movement common to swimming mayflies. As GBT became more severe, response to disturbance was greatly reduced, gills were flared, the head protruded from the thorax, and the abdomen often became distended (segments separated making the membranes joining them clearly visible). In the latter stages, wing pads filled with air and some gills ceased to move. Movement at this stage was sluggish, principally restricted to the appendages, and the organism was near death.

Decreases in the number of individuals classified as stranded (i.e. in tank 5 at $h 60$, Figure 24) does not suggest that buoyant organisms recovered. Once on the surface, Ephemerella were carried to the standpipe by current. Most remained, but some crawled down the standpipe to the floor of the tank. These individuals were indistinguishable from those unaffected. At the conclusion of the test, all nymphs remaining on tank floors were examined. Two were buoyant in tank 6 and one in $\operatorname{tank} 4$.

six partially eaten nymphs (three buoyant) were recovered 
during the test: one in each of tanks 1, 4, 5 and 6 , and two in tank 3. Four of these were tied to Amiocentrus cases, but it is unknown whether they were preyed upon or scavenged.

\section{Isoperla quinguepunctata}

Gas bubble trauma symptoms exhibited by I. quinquepunctata were similar to those of $\mathrm{E}$. inermis. Stranded nymphs floated ventral side up and response to disturbance declined as symptoms became more acute. As GBT progressed, movement of the abdomen was restricted, limiting nymphs' ability to swim. Wing pad inflation, however, did not occur in this species.

I. quinquepunctata's posture also changed with increasing severity of GBT. Unaffected stonefies maintained a low vertical profile, with theix entire thorax and abdomen resting against the tank bottom. The profile of nymphs with advanced GBT varied from the abdomen being arched away from the attachment surface to having the entire thorax and abdomen lifted off the surface. This could be detrimental in running water by subjecting organisms to greater water velocities.

During the first $24 \mathrm{~h}$ of the test, some I. quinquepunctata nymphs at the three highest gas levels (delta $\mathrm{P}>181.6 \mathrm{~mm} \mathrm{Hg}$ ) became temporarily stranded at the water surface, due to external bubble formation. External bubbles were observed on nymphs later in the tests, but fewer individuals became stranded. I. quinquepunctata, as well as individuals of other taxa tested, were most active early in the test, likely due to unfamiliarity with their surroundings. Externally induced buoyancy may not have forced these stonefly nymphs to become detached from the 
tank bottom or sides, but likely became a factor when they released their foothold to move.

Reductions in the number of stranded Isoperla nymphs occurred several times during the test. This was the most mobile taxa tested and some individuals suffering from Gas bubble trauma apparently crawled or swam to the tank bottom. At the conclusion of the test, six stonefly nymphs on the bottom of tank 5 were strongly buoyant. Thus, stoneflies classified as stranded during the bioassay likely remained buoyant throughout the test. When buoyant, Isoperla's agility may be an advantage compared to less mobile taxa.

\section{Hydropsyche spp.}

Hydropsyche with GBT experienced gas accumulation severe enough to cause aistension; sometimes to the extent that the abdomen and thorax became translucent. A hole was noted behind the head of one dead larvae. It is unknown if this resulted from the organism bursting or if it had been preyed upon. Gas bubbles were also noted in the anal claws, meso- and metathoracic legs, and the metathorax, of various individuals.

Predation on Hydropsyche was not documented, however one Amiocentrus and one Isoperla were seen eating dead larvae. Because larvae often remained inside their nets until they were near death, it seems more likely that they were scavenged. Amiocentrus aspilis

Amiocentrus was quite susceptible to buoyancy from external gas bubbles, particularly during the first day of the bioassay. Seventeen larvae with numerous external gas bubbles were stranded 
in tank 6 during the first $8 \mathrm{~h}$ of the test. Their buoyancy differed from that of other organisms tested in that gas bubbles were attached to the caddis case and frequently became lodged in the posterior opening of the case. The case surface likely provided numerous nucleation sites for gas bubble formation and growth. Once present, there was little the larvae could do to dislodge them. By $h 20$, all larvae had attached themselves to the tank, preventing surface stranding.

External gas bubbles primarily occurred when the average delta $\mathrm{P}$ was $120.3 \mathrm{~mm} \mathrm{Hg}$ and greater (tanks 3 - 6). Larvae began to develop GBT sometime prior to $\mathrm{h}$ 104. When GBT became severe, bloating prevented them from retreating into their cases when disturbed. Like in Hydropsyche, inflation became severe enough to make their thorax nearly transparent.

\section{Bioassay \#2}

\section{Gammarus lacustrus}

Gas bubble trauma in Gammarus was first observed in tank 4 at $h$ 52; a bubble had formed in the marsupium of a female. As the test progressed, many amphipods occupying positions in the upper portion of tanks 4,5 and 6 suffered this affliction. Distally hooked stiff hairs border amphipod oostegites, helping to retain eggs in the marsupium (Pennak 1978). These hairs may act as nucleation sites for bubble formation and likely inhibit

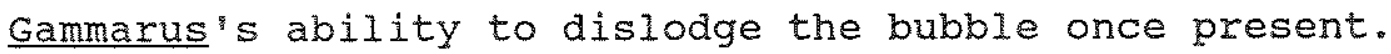

Gas bubbles in the marsupium affected buoyancy, but did not appear to affect survival. More severe GBT was indicated by gas 
accumulation in the gut. All Gammarus that died had a bubble or bubbles running the length of the gut. In one individual, bubbles were also present in the head. By the end of the test, most Gammarus in tanks 5 and 6 had bubbles in the marsupium or gut.

Short term stranding due to external bubbles occurred in several individuals during the test. When bubbles broke or were dislodged, they returned to the tank bottom. Externally induced buoyancy occurred with increasing frequency in tanks 4, 5, and 6, but Gammarus exposed to lower gas levels ( $\leq 113.1 \mathrm{~mm} \mathrm{Hg}$ ) were unaffected.

\section{Tricorythodes minutus}

Tricorythodes, like Ephemerella and Isoperla, floated ventral side up. Response to disturbance was good initially, but degraded as GBT symptoms became more severe. Gas accumulation in the body cavity was the primary avenue of GBT affliction. Separation of abdominal segments and protraction of the head away from the body were observed in affected nymphs. In addition, later stages of GBT were characterized by inflation of the wing pads, and by flared gills. Gas bubbles were seen dorsally in the thorax, in the wings, and posteriorly in the abdomen. In one individual, a bubble ran the entire length of the body cavity.

A decreasing trend in the number of stranded Tricorythodes occurred in tank 5 during the fifth day of the bioassay. This was probably due to predation and to accidental transfer of nymphs. At the conclusion of the test only 14 of 20 nymphs in tank 5 were accounted for, however, three extra nymphs were found 
in tank 6. Gas supersaturation levels were measured in sequence from tank 1 through tank 6. Although the Bouck gasometer was examined after each measurement, it appears that some organisms were not detected and some transfer occurred.

\section{Baetis tricaudatis}

Gas bubble trauma symptoms in Baetis were the same as those exhibited by Ephemerella and Tricorythodes. Mortality, unrelated to GBT, was high. Nineteen non-gas mortalities occurred during the test. Seven, five, and four of these occurred in tanks 3, 1, and 2 , respectively. Seven mortalities occurred in the first $8 \mathrm{~h}$ and may have been due to handling. Baetis's size and mobility made handing more difficult than other taxa.

\section{Physella sp.}

Single non-gas mortalities occurred in tanks $1,2,3,4$, and 6. All organisms except the one in tank 3 had crushed shells when they were discovered. Injuries probably occurred when the Bouck gasometer was placed in the tanks to measure gas saturation levels. 


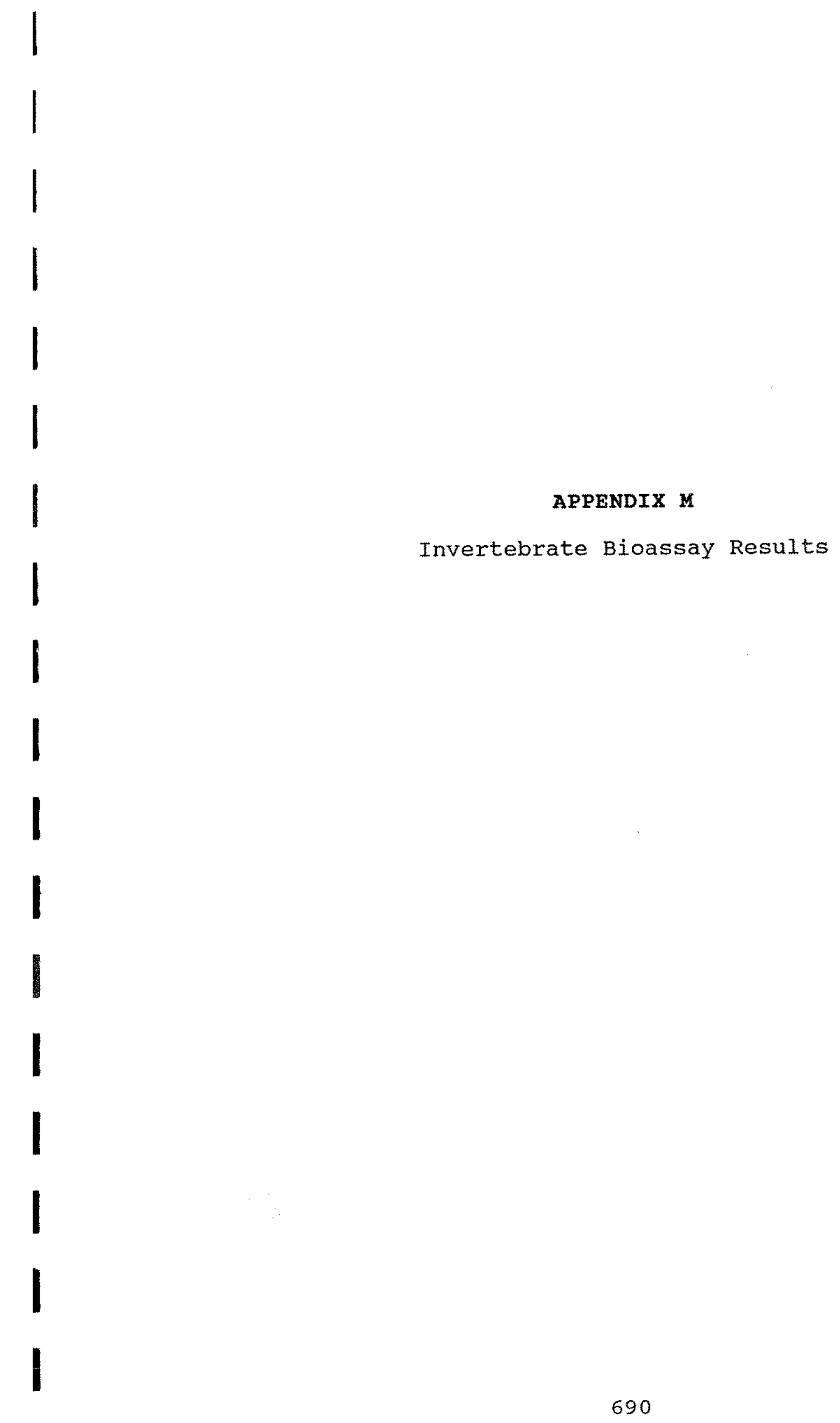




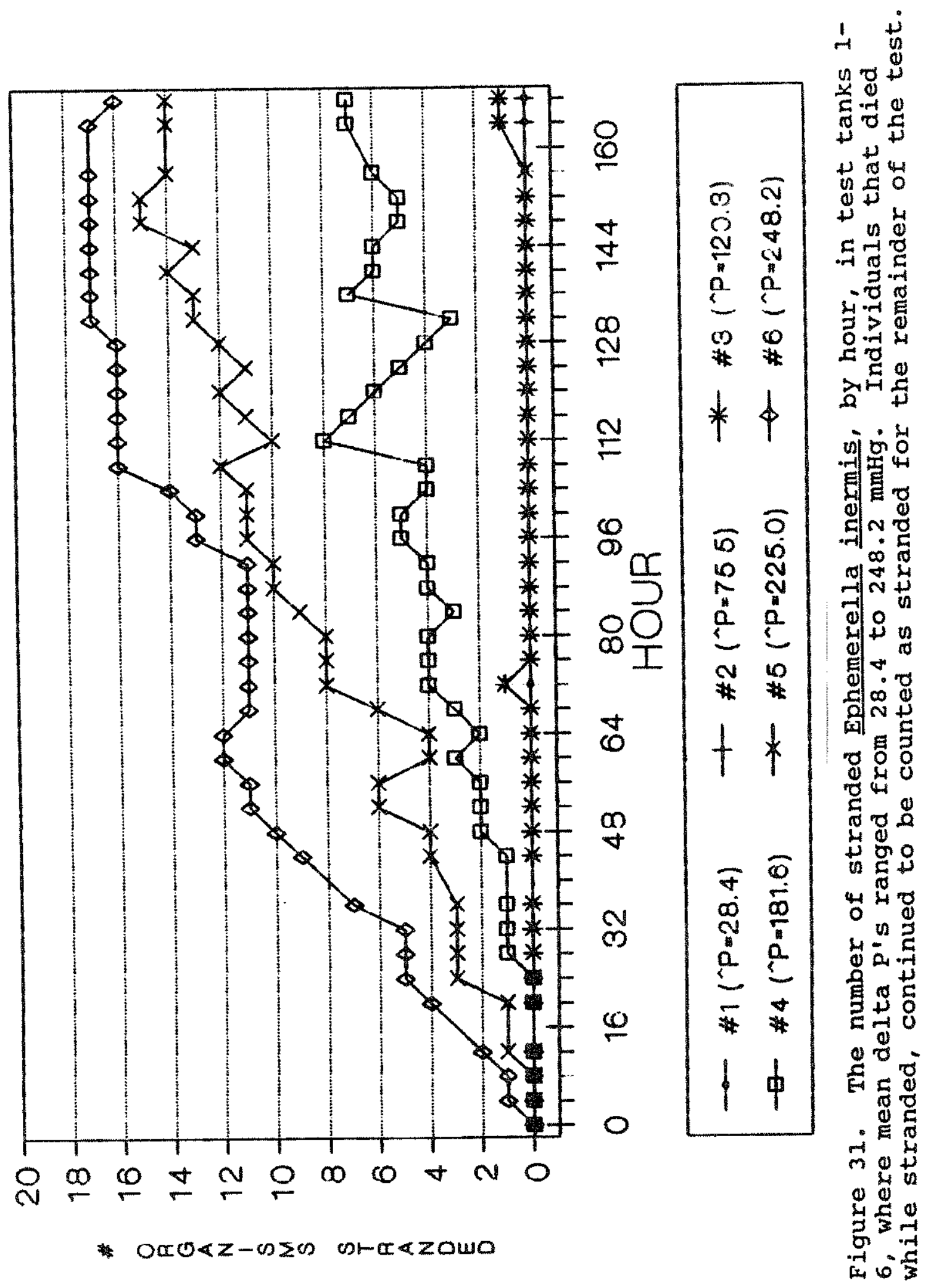




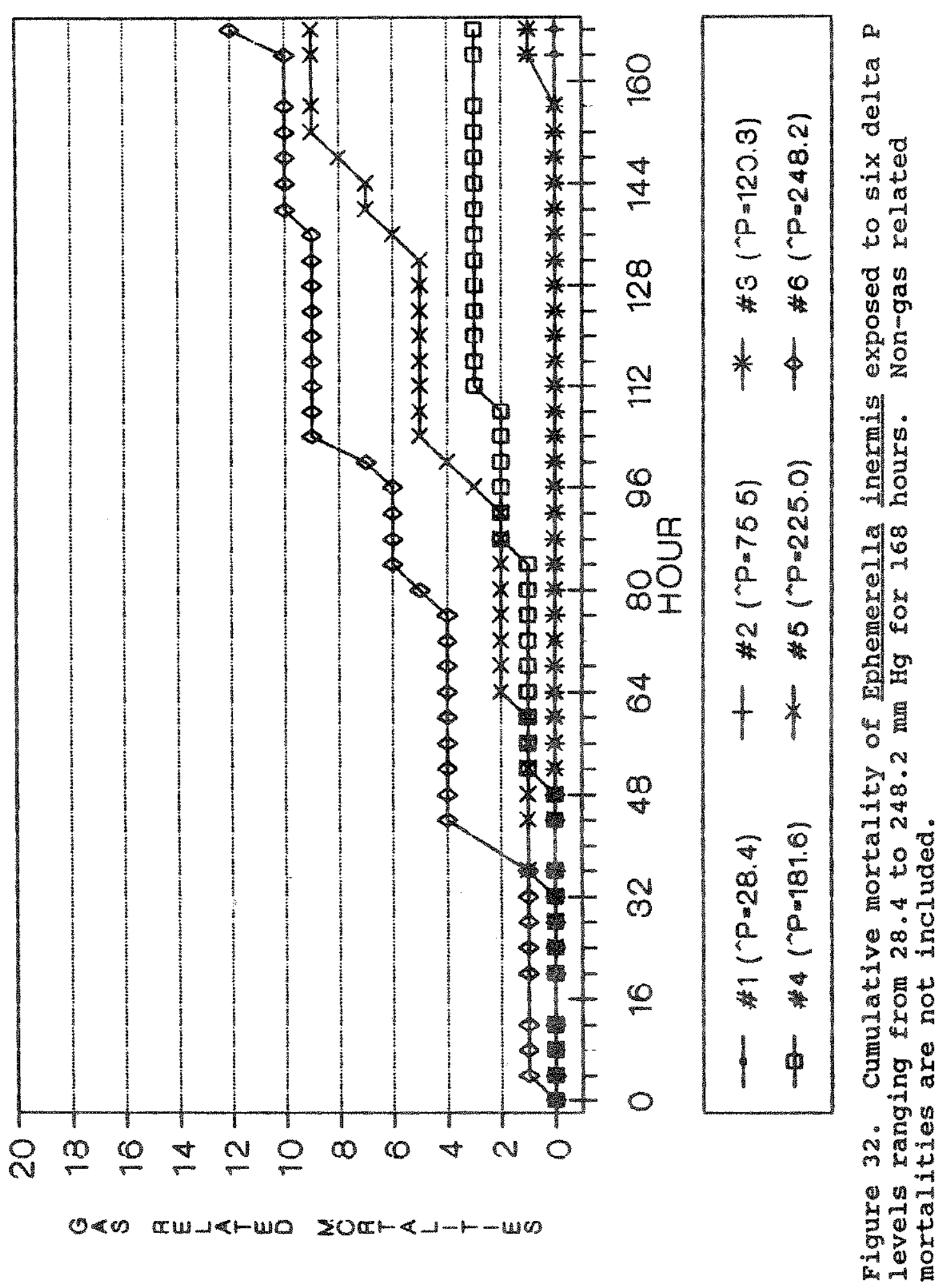




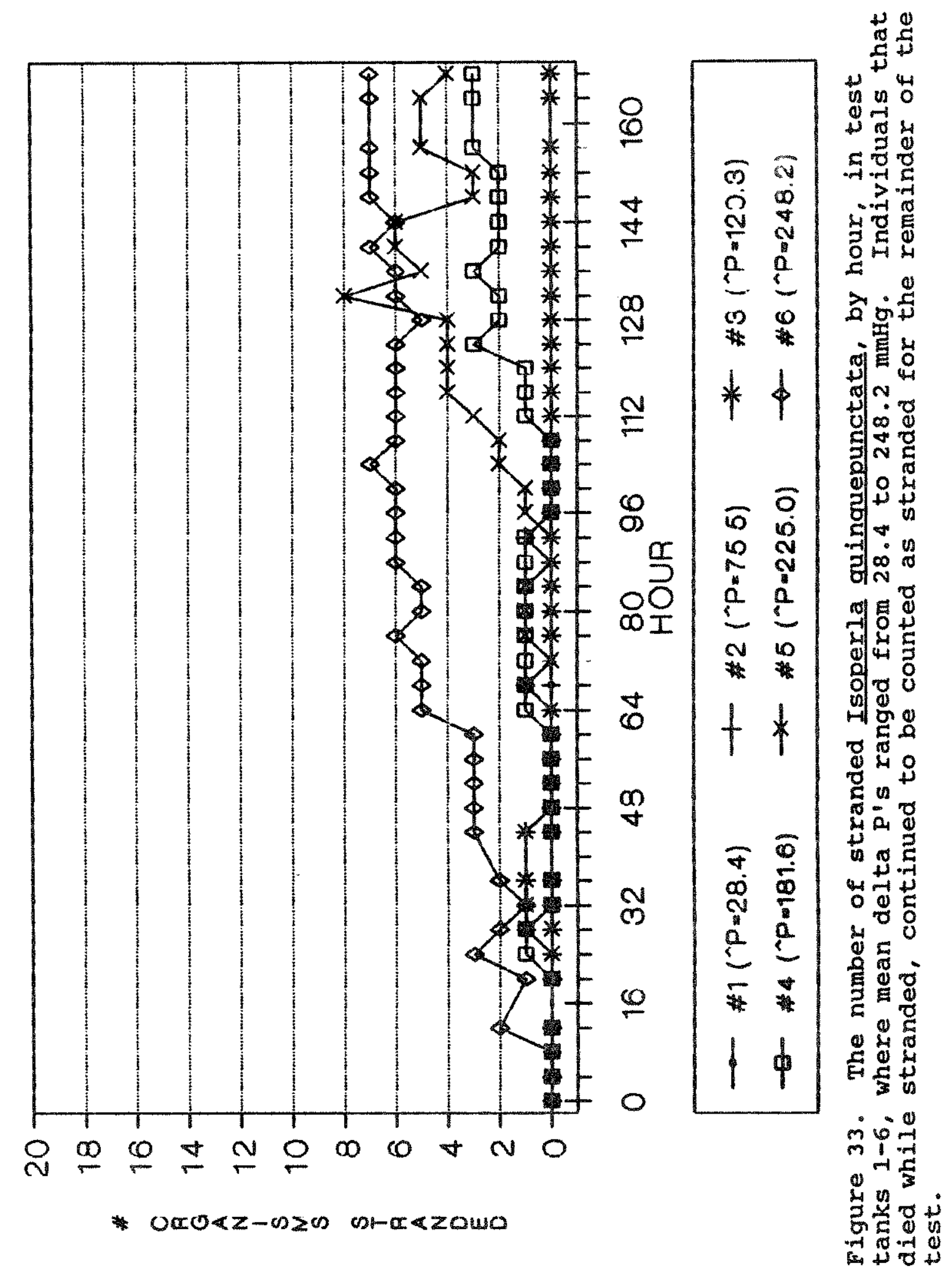




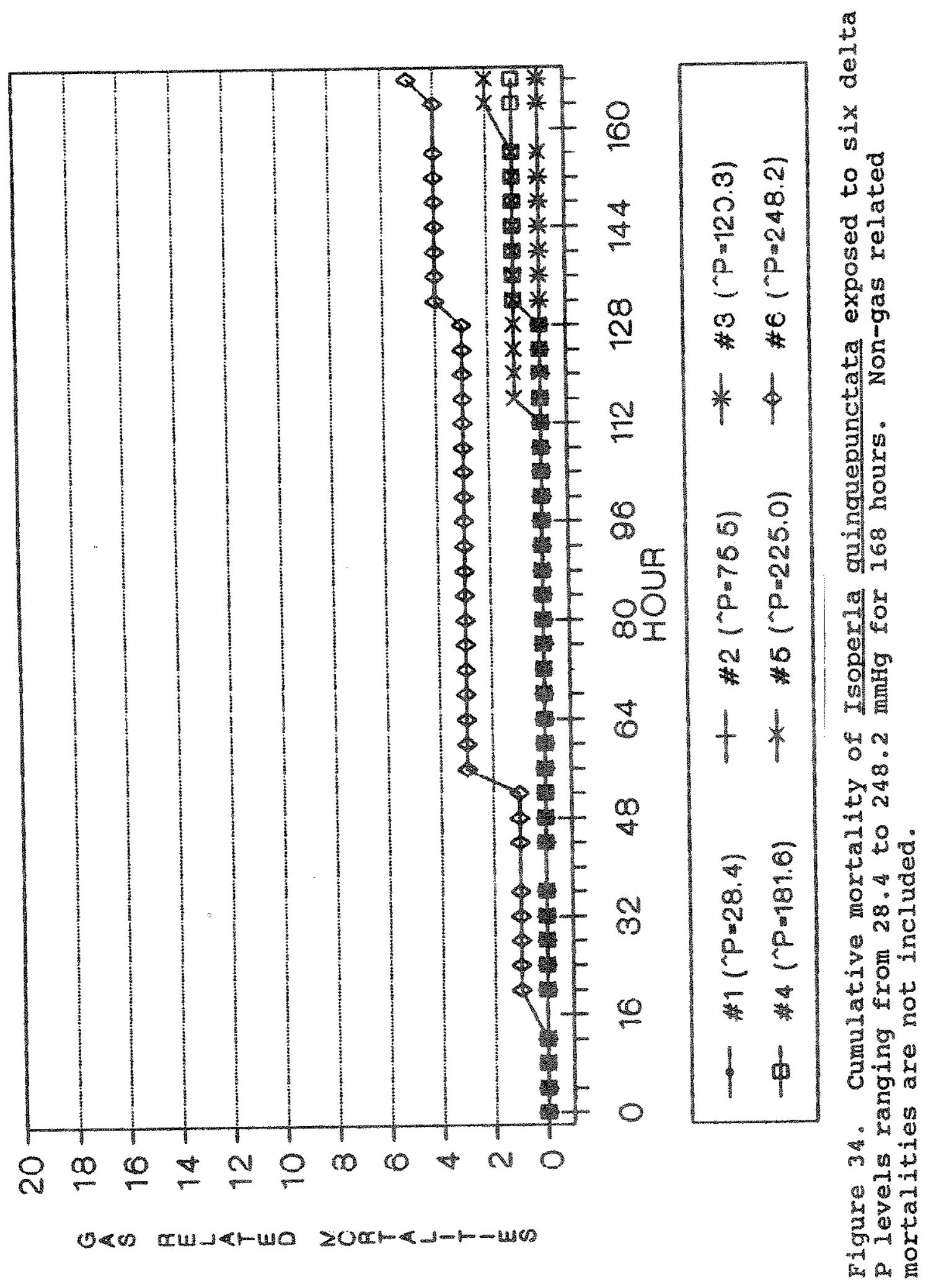




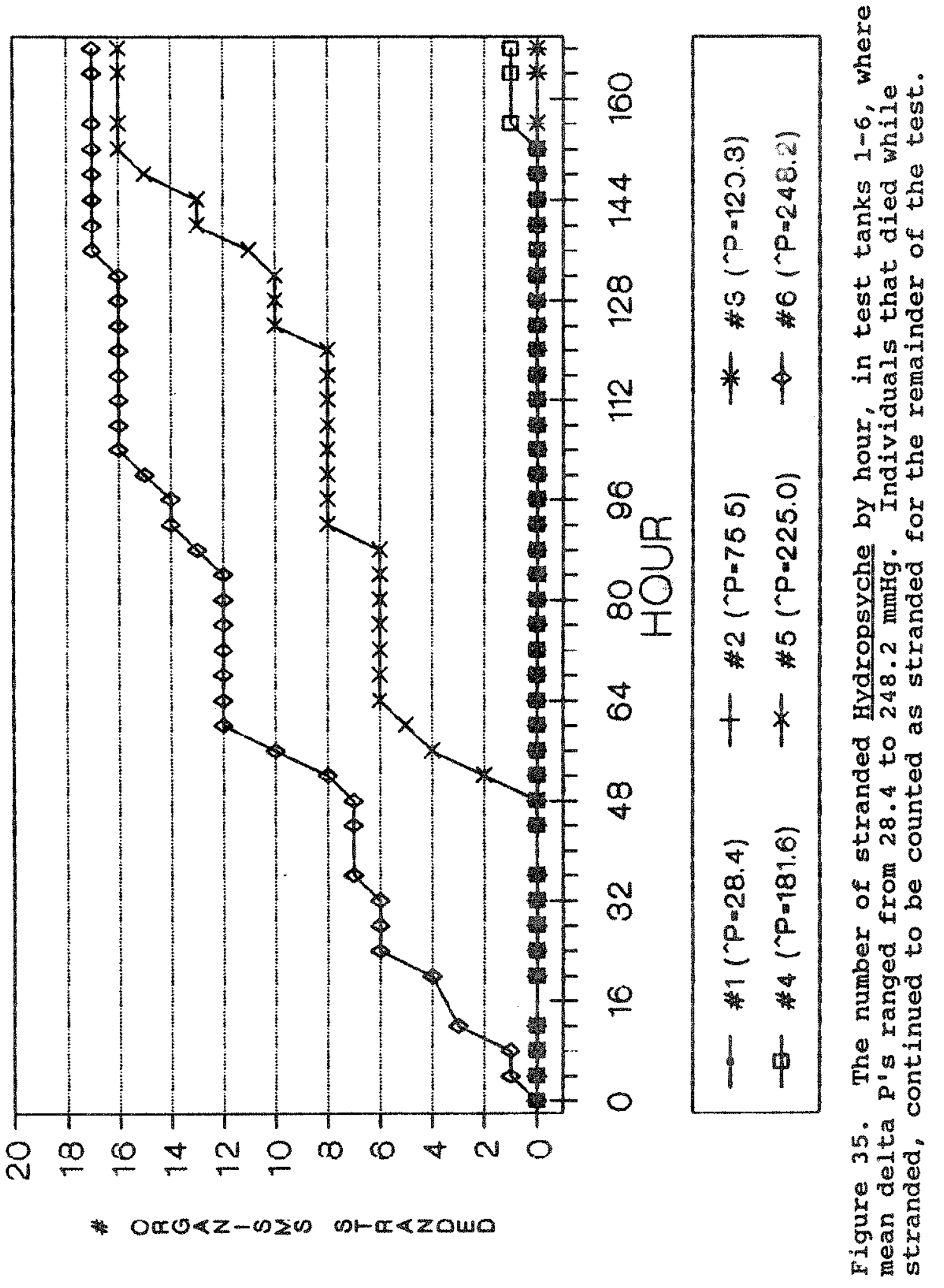




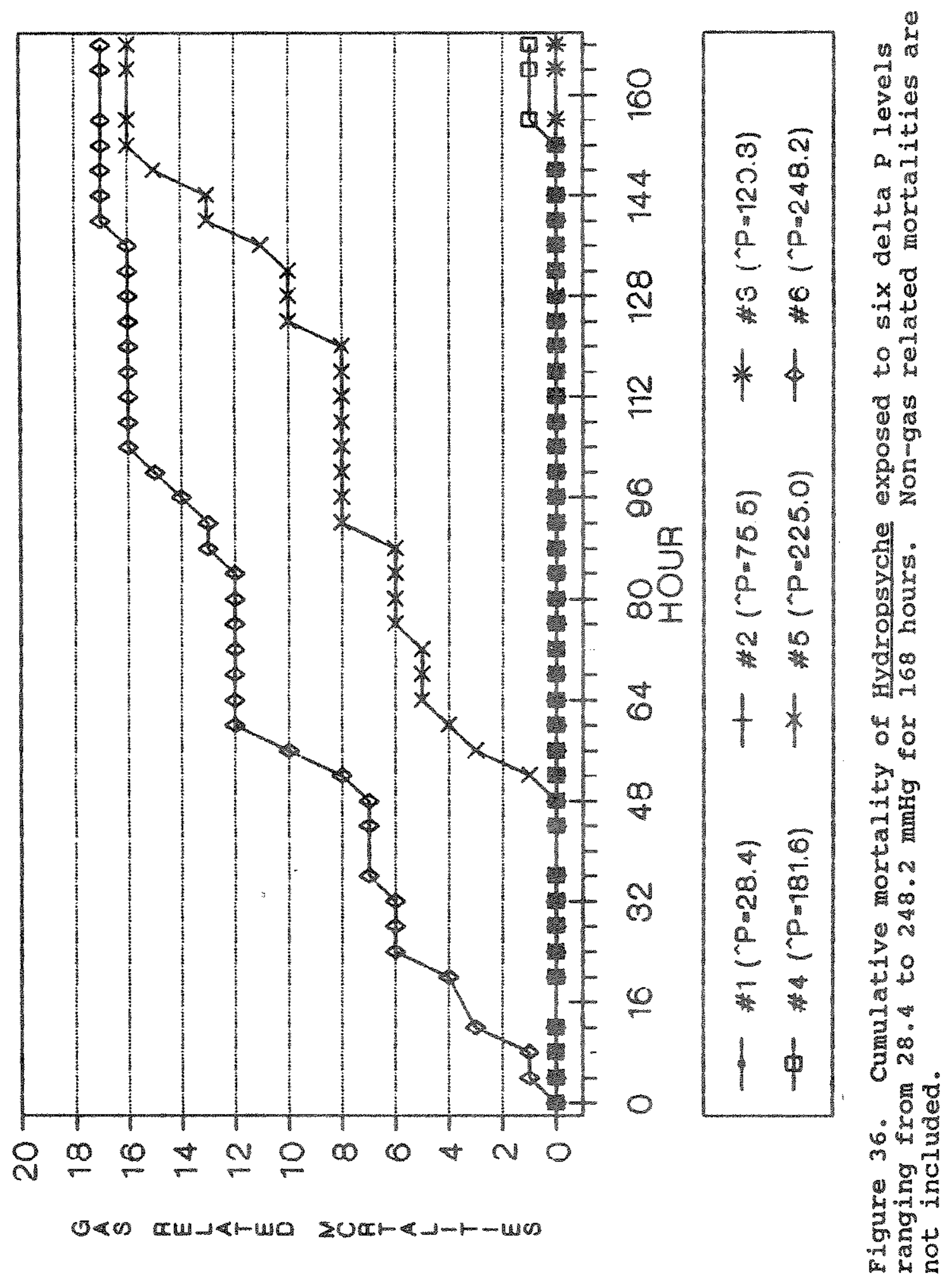




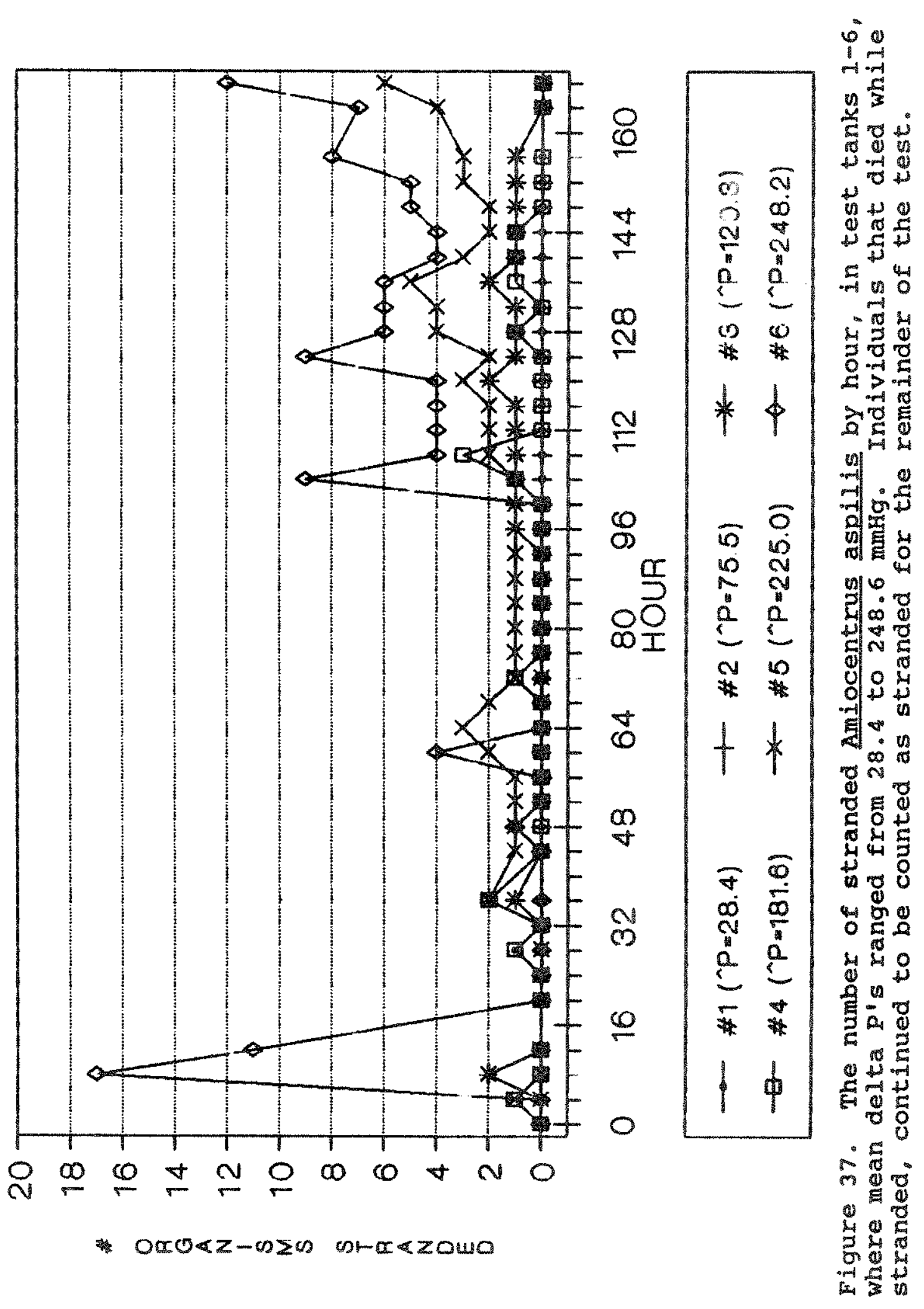



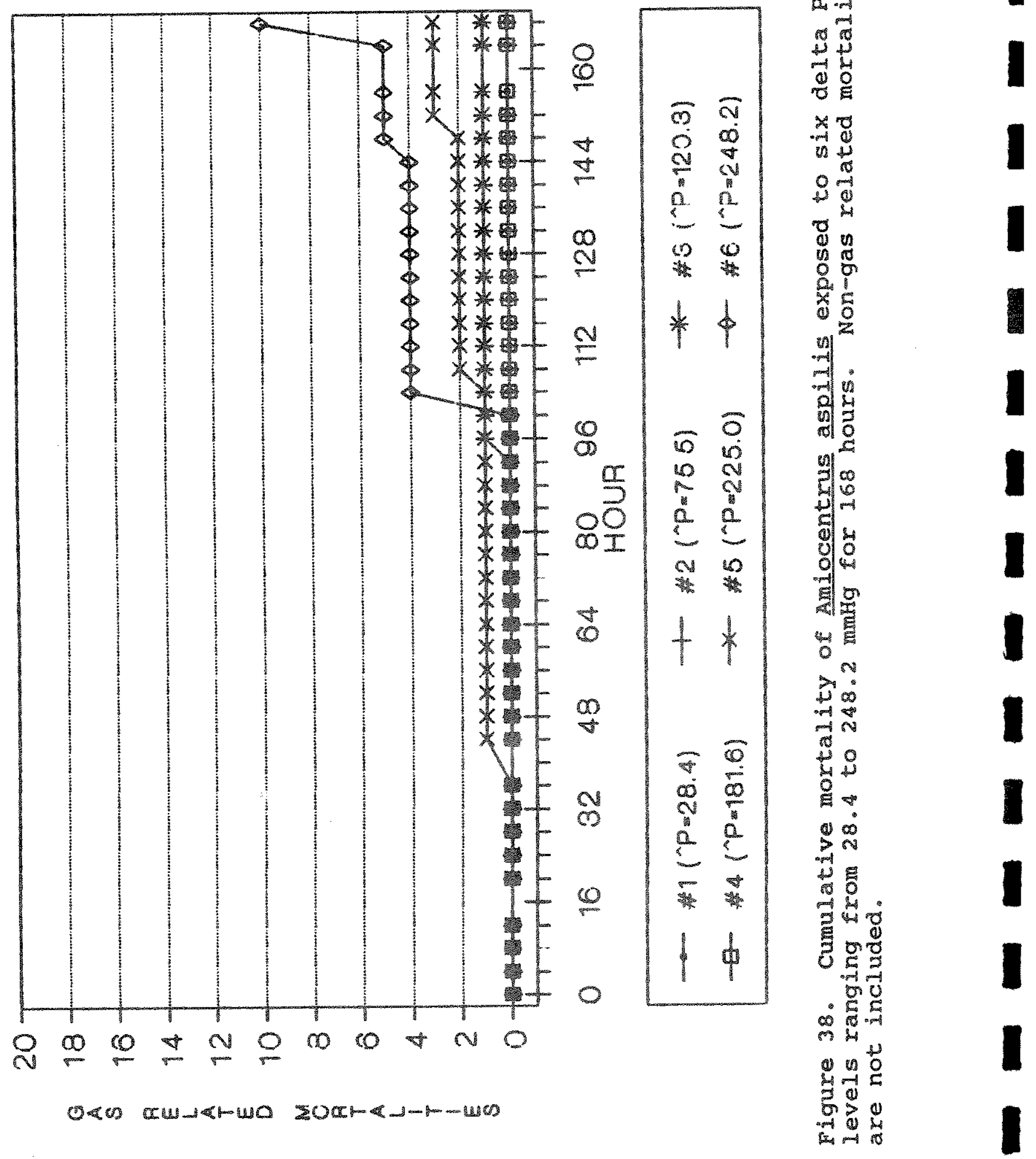


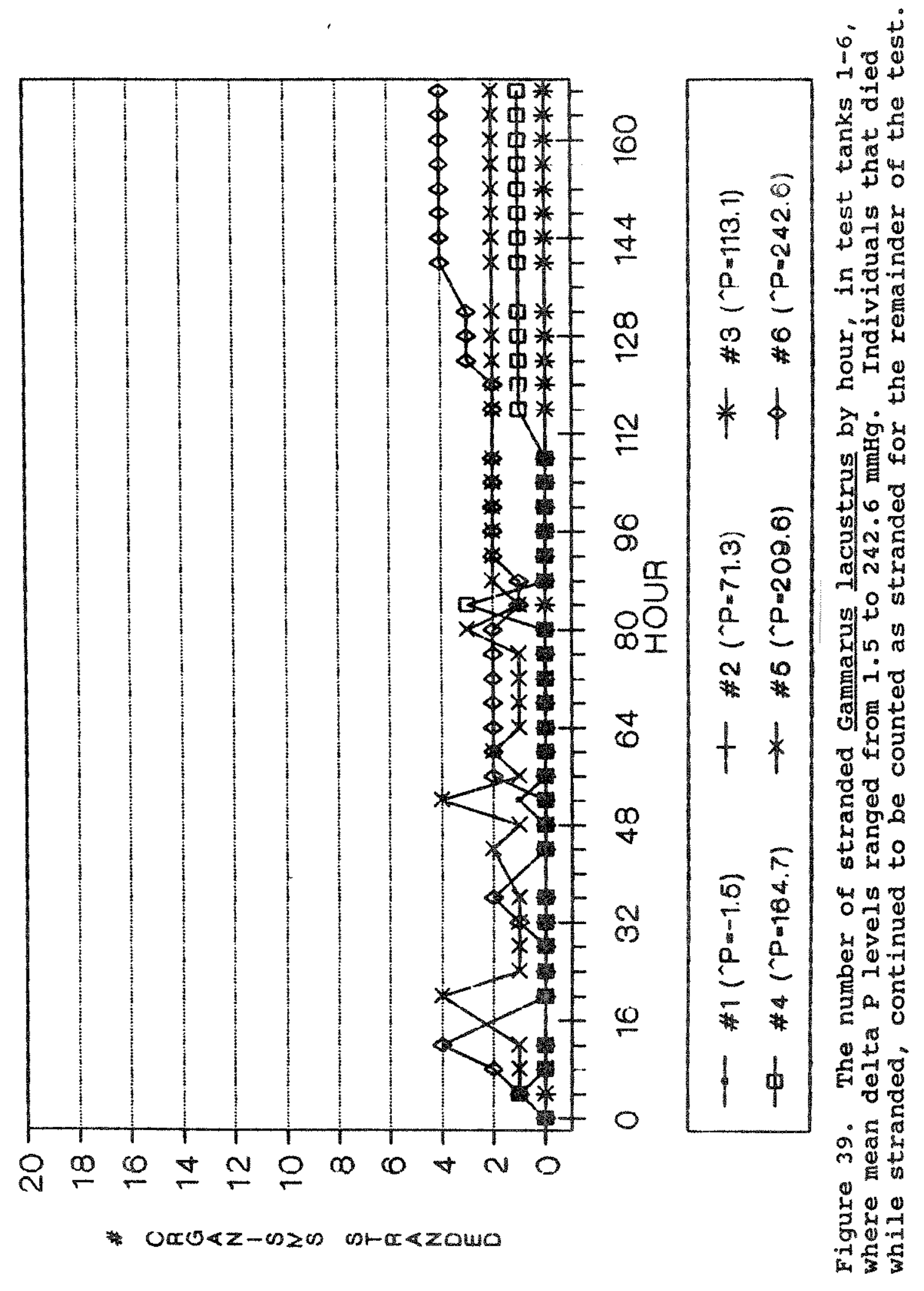




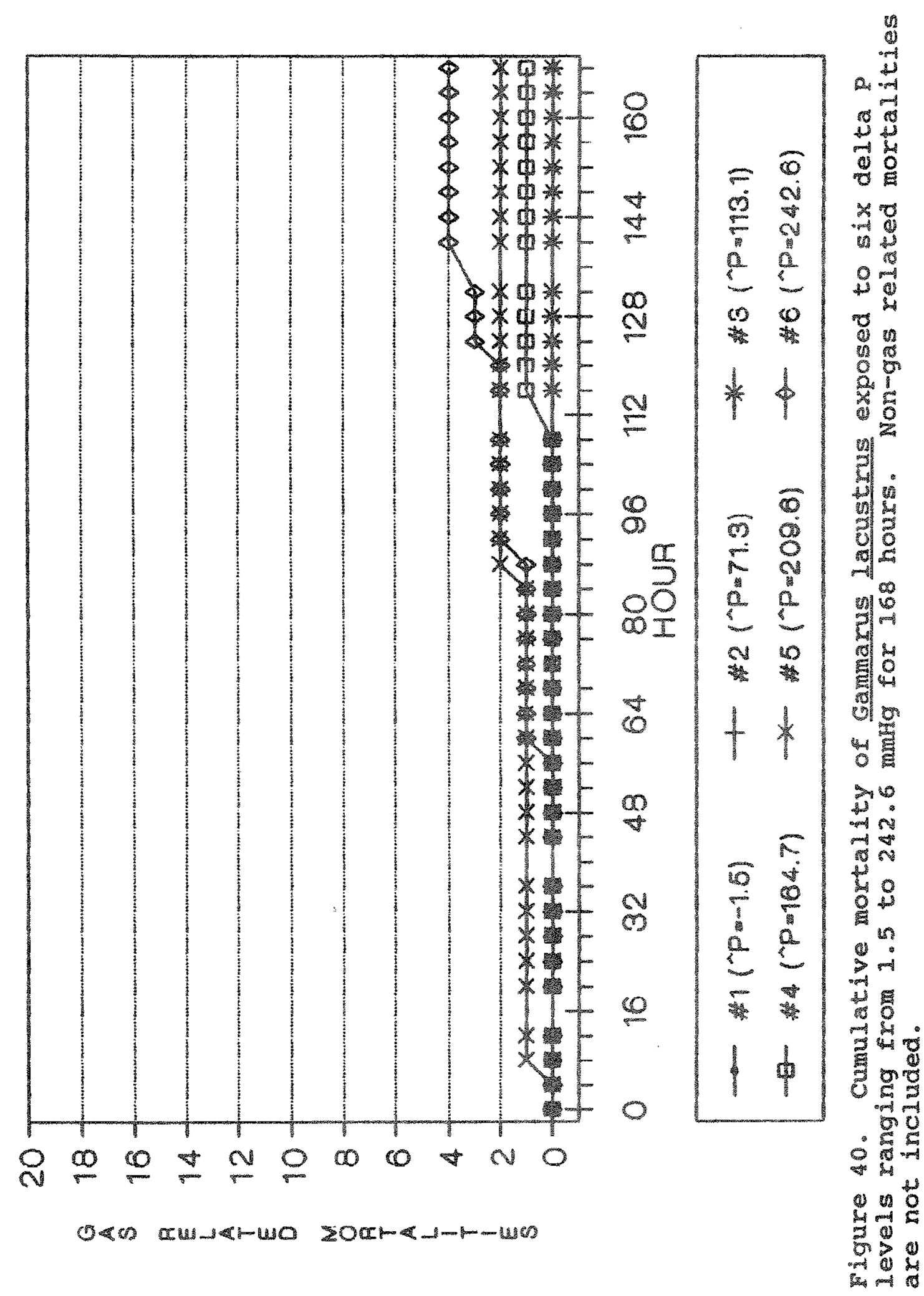




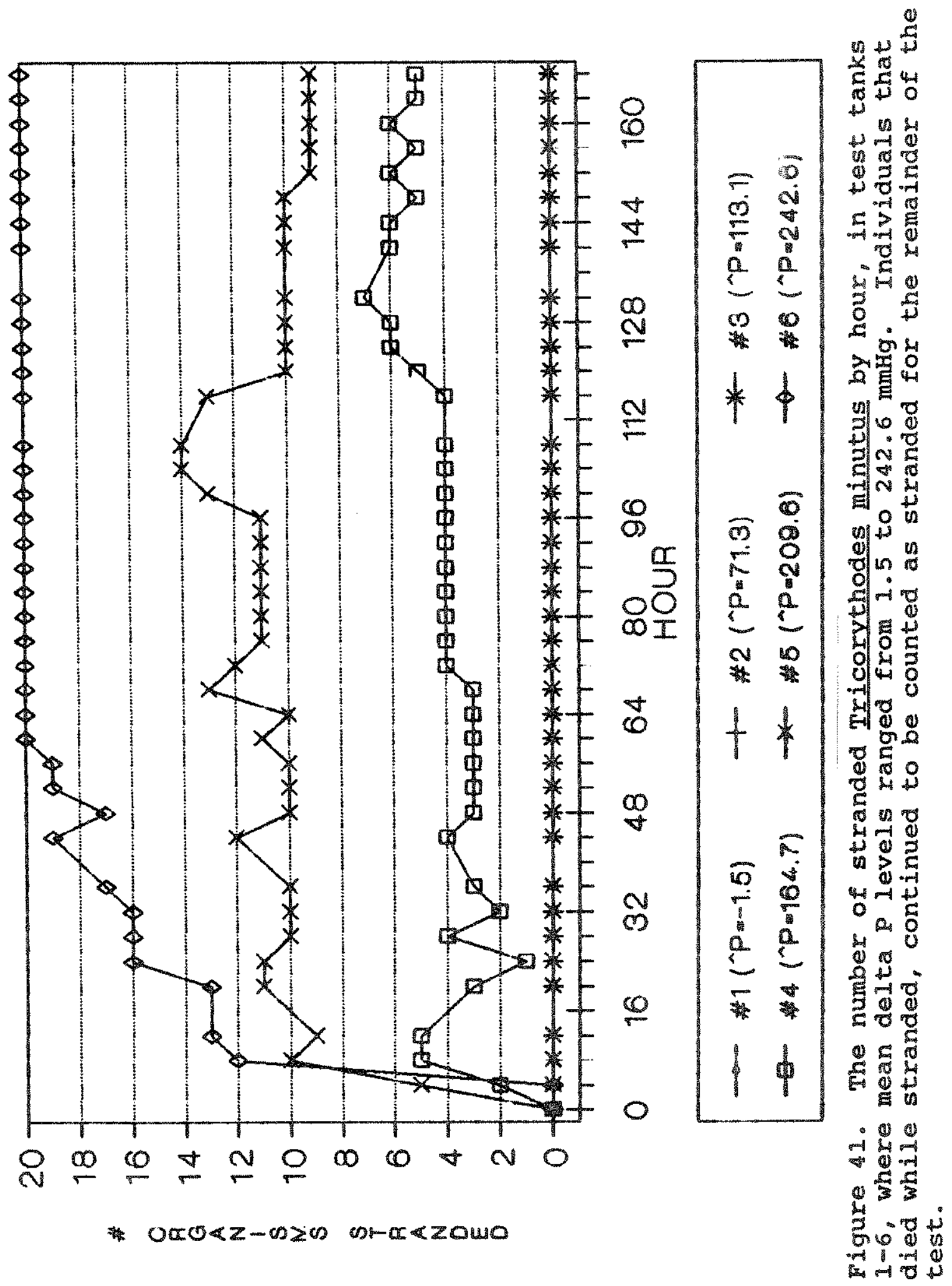




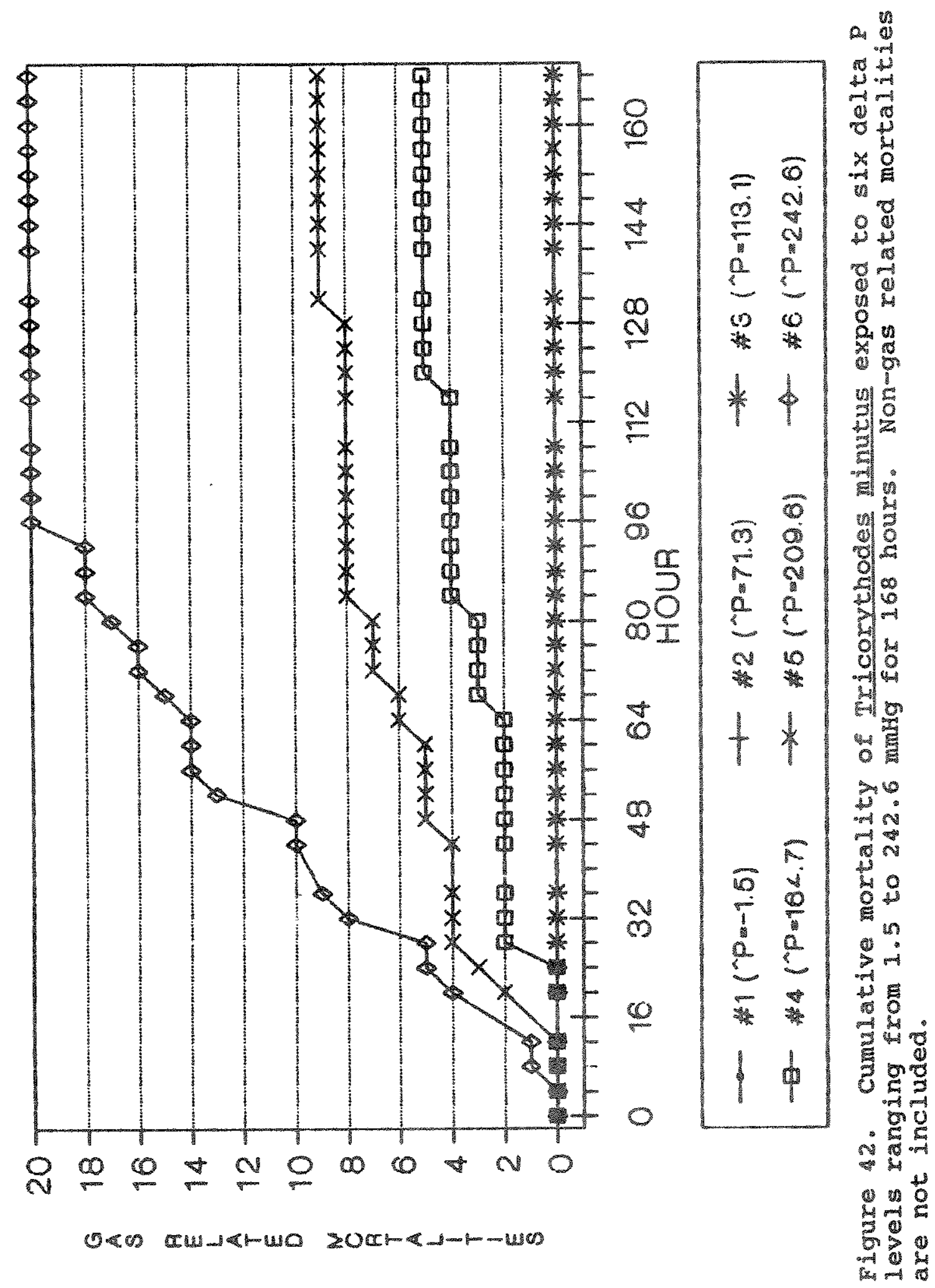




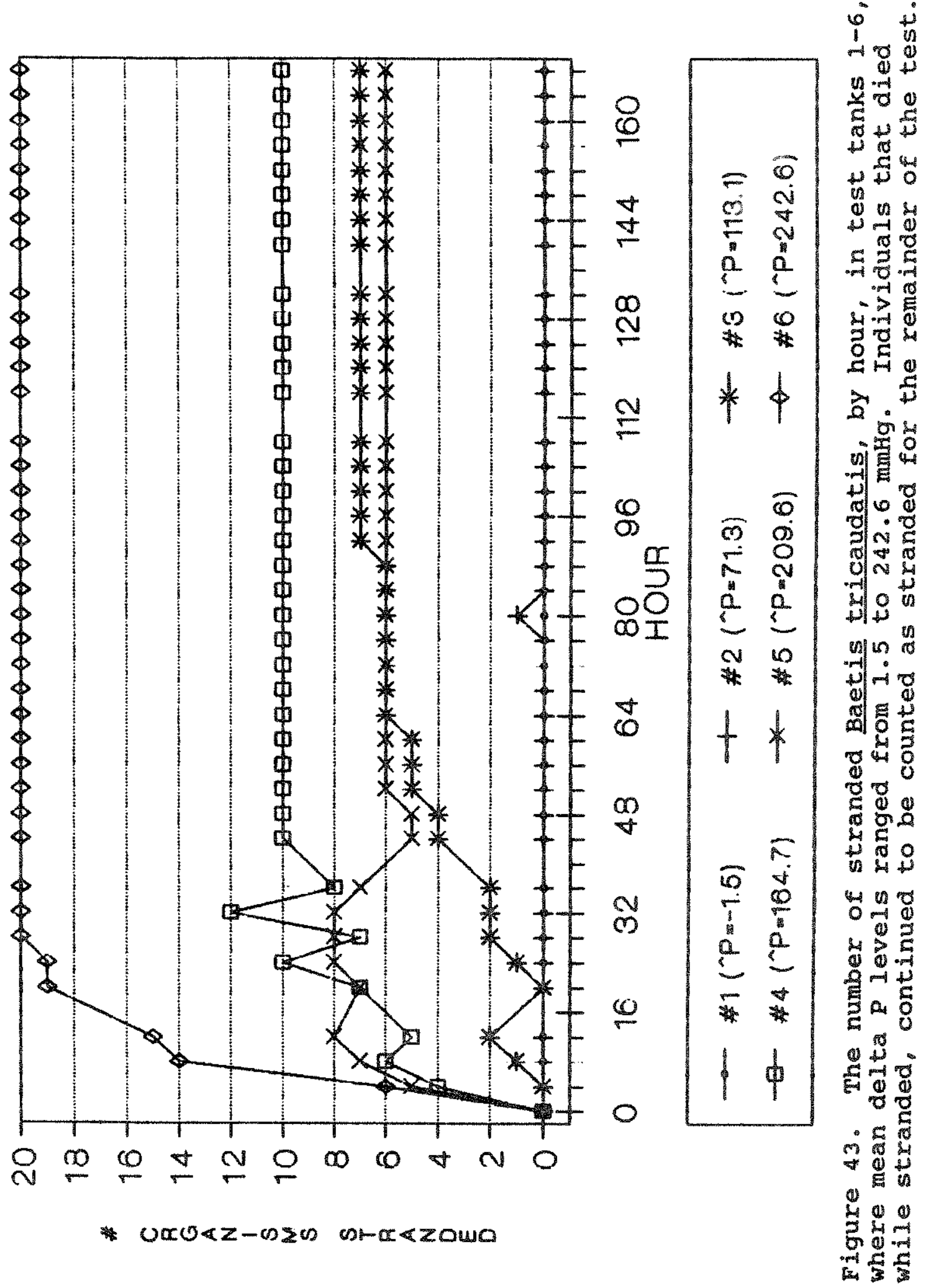




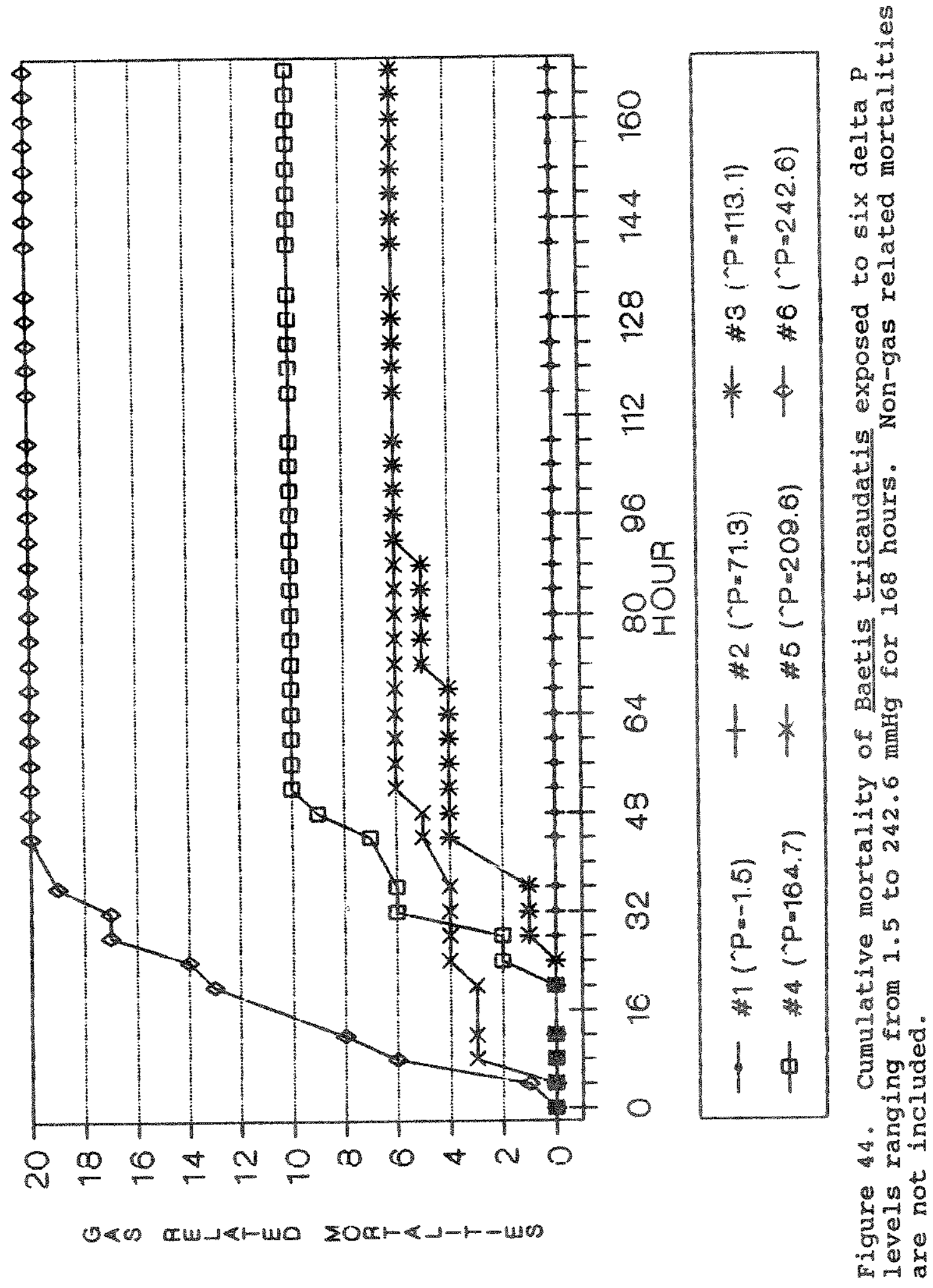




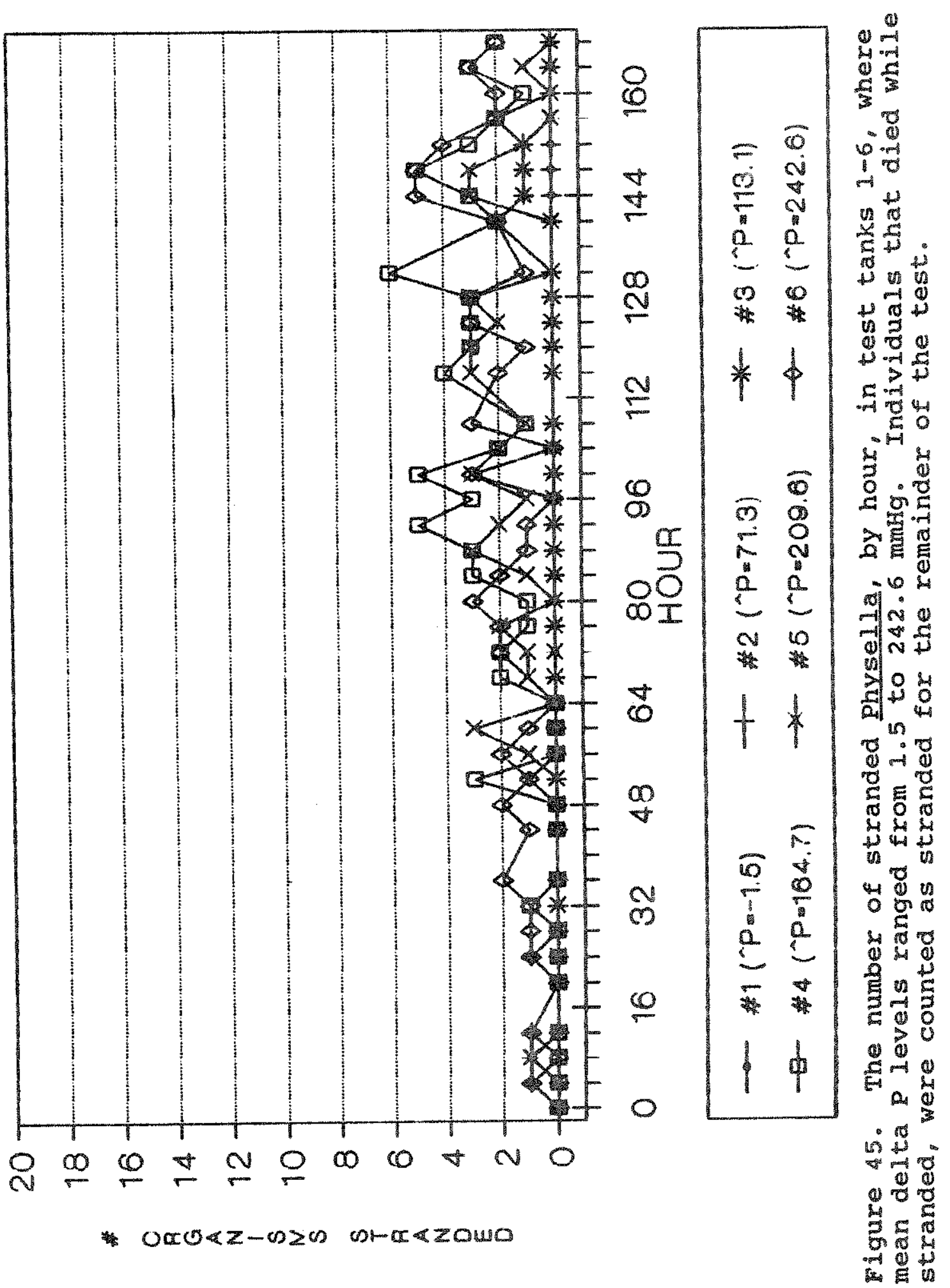


I
I
I
I

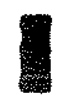

I

I

1

I

I

I

I

I

I

I

I 


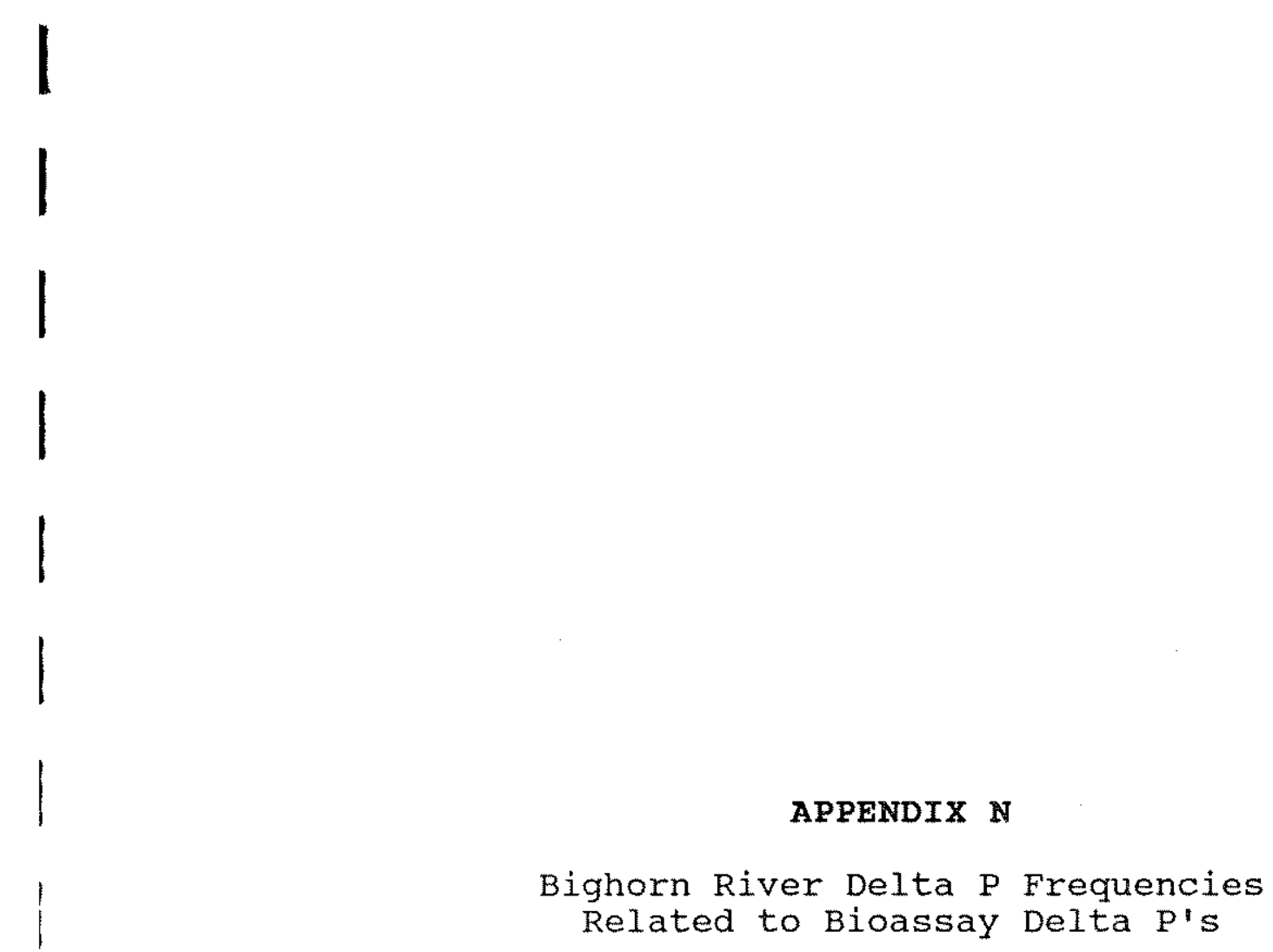

\begin{abstract}
Related to Bioassay Delta P's
\end{abstract}


Table 57. The frequencies (percent of measurements taken) that Bighorn River delta P's at rkm 2.4 equaled or exceeded mean delta $P$ levels ( $\mathrm{mmHg}$ ) in test tanks 2-6 during bioassay \#1. Results are provided for 1986 and 1987 by month.

\begin{tabular}{|c|c|c|c|c|c|}
\hline Year/month & 75 & $\begin{array}{r}\text { Test } \\
120\end{array}$ & $\begin{array}{c}\text { tank delta } \\
181\end{array}$ & $\begin{array}{c}\text { P levels } \\
225\end{array}$ & 248 \\
\hline \multicolumn{6}{|l|}{1986} \\
\hline January & 79 & 0 & 0 & 0 & 0 \\
\hline February & 90 & 0 & 0 & 0 & 0 \\
\hline March & 100 & 50 & 0 & 0 & 0 \\
\hline April & 100 & 63 & 0 & 0 & 0 \\
\hline May & 100 & 55 & 0 & 0 & 0 \\
\hline June & 100 & 85 & 0 & 0 & 0 \\
\hline July & 73 & 27 & 0 & 0 & 0 \\
\hline August & 80 & 0 & 0 & 0 & 0 \\
\hline September & 100 & 0 & 0 & 0 & 0 \\
\hline October & 100 & 36 & 0 & 0 & 0 \\
\hline November & 100 & 0 & 0 & 0 & 0 \\
\hline December & 63 & 0 & 0 & 0 & 0 \\
\hline \multicolumn{6}{|l|}{1987} \\
\hline January & 92 & 0 & 0 & 0 & 0 \\
\hline February & 91 & 0 & 0 & 0 & 0 \\
\hline March & 100 & 8 & 0 & 0 & 0 \\
\hline April & 100 & 36 & 0 & 0 & 0 \\
\hline May & 100 & 70 & 0 & 0 & 0 \\
\hline June & 100 & 92 & 0 & 0 & 0 \\
\hline July & 100 & 85 & 0 & 0 & 0 \\
\hline August & 100 & 25 & 0 & 0 & 0 \\
\hline september & 100 & 0 & 0 & 0 & 0 \\
\hline October & 100 & 0 & 0 & 0 & 0 \\
\hline November & 86 & 40 & 0 & 0 & 0 \\
\hline December & 83 & 0 & 0 & 0 & 0 \\
\hline
\end{tabular}


Table 58. The frequencies (percent of measurements taken) that Bighorn River delta $\mathrm{P}^{\prime} \mathrm{s}$ at $\mathrm{rkm} 14.5$ equaled or exceeded mean delta $P$ levels (mmHg) in test tanks 2-6 during bioassay \#1. Results are provided for 1986 and 1987 by month.

\begin{tabular}{|c|c|c|c|c|c|}
\hline Year/month & 75 & $\begin{array}{r}\text { Test } \\
120\end{array}$ & $\begin{array}{c}\text { tank delta } \\
181\end{array}$ & $\begin{array}{c}\mathrm{P} \text { levels } \\
225\end{array}$ & 248 \\
\hline \multicolumn{6}{|l|}{1986} \\
\hline January & 0 & 0 & 0 & 0 & 0 \\
\hline February & 10 & 0 & 0 & 0 & 0 \\
\hline March & 75 & 0 & 0 & 0 & 0 \\
\hline April & 88 & 13 & 0 & 0 & 0 \\
\hline May & 82 & 27 & 0 & 0 & 0 \\
\hline June & 92 & 77 & 0 & 0 & 0 \\
\hline July & 60 & 18 & 0 & 0 & 0 \\
\hline August & 50 & 0 & 0 & 0 & 0 \\
\hline september & 9 & 0 & 0 & 0 & 0 \\
\hline October & 43 & 0 & 0 & 0 & 0 \\
\hline November & 38 & 0 & 0 & 0 & 0 \\
\hline December & 63 & 0 & 0 & 0 & 0 \\
\hline \multicolumn{6}{|l|}{1987} \\
\hline January & 75 & 0 & 0 & 0 & 0 \\
\hline February & 90 & 0 & 0 & 0 & 0 \\
\hline March & 70 & 50 & 0 & 0 & 0 \\
\hline April & 100 & 40 & 0 & 0 & 0 \\
\hline May & 88 & 50 & 0 & 0 & 0 \\
\hline June & 100 & 69 & 0 & 0 & 0 \\
\hline July & 93 & 71 & 21 & 0 & 0 \\
\hline August & 92 & 25 & 0 & 0 & 0 \\
\hline September & 33 & 0 & 0 & 0 & 0 \\
\hline October & 90 & 0 & 0 & 0 & 0 \\
\hline November & 60 & 0 & 0 & 0 & 0 \\
\hline December & 17 & 0 & 0 & 0 & 0 \\
\hline
\end{tabular}


Table 59. The frequencies (percent of measurements taken) that Bighorn River delta $\mathrm{P}^{\prime} \mathrm{s}$ at rkm 2.4 equaled or exceeded mean delta $p$ levels (mmHg) in test tanks 2-6 during bioassay \#2. Results are provided for 1986 and 1987 by month.

\begin{tabular}{|c|c|c|c|c|c|}
\hline Year/month & 71 & $\begin{array}{r}\text { Test } \\
113\end{array}$ & $\begin{array}{c}\text { tank delta } \\
164\end{array}$ & $\begin{array}{c}\text { P levels } \\
209\end{array}$ & 242 \\
\hline \multicolumn{6}{|l|}{1986} \\
\hline January & 85 & 0 & 0 & 0 & 0 \\
\hline February & 100 & 0 & 0 & 0 & 0 \\
\hline March & 100 & 80 & 0 & 0 & 0 \\
\hline April & 100 & 88 & 0 & 0 & 0 \\
\hline May & 100 & 82 & 0 & 0 & 0 \\
\hline June & 100 & 100 & 0 & 0 & 0 \\
\hline July & 73 & 36 & 0 & 0 & 0 \\
\hline August & 80 & 0 & 0 & 0 & 0 \\
\hline September & 100 & 10 & 0 & 0 & 0 \\
\hline october & 100 & 36 & 0 & 0 & 0 \\
\hline November & 100 & 29 & 0 & 0 & 0 \\
\hline December & 63 & 0 & 0 & 0 & 0 \\
\hline \multicolumn{6}{|l|}{1987} \\
\hline January & 92 & 0 & 0 & 0 & 0 \\
\hline February & 100 & 18 & 0 & 0 & 0 \\
\hline March & 100 & 23 & 0 & 0 & 0 \\
\hline April & 100 & 56 & 0 & 0 & 0 \\
\hline May & 100 & 80 & 0 & 0 & 0 \\
\hline June & 100 & 92 & 0 & 0 & 0 \\
\hline July & 100 & 85 & 0 & 0 & 0 \\
\hline August & 100 & 33 & 0 & 0 & 0 \\
\hline September & 100 & 0 & 0 & 0 & 0 \\
\hline October & 100 & 30 & 0 & 0 & 0 \\
\hline November & 90 & 70 & 0 & 0 & 0 \\
\hline December & 100 & 17 & 0 & 0 & 0 \\
\hline
\end{tabular}


Table 60. The frequencies (percent of measurements taken) that Bighorn River delta P's at rkm 14.5 equaled or exceeded mean delta $\mathrm{p}$ levels ( $\mathrm{mmHg}$ ) in test tanks 2-6 during bioassay \#2. Results are provided for 1986 and 1987 by month.

\begin{tabular}{|c|c|c|c|c|c|}
\hline Year/month & 71 & $\begin{array}{r}\text { Test } \\
113\end{array}$ & $\begin{array}{c}\text { tank delta } \\
164\end{array}$ & $\begin{array}{c}\text { P levels } \\
209\end{array}$ & 242 \\
\hline \multicolumn{6}{|l|}{1986} \\
\hline January & 0 & 0 & 0 & 0 & 0 \\
\hline February & 10 & 0 & 0 & 0 & 0 \\
\hline March & 88 & 0 & 0 & 0 & 0 \\
\hline Apri1 & 88 & 50 & 0 & 0 & 0 \\
\hline May & 82 & 56 & 0 & 0 & 0 \\
\hline June & 100 & 77 & 0 & 0 & 0 \\
\hline July & 91 & 27 & 0 & 0 & 0 \\
\hline August & 80 & 0 & 0 & 0 & 0 \\
\hline september & 50 & 0 & 0 & 0 & 0 \\
\hline October & 55 & 18 & 0 & 0 & 0 \\
\hline November & 57 & 0 & 0 & 0 & 0 \\
\hline December & 38 & 0 & 0 & 0 & 0 \\
\hline \multicolumn{6}{|l|}{1987} \\
\hline January & 75 & 0 & 0 & 0 & 0 \\
\hline February & 100 & 27 & 0 & 0 & 0 \\
\hline March & 70 & 50 & 0 & 0 & 0 \\
\hline Apri1 & 100 & 50 & 0 & 0 & 0 \\
\hline May & 100 & 50 & 0 & 0 & 0 \\
\hline June & 100 & 85 & 0 & 0 & 0 \\
\hline July & 100 & 79 & 21 & 0 & 0 \\
\hline August & 92 & 25 & 0 & 0 & 0 \\
\hline September & 43 & 0 & 0 & 0 & 0 \\
\hline October & 90 & 0 & 0 & 0 & 0 \\
\hline November & 60 & 0 & 0 & 0 & 0 \\
\hline December & 20 & 0 & 0 & 0 & 0 \\
\hline
\end{tabular}

